Supporting Information

\title{
Copper-Photocatalyzed contra-Thermodynamic Isomerization of Polarized Alkenes
}

\author{
Thibaud Brégent ${ }^{\dagger}$, Jean-Philippe Bouillon ${ }^{\dagger}$, Thomas Poisson ${ }^{\dagger, \neq *}$ \\ ${ }^{\dagger}$ Normandie Univ, INSA Rouen, UNIROUEN, CNRS, COBRA (UMR 6014), 76000 Rouen, France. \\ ${ }^{\ddagger}$ Institut Universitaire de France, 1 rue Descartes, 75231 Paris, France.
}




\title{
Supporting Information
}

\author{
Table of contents
}

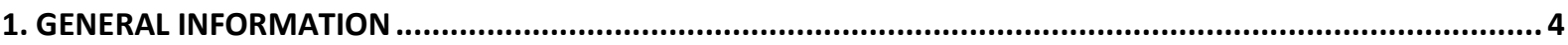

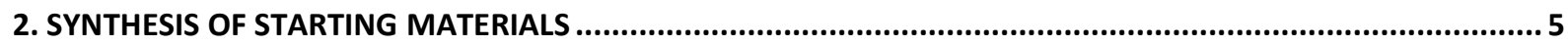

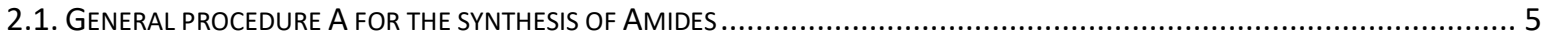

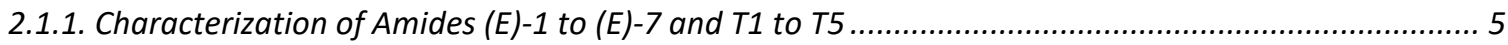

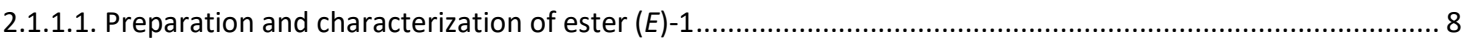

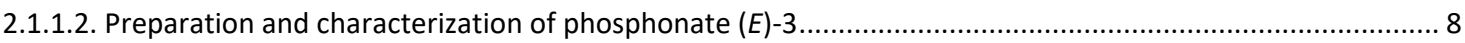

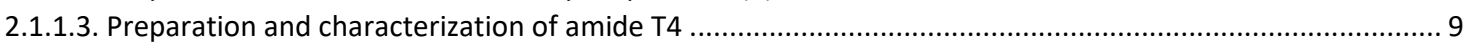

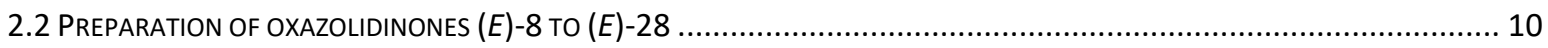

2.2.1 General Procedure $B$ for the preparation of esters................................................................... 10

2.2.2 General Procedure $C$ for the preparation of acids .................................................................... 10

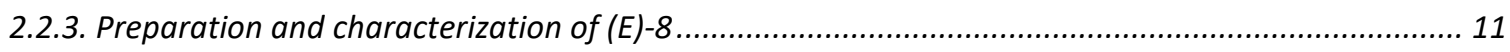

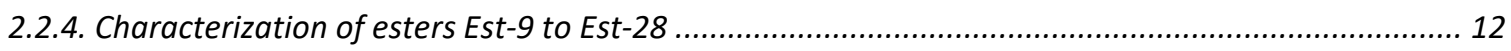

2.2.5. Preparation and characterization of ester Est-26............................................................... 17

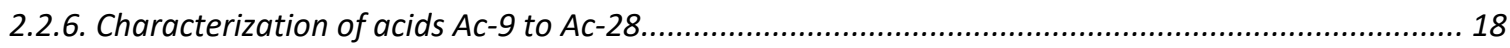

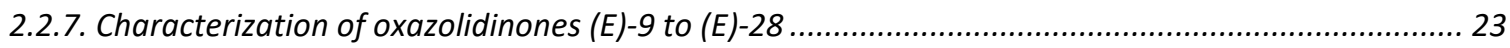

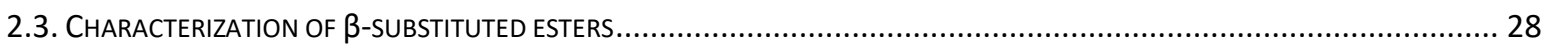

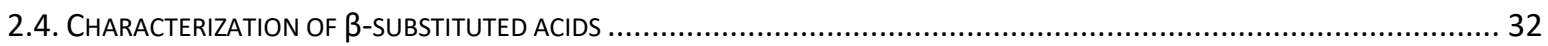

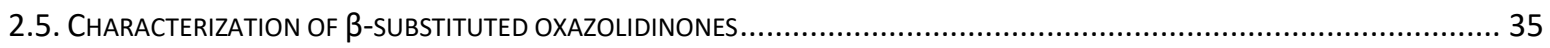

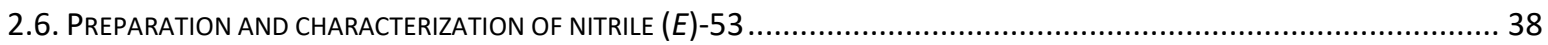

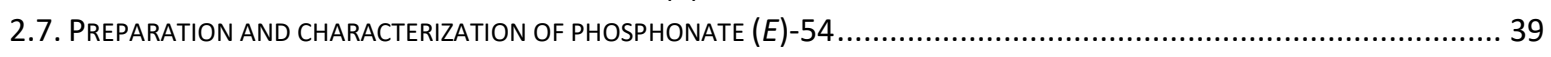

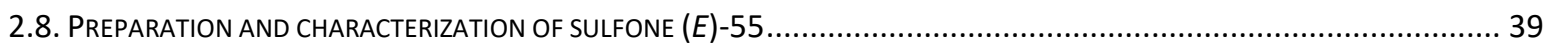

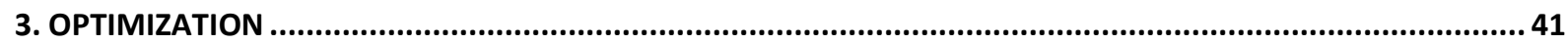

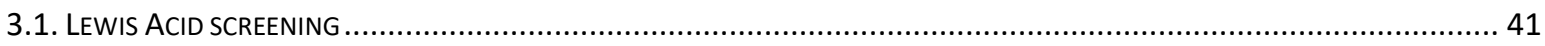

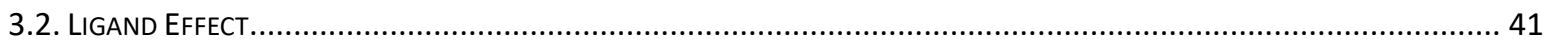

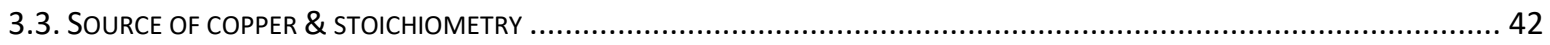

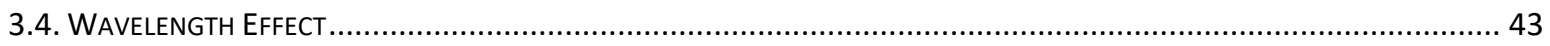

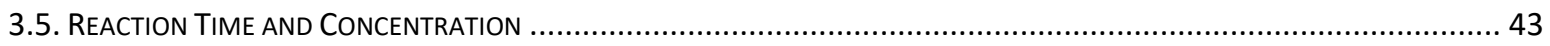

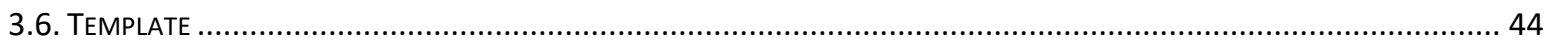

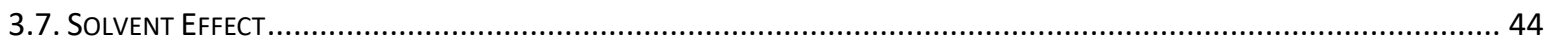

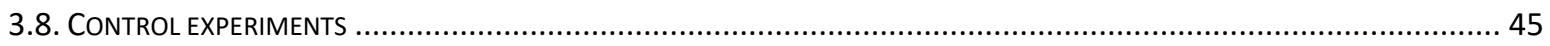

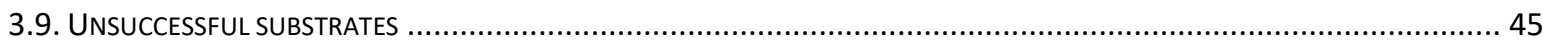

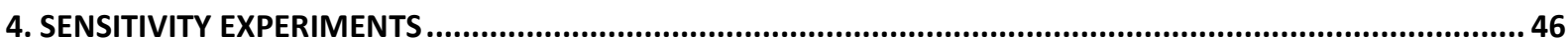

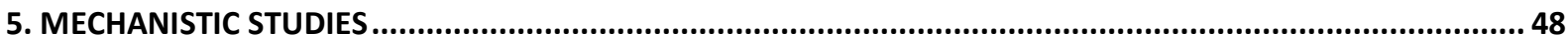

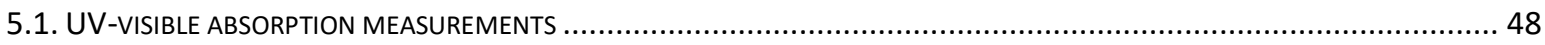

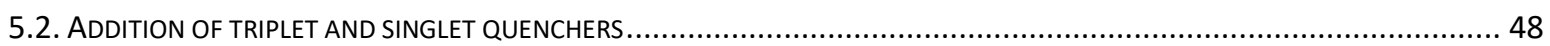

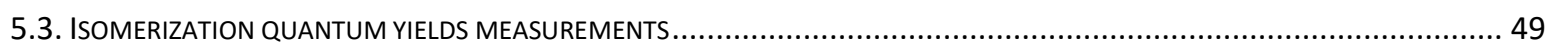

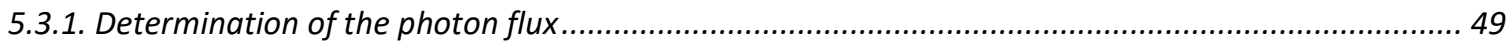

5.3.2. Determination of isomerization quantum yield ..................................................................... 50

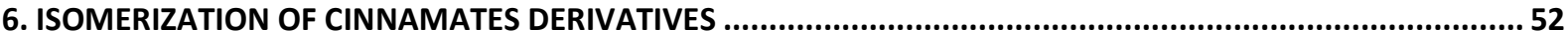

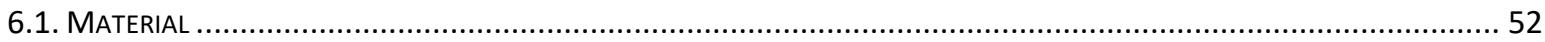


6.2. General Procedure D

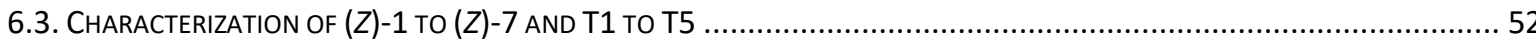

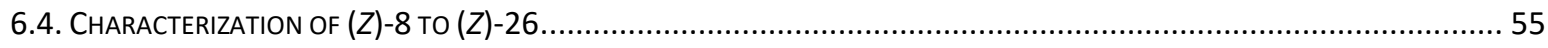

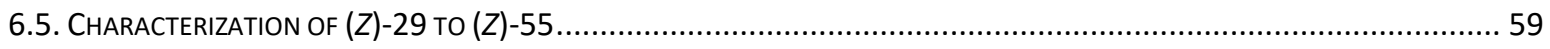

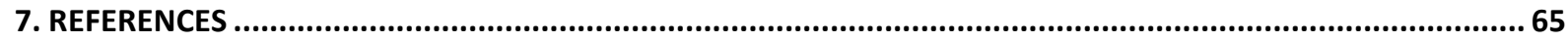

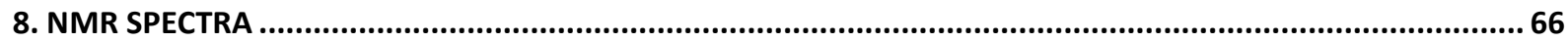

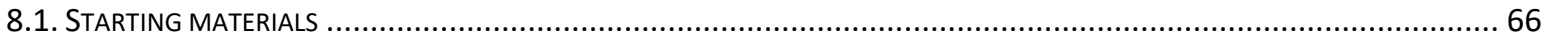

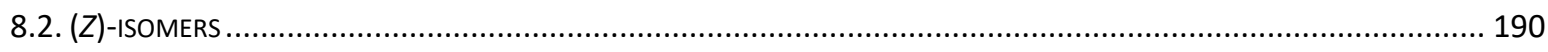

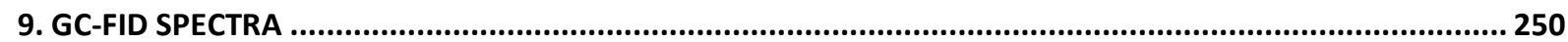




\section{General Information}

All reactions were carried out using oven dried glassware and magnetic stirring under an atmosphere of argon unless otherwise stated. Flash chromatography was performed with silica gel $(40-63 \mu \mathrm{m})$ supplied by VWR. Analytical thin layer chromatography was performed on silica gel aluminum plates with F-254 indicator and visualized by UV light ( $254 \mathrm{~nm}$ ) and/or chemical staining with a $\mathrm{KMnO}_{4}$ solution. All reagents were purchased from commercial suppliers (Sigma Aldrich, Fisher Scientific, TCI, VWR and Fluorochem) and used as received except the following: THF and toluene were distilled over sodium and benzophenone under argon atmosphere, EtOAc and DCM over $\mathrm{CaH}_{2}$ under argon. Technical grade solvents for extraction and purification (cyclohexane, dichloromethane, $n$-pentane, ethyl acetate, diethyl ether, ethanol and petroleum ether) were used without purification.

${ }^{1} \mathrm{H}$ NMR spectra were recorded on a Brucker DXP 300 instrument at $300 \mathrm{MHz}\left(75 \mathrm{MHz}\right.$ for ${ }^{13} \mathrm{C}$, $96 \mathrm{MHz}$ for ${ }^{11} \mathrm{~B}, 282 \mathrm{MHz}$ for ${ }^{19} \mathrm{~F}$ and $121 \mathrm{MHz}$ for ${ }^{31} \mathrm{P}$ ) in $\mathrm{CDCl}_{3}$ at room temperature unless otherwise stated. Chemical shifts $(\delta)$ were quoted in parts per million (ppm) relative to the residual peak of $\mathrm{CDCl}_{3}\left(\delta_{\mathrm{H}}=7.267 \mathrm{ppm}\right.$ and $\delta_{\mathrm{C}}=77.16 \mathrm{ppm}$; or relative to external $\mathrm{CFCl}_{3}: \delta=$ $0.00 \mathrm{ppm})$. Spectra are reported as follows: chemical shift $\delta(\mathrm{ppm})$, multiplicity ( $\mathrm{s}=$ singlet, $\mathrm{d}$ $=$ doublet, $\mathrm{dd}=$ doublet of doublets, $\mathrm{dt}=$ doublet of triplets, $\mathrm{t}=$ triplet, $\mathrm{td}=$ triplet of doublets, $q=$ quartet, $p=$ quintuplet, $m=$ multiplet and $b r=$ broad signal), coupling constant and integration.

High-resolution mass spectra (HRMS) were recorded on Waters LCT Premier spectrometer. IR spectra were recorded on a PerkinElmer Spectrum 100 (br = broad, $\mathrm{w}=$ weak, $\mathrm{m}=$ medium, $\mathrm{s}$ = strong and vs = very strong). Melting points were measured on a STUART SMP3 meltingpoint apparatus in open capillaries. GC-FID analysis were obtained on a SCION 436-GC apparatus equipped with a RXI 5MS RESTEK column (internal diameter $30 \times 0.25 \mathrm{~mm}, 0.25 \mu \mathrm{m}$ deposit). Absorption spectra were recorded on UV-Visible Agilent Cary 60 spectrophotometer.

The lamp used for isomerization was KESSIL ${ }^{\mathrm{TM}} \mathrm{H} 150-\mathrm{BLUE} 34 \mathrm{~W}$ with $\lambda_{\max 1}=450 \mathrm{~nm}$ and $\lambda_{\max 2}$ $=480 \mathrm{~nm}$.

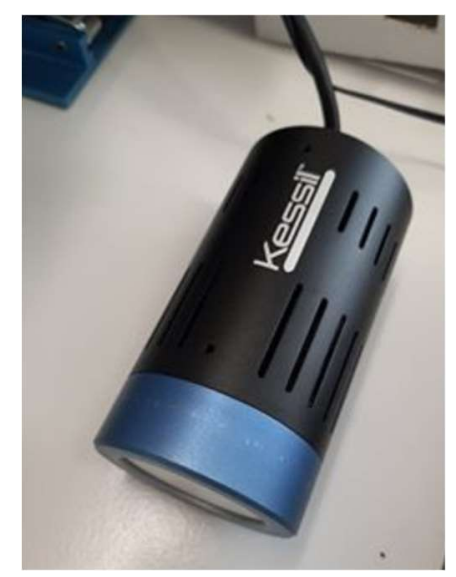

Photo 1 : KESSIL ${ }^{\text {TM }}$ H150-BLUE $34 W$ 


\section{Synthesis of starting materials}

$\alpha$-Methyl cinnamic acid was purchased from Alfa Aesar. Starting amines, aldehydes and ketones were purchased from Sigma Aldrich, Fisher scientific, Apollo or Fluorochem and used as supplied.

\subsection{General procedure A for the synthesis of Amides}

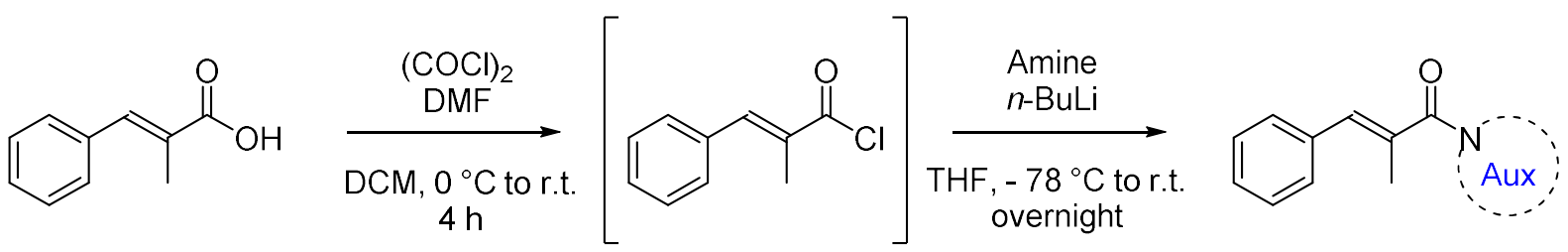

In an oven-dried flask were added the corresponding carboxylic acid (12 mmol, 1 equiv.) and DCM $(35 \mathrm{~mL})$ under inert atmosphere. At $0{ }^{\circ} \mathrm{C}$ were added $(\mathrm{COCl})_{2}(30 \mathrm{mmol}, 2.5$ equiv. $)$ and DMF (10 drops). The reaction mixture was allowed to warm to r.t. and stirred for $4 \mathrm{~h}$. Then, all volatiles were removed under reduced pressure to afford the corresponding acyl chloride. In an other oven-dried flask were added the corresponding amine (12 mmol, 1 equiv.) and THF (70 mL). Then, $n$-BuLi solution (12 mmol, 1 equiv., $2.5 \mathrm{M}$ in hexanes) was slowly added at -78 ${ }^{\circ} \mathrm{C}$. The mixture was stirred for $1 \mathrm{~h}$ at $-78^{\circ} \mathrm{C}$. Then, the freshly prepared solution of acyl chloride in THF $(10 \mathrm{~mL})$ was slowly added at $-78^{\circ} \mathrm{C}$ and the reaction mixture was stirred for $1 \mathrm{~h}$ at -78 ${ }^{\circ} \mathrm{C}$ and allowed to warm to r.t. and stirred overnight. The reaction mixture was quenched with $\mathrm{NH}_{4} \mathrm{Cl}$ (saturated aqueous solution, $40 \mathrm{~mL}$ ) and the aqueous layer was extracted with EtOAc $(3 \times 15 \mathrm{~mL})$. The combined organic layers were washed with brine, dried over $\mathrm{Na}_{2} \mathrm{SO}_{4}$ and concentrated under vacuum to give the corresponding amide.

\subsubsection{Characterization of Amides $(E)-1$ to $(E)-7$ and T1 to T5}

(E)-N,N,2-trimethyl-3-phenylacrylamide ((E)-2):<smiles>C/C(=C\c1ccccc1)C(=O)N(C)C</smiles>

$(E)-2$

Obtained as a slightly orange oil (1.79 g, 79\% yield) after column chromatography on silica gel (PE:EtOAc from 9:1 to 6:4). Rf: 0.33 (PE:EtOAC ; 7:3); ${ }^{1} \mathrm{H}$ NMR (300 MHz, $\left.\mathrm{CDCl}_{3}\right): \delta 7.40-7.27(\mathrm{~m}, 5 \mathrm{H}), 6.54$ (s, $1 \mathrm{H}), 3.07(\mathrm{~s}, 6 \mathrm{H}), 2.11(\mathrm{~s}, 3 \mathrm{H}) ;{ }^{13} \mathrm{C}\left\{{ }^{1} \mathrm{H}\right\}$ NMR (75 MHz, $\left.\mathrm{CDCl}_{3}\right): \delta 173.6$, 136.0, 133.3, 129.5, 128.9 (2C), 128.2 (2C), 127.3, 38.7, 34.8, 15.9; IR (neat): v 3473 (br), 2922 (br), 1615 (vs), 1493 (m), 1391 (s), 1093 (s), 697 (vs), 573 (m), 510 (s) cm ${ }^{-1}$; HRMS (TOF-ESI ) $\mathrm{m} / \mathbf{z}$ : calcd for $[\mathrm{M}+\mathrm{H}]^{+} \mathrm{C}_{12} \mathrm{H}_{16} \mathrm{NO}: 190.1232$, found: 190.1226 (- $\left.3.2 \mathrm{ppm}\right)$.

(E)-3-(2-methyl-3-phenylacryloyl)oxazolidin-2-one ((E)-4):<smiles>C/C(=C\c1ccccc1)C(=O)N1CCOC1=O</smiles>

(E)-4

Obtained a white solid (2.19 g, 79\% yield) after recrystallization in a mixture DCM:PE. Rf: 0.23 (PE:EtOAC ; 7:3); Mp: $113-114{ }^{\circ} \mathrm{C}(\mathrm{DCM}: \mathrm{PE})$; ${ }^{1} \mathrm{H}$ NMR (300 MHz, $\left.\mathrm{CDCl}_{3}\right): \delta 7.43-7.31(\mathrm{~m}, 5 \mathrm{H}), 6.97(\mathrm{~s}, 1 \mathrm{H}), 4.48(\mathrm{t}$, $2 \mathrm{H}, J=7.5 \mathrm{~Hz}), 4.09(\mathrm{t}, 2 \mathrm{H}, J=7.5 \mathrm{~Hz}), 2.19(\mathrm{~s}, 3 \mathrm{H}) ;{ }^{13} \mathrm{C}\left\{{ }^{1} \mathrm{H}\right\}$ NMR (75 $\mathrm{MHz}_{\mathrm{CDCl}}$ ): $\delta$ 172.2, 153.1, 135.8, 135.6, 131.5, 129.6, 128.4, 128.2, 62.4, 43.4, 15.6; IR (neat): v 2917 (br), 1765 (s), 1674 (vs), 1386 (s), 1305 (vs), 1203 (vs), 1014 
(s), 761 (vs), 698 (vs) cm ${ }^{-1}$; HRMS (TOF-ESI ${ }^{+}$) m/z: calcd for $[\mathrm{M}+\mathrm{H}]^{+} \mathrm{C}_{13} \mathrm{H}_{14} \mathrm{NO}_{3}: 232.0974$, found: 232.0984 (+ $4.3 \mathrm{ppm})$.

(E)-N,2-dimethyl-3-phenyl-N-(pyridin-2-yl)acrylamide ((E)-5):

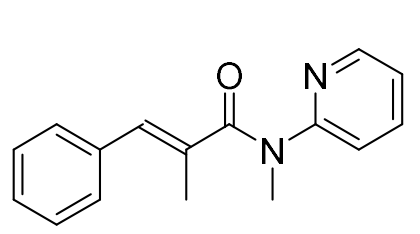

$(E)-5$

Obtained as a beige solid (2.65 $\mathrm{g}, 88 \%$ yield) after column chromatography on silica gel (PE:EtOAC; 7:3). Rf: 0.25 (PE:EtOAC ; 7:3); Mp: $58-59{ }^{\circ} \mathrm{C} ;{ }^{1} \mathrm{H}$ NMR (300 MHz, $\left.\mathrm{CDCl}_{3}\right): \delta 8.48(\mathrm{dd}, 1 \mathrm{H}, \mathrm{J}=$ $4.6 \& 1.4 \mathrm{~Hz}), 7.65(\mathrm{td}, 1 \mathrm{H}, J=7.8 \& 1.9 \mathrm{~Hz}), 7.29-7.08(\mathrm{~m}, 7 \mathrm{H}), 6.57$ $(\mathrm{s}, 1 \mathrm{H}), 3.53(\mathrm{~s}, 3 \mathrm{H}), 2.00(\mathrm{~d}, 3 \mathrm{H}, \mathrm{J}=1.3 \mathrm{~Hz}) ;{ }^{13} \mathrm{C}\left\{{ }^{1} \mathrm{H}\right\}$ NMR $(75 \mathrm{MHz}$, $\left.\mathrm{CDCl}_{3}\right): \delta 173.6,157.0,148.8,137.7,136.0,133.7,133.6,128.9$ (2C), 128.2 (2C), 127.5, 121.1, 120.3, 35.5, 16.1; IR (neat): v 3024 (br), 2920 (br), 1646 (s), 1584 (s), 1469 (s), 1435 (s), 1343 (s), 745 (s), 697 (vs) cm ${ }^{-1}$; HRMS (TOF-ESI+) m/z: calcd for [M+H] ${ }^{+}$ $\mathrm{C}_{16} \mathrm{H}_{17} \mathrm{~N}_{2} \mathrm{O}: 253.1341$, found: 253.1351 (+ $4.0 \mathrm{ppm}$ ).

(E)-1-(2,3-dihydro-1H-pyrrolo[2,3-b]pyridin-1-yl)-2-methyl-3-phenylprop-2-en-1-one ((E)6):<smiles>CC(=Cc1ccccc1)C(=O)N1CCc2cccnc21</smiles>

$(E)-6$

Obtained as a white solid (2.47 $\mathrm{g}, 78 \%$ yield) after column chromatography on silica gel (PE:EtOAC ; 7:3). Rf: 0.25 (PE:EtOAC ; 7:3); Mp: $87-88{ }^{\circ} \mathrm{C} ;{ }^{1} \mathbf{H}$ NMR (300 MHz, $\left.\mathrm{CDCl}_{3}\right): \delta 8.08(\mathrm{~d}, 1 \mathrm{H}, \mathrm{J}=$ $4.9 \mathrm{~Hz}), 7.48-7.35(\mathrm{~m}, 6 \mathrm{H}), 6.86(\mathrm{dd}, 1 \mathrm{H}, J=7.3 \& 5.1 \mathrm{~Hz}), 6.77(\mathrm{~s}$, $1 \mathrm{H}), 4.17(\mathrm{t}, 2 \mathrm{H}, J=8.3 \mathrm{~Hz}), 3.12(\mathrm{t}, 2 \mathrm{H}, J=8.3 \mathrm{~Hz}), 2.20(\mathrm{~s}, 3 \mathrm{H}) ;{ }^{1} \mathrm{H}$ NMR (300 MHz, benzene-d6): $\delta 7.87(\mathrm{~d}, 1 \mathrm{H}, J=5.2 \mathrm{~Hz}), 7.40(\mathrm{~d}, 2 \mathrm{H}, J=7.4 \mathrm{~Hz}), 7.19-7.01(\mathrm{~m}$, $4 \mathrm{H}), 6.66(\mathrm{dd}, 1 \mathrm{H}, J=7.3 \& 1.3 \mathrm{~Hz}), 6.28(\mathrm{dd}, 1 \mathrm{H}, J=7.3 \& 5.1 \mathrm{~Hz}), 3.78(\mathrm{t}, 2 \mathrm{H}, J=8.4 \mathrm{~Hz}), 2.34$ (d, $3 \mathrm{H}, J=1.4 \mathrm{~Hz}$ ), $2.17(\mathrm{t}, 2 \mathrm{H}, J=8.4 \mathrm{~Hz}) ;{ }^{13} \mathrm{C}\left\{{ }^{1} \mathrm{H}\right\} \mathbf{N M R}\left(75 \mathrm{MHz}, \mathrm{CDCl}_{3}\right): \delta 171.7,155.8,146.6$, 136.7, 135.1, 133.1, 130.9, 129.3 (2C), 128.2 (2C), 127.3, 125.6, 118.2, 46.7, 24.9, 16.2; IR (neat): v 3023 (w), 2960 (w), 2918 (w), 2245 (w), 1624 (s), 1587 (s), 1414 (vs), 1376 (s), 1340 (vs), 1237 (m), 783 (m), 729 (s), 696 (vs) cm ${ }^{-1}$; HRMS (TOF-ESI') m/z: calcd for [M+H] ${ }^{+}$ $\mathrm{C}_{17} \mathrm{H}_{17} \mathrm{~N}_{2} \mathrm{O}: 265.1341$, found: 265.1350 (+3.4 ppm).

(E)-2-methyl-3-phenyl-1-(1H-pyrazol-1-yl)prop-2-en-1-one ((E)-7):<smiles>C/C(=C\c1ccccc1)C(=O)n1cccn1</smiles>

$(E)-7$

Obtained as a white solid $(2.42 \mathrm{~g}$, 95\% yield) after column chromatography on silica gel (PE:EtOAc ; 9:1). Rf: 0.41 (PE:EtOAc ; 9:1); Mp: $55-56{ }^{\circ} \mathrm{C} ;{ }^{1} \mathrm{H}$ NMR (300 MHz, $\left.\mathrm{CDCl}_{3}\right): \delta 8.34(\mathrm{~d}, 1 \mathrm{H}, \mathrm{J}=2.7 \mathrm{~Hz})$, $7.78(\mathrm{~s}, 1 \mathrm{H}), 7.57(\mathrm{~s}, 1 \mathrm{H}), 7.50-7.35(\mathrm{~m}, 5 \mathrm{H}), 6.50(\mathrm{~s}, 1 \mathrm{H}), 2.35(\mathrm{~s}, 3 \mathrm{H})$; ${ }^{13} \mathrm{C}\left\{{ }^{1} \mathrm{H}\right\}$ NMR (75 MHz, CDCl 3$): \delta$ 168.9, 144.0, 141.8, 135.4, 130.4, 129.9 (2C), 129.8, 128.7, $128.4(2 \mathrm{C}), 109.2,16.2$; IR (neat): v $3142(\mathrm{w}), 1698(\mathrm{~m}), 1391(\mathrm{~m}), 1343(\mathrm{~s}), 1084(\mathrm{~m}), 915(\mathrm{~m})$, 766 (vs), 696 (s), 521 (m) cm ${ }^{-1}$; HRMS (TOF-ESI ${ }^{+}$) m/z: calcd for $[\mathrm{M}+\mathrm{H}]^{+} \mathrm{C}_{13} \mathrm{H}_{13} \mathrm{~N}_{2} \mathrm{O}: 213.1028$, found: 213.1022 (- $2.8 \mathrm{ppm})$. 
(E)-3-(2-methyl-3-phenylacryloyl)benzo[d]oxazol-2(3H)-one (T1):<smiles>C/C(=C\c1ccccc1)C(=O)n1c(=O)oc2ccccc21</smiles>

T1

Obtained as a white solid ( $1.77 \mathrm{~g}, 53 \%$ yield) after recrystallization in a mixture DCM:PE. Rf: 0.78 (PE:EtOAC ; 8:2); Mp: $96-97{ }^{\circ} \mathrm{C}$ (DCM:PE); ${ }^{1} \mathrm{H}$ NMR (300 MHz, $\left.\mathrm{CDCl}_{3}\right): \delta 7.85-7.82(\mathrm{~m}, 1 \mathrm{H}), 7.49-7.36(\mathrm{~m}, 7 \mathrm{H})$, $7.23(\mathrm{~s}, 1 \mathrm{H}), 7.17-7.03(\mathrm{~m}, 1 \mathrm{H}), 2.30(\mathrm{~d}, 3 \mathrm{H}, J=1.1 \mathrm{~Hz}) ;{ }^{13} \mathrm{C}\left\{{ }^{1} \mathrm{H}\right\}$ NMR (75 MHz, $\mathrm{CDCl}_{3}$ ): $\delta 170.1,150.8,142.8,139.1,135.2,130.8,129.9$ (2C), $128.9,128.6$ (2C), 128.4, 125.1, 124.7, 115.0, 110.2, 15.2; IR (neat): v 1804 (vs), 1699 (vs), 1479 (m), 1306 (vs), 1140 (vs), 752 (vs), 693 (s) cm ${ }^{-1}$; HRMS (TOF-ESI') $\mathrm{m} / \mathrm{z}$ : calcd for $\left[\mathrm{M}+\mathrm{NH}_{4}\right]^{+} \mathrm{C}_{17} \mathrm{H}_{17} \mathrm{~N}_{2} \mathrm{O}_{3}: 297.1239$, found: 297.1248 (+ $3.0 \mathrm{ppm}$ ).

(E)-1-(2-methyl-3-phenylacryloyl)pyrrolidin-2-one (T2):<smiles>C/C(=C\c1ccccc1)C(=O)N1CCCC1=O</smiles>

T2

Obtained as a white solid (2.21 $\mathrm{g}, 80 \%$ yield) after column chromatography on silica gel (PE:EtOAC ; 8:2). Rf: 0.18 (PE:EtOAC; 8:2); Mp: $70-71{ }^{\circ} \mathrm{C} ;{ }^{1} \mathrm{H}$ NMR (300 MHz, $\left.\mathrm{CDCl}_{3}\right): \delta 7.43-7.29(\mathrm{~m}, 5 \mathrm{H}), 6.86$ $(\mathrm{s}, 1 \mathrm{H}), 3.87(\mathrm{t}, 2 \mathrm{H}, J=6.8 \mathrm{~Hz}), 2.61(\mathrm{t}, 2 \mathrm{H}, J=6.8 \mathrm{~Hz}), 2.18-2.05(\mathrm{~m}$, $2 \mathrm{H}), 2.14(\mathrm{~d}, 3 \mathrm{H}, J=1.3 \mathrm{~Hz}) ;{ }^{13} \mathrm{C}\left\{{ }^{1} \mathrm{H}\right\}$ NMR (75 MHz, $\left.\mathrm{CDCl}_{3}\right): \delta 174.2$, 172.4, 135.6, 133.6, 133.2, 129.1 (2C), 128.0 (2C), 127.5, 45.6, 32.7, 17.3, 15.2; IR (neat): v 2981 (br), 1741 (s), 1656 (vs), 1361 (s), 1277 (vs), 1227 (vs), 1190 (vs), 919 (s), 693 (vs) $\mathrm{cm}^{-1}$; HRMS (TOF-ESI ${ }^{+}$) m/z: calcd for $\left[\mathrm{M}+\mathrm{H}^{+}{ }^{+} \mathrm{C}_{14} \mathrm{H}_{16} \mathrm{NO}_{2}: 230.1181\right.$, found: 230.1180 (- $0.4 \mathrm{ppm})$.

(E)-1-(2-methyl-3-phenylacryloyl)piperidin-2-one (T3):<smiles>C/C(=C\c1ccccc1)C(=O)N1CCCCC1=O</smiles>

T3

Obtained as a white solid (2.42 $\mathrm{g}, 83 \%$ yield) after column chromatography on silica gel (PE:EtOAC ; 8:2). Rf: 0.28 (PE:EtOAC ; 8:2); Mp: $37-38{ }^{\circ} \mathrm{C} ;{ }^{1} \mathbf{H}$ NMR (300 MHz, $\left.\mathrm{CDCl}_{3}\right): \delta 7.38-7.30(\mathrm{~m}, 5 \mathrm{H}), 6.86$ $(\mathrm{s}, 1 \mathrm{H}), 3.73(\mathrm{t}, 2 \mathrm{H}, J=5.6 \mathrm{~Hz}), 2.55(\mathrm{t}, 2 \mathrm{H}, J=6.0 \mathrm{~Hz}), 2.10(\mathrm{~d}, 3 \mathrm{H}, J=$ $1.2 \mathrm{~Hz}), 1.94-1.92(\mathrm{~m}, 4 \mathrm{H}) ;{ }^{13} \mathrm{C}\left\{{ }^{1} \mathrm{H}\right\}$ NMR (75 MHz, $\left.\mathrm{CDCl}_{3}\right): \delta 176.6$, $172.8,135.8,135.0,131.9,129.1$ (2C), 128.0 (2C), 127.5, 45.4, 34.2, 22.3, 20.9, 15.2; IR (neat): v 2961 (br), 2250 (w), 1672 (vs), 1288 (s), 1243 (vs), 1149 (s), 728 (vs), 694 (s) $\mathrm{cm}^{-1}$; HRMS (TOF-ESI ${ }^{+}$) m/z: calcd for $[\mathrm{M}+\mathrm{H}]^{+} \mathrm{C}_{15} \mathrm{H}_{18} \mathrm{NO}_{2}: 244.1338$, found: $244.1333(-2.0 \mathrm{ppm})$.

(E)-2-(2-methyl-3-phenylacryloyl)-1-phenylpyrazolidin-3-one (T5):<smiles>C/C(=C\c1ccccc1)C(=O)N1C(=O)CCN1Pc1ccccc1</smiles>

T5

Obtained as a pale yellow solid (2.13 g, 58\% yield) after column chromatography on silica gel (PE:EtOAc from 8:2 to 7:3). Rf: 0.29 (PE:EtOAC; 7:3); Mp: $76-77^{\circ} \mathrm{C} ;{ }^{1} \mathbf{H}$ NMR (300 MHz, $\left.\mathrm{CDCl}_{3}\right): \delta 7.44-$ $7.29(\mathrm{~m}, 7 \mathrm{H}), 7.13-7.00(\mathrm{~m}, 4 \mathrm{H}), 4.00(\mathrm{t}, 2 \mathrm{H}, \mathrm{J}=7.4 \mathrm{~Hz}), 2.74(\mathrm{t}, 2 \mathrm{H}, \mathrm{J}=$ $7.3 \mathrm{~Hz}), 2.21(\mathrm{~s}, 3 \mathrm{H}) ;{ }^{13} \mathrm{C}\left\{{ }^{1} \mathrm{H}\right\}$ NMR (75 $\left.\mathrm{MHz} \mathrm{CDCl}_{3}\right): \delta 172.9,168.5$, $149.8,135.6,135.5,132.2,129.5$ (2C), 129.5 (2C), 128.4 (2C), 128.2, 123.9, 117.5 (2C), 54.1, 31.3, 15.6; IR (neat): v 3026 (br), 2251 (w), 1755 (s), 1683 (s), 1489 (m), 1273 (s), 1221 (s), 1180 (s), 758 (s), 693 (vs) cm ${ }^{-1}$; HRMS (TOF-ESI ${ }^{+}$) m/z: calcd for $[\mathrm{M}+\mathrm{H}]^{+} \mathrm{C}_{19} \mathrm{H}_{19} \mathrm{~N}_{2} \mathrm{O}_{2}$ : 307.1447, found: $307.1443(-1.3 \mathrm{ppm})$. 


\subsubsection{Preparation and characterization of ester $(E)-1$}<smiles>C/C(=C\c1ccccc1)C(=O)O</smiles>

1) $\mathrm{SOCl}_{2}$ (20 equiv.), reflux, $2 \mathrm{~h}$

2) $\mathrm{MeOH}$ dry, $E \mathrm{t}_{3} \mathrm{~N}(1.5$ equiv.) DMAP (25 mol\%)

DCM dry, $0^{\circ} \mathrm{C}$ to r.t., overnight<smiles>COC(=O)/C(C)=C/c1ccccc1</smiles>

$(E)-1$

In an oven-dried flask were added acid $\mathbf{A}\left(2.46 \mathrm{~g}, 15.2 \mathrm{mmol}, 1.1\right.$ equiv.) and $\mathrm{SOCl}_{2}(21 \mathrm{~mL})$ under an inert atmosphere. The reaction mixture was stirred and heated using an oil bath at reflux for $2 \mathrm{~h}$. Then, excess of $\mathrm{SOCl}_{2}$ was removed under reduced pressure to afford the corresponding acyl chloride. In an other oven-dried flask were added DMAP (435 mg, 3.45 mmol, 0.25 equiv.), DCM dry $(90 \mathrm{~mL}), \mathrm{MeOH}$ dry $(0.60 \mathrm{~mL}, 14.8 \mathrm{mmol}, 1$ equiv.) and triethylamine $\left(2.89 \mathrm{~mL}, 20.7 \mathrm{mmol}, 1.5\right.$ equiv.). The resulting solution was stirred at $0{ }^{\circ} \mathrm{C}$. Then, the freshly prepared solution of acyl chloride was dissolved in DCM dry (10 mL) and was slowly added at $0{ }^{\circ} \mathrm{C}$. Then, the reaction mixture was allowed to warm to r.t. and stirred overnight. The reaction mixture was quenched with saturated aqueous $\mathrm{NaHCO}_{3}$ solution $(50 \mathrm{~mL})$ and the aqueous layer was extracted with DCM $(3 \times 25 \mathrm{~mL})$. The combined organic layers were washed with water, brine, dried over $\mathrm{Na}_{2} \mathrm{SO}_{4}$ and concentrated under vacuum.

methyl (E)-2-methyl-3-phenylacrylate $((E)-1)$ :<smiles>COC(=O)/C(C)=C/c1ccccc1</smiles>

$(E)-1$

Obtained as a yellow oil $(2.57 \mathrm{~g}$, 99\% yield) after column chromatography on silica gel (PE:EtOAC; 95:5). Rf: 0.69 (PE:EtOAC; 8:2); ${ }^{1} \mathrm{H}$ NMR (300 MHz, CDCl$): ~ \delta 7.70(s, 1 \mathrm{H}), 7.41(\mathrm{~d}, 4 \mathrm{H}, J=4.1 \mathrm{~Hz}), 7.37-$ $7.32(\mathrm{~m}, 1 \mathrm{H}), 3.83(\mathrm{~s}, 3 \mathrm{H}), 2.13(\mathrm{~s}, 3 \mathrm{H}) ;{ }^{13} \mathrm{C}\left\{{ }^{1} \mathrm{H}\right\}$ NMR (75 MHz, $\left.\mathrm{CDCl}_{3}\right): \delta$ 169.1, 138.9, 135.8, 129.6 (2C), 128.4 (2C), 128.3, 128.3, 52.0, 14.1; IR (neat): v 2950 (br), 1706 (vs), 1434 (s), 1250 (vs), 1112 (vs), 764 (vs), 691 (vs) cm-1; HRMS (Cl+) $\mathrm{m} / \mathrm{z}$ : calcd for $[\mathrm{M}+\mathrm{H}]^{+} \mathrm{C}_{11} \mathrm{H}_{13} \mathrm{O}_{2}: 177.0915$, found: 177.0916 (+ $0.2 \mathrm{ppm}$ ).

\subsubsection{Preparation and characterization of phosphonate $(E)-3$}<smiles>C/C(=C\c1ccccc1)C(=O)O</smiles>
$(\mathrm{COCl})_{2}(1.2$ equiv. $)$

$$
\text { DMF cat. }
$$

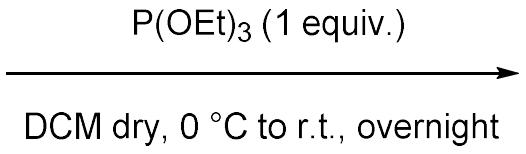<smiles>CCOP(=O)(OCC)C(=O)/C(C)=C/c1ccccc1</smiles>

(E)-3

diethyl (E)-(2-methyl-3-phenylacryloyl)phosphonate $(E)-3$ was prepared according to the reported method. ${ }^{[1]}$

In an oven-dried flask was dissolved $\alpha$-methyl cinnamic acid (1.95 g, $12 \mathrm{mmol}, 1.2$ equiv.) in DCM dry $(5 \mathrm{~mL})$. The resulting solution was cooled down to $0{ }^{\circ} \mathrm{C}$ and $(\mathrm{COCl})_{2}(1.05 \mathrm{~mL}, 12$ mmol, 1.2 equiv.) was added followed by the addition of DMF (4 drops). The reaction mixture was stirred at $0{ }^{\circ} \mathrm{C}$ for $10 \mathrm{~min}$ and allowed to warm to r.t. and stirred for $3 \mathrm{~h}$. The reaction mixture was again cooled down to $0{ }^{\circ} \mathrm{C}$ and $\mathrm{P}(\mathrm{OEt})_{3}(1.70 \mathrm{~mL}, 10 \mathrm{mmol}, 1$ equiv.) was added 
via syringe over $1 \mathrm{~h}$. Then, the reaction mixture was allowed to warm to r.t. and stirred overnight. Then, the mixture was concentrated in vacuo.

diethyl (E)-(2-methyl-3-phenylacryloyl)phosphonate ((E)-3):<smiles>CCOP(=O)(OCC)C(=O)/C(C)=C/c1ccccc1</smiles>

$(E)-3$

Obtained as a yellow oil $(0.84 \mathrm{~g}, 30 \%$ yield) after column chromatography on silica gel (PE:EtOAc from 100:0 to 80:20). Rf: 0.17 (PE:EtOAC ; 9:1); ${ }^{1} \mathbf{H}$ NMR (300 MHz, $\left.\mathbf{C D C l}_{3}\right): \delta 8.39(\mathrm{~s}, 1 \mathrm{H}), 7.51-7.35$ $(\mathrm{m}, 5 \mathrm{H}), 4.24(\mathrm{p}, 4 \mathrm{H}, J=7.1 \mathrm{~Hz}), 2.06(\mathrm{~s}, 3 \mathrm{H}), 1.37(\mathrm{t}, 6 \mathrm{H}, J=7.0 \mathrm{~Hz})$; ${ }^{13} \mathrm{C}\left\{{ }^{1} \mathrm{H}\right\}$ NMR $\left(75 \mathrm{MHz}, \mathrm{CDCl}_{3}\right): \delta 200.2(\mathrm{~d}, J=170.5 \mathrm{~Hz}), 148.5(\mathrm{~d}, J=$ $2.7 \mathrm{~Hz}), 137.1(\mathrm{~d}, J=62.3 \mathrm{~Hz}), 135.2,130.4(2 \mathrm{C}), 129.7,128.6(2 \mathrm{C}), 63.9$ (d, 2C, J = 7.4 Hz), 16.4 (d, 2C, J = 5.9 Hz), 12.4 (d, J=5.0 Hz); ${ }^{1} \mathrm{P}\left\{{ }^{1} \mathrm{H}\right\}$ NMR (121 MHz, CDCl 3 ): $\delta$ - 0.43; IR (neat): v 2988 (br), 2243 (w), 1609 (m), 1248 (m), 1016 (vs), 973 (s), 728 (vs), 567 (s) $\mathrm{cm}^{-1}$; HRMS (TOF-ESI+) m/z: calcd for $[\mathrm{M}+\mathrm{H}]^{+} \mathrm{C}_{14} \mathrm{H}_{20} \mathrm{O}_{4} \mathrm{P}: 283.1099$, found: 283.1102 (+ 1.1 ppm).

2.1.1.3. Preparation and characterization of amide $\mathrm{T} 4$

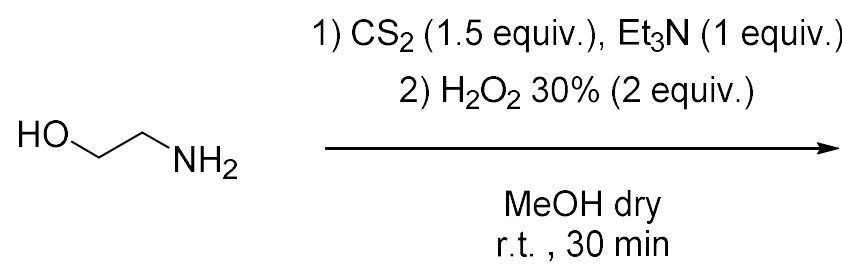

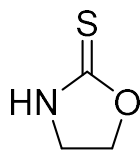

A

Oxazolidine-2-thione $\mathbf{A}$ was prepared according to the reported method. ${ }^{[2]}$

A solution of ethanolamine $(3.01 \mathrm{~mL}, 50 \mathrm{mmol})$ in methanol $(50 \mathrm{~mL})$, was treated successively with triethylamine $(6.95 \mathrm{~mL}, 50 \mathrm{mmol})$ and carbon disulphide $(4.51 \mathrm{~mL}, 75 \mathrm{mmol})$, at $0{ }^{\circ} \mathrm{C}$ under an argon atmosphere. The resulting solution was stirred at room temperature for 30 min, then hydrogen peroxide $(30 \%, 10 \mathrm{~mL}, 100 \mathrm{mmol})$ was added at such a rate that reflux of the solvent was observed until the upper solution of the reaction mixture became no longer cloudy by addition of extra hydrogen peroxide. The reaction mixture was then cooled down to room temperature, filtered and concentrated under reduced pressure. An aqueous $1 \mathrm{M}$ $\mathrm{NaOH}$ solution $(20 \mathrm{~mL})$ was added to this residue and the volatiles were removed in vacuo. Same operation was repeated twice. The mixture was finally neutralized $(\mathrm{pH} 7)$ with $6 \mathrm{M} \mathrm{HCl}$ solution. DCM $(50 \mathrm{~mL})$ was added and the resulting organic solution was washed with brine $(50 \mathrm{~mL})$ and concentrated under reduced pressure.

\section{oxazolidine-2-thione (A):}

Obtained as a white solid $(2.54 \mathrm{~g}, 54 \%$ yield) after recrystallization in a mixture<smiles>S=C1NCCO1</smiles>

A DCM:PE. Rf: 0.13 (PE:EtOAC ; 1:1); Mp: $97-98{ }^{\circ} \mathrm{C}$ (DCM:PE); ${ }^{1} \mathbf{H}$ NMR (300 MHz, $\left.\mathrm{CDCl}_{3}\right): \delta 4.73(\mathrm{t}, 2 \mathrm{H}, J=8.6 \mathrm{~Hz}), 3.83(\mathrm{t}, 2 \mathrm{H}, J=8.6 \mathrm{~Hz}) ;{ }^{13} \mathrm{C}\left\{{ }^{1} \mathrm{H}\right\} \mathrm{NMR}\left(75 \mathrm{MHz}, \mathrm{CDCl}_{3}\right)$ : $\delta$ 190.1, 70.5, 44.3; IR (neat): v 3199 (br), 2898 (br), 1680 (w), 1163 (s), 912 (s), 629 (vs) $\mathrm{cm}^{-1}$; HRMS (El ${ }^{+}$) m/z: calcd for $[\mathrm{M}]^{+} \mathrm{C}_{3} \mathrm{H}_{5} \mathrm{NOS}: 103.0092$, found: 103.0094 (+ $1.9 \mathrm{ppm})$. 
Prepared according to general procedure $\mathbf{A}$ from oxazolidine-2-thione<smiles>C/C(=C\c1ccccc1)C(=O)N1CCOC1=S</smiles>

T4

A. Obtained as a yellow solid ( $1.04 \mathrm{~g}, 35 \%$ yield) after recrystallization in a mixture DCM:PE. Rf: 0.29 (PE:EtOAC; 8:2); Mp: $114-115{ }^{\circ} \mathrm{C}$ (DCM:PE); ${ }^{1} \mathrm{H}$ NMR (300 MHz, $\left.\mathrm{CDCl}_{3}\right): \delta 7.41(\mathrm{~d}, 4 \mathrm{H}, J=4.3 \mathrm{~Hz}), 7.34$ (dt, $1 \mathrm{H}, J=9.2 \& 4.5 \mathrm{~Hz}), 7.04(\mathrm{~s}, 1 \mathrm{H}), 4.65(\mathrm{t}, 2 \mathrm{H}, J=8.1 \mathrm{~Hz}), 4.23(\mathrm{t}$, $2 \mathrm{H}, J=8.1 \mathrm{~Hz}), 2.22(\mathrm{~d}, 3 \mathrm{H}, J=1.0 \mathrm{~Hz}) ;{ }^{13} \mathrm{C}\left\{{ }^{1} \mathrm{H}\right\} \mathrm{NMR}\left(75 \mathrm{MHz}, \mathrm{CDCl}_{3}\right)$ : $\delta 186.3,173.3,136.7,135.5,131.6,129.4$ (2C), 128.5 (2C), 128.4, 67.3, 47.6, 15.8; IR (neat): v 2981 (br), 1680 (s), 1335 (s), 1247 (vs), 1222 (s), 1175 (vs), 698 (s) cm ${ }^{-1}$; HRMS (TOF-ESI') m/z: calcd for $[\mathrm{M}+\mathrm{H}]^{+} \mathrm{C}_{13} \mathrm{H}_{14} \mathrm{NO}_{2} \mathrm{~S}: 248.0745$, found: 248.0754 (+ $\left.3.6 \mathrm{ppm}\right)$.

\subsection{Preparation of oxazolidinones $(E)-8$ to $(E)-28$}
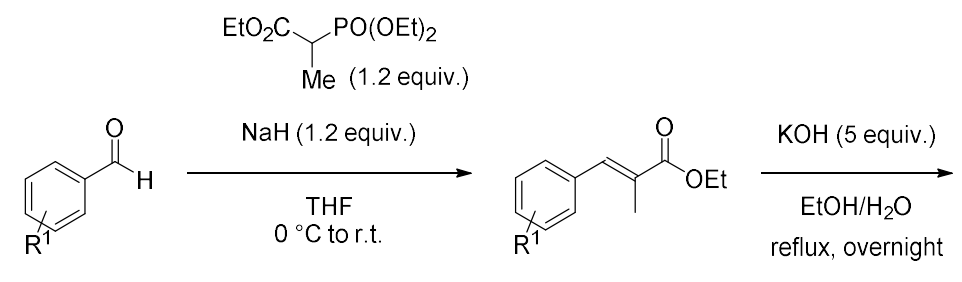

Est-9-28

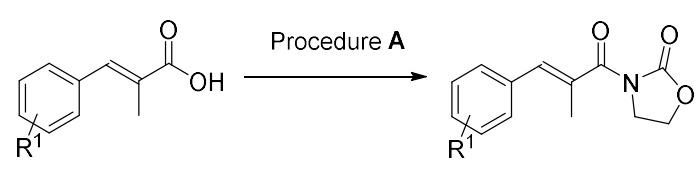

Ac-9-28

(E)-9-28

\subsubsection{General Procedure B for the preparation of esters}

In an oven-dried flask was dissolved $\mathrm{NaH}$ (60\% in mineral oil, $19.2 \mathrm{mmol}, 1.2$ equiv.) in THF $(30 \mathrm{~mL})$. Then, triethylphosphonopropionate $(19.2 \mathrm{mmol}, 1.2$ equiv.) was added dropwise at $0{ }^{\circ} \mathrm{C}$ and the reaction mixture was stirred for $30 \mathrm{~min}$. Then a solution of the corresponding aldehyde (16 mmol, 1 equiv.) in THF $(6 \mathrm{~mL})$ was added. The reaction mixture was allowed to warm to r.t. and stirred overnight. The reaction mixture was quenched with saturated aqueous $\mathrm{NH}_{4} \mathrm{Cl}$ solution $(30 \mathrm{~mL}$ ) and the aqueous layer was extracted with EtOAc $(3 \times 40 \mathrm{~mL})$. The combined organic layers were washed with brine $(40 \mathrm{~mL})$, dried over $\mathrm{Na}_{2} \mathrm{SO}_{4}$ and concentrated under vacuum to afford the corresponding ester.

\subsubsection{General Procedure $\mathrm{C}$ for the preparation of acids}

In an oven-dried flask was dissolved the corresponding ester (1 equiv.) in a mixture of EtOH: $\mathrm{H}_{2} \mathrm{O}(7: 3)(3 \mathrm{~mL} / \mathrm{mmol})$. Then, pellets of $\mathrm{KOH}$ (5 equiv.) were added and the reaction mixture was stirred and heated at reflux using an oil bath overnight. Then, the solution was acidified with $\mathrm{HCl} 1 \mathrm{M}$ solution (until $\mathrm{pH} 2$, unless otherwise stated). The resulting mixture was extracted with EtOAc $(3 \times 20 \mathrm{~mL})$. The combined organic layers were washed with brine (30 $\mathrm{mL}$ ), dried over $\mathrm{Na}_{2} \mathrm{SO}_{4}$ and concentrated under vacuum to afford corresponding carboxylic acid, used in the next step without further purification. 


\subsubsection{Preparation and characterization of $(E)-8$}

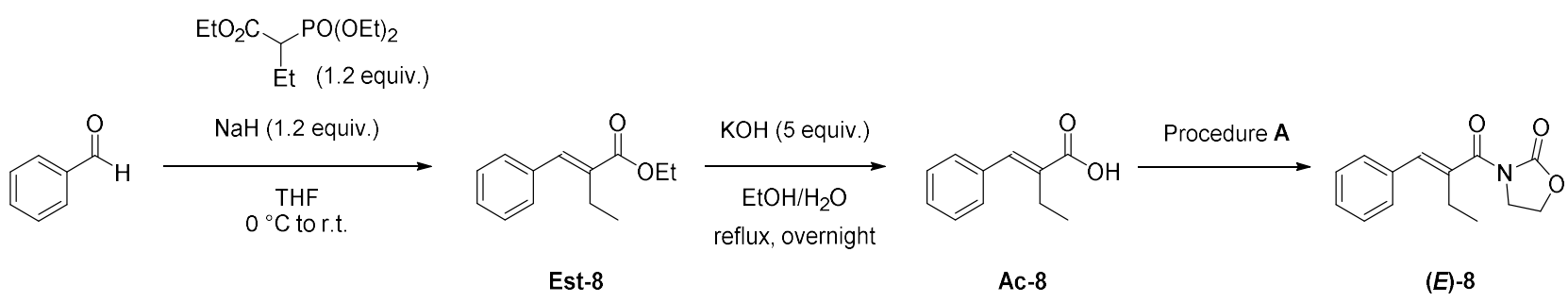

ethyl (E)-2-benzylidenebutanoate (Est-8):<smiles>CCOC(=O)/C(=C/c1ccccc1)CC</smiles>

Est-8

Prepared according to general procedure B. Obtained as a colorless oil $(2.05 \mathrm{~g}, 62 \%$ yield) after column chromatography on silica gel (PE:EtOAc from 100:0 to 99:1). Rf: 0.44 (PE:EtOAC ; 95:5); ${ }^{1} \mathbf{H ~ N M R ~ ( 3 0 0 ~ M H z , ~} \mathbf{C D C l}_{3}$ ): $\delta 7.65(\mathrm{~s}, 1 \mathrm{H}), 7.43-7.33(\mathrm{~m}, 5 \mathrm{H}), 4,29(\mathrm{q}, 2 \mathrm{H}, J=7.1 \mathrm{~Hz}), 2.55(\mathrm{q}, 2 \mathrm{H}, J=$ $7.4 \mathrm{~Hz}), 1.36(\mathrm{t}, 3 \mathrm{H}, J=7.1 \mathrm{~Hz}), 1.18(\mathrm{t}, 3 \mathrm{H}, J=7.4 \mathrm{~Hz}) ;{ }^{13} \mathrm{C}\left\{{ }^{1} \mathrm{H}\right\}$ NMR (75 MHz, $\mathrm{CDCl}_{3}$ ): $\delta$ 168.4, 138.4, 135.9, 135.1, 129.3 (2C), 128.5 (2C), 128.4, 60.8, 20.9, 14.4, 14.0; IR (neat): v 2977 (w, br), 1703 (s), 1230 (vs), 1200 (s), 1123 (s), 1045 (m), 763 (m), 698 (s) cm 1; HRMS (EI+) m/z: calcd for $[M]^{+} \mathrm{C}_{13} \mathrm{H}_{16} \mathrm{O}_{2}: 204.1150$, found: 204.1148 (- 0.9 ppm).

\section{(E)-2-benzylidenebutanoic acid (Ac-8):}<smiles>CC/C(=C\c1ccccc1)C(=O)O</smiles>

Ac-8

Prepared according to general procedure $\mathbf{C}$. Obtained as a beige solid (1.75 g, 99\% yield, on $10 \mathrm{mmol}$ scale). Mp: $109-110{ }^{\circ} \mathrm{C} ;{ }^{1} \mathrm{H}$ NMR (300 $\left.\mathrm{MHz}, \mathrm{CDCl}_{3}\right): \delta 11.41$ (br s, $\left.1 \mathrm{H}\right), 7.80(\mathrm{~s}, 1 \mathrm{H}), 7.43-7.36(\mathrm{~m}, 5 \mathrm{H}), 2.59$ (q, $2 \mathrm{H}, J=7.4 \mathrm{~Hz}$ ), 1.23 (t, 3H, $J=7.4 \mathrm{~Hz}$ ); ${ }^{13} \mathrm{C}\left\{{ }^{1} \mathrm{H}\right\}$ NMR (75 MHz, DMSO-d6): ठ 169.0, 137.4, 135.4, 135.1, 129.1 (2C), 128.7 (2C), 128.5, 20.4, 13.7; IR (neat): v 2963 (w), 2927 (w), 2873 (w), 2492 (br), 1668 (s), 1493 (w), 1422 (m), 1313 (m), 1295 (m), 1259 (s), 1141 (m), 921 (s), 763 (s), 746 (s), 693 (vs), 602 (m), $568(\mathrm{~m}), 514$ (s) cm ; $^{-1}$ HRMS (TOF-ESI') m/z: calcd for [M-H] ${ }^{-} \mathrm{C}_{11} \mathrm{H}_{11} \mathrm{O}_{2}: 175.0759$, found: 175.0759 (+ $0.0 \mathrm{ppm}$ ).

\section{(E)-3-(2-benzylidenebutanoyl)oxazolidin-2-one ((E)-8):}<smiles>CC/C(=C\c1ccccc1)C(=O)N1CCOC1=O</smiles>

(E)-8

Prepared according to general procedure A. Obtained as a white solid (1.13 g, 81\% yield, on $5.7 \mathrm{mmol}$ scale) after column chromatography on silica gel (PE:EtOAC ; 7:3). Rf: 0.28 (PE:EtOAC; 7:3); Mp: $106-107$ ${ }^{\circ} \mathrm{C} ;{ }^{1} \mathrm{H}$ NMR (300 MHz, $\left.\mathrm{CDCl}_{3}\right): \delta 7.42-7.29(\mathrm{~m}, 5 \mathrm{H}), 6.84(\mathrm{~s}, 1 \mathrm{H}), 4.48$ $(\mathrm{t}, 2 \mathrm{H}, J=7.8 \mathrm{~Hz}), 4.10(\mathrm{t}, 2 \mathrm{H}, J=7.8 \mathrm{~Hz}), 2.67(\mathrm{q}, 2 \mathrm{H}, J=7.5 \mathrm{~Hz}), 1.09$

$(\mathrm{t}, 3 \mathrm{H}, J=7.5 \mathrm{~Hz}) ;{ }^{13} \mathrm{C}\left\{{ }^{1} \mathrm{H}\right\}$ NMR $\left(75 \mathrm{MHz}, \mathbf{C D C l}_{3}\right): \delta 171.8,153.1,137.9,135.5,134.1,129.1$ (2C), 128.4 (2C), 128.0, 62.3, 43.2, 22.1, 12.9; IR (neat): v 3329 (br), 2974 (w), 2933 (w), 2880 (w), $1770(w), 1651(\mathrm{~m}), 1478(\mathrm{w}), 1382(\mathrm{~m}), 1331(\mathrm{~m}), 1313(\mathrm{~m}), 1266(\mathrm{w}), 1201(\mathrm{~m}), 1089$ (m), 1043 (vs), 881 (m), 803 (w), 752 (m), 702 (s), $644(\mathrm{w}), 485(\mathrm{~m}) \mathrm{cm}^{-1}$; HRMS (TOF-ESI ${ }^{+}$) m/z: calcd for $[\mathrm{M}+\mathrm{H}]^{+} \mathrm{C}_{14} \mathrm{H}_{16} \mathrm{NO}_{3}: 246.1130$, found: 246.1121 (- $\left.3.7 \mathrm{ppm}\right)$. 


\subsubsection{Characterization of esters Est-9 to Est-28}

ethyl (E)-2-methyl-3-(p-tolyl)acrylate (Est-9):<smiles>CCOC(=O)/C(C)=C/c1ccc(C)cc1</smiles>

Est-9

Prepared according to general procedure B. Obtained as a colorless oil (3.24 g, 99\% yield) after column chromatography on silica gel (PE:EtOAC ; 95:5); Rf: 0.42 (PE:EtOAC ; 95:5). ${ }^{1} \mathbf{H}$ NMR (300 MHz, $\mathbf{C D C l}_{3}$ ): $\delta 7.67(\mathrm{~m}, 1 \mathrm{H}), 7.32(\mathrm{~d}, 2 \mathrm{H}, J=7.9 \mathrm{~Hz}), 7.21(\mathrm{~d}, 2 \mathrm{H}, J=7.9 \mathrm{~Hz}), 4.27(\mathrm{q}$, $2 \mathrm{H}, J=7.1 \mathrm{~Hz}), 2.38(\mathrm{~s}, 3 \mathrm{H}), 2.13(\mathrm{~s}, 3 \mathrm{H}), 1.35(\mathrm{t}, 3 \mathrm{H}, J=7.1 \mathrm{~Hz}) ;{ }^{13} \mathrm{C}\left\{{ }^{1} \mathrm{H}\right\}$ NMR (75 MHz, CDCl 3 ): $\delta$ 168.9, 138.7, 138.4, 133.2, 129.8 (2C), 129.2 (2C), 127.8, 60.8, 21.4, 14.4, 14.1; IR (neat): v 2985 (w), 2921 (w), 2862 (w), 1702 (vs), 1633 (w), 1512 (w), 1446 (w), 1366 (w), 1247 (vs), 1200 (s), 1106 (vs), 1031 (m), 995 (w), 914 (w), $811(\mathrm{~m}), 747(\mathrm{~m}), 711(\mathrm{w})$, 516 (s) $\mathrm{cm}^{-1}$; HRMS (TOF-API ${ }^{+}$) m/z: calcd for $[\mathrm{M}+\mathrm{H}]^{+} \mathrm{C}_{13} \mathrm{H}_{17} \mathrm{O}_{2}: 205.1229$, found: 205.1223 ($2.9 \mathrm{ppm})$.

ethyl (E)-2-methyl-3-(m-tolyl)acrylate (Est-10):<smiles>CCOC(=O)/C(C)=C/c1cccc(C)c1</smiles>

Est-10

Prepared according to general procedure B. Obtained as a colorless oil (3.30 g, 99\% yield) after column chromatography on silica gel (PE:EtOAC ; 95:5). Rf: 0.35 (PE:EtOAC; 95:5); ${ }^{1} \mathbf{H} \mathbf{~ N M R ~ ( 3 0 0 ~ M H z , ~} \mathbf{C D C l}_{3}$ ): $\delta 7.67(\mathrm{~m}, 1 \mathrm{H}), 7.31(\mathrm{~d}, 1 \mathrm{H}, J=7.9 \mathrm{~Hz}), 7.21(\mathrm{br} \mathrm{m}, 2 \mathrm{H}), 7.14(\mathrm{~d}, 1 \mathrm{H}, J=$ $7.4 \mathrm{~Hz}), 4.28(\mathrm{q}, 2 \mathrm{H}, J=7.1 \mathrm{~Hz}), 2.38(\mathrm{~s}, 3 \mathrm{H}), 2.12(\mathrm{~s}, 3 \mathrm{H}), 1.36(\mathrm{t}, 3 \mathrm{H}, J$ $=7.1 \mathrm{~Hz}) ;{ }^{13} \mathrm{C}\left\{{ }^{1} \mathrm{H}\right\}$ NMR (75 MHz, $\left.\mathrm{CDCl}_{3}\right): \delta$ 168.8, 138.9, 138.0, 136.0, 130.4, 129.1, 128.5, 128.3, 126.8, 60.9, 21.5, 14.4, 14.2; IR (neat): v 2981 (br), 1703 (vs), 1262 (s), 1224 (vs), 1108 (vs), $1032(\mathrm{~m}), 791(\mathrm{~m}), 688(\mathrm{~m}) \mathrm{cm}^{-1}$; HRMS (TOF-API ${ }^{+}$) m/z: calcd for $[\mathrm{M}+\mathrm{H}]^{+} \mathrm{C}_{13} \mathrm{H}_{17} \mathrm{O}_{2}$ : 205.1229, found: 205.1225 (- $2.0 \mathrm{ppm})$.

ethyl (E)-2-methyl-3-(o-tolyl)acrylate (Est-11):<smiles>CCOC(=O)/C(C)=C/c1ccccc1C</smiles>

Est-11

Prepared according to general procedure B. Obtained as a colorless oil ( $3.10 \mathrm{~g}, 95 \%$ yield) after column chromatography on silica gel (PE:EtOAc ; 95:5). Rf: 0.42 (PE:EtOAC ; 95:5); ${ }^{1} \mathbf{H}$ NMR (300 MHz, $\left.\mathbf{C D C l}_{3}\right): \delta 7.75$ (m, $1 \mathrm{H}), 7.21(\mathrm{br} \mathrm{m}, 4 \mathrm{H}), 4.29$ (q, $2 \mathrm{H}, J=7.1 \mathrm{~Hz}), 2.29(\mathrm{~s}, 3 \mathrm{H}), 1.96(\mathrm{~s}, 3 \mathrm{H}), 1.36$ $(\mathrm{t}, 3 \mathrm{H}, J=7.1 \mathrm{~Hz}) ;{ }^{13} \mathrm{C}\left\{{ }^{1} \mathrm{H}\right\}$ NMR $\left(75 \mathrm{MHz}, \mathrm{CDCl}_{3}\right): \delta 168.6,138.2,136.9$, 135.3, 130.1, 129.4, 128.9, 128.2, 125.6, 60.9, 19.9, 14.4, 14.0; IR (neat): v 3064 (w), 2981 (w), 2927 (w), 1706 (vs), 1638 (w), 1484 (w), 1446 (w), $1366(\mathrm{~m}), 1288$ (w), 1249 (vs), 1219 (s), 1186 (m), 1116 (s), 1102 (vs), 1031 (m), $994(w), 793(w), 756$ (s), 741 (s), $719(\mathrm{~m}), 542(\mathrm{w}), 464(\mathrm{~m})$ $\mathrm{cm}^{-1}$; HRMS (TOF-API ${ }^{+}$) m/z: calcd for $[\mathrm{M}+\mathrm{H}]^{+} \mathrm{C}_{13} \mathrm{H}_{17} \mathrm{O}_{2}: 205.1229$, found: 205.1222 (- 3.4 ppm).

ethyl (E)-3-(4-(tert-butyl)phenyl)-2-methylacrylate (Est-12):<smiles>CCOC(=O)/C(C)=C/c1ccc(C(C)(C)C)cc1</smiles>

Est-12

Prepared according to general procedure B. Obtained as a colorless oil (3.97 g, 99\% yield) after column chromatography on silica gel (PE:EtOAc from 100:0 to 95:5). Rf: 0.40 (PE:EtOAC; 95:5); ${ }^{1} \mathbf{H}$ NMR (300 MHz, $\left.\mathrm{CDCl}_{3}\right)$ : $\delta 7.67(\mathrm{~m}, 1 \mathrm{H}), 7.40(\mathrm{~m}, 4 \mathrm{H}), 4.28(\mathrm{q}, 2 \mathrm{H}, J=7.1$ $\mathrm{Hz}), 2.14(\mathrm{~d}, 3 \mathrm{H}, \mathrm{J}=1.0 \mathrm{~Hz}), 1.38-1.34(\mathrm{~m}, 12 \mathrm{H}) ;{ }^{13} \mathrm{C}\left\{{ }^{1} \mathrm{H}\right\}$ NMR $(75$ $\mathrm{MHz} \mathrm{CDCl}_{3}$ ): $\delta$ 168.9, 151.6, 138.7, 133.2, 129.7 (2C), 127.9, 125.4 
(2C), 60.9, 34.8, 31.3 (3C), 14.5, 14.2; IR (neat): v 2962 (m, br), 1703 (vs), 1247 (vs), 1210 (s), 1105 (vs), 561 (s) cm ${ }^{-1}$; HRMS (El+) m/z: calcd for [M] ${ }^{+} \mathrm{C}_{16} \mathrm{H}_{22} \mathrm{O}_{2}: 246.1620$, found: 246.1616 ($1.5 \mathrm{ppm})$.

ethyl (E)-3-([1,1'-biphenyl]-4-yl)-2-methylacrylate (Est-13):<smiles>CCOC(=O)/C(C)=C/c1ccc(-c2ccccc2)cc1</smiles>

Est-13

Prepared according to general procedure B. Obtained as a white solid ( $3.46 \mathrm{~g}, 81 \%$ yield) after column chromatography on silica gel (PE:EtOAC ; 9:1). Rf: 0.55 (PE:EtOAC ; 9:1); Mp: $102-103^{\circ} \mathrm{C} ;{ }^{1} \mathbf{H}$ NMR (300 MHz, $\left.\mathrm{CDCl}_{3}\right)$ : $\delta 7.73(\mathrm{~m}, 1 \mathrm{H}), 7.65-7.63(\mathrm{~m}, 4 \mathrm{H}), 7.51-7.37(\mathrm{~m}$, $5 \mathrm{H}), 4.30(\mathrm{q}, 2 \mathrm{H}, J=7.1 \mathrm{~Hz}), 2.18(\mathrm{~s}, 3 \mathrm{H}), 1.37(\mathrm{t}, 3 \mathrm{H}, J=7.1 \mathrm{~Hz})$; ${ }^{13} \mathrm{C}\left\{{ }^{1} \mathrm{H}\right\}$ NMR (75 MHz, CDCl 3 ): $\delta 168.8,141.1,140.5,138.3,135.0,130.3(2 \mathrm{C}), 128.9$ (2C), 128.7, 127.7, 127.1 (2C), 127.1 (2C), 61.0, 14.4, 14.3; IR (neat): v 2924 (br), 1688 (m), 1260 (s), 1209 (m), 1193 (m), 1109 (m), 847 (m), 773 (vs), 702 (s), 510 (s) cm ${ }^{-1}$; HRMS (TOF-API ${ }^{+}$) m/z: calcd for $[\mathrm{M}+\mathrm{H}]^{+} \mathrm{C}_{18} \mathrm{H}_{19} \mathrm{O}_{2}: 267.1385$, found: 267.1388 (+ $1.1 \mathrm{ppm}$ ).

ethyl (E)-3-(4-methoxyphenyl)-2-methylacrylate (Est-14):<smiles>CCOC(=O)/C(C)=C/c1ccc(OC)cc1</smiles>

Est-14

Prepared according to general procedure B. Obtained as a colorless oil (3.37 g, 96\% yield) after column chromatography on silica gel (PE:EtOAC ; 9:1). Rf: 0.46 (PE:EtOAC; 95:5); ${ }^{1} \mathbf{H}$ NMR (300 MHz, $\left.\mathrm{CDCl}_{3}\right): \delta 7.65(\mathrm{~m}, 1 \mathrm{H}), 7.39(\mathrm{~d}, 2 \mathrm{H}, J=8.6 \mathrm{~Hz}), 6.93(\mathrm{~d}, 2 \mathrm{H}, J=8.6$ $\mathrm{Hz}), 4.27(\mathrm{q}, 2 \mathrm{H}, J=7.1 \mathrm{~Hz}), 3.85(\mathrm{~s}, 3 \mathrm{H}), 2.14(\mathrm{~s}, 3 \mathrm{H}), 1.35(\mathrm{t}, 3 \mathrm{H}, J$ $=7.1 \mathrm{~Hz}) ;{ }^{13} \mathrm{C}\left\{{ }^{1} \mathrm{H}\right\}$ NMR $\left(75 \mathrm{MHz}, \mathrm{CDCl}_{3}\right): \delta 168.9,159.6,138.3$, $131.4(2 \mathrm{C}), 128.5,126.3,113.8$ (2C), 60.7, 55.2, 14.3, 14.1; IR (neat): v 2988 (br), 2840 (w), 1698 (s), 1604 (s), 1510 (s), 1245 (vs), 1174 (vs), 1103 (vs), 1031 (s), 823 (m) cm ; HRMS (Cl+) $\mathrm{m} / \mathrm{z}$ : calcd for $[\mathrm{M}+\mathrm{H}]^{+} \mathrm{C}_{13} \mathrm{H}_{17} \mathrm{O}_{3}: 221.1178$, found: 221.1180 (+1.1 ppm).

ethyl (E)-2-methyl-3-(naphthalen-2-yl)acrylate (Est-15):<smiles>CCOC(=O)/C(C)=C/c1ccc2ccccc2c1</smiles>

Est-15

Prepared according to general procedure B. Obtained as a white solid (4.08 g, 99\% yield) after column chromatography on silica gel (PE:EtOAC ; 9:1). Rf: 0.65 (PE:EtOAC; 9:1); Mp: $43-44{ }^{\circ} \mathrm{C} ;{ }^{1} \mathbf{H}$ NMR (300 MHz, $\left.\mathrm{CDCl}_{3}\right): \delta 7.86(\mathrm{~m}, 5 \mathrm{H}), 7.54-7.50(\mathrm{~m}, 3 \mathrm{H}), 4.31$ (q, $2 \mathrm{H}, \mathrm{J}$ $=7.0 \mathrm{~Hz}), 2.21(\mathrm{~s}, 3 \mathrm{H}), 1.38(\mathrm{t}, 3 \mathrm{H}, J=7.1 \mathrm{~Hz}) ;{ }^{13} \mathrm{C}\left\{{ }^{1} \mathrm{H}\right\}$ NMR $(75 \mathrm{MHz}$, $\left.\mathrm{CDCl}_{3}\right): \delta 168.8,133.6,133.2,133.0,129.4,129.0,128.4,128.0,127.7,127.2,126.7,126.5$, 61.0, 14.5, 14.3; IR (neat): v 3056 (w), 2925 (br), 1700 (s), 1633 (w), 1366 (m), 1261 (m), 1237 (vs), 1106 (vs), 817 (m), 737 (s), 475 (vs) cm ${ }^{-1}$; HRMS (TOF-API+) m/z: calcd for [M] ${ }^{+} \mathrm{C}_{16} \mathrm{H}_{17} \mathrm{O}_{2}$ : 241.1229, found: 241.1230 (+0.4 ppm).

ethyl (E)-3-(4-fluorophenyl)-2-methylacrylate (Est-16):<smiles>CCOC(=O)/C(C)=C/c1ccc(F)cc1</smiles>

Est-16

Prepared according to general procedure B. Obtained as a colorless oil (3.07 g, 92\% yield) after column chromatography on silica gel (PE:EtOAC; 95:5). Rf: 0.33 (PE:EtOAC; 95:5); ${ }^{1} \mathbf{H}$ NMR (300 MHz, $\left.\mathrm{CDCl}_{3}\right): \delta 7.65(\mathrm{~m}, 1 \mathrm{H}), 7.41-7.36(\mathrm{~m}, 2 \mathrm{H}), 7.09(\mathrm{~m}, 2 \mathrm{H}), 4.28(\mathrm{q}, 2 \mathrm{H}, J$ $=7.1 \mathrm{~Hz}), 2.11(\mathrm{~s}, 3 \mathrm{H}), 1.36(\mathrm{t}, 3 \mathrm{H}, J=7.1 \mathrm{~Hz}) ;{ }^{13} \mathrm{C}\left\{{ }^{1} \mathrm{H}\right\} \mathbf{N M R}(75 \mathrm{MHz}$, $\left.\mathrm{CDCl}_{3}\right): \delta 168.6,162.5(\mathrm{~d}, J=249.1 \mathrm{~Hz}), 137.5,132.1(\mathrm{~d}, J=3.4 \mathrm{~Hz}), 131.5(\mathrm{~d}, 2 \mathrm{C}, J=8.2 \mathrm{~Hz})$, 
$128.5(\mathrm{~d}, J=1.4 \mathrm{~Hz}), 115.5$ (d, 2C, J=21.6 Hz), 61.0, 14.4, 14.0; ${ }^{19} \mathrm{~F}\left\{{ }^{1} \mathrm{H}\right\}$ NMR (282 MHz, CDCl 3$)$ : $\delta$ - 113.0; IR (neat): v 2983 (br), 1703 (s), 1600 (w), 1508 (s), 1250 (m), 1224 (vs), 1199 (s), 1159 (m), 1109 (vs), 829 (m), 748 (m), 524 (s) cm ${ }^{-1}$; HRMS (TOF-API ${ }^{+}$) m/z: calcd for [M+H] ${ }^{+}$ $\mathrm{C}_{12} \mathrm{H}_{14} \mathrm{FO}_{2}: 209.0978$, found: 209.0978 (+ $0.0 \mathrm{ppm}$ ).

ethyl (E)-3-(4-chlorophenyl)-2-methylacrylate (Est-17):<smiles>CCOC(=O)/C(C)=C/c1ccc(Cl)cc1</smiles>

Est-17

Prepared according to general procedure B. Obtained as a colorless oil (3.45 g, 96\% yield) after column chromatography on silica gel (PE:EtOAC ; 9:1). Rf: 0.55 (PE:EtOAC ; 9:1); ${ }^{1} \mathrm{H}$ NMR (300 MHz, $\mathrm{CDCl}_{3}$ ): $\delta 7.63(\mathrm{~m}, 1 \mathrm{H}), 7.35(\mathrm{~m}, 4 \mathrm{H}), 4.28(\mathrm{q}, 2 \mathrm{H}, J=7.1 \mathrm{~Hz}), 2.10(\mathrm{~s}, 3 \mathrm{H}), 1.36$ $(\mathrm{t}, 3 \mathrm{H}, J=7.1 \mathrm{~Hz}) ;{ }^{13} \mathrm{C}\left\{{ }^{1} \mathrm{H}\right\}$ NMR $\left(75 \mathrm{MHz}, \mathrm{CDCl}_{3}\right): \delta 168.5,137.3$, 134.4, 134.2, 131.0 (2C), 129.3, 128.7 (2C), 61.1, 14.4, 14.1; IR (neat): v 2981 (br), 1704 (vs), 1491 (m), 1246 (vs), 1199 (s), 1110 (vs), 1090 (vs), 1013 (m), 808 (m), 515 (vs) cm-1; HRMS (TOF-API ${ }^{+}$) m/z: calcd for $[\mathrm{M}+\mathrm{H}]^{+} \mathrm{C}_{12} \mathrm{H}_{14} \mathrm{ClO}_{2}: 225.0682$, found: 225.0683 (+ 0.4 ppm).

ethyl (E)-3-(4-bromophenyl)-2-methylacrylate (Est-18):<smiles>CCOC(=O)/C(C)=C/c1ccc(Br)cc1</smiles>

Est-18

Prepared according to general procedure B. Obtained as a colorless oil (4.08 g, 95\% yield) after column chromatography on silica gel (PE:EtOAc from 100:0 to 95:5). Rf: 0.36 (PE:EtOAc; 95:5); ${ }^{\mathbf{1}} \mathbf{H}$ NMR (300 MHz, $\left.\mathrm{CDCl}_{3}\right): \delta 7.61(\mathrm{~m}, 1 \mathrm{H}), 7.53(\mathrm{~d}, 2 \mathrm{H}, J=8.4 \mathrm{~Hz}), 7.27(\mathrm{~d}, 2 \mathrm{H}$, $J=8.3 \mathrm{~Hz}), 4.28(\mathrm{q}, 2 \mathrm{H}, J=7.1 \mathrm{~Hz}), 2.09(\mathrm{~s}, 3 \mathrm{H}), 1.36(\mathrm{t}, 3 \mathrm{H}, J=7.1 \mathrm{~Hz})$; ${ }^{13} \mathrm{C}\left\{{ }^{1} \mathrm{H}\right\}$ NMR (75 MHz, $\left.\mathrm{CDCl}_{3}\right): \delta 168.5,137.4,134.9,131.7(2 \mathrm{C})$, 131.2 (2C), 129.4, 122.5, 61.1, 14.4, 14.2; IR (neat): v 2980 (w, br), 1703 (vs), 1487 (m), 1243 (vs), 1109 (vs), 1009 (s) cm ${ }^{-1}$; HRMS (El+) m/z: calcd for [M] ${ }^{+} \mathrm{C}_{12} \mathrm{H}_{13} \mathrm{BrO}_{2}: 268.0099$, found: $268.0100(+0.3 \mathrm{ppm})$.

ethyl (E)-3-(4-iodophenyl)-2-methylacrylate (Est-19):<smiles>CCOC(=O)/C(C)=C/c1ccc(I)cc1</smiles>

Est-19

Prepared according to general procedure B. Obtained as a yellow oil (4.2 $\mathrm{g}, 83 \%$ yield) after column chromatography on silica gel (PE:EtOAC ; 9:1). Rf: 0.67 (PE:EtOAC ; 9:1); ${ }^{1} \mathbf{H}$ NMR (300 MHz, $\mathrm{CDCl}_{3}$ ): $\delta 7.73(\mathrm{~d}, 2 \mathrm{H}, J=8.1 \mathrm{~Hz}), 7.59(\mathrm{~m}, 1 \mathrm{H}), 7.13(\mathrm{~d}, 2 \mathrm{H}, J=8.1 \mathrm{~Hz}), 4.28(\mathrm{q}$, $2 \mathrm{H}, J=7.1 \mathrm{~Hz}), 2.09(\mathrm{~s}, 3 \mathrm{H}), 1.35(\mathrm{t}, 3 \mathrm{H}, J=7.1 \mathrm{~Hz}) ;{ }^{13} \mathrm{C}\left\{{ }^{1} \mathrm{H}\right\}$ NMR (75 $\mathrm{MHz}_{1} \mathrm{CDCl}_{3}$ ): $\delta$ 168.4, 137.6 (2C), 137.5, 135.4, 131.3 (2C), 129.5, 94.3, 61.1, 14.4, 14.2; IR (neat): v 2979 (br), 1701 (vs), 1483 (m), 1242 (vs), 1199 (s), 1106 (vs), 1004 (s), 802 (m), 747 (m), 510 (vs) $\mathrm{cm}^{-1}$; HRMS (TOF-API ${ }^{+}$) m/z: calcd for $[\mathrm{M}+\mathrm{H}]^{+} \mathrm{C}_{12} \mathrm{H}_{14} \mathrm{IO}_{2}: 317.0038$, found: 317.0049 (+ $3.5 \mathrm{ppm})$.

ethyl (E)-2-methyl-3-(4-(trifluoromethyl)phenyl)acrylate (Est-20):<smiles>CCOC(=O)/C(C)=C/c1ccc(C(F)(F)F)cc1</smiles>

Est-20

Prepared according to general procedure B. Obtained as a colorless oil (4.07 g, 98\% yield) after column chromatography on silica gel (PE:EtOAc from 100:0 to 95:5). Rf: 0.48 (PE:EtOAc; 95:5); ${ }^{1} \mathbf{H}$ NMR (300 MHz, $\mathrm{CDCl}_{3}$ ): $\delta 7.69(\mathrm{~m}, 1 \mathrm{H}), 7.66(\mathrm{~d}, 2 \mathrm{H}, J=8.3 \mathrm{~Hz}$ ), 7.49 (d, $2 \mathrm{H}, J=8.1 \mathrm{~Hz}$ ), $4.30(\mathrm{q}, 2 \mathrm{H}, J=7.1 \mathrm{~Hz}), 2.11(\mathrm{~d}, 3 \mathrm{H}, J=1.2 \mathrm{~Hz}), 1.37$ $(\mathrm{t}, 3 \mathrm{H}, J=7.1 \mathrm{~Hz}) ;{ }^{13} \mathrm{C}\left\{{ }^{1} \mathrm{H}\right\}$ NMR $\left(75 \mathrm{MHz}, \mathrm{CDCl}_{3}\right): \delta 168.3,139.6$, 
137.0, 131.0, 130.3, 129.8 (3C), 125.4 (q, J = 3.8 Hz), 124.1 (q, $J=272.0 \mathrm{~Hz}$ ), 61.2, 14.4, 14.1; ${ }^{19} \mathrm{~F}\left\{{ }^{1} \mathrm{H}\right\}$ NMR (282 MHz, CDCl 3 ): $\delta$ - 63.2; IR (neat): v 2985 (w, br), 1708 (s), 1321 (vs), 1247 (s), 1107 (vs), 1066 (vs), 847 (m) cm ${ }^{-1}$; HRMS (EI+) m/z: calcd for [M] ${ }^{+} \mathrm{C}_{13} \mathrm{H}_{13} \mathrm{~F}_{3} \mathrm{O}_{2}: 258.0868$, found: 258.0867 (- $0.1 \mathrm{ppm})$.

ethyl (E)-2-methyl-3-(4-(4,4,5,5-tetramethyl-1,3,2-dioxaborolan-2-yl)phenyl)acrylate (Est21):

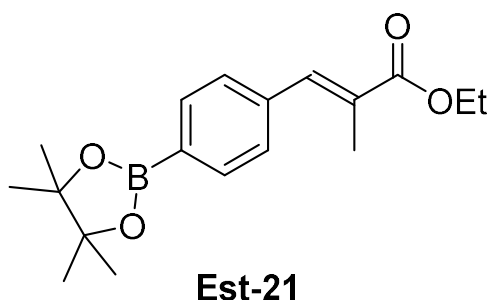

Prepared according to general procedure B. Obtained as a white solid ( $4.34 \mathrm{~g}, 86 \%$ yield) after column chromatography on silica gel (PE:EtOAc from 100:0 to 95:5). Rf: 0.26 (PE:EtOAC ; 95:5); Mp: $55-56{ }^{\circ} \mathrm{C} ;{ }^{1} \mathbf{H}$ NMR (300 MHz, $\left.\mathrm{CDCl}_{3}\right): \delta 7.83$ (d, $2 \mathrm{H}, J=8.0 \mathrm{~Hz}), 7.69(\mathrm{~m}, 1 \mathrm{H}), 7.40(\mathrm{~d}, 2 \mathrm{H}, J=7.9 \mathrm{~Hz}), 4.28(\mathrm{q}, 2 \mathrm{H}$, $J=7.1 \mathrm{~Hz}), 2.12(\mathrm{~d}, 3 \mathrm{H}, J=1.3 \mathrm{~Hz}), 1.38-1.33(\mathrm{~m}, 15 \mathrm{H}) ;{ }^{13} \mathrm{C}\left\{{ }^{1} \mathrm{H}\right\}$ NMR (75 MHz, $\left.\mathrm{CDCl}_{3}\right): \delta$ 168.3, 138.6, 138.4, 134.7 (2C), 129.3, $128.8(2 \mathrm{C}), 83.8(2 \mathrm{C}), 60.8,24.8(4 \mathrm{C}), 14.3,14.0$. The carbon bearing boron was not observed; ${ }^{11}$ B NMR (96 MHz, CDCl $)$ ): $\delta$ 31.1; IR (neat): v 2981 (br), 2250 (w), 1706 (s), 1618 (m), 1357 (vs), 1250 (s), 1143 (s), 1089 (vs), 731 (s), 654 (s) cm ${ }^{-1}$; HRMS (TOF-API ${ }^{+}$) m/z: calcd for [M+H] ${ }^{+}$ $\mathrm{C}_{18} \mathrm{H}_{26} \mathrm{BO}_{4}: 317.1924$, found: 317.1925 (+ 0.3 ppm).

ethyl (E)-2-methyl-3-(4-(methylsulfonyl)phenyl)acrylate (Est-22):<smiles>CCOC(=O)/C(C)=C/c1ccc(S(C)(=O)=O)cc1</smiles>

Est-22

Prepared according to general procedure B. Obtained as a white solid ( $2.98 \mathrm{~g}, 69 \%$ yield) after column chromatography on silica gel (PE:EtOAc ; 7:3). Rf: 0.45 (PE:EtOAC; 7:3); Mp: $73-74{ }^{\circ} \mathrm{C} ;{ }^{1} \mathbf{H}$ NMR (300 MHz, $\left.\mathrm{CDCl}_{3}\right): \delta 7.97(\mathrm{~d}, 2 \mathrm{H}, J=8.1 \mathrm{~Hz}), 7.69(\mathrm{~m}, 1 \mathrm{H})$, $7.56(\mathrm{~d}, 2 \mathrm{H}, J=8.1 \mathrm{~Hz}), 4.30(\mathrm{q}, 2 \mathrm{H}, J=7.3 \mathrm{~Hz}), 3.09(\mathrm{~s}, 3 \mathrm{H}), 2.11$ (s, 3H), $1.37(\mathrm{t}, 3 \mathrm{H}, J=7.1 \mathrm{~Hz}) ;{ }^{13} \mathrm{C}\left\{{ }^{1} \mathrm{H}\right\}$ NMR (75 MHz, $\left.\mathrm{CDCl}_{3}\right): \delta$ 167.9, 141.5, 139.7, 136.3, 131.9, 130.2 (2C), 127.4 (2C), 61.2, 44.4, 14.3, 14.1; IR (neat): v 2973 (br), 1691 (m), 1302 (m), 1257 (s), 1147 (vs), 772 (s), 526 (vs) cm ${ }^{-1}$; HRMS (EI+) m/z: calcd for $[\mathrm{M}]^{+} \mathrm{C}_{13} \mathrm{H}_{16} \mathrm{O}_{4} \mathrm{~S}: 268.0769$, found: 268.0767 (- $\left.0.9 \mathrm{ppm}\right)$.

ethyl (E)-3-(4-acetylphenyl)-2-methylacrylate (Est-23):<smiles>CCOC(=O)/C(C)=C/c1ccc(C(C)=O)cc1</smiles>

Prepared according to general procedure B. Obtained as a colorless oil (3.49 g, 67\% yield, on $22.3 \mathrm{mmol}$ scale) after column chromatography (PE:EtOAc from 100:0 to 95:5). Rf: 0.31 (PE:EtOAc ; 9:1); ${ }^{1} \mathrm{H}$ NMR (300 MHz, $\left.\mathrm{CDCl}_{3}\right): \delta 7.99$ (d, $\left.2 \mathrm{H}, J=8.3 \mathrm{~Hz}\right), 7.69(\mathrm{~m}$, $1 \mathrm{H}), 7.48(\mathrm{~d}, 2 \mathrm{H}, J=8.2 \mathrm{~Hz}), 4.29(\mathrm{q}, 2 \mathrm{H}, J=7.1 \mathrm{~Hz}), 2.63(\mathrm{~s}, 3 \mathrm{H}), 2.13$ $(\mathrm{d}, 3 \mathrm{H}, J=1.3 \mathrm{~Hz}), 1.36(\mathrm{t}, 3 \mathrm{H}, J=7.1 \mathrm{~Hz}) ;{ }^{13} \mathrm{C}\left\{{ }^{1} \mathrm{H}\right\}$ NMR (75 MHz, $\left.\mathrm{CDCl}_{3}\right): \delta 197.6,168.3,140.7$, 137.3, 136.4, 130.8, 129.7 (2C), 128.4 (2C), 61.2, 26.7, 14.4, 14.2; IR (neat): v 2982 (br), 1704 (m), 1681 (s), 1359 (w), 1245 (vs), 1202 (s), 1107 (s), 593 (s) cm ${ }^{-1}$; HRMS (TOF-API ) m/z: calcd for $[\mathrm{M}+\mathrm{H}]^{+} \mathrm{C}_{14} \mathrm{H}_{17} \mathrm{O}_{3}: 233.1178$, found: 233.1185 (+ $3.0 \mathrm{ppm}$ ). 
ethyl (E)-3-(furan-2-yl)-2-methylacrylate (Est-24):

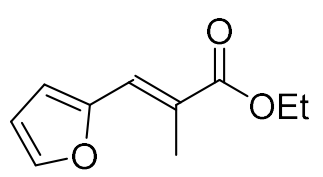

Est-24

Prepared according to general procedure B. Obtained as a colorless to orange oil [caution: the product is light sensitive] ( $2.87 \mathrm{~g}, 99 \%$ yield) after column chromatography on silica gel (PE:EtOAc from 100:0 to 90:10). Rf: 0.51 (PE:EtOAC ; 9:1); ${ }^{1} \mathbf{H}$ NMR (300 MHz, $\left.\mathrm{CDCl}_{3}\right): \delta 7.54(\mathrm{~m}, 1 \mathrm{H}), 7.45(\mathrm{~m}$, $1 \mathrm{H}), 6.61(\mathrm{~d}, 1 \mathrm{H}, J=3.3 \mathrm{~Hz}), 6.51-6.50(\mathrm{~m}, 1 \mathrm{H}), 4.26(\mathrm{q}, 2 \mathrm{H}, J=7.1 \mathrm{~Hz})$, $2.22(\mathrm{~s}, 3 \mathrm{H}), 1.34(\mathrm{t}, 3 \mathrm{H}, \mathrm{J}=7.1 \mathrm{~Hz}) ;{ }^{13} \mathrm{C}\left\{{ }^{1} \mathrm{H}\right\} \mathrm{NMR}\left(75 \mathrm{MHz}, \mathrm{CDCl}_{3}\right): \delta$ 168.4, 152.0, 143.9, 125.5, 125.0, 114.6, 112.0, 60.8, 14.3, 14.0; IR (neat): v 3115 (w), 2981 (br, m), 1698 (vs), 1252 (vs), 1206 (vs), 1106 (s), 739 (vs) cm${ }^{-1}$; HRMS (Cl+) m/z: calcd for [M+H] ${ }^{+}$ $\mathrm{C}_{10} \mathrm{H}_{13} \mathrm{O}_{3}: 181.0865$, found: 181.0861 (- $\left.1.8 \mathrm{ppm}\right)$.

ethyl (E)-2-methyl-3-(thiophen-2-yl)acrylate (Est-25):<smiles>CCOC(=O)/C(C)=C/c1cccs1</smiles>

Est-25

Prepared according to general procedure B. Obtained as a colorless oil (3.14 g, 99\% yield) after column chromatography on silica gel (PE:EtOAc from 100:0 to 90:10). Rf: 0.69 (PE:EtOAC ; 9:1); ${ }^{1} \mathbf{H}$ NMR (300 MHz, $\mathbf{C D C l}_{3}$ ): $\delta 7.86(\mathrm{~s}, 1 \mathrm{H}), 7.50(\mathrm{~d}, 1 \mathrm{H}, J=5.0 \mathrm{~Hz}), 7.29(\mathrm{~d}, 1 \mathrm{H}, J=3.4 \mathrm{~Hz}), 7.12(\mathrm{dd}, 1 \mathrm{H}$, $J=5.0 \& 3.8 \mathrm{~Hz}), 4.27(\mathrm{q}, 2 \mathrm{H}, J=7.1 \mathrm{~Hz}), 2.22(\mathrm{~s}, 3 \mathrm{H}), 1.35(\mathrm{t}, 3 \mathrm{H}, J=7.1$ $\mathrm{Hz}) ;{ }^{13} \mathrm{C}\left\{{ }^{1} \mathrm{H}\right\}$ NMR (75 MHz, $\left.\mathrm{CDCl}_{3}\right): \delta 168.6,139.4,131.7,131.5,129.1,127.4,125.0,61.0,14.4$, 14.4; IR (neat): v 3105 (br), 2980 (w), 1695 (s), 1620 (m), 1365 (m), 1254 (s), 1200 (vs), 1104 (vs), 1050 (m), 699 (vs), 514 (m) cm ${ }^{-1}$; HRMS (TOF-API ${ }^{+}$) m/z: calcd for $[\mathrm{M}+\mathrm{H}]^{+} \mathrm{C}_{10} \mathrm{H}_{13} \mathrm{O}_{2} \mathrm{~S}$ : 197.0636, found: 197.0636 (+ $0.0 \mathrm{ppm})$.

ethyl (E)-3-mesityl-2-methylacrylate (Est-27):<smiles>CCOC(=O)/C(C)=C/c1c(C)cc(C)cc1C</smiles>

Est-27

Prepared according to general procedure B. Obtained as a colorless oil (3.22 g, 87\% yield) after column chromatography on silica gel (PE:EtOAC ; 95:5). Rf: 0.43 (PE:EtOAC ; 95:5); ${ }^{1} \mathbf{H}$ NMR (300 $\mathbf{~ M H z , ~} \mathbf{C D C l}_{3}$ ): $\delta 7.61(\mathrm{~s}, 1 \mathrm{H}), 6.88(\mathrm{~s}, 2 \mathrm{H}), 4.28(\mathrm{q}, 2 \mathrm{H}, \mathrm{J}=7.1 \mathrm{~Hz}), 2.29(\mathrm{~s}, 3 \mathrm{H}), 2.13$ (s, $3 \mathrm{H}), 1.68(\mathrm{~s}, 3 \mathrm{H}), 1.36(\mathrm{t}, 3 \mathrm{H}, J=7.1 \mathrm{~Hz}) ;{ }^{13} \mathrm{C}\left\{{ }^{1} \mathrm{H}\right\}$ NMR $\left(75 \mathrm{MHz}, \mathrm{CDCl}_{3}\right)$ : $\delta$ 168.0, 139.0, 136.9, 135.3, 132.3, 130.6, 128.1 (3C), 60.7, 21.0, 20.0 (2C), 14.3, 13.8; IR (neat): v 2980 (w), 2921 (w), 2862 (w), 1709 (vs), 1644 (w), 1613 (w), 1444 (w), 1367 (w), 1343 (w), 1251 (vs), 1206 (m), 1110 (vs), 1032 (m), 993 (w), 851 (m), 747 (m), 559 (w) cm-1; HRMS (TOF-API ${ }^{+}$) m/z: calcd for $[\mathrm{M}+\mathrm{H}]^{+} \mathrm{C}_{15} \mathrm{H}_{21} \mathrm{O}_{2}: 233.1542$, found: 233.1546 (+ $1.7 \mathrm{ppm}$ ).

ethyl (E)-2-methyloct-2-enoate (Est-28):

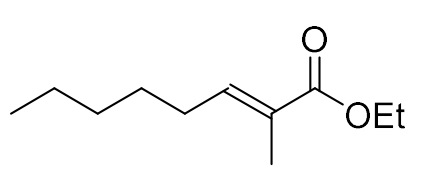

Est-28

Prepared according to general procedure B. Obtained as a colorless oil (1.94 g, 66\% yield) after column chromatography on silica gel (PE:EtOAc from 100:0 to 98:2). Rf: 0.51 (PE:EtOAC; 95:5); ${ }^{\mathbf{1}} \mathbf{H}$ NMR (300 MHz, $\mathrm{CDCl}_{3}$ ): $\delta 6.77(\mathrm{t}, 1 \mathrm{H}, J=7.4 \mathrm{~Hz}$ ), $4.19(\mathrm{q}, 2 \mathrm{H}, J=7.1 \mathrm{~Hz}$ ), $2.17(\mathrm{q}, 2 \mathrm{H}, J=7.4 \mathrm{~Hz}), 1.83(\mathrm{~s}, 3 \mathrm{H}), 1.45(\mathrm{dt}, 2 \mathrm{H}, J=7.1 \mathrm{~Hz}), 1.30(\mathrm{~m}$, 7H), $0.90(\mathrm{t}, 3 \mathrm{H}, J=6.7 \mathrm{~Hz}) ;{ }^{13} \mathrm{C}\left\{{ }^{1} \mathrm{H}\right\} \mathbf{N M R}\left(75 \mathrm{MHz}, \mathrm{CDCl}_{3}\right): \delta 168.2,142.4,127.7,60.3,31.6$, 28.6, 28.3, 22.5, 14.3, 14.0, 12.3; IR (neat): v 2928 (br, m), 2860 (w), 1709 (vs), 1651 (w), 1367 (w), 1253 (s), $1142(\mathrm{~m}), 1096$ (s), $744(\mathrm{~m}) \mathrm{cm}^{-1}$; HRMS (Cl+) m/z: calcd for $\left[\mathrm{M}+\mathrm{H}^{+} \mathrm{C}_{11} \mathrm{H}_{21} \mathrm{O}_{2}\right.$ : 185.1540, found: 185.1531 (- $4.9 \mathrm{ppm})$. 


\subsubsection{Preparation and characterization of ester Est-26}<smiles>CCOC(=O)C(C)=Cc1ccc(OCC=Cc2ccc(O)cc2)cc1</smiles>

Ethyl (E)-3-(4-(cinnamyloxy)phenyl)-2-methylacrylate Est-26 was prepared according to the reported method. ${ }^{[3]}$

In an oven-dried flask was dissolved $\mathrm{NaH}$ ( $60 \%$ in mineral oil, $1.55 \mathrm{~g}, 38.8 \mathrm{mmol}, 4$ equiv.) in THF (40 mL). Then, B (prepared from procedure B, $2.00 \mathrm{~g}, 9.7 \mathrm{mmol}, 1$ equiv.) was portionwised added at $0{ }^{\circ} \mathrm{C}$ and the reaction mixture was stirred for $30 \mathrm{~min}$. Then, a solution of cinnamyl bromide (3.82 g, $19.4 \mathrm{mmol}, 2$ equiv.) in THF $(10 \mathrm{~mL})$ was added. The reaction mixture was allowed to warm to r.t. and stirred overnight. The reaction mixture was quenched at $0{ }^{\circ} \mathrm{C}$ with saturated aqueous $\mathrm{NH}_{4} \mathrm{Cl}$ solution $(30 \mathrm{~mL})$ and the aqueous layer was extracted with EtOAc ( $3 \times 40 \mathrm{~mL})$. The combined organic layers were washed with brine (40 mL), dried over $\mathrm{Na}_{2} \mathrm{SO}_{4}$ and concentrated under vacuum.

ethyl (E)-3-(4-hydroxyphenyl)-2-methylacrylate (B):<smiles>CCOC(=O)/C(C)=C/c1ccc(O)cc1</smiles>

B

Prepared according to general procedure B. Obtained as a white solid (1.12 g, 34\% yield) after column chromatography on silica gel (PE:EtOAC ; 95:5). Rf: 0.28 (PE:EtOAC; 8:2); Mp: $89-90{ }^{\circ} \mathrm{C} ;{ }^{1}$ H NMR (300 MHz, $\left.\mathrm{CDCl}_{3}\right): \delta 7.63(\mathrm{~m}, 1 \mathrm{H}), 7.34(\mathrm{~d}, 2 \mathrm{H}, J=8.6 \mathrm{~Hz}), 6.86(\mathrm{~d}$, $2 \mathrm{H}, J=8.6 \mathrm{~Hz}), 4.88(\mathrm{~s}, 1 \mathrm{H}), 4.27(\mathrm{q}, 2 \mathrm{H}, J=7.1 \mathrm{~Hz}), 2.13(\mathrm{~d}, 3 \mathrm{H}, \mathrm{J}=$ $1.1 \mathrm{~Hz}), 1.35(\mathrm{t}, 3 \mathrm{H}, J=7.2 \mathrm{~Hz}) ;{ }^{13} \mathrm{C}\left\{{ }^{1} \mathrm{H}\right\}$ NMR (75 MHz, $\left.\mathrm{CDCl}_{3}\right): \delta$ 170.1, 156.7, 139.5, 131.8 (2C), 128.1, 125.7, 115.6 (2C), 61.3, 14.4, 14.1; IR (neat): v 3224 (br), $2979(\mathrm{w}), 1676(\mathrm{~m}), 1606(\mathrm{~m}), 1585(\mathrm{~m}), 1510(\mathrm{~s}), 1369(\mathrm{~m}), 1271(\mathrm{~s}), 1233$ (vs), 1207 (vs), 1173 (s), 1134 (s), 1003 (m), 829 (m), 526 (vs) cm ${ }^{-1}$; HRMS (TOF-API ${ }^{+}$m/z: calcd for [M+H] ${ }^{+}$ $\mathrm{C}_{12} \mathrm{H}_{15} \mathrm{O}_{3}: 207.1021$, found: 207.1024 (+ 1.4 ppm).

ethyl (E)-3-(4-(cinnamyloxy)phenyl)-2-methylacrylate (Est-26):<smiles>CCOC(=O)/C(C)=C/c1ccc(OC/C=C/c2ccccc2)cc1</smiles>

$2 x$

Obtained as a beige solid ( $1.85 \mathrm{~g}, 59 \%$ yield) after column chromatography (PE:EtOAC; from 100:0 to 94:6); Rf: 0.19 (PE:EtOAC; 95:5); Mp: $56-57{ }^{\circ} \mathrm{C}$; ${ }^{1} \mathbf{H}$ NMR (300 MHz, $\left.\mathrm{CDCl}_{3}\right): \delta 7.65(\mathrm{~s}, 1 \mathrm{H}), 7.44-7.32(\mathrm{~m}, 7 \mathrm{H}), 6.99(\mathrm{~d}, 2 \mathrm{H}, J=$ $8.4 \mathrm{~Hz}), 6.75(\mathrm{~d}, 1 \mathrm{H}, J=16.2 \mathrm{~Hz}), 6.43(\mathrm{dt}, 1 \mathrm{H}, J=11.6$ \& $5.4 \mathrm{~Hz}), 4.74(\mathrm{~d}, 2 \mathrm{H}, J=5.5 \mathrm{~Hz}), 4.27(\mathrm{q}, 2 \mathrm{H}, J=7.0 \mathrm{~Hz})$, $2.14(\mathrm{~s}, 3 \mathrm{H}), 1.35(\mathrm{t}, 3 \mathrm{H}, J=7.0 \mathrm{~Hz}) ;{ }^{13} \mathrm{C}\left\{{ }^{1} \mathrm{H}\right\}$ NMR $(75 \mathrm{MHz}$, $\left.\mathrm{CDCl}_{3}\right): \delta 169.1,158.8,138.5,136.4,133.4,131.6$ (2C), 128.8, 128.7 (2C), 128.1, 126.7 (2C), 126.6, 124.1, 114.7 (2C), 68.7, 60.9, 14.5, 14.2; IR (neat): v 2983 (br), 2862 (w), 1705 (m), 1603 (m), 1509 (m), 1251 (vs), 1201 (s), 1177 (vs), 1109 (m), 967 (m), 839 (s), 744 (s), 692 (s), 535 (m) $\mathrm{cm}^{-1} ;$ HRMS (TOF-API ${ }^{+}$) m/z: calcd for $[\mathrm{M}+\mathrm{H}]^{+} \mathrm{C}_{21} \mathrm{H}_{23} \mathrm{O}_{3}: 323.1647$, found: 323.1650 (+ 0.9 ppm). 


\subsubsection{Characterization of acids Ac-9 to Ac- 28}

(E)-2-methyl-3-(p-tolyl)acrylic acid (Ac-9):<smiles>C/C(=C\c1ccc(C)cc1)C(=O)O</smiles>

Ac-9

Prepared according to general procedure $\mathbf{C}$. Obtained as a green solid (1.69 g, 98\% yield, on $9.8 \mathrm{mmol}$ scale). Mp: $170-171{ }^{\circ} \mathrm{C} ;{ }^{1} \mathrm{H}$ NMR (300 $\left.\mathbf{M H z} \mathrm{CDCl}_{3}\right): \delta 10.64(\mathrm{br} \mathrm{s}, 1 \mathrm{H}), 7.80(\mathrm{~m}, 1 \mathrm{H}), 7.36(\mathrm{~d}, 2 \mathrm{H}, J=7.9 \mathrm{~Hz})$, $7.23(\mathrm{~d}, 2 \mathrm{H}, \mathrm{J}=7.9 \mathrm{~Hz}), 2.39(\mathrm{~s}, 3 \mathrm{H}), 2.16(\mathrm{~s}, 3 \mathrm{H}) ;{ }^{13} \mathrm{C}\left\{{ }^{1} \mathrm{H}\right\}$ NMR $(75 \mathrm{MHz}$, DMSO-d6): $\delta$ 169.5, 138.1, 137.8, 132.8, 129.7 (2C), 129.2 (2C), 127.8, 20.9, 14.0; IR (neat): v 2922 (w), 2629 (br), 2510 (br), 1659 (s), 1604 (m), 1417 (m), 1316 (w), 1267 (s), 1206 (m), 1126 (m), 918 (m), 805 (m), 560 (m), 518 (vs) cm ${ }^{-1}$; HRMS (TOF-ESI) m/z: calcd for $[\mathrm{M}-\mathrm{H}]^{-} \mathrm{C}_{11} \mathrm{H}_{11} \mathrm{O}_{2}: 175.0759$, found: 175.0762 (+ $\left.1.7 \mathrm{ppm}\right)$.

(E)-2-methyl-3-(m-tolyl)acrylic acid (Ac-10):<smiles>C/C(=C\c1cccc(C)c1)C(=O)O</smiles>

Ac-10

Prepared according to general procedure $\mathbf{C}$. Obtained as a white solid (1.63 g, 95\% yield, on $9.8 \mathrm{mmol}$ scale). Mp: $59-60{ }^{\circ} \mathrm{C} ;{ }^{1} \mathbf{H}$ NMR (300 $\left.\mathrm{MHz}, \mathrm{CDCl}_{3}\right): \delta 11.25$ (br s, $\left.1 \mathrm{H}\right), 7.81(\mathrm{~m}, 1 \mathrm{H}), 7.30(\mathrm{~d}, 1 \mathrm{H}, J=7.5 \mathrm{~Hz})$, $7.25(\mathrm{~m}, 2 \mathrm{H}), 7.17(\mathrm{~d}, 1 \mathrm{H}, J=7.0 \mathrm{~Hz}), 2.40(\mathrm{~s}, 3 \mathrm{H}), 2.15(\mathrm{~s}, 3 \mathrm{H}) ;{ }^{13} \mathrm{C}\left\{{ }^{1} \mathrm{H}\right\}$ NMR (75 MHz, DMSO-d6): $\delta$ 169.5, 137.9, 137.7, 135.6, 130.2, 129.1, 128.6, 128.4, 126.7, 21.0, 14.0; IR (neat): v 2957 (m), 2922 (m), 2854 (m), 2617 (w), 1678 (vs), 1609 (m), 1412 (m), 1273 (vs), 1242 (vs), 1127 (m), 914 (s), 786 (vs), 686 (vs), 559 (s), 446 (s), $405(\mathrm{~m}) \mathrm{cm}^{-1}$; HRMS (TOF-ESI') m/z: calcd for [M-H] ${ }^{-} \mathrm{C}_{11} \mathrm{H}_{11} \mathrm{O}_{2}: 175.0759$, found: 175.0770 (+ $6.3 \mathrm{ppm})$.

(E)-2-methyl-3-(o-tolyl)acrylic acid (Ac-11):<smiles>C/C(=C\c1ccccc1C)C(=O)O</smiles>

Ac-11

Prepared according to general procedure C. Obtained as a yellow solid (1.65 g, 96\% yield, on $9.8 \mathrm{mmol}$ scale). $\mathrm{Mp:} 99-100{ }^{\circ} \mathrm{C} ;{ }^{1} \mathbf{H}$ NMR (300 $\left.\mathrm{MHz} \mathrm{CDCl}_{3}\right): \delta 11.26$ (br s, $\left.1 \mathrm{H}\right), 7.91(\mathrm{~m}, 1 \mathrm{H}), 7.24-7.19(\mathrm{~m}, 4 \mathrm{H}), 2.31(\mathrm{~s}$, 3H), 2.00 (s, 3H); ${ }^{13} \mathrm{C}\left\{{ }^{1} \mathrm{H}\right\}$ NMR (75 MHz, DMSO-d6): $\delta$ 169.2, 136.9, 136.4, 134.8, 130.0, 129.4, 128.7, 128.2, 125.7, 19.5, 13.9; IR (neat): v 2924 (w), 2850 (br), 2611 (br), 2510 (br), 1674 (vs), 1617 (m), 1480 (w), 1415 (m), $1267(\mathrm{~s}), 1226(\mathrm{~m}), 1133(\mathrm{~m}), 985(\mathrm{~m}), 920(\mathrm{~m}), 814(\mathrm{w}), 756(\mathrm{~s}), 742(\mathrm{vs}), 717(\mathrm{~s}), 605(\mathrm{~m}), 557$ (m), $537(\mathrm{~m}), 464(\mathrm{~s}), 448(\mathrm{~m}) \mathrm{cm}^{-1}$; HRMS (TOF-ESI') m/z: calcd for [M-H] ${ }^{-} \mathrm{C}_{11} \mathrm{H}_{11} \mathrm{O}_{2}: 175.0759$, found: $175.0753(-3.4 \mathrm{ppm})$.

\section{(E)-3-(4-(tert-butyl)phenyl)-2-methylacrylic acid (Ac-12):}

Prepared according to general procedure $\mathbf{C}$. Obtained as a white<smiles>CC(=Cc1ccc(C(C)(C)C)cc1)C(=O)O</smiles>

Ac-12 solid (1.60 g, 91\% yield, on $8.1 \mathrm{mmol}$ scale). Mp: $130-131{ }^{\circ} \mathrm{C} ;{ }^{1} \mathbf{H}$ NMR (300 MHz, CDCl $)$ : $\delta 10.54$ (br s, $1 \mathrm{H}), 7.80(\mathrm{~m}, 1 \mathrm{H}), 7.43(\mathrm{~m}, 4 \mathrm{H})$, $2.18(\mathrm{~s}, 3 \mathrm{H}), 1.35$ (s, 9H); ${ }^{13} \mathrm{C}\left\{{ }^{1} \mathrm{H}\right\}$ NMR (75 MHz, DMSO-d6): $\delta$ 169.5, 151.1, 137.6, 132.8, 129.6 (2C), 127.9, 125.3 (2C), 34.4, 31.0 (3C), 14.0; IR (neat): v 2956 (m), 2867 (w), 2611 (br), 2516 (br), 1670 (vs), $1620(\mathrm{~m}), 1507(\mathrm{w}), 1418(\mathrm{~m}), 1362(\mathrm{~m}), 1287(\mathrm{~s}), 1267(\mathrm{~s}), 1132(\mathrm{~m}), 1109(\mathrm{~m}), 991(\mathrm{~m}), 916$ (m), 837 (s), 562 (vs) cm ${ }^{-1}$; HRMS (TOF-ESI) m/z: calcd for [M-H] ${ }^{-} \mathrm{C}_{14} \mathrm{H}_{17} \mathrm{O}_{2}: 217.1229$, found: $217.1226(-1.4 \mathrm{ppm})$. 
(E)-3-([1,1'-biphenyl]-4-yl)-2-methylacrylic acid (Ac-13):<smiles>C/C(=C\c1ccc(-c2ccccc2)cc1)C(=O)O</smiles>

Ac-13

Prepared according to general procedure $\mathbf{C}$. Obtained as a white solid (1.73 g, 97\% yield, on $7.5 \mathrm{mmol}$ scale). Mp: $\mathrm{n}$.d. decomposition of the product; ${ }^{1} \mathbf{H}$ NMR (300 MHz, $\left.\mathrm{CDCl}_{3}\right): \delta 10.92$ (br s, $\left.1 \mathrm{H}\right), 7.88(\mathrm{~m}, 1 \mathrm{H})$, $7.67(\mathrm{~m}, 4 \mathrm{H}), 7.56(\mathrm{~d}, 2 \mathrm{H}, \mathrm{J}=8.2 \mathrm{~Hz}), 7.49(\mathrm{~m}, 2 \mathrm{H}), 7.41(\mathrm{~m}, 1 \mathrm{H}), 2.23$ (s, 3H); ${ }^{13} \mathrm{C}\left\{{ }^{1} \mathrm{H}\right\}$ NMR (75 MHz, DMSO-d6): $\delta$ 169.5, 140.0, 139.4, $137.2,134.7,130.4(2 \mathrm{C}), 129.0(2 \mathrm{C}), 128.8,127.8,126.7$ (2C), 126.7 (2C), 14.1; IR (neat): v 3028 (w), 2928 (w), 1682 (m), 1628 (m), 1487 (w), 1365 (w), 1222 (m), $995(\mathrm{~m}), 846(\mathrm{~m}), 770$ (vs), 739 (s), $686(\mathrm{~s}), 498(\mathrm{~m}) \mathrm{cm}^{-1}$; HRMS (TOF-ESI') m/z: calcd for [M$\mathrm{H}^{-} \mathrm{C}_{16} \mathrm{H}_{13} \mathrm{O}_{2}: 237.0916$, found: 237.0918 (+ $\left.0.8 \mathrm{ppm}\right)$.

(E)-3-(4-methoxyphenyl)-2-methylacrylic acid (Ac-14):<smiles>COc1ccc(/C=C(\C)C(=O)O)cc1</smiles>

Ac-14

Prepared according to general procedure $\mathbf{C}$. Obtained as a white solid (1.66 g, 95\% yield, on $9.1 \mathrm{mmol}$ scale). Mp: $153-154{ }^{\circ} \mathrm{C} ;{ }^{1} \mathrm{H}$ NMR (300 MHz, $\left.\mathrm{CDCl}_{3}\right)$ : $\delta 11.11$ (br s, 1H), $7.78(\mathrm{~m}, 1 \mathrm{H}), 7.44$ (d, $2 \mathrm{H}, J=8.5 \mathrm{~Hz}$ ), $6.95(\mathrm{~d}, 2 \mathrm{H}, J=8.5 \mathrm{~Hz}), 3.86(\mathrm{~s}, 3 \mathrm{H}), 2.17(\mathrm{~s}, 3 \mathrm{H})$; ${ }^{13} \mathrm{C}\left\{{ }^{1} \mathrm{H}\right\}$ NMR (75 MHz, DMSO-d6): $\delta$ 169.7, 159.5, 137.6, 131.5 (2C), 128.1, 126.3, 114.0 (2C), 55.2, 14.0; IR (neat): v 2952 (br), 2838 (w), 2511 (br), 1708 (w), 1661 (m), 1601 (s), 1568 (w), 1424 (m), 1319 (w), 1250 (vs), 1177 (vs), 1120 (s), 1029 (m), 917 (m), 828 (s), 566 (m), 529 (vs) cm ${ }^{-1}$; HRMS (TOF-ESI) m/z: calcd for [M$\mathrm{H}^{-} \mathrm{C}_{11} \mathrm{H}_{11} \mathrm{O}_{3}: 191.0708$, found: 191.0714 (+ $\left.3.1 \mathrm{ppm}\right)$.

(E)-2-methyl-3-(naphthalen-2-yl)acrylic acid (Ac-15):<smiles>C/C(=C\c1ccc2ccccc2c1)C(=O)O</smiles>

Ac-15

Prepared according to general procedure C. Obtained as a white solid (1.62 g, 92\% yield, on $8.3 \mathrm{mmol}$ scale). Mp: $147-148{ }^{\circ} \mathrm{C} ;{ }^{1} \mathrm{H}$ NMR (300 MHz, $\left.\mathrm{CDCl}_{3}\right)$ : $\delta 10.44$ (br s, 1H), $7.99(\mathrm{~m}, 1 \mathrm{H}), 7.91$ (d, 2H, $J=8.9 \mathrm{~Hz}), 7.88-7.85(\mathrm{~m}, 2 \mathrm{H}), 7.58-7.52(\mathrm{~m}, 3 \mathrm{H}), 2.26(\mathrm{~d}, 3 \mathrm{H}, J=$ $1.2 \mathrm{~Hz}) ; \mathrm{NMR}{ }^{13} \mathrm{C}\left\{{ }^{1} \mathrm{H}\right\}$ (75 MHz, DMSO-d6): $\delta$ 169.5, 137.7, 133.2, $132.8,132.5,129.2,129.1,128.3,127.9,127.6,127.2,126.8,126.5,14.2$; IR (neat): v 3052 (w), 2922 (w), 2853 (w), 2610 (br), 2515 (br), 1670 (vs), 1503 (w), 1416 (m), 1348 (m), 1279 (vs), $1251(\mathrm{~m}), 1124(\mathrm{~m}), 917(\mathrm{~m}), 853(\mathrm{w}), 818(\mathrm{~m}), 735(\mathrm{~s}), 689(\mathrm{w}), 561(\mathrm{~m}), 472(\mathrm{~s}), 424(\mathrm{~m}) \mathrm{cm}^{-}$ 1; HRMS (TOF-ESI') m/z: calcd for [M-H] ${ }^{-} \mathrm{C}_{14} \mathrm{H}_{11} \mathrm{O}_{2}: 211.0759$, found: 211.0753 (- $2.8 \mathrm{ppm}$ ).

(E)-3-(4-fluorophenyl)-2-methylacrylic acid (Ac-16):<smiles>CC(=Cc1ccc(F)cc1)C(=O)O</smiles>

Ac-16

Prepared according to general procedure $\mathbf{C}$. Obtained as a white solid (1.64 g, 97\% yield, on $9.6 \mathrm{mmol}$ scale). Mp: $151-152{ }^{\circ} \mathrm{C} ;{ }^{1} \mathrm{H}$ NMR (300 $\left.\mathrm{MHz} \mathrm{CDCl}_{3}\right): \delta 11.32$ (br s, $\left.1 \mathrm{H}\right), 7.80(\mathrm{~m}, 1 \mathrm{H}), 7.45$ (dd, $2 \mathrm{H}, J=8.1 \& 5.8$ $\mathrm{Hz}), 7.13(\mathrm{~m}, 2 \mathrm{H}), 2.16(\mathrm{~s}, 3 \mathrm{H}) ;{ }^{13} \mathrm{C}\left\{{ }^{1} \mathrm{H}\right\}$ NMR (75 MHz, DMSO-d6): $\delta$ $169.4,161.9$ (d, $J=246.8 \mathrm{~Hz}), 136.6,132.1$ (d, $J=3.2 \mathrm{~Hz}), 131.9$ (d, 2C, $J=8.3 \mathrm{~Hz}), 128.6(\mathrm{~d}, J=1.4 \mathrm{~Hz}), 115.5(\mathrm{~d}, 2 \mathrm{C}, J=21.5 \mathrm{~Hz}), 13.9 ;{ }^{19} \mathrm{~F}\left\{{ }^{1} \mathrm{H}\right\}$ NMR $\left(282 \mathrm{MHz}, \mathrm{CDCl}_{3}\right)$ : - 112.1; IR (neat): v 3076 (w), 2836 (br), 2521 (w), 1662 (s), 1595 (m), 1508 (m), 1424 (m), 1312 (m), $1268(\mathrm{~m}), 1224(\mathrm{~s}), 1165(\mathrm{~m}), 1126(\mathrm{~m}), 914$ (s), 833 (vs), 816 (s), 558 (s), 527 (vs) cm-1; HRMS (TOF-ESI') m/z: calcd for [M-H] ${ }^{-} \mathrm{C}_{10} \mathrm{H}_{8} \mathrm{FO}_{2}: 179.0508$, found: 179.0510 (+ $1.1 \mathrm{ppm}$ ). 
(E)-3-(4-chlorophenyl)-2-methylacrylic acid (Ac-17):<smiles>C/C(=C\c1ccc(Cl)cc1)C(=O)O</smiles>

Ac-17

Prepared according to general procedure $\mathbf{C}$. Obtained as a beige solid (1.68 g, 96\% yield, on $8.9 \mathrm{mmol}$ scale). Mp: $166-167{ }^{\circ} \mathrm{C} ;{ }^{1} \mathbf{H}$ NMR (300 MHz, $\left.\mathrm{CDCl}_{3}\right): \delta 10.61$ (br s, 1H), $7.76(\mathrm{~m}, 1 \mathrm{H}), 7.42-7.35(\mathrm{~m}, 4 \mathrm{H})$, $2.14(\mathrm{~s}, 3 \mathrm{H}) ;{ }^{13} \mathrm{C}\left\{{ }^{1} \mathrm{H}\right\}$ NMR (75 MHz, DMSO-d6): $\delta$ 169.2, 136.4, 134.4, 133.1, 131.4 (2C), 129.5, 128.5 (2C), 14.0; IR (neat): v 2951 (w), 2923 $(\mathrm{m}), 2853(\mathrm{~m}), 2510(\mathrm{br}), 1669(\mathrm{~m}), 1491(\mathrm{w}), 1444(\mathrm{w}), 1420(\mathrm{w}), 1286(\mathrm{~m}), 1260(\mathrm{~m}), 1129$ (m), $1088(\mathrm{~m}), 986(\mathrm{w}), 910(\mathrm{~m}), 833$ (s), 802 (s), $721(\mathrm{~m}), 546(\mathrm{~m}), 514(\mathrm{vs}), 459$ (s) cm-1; HRMS (TOF-ESI') m/z: calcd for [M-H] ${ }^{-} \mathrm{C}_{10} \mathrm{H}_{8} \mathrm{ClO}_{2}: 195.0213$, found: 195.0213 (+ 0.0 ppm).

(E)-3-(4-bromophenyl)-2-methylacrylic acid (Ac-18):<smiles>CC(=Cc1ccc(Br)cc1)C(=O)O</smiles>

Ac-18

Prepared according to general procedure $\mathbf{C}$. Obtained as a white solid (1.66 g, 93\% yield, on $7.4 \mathrm{mmol}$ scale). Mp: $179-180{ }^{\circ} \mathrm{C} ;{ }^{1} \mathbf{H}$ NMR (300 MHz, $\mathrm{CDCl}_{3}$ ): $\delta 9.75$ (br s, $\left.1 \mathrm{H}\right), 7.73(\mathrm{~m}, 1 \mathrm{H}), 7.55(\mathrm{~d}, 2 \mathrm{H}, J=8.4$ $\mathrm{Hz}), 7.30(\mathrm{~d}, 2 \mathrm{H}, J=8.5 \mathrm{~Hz}), 2.13(\mathrm{~d}, 3 \mathrm{H}, J=1.0 \mathrm{~Hz}) ;{ }^{13} \mathrm{C}\left\{{ }^{1} \mathrm{H}\right\}$ NMR $(75$ MHz, CD ${ }_{3}$ OD-d4): $\delta$ 171.6, 138.6, 136.3, 132.7 (2C), 132.4 (2C), 130.6, 123.4, 14.2; IR (neat): v 2200 (br), 2080 (br), 1667 (vs), 1585 (w), 1488 (w), $1444(w), 1418(w), 1368(m), 1286(m), 1263(m), 1129(m), 1072(m), 1009(m), 986(m)$, 910 (m), 831 (m), 514 (vs), 406 (m) cm ${ }^{-1}$; HRMS (TOF-ESI') m/z: calcd for [M-H] ${ }^{-} \mathrm{C}_{10} \mathrm{H}_{8} \mathrm{BrO}_{2}$ : 238.9708, found: 238.9708 (+0.0 ppm).

(E)-3-(4-iodophenyl)-2-methylacrylic acid (Ac-19):<smiles>CC(=Cc1ccc(I)cc1)C(=O)O</smiles>

Ac-19

Prepared according to general procedure $\mathbf{C}$. Obtained as a brown solid ( $2.15 \mathrm{~g}, 94 \%$ yield, on $7.9 \mathrm{mmol}$ scale) after a short filtration on silica gel (PE:EtOAC ; 1:1). Rf: 0.31 (PE:EtOAc ; 6:4); Mp: $173-174{ }^{\circ} \mathrm{C} ;{ }^{1} \mathbf{H}$ NMR (300 MHz, $\left.\mathrm{CDCl}_{3}\right): \delta 11.37$ (br s, $\left.1 \mathrm{H}\right), 7.76$ (d, 2H, J = $\left.8.1 \mathrm{~Hz}\right), 7.72$ $(\mathrm{m}, 1 \mathrm{H}), 7.17(\mathrm{~d}, 2 \mathrm{H}, \mathrm{J}=8.1 \mathrm{~Hz}), 2.12(\mathrm{~s}, 3 \mathrm{H}) ;{ }^{13} \mathrm{C}\left\{{ }^{1} \mathrm{H}\right\}$ NMR $(75 \mathrm{MHz}$, $\mathrm{CDCl}_{3}$ ): $\delta$ 169.2, 137.3 (2C), 136.6, 135.0, 131.6 (2C), 129.5, 95.1, 14.0; IR (neat): v $3076(\mathrm{w})$, 2969 (br), 2824 (br), 2510 (br), 1668 (vs), 1620 (m), $1484(\mathrm{w}), 1417(\mathrm{~m}), 1281$ (s), 1260 (s), $1186(\mathrm{w}), 1128(\mathrm{~m}), 1060(\mathrm{w}), 1003(\mathrm{~m}), 986(\mathrm{~m}), 908(\mathrm{~s}), 830(\mathrm{~s}), 798(\mathrm{~s}), 684(\mathrm{~s}), 541(\mathrm{w}), 512$ (vs) $\mathrm{cm}^{-1}$; HRMS (TOF-ESI) $\mathrm{m} / \mathrm{z}$ : calcd for [M-H] ${ }^{-} \mathrm{C}_{10} \mathrm{H}_{8} \mathrm{IO}_{2}: 286.9569$, found: 286.9575 (+ 2.1 ppm).

(E)-2-methyl-3-(4-(trifluoromethyl)phenyl)acrylic acid (Ac-20):<smiles>C/C(=C\c1ccc(C(F)(F)F)cc1)C(=O)O</smiles>

Ac-20

Prepared according to general procedure C. Obtained as a beige solid (1.72 g, 97\% yield, on $7.7 \mathrm{mmol}$ scale). $\mathrm{Mp}$ : $157-158{ }^{\circ} \mathrm{C} ;{ }^{1} \mathrm{H}$ NMR (300 MHz, $\left.\mathrm{CDCl}_{3}\right): \delta 10.23$ (br s, $\left.1 \mathrm{H}\right), 7.82(\mathrm{~m}, 1 \mathrm{H}), 7.68$ (d, $2 \mathrm{H}$, $J=8.5 \mathrm{~Hz}), 7.53(\mathrm{~d}, 2 \mathrm{H}, J=8.1 \mathrm{~Hz}), 2.15(\mathrm{~d}, 3 \mathrm{H}, J=1.2 \mathrm{~Hz}) ;{ }^{13} \mathrm{C}\left\{{ }^{1} \mathrm{H}\right\}$ NMR (75 MHz, DMSO-d6): $\delta$ 169.1, 136.0, 131.3, 130.2 (3C), 128.6, $128.2,125.3(q, J=3.7 \mathrm{~Hz}), 124.2(q, J=272.0 \mathrm{~Hz}), 13.9 ;{ }^{19} \mathrm{~F}\left\{{ }^{1} \mathrm{H}\right\}$ NMR (282 MHz, DMSO-d6): $\delta$ - 61.3; IR (neat): v 2951 (br), 2510 (br), 1675 (m), 1616 (w), 1410 (w), $1320(\mathrm{~m}), 1263(\mathrm{~m}), 1167$ (s), 1127 (vs), 1110 (vs), $1066(\mathrm{~s}), 913(\mathrm{~m}), 844(\mathrm{~s}), 808(\mathrm{~m}), 600(\mathrm{~m})$, 
$542(\mathrm{~m}), 515(\mathrm{~m}), 425(\mathrm{~m}) \mathrm{cm}^{-1}$; HRMS (TOF-ESI) m/z: calcd for [M-H] ${ }^{-} \mathrm{C}_{11} \mathrm{H}_{8} \mathrm{~F}_{3} \mathrm{O}_{2}: 229.0476$, found: 229.0469 (- $3.1 \mathrm{ppm})$.

(E)-2-methyl-3-(4-(4,4,5,5-tetramethyl-1,3,2-dioxaborolan-2-yl)phenyl)acrylic acid (Ac-21):

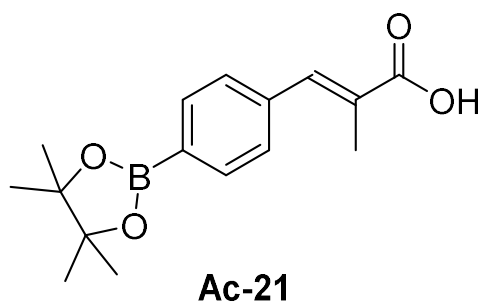

Ac-21

Prepared according to general procedure C. Obtained as a white solid ( $0.87 \mathrm{~g}, 96 \%$ yield, on $3.2 \mathrm{mmol}$ scale). Mp: 157 $158{ }^{\circ} \mathrm{C} ;{ }^{1} \mathbf{H}$ NMR (300 MHz, $\left.\mathrm{CDCl}_{3}\right): \delta 9.57$ (br s, $1 \mathrm{H}$ ), $7.86-7.81$ $(\mathrm{m}, 3 \mathrm{H}), 7.43(\mathrm{~d}, 2 \mathrm{H}, \mathrm{J}=7.4 \mathrm{~Hz}), 2.15(\mathrm{~s}, 3 \mathrm{H}), 1.36(\mathrm{~s}, 12 \mathrm{H})$; ${ }^{13} \mathrm{C}\left\{{ }^{1} \mathrm{H}\right\}$ NMR (75 MHz, CD $\mathrm{OD}$ ): $\delta 171.7,139.8,135.6(2 \mathrm{C})$, $134.8,130.5,129.8(2 \mathrm{C}), 85.1(2 \mathrm{C}), 25.2$ (4C), 14.3. The carbon bearing boron was not observed; ${ }^{11} \mathrm{~B}$ NMR $\left(96 \mathrm{MHz}, \mathrm{CD}_{3} \mathrm{OD}-\right.$

d4): $\delta$ - 22.7; IR (neat): v 3347 (br), 2976 (m), 2894 (w), 2517 (m), 1674 (m), 1608 (w), 1356 (s), 1323 (m), 1267 (m), 1142 (m), 1045 (s), 1088 (vs), 879 (m), 655 (s) cm ${ }^{-1}$; HRMS (TOF-ESI) $\mathrm{m} / \mathrm{z}$ : calcd for $[\mathrm{M}-\mathrm{H}]^{-} \mathrm{C}_{16} \mathrm{H}_{20} \mathrm{BO}_{4}: 287.1455$, found: 287.1454 (- $0.3 \mathrm{ppm}$ ).

(E)-2-methyl-3-(4-(methylsulfonyl)phenyl)acrylic acid (Ac-22):<smiles>COS(=O)(=O)c1ccc(/C=C(\C)C(=O)O)cc1</smiles>

Ac-22

Prepared according to general procedure $\mathbf{C}$. Obtained as a white solid (1.65 g, 92\% yield, on $7.5 \mathrm{mmol}$ scale). $\mathrm{Mp:} 186-187^{\circ} \mathrm{C} ;{ }^{1} \mathrm{H}$ NMR (300 MHz, CDCl $)$ : $\delta 10.64$ (br s, $1 \mathrm{H}), 8.00$ (d, $2 \mathrm{H}, J=8.1 \mathrm{~Hz})$, $7.82(\mathrm{~m}, 1 \mathrm{H}), 7.60(\mathrm{~d}, 2 \mathrm{H}, J=8.1 \mathrm{~Hz}), 3.10(\mathrm{~s}, 3 \mathrm{H}), 2.15(\mathrm{~s}, 3 \mathrm{H})$; ${ }^{13} \mathrm{C}\left\{{ }^{1} \mathrm{H}\right\}$ NMR (75 MHz, $\left.\mathrm{CDCl}_{3}\right): \delta 169.0,140.7,140.1,135.9$, 131.7, 130.3 (2C), 127.1 (2C), 43.5, 14.0; IR (neat): v 3017 (br), 2929 (w), 2516 (br), 1672 (s), 1421 (w), 1306 (m), 1269 (m), 1147 (vs), 1088 (w), 955 (m), 766 (m), 545 (m), 526 (vs) cm ${ }^{-1}$; HRMS (TOF-ESI) m/z: calcd for [M-H] ${ }^{-} \mathrm{C}_{11} \mathrm{H}_{11} \mathrm{O}_{4} \mathrm{~S}: 239.0378$, found: 239.0377 (- $0.4 \mathrm{ppm})$.

(E)-3-(4-acetylphenyl)-2-methylacrylic acid (Ac-23):<smiles>CC(=O)c1ccc(/C=C(\C)C(=O)O)cc1</smiles>

Ac-23

Prepared according to general procedure $\mathbf{C}$. Obtained as a brown solid (2.03 g, 93\% yield, on $10.8 \mathrm{mmol}$ scale). Mp: $102-103{ }^{\circ} \mathrm{C} ;{ }^{1} \mathrm{H}$ NMR (300 MHz, $\left.\mathrm{CDCl}_{3}\right): \delta 11.38$ (br s, $\left.1 \mathrm{H}\right), 8.01(\mathrm{~d}, 2 \mathrm{H}, J=8.3 \mathrm{~Hz})$, $7.84(\mathrm{~m}, 1 \mathrm{H}), 7.52(\mathrm{~d}, 2 \mathrm{H}, J=8.2 \mathrm{~Hz}), 2.64(\mathrm{~s}, 3 \mathrm{H}), 2.16(\mathrm{~s}, 3 \mathrm{H}) ;{ }^{13} \mathrm{C}\left\{{ }^{1} \mathrm{H}\right\}$ NMR (75 MHz, DMSO-d6): $\delta$ 197.4, 169.2, 140.2, 136.6, 136.1, 130.9, 129.8 (2C), 128.3 (2C), 26.8, 14.1; IR (neat): v 2966 (br), 2623 (br), 2515 (br), 1673 (vs), $1601(\mathrm{~m}), 1410(\mathrm{~m}), 1360(\mathrm{~m}), 1262(\mathrm{vs}), 1209(\mathrm{~s}), 1129(\mathrm{~m}), 992(\mathrm{~m}), 916(\mathrm{~m}), 837(\mathrm{~m}), 804$ (m), $594(\mathrm{~m}), 545(\mathrm{~m}), 507$ (s) cm ${ }^{-1}$; HRMS (TOF-ESI') m/z: calcd for [M-H] ${ }^{-} \mathrm{C}_{12} \mathrm{H}_{11} \mathrm{O}_{3}: 203.0708$, found: $203.0716(+3.9 \mathrm{ppm})$.

\section{(E)-3-(furan-2-yl)-2-methylacrylic acid (Ac-24):}

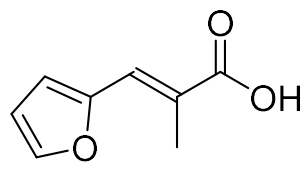

Ac-24

Prepared according to general procedure $\mathbf{C}$. Obtained as an orange solid (1.55 g, 93\% yield, on $11.1 \mathrm{mmol}$ scale). Mp: $117-118{ }^{\circ} \mathrm{C} ;{ }^{1} \mathbf{H}$ NMR (300 $\mathrm{MHz}_{\mathrm{CDCl}}$ ): $\delta 11.27$ (br s, $\left.1 \mathrm{H}\right), 7.57(\mathrm{~s}, 2 \mathrm{H}), 6.68(\mathrm{~d}, 1 \mathrm{H}, J=3.3 \mathrm{~Hz}), 6.53$ (m, 1H), 2.25 (s, 3H); ${ }^{13} \mathrm{C}\left\{{ }^{1} \mathrm{H}\right\}$ NMR (75 MHz, DMSO-d6): $\delta$ 169.3, 151.3, 145.0, 124.9, 124.9, 115.3, 112.5, 14.0; IR (neat): v 3130 (w), 2917 (br), 2605 (br), 2541 (w), 1668 (s), 1620 (s), 1552 (w), 1423 (m), 1287 (vs), 1267 (vs), 1220 (s), 1131 
(w), 1021 (s), $926(\mathrm{~m}), 820(\mathrm{~m}), 758(\mathrm{~m}), 745$ (vs), $593(\mathrm{~m}), 558(\mathrm{~m}) \mathrm{cm}^{-1}$; HRMS (TOF-ESI) m/z: calcd for $[\mathrm{M}-\mathrm{H}]^{-} \mathrm{C}_{8} \mathrm{H}_{7} \mathrm{O}_{3}: 151.0395$, found: 151.0391 (- $\left.2.6 \mathrm{ppm}\right)$.

(E)-2-methyl-3-(thiophen-2-yl)acrylic acid (Ac-25):<smiles>C/C(=C\c1cccs1)C(=O)O</smiles>

Ac-25

Prepared according to general procedure C. Obtained as a white solid (1.67 g, 98\% yield, on $10.2 \mathrm{mmol}$ scale). Mp: $148-149{ }^{\circ} \mathrm{C} ;{ }^{1} \mathrm{H}$ NMR (300 $\left.\mathrm{MHz} \mathrm{CDCl}_{3}\right): \delta 10.92(\mathrm{br} \mathrm{s}, 1 \mathrm{H}), 8.00(\mathrm{~s}, 1 \mathrm{H}), 7.56(\mathrm{~d}, 1 \mathrm{H}, J=5.1 \mathrm{~Hz}), 7.34$ (d, $1 \mathrm{H}, J=3.3 \mathrm{~Hz}$ ), $7.15(\mathrm{~m}, 1 \mathrm{H}), 2.24(\mathrm{~s}, 3 \mathrm{H}) ;{ }^{13} \mathrm{C}\left\{{ }^{1} \mathrm{H}\right\}$ NMR (75 MHz, DMSOd6): $\delta 169.3,138.7,132.5,130.9,130.3,127.8,124.7,14.1$; IR (film): v 3100 (w), 2921 (br), 2820 (br), 2605 (br), 2540 (w), 1666 (s), $1617(\mathrm{~m}), 1417(\mathrm{~m}), 1339(\mathrm{w})$, 1281 (vs), 1219 (s), 1125 (w), 1053 (w), 936 (w), $914(\mathrm{~m}), 858$ (m), 827 (w), 717 (s), 704 (vs), $605(\mathrm{~m}), 543(\mathrm{w}), 512(\mathrm{~s}), 404(\mathrm{~m}) \mathrm{cm}^{-1}$; HRMS (TOF-ESI) m/z: calcd for [M-H] ${ }^{-} \mathrm{C}_{8} \mathrm{H}_{7} \mathrm{O}_{2} \mathrm{~S}$ : 167.0167, found: 167.0153 (-8.4 ppm).

(E)-3-(4-(cinnamyloxy)phenyl)-2-methylacrylic acid (Ac-26):

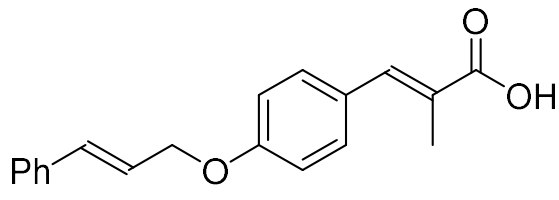

Ac-26

Prepared according to general procedure $\mathbf{C}$. Obtained as a beige solid ( $0.99 \mathrm{~g}, 73 \%$ yield, on $4.7 \mathrm{mmol}$ scale). Mp: 151 $-152{ }^{\circ} \mathrm{C} ;{ }^{1} \mathrm{H}$ NMR (300 MHz, $\left.\mathrm{CDCl}_{3}\right): \delta 10.87$ (br s, $\left.1 \mathrm{H}\right), 7.78$ $(\mathrm{s}, 1 \mathrm{H}), 7.46-7.30(\mathrm{~m}, 7 \mathrm{H}), 7.01(\mathrm{~d}, 2 \mathrm{H}, J=8.7 \mathrm{~Hz}), 6.76(\mathrm{~d}$, $1 \mathrm{H}, J=16.1 \mathrm{~Hz}), 6.43(\mathrm{dt}, 1 \mathrm{H}, J=16.1 \& 5.8 \mathrm{~Hz}), 4.75(\mathrm{~d}, 2 \mathrm{H}$, $J=5.8 \mathrm{~Hz}), 2.17(\mathrm{~s}, 3 \mathrm{H}) ;{ }^{13} \mathrm{C}\left\{{ }^{1} \mathrm{H}\right\}$ NMR $\left(75 \mathrm{MHz}, \mathrm{CDCl}_{3}\right): \delta$ $174.5,159.2,141.0,136.4,133.5,132.0$ (2C), 128.8 (2C), 128.5, 128.2, 126.7 (2C), 125.3, 124.1, 114.9 (2C), 68.8, 13.9; IR (neat): v 3028 (w), 2906 (br), 2856 (w), 2521 (br), 2104 (br), 1908 (w), $1683(\mathrm{~s}), 1603$ (s), 1509 (m), 1425 (m), 1383 (m), 1296 (s), 1246 (vs), 1180 (s), 1131 (s), $1013(\mathrm{~m}), 998(\mathrm{~m}), 962$ (vs), $911(\mathrm{~m}), 840(\mathrm{~s}), 812(\mathrm{~m}), 740(\mathrm{~s}), 690(\mathrm{vs}), 589(\mathrm{~m}), 537(\mathrm{~s}) \mathrm{cm}^{-1}$; HRMS (TOF-ESI') m/z: calcd for [M-H] ${ }^{-} \mathrm{C}_{19} \mathrm{H}_{17} \mathrm{O}_{3}: 293.1178$, found: 293.1187 (+ 3.1 ppm).

(E)-3-mesityl-2-methylacrylic acid (Ac-27):<smiles>CC(=Cc1c(C)cc(C)cc1C)C(=O)O</smiles>

Ac-27

Prepared according to general procedure $\mathbf{C}$. Obtained as a beige solid (1.64 g, 94\% yield, on $8.6 \mathrm{mmol}$ scale). Mp: $129-130{ }^{\circ} \mathrm{C} ;{ }^{1} \mathrm{H}$ NMR (300 $\left.\mathrm{MHz}, \mathrm{CDCl}_{3}\right): \delta 10.50$ (br s, $\left.1 \mathrm{H}\right), 7.76(\mathrm{~s}, 1 \mathrm{H}), 6.90(\mathrm{~s}, 2 \mathrm{H}), 2.30(\mathrm{~s}, 3 \mathrm{H})$, $2.15(\mathrm{~s}, 6 \mathrm{H}), 1.71(\mathrm{~s}, 3 \mathrm{H}) ;{ }^{13} \mathrm{C}\left\{{ }^{1} \mathrm{H}\right\}$ NMR (75 MHz, CD $\left.\mathrm{OD}\right): \delta 171.2,140.5$, 138.1, 136.1, 133.3, 131.8, 129.1 (3C), 21.1, 20.1 (2C), 14.0; IR (neat): v 2918 (w), 2862 (w), 2604 (w), 2526 (w), 2224 (br), 2072 (br), 1681 (vs), 1632 (m), $1480(w)$, $1427(\mathrm{~m}), 1371$ (w), 1268 (s), 1134 (m), 987 (m), 854 (s), 750 (w), 561 (s) cm ${ }^{-1}$; HRMS (TOF-ESI) $\mathrm{m} / \mathrm{z}$ : calcd for [M-H] ${ }^{-} \mathrm{C}_{13} \mathrm{H}_{15} \mathrm{O}_{2}:$ :203.1072, found: 203.1065 (-3.4 ppm).

(E)-2-methyloct-2-enoic acid (AC-28):

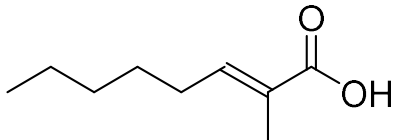

Ac-28

Prepared according to general procedure $\mathbf{C}$. Obtained as an orange oil (1.44 g, 99\% yield, on $8.9 \mathrm{mmol}$ scale). ${ }^{1} \mathrm{H}$ NMR (300 MHz, $\mathrm{CDCl}_{3}$ ): $\delta 11.18(\mathrm{br} \mathrm{s}, 1 \mathrm{H}), 6.92(\mathrm{t}, 1 \mathrm{H}, J=6.9 \mathrm{~Hz}), 2.20(\mathrm{q}, 2 \mathrm{H}, J=7.4 \mathrm{~Hz}), 1.84$ $(\mathrm{s}, 3 \mathrm{H}), 1.46(\mathrm{~m}, 2 \mathrm{H}), 1.31(\mathrm{~m}, 4 \mathrm{H}), 0.90(\mathrm{t}, 3 \mathrm{H}, J=6.8 \mathrm{~Hz}) ;{ }^{13} \mathrm{C}\left\{{ }^{1} \mathrm{H}\right\}$ NMR (75 MHz, DMSO-d6): $\delta$ 168.9, 141.5, 127.8, 31.1, 28.1, 27.9, 22.1, 13.9, 12.2; IR (neat): v 2957 (m), 2928 (m), 2860 (m), 2667 (br), 2551 (br), 1682 (vs), 1642 
(m), $1420(\mathrm{~m}), 1281$ (s), $934(\mathrm{w}), 563(\mathrm{~m}) \mathrm{cm}^{-1}$; HRMS (TOF-ESI') m/z: calcd for [M-H] ${ }^{-} \mathrm{C}_{9} \mathrm{H}_{15} \mathrm{O}_{2}$ : 155.1072, found: $155.1079(+4.5 \mathrm{ppm})$.

\subsubsection{Characterization of oxazolidinones $(E)-9$ to $(E)-28$}

(E)-3-(2-methyl-3-(p-tolyl)acryloyl)oxazolidin-2-one ((E)-9):<smiles>CC(=Cc1ccc(C)cc1)C(=O)N1CCOC1=O</smiles>

$(E)-9$

Prepared according to general procedure A. Obtained as a white solid (0.91 g, 65\% yield, on $5.7 \mathrm{mmol}$ scale) after column chromatography on silica gel (PE:EtOAc ; 7:3). Rf: 0.28 (PE:EtOAC ; 7:3); Mp: $97-98{ }^{\circ} \mathrm{C} ;{ }^{1} \mathrm{H}$ NMR (300 MHz, $\left.\mathrm{CDCl}_{3}\right): \delta 7.33$ (d, $2 \mathrm{H}, J=$ $7.7 \mathrm{~Hz}), 7.20(\mathrm{~d}, 2 \mathrm{H}, J=7.6 \mathrm{~Hz}), 6.97(\mathrm{~m}, 1 \mathrm{H}), 4.48(\mathrm{t}, 2 \mathrm{H}, J=7.7 \mathrm{~Hz})$, $4.08(\mathrm{t}, 2 \mathrm{H}, J=7.9 \mathrm{~Hz}), 2.37(\mathrm{~s}, 3 \mathrm{H}), 2.19(\mathrm{~s}, 3 \mathrm{H}) ;{ }^{13} \mathrm{C}\left\{{ }^{1} \mathrm{H}\right\}$ NMR $\left(75 \mathrm{MHz}_{2} \mathrm{CDCl}_{3}\right): \delta$ 172.3, 153.1, 138.3, 136.3, 132.7, 130.4, 129.7 (2C), 129.1 (2C), 62.4, 43.4, 21.4, 15.6; IR (neat): v 2963 (w), 2915 (w), 1775 (s), 1704 (m), 1672 (vs), $1511(w), 1481(w), 1448(w), 1370(\mathrm{~m}), 1325$ (m), 1303 (s), 1187 (vs), $1091(\mathrm{~m}), 1029(\mathrm{~m}), 1012$ (s), $917(\mathrm{~m}), 759$ (s), 519 (s) cm $\mathrm{cm}^{-1}$

(E)-3-(2-methyl-3-(m-tolyl)acryloyl)oxazolidin-2-one ((E)-10):<smiles>C/C(=C\c1cccc(C)c1)C(=O)N1CCOC1=O</smiles>

(E)-10

Prepared according to general procedure A. Obtained as a white solid (0.99 $\mathrm{g}, 71 \%$ yield, on $5.7 \mathrm{mmol}$ scale) after column chromatography on silica gel (PE:EtOAC; 7:3). Rf: 0.28 (PE:EtOAC ; 7:3); $\mathrm{Mp:} 78-79{ }^{\circ} \mathrm{C} ;{ }^{1} \mathrm{H}$ NMR (300 MHz, $\left.\mathrm{CDCl}_{3}\right): \delta 7.31-7.23(\mathrm{~m}$, $3 \mathrm{H}), 7.13(\mathrm{~d}, 1 \mathrm{H}, J=7.1 \mathrm{~Hz}), 6.95(\mathrm{~m}, 1 \mathrm{H}), 4.48(\mathrm{t}, 2 \mathrm{H}, J=7.8 \mathrm{~Hz}), 4.09$ $(\mathrm{t}, 2 \mathrm{H}, J=7.8 \mathrm{~Hz}), 2.37(\mathrm{~s}, 3 \mathrm{H}), 2.18(\mathrm{~s}, 3 \mathrm{H}) ;{ }^{13} \mathrm{C}\left\{{ }^{1} \mathrm{H}\right\} \mathrm{NMR}\left(75 \mathrm{MHz}, \mathrm{CDCl}_{3}\right): \delta 172.2,153.1,138.0$, 136.1, 135.5, 131.2, 130.3, 129.0, 128.3, 126.6, 62.4, 43.4, 21.5, 15.6; IR (neat): v 2993 (w), 2912 (w), 1780 (m), 1676 (vs), 1631 (w), 1476 (w), 1384 (m), 1307 (vs), 1204 (vs), 1109 (m), $1042(\mathrm{~m}), 933(\mathrm{w}), 896(\mathrm{w}), 799(\mathrm{~m}), 776(\mathrm{w}), 761(\mathrm{~s}), 697(\mathrm{~s}), 648(\mathrm{w}), 571(\mathrm{w}) \mathrm{cm}^{-1}$.

(E)-3-(2-methyl-3-(o-tolyl)acryloyl)oxazolidin-2-one ((E)-11):<smiles>C/C(=C\c1ccccc1C)C(=O)N1CCOC1=O</smiles>

(E)-11

Prepared according to general procedure A. Obtained as a white solid ( $0.84 \mathrm{~g}, 60 \%$ yield, on $5.7 \mathrm{mmol}$ scale) after column chromatography on silica gel (PE:EtOAC; 7:3). Rf: 0.30 (PE:EtOAC; 7:3); Mp: $60-61^{\circ} \mathrm{C}$; ${ }^{1} \mathrm{H}$ NMR (300 MHz, $\left.\mathrm{CDCl}_{3}\right): \delta 7.30-7.29(\mathrm{~m}, 1 \mathrm{H}), 7.21(\mathrm{br} \mathrm{m}, 3 \mathrm{H}), 6.98$ $(\mathrm{m}, 1 \mathrm{H}), 4.49(\mathrm{t}, 2 \mathrm{H}, J=7.8 \mathrm{~Hz}), 4.10(\mathrm{t}, 2 \mathrm{H}, J=7.8 \mathrm{~Hz}), 2.30(\mathrm{~s}, 3 \mathrm{H}), 2.01$ (s, 3H); ${ }^{13} \mathrm{C}\left\{{ }^{1} \mathrm{H}\right\}$ NMR (75 MHz, $\left.\mathrm{CDCl}_{3}\right): \delta 172.1,153.1,137.1,134.6,134.6,132.1,130.1,128.9$, 128.1, 125.5, 62.4, 43.3, 19.8, 15.1; IR (neat): v 3018 (w), 2920 (w), 1777 (vs), 1674 (s), 1479 (w), $1382(\mathrm{~m}), 1355(\mathrm{~m}), 1305$ (s), $1190(\mathrm{~s}), 1101(\mathrm{~m}), 1034(\mathrm{~m}), 1009(\mathrm{~m}), 917(\mathrm{~m}), 757$ (s), 742 (s), $677(\mathrm{~m}), 587(\mathrm{w}) \mathrm{cm}^{-1}$.

(E)-3-(3-(4-(tert-butyl)phenyl)-2-methylacryloyl)oxazolidin-2-one ((E)-12):

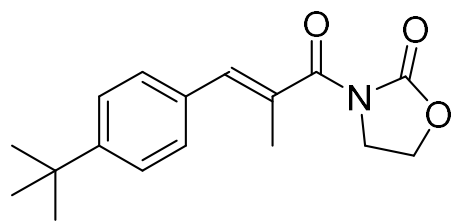

(E)-12

Prepared according to general procedure A. Obtained as a white solid ( $0.69 \mathrm{~g}, 53 \%$ yield, on $4.6 \mathrm{mmol}$ scale) after column chromatography on silica gel (PE:EtOAC; 8:2). Rf: 0.15 (PE:EtOAc ; 8:2); $\mathrm{Mp:} 96-97{ }^{\circ} \mathrm{C} ;{ }^{1} \mathrm{H}$ NMR (300 MHz, $\left.\mathrm{CDCl}_{3}\right): \delta 7.40(\mathrm{~m}, 4 \mathrm{H})$, $6.97(\mathrm{~m}, 1 \mathrm{H}), 4.47(\mathrm{t}, 2 \mathrm{H}, J=7.8 \mathrm{~Hz}), 4.08(\mathrm{t}, 2 \mathrm{H}, J=8.0 \mathrm{~Hz}), 2.20$ 
(s, 3H), $1.33(\mathrm{~s}, 9 \mathrm{H}) ;{ }^{13} \mathrm{C}\left\{{ }^{1} \mathrm{H}\right\}$ NMR (75 MHz, $\left.\mathrm{CDCl}_{3}\right): \delta 172.3,153.1,151.5,136.2,132.7,130.5$, 129.5 (2C), 125.4 (2C), 62.4, 43.5, 34.7, 31.3 (3C), 15.7; IR (neat): v 2957 (m), 2927 (w), 2903 (w), 2867 (w), $1786(\mathrm{~m}), 1765$ (vs), 1682 (vs), $1480(\mathrm{~m}), 1402(\mathrm{~m}), 1363(\mathrm{~m}), 1321(\mathrm{~m}), 1299$ (s), $1249(\mathrm{~s}), 1222(\mathrm{~s}), 1112(\mathrm{~m}), 1039(\mathrm{~m}), 1009(\mathrm{~s}), 891(\mathrm{~m}), 851(\mathrm{w}), 756(\mathrm{~s}), 704(\mathrm{~m}), 655(\mathrm{w})$, 562 (s) $\mathrm{cm}^{-1}$; HRMS (TOF-ESI ${ }^{+}$) m/z: calcd for $[\mathrm{M}+\mathrm{H}]^{+} \mathrm{C}_{17} \mathrm{H}_{22} \mathrm{NO}_{3}: 288.1600$, found: 288.1605 (+ $1.7 \mathrm{ppm})$.

(E)-3-(3-([1,1'-biphenyl]-4-yl)-2-methylacryloyl)oxazolidin-2-one ((E)-13):<smiles>C/C(=C\c1ccc(-c2ccccc2)cc1)C(=O)N1CCOC1=O</smiles>

$(E)-13$

Prepared according to general procedure A. Obtained as a white solid ( $1.18 \mathrm{~g}, 76 \%$ yield) without any purification. [The product was poorly soluble in most of the usual organic solvents]. Rf: 0.29 (PE:EtOAC ; 7:3); Mp: $197-198{ }^{\circ} \mathrm{C} ;{ }^{1} \mathbf{H}$ NMR (300 MHz, CDCl 3 ): $\delta$ $7.70-7.36(\mathrm{~m}, 9 \mathrm{H}), 7.03(\mathrm{~m}, 1 \mathrm{H}), 4.51(\mathrm{t}, 2 \mathrm{H}, J=7.8 \mathrm{~Hz}) 4.12(\mathrm{t}$, $2 \mathrm{H}, J=7.8 \mathrm{~Hz}$ ), 2.25 (s, 3H); ${ }^{13} \mathrm{C}\left\{{ }^{1} \mathrm{H}\right\}$ NMR (75 MHz, CD 3 OD-d4): $\delta$ 169.6, 141.7, 141.0, 138.8, 135.9, 134.3, 131.2 (2C), 130.9, 129.8 (2C), 128.5, 127.7 (2C), 127.7 (2C), 63.5, 44.1, 15.9; IR (neat): v $2921(\mathrm{w}), 2850(\mathrm{w}), 1780(\mathrm{~m}), 1673(\mathrm{~s}), 1387(\mathrm{~m}), 1322(\mathrm{~m}), 1303(\mathrm{~m}), 1201(\mathrm{~s}), 1110$ (m), $1042(\mathrm{~m}), 1014(\mathrm{~m}), 915(\mathrm{~m}), 843(\mathrm{~m}), 761(\mathrm{vs}), 692(\mathrm{~s}), 675(\mathrm{~s}), 499(\mathrm{~s}) \mathrm{cm}^{-1}$.

(E)-3-(3-(4-methoxyphenyl)-2-methylacryloyl)oxazolidin-2-one ((E)-14):<smiles>COc1ccc(/C=C(\C)C(=O)N2CCOC2=O)cc1</smiles>

$(E)-14$

Prepared according to general procedure $\mathbf{A}$. Obtained as a white solid $(1.20 \mathrm{~g}, 74 \%$ yield, on $6.2 \mathrm{mmol}$ scale) after column chromatography on silica gel (PE:EtOAc ; 7:3). Rf: 0.21 (PE:EtOAc ; 7:3); Mp: $110-111^{\circ} \mathrm{C} ;{ }^{1} \mathrm{H}$ NMR (300 $\mathrm{MHz}^{\mathrm{CDCl}}$ ): $\delta 7.40$ (d, $2 \mathrm{H}, J=8.4 \mathrm{~Hz}), 6.98(\mathrm{~m}, 1 \mathrm{H}), 6.92(\mathrm{~d}, 2 \mathrm{H}, J=8.4 \mathrm{~Hz}), 4.47(\mathrm{t}, 2 \mathrm{H}$, $J=7.8 \mathrm{~Hz}), 4.08(\mathrm{t}, 2 \mathrm{H}, J=7.8 \mathrm{~Hz}), 3.84(\mathrm{~s}, 3 \mathrm{H}), 2.19(\mathrm{~s}, 3 \mathrm{H})$; ${ }^{13} \mathrm{C}\left\{{ }^{1} \mathrm{H}\right\}$ NMR (75 MHz, $\left.\mathrm{CDCl}_{3}\right): \delta$ 172.4, 159.6, 153.2, 136.4, 131.4 (2C), 129.0, 128.2, 113.9 (2C), 62.4, 55.3, 43.5, 15.7; IR (neat): v 2965 (w), 2832 (w), 1774 (s), 1671 (vs), 1604 (m), 1510 $(\mathrm{m}), 1374(\mathrm{~m}), 1323(\mathrm{~m}), 1287(\mathrm{~m}), 1255(\mathrm{~s}), 1188(\mathrm{~s}), 1176(\mathrm{vs}), 1034(\mathrm{~s}), 1020(\mathrm{~s}), 760(\mathrm{~s}) \mathrm{cm}^{-}$ 1; HRMS (TOF-ESI ${ }^{+}$) m/z: calcd for $[\mathrm{M}+\mathrm{H}]^{+} \mathrm{C}_{14} \mathrm{H}_{16} \mathrm{NO}_{4}: 262.1079$, found: 262.1078 (- 0.4 ppm).

(E)-3-(2-methyl-3-(naphthalen-2-yl)acryloyl)oxazolidin-2-one ((E)-15):

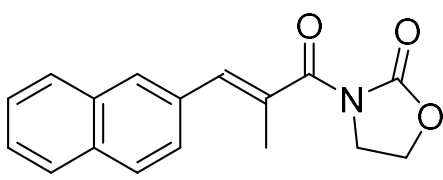

$(E)-15$

Prepared according to general procedure A. Obtained as a white solid $(0.7 \mathrm{~g}, 53 \%$ yield, on $4.7 \mathrm{mmol}$ scale) after column chromatography on silica gel (PE:EtOAc from 7:3 to 6:4). Rf: 0.08 (PE:EtOAC ; 8:2); Mp: $154-155^{\circ} \mathrm{C} ;{ }^{1} \mathbf{H}$ NMR (300 MHz, $\left.\mathrm{CDCl}_{3}\right): \delta$ $7.90-7.82(\mathrm{~m}, 4 \mathrm{H}), 7.55-7.48(\mathrm{~m}, 3 \mathrm{H}), 7.13(\mathrm{~m}, 1 \mathrm{H}), 4.47(\mathrm{t}, 2 \mathrm{H}$, $J=7.8 \mathrm{~Hz}), 4.09(\mathrm{t}, 2 \mathrm{H}, J=7.8 \mathrm{~Hz}), 2.28(\mathrm{~d}, 3 \mathrm{H}, J=0.8 \mathrm{~Hz}) ;{ }^{13} \mathrm{C}\left\{{ }^{1} \mathrm{H}\right\}$ NMR $\left(75 \mathrm{MHz}, \mathrm{CDCl}_{3}\right): 172.3$, 153.2, 136.0, 133.2, 133.1, 133.0, 131.7, 129.2, 128.4, 128.0, 127.7, 127.1, 126.7, 126.4, 62.5, 43.5, 15.8; IR (neat): v 2975 (w), 2916 (w), 1768 (s), 1664 (vs), 1377 (m), 1345 (m), 1291 (m), 1197 (s), 1100 (m), 1040 (m), 827 (m), 750 (s), 482 (m) cm ${ }^{-1}$; HRMS (TOF-ESI ${ }^{+}$) m/z: calcd for $[\mathrm{M}+\mathrm{H}]^{+} \mathrm{C}_{17} \mathrm{H}_{16} \mathrm{NO}_{3}: 282.1130$, found: 282.1131 (+ $0.4 \mathrm{ppm}$ ). 
(E)-3-(3-(4-fluorophenyl)-2-methylacryloyl)oxazolidin-2-one ((E)-16):<smiles>C/C(=C\c1ccc(F)cc1)C(=O)N1CCOC1=O</smiles>

$(E)-16$

Prepared according to general procedure A. Obtained as a beige solid (1.26 g, 91\% yield, on $5.5 \mathrm{mmol}$ scale) without any purification. Rf: 0.26 (PE:EtOAC; 7:3); Mp: $121-122{ }^{\circ} \mathrm{C} ;{ }^{1} \mathbf{H}$ NMR (300 MHz, $\left.\mathrm{CDCl}_{3}\right): \delta 7.42-7.37(\mathrm{~m}, 2 \mathrm{H}), 7.08(\mathrm{~m}, 2 \mathrm{H}), 6.92(\mathrm{~s}, 1 \mathrm{H})$, $4.49(\mathrm{t}, 2 \mathrm{H}, J=7.8 \mathrm{~Hz}), 4.09(\mathrm{t}, 2 \mathrm{H}, J=7.8 \mathrm{~Hz}), 2.16(\mathrm{~s}, 3 \mathrm{H}) ;{ }^{13} \mathrm{C}\left\{{ }^{1} \mathrm{H}\right\}$ NMR (75 MHz, CDCl 3 ): $\delta$ 172.1, 162.4 (d, $J=248.7 \mathrm{~Hz}$ ), 153.1, 134.6, 131.7 (d, J=3.4 Hz), 131.4 $(\mathrm{d}, 2 \mathrm{C}, J=8.2 \mathrm{~Hz}), 131.3(\mathrm{~d}, J=1.6 \mathrm{~Hz}), 115.5(\mathrm{~d}, 2 \mathrm{C}, J=21.6 \mathrm{~Hz}), 62.5,43.4,15.5 ;{ }^{19} \mathbf{F}\left\{{ }^{1} \mathrm{H}\right\} \mathrm{NMR}$ (282 MHz, CDCl $)$ : - 113.2; IR (neat): v 2962 (w), 2927 (w), 1772 (m), 1706 (w), 1669 (s), 1597 (w), 1506 (m), $1474(\mathrm{w}), 1373(\mathrm{~m}), 1319$ (m), 1285 (m), 1227 (vs), 1186 (vs), 1159 (vs), 1097 (s), $1023(\mathrm{~s}), 905(\mathrm{~m}), 836(\mathrm{~s}), 788(\mathrm{~m}), 760(\mathrm{~s}), 637(\mathrm{w}), 531(\mathrm{vs}) \mathrm{cm}^{-1}$.

(E)-3-(3-(4-chlorophenyl)-2-methylacryloyl)oxazolidin-2-one ((E)-17):<smiles>C/C(=C\c1ccc(Cl)cc1)C(=O)N1CCOC1=O</smiles>

$(E)-17$

Prepared according to general procedure $\mathbf{A}$. Obtained as a beige solid (1.20 g, $88 \%$ yield, on $5.1 \mathrm{mmol}$ scale) without any purification. Rf: 0.29 (PE:EtOAC; 7:3); Mp: $123-124{ }^{\circ} \mathrm{C} ;{ }^{1} \mathbf{H}$ NMR (300 MHz, $\left.\mathrm{CDCl}_{3}\right): \delta 7.35(\mathrm{~m}, 4 \mathrm{H}), 6.88(\mathrm{~m}, 1 \mathrm{H}), 4.49(\mathrm{t}, 2 \mathrm{H}, J=7.8$ $\mathrm{Hz}), 4.09(\mathrm{t}, 2 \mathrm{H}, J=7.8 \mathrm{~Hz}), 2.16(\mathrm{~s}, 3 \mathrm{H}) ;{ }^{13} \mathrm{C}\left\{{ }^{1} \mathrm{H}\right\}$ NMR $(75 \mathrm{MHz}$, $\left.\mathrm{CDCl}_{3}\right): \delta 171.9,153.1,134.2,134.0,134.0,132.2,130.8$ (2C), 128.7 (2C), 62.5, 43.3, 15.6; IR (neat): v 2919 (w), 2856 (w), 1778 (s), 1676 (m), 1489 (m), 1357 (s), 1318 (m), 1188 (vs), 1091

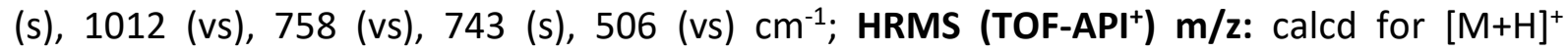
$\mathrm{C}_{13} \mathrm{H}_{13} \mathrm{NClO}_{3}: 266.0584$, found: 266.0588 (+ $1.5 \mathrm{ppm}$ ).

(E)-3-(3-(4-bromophenyl)-2-methylacryloyl)oxazolidin-2-one ((E)-18):<smiles>C/C(=C\c1ccc(Br)cc1)C(=O)N1CCOC1=O</smiles>

$(E)-18$

Prepared according to general procedure A. Obtained as a beige solid (0.96 g, 74\% yield, on $4.1 \mathrm{mmol}$ scale) without any purification. $\mathbf{R f :} 0.34$ (PE:EtOAC; 6:4); $\mathbf{M p : ~} 107-108{ }^{\circ} \mathrm{C} ;{ }^{1} \mathbf{H}$ NMR (300 MHz, $\left.\mathrm{CDCl}_{3}\right): \delta 7.52(\mathrm{~d}, 2 \mathrm{H}, J=8.2 \mathrm{~Hz}), 7.29(\mathrm{~d}, 2 \mathrm{H}, J=8.2 \mathrm{~Hz})$, $6.86(\mathrm{~m}, 1 \mathrm{H}), 4.49(\mathrm{t}, 2 \mathrm{H}, J=7.7 \mathrm{~Hz}), 4.09(\mathrm{t}, 2 \mathrm{H}, J=7.8 \mathrm{~Hz}), 2.15$ $(\mathrm{s}, 3 \mathrm{H}) ;{ }^{13} \mathrm{C}\left\{{ }^{1} \mathrm{H}\right\}$ NMR $\left(75 \mathrm{MHz}, \mathrm{CDCl}_{3}\right): \delta$ 171.9, 153.1, 134.5, 134.2, 132.3, 131.6 (2C), 131.1 (2C), 122.3, 62.5, 43.3, 15.6; IR (neat): v 3338 (br), 2963 (w), 2916 (w), $2862(\mathrm{w}), 1773$ (s), 1675 (s), $1527(\mathrm{w}), 1482(\mathrm{~m}), 1449(\mathrm{w}), 1377(\mathrm{~m}), 1357(\mathrm{~m}), 1317$ (s), 1297 (s), $1239(\mathrm{~m}), 1219(\mathrm{~m}), 1193(\mathrm{vs}), 1093(\mathrm{~m}), 1074(\mathrm{~s}), 1008(\mathrm{vs}), 920(\mathrm{~m}), 812(\mathrm{~m}), 761(\mathrm{~s}), 603$ (w), $516(\mathrm{~m}), 504(\mathrm{~s}) \mathrm{cm}^{-1}$.

(E)-3-(3-(4-iodophenyl)-2-methylacryloyl)oxazolidin-2-one ((E)-19):<smiles>C/C(=C\c1ccc(I)cc1)C(=O)N1CCOC1=O</smiles>

$(E)-19$

Prepared according to general procedure A. Obtained as a beige solid $(0.88 \mathrm{~g}, 59 \%$ yield, on $4.2 \mathrm{mmol}$ scale) after column chromatography on silica gel (PE:EtOAC ; 7:3). Rf: 0.24 (PE/EtOAC ; 7:3); Mp: $128-129{ }^{\circ} \mathrm{C} ;{ }^{1} \mathrm{H}$ NMR (300 MHz, $\mathrm{CDCl}_{3}$ ): $\delta 7.72$ (d, $2 \mathrm{H}, \mathrm{J}=$ $8.0 \mathrm{~Hz}), 7.14(\mathrm{~d}, 2 \mathrm{H}, J=8.0 \mathrm{~Hz}), 6.84(\mathrm{~m}, 1 \mathrm{H}), 4.48(\mathrm{t}, 2 \mathrm{H}, J=7.8 \mathrm{~Hz})$, $4.08(\mathrm{t}, 2 \mathrm{H}, J=7.8 \mathrm{~Hz}), 2.15(\mathrm{~s}, 3 \mathrm{H}) ;{ }^{13} \mathrm{C}\left\{{ }^{1} \mathrm{H}\right\}$ NMR (75 MHz, CDCl 3 ): $\delta 171.9,153.1,137.6(2 \mathrm{C})$, 135.0, 134.4, 132.4, 131.2 (2C), 94.1, 62.5, 43.3, 15.6; IR (neat): v 2969 (w), 2914 (w), 1767 (s), 
1675 (vs), 1478 (m), 1446 (w), 1377 (m), 1358 (m), 1314 (s), 1295 (s), 1219 (m), 1191 (vs), 1094 $(\mathrm{m}), 1037(\mathrm{~m}), 1004(\mathrm{~m}), 919(\mathrm{w}), 808(\mathrm{w}), 761(\mathrm{~s}), 597(\mathrm{w}), 512(\mathrm{~m}) \mathrm{cm}^{-1}$.

(E)-3-(2-methyl-3-(4-(trifluoromethyl)phenyl)acryloyl)oxazolidin-2-one ((E)-20):<smiles>C/C(=C\c1ccc(C(F)(F)F)cc1)C(=O)N1CCOC1=O</smiles>

$(E)-20$

Prepared according to general procedure A. Obtained as a white solid $(0.86 \mathrm{~g}, 67 \%$ yield, on $4.3 \mathrm{mmol}$ scale) after column chromatography on silica gel (PE:EtOAc ; 7:3). Rf: 0.15 (PE:EtOAC ; 8:2); $\mathrm{Mp:} 108-109{ }^{\circ} \mathrm{C} ;{ }^{1} \mathbf{H}$ NMR (300 $\left.\mathbf{M H z} \mathrm{CDCl}_{3}\right): \delta 7.58(\mathrm{~m}$, $4 \mathrm{H}), 6.91(\mathrm{~m}, 1 \mathrm{H}), 4.49(\mathrm{t}, 2 \mathrm{H}, J=7.8 \mathrm{~Hz}), 4.10(\mathrm{t}, 2 \mathrm{H}, J=7.8 \mathrm{~Hz})$, $2.17(\mathrm{~s}, 3 \mathrm{H}) ;{ }^{13} \mathrm{C}\left\{{ }^{1} \mathrm{H}\right\}$ NMR (75 MHz, $\left.\mathrm{CDCl}_{3}\right): \delta 171.7,153.0,139.1$, 133.9, 133.3, 130.1, 129.6 (3C), 125.4 (q, $J=3.8 \mathrm{~Hz}$ ), 124.1 (q, $J=272.0 \mathrm{~Hz}$ ), 62.5, 43.2, 15.6; ${ }^{19} \mathrm{~F}\left\{{ }^{1} \mathrm{H}\right\}$ NMR (282 MHz, CDCl 3 ): - 63.2; IR (neat): v $2993(\mathrm{w}), 2923(\mathrm{w}), 1789(\mathrm{~m}), 1772(\mathrm{w})$, $1676(\mathrm{~m}), 1615(\mathrm{w}), 1476(\mathrm{w}), 1386(\mathrm{w}), 1324(\mathrm{~s}), 1311(\mathrm{~m}), 1221(\mathrm{~m}), 1201(\mathrm{~m}), 1163(\mathrm{~m}), 1111$ (vs), 1068 (s), $1013(\mathrm{~m}), 918(\mathrm{~m}), 834(\mathrm{~m}), 758(\mathrm{~m}), 697(\mathrm{w}), 595(\mathrm{w}), 517(\mathrm{w}) \mathrm{cm}^{-1}$; HRMS (TOF$\left.\mathrm{API}^{+}\right) \mathrm{m} / \mathrm{z}$ : calcd for $[\mathrm{M}+\mathrm{H}]^{+} \mathrm{C}_{14} \mathrm{H}_{13} \mathrm{NF}_{3} \mathrm{O}_{3}: 300.0848$, found: 300.0845 (- $1.0 \mathrm{ppm}$ ).

(E)-3-(2-methyl-3-(4-(4,4,5,5-tetramethyl-1,3,2-dioxaborolan-2yl)phenyl)acryloyl)oxazolidin-2-one ((E)-21):

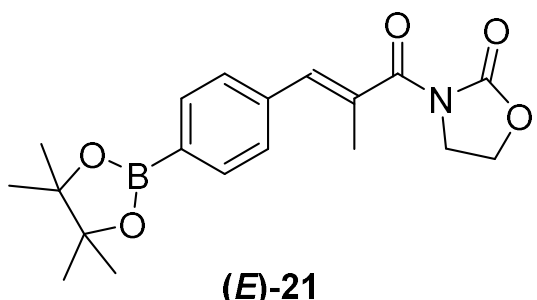

$(E)-21$

Prepared according to general procedure A. Obtained as a white solid (0.95 g, 64\% yield, on $4.2 \mathrm{mmol}$ scale) after column chromatography on silica gel (PE:EtOAC ; 7:3). Rf: 0.20 (PE:EtOAC ; 7:3); Mp: $111-112{ }^{\circ} \mathrm{C} ;{ }^{1} \mathbf{H}$ NMR (300 MHz, $\left.\mathrm{CDCl}_{3}\right): \delta 7.81(\mathrm{~d}, 2 \mathrm{H}, J=7.7 \mathrm{~Hz}), 7.40(\mathrm{~d}, 2 \mathrm{H}, J=7.7 \mathrm{~Hz}), 6.94$ $(\mathrm{m}, 1 \mathrm{H}), 4.44(\mathrm{t}, 2 \mathrm{H}, J=7.8 \mathrm{~Hz}), 4.05(\mathrm{t}, 2 \mathrm{H}, J=7.8 \mathrm{~Hz}), 2.16$ (s, 3H), $1.34(\mathrm{~s}, 12 \mathrm{H}) ;{ }^{13} \mathrm{C}\left\{{ }^{1} \mathrm{H}\right\}$ NMR (75 MHz, $\left.\mathrm{CDCl}_{3}\right): \delta 172.1$, $153.1,138.3,135.6,134.8$ (2C), 132.2, 128.8 (2C), 84.0 (2C), 62.5, 43.4, 24.9 (4C), 15.6. The carbon bearing boron was not observed; ${ }^{11} \mathrm{~B}$ NMR (96 MHz, CDCl 3$): \delta 23.5$; IR (neat): v 2982 (w), 2933 (w), 1789 (s), 1687 (s), 1515 (w), 1484 (w), 1390 (m), 1351 (vs), 1314 (vs), 1270 (m), 1209 (s), 1142 (vs), $1108(\mathrm{~m}), 1088$ (s), $1046(\mathrm{~m}), 1014(\mathrm{~s}), 965(\mathrm{w}), 877(\mathrm{~m}), 858(\mathrm{~s}), 756(\mathrm{~m})$, 690 (m), 656 (vs), 516 (w) cm ${ }^{-1}$; HRMS (TOF-ESI ${ }^{+}$) m/z: calcd for $[\mathrm{M}+\mathrm{H}]^{+} \mathrm{C}_{19} \mathrm{H}_{25} \mathrm{BNO}_{5}: 358.1826$, found: $358.1837(+3.1 \mathrm{ppm})$.

(E)-3-(2-methyl-3-(4-(methylsulfonyl)phenyl)acryloyl)oxazolidin-2-one ((E)-22):<smiles>COS(=O)(=O)c1ccc(/C=C(\C)C(=O)N2CCOC2=O)cc1</smiles>

(E)-22

Prepared according to general procedure A. Obtained as a white solid ( $1.11 \mathrm{~g}$, 78\% yield, on $4.6 \mathrm{mmol}$ scale) without any purification. Rf: 0.04 (PE:EtOAC ; 7:3); Mp: $152-153{ }^{\circ} \mathrm{C} ;{ }^{1} \mathbf{H}$ NMR (300 MHz, $\left.\mathrm{CDCl}_{3}\right): \delta 7.96(\mathrm{~d}, 2 \mathrm{H}, J=8.2 \mathrm{~Hz}), 7.58(\mathrm{~d}, 2 \mathrm{H}$, $J=8.1 \mathrm{~Hz}), 6.89(\mathrm{~m}, 1 \mathrm{H}), 4.51(\mathrm{t}, 2 \mathrm{H}, J=7.8 \mathrm{~Hz}), 4.11(\mathrm{t}, 2 \mathrm{H}, J=$ $7.8 \mathrm{~Hz}), 3.08(\mathrm{~s}, 3 \mathrm{H}), 2.17(\mathrm{~s}, 3 \mathrm{H}) ;{ }^{13} \mathrm{C}\left\{{ }^{1} \mathrm{H}\right\}$ NMR (75 MHz, $\left.\mathrm{CDCl}_{3}\right): \delta 171.4,153.0,141.2,139.6,135.1,132.3,130.1$ (2C), 127.5 (2C), 62.6, 44.6, 43.1, 15.7; IR (neat): v 2995 (w), 2919 (w), 1781 (m), 1672 (m), 1319 (m), 1295 (s), 1192 (m), 1146 (vs), $1104(\mathrm{~m}), 756(\mathrm{~m}), 767$ (s), $619(\mathrm{~m}), 535$ (vs) cm $\mathrm{cm}^{-1}$; HRMS (TOF-API ${ }^{+}$) m/z: calcd for [M+H] ${ }^{+}$ $\mathrm{C}_{14} \mathrm{H}_{16} \mathrm{NO}_{5} \mathrm{~S}: 310.0749$, found: 310.0762 (+ $\left.4.2 \mathrm{ppm}\right)$. 
(E)-3-(3-(4-acetylphenyl)-2-methylacryloyl)oxazolidin-2-one ((E)-23):<smiles>CC(=O)c1ccc(/C=C(\C)C(=O)N2CCOC2=O)cc1</smiles>

Prepared according to general procedure A. Obtained as a beige solid ( $0.64 \mathrm{~g}, 44 \%$ yield, on $5.4 \mathrm{mmol}$ scale) after column chromatography on silica gel (PE:EtOAc; 70:30). Rf: 0.11 (PE:EtOAC ; 7:3); Mp: $103-104{ }^{\circ} \mathrm{C} ;{ }^{1} \mathrm{H}$ NMR (300 MHz, $\left.\mathrm{CDCl}_{3}\right): \delta$ $7.97(\mathrm{~d}, 2 \mathrm{H}, J=8.3 \mathrm{~Hz}), 7.49(\mathrm{~d}, 2 \mathrm{H}, J=8.3 \mathrm{~Hz}), 6.92(\mathrm{~m}, 1 \mathrm{H}), 4.50$

$(\mathrm{t}, 2 \mathrm{H}, J=7.9 \mathrm{~Hz}), 4.10(\mathrm{t}, 2 \mathrm{H}, J=7.9 \mathrm{~Hz}), 2.62(\mathrm{~s}, 3 \mathrm{H}), 2.19(\mathrm{~s}, 3 \mathrm{H}) ;{ }^{13} \mathrm{C}\left\{{ }^{1} \mathrm{H}\right\} \mathbf{N M R}(75 \mathrm{MHz}$, $\left.\mathrm{CDCl}_{3}\right): \delta$ 197.8, 171.7, 153.0, 140.3, 136.2, 133.8, 133.7, 129.6 (2C), 128.4 (2C), 62.5, 43.2, 26.7, 15.7; IR (neat): v 3338 (w), 2978 (w), 2918 (w), 2873 (w), 1784 (s), 1673 (vs), 1598 (m), $1473(w), 1415$ (w), 1382 (w), 1361 (w), 1320 (s), 1268 (s), 1203 (vs), $1111(\mathrm{~s}), 1041$ (m), 1012 (m), $955(\mathrm{w}), 916(\mathrm{~m}), 829(\mathrm{~m}), 758(\mathrm{~s}), 661(\mathrm{~s}), 593(\mathrm{~m}) \mathrm{cm}^{-1}$; HRMS (TOF-ESI ${ }^{+}$) m/z: calcd for $[\mathrm{M}+\mathrm{H}]^{+} \mathrm{C}_{15} \mathrm{H}_{16} \mathrm{NO}_{4}: 274.1079$, found: 274.1092 (+ $\left.4.7 \mathrm{ppm}\right)$.

\section{(E)-3-(3-(furan-2-yl)-2-methylacryloyl)oxazolidin-2-one ((E)-24):}<smiles>C/C(=C\c1ccco1)C(=O)N1CCOC1=O</smiles>

$(E)-24$

Prepared according to general procedure A. Obtained as a beige solid (1.29 g, 89\% yield, on $6.6 \mathrm{mmol}$ scale) after column chromatography on silica gel (PE:EtOAc; 1:1). Rf: 0.34 (PE:EtOAc; 6:4); Mp: $115-116$ ${ }^{\circ} \mathrm{C} ;{ }^{1} \mathrm{H}$ NMR (300 MHz, $\left.\mathrm{CDCl}_{3}\right): \delta 7.52(\mathrm{~m}, 1 \mathrm{H}), 6.89(\mathrm{~m}, 1 \mathrm{H}), 6.59(\mathrm{~d}, 1 \mathrm{H}$, $J=3.1 \mathrm{~Hz}), 6.50(\mathrm{~m}, 1 \mathrm{H}), 4.47(\mathrm{t}, 2 \mathrm{H}, J=7.8 \mathrm{~Hz}), 4.07(\mathrm{t}, 2 \mathrm{H}, J=7.8 \mathrm{~Hz})$, $2.26(\mathrm{~s}, 3 \mathrm{H}) ;{ }^{13} \mathrm{C}\left\{{ }^{1} \mathrm{H}\right\} \mathrm{NMR}\left(75 \mathrm{MHz}, \mathrm{CDCl}_{3}\right): \delta 171.7,153.2,151.6,144.0$, 127.8, 124.9, 114.5, 112.1, 62.5, 43.6, 16.0; IR (neat): v 3148 (w), 2921 (w), 1772 (vs), 1668 (vs), $1475(\mathrm{w}), 1375(\mathrm{~m}), 1358(\mathrm{~s}), 1309(\mathrm{~s}), 1239(\mathrm{~m}), 1210(\mathrm{~m}), 1186(\mathrm{vs}), 1104(\mathrm{~m}), 1026(\mathrm{~s})$, $946(\mathrm{~m}), 898(\mathrm{w}), 748(\mathrm{~s}), 718(\mathrm{~m}), 696(\mathrm{~m}), 593(\mathrm{~m}) \mathrm{cm}^{-1}$.

(E)-3-(2-methyl-3-(thiophen-2-yl)acryloyl)oxazolidin-2-one ((E)-25):<smiles>C/C(=C\c1cccs1)C(=O)N1CCOC1=O</smiles>

$(E)-25$

Prepared according to general procedure A. Obtained as a beige solid ( $0.85 \mathrm{~g}, 61 \%$ yield, on $5.9 \mathrm{mmol}$ scale) after column chromatography on silica gel (PE:EtOAC; 7:3). Rf: 0.22 (PE:EtOAc ; 7:3); Mp: $117-118$ ${ }^{\circ} \mathrm{C} ;{ }^{1} \mathrm{H}$ NMR (300 MHz, $\left.\mathrm{CDCl}_{3}\right): \delta 7.50(\mathrm{~d}, 1 \mathrm{H}, J=4.7 \mathrm{~Hz}), 7.29(\mathrm{~m}, 2 \mathrm{H})$, $7.11(\mathrm{~m}, 1 \mathrm{H}), 4.48(\mathrm{t}, 2 \mathrm{H}, J=7.8 \mathrm{~Hz}), 4.08(\mathrm{t}, 2 \mathrm{H}, J=7.9 \mathrm{~Hz}), 2.26(\mathrm{~s}, 3 \mathrm{H})$; ${ }^{13} \mathrm{C}\left\{{ }^{1} \mathrm{H}\right\}$ NMR (75 MHz, $\left.\mathrm{CDCl}_{3}\right): \delta 171.8,153.2,138.9,131.5,130.7,129.2,127.7,127.4,62.5$, 43.7, 16.3; IR (neat): v 3102 (w), 2981 (br), 2917 (w), 1777 (m), 1763 (m), 1670 (s), 1384 (m), 1298 (vs), 1207 (vs), 1016 (m), 910 (m), 760 (m), 718 (vs), 701 (vs), 578 (m) cm-1; HRMS (TOF$\mathrm{API}^{+}$) m/z: calcd for $[\mathrm{M}+\mathrm{H}]^{+} \mathrm{C}_{11} \mathrm{H}_{12} \mathrm{NO}_{3} \mathrm{~S}: 238.0538$, found: 238.0541 (+ $1.3 \mathrm{ppm}$ ).

\section{3-((E)-3-(4-(cinnamyloxy)phenyl)-2-methylacryloyl)oxazolidin-2-one ((E)-26):}

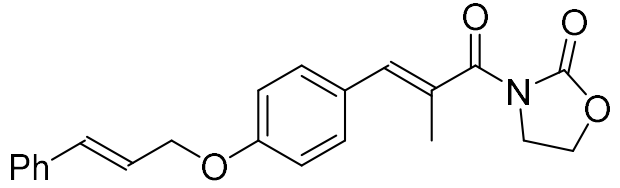

$(E)-26$

Prepared according to general procedure A. Obtained as a beige solid $(0.21 \mathrm{~g}, 19 \%$ yield, on $3.1 \mathrm{mmol}$ scale) after column chromatography on silica gel (PE:EtOAc ; 7:3). Rf: 0.23 (PE:EtOAC ; 7:3); Mp: $144-145{ }^{\circ} \mathrm{C} ;{ }^{1} \mathbf{H}$ NMR (300 MHz, $\left.\mathrm{CDCl}_{3}\right): \delta 7.44-7.29(\mathrm{~m}, 7 \mathrm{H}), 6.99-$ $6.96(\mathrm{~m}, 3 \mathrm{H}), 6.75(\mathrm{~d}, 1 \mathrm{H}, J=16.0 \mathrm{~Hz}), 6.42(\mathrm{dt}, 1 \mathrm{H}, J=$ 16.0 \& $5.8 \mathrm{~Hz}), 4.73(\mathrm{~d}, 2 \mathrm{H}, J=5.5 \mathrm{~Hz}), 4.46(\mathrm{t}, 2 \mathrm{H}, J=7.8 \mathrm{~Hz}), 4.07(\mathrm{t}, 2 \mathrm{H}, J=7.8 \mathrm{~Hz}), 2.20(\mathrm{~s}$, 
$3 \mathrm{H}) ;{ }^{13} \mathrm{C}\left\{{ }^{1} \mathrm{H}\right\}$ NMR (75 MHz, $\left.\mathrm{CDCl}_{3}\right): \delta$ 172.5, 158.8, 153.2, 136.6, 136.4, 133.4, 131.5 (2C), 129.2, 128.7 (2C), 128.5, 128.1, 126.7 (2C), 124.2, 114.8 (2C), 68.8, 62.4, 43.6, 15.8; IR (neat): v 2993 (w), 2924 (w), 2867 (w), 1781 (vs), 1674 (vs), 1629 (w), 1602 (m), 1509 (m), 1476 (w), 1385 (s), 1319 (s), 1307 (s), 1244 (vs), 1223 (vs), 1205 (vs), 1189 (vs), 1112 (m), 1042 (m), 1006 (vs), $962(\mathrm{~s}), 942(\mathrm{w}), 914(\mathrm{w}), 834(\mathrm{~m}), 762(\mathrm{vs}), 748(\mathrm{~m}), 696(\mathrm{~m}), 652(\mathrm{w}), 569(\mathrm{~m}), 535(\mathrm{~m})$ $\mathrm{cm}^{-1}$; HRMS (TOF-ESI ${ }^{+}$) m/z: calcd for $[\mathrm{M}+\mathrm{H}]^{+} \mathrm{C}_{22} \mathrm{H}_{22} \mathrm{NO}_{4}: 364.1549$, found: 364.1551 (+ 0.5 ppm).

(E)-3-(3-mesityl-2-methylacryloyl)oxazolidin-2-one ((E)-27):

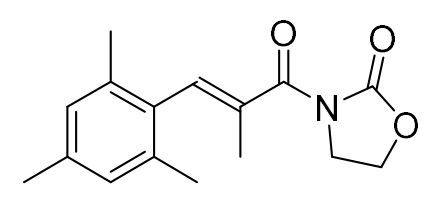

(E)-27

Prepared according to general procedure B. Obtained as a white solid $(0.97 \mathrm{~g}, 73 \%$ yield, on $4.9 \mathrm{mmol}$ scale) after column chromatography on silica gel (PE:EtOAc ; 7:3). Rf: 0.48 (PE:EtOAC ; 6:4); $\mathrm{Mp:} 90-91{ }^{\circ} \mathrm{C} ;{ }^{1} \mathrm{H}$ NMR (300 MHz, $\left.\mathrm{CDCl}_{3}\right): \delta 6.88(\mathrm{~m}, 3 \mathrm{H}), 4.48$ $(\mathrm{t}, 2 \mathrm{H}, J=7.7 \mathrm{~Hz}), 4.10(\mathrm{t}, 2 \mathrm{H}, J=7.8 \mathrm{~Hz}), 2.29(\mathrm{~s}, 3 \mathrm{H}), 2.22(\mathrm{~s}, 6 \mathrm{H})$, $1.76(\mathrm{~s}, 3 \mathrm{H}) ;{ }^{13} \mathrm{C}\left\{{ }^{1} \mathrm{H}\right\}$ NMR (75 MHz, $\left.\mathrm{CDCl}_{3}\right): \delta 171.5,153.1,137.1,136.1,135.5,133.7,131.4$, 128.1 (3C), 62.4, 43.4, 21.0, 19.9 (2C), 14.7; IR (neat): v 2963 (w), 2920 (w), 2856 (w), 1784 (vs), 1679 (vs), $1478(\mathrm{w}), 1381(\mathrm{~m}), 1344(\mathrm{~m}), 1300(\mathrm{~s}), 1240(\mathrm{w}), 1217(\mathrm{~m}), 1197(\mathrm{~s}), 1099(\mathrm{~m})$, $1033(w), 1004(s), 928(w), 856(m), 758(s), 731(w), 665(m), 560(w) \mathrm{cm}^{-1}$.

(E)-3-(2-methyloct-2-enoyl)oxazolidin-2-one ((E)-28):

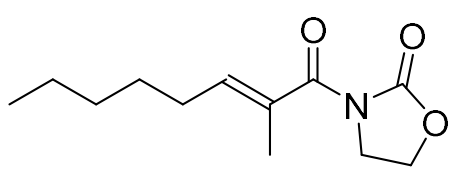

$(E)-28$

Prepared according to general procedure A. Obtained as a colorless oil ( $1.00 \mathrm{~g}, 69 \%$ yield, on $6.4 \mathrm{mmol}$ scale) after column chromatography on silica gel (PE:EtOAc ; 8:2). Rf: 0.35 (PE:EtOAC ; 7:3); ${ }^{1} \mathrm{H}$ NMR (300 MHz, $\left.\mathrm{CDCl}_{3}\right): \delta 6.10(\mathrm{t}, 1 \mathrm{H}, \mathrm{J}=7.4 \mathrm{~Hz}), 4.42$ $(\mathrm{t}, 2 \mathrm{H}, J=7.8 \mathrm{~Hz}), 4.02(\mathrm{t}, 2 \mathrm{H}, J=7.8 \mathrm{~Hz}), 2.18(\mathrm{~m}, 2 \mathrm{H}), 1.90(\mathrm{~s}$, $3 \mathrm{H}), 1.49-1.40(\mathrm{~m}, 2 \mathrm{H}), 1.33-1.31(\mathrm{~m}, 4 \mathrm{H}), 0.89(\mathrm{t}, 3 \mathrm{H}, J=6.1 \mathrm{~Hz}) ;{ }^{13} \mathrm{C}\left\{{ }^{1} \mathrm{H}\right\}$ NMR $(75 \mathrm{MHz}$, $\mathrm{CDCl}_{3}$ ): $\delta$ 172.0, 153.2, 140.3, 129.9, 62.3, 43.5, 31.5, 28.4, 28.1, 22.5, 14.1, 13.6; IR (neat): $\mathrm{V}$ $2957(w), 2929$ (w), 2861 (w), $1780(v s), 1678(s), 1478(w), 1383(s), 1360(\mathrm{~m}), 1308$ (s), 1194 (vs), $1099(\mathrm{~m}), 1037(\mathrm{~m}), 1009(\mathrm{~m}), 759(\mathrm{~m}), 670(\mathrm{w}) \mathrm{cm}^{-1}$.

\subsection{Characterization of $\beta$-substituted esters}

ethyl (E)-3-phenylbut-2-enoate ((E)-30):

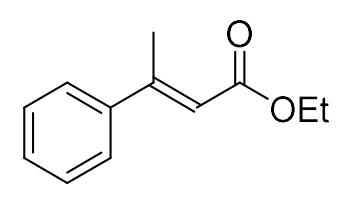

$(E)-30$

Prepared according to general procedure B. Obtained as a slightly yellow oil (1.71 g, 56\% yield) after column chromatography on silica gel (PE:EtOAc from 100:0 to 98:2). Rf: 0.44 (PE:EtOAC; 95:5); ${ }^{1} \mathbf{H}$ NMR (300 $\left.\mathrm{MHz} \mathrm{CDCl}_{3}\right): \delta 7.49-7.47(\mathrm{~m}, 2 \mathrm{H}), 7.38-7.37(\mathrm{~m}, 3 \mathrm{H}), 6.14(\mathrm{~m}, 1 \mathrm{H}), 4.22$ (q, $2 \mathrm{H}, J=7.1 \mathrm{~Hz}), 2.59(\mathrm{~s}, 3 \mathrm{H}), 1.33(\mathrm{t}, 3 \mathrm{H}, J=7.1 \mathrm{~Hz}) ;{ }^{13} \mathrm{C}\left\{{ }^{1} \mathrm{H}\right\}$ NMR $(75$ $\mathrm{MHz}_{2} \mathrm{CDCl}_{3}$ ): $\delta$ 166.9, 155.6, 142.3, 129.1, 128.6 (2C), 126.4 (2C), 117.3, 59.9, 18.0, 14.4; IR (neat): v 2981 (w, br), 1709 (s), 1626 (m), 1271 (m), 1154 (vs), 1041 (m), $764(\mathrm{~m}), 692(\mathrm{~m}) \mathrm{cm}^{-1}$; HRMS (EI+) m/z: calcd for [M] ${ }^{+} \mathrm{C}_{12} \mathrm{H}_{14} \mathrm{O}_{2}:$ 190.0994, found: 190.0994 (+ $0.3 \mathrm{ppm})$. 
ethyl (E)-3-phenylpent-2-enoate ((E)-32):

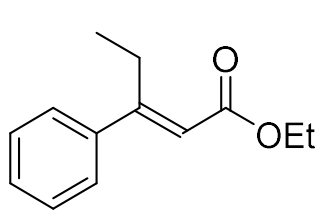

$(E)-32$

Prepared according to general procedure B. Obtained as a slightly yellow oil (1.46 g, 45\% yield) after column chromatography on silica gel (PE:EtOAc from 100:0 to 97:3). Rf: 0.51 (PE:EtOAC ; 95:5); ${ }^{1} \mathbf{H}$ NMR (300 $\left.\mathrm{MHz} \mathrm{CDCl}_{3}\right): \delta 7.45-7.36(\mathrm{~m}, 5 \mathrm{H}), 6.02(\mathrm{~m}, 1 \mathrm{H}), 4.22(\mathrm{q}, 2 \mathrm{H}, J=7.1 \mathrm{~Hz})$, $3.11(\mathrm{q}, 2 \mathrm{H}, J=7.5 \mathrm{~Hz}), 1.32(\mathrm{t}, 3 \mathrm{H}, J=7.1 \mathrm{~Hz}), 1.08(\mathrm{t}, 3 \mathrm{H}, J=7.5 \mathrm{~Hz})$; ${ }^{13} \mathrm{C}\left\{{ }^{1} \mathrm{H}\right\}$ NMR (75 MHz, $\left.\mathrm{CDCl}_{3}\right): \delta 166.5,162.1,141.2,128.9,128.6(2 \mathrm{C})$, 126.8 (2C), 116.9, 59.9, 24.4, 14.4, 13.6; IR (neat): v 3058 (w), 2976 (w), 2937 (w), 2976 (w), $1710(\mathrm{~s}), 1623(\mathrm{~m}), 1576(\mathrm{w}), 1446(\mathrm{w}), 1367(\mathrm{w}), 1349(\mathrm{w}), 1289(\mathrm{~m}), 1238(\mathrm{w}), 1154$ (vs), 1042 (m), $874(\mathrm{~m}), 770$ (s), 695 (s) cm ${ }^{-1}$; HRMS (TOF-API ${ }^{+}$) m/z: calcd for $[\mathrm{M}+\mathrm{H}]^{+} \mathrm{C}_{13} \mathrm{H}_{17} \mathrm{O}_{2}:$ : 205.1229, found: 205.1225 (- $2.0 \mathrm{ppm})$.

\section{ethyl (E)-3-(p-tolyl)but-2-enoate ((E)-34):}

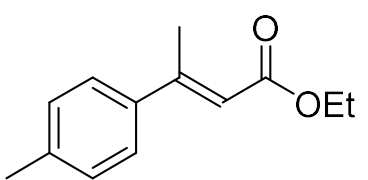

$(E)-34$

Prepared according to general procedure B. Obtained as a yellow oil (1.53 g, 47\% yield) after column chromatography on silica gel (PE:EtOAc from 100:0 to 98:2). Rf: 0.40 (PE:EtOAC ; 95:5); ${ }^{1} \mathbf{H}$ NMR (300 $\left.\mathrm{MHz}^{\mathrm{CDCl}} \mathrm{CD}_{3}\right): \delta 7.40(\mathrm{~d}, 2 \mathrm{H}, J=7.9 \mathrm{~Hz}), 7.19(\mathrm{~d}, 2 \mathrm{H}, J=7.9 \mathrm{~Hz}), 6.13(\mathrm{~m}$, $1 \mathrm{H}), 4.22(\mathrm{q}, 2 \mathrm{H}, J=7.1 \mathrm{~Hz}), 2.57(\mathrm{~s}, 3 \mathrm{H}), 2.37(\mathrm{~s}, 3 \mathrm{H}), 1.32(\mathrm{t}, 3 \mathrm{H}, J=7.1$ $\mathrm{Hz}) ;{ }^{13} \mathrm{C}\left\{{ }^{1} \mathrm{H}\right\}$ NMR $\left(75 \mathrm{MHz} \mathrm{CDCl}_{3}\right): \delta$ 167.0, 155.4, 139.3, 139.2, $129.2(2 \mathrm{C}), 126.3(2 \mathrm{C}), 116.3$, 59.8, 21.2, 17.8, 14.4; IR (neat): v 3034 (w), 2980 (w), 1908 (w), 1710 (s), 1626 (m), 1570 (w), 1513 (w), 1443 (w), 1366 (w), 1341 (w), 1274 (m), 1154 (vs), 1042 (m), 873 (w), 814 (s) cm $\mathrm{cm}^{-1}$ HRMS (TOF-API ${ }^{+}$) m/z: calcd for $[\mathrm{M}+\mathrm{H}]^{+} \mathrm{C}_{13} \mathrm{H}_{17} \mathrm{O}_{2}: 205.1229$, found: 205.1226 (- $1.5 \mathrm{ppm}$ ).

ethyl (E)-3-(m-tolyl)but-2-enoate ((E)-36):<smiles>CCOC(=O)/C=C(\C)c1cccc(C)c1</smiles>

$(E)-36$

Prepared according to general procedure B. Obtained as a yellow oil (2.01 g, 62\% yield) after column chromatography on silica gel (PE:EtOAc from 100:0 to 99:1). Rf: 0.36 (PE:EtOAC ; 95:5); ${ }^{1}$ H NMR (300 $\left.\mathrm{MHz} \mathrm{CDCl}_{3}\right): \delta 7.33-7.29(\mathrm{~m}, 3 \mathrm{H}), 7.22-7.28(\mathrm{~m}, 1 \mathrm{H}), 6.17(\mathrm{~m}, 1 \mathrm{H})$, $4.26(\mathrm{q}, 2 \mathrm{H}, \mathrm{J}=7.1 \mathrm{~Hz}), 2.61(\mathrm{~s}, 3 \mathrm{H}), 2.41(\mathrm{~s}, 3 \mathrm{H}), 1.36(\mathrm{t}, 3 \mathrm{H}, \mathrm{J}=7.1 \mathrm{~Hz})$; ${ }^{13} \mathrm{C}\left\{{ }^{1} \mathrm{H}\right\}$ NMR (75 MHz, $\left.\mathrm{CDCl}_{3}\right): \delta 167.0,155.8,142.4,138.2,129.8$, 128.5, 127.1, 123.5, 117.1, 59.9, 21.5, 18.1, 14.4; IR (neat): v 2980 (w), 1709 (s), 1626 (m), 1444 (w), 1366 (w), 1340 (m), 1285 (m), 1199 (m), 1153 (vs), 1095 (w), 1042 (m), 870 (m), 785 (s), $695(\mathrm{~m}) \mathrm{cm}^{-1}$; HRMS (TOF-API ${ }^{+}$) m/z: calcd for $[\mathrm{M}+\mathrm{H}]^{+} \mathrm{C}_{13} \mathrm{H}_{17} \mathrm{O}_{2}:$ 205.1229, found: $205.1222(-3.4 \mathrm{ppm})$.

ethyl (E)-3-(o-tolyl)but-2-enoate ((E)-38):<smiles>CCOC(=O)/C=C(\C)c1ccccc1C</smiles>

(E)-38

Prepared according to general procedure B. Obtained as a colorless oil (0.95 g, 29\% yield) after column chromatography on silica gel (PE:EtOAc from 100:0 to 99:1). Rf: 0.37 (PE:EtOAC; 95:5); ${ }^{1} \mathbf{H}$ NMR (300 $\left.\mathrm{MHz}, \mathrm{CDCl}_{3}\right): \delta 7.22-7.06(\mathrm{~m}, 4 \mathrm{H}), 5.77(\mathrm{q}, 1 \mathrm{H}, J=1.4 \mathrm{~Hz}), 4.22(\mathrm{q}, 2 \mathrm{H}, J$ $=7.1 \mathrm{~Hz}), 2.45(\mathrm{~d}, 3 \mathrm{H}, J=1.4 \mathrm{~Hz}), 2.30(\mathrm{~s}, 3 \mathrm{H}), 1.32(\mathrm{t}, 3 \mathrm{H}, J=7.1 \mathrm{~Hz})$; ${ }^{13} \mathrm{C}\left\{{ }^{1} \mathrm{H}\right\}$ NMR $\left(75 \mathrm{MHz}, \mathrm{CDCl}_{3}\right): \delta$ 166.7, 158.3, 144.0, 133.9, 130.5, 127.8, 127.2, 125.8, 119.5, 59.9, 20.9, 19.8, 14.4; IR (neat): v 3064 (w), 2981 (w), 1713 (s), 1640 (m), 1486 (w), 1446 (w), 
1366 (w), 1338 (w), 1262 (m), 1159 (vs), 1040 (m), 877 (w), 760 (m), 727 (m), 454 (w) cm-1; HRMS (TOF-API ${ }^{+}$) m/z: calcd for $[\mathrm{M}+\mathrm{H}]^{+} \mathrm{C}_{13} \mathrm{H}_{17} \mathrm{O}_{2}: 205.1229$, found: 205.1233 (+ $2.0 \mathrm{ppm}$ ).

ethyl (E)-3-(4-(tert-butyl)phenyl)but-2-enoate ((E)-40):<smiles>CCOC(=O)/C=C(\C)c1ccc(C(C)(C)C)cc1</smiles>

$(E)-40$

Prepared according to general procedure B. Obtained as a colorless oil (1.12 g, 28\% yield) after column chromatography on silica gel (PE:EtOAc from 100:0 to 99:1). Rf: 0.58 (PE:EtOAC; 95:5); ${ }^{1} \mathbf{H}$ NMR (300 MHz, $\mathrm{CDCl}_{3}$ ): $\delta 7.46-7.38(\mathrm{~m}, 4 \mathrm{H}), 6.15(\mathrm{~m}, 1 \mathrm{H}), 4.22(\mathrm{q}, 2 \mathrm{H}, \mathrm{J}$ $=7.1 \mathrm{~Hz}), 2.58(\mathrm{~d}, 3 \mathrm{H}, J=1.1 \mathrm{~Hz}), 1.35-1.33(\mathrm{~m}, 12 \mathrm{H}) ;{ }^{13} \mathrm{C}\left\{{ }^{1} \mathrm{H}\right\} \mathrm{NMR}$

(75 MHz, $\left.\mathrm{CDCl}_{3}\right): \delta 167.2,155.5,152.5,139.3,126.2(2 \mathrm{C}), 125.6(2 \mathrm{C})$, 116.5, 59.9, 34.8, 31.4 (3C), 17.9, 14.5; IR (neat): v 2963 (m), 2905 (w), $2870(w), 1711(\mathrm{~s})$, $1626(\mathrm{~m}), 1510(\mathrm{w}), 1462(\mathrm{w}), 1444(\mathrm{w}), 1365(\mathrm{~m}), 1341(\mathrm{~m}), 1269(\mathrm{~m}), 1158(\mathrm{vs}), 1114(\mathrm{~s})$, $1043(\mathrm{~m}), 875(\mathrm{w}), 831(\mathrm{~s}), 578(\mathrm{w}) \mathrm{cm}^{-1}$.

ethyl (E)-3-(4-methoxyphenyl)but-2-enoate ((E)-42):<smiles>CCOC(=O)/C=C(\C)c1ccc(OC)cc1</smiles>

$(E)-42$

Prepared according to general procedure B. Obtained as a slightly yellow oil ( $1.48 \mathrm{~g}, 42 \%$ yield) after column chromatography on silica gel (PE:EtOAc from 100:0 to 97:3). Rf: 0.46 (PE:EtOAc ; 9:1); ${ }^{1} \mathrm{H}$ NMR (300 MHz, CDCl 3 ): $\delta 7.46(\mathrm{~d}, 2 \mathrm{H}, J=8.5 \mathrm{~Hz}), 6.90(\mathrm{~d}, 2 \mathrm{H}, J$ $=8.5 \mathrm{~Hz}), 6.11(\mathrm{~m}, 1 \mathrm{H}), 4.21(\mathrm{q}, 2 \mathrm{H}, J=7.1 \mathrm{~Hz}), 3.84(\mathrm{~s}, 3 \mathrm{H}), 2.57(\mathrm{~s}$, $3 \mathrm{H}), 1.32(\mathrm{t}, 3 \mathrm{H}, J=7.1 \mathrm{~Hz}) ;{ }^{13} \mathrm{C}\left\{{ }^{1} \mathrm{H}\right\}$ NMR $\left(75 \mathrm{MHz}, \mathrm{CDCl}_{3}\right): \delta 167.1$, 160.5, 154.9, 134.3, 127.7 (2C), 115.3, 113.9 (2C), 59.7, 55.3, 17.6, 14.4; IR (neat): v 2991 (w), $2956(w), 2838(w), 1706(m), 1602(w), 1512(m), 1440(w), 1366(w), 1343(w), 1249(m)$, 1151 (vs), 1030 (s), 870 (w), 828 (s) cm ${ }^{-1}$; HRMS (TOF-API ) m/z: calcd for [M+H] ${ }^{+} \mathrm{C}_{13} \mathrm{H}_{17} \mathrm{O}_{3}$ : 221.1178, found: 221.1175 (- $1.4 \mathrm{ppm})$.

ethyl (E)-3-(4-fluorophenyl)but-2-enoate ((E)-44):<smiles>CCOC(=O)/C=C(\C)c1ccc(F)cc1</smiles>

$(E)-44$

Prepared according to general procedure B. Obtained as a colorless oil (2.29 g, 69\% yield) after column chromatography on silica gel (PE:EtOAc from 100:0 to 99:1). Rf: 0.31 (PE:EtOAc; 95:5); ${ }^{1} \mathbf{H}$ NMR (300 MHz, $\left.\mathrm{CDCl}_{3}\right): \delta 7.47$ (dd, $2 \mathrm{H}, J=8.9 \& 5.3 \mathrm{~Hz}$ ), $7.06(\mathrm{~m}, 2 \mathrm{H}), 6.10$ $(\mathrm{q}, 1 \mathrm{H}, J=1.1 \mathrm{~Hz}), 4.22(\mathrm{q}, 2 \mathrm{H}, J=7.1 \mathrm{~Hz}), 2.56(\mathrm{~d}, 3 \mathrm{H}, J=1.2 \mathrm{~Hz}), 1.32$ $(\mathrm{t}, 3 \mathrm{H}, J=7.1 \mathrm{~Hz}) ;{ }^{13} \mathrm{C}\left\{{ }^{1} \mathrm{H}\right\}$ NMR $\left(75 \mathrm{MHz}, \mathrm{CDCl}_{3}\right): \delta 166.7,163.2(\mathrm{~d}, J=$ $249.0 \mathrm{~Hz}$ ), 154.2, 138.2 (d, J = 3.3 Hz), 128.1 (d, 2C, J = 8.1 Hz), 117.1, 115.5 (d, 2C, J = 21.5 Hz), 59.9, 17.9, 14.4; ${ }^{19} \mathrm{~F}\left\{{ }^{1} \mathrm{H}\right\}$ NMR (282 MHz, CDCl 3 ): $\delta$ - 113.1; IR (neat): v 2982 (w), 1709 (s), 1630 (m), 1601 (m), $1509(\mathrm{~m}), 1443(\mathrm{w}), 1367$ (w), 1342 (w), $1272(\mathrm{~m}), 1233$ (m), 1157 (vs), 1042 (s), $876(w), 832$ (vs), 557 (w), $500(w), 451(w) \mathrm{cm}^{-1}$; HRMS (TOF-API ${ }^{+}$m/z: calcd for [M+H] ${ }^{+}$ $\mathrm{C}_{12} \mathrm{H}_{14} \mathrm{FO}_{2}: 209.0978$, found: 209.0979 (+ 0.5 ppm). 
ethyl (E)-3-(4-bromophenyl)but-2-enoate ((E)-46):<smiles>CCOC(=O)/C=C(\C)c1ccc(Br)cc1</smiles>

$(E)-46$

Prepared according to general procedure B. Obtained as a colorless oil (1.15 g, 27\% yield) after column chromatography on silica gel (PE:EtOAc from 100:0 to 99:1). Rf: 0.62 (PE:EtOAC; 95:5); ${ }^{\mathbf{1}} \mathbf{H}$ NMR (300 MHz, $\mathrm{CDCl}_{3}$ ): $\delta 7.51$ (d, $2 \mathrm{H}, J=8.5 \mathrm{~Hz}$ ), $7.35(\mathrm{~d}, 2 \mathrm{H}, J=8.6 \mathrm{~Hz}$ ), $6.12(\mathrm{q}, 1 \mathrm{H}, J=1.0 \mathrm{~Hz}), 4.22(\mathrm{q}, 2 \mathrm{H}, J=7.1 \mathrm{~Hz}), 2.55(\mathrm{~d}, 3 \mathrm{H}, J=0.9 \mathrm{~Hz})$, $1.32(\mathrm{t}, 3 \mathrm{H}, J=7.1 \mathrm{~Hz}) ;{ }^{13} \mathrm{C}\left\{{ }^{1} \mathrm{H}\right\}$ NMR $\left(75 \mathrm{MHz}, \mathrm{CDCl}_{3}\right): \delta 166.8,154.2$, 141.2, 131.8 (2C), 128.0 (2C), 123.4, 117.7, 60.1, 17.9, 14.5; IR (neat): v $2980(w), 2903(w)$, $1710(\mathrm{~s}), 1627(\mathrm{~m}), 1586(\mathrm{w}), 1487(\mathrm{w}), 1440(\mathrm{w}), 1401(\mathrm{w}), 1366(\mathrm{w}), 1341(\mathrm{~m}), 1273(\mathrm{~m}), 1159$ (vs), 1080 (s), 1041 (m), 1007 (s), 876 (w), 821 (s), 747 (w), $554(w) \mathrm{cm}^{-1}$. The obtained special data were in agreement with the literature. ${ }^{[7]}$

ethyl (E)-3-(4-iodophenyl)but-2-enoate ((E)-48):<smiles>CCOC(=O)/C=C(\C)c1ccc(I)cc1</smiles>

(E)-48

Prepared according to general procedure B. Obtained as an orange oil (1.36 g, 27\% yield) after column chromatography on silica gel (PE:EtOAc from 100:0 to 99:1). Rf: 0.30 (PE:EtOAC ; 95:5); ${ }^{1} \mathrm{H}$ NMR (300 $\left.\mathrm{MHz} \mathrm{CDCl}_{3}\right): \delta 7.71(\mathrm{~d}, 2 \mathrm{H}, J=8.3 \mathrm{~Hz}), 7.21(\mathrm{~d}, 2 \mathrm{H}, J=8.3 \mathrm{~Hz}), 6.12(\mathrm{~m}$, $1 \mathrm{H}), 4.22(\mathrm{q}, 2 \mathrm{H}, J=7.1 \mathrm{~Hz}), 2.54(\mathrm{~s}, 3 \mathrm{H}), 1.32(\mathrm{t}, 3 \mathrm{H}, J=7.1 \mathrm{~Hz}) ;{ }^{13} \mathrm{C}\left\{{ }^{1} \mathrm{H}\right\}$ NMR (75 MHz, CDCl $): \delta 166.5,154.1,141.5,137.6$ (2C), 128.0 (2C), 117.5, 95.1, 59.9, 17.7, 14.4; IR (neat): v 2078 (w), 2897 (w), 1904 (w), 1708 (s), 1626 (m), 1581 (w), 1483 (w), 1441 (w), $1398(w), 1365(w), 1340(w), 1273(\mathrm{~m}), 1158(\mathrm{vs}), 1078(\mathrm{~m}), 1041(\mathrm{w}), 1002(\mathrm{~s}), 875(\mathrm{w})$, 817 (s) $\mathrm{cm}^{-1}$; HRMS (TOF-API ${ }^{+}$) m/z: calcd for [M+H] ${ }^{+} \mathrm{C}_{12} \mathrm{H}_{14} \mathrm{IO}_{2}: 317.0039$, found: 317.0053 (+ $4.4 \mathrm{ppm})$.

ethyl (E)-3-(4-nitrophenyl)but-2-enoate ((E)-50):<smiles>CCOC(=O)/C=C(\C)c1ccc([N+](=O)[O-])cc1</smiles>

$(E)-50$

Prepared according to general procedure B. Obtained as a white solid ( $1.72 \mathrm{~g}, 45 \%$ yield) after column chromatography on silica gel (PE:EtOAc from 100:0 to 98:2). Rf: 0.21 (PE:EtOAc ; 95:5); Mp: 73 $74{ }^{\circ} \mathrm{C} ;{ }^{1} \mathrm{H}$ NMR (300 MHz, $\left.\mathrm{CDCl}_{3}\right): \delta 8.24(\mathrm{~d}, 2 \mathrm{H}, \mathrm{J}=8.6 \mathrm{~Hz}), 7.62$ (d, $2 \mathrm{H}, J=8.6 \mathrm{~Hz}), 6.19(\mathrm{~m}, 1 \mathrm{H}), 4.25(\mathrm{q}, 2 \mathrm{H}, J=7.1 \mathrm{~Hz}), 2.59(\mathrm{~s}, 3 \mathrm{H})$, $1.34(\mathrm{t}, 3 \mathrm{H}, J=7.1 \mathrm{~Hz}) ;{ }^{13} \mathrm{C}\left\{{ }^{1} \mathrm{H}\right\}$ NMR $\left(75 \mathrm{MHz}, \mathrm{CDCl}_{3}\right): \delta$ 166.2, 152.7, 148.6, 147.9, 127.3 (2C), 123.8 (2C), 120.2, 60.3, 17.9, 14.3; IR (neat): v 3118 (w), 2983 (w), 2903 (w), 2229 (w), 1712 (s), $1633(\mathrm{~m}), 1596(\mathrm{~m}), 1516$ (s), 1443 (w), 1342 (vs), $1274(\mathrm{~m}), 1235$ (m), 1163 (vs), $1108(\mathrm{~m})$, 1041 (s), 849 (vs), $757(\mathrm{~m}), 692(\mathrm{~m}) \mathrm{cm}^{-1}$; HRMS (TOF-API ${ }^{+}$) m/z: calcd for [M+H] ${ }^{+} \mathrm{C}_{12} \mathrm{H}_{14} \mathrm{NO}_{4}$ : 236.0923, found: 236.0918 (- $2.1 \mathrm{ppm})$.

ethyl (E)-4,4,4-trifluoro-3-phenylbut-2-enoate ((E)-52):<smiles>CCOC(=O)/C=C(/c1ccccc1)C(F)(F)F</smiles>

$(E)-52$

Prepared according to general procedure B. Obtained as a colorless oil (1.46 $\mathrm{g}, 37 \%$ yield) after column chromatography on silica gel (PE:EtOAc from 100:0 to 99:1). $\mathbf{R f :} 0.32$ (PE:EtOAC ; 95:5); ${ }^{1} \mathbf{H ~ N M R ~ ( 3 0 0 ~ M H z , ~} \mathrm{CDCl}_{3}$ ): $\delta 7.44$ $-7.38(\mathrm{~m}, 3 \mathrm{H}), 7.32-7.29(\mathrm{~m}, 2 \mathrm{H}), 6.63(\mathrm{~d}, 1 \mathrm{H}, J=1.1 \mathrm{~Hz}), 4.06(\mathrm{q}, 2 \mathrm{H}, J=$ $7.1 \mathrm{~Hz}), 1.07(\mathrm{t}, 3 \mathrm{H}, J=7.1 \mathrm{~Hz}) ;{ }^{13} \mathrm{C}\left\{{ }^{1} \mathrm{H}\right\} \mathrm{NMR}\left(75 \mathrm{MHz}, \mathrm{CDCl}_{3}\right): \delta 164.3,142.5$ $(\mathrm{d}, J=30.8 \mathrm{~Hz}), 131.2,129.4,128.8(2 \mathrm{C}), 128.3(2 \mathrm{C}), 124.7(\mathrm{q}, J=5.5 \mathrm{~Hz})$, 
$122.7\left(q, J=274.8 \mathrm{~Hz}\right.$ ), 61.2, 13.8; ${ }^{19} \mathrm{~F}\left\{{ }^{1} \mathrm{H}\right\}$ NMR (282 MHz, $\left.\mathrm{CDCl}_{3}\right): \delta$ - 68.1; IR (neat): v 3064 $(w), 2987(w), 1727(m), 1446(w), 1282(s), 1255$ (s), $1174(s), 1127$ (vs), $1027(\mathrm{~m}), 901(w)$, 697 (s), $658(\mathrm{~m}) \mathrm{cm}^{-1}$; HRMS (TOF-API ${ }^{+}$) m/z: calcd for $[\mathrm{M}+\mathrm{H}]^{+} \mathrm{C}_{12} \mathrm{H}_{12} \mathrm{~F}_{3} \mathrm{O}_{2}: 245.0789$, found: 245.0793 (+ $1.6 \mathrm{ppm})$.

\subsection{Characterization of $\beta$-substituted acids}

(E)-3-phenylbut-2-enoic acid (Ac-29):<smiles>C/C(=C\C(=O)O)c1ccccc1</smiles>

Ac-29

Prepared according to general procedure C. Obtained as a white solid (0.72 g, 83\% yield, on $5.4 \mathrm{mmol}$ scale). Mp: $91-92{ }^{\circ} \mathrm{C} ;{ }^{1} \mathrm{H}$ NMR (300 MHz, $\left.\mathrm{CDCl}_{3}\right): \delta 12.18(\mathrm{br} \mathrm{s}, 1 \mathrm{H}), 7.54-7.51(\mathrm{~m}, 2 \mathrm{H}), 7.43-7.41(\mathrm{~m}, 3 \mathrm{H}), 6.21$ $(\mathrm{m}, 1 \mathrm{H}), 2.64(\mathrm{~d}, 3 \mathrm{H}, J=1.1 \mathrm{~Hz}) ;{ }^{13} \mathrm{C}\left\{{ }^{1} \mathrm{H}\right\}$ NMR (75 MHz, $\left.\mathrm{CDCl}_{3}\right): \delta 172.8$, 158.7, 142.1, 129.5, 128.7 (2C), 126.5 (2C), 116.6, 18.4; IR (neat): v 3963 (w), 2911 (br), 2675 (br), 2596 br), 2537 (br), $1674(\mathrm{~s}), 1617(\mathrm{~s}), 1575(\mathrm{~m})$, $1496(w), 1448$ (w), $1409(\mathrm{~m}), 1340(\mathrm{~m}), 1283$ (s), 1221 (vs), $1074(\mathrm{w}), 910$ (m), 865 (vs), 763 (vs), 719 (s), 694 (vs), 683 (vs), 594 (m), 478 (m), 441 (m) cm ${ }^{-1}$; HRMS (TOF-ESI') m/z: calcd for $[\mathrm{M}-\mathrm{H}]^{-} \mathrm{C}_{10} \mathrm{H}_{9} \mathrm{O}_{2}:$ : 161.0603, found: 161.0599 (- $\left.2.5 \mathrm{ppm}\right)$.

(E)-3-phenylpent-2-enoic acid (Ac-31):

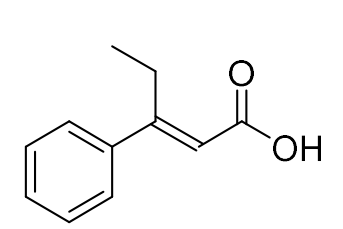

Ac-31

Prepared according to general procedure $\mathbf{C}$. Obtained as a white solid (1.43 g, 99\% yield, on $8.2 \mathrm{mmol}$ scale). Mp: $95-96{ }^{\circ} \mathrm{C} ;{ }^{1} \mathrm{H} \mathrm{NMR} \mathrm{(300} \mathrm{MHz,}$ $\left.\mathrm{CDCl}_{3}\right): \delta 10.97$ (br s, 1H), $7.47-7.39(\mathrm{~m}, 5 \mathrm{H}), 6.06(\mathrm{~m}, 1 \mathrm{H}), 3.13(\mathrm{q}, 2 \mathrm{H}, J$ $=7.4 \mathrm{~Hz}$ ), 1.09 (t, 3H, $J=7.4 \mathrm{~Hz}) ;{ }^{13} \mathrm{C}\left\{{ }^{1} \mathrm{H}\right\}$ NMR (75 MHz, DMSO-d6): $\delta$ 167.3, 160.2, 140.3, 129.0, 128.7 (2C), 126.6 (2C), 117.4, 23.2, 13.6; IR (neat): v 2973 (w), 2933 (br), 2873 (w), 2558 (br), 1682 (s), 1612 (s), 1575 (m), $1413(\mathrm{~m}), 1292(\mathrm{~m}), 1243(\mathrm{~m}), 1212$ (vs), $932(\mathrm{~m}), 885(\mathrm{~m}), 771(\mathrm{~m}), 751(\mathrm{~m}), 693(\mathrm{vs}) \mathrm{cm}^{-}$ 1; HRMS (TOF-ESI') m/z: calcd for [M-H] ${ }^{-} \mathrm{C}_{11} \mathrm{H}_{11} \mathrm{O}_{2}: 175.0759$, found: 175.0752 (- $4.0 \mathrm{ppm}$ ).

\section{(E)-3-(p-tolyl)but-2-enoic acid (Ac-33):}<smiles>C/C(=C\C(=O)O)c1ccc(C)cc1</smiles>

Ac-33

Prepared according to general procedure $\mathbf{B}$. Obtained as a white solid ( $0.90 \mathrm{~g}, 89 \%$ yield, on $5.8 \mathrm{mmol}$ scale). Mp: $128-129^{\circ} \mathrm{C} ;{ }^{1} \mathrm{H}$ NMR (300 $\mathrm{MHz}^{\mathrm{CDCl}} \mathrm{CD}_{3}: \delta 7.42(\mathrm{~d}, 2 \mathrm{H}, J=7.9 \mathrm{~Hz}), 7.21(\mathrm{~d}, 2 \mathrm{H}, J=7.9 \mathrm{~Hz}), 6.18(\mathrm{~m}$, $1 \mathrm{H}), 2.60(\mathrm{~s}, 3 \mathrm{H}), 2.39(\mathrm{~s}, 3 \mathrm{H})$. Proton of carboxylic acid was not observed; ${ }^{13} \mathrm{C}\left\{{ }^{1} \mathrm{H}\right\}$ NMR (75 MHz, $\mathrm{CDCl}_{3}$ ): $\delta 172.1,158.4,139.6,139.0$, 129.3 (2C), 126.3 (2C), 115.4, 21.2, 18.2; IR (neat): v 2915 (br), 2675 (w), 2595 (br), 2188 (w), 1684 (s), 1616 (vs), 1605 (vs), 1568 (m), 1513 (w), 1440 (m), 1370 (w), 1343 (m), 1280 (vs), 1224 (s), 1205 (vs), 1130 (m), 1075 (w), 1018 (w), 925 (m), 868 (m), 811 (vs), 685 (s), $558(\mathrm{w})$, 454 (s) $\mathrm{cm}^{-1}$; HRMS (TOF-ESI) m/z: calcd for [M-H] ${ }^{-} \mathrm{C}_{11} \mathrm{H}_{11} \mathrm{O}_{2}: 175.0759$, found: 175.0769 (+ $5.7 \mathrm{ppm})$. 
(E)-3-(m-tolyl)but-2-enoic acid (Ac-35):

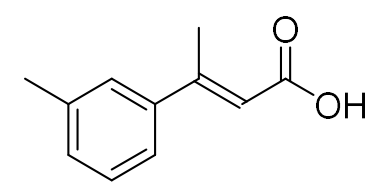

Ac-35

Prepared according to general procedure B. Obtained as a beige solid (1.43 g, 91\% yield, on $8.9 \mathrm{mmol}$ scale). $\mathrm{Mp}: 106-107^{\circ} \mathrm{C} ;{ }^{1} \mathrm{H}$ NMR (300 $\left.\mathrm{MHz}, \mathrm{CDCl}_{3}\right): \delta 11.45(\mathrm{brs}, 1 \mathrm{H}), 7.32(\mathrm{~m}, 3 \mathrm{H}), 7.23(\mathrm{~m}, 1 \mathrm{H}), 6.18(\mathrm{~m}, 1 \mathrm{H})$, $2.61(\mathrm{~s}, 3 \mathrm{H}), 2.41(\mathrm{~s}, 3 \mathrm{H}) ;{ }^{13} \mathrm{C}\left\{{ }^{1} \mathrm{H}\right\}$ NMR (75 MHz, $\left.\mathrm{CDCl}_{3}\right): \delta 172.7,158.9$, 142.2, 138.3, 130.2, 128.6, 127.2, 123.7, 116.4, 21.6, 18.5; IR (neat): v 2921 (br), 2552 (br), 1679 (s), $1616(\mathrm{~m}), 1422$ (w), $1373(\mathrm{w}), 1341(\mathrm{w})$, $1286(\mathrm{~m}), 1220(\mathrm{vs}), 1187(\mathrm{~m}), 1174(\mathrm{~m}), 933(\mathrm{~m}), 858(\mathrm{~m}), 867(\mathrm{~m}), 787(\mathrm{vs}), 694(\mathrm{~s}), 459(\mathrm{~m})$, 430 (s) cm ${ }^{-1}$; HRMS (TOF-ESI') m/z: calcd for [M-H] ${ }^{-} \mathrm{C}_{11} \mathrm{H}_{11} \mathrm{O}_{2}: 175.0759$, found: 175.0754 ($2.9 \mathrm{ppm})$.

(E)-3-(o-tolyl)but-2-enoic acid (Ac-37):<smiles>C/C(=C\C(=O)O)c1ccccc1C</smiles>

Ac-37

Prepared according to general procedure B. Obtained as an orange oil (0.89 g, 90\% yield, on $5.6 \mathrm{mmol}$ scale). ${ }^{1} \mathrm{H}$ NMR (300 MHz, $\left.\mathrm{CDCl}_{3}\right): \delta 11.27$ (br s, 1H), $7.24-7.17(\mathrm{~m}, 3 \mathrm{H}), 7.10-7.08(\mathrm{~m}, 1 \mathrm{H}), 5.82(\mathrm{~m}, 1 \mathrm{H}), 2.47(\mathrm{~s}$, $3 \mathrm{H}), 2.31(\mathrm{~s}, 3 \mathrm{H}) ;{ }^{13} \mathrm{C}\left\{{ }^{1} \mathrm{H}\right\}$ NMR (75 MHz, $\left.\mathrm{CDCl}_{3}\right): \delta$ 172.0, 161.6, 143.8, 133.9, 130.6, 128.1, 127.1, 125.9, 118.9, 21.3, 19.9; IR (neat): v 3017 (br), 2925 (br), 2546 (br), 1686 (vs), 1633 (s), 1486 (w), 1409 (m), 1336 (w), 1291 (m), 1267 (m), $1211(\mathrm{~s}), 879(\mathrm{~m}), 759(\mathrm{~s}), 726(\mathrm{~m}), 689(\mathrm{~m}), 605(\mathrm{w}), 458(\mathrm{~m}) \mathrm{cm}^{-1}$; HRMS (TOF-ESI) m/z: calcd for $[\mathrm{M}-\mathrm{H}]^{-} \mathrm{C}_{11} \mathrm{H}_{11} \mathrm{O}_{2}: 175.0759$, found: 175.0756 (- $\left.1.7 \mathrm{ppm}\right)$.

(E)-3-(4-(tert-butyl)phenyl)but-2-enoic acid (Ac-39):<smiles>C/C(=C\C(=O)O)c1ccc(C(C)(C)C)cc1</smiles>

Ac-39

Prepared according to general procedure B. Obtained as a white solid (0.74 g, 99\% yield, on $3.4 \mathrm{mmol}$ scale). Mp: $132-133{ }^{\circ} \mathrm{C} ;{ }^{1} \mathrm{H}$ NMR (300 MHz, CDCl $): \delta 11.41$ (br s, $1 \mathrm{H}), 7.49-7.41(\mathrm{~m}, 4 \mathrm{H}), 6.20$ $(\mathrm{m}, 1 \mathrm{H}), 2.62(\mathrm{~d}, 3 \mathrm{H}, \mathrm{J}=1.0 \mathrm{~Hz}), 1.35(\mathrm{~s}, 9 \mathrm{H}) ;{ }^{13} \mathrm{C}\left\{{ }^{1} \mathrm{H}\right\}$ NMR $(75 \mathrm{MHz}$, $\left.\mathrm{CDCl}_{3}\right): \delta 172.6,158.5,152.9,139.1,126.3(2 \mathrm{C}), 125.7$ (2C), 115.8, 34.8, 31.3 (3C), 18.2; IR (neat): v 2953 (m), 2872 (w), 2531 (br), 1682 (s), $1614(\mathrm{~s}), 1512(\mathrm{w}), 1396(\mathrm{w}), 1364(\mathrm{w}), 1339$ (w), $1268(\mathrm{~m}), 1215$ (vs), 1201 (vs), 1113 (m), $1013(\mathrm{w}), 930(\mathrm{~m}), 872(\mathrm{~m}), 833(\mathrm{vs}), 820(\mathrm{~s}), 718(\mathrm{w}), 654(\mathrm{w}), 575(\mathrm{~m}), 522(\mathrm{~m}), 484(\mathrm{~m}) \mathrm{cm}^{-1}$; HRMS (TOF-ESI') m/z: calcd for [M-H] ${ }^{-} \mathrm{C}_{14} \mathrm{H}_{17} \mathrm{O}_{2}: 217.1229$, found: 217.1227 (- 0.9 ppm).

(E)-3-(4-methoxyphenyl)but-2-enoic acid (Ac-41):<smiles>COc1ccc(/C(C)=C/C(=O)O)cc1</smiles>

Ac-41

Prepared according to general procedure B. Obtained as a white solid (0.94 g, 91\% yield, on $5.4 \mathrm{mmol}$ scale). Mp: $157-158{ }^{\circ} \mathrm{C} ;{ }^{1} \mathrm{H}$ NMR (300 MHz, $\left.\mathrm{CDCl}_{3}\right): \delta 10.48$ (br s, $\left.1 \mathrm{H}\right), 7.49(\mathrm{~d}, 2 \mathrm{H}, J=8.8 \mathrm{~Hz})$, $6.92(\mathrm{~d}, 2 \mathrm{H}, \mathrm{J}=8.9 \mathrm{~Hz}), 6.16(\mathrm{~m}, 1 \mathrm{H}), 3.85(\mathrm{~s}, 3 \mathrm{H}), 2.59(\mathrm{~s}, 3 \mathrm{H})$; NMR ${ }^{13} \mathrm{C}\left\{{ }^{1} \mathrm{H}\right\}$ (75 MHz, $\left.\mathrm{CDCl}_{3}\right): \delta 172.6,160.9,158.0,134.2,128.0(2 \mathrm{C})$, 114.6, 114.0 (2C), 55.5, 18.1; IR (neat): v 2943 (br), 2842 (w), 2536 (br), $1682(\mathrm{~m}), 1587(\mathrm{~s}), 1515(\mathrm{~m}), 1440(\mathrm{~m}), 1376(\mathrm{w}), 1348(\mathrm{~m}), 1286(\mathrm{~m}), 1257(\mathrm{~m}), 1215(\mathrm{~s})$, 1188 (vs), 1081 (w), 1027 (m), 928 (m), 862 (m), 822 (vs), 759 (m), 489 (s), 455 (vs) cm-1; HRMS (TOF-ESI') m/z: calcd for [M-H] ${ }^{-} \mathrm{C}_{11} \mathrm{H}_{11} \mathrm{O}_{3}: 191.0708$, found: 191.0704 (- $2.1 \mathrm{ppm}$ ). 
(E)-3-(4-fluorophenyl)but-2-enoic acid (Ac-43):<smiles>C/C(=C\C(=O)O)c1ccc(F)cc1</smiles>

Ac-43

Prepared according to general procedure B. Obtained as a brown solid (1.66 g, 93\% yield, on $9.9 \mathrm{mmol}$ scale). $\mathrm{Mp}: 138-139{ }^{\circ} \mathrm{C} ;{ }^{1} \mathrm{H}$ NMR (300 $\left.\mathrm{MHz}, \mathrm{CDCl}_{3}\right): \delta 11.05$ (br s, $\left.1 \mathrm{H}\right), 7.51-7.47(\mathrm{~m}, 2 \mathrm{H}), 7.11-7.06(\mathrm{~m}$, $2 \mathrm{H}), 6.14(\mathrm{~m}, 1 \mathrm{H}), 2.59(\mathrm{~s}, 3 \mathrm{H}) ;{ }^{13} \mathrm{C}\left\{{ }^{1} \mathrm{H}\right\}$ NMR (75 MHz, $\left.\mathrm{CDCl}_{3}\right): \delta 172.5$, $163.6(\mathrm{~d}, J=250.3 \mathrm{~Hz}), 157.5,138.1(\mathrm{~d}, J=3.0 \mathrm{~Hz}), 128.4(\mathrm{~d}, 2 \mathrm{C}, J=8.4$ $\mathrm{Hz}), 116.4,115.7(\mathrm{~d}, 2 \mathrm{C}, J=21.6 \mathrm{~Hz}), 18.4 ;{ }^{19} \mathrm{~F}\left\{{ }^{1} \mathrm{H}\right\}$ NMR $(282 \mathrm{MHz}$, $\mathrm{CDCl}_{3}$ ): $\delta$-112.3; IR (neat): v 2917 (br), 2593 (br), 1685 (m), $1616(\mathrm{~m}), 1600$ (m), $1592(\mathrm{~m})$, $1509(\mathrm{~s}), 1440(\mathrm{w}), 1340(\mathrm{w}), 1281(\mathrm{~m}), 1214(\mathrm{~s}), 1166(\mathrm{~s}), 1011(\mathrm{w}), 903(\mathrm{~m}), 869(\mathrm{~m}), 829$ (vs), 763 (m), 739 (w), 685 (w), 556 (w), 463 (s), 424 (m) cm cm $^{-1}$ HRMS (TOF-ESI) m/z: calcd for [M$\mathrm{H}^{-} \mathrm{C}_{10} \mathrm{H}_{8} \mathrm{FO}_{2}:$ : 179.0508, found: 179.0504 (- $\left.2.2 \mathrm{ppm}\right)$.

\section{(E)-3-(4-bromophenyl)but-2-enoic acid (AC-45):}<smiles>C/C(=C\C(=O)O)c1ccc(Br)cc1</smiles>

Ac-45

Prepared according to general procedure $\mathbf{B}$. Obtained as a white solid (0.72 g, 88\% yield, on $3.4 \mathrm{mmol}$ scale). Mp: $140-141{ }^{\circ} \mathrm{C} ;{ }^{1} \mathbf{H}$ NMR (300 MHz, $\mathrm{CDCl}_{3}$ ): $\delta 10.91$ (br s, $\left.1 \mathrm{H}\right), 7.53(\mathrm{~d}, 2 \mathrm{H}, J=8.4 \mathrm{~Hz}$ ), 7.36 (d, $2 \mathrm{H}, J=8.5 \mathrm{~Hz}), 6.15(\mathrm{~m}, 1 \mathrm{H}), 2.57(\mathrm{~s}, 3 \mathrm{H}) ;{ }^{13} \mathrm{C}\left\{{ }^{1} \mathrm{H}\right\}$ NMR $(75 \mathrm{MHz}$, $\left.\mathrm{CDCl}_{3}\right): \delta 172.2,157.3,140.9,131.9(2 \mathrm{C}), 128.1(2 \mathrm{C}), 123.8,116.9$, 18.3; IR (neat): v 2925 (br), 2848 (br), 2539 (br), 1676 (s), 1618 (s), $1488(w), 1434(w), 1400(w), 1343(w), 1296(m), 1213(s), 1080(m), 1005(m), 914(m), 875$ (m), 819 (vs), $717(\mathrm{~m}), 507(\mathrm{~m}), 461(\mathrm{~m}) \mathrm{cm}^{-1}$; HRMS (TOF-ESI') m/z: calcd for [M-H] ${ }^{-} \mathrm{C}_{10} \mathrm{H}_{8} \mathrm{BrO}_{2}$ : 238.9708, found: $238.9713(+2.1 \mathrm{ppm})$.

(E)-3-(4-iodophenyl)but-2-enoic acid (Ac-47):<smiles>C/C(=C\C(=O)O)c1ccc(I)cc1</smiles>

Ac-47

Prepared according to general procedure B. Obtained as a beige solid ( $0.78 \mathrm{~g}$, 91\% yield, on $2.9 \mathrm{mmol}$ scale). $\mathrm{Mp:} 139-140{ }^{\circ} \mathrm{C} ;{ }^{1} \mathrm{H}$ NMR (300 $\left.\mathrm{MHz} \mathrm{CDCl}_{3}\right): \delta 10.85$ (br s, $\left.1 \mathrm{H}\right), 7.76(\mathrm{~d}, 2 \mathrm{H}, J=8.2 \mathrm{~Hz}), 7.25(\mathrm{~d}, 2 \mathrm{H}, J=$ $8.1 \mathrm{~Hz}), 6.18(\mathrm{~m}, 1 \mathrm{H}), 2.59(\mathrm{~s}, 3 \mathrm{H}) ;{ }^{13} \mathrm{C}\left\{{ }^{1} \mathrm{H}\right\}$ NMR (75 MHz, $\left.\mathrm{CDCl}_{3}\right): \delta$ $172.2,157.5,141.5,137.9$ (2C), 128.3 (2C), 116.9, 95.7, 18.2; IR (neat): v 2923 (br), 2534 (br), $1680(\mathrm{~s}), 1618(\mathrm{~m}), 1578(\mathrm{w}), 1484(\mathrm{w}), 1389(\mathrm{w}), 1338(\mathrm{w}), 1279(\mathrm{~m})$, 1212 (s), 1078 (w), 1002 (m), 907 (w), 871 (w), 815 (vs), 713 (m), 494 (m), 449 (m) cm' ; HRMS (TOF-ESI) $\mathrm{m} / \mathrm{z}$ : calcd for [M-H] ${ }^{-} \mathrm{C}_{10} \mathrm{H}_{8} \mathrm{IO}_{2}: 286.9569$, found: 286.9569 (+0.0 ppm).

(E)-3-(4-nitrophenyl)but-2-enoic acid (Ac-49):<smiles>C/C(=C\C(=O)O)c1ccc([N+](=O)[O-])cc1</smiles>

Ac-49

Prepared according to general procedure B. Obtained as a beige solid (0.98 g, 91\% yield, on $5.2 \mathrm{mmol}$ scale). Mp: $150-151{ }^{\circ} \mathrm{C} ;{ }^{1} \mathbf{H}$ NMR (300 MHz, CDCl 3 ): $\delta 10.73(\mathrm{br} \mathrm{s}, 1 \mathrm{H}), 8.26(\mathrm{~d}, 2 \mathrm{H}, J=8.9 \mathrm{~Hz})$, $7.64(\mathrm{~d}, 2 \mathrm{H}, J=8.8 \mathrm{~Hz}), 6.22(\mathrm{q}, 1 \mathrm{H}, J=1.2 \mathrm{~Hz}), 2.62(\mathrm{~d}, 3 \mathrm{H}, J=1.2$ $\mathrm{Hz}) ;{ }^{13} \mathrm{C}\left\{{ }^{1} \mathrm{H}\right\}$ NMR (75 MHz, $\left.\mathrm{CDCl}_{3}\right): \delta 171.6,156.1,148.3,127.5(2 \mathrm{C})$, 126.8, 124.0 (2C), 119.3, 18.5; IR (neat): v 3082 (br), 2981 (br), 2928 (br), 1700 (m), 1597 (m), $1514(\mathrm{~s}), 1411$ (w), 1343 (vs), $1261(\mathrm{~m}), 1192(\mathrm{~m}), 1109(\mathrm{~m}), 1013(\mathrm{w}), 925(\mathrm{w}), 854(\mathrm{~s}), 755$ (w), $692(\mathrm{~m}) \mathrm{cm}^{-1}$; HRMS (TOF-ESI) $\mathrm{m} / \mathrm{z}$ : calcd for [M-H] ${ }^{-} \mathrm{C}_{10} \mathrm{H}_{8} \mathrm{NO}_{4}: 206.0453$, found: 206.0454 (+ $0.5 \mathrm{ppm})$. 
(E)-4,4,4-trifluoro-3-phenylbut-2-enoic acid (Ac-51):<smiles>O=C(O)/C=C(\c1ccccc1)C(F)(F)F</smiles>

Ac-51

Prepared according to general procedure C. Obtained as a white solid (1.08 g, $85 \%$ yield, on $5.9 \mathrm{mmol}$ scale). $\mathrm{Mp}$ : $96-97^{\circ} \mathrm{C} ;{ }^{1} \mathrm{H}$ NMR (300 $\mathrm{MHz}, \mathrm{CDCl}_{3}$ ): $\delta 9.86$ (br s, 1H), $7.42-7.29(\mathrm{~m}, 4 \mathrm{H}), 6.58(\mathrm{~m}, 1 \mathrm{H}) ;{ }^{13} \mathrm{C}\left\{{ }^{1} \mathrm{H}\right\}$ NMR $(75 \mathrm{MHz}$, $\mathrm{CDCl}_{3}$ ): $\delta 169.5,145.0,130.5,129.8,128.6(2 \mathrm{C}), 128.4(2 \mathrm{C}), 123.4$ (q, $J=5.5$ $\mathrm{Hz}), 122.4(\mathrm{q}, J=275.4 \mathrm{~Hz}) ;{ }^{19} \mathrm{~F}\left\{{ }^{1} \mathrm{H}\right\}$ NMR $\left(282 \mathrm{MHz} \mathrm{CDCl}_{3}\right): \delta-68.2 ; \mathrm{IR}$ (neat): v 2851 (br), 2552 (br), $1717(\mathrm{~m}), 1699(\mathrm{~m}), 1648(\mathrm{w}), 1439(\mathrm{w}), 1419$ (w), 1282 (m), 1257 (m), 1240 (s), 1178 (vs), 1137 (vs), 914 (m), 699 (s), 657 (s) cm

\subsection{Characterization of $\beta$-substituted oxazolidinones}

(E)-3-(3-phenylbut-2-enoyl)oxazolidin-2-one ((E)-29):<smiles>C/C(=C\C(=O)N1CCOC1=O)c1ccccc1</smiles>

$(E)-29$

Prepared according to general procedure $\mathbf{A}$. Obtained as a yellow solid ( $0.54 \mathrm{~g}, 53 \%$ yield, on $4.4 \mathrm{mmol}$ scale) after recrystallization in a mixture DCM:PE. Mp: $139-140{ }^{\circ} \mathrm{C}$ (DCM:PE); ${ }^{1} \mathbf{H}$ NMR (300 MHz, $\left.\mathrm{CDCl}_{3}\right): \delta 7.60-7.56(\mathrm{~m}, 2 \mathrm{H}), 7.43-7.38(\mathrm{~m}, 4 \mathrm{H}), 4.45(\mathrm{t}, 2 \mathrm{H}, J=8.0$ $\mathrm{Hz}), 4.12(\mathrm{t}, 2 \mathrm{H}, J=8.0 \mathrm{~Hz}), 2.58(\mathrm{~d}, 3 \mathrm{H}, J=1.1 \mathrm{~Hz}) ;{ }^{13} \mathrm{C}\left\{{ }^{1} \mathrm{H}\right\}$ NMR $(75$ MHz, CDCl 3 ): $\delta$ 165.4, 156.8, 153.6, 142.2, 129.4, 128.6 (2C), 126.6 (2C), 116.4, 62.0, 42.7, 18.8; IR (neat): v 2993 (w), 2920 (w), 1764 (s), 1667 (s), 1609 (m), 1480 (w), 1449 (w), 1383 (m), 1359 (s), 1287 (m), 1209 (vs), 1116 (s), 1042 (s), 942 (m), 869 (m), 755 (vs), 703 (vs), 690 (vs), $560(\mathrm{~m}) \mathrm{cm}^{-1}$.

(E)-3-(3-phenylpent-2-enoyl)oxazolidin-2-one ((E)-31):<smiles>CC/C(=C\C(=O)N1CCOC1=O)c1ccccc1</smiles>

$(E)-31$

Prepared according to general procedure $\mathbf{A}$. Obtained as a white solid ( $1.07 \mathrm{~g}, 77 \%$ yield, on $5.7 \mathrm{mmol}$ scale) after column chromatography on silica gel (PE:EtOAC ; 8:2). Rf: 0.23 (PE:EtOAC ; 8:2); Mp: $62-63{ }^{\circ} \mathrm{C}$; ${ }^{1} \mathrm{H}$ NMR $\left(300 \mathrm{MHz}, \mathrm{CDCl}_{3}\right): \delta 7.56-7.53(\mathrm{~m}, 2 \mathrm{H}), 7.40-7.38(\mathrm{~m}, 3 \mathrm{H})$, $7.31(\mathrm{~s}, 1 \mathrm{H}), 4.44(\mathrm{t}, 2 \mathrm{H}, \mathrm{J}=8.0 \mathrm{~Hz}), 4.12(\mathrm{t}, 2 \mathrm{H}, \mathrm{J}=8.0 \mathrm{~Hz}), 3.07(\mathrm{q}, 2 \mathrm{H}$, $J=7.4 \mathrm{~Hz}$ ), $1.12(\mathrm{t}, 3 \mathrm{H}, J=7.4 \mathrm{~Hz}) ;{ }^{13} \mathrm{C}\left\{{ }^{1} \mathrm{H}\right\}$ NMR $\left(75 \mathrm{MHz}, \mathrm{CDCl}_{3}\right): \delta$ 165.0, 163.3, 153.6, 141.0, 129.3, 128.6 (2C), 127.0 (2C), 115.9, 61.9, 42.8, 25.1, 13.7; IR (neat): v $3106(w), 2978(w), 2921(w), 2885(w), 1767(s), 1669(s), 1604(s), 1471(w), 1382$ (s), 1359 (m), 1292 (m), 1200 (vs), $1112(\mathrm{~m}), 1069(\mathrm{~m}), 1041$ (s), 932 (w), 875 (w), 772 (vs), 756 (vs), 703 (vs), 689 (vs), $561(\mathrm{~m}) \mathrm{cm}^{-1}$.

(E)-3-(3-(p-tolyl)but-2-enoyl)oxazolidin-2-one ((E)-33):<smiles>C/C(=C\C(=O)N1CCOC1=O)c1ccc(C)cc1</smiles>

$(E)-33$

Prepared according to general procedure A. Obtained as a white solid $(0.53 \mathrm{~g}, 48 \%$ yield, on $4.5 \mathrm{mmol}$ scale) after column chromatography on silica gel (PE:EtOAc; 75:25) . Rf: 0.39 (PE:EtOAc ; 7:3); Mp: $100-101{ }^{\circ} \mathrm{C} ;{ }^{1} \mathrm{H}$ NMR (300 MHz, $\mathrm{CDCl}_{3}$ ): $\delta 7.49$ (d, $2 \mathrm{H}, J$ $=8.1 \mathrm{~Hz}), 7.43(\mathrm{~m}, 1 \mathrm{H}), 7.20(\mathrm{~d}, 2 \mathrm{H}, J=8.2 \mathrm{~Hz}), 4.44(\mathrm{t}, 2 \mathrm{H}, J=8.0$ $\mathrm{Hz}), 4.12(\mathrm{t}, 2 \mathrm{H}, J=7.9 \mathrm{~Hz}), 2.57(\mathrm{~d}, 3 \mathrm{H}, J=0.9 \mathrm{~Hz}), 2.38(\mathrm{~s}, 3 \mathrm{H}) ;{ }^{13} \mathrm{C}\left\{{ }^{1} \mathrm{H}\right\}$ NMR $\left(75 \mathrm{MHz} \mathrm{CDCl}_{3}\right)$ : ठ 165.6, 157.1, 153.7, 139.7, 139.3, 129.4 (2C), 126.7 (2C), 115.5, 62.0, 42.9, 21.4, 18.8; IR (neat): v 2985 (w), 2922 (w), 1760 (vs), 1675 (s), 1605 (m), 1511 (w), 1477 (w), 1385 (s), 1359 
(s), $1289(\mathrm{~m}), 1223(\mathrm{~m}), 1191(\mathrm{vs}), 1108(\mathrm{~m}), 1038(\mathrm{~s}), 944(\mathrm{~m}), 824(\mathrm{~s}), 760(\mathrm{~m}), 675(\mathrm{~m}), 485$ (m) $\mathrm{cm}^{-1}$.

(E)-3-(3-(m-tolyl)but-2-enoyl)oxazolidin-2-one ((E)-35):<smiles>C/C(=C\C(=O)N1CCOC1=O)c1cccc(C)c1</smiles>

(E)-35

Prepared according to general procedure A. Obtained as a white solid $(0.76 \mathrm{~g}, 46 \%$ yield, on $6.8 \mathrm{mmol}$ scale) after column chromatography on silica gel (PE:EtOAc from 100:0 to 85:15). Rf: 0.5 (PE:EtOAc ; 7:3); Mp: $68-69{ }^{\circ} \mathrm{C} ;{ }^{1} \mathrm{H}$ NMR (300 MHz, $\left.\mathrm{CDCl}_{3}\right): \delta 7.30$ $-7.27(\mathrm{~m}, 3 \mathrm{H}), 7.21-7.15(\mathrm{~m}, 1 \mathrm{H}), 7.11-7.08(\mathrm{~m}, 1 \mathrm{H}), 4.32(\mathrm{t}, 2 \mathrm{H}$, $J=8.0 \mathrm{~Hz}), 4.00(\mathrm{t}, 2 \mathrm{H}, J=8.0 \mathrm{~Hz}), 2.47(\mathrm{~s}, 3 \mathrm{H}), 2.29(\mathrm{~s}, 3 \mathrm{H}) ;{ }^{13} \mathrm{C}\left\{{ }^{1} \mathrm{H}\right\}$ NMR (75 MHz, $\left.\mathrm{CDCl}_{3}\right): \delta$ 165.5, 157.3, 153.7, 142.3, 138.2, 130.2, 128.5, 127.4, 123.9, 116.2, 62.0, 42.8, 21.6, 19.0; IR (neat): v 3104 (w), 2985 (br), 2918 (w), 1762 (vs), 1672 (s), 1614 (m), $1473(\mathrm{w}), 1438(\mathrm{w}), 1379(\mathrm{~m}), 1358(\mathrm{~s}), 1293(\mathrm{~m}), 1242(\mathrm{~m}), 1211$ (vs), $1112(\mathrm{~m}), 1045$ (s), 957 (w), $866(\mathrm{~m}), 788(\mathrm{vs}), 757(\mathrm{~s}), 691(\mathrm{vs}), 571(\mathrm{~m}), 456(\mathrm{w}), 418(\mathrm{w}) \mathrm{cm}^{-1}$.

(E)-3-(3-(o-tolyl)but-2-enoyl)oxazolidin-2-one ((E)-37):<smiles>C/C(=C\C(=O)N1CCOC1=O)c1ccccc1C</smiles>

(E)-37

Prepared according to general procedure $\mathbf{A}$. Obtained as an orange oil ( $0.78 \mathrm{~g}, 66 \%$ yield, on $4.8 \mathrm{mmol}$ scale) after column chromatography on silica gel (PE:EtOAC; 8:2). Rf: 0.44 (PE:EtOAC; 7:3); ${ }^{\mathbf{H}}$ NMR (300 $\left.\mathrm{MHz} \mathrm{CDCl}_{3}\right): \delta 7.22-7.11(\mathrm{~m}, 5 \mathrm{H}), 7.03(\mathrm{~m}, 1 \mathrm{H}), 4.41(\mathrm{t}, 3 \mathrm{H}, J=8.0$ $\mathrm{Hz}), 4.10(\mathrm{t}, 3 \mathrm{H}, J=8.0 \mathrm{~Hz}), 2.47(\mathrm{~d}, 3 \mathrm{H}, J=1.3 \mathrm{~Hz}), 2.33(\mathrm{~s}, 3 \mathrm{H}) ;{ }^{13} \mathrm{C}\left\{{ }^{1} \mathrm{H}\right\}$ NMR (75 MHz, CDCl $\left.{ }_{3}\right): \delta 165.3,160.0,153.4,144.0,134.1,130.6,127.9,127.3,125.8,118.7$, $62.0,42.7,21.9,20.0$.

(E)-3-(3-(4-(tert-butyl)phenyl)but-2-enoyl)oxazolidin-2-one ((E)-39):<smiles>C/C(=C\C(=O)N1CCOC1=O)c1ccc(C(C)(C)C)cc1</smiles>

(E) -39

Prepared according to general procedure $\mathbf{A}$. Obtained as a white solid ( $0.60 \mathrm{~g}, 84 \%$ yield, on $2.9 \mathrm{mmol}$ scale) after column chromatography on silica gel (PE:EtOAc from 9:1 to 8:2). Rf: 0.50 (PE:EtOAC ; 7:3); Mp: $111-112{ }^{\circ} \mathrm{C} ;{ }^{1} \mathbf{H}$ NMR (300 MHz, $\mathrm{CDCl}_{3}$ ): $\delta$ $7.55-7.52(\mathrm{~m}, 2 \mathrm{H}), 7.45-7.40(\mathrm{~m}, 3 \mathrm{H}), 4.42(\mathrm{t}, 2 \mathrm{H}, J=7.9 \mathrm{~Hz})$, $4.11(\mathrm{t}, 2 \mathrm{H}, J=7.9 \mathrm{~Hz}), 2.58(\mathrm{~s}, 3 \mathrm{H}), 1.34(\mathrm{~s}, 9 \mathrm{H}) ;{ }^{13} \mathrm{C}\left\{{ }^{1} \mathrm{H}\right\}$ NMR $\left(75 \mathrm{MHz}, \mathrm{CDCl}_{3}\right): \delta$ 165.6, 157.0, $153.7,152.8,139.2,126.5$ (2C), 125.6 (2C), 115.6, 62.0, 42.8, 34.8, 31.3 (3C), 18.7.

(E)-3-(3-(4-methoxyphenyl)but-2-enoyl)oxazolidin-2-one ((E)-41):<smiles>COc1ccc(/C(C)=C/C(=O)N2CCOC2=O)cc1</smiles>

$(E)-41$

Prepared according to general procedure A. Obtained as a yellow solid $(0.74 \mathrm{~g}, 69 \%$ yield, on $4.1 \mathrm{mmol}$ scale) after column chromatography on silica gel (PE:EtOAC ; 7:3). Rf: 0.27 (PE:EtOAC ; 7:3); Mp: $135-136{ }^{\circ} \mathrm{C}$; ${ }^{1} \mathrm{H}$ NMR (300 MHz, $\mathrm{CDCl}_{3}$ ): $\delta 7.55$ (d, $2 \mathrm{H}, J=8.8 \mathrm{~Hz}), 7.41(\mathrm{~m}, 1 \mathrm{H}), 6.90(\mathrm{~d}, 2 \mathrm{H}, J=8.8 \mathrm{~Hz}), 4.40(\mathrm{t}, 2 \mathrm{H}$, $J=8.0 \mathrm{~Hz}), 4.08(\mathrm{t}, 2 \mathrm{H}, J=8.0 \mathrm{~Hz}), 3.82(\mathrm{~s}, 3 \mathrm{H}), 2.56(\mathrm{~s}, 3 \mathrm{H})$; ${ }^{13} \mathrm{C}\left\{{ }^{1} \mathrm{H}\right\}$ NMR (75 MHz, $\left.\mathrm{CDCl}_{3}\right): \delta$ 165.6, 160.8, 156.5, 153.7, 134.3, $128.2(2 \mathrm{C}), 114.4,114.0$ (2C), 61.9, 55.4, 42.8, 18.6; IR (neat): v 2915 (w), 2981 (w), 2844 (w), 1771 (s), 1707 (w), 1660 $(\mathrm{m}), 1600(\mathrm{~s}), 1474(\mathrm{w}), 1438(\mathrm{w}), 1384(\mathrm{~m}), 1358(\mathrm{~m}), 1297(\mathrm{~m}), 1240(\mathrm{~m}), 1216(\mathrm{vs}), 1179(\mathrm{vs})$, $1115(\mathrm{~m}), 1045(\mathrm{~m}), 1022(\mathrm{~s}), 945(\mathrm{~m}), 828(\mathrm{vs}), 752(\mathrm{~m}), 701(\mathrm{~m}), 548(\mathrm{~m}), 501(\mathrm{~m}) \mathrm{cm}^{-1}$. 
(E)-3-(3-(4-fluorophenyl)but-2-enoyl)oxazolidin-2-one ((E)-43):<smiles>C/C(=C\C(=O)N1CCOC1=O)c1ccc(F)cc1</smiles>

(E)-43

Prepared according to general procedure A. Obtained as a white solid (1.09 g, $66 \%$ yield, on $6.6 \mathrm{mmol}$ scale) after column chromatography on silica gel (PE:EtOAc from 9:1 to 8:2). Rf: 0.37 (PE:EtOAC ; 7:3); Mp: $121-122{ }^{\circ} \mathrm{C} ;{ }^{1} \mathbf{H}$ NMR (300 MHz, $\left.\mathrm{CDCl}_{3}\right): \delta$ $7.57-7.52(\mathrm{~m}, 2 \mathrm{H}), 7.36(\mathrm{~m}, 1 \mathrm{H}), 7.09-7.03(\mathrm{~m}, 2 \mathrm{H}), 4.43(\mathrm{t}, 2 \mathrm{H}, J$ $=8.0 \mathrm{~Hz}), 4.10(\mathrm{t}, 2 \mathrm{H}, \mathrm{J}=8.0 \mathrm{~Hz}), 2,54(\mathrm{~s}, 3 \mathrm{H}) ;{ }^{13} \mathrm{C}\left\{{ }^{1} \mathrm{H}\right\}$ NMR $(75 \mathrm{MHz}$, $\left.\mathrm{CDCl}_{3}\right): \delta 165.4,163.5(\mathrm{~d}, J=249.2 \mathrm{~Hz}), 155.5,153.7,138.2(\mathrm{~d}, J=3.3 \mathrm{~Hz}), 128.6(\mathrm{~d}, 2 \mathrm{C}, J=8.4$ $\mathrm{Hz}), 116.3,115.6(\mathrm{~d}, 2 \mathrm{C}, J=21.5 \mathrm{~Hz}), 62.0,42.8,18.9 ;{ }^{19} \mathrm{~F}\left\{{ }^{1} \mathrm{H}\right\}$ NMR $\left(282 \mathrm{MHz}, \mathrm{CDCl}_{3}\right): \delta$ - 112.4; IR (neat): v 2977 (w), 2925 (w), 1770 (s), $1666(\mathrm{~m}), 1602(\mathrm{~m}), 1509(\mathrm{~m}), 1476(\mathrm{w}), 1382$ (m), 1359 (s), 1284 (m), 1222 (vs), 1200 (vs), 1159 (s), 1110 (m), 1043 (s), 944 (m), 833 (vs), 747 (m), $677(\mathrm{~m}), 524(\mathrm{~m}), 494(\mathrm{~m}), 456(\mathrm{~m}) \mathrm{cm}^{-1}$.

(E)-3-(3-(4-bromophenyl)but-2-enoyl)oxazolidin-2-one ((E)-45):<smiles>C/C(=C\C(=O)N1CCOC1=O)c1ccc(Br)cc1</smiles>

$(E)-45$

Prepared according to general procedure A. Obtained as a white solid (0.53 g, 66\% yield, on $2.6 \mathrm{mmol}$ scale) after column chromatography on silica gel (PE:EtOAc from 80:20 to 75:25). Rf: 0.34 (PE:EtOAC; 7:3); $\mathbf{M p : ~} 119-120{ }^{\circ} \mathrm{C} ;{ }^{1} \mathrm{H} \mathrm{NMR} \mathrm{(300} \mathrm{MHz,} \mathrm{CDCl}_{3}$ ): $\delta 7.52-7.41(\mathrm{~m}, 4 \mathrm{H}), 7.38(\mathrm{~m}, 1 \mathrm{H}), 4.43(\mathrm{t}, 2 \mathrm{H}, J=8.0 \mathrm{~Hz}), 4.10(\mathrm{t}$, $2 \mathrm{H}, J=8.0 \mathrm{~Hz}), 2.53(\mathrm{~s}, 3 \mathrm{H}) ;{ }^{13} \mathrm{C}\left\{{ }^{1} \mathrm{H}\right\}$ NMR $\left(75 \mathrm{MHz}, \mathrm{CDCl}_{3}\right): \delta$ 165.3, 155.3, 153.7, 141.1, 131.8 (2C), 128.3 (2C), 123.7, 116.8, 62.1, 42.8, 18.7; IR (neat): v 3098 (w), 2973 (br), 2925 (w), 1769 (vs), 1666 (m), 1612 (m), 1488 (w), 1435 (w), 1381 (s), 1359 (s), 1286 (m), 1197 (vs), 1112 (s), 1042 (vs), 1006 (s), 944 (m), 874 (w), 820 (vs), 753 (m), 711 (s), 601 (m), $559(\mathrm{w}), 438(\mathrm{~m}) \mathrm{cm}^{-1}$.

(E)-3-(3-(4-iodophenyl)but-2-enoyl)oxazolidin-2-one ((E)-47):<smiles>C/C(=C\C(=O)N1CCOC1=O)c1ccc(I)cc1</smiles>

(E)-47

Prepared according to general procedure A. Obtained as a beige solid (0.59 g, 69\% yield, on $2.4 \mathrm{mmol}$ scale) after column chromatography on silica gel (PE:EtOAc from 80:20 to 75:25). Rf: 0.33 (PE:EtOAC ; 7:3); $\mathrm{Mp:} 148-149{ }^{\circ} \mathrm{C} ;{ }^{1} \mathrm{H}$ NMR (300 MHz, $\mathrm{CDCl}_{3}$ ): $\delta 7.73(\mathrm{~d}, 2 \mathrm{H}, J=8.6 \mathrm{~Hz}), 7.39(\mathrm{~m}, 1 \mathrm{H}), 7.30(\mathrm{~d}, 2 \mathrm{H}, J=8.6 \mathrm{~Hz}), 4.45$ $(\mathrm{t}, 2 \mathrm{H}, J=8.1 \mathrm{~Hz}), 4.11(\mathrm{t}, 2 \mathrm{H}, J=8.1 \mathrm{~Hz}), 2.53(\mathrm{~d}, 3 \mathrm{H}, J=1.1 \mathrm{~Hz}) ;{ }^{13} \mathrm{C}\left\{{ }^{1} \mathrm{H}\right\} \mathbf{N M R}\left(75 \mathrm{MHz}, \mathrm{CDCl}_{3}\right)$ : $\delta 165.1,155.1,153.5,141.5,137.7$ (2C), 128.2 (2C), 116.7, 95.5, 62.0, 42.7, 18.5; IR (neat): $v$ 2991 (w), 2919 (br), 1754 (vs), 1665 (s), 1608 (m), 1480 (w), 1436 (w), 1392 (s), 1361 (vs), 1294 (m), 1220 (vs), $1120(\mathrm{~m}), 1043(\mathrm{~s}), 1003(\mathrm{~s}), 944(\mathrm{~m}), 884(\mathrm{w}), 817$ (vs), $758(\mathrm{~s}), 715$ (s), $699(\mathrm{~m})$, $621(w), 594(\mathrm{~m}), 560(\mathrm{w}), 500(\mathrm{~m}), 428(\mathrm{~m}) \mathrm{cm}^{-1}$.

(E)-3-(3-(4-nitrophenyl)but-2-enoyl)oxazolidin-2-one ((E)-49):<smiles>C/C(=C\C(=O)N1CCOC1=O)c1ccc([N+](=O)[O-])cc1</smiles>

$(E)-49$

Prepared according to general procedure A. Obtained as a yellow solid ( $0.62 \mathrm{~g}, 55 \%$ yield, on $4.1 \mathrm{mmol}$ scale) after column chromatography on silica gel (PE:EtOAc ; 7:3) . Rf: 0.21 (PE:EtOAc ; 7:3); Mp: $136-137{ }^{\circ} \mathrm{C}$; ${ }^{1} \mathbf{H}$ NMR (300 MHz, $\mathrm{CDCl}_{3}$ ): $\delta 8.11$ (d, $2 \mathrm{H}, J=8.5 \mathrm{~Hz}$ ), $7.59(\mathrm{~d}, 2 \mathrm{H}, J=8.5 \mathrm{~Hz}), 7.30(\mathrm{~m}, 1 \mathrm{H}), 4.38(\mathrm{t}, 2 \mathrm{H}$, 
$J=7.9 \mathrm{~Hz}), 4.03(\mathrm{t}, 2 \mathrm{H}, J=7.9 \mathrm{~Hz}), 2.44(\mathrm{~s}, 3 \mathrm{H}) ;{ }^{13} \mathrm{C}\left\{{ }^{1} \mathrm{H}\right\}$ NMR $\left(75 \mathrm{MHz}, \mathrm{CDCl}_{3}\right): \delta 164.8,153.6$, 152.9, 148.4, 147.9, 127.5 (2C), 123.8 (2C), 119.5, 62.2, 42.6, 18.5; IR (neat): v 2926 (w), 2256 (w), $1769(\mathrm{~m}), 1676(\mathrm{~m}), 1627(\mathrm{w}), 1596(\mathrm{w}), 1516(\mathrm{~s}), 1477(\mathrm{w}), 1388(\mathrm{~m}), 1344(\mathrm{vs}), 1290(\mathrm{~m})$, $1217(\mathrm{~s}), 1110(\mathrm{~m}), 1040(\mathrm{~m}), 908(\mathrm{~s}), 850(\mathrm{~m}), 727(\mathrm{vs}), 698(\mathrm{~s}), 684(\mathrm{~m}) \mathrm{cm}^{-1}$.

\section{(E)-3-(4,4,4-trifluoro-3-phenylbut-2-enoyl)oxazolidin-2-one ((E)-51):}<smiles>O=C(/C=C(/c1ccccc1)C(F)(F)F)N1CCOC1=O</smiles>

$(E)-51$

Prepared according to general procedure $\mathbf{A}$. Obtained as a white solid ( $0.78 \mathrm{~g}, 71 \%$ yield, on $3.8 \mathrm{mmol}$ scale) after column chromatography on silica gel (PE:EtOAC ; 7:3). Rf: 0.28 (PE:EtOAC; 7:3); Mp: $139-140{ }^{\circ} \mathrm{C} ;{ }^{1} \mathrm{H}$ NMR (300 MHz, $\left.\mathrm{CDCl}_{3}\right): \delta 7.43-7.38(\mathrm{~m}, 4 \mathrm{H}), 7.32-7.30(\mathrm{~m}, 2 \mathrm{H}), 4.36$ $(\mathrm{t}, 2 \mathrm{H}, J=8.0 \mathrm{~Hz}), 3.88(\mathrm{t}, 2 \mathrm{H}, J=8.0 \mathrm{~Hz}) ;{ }^{13} \mathrm{C}\left\{{ }^{1} \mathrm{H}\right\}$ NMR $\left(75 \mathrm{MHz}, \mathrm{CDCl}_{3}\right)$ : $\delta 163.3,153.1,141.0(q, J=31.1 \mathrm{~Hz}), 131.1,129.5,128.8(2 \mathrm{C}), 128.4(2 \mathrm{C})$, $125.0(q, J=5.8 \mathrm{~Hz}), 122.5(\mathrm{q}, J=274.5 \mathrm{~Hz}), 62.6,42.2 ;{ }^{19} \mathrm{~F}\left\{{ }^{1} \mathrm{H}\right\}$ NMR $(282$

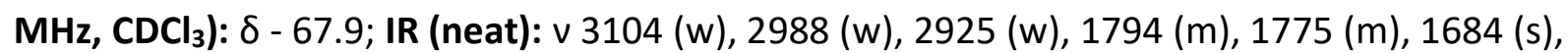
$1473(\mathrm{w}), 1392(\mathrm{~m}), 1363$ (w), $1311(\mathrm{~m}), 1269$ (s), 1222 (m), 1171 (vs), 1120 (vs), 1039 (vs), 960 $(\mathrm{m}), 881(\mathrm{~m}), 780(\mathrm{w}), 754(\mathrm{~s}), 703(\mathrm{~s}), 672(\mathrm{~m}), 631(\mathrm{~s}), 511(\mathrm{w}) \mathrm{cm}^{-1}$.

\subsection{Preparation and characterization of nitrile $(E)-53$}

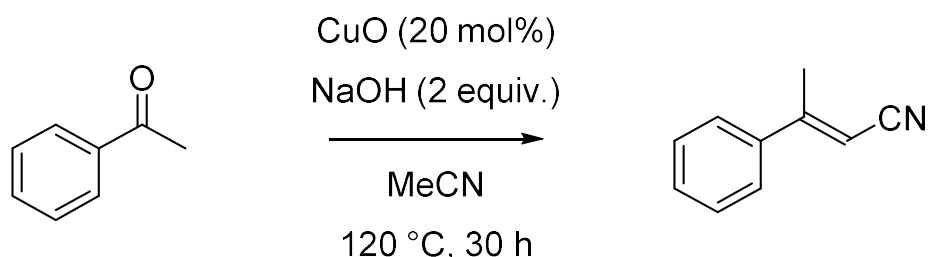

$(E)-53$

(E)-3-phenylbut-2-enenitrile $(E)$-53 was prepared according to the reported method. ${ }^{[4]}$

An oven-dried schlenk tube was charged with $\mathrm{CuO}(191 \mathrm{mg}, 0.0024 \mathrm{mmol}, 0.2$ equiv.) and $\mathrm{NaOH}$ ( $960 \mathrm{mg}, 24 \mathrm{mmol}, 2$ equiv.). Then, acetophenone $(1.4 \mathrm{~mL}, 12 \mathrm{mmol}, 1$ equiv.) and dried acetonitrile $(24 \mathrm{~mL})$ were added. The reaction mixture was stirred and heated at $120^{\circ} \mathrm{C}$ for 30 $\mathrm{h}$ under Ar atmosphere. After completion of the reaction, the reaction mixture was cooled down to room temperature and then washed with a saturated aqueous $\mathrm{NH}_{4} \mathrm{Cl}$ solution (75 $\mathrm{mL})$ and extracted with EtOAc $(3 \times 100 \mathrm{~mL})$. The combined organic layers were washed with brine $(50 \mathrm{~mL})$, dried over anhydrous $\mathrm{Na}_{2} \mathrm{SO}_{4}$ and concentrated in vacuo.

(E)-3-phenylbut-2-enenitrile ((E)-53):<smiles>C/C(=C\C#N)c1ccccc1</smiles>

$(E)-53$

Obtained as an orange oil ( $0.21 \mathrm{~g}, 12 \%$ yield) after column chromatography on silica gel (PE :EtOAc from 100:0 to 95:5). Rf: 0.57 (PE:EtOAC; 9:1); ${ }^{1}$ H NMR (300 MHz, $\left.\mathrm{CDCl}_{3}\right): \delta 7.49-7.41(\mathrm{~m}, 5 \mathrm{H}), 5.63(\mathrm{~m}, 1 \mathrm{H}), 2.48(\mathrm{~s}, 3 \mathrm{H}) ;{ }^{13} \mathrm{C}\left\{{ }^{1} \mathrm{H}\right\}$ NMR (75 MHz, CDCl $): \delta$ 159.9, 138.4, 130.4, 129.0 (2C), 126.0 (2C), 117.8, 95.7, 20.4; IR (neat): v 3063 (w), 2981 (w), $2213(\mathrm{~s}), 1608$ (m), 1575 (w), 1496 (w), $1446(\mathrm{~m}), 1381(\mathrm{~m}), 1333(\mathrm{w}), 1261(w), 1159(w), 1079(w), 1027(w), 921(w), 826(\mathrm{~m})$, 754 (vs), 690 (vs), $636(\mathrm{w}), 552(\mathrm{~m}) \mathrm{cm}^{-1}$. The obtained special data were in agreement with the literature. ${ }^{[4]}$ 


\subsection{Preparation and characterization of phosphonate $(E)-54$}<smiles>CC(=O)c1ccccc1</smiles><smiles>CCOCOPCOCC</smiles>

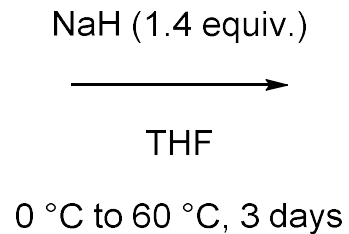

$0^{\circ} \mathrm{C}$ to $60^{\circ} \mathrm{C}, 3$ days<smiles>CCOC=C(C)c1ccccc1</smiles>

$(E)-54$

diethyl $(E)$-(2-phenylprop-1-en-1-yl)phosphonate $(E)$-54 was prepared according to the reported method. ${ }^{[5]}$

In a oven-dried schlenk flask under an argon atmosphere, sodium hydride (60\% in mineral oil, $355 \mathrm{mg}, 8.89 \mathrm{mmol}, 1.4$ equiv.) was dissolved in dry THF $(60 \mathrm{~mL})$ at $0{ }^{\circ} \mathrm{C}$. Tetraalkyl methylenediphosphonate $(2.0 \mathrm{~mL}, 8.07 \mathrm{mmol}, 1.27$ equiv.) was added dropwise and the solution was stirred under an argon atmosphere at $0{ }^{\circ} \mathrm{C}$ for $1 \mathrm{~h}$. Acetophenone $(0.74 \mathrm{~mL}, 6.35$ mmol, 1 equiv.) was added via syringe and the solution was heated at $60{ }^{\circ} \mathrm{C}$ and stirred for 3 days. Then, the mixture was cooled to room temperature, water $(50 \mathrm{~mL})$ and EtOAc $(60 \mathrm{~mL})$ were added. The layers were separated and the aqueous layer was extracted with EtOAc ( $3 \mathrm{x}$ $50 \mathrm{~mL})$. The combined organic layers were washed with brine $(50 \mathrm{~mL})$, dried over anhydrous $\mathrm{Na}_{2} \mathrm{SO}_{4}$ and the solvent was removed in vacuo.

diethyl (E)-(2-phenylprop-1-en-1-yl)phosphonate ((E)-54):<smiles>CCOC=C(C)c1ccccc1</smiles>

$(E)-54$

Obtained as a colorless oil $(0.64 \mathrm{~g}, 40 \%$ yield) after column chromatography on silica gel (PE :EtOAC; 6:4). Rf: 0.25 (PE:EtOAc ; 6:4); ${ }^{1} \mathrm{H}$ NMR (300 MHz, $\left.\mathrm{CDCl}_{3}\right): \delta 7.50-7.36(\mathrm{~m}, 5 \mathrm{H}), 5.91$ (d, $J=16.3$ $\mathrm{Hz}, 1 \mathrm{H}), 4.14(\mathrm{dq}, 4 \mathrm{H}, J=7.9 \& 7.1 \mathrm{~Hz}), 2.51(\mathrm{~d}, 3 \mathrm{H}, J=2.7 \mathrm{~Hz}), 1.36(\mathrm{t}$, $6 \mathrm{H}, J=7.1 \mathrm{~Hz}) ;{ }^{13} \mathrm{C}\left\{{ }^{1} \mathrm{H}\right\}$ NMR $\left(75 \mathrm{MHz}, \mathrm{CDCl}_{3}\right): \delta 158.2(\mathrm{~d}, J=8.2 \mathrm{~Hz})$, $141.8(\mathrm{~d}, J=23.7 \mathrm{~Hz}), 129.2,128.5(2 \mathrm{C}), 126.0(2 \mathrm{C}), 113.5$ (d, $J=190.3 \mathrm{~Hz}), 61.5(\mathrm{~d}, 2 \mathrm{C}, J=5.6$ $\mathrm{Hz}), 19.3(\mathrm{~d}, J=7.0 \mathrm{~Hz}), 16.4(\mathrm{~d}, 2 \mathrm{C}, J=6.5 \mathrm{~Hz}) ;{ }^{31} \mathbf{P}\left\{{ }^{1} \mathrm{H}\right\} \mathbf{N M R}\left(121 \mathrm{MHz}, \mathrm{CDCl}_{3}\right): \delta$ 18.3; IR (neat): v 3464 (br), 2982 (w), 1712 (w), 1609 (m), 1575 (w), 1445 (m), 1392 (w), 1245 (s), 1164 (m), 1050 (s), 1024 (vs), $958(\mathrm{~s}), 835(\mathrm{~s}), 790(\mathrm{~m}), 753(\mathrm{~s}), 695(\mathrm{~m}), 612(\mathrm{~m}), 531(\mathrm{~m}) \mathrm{cm}^{-1}$. The obtained special data were in agreement with the literature. ${ }^{[5]}$

\subsection{Preparation and characterization of sulfone $(E)-55$}<smiles>CC(=O)c1ccccc1</smiles><smiles>CS(=O)(=O)c1ccccc1</smiles>

$$
\begin{aligned}
& \text { 1) } n \text {-BuLi (1.1 equiv.) } \\
& \text { THF, }-78^{\circ} \mathrm{C} \\
& \text { 2) DMAP (10 mol\%) } \\
& \mathrm{Et}_{3} \mathrm{~N} \text { (2 equiv.) } \\
& \text { TFAA (1.2 equiv.) } \\
& \text { DCM, } 0{ }^{\circ} \mathrm{C} \text { to r.t. }
\end{aligned}
$$<smiles>CC(=CS(=O)(=O)c1ccccc1)c1ccccc1</smiles>

$(E)-55$

(E)-((2-phenylprop-1-en-1-yl)sulfonyl)benzene $(E)-55$ was prepared according to the reported method. ${ }^{[6]}$ 
In an oven-dried $250 \mathrm{~mL}$ round-bottom flask was charged methyl phenyl sulfone $(1.72 \mathrm{~g}, 11$ mmol, 1.1 equiv.). Dry THF was added $(60 \mathrm{~mL})$ under an argon atmosphere and the reaction mixture was cooled to $-78^{\circ} \mathrm{C}$. Then, $n$-BuLi $(2.5 \mathrm{M}$ in hexanes, $4.4 \mathrm{~mL}, 1.1$ equiv.) was added dropwise. The reaction mixture was stirred for $30 \mathrm{~min}$ and acetophenone $(1.17 \mathrm{~mL}, 10 \mathrm{mmol}$, 1 equiv.) was added dropwise at $-78^{\circ} \mathrm{C}$. The reaction mixture was stirred for $1 \mathrm{~h}$ at $-78^{\circ} \mathrm{C}$, and the mixture was quenched with a saturated aqueous $\mathrm{NH}_{4} \mathrm{Cl}$ solution $(30 \mathrm{~mL})$. Aqueous layer was extracted with EtOAc $(3 \times 40 \mathrm{~mL})$ and the combined organic layers were washed with brine $(50 \mathrm{~mL})$, dried over $\mathrm{Na}_{2} \mathrm{SO}_{4}$ and concentrated in vacuo. The crude residue and DMAP (122 mg, $1 \mathrm{mmol}, 10 \mathrm{~mol} \%)$ were dissolved in dry $\mathrm{DCM}(30 \mathrm{~mL})$ and cooled to $0{ }^{\circ} \mathrm{C}$. Distilled Triethylamine ( $2.7 \mathrm{~mL}, 20 \mathrm{mmol}, 2$ equiv.) and TFAA (1.67 mL, $12 \mathrm{mmol}, 1.2$ equiv.) were added. The reaction mixture was allowed to warm to room temperature and stirred overnight. Then, a saturated aqueous $\mathrm{NH}_{4} \mathrm{Cl}$ solution $(20 \mathrm{~mL})$ was added. The aqueous layer was extracted with DCM $(3 \times 30 \mathrm{~mL})$. The combined organic layers were washed with brine $(50$ $\mathrm{mL}$ ), dried over $\mathrm{Na}_{2} \mathrm{SO}_{4}$ and concentrated in vacuo.

\section{(E)-((2-phenylprop-1-en-1-yl)sulfonyl)benzene ((E)-55):}

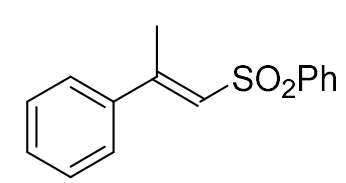

$(E)-55$

Obtained as a white solid (1.55 g, 60\% yield) after column chromatography on silica gel (PE :EtOAc from 100:0 to 93:7). Rf: 0.12 (PE:EtOAC ; 95:5); Mp: $83-84{ }^{\circ} \mathrm{C} ;{ }^{1} \mathrm{H}$ NMR (300 MHz, $\left.\mathrm{CDCl}_{3}\right): \delta 8.00-$ $7.97(\mathrm{~m}, 2 \mathrm{H}), 7.66-7.54(\mathrm{~m}, 3 \mathrm{H}), 7.43-7.33(\mathrm{~m}, 5 \mathrm{H}), 6.62(\mathrm{~m}, 1 \mathrm{H}), 2.54$ $(\mathrm{d}, 3 \mathrm{H}, J=1.0 \mathrm{~Hz}) ;{ }^{13} \mathrm{C}\left\{{ }^{1} \mathrm{H}\right\}$ NMR $\left(75 \mathrm{MHz}, \mathrm{CDCl}_{3}\right): \delta 153.6,142.2,140.1$, 133.3, 130.0, $129.3(2 \mathrm{C}), 128.8(2 \mathrm{C}), 127.5,127.3(2 \mathrm{C}), 126.4(2 \mathrm{C}), 17.3$; IR (neat): v 3060 (w), $1607(w), 1572(w), 1446(w), 1303(\mathrm{~m}), 1147(\mathrm{~s}), 1086(\mathrm{~m}), 847(\mathrm{~m}), 802(\mathrm{~m}), 759(\mathrm{~s}), 750(\mathrm{~s})$, $714(\mathrm{~m}), 683(\mathrm{~s}), 624(\mathrm{~s}), 572(\mathrm{~s}), 555(\mathrm{~m}), 539(\mathrm{vs}), 449(\mathrm{w}) \mathrm{cm}^{-1}$. The obtained special data were in agreement with the literature. ${ }^{[6]}$ 


\section{Optimization}

\subsection{Lewis Acid screening}<smiles>CC(=Cc1ccccc1)C(=O)N1CCOC1=O</smiles>

(E)-4

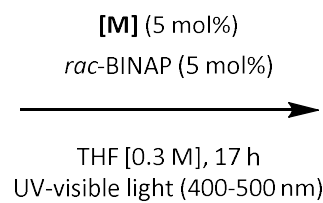

UV-visible light (400-500 nm

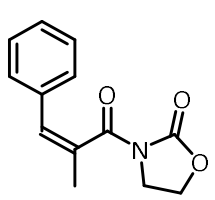

(Z)-4

\begin{tabular}{ccc}
\hline Entry & {$[\mathrm{M}]$} & Ratio $E / Z$ \\
\hline 1 & $\mathrm{Cu}(\mathrm{OAc})_{2}$ & $15: 85$ \\
2 & $\mathrm{PdCl}{ }_{2}$ & $100: 0$ \\
3 & $\mathrm{Pd}(\mathrm{OAc})_{2}$ & $100: 0$ \\
4 & $\mathrm{ZnCl}$ & $99: 1$ \\
5 & $\mathrm{Ni}(\mathrm{OAc})_{2} \cdot 4 \mathrm{H}_{2} \mathrm{O}$ & $100: 0$ \\
6 & $\mathrm{Mn}(\mathrm{OAC})_{2} .2 \mathrm{H}_{2} \mathrm{O}$ & $100: 0$ \\
\hline \multicolumn{3}{c}{ Table $1:$ Study of the metal effect }
\end{tabular}

[Isomerization using Ribolfavin as organophotocatalyst] ${ }^{[7]}$<smiles>CC(=Cc1ccccc1)C(=O)N1CCOC1=O</smiles>

(E)-4

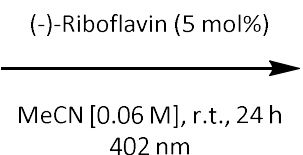

$402 \mathrm{~nm}$

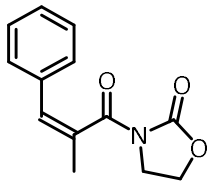

(Z)-4

\subsection{Ligand Effect}<smiles>CC(=Cc1ccccc1)C(=O)N1CCOC1=O</smiles>

(E)-4

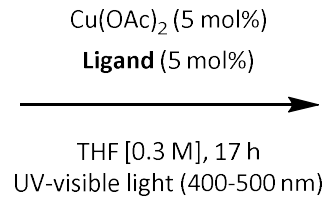

UV-visible light (400-500 $\mathrm{nm}$ )

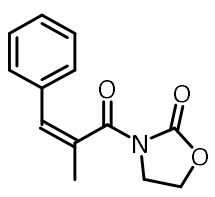

(Z) -4

\begin{tabular}{ccc}
\hline Entry & Ligand & Ratio $E / Z$ \\
\hline 1 & BINAP-rac & $15: 85$ \\
2 & XantPhos & $100: 0$ \\
3 & Dppe & $100: 0$ \\
4 & Dppf & $100: 0$ \\
5 & DpePhos & $100: 0$ \\
6 & (S)-p-tol-BINAP & $17: 83$ \\
7 & Dppbz & $36: 64$ \\
8 & $1,10-$ Phenanthroline & $100: 0$ \\
9 & Bathocuproin & $100: 0$ \\
10 & (R)-DM-SEGPHOS & $73: 27$ \\
$11^{*}$ & PPh 3 & $100: 0$ \\
$12^{*}$ & L1 & $81: 19$ \\
$13^{*}$ & L2 & $100: 0$ \\
14 & - & $100: 0$ \\
\hline \multicolumn{3}{r}{ 10 mol\% of ligand was used }
\end{tabular}




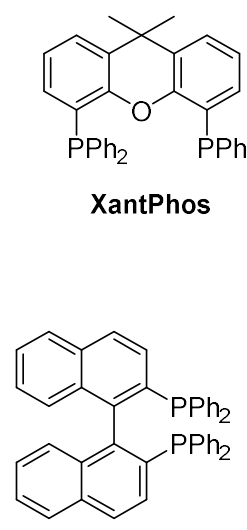

rac-BINAP

$$
\mathrm{Ph}_{2} \mathrm{P} \frown \mathrm{PPh}_{2}
$$

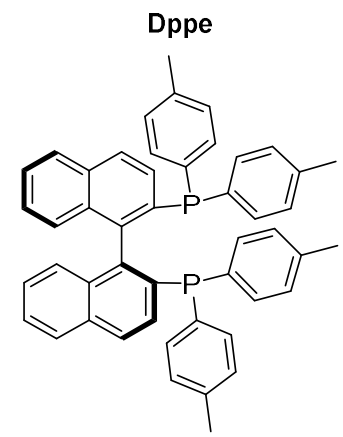

(S)-Tol-BINAP

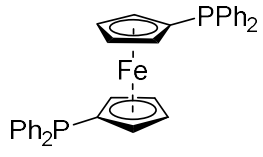

Dppf<smiles>Pc1ccccc1Pc1cccc(P)c1P</smiles>

DpePhos<smiles>C=C(/C(=C(\C)OCC)c1c(P(c2cc(C)cc(C)c2)c2ccc3c(c2)OCO3)ccc2c1OCO2)P(c1cc(C)cc(C)c1)C(C)C</smiles>

(R)-DM-SEGPHOS Dppbz<smiles></smiles>

L2<smiles></smiles>

L1<smiles>c1cnc2c(c1)ccc1cccnc12</smiles><smiles>Cc1cc(P)c2ccc3c(P)cc(P)nc3c2n1</smiles>

Bathocuproine

Table 2 : Study of the ligand

\subsection{Source of copper \& stoichiometry}

\begin{tabular}{|c|c|c|c|}
\hline & $\frac{\text { [C }}{r a c-1}$ & 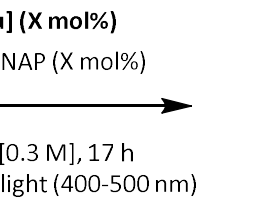 & \\
\hline Entry & {$[\mathrm{Cu}]$} & Catalyst loading & Ratio $E / Z$ \\
\hline 1 & & $5 \mathrm{~mol} \%$ & $15: 85$ \\
\hline 2 & $\mathrm{Cu}(\mathrm{OAc})_{2}$ & $2.5 \mathrm{~mol} \%$ & $17: 83$ \\
\hline 3 & & $10 \mathrm{~mol} \%$ & $16: 84$ \\
\hline 4 & $\mathrm{Cu}(\mathrm{OTf})_{2}$ & $5 \mathrm{~mol} \%$ & $20: 80$ \\
\hline 5 & {$\left[\mathrm{CuPF}_{6} \cdot(\mathrm{MeCN})_{4}\right]$} & $5 \mathrm{~mol} \%$ & $15: 85$ \\
\hline 6 & CuOAc & 5 mol\% & $18: 82$ \\
\hline 7 & $\mathrm{CuCl}$ & $5 \mathrm{~mol} \%$ & $18: 82$ \\
\hline 8 & $\mathrm{CuCl}_{2}$ & $5 \mathrm{~mol} \%$ & $93: 7$ \\
\hline 9 & CuSCN & $5 \mathrm{~mol} \%$ & 19:81 \\
\hline 10 & {$\left[\mathrm{CuBF}_{4 \cdot} \cdot(\mathrm{MeCN})_{4}\right]$} & $5 \mathrm{~mol} \%$ & $16: 84$ \\
\hline
\end{tabular}




\subsection{Wavelength Effect}<smiles>CC(=Cc1ccccc1)C(=O)N1CCOC1=O</smiles>

(E)-4

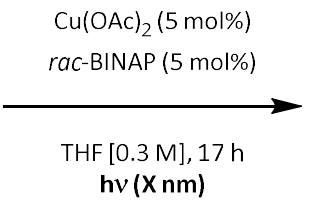

$\mathrm{h} v(\mathrm{Xnm})$

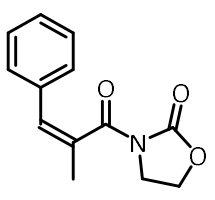

(Z)-4

\begin{tabular}{ccc}
\hline Entry & Wavelength & Ratio $E / Z$ \\
\hline 1 & $400-500 \mathrm{~nm}$ & $15: 85$ \\
2 & $405 \mathrm{~nm}$ & $23: 77$ \\
3 & $425 \mathrm{~nm}$ & $16: 84$ \\
4 & $450 \mathrm{~nm}$ & $18: 82$ \\
5 & $475-480 \mathrm{~nm}$ & $25: 75$ \\
6 & $365 \mathrm{~nm}$ & $14: 86$ \\
7 & White lamps & $50: 50$ \\
\hline \multicolumn{3}{l}{ Table 4: Study of the wavelength }
\end{tabular}

\subsection{Reaction Time and Concentration}

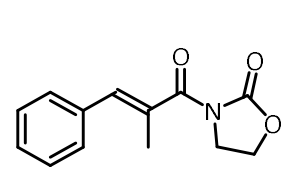

(E)-4

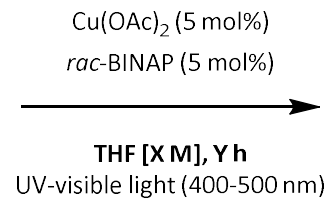

UV-visible light (400-500 nm)

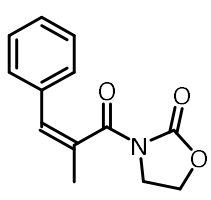

(Z)-4

\begin{tabular}{ccc}
\hline Entry & Reaction Time & Ratio $E / Z$ \\
\hline 1 & $2 \mathrm{~h}$ & $48: 52$ \\
2 & $4 \mathrm{~h}$ & $34: 66$ \\
3 & $6 \mathrm{~h}$ & $18: 82$ \\
4 & $8 \mathrm{~h}$ & $16: 84$ \\
5 & $12 \mathrm{~h}$ & $16: 84$ \\
6 & $17 \mathrm{~h}$ & $15: 85$ \\
7 & $24 \mathrm{~h}$ & $15: 85$ \\
\hline Table 5: Optimization of the reaction time
\end{tabular}

\begin{tabular}{ccc}
\hline Entry & Concentration & Ratio $E / Z$ \\
\hline 1 & $0.05 \mathrm{M}$ & $45: 55$ \\
2 & $0.15 \mathrm{M}$ & $33: 67$ \\
3 & $0.3 \mathrm{M}$ & $15: 85$ \\
4 & $0.6 \mathrm{M}$ & $16: 84$ \\
5 & $1 \mathrm{M}$ & $15: 85$ \\
\hline Table 6: Optimization of the concentration
\end{tabular}




\subsection{Template}

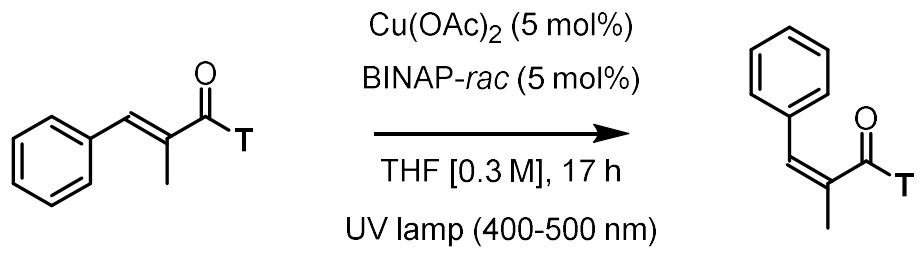<smiles>COC(=O)/C(C)=C/c1ccccc1</smiles>

$(E)-1$

Ratio $E: Z=29: 71$<smiles>C/C(=C\c1ccccc1)C(=O)N(C)C</smiles>

(E)-2<smiles>CCOP(=O)(OCC)C(=O)/C(C)=C/c1ccccc1</smiles>

(E)-3<smiles>C/C(=C\c1ccccc1)C(=O)N1CCOC1=O</smiles>

(E)-4

Ratio $E: Z=46: 54$<smiles>CC(=Cc1ccccc1)C(=O)N(C)c1ccccn1</smiles>

(E)-5<smiles>CC(=Cc1ccccc1)C(=O)N1CCc2cccnc21</smiles>

(E)-6<smiles>CC(=Cc1ccccc1)C(=O)n1cccn1</smiles>

(E)-7

Ratio $E: Z=17: 83$

Ratio $E: Z=22: 78$

Ratio $E: Z=24: 76$<smiles>CC(=Cc1ccccc1)C(=O)n1c(=O)oc2ccccc21</smiles>

$\mathrm{T} 1$

Ratio $E: Z=17: 83$<smiles>CC(=Cc1ccccc1)C(=O)N1CCCC1=O</smiles>

T2<smiles>CC(=Cc1ccccc1)C(=O)N1CCCCC1=O</smiles>

T3

Ratio $E: Z=18: 82$

Ratio $E: Z=15: 85$<smiles>CC(=Cc1ccccc1)C(=O)N1CCOC1=S</smiles>

T4

Ratio $E: Z=34: 66$<smiles>C/C(=C\c1ccccc1)C(=O)N1C(=O)CCN1Pc1ccccc1</smiles>

T5

Ratio $E: Z=20: 80$

\subsection{Solvent Effect}<smiles>CC(=Cc1ccccc1)C(=O)N1CCOC1=O</smiles>

(E)-4

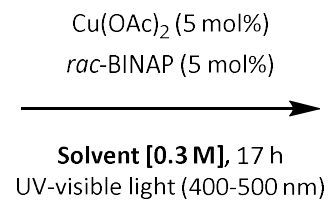

UV-visible light (400-500 nm)

\begin{tabular}{ccc}
\hline Entry & Solvent & Ratio $E / Z$ \\
\hline 1 & THF $^{*}$ & $15: 85$ \\
2 & MeCN $^{*}$ & $20: 80$ \\
3 & Toluene $^{*}$ & $15: 85$ \\
\hline
\end{tabular}

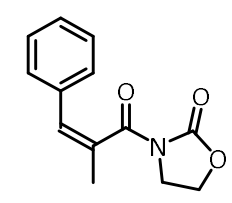

(Z)-4 


\begin{tabular}{ccc}
4 & THF/ $\mathrm{H}_{2} \mathrm{O} 9: 1$ & $19: 81$ \\
5 & Dimethylcarbonate & $14: 86$ \\
6 & DMF & $17: 83$ \\
7 & AcOEt $^{*}$ & $12: 88$ \\
8 & MTBE & $15: 85$ \\
9 & Chloroforme & $14: 86$ \\
10 & MeTHF & $16: 84$ \\
11 & iPrOAc & $15: 85$ \\
12 & DMF & $17: 83$ \\
13 & Acetone & $18: 82$ \\
14 & Dioxane & $20: 80$ \\
15 & DMSO & $20: 80$ \\
16 & Et ${ }_{2} \mathrm{O}$ & $24: 76$ \\
17 & DCM & $32: 68$ \\
\hline 18 & HFIP & $88: 12$ \\
19 & iPrOH & $49: 51$ \\
20 & Cyclohexane & $65: 35$ \\
21 & Cumene & $55: 45$ \\
22 & iPr ${ }_{2} \mathrm{O}$ & $72: 28$ \\
\hline \multicolumn{3}{c}{}
\end{tabular}

Table 7 : Optimization of the solvent

\subsection{Control experiments}<smiles>CC(=Cc1ccccc1)C(=O)N1CCCC1=O</smiles>

(E)-4

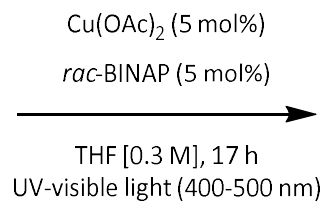

UV-visible light (400-500 nm)

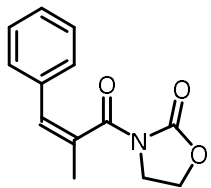

(Z)-4

\begin{tabular}{ccc}
\hline Entry & Deviation from the standard conditions & Ratio $E / Z$ \\
\hline 1 & - & $15: 85$ \\
2 & Dark & $100: 0$ \\
3 & Dark, $80^{\circ} \mathrm{C}$ & $100: 0$ \\
4 & Under air & $18: 82$ \\
5 & Degassed solvent & $16: 84$ \\
6 & No catalyst & $100: 0$ \\
\hline \multicolumn{3}{c}{}
\end{tabular}

\subsection{Unsuccessful substrates}<smiles>CC(=Cc1c(C)cc(C)cc1C)C(=O)N1CCOC1=O</smiles><smiles>CCCCCC=C(C)C(=O)N1CCOC1=O</smiles><smiles>CCOC(=O)/C=C(\C)c1cccnc1</smiles><smiles>O=C(/C=C/c1ccccc1)N1CCOC1=O</smiles>

$E: Z=92: 8$ $E: Z=40: 60$ 


\section{Sensitivity experiments}<smiles>C/C(=C\c1ccccc1)C(=O)N1CCOC1=O</smiles>

$($ E) -4

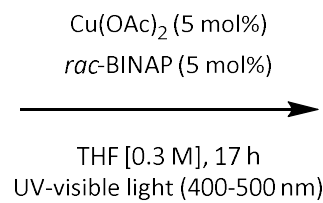

UV-visible light $(400-500 \mathrm{~nm})$

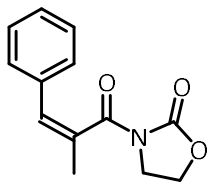

(Z)-4

The sensitivity assessment was conducted as described in Pitzer, L.; Schäfers, F.; Glorius, F. Angew. Chem. Int. Ed. 2019, 58, 8572.

The influence of water, oxygen, light intensity, temperature and concentration was investigated, as well as the scalability. While one parameter was varied, all other parameters were kept constant. The $E / Z$ isomer ratio was determined by integration of the ${ }^{1} \mathrm{H} N M R$ spectra of the crude mixture. The respective deviation was calculated in reference to a control reaction (ratio $E / Z=15: 85$ on $0.3 \mathrm{mmol}$ scale)

The results are shown in Table 9 and the respective Figure 1. The reaction showed to be overall rather robust. The diminution of the concentration appeared to have a negative effect (- $35 \%)$.

In summary, to reproduce the reaction, working in rather concentrated medium is important, while the reaction is not sensitive towards temperature change (Photo 2), lower light intensity (Photo 3), water, oxygen and also works at larger scale (Photo 4).

\begin{tabular}{|c|c|c|c|c|c|}
\hline Entry & Parameters & Conditions standards & Conditions studied & $(Z)-1 a$ & Deviation (\%) \\
\hline 1 & \multirow{2}{*}{ Concentration } & \multirow{2}{*}{$0.3 \mathrm{M}$} & High : $1 \mathrm{M}$ & $85 \%$ & 0 \\
\hline 2 & & & Low : $0.05 \mathrm{M}$ & $55 \%$ & -35 \\
\hline 3 & Medium & THF anhydrous & $\mathrm{THF} / \mathrm{H}_{2} \mathrm{O} 9: 1$ & $81 \%$ & -5 \\
\hline 4 & \multirow{2}{*}{ Atmosphere } & \multirow{2}{*}{ Under argon } & THF degassed & $84 \%$ & -1 \\
\hline 5 & & & Under air & $82 \%$ & -3 \\
\hline 6 & \multirow{3}{*}{ Temperature } & \multirow{3}{*}{$28^{\circ} \mathrm{C}$} & $15^{\circ} \mathrm{C}^{(\mathrm{a})}$ & $87 \%$ & 2 \\
\hline 7 & & & $40^{\circ} \mathrm{C}$ & $83 \%$ & -2 \\
\hline 8 & & & Control $^{(b)}$ & $85 \%$ & 0 \\
\hline 9 & \multirow{2}{*}{ Intensity (W) } & \multirow{2}{*}{$\mathrm{d}=2 \mathrm{~cm}$} & High : $d=1 \mathrm{~cm}$ & $85 \%$ & 0 \\
\hline 10 & & & Low : $\mathrm{d}=8 \mathrm{~cm}$ & $79 \%$ & -7 \\
\hline 11 & Scale & $0.3 \mathrm{mmol} / 1 \mathrm{~mL}$ & $3 \mathrm{mmol} / 10 \mathrm{~mL}$ & $86 \%$ & 1 \\
\hline
\end{tabular}

(a) The same result was obtained at $0{ }^{\circ} \mathrm{C}$. (b) A control experiment was crucial to see if the cryogenic liquid (water or propylene glycol) was changing the efficency of the reaction. This reaction was set up with double sided tube and water without cooling flow. 


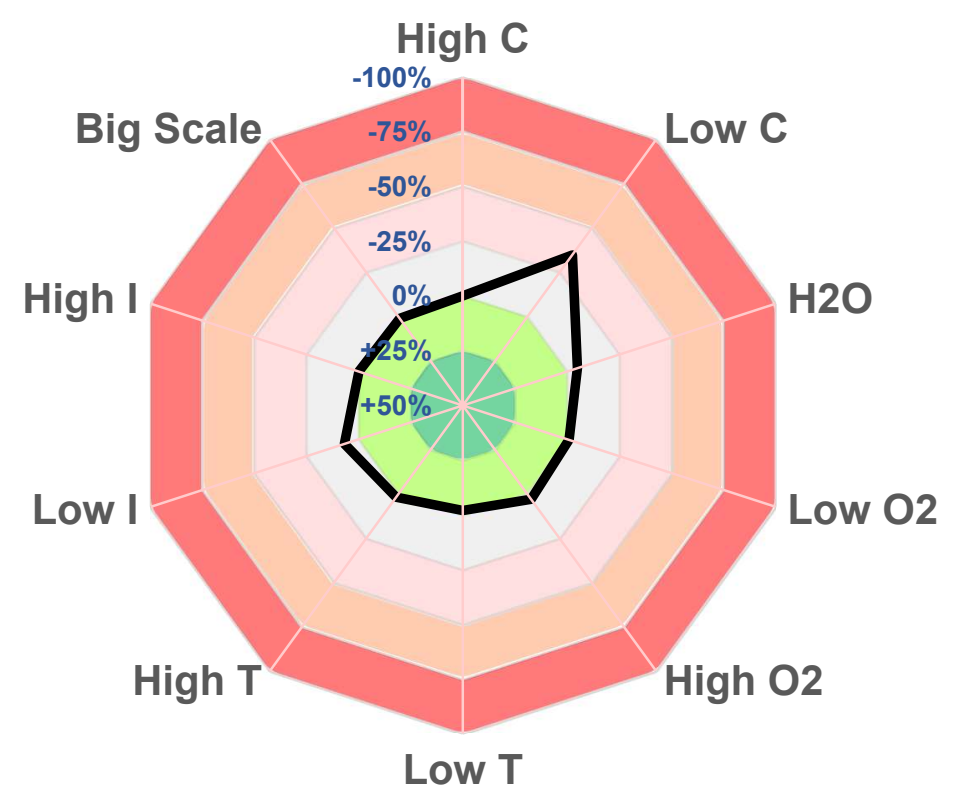

Figure 1 : Sensitivity assessment
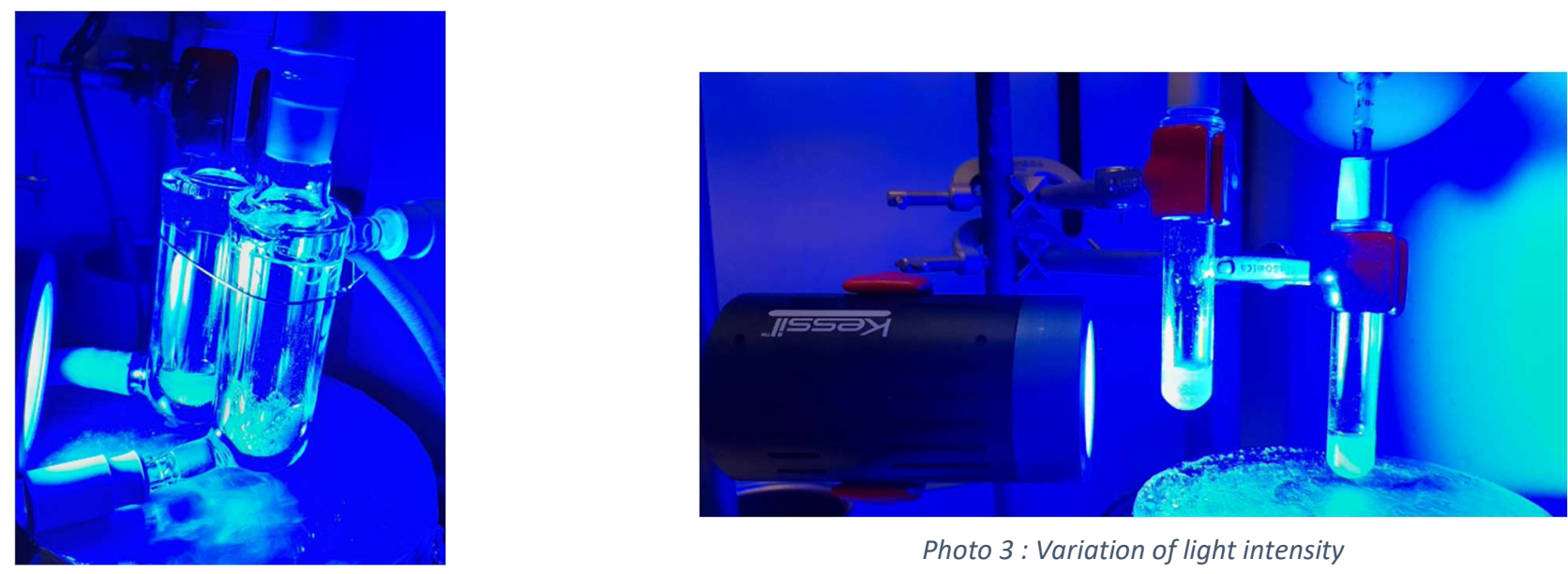

Photo 3 : Variation of light intensity

Photo 2 : Double sided tubes

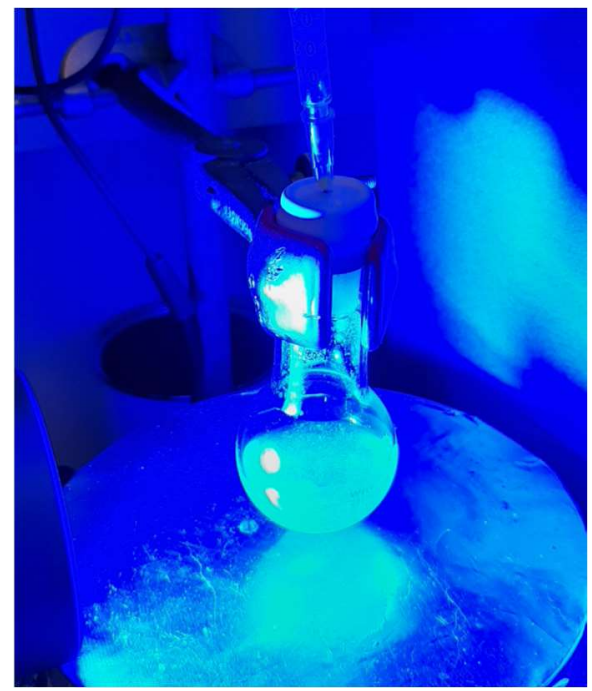

Photo 4 : Larger scale isomerization 


\section{Mechanistic Studies}

\subsection{UV-visible absorption measurements}

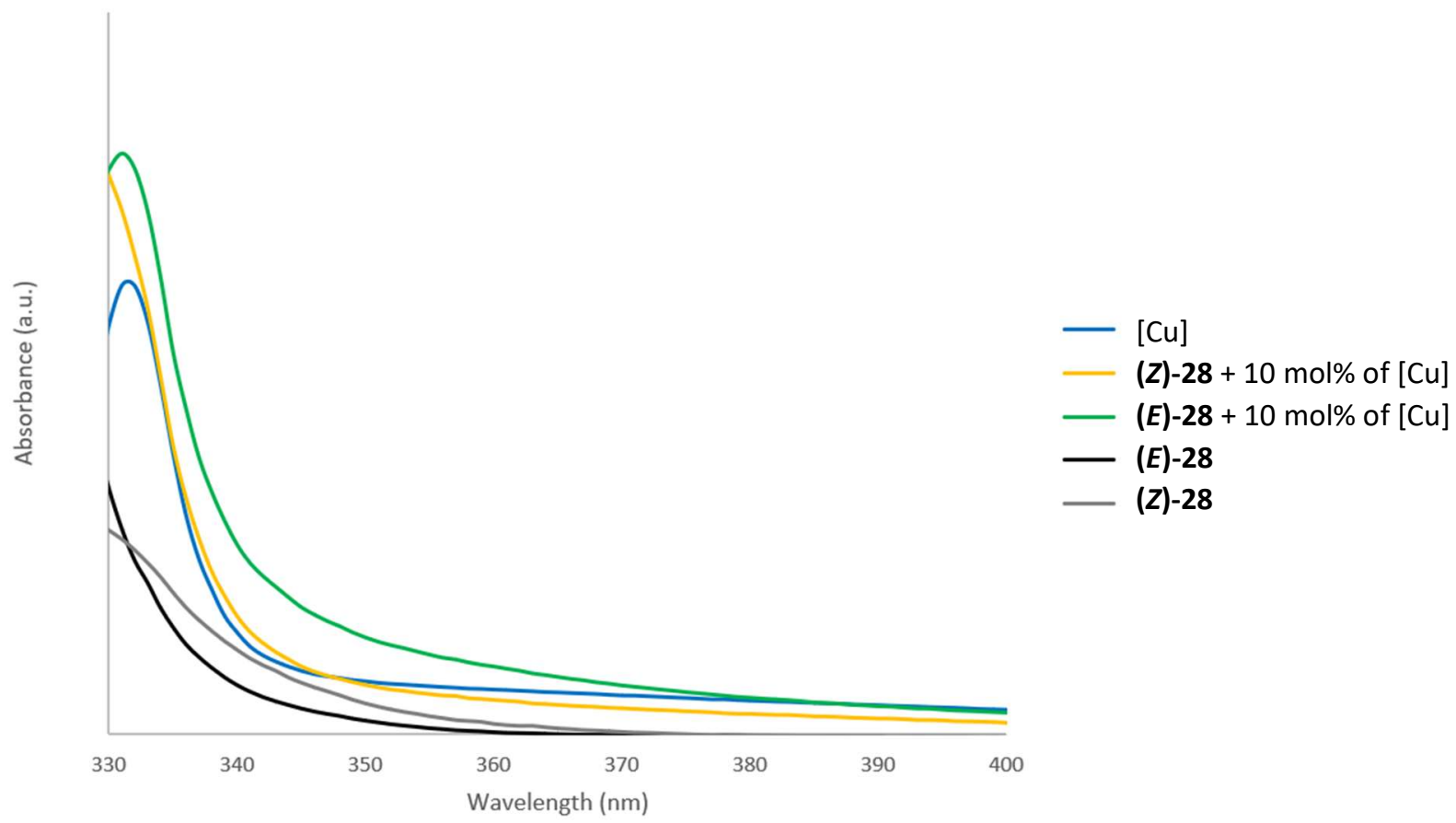

Figure 2 : UV-visible absorption spectra

The concentration of $(E)$ and $(Z)$-isomers was $1 \mathrm{mM}$ in THF. $[\mathrm{Cu}]=\mathrm{Cu}(\mathrm{OAc})_{2} / \mathrm{rac}$-BINAP (1:1).

\subsection{Addition of triplet and singlet quenchers}

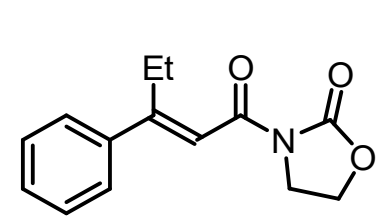

$(E)-31$

$$
\mathrm{Cu}(\mathrm{OAc})_{2}(5 \mathrm{~mol} \%)
$$

BINAP-rac (5 mol\%)

Additive

EtOAc $[0.3 \mathrm{M}]$

Blue light<smiles>CC/C(=C/C(=O)N1CCOC1=O)c1ccccc1</smiles>

$(Z)-31$

\begin{tabular}{cc}
\hline Additive & Ratio E: $\boldsymbol{Z}^{(a)}$ \\
\hline none & $5: 95$ \\
$\mathrm{O}_{2}$ & $5: 95$ \\
1,3-cyclohexadiene (1 equiv.) & $5: 95$ \\
Azulene (1 equiv.) & $88: 12$ \\
\hline
\end{tabular}

${ }^{(a)}$ Determined on the crude mixture by ${ }^{1} H N M R$

1,3-cyclohexadiene and $\mathrm{O}_{2}$ are known to quench triplet states and azulene is known to quench both singlet and triplet states. With these experiments, we can suggest a singlet state mechanism. 


\subsection{Isomerization quantum yields measurements}

\subsubsection{Determination of the photon flux}

Following literature procedures of Yoon ${ }^{[8]}$ and Glorius ${ }^{[9]}$, the photon flux of the UV-lamp KESSIL ${ }^{\mathrm{TM}} \mathrm{H} 150\left(34 \mathrm{~W}, \lambda_{\max }=450 \mathrm{~nm}\right)$ was determined by standard ferrioxalate actinometry. A solution of ferrioxalate $(0.15 \mathrm{M})$ was prepared by dissolving potassium ferrioxalate hydrate (737 mg) in $\mathrm{H}_{2} \mathrm{SO}_{4}$ aq. solution $(0.05 \mathrm{M}, 10 \mathrm{~mL}$ ). A buffered solution of 1,10-phenanthroline was prepared by dissolving 1,10-phenanthroline $(20 \mathrm{mg})$ and sodium acetate $(4.5 \mathrm{~g})$ in $\mathrm{H}_{2} \mathrm{SO}_{4}$ aq. solution $(0.5 \mathrm{M}, 20 \mathrm{~mL})$. Both solutions were stored in the dark.

To determine the photon flux of the lamp, the ferrioxalate solution $(1000 \mu \mathrm{L})$ was placed in a microwave tube and irradiated for $90 \mathrm{~s}$ at $\lambda_{\max }=450 \mathrm{~nm}$. After irradiation, the phenanthroline solution $(175 \mu \mathrm{L})$ was added and the mixture was stirred for $1 \mathrm{~h}$ in the dark. The solution was transfered to a quartz cuvette and the absorption of the solution was measured at $510 \mathrm{~nm}$. A non-irradiated sample was prepared and the absorption was measured. The average of the absorption of 3 experiments (irradiated and non-irradiated) was determined and used to calculate the conversion using (eq. A).

$$
n\left(F e^{2+}\right)=\frac{V . \Delta A(510 \mathrm{~nm})}{l . \varepsilon} \quad \text { (eq.A) }
$$

In our case, $V$ is the total volume $(0.001175 \mathrm{~L})$ of the solution after addition of buffered solution. $\Delta A$ is the difference in absorption at $510 \mathrm{~nm}$ between the irradiated and nonirradiated solutions, $I$ is the path length $(1.0 \mathrm{~cm})$, and $\varepsilon$ is the molar absorption coefficient of the ferrioxalate actinometer at $510 \mathrm{~nm}\left(11100 \mathrm{~L}^{-\mathrm{cm}^{-1}} \cdot \mathrm{mol}^{-1}\right) \cdot \mathrm{n}\left(\mathrm{Fe}^{2+}\right)$ was calculated to be 2.57 $\times 10^{-7} \mathrm{~mol}$. The photon flux $\left(\phi_{\mathrm{q}}\right)$ can be calculated using (eq. $\left.B\right)$.

$$
\varphi_{q}=\frac{n\left(F e^{2+}\right)}{\varphi_{F e r} . t . f} \quad \text { (eq.B) }
$$

Here, $\phi_{\mathrm{Fer}}$ is the photoreaction quantum yield for the ferrioxalate actinometer (1.01 at $\lambda_{\mathrm{ex}}=$ $450 \mathrm{~nm}), \mathrm{t}$ is the irradiation time $(90 \mathrm{~s})$, and $\mathrm{f}$ is the fraction of light absorbed at $\lambda_{\mathrm{ex}}=450 \mathrm{~nm}$ by the ferrioxalate actinometer. This value is calculated using (eq. C) where $A(450 \mathrm{~nm})$ is the absorption of the ferrioxalate solution at $450 \mathrm{~nm}$. An absorption spectrum gave an $\mathrm{A}(450 \mathrm{~nm})$ value of 1.55 , indicating that the fraction of absorbed light (f) is 0.972 .

$$
\left.f=1-10^{-A(450 \mathrm{~nm})} \quad \text { (eq. } \mathrm{C}\right)
$$




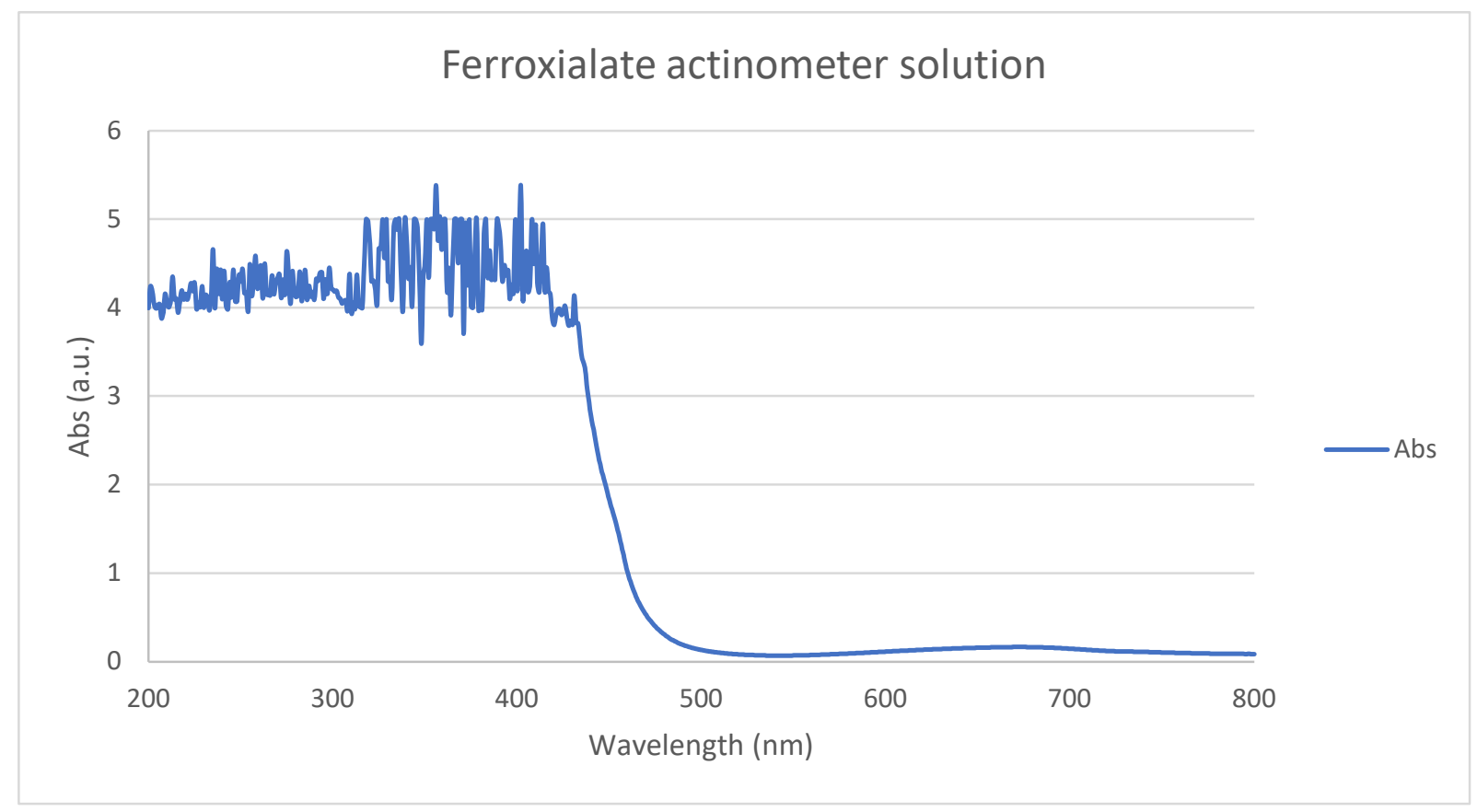

Figure 3 : UV-visible absorption spectra of ferrioxalate actinometer solution

Finally, the photon flux was determined (average of 3 experiments) to be $\mathbf{2 . 9 1} \times \mathbf{1 0 ^ { - 9 }}$ einstein. $s^{-1}$

\subsubsection{Determination of isomerization quantum yield}

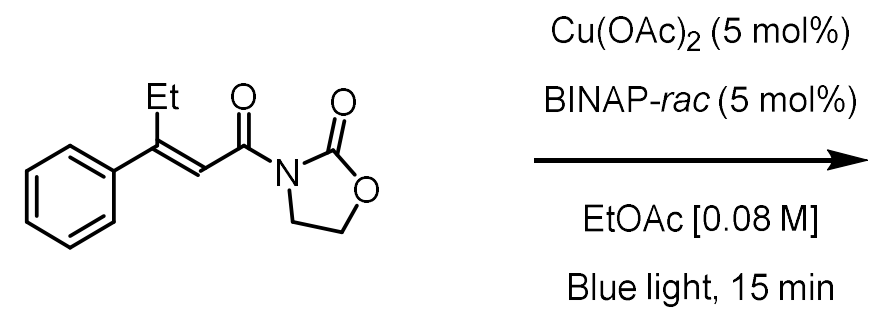

(E)-31<smiles>CC/C(=C/C(=O)N1CCOC1=O)c1ccccc1</smiles>

$(Z)-31$

The quantum yield was calculated following general procedure $\mathbf{D}$ at $0.08 \mathrm{M}$ for $15 \mathrm{~min}$. After irradiation, the yield was determined by GC-FID analysis. The yield was determined to be 2.84 \% (average from 3 parallel experiments giving $2.83 \%, 2.57 \%$ and $3.13 \%$ ) meaning $2.72 \times 10^{-6}$ mol.

The quantum yield was determined using (eq. D) where the photon flux $\phi_{\mathrm{q}}$ was $2.91 \times 10^{-9}$ einstein. $\mathrm{s}^{-1}, t$ was the reaction time $(900 \mathrm{~s})$, and $f_{\mathrm{R}}$ the fraction of absorbed incident light (determined using eq. C). At $450 \mathrm{~nm}$, the measured absorption was 1.45 a.u. giving 0.964 for $f_{\mathrm{R}}$.

$$
\varphi=\frac{n(\text { product })}{\varphi_{q} \cdot t \cdot f_{R}} \quad(\text { eq.D) }
$$

Finally, the reaction quantum yield $\phi_{\mathrm{E} \rightarrow \mathrm{z}}$ was determined to be $\phi_{\mathrm{E} \rightarrow \mathrm{Z}}=\mathbf{0 . 9 0}$.

The same procedure was repeated starting from the Z-isomer to calculate the reaction quantum yield $\phi_{\mathrm{Z} \rightarrow \mathrm{E}}$. 
After 3 experiments, the reaction quantum yield $\phi_{Z \rightarrow E}$ was determined to be $\phi_{Z \rightarrow E}=\mathbf{0}$. (no conversion was observed, even after prolonged reaction time: $24 \mathrm{~h}$ ) 


\section{Isomerization of cinnamates derivatives}

\subsection{Material}

$\mathrm{Cu}(\mathrm{OAc})_{2}$ was purchased from Sigma-Aldrich and rac-BINAP from Acros Organics. Both were stored in a glovebox and used as received.

\subsection{General Procedure D}

An oven-dried microwave tube equipped with a magnetic stir bar was charged with $\mathrm{Cu}(\mathrm{OAc})_{2}$ ( $2.7 \mathrm{mg}, 0.015 \mathrm{mmol}, 0.05$ equiv.) and rac-BINAP ( $9.3 \mathrm{mg}, 0.015 \mathrm{mmol}, 0.05$ equiv.) in a glovebox and sealed with a septum. Then, the corresponding unsaturated compound $(0.3$ mmol, 1 equiv.) was added and the tube was evacuated and back-filled with argon 3 times. Then, dry EtOAc ( $1 \mathrm{~mL}$ ) was added by syringe and the reaction mixture was stirred for $17 \mathrm{~h}$ (unless otherwise stated) under visible light irradiation $(400-500 \mathrm{~nm})$. Then, the residual catalyst was removed by filtration through a short plug of silica and silica was rinsed with $\mathrm{Et}_{2} \mathrm{O}$ and the solution was concentrated in vacuo. The yield was determined by mass recovery based on NMR-purity and the E-/Z-isomer ratio was determined by GC-FID analysis (unless otherwise stated).

\section{[Large-scale, $3 \mathrm{mmol}]$}

An oven-dried $25 \mathrm{~mL}$ round-bottom flask equipped with a magnetic stir bar was charged with $\mathrm{Cu}(\mathrm{OAc})_{2}$ (27.2 mg, $0.15 \mathrm{mmol}, 0.05$ equiv.) and rac-BINAP (93.4 mg, $0.015 \mathrm{mmol}, 0.05$ equiv.) in a glovebox and sealed with a septum. Then, the amide (E)-4 (693 mg, 3 mmol, 1 equiv.) was added and the tube was evacuated and back-filled with argon 3 times. Then, dry THF $(10 \mathrm{~mL})$ was added by syringe and the reaction mixture was stirred for $17 \mathrm{~h}$ (unless otherwise stated) under visible light irradiation (400-500 $\mathrm{nm}$ ). Then, the residual catalyst was removed by filtration through a short plug of silica and silica was rinsed with $\mathrm{Et}_{2} \mathrm{O}$ and the solution was concentrated in vacuo. The yield was determined by mass recovery based on NMR-purity and the $E$-/Z-isomer ratio was determined by GC-FID analysis (unless otherwise stated).

\subsection{Characterization of (Z)-1 to (Z)-7 and T1 to T5}

\section{methyl (Z)-2-methyl-3-phenylacrylate ((Z)-1):}

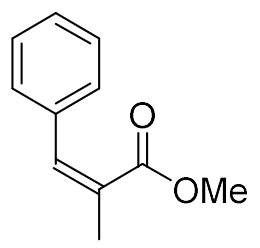

$(Z)-1$

Prepared according to general procedure $\mathbf{D}$. Obtained as a slightly yellow oil (52 mg, quant. yield, $E / Z=29: 71$ determined by ${ }^{1} \mathrm{H} N M R$ ) after filtration over a plug of silica $\left(\mathrm{Et}_{2} \mathrm{O}\right) .{ }^{1} \mathbf{H}$ NMR $\left(300 \mathrm{MHz}, \mathrm{CDCl}_{3}\right): \delta 7.36-7.23(\mathrm{~m}, 5 \mathrm{H}), 6.72$ $(\mathrm{m}, 1 \mathrm{H}), 3.66(\mathrm{~s}, 3 \mathrm{H}), 2.11(\mathrm{~d}, 3 \mathrm{H}, J=1.4 \mathrm{~Hz}) ;{ }^{13} \mathrm{C}\left\{{ }^{1} \mathrm{H}\right\}$ NMR $\left(75 \mathrm{MHz}, \mathrm{CDCl}_{3}\right): \delta$ $170.2,134.7,129.7,128.5,128.2$ (2C), 128.1 (2C), 127.7, 51.7, 21.6. 
(Z)-N,N,2-trimethyl-3-phenylacrylamide ((Z)-2):<smiles>C/C(=C/c1ccccc1)C(=O)N(C)C</smiles>

$(Z)-2$

Prepared according to general procedure D. Obtained as an orange oil (56 mg, quant. yield, $E / Z=46: 54$ determined by ${ }^{1} \mathrm{H} N \mathrm{NMR}$ ) after filtration over a plug of silica (Et $\left.{ }_{2} \mathrm{O}\right) .{ }^{1} \mathbf{H}$ NMR (300 MHz, $\left.\mathrm{CDCl}_{3}\right): \delta 7.32-7.19(\mathrm{~m}, 5 \mathrm{H}), 6.38(\mathrm{~m}, 1 \mathrm{H})$, $2.95(\mathrm{~s}, 3 \mathrm{H}), 2.72(\mathrm{~s}, 3 \mathrm{H}), 2.09(\mathrm{~s}, 3 \mathrm{H}) ;{ }^{13} \mathrm{C}\left\{{ }^{1} \mathrm{H}\right\}$ NMR (75 MHz, $\left.\mathrm{CDCl}_{3}\right): \delta$ 172.3, $136.2,133.0,128.4$ (2C), 127.8, 127.5 (2C), 127.5, 37.2, 34.3, 22.0.

diethyl (Z)-(2-methyl-3-phenylacryloyl)phosphonate ((Z)-3):<smiles>CCOP(=O)(OCC)C(=O)/C(C)=C\c1ccccc1</smiles>

(Z)-3

Prepared according to general procedure $D$. Obtained as a slightly yellow oil (84 mg, quant. yield, $E / Z=28: 72$ determined by ${ }^{1} \mathrm{H} N M R$ ) after filtration over a plug of silica $\left(\mathrm{Et}_{2} \mathrm{O}\right) .{ }^{1} \mathrm{H} \mathbf{~ N M R}\left(300 \mathrm{MHz} \mathrm{CDCl}_{3}\right)$ : $\delta 7.35-7.30(\mathrm{~m}, 3 \mathrm{H})$, $7.24-7.22(\mathrm{~m}, 2 \mathrm{H}), 6.82(\mathrm{~m}, 1 \mathrm{H}), 4.11-3.93(\mathrm{~m}, 4 \mathrm{H}), 2.16(\mathrm{~s}, 3 \mathrm{H}), 1.23(\mathrm{t}$, $6 \mathrm{H}, J=7.0 \mathrm{~Hz}) ;{ }^{13} \mathrm{C}\left\{{ }^{1} \mathrm{H}\right\}$ NMR $\left(75 \mathrm{MHz}, \mathrm{CDCl}_{3}\right): \delta 174.2(\mathrm{~d}, J=13.3 \mathrm{~Hz}), 141.2$, $137.5,135.9$ (d, $J=28.9 \mathrm{~Hz}), 130.0,128.8(\mathrm{~d}, J=2.8 \mathrm{~Hz}), 128.5(\mathrm{~d}, 2 \mathrm{C}, J=2.0$ $\mathrm{Hz}), 128.2(2 \mathrm{C}), 21.6,13.9 ;{ }^{31} \mathrm{P}\left\{{ }^{1} \mathrm{H}\right\} \mathrm{NMR}\left(121 \mathrm{MHz}, \mathrm{CDCl}_{3}\right): \delta 22.7$.

(Z)-3-(2-methyl-3-phenylacryloyl)oxazolidin-2-one ((Z)-4):<smiles>C/C(=C/c1ccccc1)C(=O)N1CCOC1=O</smiles>

$(Z)-4$

Prepared according to general procedure D. Obtained as a white solid (67 $\mathrm{mg}$, quant. yield, $E / Z=15: 85$ in THF determined by ${ }^{1} \mathrm{H} N M R$ and 11:89 in EtOAc) after filtration over a plug of silica $\left(\mathrm{Et}_{2} \mathrm{O}\right)$. Rf: 0.24 (PE:EtOAc ; 7:3); ${ }^{1} \mathbf{H}$ NMR (300 MHz, $\left.\mathrm{CDCl}_{3}\right): \delta 7.31-7.24(\mathrm{~m}, 3 \mathrm{H}), 7.20-7.17(\mathrm{~m}, 2 \mathrm{H}), 6.61$ $(\mathrm{m}, 1 \mathrm{H}), 4.17(\mathrm{t}, 2 \mathrm{H}, J=7.7 \mathrm{~Hz}), 3.92(\mathrm{t}, 2 \mathrm{H}, J=7.8 \mathrm{~Hz}), 2.16(\mathrm{~d}, 3 \mathrm{H}, J=1.1$ $\mathrm{Hz}) ;{ }^{13} \mathrm{C}\left\{{ }^{1} \mathrm{H}\right\}$ NMR (75 MHz, $\left.\mathrm{CDCl}_{3}\right): \delta 171.2,152.0,136.2,132.0,130.6$, $128.4(2 \mathrm{C}), 127.7,127.6(2 \mathrm{C}), 62.3,42.6,21.0$.

(Z)-N,2-dimethyl-3-phenyl-N-(pyridin-2-yl)acrylamide ((Z)-5):

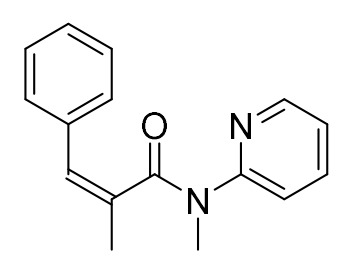

$(Z)-5$

Prepared according to general procedure $\mathbf{D}$. Obtained as a colorless oil (75 mg, quant. yield, $E / Z=17: 83$ determined by ${ }^{1} \mathrm{H} N M R$ ) after filtration over a plug of silica $\left(\mathrm{Et}_{2} \mathrm{O}\right) .{ }^{1} \mathbf{H}$ NMR (300 MHz, $\left.\mathrm{CDCl}_{3}\right)$ : $\delta 8.31-8.26(\mathrm{~m}$, $1 \mathrm{H}), 7.47-7.38(\mathrm{~m}, 1 \mathrm{H}), 7.30-7.03(\mathrm{~m}, 7 \mathrm{H}), 6.34-6.09(\mathrm{~m}, 1 \mathrm{H}), 3.29(\mathrm{~s}$, $3 \mathrm{H}), 2.15(\mathrm{~s}, 3 \mathrm{H}) ;{ }^{13} \mathrm{C}\left\{{ }^{1} \mathrm{H}\right\}$ NMR (75 MHz, $\left.\mathrm{CDCl}_{3}\right): \delta 161.5,154.3,147.8$, $137.1,136.2,133.6,128.9,128.2,127.6$ (2C), 127.4 (2C), 121.1, 120.3, 34.7, 22.3.

\section{(Z)-1-(2,3-dihydro-1H-pyrrolo[2,3-b]pyridin-1-yl)-2-methyl-3-phenylprop-2-en-1-one ((Z)-} 6):

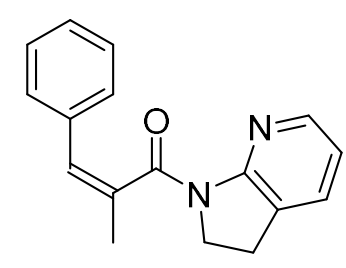

(Z)-6

Prepared according to general procedure $\mathbf{D}$. Obtained as a white solid (79 mg, quant. yield, $E / Z=22: 78$ determined by ${ }^{1} \mathrm{H} N M R$ ) after filtration over a plug of silica (Et $\left.{ }_{2} \mathrm{O}\right) .{ }^{1} \mathbf{H}$ NMR (300 MHz, benzene-d6): $\delta 7.91(\mathrm{~d}$, $1 \mathrm{H}, J=5.0 \mathrm{~Hz}), 7.31(\mathrm{~d}, 2 \mathrm{H}, J=7.3 \mathrm{~Hz}), 7.01-6.96(\mathrm{~m}, 2 \mathrm{H}), 6.90-6.87$ $(\mathrm{m}, 1 \mathrm{H}), 6.49(\mathrm{dd}, 1 \mathrm{H}, J=7.2 \& 1.2 \mathrm{~Hz}), 6.40(\mathrm{~m}, 1 \mathrm{H}), 6.21(\mathrm{dd}, 1 \mathrm{H}, J=7.4$ \& $5.1 \mathrm{~Hz}), 3.78(\mathrm{~m}, 2 \mathrm{H}), 2.34(\mathrm{~d}, 3 \mathrm{H}, J=1.3 \mathrm{~Hz}), 1.99(\mathrm{t}, 2 \mathrm{H}, J=8.3 \mathrm{~Hz})$; 
${ }^{13} \mathrm{C}\left\{{ }^{1} \mathrm{H}\right\}$ NMR (75 MHz, $\left.\mathrm{CDCl}_{3}\right): \delta 170.1,146.6,134.7,132.9(2 \mathrm{C}), 129.4,128.2,128.1,127.3$ (2C), 126.9, 125.6, 118.5, 46.0, 24.7, 21.7.

(Z)-2-methyl-3-phenyl-1-(1H-pyrazol-1-yl)prop-2-en-1-one ((Z)-7):<smiles>C/C(=C/c1ccccc1)C(=O)n1cccn1</smiles>

(Z)-7

Prepared according to general procedure D. Obtained as a colorless oil (63 $\mathrm{mg}$, quant. yield, $E / Z=24: 76$ determined by ${ }^{1} \mathrm{H} N M R$ ) after filtration over a plug of silica (Et $\left.{ }_{2} \mathrm{O}\right) .{ }^{1} \mathrm{H}$ NMR (300 MHz, $\left.\mathrm{CDCl}_{3}\right): \delta 8.22(\mathrm{~d}, 1 \mathrm{H}, J=2.6 \mathrm{~Hz}), 7.66$ $(\mathrm{m}, 1 \mathrm{H}), 7.20-7.12(\mathrm{~m}, 5 \mathrm{H}), 6.80(\mathrm{~m}, 1 \mathrm{H}), 6.41(\mathrm{~s}, 1 \mathrm{H}), 2.29(\mathrm{~d}, 3 \mathrm{H}, J=1.5$ $\mathrm{Hz}) ;{ }^{13} \mathrm{C}\left\{{ }^{1} \mathrm{H}\right\}$ NMR $\left(75 \mathrm{MHz}, \mathrm{CDCl}_{3}\right): \delta 169.8,145.0,135.5,132.9,131.0,128.9$, $128.5(2 \mathrm{C}), 127.9,127.7(2 \mathrm{C}), 110.0,22.3$.

(Z)-3-(2-methyl-3-phenylacryloyl)benzo[d]oxazol-2(3H)-one (T1):<smiles>C/C(=C/c1ccccc1)C(=O)n1c(=O)oc2ccc(I)cc21</smiles>

$\mathrm{T1}$

Prepared according to general procedure D. Obtained as a beige solid (85 mg, quant. yield, $E / Z=17: 83$ determined by ${ }^{1} \mathrm{H} N M R$ ) after filtration over a plug of silica (Et $\left.{ }_{2} \mathrm{O}\right) .{ }^{1} \mathbf{H}$ NMR (300 MHz, $\left.\mathrm{CDCl}_{3}\right): \delta 8.03-8.0(\mathrm{~m}, 1 \mathrm{H}), 7.24-$ $7.11(\mathrm{~m}, 8 \mathrm{H}), 6.76(\mathrm{q}, 1 \mathrm{H}, J=1.5 \mathrm{~Hz}), 2.26(\mathrm{~d}, 3 \mathrm{H}, J=1.5 \mathrm{~Hz}) ;{ }^{13} \mathrm{C}\left\{{ }^{1} \mathrm{H}\right\}$ NMR (75 MHz, $\mathrm{CDCl}_{3}$ ): $\delta$ 169.9, 149.8, 142.4, 135.4, 132.6, 131.0, 129.9, 128.5 (2C), 128.0, 127.6 (2C), 125.4, 124.8, 115.2, 109.9, 20.9.

(Z)-1-(2-methyl-3-phenylacryloyl)pyrrolidin-2-one (T2):<smiles>C/C(=C/c1ccccc1)C(=O)N1CCCC1=O</smiles>

T2

Prepared according to general procedure $\mathbf{D}$. Obtained as a colorless solid (68 $\mathrm{mg}$, quant. yield, $E / Z=18: 82$ determined by ${ }^{1} \mathrm{H} N M R$ ) after filtration over a plug of silica ( $\left.\mathrm{Et}_{2} \mathrm{O}\right) .{ }^{1} \mathbf{H} \mathbf{~ N M R}\left(\mathbf{3 0 0} \mathbf{~ M H z}, \mathrm{CDCl}_{3}\right)$ : $\delta 7.43-7.35(\mathrm{~m}, 1 \mathrm{H}), 7.24-$ $7.13(\mathrm{~m}, 4 \mathrm{H}), 6.51(\mathrm{~m}, 1 \mathrm{H}), 3.71(\mathrm{t}, 2 \mathrm{H}, J=7.1 \mathrm{~Hz}), 2.29(\mathrm{t}, 2 \mathrm{H}, J=8.0 \mathrm{~Hz})$, $2.13(\mathrm{~d}, 3 \mathrm{H}, J=1.4 \mathrm{~Hz}), 1.88-1.78(\mathrm{~m}, 2 \mathrm{H}) ;{ }^{13} \mathrm{C}\left\{{ }^{1} \mathrm{H}\right\}$ NMR $\left(75 \mathrm{MHz}, \mathrm{CDCl}_{3}\right): \delta$ 174.0, 171.8, 136.5, 133.7, 129.0, 128.3 (2C), 127.5 (2C), 127.4, 45.3, 32.8, 21.0, 17.5 .

\section{(Z)-1-(2-methyl-3-phenylacryloyl)piperidin-2-one (T3):}<smiles>C/C(=C/c1ccccc1)C(=O)N1CCCCC1=O</smiles>

T3

Prepared according to general procedure D. Obtained as a yellow solid (73 $\mathrm{mg}$, quant. yield, $E / Z=15: 85$ determined by ${ }^{1} \mathrm{H}$ NMR) after filtration over a plug of silica $\left(\mathrm{Et}_{2} \mathrm{O}\right) .{ }^{1} \mathbf{H}$ NMR $\left(300 \mathrm{MHz}, \mathrm{CDCl}_{3}\right): \delta 7.43-7.20(\mathrm{~m}, 3 \mathrm{H}), 7.09$ $(\mathrm{d}, 2 \mathrm{H}, J=6.8 \mathrm{~Hz}), 6.38(\mathrm{~s}, 1 \mathrm{H}), 3.54(\mathrm{~m}, 2 \mathrm{H}), 2.16(\mathrm{~d}, 3 \mathrm{H}, J=1.2 \mathrm{~Hz}), 1.99(\mathrm{~m}$, $2 \mathrm{H}), 1.46(\mathrm{~m}, 4 \mathrm{H}) ;{ }^{13} \mathrm{C}\left\{{ }^{1} \mathrm{H}\right\}$ NMR $\left(75 \mathrm{MHz} \mathrm{CDCl}_{3}\right): \delta 174.9,173.1,137.0$, 136.0, 128.3 (2C), 127.5 (2C), 127.2, 126.3, 44.7, 34.0, 21.9, 21.0, 20.7. 
(Z)-2-methyl-3-phenyl-1-(2-thioxooxazolidin-3-yl)prop-2-en-1-one (T4):

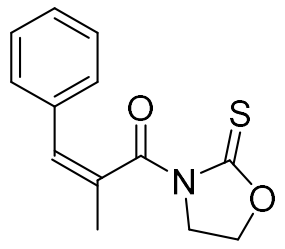

Prepared according to general procedure $\mathbf{D}$. Obtained as a colorless oil (74 $\mathrm{mg}$, quant. yield, $E / Z=34: 66$ determined by ${ }^{1} \mathrm{H} N M R$ ) after filtration over a plug of silica (Et $\left.{ }_{2} \mathrm{O}\right) .{ }^{1} \mathbf{H}$ NMR (300 MHz, $\left.\mathbf{C D C l}_{3}\right): \delta 7.29-7.23(\mathrm{~m}, 3 \mathrm{H}), 7.18$ - $7.15(\mathrm{~m}, 2 \mathrm{H}), 6.59(\mathrm{~m}, 1 \mathrm{H}), 4.13(\mathrm{t}, 2 \mathrm{H}, J=7.2 \mathrm{~Hz}), 3.98(\mathrm{t}, 2 \mathrm{H}, J=7.7 \mathrm{~Hz})$, $2.21(\mathrm{~d}, 3 \mathrm{H}, J=1.4 \mathrm{~Hz}) ;{ }^{13} \mathrm{C}\left\{{ }^{1} \mathrm{H}\right\} \mathrm{NMR}\left(75 \mathrm{MHz} \mathrm{CDCl}_{3}\right): \delta 184.4,172.0,136.5$, T4 $132.3,131.7,128.3(2 \mathrm{C}), 127.8,127.4$ (2C), 67.0, 47.0, 21.1.

(Z)-2-(2-methyl-3-phenylacryloyl)-1-phenylpyrazolidin-3-one (T5):<smiles>C/C(=C/c1ccccc1)C(=O)N1C(=O)CCN1c1ccccc1</smiles>

T5

Prepared according to general procedure D. Obtained as a yellow oil (91 mg, quant. yield, $E / Z=20: 80$ determined by ${ }^{1} \mathrm{H} N M R$ ) after filtration over a plug of silica $\left(\mathrm{Et}_{2} \mathrm{O}\right) .{ }^{1} \mathbf{H}$ NMR (300 MHz, $\left.\mathrm{CDCl}_{3}\right): \delta 7.33-7.32(\mathrm{~m}, 6 \mathrm{H}), 7.00(\mathrm{~m}$, $2 \mathrm{H}), 6.79-6.76(\mathrm{~m}, 2 \mathrm{H}), 6.55(\mathrm{~m}, 1 \mathrm{H}), 3.92(\mathrm{t}, 2 \mathrm{H}, J=7.3 \mathrm{~Hz}), 2.66(\mathrm{t}, 2 \mathrm{H}, J=$ $7.3 \mathrm{~Hz}), 2.16(\mathrm{~d}, 3 \mathrm{H}, J=1.0 \mathrm{~Hz}) ;{ }^{13} \mathrm{C}\left\{{ }^{1} \mathrm{H}\right\}$ NMR $\left(75 \mathrm{MHz} \mathrm{CDCl}_{3}\right): \delta$ 172.6, 168.6, 149.5, 130.2, 129.9, 129.7, 129.3 (2C), 128.5 (2C), 128.0 (2C), 127.7, 123.9, $117.4(2 \mathrm{C}), 53.9,30.7,21.2$.

\subsection{Characterization of (Z)-8 to (Z)-26}

(Z)-3-(2-benzylidenebutanoyl)oxazolidin-2-one ((Z)-8):<smiles>CC/C(=C/c1ccccc1)C(=O)N1CCOC1=O</smiles>

(Z)-8

Prepared according to general procedure $\mathbf{D}$. Obtained as a yellow oil (74 $\mathrm{mg}$, quant. yield, $E / Z=22: 78)$ after filtration over a plug of silica $\left(\mathrm{Et}_{2} \mathrm{O}\right) .{ }^{1} \mathrm{H}$ NMR (300 MHz, $\left.\mathrm{CDCl}_{3}\right): \delta 7.35-7.24(\mathrm{~m}, 3 \mathrm{H}), 7.19(\mathrm{~d}, 2 \mathrm{H}, J=7.2 \mathrm{~Hz}), 6.60$ $(\mathrm{m}, 1 \mathrm{H}), 4.14(\mathrm{t}, 2 \mathrm{H}, J=7.7 \mathrm{~Hz}), 3.91(\mathrm{t}, 2 \mathrm{H}, J=7.7 \mathrm{~Hz}), 2.51(\mathrm{q}, 2 \mathrm{H}, J=7.2$ $\mathrm{Hz}), 1.21(\mathrm{t}, 3 \mathrm{H}, J=7.5 \mathrm{~Hz}) ;{ }^{13} \mathrm{C}\left\{{ }^{1} \mathrm{H}\right\}$ NMR $\left(75 \mathrm{MHz}, \mathrm{CDCl}_{3}\right): \delta 171.1,151.9$, $137.8,136.3,134.1,128.8,128.4$ (2C), 127.6 (2C), 62.1, 42.6, 27.9, 12.0.

(Z)-3-(2-methyl-3-(p-tolyl)acryloyl)oxazolidin-2-one ((Z)-9):<smiles>C/C(=C/c1ccc(C)cc1)C(=O)N1CCOC1=O</smiles>

$(Z)-9$

Prepared according to general procedure $\mathbf{D}$. Obtained as a white solid (73 $\mathrm{mg}$, quant. yield, $E / Z=7: 93$ ) after filtration over a plug of silica (Et $\left.{ }_{2} \mathrm{O}\right) .{ }^{1} \mathbf{H}$ NMR (300 MHz, $\left.\mathrm{CDCl}_{3}\right): \delta 7.08(\mathrm{~m}, 4 \mathrm{H}), 6.57(\mathrm{~m}, 1 \mathrm{H}), 4.21(\mathrm{t}$, $2 \mathrm{H}, J=7.8 \mathrm{~Hz}), 3.95(\mathrm{t}, 2 \mathrm{H}, J=7.8 \mathrm{~Hz}), 2.32(\mathrm{~s}, 3 \mathrm{H}), 2.14(\mathrm{~s}, 3 \mathrm{H}) ;{ }^{13} \mathrm{C}\left\{{ }^{1} \mathrm{H}\right\}$ NMR (75 MHz, $\left.\mathrm{CDCl}_{3}\right): \delta$ 171.3, 152.0, 137.5, 133.2, 131.0, 130.5, 129.1 (2C), $127.4(2 \mathrm{C}), 62.2,42.6,21.2,21.0$.

(Z)-3-(2-methyl-3-(m-tolyl)acryloyl)oxazolidin-2-one ((Z)-10):<smiles>C/C(=C/c1cccc(C)c1)C(=O)N1CCOC1=O</smiles>

(Z)-10

Prepared according to general procedure D. Obtained as a yellow oil (73 $\mathrm{mg}$, quant. yield, $E / Z=15: 85$ ) after filtration over a plug of silica (Et $\left.{ }_{2} \mathrm{O}\right) .{ }^{1} \mathrm{H} \mathrm{NMR}\left(300 \mathrm{MHz}, \mathrm{CDCl}_{3}\right): \delta 7.17(\mathrm{~d}, 1 \mathrm{H}, J=7.5 \mathrm{~Hz}), 7.05(\mathrm{~d}, 1 \mathrm{H}$, $J=7.6 \mathrm{~Hz}), 6.98(\mathrm{~m}, 2 \mathrm{H}), 6.58(\mathrm{~m}, 1 \mathrm{H}), 4.19(\mathrm{t}, 2 \mathrm{H}, J=7.6 \mathrm{~Hz}), 3.93(\mathrm{t}$, $2 \mathrm{H}, J=7.8 \mathrm{~Hz}$ ), $2.31(\mathrm{~s}, 3 \mathrm{H}), 2.15(\mathrm{~d}, 3 \mathrm{H}, J=1.6 \mathrm{~Hz}) ;{ }^{13} \mathrm{C}\left\{{ }^{1} \mathrm{H}\right\}$ NMR (75 $\left.\mathrm{MHz}, \mathrm{CDCl}_{3}\right): \delta 171.2,152.0,138.0,136.1,131.6,130.7,128.5,128.4$, $128.3,124.4,62.2,42.6,21.4,21.0$. 
(Z)-3-(2-methyl-3-(o-tolyl)acryloyl)oxazolidin-2-one ((Z)-11):<smiles>C/C(=C/c1ccccc1C)C(=O)N1CCOC1=O</smiles>

(Z)-11

Prepared according to general procedure D. Obtained as a colorless oil (73 mg, quant. yield, $E / Z=29: 71)$ after filtration over a plug of silica $\left(\mathrm{Et}_{2} \mathrm{O}\right)$.

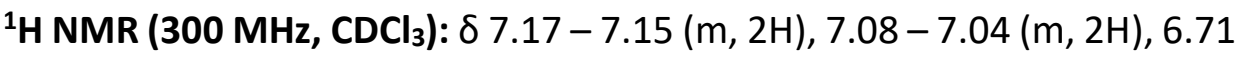
$(\mathrm{m}, 1 \mathrm{H}), 4.03(\mathrm{t}, 2 \mathrm{H}, J=7.6 \mathrm{~Hz}), 3.79(\mathrm{t}, 2 \mathrm{H}, J=7.8 \mathrm{~Hz}), 2.30(\mathrm{~s}, 3 \mathrm{H}), 2.18$ $(\mathrm{d}, 3 \mathrm{H}, J=1.6 \mathrm{~Hz}) ;{ }^{13} \mathrm{C}\left\{{ }^{1} \mathrm{H}\right\}$ NMR $\left(75 \mathrm{MHz}, \mathrm{CDCl}_{3}\right): \delta 171.1,152.2,136.8$, 135.7, 132.4, 130.3, 130.2, 127.9, 127.6, 125.4, 62.3, 42.6, 20.6, 19.7.

(Z)-3-(3-(4-(tert-butyl)phenyl)-2-methylacryloyl)oxazolidin-2-one ((Z)-12):<smiles>C/C(=C/c1ccc(C(C)(C)C)cc1)C(=O)N1CCOC1=O</smiles>

(Z)-12

Prepared according to general procedure $\mathbf{D}$. Obtained as a white solid (86 mg, quant. yield, $E / Z=10: 90$ ) after filtration over a plug of silica ( $\left.\mathrm{Et}_{2} \mathrm{O}\right) .{ }^{1} \mathbf{H}$ NMR (300 MHz, $\left.\mathrm{CDCl}_{3}\right): \delta 7.30(\mathrm{~d}, 2 \mathrm{H}, J=8.3 \mathrm{~Hz}), 7.11$ $(\mathrm{d}, 2 \mathrm{H}, J=8.2 \mathrm{~Hz}), 6.58(\mathrm{~m}, 1 \mathrm{H}), 4.18(\mathrm{t}, 2 \mathrm{H}, J=7.9 \mathrm{~Hz}), 3.95(\mathrm{t}, 2 \mathrm{H}, J$ $=7.9 \mathrm{~Hz}), 2.14(\mathrm{~s}, 3 \mathrm{H}), 1.30(\mathrm{~s}, 9 \mathrm{H}) ;{ }^{13} \mathrm{C}\left\{{ }^{1} \mathrm{H}\right\} \mathrm{NMR}\left(75 \mathrm{MHz}, \mathrm{CDCl}_{3}\right): \delta$ $171.3,152.0,150.8,133.2,131.0,130.5,127.3(2 \mathrm{C}), 125.3(2 \mathrm{C}), 62.2$, $42.6,34.6,31.3,21.1$.

(Z)-3-(3-([1,1'-biphenyl]-4-yl)-2-methylacryloyl)oxazolidin-2-one ((Z)-13):<smiles>C/C(=C/c1ccc(-c2ccccc2)cc1)C(=O)N1CCOC1=O</smiles>

(Z)-13

Prepared according to general procedure $\mathbf{D}$. Obtained as a white solid (50 mg, 54\% yield, $E / Z=27: 73$ determined by ${ }^{1} \mathrm{H} N M R$ ) after filtration over a plug of silica ( $\left.\mathrm{Et}_{2} \mathrm{O}\right) .{ }^{1} \mathrm{H}$ NMR $\left(300 \mathbf{M H z} \mathrm{CDCl}_{3}\right): \delta 7.84(\mathrm{~m}, 1 \mathrm{H})$, $7.57(\mathrm{~m}, 7 \mathrm{H}), 6.89(\mathrm{~m}, 1 \mathrm{H}), 6.64(\mathrm{~m}, 1 \mathrm{H}), 4.22(\mathrm{t}, 2 \mathrm{H}, J=7.2 \mathrm{~Hz}) 3.98$ $(\mathrm{t}, 2 \mathrm{H}, J=7.8 \mathrm{~Hz}), 2.21(\mathrm{~s}, 3 \mathrm{H})$. Due to solubility issues, the product was not characterized on ${ }^{13} \mathrm{C}$ NMR.

(Z)-3-(3-(4-methoxyphenyl)-2-methylacryloyl)oxazolidin-2-one ((Z)-14):<smiles>COc1ccc(/C=C(/C)C(=O)N2CCOC2=O)cc1</smiles>

(Z)-14

Prepared according to general procedure D. Obtained as a beige solid (73 $\mathrm{mg}, 93 \%$ yield, $E / Z=7: 93$ ) after filtration over a plug of silica (Et $\left.{ }_{2} \mathrm{O}\right) .{ }^{1} \mathbf{H}$ NMR (300 MHz, $\left.\mathrm{CDCl}_{3}\right): \delta 7.12(\mathrm{~d}, 2 \mathrm{H}, J=8.5 \mathrm{~Hz})$, $6.82(\mathrm{~d}, 2 \mathrm{H}, J=8.5 \mathrm{~Hz}), 6.55(\mathrm{~m}, 1 \mathrm{H}), 4.23(\mathrm{t}, 2 \mathrm{H}, J=7.8 \mathrm{~Hz}), 3.96(\mathrm{t}$, $2 \mathrm{H}, J=7.8 \mathrm{~Hz}), 3.80(\mathrm{~s}, 3 \mathrm{H}), 2.13(\mathrm{~s}, 3 \mathrm{H}) ;{ }^{13} \mathrm{C}\left\{{ }^{1} \mathrm{H}\right\}$ NMR $(75 \mathrm{MHz}$, $\left.\mathrm{CDCl}_{3}\right): \delta 171.4,159.2,152.0,130.2,130.0,128.8(2 \mathrm{C}), 128.7,113.9$

(2C), 62.2, 55.3, 42.6, 21.0.

(Z)-3-(2-methyl-3-(naphthalen-2-yl)acryloyl)oxazolidin-2-one ((Z)-15):

Prepared according to general procedure $\mathbf{D}$. Obtained as a beige<smiles>C/C(=C/c1ccc2ccccc2c1)C(=O)N1CCOC1=O</smiles>
solid (49 mg, 58\% yield, $E / Z=23: 77$ determined by ${ }^{1} \mathrm{H} N M R$ ) after filtration over a plug of silica ( $\left.\mathrm{Et}_{2} \mathrm{O}\right) .{ }^{1} \mathrm{H} \mathbf{~ N M R}\left(300 \mathrm{MHz}, \mathrm{CDCl}_{3}\right): \delta 7.81$ $-7.74(\mathrm{~m}, 2 \mathrm{H}), 7.66(\mathrm{~m}, 1 \mathrm{H}), 7.48-7.45(\mathrm{~m}, 2 \mathrm{H}), 7.31(\mathrm{~d}, 2 \mathrm{H}, \mathrm{J}=8.2$ (Z)-15 $\mathrm{Hz}), 6.77(\mathrm{~m}, 1 \mathrm{H}), 4.08(\mathrm{~m}, 2 \mathrm{H}), 3.93(\mathrm{t}, 2 \mathrm{H}, J=8.0 \mathrm{~Hz}), 2.21(\mathrm{~s}, 3 \mathrm{H})$; ${ }^{13} \mathrm{C}\left\{{ }^{1} \mathrm{H}\right\}$ NMR $\left(75 \mathrm{MHz}^{\mathrm{C}} \mathrm{CDCl}_{3}\right): \delta 171.2,152.0,133.7,133.3,132.7$, 132.3, 130.6, 128.1, 128.1, 127.7, 126.8, 126.4, 126.3, 125.4, 62.2, 42.6, 21.1. 
(Z)-3-(3-(4-fluorophenyl)-2-methylacryloyl)oxazolidin-2-one ((Z)-16):<smiles>C/C(=C/c1ccc(F)cc1)C(=O)N1CCOC1=O</smiles>

(Z)-16

Prepared according to general procedure $\mathbf{D}$. Obtained as a colorless oil (74 $\mathrm{mg}$, quant. yield, $E / Z=10: 90$ ) after filtration over a plug of silica ( $\left.\mathrm{Et}_{2} \mathrm{O}\right) .{ }^{1} \mathrm{H}$ NMR (300 MHz, $\mathrm{CDCl}_{3}$ ): $\delta 7.16$ (dd, $\left.2 \mathrm{H}, J=8.5 \& 5.5 \mathrm{~Hz}\right), 6.98$ (dd, $2 \mathrm{H}, J=8.7 \mathrm{~Hz}), 6.56(\mathrm{~m}, 1 \mathrm{H}), 4.25(\mathrm{t}, 2 \mathrm{H}, J=7.9 \mathrm{~Hz}), 3.95(\mathrm{t}, 2 \mathrm{H}, J=$ $7.9 \mathrm{~Hz}), 2.14(\mathrm{~d}, 3 \mathrm{H}, J=1.6 \mathrm{~Hz}) ;{ }^{13} \mathrm{C}\left\{{ }^{1} \mathrm{H}\right\} \mathrm{NMR}\left(75 \mathrm{MHz}, \mathrm{CDCl}_{3}\right): \delta 171.0$, $162.2(\mathrm{~d}, J=247.4 \mathrm{~Hz}$ ), 152.0, $132.3(\mathrm{~d}, J=3.4 \mathrm{~Hz}), 132.1$ (d, $J=1.4 \mathrm{~Hz}$ ), 129.4, 129.3 (d, 2C, J = 7.9 Hz), 115.4 (d, 2C, $J=21.5 \mathrm{~Hz}), 62.2,42.5,21.0 ;{ }^{19} \mathrm{~F}\left\{{ }^{1} \mathrm{H}\right\}$ NMR (282 $\left.\mathrm{MHz}, \mathrm{CDCl}_{3}\right):-114.4$.

(Z)-3-(3-(4-chlorophenyl)-2-methylacryloyl)oxazolidin-2-one ((Z)-17):<smiles>C/C(=C/c1ccc(Cl)cc1)C(=O)N1CCOC1=O</smiles>

$(Z)-17$

Prepared according to general procedure $\mathbf{D}$. Obtained as a beige solid (76 $\mathrm{mg}, 96 \%$ yield, $E / Z=8: 92$ ) after filtration over a plug of silica (Et $\left.{ }_{2} \mathrm{O}\right) .{ }^{1} \mathrm{H}$ NMR (300 MHz, CDCl$): \delta 7.24(\mathrm{~m}, 2 \mathrm{H}), 7.12(\mathrm{~d}, 2 \mathrm{H}, J=8.3$ $\mathrm{Hz}), 6.54(\mathrm{~m}, 1 \mathrm{H}), 4.27(\mathrm{t}, 2 \mathrm{H}, J=7.8 \mathrm{~Hz}), 3.95(\mathrm{t}, 2 \mathrm{H}, J=7.9 \mathrm{~Hz}), 2.14$ $(\mathrm{s}, 3 \mathrm{H}) ;{ }^{13} \mathrm{C}\left\{{ }^{1} \mathrm{H}\right\}$ NMR $\left(75 \mathrm{MHz}, \mathrm{CDCl}_{3}\right): \delta 170.8,151.9,134.6,133.5$, $132.8,129.1,128.9$ (2C), 128.7 (2C), 62.3, 42.4, 21.1.

(Z)-3-(3-(4-bromophenyl)-2-methylacryloyl)oxazolidin-2-one ((Z)-18):<smiles>C/C(=C/c1ccc(Br)cc1)C(=O)N1CCOC1=O</smiles>

$(Z)-18$

Prepared according to general procedure $\mathbf{D}$. Obtained as a white solid (90 $\mathrm{mg}, 97 \%$ yield, $E / Z=10: 90$ ) after filtration over a plug of silica (Et $\left.{ }_{2} \mathrm{O}\right) .{ }^{1} \mathrm{H}$ NMR (300 MHz, CDCl$\left.)_{3}\right): \delta 7.41(\mathrm{~d}, 2 \mathrm{H}, J=8.3 \mathrm{~Hz}), 7.06(\mathrm{~d}$, $2 \mathrm{H}, J=8.3 \mathrm{~Hz}), 6.52(\mathrm{~m}, 1 \mathrm{H}), 4.27(\mathrm{t}, 2 \mathrm{H}, J=7.9 \mathrm{~Hz}), 3.95(\mathrm{t}, 2 \mathrm{H}, J=7.9$ $\mathrm{Hz}), 2.14(\mathrm{~s}, 3 \mathrm{H}) ;{ }^{13} \mathrm{C}\left\{{ }^{1} \mathrm{H}\right\}$ NMR (75 MHz, $\left.\mathrm{CDCl}_{3}\right): \delta 170.8,151.9,135.1$, 132.9, 131.6 (2C), 129.2 (2C), 129.2, 121.7, 62.3, 42.4, 21.2.

(Z)-3-(3-(4-iodophenyl)-2-methylacryloyl)oxazolidin-2-one ((Z)-19):<smiles>C/C(=C/c1ccc(I)cc1)C(=O)N1CCOC1=O</smiles>

$(Z)-19$

Prepared according to general procedure D. Obtained as a white solid (96 mg, 90\% yield, $E / Z=5: 95$ ) after filtration over a plug of silica $\left(\mathrm{Et}_{2} \mathrm{O}\right)$. ${ }^{1} \mathrm{H}$ NMR $\left(300 \mathrm{MHz}, \mathrm{CDCl}_{3}\right): \delta 7.61(\mathrm{~d}, 2 \mathrm{H}, J=8.1 \mathrm{~Hz}), 6.93(\mathrm{~d}, 2 \mathrm{H}, J=8.0$ $\mathrm{Hz}), 6.50(\mathrm{~m}, 1 \mathrm{H}), 4.27(\mathrm{t}, 2 \mathrm{H}, J=7.8 \mathrm{~Hz}), 3.95(\mathrm{t}, 2 \mathrm{H}, J=7.9 \mathrm{~Hz}), 2.13(\mathrm{~s}$, $3 \mathrm{H}) ;{ }^{13} \mathrm{C}\left\{{ }^{1} \mathrm{H}\right\}$ NMR (75 MHz, $\left.\mathrm{CDCl}_{3}\right): \delta 170.8,151.9,137.6(2 \mathrm{C}), 135.6$, $132.9,129.4$ (2C), 129.3, 93.3, 62.3, 42.4, 21.2 .

(Z)-3-(2-methyl-3-(4-(trifluoromethyl)phenyl)acryloyl)oxazolidin-2-one ((Z)-20):<smiles>C/C(=C/c1ccc(C(F)(F)F)cc1)C(=O)N1CCOC1=O</smiles>

$(Z)-20$

Prepared according to general procedure $\mathbf{D}$. Obtained as a white solid (89 $\mathrm{mg}$, quant. yield, $E / Z=12: 88$ ) after filtration over a plug of silica (Et $\left.{ }_{2} \mathrm{O}\right) .{ }^{1} \mathrm{H}$ NMR $\left(300 \mathrm{MHz}, \mathrm{CDCl}_{3}\right): \delta 7.54(\mathrm{~d}, 2 \mathrm{H}, J=8.0 \mathrm{~Hz})$, $7.30(\mathrm{~d}, 2 \mathrm{H}, J=8.1 \mathrm{~Hz}), 6.61(\mathrm{~m}, 1 \mathrm{H}), 4.27(\mathrm{t}, 2 \mathrm{H}, J=7.8 \mathrm{~Hz}), 3.96(\mathrm{t}$, $2 \mathrm{H}, J=7.8 \mathrm{~Hz}), 2.17(\mathrm{~s}, 3 \mathrm{H}) ;{ }^{13} \mathrm{C}\left\{{ }^{1} \mathrm{H}\right\}$ NMR $\left(75 \mathrm{MHz}, \mathrm{CDCl}_{3}\right): \delta 170.5$, 152.0, 139.8, 134.4, 129.4, 128.9, 127.9 (3C), 125.4 (q, $J=3.7 \mathrm{~Hz})$, $124.1(q, J=272.0 \mathrm{~Hz}), 62.3,42.4,21.3 ;{ }^{19} \mathrm{~F}\left\{{ }^{1} \mathrm{H}\right\}$ NMR $(282 \mathrm{MHz}$,

$\left.\mathrm{CDCl}_{3}\right): \delta-63.1$ 
(Z)-3-(2-methyl-3-(4-(4,4,5,5-tetramethyl-1,3,2-dioxaborolan-2yl)phenyl)acryloyl)oxazolidin-2-one ((Z)-21):

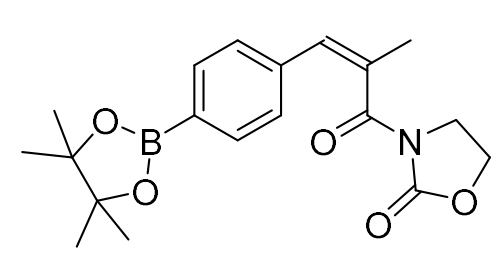

$(Z)-21$

Prepared according to general procedure $\mathbf{D}$. Obtained as a white solid ( $85 \mathrm{mg}, 79 \%$ yield, $E / Z=5: 95$ ) after filtration over a plug of silica (Et $\left.{ }_{2} \mathrm{O}\right) .{ }^{1} \mathrm{H}$ NMR (300 MHz, $\left.\mathrm{CDCl}_{3}\right): \delta 7.72(\mathrm{~d}, 2 \mathrm{H}, \mathrm{J}$ $=8.0 \mathrm{~Hz}), 7.18(\mathrm{~d}, 2 \mathrm{H}, J=7.9 \mathrm{~Hz}), 6.61(\mathrm{~m}, 1 \mathrm{H}), 4.19(\mathrm{t}, 2 \mathrm{H}, J=$ $8.0 \mathrm{~Hz}), 3.93(\mathrm{t}, 2 \mathrm{H}, J=7.9 \mathrm{~Hz}), 2.16(\mathrm{~d}, 3 \mathrm{H}, J=1.5 \mathrm{~Hz}), 1.34(\mathrm{~s}$, $12 \mathrm{H}) ;{ }^{13} \mathrm{C}\left\{{ }^{1} \mathrm{H}\right\}$ NMR (75 MHz, $\left.\mathrm{CDCl}_{3}\right): \delta 171.1,152.0,138.9$, $134.9(2 \mathrm{C}), 132.6,130.4,126.8(2 \mathrm{C}), 84.0(2 \mathrm{C}), 62.3,42.5,25.0$ (4C), 21.2. The carbon bearing boron was not observed; ${ }^{11} \mathrm{~B} \mathrm{NMR}\left(96 \mathrm{MHz}, \mathrm{CDCl}_{3}\right): \delta 31.2$.

(Z)-3-(2-methyl-3-(4-(methylsulfonyl)phenyl)acryloyl)oxazolidin-2-one ((Z)-22):<smiles>COS(=O)(=O)c1ccc(/C=C(/C)C(=O)N2CCOC2=O)cc1</smiles>

(Z)-22

Prepared according to general procedure $\mathbf{D}$. Obtained as a yellow solid (75 $\mathrm{mg}, 81 \%$ yield, $E / Z=2: 98$ ) after filtration over a plug of silica (Et $\left.{ }_{2} \mathrm{O}\right) .{ }^{1} \mathrm{H}$ NMR (300 MHz, $\left.\mathrm{CDCl}_{3}\right): \delta 7.86(\mathrm{~d}, 2 \mathrm{H}, J=8.1 \mathrm{~Hz})$, $7.38(\mathrm{~d}, 2 \mathrm{H}, J=8.1 \mathrm{~Hz}), 6.60(\mathrm{~m}, 1 \mathrm{H}), 4.33(\mathrm{t}, 2 \mathrm{H}, J=7.8 \mathrm{~Hz}), 3.99$ $(\mathrm{t}, 2 \mathrm{H}, J=8.0 \mathrm{~Hz}), 3.06(\mathrm{~s}, 3 \mathrm{H}), 2.19(\mathrm{~s}, 3 \mathrm{H}) ;{ }^{13} \mathrm{C}\left\{{ }^{1} \mathrm{H}\right\} \mathrm{NMR}(75 \mathrm{MHz}$, $\left.\mathrm{CDCl}_{3}\right): \delta 170.3,152.0,141.7,139.3,135.7,128.5$ (2C), 128.2,

$127.7(2 \mathrm{C}), 62.4,44.5,42.3,21.5$.

(Z)-3-(3-(4-acetylphenyl)-2-methylacryloyl)oxazolidin-2-one ((Z)-23):<smiles>CC(=O)c1ccc(/C=C(/C)C(=O)N2CCOC2=O)cc1</smiles>

(Z)-23

Prepared according to general procedure D. Obtained as a colorless oil (72 mg, 88\% yield, $E / Z=60: 40$ ) after filtration over a plug of silica ( $\left.\mathrm{Et}_{2} \mathrm{O}\right) .{ }^{1} \mathrm{H}$ NMR $\left(300 \mathrm{MHz}, \mathrm{CDCl}_{3}\right): \delta 7.89(\mathrm{~d}, 2 \mathrm{H}, \mathrm{J}=8.3 \mathrm{~Hz}), 7.28(\mathrm{~d}$, $2 \mathrm{H}, J=8.2 \mathrm{~Hz}), 6.61(\mathrm{~m}, 1 \mathrm{H}), 4.28(\mathrm{t}, 2 \mathrm{H}, J=7.9 \mathrm{~Hz}), 3.99(\mathrm{t}, 2 \mathrm{H}, J=$ $7.9 \mathrm{~Hz}), 2.59(\mathrm{~s}, 3 \mathrm{H}), 2.18(\mathrm{~m}, 3 \mathrm{H}) ;{ }^{13} \mathrm{C}\left\{{ }^{1} \mathrm{H}\right\}$ NMR (75 MHz, $\left.\mathrm{CDCl}_{3}\right): \delta$ $197.7,171.3,150.5,140.9,136.1,134.3,129.3$ (2C), 128.6 (2C),

$127.7,62.3,42.4,26.7,21.4$.

(Z)-3-(3-(furan-2-yl)-2-methylacryloyl)oxazolidin-2-one ((Z)-24):

Prepared according to general procedure $\mathbf{D}$. Obtained as an orange oil (66<smiles>C/C(=C/c1ccco1)C(=O)N1CCOC1=O</smiles>
$\mathrm{mg}$, quant. yield, $E / Z=40: 60$ ) after filtration over a plug of silica $\left(E t_{2} \mathrm{O}\right)$. Rf: 0.34 (PE:EtOAC ; 6:4); ${ }^{1} \mathbf{H}$ NMR (300 MHz, $\left.\mathrm{CDCl}_{3}\right)$ : $\delta 7.28(\mathrm{~m}, 1 \mathrm{H}), 6.34(\mathrm{~m}$, $1 \mathrm{H}), 6.23(\mathrm{~d}, 2 \mathrm{H}, J=3.4 \mathrm{~Hz}), 4.46(\mathrm{t}, 2 \mathrm{H}, J=7.9 \mathrm{~Hz}), 4.16(\mathrm{t}, 2 \mathrm{H}, J=7.9 \mathrm{~Hz})$, $2.13(\mathrm{~s}, 3 \mathrm{H}) ;{ }^{13} \mathrm{C}\left\{{ }^{1} \mathrm{H}\right\}$ NMR $\left(75 \mathrm{MHz}, \mathrm{CDCl}_{3}\right): \delta 170.4,152.5,150.8,142.7$, (Z)-24 128.6, 125.1, 117.2, 110.0, 62.6, 42.5, 21.1.

(Z)-3-(2-methyl-3-(thiophen-2-yl)acryloyl)oxazolidin-2-one ((Z)-25):<smiles>C/C(=C/c1cccs1)C(=O)N1CCOC1=O</smiles>

Prepared according to general procedure $\mathbf{D}$. Obtained as a beige solid (70 $\mathrm{mg}$, quant. yield, $E / Z=24: 76)$ after filtration over a plug of silica $\left(\mathrm{Et}_{2} \mathrm{O}\right) .{ }^{1} \mathbf{H}$ NMR (300 MHz, $\left.\mathrm{CDCl}_{3}\right): \delta 7.19(\mathrm{~d}, 1 \mathrm{H}, J=4.5 \mathrm{~Hz}), 6.98-6.91(\mathrm{~m}, 2 \mathrm{H}), 6.61$ $(\mathrm{m}, 1 \mathrm{H}), 4.43(\mathrm{t}, 2 \mathrm{H}, J=7.5 \mathrm{~Hz}), 4.12(\mathrm{t}, 2 \mathrm{H}, J=7.5 \mathrm{~Hz}), 2.14(\mathrm{~s}, 3 \mathrm{H}) ;{ }^{13} \mathrm{C}\left\{{ }^{1} \mathrm{H}\right\}$

$(Z)-25$ 
NMR (75 MHz, $\left.\mathrm{CDCl}_{3}\right): \delta$ 170.7, 152.1, 138.3, 130.0, 127.9, 127.3, 125.7, 122.6, 62.4, 42.5, 21.2 .

3-((Z)-3-(4-(cinnamyloxy)phenyl)-2-methylacryloyl)oxazolidin-2-one ((Z)-26):<smiles>C/C(=C/c1ccc(OC/C=C/c2ccccc2)cc1)C(=O)N1CCOC1=O</smiles>

(Z)-26

Prepared according to general procedure $\mathbf{D}$. Obtained as a beige solid (45 $\mathrm{mg}, 83 \%$ yield, on $0.15 \mathrm{mmol}$ scale, $E / Z=$ 37:63 determined by $\left.{ }^{1} \mathrm{H} N M R\right)$ after filtration over a plug of silica (Et $\left.{ }_{2} \mathrm{O}\right) .{ }^{1} \mathbf{H} \mathbf{~ N M R}\left(300 \mathrm{MHz}, \mathrm{CDCl}_{3}\right): \delta 7.41-7.34(\mathrm{~m}$, $5 \mathrm{H}), 7.12(\mathrm{~d}, 2 \mathrm{H}, J=8.6 \mathrm{~Hz}), 6.87(\mathrm{~d}, 2 \mathrm{H}, J=8.7 \mathrm{~Hz}), 6.72(\mathrm{~d}$, $1 \mathrm{H}, J=16.1 \mathrm{~Hz}), 6.55(\mathrm{~m}, 1 \mathrm{H}), 6.39(\mathrm{dt}, 1 \mathrm{H}, J=15.9 \& 5.8 \mathrm{~Hz}), 4.69(\mathrm{~d}, 2 \mathrm{H}, J=5.8 \mathrm{~Hz}), 4.53(\mathrm{~m}$, $2 \mathrm{H}), 4.17(\mathrm{~m}, 2 \mathrm{H}), 2.13(\mathrm{~d}, 3 \mathrm{H}, J=1.2 \mathrm{~Hz}) ;{ }^{13} \mathrm{C}\left\{{ }^{1} \mathrm{H}\right\}$ NMR $\left(75 \mathrm{MHz}, \mathrm{CDCl}_{3}\right): \delta 171.5,158.3,154.1$, $136.6,133.4,131.5,130.4,130.3,129.0$ (2C), 128.7 (2C), 128.1, 126.7 (2C), 124.3, 114.8 (2C), $68.7,62.2,42.7,29.8$.

\subsection{Characterization of (Z)-29 to (Z)-55}

(Z)-3-(3-phenylbut-2-enoyl)oxazolidin-2-one ((Z)-29):<smiles>C/C(=C/C(=O)N1CCOC1=O)c1ccccc1</smiles>

(Z)-29

Prepared according to general procedure D. Obtained as a white solid (69 $\mathrm{mg}$, quant. yield, $E / Z>95: 5)$ after filtration over a plug of silica $\left(\mathrm{Et}_{2} \mathrm{O}\right) .{ }^{1} \mathbf{H}$ NMR (300 MHz, $\left.\mathbf{C D C l}_{3}\right): \delta 7.37-7.32(\mathrm{~m}, 3 \mathrm{H}), 7.23-7.20(\mathrm{~m}, 2 \mathrm{H}), 7.00$ $(\mathrm{q}, 1 \mathrm{H}, J=1.2 \mathrm{~Hz}), 4.35(\mathrm{t}, 2 \mathrm{H}, J=8.0 \mathrm{~Hz}), 3.93(\mathrm{t}, 2 \mathrm{H}, J=8.0 \mathrm{~Hz}), 2.26(\mathrm{~d}$, $3 \mathrm{H}, J=1.3 \mathrm{~Hz}) ;{ }^{13} \mathrm{C}\left\{{ }^{1} \mathrm{H}\right\}$ NMR $\left(75 \mathrm{MHz}, \mathrm{CDCl}_{3}\right): \delta 164.6,157.2,153.6,141.3$, $128.1(2 \mathrm{C}), 127.9,126.7$ (2C), 117.0, 62.1, 42.6, 27.7.

ethyl (Z)-3-phenylbut-2-enoate ((Z)-30):<smiles>CCOC(=O)/C=C(/C)c1ccccc1</smiles>

(Z)-30

Prepared according to general procedure D. Obtained as a colorless oil (61 $\mathrm{mg}, 96 \%$ yield, $E / Z=3: 97)$ after filtration over a plug of silica $\left(E_{2} \mathrm{O}\right) .{ }^{1} \mathbf{H}$ NMR (300 MHz, $\mathrm{CDCl}_{3}$ ): $\delta 7.40-7.35(\mathrm{~m}, 3 \mathrm{H}), 7.27-7.24(\mathrm{~m}, 2 \mathrm{H}), 5.96(\mathrm{q}, 1 \mathrm{H}, J$ $=1.4 \mathrm{~Hz}), 4.05(\mathrm{q}, 2 \mathrm{H}, J=7.1 \mathrm{~Hz}), 2.23(\mathrm{~d}, 3 \mathrm{H}, J=1.4 \mathrm{~Hz}), 1.13(\mathrm{~d}, 3 \mathrm{H}, J=7.1$ $\mathrm{Hz}$ ); ${ }^{13} \mathrm{C}\left\{{ }^{1} \mathrm{H}\right\}$ NMR (75 MHz, $\left.\mathrm{CDCl}_{3}\right): \delta 166.1,155.5,141.0,128.0(2 \mathrm{C}), 127.8$, $126.9(2 \mathrm{C}), 117.9,59.9,27.3,14.1$. The obtained special data were in agreement with the literature. ${ }^{[7]}$

(Z)-3-(3-phenylpent-2-enoyl)oxazolidin-2-one ((Z)-31):<smiles>CC/C(=C/C(=O)N1CCOC1=O)c1ccccc1</smiles>

(Z)-31

Prepared according to general procedure $\mathbf{D}$. Obtained as a yellow solid (73 mg, quant. yield, $E / Z=3: 97$ ) after filtration over a plug of silica $\left(\mathrm{Et}_{2} \mathrm{O}\right)$. ${ }^{1} \mathrm{H}$ NMR (300 MHz, CDCl 3 ): $\delta 7.35(\mathrm{~m}, 3 \mathrm{H}), 7.17(\mathrm{~d}, 2 \mathrm{H}, J=7.0 \mathrm{~Hz}), 6.99(\mathrm{~m}$, $1 \mathrm{H}), 4.34(\mathrm{t}, 2 \mathrm{H}, J=8.0 \mathrm{~Hz}), 3.92(\mathrm{t}, 2 \mathrm{H}, J=8.0 \mathrm{~Hz}), 2.54(\mathrm{q}, 2 \mathrm{H}, J=7.3 \mathrm{~Hz})$, $1.11(\mathrm{t}, 3 \mathrm{H}, J=7.3 \mathrm{~Hz}) ;{ }^{13} \mathrm{C}\left\{{ }^{1} \mathrm{H}\right\}$ NMR $\left(75 \mathrm{MHz}, \mathrm{CDCl}_{3}\right): \delta 164.8,162.7$, $153.6,140.8,128.0(2 \mathrm{C}), 127.7,126.9$ (2C), 115.8, 62.0, 42.6, 33.8, 12.1. 
ethyl (Z)-3-phenylpent-2-enoate ((Z)-32):<smiles>CCOC(=O)/C=C(/CC)c1ccccc1</smiles>

(Z)-32

Prepared according to general procedure D. Obtained as a colorless oil (61 $\mathrm{mg}$, quant. yield, $E / Z=62: 38)$ after filtration over a plug of silica $\left(\mathrm{Et}_{2} \mathrm{O}\right) .{ }^{1} \mathbf{H}$ NMR (300 MHz, $\left.\mathrm{CDCl}_{3}\right): \delta 7.33-7.30(\mathrm{~m}, 3 \mathrm{H}), 7.16(\mathrm{~d}, 2 \mathrm{H}, J=7.1 \mathrm{~Hz}), 5.87$ $(\mathrm{m}, 1 \mathrm{H}), 3.99(\mathrm{q}, 2 \mathrm{H}, J=7.1 \mathrm{~Hz}), 2.47(\mathrm{q}, 2 \mathrm{H}, J=7.3 \mathrm{~Hz}), 1.06(\mathrm{~m}, 6 \mathrm{H}) ;{ }^{13} \mathrm{C}\left\{{ }^{1} \mathrm{H}\right\}$ NMR (75 MHz, $\left.\mathrm{CDCl}_{3}\right): \delta 166.3,161.2,140.6,127.9$ (2C), 127.6, 127.1 (2C), with the literature. ${ }^{[7]}$

$116.4,59.9,33.5,14.1,12.2$. The obtained special data were in agreement (Z)-3-(3-(p-tolyl)but-2-enoyl)oxazolidin-2-one ((Z)-33):<smiles>C/C(=C/C(=O)N1CCOC1=O)c1ccc(C)cc1</smiles>

$(Z)-33$

Prepared according to general procedure $\mathbf{D}$. Obtained as a colorless oil (73 $\mathrm{mg}$, quant. yield, $E / Z=10: 90$ ) after filtration over a plug of silica (Et $\left.{ }_{2} \mathrm{O}\right) .{ }^{1} \mathrm{H}$ NMR (300 MHz, $\left.\mathrm{CDCl}_{3}\right): \delta 7.18-7.11(\mathrm{~m}, 4 \mathrm{H}), 6.97(\mathrm{~m}, 1 \mathrm{H})$, $4.33(\mathrm{t}, 2 \mathrm{H}, J=8.0 \mathrm{~Hz}), 3.92(\mathrm{t}, 2 \mathrm{H}, J=8.0 \mathrm{~Hz}), 2.36(\mathrm{~s}, 3 \mathrm{H}), 2.25(\mathrm{~d}, 3 \mathrm{H}$, $J=1.1 \mathrm{~Hz}) ;{ }^{13} \mathrm{C}\left\{{ }^{1} \mathrm{H}\right\}$ NMR $\left(75 \mathrm{MHz}, \mathrm{CDCl}_{3}\right): \delta$ 164.6, 157.4, 153.6, 138.2, $137.8,128.8(2 \mathrm{C}), 126.7(2 \mathrm{C}), 116.7,62.0,42.6,27.7,21.3$.

ethyl (Z)-3-(p-tolyl)but-2-enoate ((Z)-34):<smiles>CCOC(=O)/C=C(/C)c1ccc(C)cc1</smiles>

(Z)-34

Prepared according to general procedure D. Obtained as a colorless oil (61 $\mathrm{mg}$, quant. yield, $E / Z=7: 93$ ) after filtration over a plug of silica $\left(E t_{2} \mathrm{O}\right)$. ${ }^{1} \mathrm{H}$ NMR (300 MHz, CDCl 3$): \delta 7.14(\mathrm{~m}, 4 \mathrm{H}), 5.89(\mathrm{q}, 1 \mathrm{H}, J=1.1 \mathrm{~Hz}), 4.02$ $(\mathrm{q}, 2 \mathrm{H}, J=7.1 \mathrm{~Hz}), 2.36(\mathrm{~s}, 3 \mathrm{H}), 2.17(\mathrm{~d}, 3 \mathrm{H}, J=1.0 \mathrm{~Hz}), 1.12(\mathrm{t}, 3 \mathrm{H}, J=7.1$ $\mathrm{Hz}) ;{ }^{13} \mathrm{C}\left\{{ }^{1} \mathrm{H}\right\}$ NMR (75 MHz, $\left.\mathrm{CDCl}_{3}\right): \delta 166.1,155.6,137.8,137.7,128.7$ (2C), 126.9 (2C), 117.4, 59.8, 27.2, 21.4, 14.1. The obtained special data were in agreement with the literature. ${ }^{[7]}$

\section{(Z)-3-(3-(m-tolyl)but-2-enoyl)oxazolidin-2-one ((Z)-35):}<smiles>C/C(=C/C(=O)N1CCOC1=O)c1cccc(C)c1</smiles>

(Z)-35

Prepared according to general procedure D. Obtained as a colorless oil (74 $\mathrm{mg}$, quant. yield, $E / Z=8: 92$ ) after filtration over a plug of silica (Et $\left.{ }_{2} \mathrm{O}\right) .{ }^{1} \mathrm{H} \mathrm{NMR}\left(300 \mathrm{MHz}, \mathrm{CDCl}_{3}\right): \delta 7.18-7.13(\mathrm{~m}, 1 \mathrm{H}), 7.05-7.03(\mathrm{~m}$, $1 \mathrm{H}), 6.93-6.88(\mathrm{~m}, 3 \mathrm{H}), 4.24(\mathrm{t}, 2 \mathrm{H}, J=8.0 \mathrm{~Hz}), 3.82(\mathrm{t}, 2 \mathrm{H}, J=8.0 \mathrm{~Hz})$, $2.27(\mathrm{~s}, 3 \mathrm{H}), 2.15(\mathrm{~d}, 3 \mathrm{H}, J=1.3 \mathrm{~Hz}) ;{ }^{13} \mathrm{C}\left\{{ }^{1} \mathrm{H}\right\} \mathbf{N M R}\left(75 \mathrm{MHz}, \mathrm{CDCl}_{3}\right): \delta$ 164.6, 157.5, 153.6, 141.3, 137.7, 128.7, 128.0, 127.1, 123.8, 116.9, $62.0,42.6,27.7,21.5$.

ethyl (Z)-3-(m-tolyl)but-2-enoate ((Z)-36):<smiles>CCOC(=O)/C=C(/C)c1cccc(C)c1</smiles>

(Z)-36

Prepared according to general procedure $\mathbf{D}$. Obtained as a colorless oil (61 $\mathrm{mg}$, quant. yield, $E / Z=43: 57$ ) after filtration over a plug of silica (Et $\left.{ }_{2} \mathrm{O}\right) .{ }^{1} \mathbf{H}$ NMR (300 MHz, $\left.\mathrm{CDCl}_{3}\right): \delta 7.27-7.25(\mathrm{~m}, 1 \mathrm{H}), 7.17-7.14(\mathrm{~m}$, $1 \mathrm{H}), 7.05-7.03(\mathrm{~m}, 2 \mathrm{H}), 5.93(\mathrm{~m}, 1 \mathrm{H}), 4.05(\mathrm{q}, 2 \mathrm{H}, J=7.1 \mathrm{~Hz}), 2.40(\mathrm{~s}, 3 \mathrm{H})$, $2.20(\mathrm{~d}, 3 \mathrm{H}, J=0.8 \mathrm{~Hz}), 1.13(\mathrm{t}, 3 \mathrm{H}, J=7.1 \mathrm{~Hz}) ;{ }^{13} \mathrm{C}\left\{{ }^{1} \mathrm{H}\right\}$ NMR $(75 \mathrm{MHz}$, $\left.\mathrm{CDCl}_{3}\right): \delta 166.1,155.6,140.9,137.5,128.6,127.9,127.5,124.0,117.7$,

$59.8,27.3,21.5,14.1$. 
(Z)-3-(3-(o-tolyl)but-2-enoyl)oxazolidin-2-one ((Z)-37):

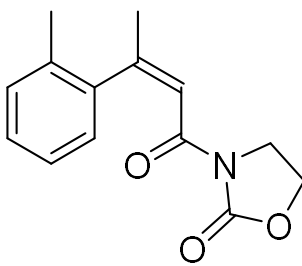

$(Z)-37$

Prepared according to general procedure D. Obtained as an orange oil (73 $\mathrm{mg}$, quant. yield, $E / Z=43: 57)$ after filtration over a plug of silica $\left(\mathrm{Et}_{2} \mathrm{O}\right) .{ }^{{ }^{1} \mathrm{H}}$ NMR (300 MHz, $\left.\mathbf{C D C l}_{3}\right): \delta 7.21-7.19(\mathrm{~m}, 3 \mathrm{H}), 7.03(\mathrm{~m}, 1 \mathrm{H}), 6.98-6.96$ $(\mathrm{m}, 1 \mathrm{H}), 4.34(\mathrm{t}, 2 \mathrm{H}, \mathrm{J}=8.0 \mathrm{~Hz}), 3.89(\mathrm{t}, 2 \mathrm{H}, \mathrm{J}=8.0 \mathrm{~Hz}), 2.23(\mathrm{~s}, 3 \mathrm{H}), 2.21$ $(\mathrm{d}, 3 \mathrm{H}, J=1.3 \mathrm{~Hz}) ;{ }^{13} \mathrm{C}\left\{{ }^{1} \mathrm{H}\right\}$ NMR $\left(75 \mathrm{MHz}, \mathrm{CDCl}_{3}\right): \delta 163.8,158.7,153.7$, $141.7,133.4,129.8,127.3,125.7,125.5,117.3,62.0,42.6,27.7,19.2$.

(Z)-3-(3-(3-(tert-butyl)phenyl)but-2-enoyl)oxazolidin-2-one ((Z)-39):<smiles>C/C(=C/C(=O)N1CCOC1=O)c1ccc(C(C)(C)C)cc1</smiles>

Prepared according to general procedure D. Obtained as a white solid (86 $\mathrm{mg}$, quant. yield, $E / Z=15: 85$ ) after filtration over a plug of silica $\left(\mathrm{Et}_{2} \mathrm{O}\right) .{ }^{1} \mathrm{H}$ NMR $\left(300 \mathrm{MHz}, \mathrm{CDCl}_{3}\right): \delta 7.37(\mathrm{~d}, 2 \mathrm{H}, J=8.4 \mathrm{~Hz}), 7.18$ $(\mathrm{d}, 2 \mathrm{H}, J=8.4 \mathrm{~Hz}), 6.96(\mathrm{~m}, 1 \mathrm{H}), 4.32(\mathrm{t}, 2 \mathrm{H}, J=8.0 \mathrm{~Hz}), 3.93(\mathrm{t}, 2 \mathrm{H}, J$ $=8.0 \mathrm{~Hz}), 2.25(\mathrm{~d}, 3 \mathrm{H}, J=1.2 \mathrm{~Hz}), 1.33(\mathrm{~s}, 9 \mathrm{H}) ;{ }^{13} \mathrm{C}\left\{{ }^{1} \mathrm{H}\right\} \mathrm{NMR}(75 \mathrm{MHz}$, $\left.\mathrm{CDCl}_{3}\right): \delta 164.7,157.1,153.6,151.0,137.9,126.7$ (2C), $125.0(2 \mathrm{C})$, $116.6,62.0,42.7,34.7,31.4(3 C), 27.7$.

ethyl (Z)-3-(3-(tert-butyl)phenyl)but-2-enoate ((Z)-40):<smiles>CCOC(=O)/C=C(/C)c1ccc(C(C)(C)C)cc1</smiles>

(Z)-40

Prepared according to general procedure $\mathbf{D}$. Obtained as a colorless oil (71 $\mathrm{mg}, 96 \%$ yield, $E / Z=8: 92$ ) after filtration over a plug of silica $\left(\mathrm{Et}_{2} \mathrm{O}\right)$. ${ }^{1} \mathrm{H}$ NMR (300 MHz, CDCl $): \delta 7.38(\mathrm{~d}, 2 \mathrm{H}, J=8.3 \mathrm{~Hz}), 7.19(\mathrm{~d}, 2 \mathrm{H}, J=8.3$ $\mathrm{Hz}), 5.91(\mathrm{~m}, 1 \mathrm{H}), 4.03(\mathrm{q}, 2 \mathrm{H}, J=7.1 \mathrm{~Hz}), 2.19(\mathrm{~d}, 3 \mathrm{H}, J=1.2 \mathrm{~Hz}), 1.35$ (s, 9H), 1.09 (t, 3H, J = $7.1 \mathrm{~Hz}) ;{ }^{13} \mathrm{C}\left\{{ }^{1} \mathrm{H}\right\}$ NMR (75 MHz, $\left.\mathrm{CDCl}_{3}\right): \delta$ 166.2, $155.4,150.8,137.7,126.8$ (2C), 124.8 (2C), 117.5, 59.8, 34.7, 31.4 (3C),

27.2, 14.0.

(Z)-3-(3-(4-methoxyphenyl)but-2-enoyl)oxazolidin-2-one ((Z)-41):<smiles>COc1ccc(/C(C)=C\C(=O)N2CCOC2=O)cc1</smiles>

42.6, 27.6.

Prepared according to general procedure $\mathbf{D}$. Obtained as a yellow solid (78 $\mathrm{mg}$, quant. yield, $E / Z=15: 85$ ) after filtration over a plug of silica (Et $\left.{ }_{2} \mathrm{O}\right) .{ }^{1} \mathbf{H} \mathbf{~ N M R}\left(300 \mathrm{MHz}, \mathrm{CDCl}_{3}\right): \delta 7.19(\mathrm{~d}, 2 \mathrm{H}, J=8.1 \mathrm{~Hz})$, $6.92-6.86(\mathrm{~m}, 3 \mathrm{H}), 4.34(\mathrm{t}, 2 \mathrm{H}, J=8.0 \mathrm{~Hz}), 3.93(\mathrm{t}, 2 \mathrm{H}, J=8.0 \mathrm{~Hz})$, $3.80(\mathrm{~s}, 3 \mathrm{H}), 2.24(\mathrm{~s}, 3 \mathrm{H}) ;{ }^{13} \mathrm{C}\left\{{ }^{1} \mathrm{H}\right\}$ NMR (75 MHz, $\left.\mathrm{CDCl}_{3}\right): \delta 164.8$, $159.5,156.8,153.6,133.0,128.5$ (2C), 116.4, 113.4 (2C), 62.0, 55.2,

ethyl (Z)-3-(4-methoxyphenyl)but-2-enoate ((Z)-42):<smiles>CCOC(=O)/C=C(/C)c1ccc(OC)cc1</smiles>

$(Z)-42$

Prepared according to general procedure $\mathbf{D}$. Obtained as a colorless oil (61 $\mathrm{mg}, 93 \%$ yield, $E / Z=33: 67$ ) after filtration over a plug of silica (Et $\left.{ }_{2} \mathrm{O}\right) .{ }^{1} \mathbf{H}$ NMR (300 MHz, $\left.\mathrm{CDCl}_{3}\right): \delta 7.20(\mathrm{~d}, 2 \mathrm{H}, J=8.7 \mathrm{~Hz}), 6.88(\mathrm{~d}$, $2 \mathrm{H}, J=8.7 \mathrm{~Hz}$ ), $5.88(\mathrm{q}, 1 \mathrm{H}, J=1.3 \mathrm{~Hz}), 4.04(\mathrm{q}, 2 \mathrm{H}, J=7.1 \mathrm{~Hz}), 3.82(\mathrm{~s}$, $3 \mathrm{H}), 2.17(\mathrm{~d}, 3 \mathrm{H}, J=1.3 \mathrm{~Hz}), 1.14(\mathrm{t}, 3 \mathrm{H}, J=7.1 \mathrm{~Hz}) ;{ }^{13} \mathrm{C}\left\{{ }^{1} \mathrm{H}\right\}$ NMR (75 $\left.\mathrm{MHz} \mathrm{CDCl}_{3}\right): \delta 166.2,159.5,155.0,132.7,128.6(2 \mathrm{C}), 117.1,113.3$ (2C), 59.8, 55.3, 27.2, 14.2. 
(Z)-3-(3-(4-fluorophenyl)but-2-enoyl)oxazolidin-2-one ((Z)-43):<smiles>C/C(=C/C(=O)N1CCOC1=O)c1ccc(F)cc1</smiles>

114.4

Prepared according to general procedure $\mathbf{D}$. Obtained as a colorless oil (75 $\mathrm{mg}$, quant. yield, $E / Z=19: 81$ ) after filtration over a plug of silica (Et $\left.{ }_{2} \mathrm{O}\right) .{ }^{1} \mathbf{H}$ NMR (300 MHz, $\left.\mathrm{CDCl}_{3}\right): \delta 7.21-7.16(\mathrm{~m}, 2 \mathrm{H}), 7.06-7.00$ $(\mathrm{m}, 3 \mathrm{H}), 4.35(\mathrm{t}, 2 \mathrm{H}, J=8.0 \mathrm{~Hz}), 3.92(\mathrm{t}, 2 \mathrm{H}, J=8.0 \mathrm{~Hz}), 2.23(\mathrm{~d}, 3 \mathrm{H}, J=$ $1.2 \mathrm{~Hz}) ;{ }^{13} \mathrm{C}\left\{{ }^{1} \mathrm{H}\right\}$ NMR $\left(75 \mathrm{MHz}, \mathrm{CDCl}_{3}\right): \delta 164.5,162.4(\mathrm{~d}, J=246.9 \mathrm{~Hz})$, $156.1,153.5,137.0$ (d, $J=3.6 \mathrm{~Hz}$ ), 128.7 (d, 2C, $J=8.1 \mathrm{~Hz}$ ), 117.3, 115.1 $(\mathrm{d}, 2 \mathrm{C}, J=21.5 \mathrm{~Hz}), 62.1,42.6,27.7 ;{ }^{19} \mathrm{~F}\left\{{ }^{1} \mathrm{H}\right\}$ NMR (282 MHz, $\left.\mathrm{CDCl}_{3}\right):-$

ethyl (Z)-3-(4-fluorophenyl)but-2-enoate ((Z)-44):<smiles>CCOC(=O)/C=C(/C)c1ccc(F)cc1</smiles>

(Z)-44

Prepared according to general procedure $\mathbf{D}$. Obtained as a colorless oil (62 $\mathrm{mg}$, quant. yield, $E / Z=69: 31$ ) after filtration over a plug of silica (Et $\left.{ }_{2} \mathrm{O}\right) .{ }^{1} \mathbf{H}$ NMR (300 MHz, CDCl 3 ): $\delta 7.19$ (dd, 2H, $J=8.6 \& 5.4 \mathrm{~Hz}$ ), 7.05 $(\mathrm{dd}, 2 \mathrm{H}, J=8.6 \mathrm{~Hz}$ ), $5.91(\mathrm{q}, 1 \mathrm{H}, J=1.1 \mathrm{~Hz}), 4.02(\mathrm{q}, 2 \mathrm{H}, J=7.1 \mathrm{~Hz}), 2.16$ (d, $3 \mathrm{H}, J=1.2 \mathrm{~Hz}), 1.12(\mathrm{t}, 3 \mathrm{H}, J=7.1 \mathrm{~Hz}) ;{ }^{13} \mathrm{C}\left\{{ }^{1} \mathrm{H}\right\}$ NMR $\left(75 \mathrm{MHz}, \mathrm{CDCl}_{3}\right)$ : $\delta 165.9,162.5(\mathrm{~d}, J=246.7 \mathrm{~Hz}), 154.4,136.6(\mathrm{~d}, J=3.5 \mathrm{~Hz}), 128.8(\mathrm{~d}, 2 \mathrm{C}$, $J=8.1 \mathrm{~Hz}), 118.2,114.9(\mathrm{~d}, 2 \mathrm{C}, J=21.5 \mathrm{~Hz}), 59.9,27.3,14.1 ;{ }^{19} \mathrm{~F}\left\{{ }^{1} \mathrm{H}\right\} \mathbf{N M R}\left(282 \mathrm{MHz}, \mathrm{CDCl}_{3}\right):-$ 114.7.

(Z)-3-(3-(4-bromophenyl)but-2-enoyl)oxazolidin-2-one ((Z)-45):<smiles>CC(=O)N1CCOC1=O</smiles>

Prepared according to general procedure $\mathbf{D}$. Obtained as a beige solid (93 $\mathrm{mg}$, quant. yield, $E / Z=9: 91$ ) after filtration over a plug of silica (Et $\left.{ }_{2} \mathrm{O}\right) .{ }^{1} \mathrm{H}$ NMR (300 MHz, $\left.\mathrm{CDCl}_{3}\right): \delta 7.47(\mathrm{~d}, 2 \mathrm{H}, J=8.4 \mathrm{~Hz}), 7.08(\mathrm{~d}$, $2 \mathrm{H}, J=8.4 \mathrm{~Hz}), 7.03(\mathrm{~m}, 1 \mathrm{H}), 4.35(\mathrm{t}, 2 \mathrm{H}, J=8.0 \mathrm{~Hz}), 3.92(\mathrm{t}, 2 \mathrm{H}, J=8.0$ $\mathrm{Hz}), 2.22(\mathrm{~d}, 3 \mathrm{H}, J=1.2 \mathrm{~Hz}) ;{ }^{13} \mathrm{C}\left\{{ }^{1} \mathrm{H}\right\}$ NMR (75 MHz, $\left.\mathrm{CDCl}_{3}\right): \delta 164.3$, 155.9, 153.5, 140.1, 131.3 (2C), 128.5 (2C), 122.0, 117.5, 62.1, 42.5,

27.5.

ethyl (Z)-3-(4-bromophenyl)but-2-enoate ((Z)-46):<smiles>CCOC(=O)/C=C(/C)c1ccc(Br)cc1</smiles>

(Z)-46

Prepared according to general procedure $\mathbf{D}$. Obtained as a colorless oil (81 $\mathrm{mg}$, quant. yield, $E / Z=5: 95$ ) after filtration over a plug of silica (Et $\left.{ }_{2} \mathrm{O}\right) .{ }^{1} \mathrm{H}$ NMR (300 MHz, CDCl 3 ): $\delta 7.48(\mathrm{~d}, 2 \mathrm{H}, J=8.4 \mathrm{~Hz}), 7.09(\mathrm{~d}, 2 \mathrm{H}$, $J=8.4 \mathrm{~Hz}), 5.93(\mathrm{~m}, 1 \mathrm{H}), 4.02(\mathrm{q}, 2 \mathrm{H}, J=7.1 \mathrm{~Hz}), 2.16(\mathrm{~d}, 3 \mathrm{H}, J=1.3 \mathrm{~Hz})$, $1.13(\mathrm{t}, 3 \mathrm{H}, J=7.1 \mathrm{~Hz}) ;{ }^{13} \mathrm{C}\left\{{ }^{1} \mathrm{H}\right\}$ NMR $\left(75 \mathrm{MHz}, \mathrm{CDCl}_{3}\right): \delta 165.7,154.3$, $139.8,131.2$ (2C), 128.7 (2C), 121.9, 118.3, 60.0, 27.1, 14.1. The obtained special data were in agreement with the literature. ${ }^{[7]}$ 
(Z)-3-(3-(4-iodophenyl)but-2-enoyl)oxazolidin-2-one ((Z)-47):<smiles>C/C(=C/C(=O)N1CCOC1=O)c1ccc(I)cc1</smiles>

(Z)-47

Prepared according to general procedure $\mathbf{D}$. Obtained as a colorless oil (92 $\mathrm{mg}, 86 \%$ yield, $E / Z=12: 88$ ) after filtration over a plug of silica (Et $\left.{ }_{2} \mathrm{O}\right) .{ }^{1} \mathrm{H}$ NMR (300 MHz, $\left.\mathrm{CDCl}_{3}\right): \delta 7.68(\mathrm{~d}, 2 \mathrm{H}, J=8.3 \mathrm{~Hz}), 7.03(\mathrm{~m}$, $1 \mathrm{H}), 6.97(\mathrm{~d}, 2 \mathrm{H}, J=8.3 \mathrm{~Hz}), 4.37(\mathrm{t}, 2 \mathrm{H}, J=8.0 \mathrm{~Hz}), 3.93(\mathrm{t}, 2 \mathrm{H}, J=8.0$ $\mathrm{Hz}), 2.23(\mathrm{~d}, 3 \mathrm{H}, J=1.2 \mathrm{~Hz}) ;{ }^{13} \mathrm{C}\left\{{ }^{1} \mathrm{H}\right\}$ NMR $\left(75 \mathrm{MHz}, \mathrm{CDCl}_{3}\right): \delta 164.2$, 156.0, 153.5, 140.7, 137.2 (2C), $128.6(2 \mathrm{C}), 117.4,93.7,62.1,42.5,27.5$.

ethyl (Z)-3-(4-iodophenyl)but-2-enoate ((Z)-48):<smiles>CCOC(=O)/C=C(/C)c1ccc(I)cc1</smiles>

(Z)-48

Prepared according to general procedure D. Obtained as a colorless oil (93 mg, 98\% yield, $E / Z=9: 91$ ) after filtration over a plug of silica $\left(\mathrm{Et}_{2} \mathrm{O}\right)$.

${ }^{1} \mathrm{H}$ NMR (300 MHz, $\left.\mathrm{CDCl}_{3}\right): \delta 7.68(\mathrm{~d}, 2 \mathrm{H}, J=8.3 \mathrm{~Hz}), 6.96(\mathrm{~d}, 2 \mathrm{H}, J=8.3$ $\mathrm{Hz}), 5.92(\mathrm{q}, 1 \mathrm{H}, J=1.0 \mathrm{~Hz}), 4.02(\mathrm{q}, 2 \mathrm{H}, J=7.1 \mathrm{~Hz}), 2.15(\mathrm{~d}, 3 \mathrm{H}, J=1.0$ $\mathrm{Hz}), 1.13(\mathrm{t}, 3 \mathrm{H}, J=7.1 \mathrm{~Hz}) ;{ }^{13} \mathrm{C}\left\{{ }^{1} \mathrm{H}\right\}$ NMR $\left(75 \mathrm{MHz}, \mathrm{CDCl}_{3}\right): \delta 165.7,154.4$, $140.4,137.1(2 \mathrm{C}), 128.9(2 \mathrm{C}), 118.3,93.6,60.0,27.1,14.1$.

(Z)-3-(3-(4-nitrophenyl)but-2-enoyl)oxazolidin-2-one ((Z)-49):<smiles>C/C(=C/C(=O)N1CCOC1=O)c1ccc([N+](=O)[O-])cc1</smiles>

Prepared according to general procedure D. Obtained as a beige solid (83 $\mathrm{mg}$, quant. yield, $E / Z=39: 61$ ) after filtration over a plug of silica (Et $\left.{ }_{2} \mathrm{O}\right) .{ }^{1} \mathrm{H}$ NMR (300 MHz, $\left.\mathrm{CDCl}_{3}\right): \delta 8.09(\mathrm{~d}, 2 \mathrm{H}, J=8.7 \mathrm{~Hz})$, $7.23(\mathrm{~d}, 2 \mathrm{H}, J=8.7 \mathrm{~Hz}), 7.06(\mathrm{~m}, 1 \mathrm{H}), 4.29(\mathrm{t}, 2 \mathrm{H}, J=8.0 \mathrm{~Hz}), 3.81(\mathrm{t}$, $2 \mathrm{H}, J=8.0 \mathrm{~Hz}), 2.15(\mathrm{~d}, 3 \mathrm{H}, J=1.2 \mathrm{~Hz}) ;{ }^{13} \mathrm{C}\left\{{ }^{1} \mathrm{H}\right\} \mathrm{NMR}\left(75 \mathrm{MHz}, \mathrm{CDCl}_{3}\right)$ : $\delta$ 163.9, 155.1, 153.5, 148.6, 147.2, 127.6 (2C), 123.6 (2C), 118.4, 62.2, 42.5, 27.3.

ethyl (Z)-3-(4-nitrophenyl)but-2-enoate ((Z)-50):<smiles>CCOC(=O)/C=C(/C)c1ccc([N+](=O)[O-])cc1</smiles>

$(Z)-50$

Prepared according to general procedure $\mathbf{D}$. Obtained as an orange oil (70 $\mathrm{mg}$, quant. yield, $E / Z=36: 64$ ) after filtration over a plug of silica $\left(\mathrm{Et}_{2} \mathrm{O}\right) .{ }^{1} \mathrm{H}$ NMR (300 MHz, $\left.\mathrm{CDCl}_{3}\right): \delta 8.21(\mathrm{~d}, 2 \mathrm{H}, J=8.6 \mathrm{~Hz}), 7.35$ (d, $2 \mathrm{H}, J=8.6 \mathrm{~Hz}), 6.00(\mathrm{~m}, 1 \mathrm{H}), 4.00(\mathrm{q}, 2 \mathrm{H}, J=7.1 \mathrm{~Hz}), 2.19(\mathrm{~d}, 3 \mathrm{H}, J=0.7$ $\mathrm{Hz}), 1.11(\mathrm{t}, 3 \mathrm{H}, J=7.1 \mathrm{~Hz}) ;{ }^{13} \mathrm{C}\left\{{ }^{1} \mathrm{H}\right\}$ NMR $\left(75 \mathrm{MHz}, \mathrm{CDCl}_{3}\right): \delta 165.2$, $153.4,148.7,148.1,127.9$ (2C), 123.4 (2C), 119.4, 60.2, 26.9, 14.1.

(Z)-3-(4,4,4-trifluoro-3-phenylbut-2-enoyl)oxazolidin-2-one ((Z)-51):<smiles>O=C(C=C(c1ccccc1)C(F)(F)F)N1CCOC1=O</smiles>

(Z)-51

Prepared according to general procedure D. Obtained as a white solid (78 $\mathrm{mg}, 91 \%$ yield, $E / Z=67: 33)$ after filtration over a plug of silica $\left(\mathrm{Et}_{2} \mathrm{O}\right) .{ }^{1} \mathbf{H}$ NMR (300 MHz, $\left.\mathrm{CDCl}_{3}\right): \delta 7.53-7.51(\mathrm{~m}, 2 \mathrm{H}), 7.43-7.41(\mathrm{~m}, 3 \mathrm{H}), 6.81(\mathrm{~m}$, $1 \mathrm{H}), 4.50(\mathrm{t}, 2 \mathrm{H}, J=8.0 \mathrm{~Hz}), 4.09(\mathrm{t}, 2 \mathrm{H}, J=8.0 \mathrm{~Hz}) ;{ }^{13} \mathrm{C}\left\{{ }^{1} \mathrm{H}\right\}$ NMR $(75 \mathrm{MHz}$, $\mathrm{CDCl}_{3}$ ): $\delta 163.9,153.5,133.6,129.7$ (2C), 128.8 (2C), 127.9, 63.0, 42.0. Due to very low conversion, carbon $\mathbf{1}, \mathbf{2}$ and $\mathbf{3}$ were not detected on NMR spectra even after prolonged analysis; ${ }^{19} \mathbf{F}\left\{{ }^{1} \mathrm{H}\right\}$ NMR $\left(282 \mathbf{M H z}, \mathrm{CDCl}_{3}\right): \delta-60.4$. 
ethyl (Z)-4,4,4-trifluoro-3-phenylbut-2-enoate ((Z)-52):<smiles>CCOC(=O)/C=C(\c1ccccc1)C(F)(F)F</smiles>

$(Z)-52$

Prepared according to general procedure D. Obtained as a colorless oil ( $73 \mathrm{mg}$, quant. yield, $E / Z=71: 29)$ after filtration over a plug of silica $\left(E_{2} \mathrm{O}\right) .{ }^{1} \mathbf{H} \mathbf{N M R}$ (300 MHz, $\left.\mathrm{CDCl}_{3}\right): \delta 7.41(\mathrm{~m}, 5 \mathrm{H}), 6.36(\mathrm{~m}, 1 \mathrm{H}), 4.32(\mathrm{q}, 2 \mathrm{H}, J=7.1 \mathrm{~Hz}), 1.36$ (t, $3 \mathrm{H}, J=7.1 \mathrm{~Hz}) ;{ }^{13} \mathrm{C}\left\{{ }^{1} \mathrm{H}\right\}$ NMR $\left(75 \mathrm{MHz}, \mathrm{CDCl}_{3}\right): \delta 164.8,143.1,137.9$ (q, $J=31.8$ $\mathrm{Hz}), 133.7,129.7(2 \mathrm{C}), 127.9(2 \mathrm{C}), 127.7(\mathrm{q}, J=3.5 \mathrm{~Hz}), 122.4(\mathrm{q}, J=275.8 \mathrm{~Hz}$ ), $61.9,14.1 ;{ }^{19} \mathbf{F}\left\{{ }^{1} \mathrm{H}\right\}$ NMR $\left(282 \mathbf{M H z} \mathbf{C D C l}_{3}\right): \delta$ - 60.6. The obtained special data were in agreement with the literature. ${ }^{[7]}$

\section{(Z)-3-phenylbut-2-enenitrile ((Z)-53):}<smiles>C/C(=C/C#N)c1ccccc1</smiles>

(Z)-53

Prepared according to general procedure D. Obtained as a colorless oil ( $43 \mathrm{mg}$, quant. yield, $E / Z=21: 79)$ after filtration over a plug of silica $\left(E_{2} \mathrm{O}\right) .{ }^{1} \mathbf{H} \mathbf{N M R}$ (300 MHz, $\left.\mathrm{CDCl}_{3}\right): \delta 7.57-7.54(\mathrm{~m}, 2 \mathrm{H}), 7.46-7.42(\mathrm{~m}, 3 \mathrm{H}), 5.41(\mathrm{q}, 1 \mathrm{H}, \mathrm{J}=1.3$ $\mathrm{Hz}), 2.29(\mathrm{~d}, 3 \mathrm{H}, J=1.3 \mathrm{~Hz}) ;{ }^{13} \mathrm{C}\left\{{ }^{1} \mathrm{H}\right\} \mathrm{NMR}\left(75 \mathrm{MHz}, \mathrm{CDCl}_{3}\right): \delta 161.1,129.9,128.9$, $128.7(2 \mathrm{C}), 127.1(2 \mathrm{C}), 125.9,95.5,24.7$.

diethyl (Z)-(2-phenylprop-1-en-1-yl)phosphonate ((Z)-54):<smiles>CCOC/C=C(/C)c1ccccc1</smiles>

(Z)-54

Prepared according to general procedure $\mathbf{D}$. Obtained as a colorless oil (76 $\mathrm{mg}$, quant. yield, $E / Z=86: 14$ ) after filtration over a plug of silica (Et $\left.{ }_{2} \mathrm{O}\right) .{ }^{1} \mathrm{H}$ NMR (300 MHz, $\left.\mathrm{CDCl}_{3}\right): \delta 7.49-7.35(\mathrm{~m}, 5 \mathrm{H}), 5.74(\mathrm{~d}, 1 \mathrm{H}, \mathrm{J}=$ $17.3 \mathrm{~Hz}), 4.14(\mathrm{dq}, 4 \mathrm{H}, J=8.5 \mathrm{~Hz}), 2.24(\mathrm{~m}, 3 \mathrm{H}), 1.08(\mathrm{t}, 6 \mathrm{H}, J=7.0 \mathrm{~Hz})$; ${ }^{31} \mathrm{P}\left\{{ }^{1} \mathrm{H}\right\}$ NMR $\left(121 \mathrm{MHz}, \mathrm{CDCl}_{3}\right): \delta 16.2$. Due to very low conversion, it was impossible to correctly characterize the $Z$-isomer on ${ }^{13} \mathrm{C}$ NMR on the crude reaction mixture.

\section{(Z)-((2-phenylprop-1-en-1-yl)sulfonyl)benzene ((Z)-55):}<smiles>C/C(=C/SOc1ccccc1)c1ccccc1</smiles>

(Z)-55

Prepared according to general procedure D. Obtained as a white solid (77 $\mathrm{mg}$, quant. yield, $E / Z=88: 12)$ after filtration over a plug of silica $\left(E_{2} \mathrm{O}\right) .{ }^{1} \mathrm{H}$ NMR (300 MHz, $\left.\mathrm{CDCl}_{3}\right)$ : $\delta 7.50-7.45(\mathrm{~m}, 3 \mathrm{H}), 7.33-7.27(\mathrm{~m}, 5 \mathrm{H}), 7.10-$ $7.07(\mathrm{~m}, 2 \mathrm{H}), 6.57(\mathrm{~m}, 1 \mathrm{H}), 2.15(\mathrm{~d}, 3 \mathrm{H}, J=1.4 \mathrm{~Hz}) ;{ }^{13} \mathrm{C}\left\{{ }^{1} \mathrm{H}\right\} \mathrm{NMR}(75 \mathrm{MHz}$, $\mathrm{CDCl}_{3}$ ): $\delta 154.7,144.1,137.5,132.8,129.1,128.7$ (2C), 128.5, 128.0 (2C), $127.6(2 \mathrm{C}), 127.3(2 \mathrm{C}), 27.8$. 


\section{References}

[1] C. F. Weise, V. H. Lauridsen, R. S. Rambo, E. H. Iversen, M.-L. Olsen, K. A. Jørgensen, J. Org. Chem. 2014, 79, 3537-3546.

[2] C. Palomo, M. Oiarbide, F. Dias, R. López, A. Linden, Angew. Chem. Int. Ed. 2004, 43, 33073310.

[3] Y.-C. Chan, Y.-Y. Yeung, Angew. Chem. Int. Ed. 2018, 57, 3483-3487.

[4] L. Su, K. Sun, N. Pan, L. Liu, M. Sun, J. Dong, Y. Zhou, S.-F. Yin, Org. Lett. 2018, 20, 33993402.

[5] C. Onneken, K. Bussmann, R. Gilmour, Angew. Chem. Int. Ed. 2020, 59, 330-334.

[6] Q. Yan, G. Xiao, Y. Wang, G. Zi, Z. Zhang, G. Hou, J. Am. Chem. Soc. 2019, 141, 1749-1756.

[7] J. B. Metternich, R. Gilmour, J. Am. Chem. Soc. 2015, 137, 11254-11257.

[8] M. A. Cismesia, T. P. Yoon, Chem. Sci. 2015, 6, 5426-5434.

[9] J. Ma, F. Schäfers, C. Daniliuc, K. Bergander, C. A. Strassert, F. Glorius, Angew. Chem. Int. Ed. 2020, 59, 9639-9645. 


\section{NMR Spectra}

\subsection{Starting materials}

methyl (E)-2-methyl-3-phenylacrylate ((E)-1):

${ }^{1} \mathrm{H}$ NMR $\left(\mathrm{CDCl}_{3}, 300 \mathrm{MHz}\right)$<smiles>CC(=O)C(C)=Cc1ccccc1</smiles>

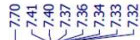

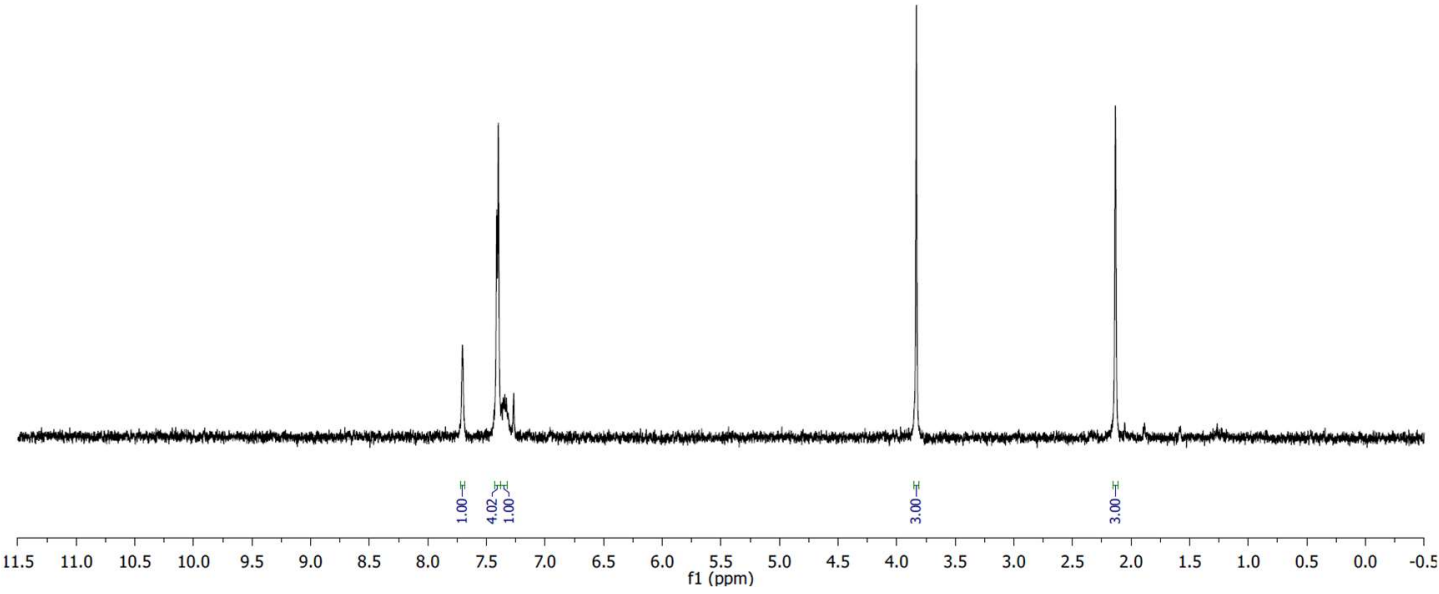

${ }^{13} \mathrm{C} \mathrm{NMR}\left(\mathrm{CDCl}_{3}, 75 \mathrm{MHz}\right)$
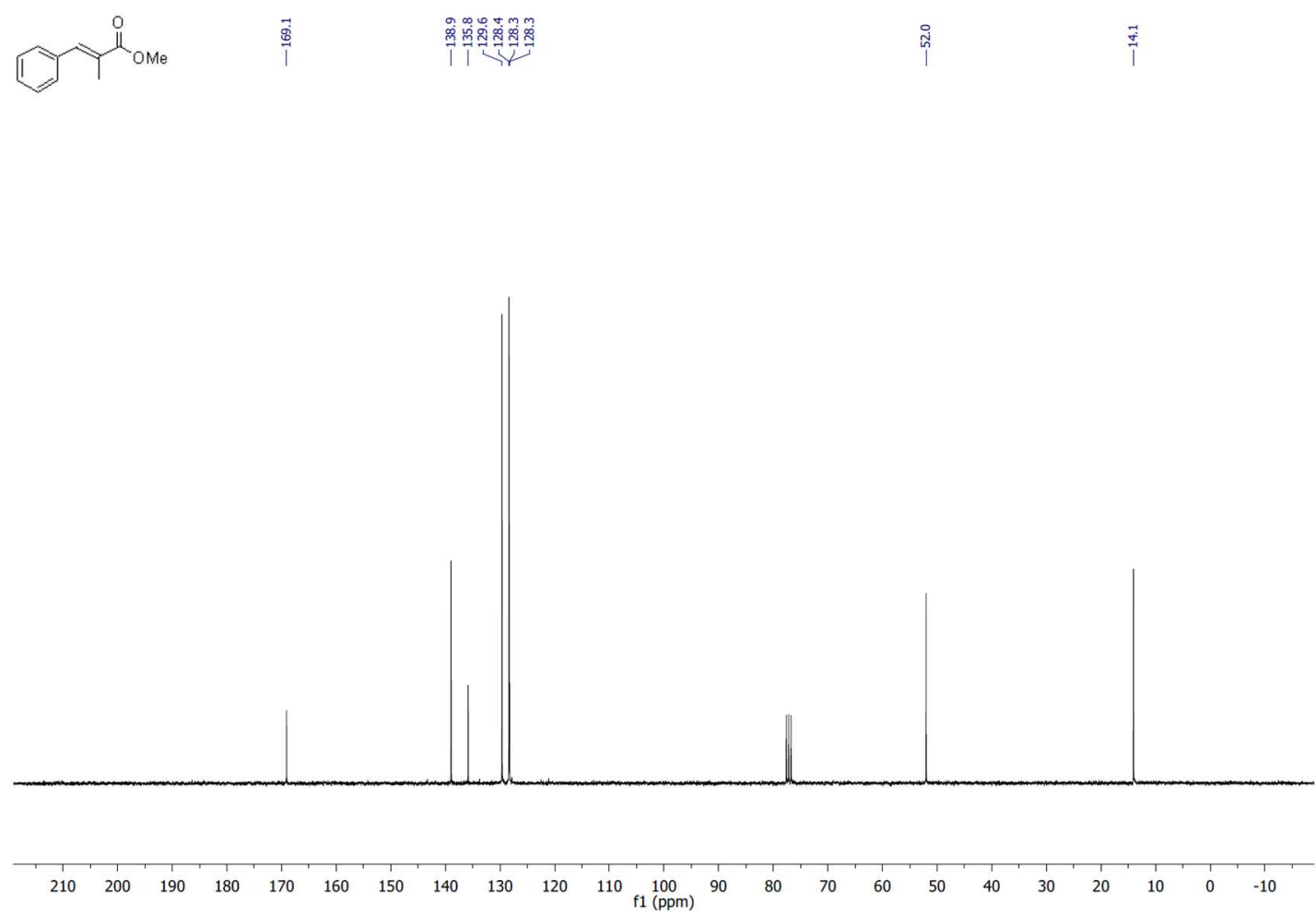
(E)-N,N,2-trimethyl-3-phenylacrylamide ((E)-2):

${ }^{1} \mathrm{H} \mathrm{NMR}\left(\mathrm{CDCl}_{3}, 300 \mathrm{MHz}\right)$

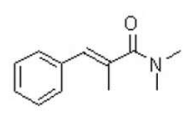

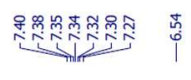

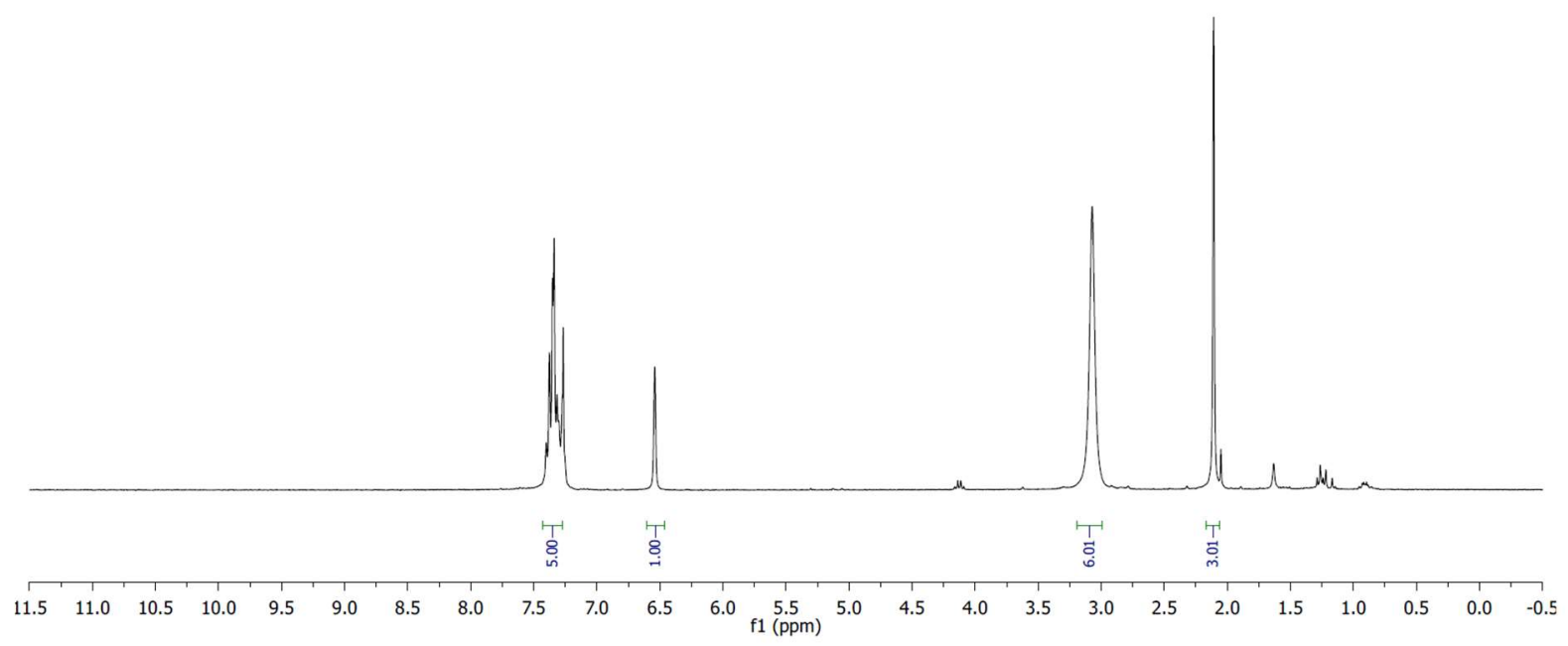

${ }^{13} \mathrm{C} \mathrm{NMR}\left(\mathrm{CDCl}_{3}, 75 \mathrm{MHz}\right)$
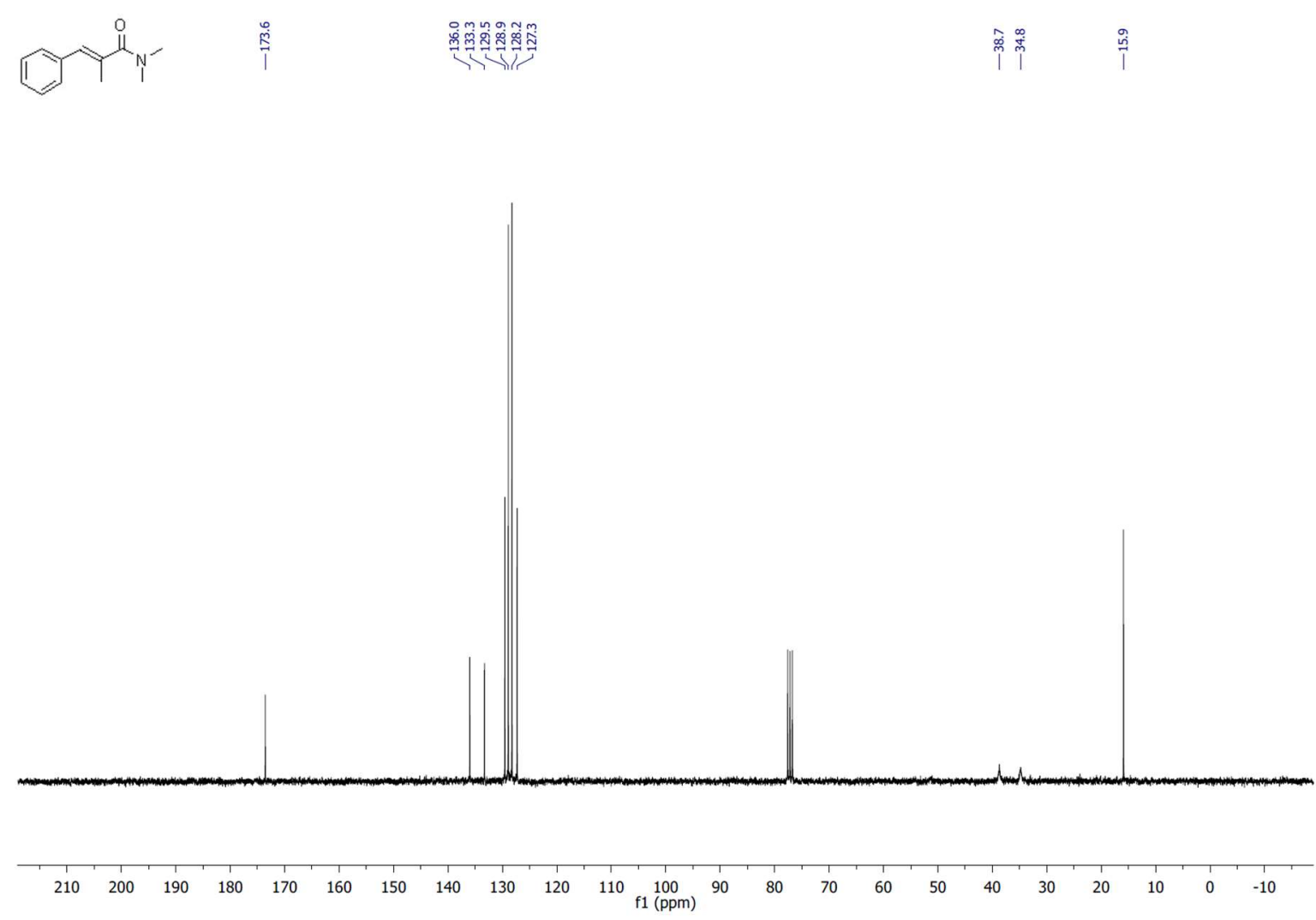
diethyl (E)-(2-methyl-3-phenylacryloyl)phosphonate ((E)-3):

${ }^{1} \mathrm{H} \mathrm{NMR}\left(\mathrm{CDCl}_{3}, 300 \mathrm{MHz}\right)$
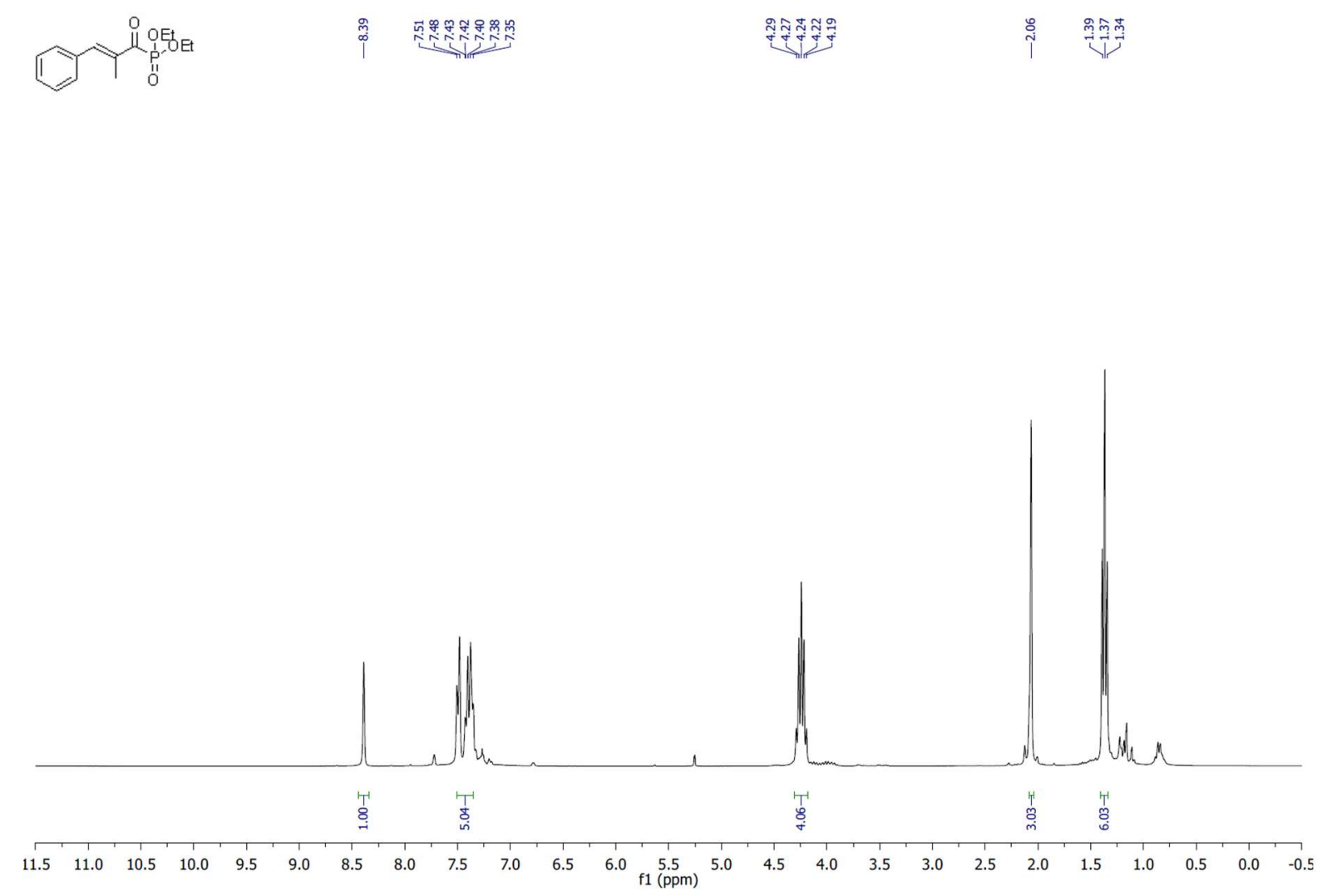

${ }^{13} \mathrm{C} \mathrm{NMR}\left(\mathrm{CDCl}_{3}, 75 \mathrm{MHz}\right)$
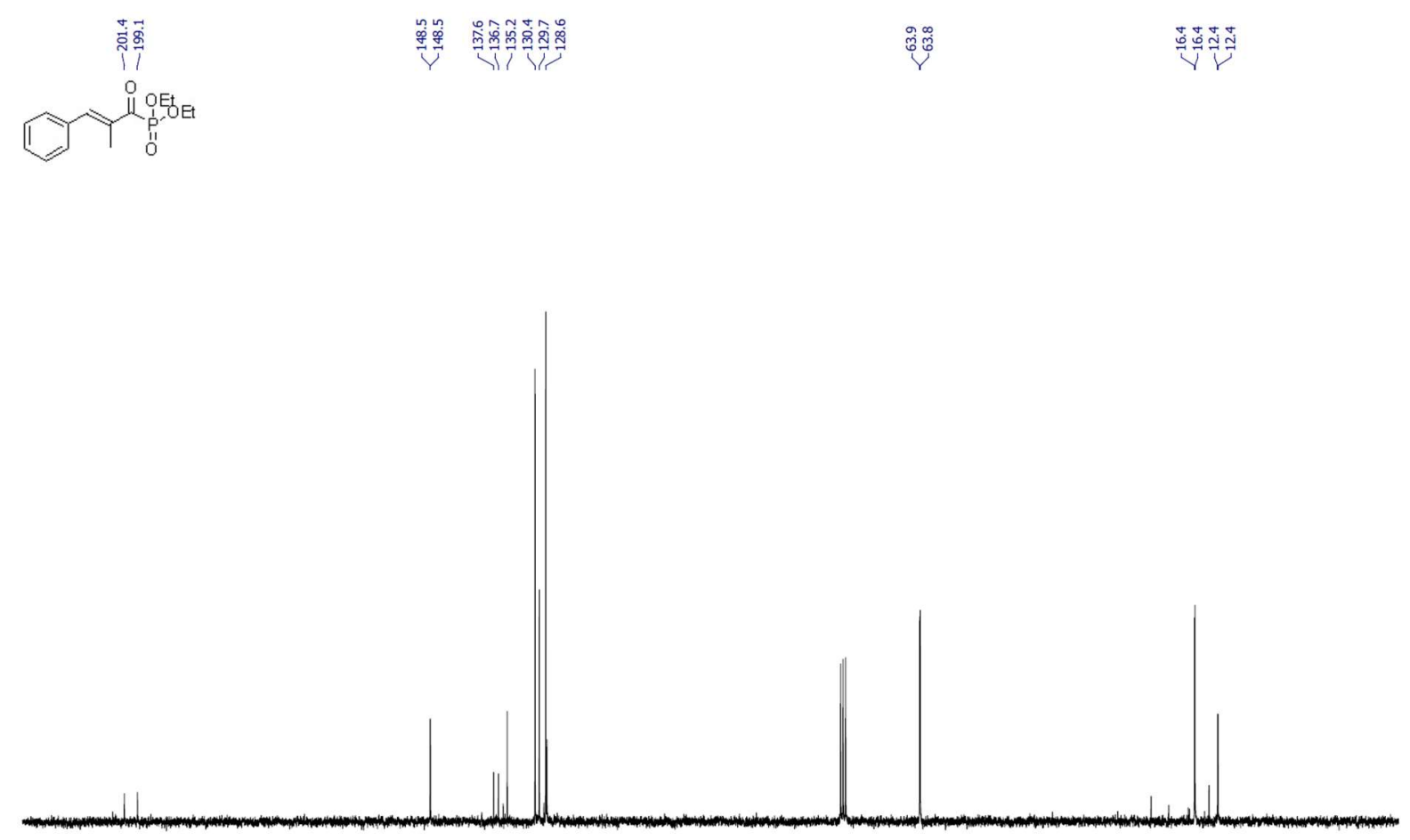

$\begin{array}{llllllllllllllllllllllllll}210 & 200 & 190 & 180 & 170 & 160 & 150 & 140 & 130 & 120 & 110 & \begin{array}{l}100 \\ \mathrm{f} 1(\mathrm{ppm})\end{array} & 90 & 80 & 70 & 60 & 50 & 40 & 30 & 20 & 10 & 0 & -10\end{array}$ 
${ }^{31} \mathrm{P} \mathrm{NMR}\left(\mathrm{CDCl}_{3}, 121 \mathrm{MHz}\right)$<smiles>CC(=O)OC(=O)C=Cc1ccccc1</smiles>

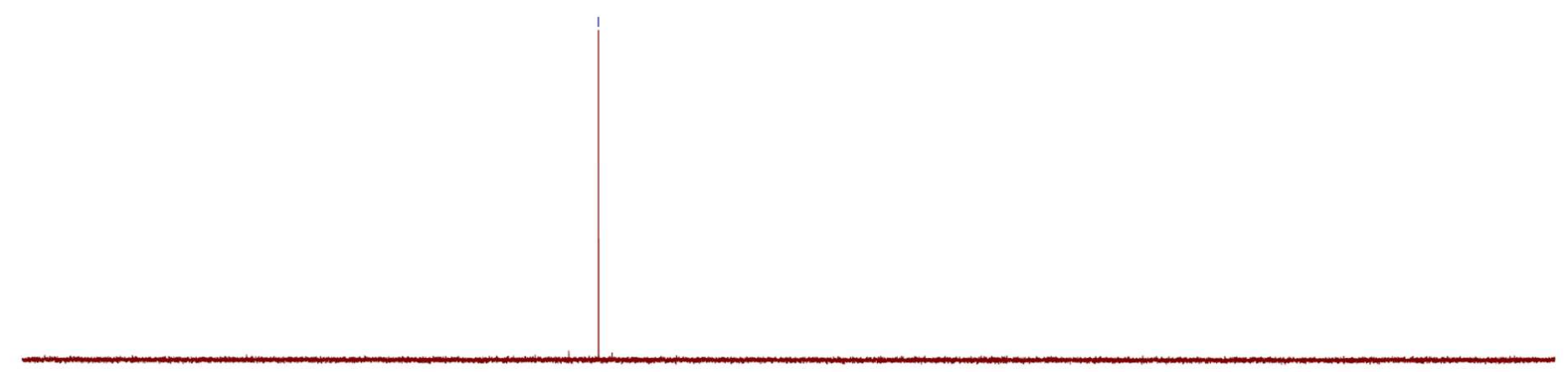

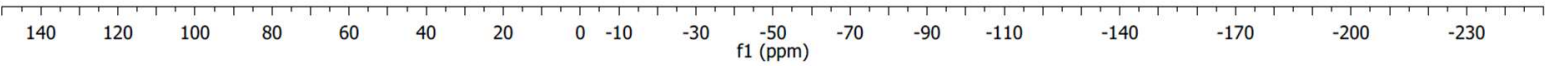

(E)-3-(2-methyl-3-phenylacryloyl)oxazolidin-2-one ((E)-4):

${ }^{1} \mathrm{H} \mathrm{NMR}\left(\mathrm{CDCl}_{3}, 300 \mathrm{MHz}\right)$

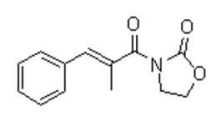

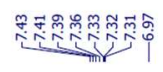

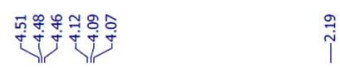

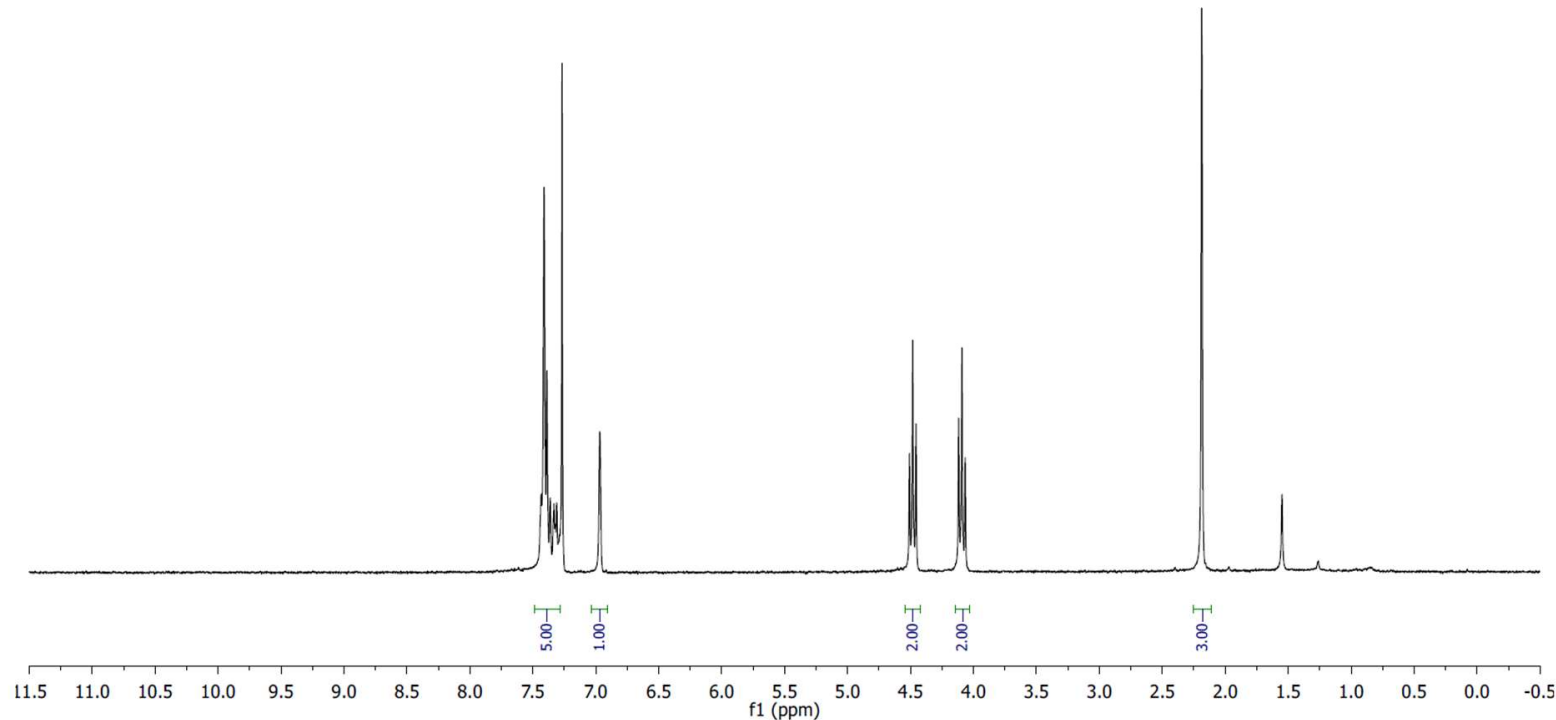


${ }^{13} \mathrm{C} \mathrm{NMR}\left(\mathrm{CDCl}_{3}, 75 \mathrm{MHz}\right)$

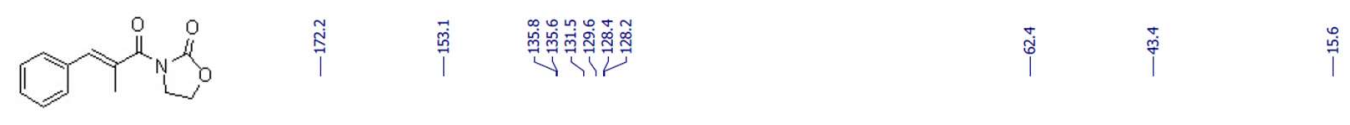

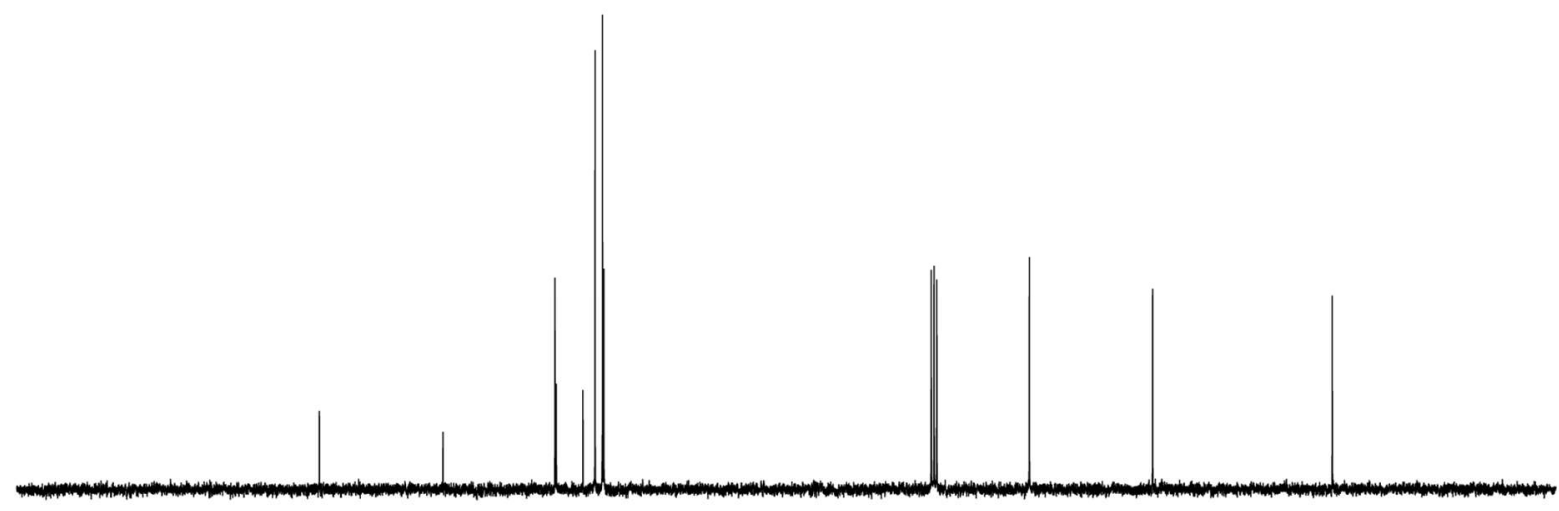

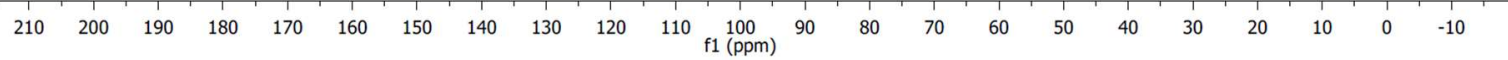

${ }^{1} \mathrm{H}$ NOESY NMR $\left(\mathrm{CDCl}_{3}, 300 \mathrm{MHz}\right)$

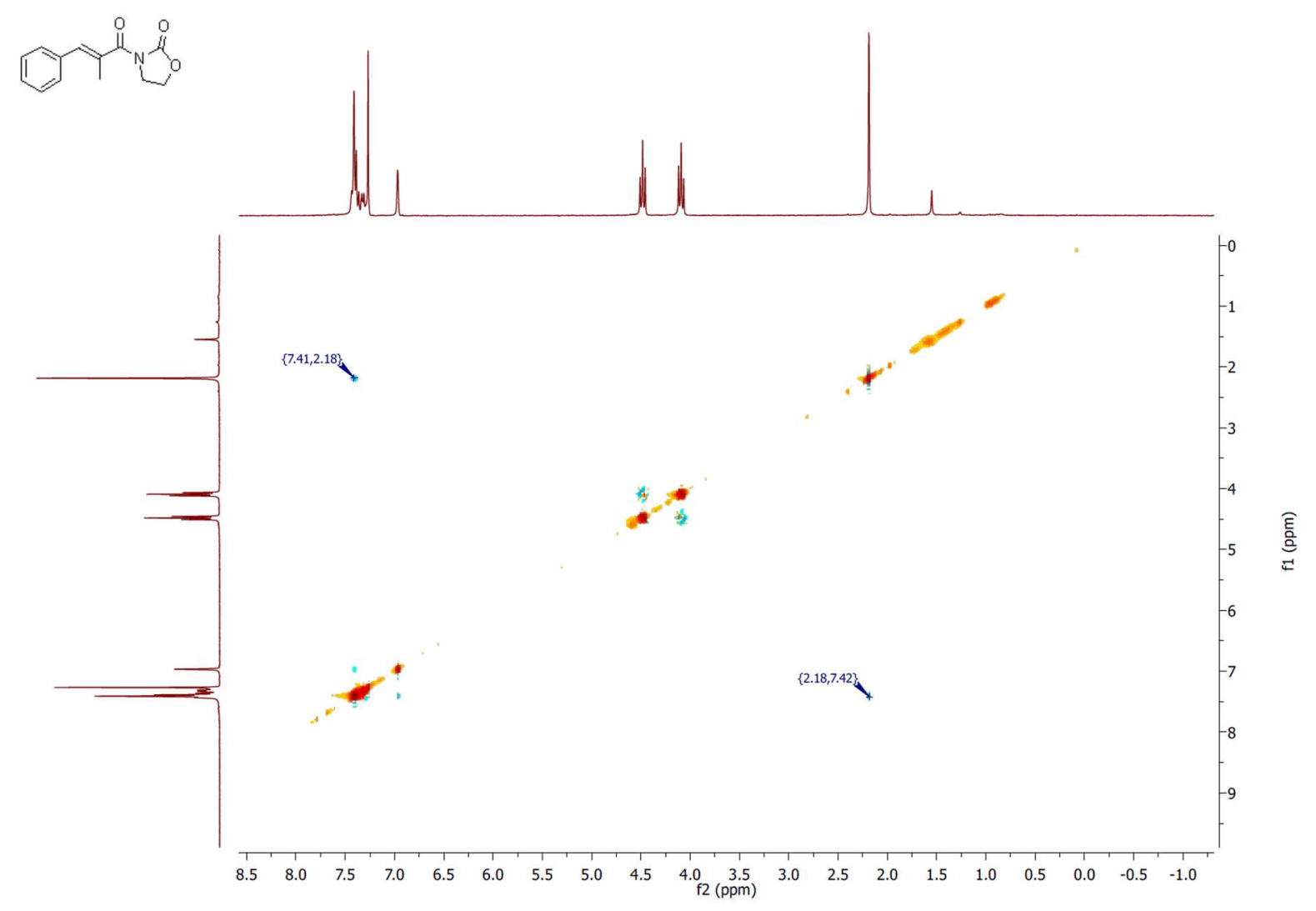


(E)-N,2-dimethyl-3-phenyl-N-(pyridin-2-yl)acrylamide ((E)-5):

${ }^{1} \mathrm{H} \mathrm{NMR}\left(\mathrm{CDCl}_{3}, 300 \mathrm{MHz}\right)$
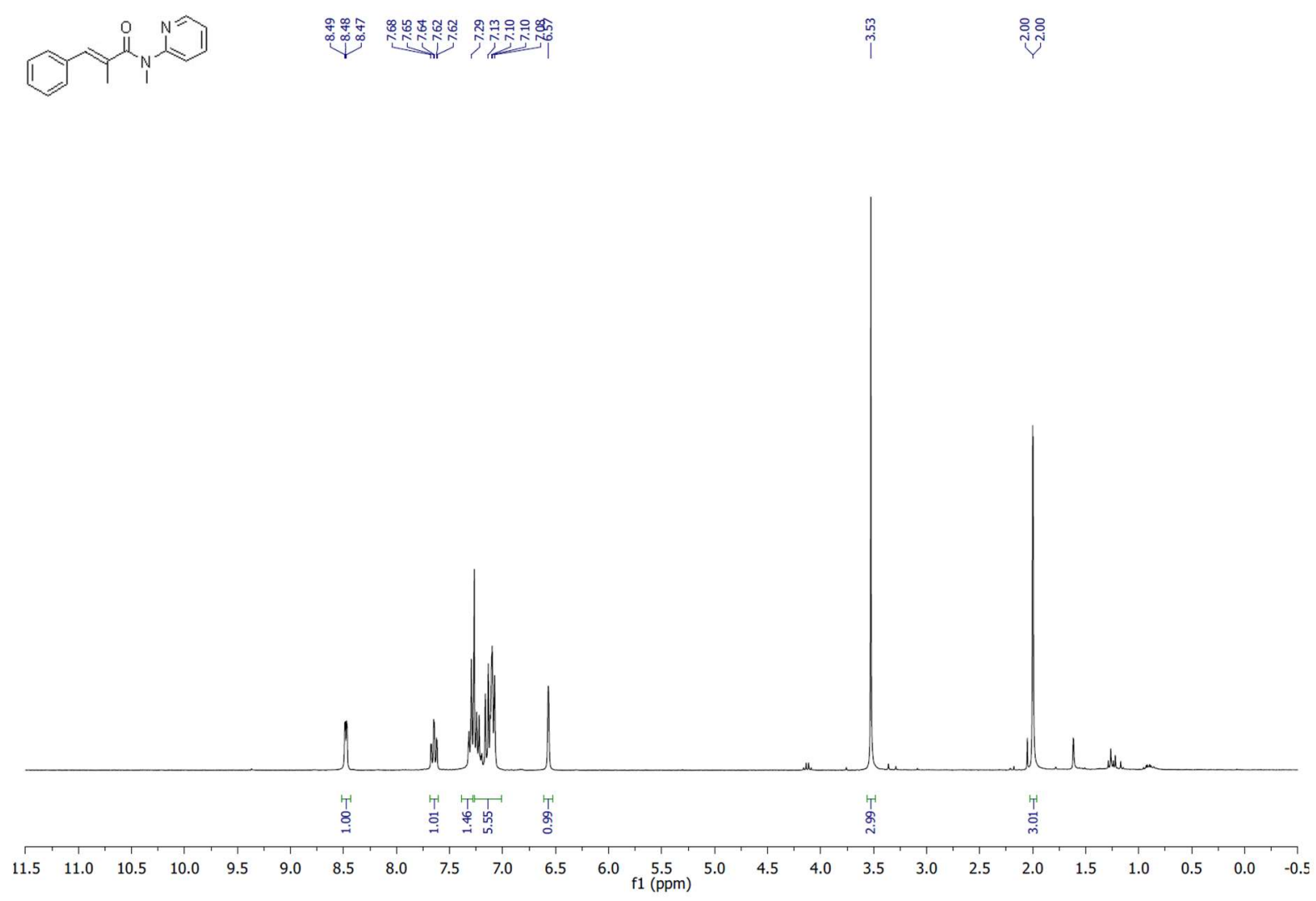

${ }^{13} \mathrm{C} \mathrm{NMR}\left(\mathrm{CDCl}_{3}, 75 \mathrm{MHz}\right)$
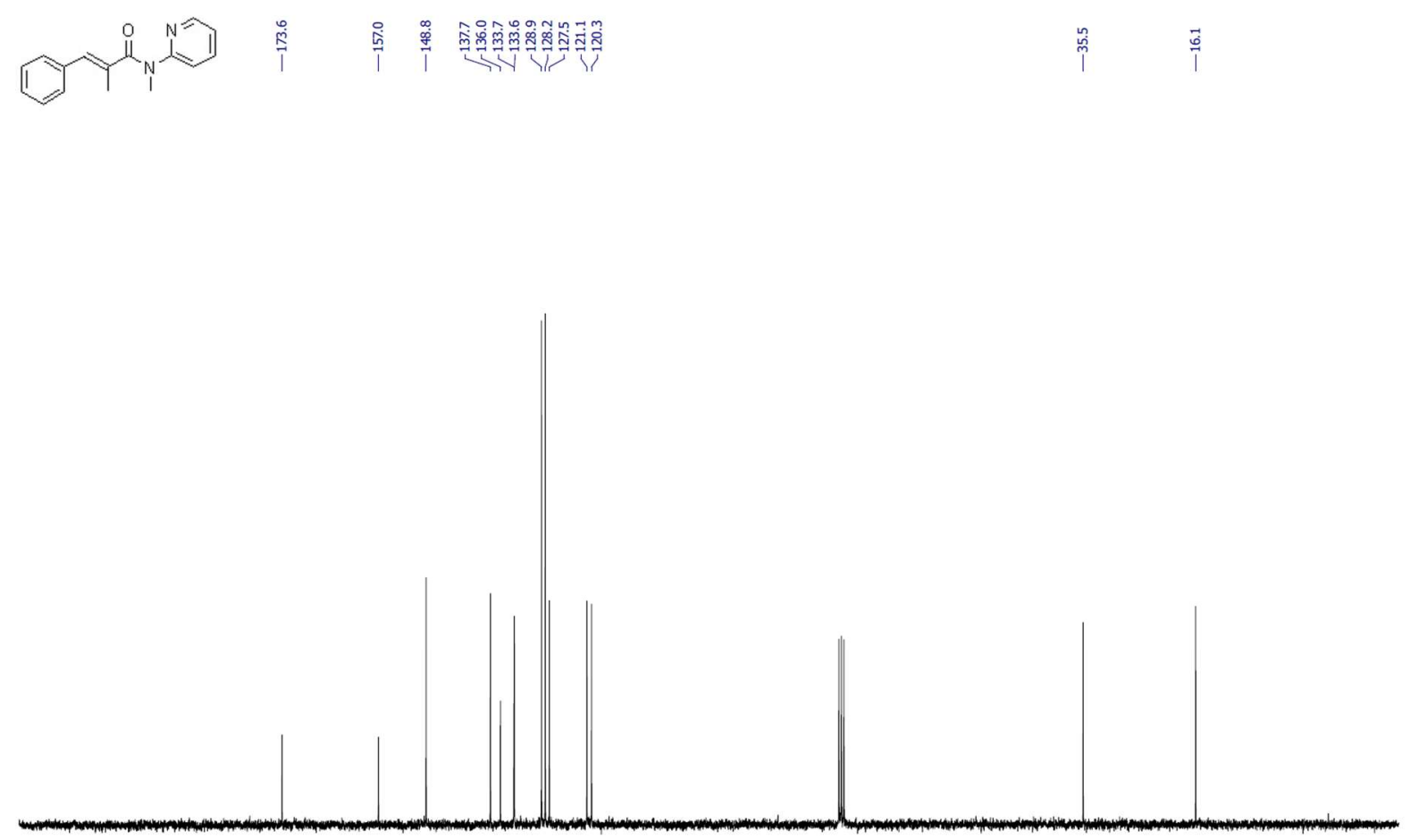

$\begin{array}{llllllllllllllllllllllll}210 & 200 & 190 & 180 & 170 & 160 & 150 & 140 & 130 & 120 & 110 & \begin{array}{c}100 \\ \mathrm{f} 1(\mathrm{ppm})\end{array} & 90 & 80 & 70 & 60 & 50 & 40 & 30 & 20 & 10 & 0 & -10\end{array}$ 
(E)-1-(2,3-dihydro-1H-pyrrolo[2,3-b]pyridin-1-yl)-2-methyl-3-phenylprop-2-en-1-one ((E)-6):

${ }^{1} \mathrm{H} \mathrm{NMR}\left(\mathrm{CDCl}_{3}, 300 \mathrm{MHz}\right)$

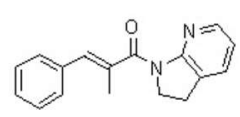

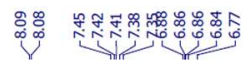

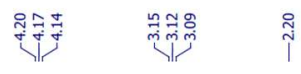

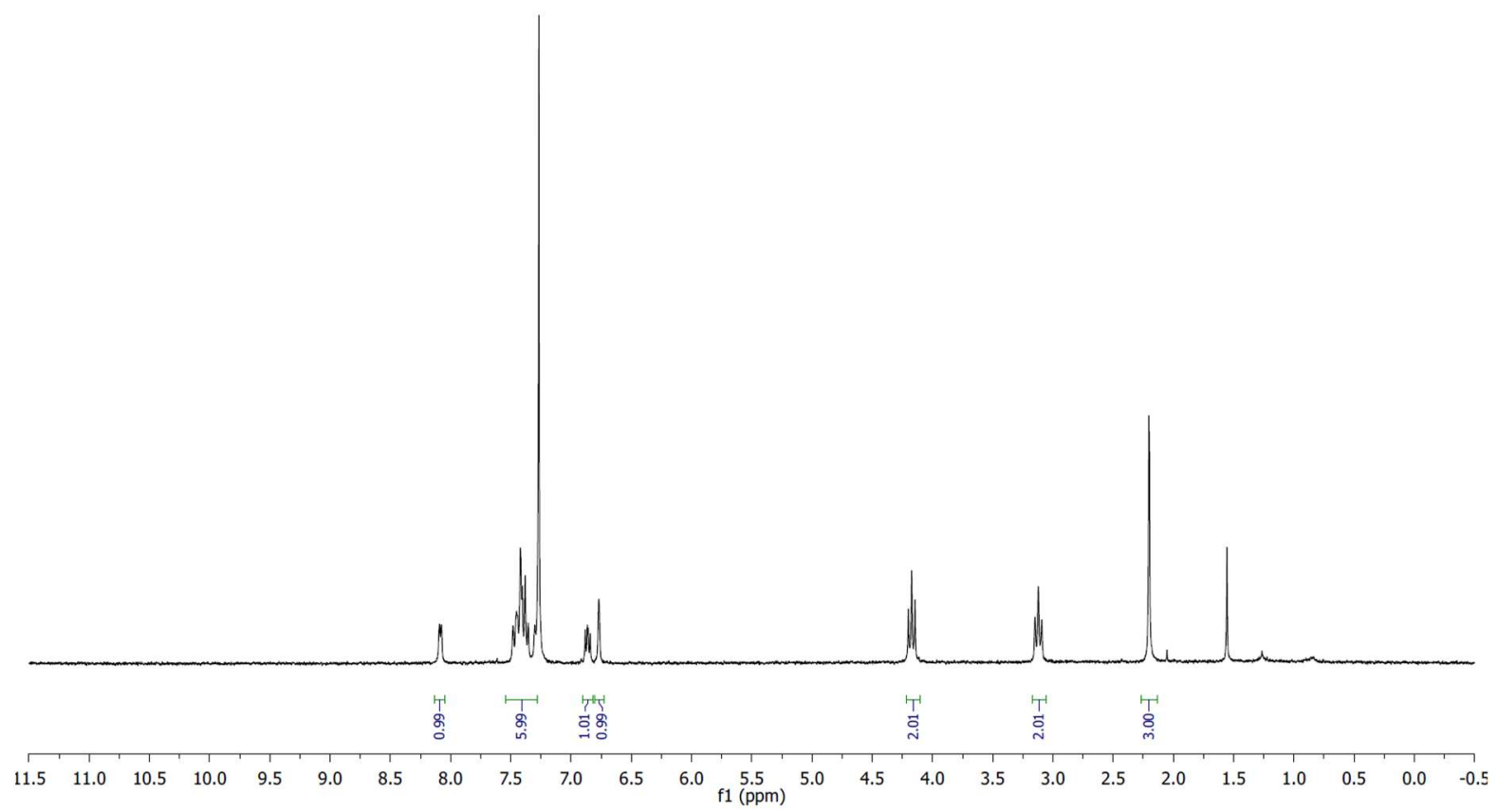

${ }^{1} \mathrm{H}$ NMR (benzene-d6, $300 \mathrm{MHz}$ )

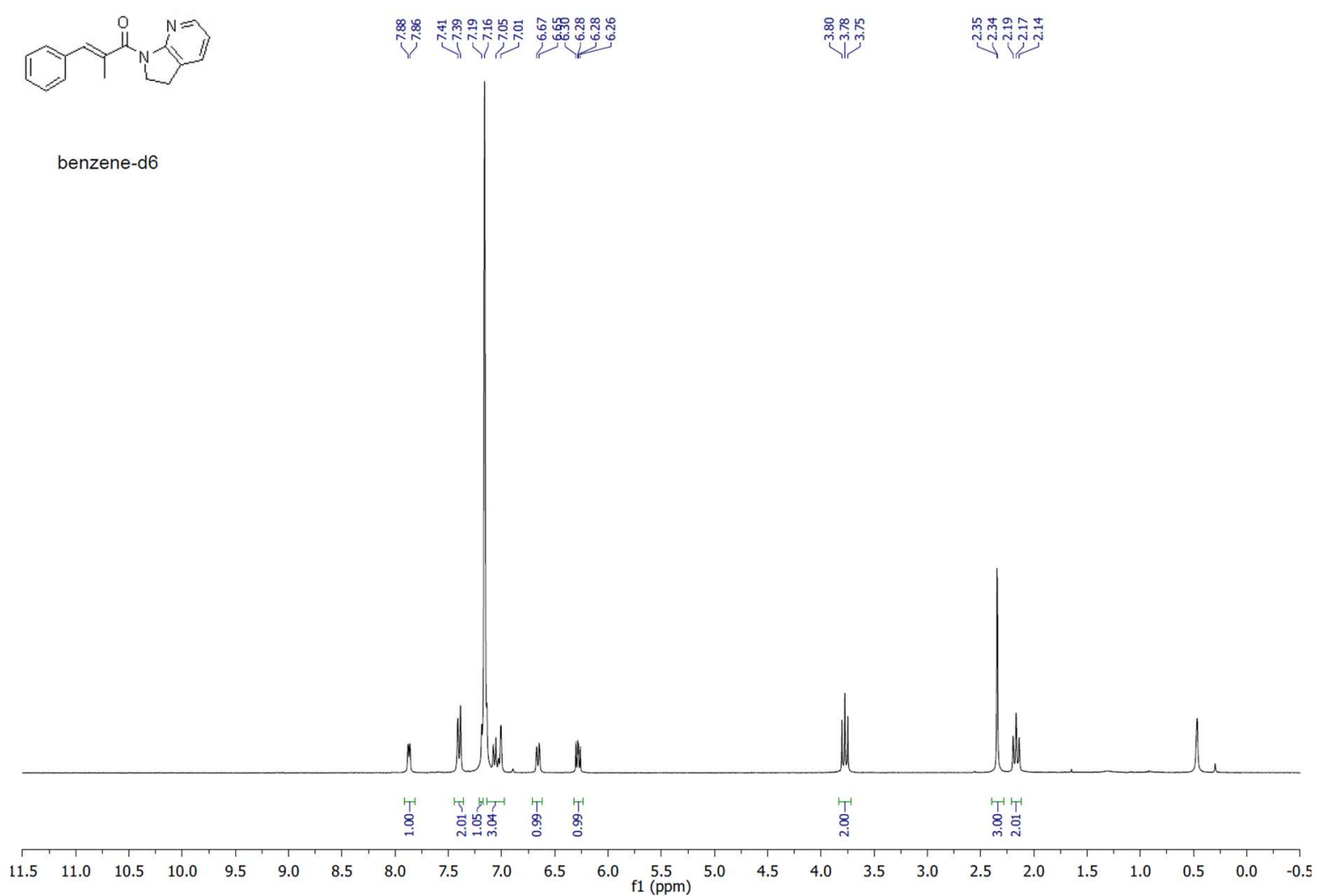


${ }^{13} \mathrm{C} \mathrm{NMR}\left(\mathrm{CDCl}_{3}, 75 \mathrm{MHz}\right)$

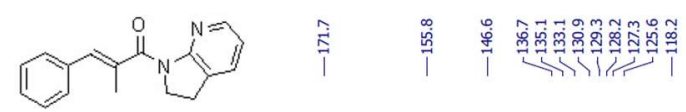

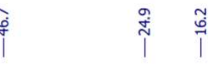

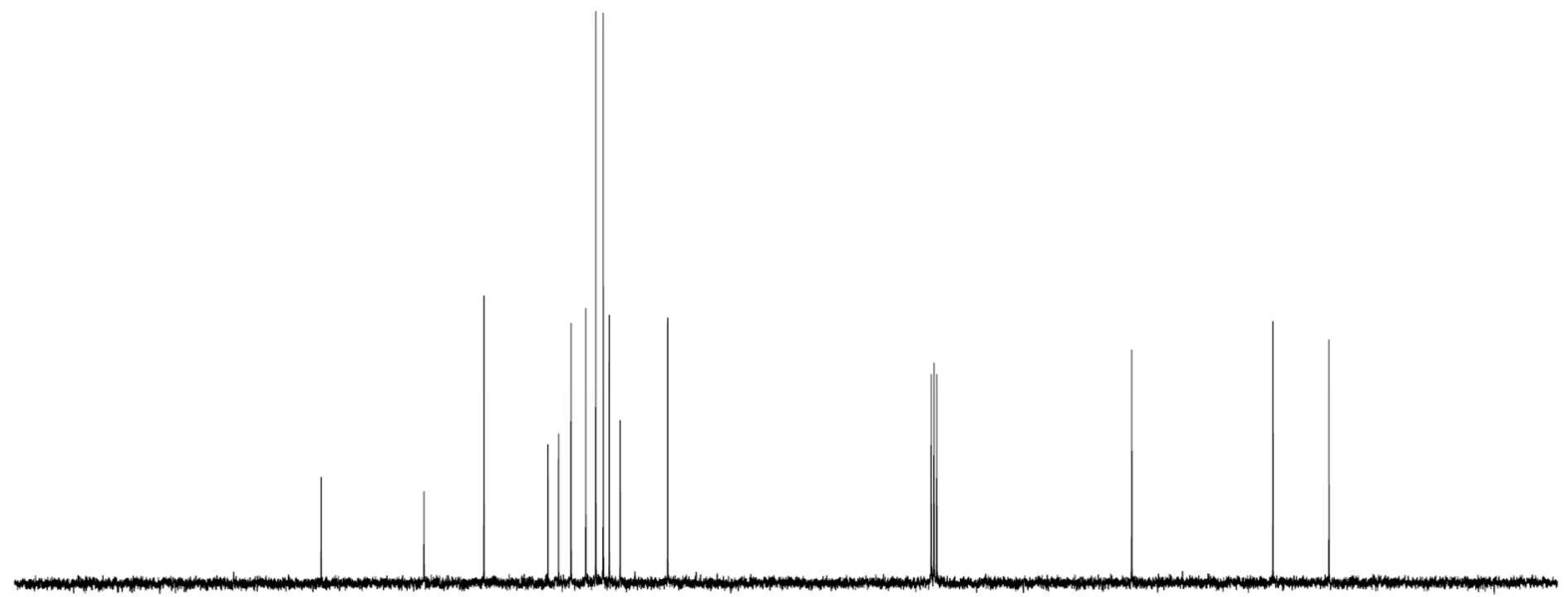

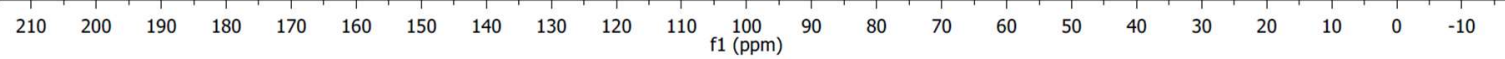

(E)-2-methyl-3-phenyl-1-(1H-pyrazol-1-yl)prop-2-en-1-one ((E)-7):

${ }^{1} \mathrm{H} \mathrm{NMR}\left(\mathrm{CDCl}_{3}, 300 \mathrm{MHz}\right)$
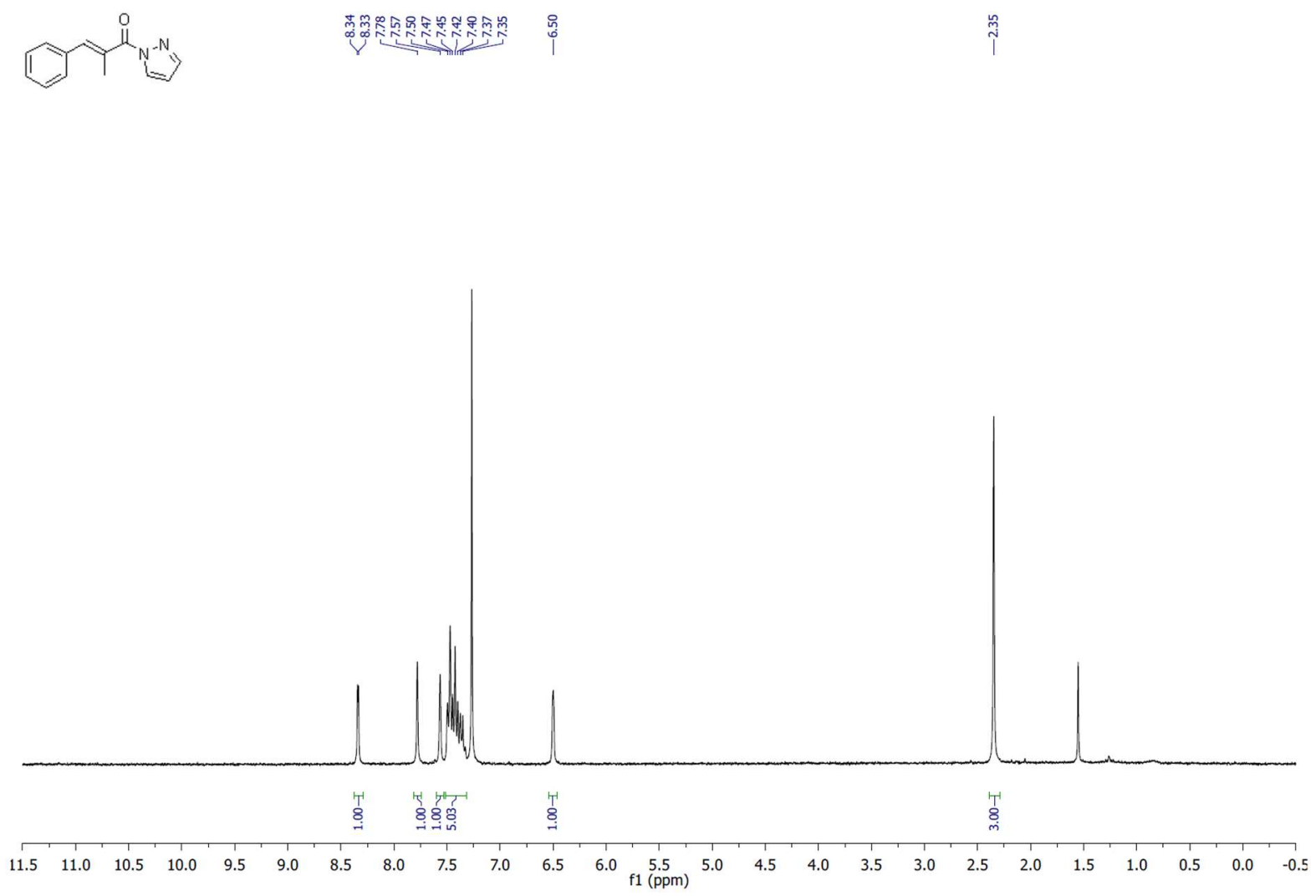
${ }^{13} \mathrm{C} \mathrm{NMR}\left(\mathrm{CDCl}_{3}, 75 \mathrm{MHz}\right)$

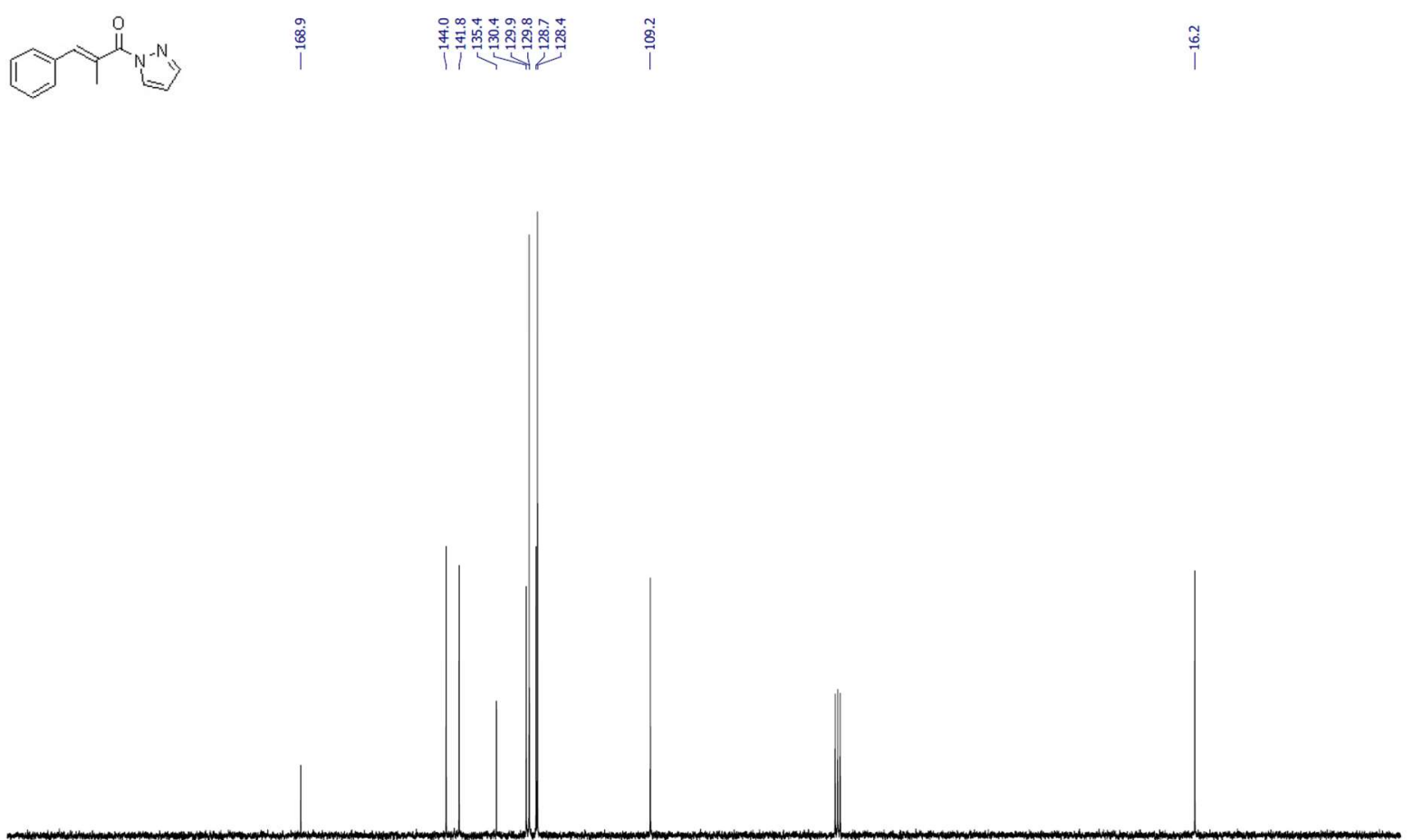

$\begin{array}{llllllllllllllllllllllllll}210 & 200 & 190 & 180 & 170 & 160 & 150 & 140 & 130 & 120 & 110 & 100 & 90 & 80 & 70 & 60 & 50 & 40 & 30 & 20 & 10 & 0 & -10\end{array}$

(E)-3-(2-methyl-3-phenylacryloyl)benzo[d]oxazol-2(3H)-one (T1):

${ }^{1} \mathrm{H} \mathrm{NMR}\left(\mathrm{CDCl}_{3}, 300 \mathrm{MHz}\right)$

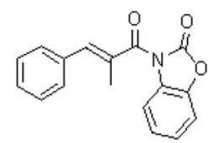

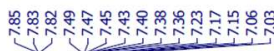

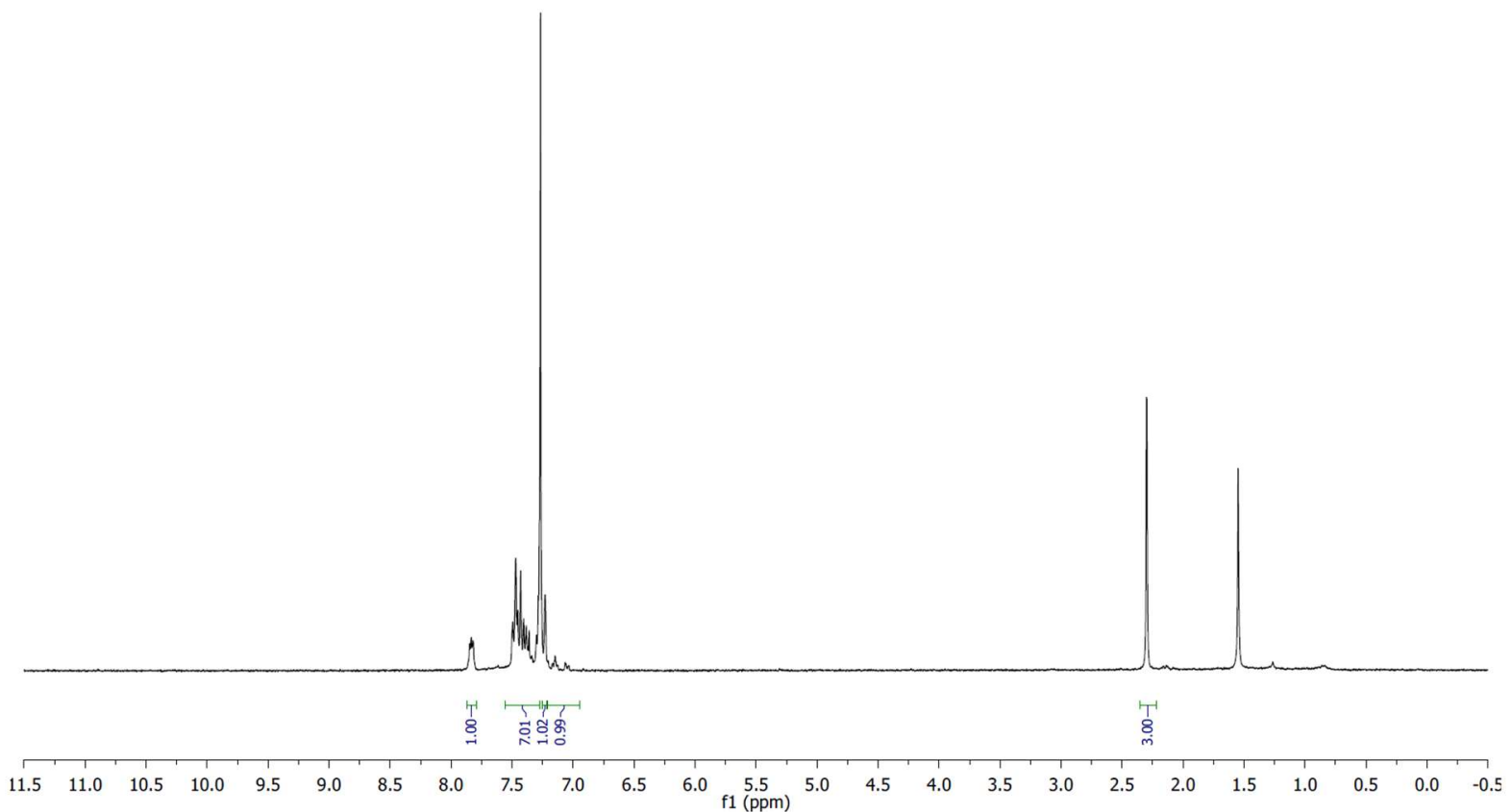


${ }^{13} \mathrm{C} \mathrm{NMR}\left(\mathrm{CDCl}_{3}, 75 \mathrm{MHz}\right)$
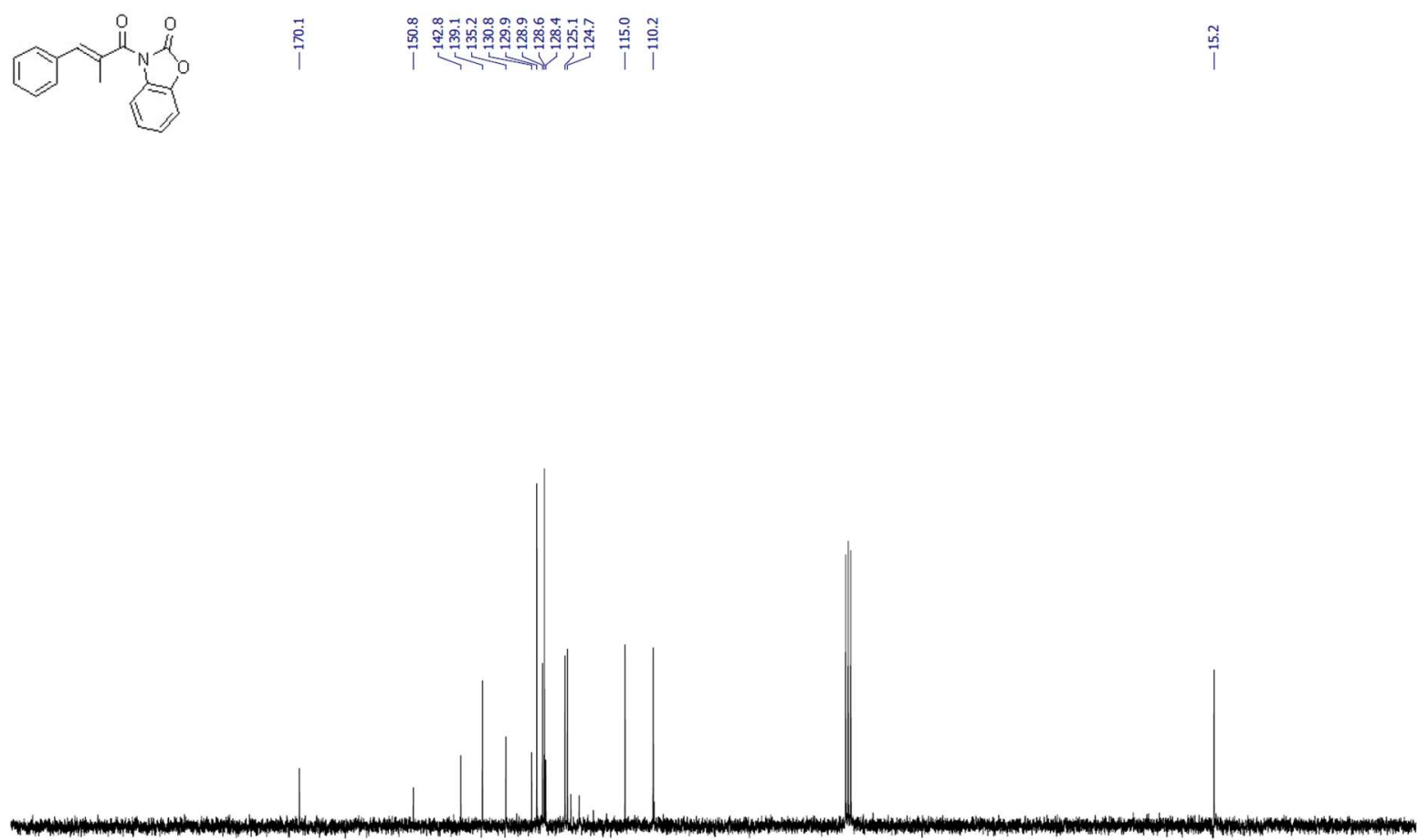

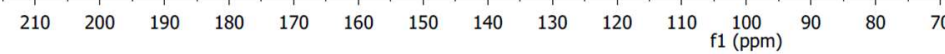

(E)-1-(2-methyl-3-phenylacryloyl)pyrrolidin-2-one (T2):

${ }^{1} \mathrm{H} \mathrm{NMR}\left(\mathrm{CDCl}_{3}, 300 \mathrm{MHz}\right)$<smiles>CC(=Cc1ccccc1)C(=O)N1CCCC1=O</smiles>

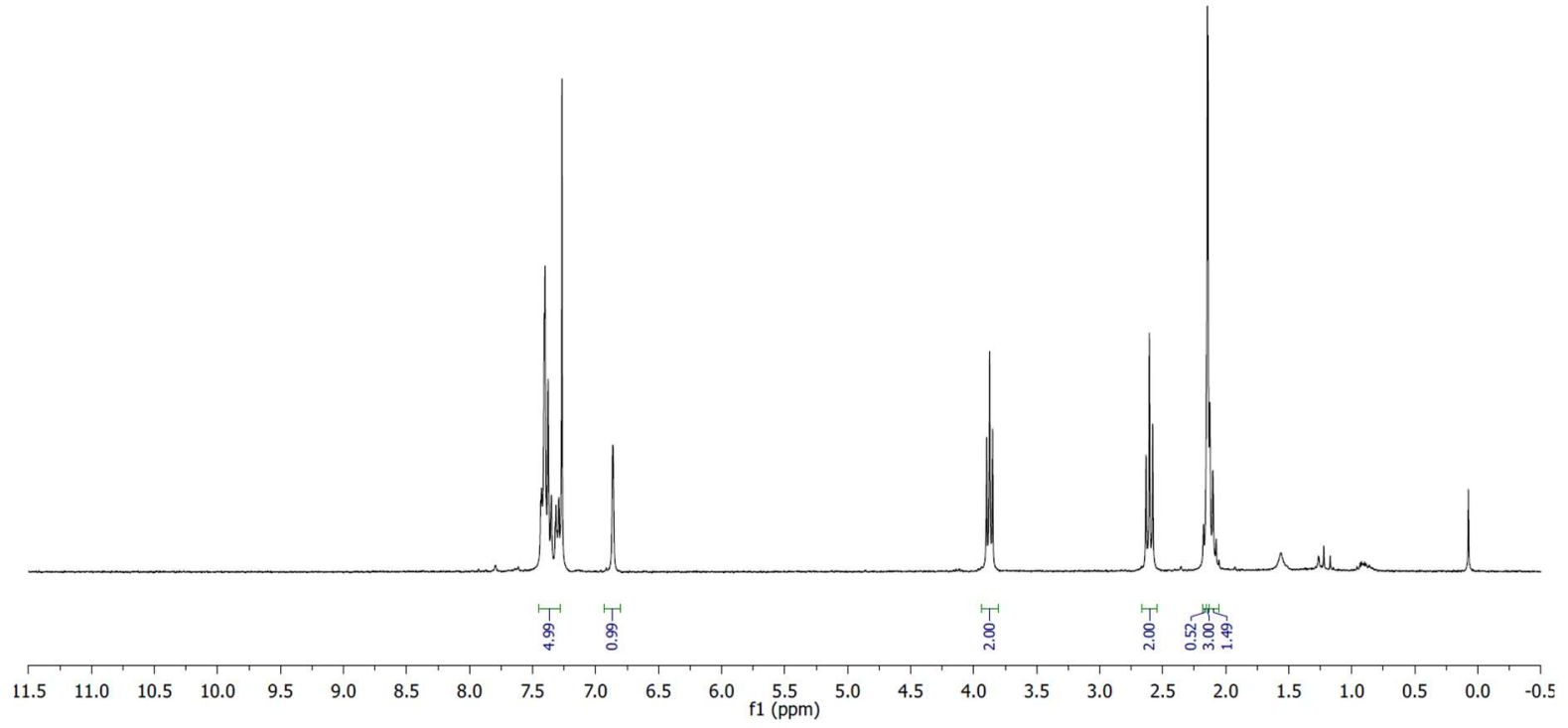


${ }^{13} \mathrm{C} \mathrm{NMR}\left(\mathrm{CDCl}_{3}, 75 \mathrm{MHz}\right)$
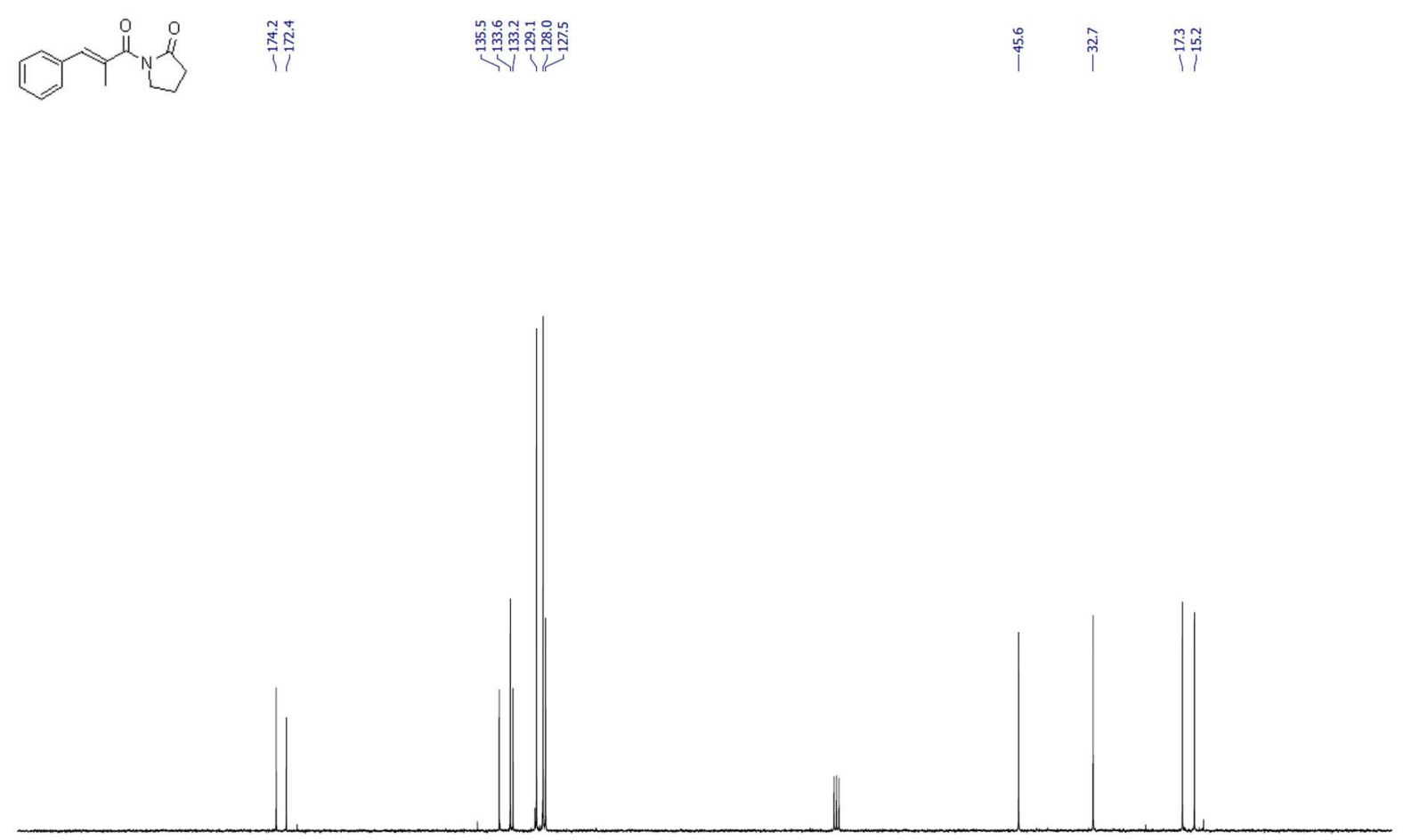

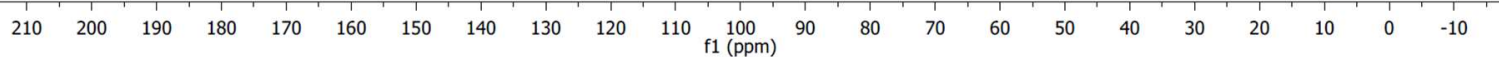

(E)-1-(2-methyl-3-phenylacryloyl)piperidin-2-one (T3):

${ }^{1} \mathrm{H} \mathrm{NMR}\left(\mathrm{CDCl}_{3}, 300 \mathrm{MHz}\right)$<smiles>CC(C)(Cc1ccccc1)N1CCCC1=O</smiles>

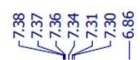
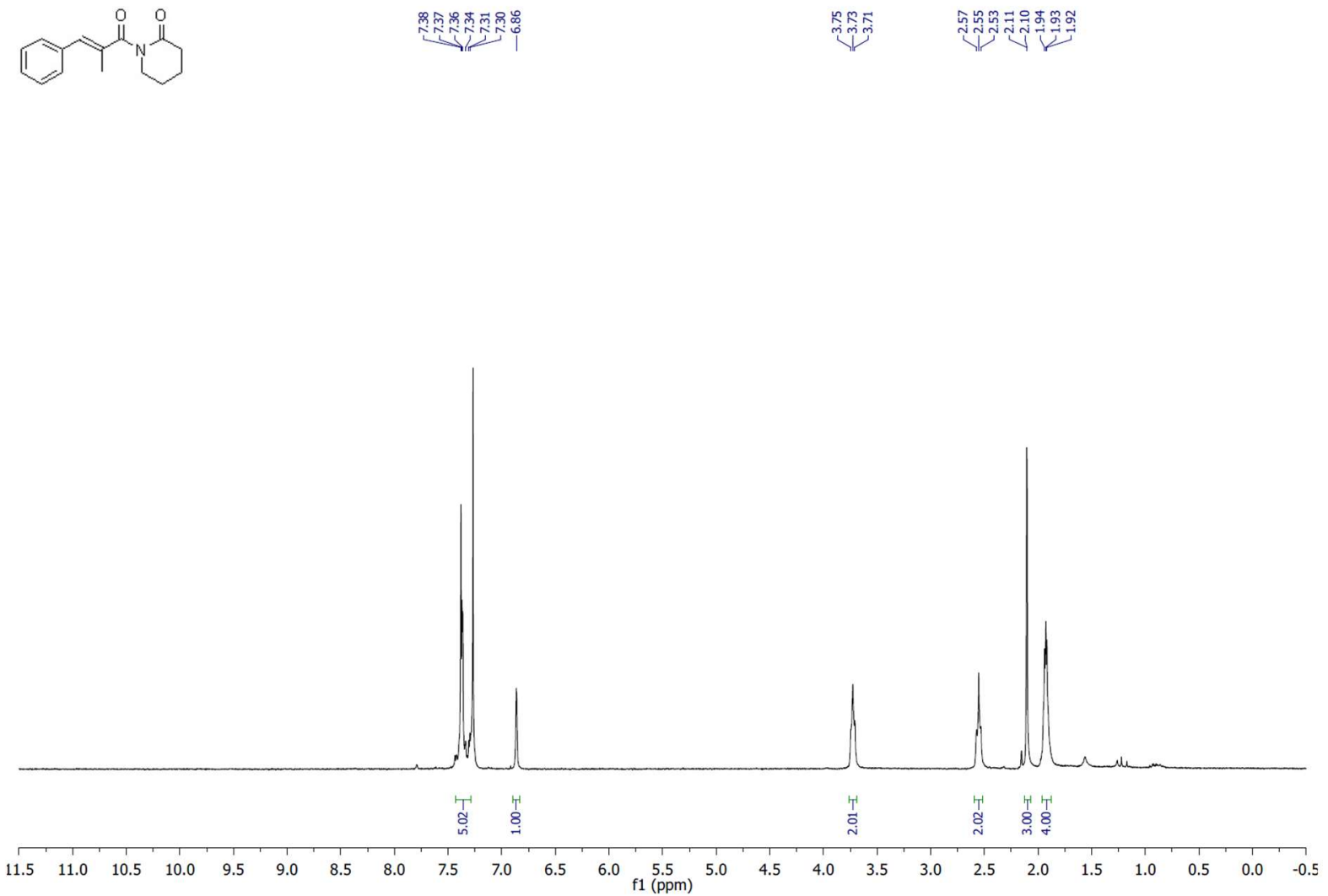
${ }^{13} \mathrm{C} \mathrm{NMR}\left(\mathrm{CDCl}_{3}, 75 \mathrm{MHz}\right)$

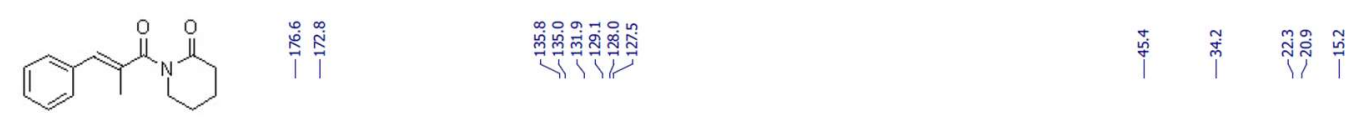

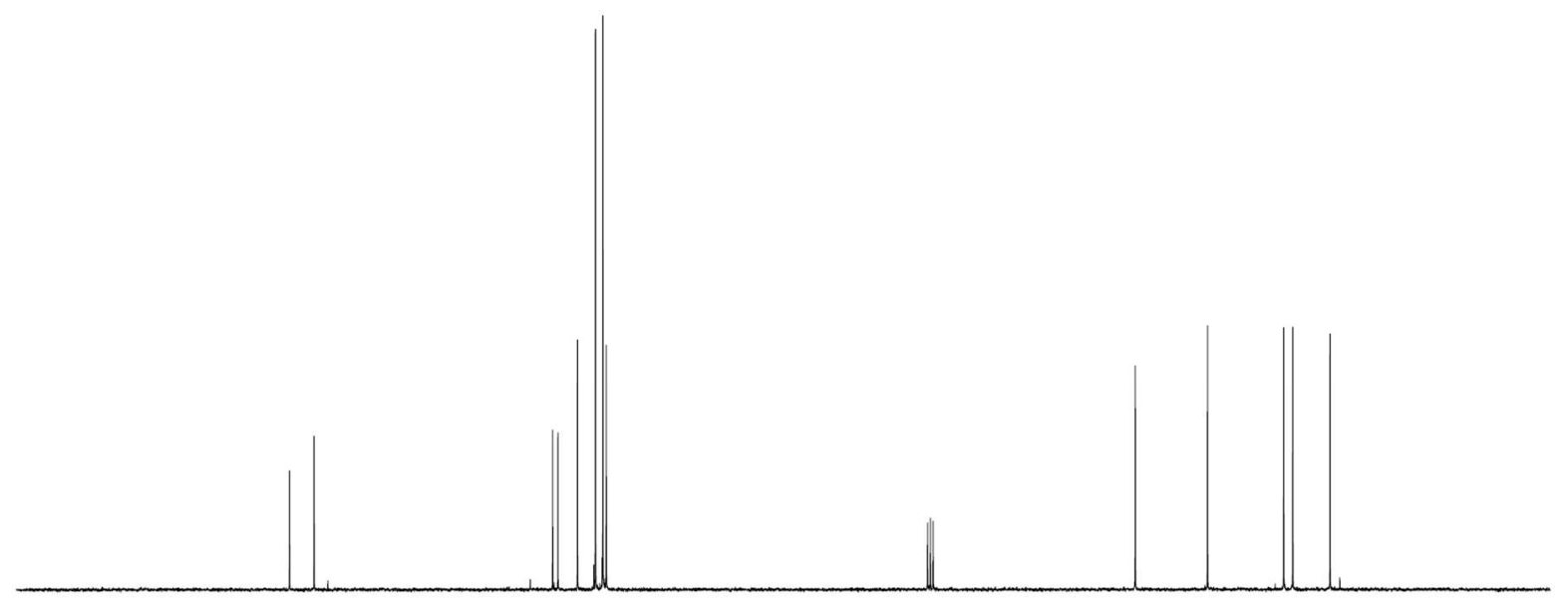

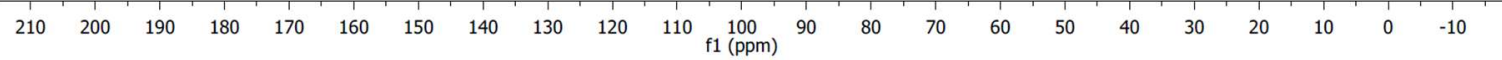

oxazolidine-2-thione (A):

${ }^{1} \mathrm{H} \mathrm{NMR}\left(\mathrm{CDCl}_{3}, 300 \mathrm{MHz}\right)$

HIN

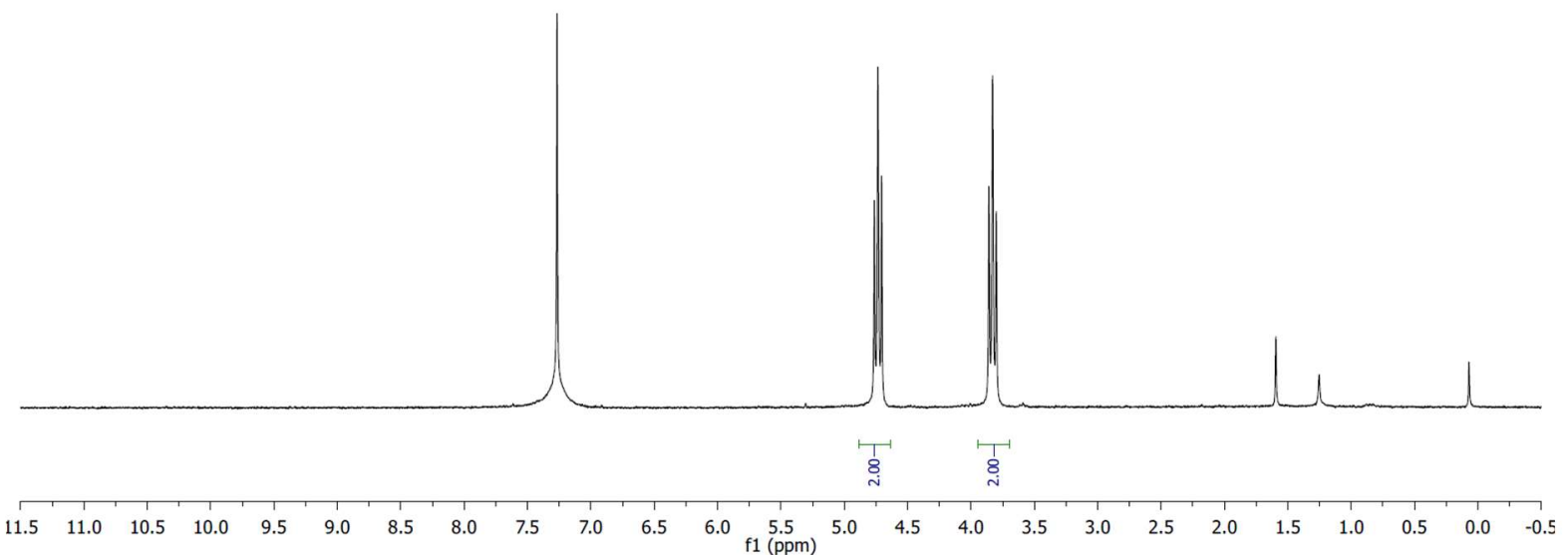


${ }^{13} \mathrm{C} \mathrm{NMR}\left(\mathrm{CDCl}_{3}, 75 \mathrm{MHz}\right)$

HIN

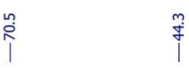

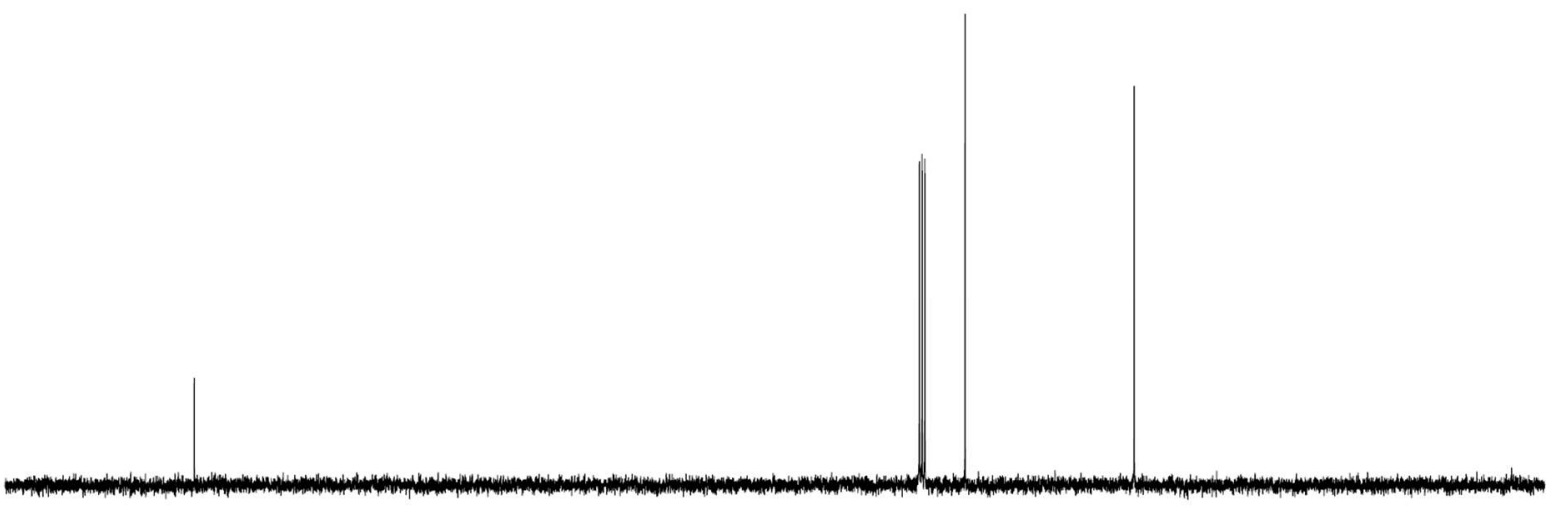

$\begin{array}{lllllllllllllllllllllll}210 & 200 & 190 & 180 & 170 & 160 & 150 & 140 & 130 & 120 & 110 & \begin{array}{c}100 \\ \mathrm{f} 1(\mathrm{ppm})\end{array} & 90 & 80 & 70 & 60 & 50 & 40 & 30 & 20 & 10 & 0 & -10\end{array}$

(E)-2-methyl-3-phenyl-1-(2-thioxooxazolidin-3-yl)prop-2-en-1-one (T4):

${ }^{1} \mathrm{H} \mathrm{NMR}\left(\mathrm{CDCl}_{3}, 300 \mathrm{MHz}\right)$<smiles>C/C(=C\c1ccccc1)C(=O)N1CCOC1=S</smiles>

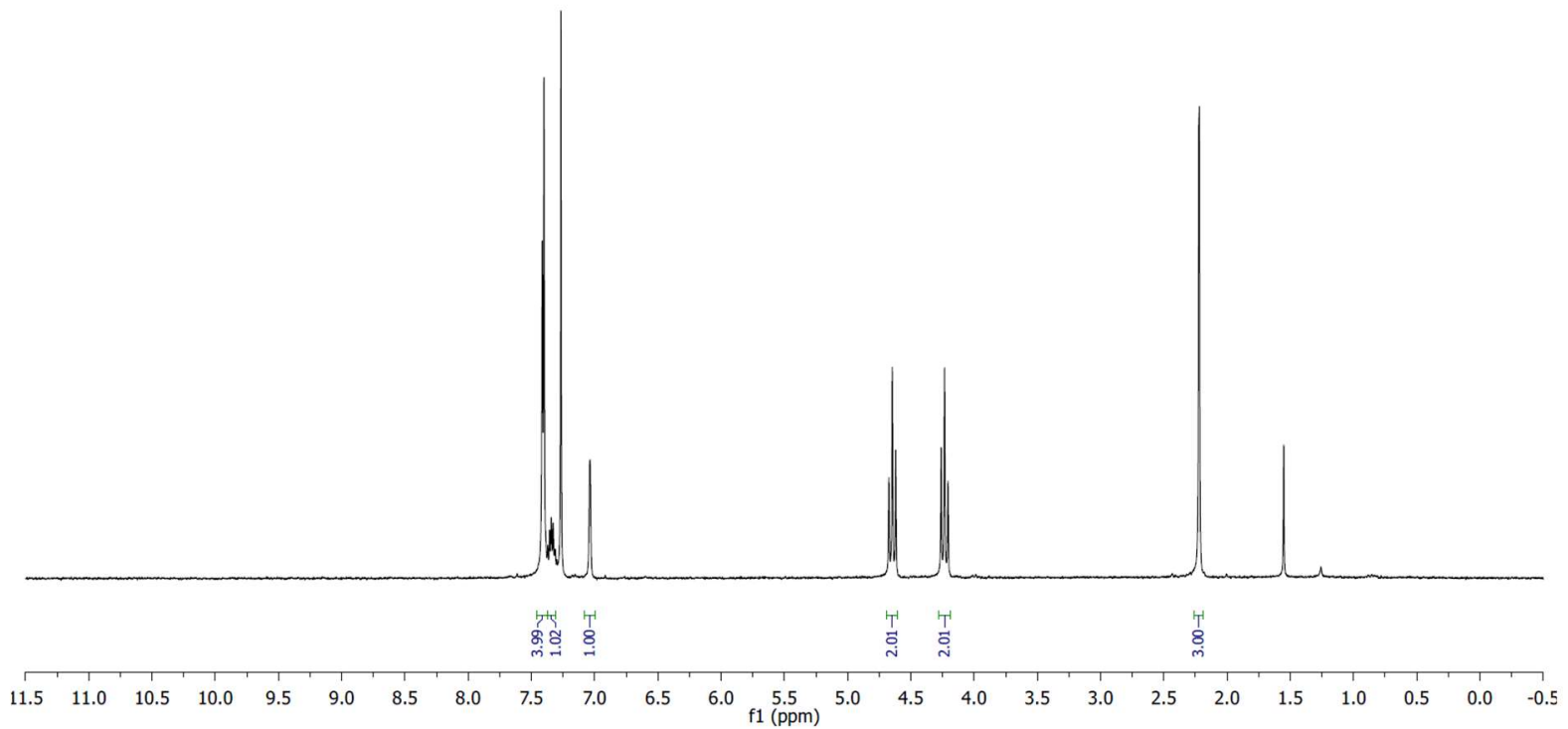


${ }^{13} \mathrm{C} \mathrm{NMR}\left(\mathrm{CDCl}_{3}, 75 \mathrm{MHz}\right)$
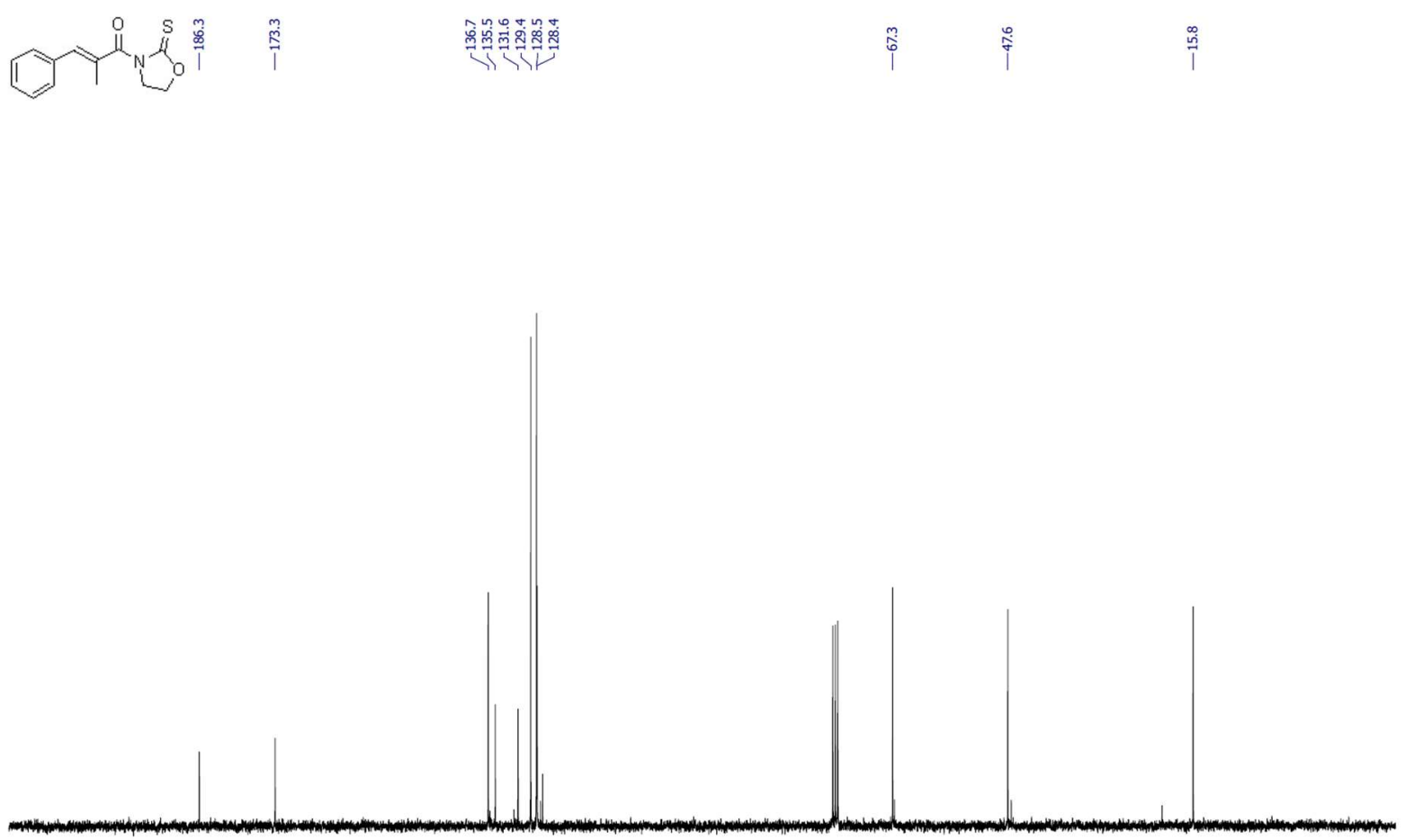

$\begin{array}{llllllll}210 & 200 & 190 & 180 & 170 & 160 & 150 & 140\end{array}$

(E)-2-(2-methyl-3-phenylacryloyl)-1-phenylpyrazolidin-3-one (T5):

${ }^{1} \mathrm{H} \mathrm{NMR}\left(\mathrm{CDCl}_{3}, 300 \mathrm{MHz}\right)$

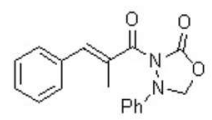

至

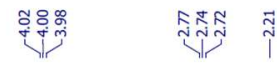

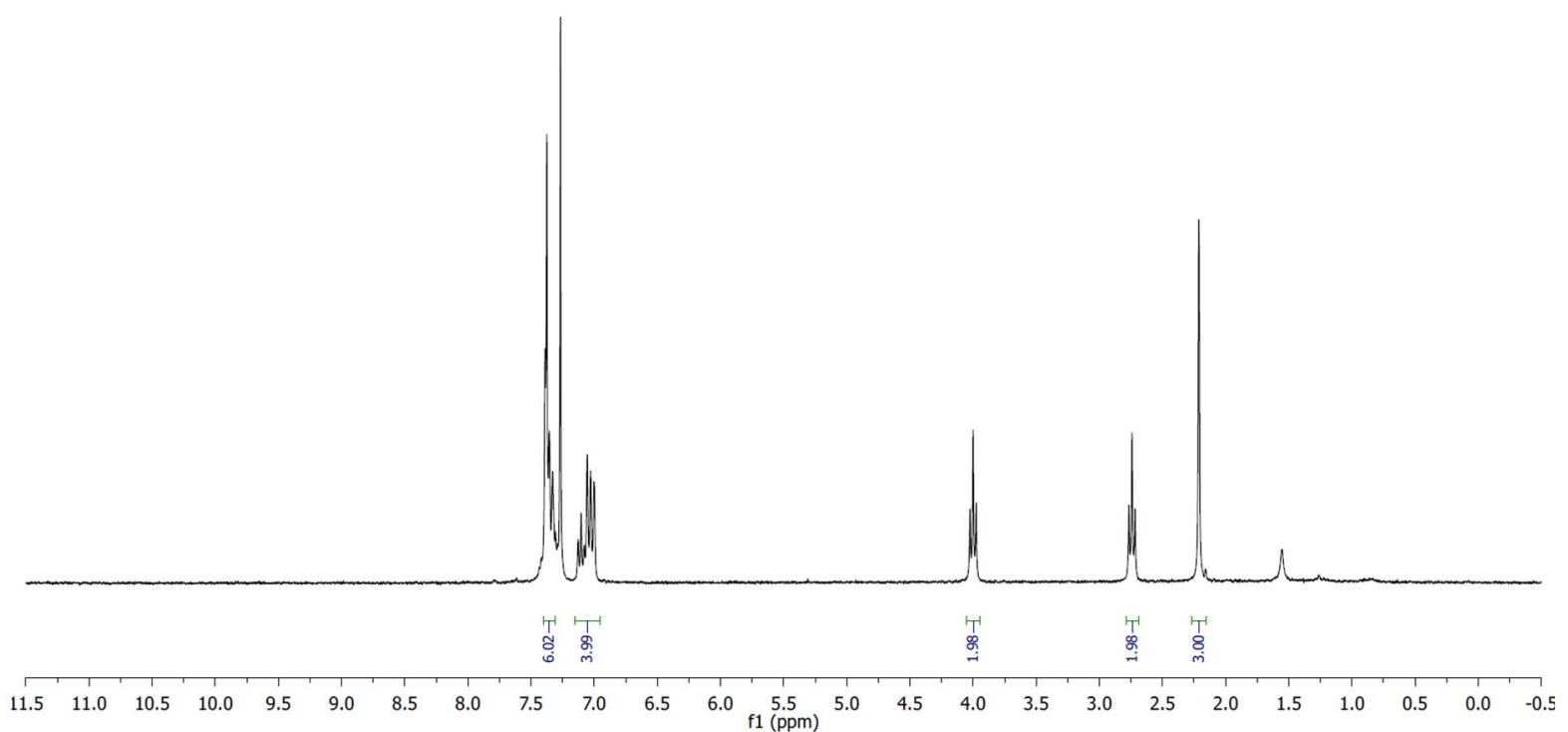


${ }^{13} \mathrm{C} \mathrm{NMR}\left(\mathrm{CDCl}_{3}, 75 \mathrm{MHz}\right)$

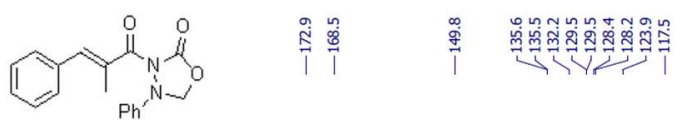

$\overrightarrow{⿱ 亠 䒑}$

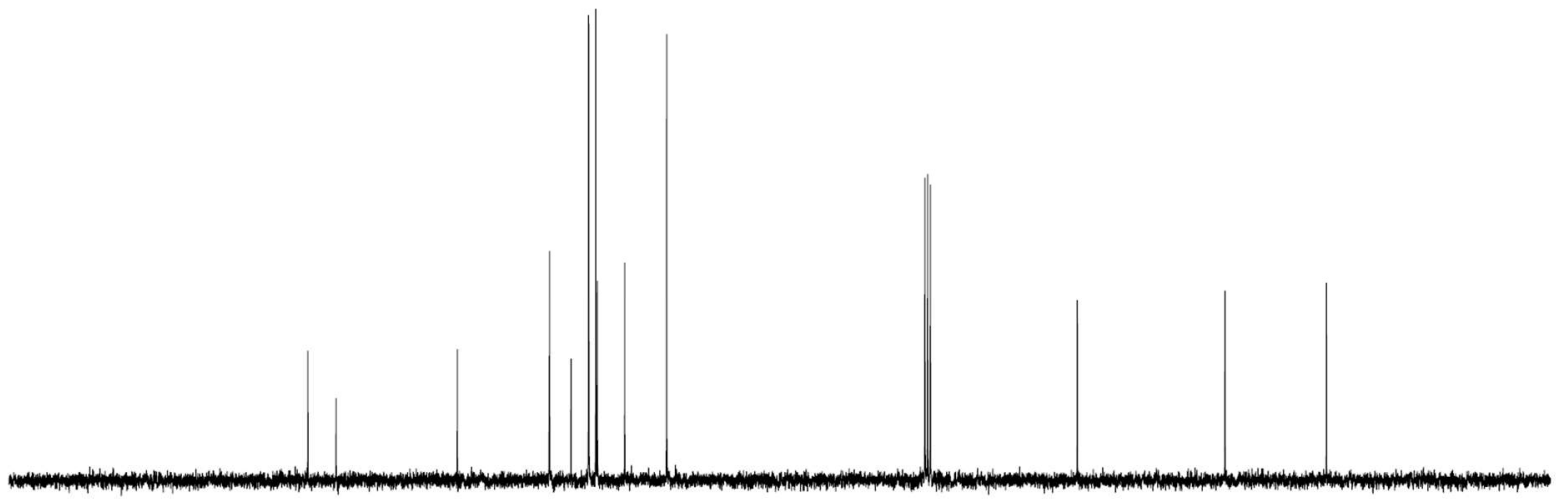

$\begin{array}{llllllllllll}210 & 200 & 190 & 180 & 170 & 160 & 150 & 140 & 130 & 120 & 110 \begin{array}{c}100 \\ \mathrm{f} 1(\mathrm{ppm})\end{array} & 90\end{array}$

ethyl (E)-2-benzylidenebutanoate (Est-8):

${ }^{1} \mathrm{H} \mathrm{NMR}\left(\mathrm{CDCl}_{3}, 300 \mathrm{MHz}\right)$

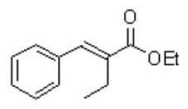

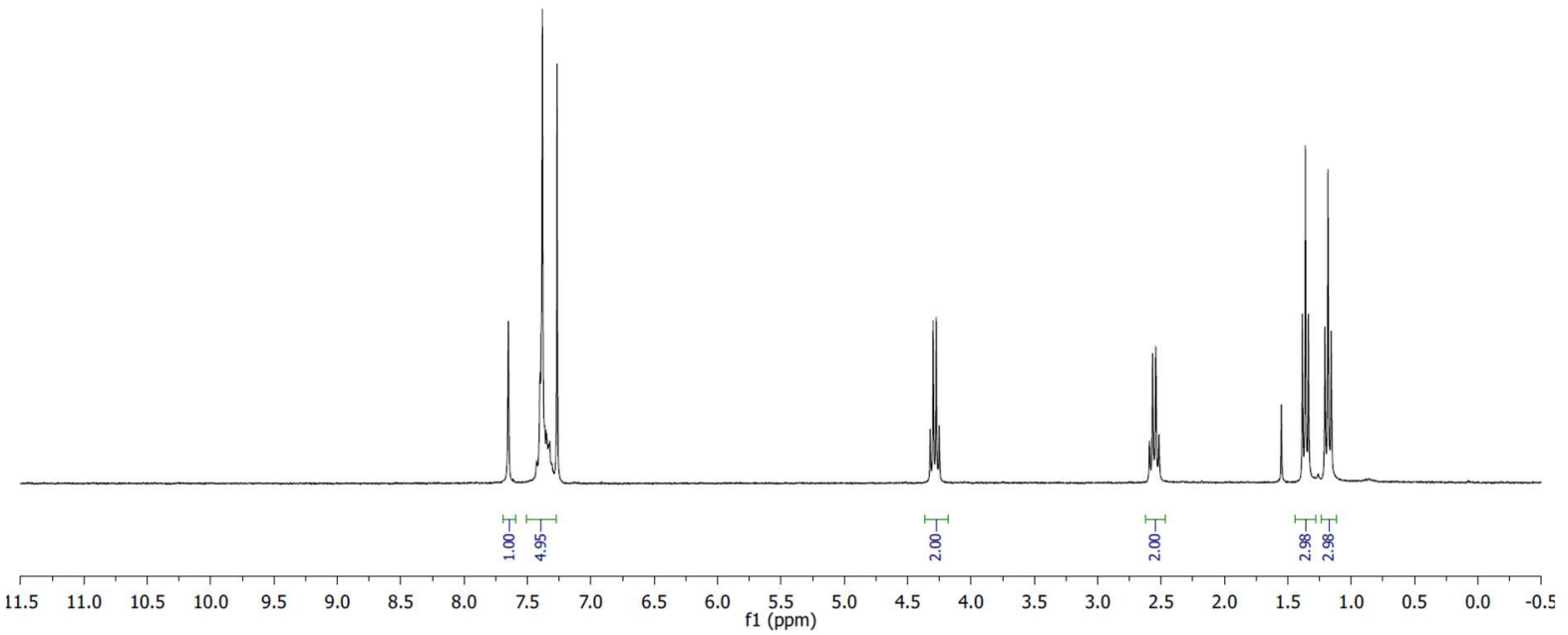


${ }^{13} \mathrm{C} \mathrm{NMR}\left(\mathrm{CDCl}_{3}, 75 \mathrm{MHz}\right)$<smiles>[13CH3]OC(=O)Cc1ccccc1</smiles>

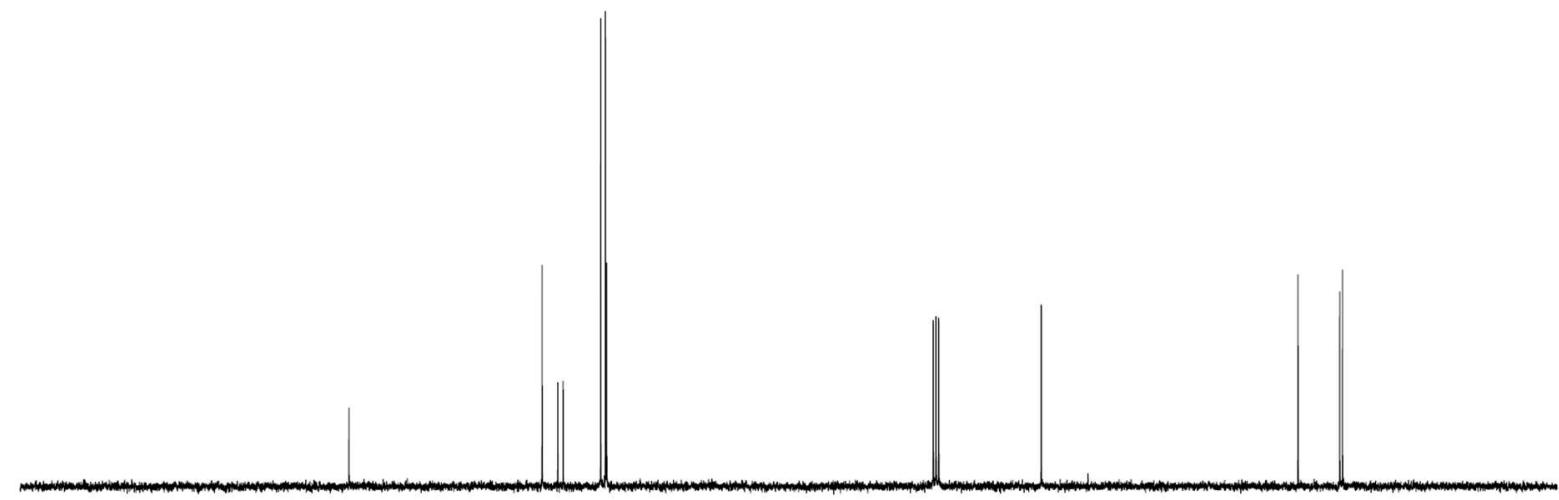

$\begin{array}{lllllllllllllllllllllll}210 & 200 & 190 & 180 & 170 & 160 & 150 & 140 & 130 & 120 & 110 & 100 & 90 & 80 & 70 & 60 & 50 & 40 & 30 & 20 & 10 & 0 & -10\end{array}$

(E)-2-benzylidenebutanoic acid (Ac-8):

${ }^{1} \mathrm{H} \mathrm{NMR}\left(\mathrm{CDCl}_{3}, 300 \mathrm{MHz}\right)$

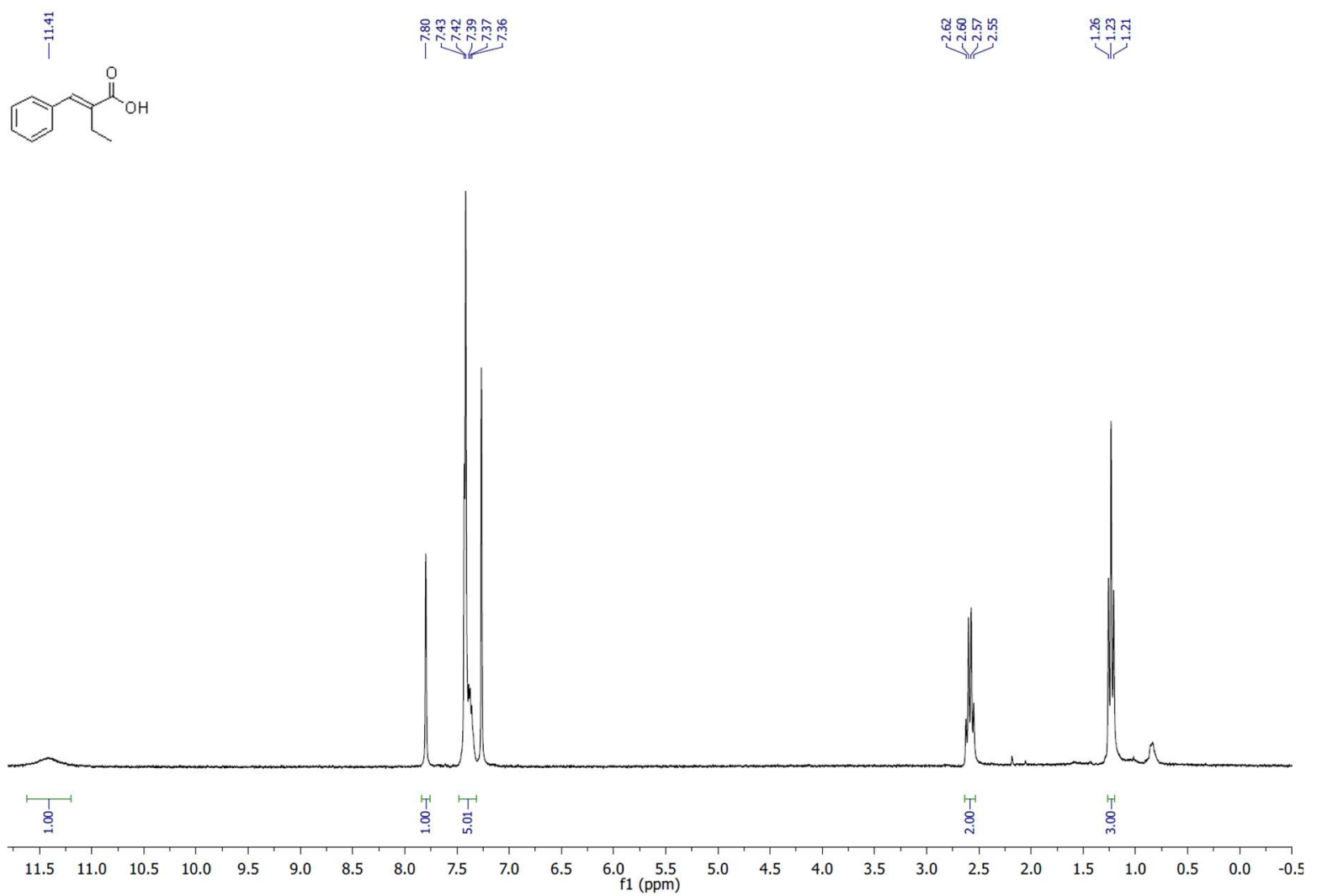


${ }^{13} \mathrm{C}$ NMR (DMSO-d6, $75 \mathrm{MHz}$ )
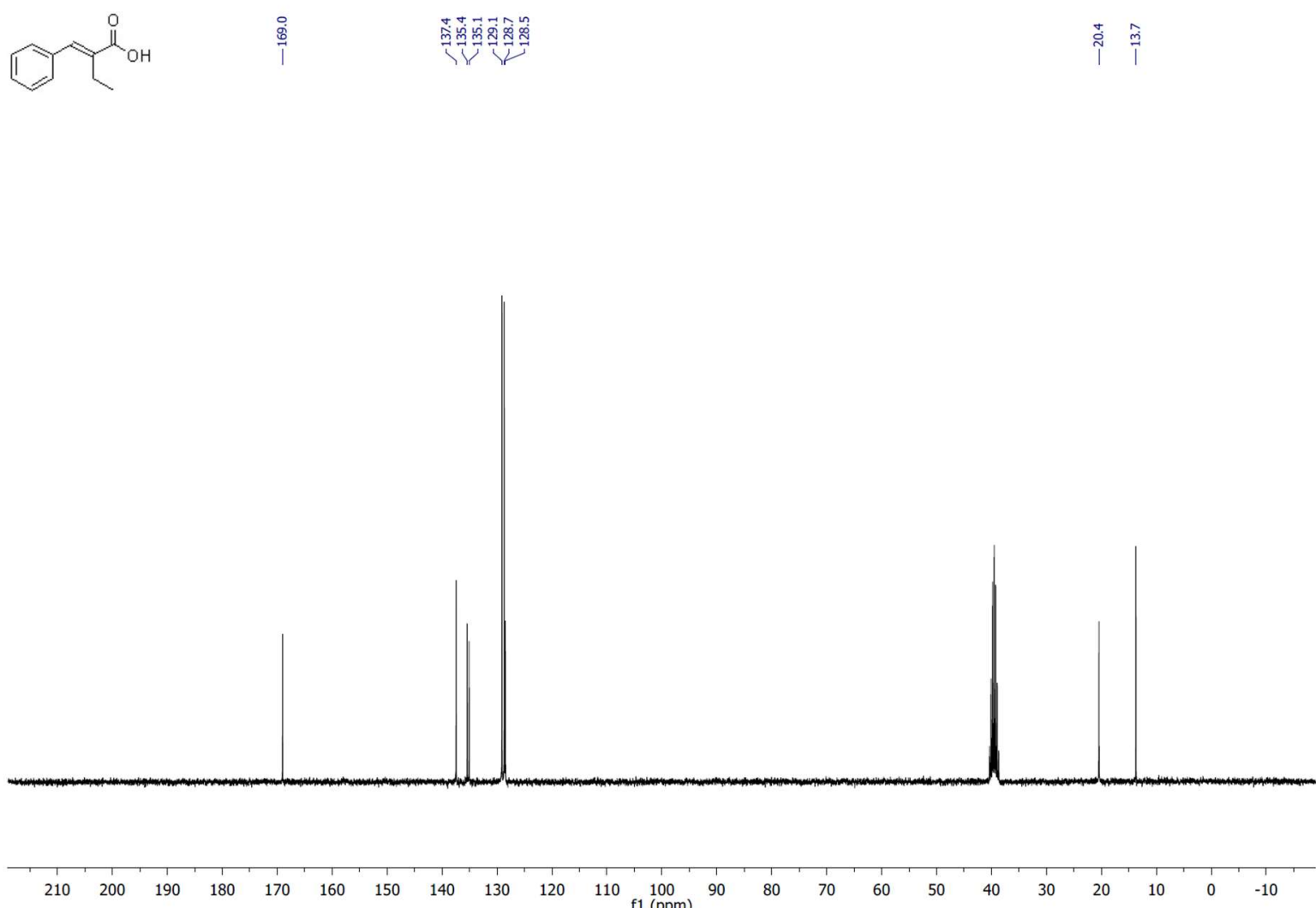

\section{(E)-3-(2-benzylidenebutanoyl)oxazolidin-2-one ((E)-8):}

${ }^{1} \mathrm{H} \mathrm{NMR}\left(\mathrm{CDCl}_{3}, 300 \mathrm{MHz}\right)$
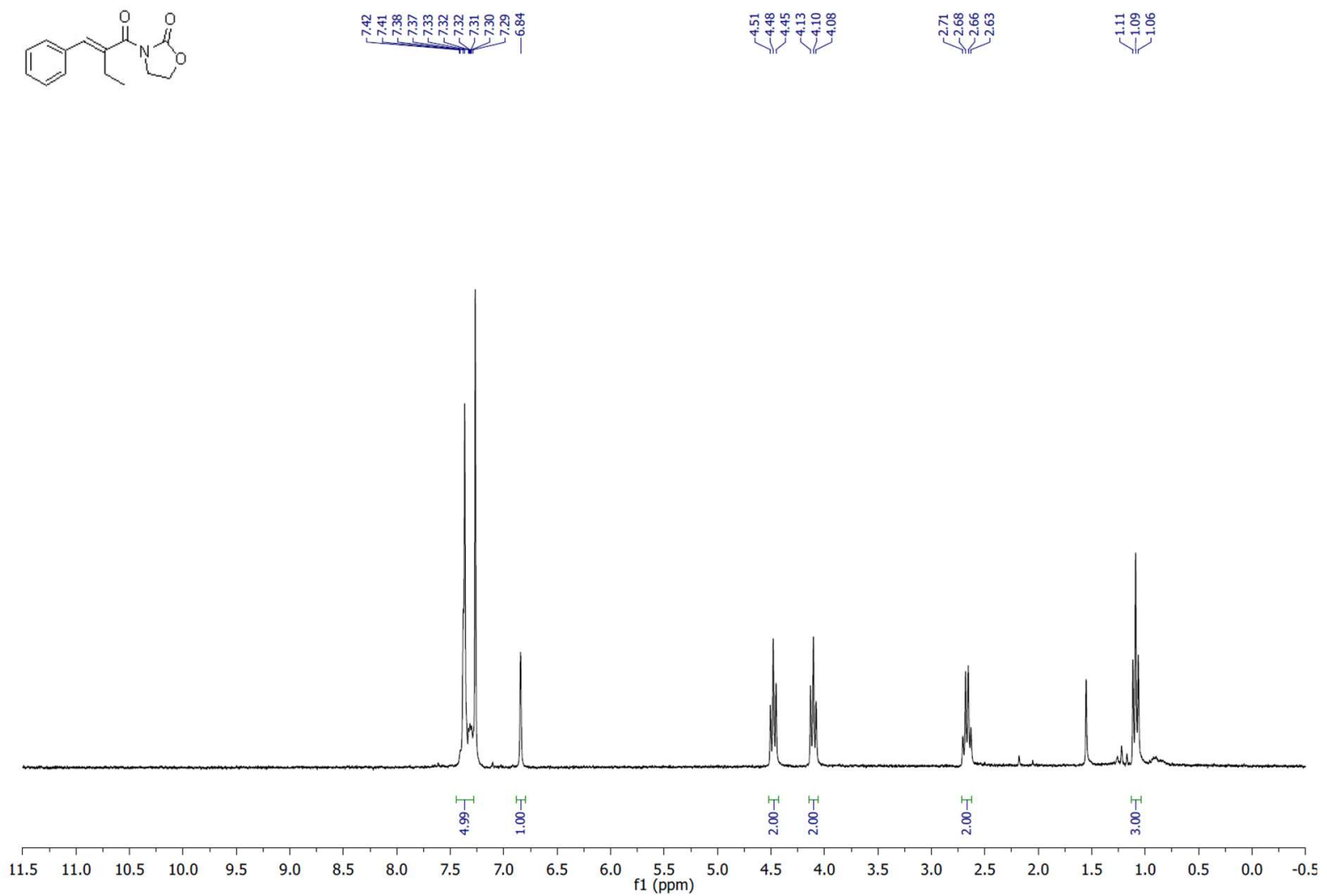
${ }^{13} \mathrm{C} \mathrm{NMR}\left(\mathrm{CDCl}_{3}, 75 \mathrm{MHz}\right)$
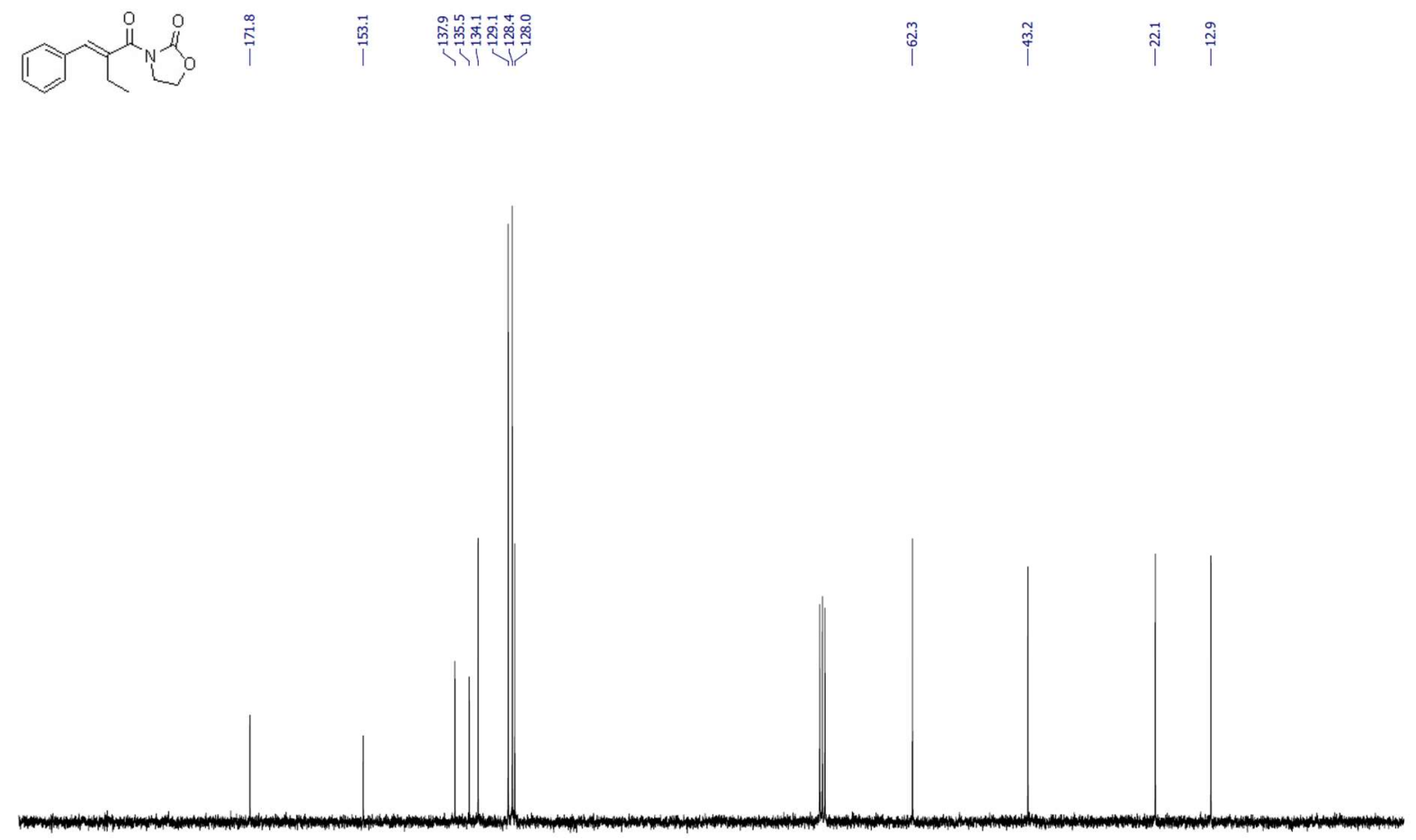

$\begin{array}{lllllllllllllllllllllllllll}210 & 200 & 190 & 180 & 170 & 160 & 150 & 140 & 130 & 120 & 110 & \underset{\mathrm{f} 1(\mathrm{ppm})}{100} & 80 & 70 & 60 & 50 & 40 & 30 & 20 & 10 & 0 & -10\end{array}$

ethyl (E)-2-methyl-3-(p-tolyl)acrylate (Est-9):

${ }^{1} \mathrm{H} \mathrm{NMR}\left(\mathrm{CDCl}_{3}, 300 \mathrm{MHz}\right)$
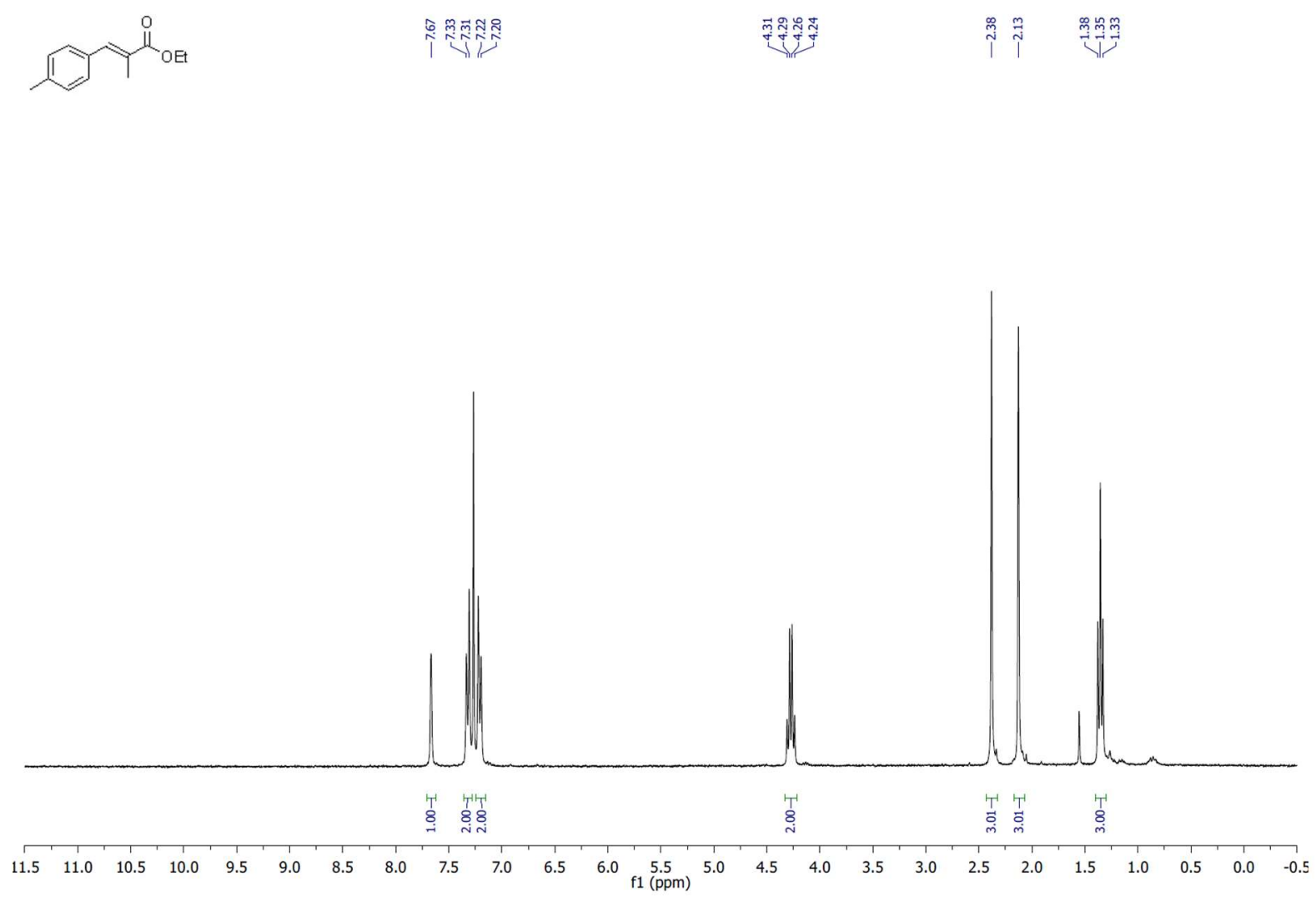
${ }^{13} \mathrm{C} \mathrm{NMR}\left(\mathrm{CDCl}_{3}, 75 \mathrm{MHz}\right)$
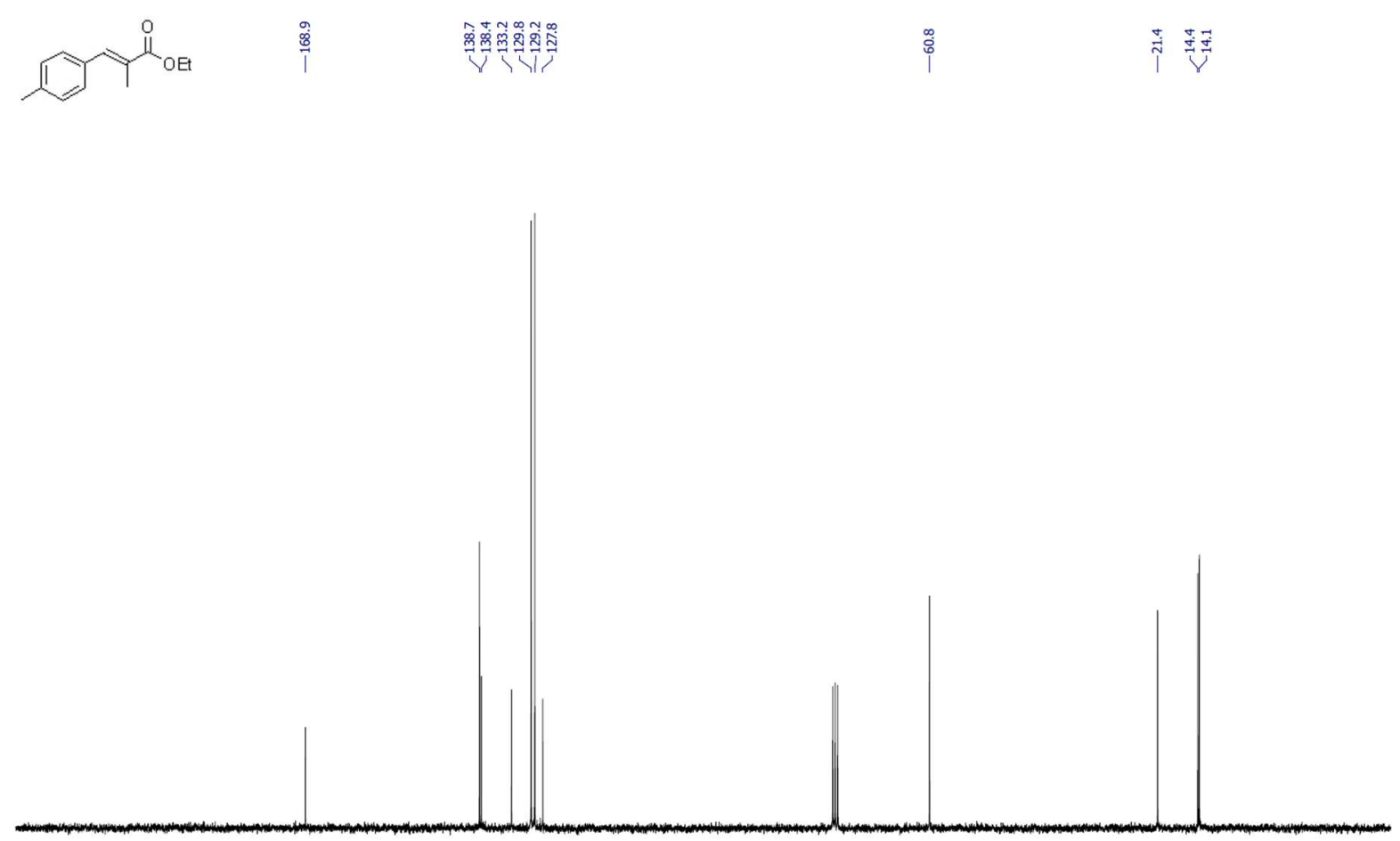

$\begin{array}{llllllllllllllllllllllllllllllll}210 & 200 & 190 & 180 & 170 & 160 & 150 & 140 & 130 & 120 & 110 & 100 & 90 & 80 & 70 & 60 & 50 & 40 & 30 & 20 & 10 & 0 & -10\end{array}$

ethyl (E)-2-methyl-3-(m-tolyl)acrylate (Est-10):

${ }^{1} \mathrm{H} \mathrm{NMR}\left(\mathrm{CDCl}_{3}, 300 \mathrm{MHz}\right)$
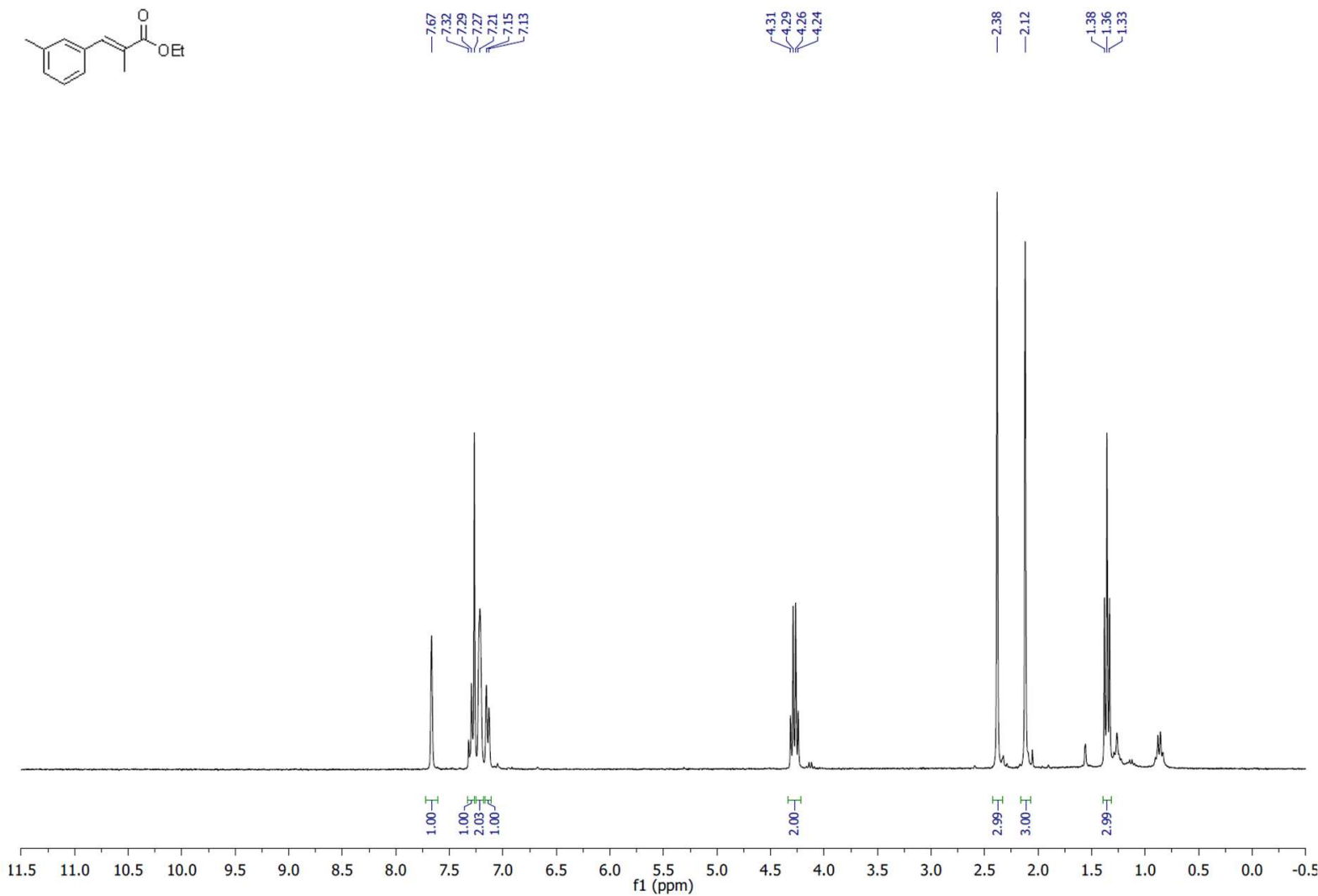
${ }^{13} \mathrm{C} \mathrm{NMR}\left(\mathrm{CDCl}_{3}, 75 \mathrm{MHz}\right)$
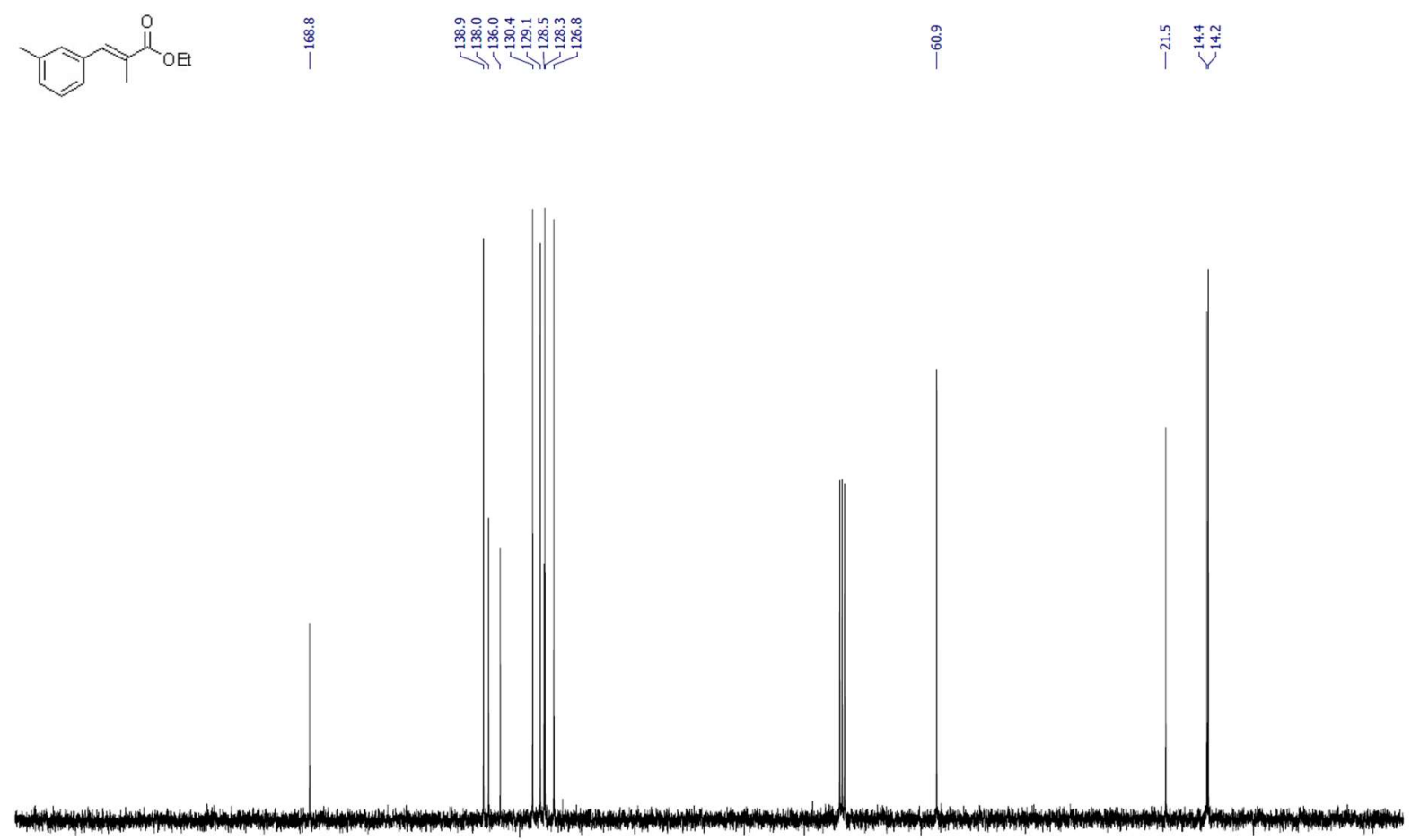

$\begin{array}{lllllllllllllllllllllllllll}210 & 200 & 190 & 180 & 170 & 160 & 150 & 140 & 130 & 120 & 110 & 100 & 90 & 80 & 70 & 60 & 50 & 40 & 30 & 20 & 10 & 0 & -10\end{array}$

ethyl (E)-2-methyl-3-(o-tolyl)acrylate (Est-11):

${ }^{1} \mathrm{H} \mathrm{NMR}\left(\mathrm{CDCl}_{3}, 300 \mathrm{MHz}\right)$
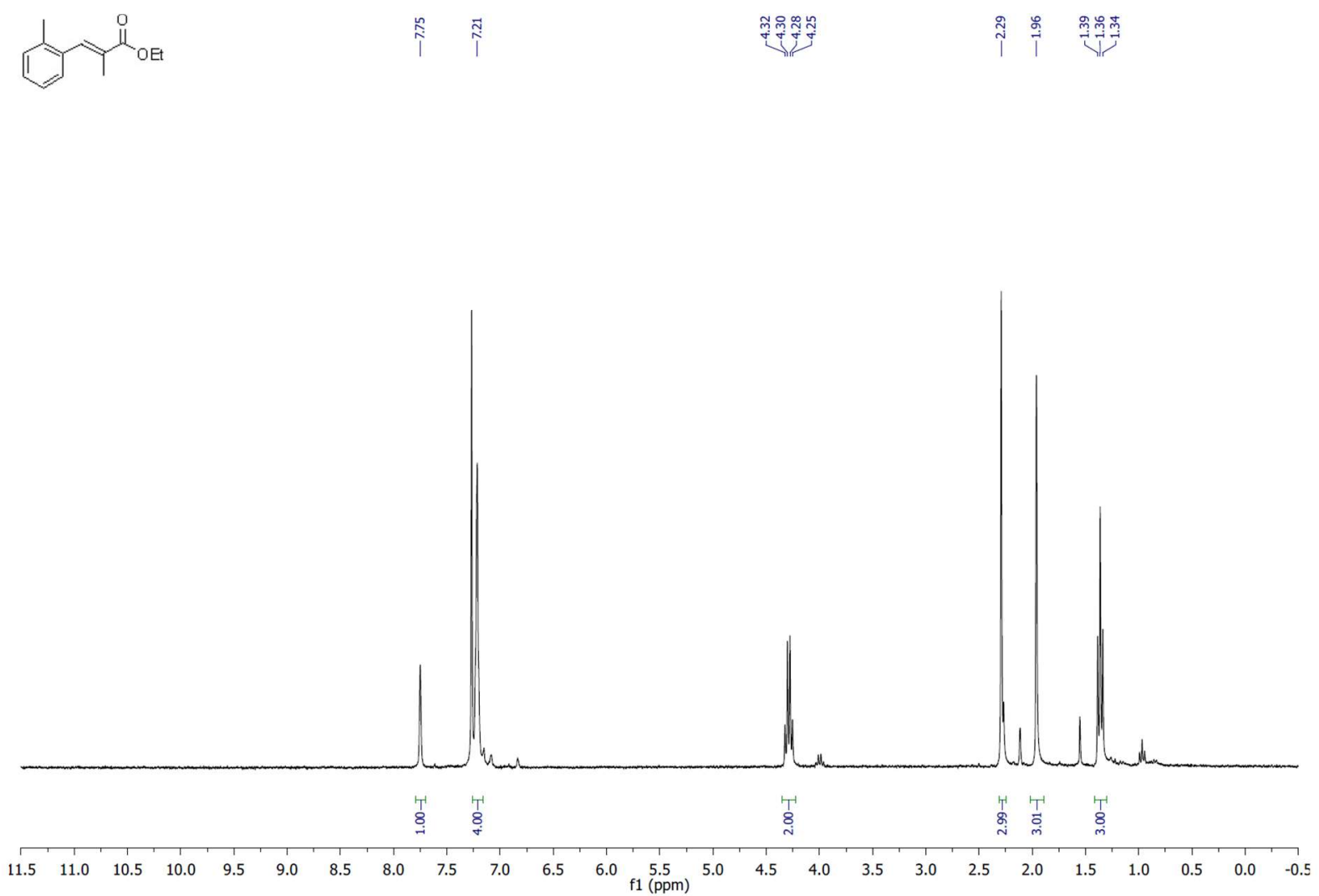
${ }^{13} \mathrm{C} \mathrm{NMR}\left(\mathrm{CDCl}_{3}, 75 \mathrm{MHz}\right)$
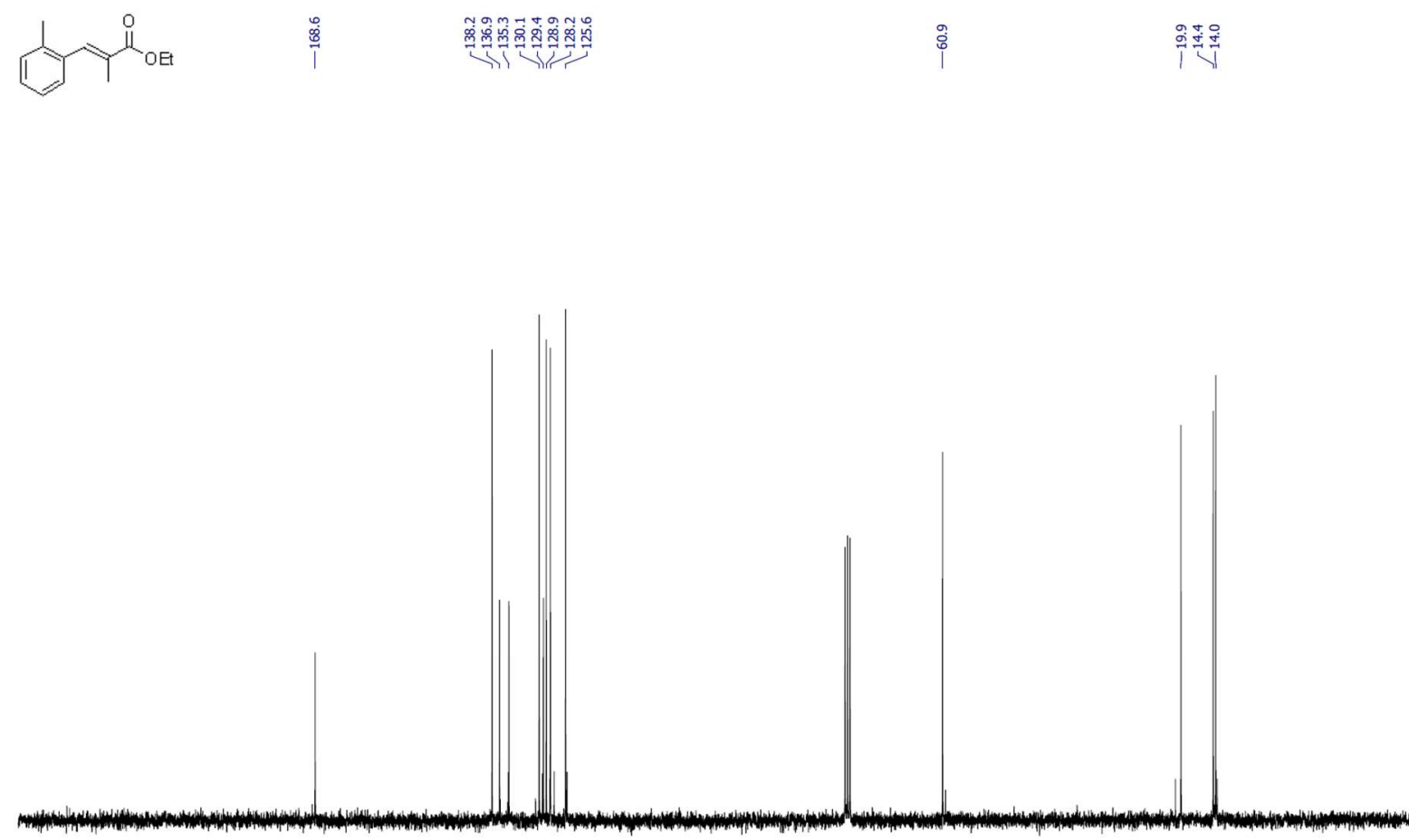

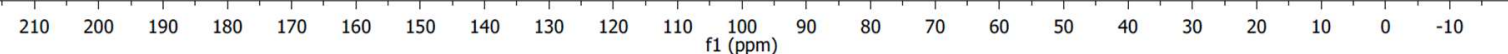

ethyl (E)-3-(4-(tert-butyl)phenyl)-2-methylacrylate (Est-12):

${ }^{1} \mathrm{H} \mathrm{NMR}\left(\mathrm{CDCl}_{3}, 300 \mathrm{MHz}\right)$<smiles>CCC(=O)C=C1C=CC2C=CC1C2(C)C</smiles>

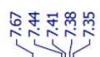

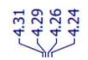

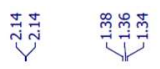

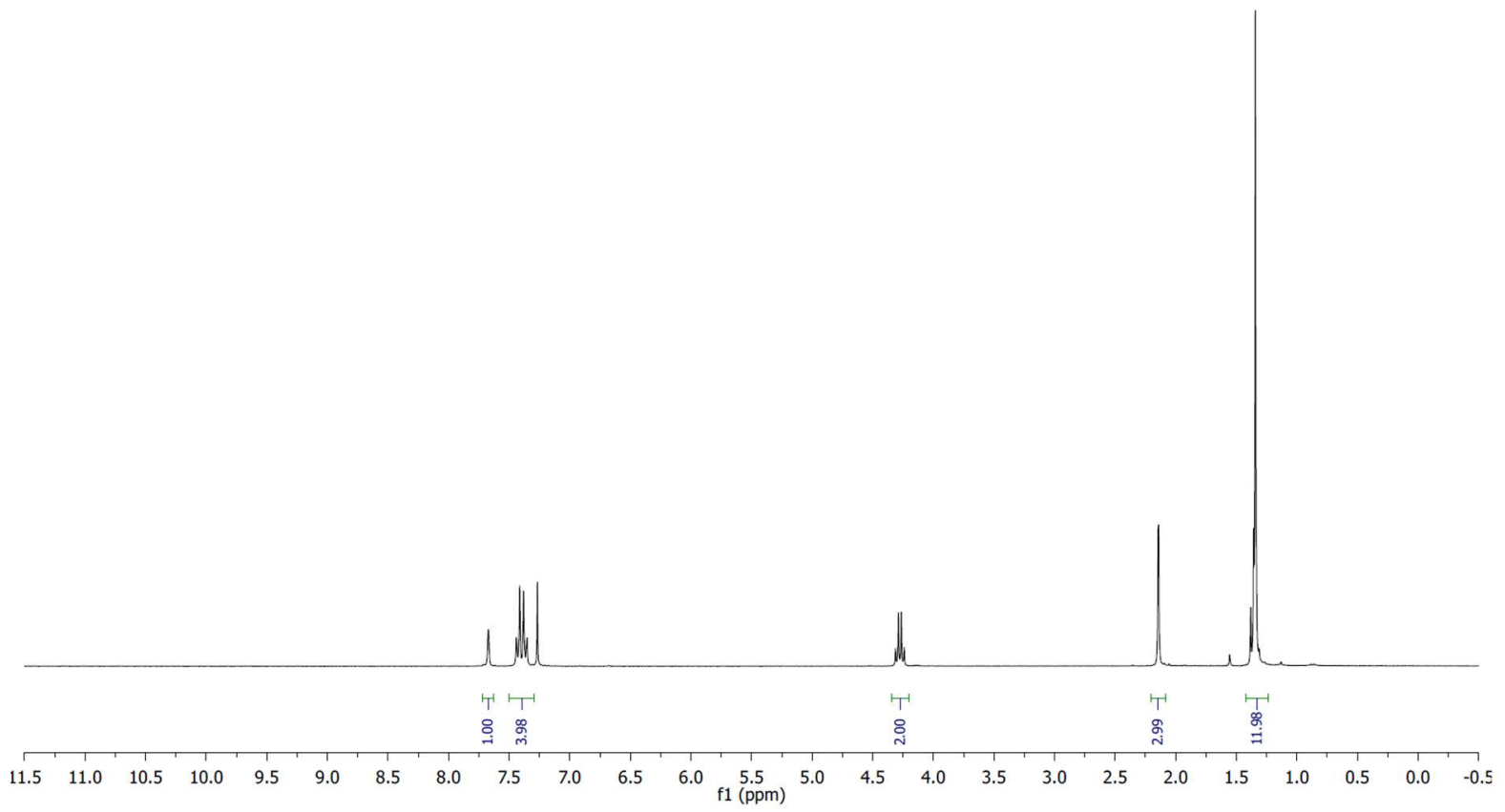


${ }^{13} \mathrm{C} \mathrm{NMR}\left(\mathrm{CDCl}_{3}, 75 \mathrm{MHz}\right)$
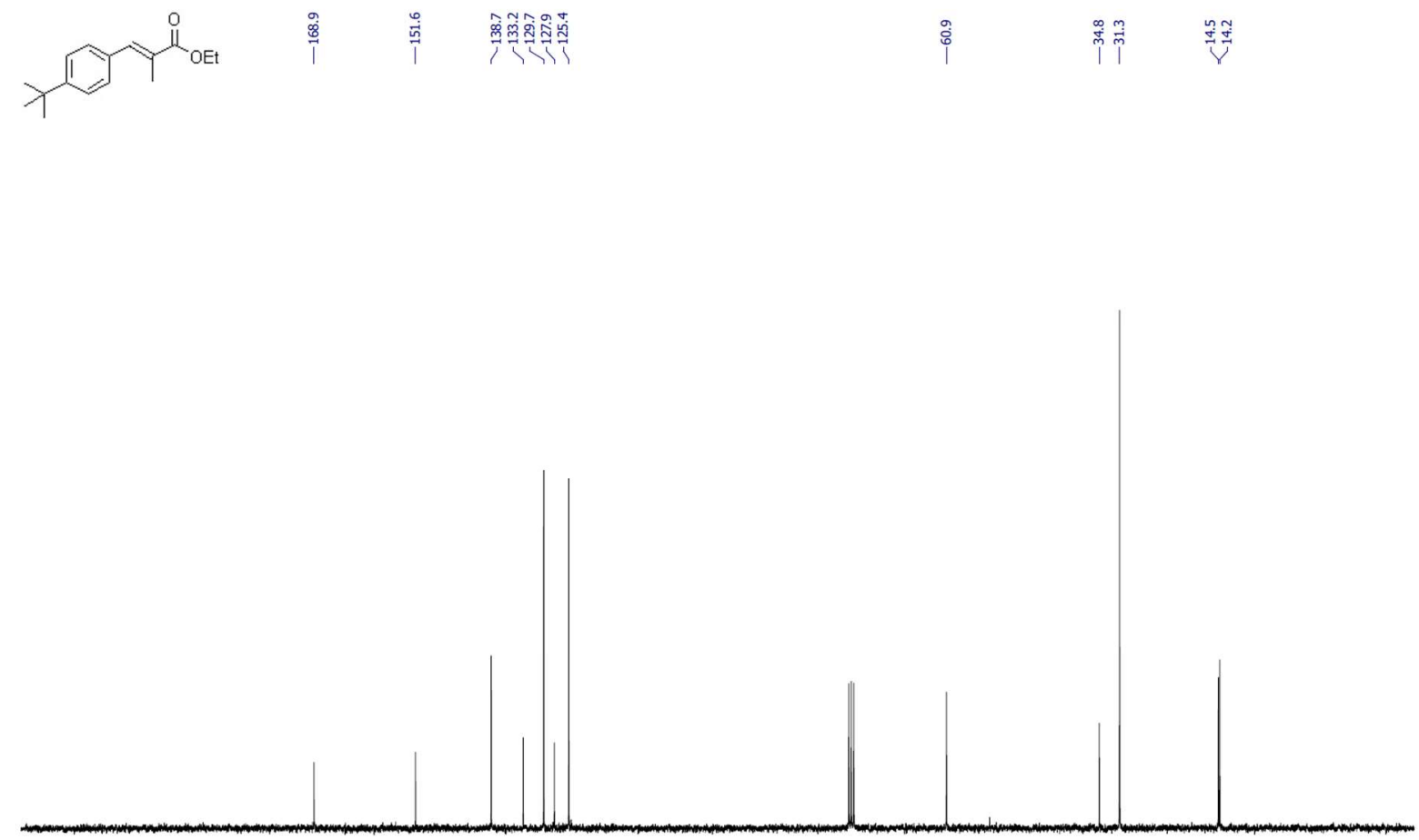

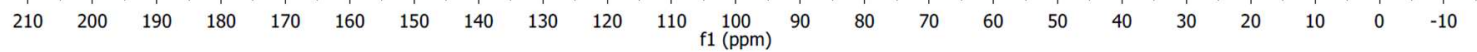

ethyl (E)-3-([1,1'-biphenyl]-4-yl)-2-methylacrylate (Est-13):

${ }^{1} \mathrm{H} \mathrm{NMR}\left(\mathrm{CDCl}_{3}, 300 \mathrm{MHz}\right)$<smiles>CCCC(=O)C(C)=CC1CCC(C)C1</smiles>

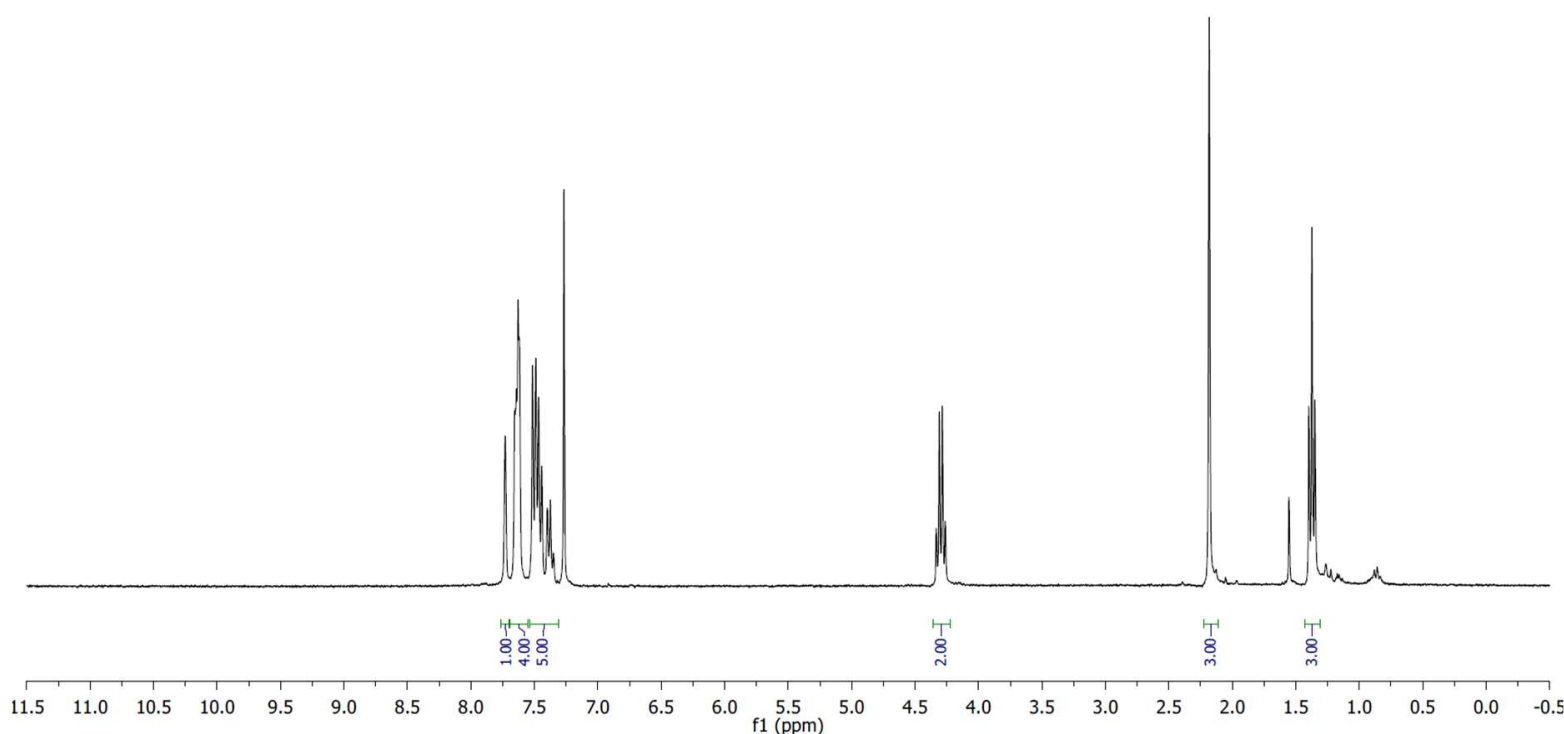


${ }^{13} \mathrm{C} \mathrm{NMR}\left(\mathrm{CDCl}_{3}, 75 \mathrm{MHz}\right)$
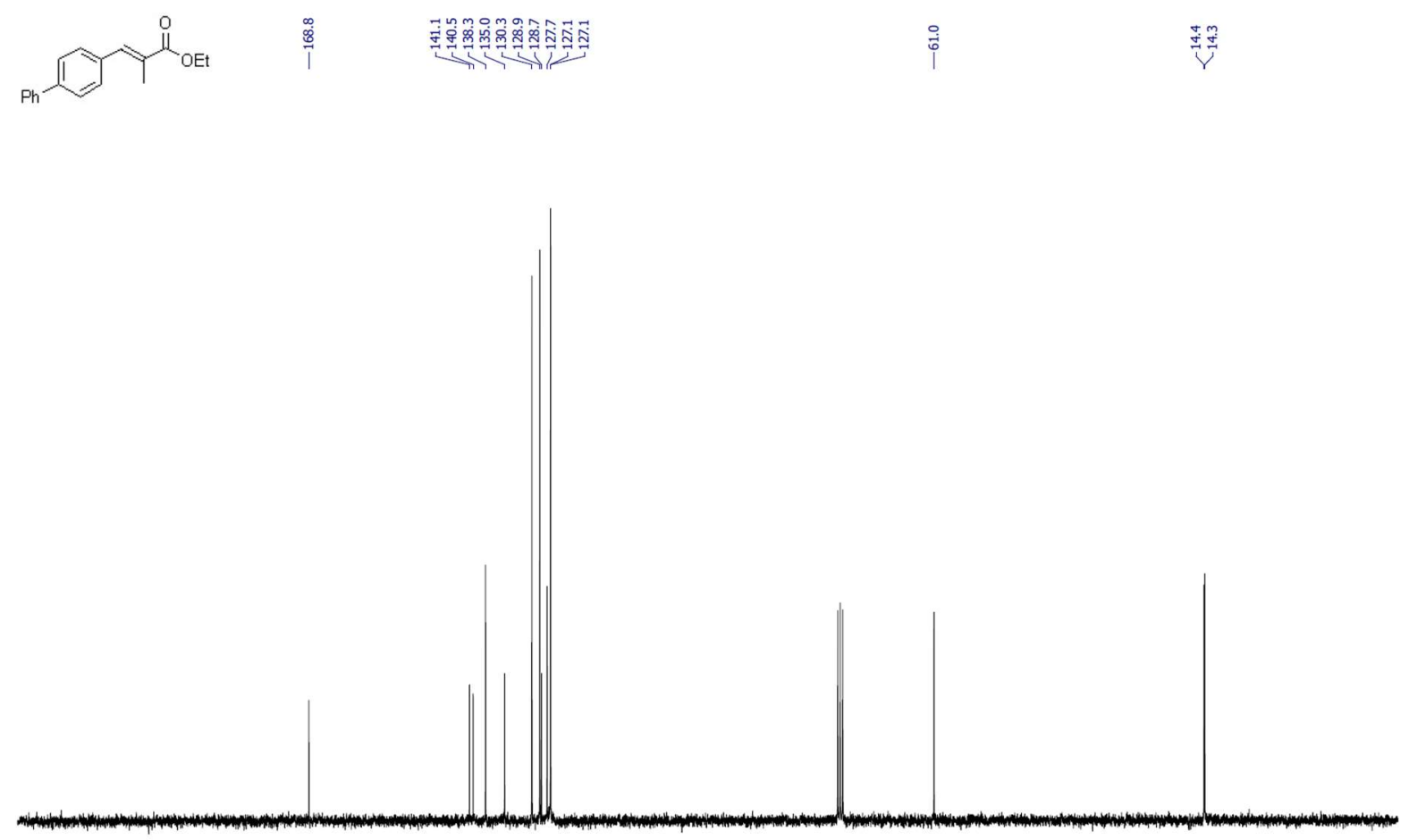

$\begin{array}{llllllllllllllllllllllllll}210 & 200 & 190 & 180 & 170 & 160 & 150 & 140 & 130 & 120 & 110 & 100 & 90 & 80 & 70 & 60 & 50 & 40 & 30 & 20 & 10 & 0 & -10\end{array}$

ethyl (E)-3-(4-methoxyphenyl)-2-methylacrylate (Est-14):

${ }^{1} \mathrm{H} \mathrm{NMR}\left(\mathrm{CDCl}_{3}, 300 \mathrm{MHz}\right)$
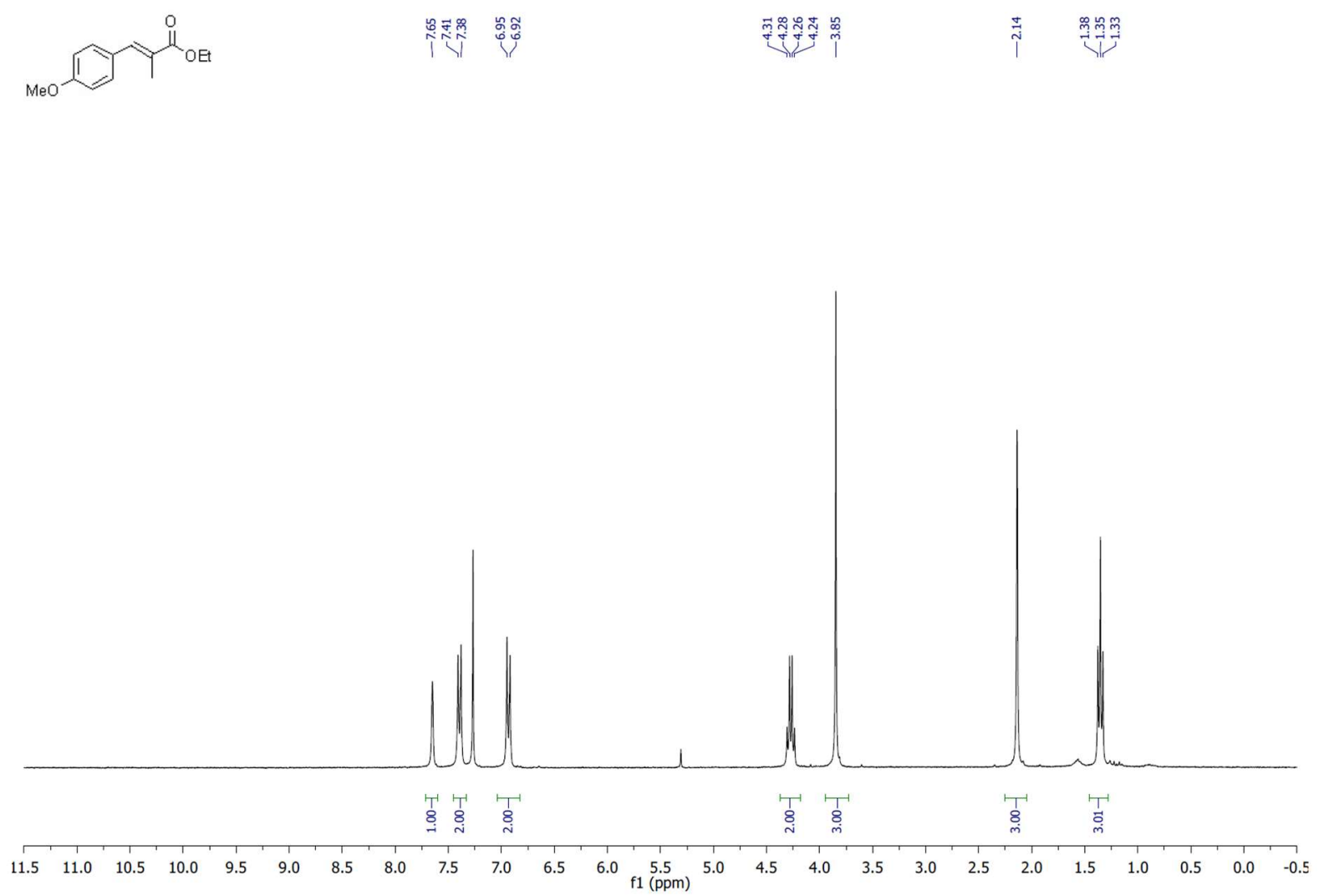
${ }^{13} \mathrm{C} \mathrm{NMR}\left(\mathrm{CDCl}_{3}, 75 \mathrm{MHz}\right)$
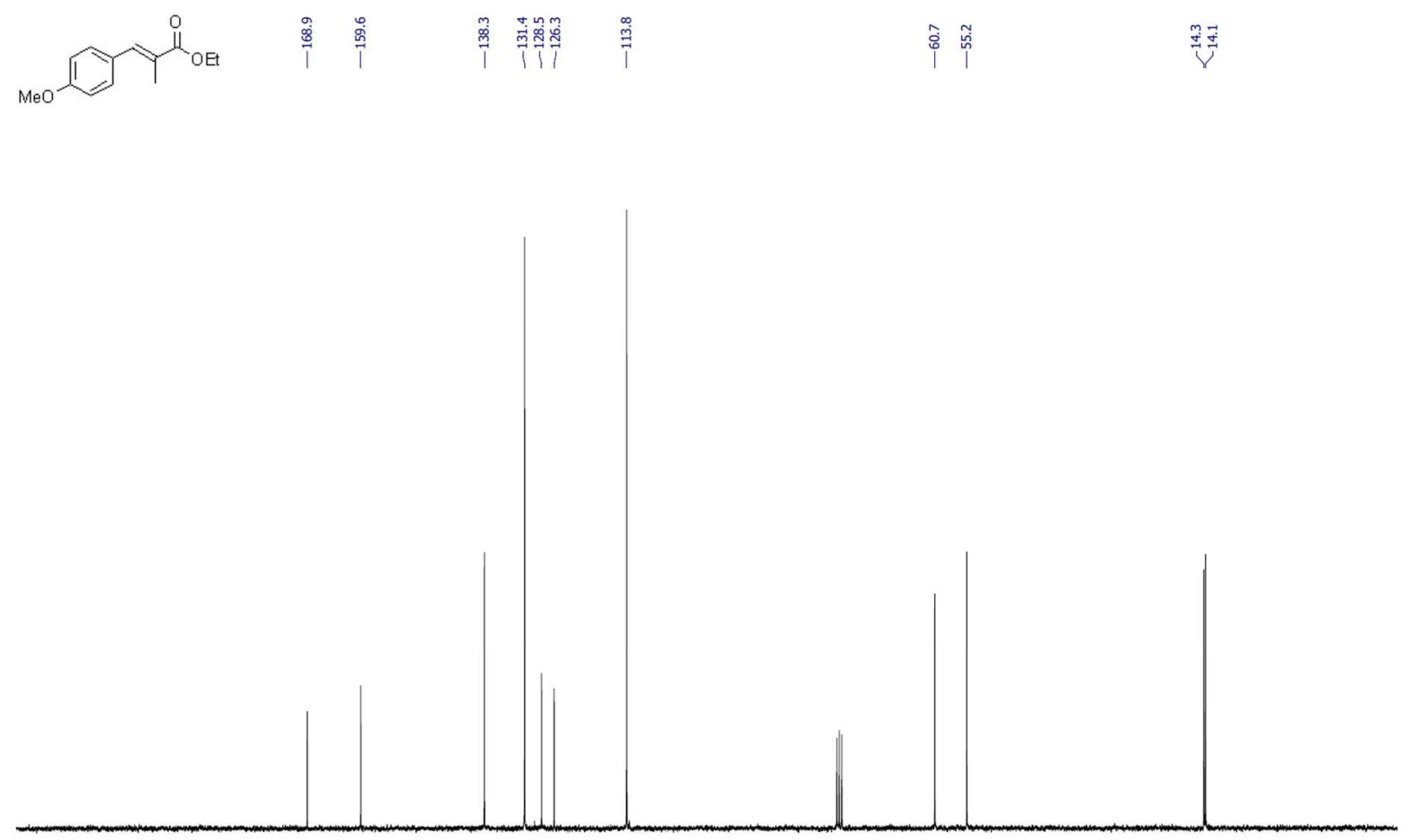

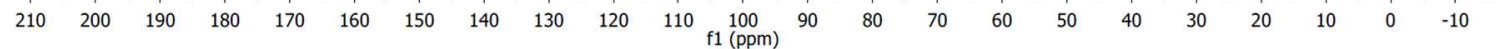

ethyl (E)-2-methyl-3-(naphthalen-2-yl)acrylate (Est-15):

${ }^{1} \mathrm{H} \mathrm{NMR}\left(\mathrm{CDCl}_{3}, 300 \mathrm{MHz}\right)$<smiles>CC(=O)C(=CC1=CC2=CC=CC2C1)C(C)=O</smiles>

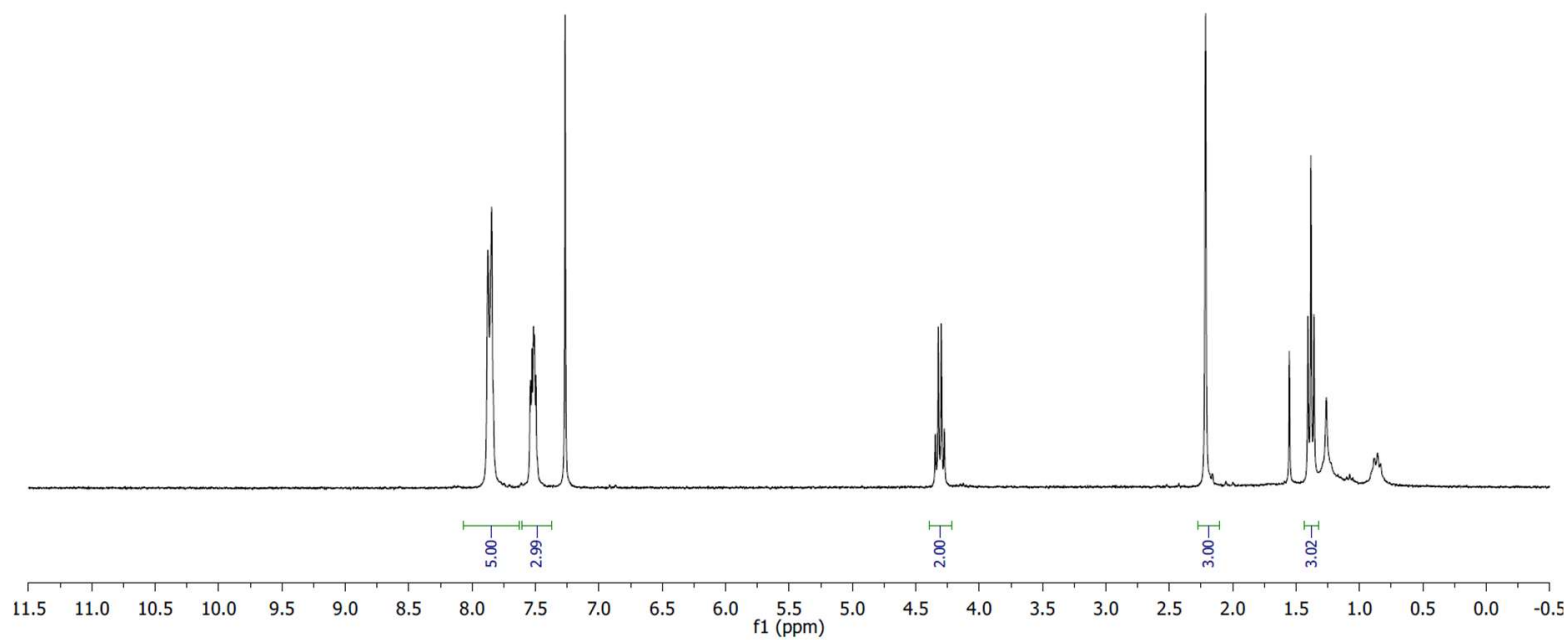


${ }^{13} \mathrm{C} \mathrm{NMR}\left(\mathrm{CDCl}_{3}, 75 \mathrm{MHz}\right)$
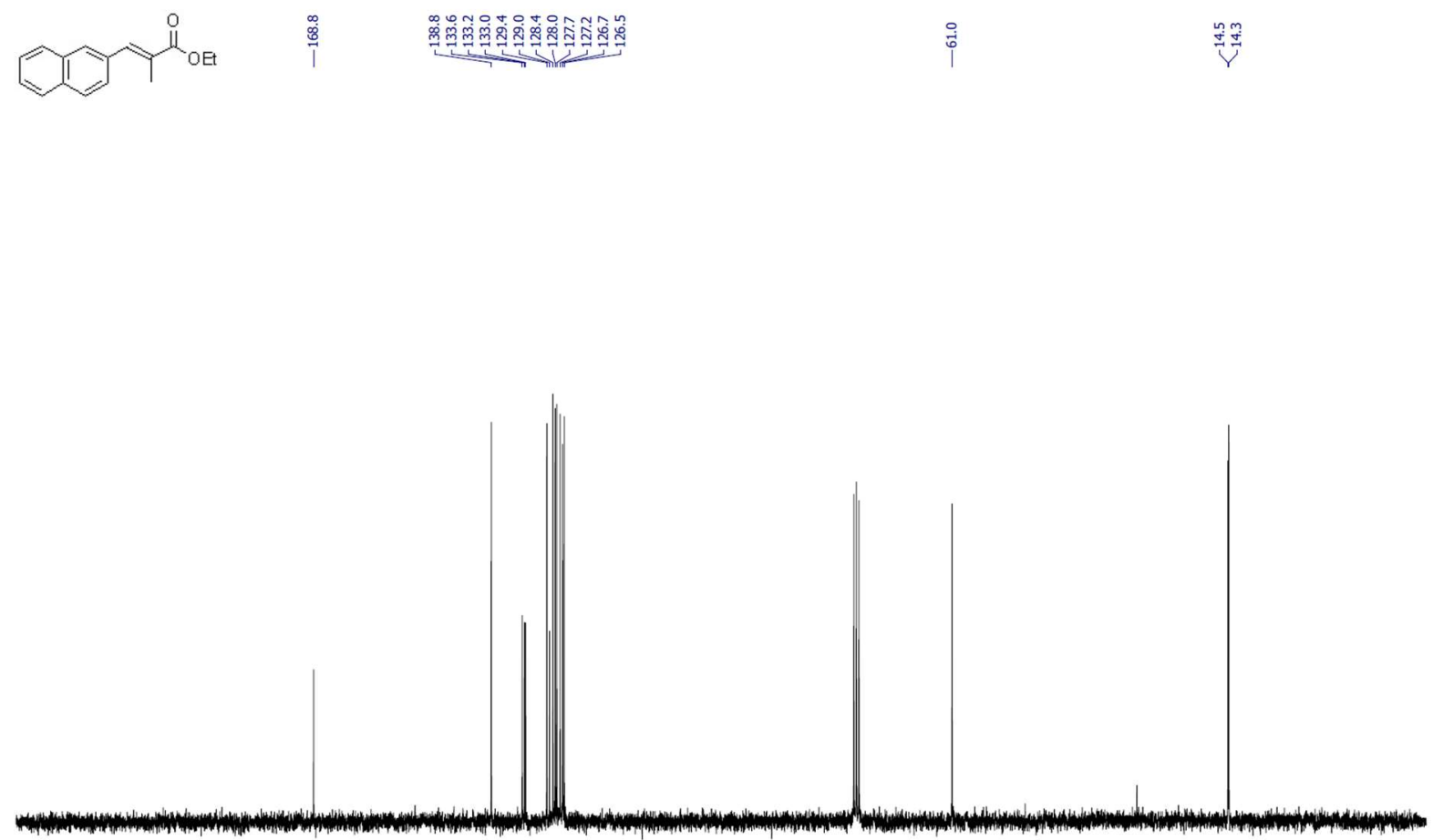

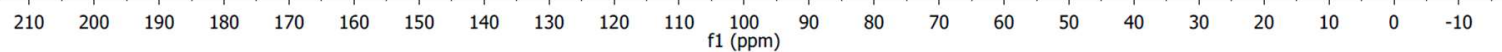

ethyl (E)-3-(4-fluorophenyl)-2-methylacrylate (Est-16):

${ }^{1} \mathrm{H} \mathrm{NMR}\left(\mathrm{CDCl}_{3}, 300 \mathrm{MHz}\right)$

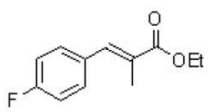

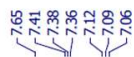

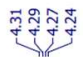

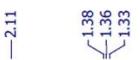

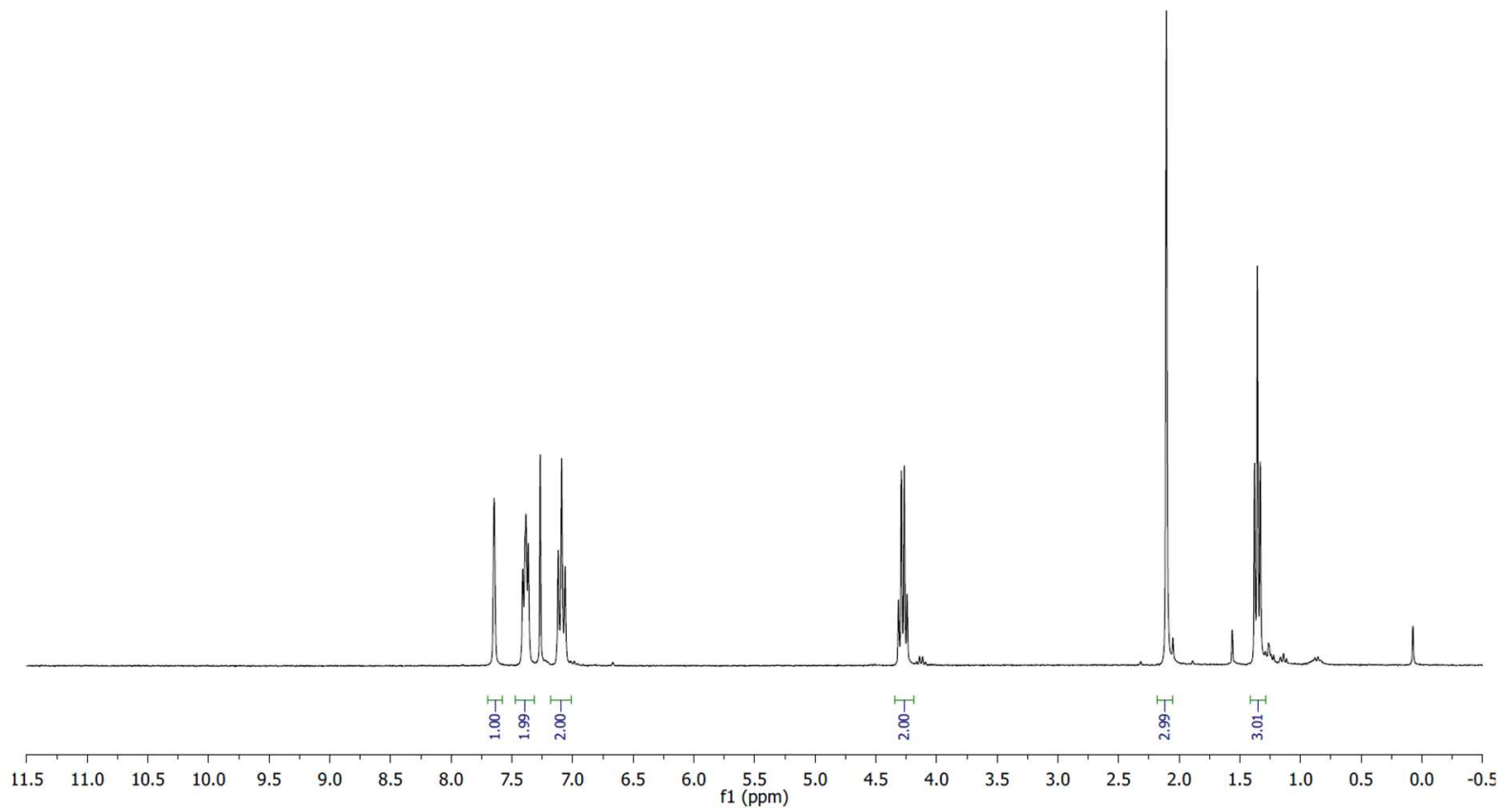


${ }^{13} \mathrm{C} \mathrm{NMR}\left(\mathrm{CDCl}_{3}, 75 \mathrm{MHz}\right)$

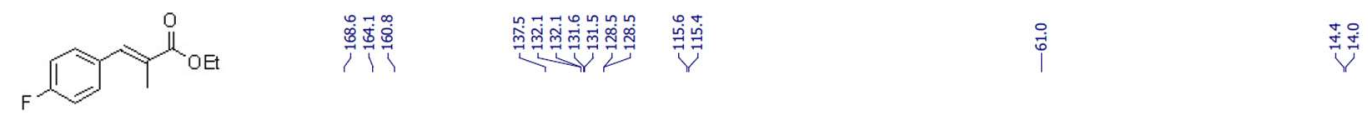
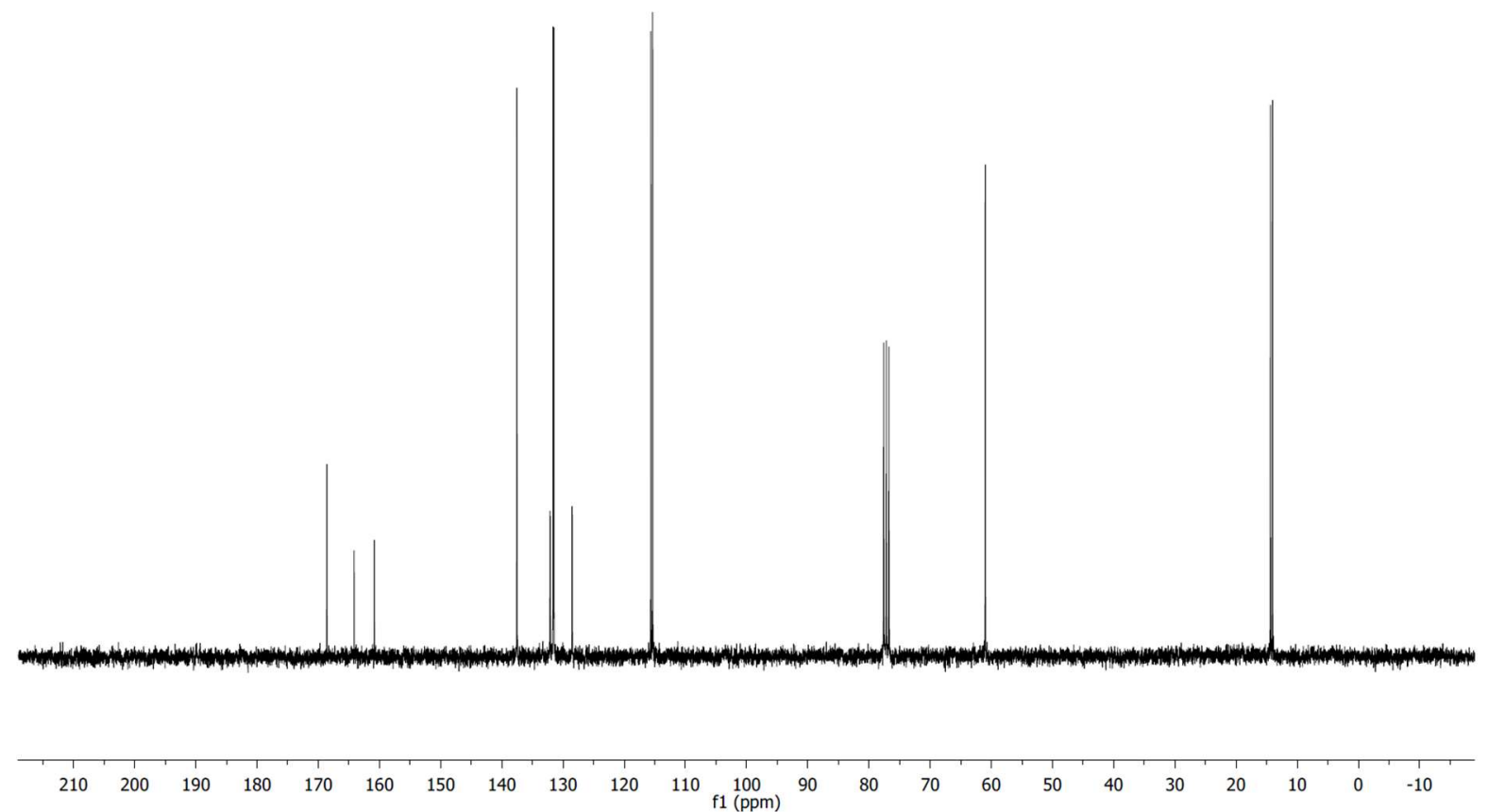

${ }^{19} \mathrm{~F} \mathrm{NMR}\left(\mathrm{CDCl}_{3}, 282 \mathrm{MHz}\right)$
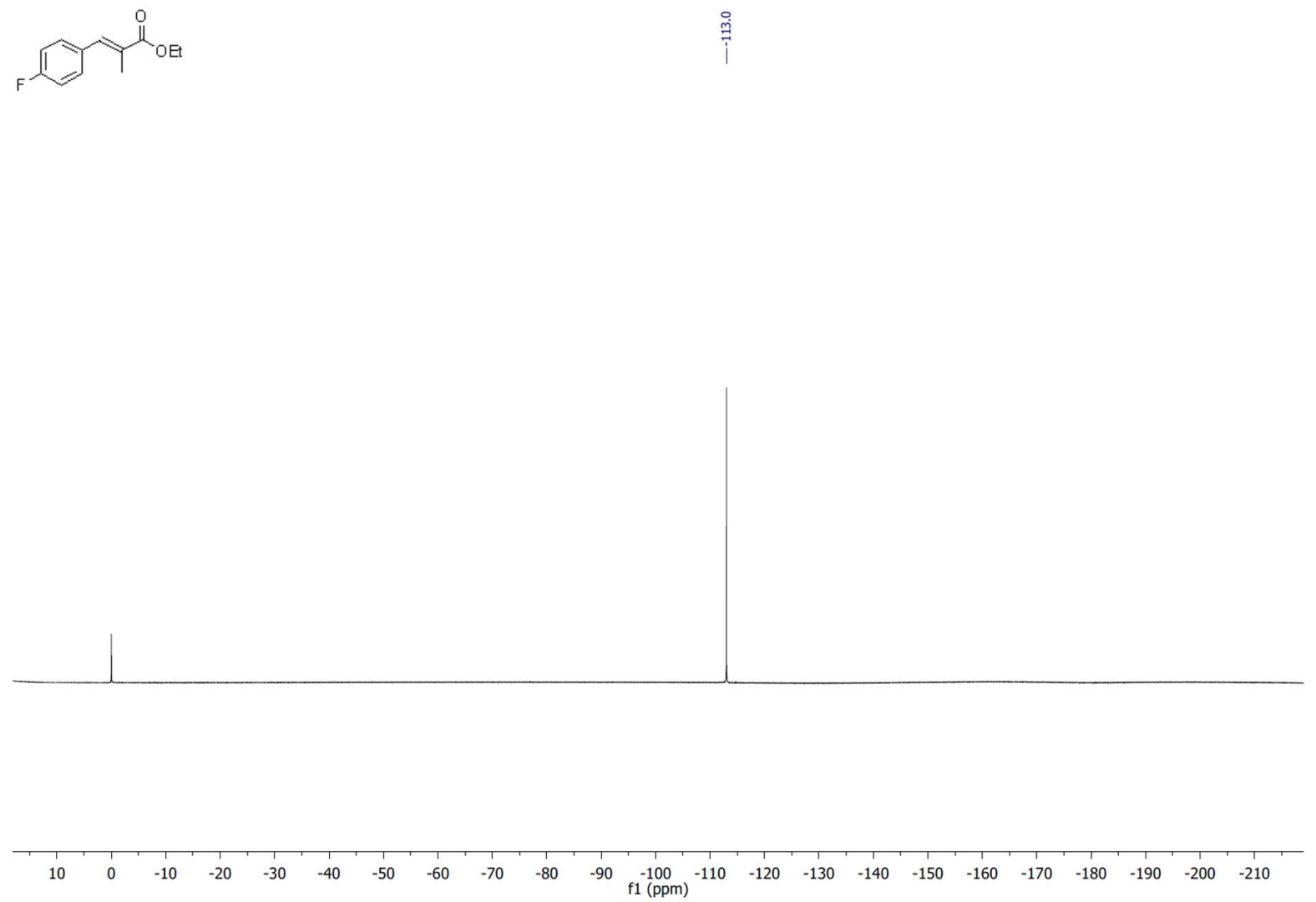
ethyl (E)-3-(4-chlorophenyl)-2-methylacrylate (Est-17):

${ }^{1} \mathrm{H} \mathrm{NMR}\left(\mathrm{CDCl}_{3}, 300 \mathrm{MHz}\right)$<smiles>CC(C)C(=O)Cc1ccc(Cl)cc1</smiles>

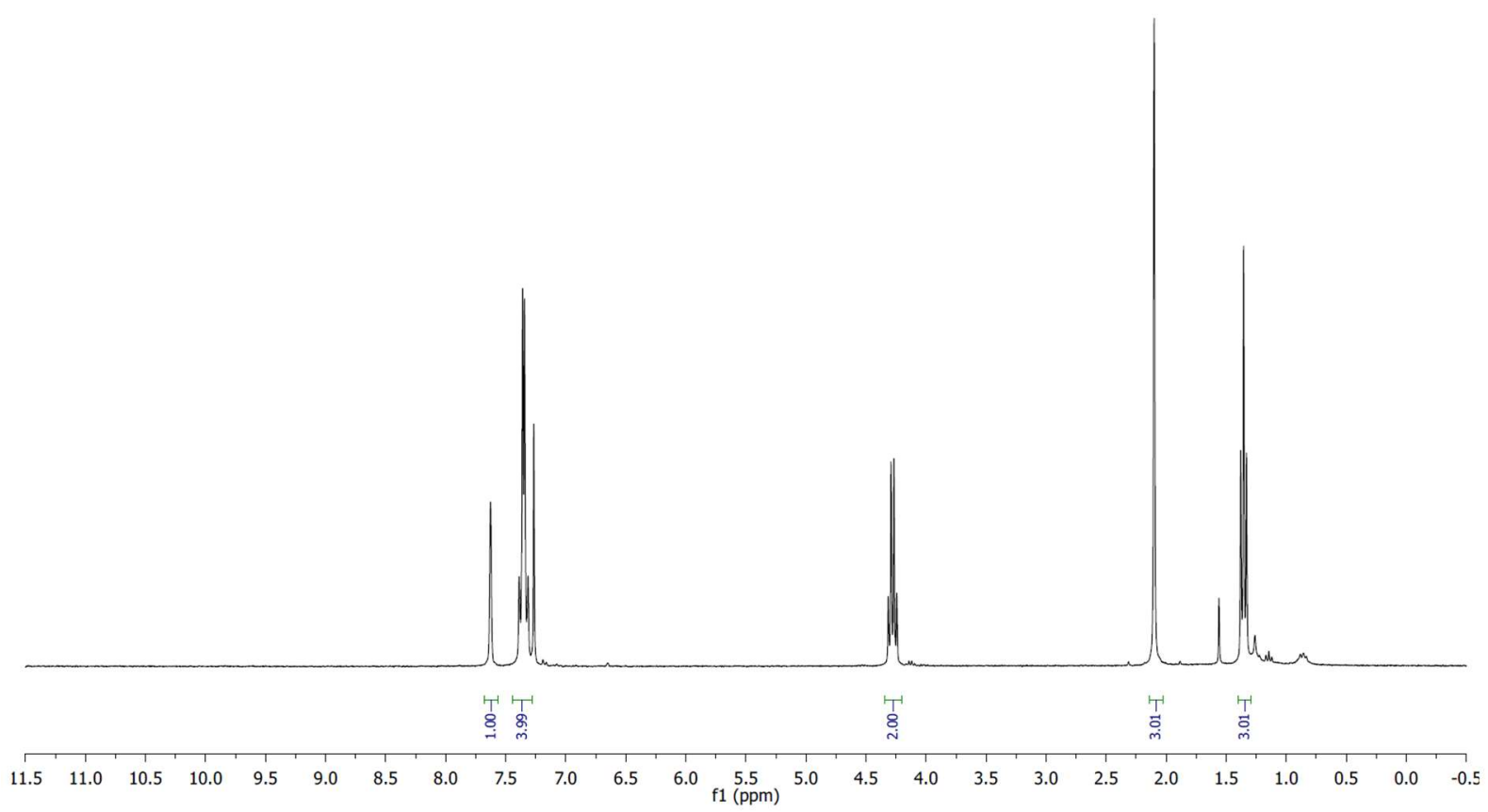

${ }^{13} \mathrm{C} \mathrm{NMR}\left(\mathrm{CDCl}_{3}, 75 \mathrm{MHz}\right)$
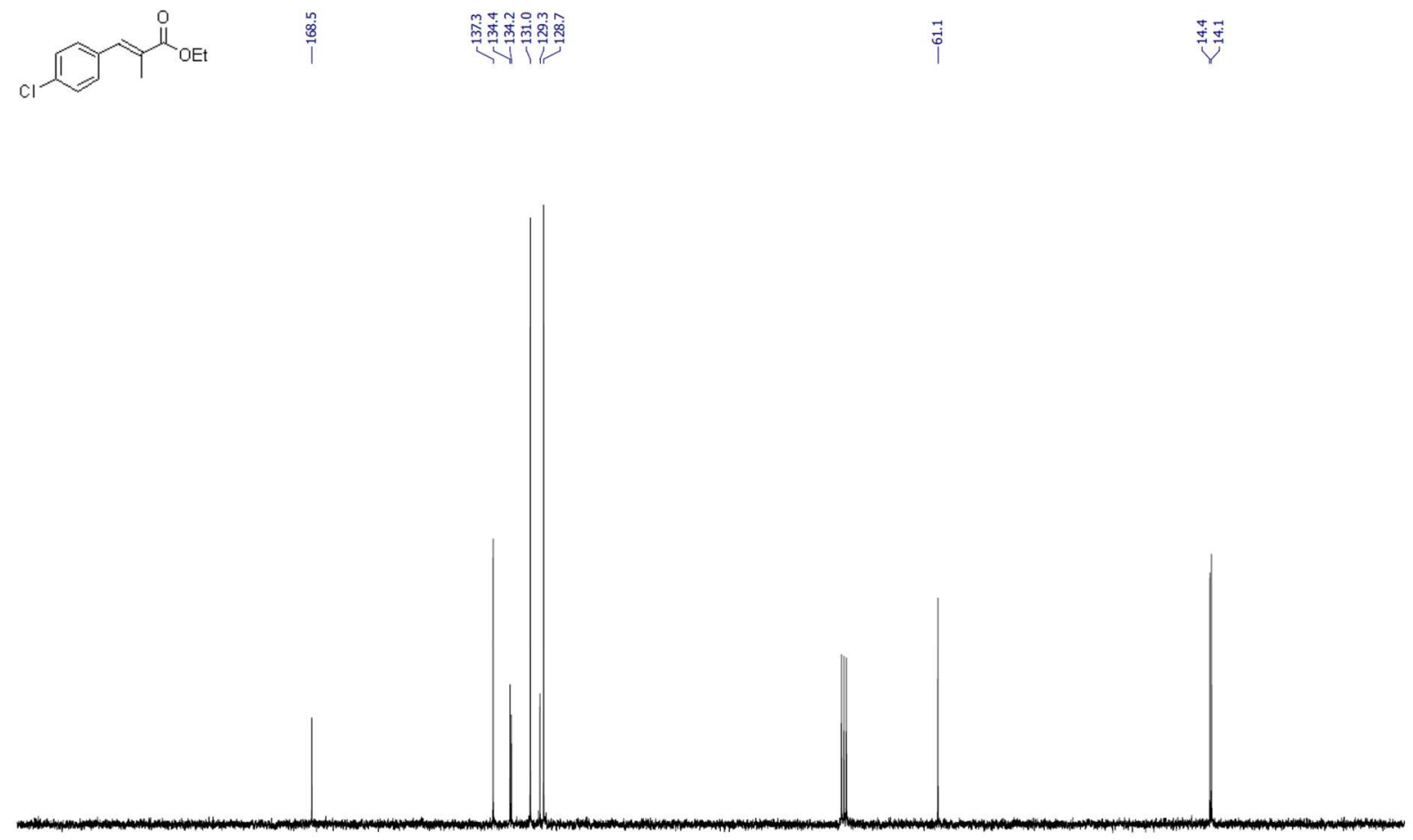

$\begin{array}{lllllllllllllllllllllllllllll}210 & 200 & 190 & 180 & 170 & 160 & 150 & 140 & 130 & 120 & 110 & 100 & 90 & 80 & 70 & 60 & 50 & 40 & 30 & 20 & 10 & 0 & -10\end{array}$ 
ethyl (E)-3-(4-bromophenyl)-2-methylacrylate (Est-18):

${ }^{1} \mathrm{H} \mathrm{NMR}\left(\mathrm{CDCl}_{3}, 300 \mathrm{MHz}\right)$
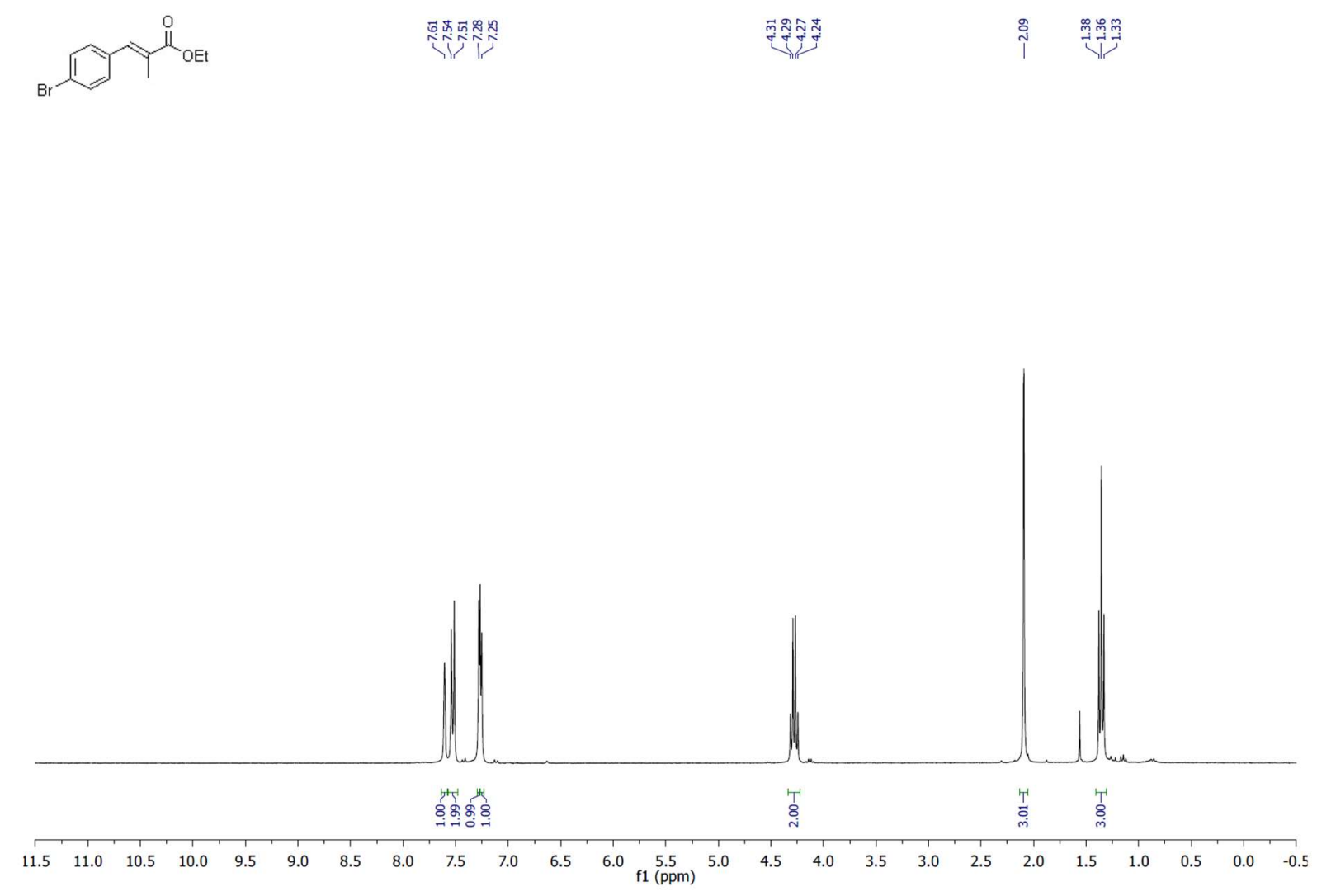

${ }^{13} \mathrm{C} \mathrm{NMR}\left(\mathrm{CDCl}_{3}, 75 \mathrm{MHz}\right)$

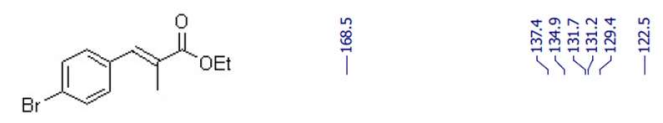

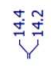

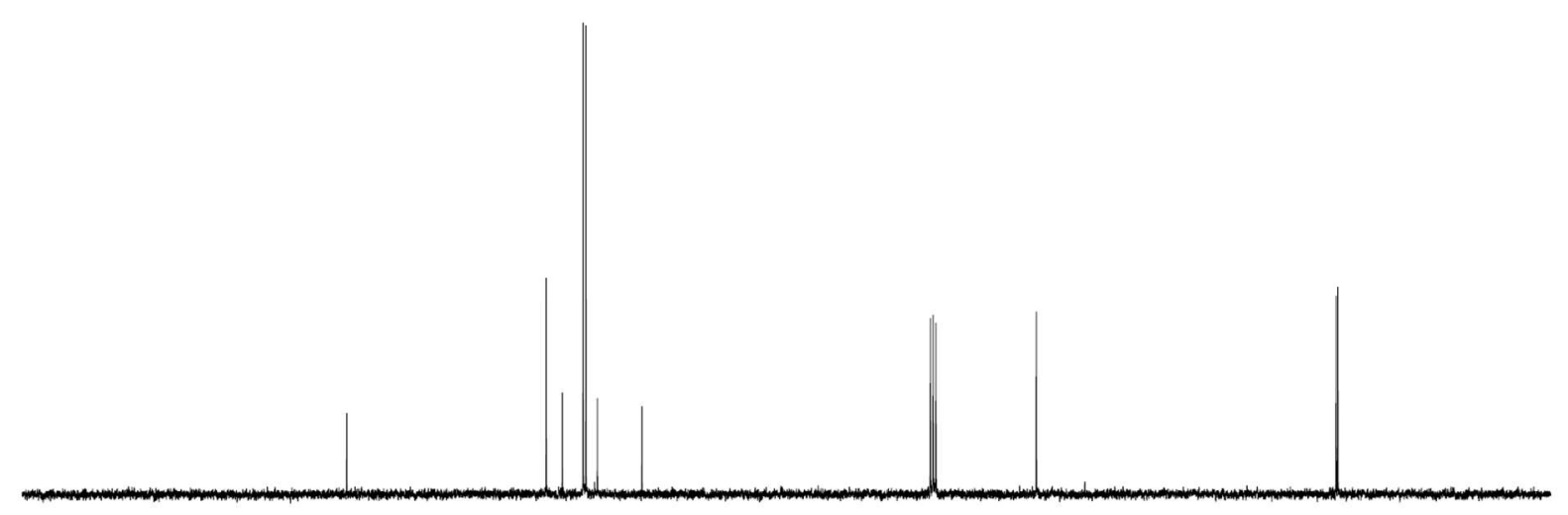

$\begin{array}{lllllllllllllllllllllllll}210 & 200 & 190 & 180 & 170 & 160 & 150 & 140 & 130 & 120 & 110 & 100 & 90 & 80 & 70 & 60 & 50 & 40 & 30 & 20 & 10 & 0 & -10\end{array}$ 
ethyl (E)-3-(4-iodophenyl)-2-methylacrylate (Est-19):

${ }^{1} \mathrm{H} \mathrm{NMR}\left(\mathrm{CDCl}_{3}, 300 \mathrm{MHz}\right)$
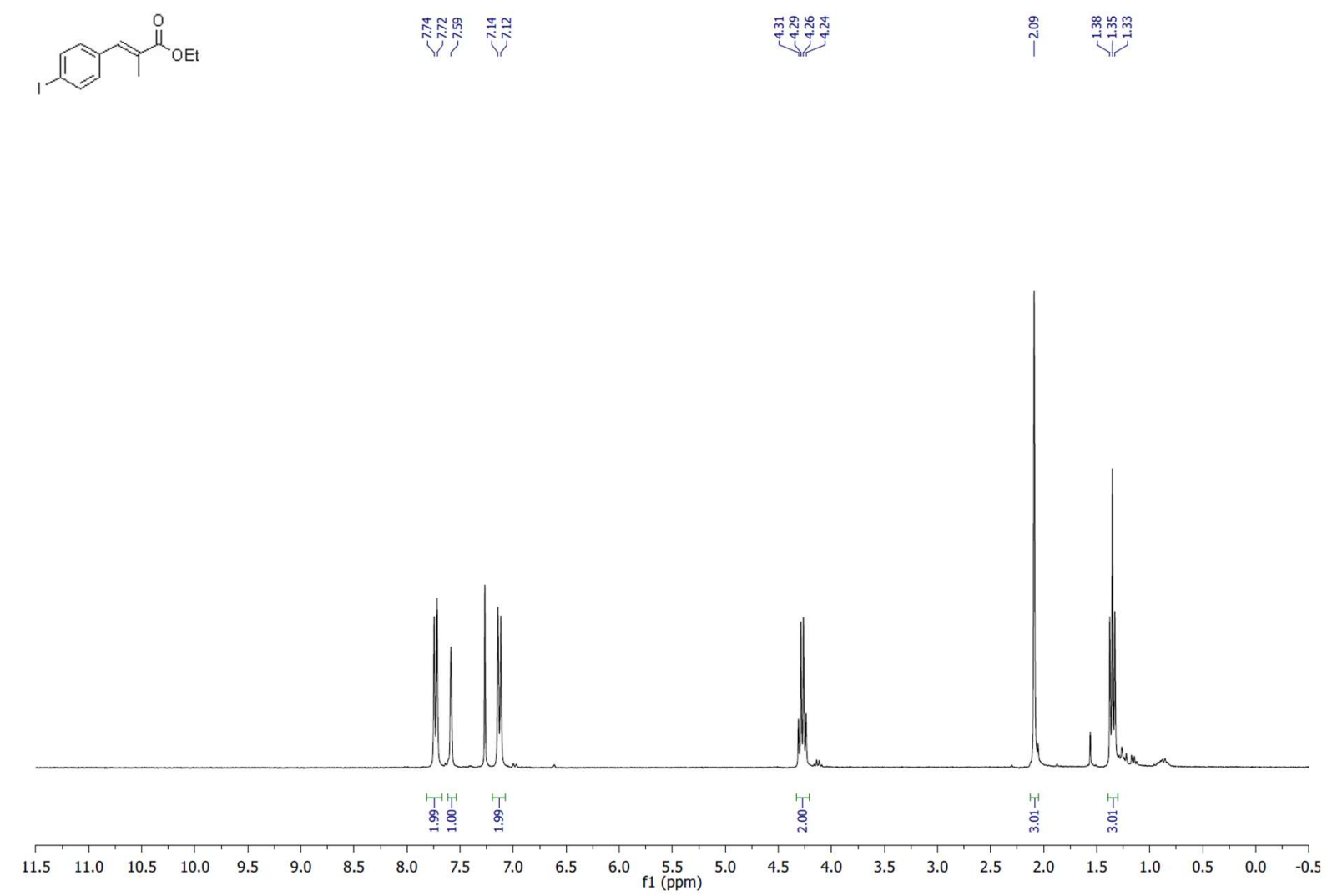

${ }^{13} \mathrm{C} \mathrm{NMR}\left(\mathrm{CDCl}_{3}, 75 \mathrm{MHz}\right)$

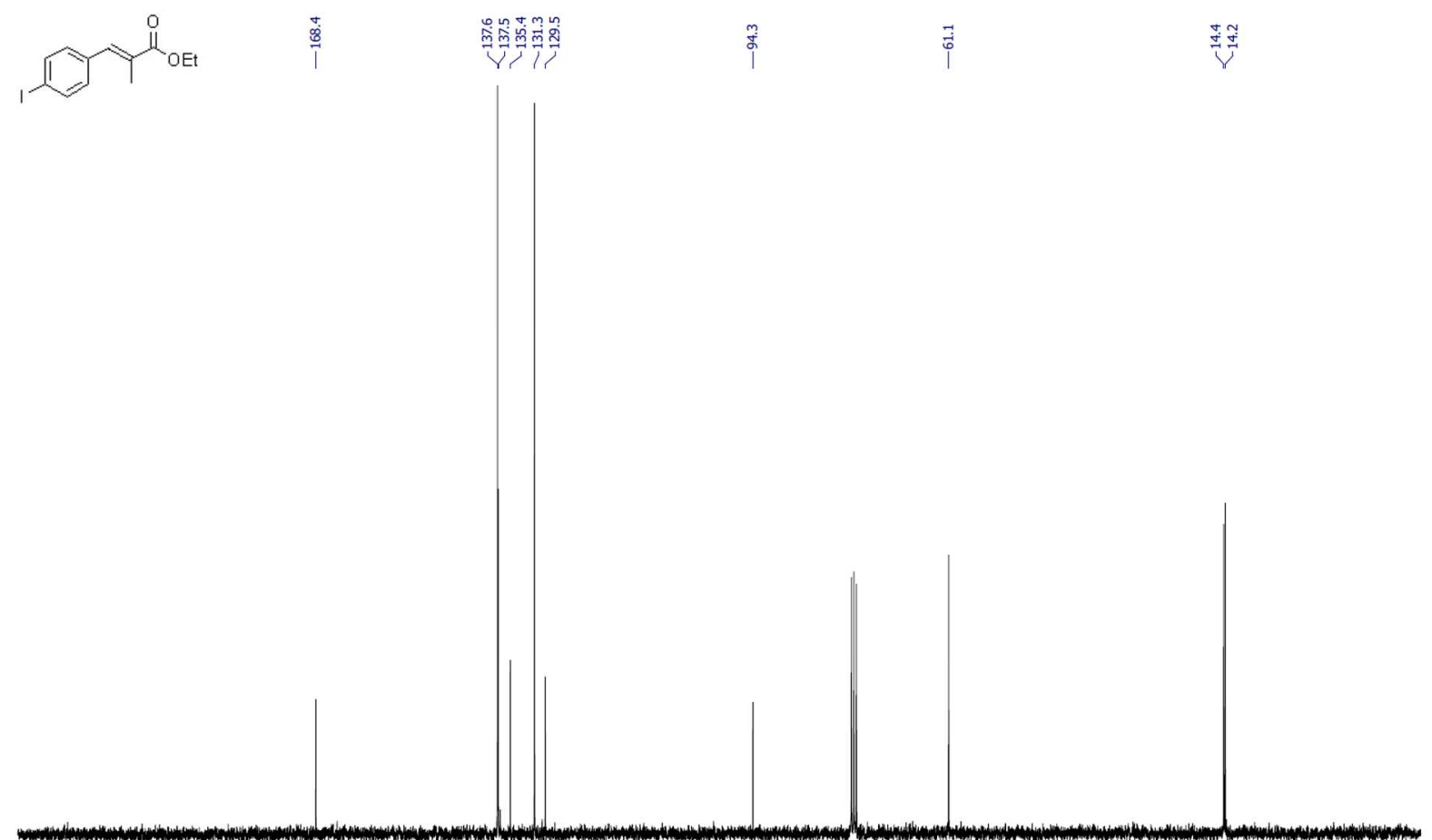

$\begin{array}{lllllllllllllllllllllll}210 & 200 & 190 & 180 & 170 & 160 & 150 & 140 & 130 & 120 & 110 & \underset{\mathrm{f} 1(\mathrm{ppm})}{100} & 90 & 80 & 70 & 60 & 50 & 40 & 30 & 20 & 10 & 0 & -10\end{array}$ 
ethyl (E)-2-methyl-3-(4-(trifluoromethyl)phenyl)acrylate (Est-20):

${ }^{1} \mathrm{H} \mathrm{NMR}\left(\mathrm{CDCl}_{3}, 300 \mathrm{MHz}\right)$<smiles>CC(=O)C=C1C=CC(C)C=C1</smiles>

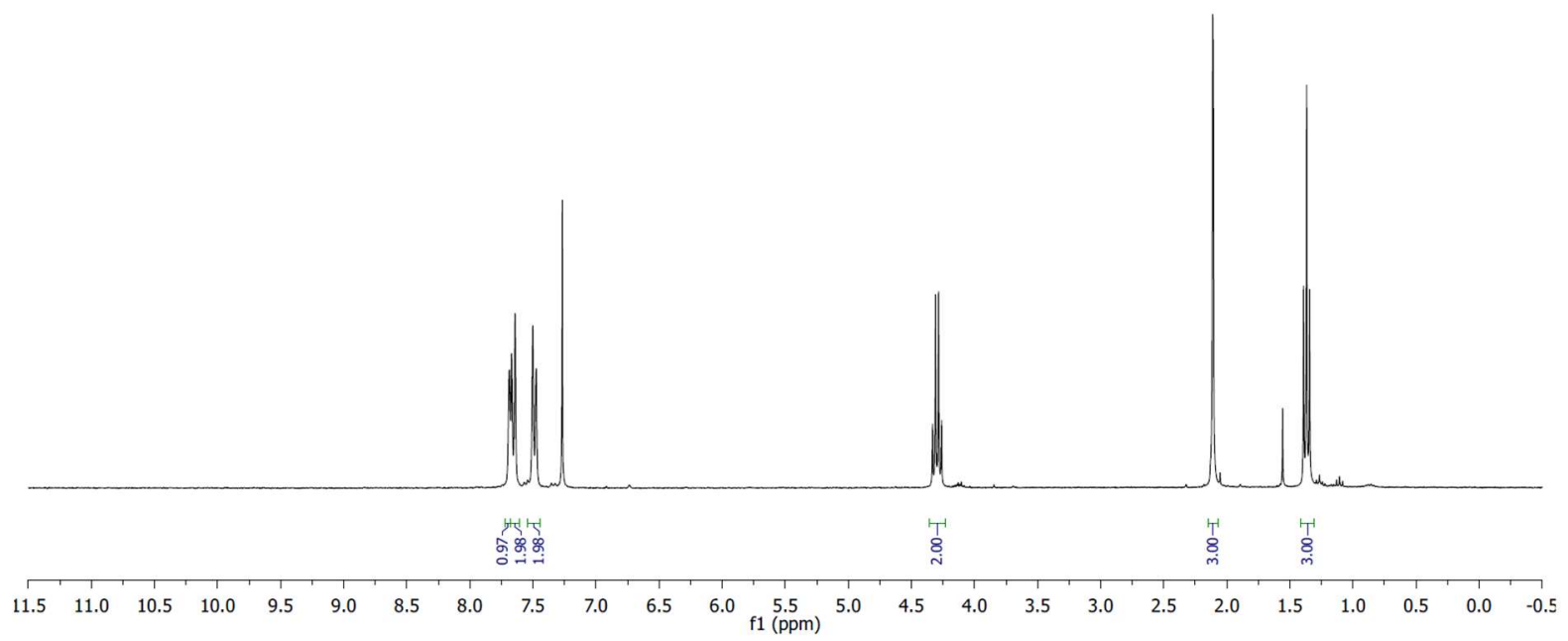

${ }^{13} \mathrm{C} \mathrm{NMR}\left(\mathrm{CDCl}_{3}, 75 \mathrm{MHz}\right)$
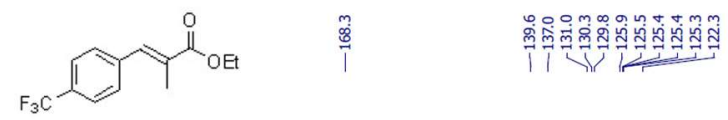

iั

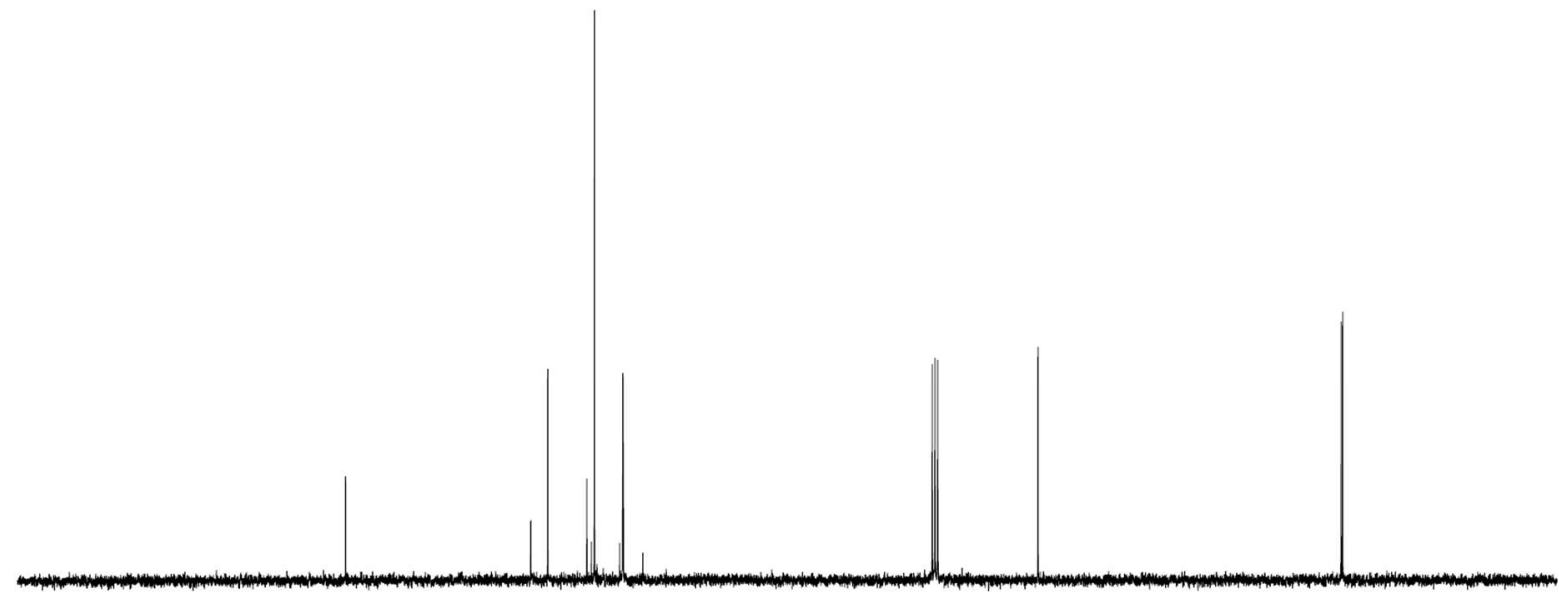

$\begin{array}{llllllllllllllllllllllllllllllll}210 & 200 & 190 & 180 & 170 & 160 & 150 & 140 & 130 & 120 & 110 & 100 & 90 & 80 & 70 & 60 & 50 & 40 & 30 & 20 & 10 & 0 & -10\end{array}$ 
${ }^{19} \mathrm{~F} \mathrm{NMR}\left(\mathrm{CDCl}_{3}, 282 \mathrm{MHz}\right)$<smiles>CC(C)=Cc1ccc(C)cc1</smiles>

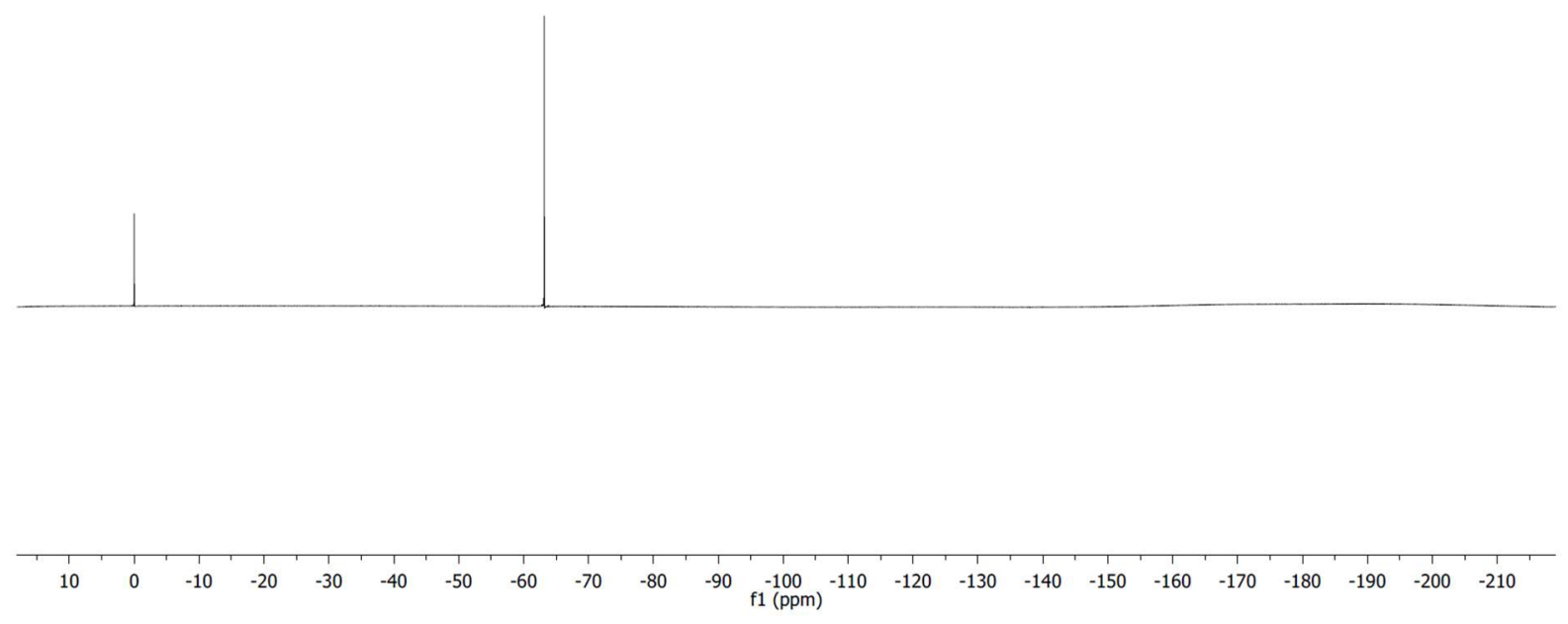

ethyl (E)-2-methyl-3-(4-(4,4,5,5-tetramethyl-1,3,2-dioxaborolan-2-yl)phenyl)acrylate (Est-21):

$\left.{ }^{1} \mathrm{H} \mathrm{NMR} \mathrm{(CDCl}, 300 \mathrm{MHz}\right)$
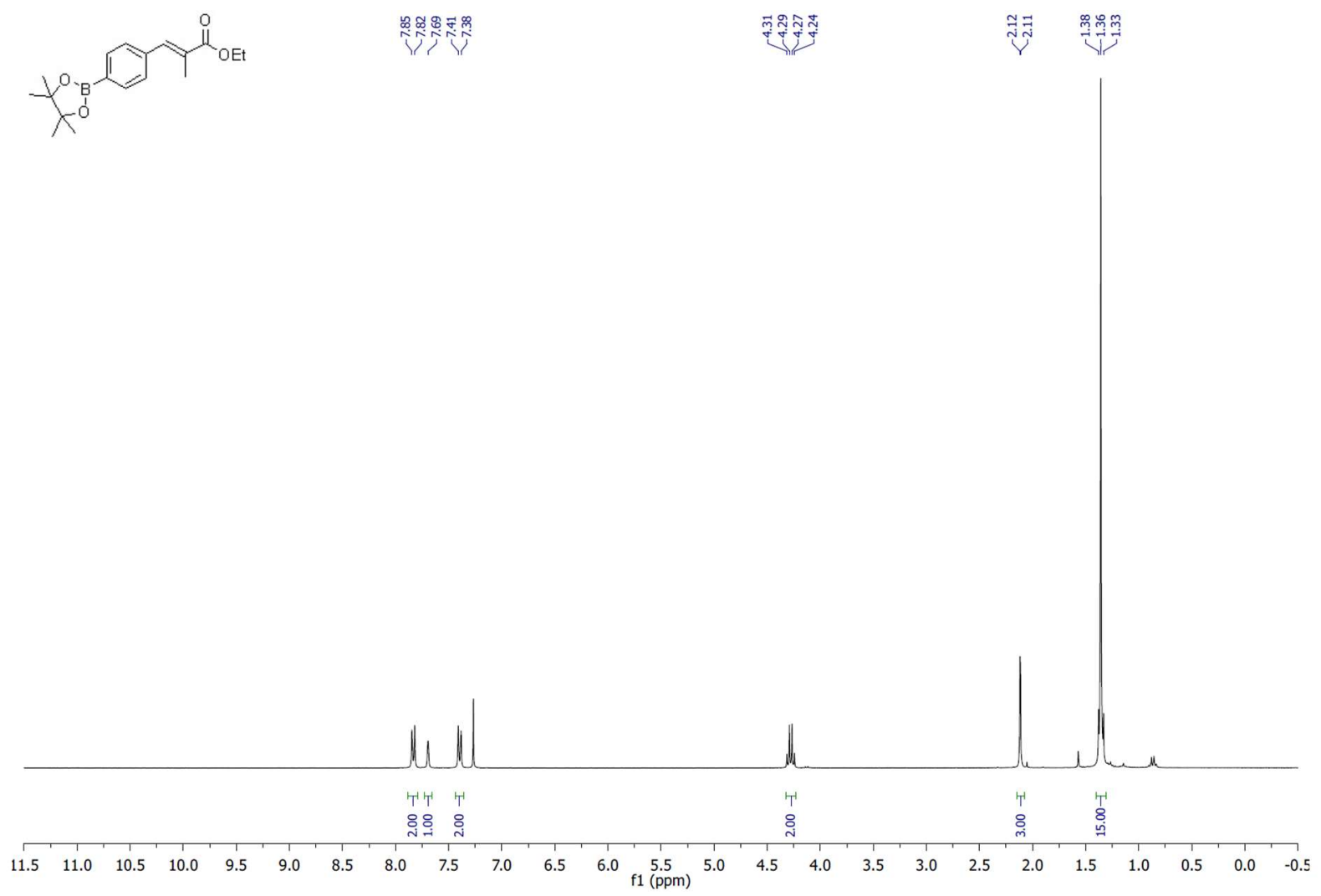
${ }^{13} \mathrm{C} \mathrm{NMR}\left(\mathrm{CDCl}_{3}, 75 \mathrm{MHz}\right)$

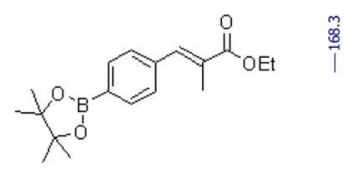

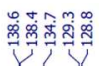
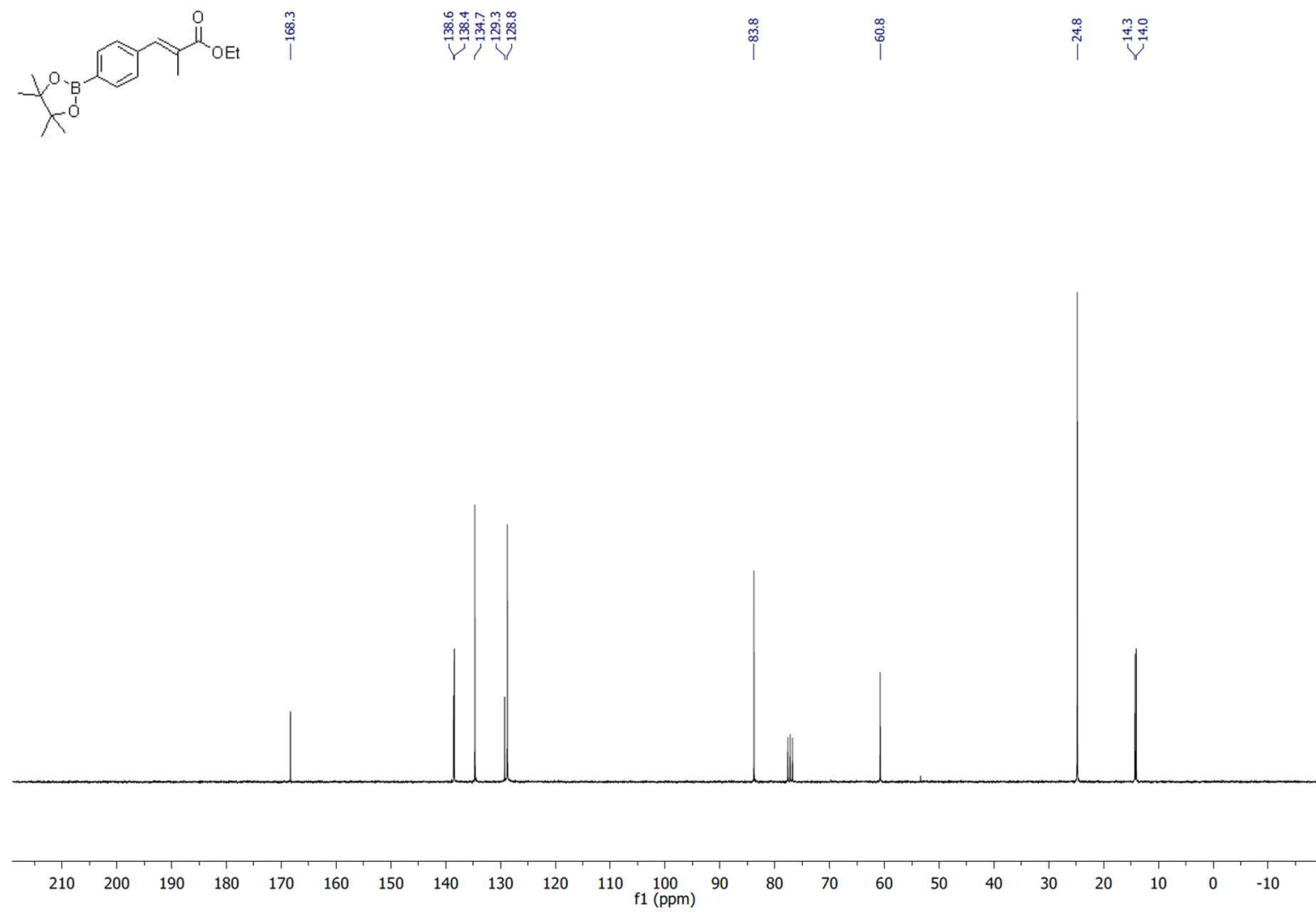

ethyl (E)-2-methyl-3-(4-(methylsulfonyl)phenyl)acrylate (Est-22):

${ }^{1} \mathrm{H} \mathrm{NMR}\left(\mathrm{CDCl}_{3}, 300 \mathrm{MHz}\right)$

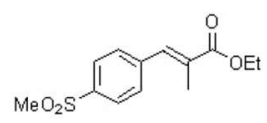

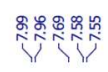

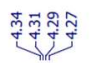

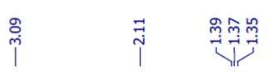

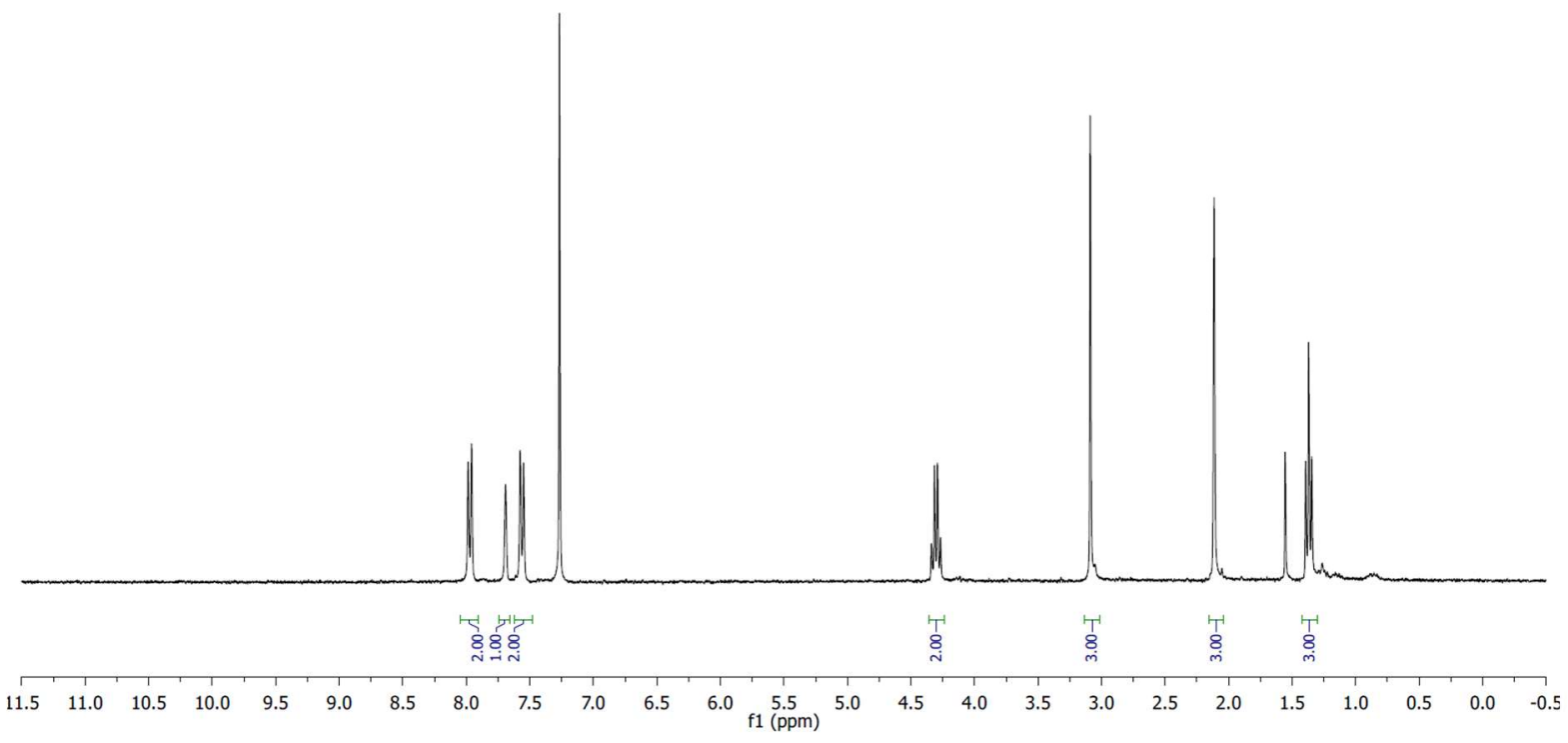

97 
${ }^{13} \mathrm{C} \mathrm{NMR}\left(\mathrm{CDCl}_{3}, 75 \mathrm{MHz}\right)$
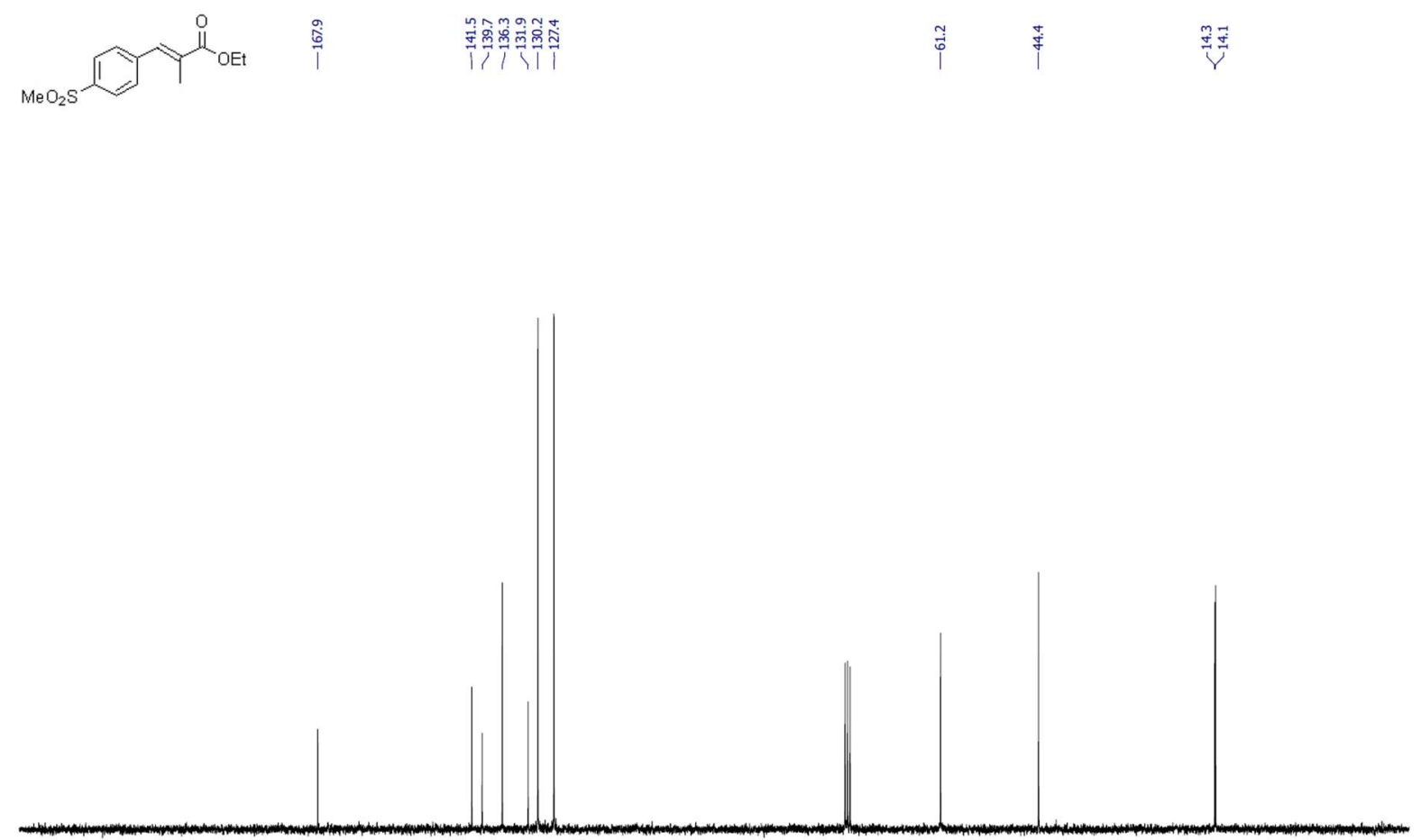

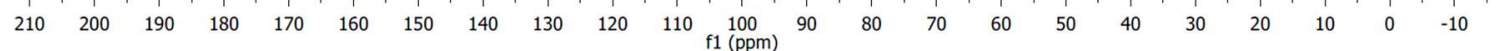

ethyl (E)-3-(4-acetylphenyl)-2-methylacrylate (Est-23):

${ }^{1} \mathrm{H} \mathrm{NMR}\left(\mathrm{CDCl}_{3}, 300 \mathrm{MHz}\right)$
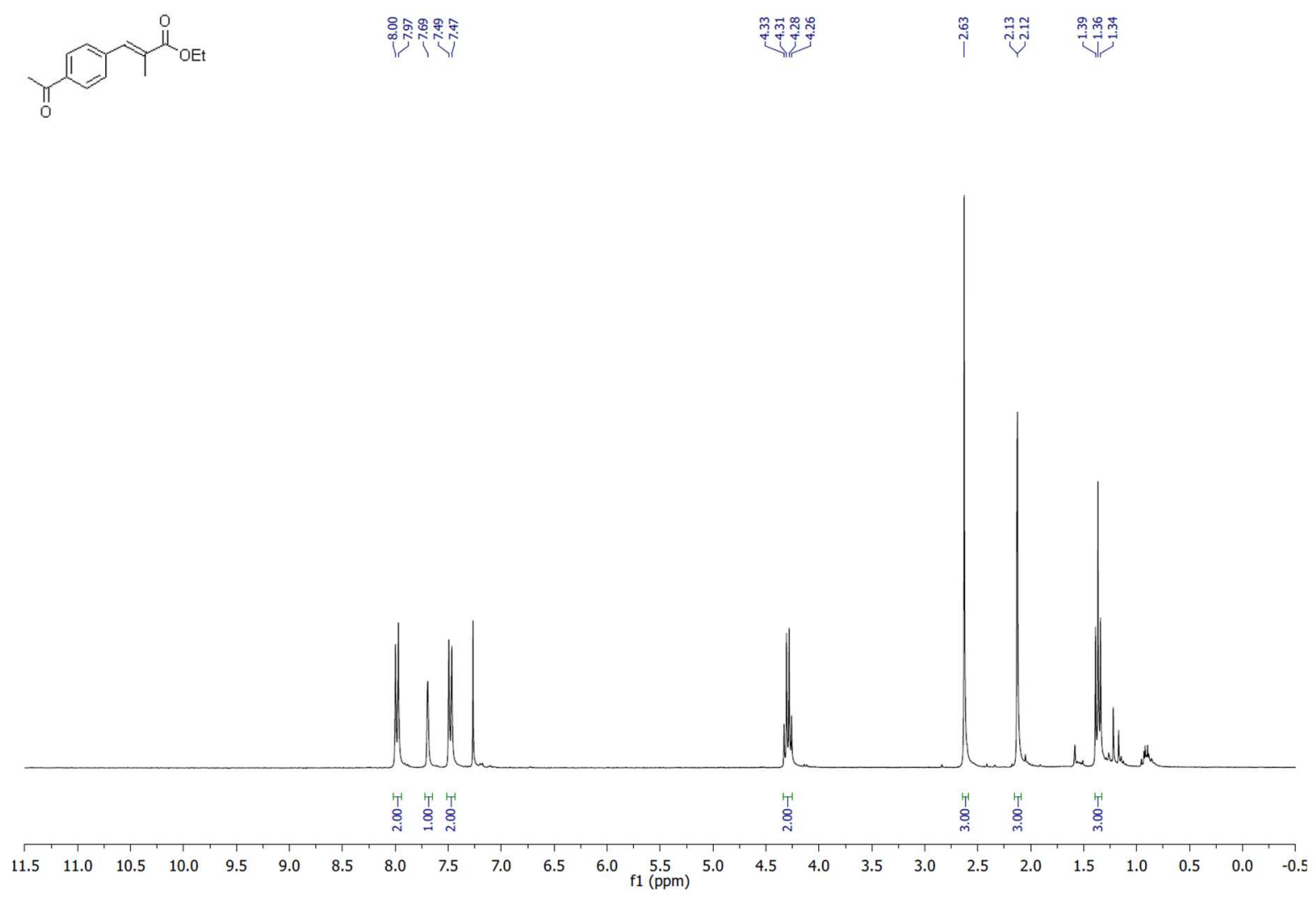
${ }^{13} \mathrm{C} \mathrm{NMR}\left(\mathrm{CDCl}_{3}, 75 \mathrm{MHz}\right)$
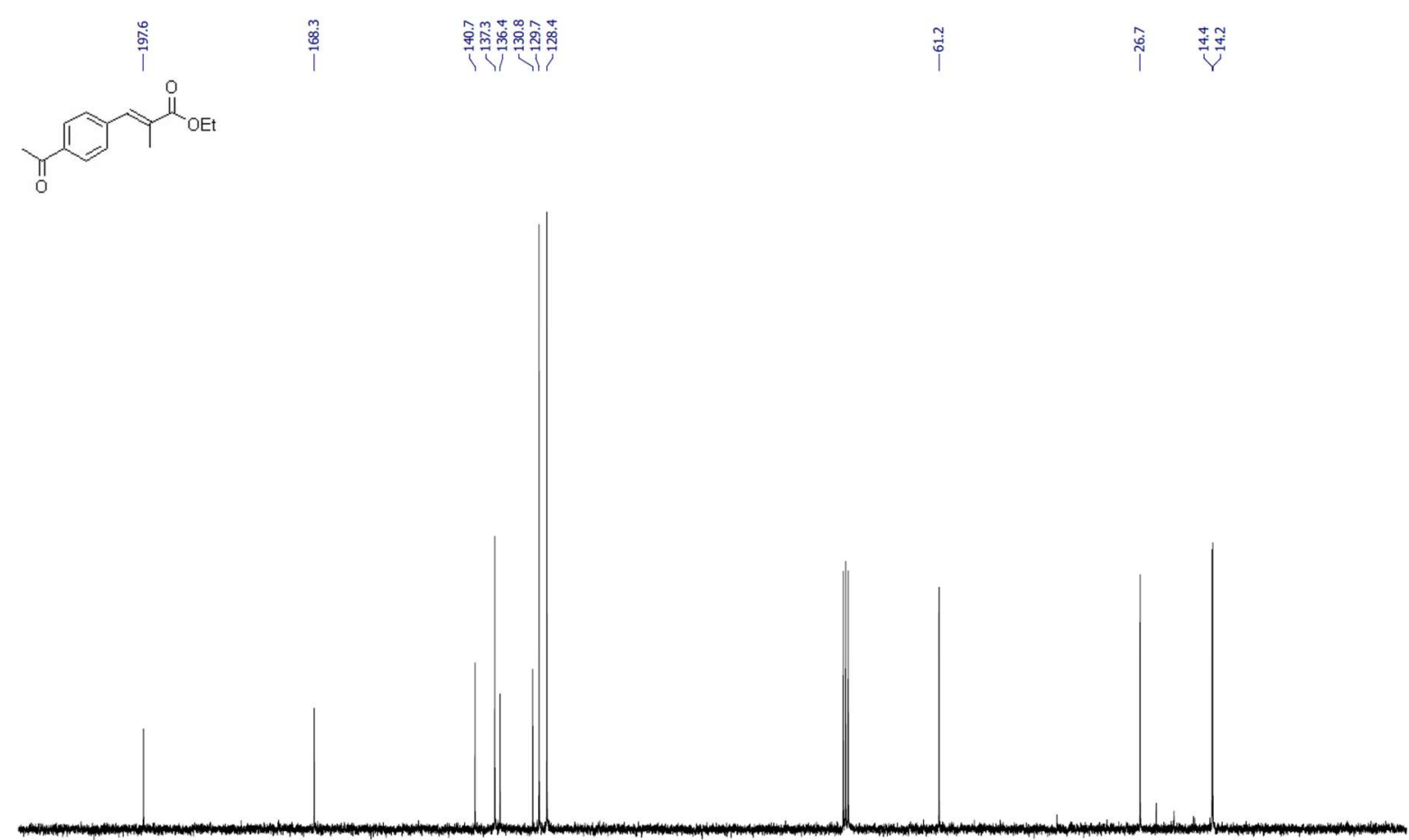

$\begin{array}{lllllllllllllllllllllll}210 & 200 & 190 & 180 & 170 & 160 & 150 & 140 & 130 & 120 & 110 & 100 & 90 & 80 & 70 & 60 & 50 & 40 & 30 & 20 & 10 & 0 & -10\end{array}$

ethyl (E)-3-(furan-2-yl)-2-methylacrylate (Est-24):

${ }^{1} \mathrm{H} \mathrm{NMR}\left(\mathrm{CDCl}_{3}, 300 \mathrm{MHz}\right)$

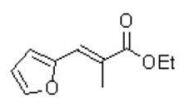

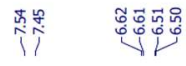

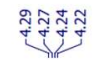

ส

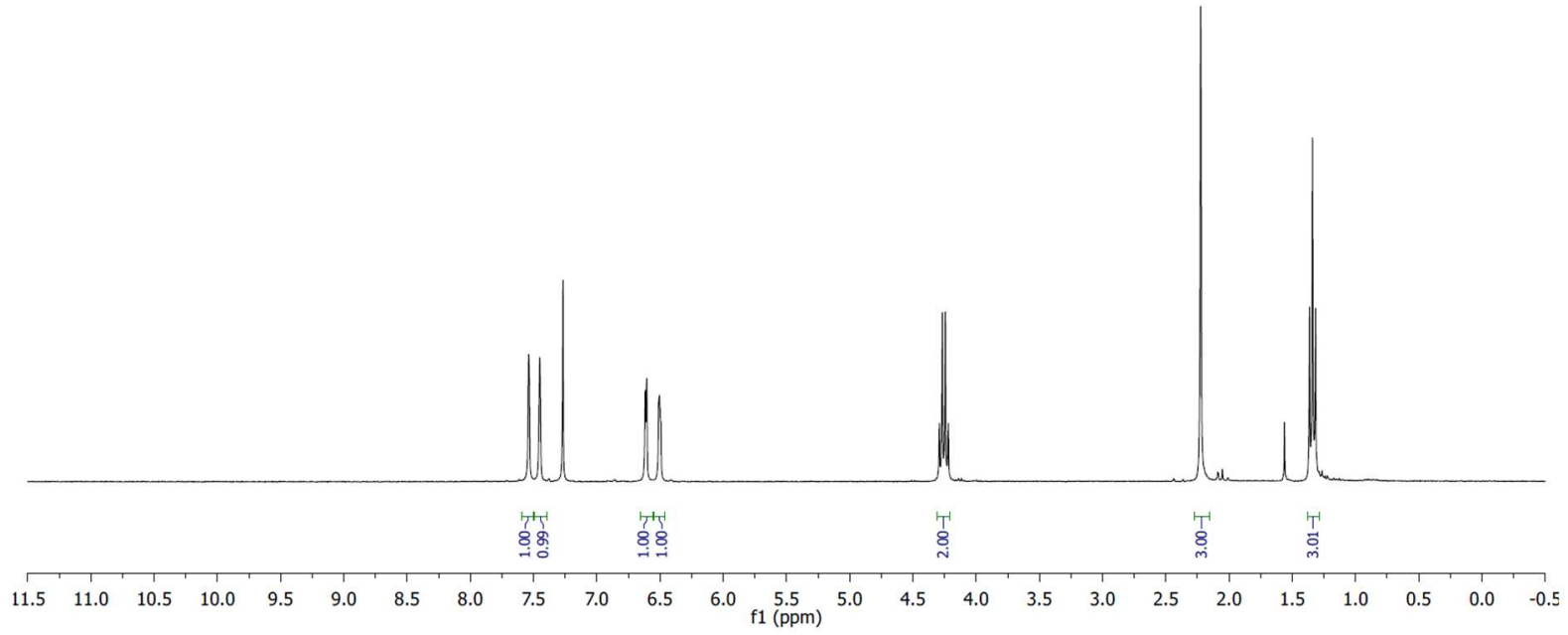


${ }^{13} \mathrm{C} \mathrm{NMR}\left(\mathrm{CDCl}_{3}, 75 \mathrm{MHz}\right)$
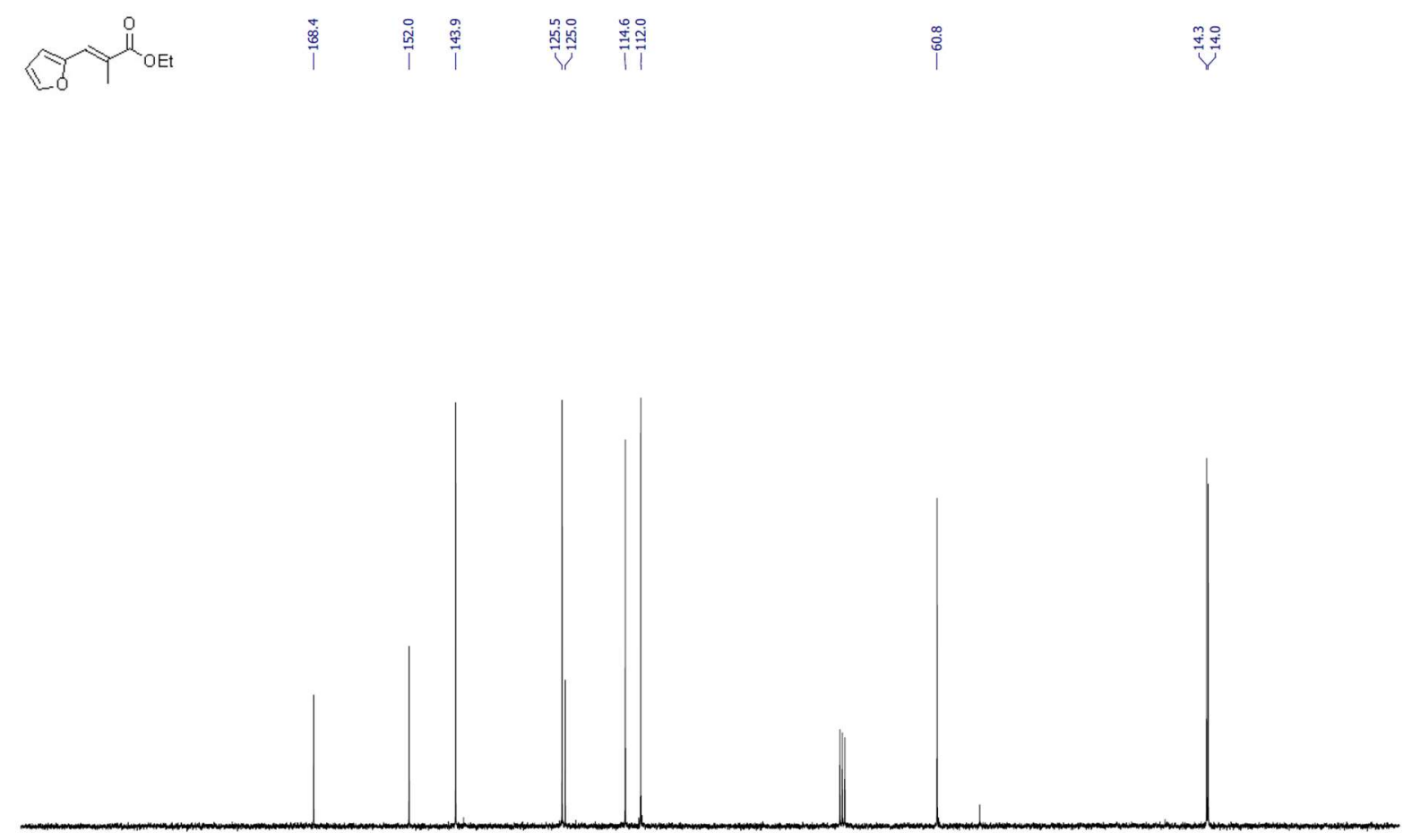

$\begin{array}{lllllllllllllllllllllll}210 & 200 & 190 & 180 & 170 & 160 & 150 & 140 & 130 & 120 & 110 & \begin{array}{c}100 \\ \mathrm{f} 1(\mathrm{ppm})\end{array} & 90 & 80 & 70 & 60 & 50 & 40 & 30 & 20 & 10 & 0 & -10\end{array}$ ethyl (E)-2-methyl-3-(thiophen-2-yl)acrylate (Est-25):

${ }^{1} \mathrm{H} \mathrm{NMR}\left(\mathrm{CDCl}_{3}, 300 \mathrm{MHz}\right)$

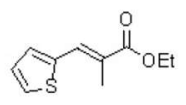

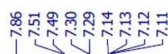

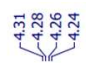

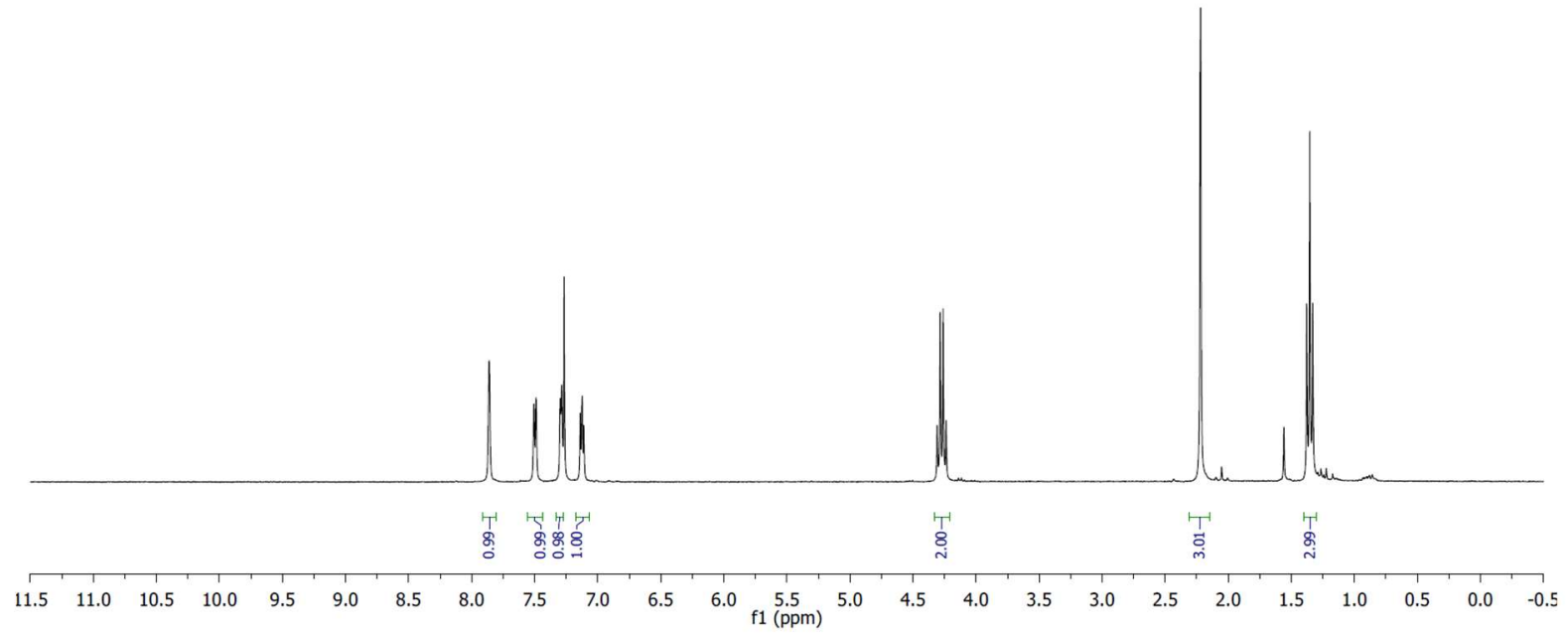


${ }^{13} \mathrm{C} \mathrm{NMR}\left(\mathrm{CDCl}_{3}, 75 \mathrm{MHz}\right)$
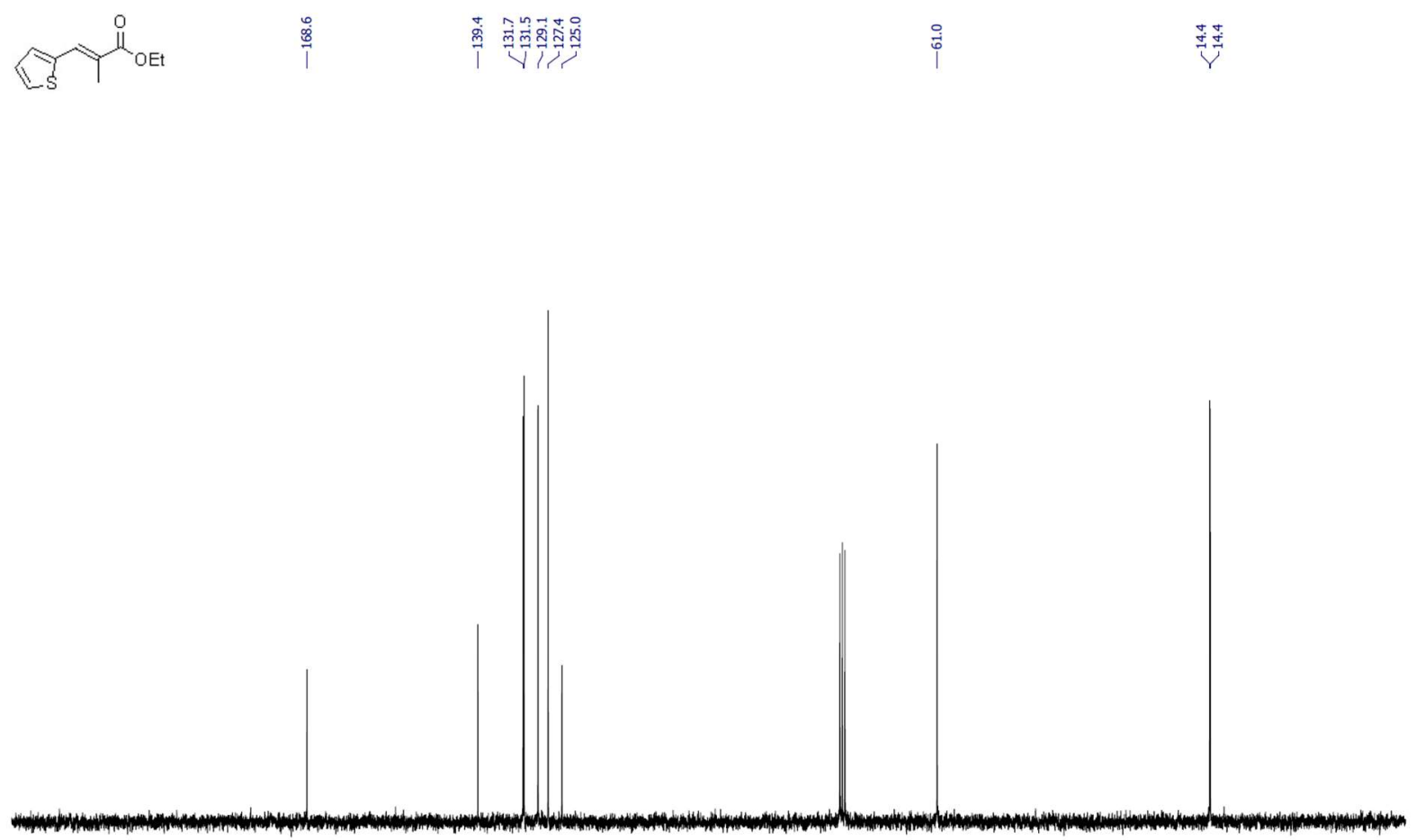

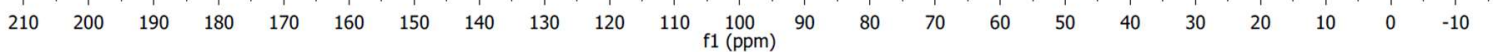

ethyl (E)-3-(4-hydroxyphenyl)-2-methylacrylate (B):

${ }^{1} \mathrm{H} \mathrm{NMR}\left(\mathrm{CDCl}_{3}, 300 \mathrm{MHz}\right)$

Ho

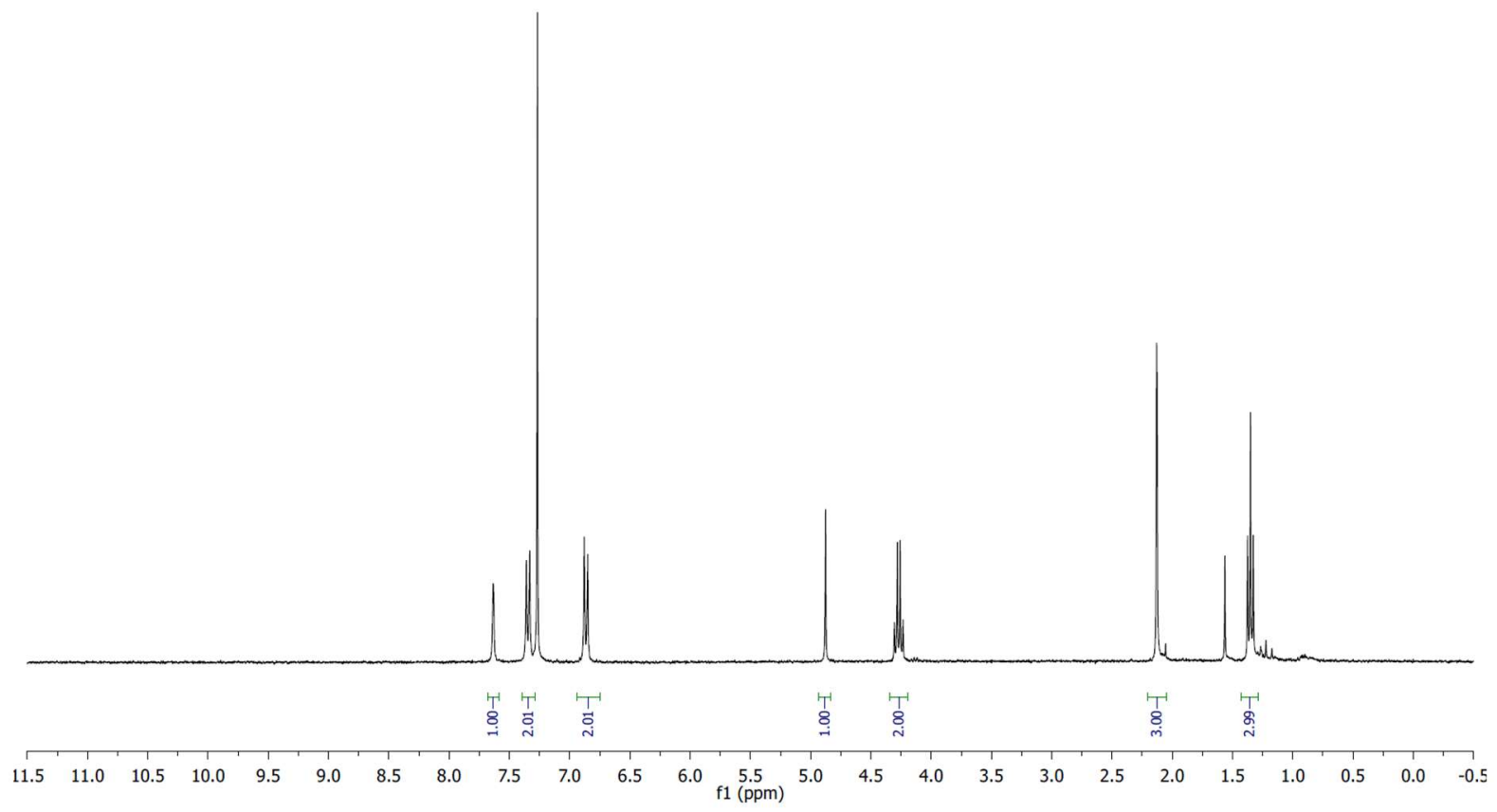


${ }^{13} \mathrm{C} \mathrm{NMR}\left(\mathrm{CDCl}_{3}, 75 \mathrm{MHz}\right)$

HO

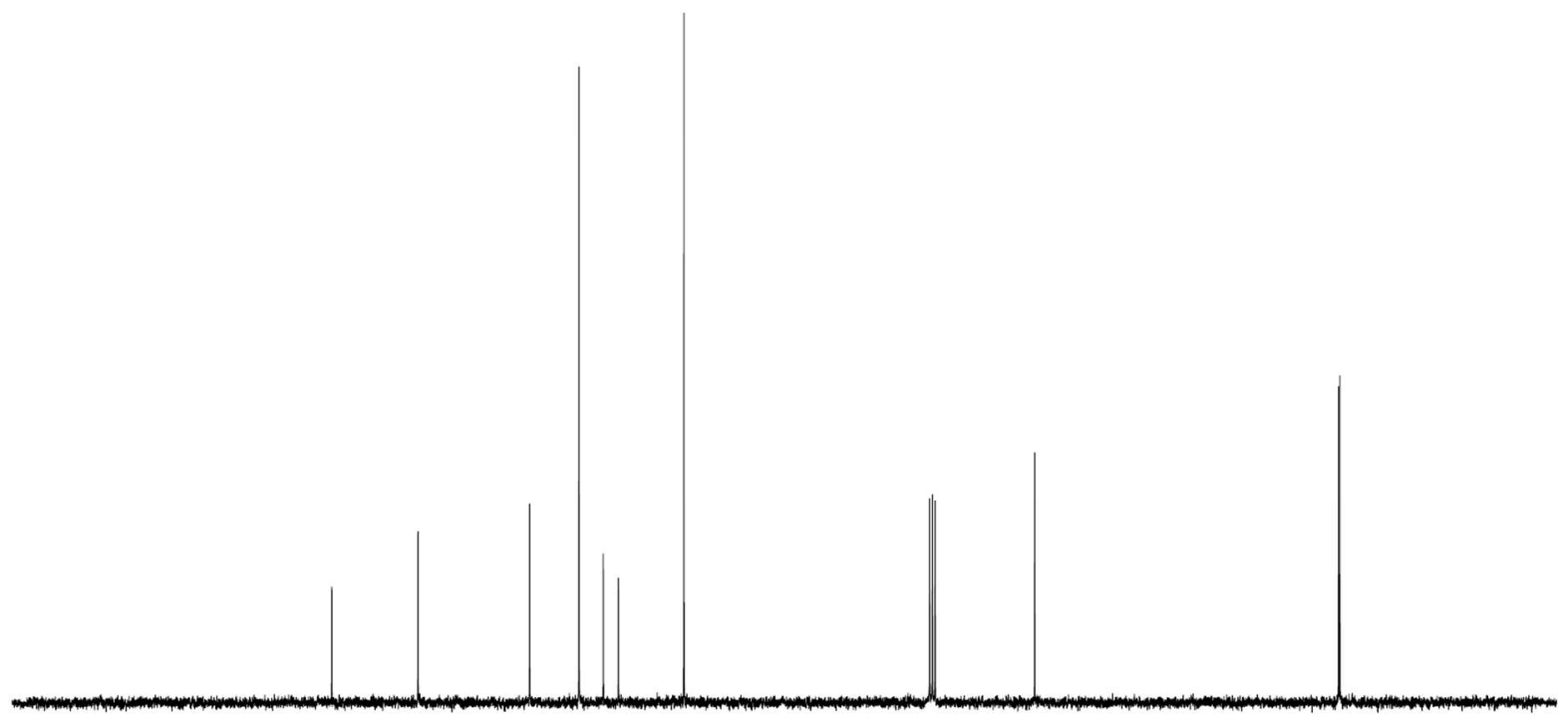

$\begin{array}{lllllllllllllllllllll}210 & 200 & 190 & 180 & 170 & 160 & 150 & 140 & 130 & 120 & 110 & \underset{\mathrm{f} 1}{100}(\mathrm{ppm}) \\ \mathbf{9 0} & 80 & 70 & 60 & 50 & 40 & 30 & 20 & 10 & 0 & -10\end{array}$

ethyl (E)-3-(4-(cinnamyloxy)phenyl)-2-methylacrylate (Est-26):

${ }^{1} \mathrm{H} \mathrm{NMR}\left(\mathrm{CDCl}_{3}, 300 \mathrm{MHz}\right)$
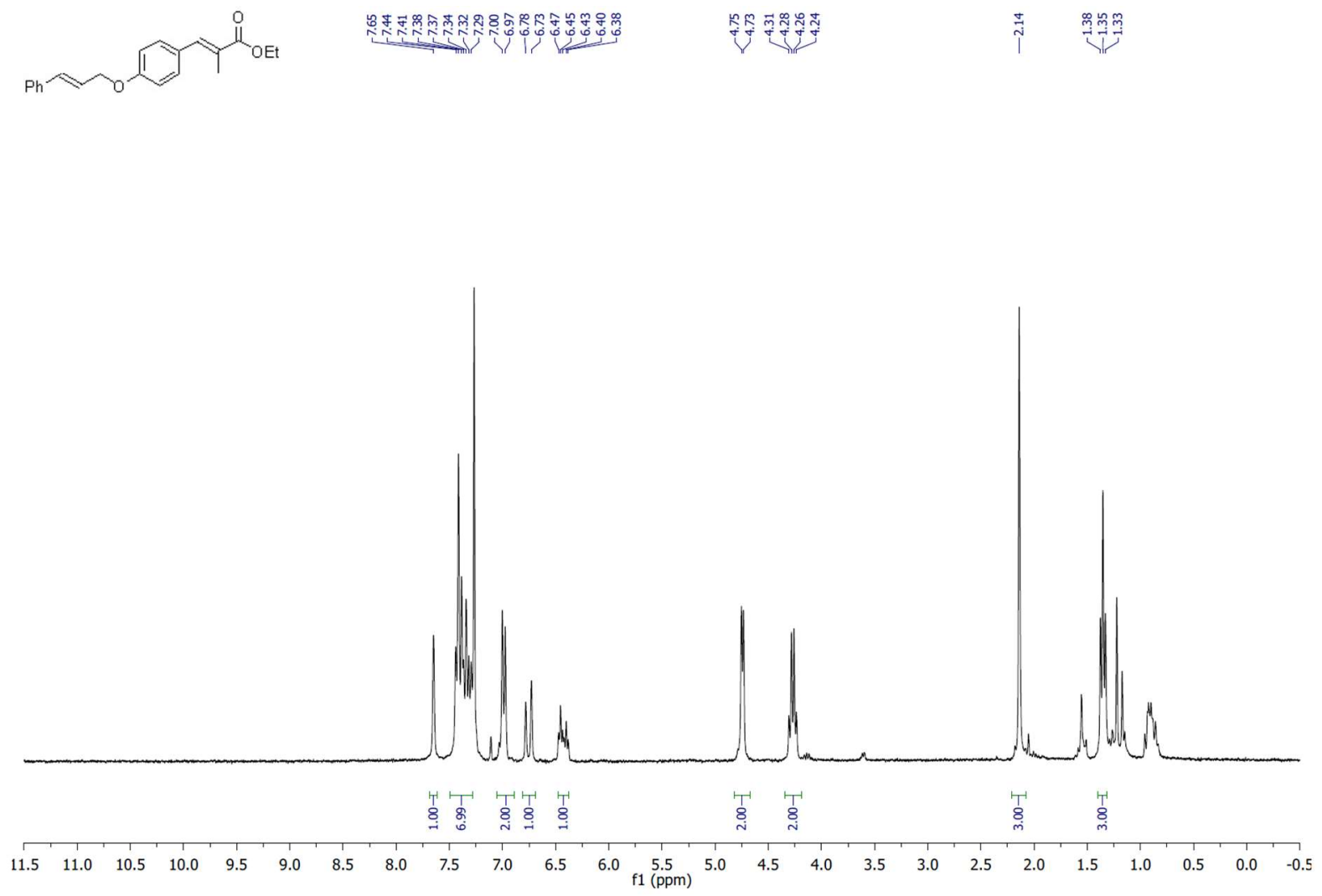
${ }^{13} \mathrm{C} \mathrm{NMR}\left(\mathrm{CDCl}_{3}, 75 \mathrm{MHz}\right)$
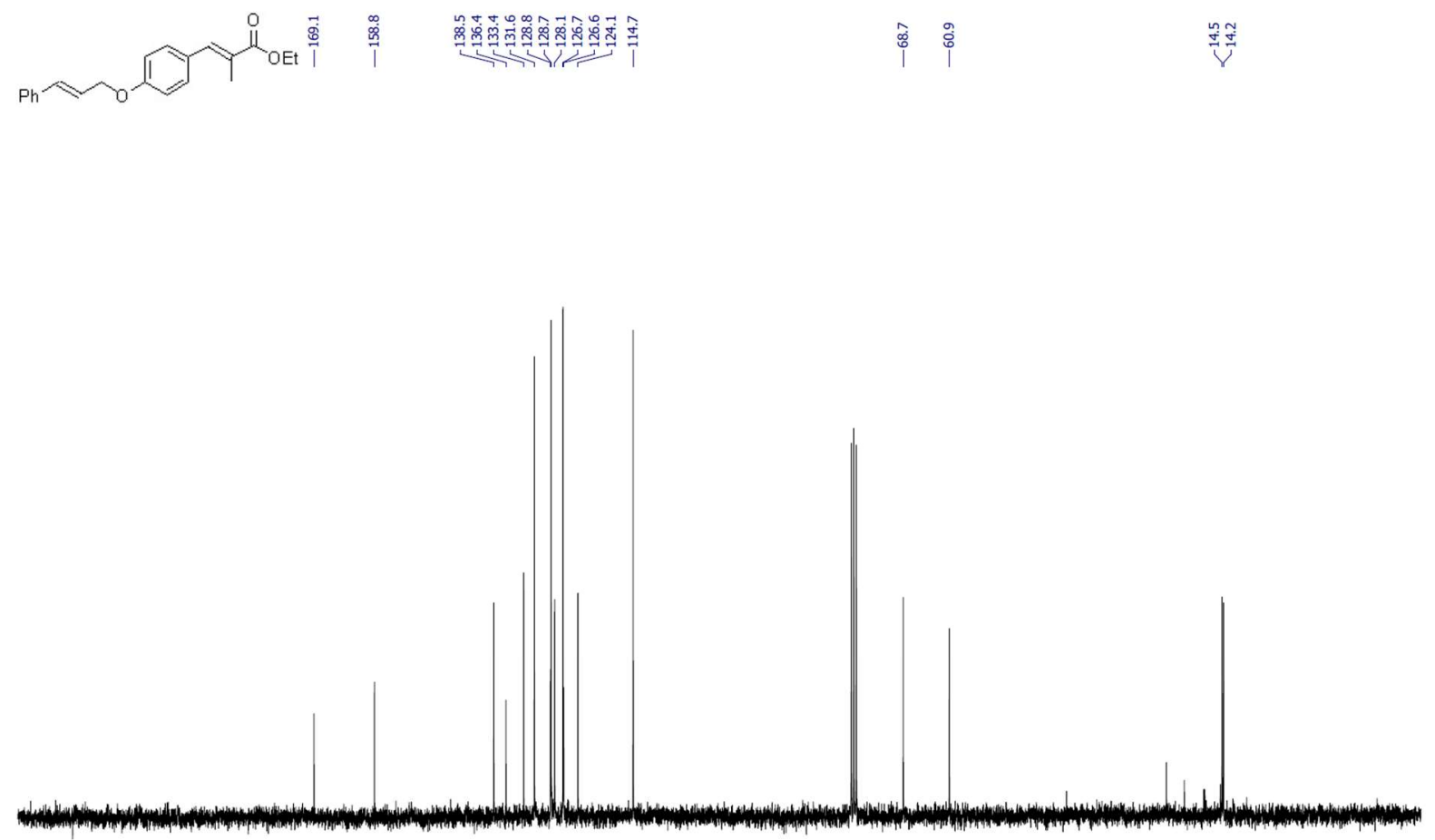

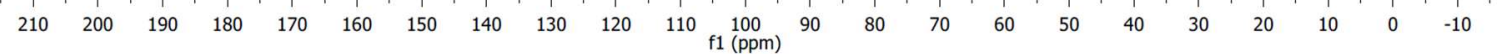

ethyl (E)-3-mesityl-2-methylacrylate (Est-27):

${ }^{1} \mathrm{H} \mathrm{NMR}\left(\mathrm{CDCl}_{3}, 300 \mathrm{MHz}\right)$

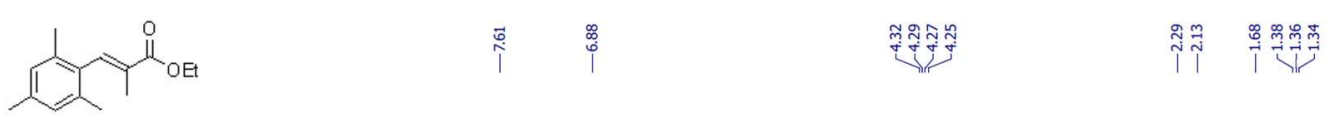

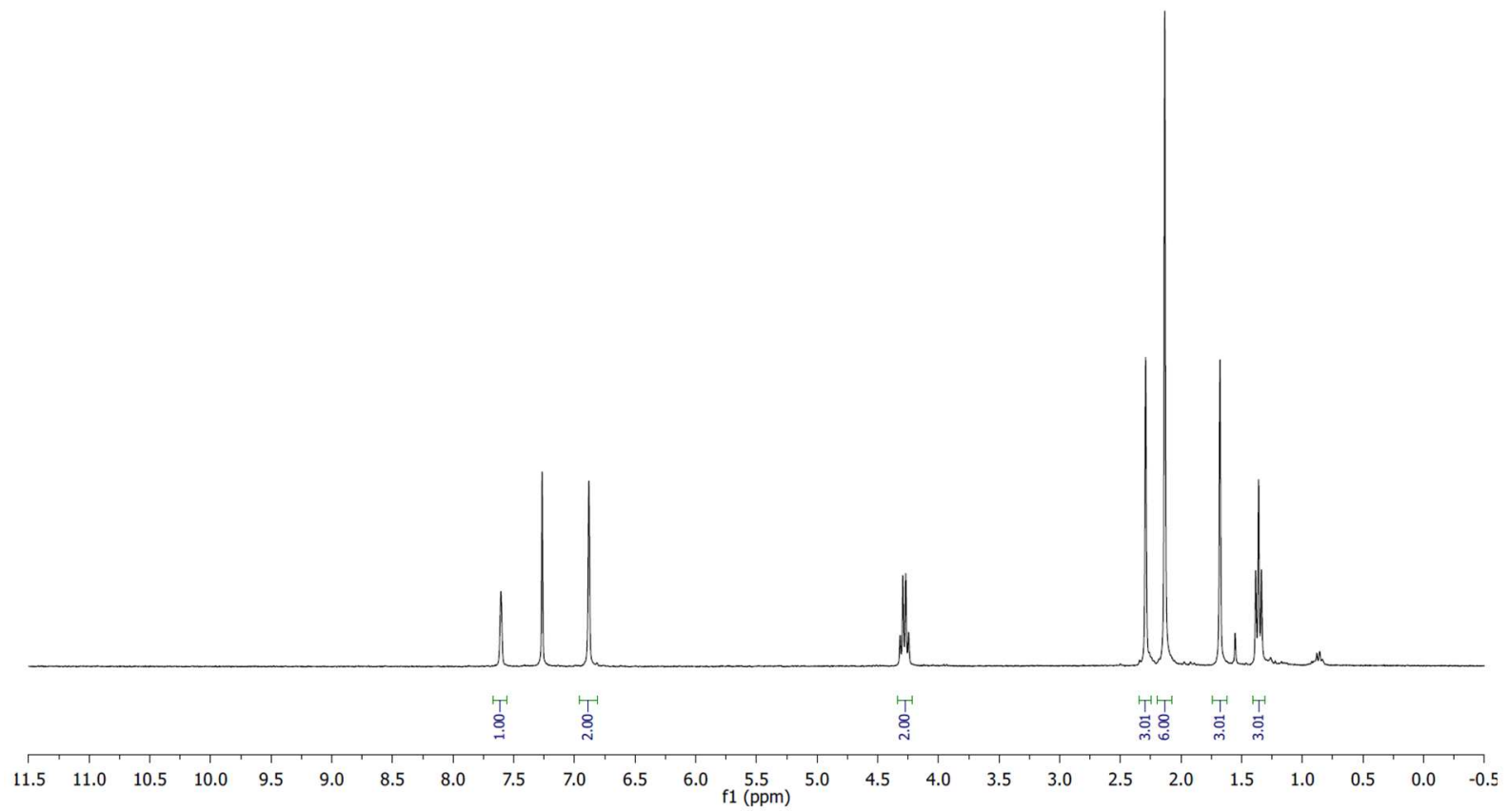


${ }^{13} \mathrm{C} \mathrm{NMR}\left(\mathrm{CDCl}_{3}, 75 \mathrm{MHz}\right)$
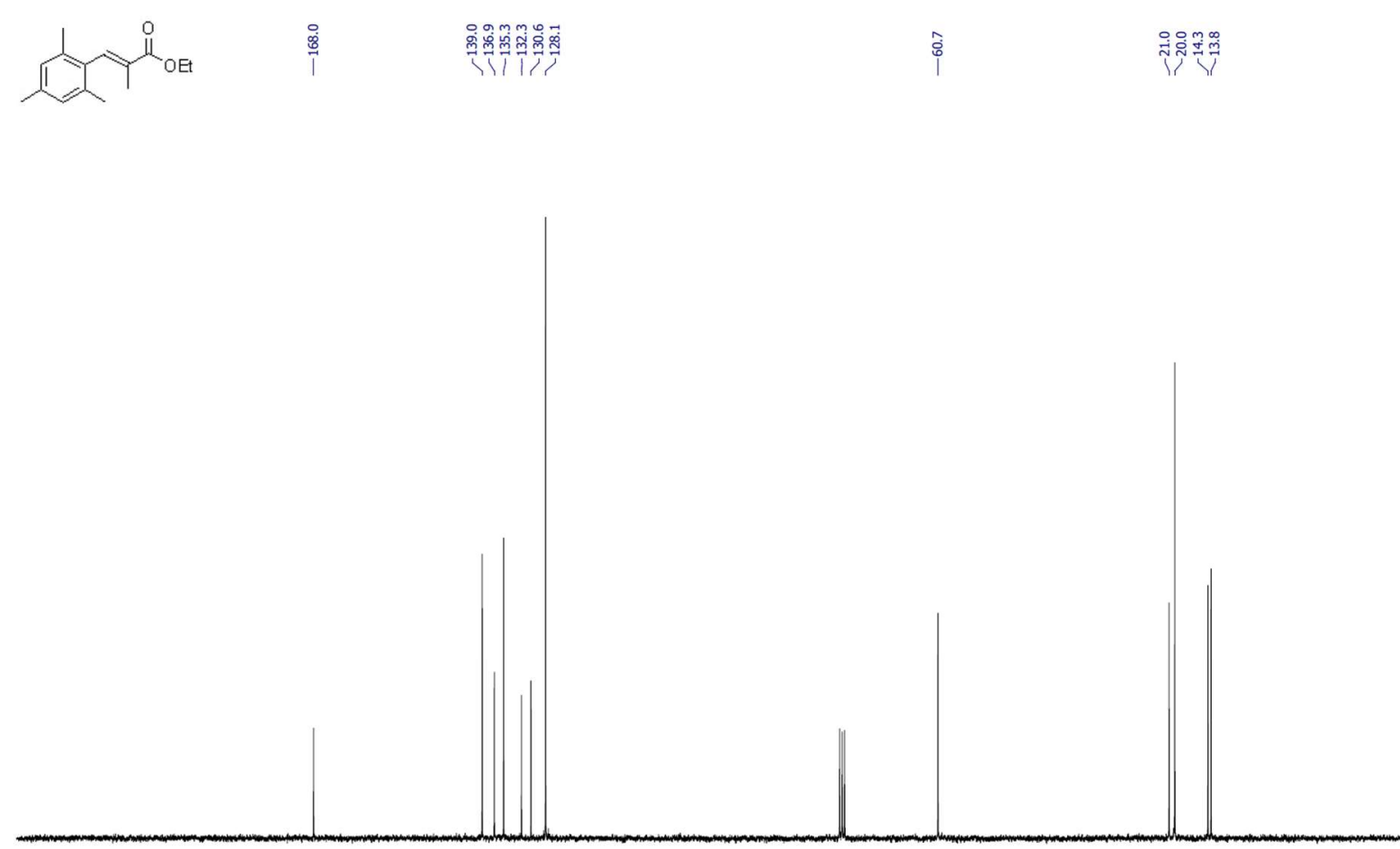

$\begin{array}{lllllllllllllllllllllll}210 & 200 & 190 & 180 & 170 & 160 & 150 & 140 & 130 & 120 & 110 & \underset{\mathrm{f} 1(\mathrm{ppm})}{100} & 90 & 80 & 70 & 60 & 50 & 40 & 30 & 20 & 10 & 0 & -10\end{array}$

ethyl (E)-2-methyloct-2-enoate (Est-28):

${ }^{1} \mathrm{H} \mathrm{NMR}\left(\mathrm{CDCl}_{3}, 300 \mathrm{MHz}\right)$
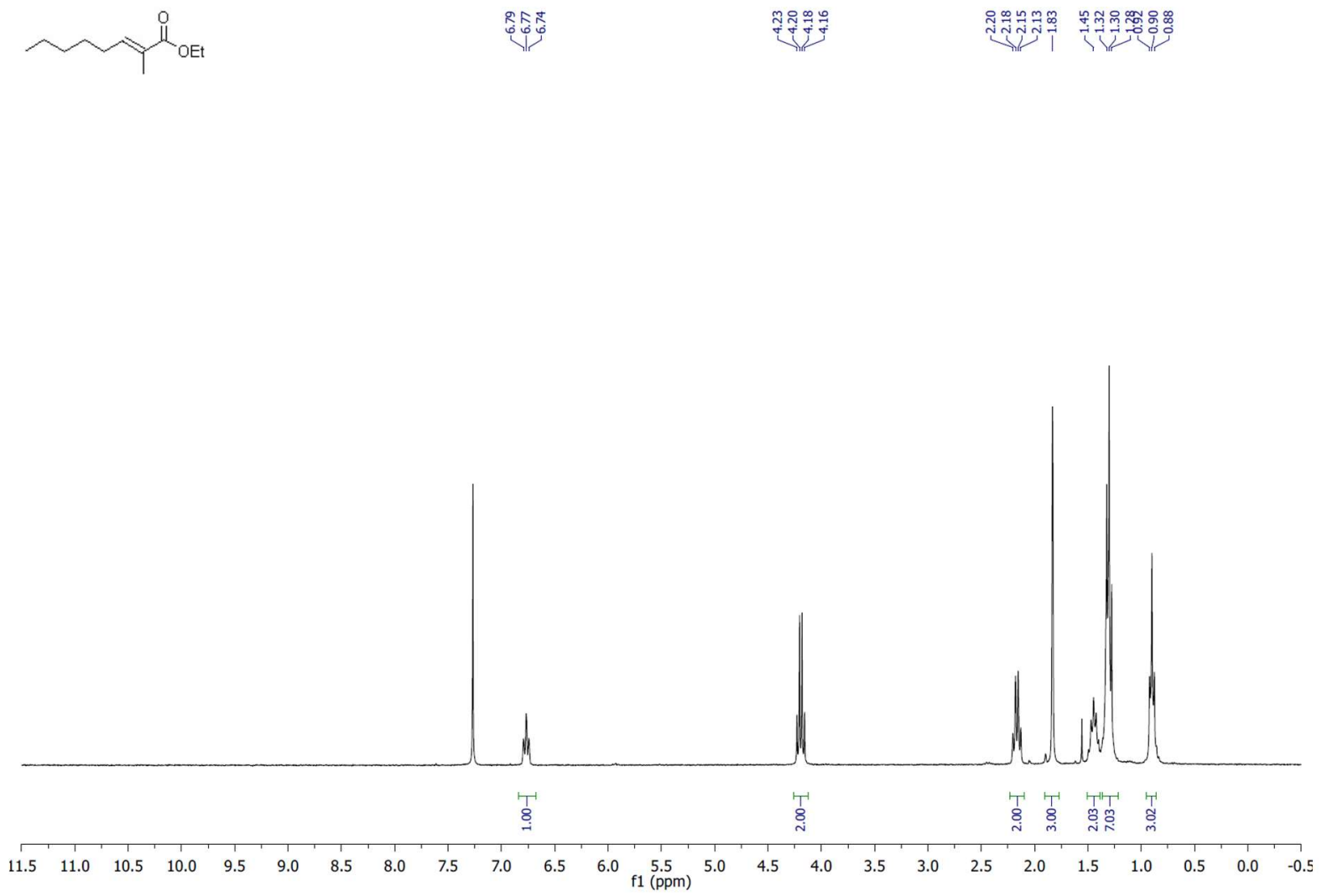
${ }^{13} \mathrm{C} \mathrm{NMR}\left(\mathrm{CDCl}_{3}, 75 \mathrm{MHz}\right)$
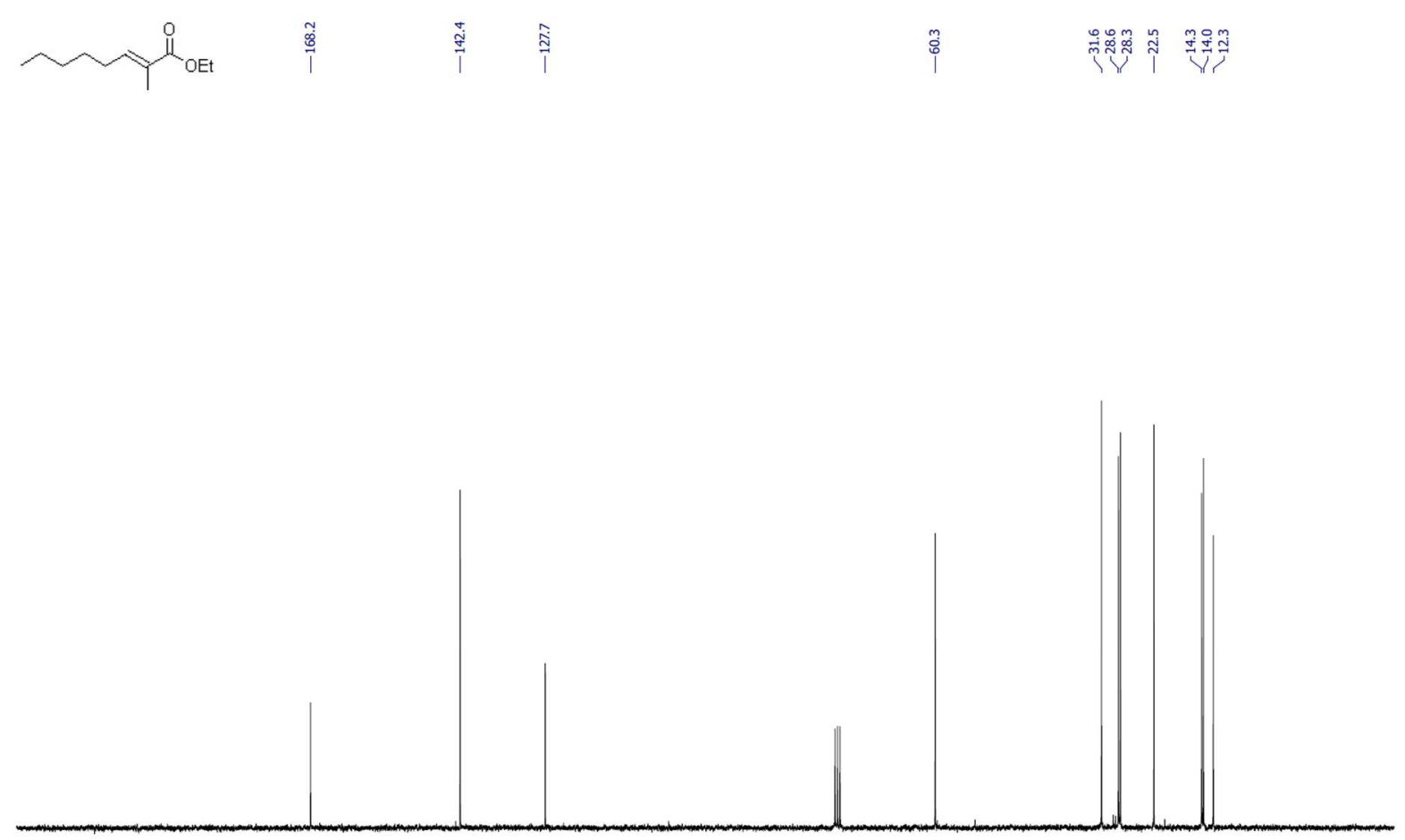

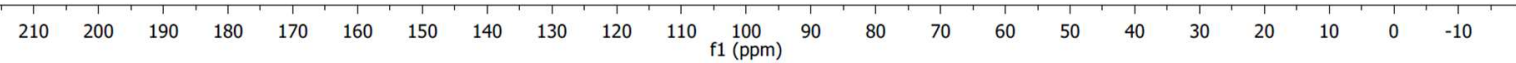

(E)-2-methyl-3-(p-tolyl)acrylic acid (Ac-9):

${ }^{1} \mathrm{H} \mathrm{NMR}\left(\mathrm{CDCl}_{3}, 300 \mathrm{MHz}\right)$
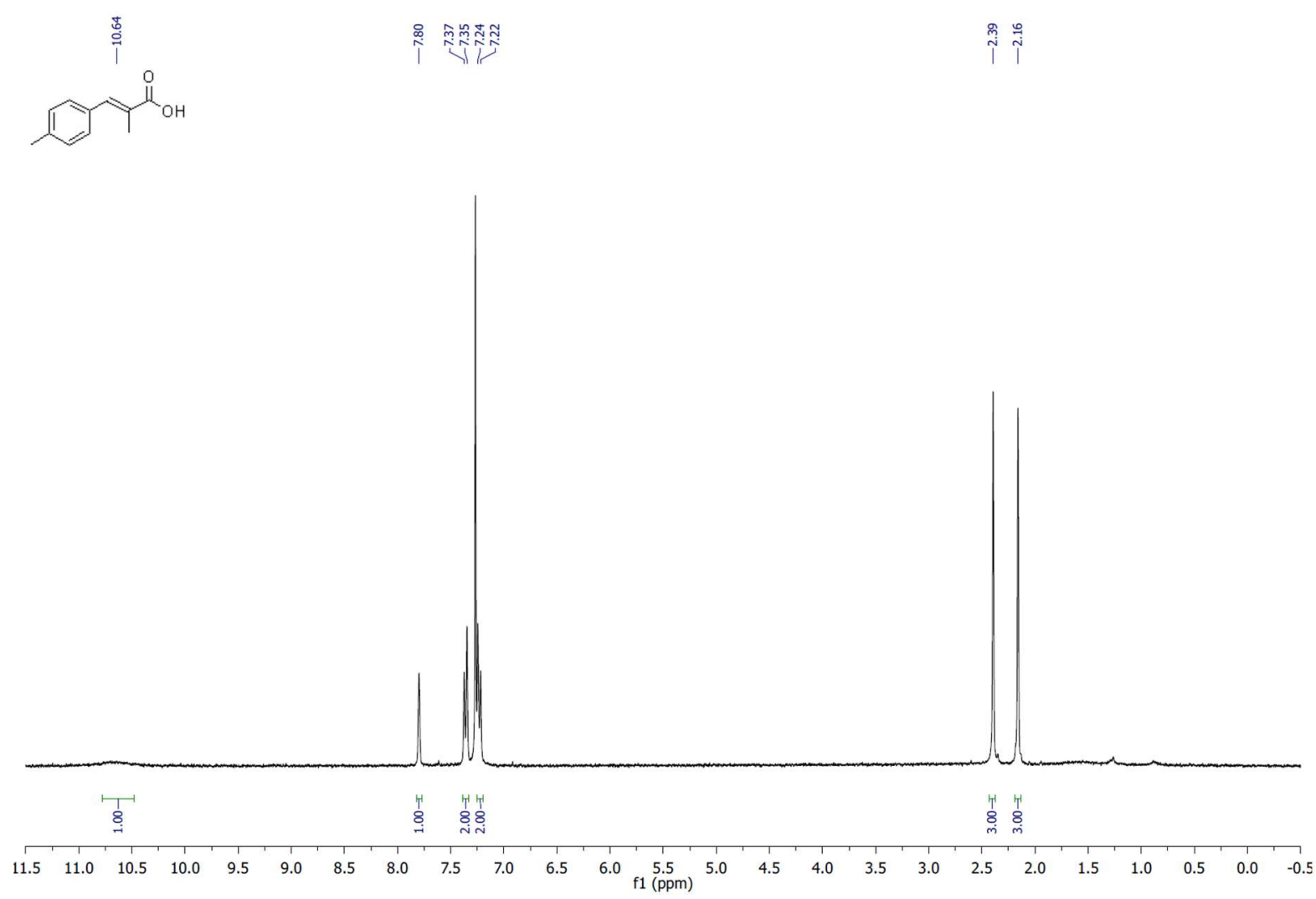
${ }^{13} \mathrm{C}$ NMR (DMSO-d6, $75 \mathrm{MHz}$ )

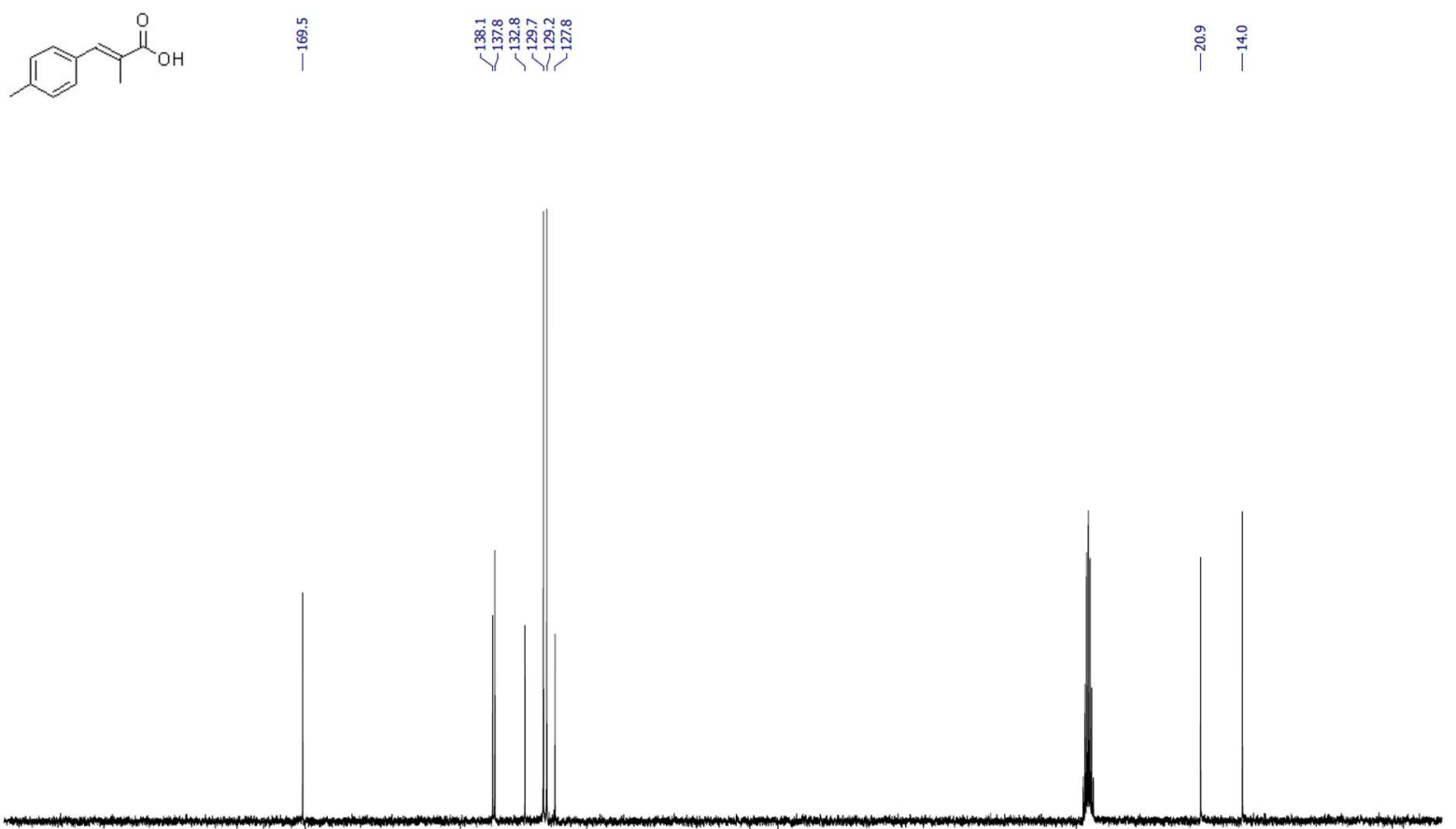

$\begin{array}{lllllllllllllllllllllll}210 & 200 & 190 & 180 & 170 & 160 & 150 & 140 & 130 & 120 & 110 & 100 & 90 & 80 & 70 & 60 & 50 & 40 & 30 & 20 & 10 & 0 & -10\end{array}$

(E)-2-methyl-3-(m-tolyl)acrylic acid (Ac-10):

${ }^{1} \mathrm{H} \mathrm{NMR}\left(\mathrm{CDCl}_{3}, 300 \mathrm{MHz}\right)$
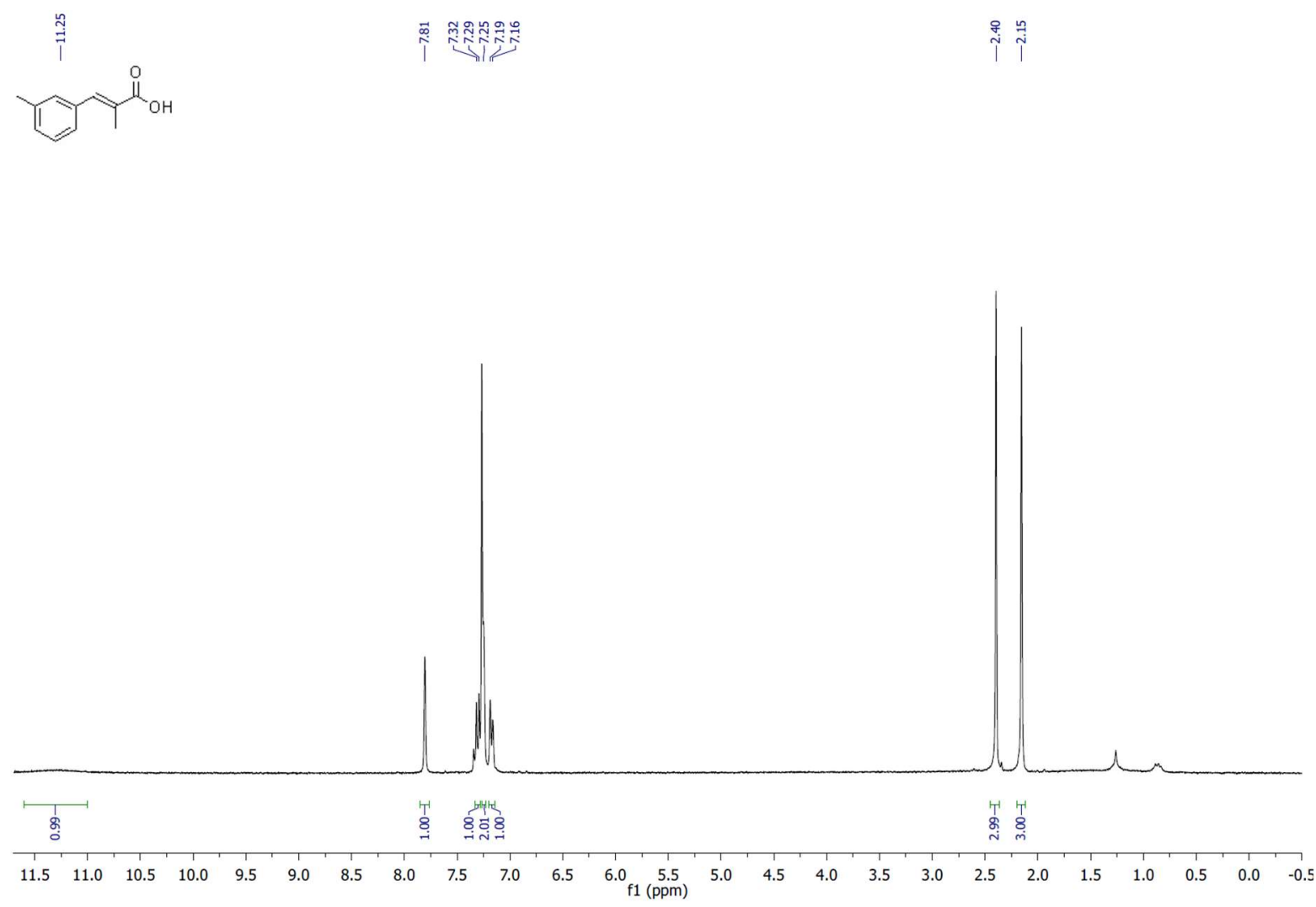
${ }^{13} \mathrm{C}$ NMR (DMSO-d6, $75 \mathrm{MHz}$ )

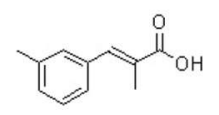

量

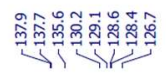

웜암
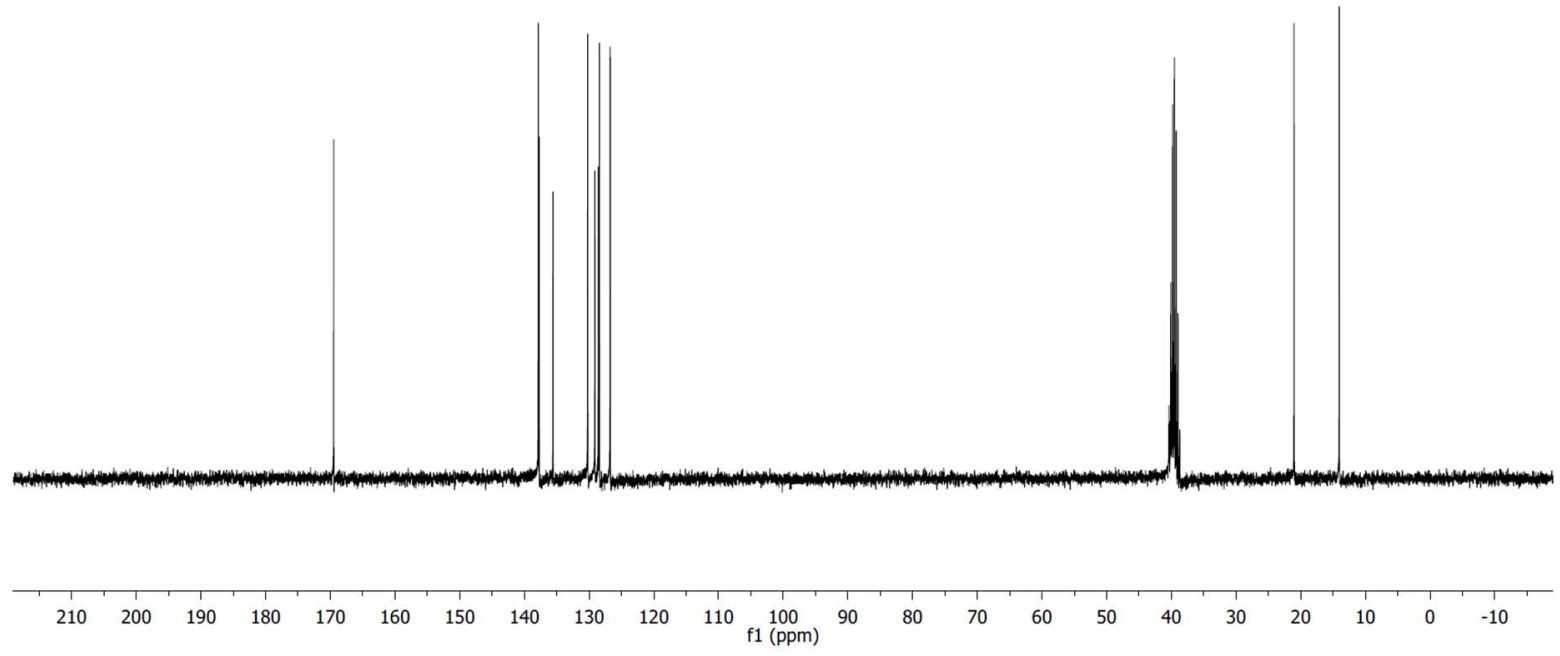

(E)-2-methyl-3-(o-tolyl)acrylic acid (Ac-11):

${ }^{1} \mathrm{H} \mathrm{NMR}\left(\mathrm{CDCl}_{3}, 300 \mathrm{MHz}\right)$
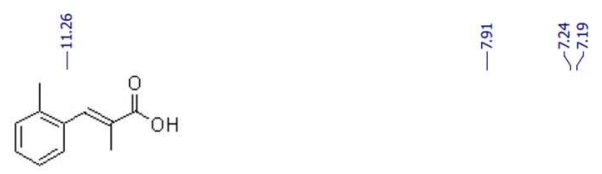

$\stackrel{\overrightarrow{7}}{i} \stackrel{8}{i}$

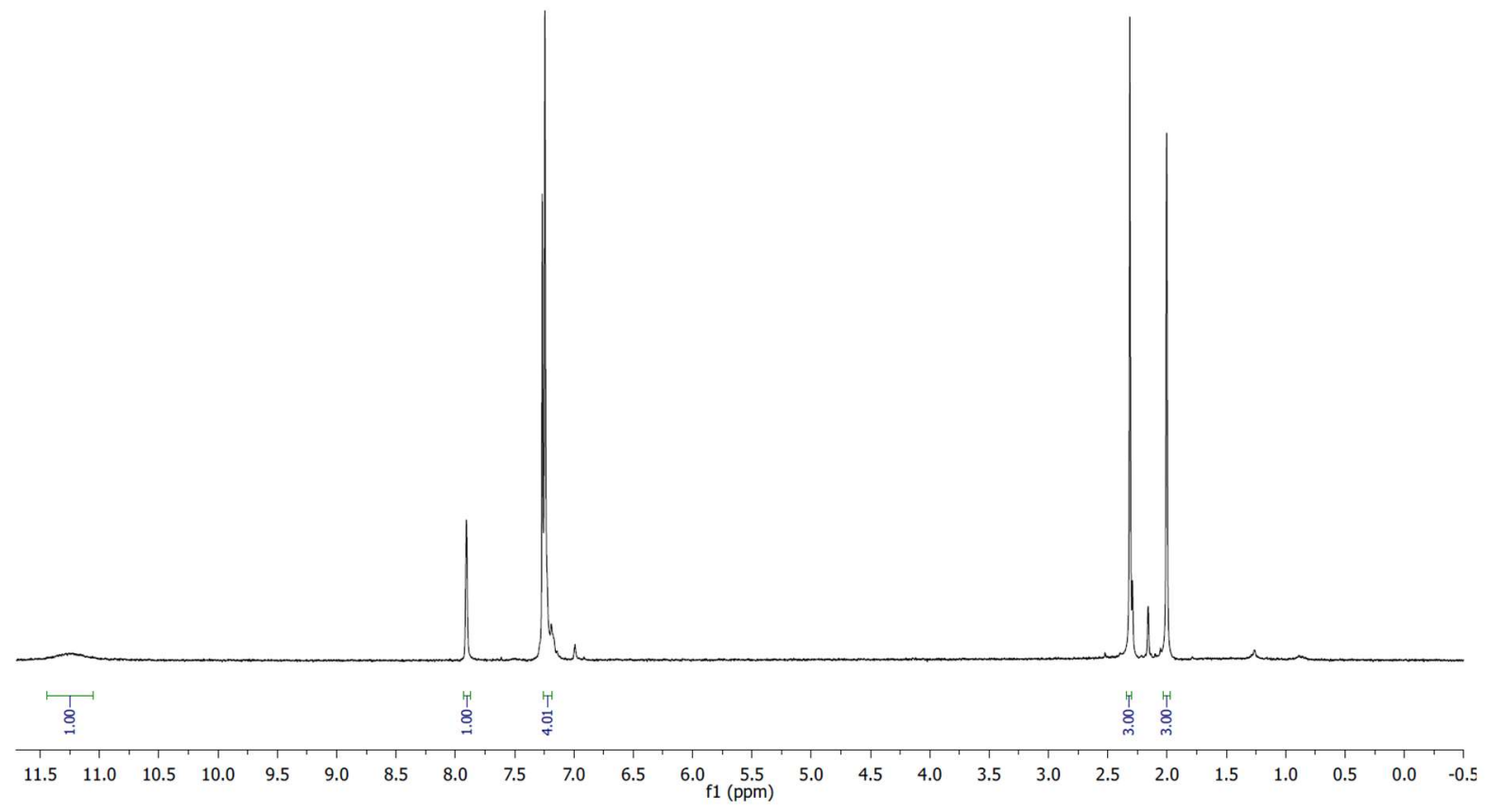

107 
${ }^{13} \mathrm{C}$ NMR (DMSO-d6, $75 \mathrm{MHz}$ )
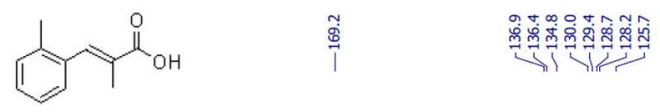

$\stackrel{n}{\stackrel{n}{1} \stackrel{9}{9}}$
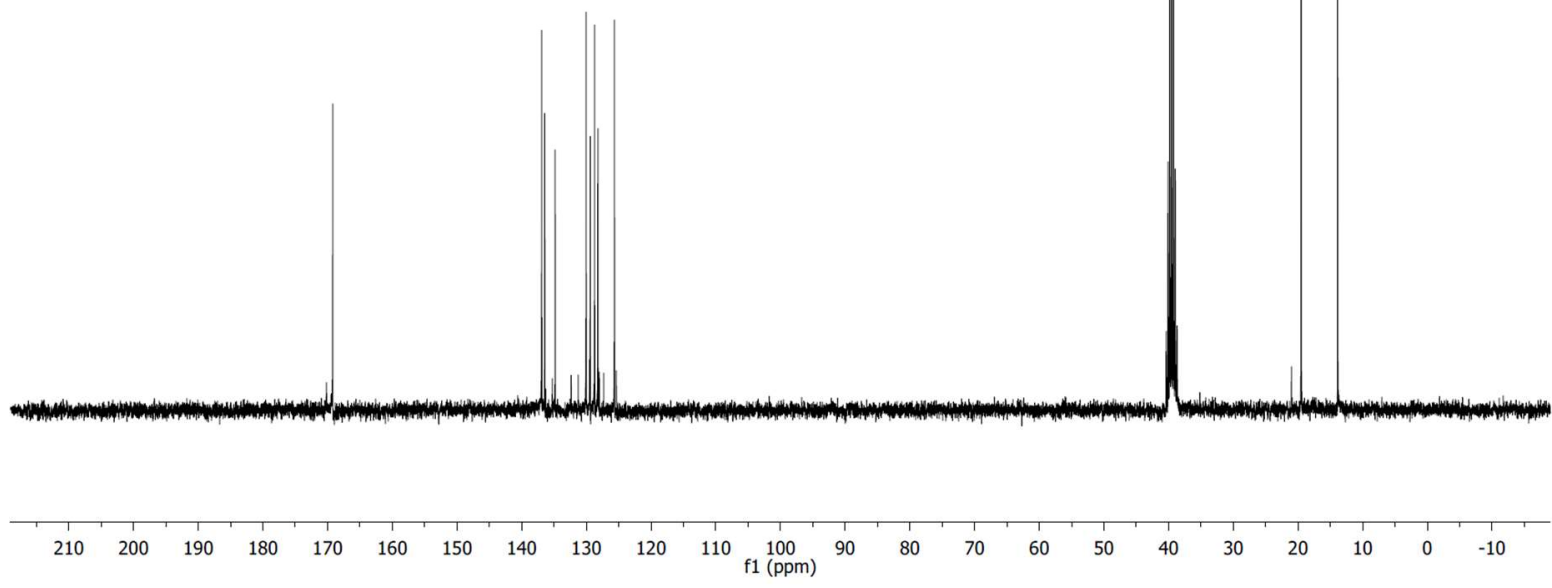

(E)-3-(4-(tert-butyl)phenyl)-2-methylacrylic acid (Ac-12):

${ }^{1} \mathrm{H} \mathrm{NMR}\left(\mathrm{CDCl}_{3}, 300 \mathrm{MHz}\right)$

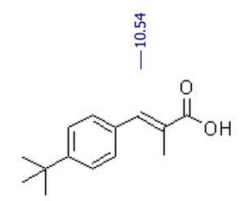

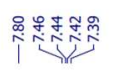
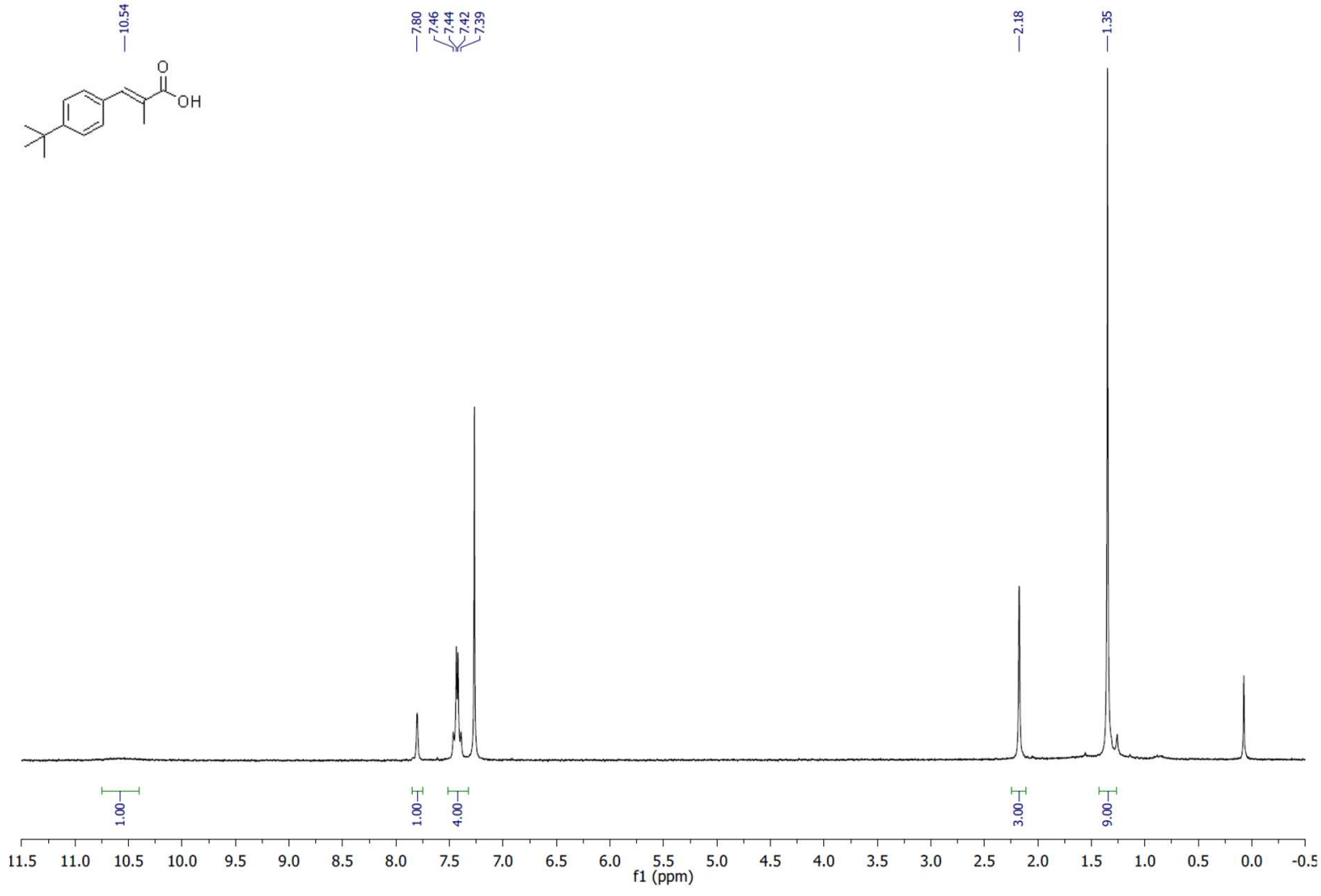

108 
${ }^{13} \mathrm{C}$ NMR (DMSO-d6, $75 \mathrm{MHz}$ )
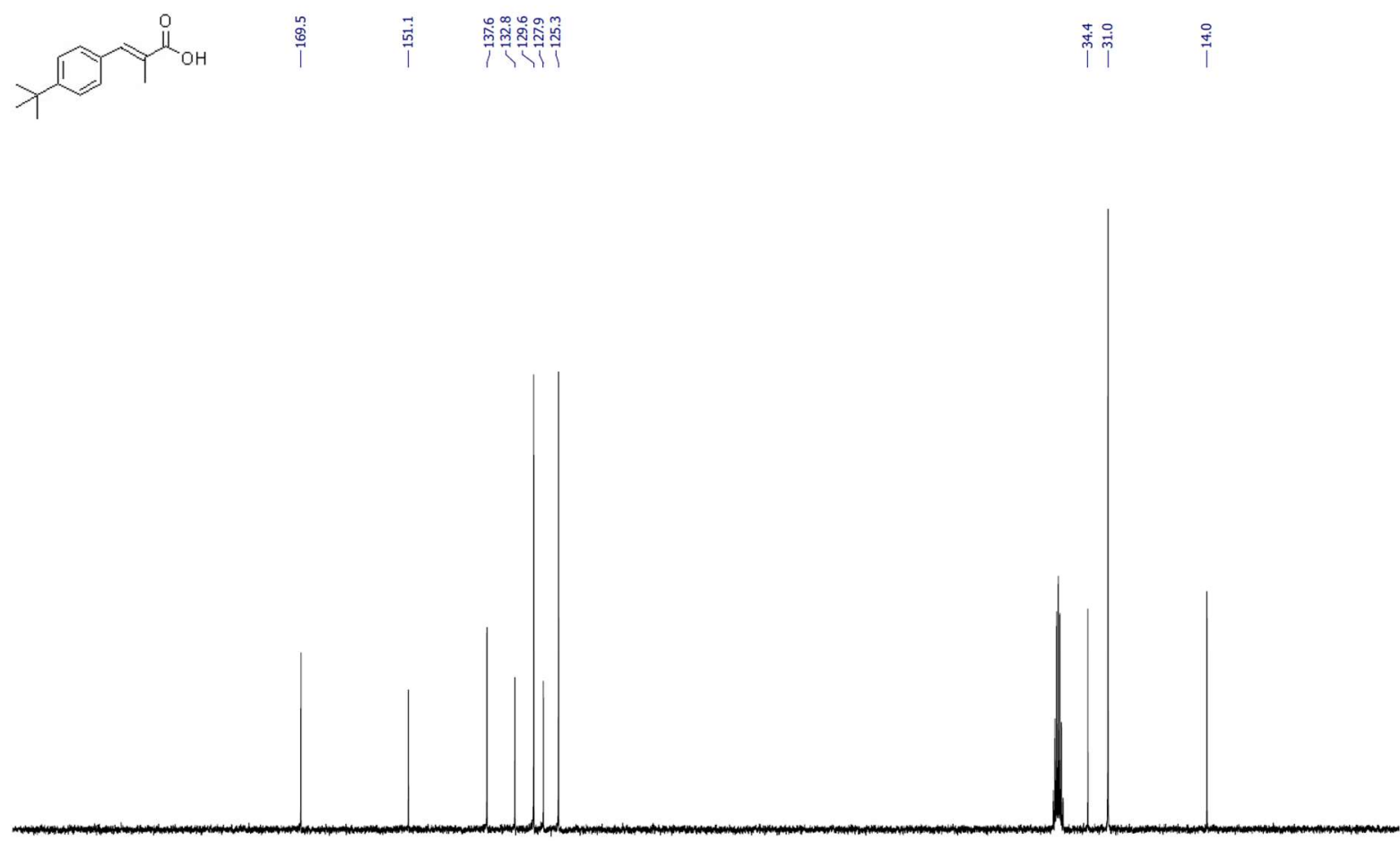

$\begin{array}{lllllllllllllllllllllll}210 & 200 & 190 & 180 & 170 & 160 & 150 & 140 & 130 & 120 & 110 & \begin{array}{c}100 \\ \mathrm{f} 1(\mathrm{ppm})\end{array} & 90 & 80 & 70 & 60 & 50 & 40 & 30 & 20 & 10 & 0 & -10\end{array}$

(E)-3-([1,1'-biphenyl]-4-yl)-2-methylacrylic acid (Ac-13):

${ }^{1} \mathrm{H}$ NMR $\left(\mathrm{CDCl}_{3}, 300 \mathrm{MHz}\right)$
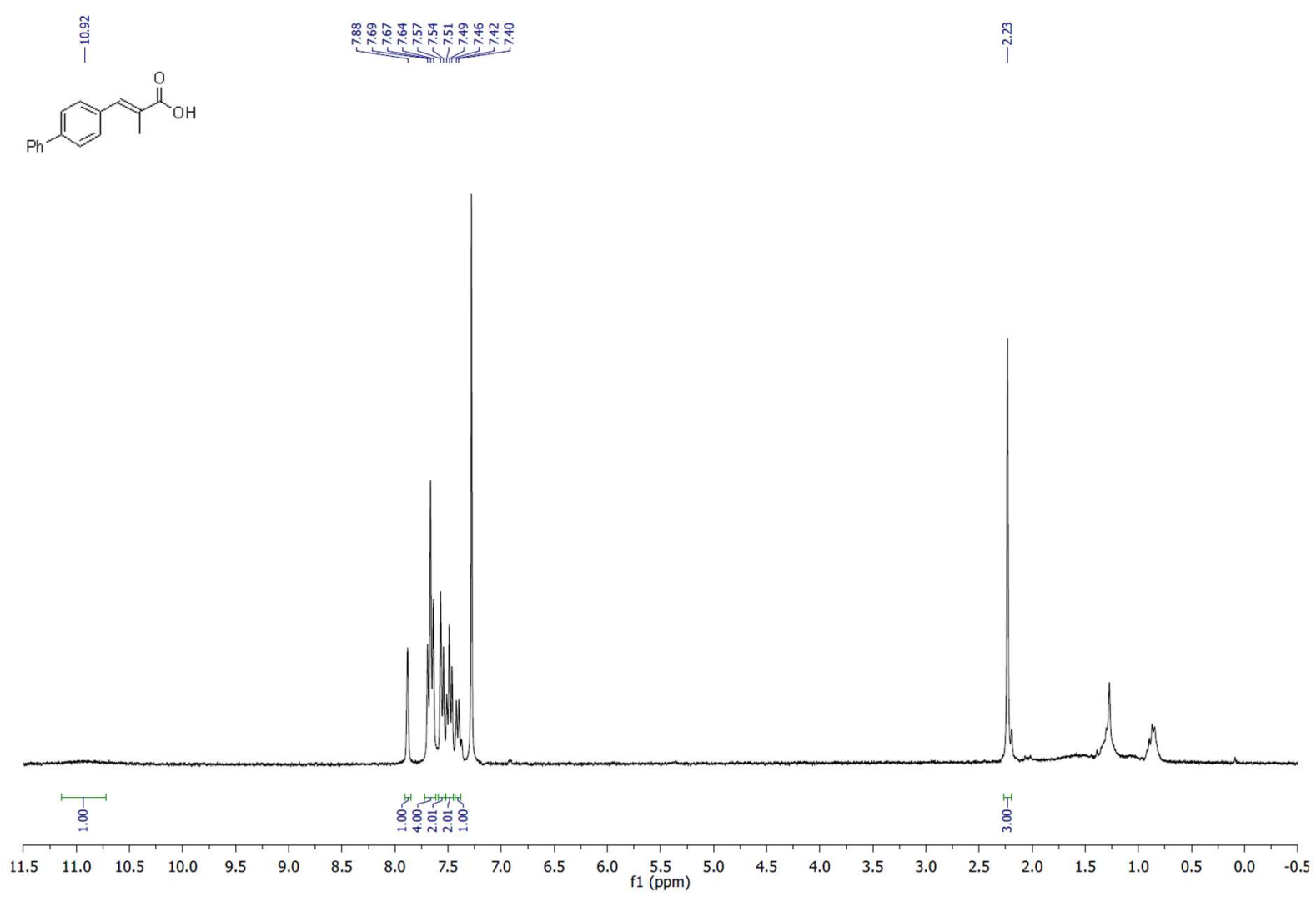
${ }^{13} \mathrm{C}$ NMR (DMSO-d6, $75 \mathrm{MHz}$ )
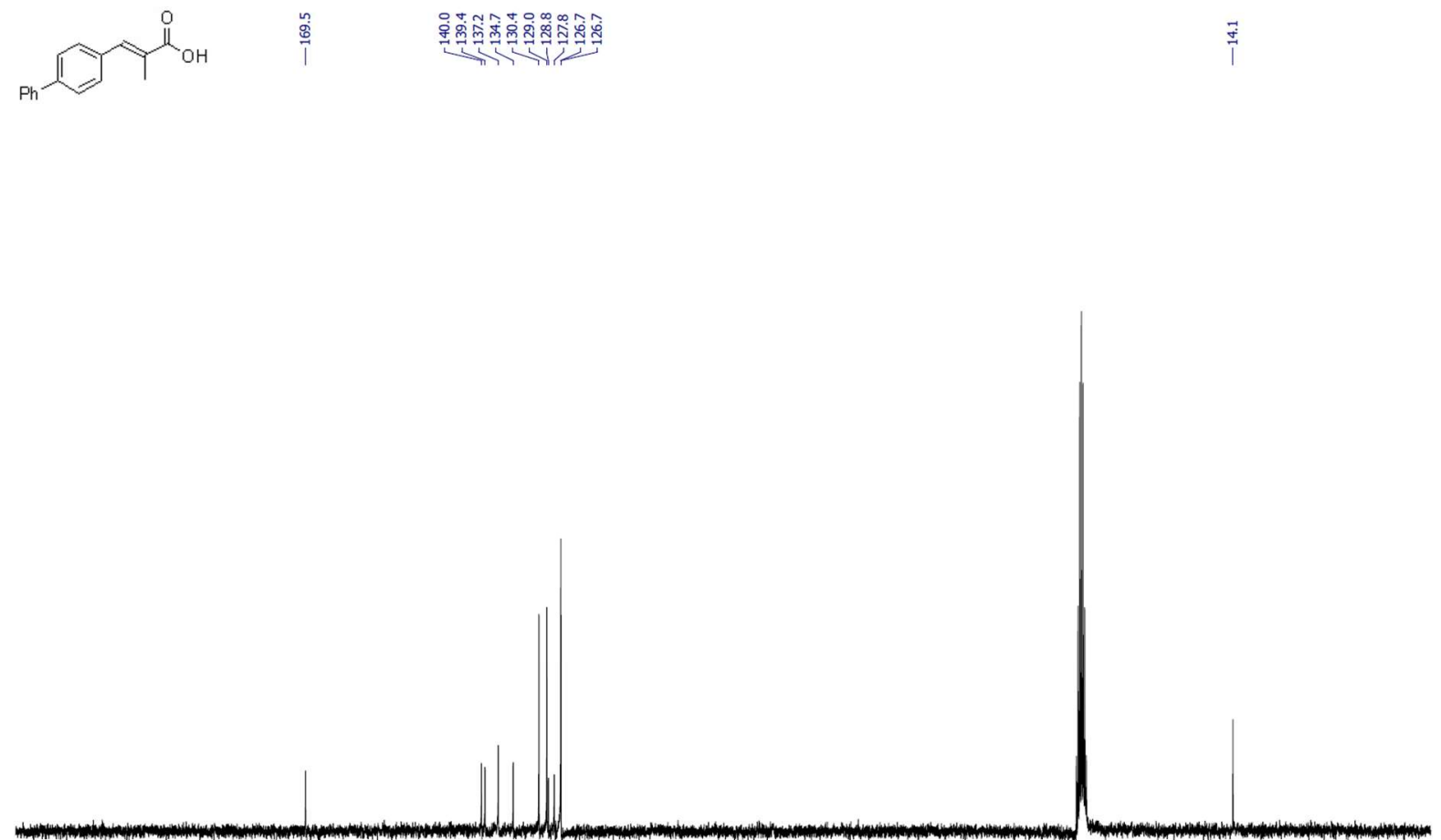

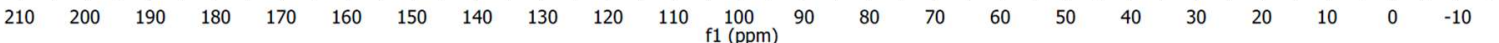

(E)-3-(4-methoxyphenyl)-2-methylacrylic acid (Ac-14):

${ }^{1} \mathrm{H} \mathrm{NMR}\left(\mathrm{CDCl}_{3}, 300 \mathrm{MHz}\right)$
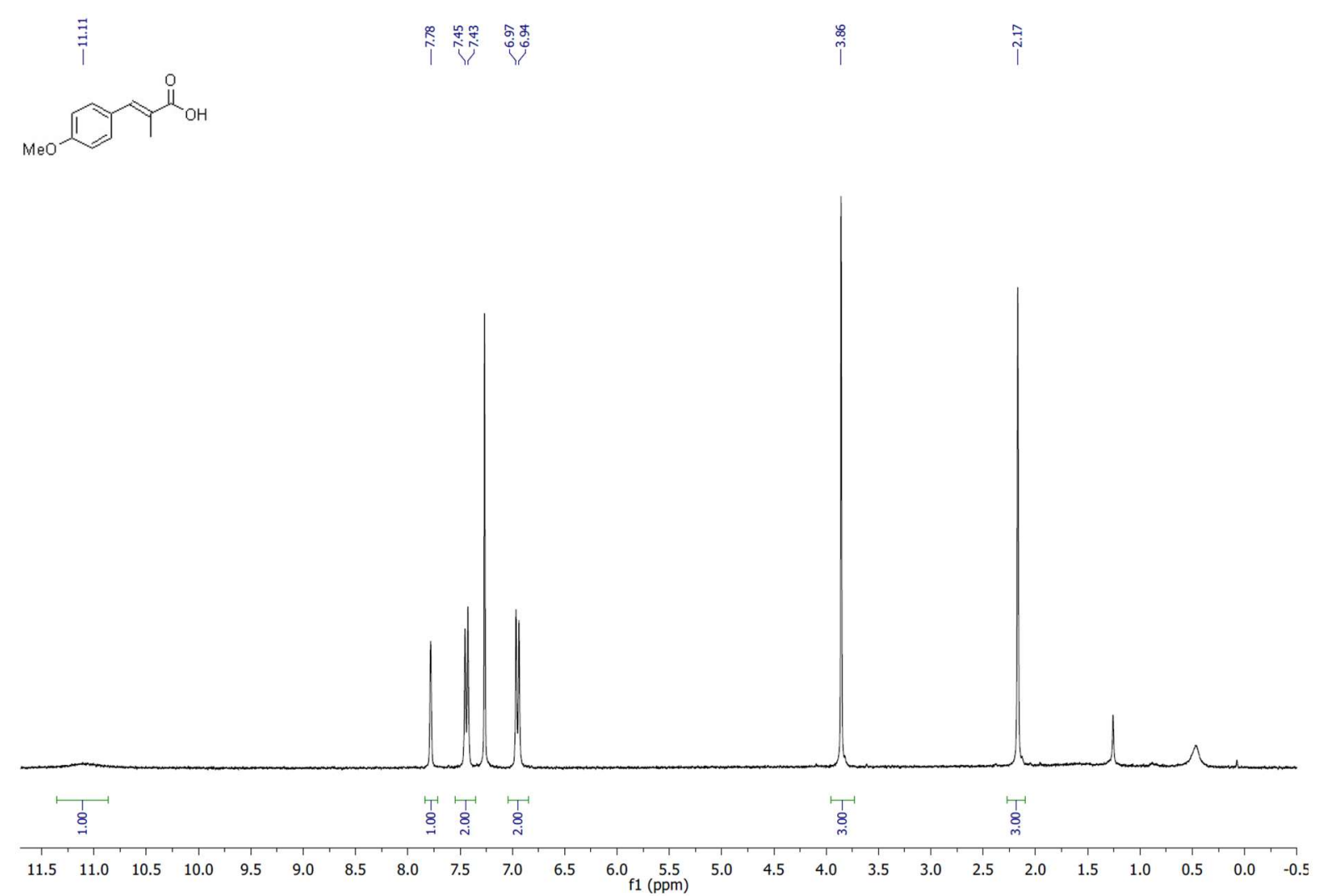
${ }^{13} \mathrm{C}$ NMR (DMSO-d6, $75 \mathrm{MHz}$ )
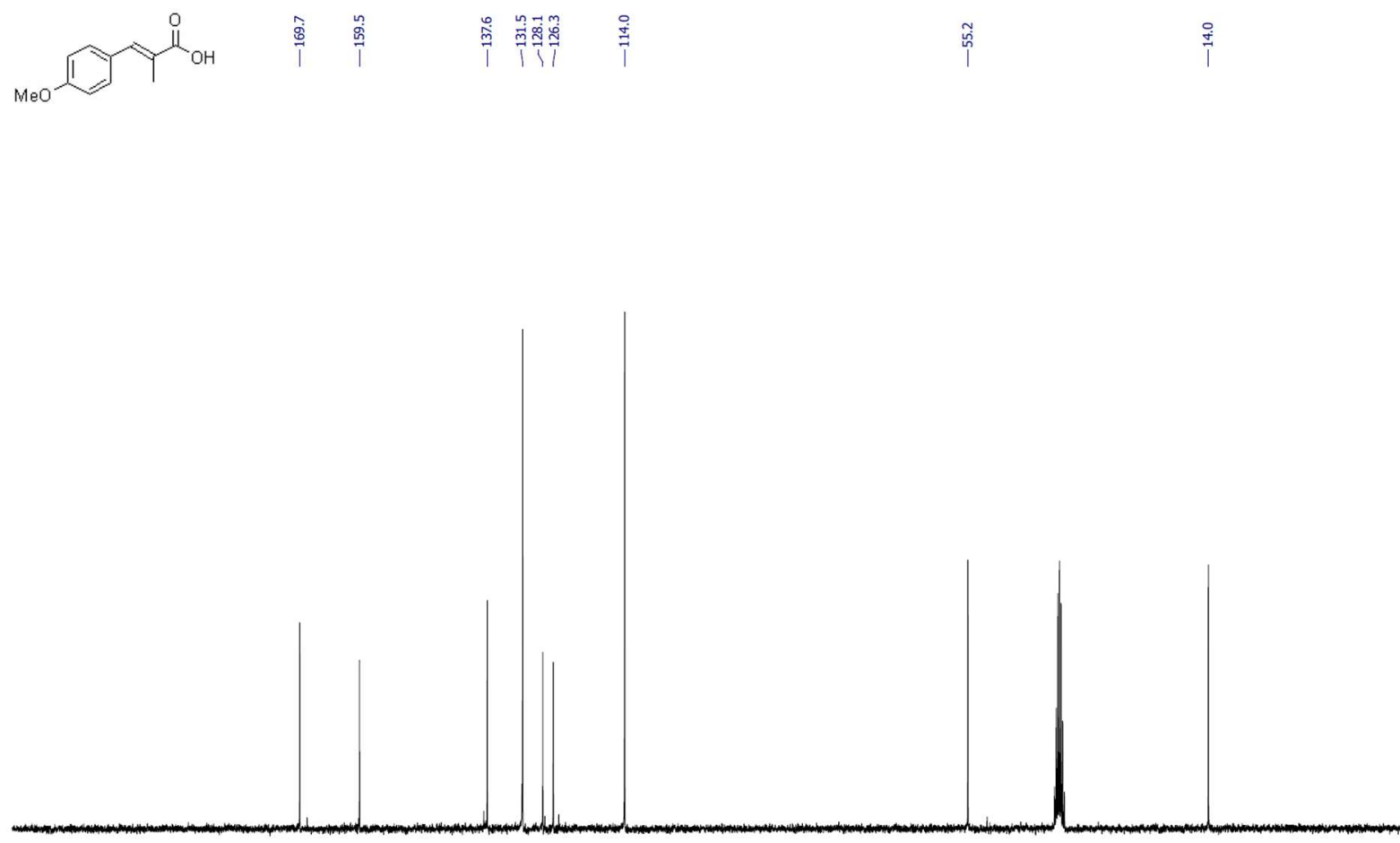

$\begin{array}{lllllllllllllllllllllll}210 & 200 & 190 & 180 & 170 & 160 & 150 & 140 & 130 & 120 & 110 & \begin{array}{c}100 \\ \mathrm{f} 1\end{array} & 90 & 80 & 70 & 60 & 50 & 40 & 30 & 20 & 10 & 0 & -10\end{array}$

(E)-2-methyl-3-(naphthalen-2-yl)acrylic acid (Ac-15):

${ }^{1} \mathrm{H} \mathrm{NMR}\left(\mathrm{CDCl}_{3}, 300 \mathrm{MHz}\right)$

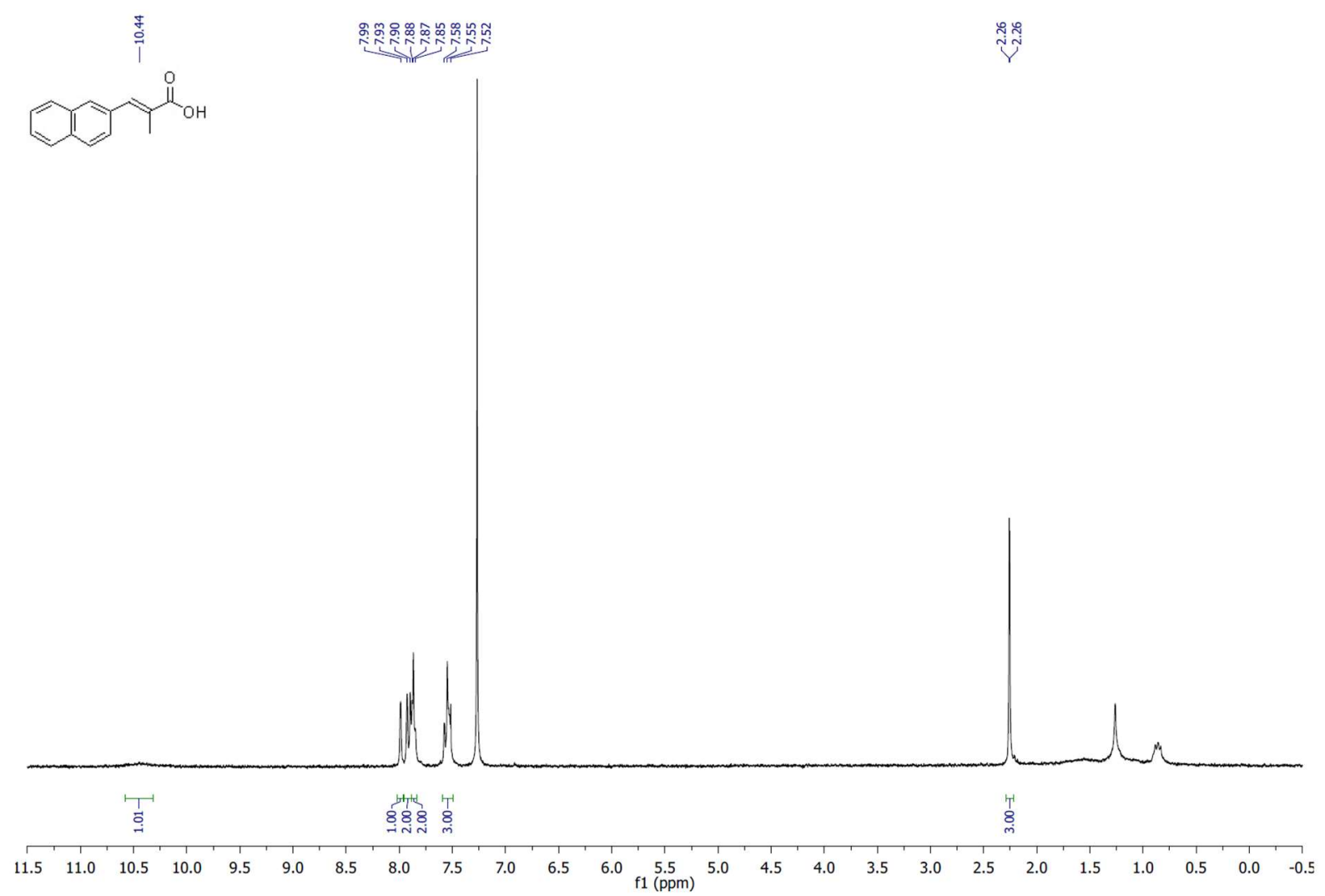


${ }^{13} \mathrm{C}$ NMR (DMSO-d6, $75 \mathrm{MHz}$ )

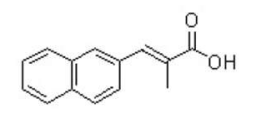

管

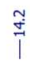

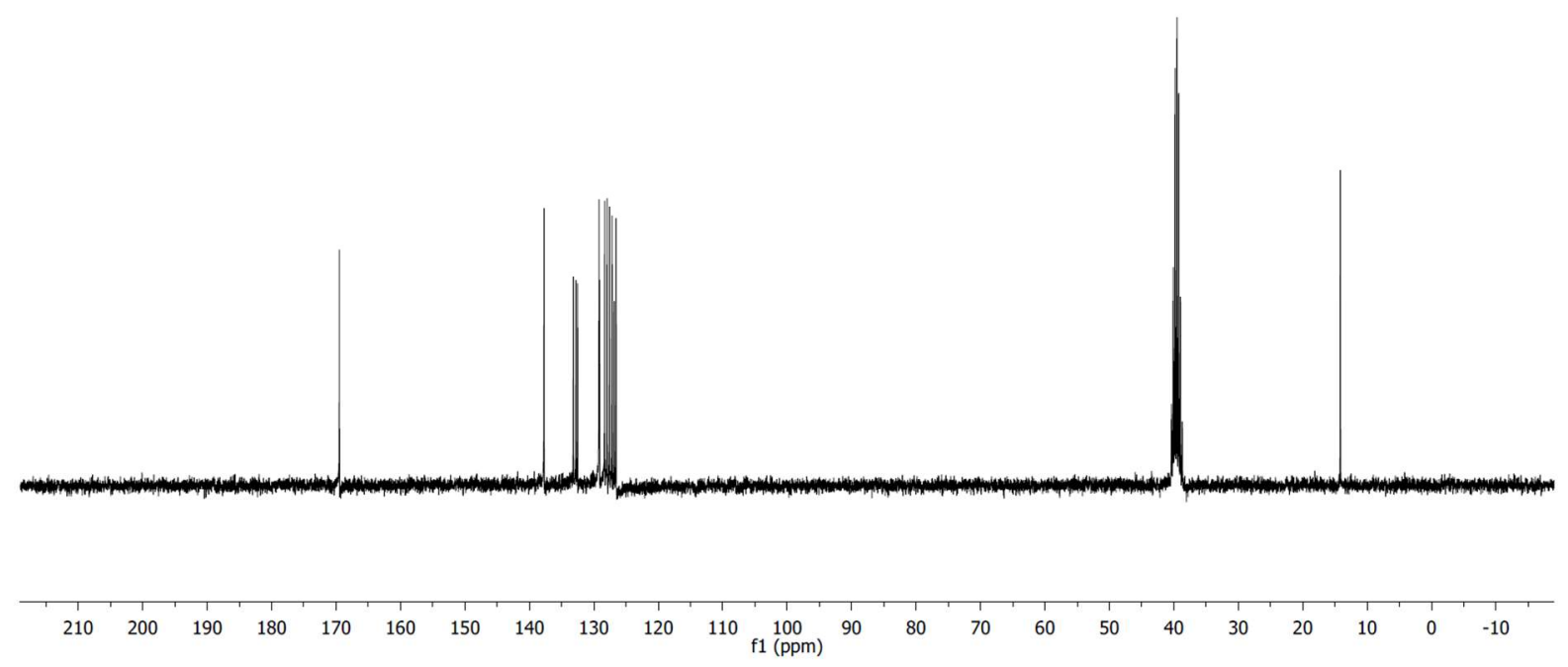

(E)-3-(4-fluorophenyl)-2-methylacrylic acid (Ac-16):

${ }^{1} \mathrm{H} \mathrm{NMR}\left(\mathrm{CDCl}_{3}, 300 \mathrm{MHz}\right)$

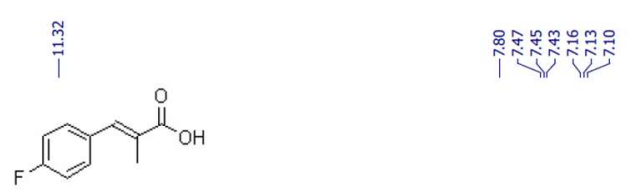

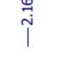

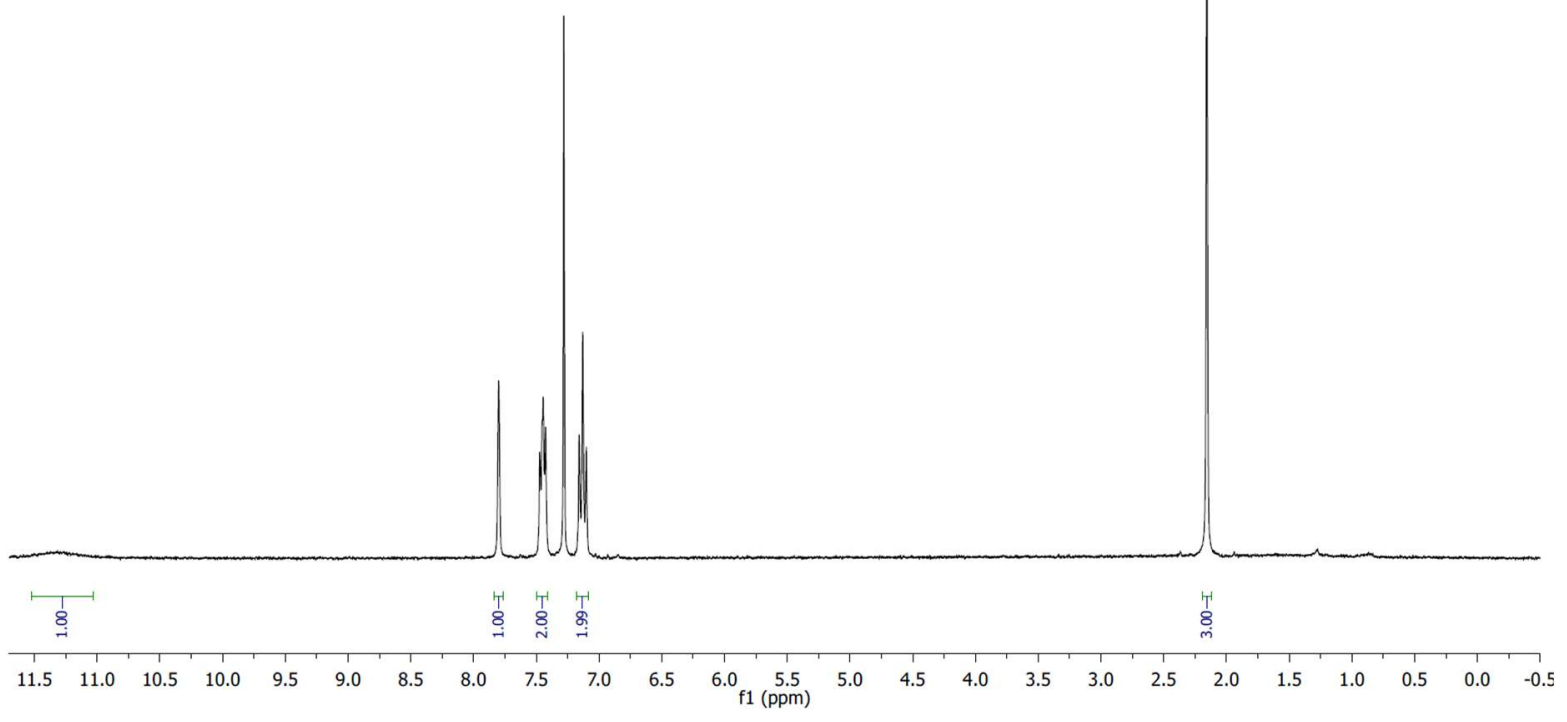

112 
${ }^{13} \mathrm{C}$ NMR (DMSO-d6, $75 \mathrm{MHz}$ )
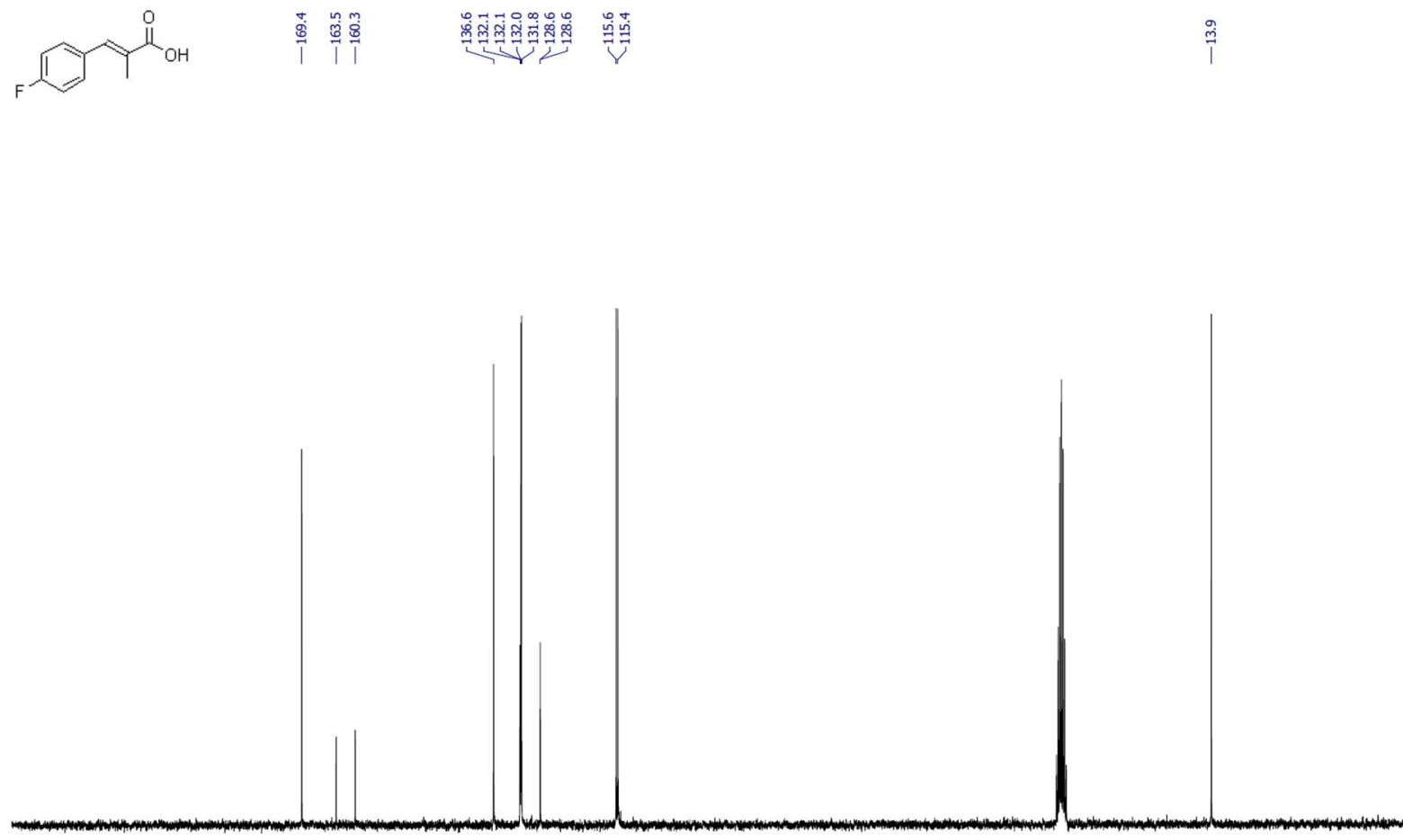

$\begin{array}{llllllllllllllllllllllll}210 & 200 & 190 & 180 & 170 & 160 & 150 & 140 & 130 & 120 & 110 & 100 & 90 & 80 & 70 & 60 & 50 & 40 & 30 & 20 & 10 & 0 & -10\end{array}$

${ }^{19} \mathrm{~F} \mathrm{NMR}\left(\mathrm{CDCl}_{3}, 282 \mathrm{MHz}\right)$

1

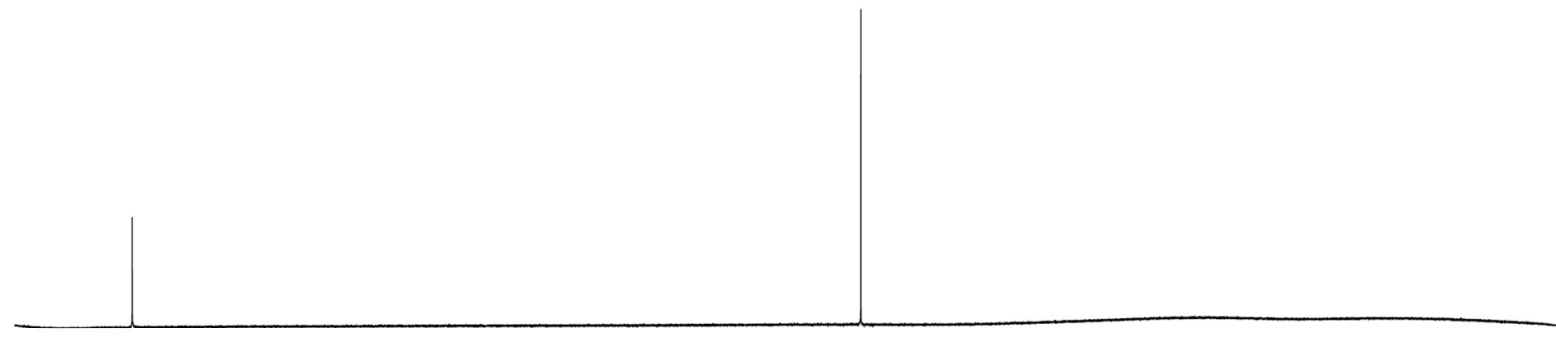


(E)-3-(4-chlorophenyl)-2-methylacrylic acid (Ac-17):

$\left.{ }^{1} \mathrm{H} \mathrm{NMR} \mathrm{(CDCl}, 300 \mathrm{MHz}\right)$
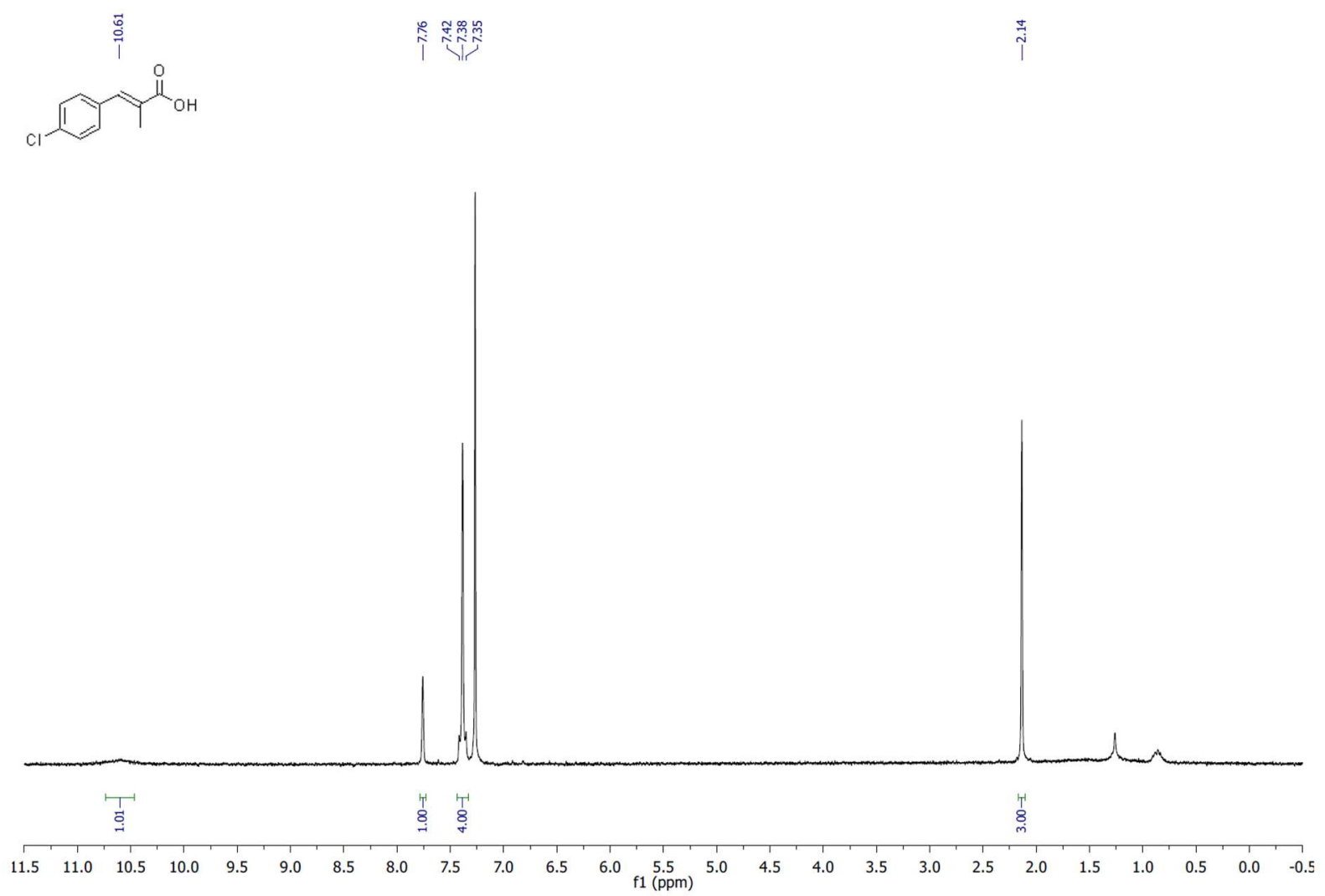

${ }^{13} \mathrm{C}$ NMR (DMSO-d6, $75 \mathrm{MHz}$ )<smiles>CC(C)=CC1=CCC(Cl)C=C1</smiles>

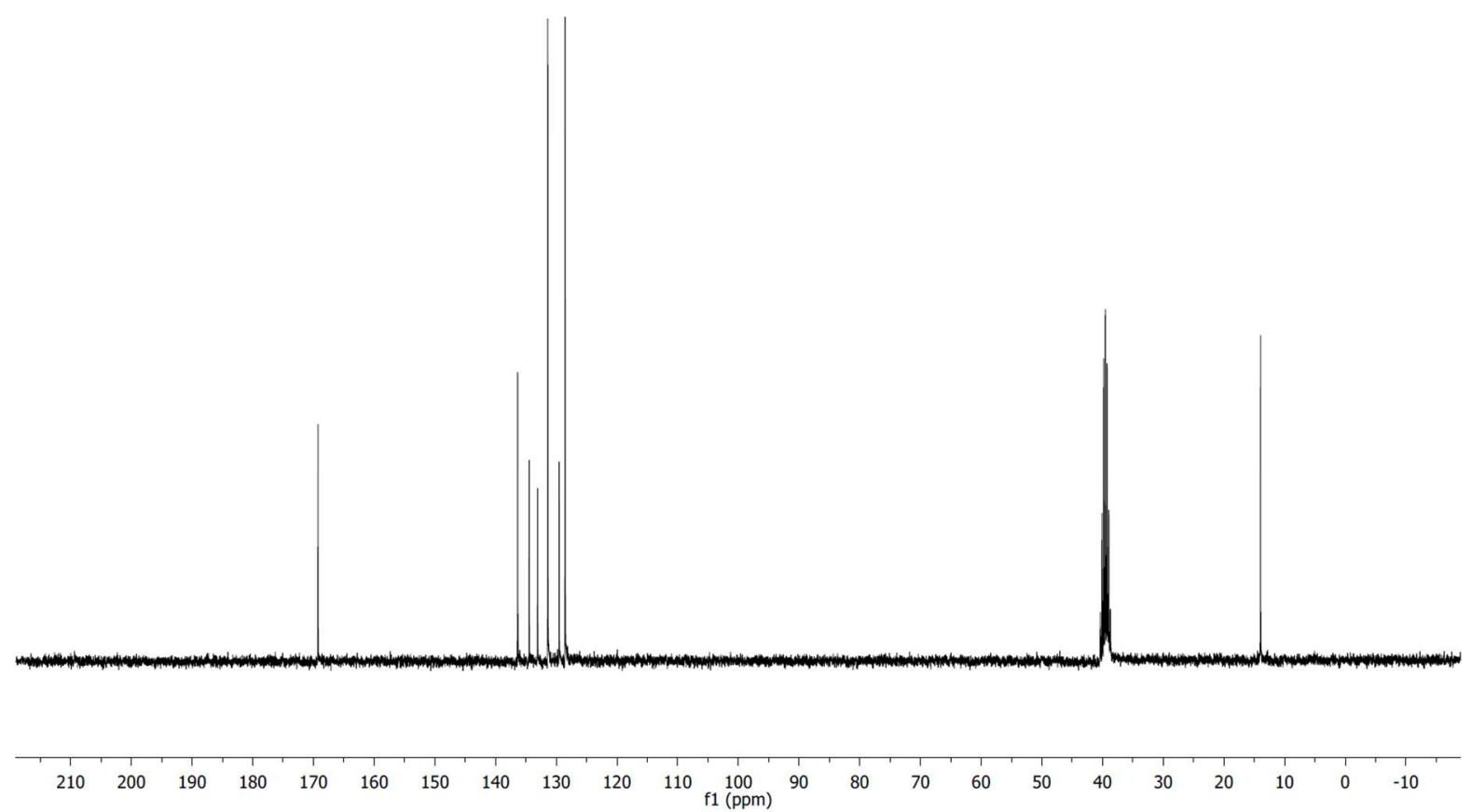


(E)-3-(4-bromophenyl)-2-methylacrylic acid (Ac-18):

${ }^{1} \mathrm{H} \mathrm{NMR}\left(\mathrm{CDCl}_{3}, 300 \mathrm{MHz}\right)$

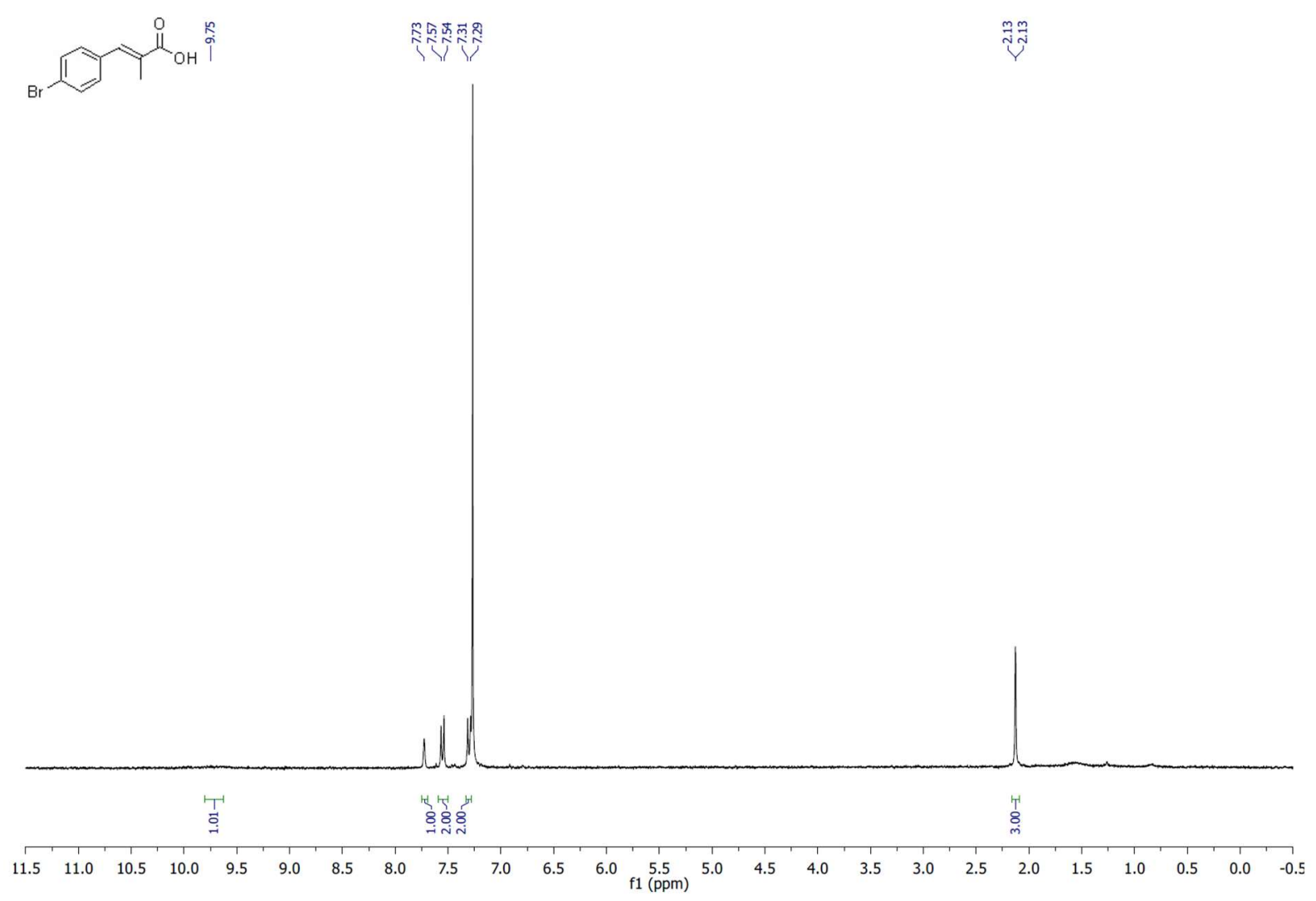

${ }^{13} \mathrm{C}$ NMR (DMSO-d6, $75 \mathrm{MHz}$ )
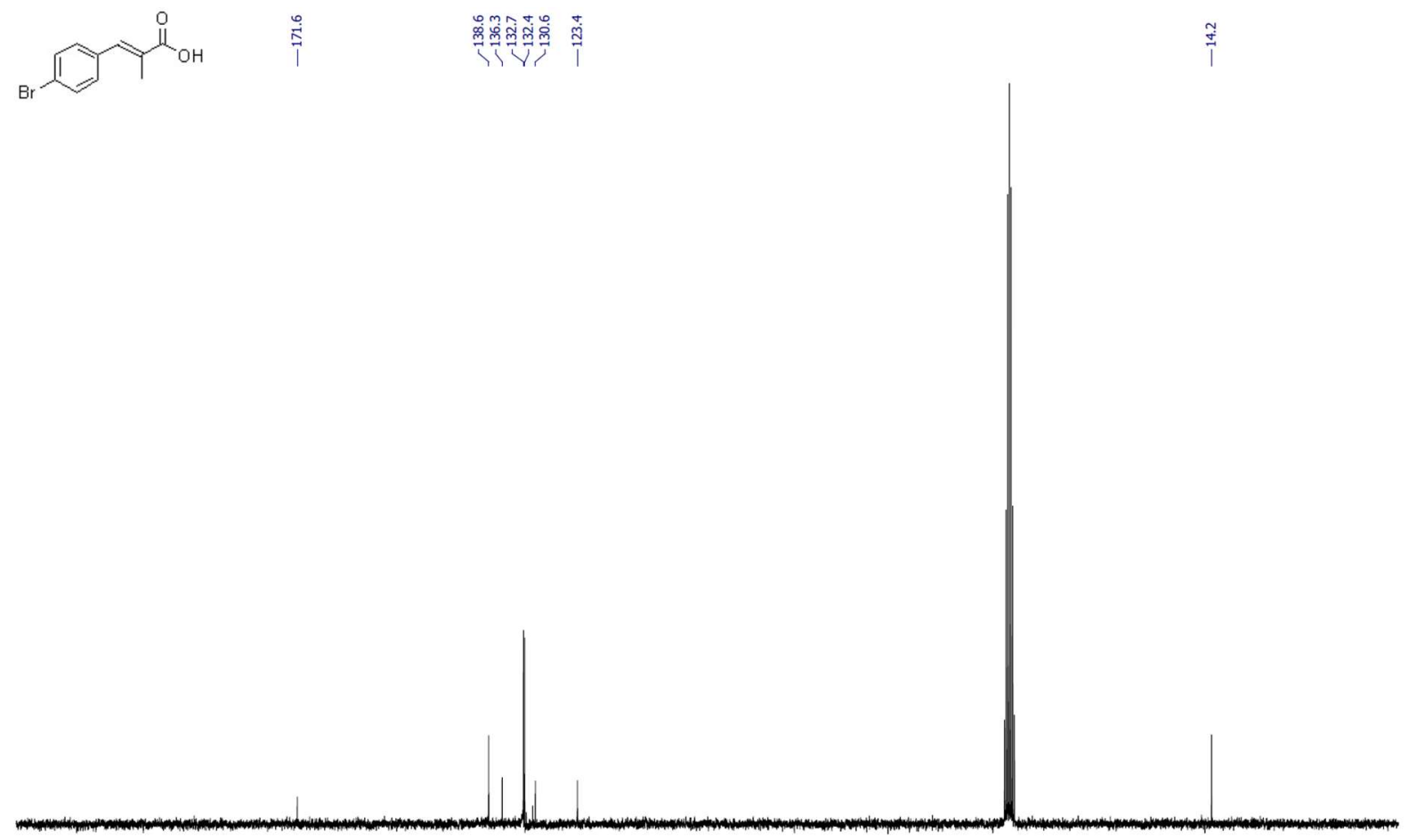

$\begin{array}{lllllllllllllllllllllllllllllll}220 & 210 & 200 & 190 & 180 & 170 & 160 & 150 & 140 & 130 & 120 & 110 & 100 & 90 & 80 & 70 & 60 & 50 & 40 & 30 & 20 & 10 & 0 & -10 & \end{array}$ 
(E)-3-(4-iodophenyl)-2-methylacrylic acid (Ac-19):

${ }^{1} \mathrm{H} \mathrm{NMR}\left(\mathrm{CDCl}_{3}, 300 \mathrm{MHz}\right)$
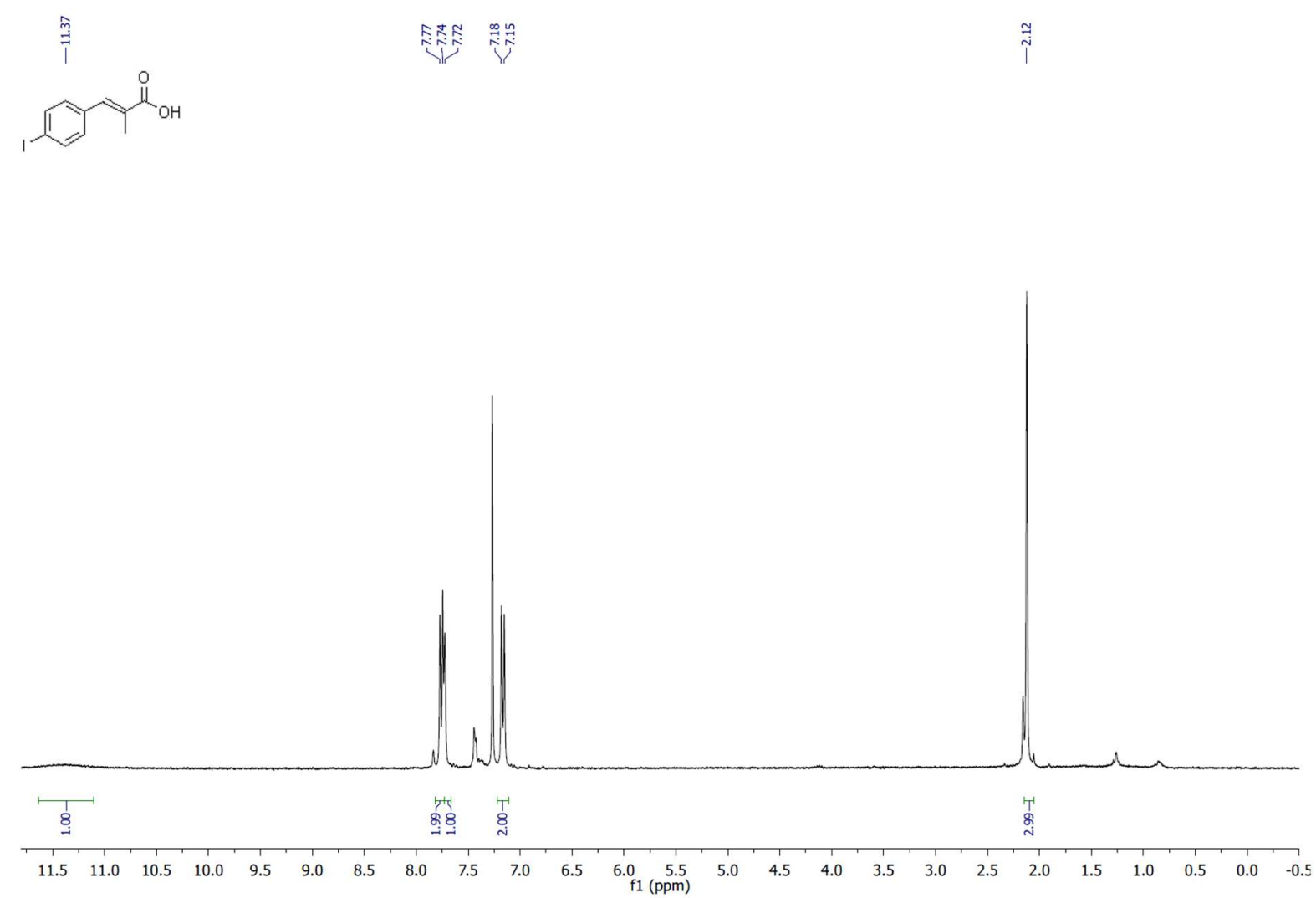

${ }^{13} \mathrm{C}$ NMR (DMSO-d6, $\left.75 \mathrm{MHz}\right)$
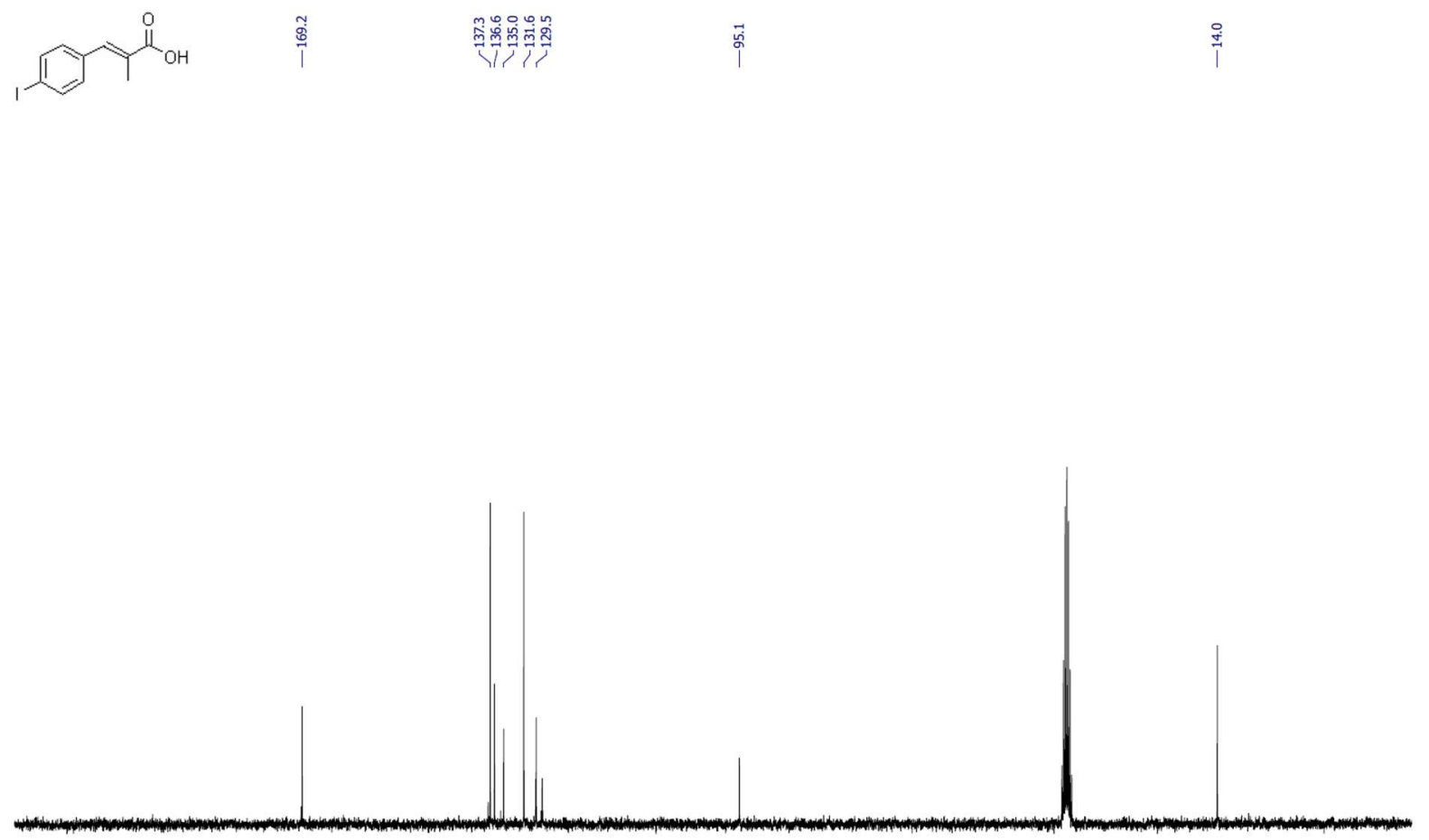

$\begin{array}{llllllllllllllllllllllll}210 & 200 & 190 & 180 & 170 & 160 & 150 & 140 & 130 & 120 & 110 & 100 & 90 & 80 & 70 & 60 & 50 & 40 & 30 & 20 & 10 & 0 & -10 & \end{array}$ 
(E)-2-methyl-3-(4-(trifluoromethyl)phenyl)acrylic acid (Ac-20):

${ }^{1} \mathrm{H} \mathrm{NMR}\left(\mathrm{CDCl}_{3}, 300 \mathrm{MHz}\right)$

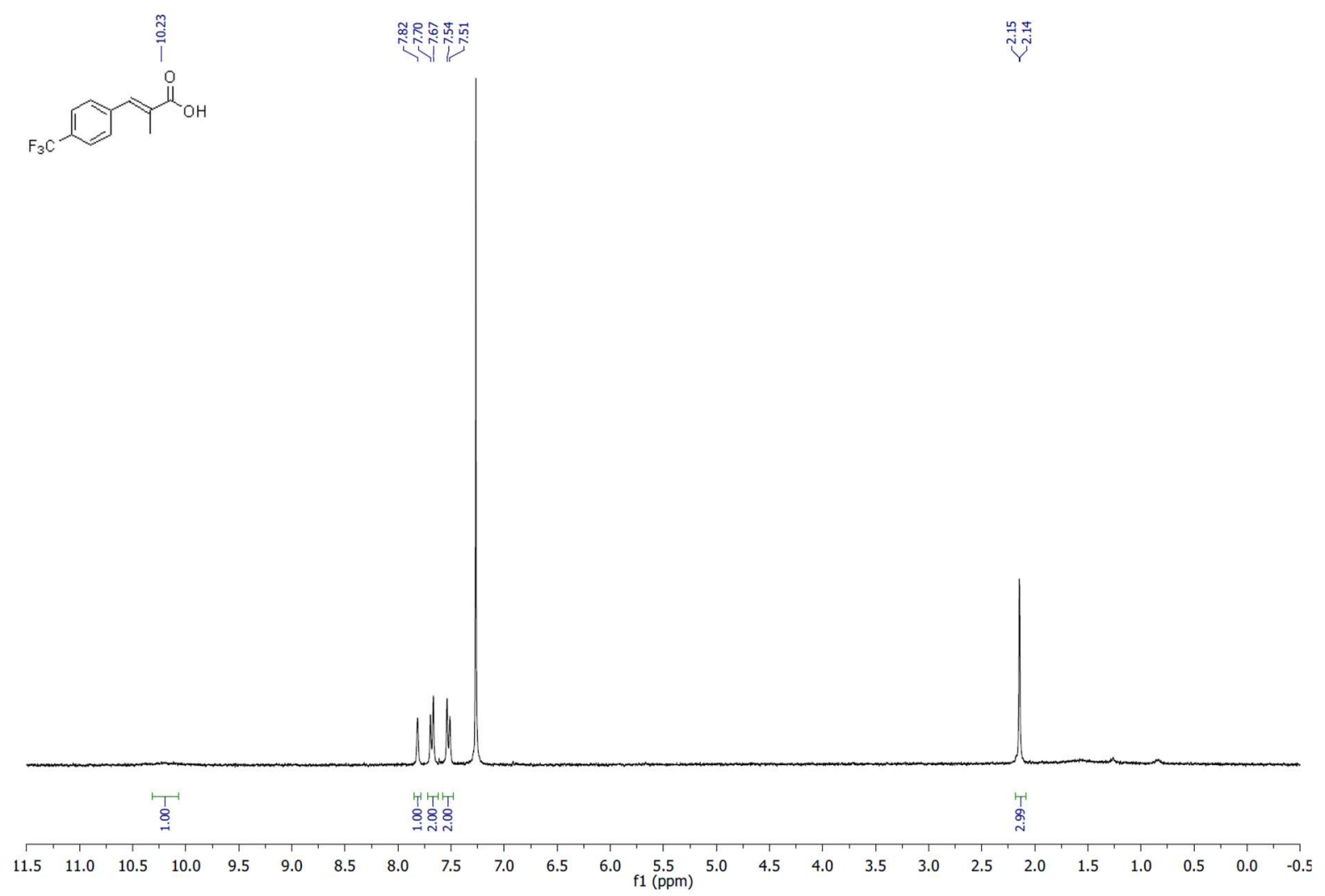

${ }^{13} \mathrm{C}$ NMR (DMSO-d6, $\left.75 \mathrm{MHz}\right)$
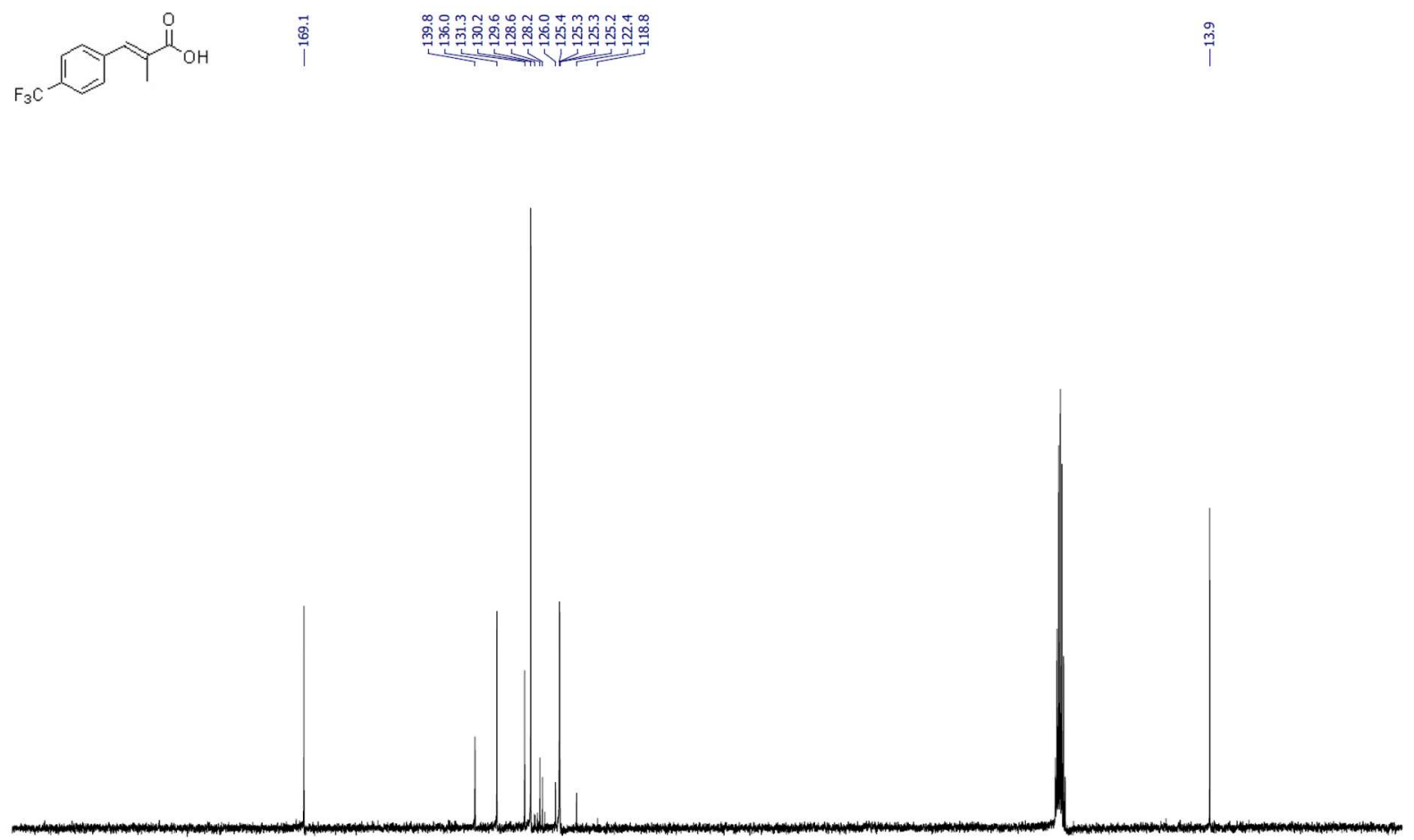

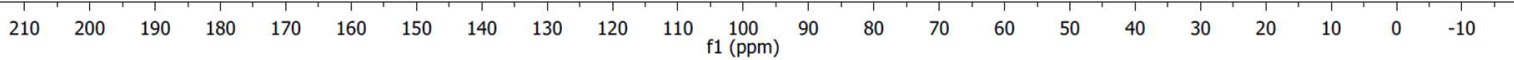


${ }^{19}$ F NMR (DMSO-d6, $282 \mathrm{MHz}$ )<smiles>CC(Cc1ccc([18F])cc1)C(=O)O</smiles>

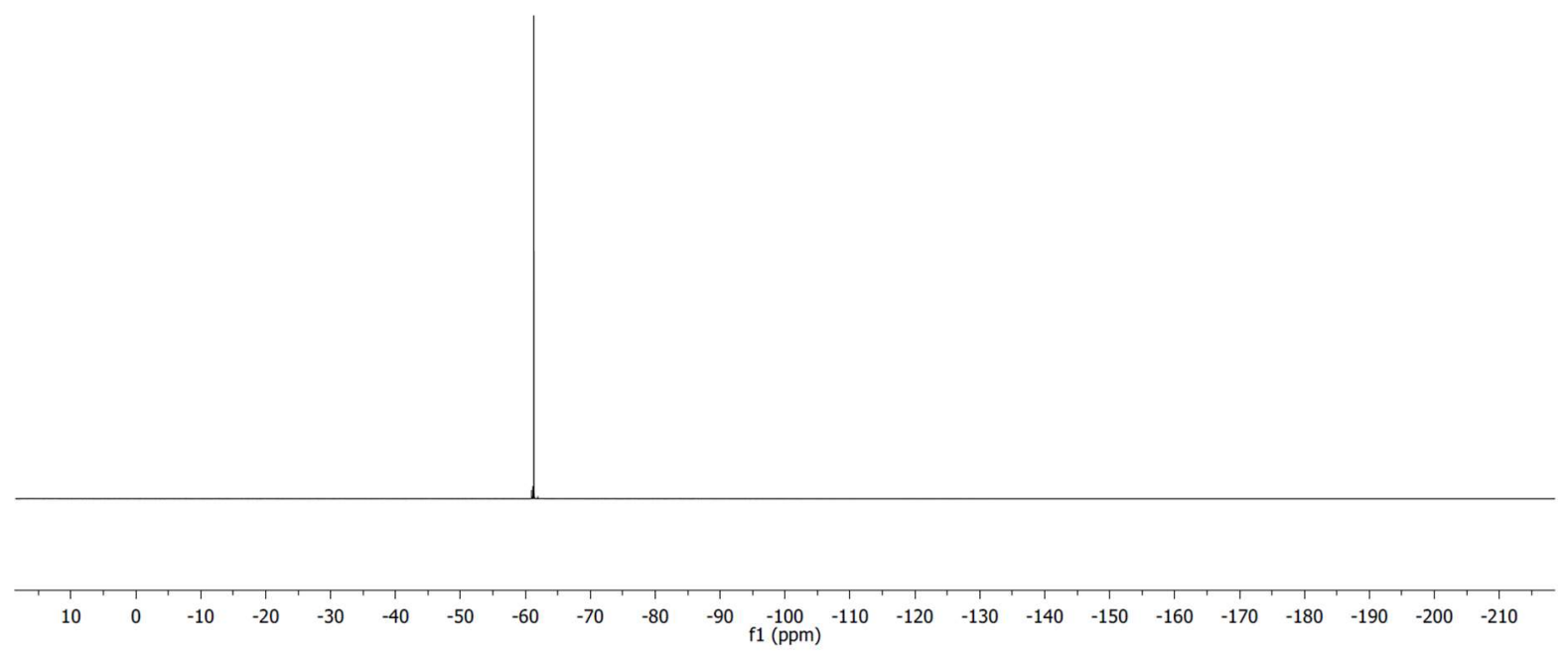

(E)-2-methyl-3-(4-(4,4,5,5-tetramethyl-1,3,2-dioxaborolan-2-yl)phenyl)acrylic acid (Ac-21):

${ }^{1} \mathrm{H} \mathrm{NMR}\left(\mathrm{CDCl}_{3}, 300 \mathrm{MHz}\right)$
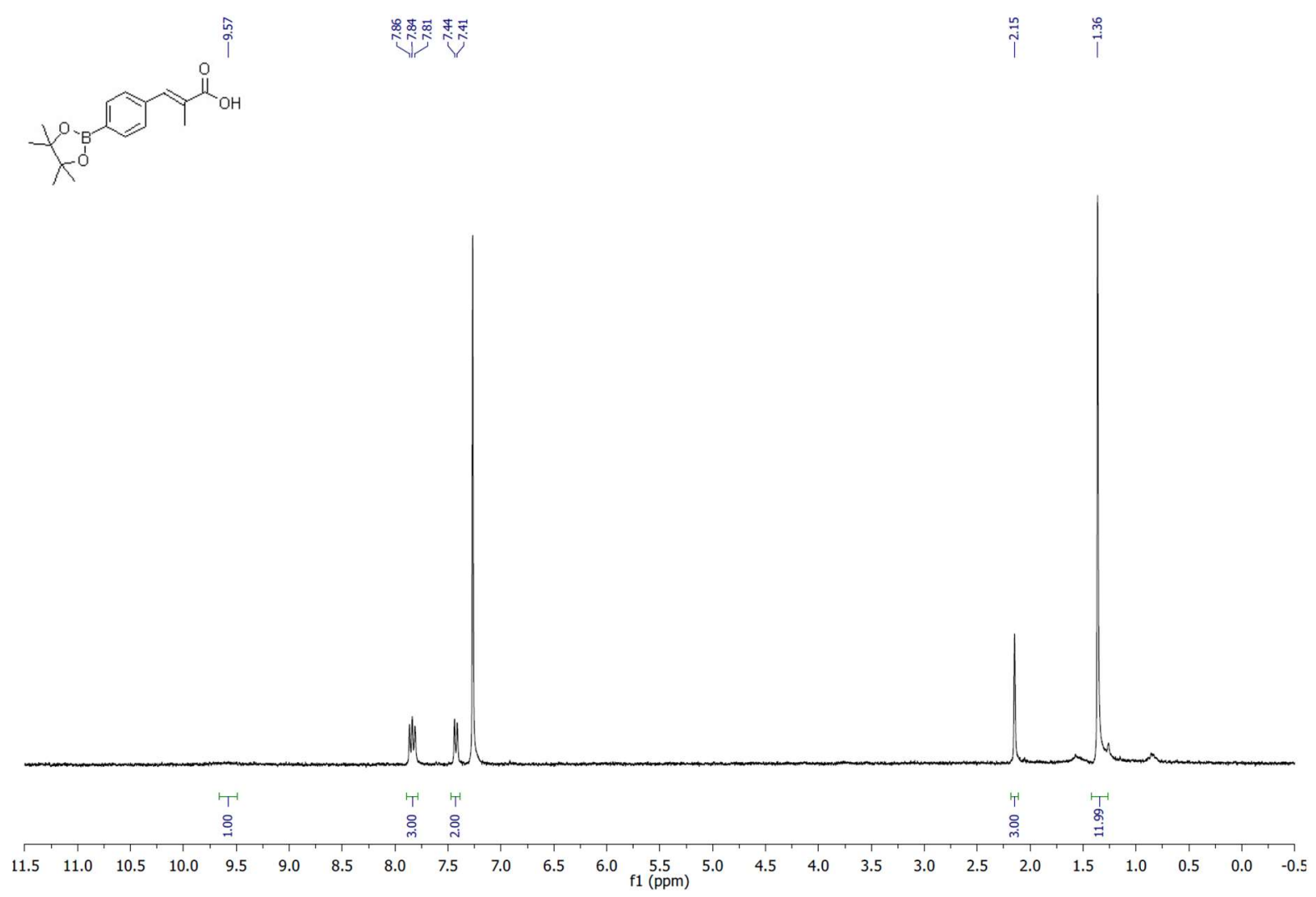
${ }^{13} \mathrm{C}$ NMR (DMSO-d6, $75 \mathrm{MHz}$ )

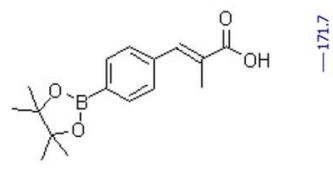

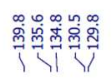

$\vec{s}$

$\stackrel{m}{\stackrel{m}{1}}$
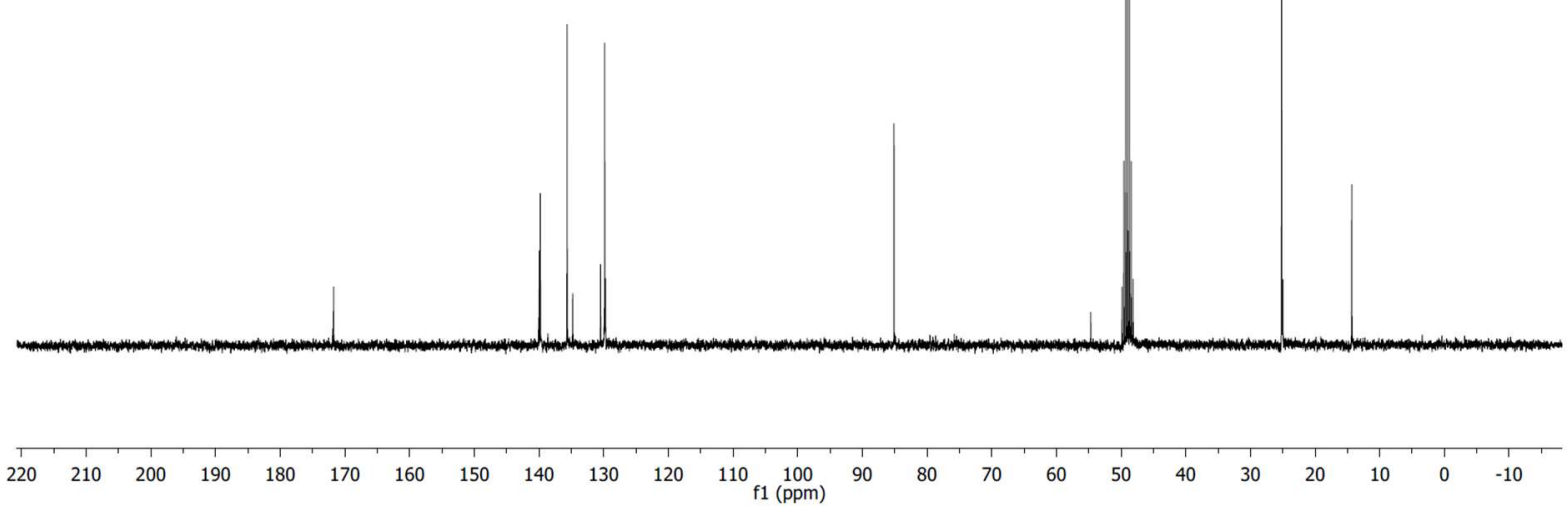

(E)-2-methyl-3-(4-(methylsulfonyl)phenyl)acrylic acid (Ac-22):

${ }^{1} \mathrm{H} \mathrm{NMR}\left(\mathrm{CDCl}_{3}, 300 \mathrm{MHz}\right)$

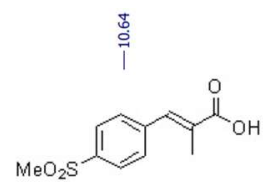

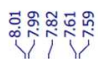

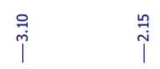

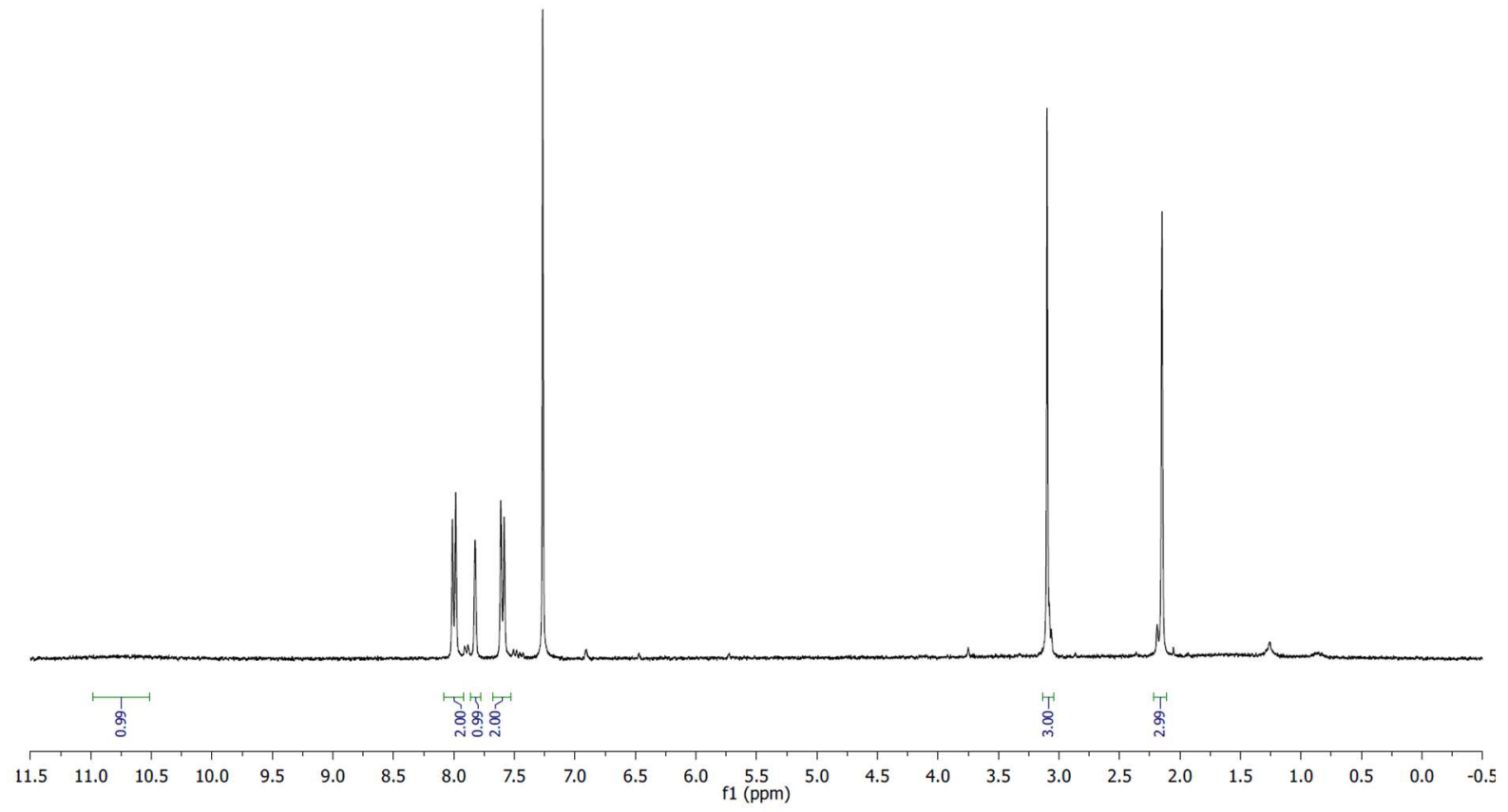

119 
${ }^{13} \mathrm{C}$ NMR (DMSO-d6, $75 \mathrm{MHz}$ )
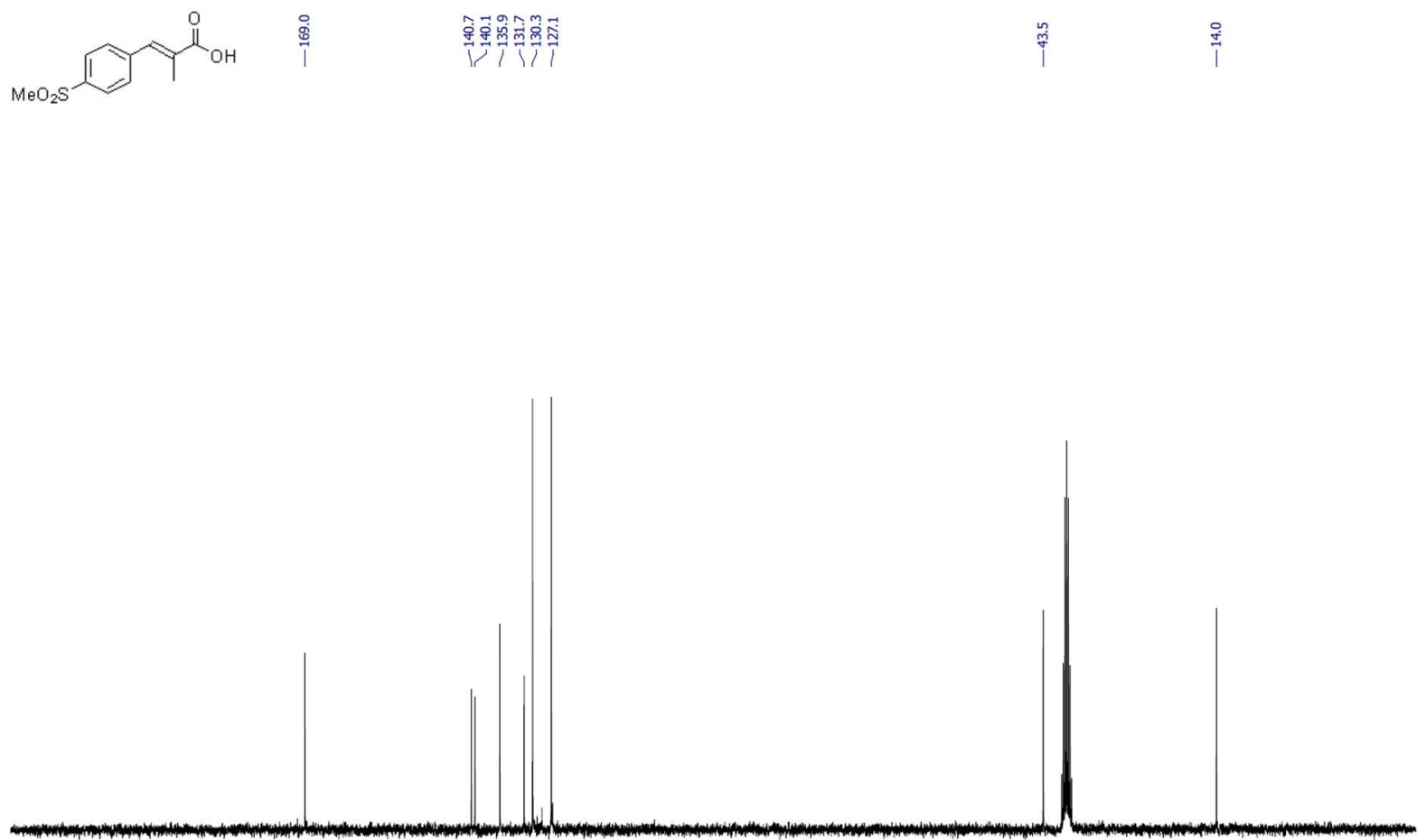

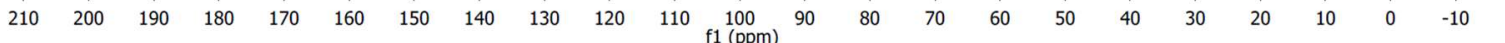

\section{(E)-3-(4-acetylphenyl)-2-methylacrylic acid (Ac-23):}

${ }^{1} \mathrm{H} \mathrm{NMR}\left(\mathrm{CDCl}_{3}, 300 \mathrm{MHz}\right)$
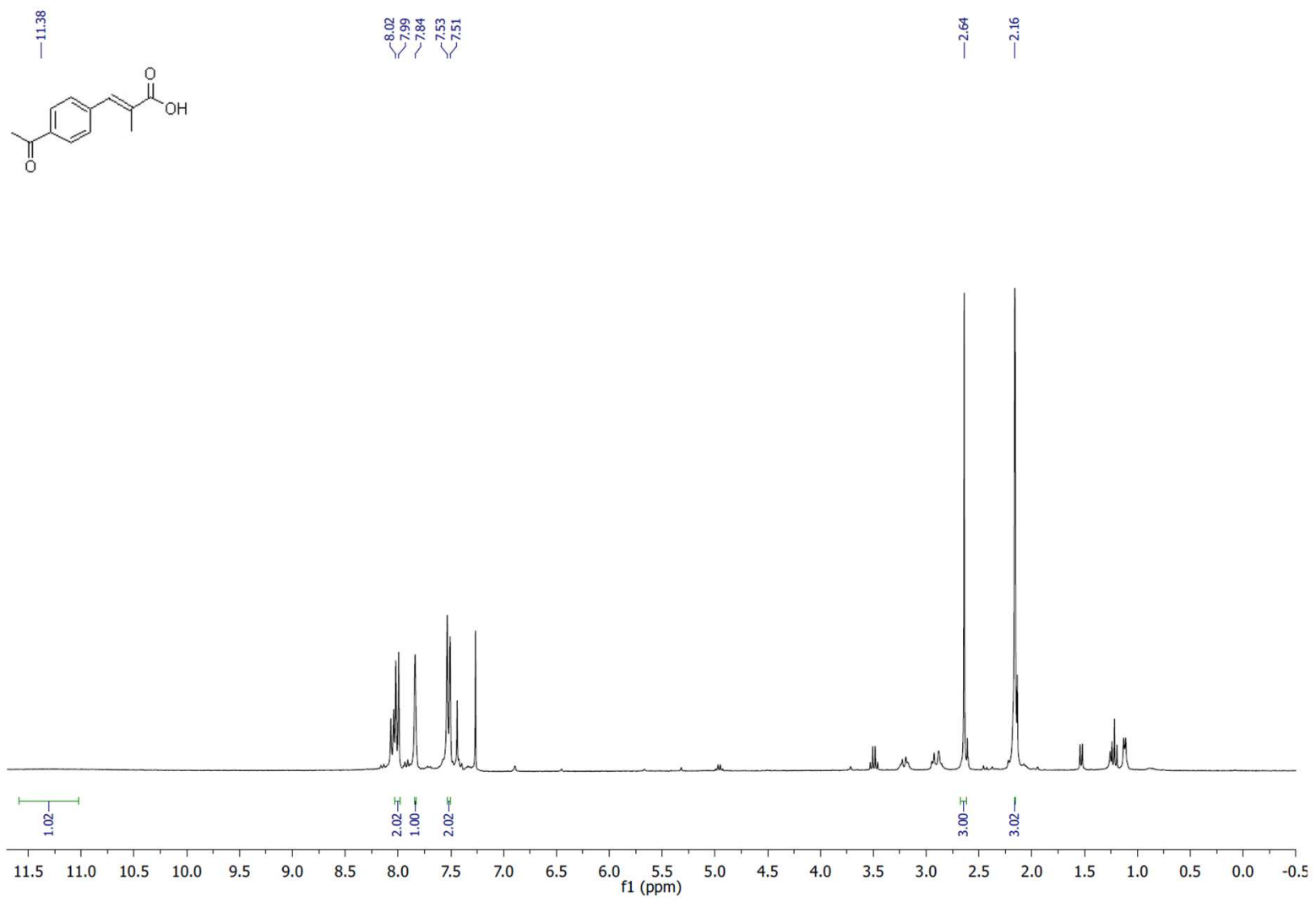
${ }^{13} \mathrm{C}$ NMR (DMSO-d6, $75 \mathrm{MHz}$ )
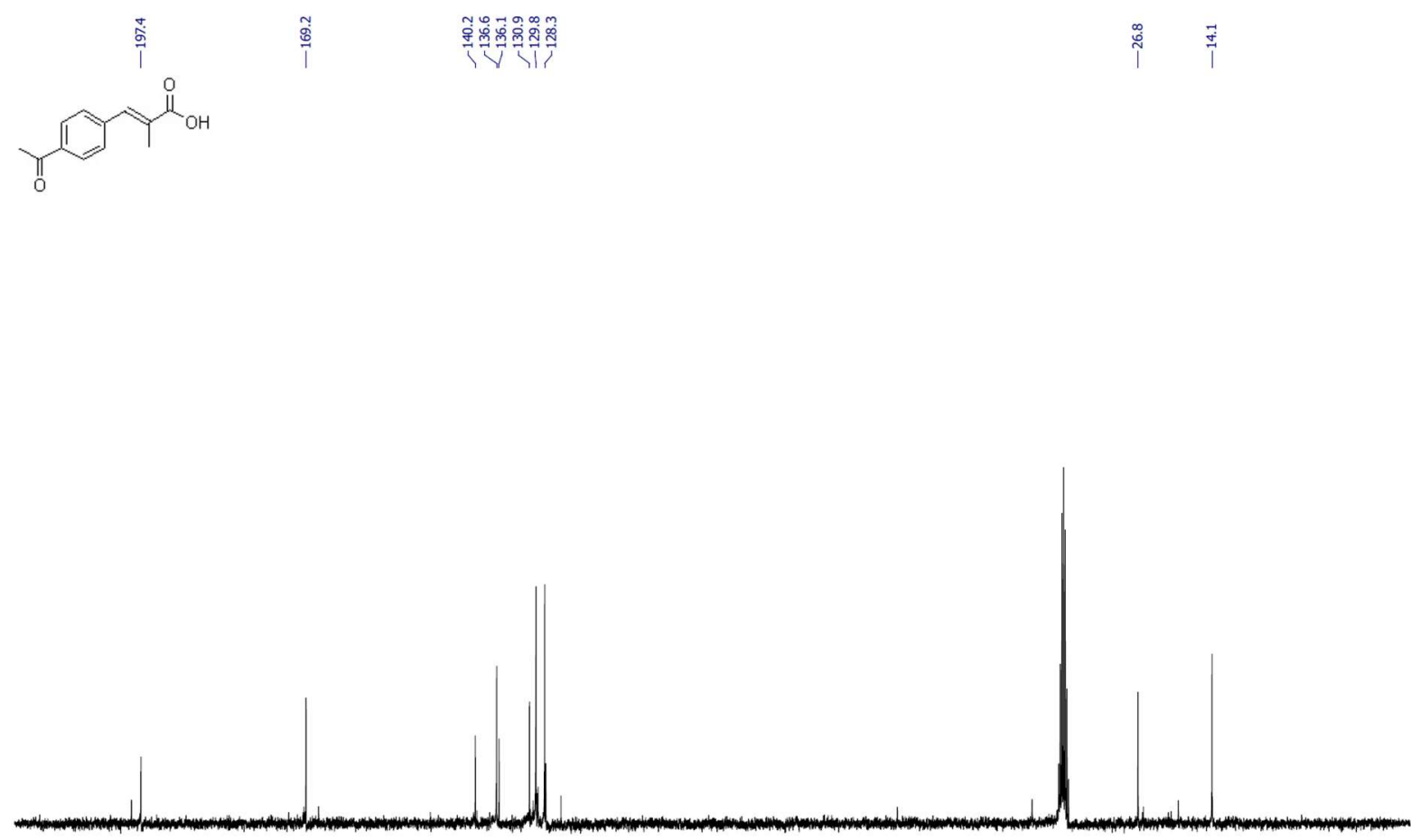

$\begin{array}{lllllllllllllllllllllllllllllll}210 & 200 & 190 & 180 & 170 & 160 & 150 & 140 & 130 & 120 & 110 & 100 & 90 & 80 & 70 & 60 & 50 & 40 & 30 & 20 & 10 & 0 & -10\end{array}$

(E)-3-(furan-2-yl)-2-methylacrylic acid (Ac-24):

${ }^{1} \mathrm{H} \mathrm{NMR}\left(\mathrm{CDCl}_{3}, 300 \mathrm{MHz}\right)$
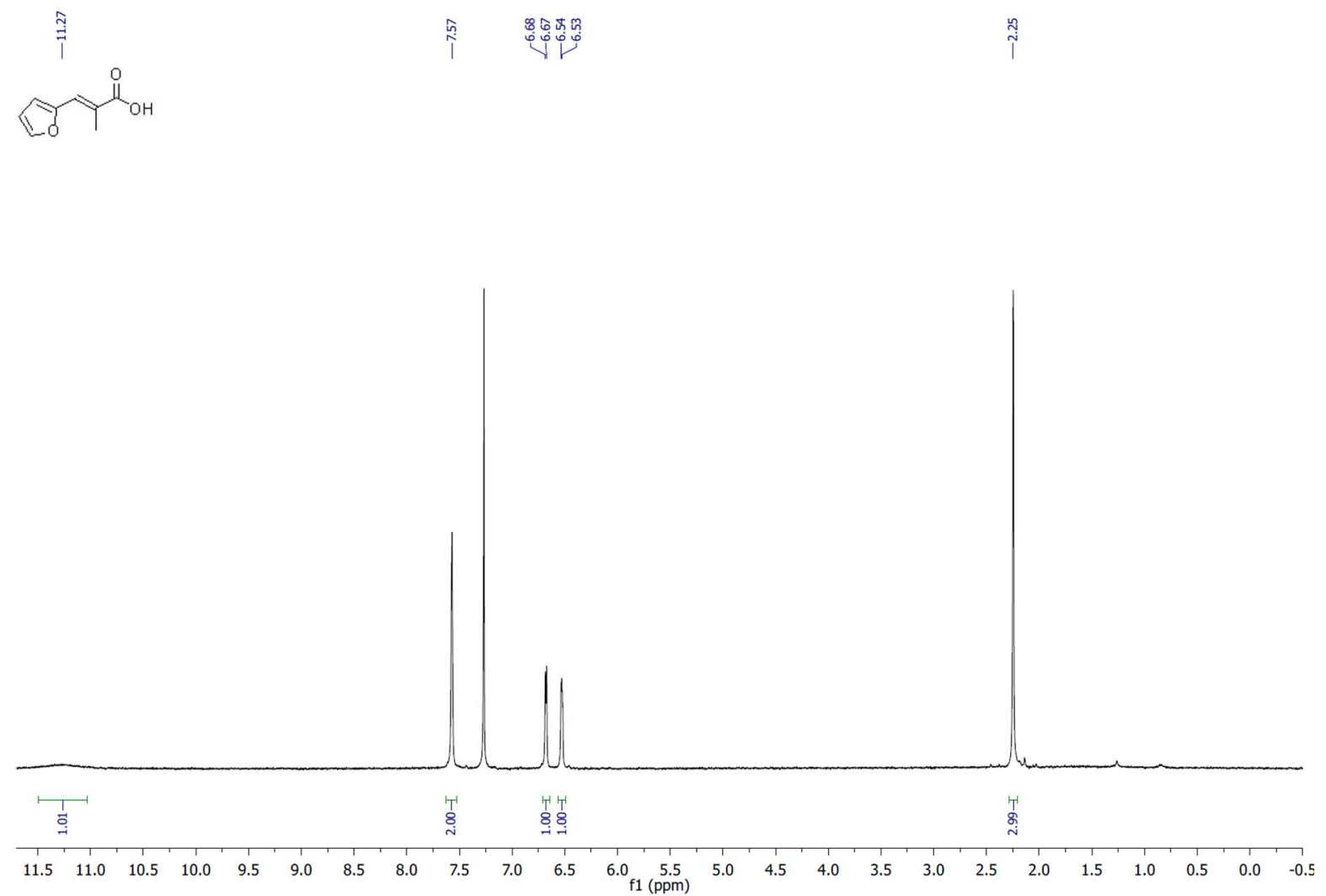
${ }^{13} \mathrm{C}$ NMR (DMSO-d6, $75 \mathrm{MHz}$ )

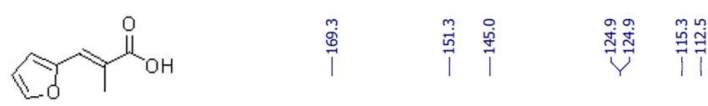

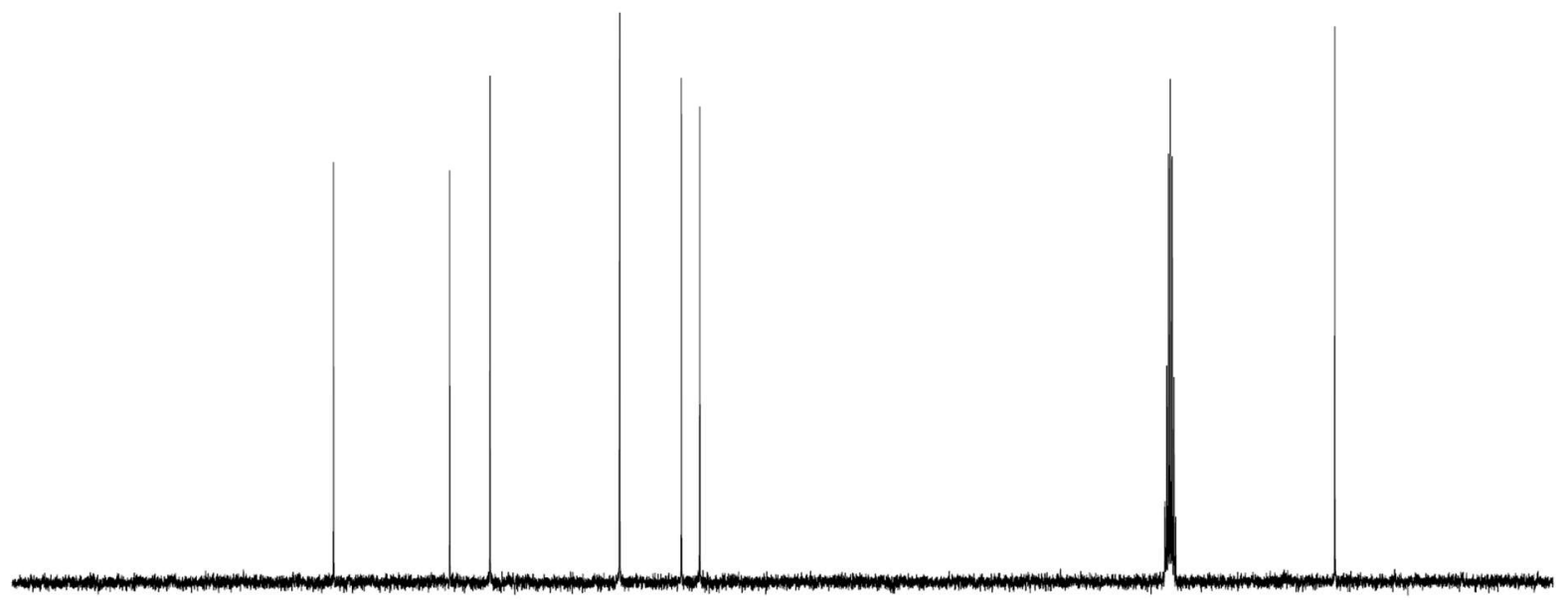

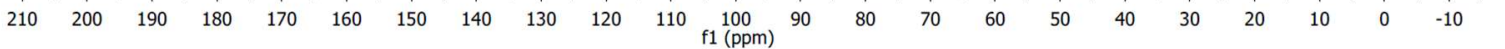

(E)-2-methyl-3-(thiophen-2-yl)acrylic acid (Ac-25):

${ }^{1} \mathrm{H} \mathrm{NMR}\left(\mathrm{CDCl}_{3}, 300 \mathrm{MHz}\right)$
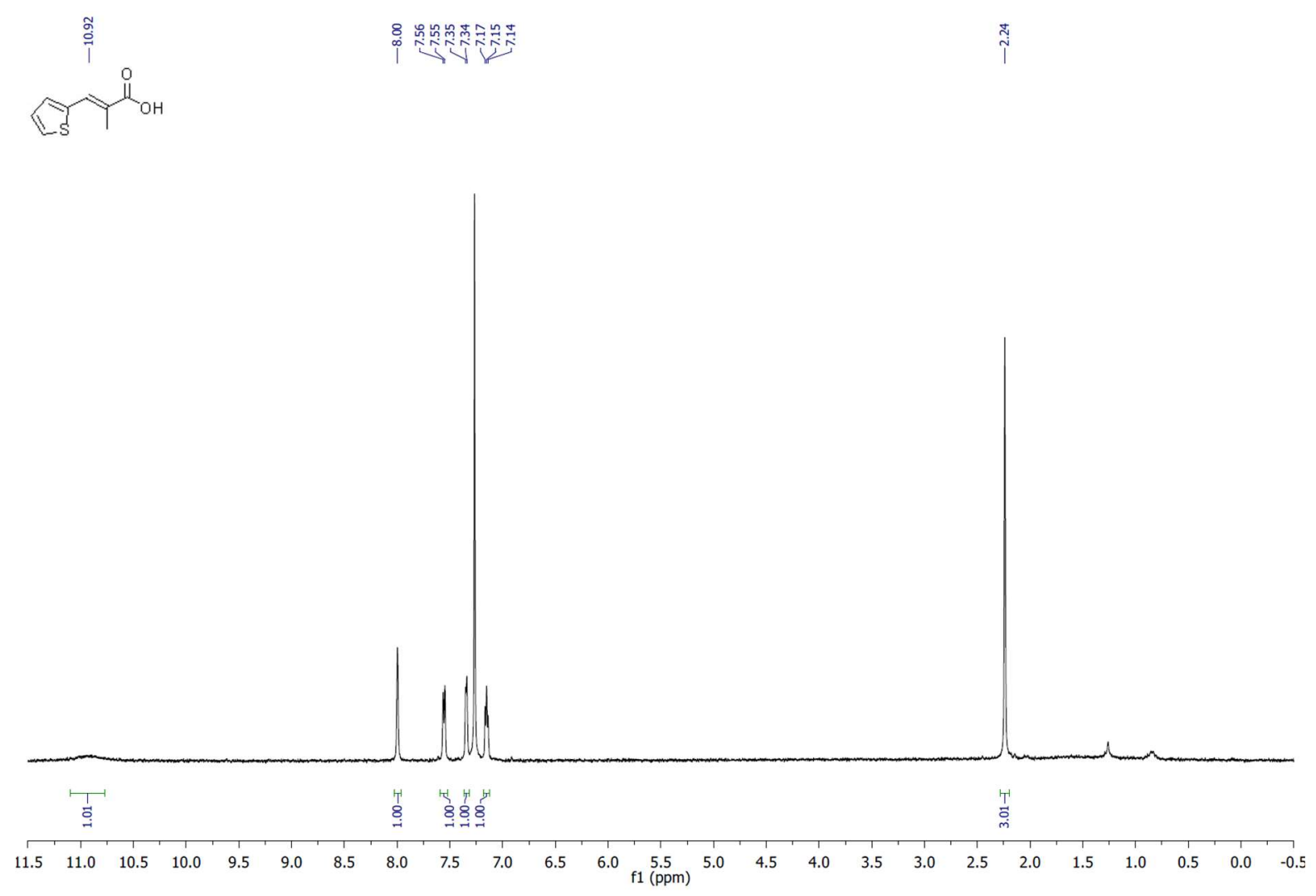
${ }^{13} \mathrm{C}$ NMR (DMSO-d6, $75 \mathrm{MHz}$ )
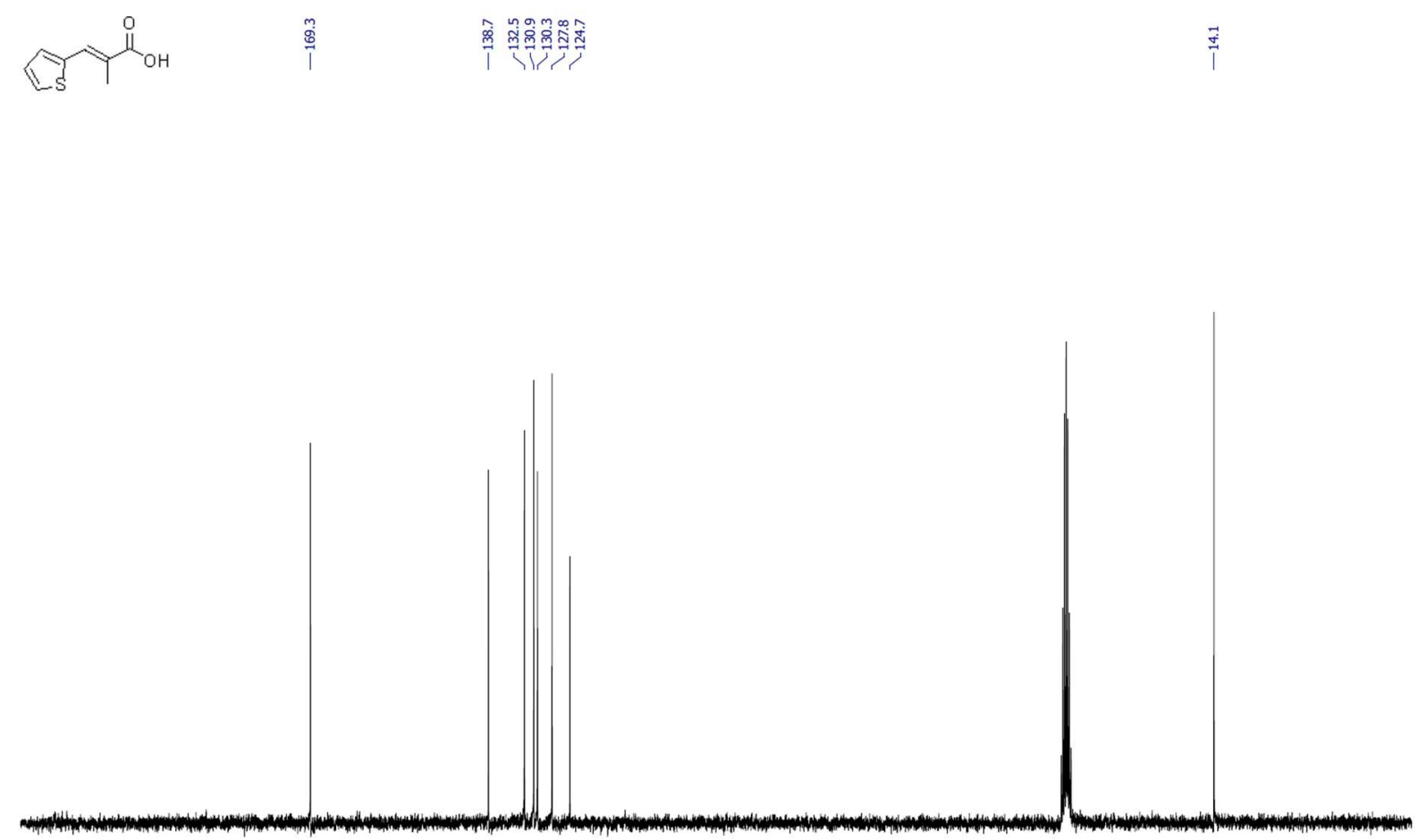

$\begin{array}{lllllllllllllllllllllll}210 & 200 & 190 & 180 & 170 & 160 & 150 & 140 & 130 & 120 & 110 & \underset{\mathrm{f} 1(\mathrm{ppm})}{100} & 90 & 80 & 70 & 60 & 50 & 40 & 30 & 20 & 10 & 0 & -10\end{array}$

(E)-3-(4-(cinnamyloxy)phenyl)-2-methylacrylic acid (Ac-26):

${ }^{1} \mathrm{H} \mathrm{NMR}\left(\mathrm{CDCl}_{3}, 300 \mathrm{MHz}\right)$

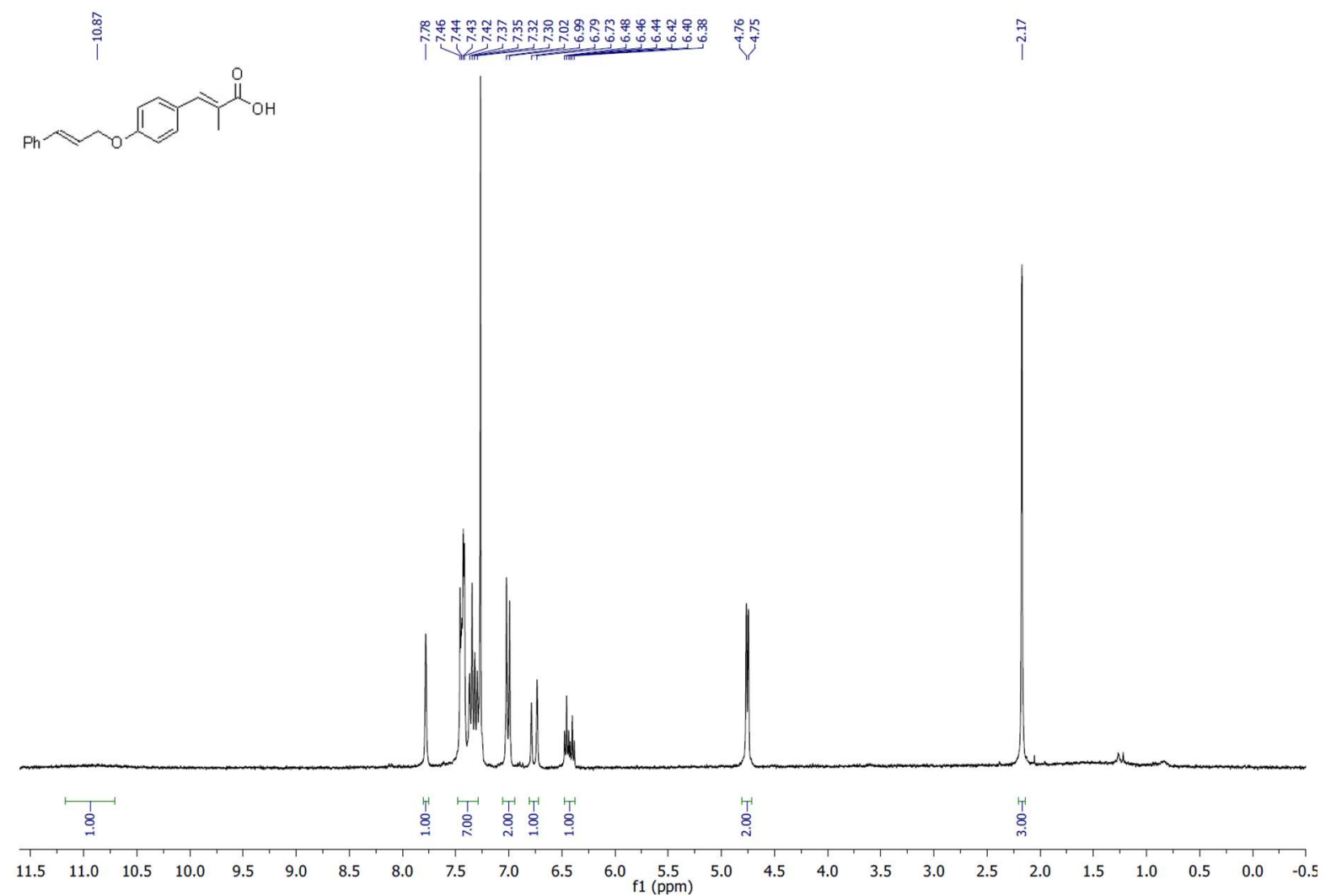


${ }^{13} \mathrm{C} \mathrm{NMR}\left(\mathrm{CDCl}_{3}, 75 \mathrm{MHz}\right)$
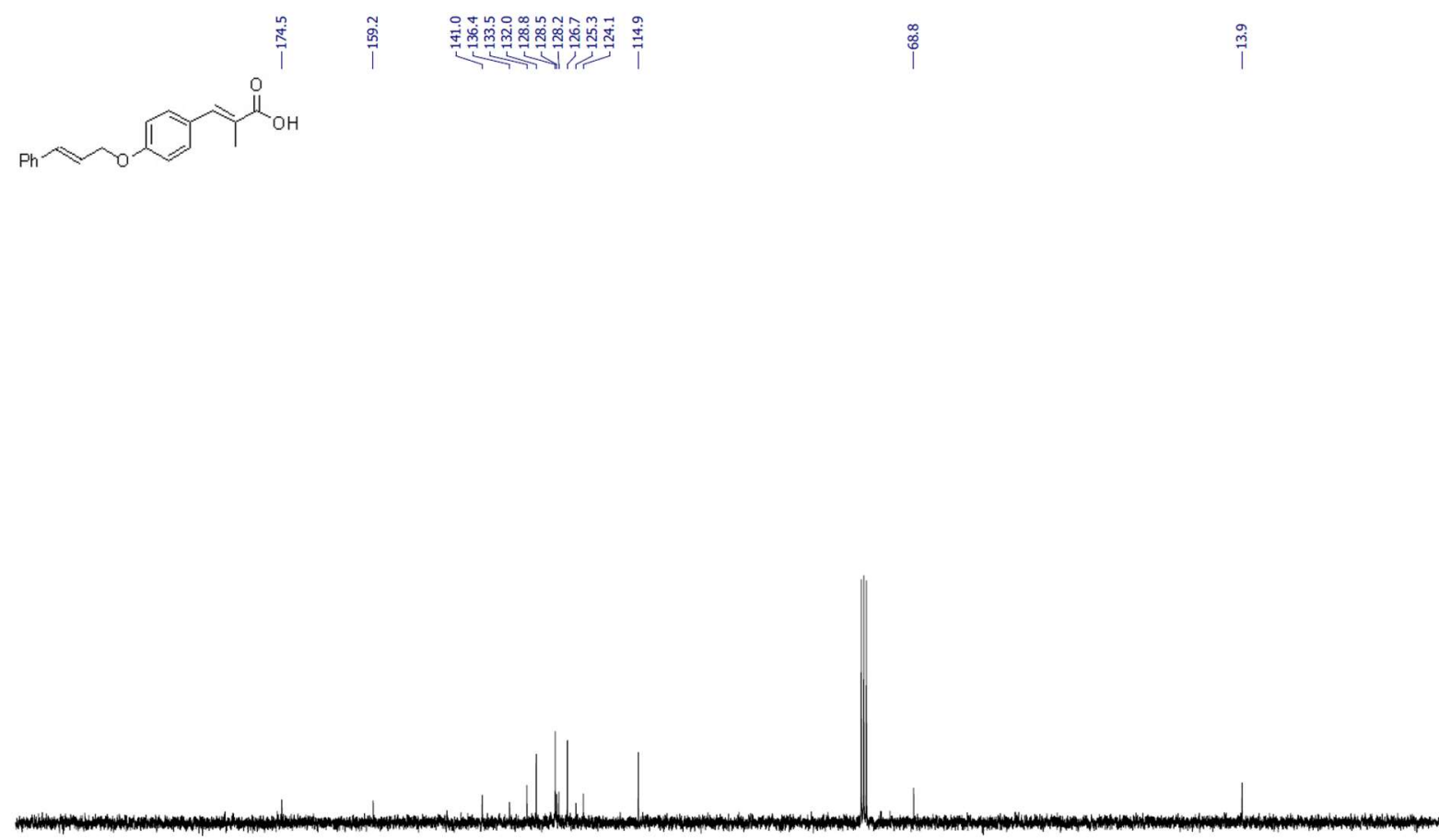

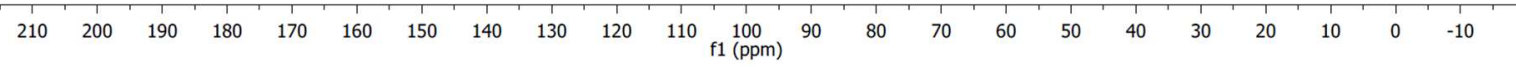

(E)-3-mesityl-2-methylacrylic acid (Ac-27):

${ }^{1} \mathrm{H}$ NMR $\left(\mathrm{CDCl}_{3}, 300 \mathrm{MHz}\right)$
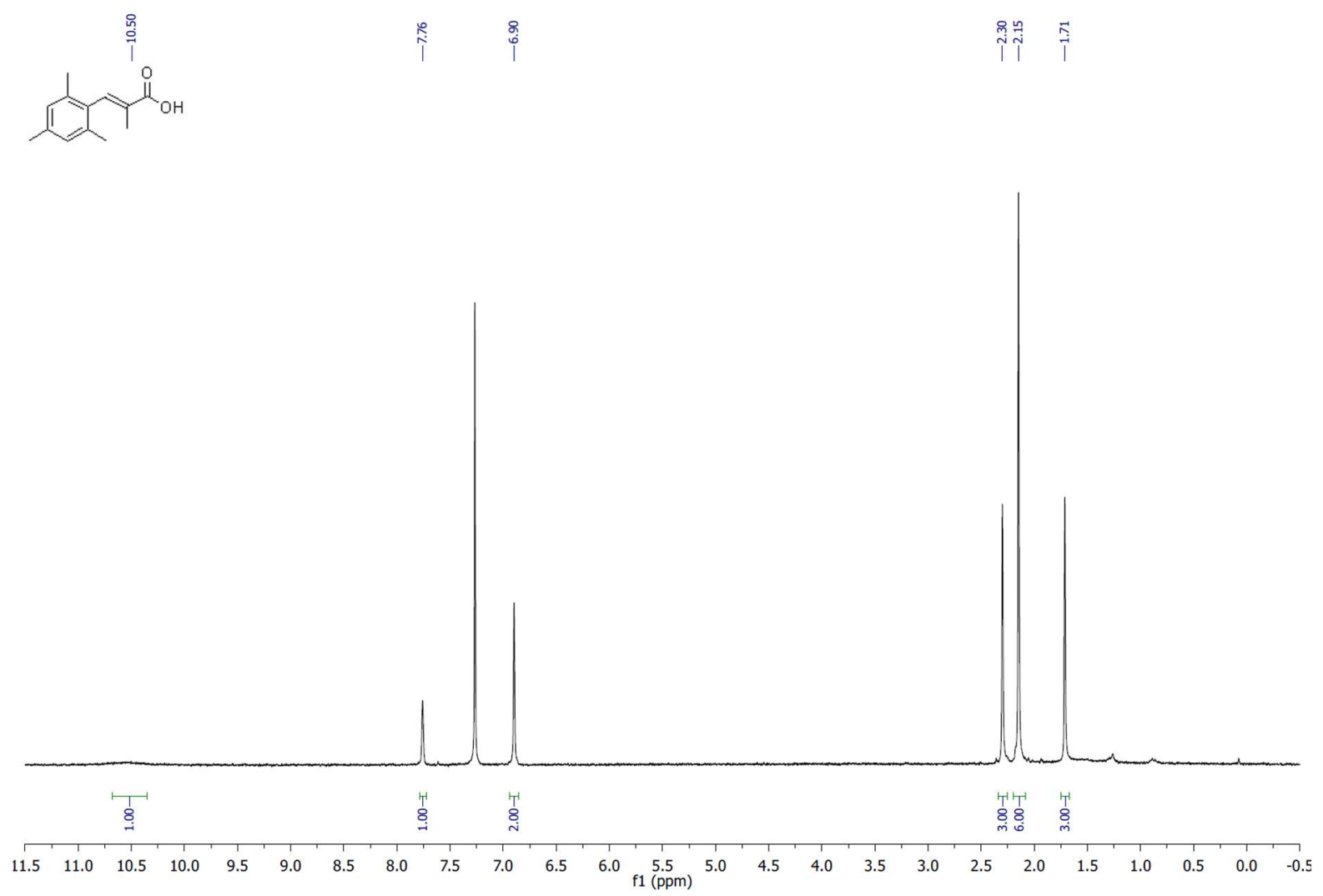
${ }^{13} \mathrm{C}$ NMR (DMSO-d6, $75 \mathrm{MHz}$ )
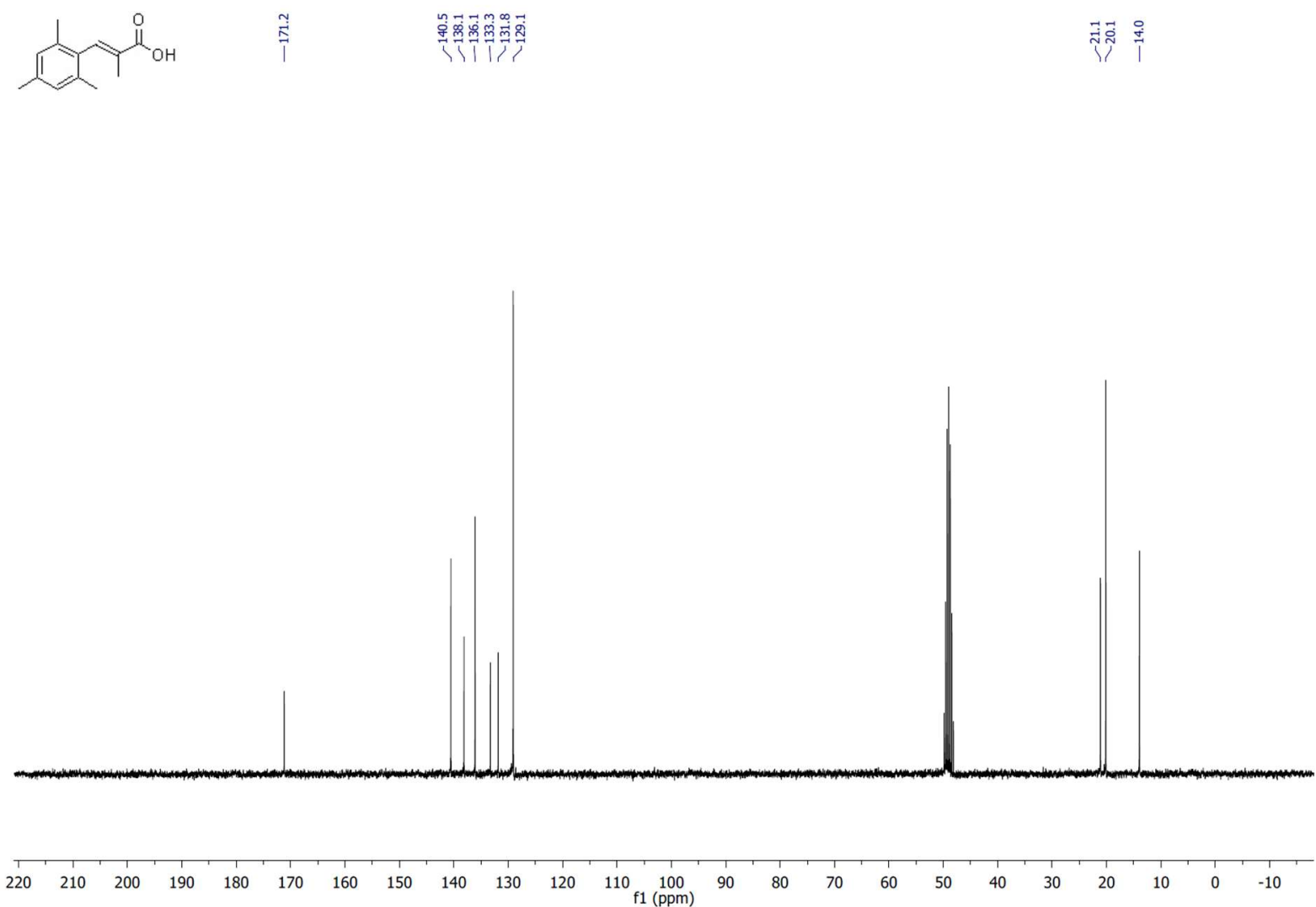

(E)-2-methyloct-2-enoic acid (Ac-28):

${ }^{1} \mathrm{H} \mathrm{NMR}\left(\mathrm{CDCl}_{3}, 300 \mathrm{MHz}\right)$
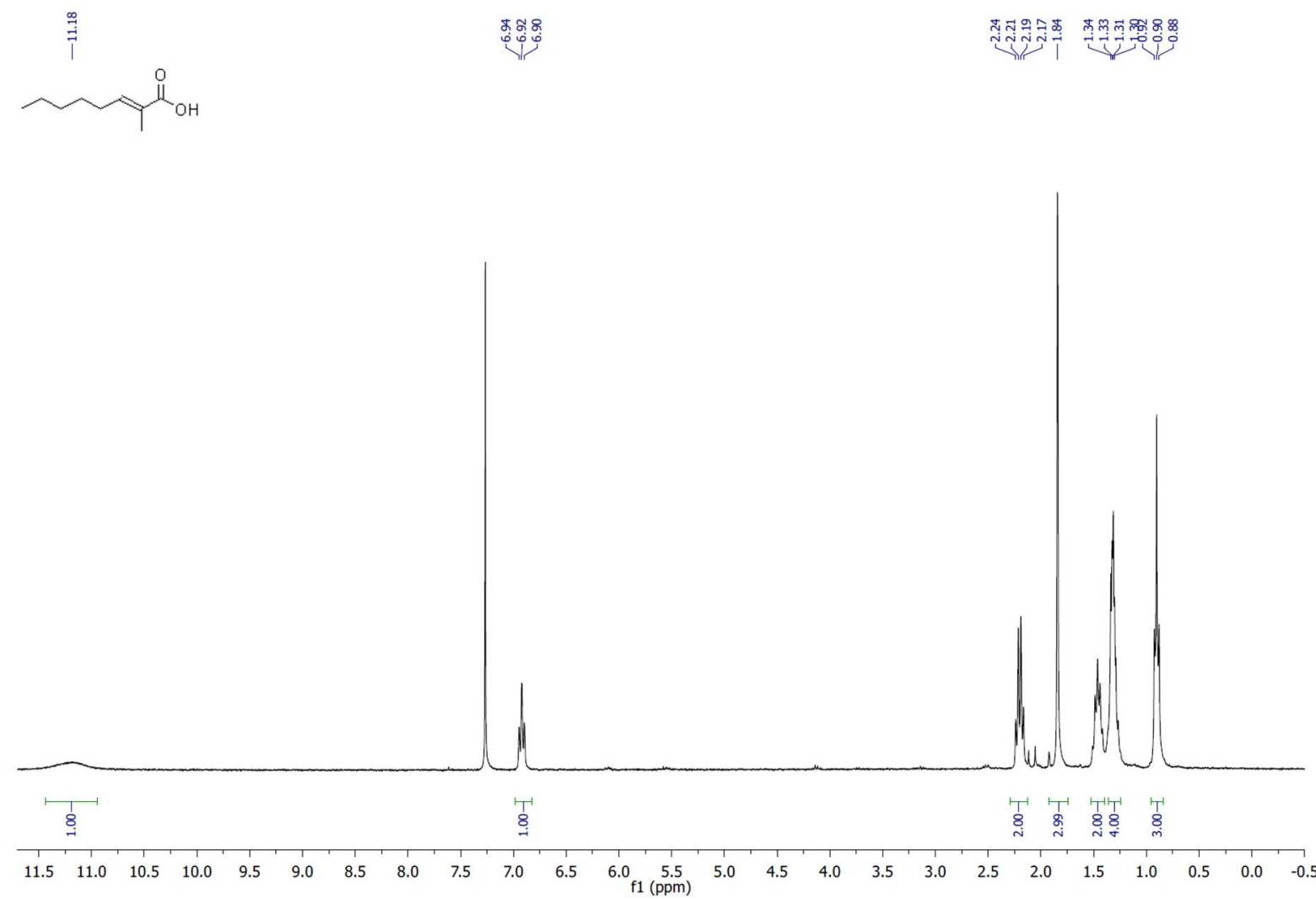
${ }^{13} \mathrm{C}$ NMR (DMSO-d6, $75 \mathrm{MHz}$ )

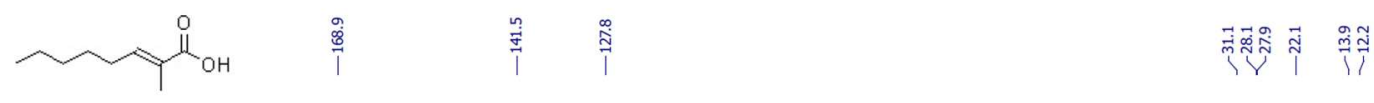

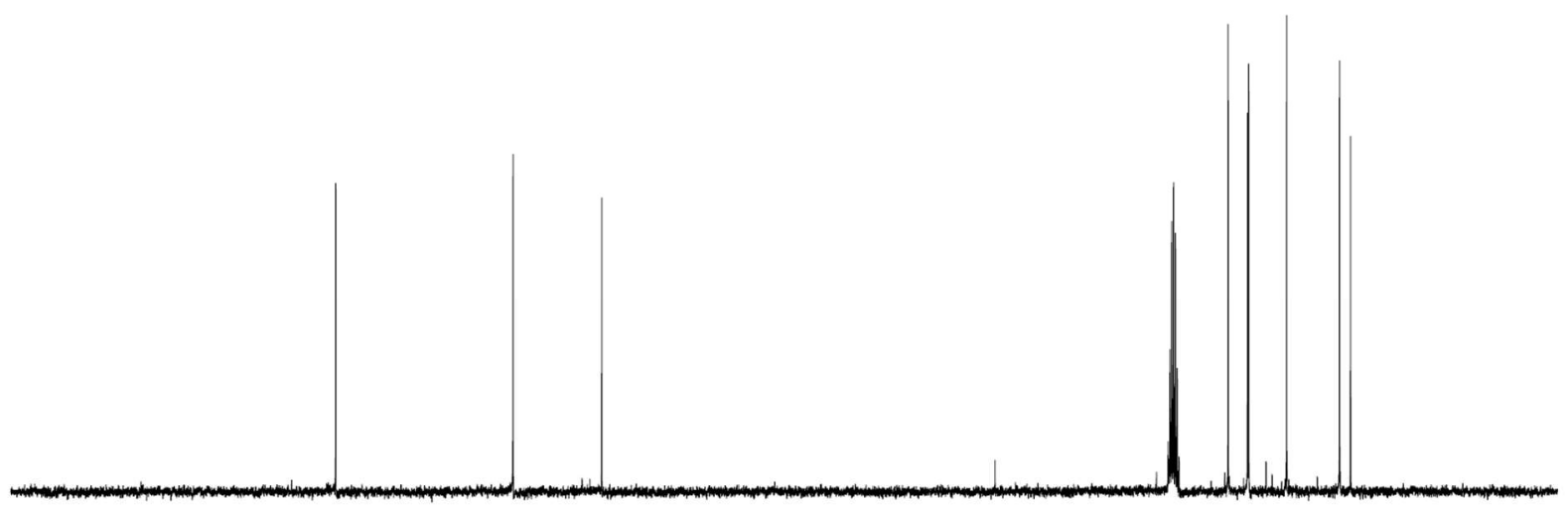

$\begin{array}{lllllllllllllllllllllll}210 & 200 & 190 & 180 & 170 & 160 & 150 & 140 & 130 & 120 & 110 & 100 & 90 & 80 & 70 & 60 & 50 & 40 & 30 & 20 & 10 & 0 & -10\end{array}$

(E)-3-(2-methyl-3-(p-tolyl)acryloyl)oxazolidin-2-one ((E)-9):

${ }^{1} \mathrm{H} \mathrm{NMR}\left(\mathrm{CDCl}_{3}, 300 \mathrm{MHz}\right)$
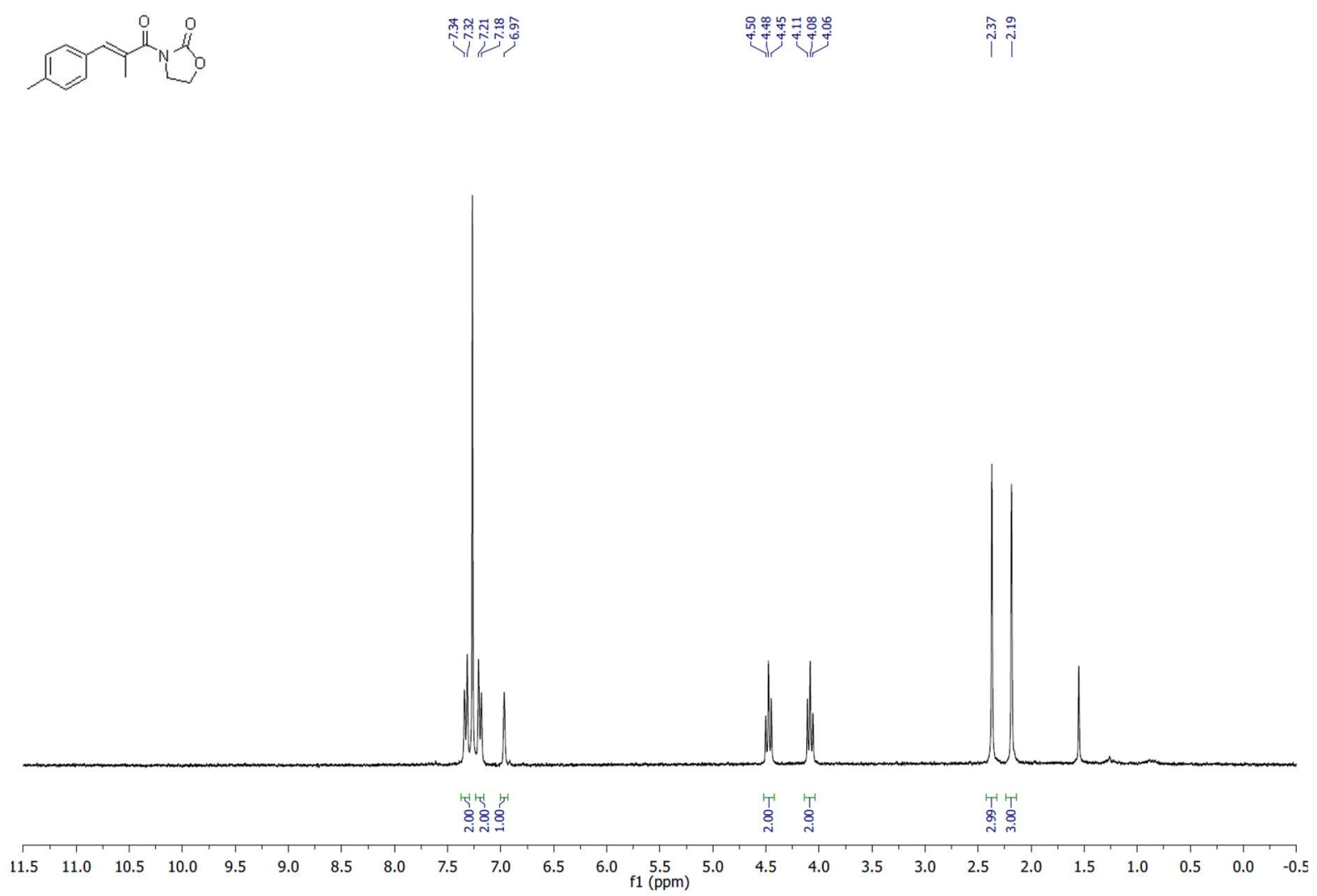
${ }^{13} \mathrm{C} \mathrm{NMR}\left(\mathrm{CDCl}_{3}, 75 \mathrm{MHz}\right)$
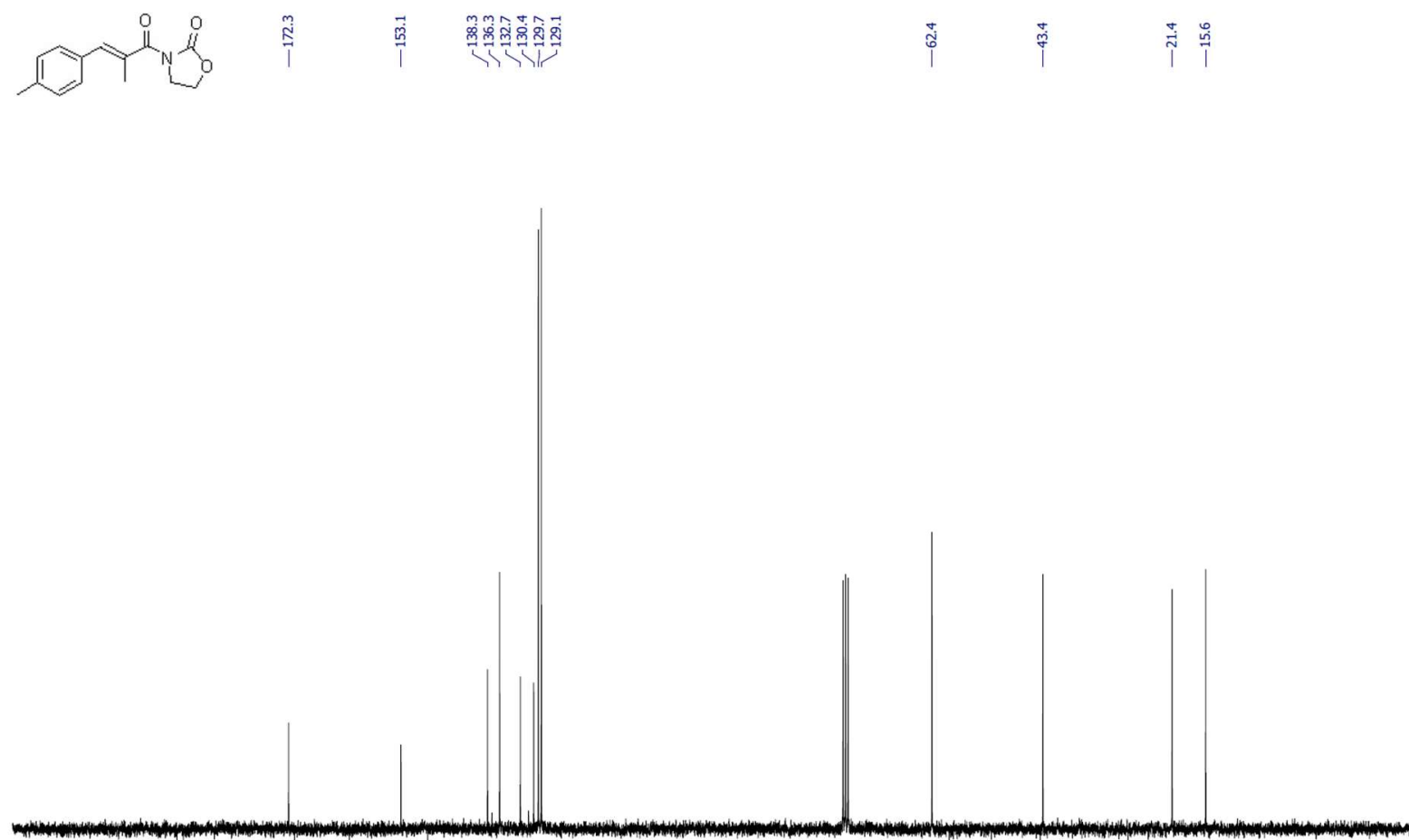

$\begin{array}{lllllllllllllllllllllll}210 & 200 & 190 & 180 & 170 & 160 & 150 & 140 & 130 & 120 & 110 & \begin{array}{l}100 \\ \mathrm{f} 1(\mathrm{ppm})\end{array} & 90 & 80 & 70 & 60 & 50 & 40 & 30 & 20 & 10 & 0 & -10\end{array}$

(E)-3-(2-methyl-3-(m-tolyl)acryloyl)oxazolidin-2-one ((E)-10):

${ }^{1} \mathrm{H} \mathrm{NMR}\left(\mathrm{CDCl}_{3}, 300 \mathrm{MHz}\right)$
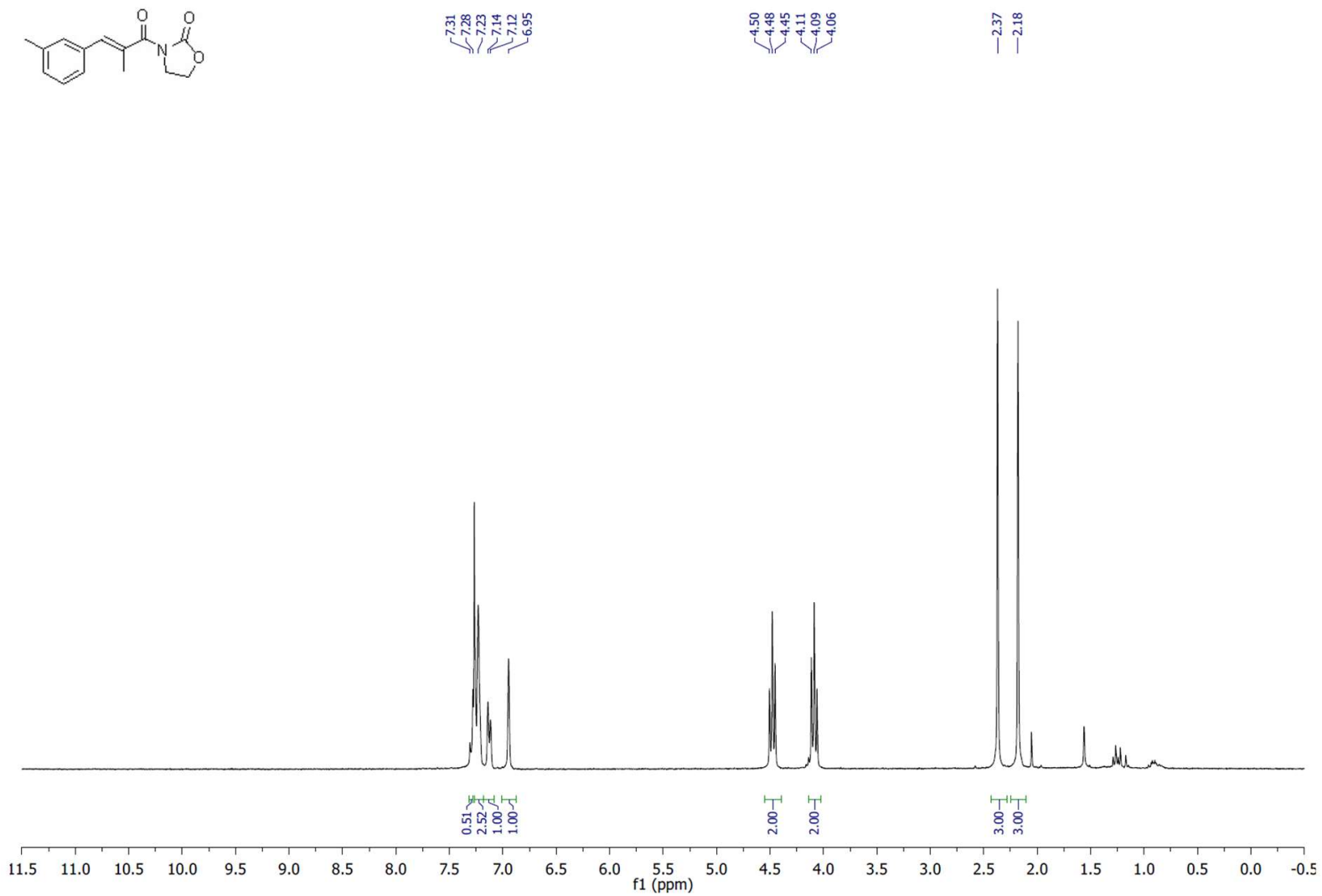
${ }^{13} \mathrm{C} \mathrm{NMR}\left(\mathrm{CDCl}_{3}, 75 \mathrm{MHz}\right)$
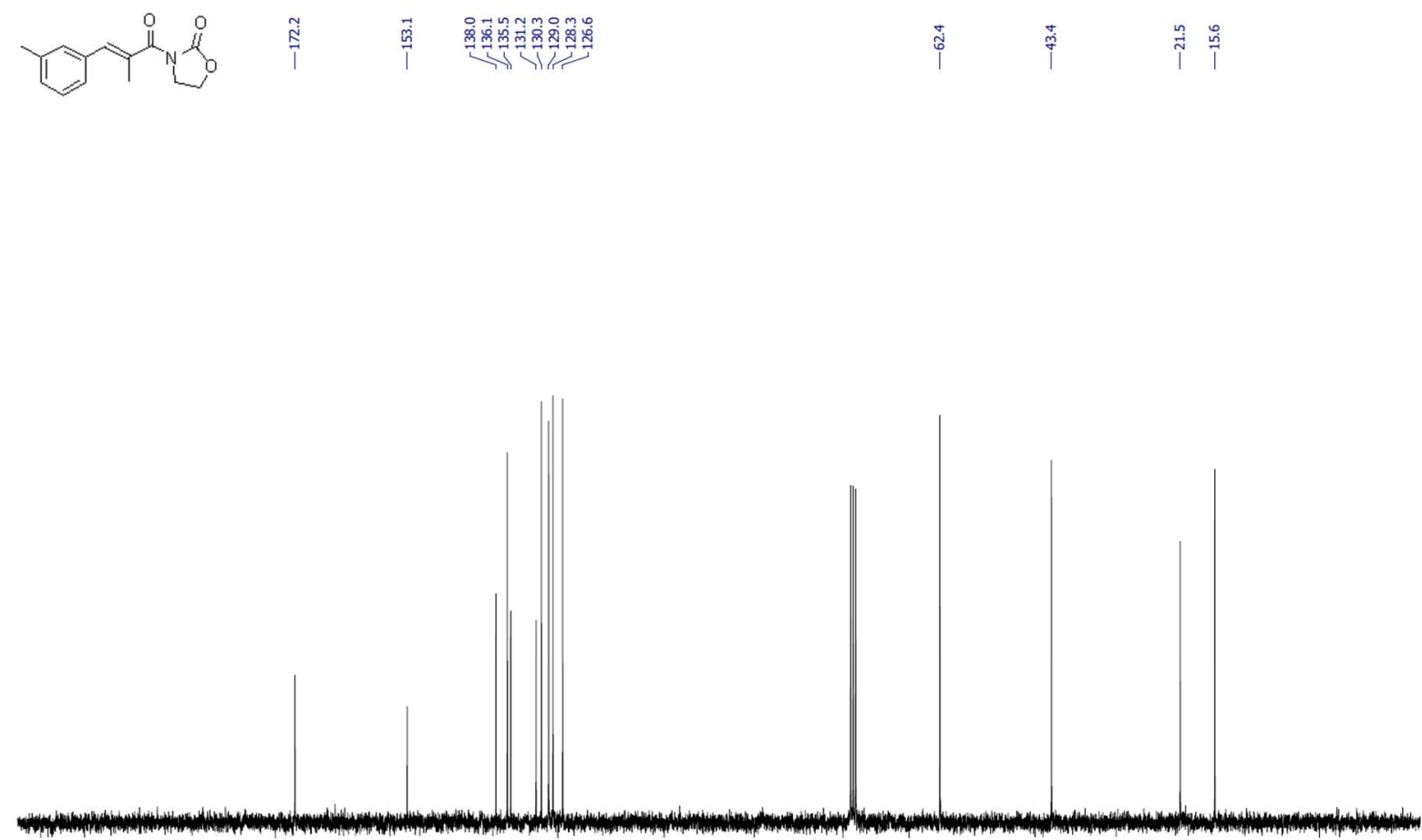

$\begin{array}{lllllllllllllllllllllll}210 & 200 & 190 & 180 & 170 & 160 & 150 & 140 & 130 & 120 & 110 & \underset{\mathrm{f} 1(\mathrm{ppm})}{100} & 90 & 80 & 70 & 60 & 50 & 40 & 30 & 20 & 10 & 0 & -10\end{array}$

(E)-3-(2-methyl-3-(o-tolyl)acryloyl)oxazolidin-2-one ((E)-11):

${ }^{1} \mathrm{H} \mathrm{NMR}\left(\mathrm{CDCl}_{3}, 300 \mathrm{MHz}\right)$
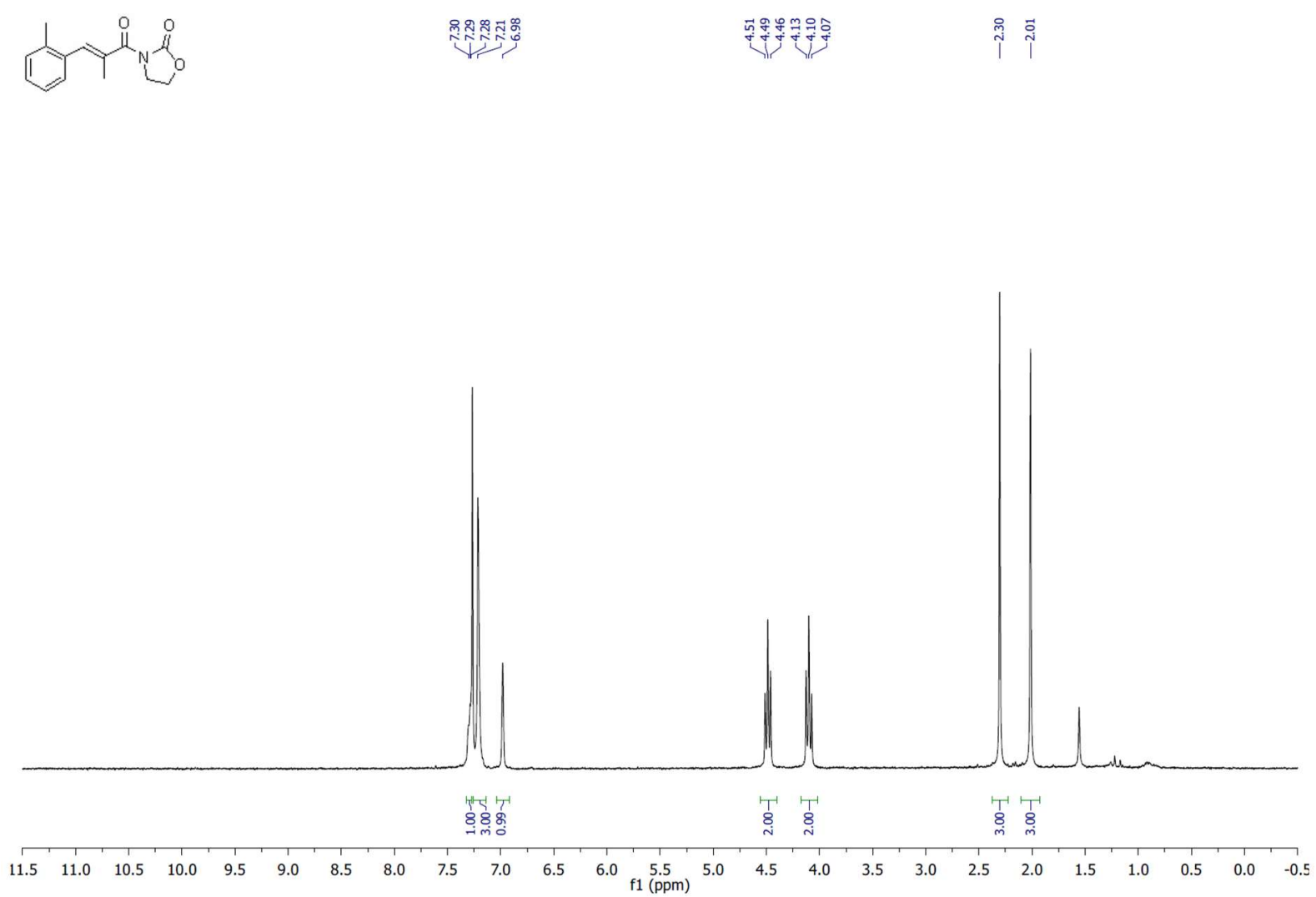
${ }^{13} \mathrm{C} \mathrm{NMR}\left(\mathrm{CDCl}_{3}, 75 \mathrm{MHz}\right)$

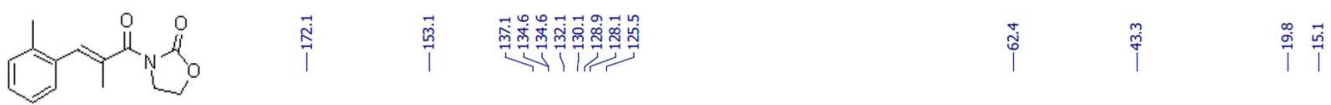

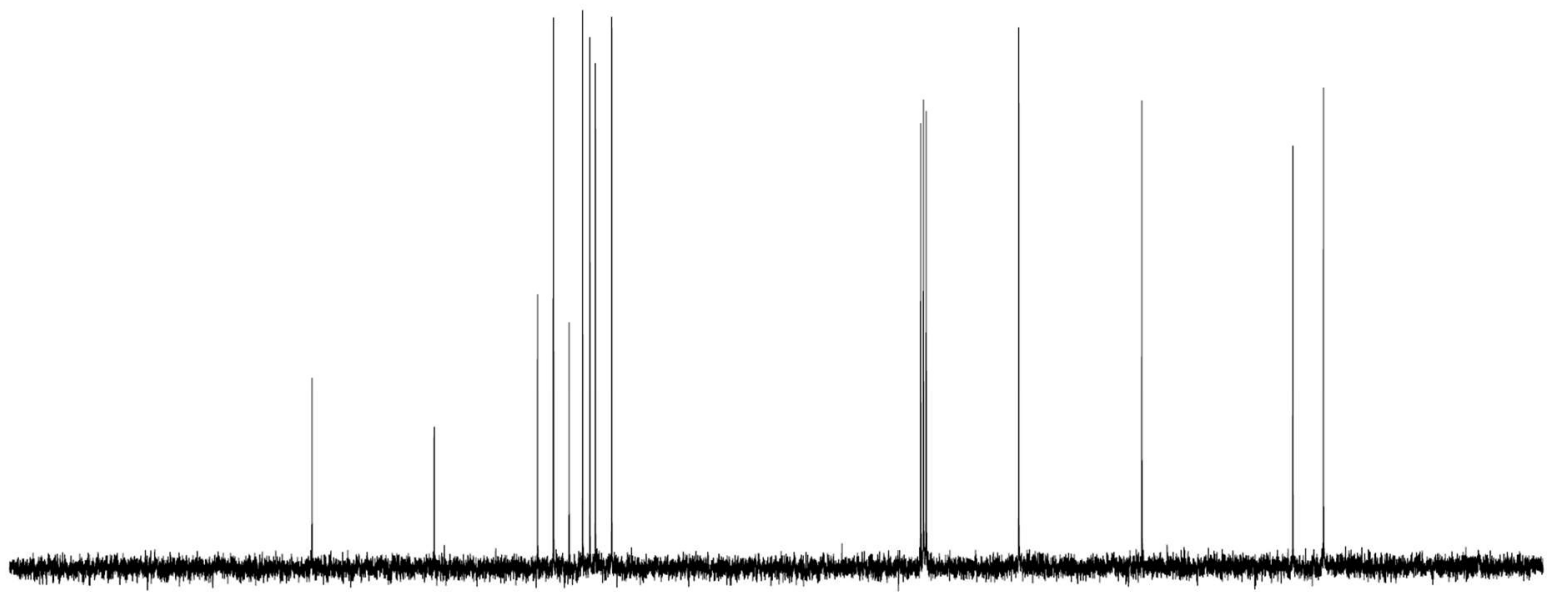

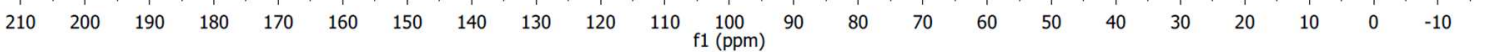

(E)-3-(3-(4-(tert-butyl)phenyl)-2-methylacryloyl)oxazolidin-2-one ((E)-12):

${ }^{1} \mathrm{H} \operatorname{NMR}\left(\mathrm{CDCl}_{3}, 300 \mathrm{MHz}\right)$
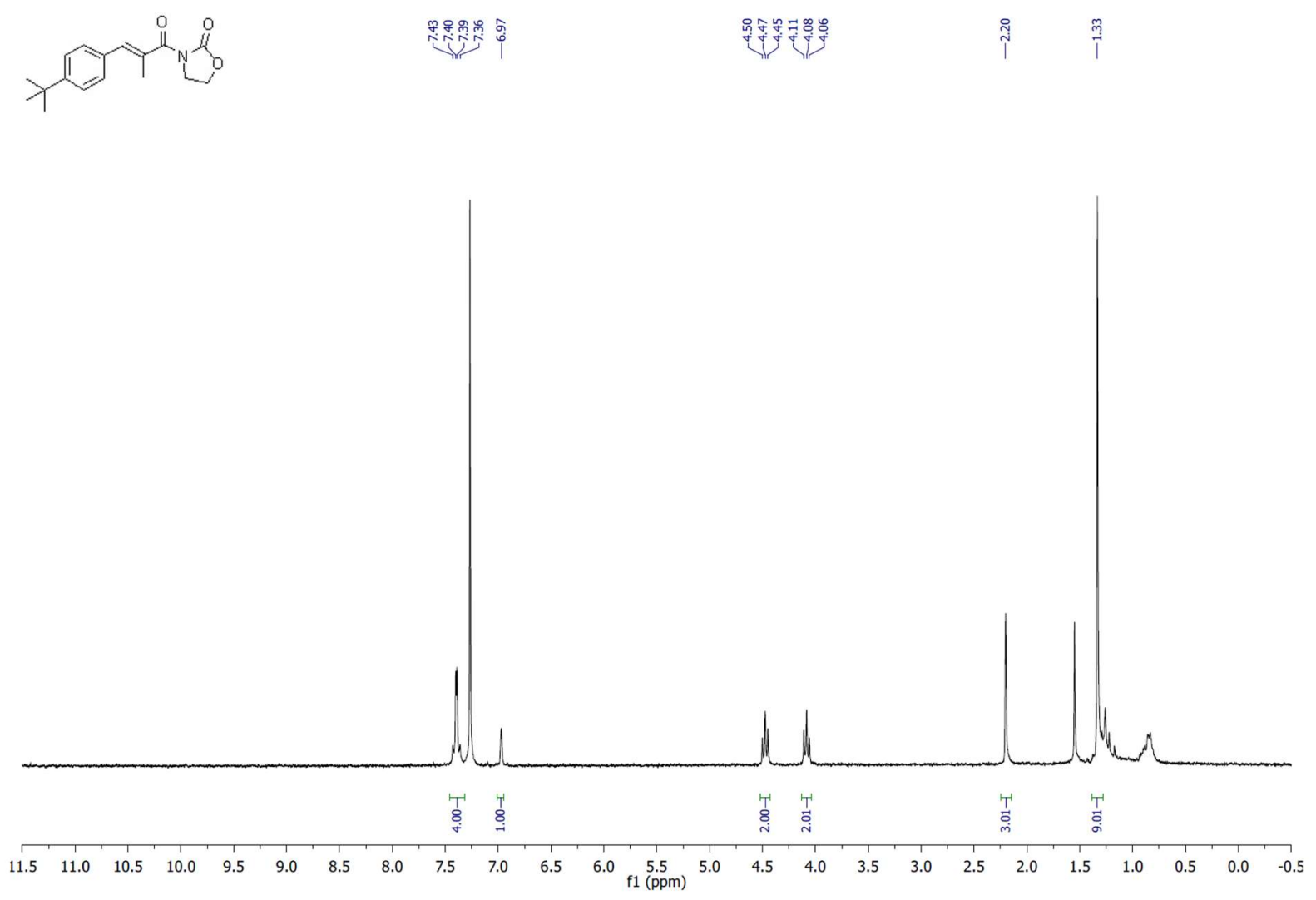
${ }^{13} \mathrm{C} \mathrm{NMR}\left(\mathrm{CDCl}_{3}, 75 \mathrm{MHz}\right)$
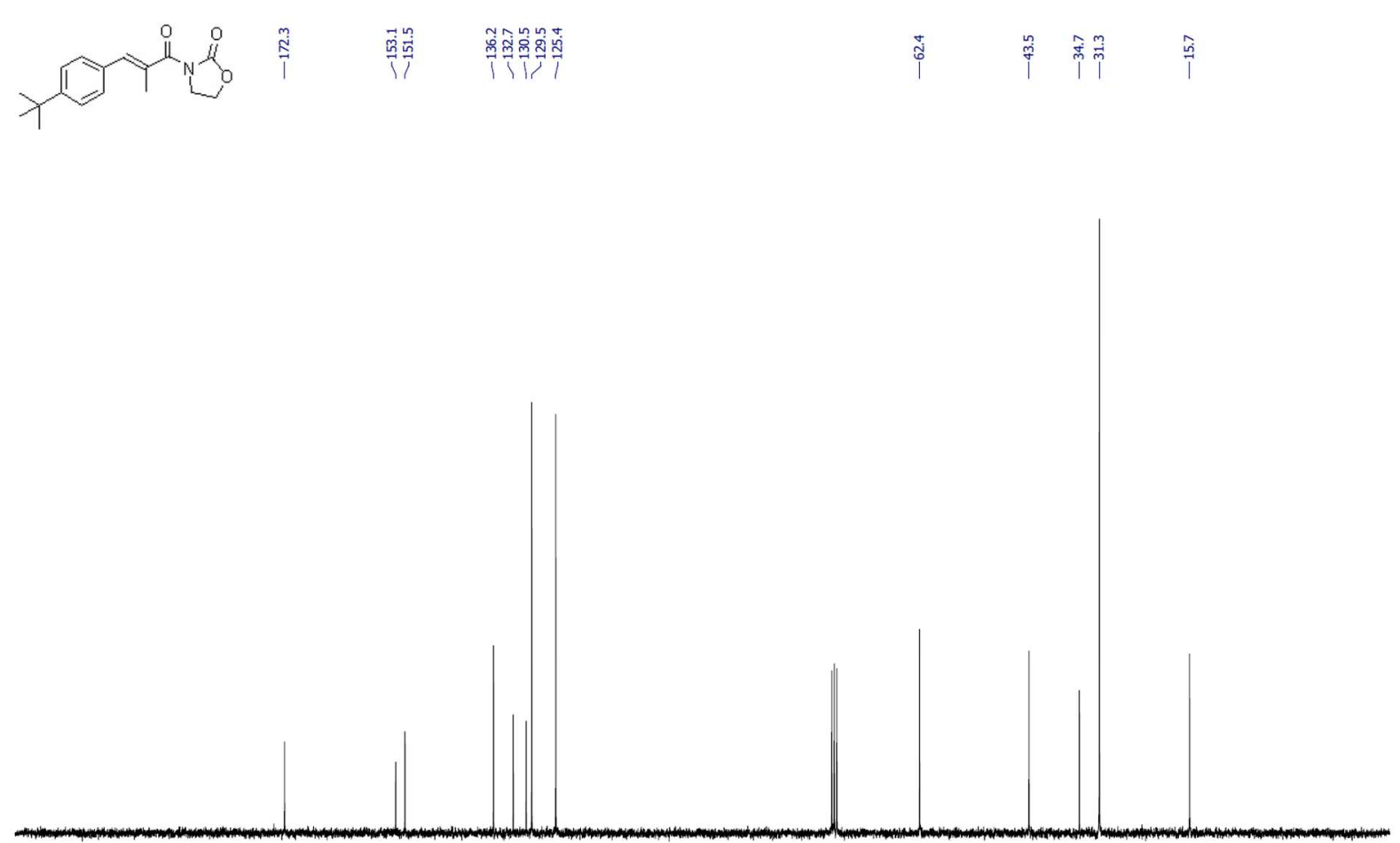

$\begin{array}{llllllllllllllllllllllllll}210 & 200 & 190 & 180 & 170 & 160 & 150 & 140 & 130 & 120 & 110 & \begin{array}{c}100 \\ \mathrm{f} 1(\mathrm{ppm})\end{array} & 90 & 80 & 70 & 60 & 50 & 40 & 30 & 20 & 10 & 0 & -10\end{array}$

(E)-3-(3-([1,1'-biphenyl]-4-yl)-2-methylacryloyl)oxazolidin-2-one ((E)-13):

${ }^{1} \mathrm{H} \mathrm{NMR}\left(\mathrm{CDCl}_{3}, 300 \mathrm{MHz}\right)$
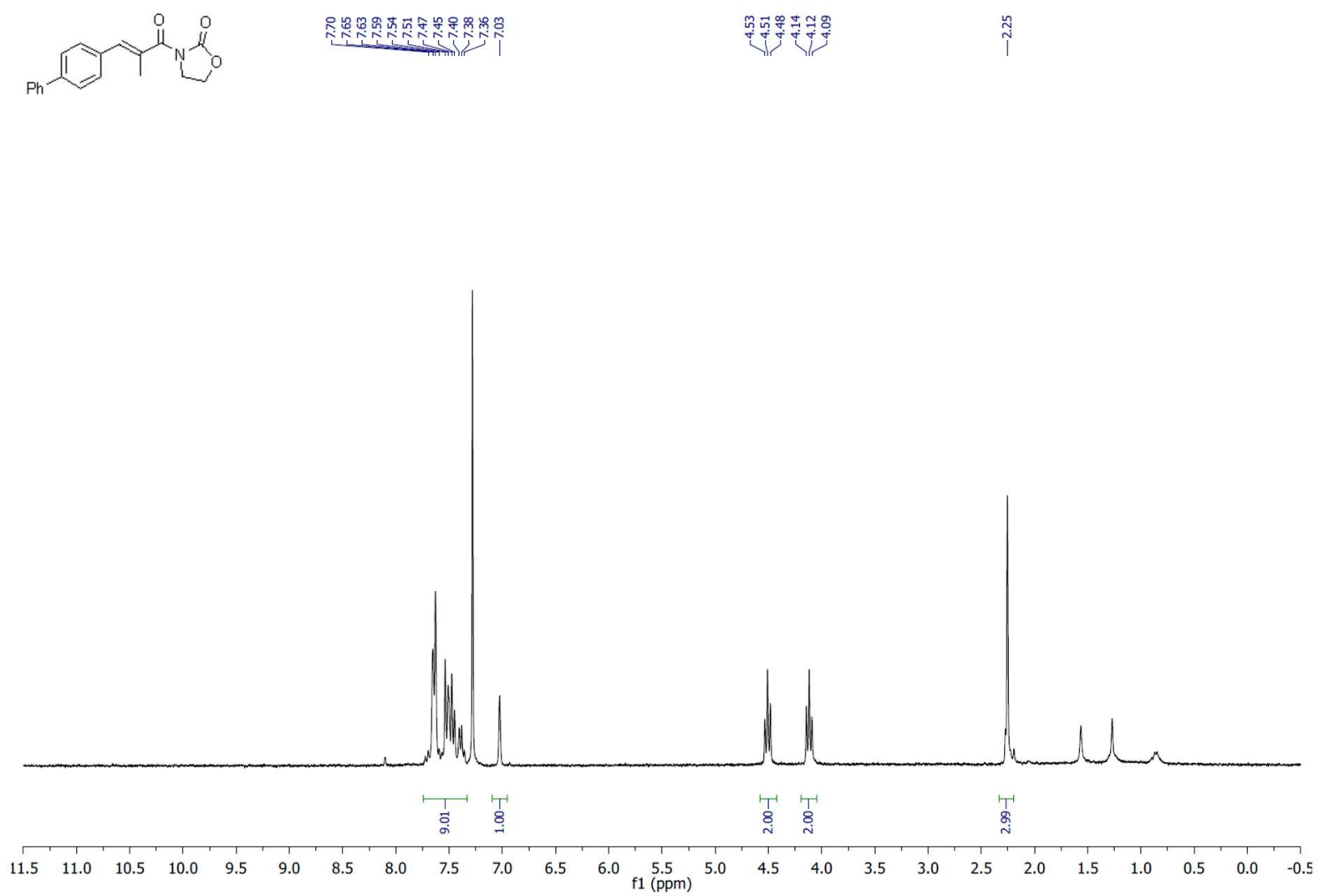
${ }^{13} \mathrm{C}$ NMR (acetone-d6, $75 \mathrm{MHz}$ )

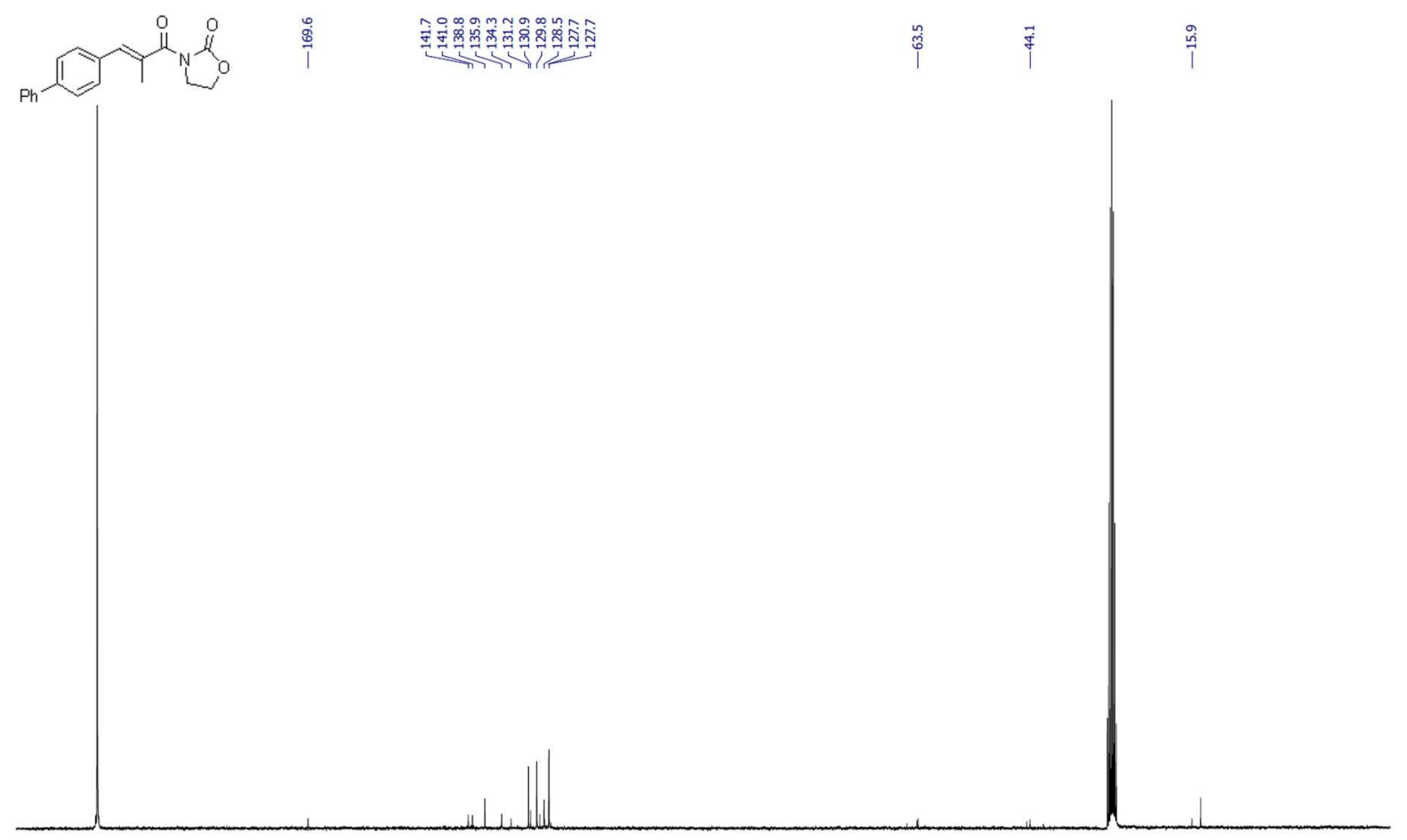

\begin{tabular}{rllllllllllllllllllllllllll}
\hline 220 & 210 & 200 & 190 & 180 & 170 & 160 & 150 & 140 & 130 & 120 & 110 & 100 & 90 & 80 & 70 & 60 & 50 & 40 & 30 & 20 & 10 & 0 & -10
\end{tabular}

(E)-3-(3-(4-methoxyphenyl)-2-methylacryloyl)oxazolidin-2-one ((E)-14):

${ }^{1} \mathrm{H} \mathrm{NMR}\left(\mathrm{CDCl}_{3}, 300 \mathrm{MHz}\right)$
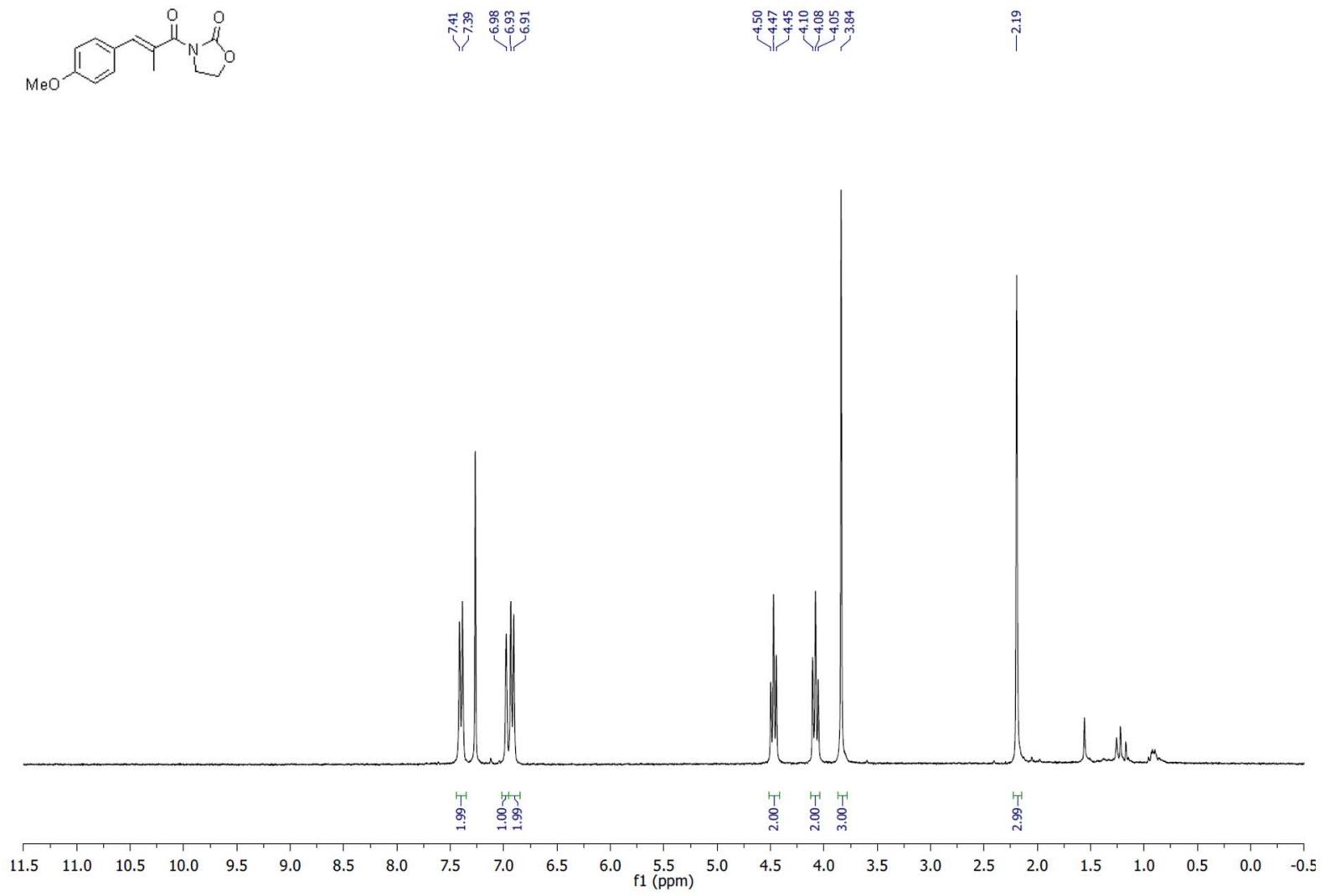
${ }^{13} \mathrm{C} \mathrm{NMR}\left(\mathrm{CDCl}_{3}, 75 \mathrm{MHz}\right)$
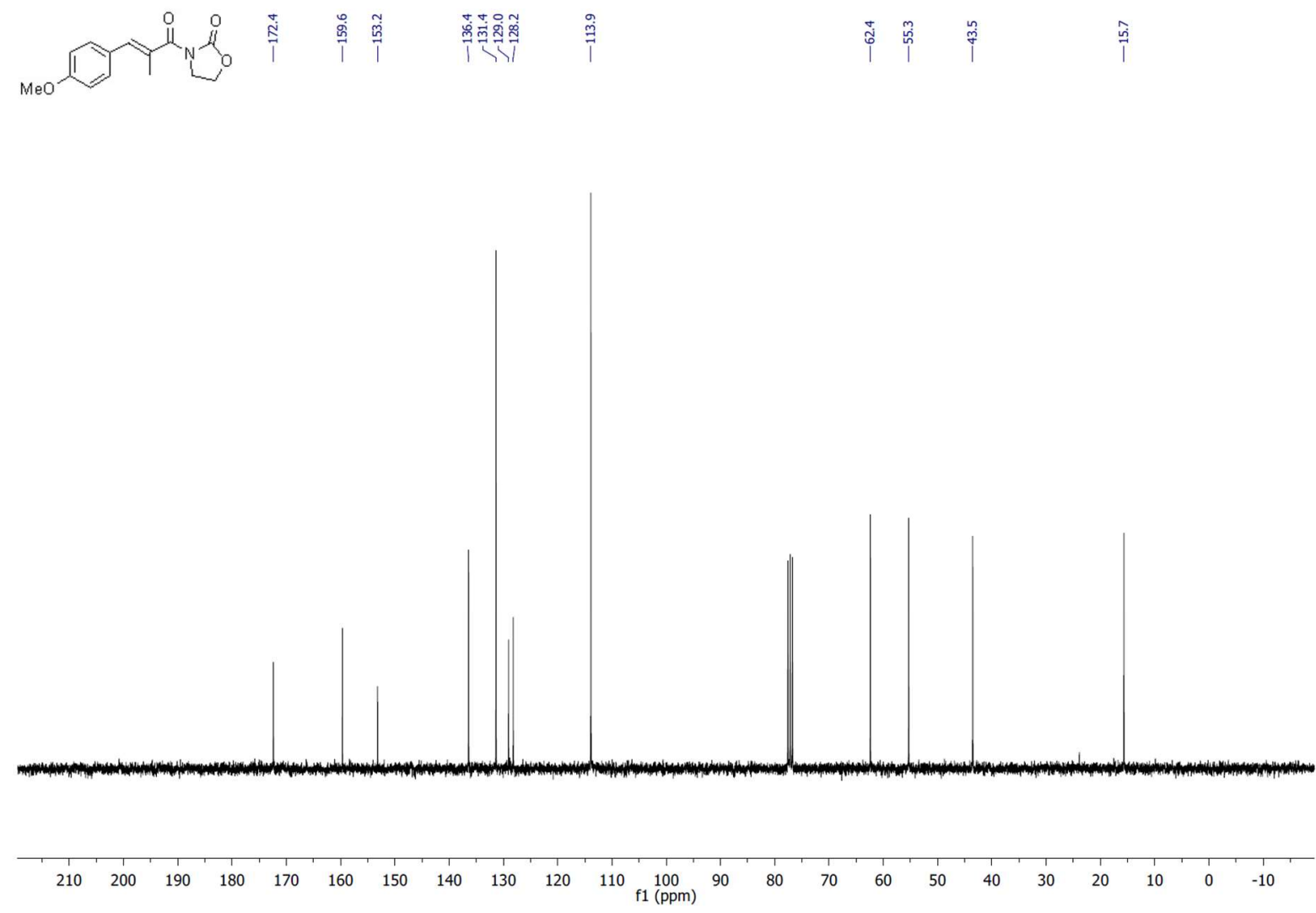

(E)-3-(2-methyl-3-(naphthalen-2-yl)acryloyl)oxazolidin-2-one ((E)-15):

${ }^{1} \mathrm{H} \mathrm{NMR}\left(\mathrm{CDCl}_{3}, 300 \mathrm{MHz}\right)$
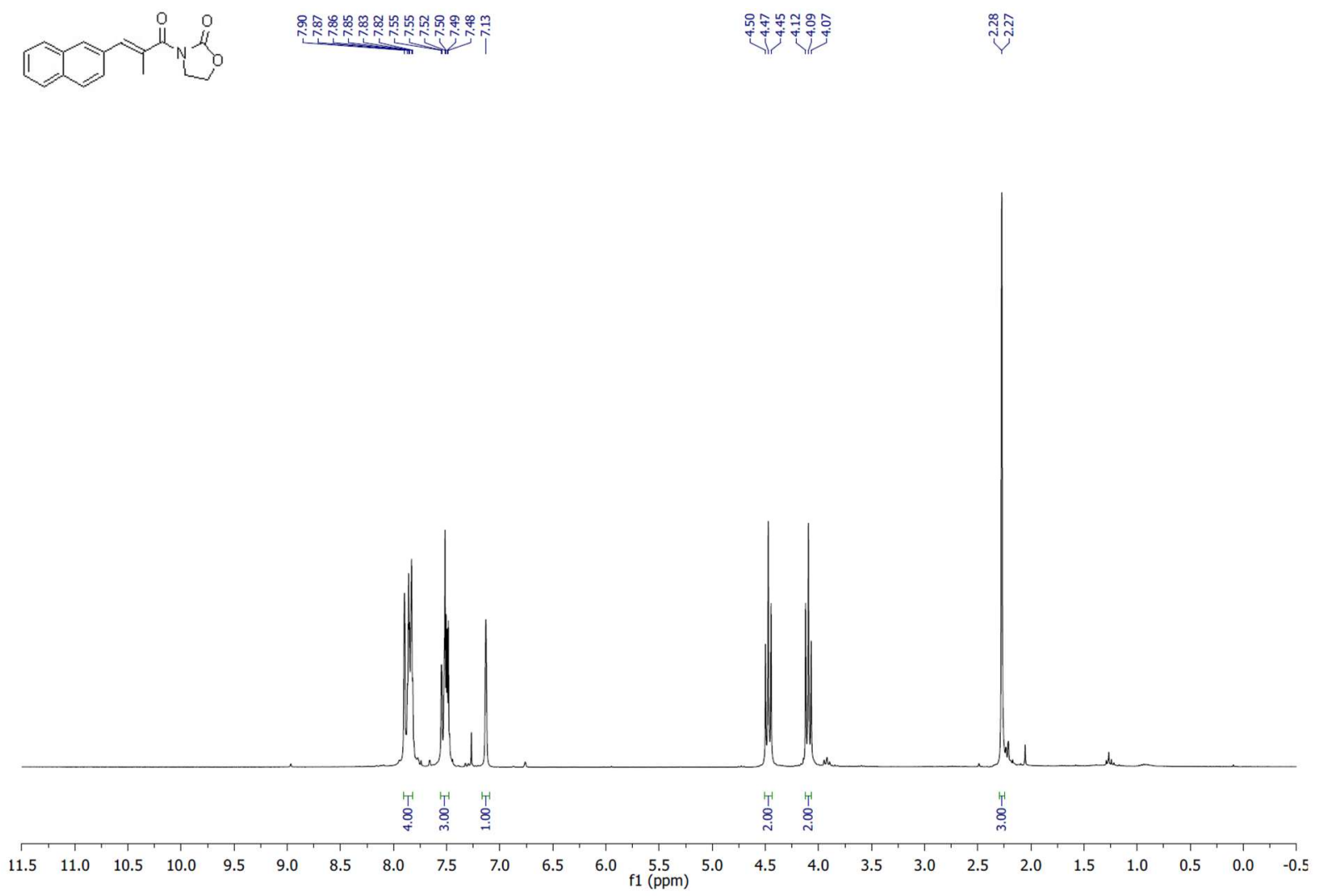
${ }^{13} \mathrm{C} \mathrm{NMR}\left(\mathrm{CDCl}_{3}, 75 \mathrm{MHz}\right)$
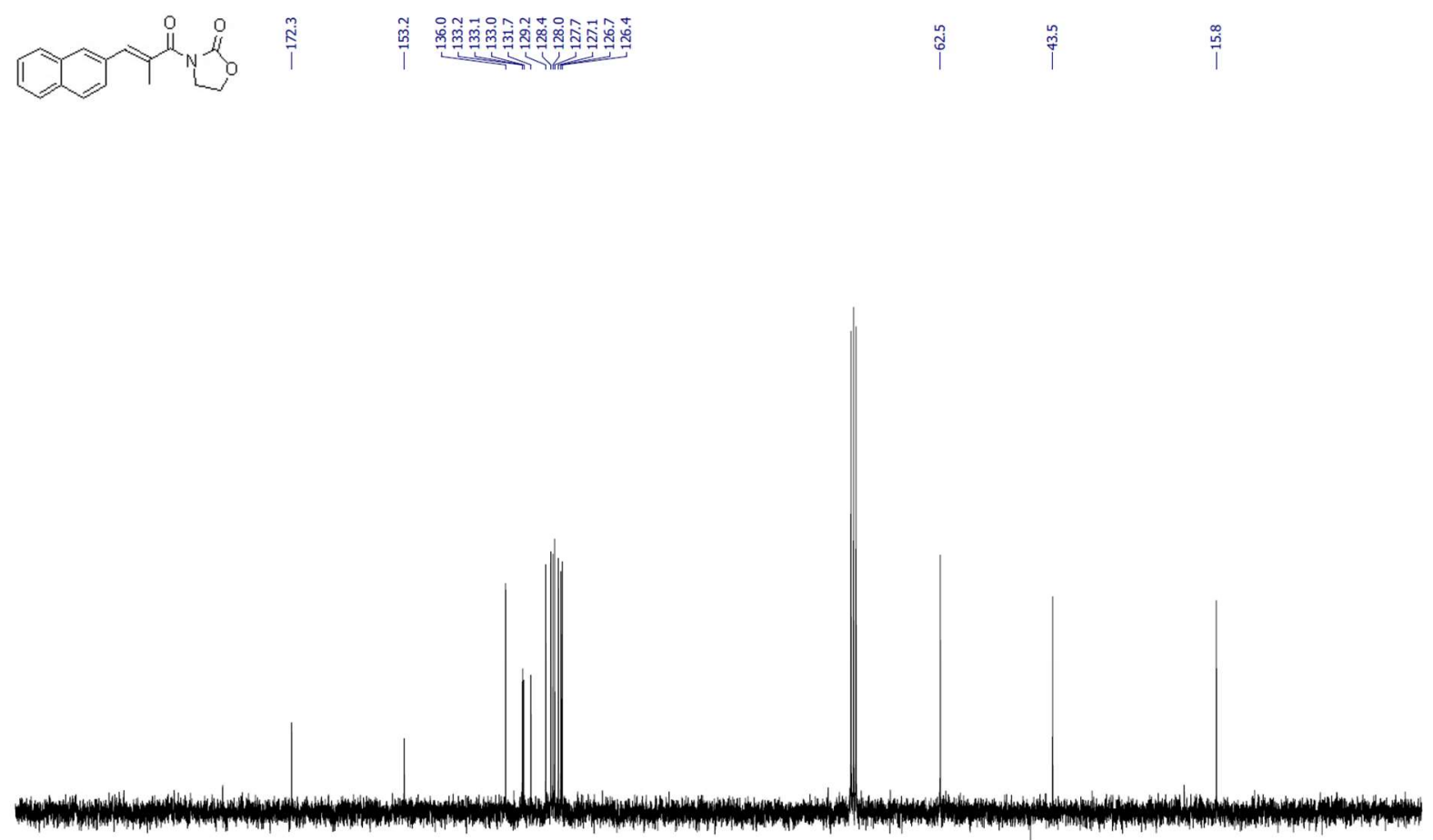

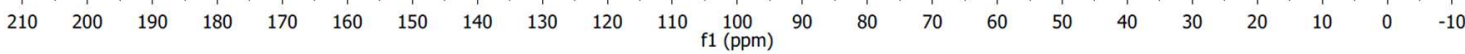

(E)-3-(3-(4-fluorophenyl)-2-methylacryloyl)oxazolidin-2-one ((E)-16):

${ }^{1} \mathrm{H} \mathrm{NMR}\left(\mathrm{CDCl}_{3}, 300 \mathrm{MHz}\right)$

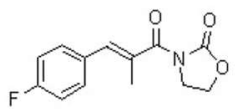

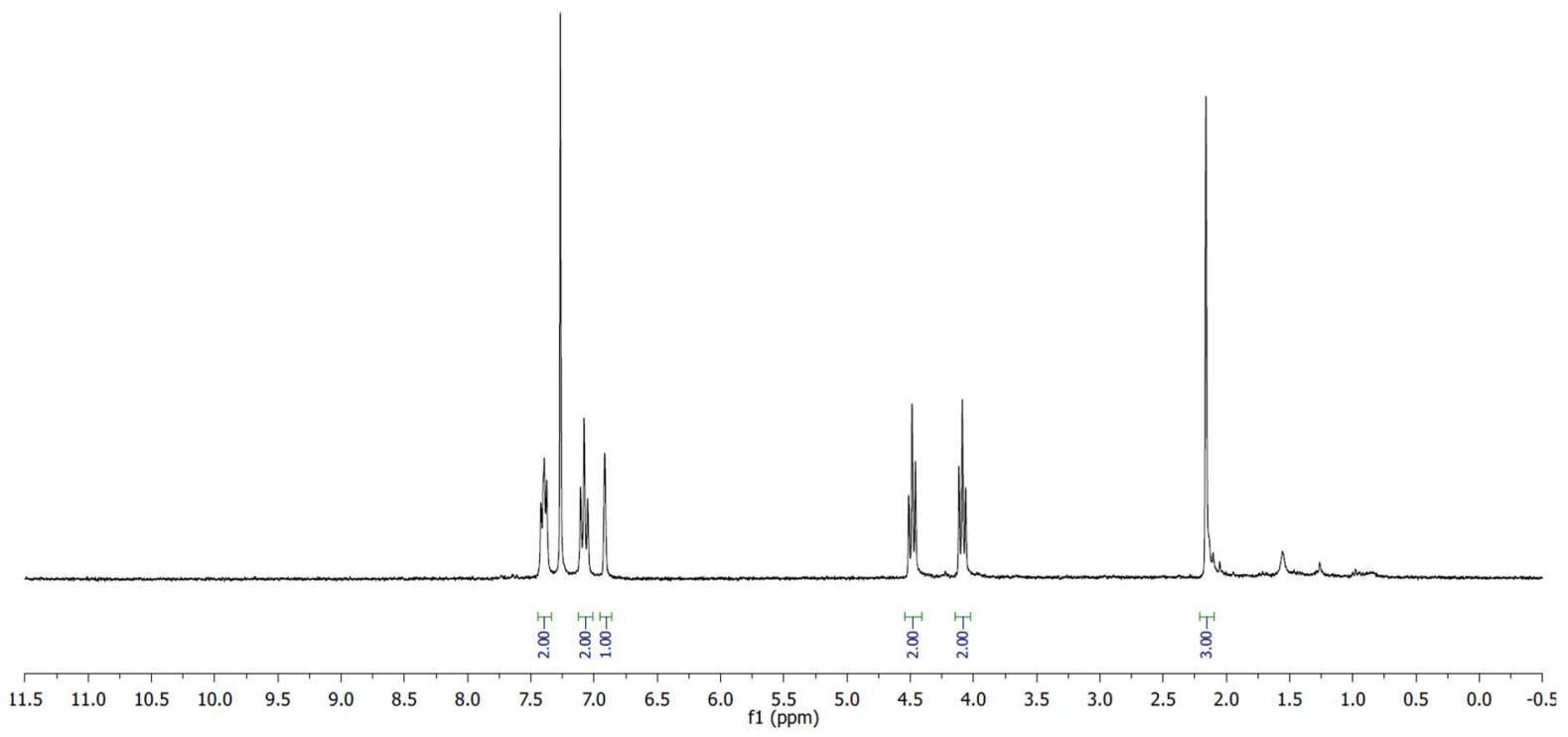


${ }^{13} \mathrm{C} \mathrm{NMR}\left(\mathrm{CDCl}_{3}, 75 \mathrm{MHz}\right)$

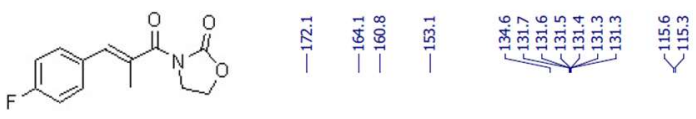

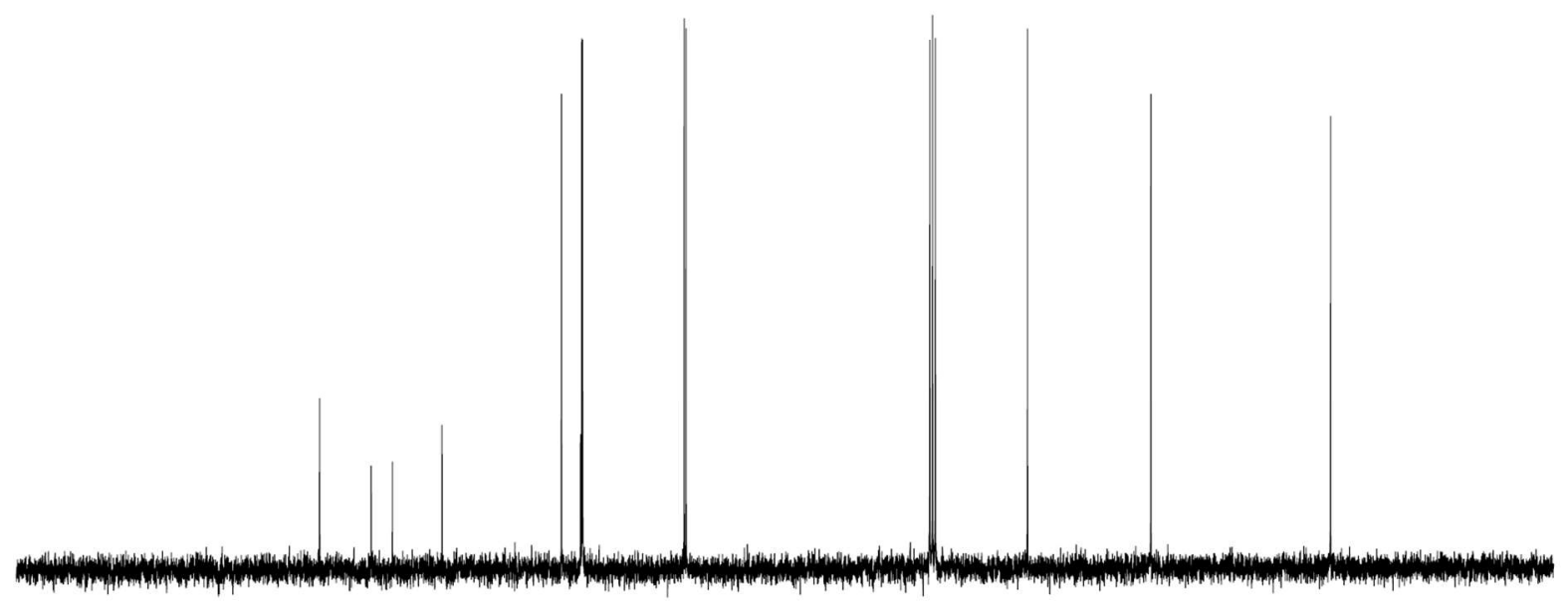

$\begin{array}{llllllllllllllllllllllllll}210 & 200 & 190 & 180 & 170 & 160 & 150 & 140 & 130 & 120 & 110 & 100 & 90 & 80 & 70 & 60 & 50 & 40 & 30 & 20 & 10 & 0 & -10\end{array}$

${ }^{19} \mathrm{~F} \mathrm{NMR}\left(\mathrm{CDCl}_{3}, 282 \mathrm{MHz}\right)$
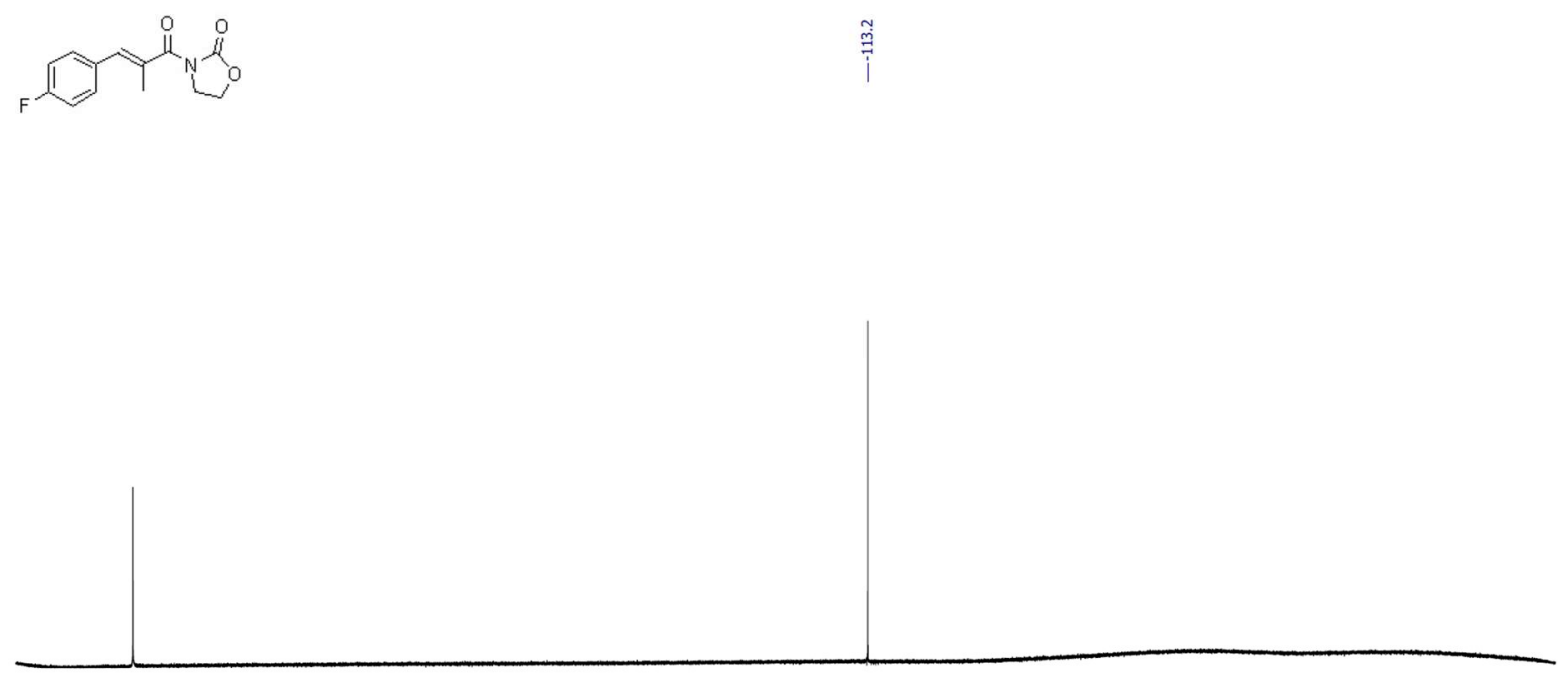

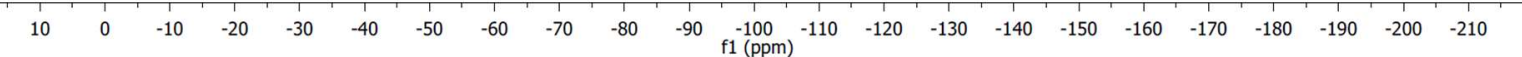


(E)-3-(3-(4-chlorophenyl)-2-methylacryloyl)oxazolidin-2-one ((E)-17):

${ }^{1} \mathrm{H} \mathrm{NMR}\left(\mathrm{CDCl}_{3}, 300 \mathrm{MHz}\right)$
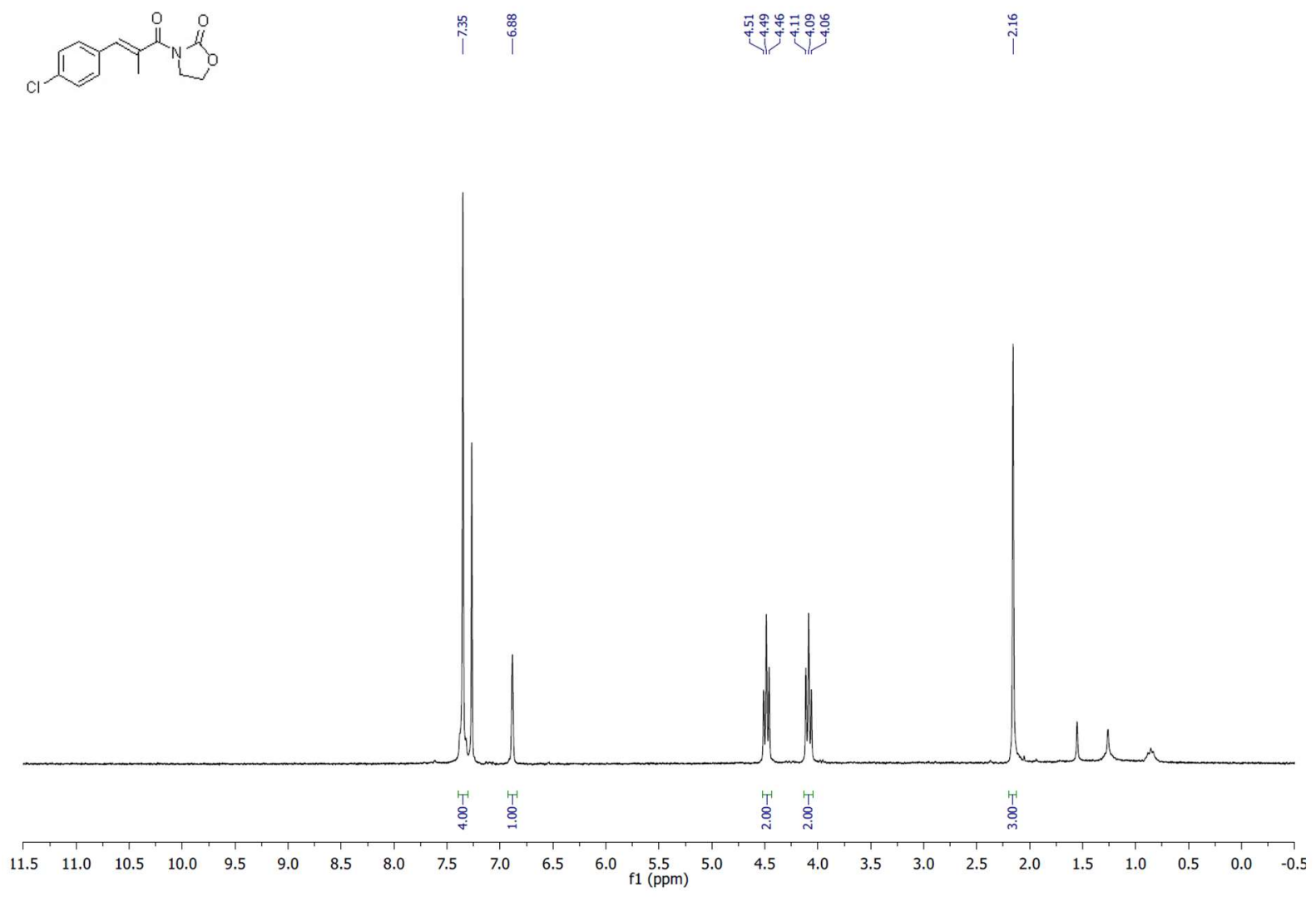

${ }^{13} \mathrm{C} \mathrm{NMR}\left(\mathrm{CDCl}_{3}, 75 \mathrm{MHz}\right)$
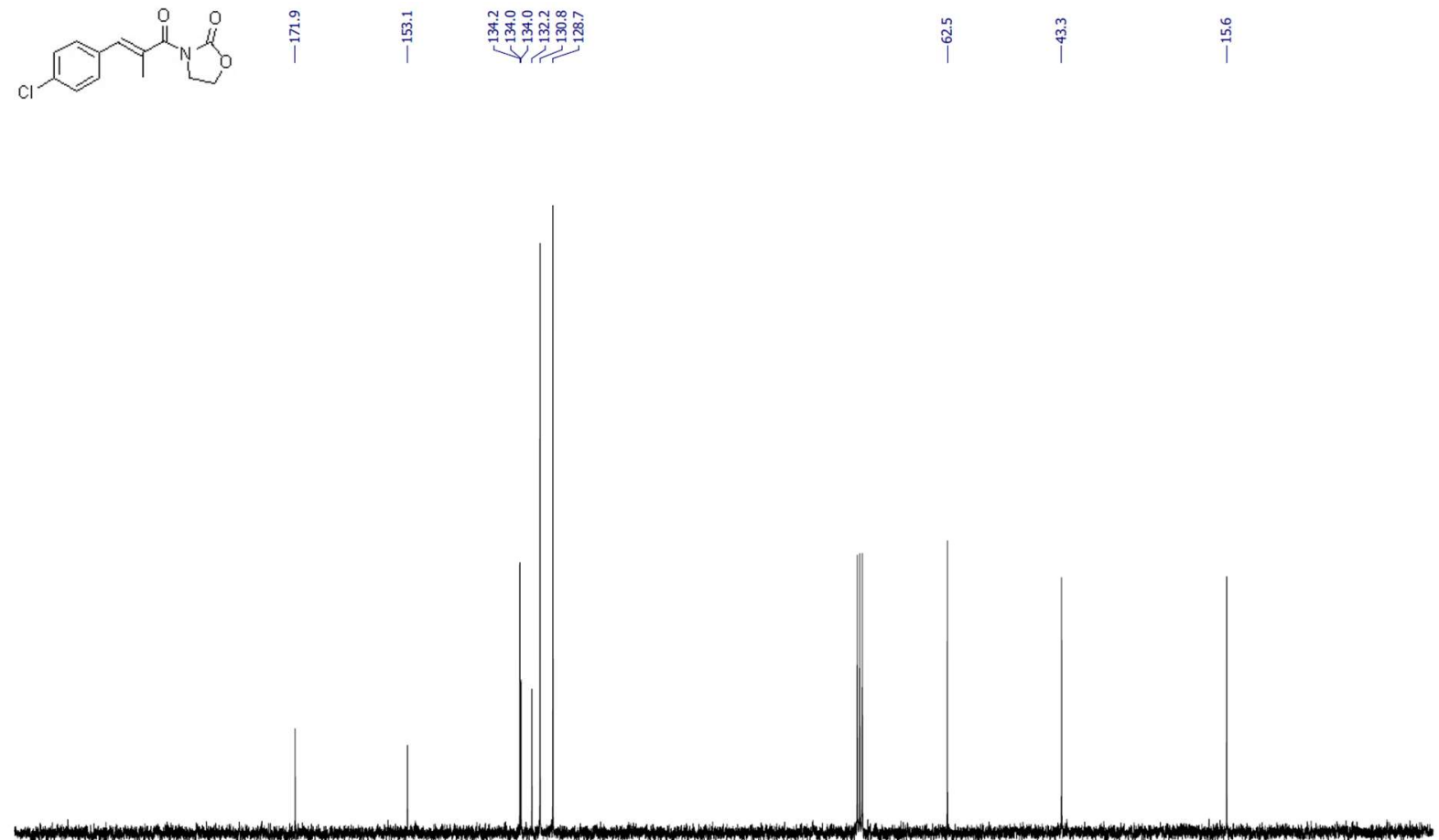

$\begin{array}{lllllllllllllllllllllll}210 & 200 & 190 & 180 & 170 & 160 & 150 & 140 & 130 & 120 & 110 & \underset{\mathrm{f} 1}{100}(\mathrm{ppm}) & 90 & 80 & 70 & 60 & 50 & 40 & 30 & 20 & 10 & 0 & -10\end{array}$ 
(E)-3-(3-(4-bromophenyl)-2-methylacryloyl)oxazolidin-2-one ((E)-18):

${ }^{1} \mathrm{H} \mathrm{NMR}\left(\mathrm{CDCl}_{3}, 300 \mathrm{MHz}\right)$<smiles>CC(=Cc1ccc(Br)cc1)N1CCCO1</smiles>

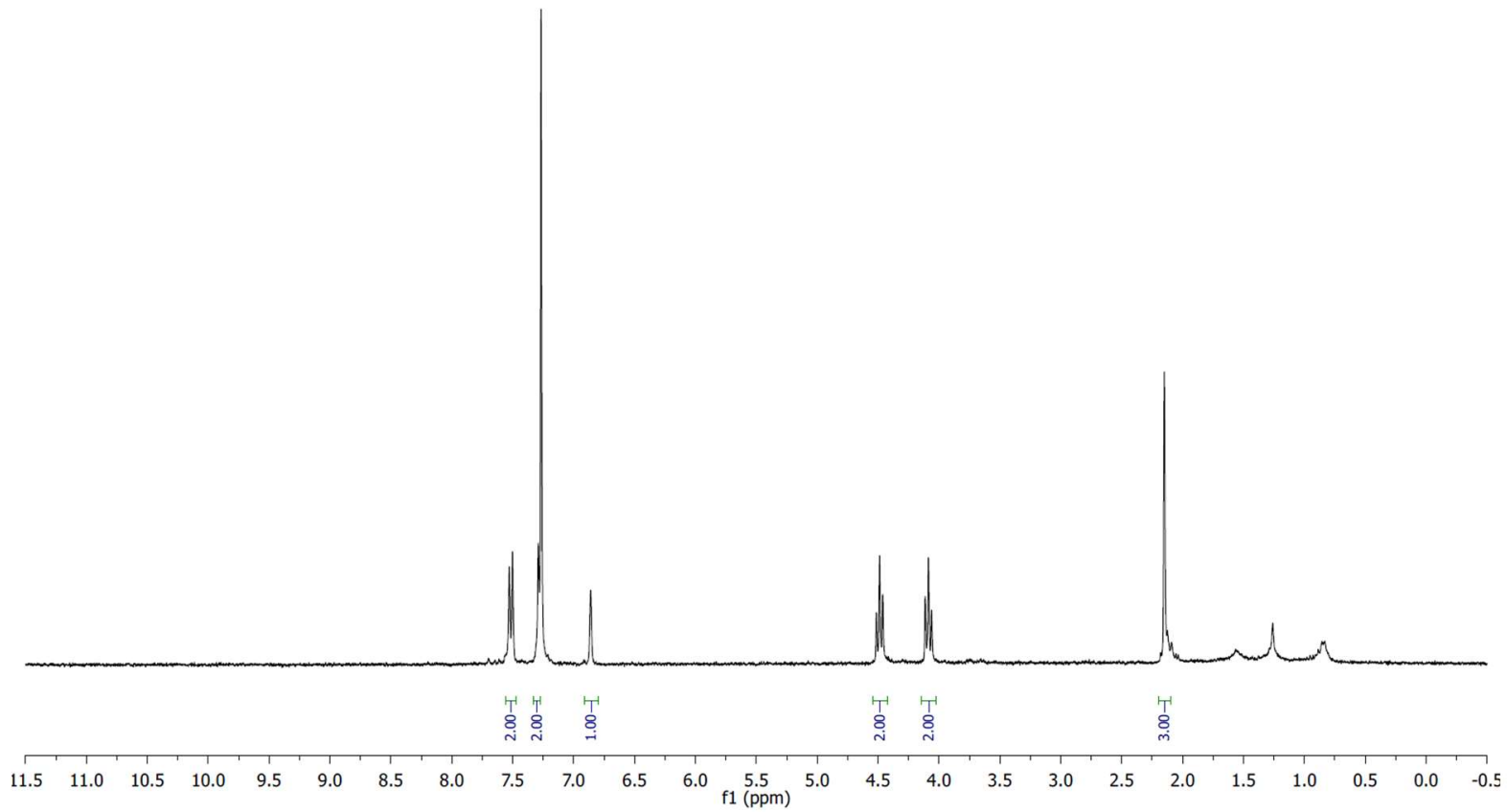

${ }^{13} \mathrm{C} \mathrm{NMR}\left(\mathrm{CDCl}_{3}, 75 \mathrm{MHz}\right)$
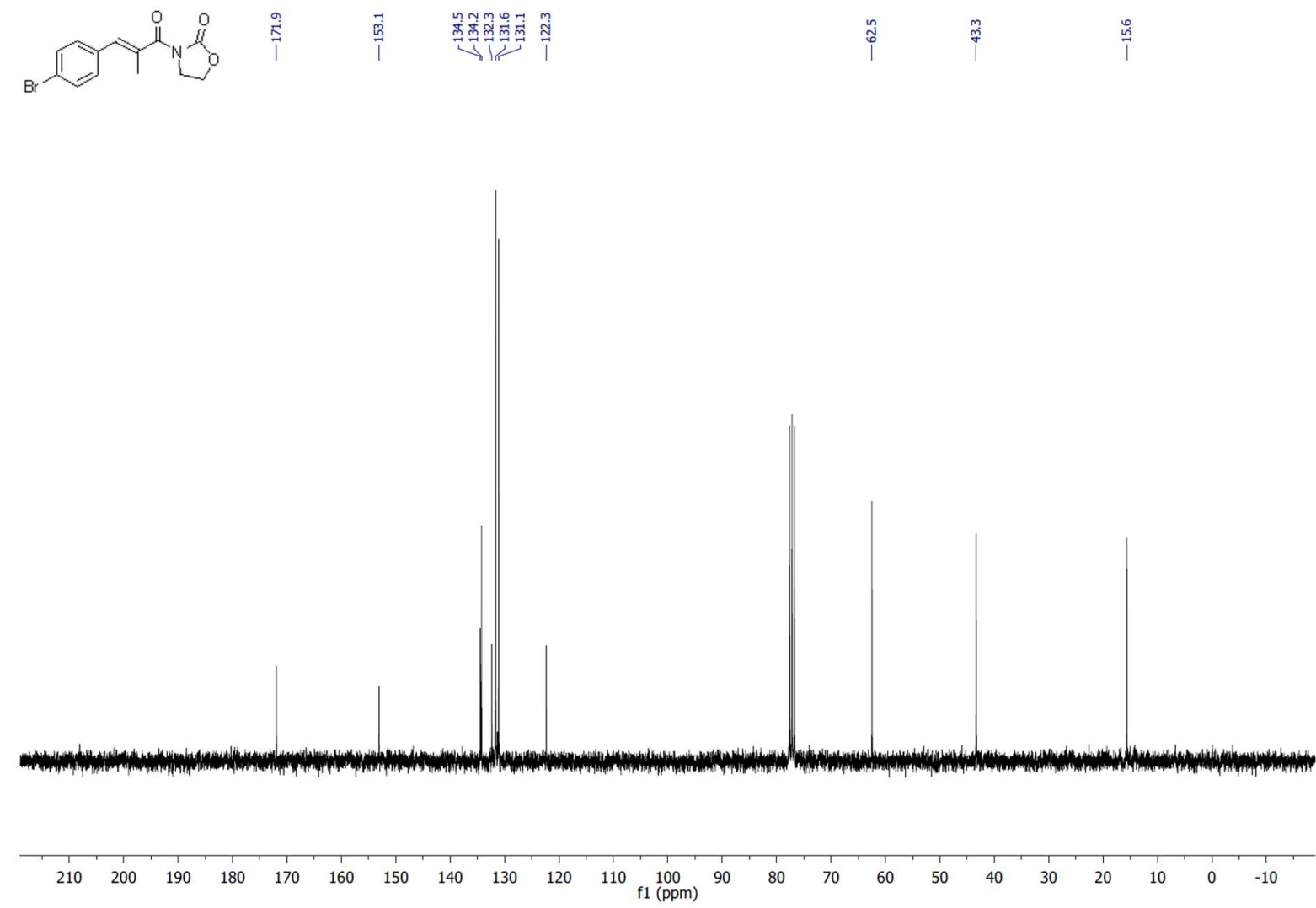
(E)-3-(3-(4-iodophenyl)-2-methylacryloyl)oxazolidin-2-one ((E)-19):

${ }^{1} \mathrm{H} \mathrm{NMR}\left(\mathrm{CDCl}_{3}, 300 \mathrm{MHz}\right)$<smiles>CCOC1OCCN1C(=O)C(C)Cc1ccc(I)cc1</smiles>

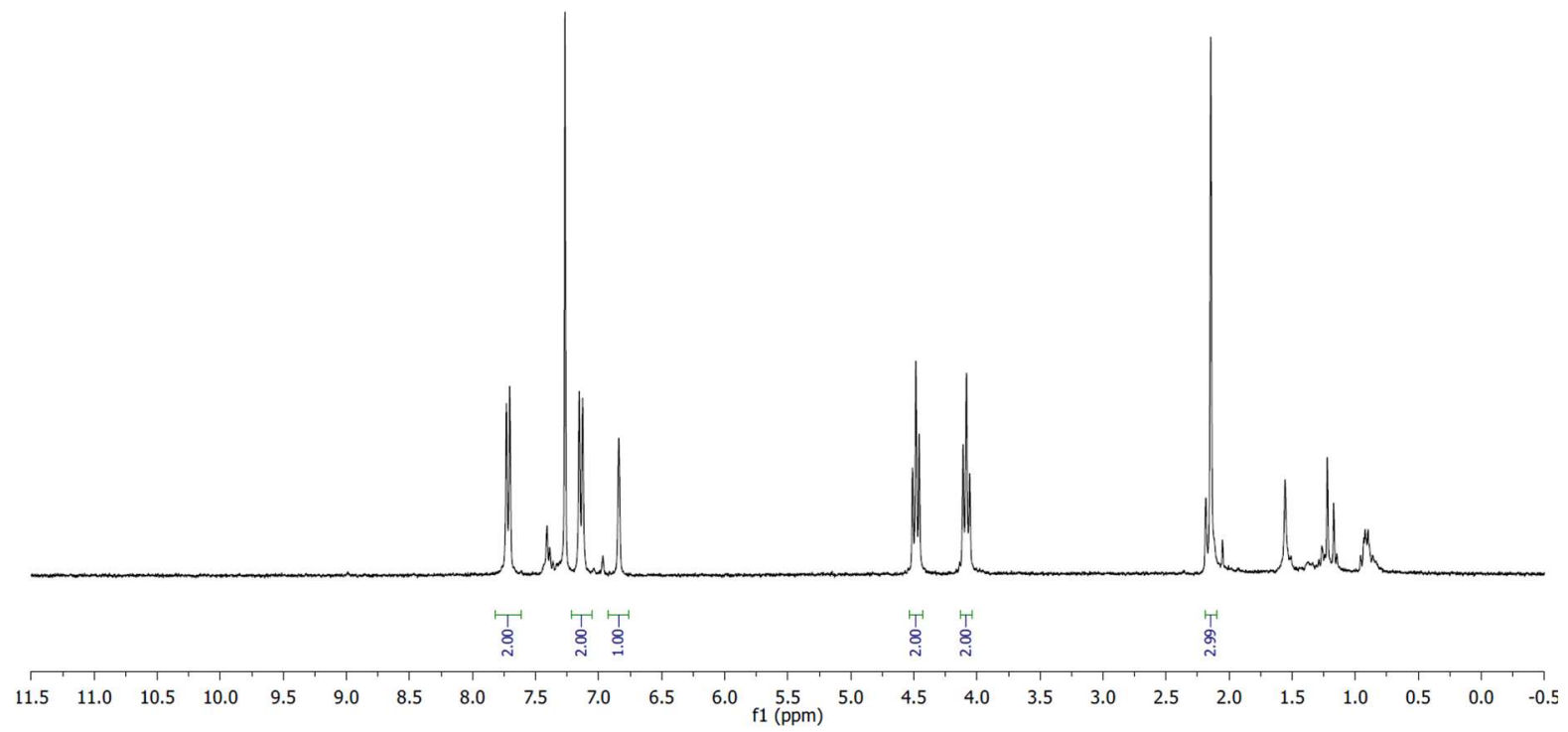

${ }^{13} \mathrm{C} \mathrm{NMR}\left(\mathrm{CDCl}_{3}, 75 \mathrm{MHz}\right)$

1

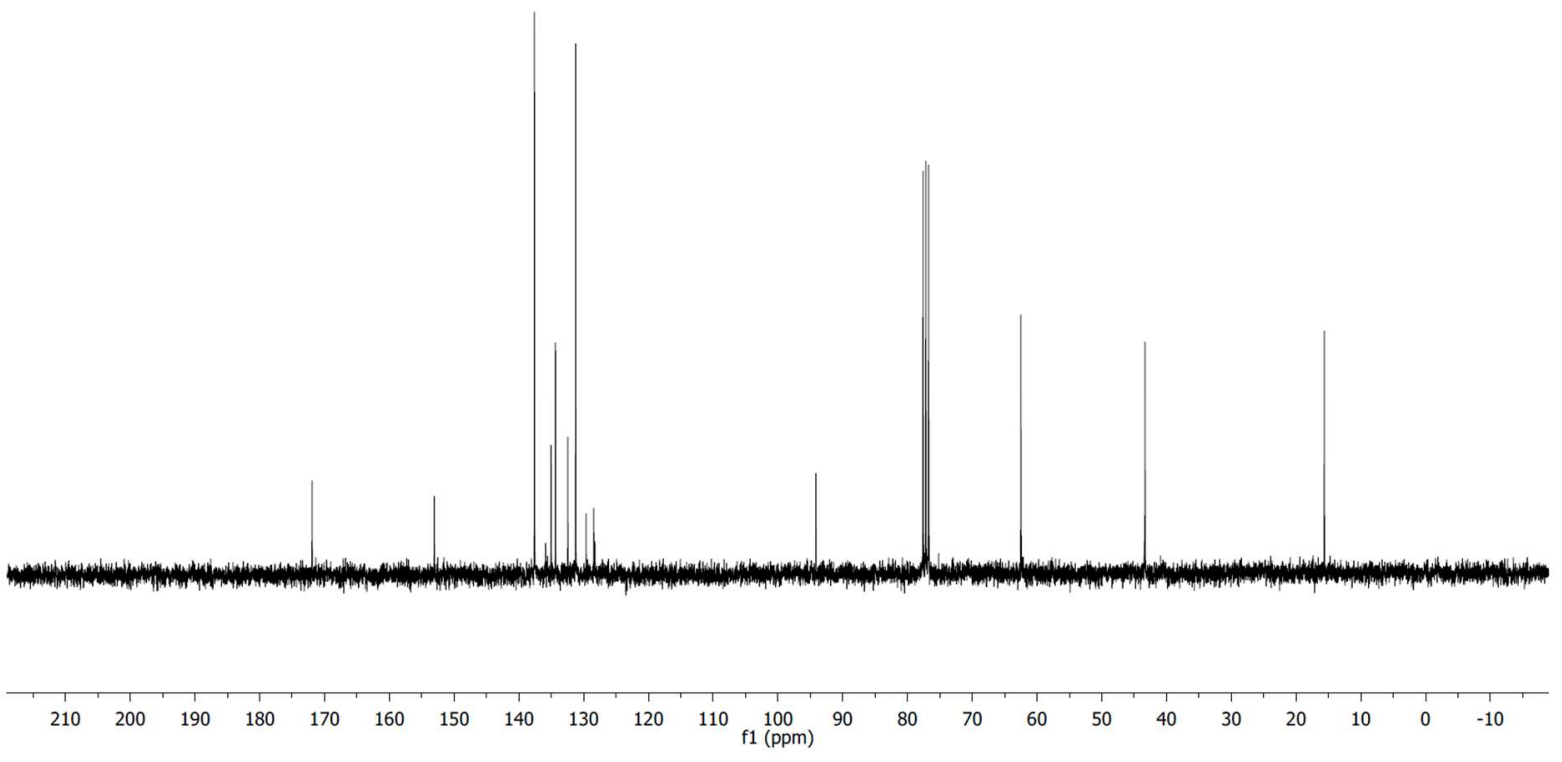


(E)-3-(2-methyl-3-(4-(trifluoromethyl)phenyl)acryloyl)oxazolidin-2-one ((E)-20):

${ }^{1} \mathrm{H} \mathrm{NMR}\left(\mathrm{CDCl}_{3}, 300 \mathrm{MHz}\right)$

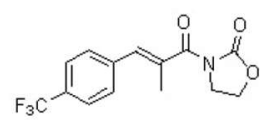

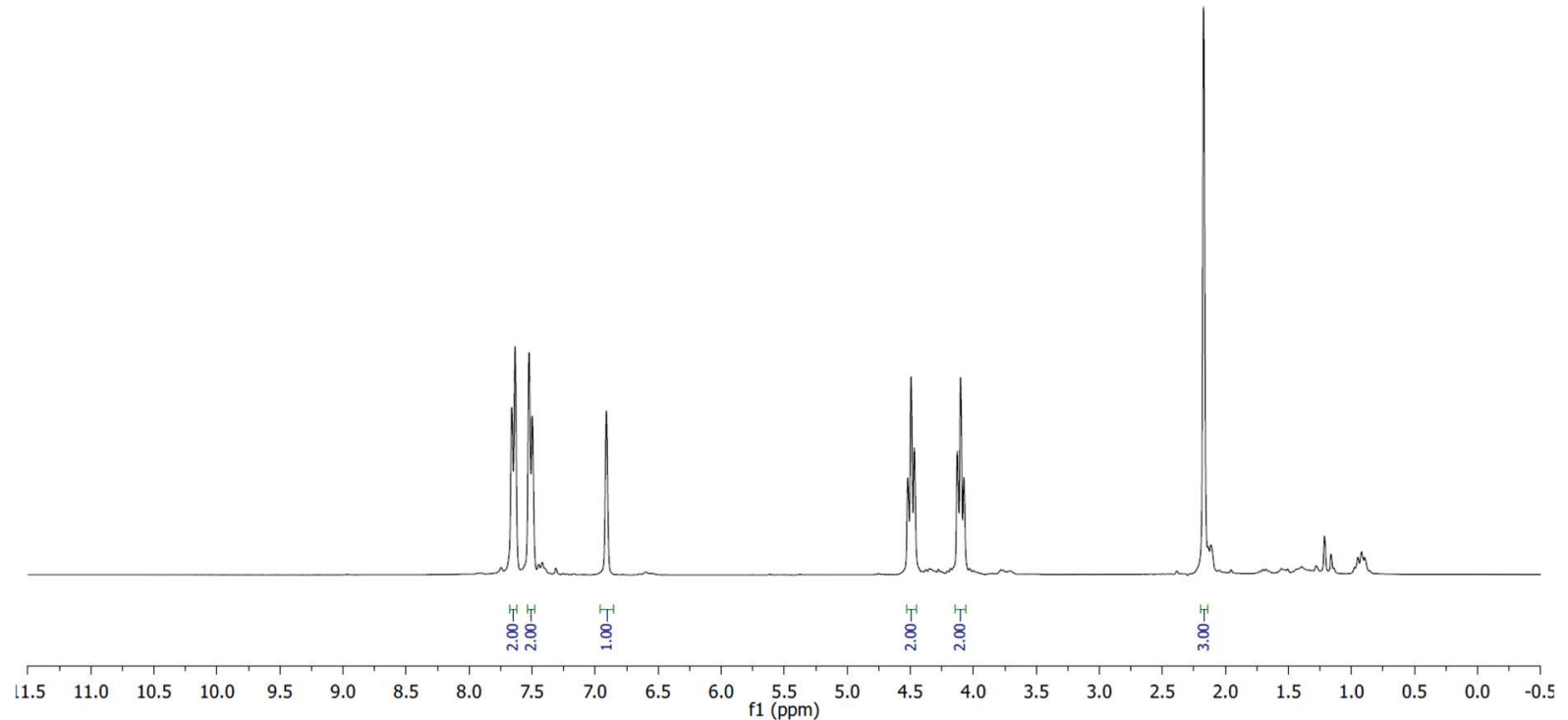

${ }^{13} \mathrm{C} \mathrm{NMR}\left(\mathrm{CDCl}_{3}, 75 \mathrm{MHz}\right)$
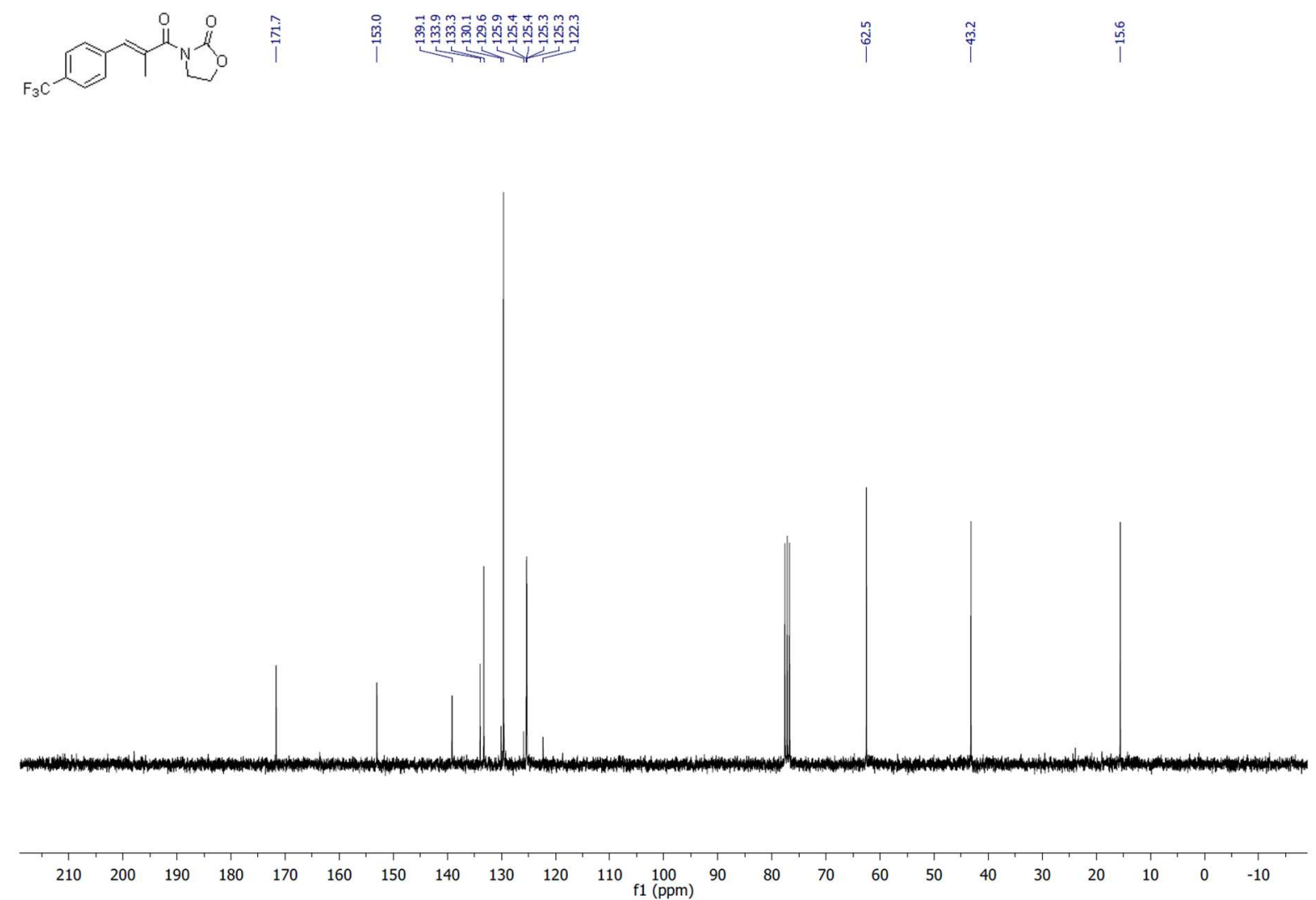
${ }^{19} \mathrm{~F} \mathrm{NMR}\left(\mathrm{CDCl}_{3}, 282 \mathrm{MHz}\right)$<smiles>CC(C)(Cc1ccc([18F])cc1)N1CCOC1=O</smiles>

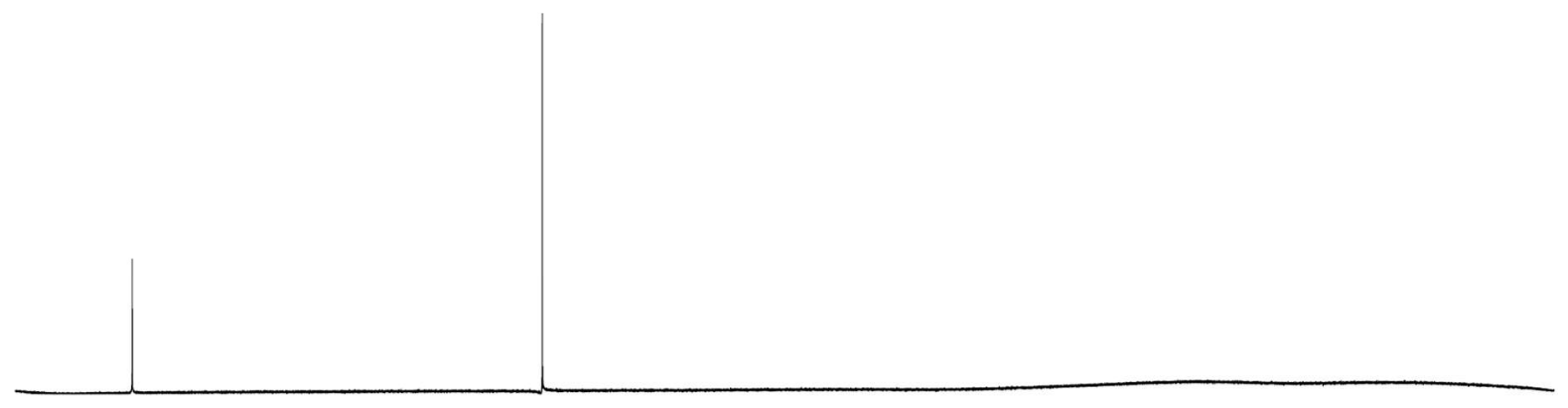

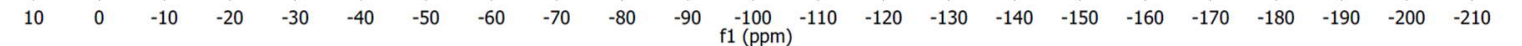

(E)-3-(2-methyl-3-(4-(4,4,5,5-tetramethyl-1,3,2-dioxaborolan-2-yl)phenyl)acryloyl)oxazolidin-2-one ((E)-21):

${ }^{1} \mathrm{H} \mathrm{NMR}\left(\mathrm{CDCl}_{3}, 300 \mathrm{MHz}\right)$

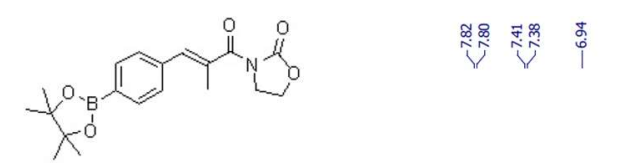

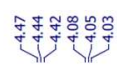

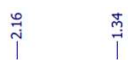

$x^{-1}$

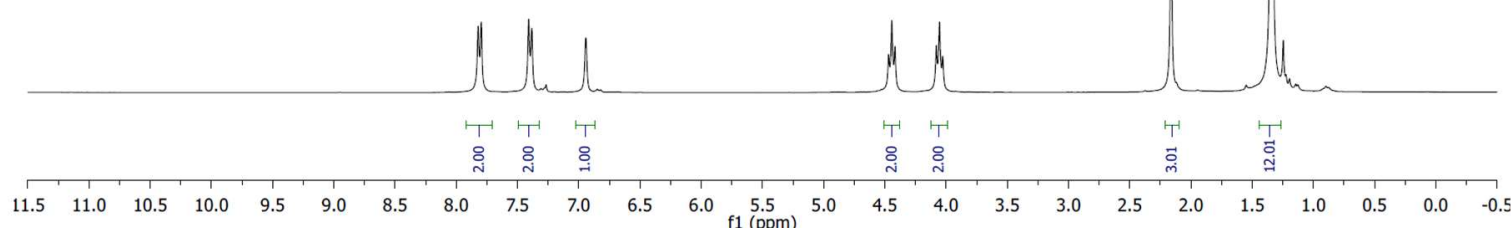


${ }^{13} \mathrm{C} \mathrm{NMR}\left(\mathrm{CDCl}_{3}, 75 \mathrm{MHz}\right)$
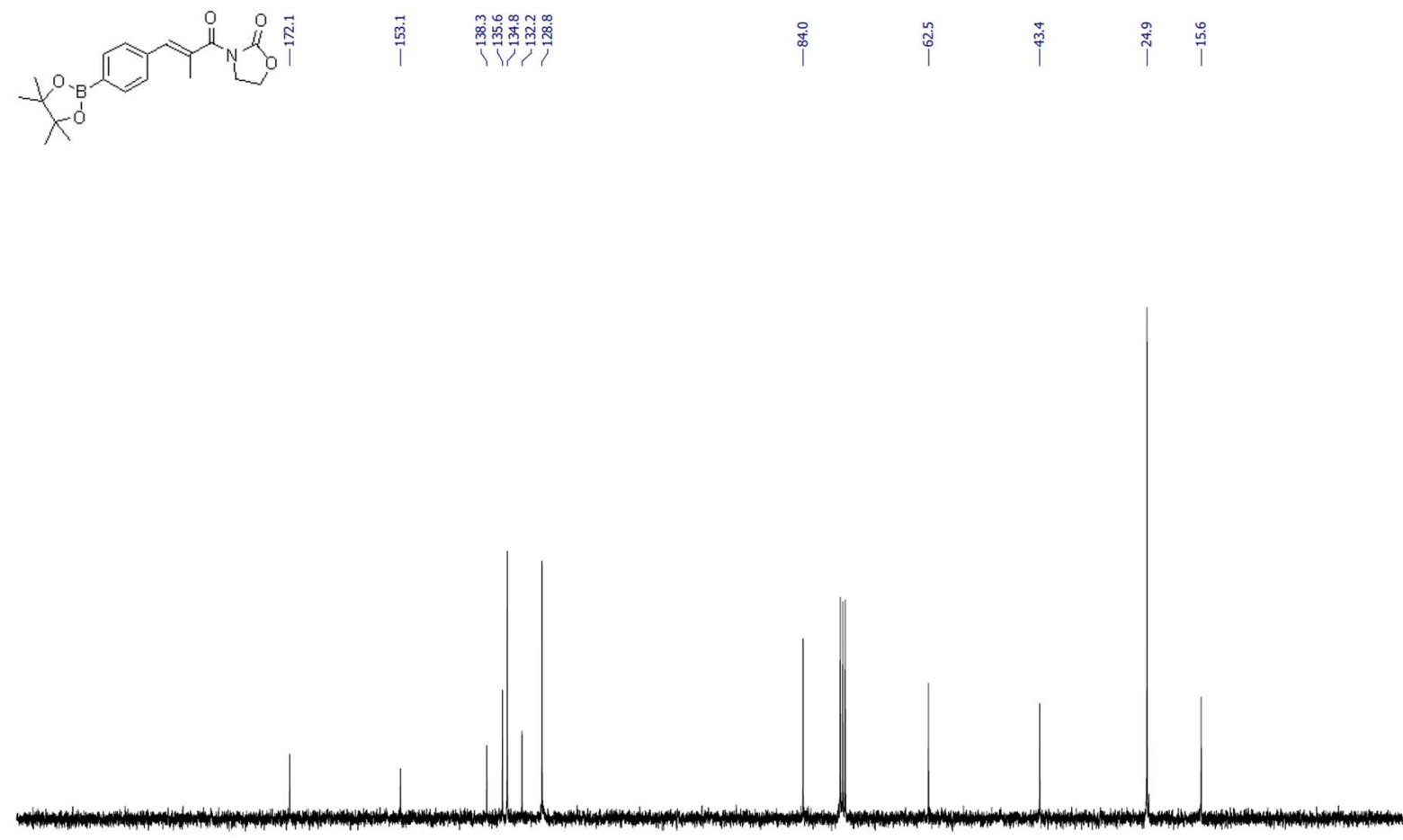

$\begin{array}{lllllllllllllllllllllll}210 & 200 & 190 & 180 & 170 & 160 & 150 & 140 & 130 & 120 & 110 & 100 & 90 & 80 & 70 & 60 & 50 & 40 & 30 & 20 & 10 & 0 & -10\end{array}$

(E)-3-(2-methyl-3-(4-(methylsulfonyl)phenyl)acryloyl)oxazolidin-2-one ((E)-22):

${ }^{1} \mathrm{H} \mathrm{NMR}\left(\mathrm{CDCl}_{3}, 300 \mathrm{MHz}\right)$
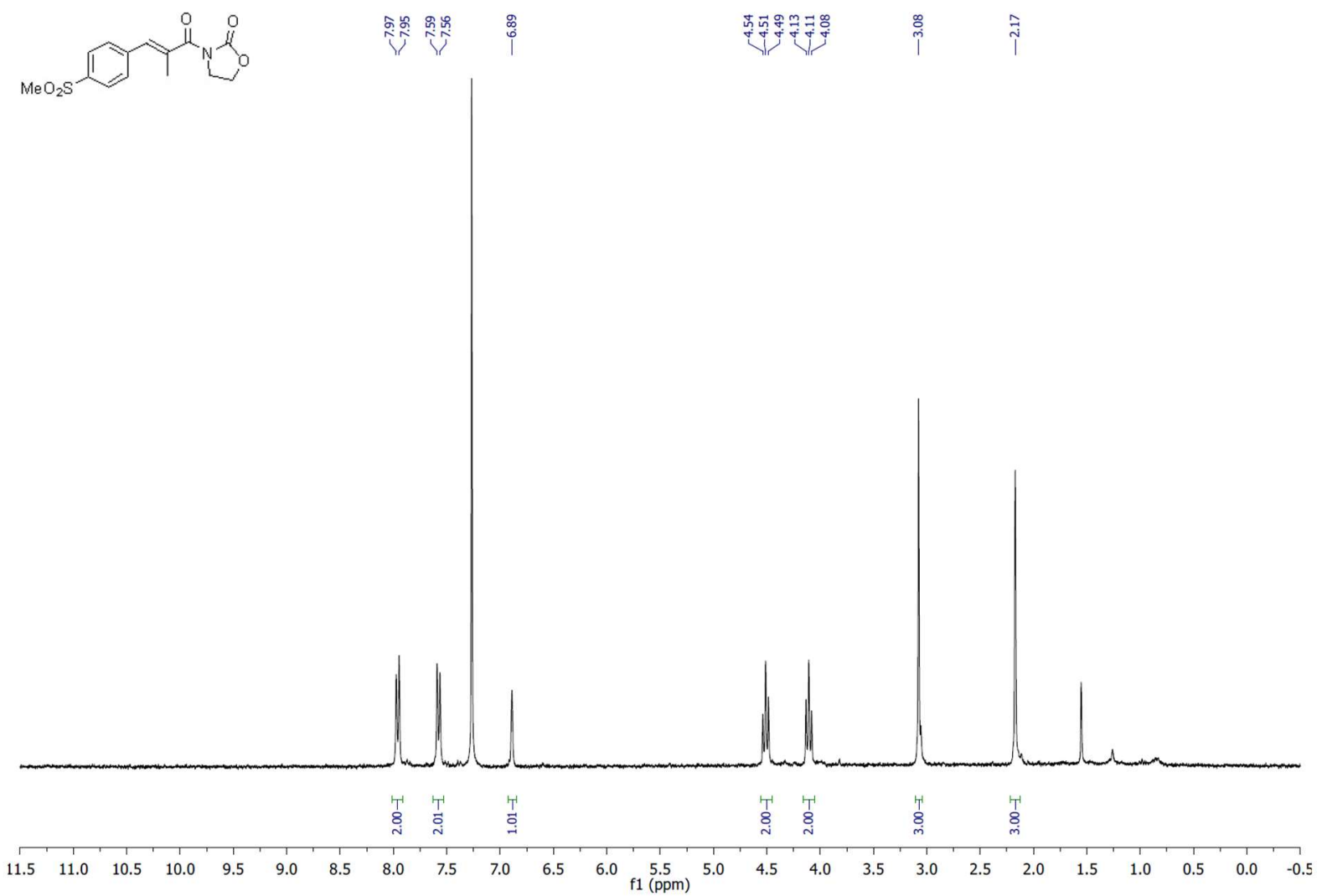
${ }^{13} \mathrm{C} \mathrm{NMR}\left(\mathrm{CDCl}_{3}, 75 \mathrm{MHz}\right)$

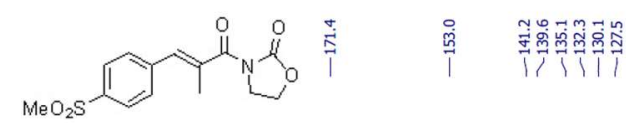

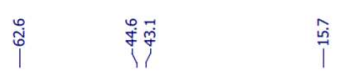
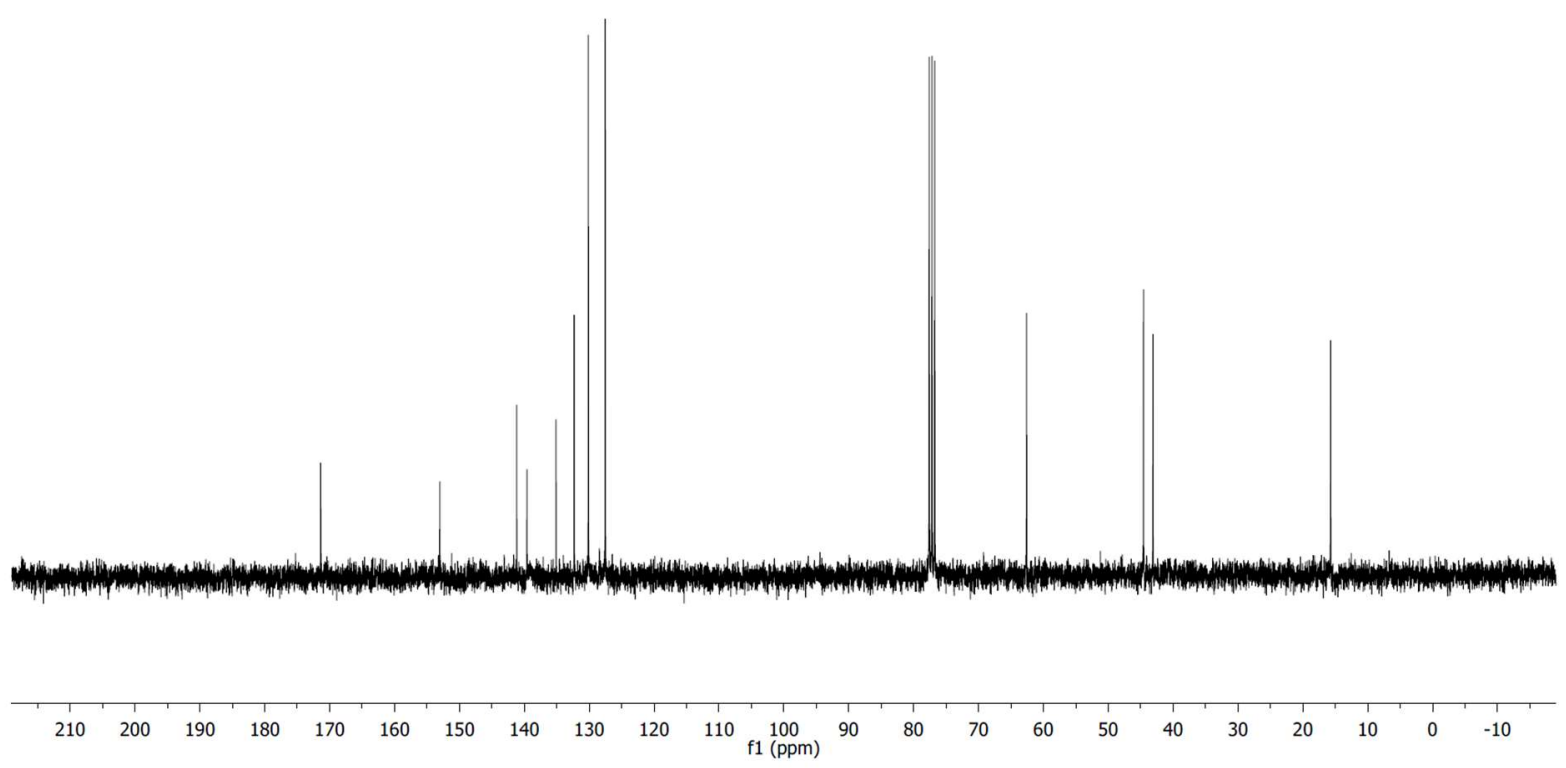

(E)-3-(3-(4-acetylphenyl)-2-methylacryloyl)oxazolidin-2-one ((E)-23):

${ }^{1} \mathrm{H} \mathrm{NMR}\left(\mathrm{CDCl}_{3}, 300 \mathrm{MHz}\right)$

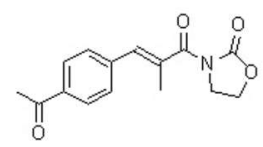

$$
\text { خ }
$$

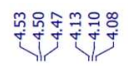

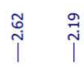

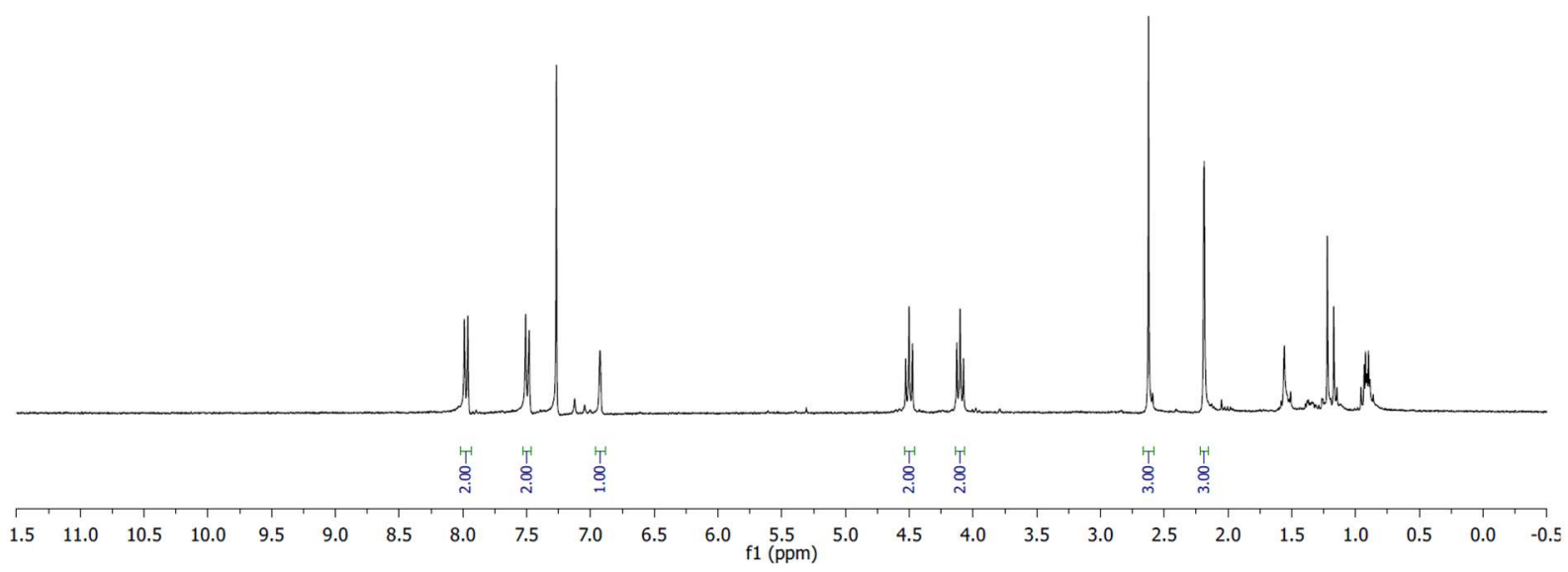

141 
${ }^{13} \mathrm{C} \mathrm{NMR}\left(\mathrm{CDCl}_{3}, 75 \mathrm{MHz}\right)$
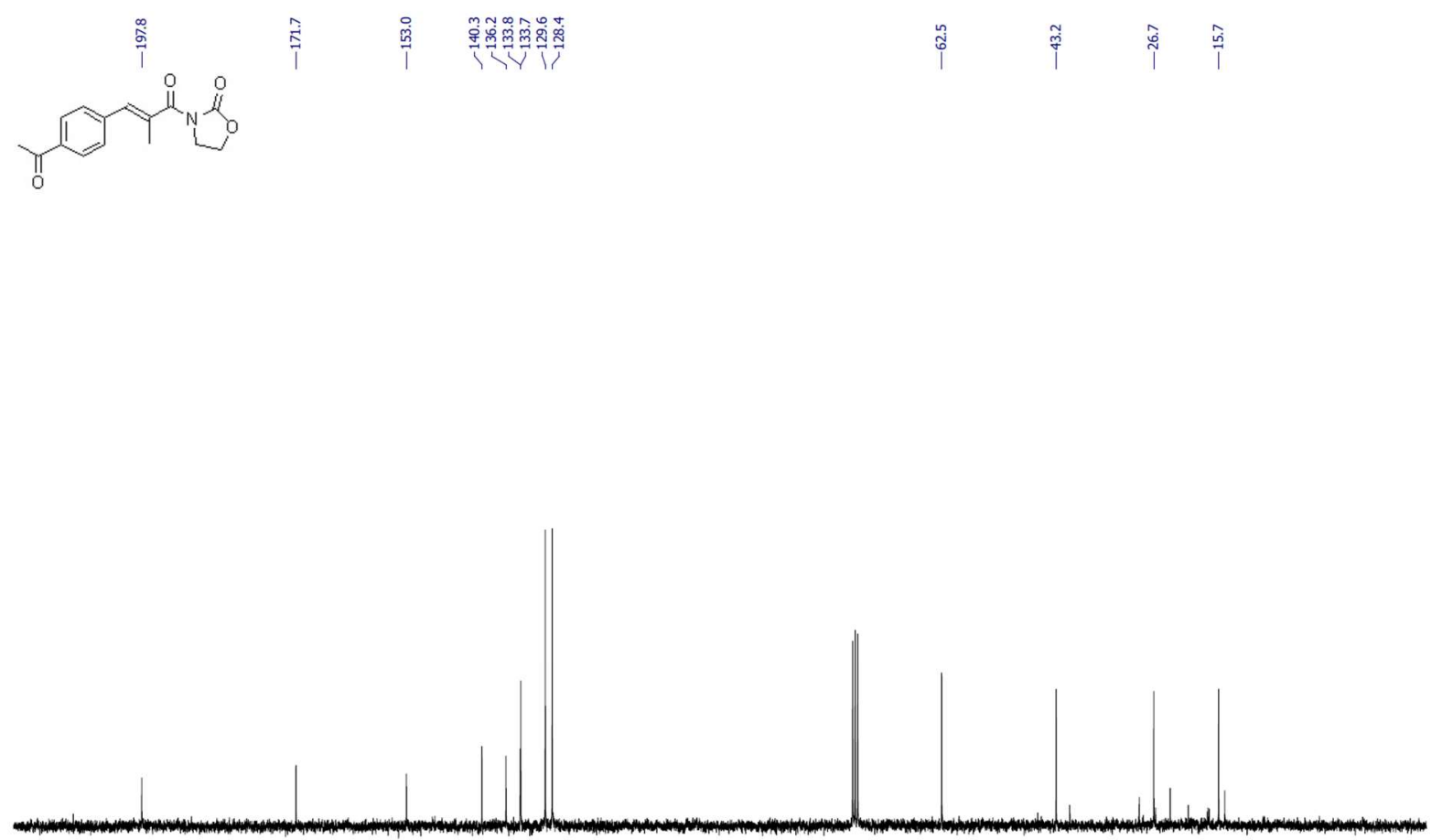

$\begin{array}{llllllllllllllllllllllllll}210 & 200 & 190 & 180 & 170 & 160 & 150 & 140 & 130 & 120 & 110 & 100 & 90 & 80 & 70 & 60 & 50 & 40 & 30 & 20 & 10 & 0 & -10\end{array}$

(E)-3-(3-(furan-2-yl)-2-methylacryloyl)oxazolidin-2-one ((E)-24):

${ }^{1} \mathrm{H} \mathrm{NMR}\left(\mathrm{CDCl}_{3}, 300 \mathrm{MHz}\right)$
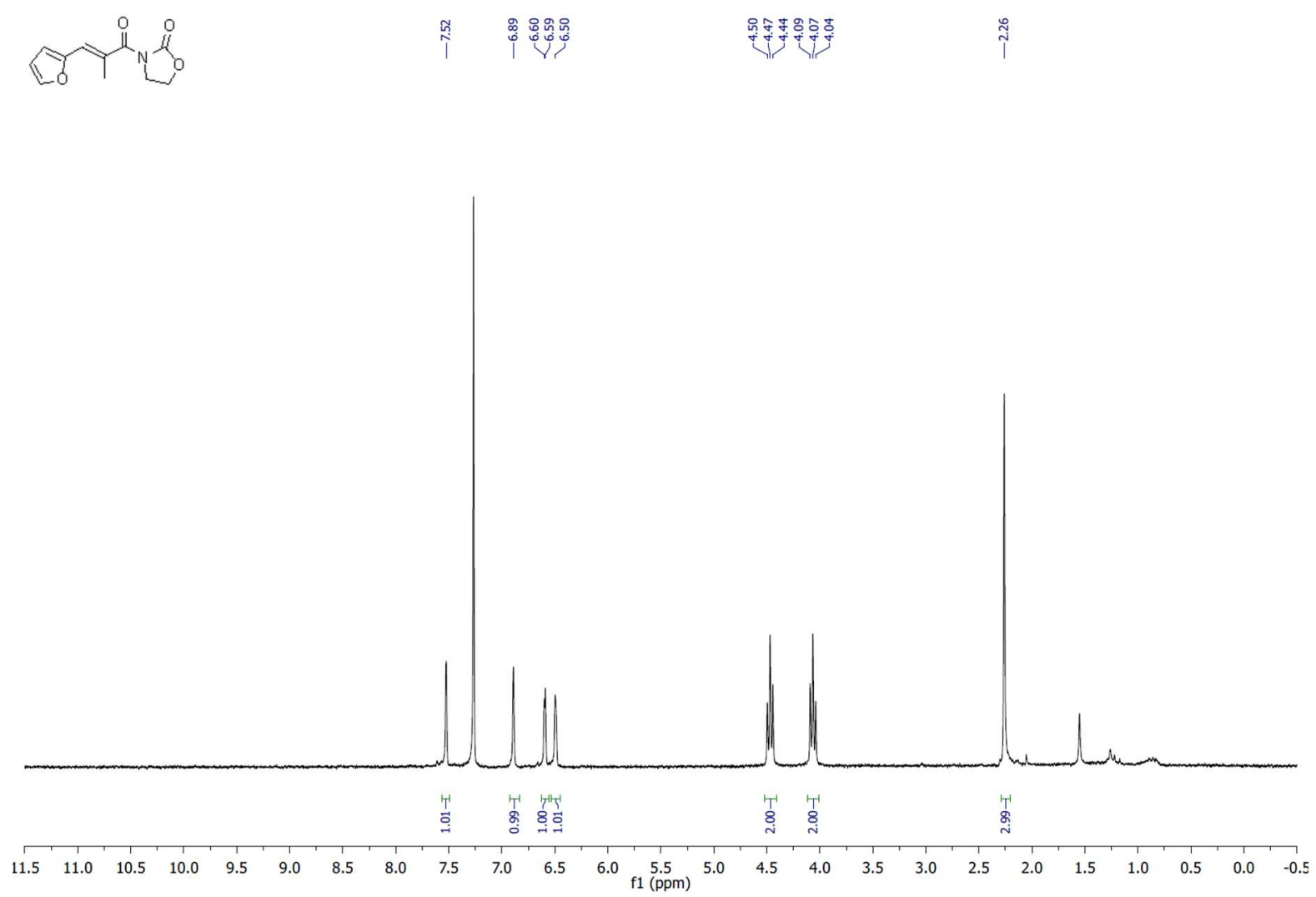
${ }^{13} \mathrm{C} \mathrm{NMR}\left(\mathrm{CDCl}_{3}, 75 \mathrm{MHz}\right)$
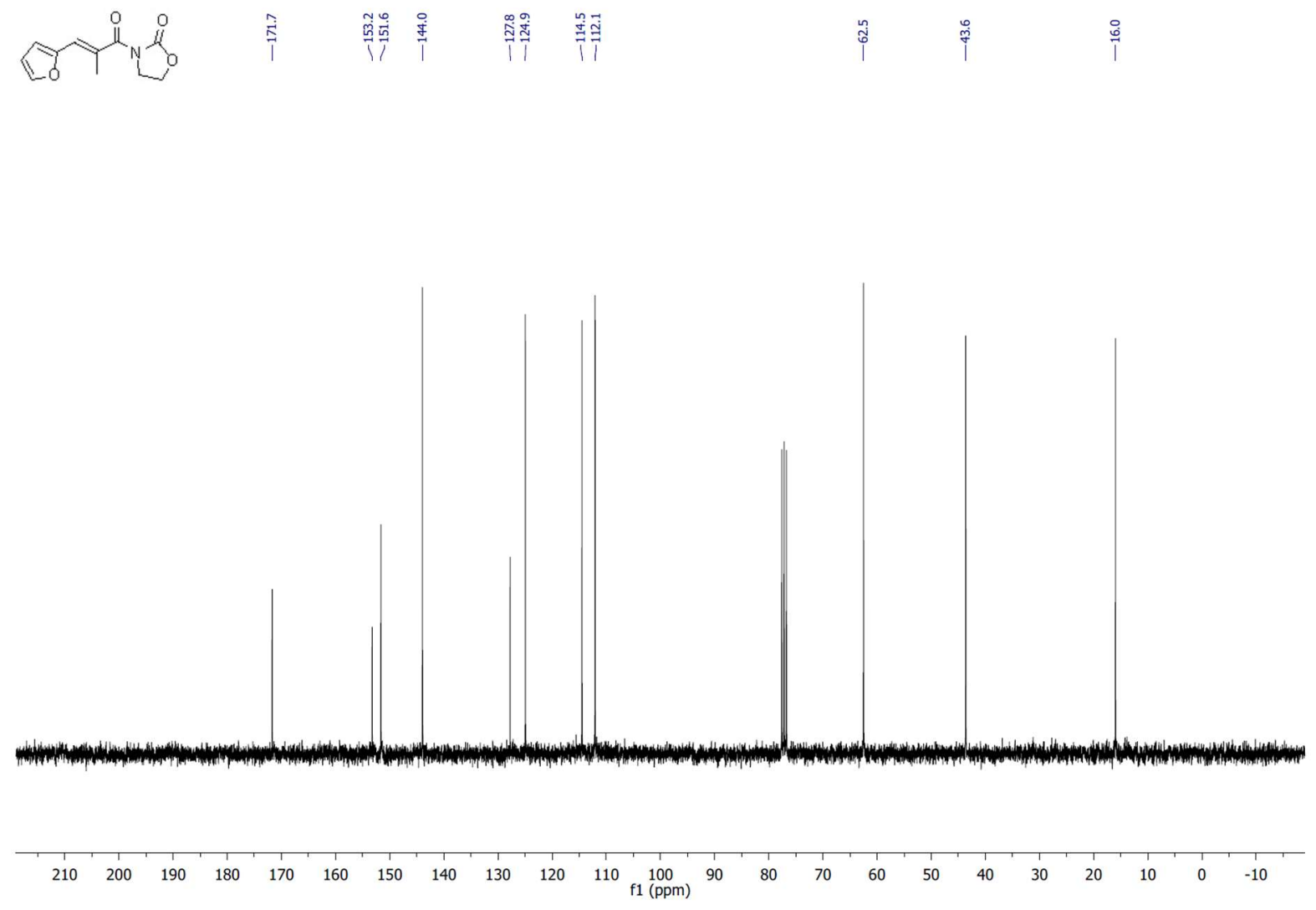

(E)-3-(2-methyl-3-(thiophen-2-yl)acryloyl)oxazolidin-2-one ((E)-25):

${ }^{1} \mathrm{H} \mathrm{NMR}\left(\mathrm{CDCl}_{3}, 300 \mathrm{MHz}\right)$

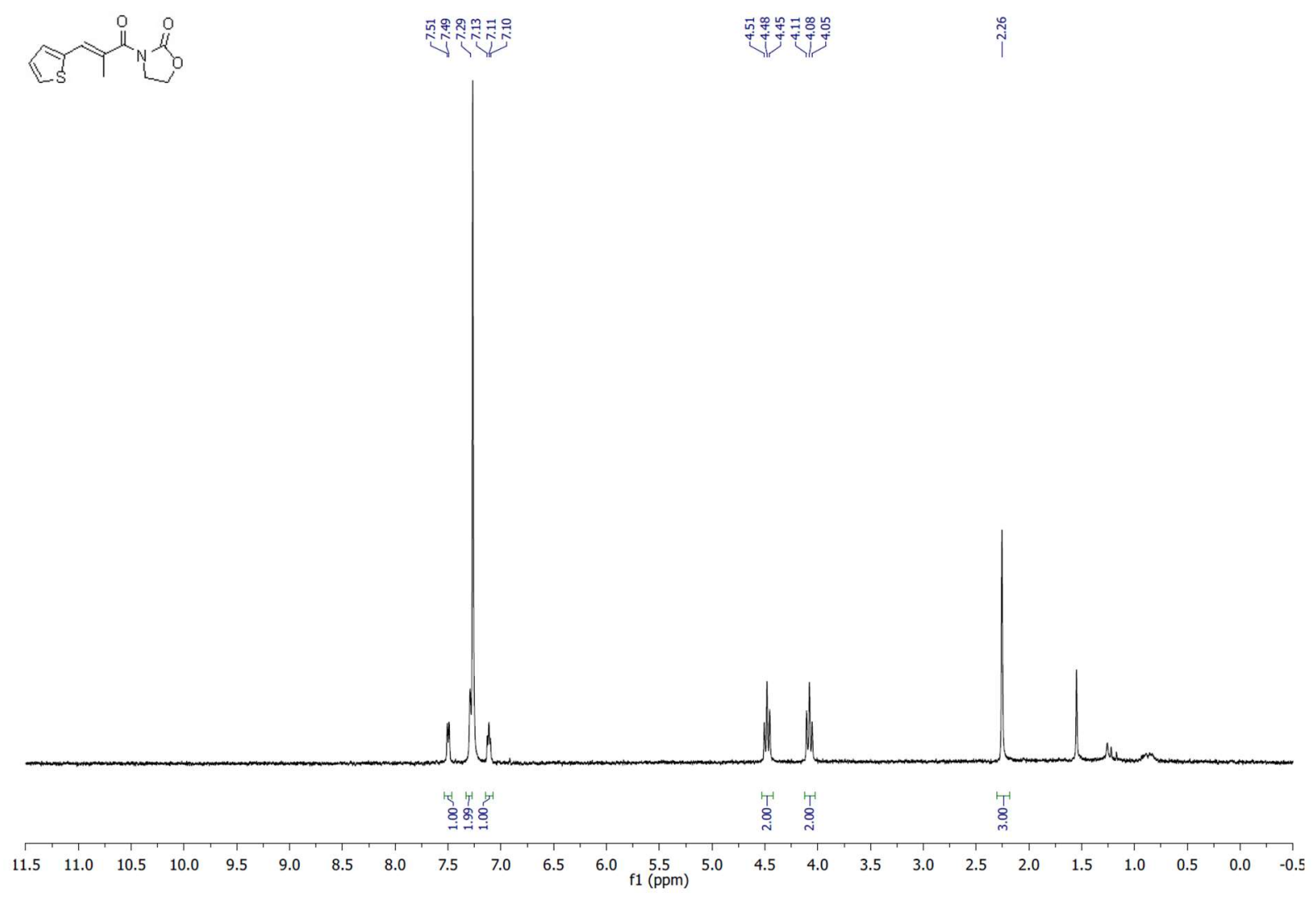


${ }^{13} \mathrm{C} \mathrm{NMR}\left(\mathrm{CDCl}_{3}, 75 \mathrm{MHz}\right)$
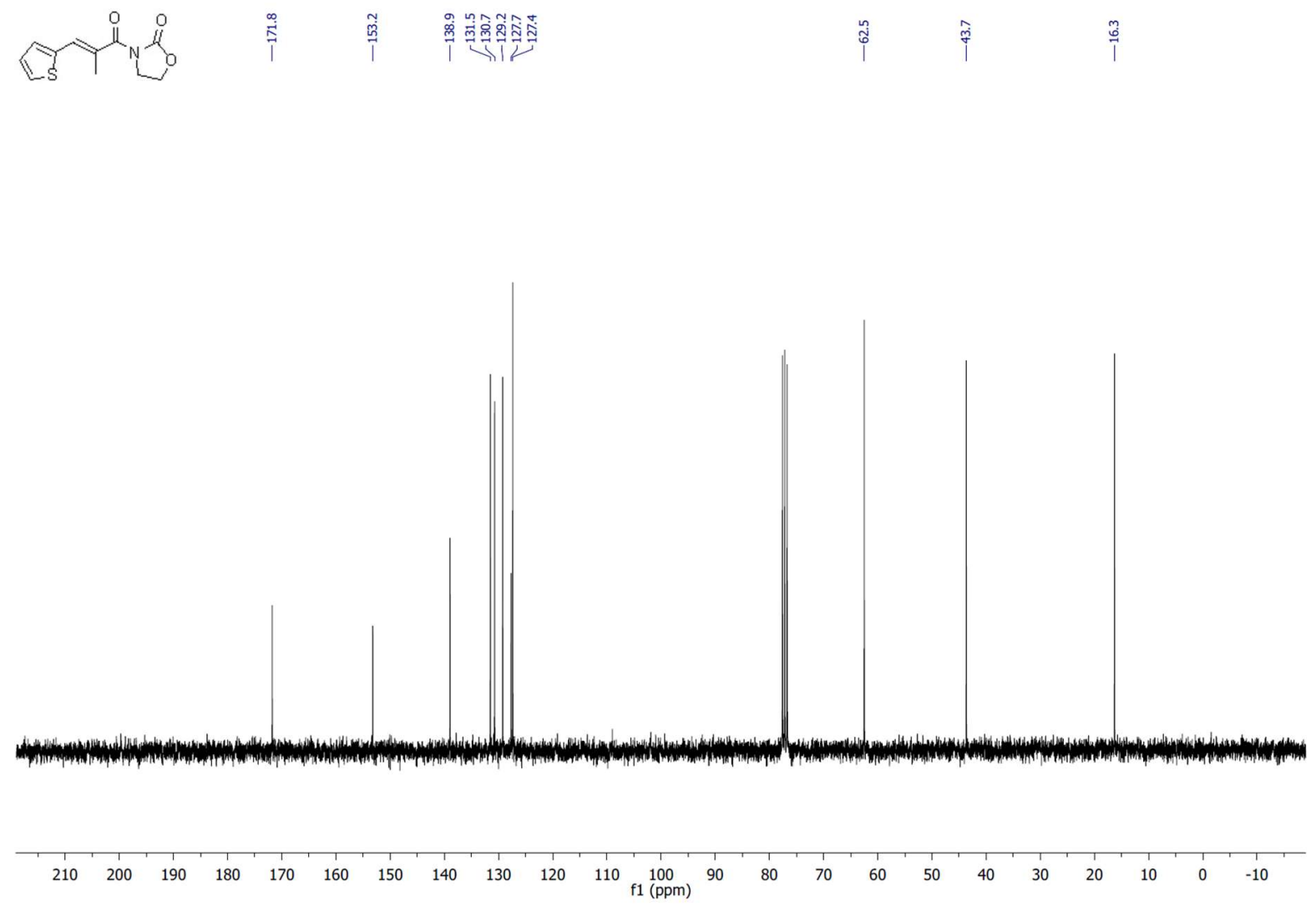

3-((E)-3-(4-(cinnamyloxy)phenyl)-2-methylacryloyl)oxazolidin-2-one ((E)-26):

${ }^{1} \mathrm{H} \mathrm{NMR}\left(\mathrm{CDCl}_{3}, 300 \mathrm{MHz}\right)$
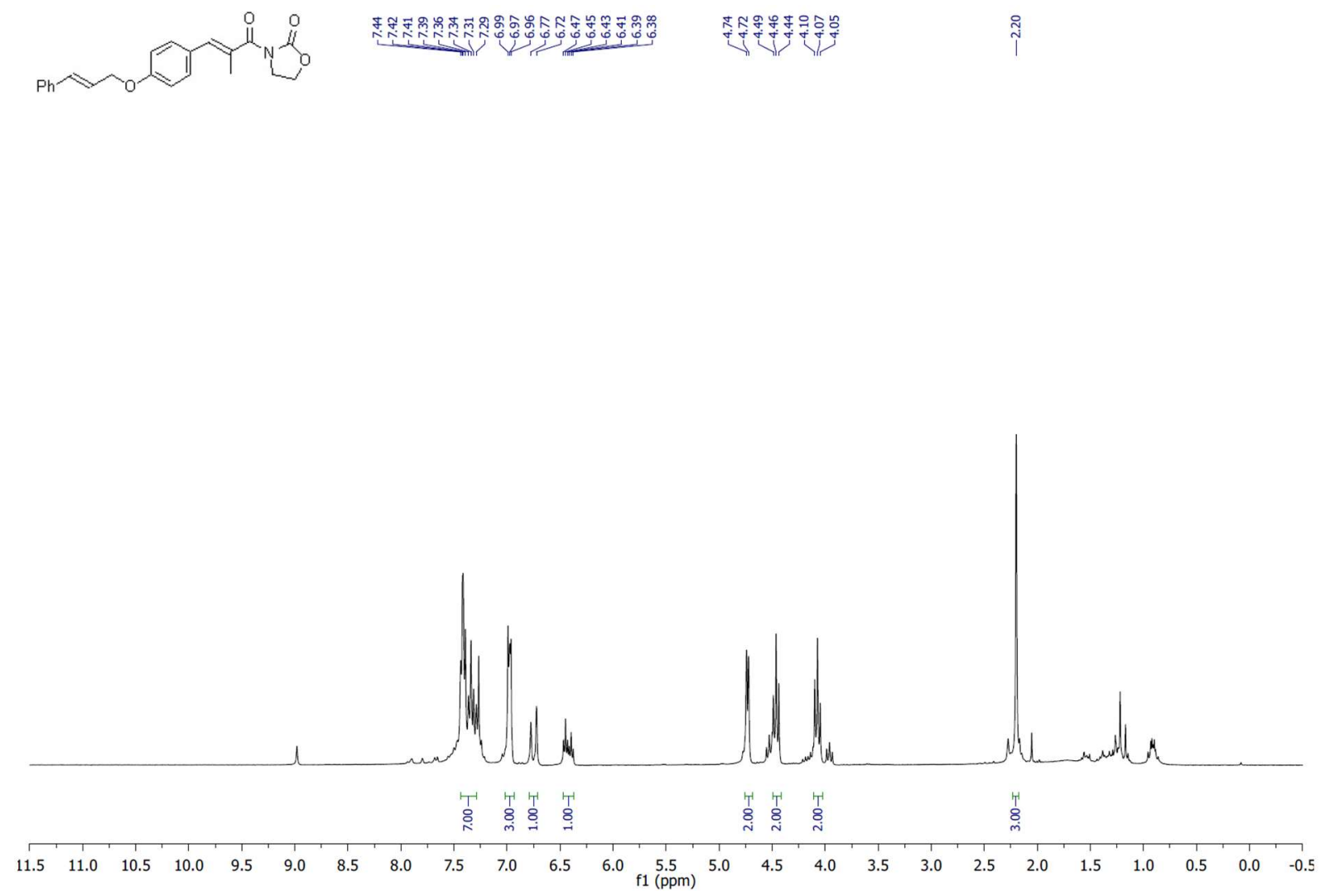
${ }^{13} \mathrm{C} \mathrm{NMR}\left(\mathrm{CDCl}_{3}, 75 \mathrm{MHz}\right)$
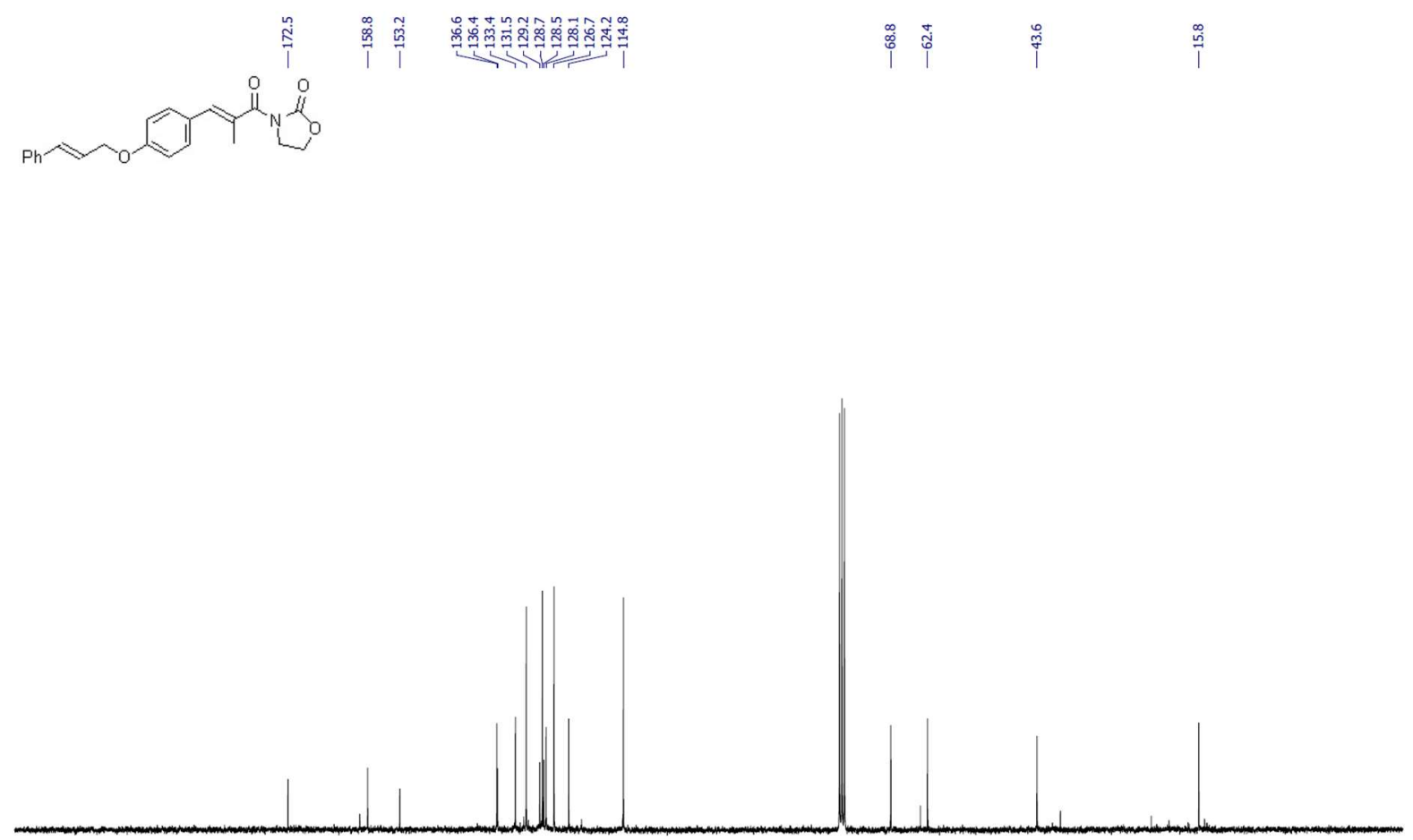

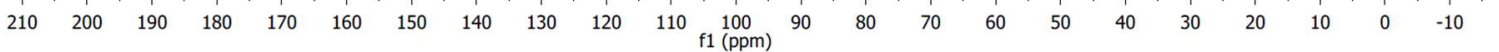

(E)-3-(3-mesityl-2-methylacryloyl)oxazolidin-2-one ((E)-27):

${ }^{1} \mathrm{H} \mathrm{NMR}\left(\mathrm{CDCl}_{3}, 300 \mathrm{MHz}\right)$
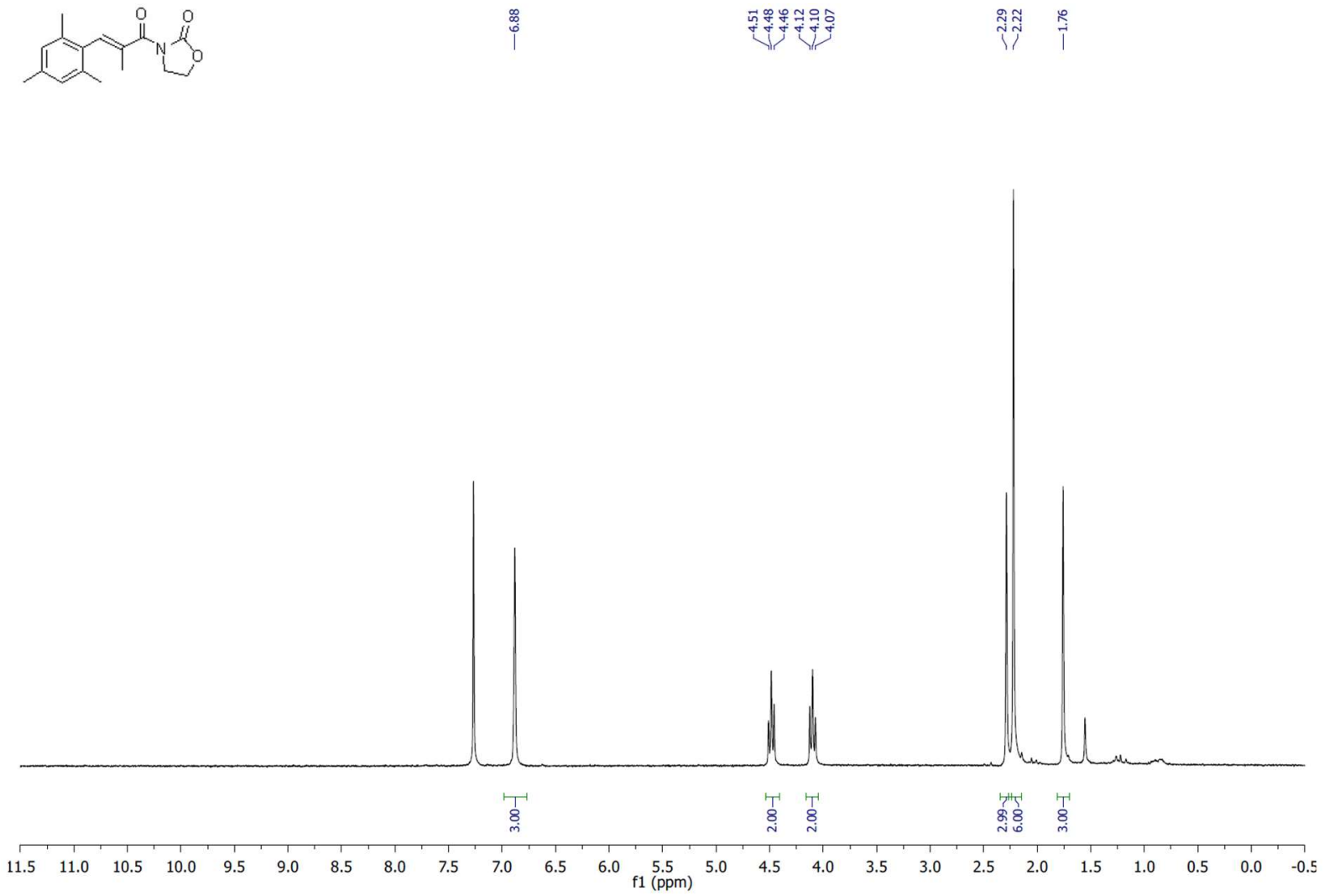
${ }^{13} \mathrm{C} \mathrm{NMR}\left(\mathrm{CDCl}_{3}, 75 \mathrm{MHz}\right)$
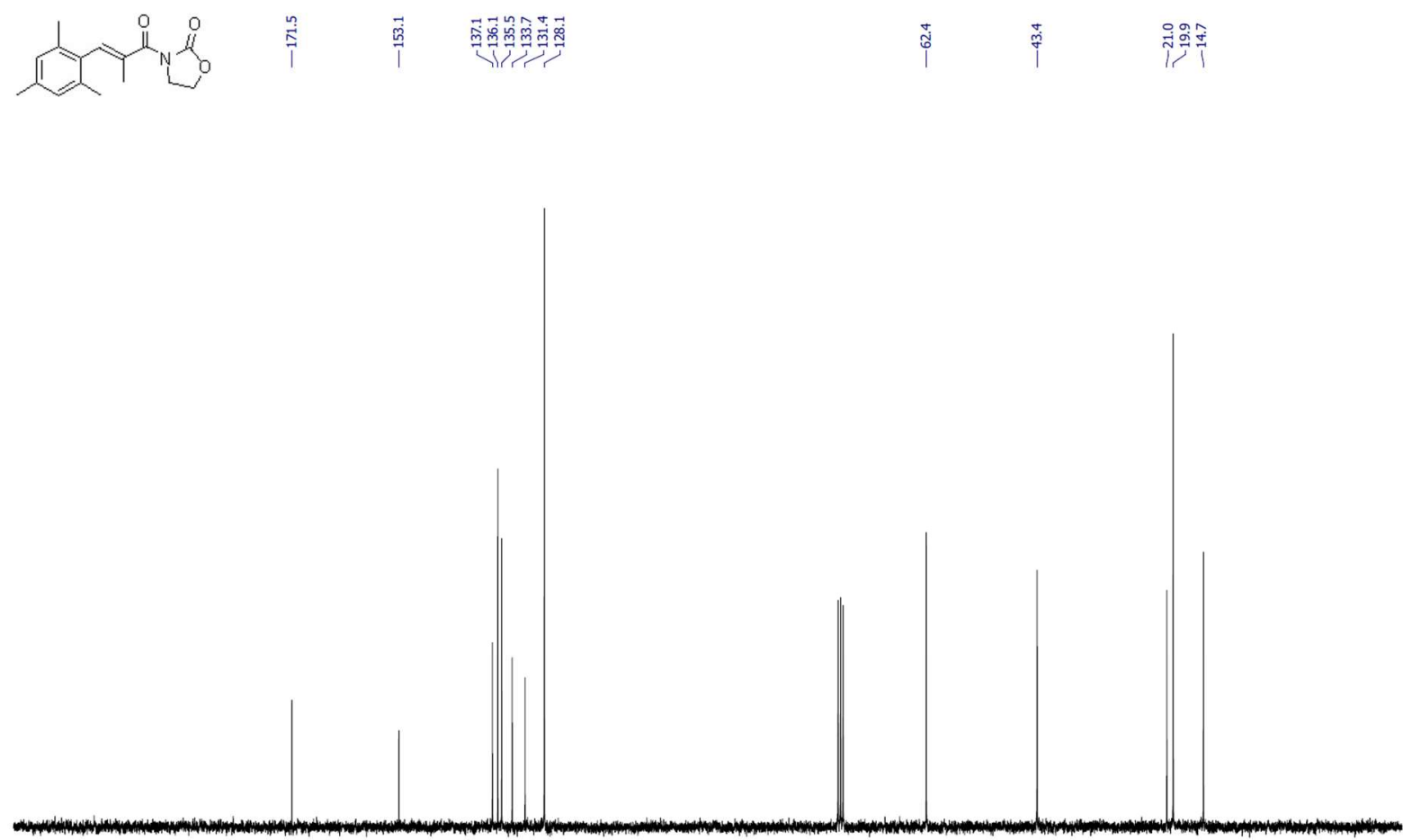

$\begin{array}{llllllllllllllllllllllll}210 & 200 & 190 & 180 & 170 & 160 & 150 & 140 & 130 & 120 & 110 & 100 & 90 & 80 & 70 & 60 & 50 & 40 & 30 & 20 & 10 & 0 & -10\end{array}$

(E)-3-(2-methyloct-2-enoyl)oxazolidin-2-one ((E)-28):

${ }^{1} \mathrm{H} \mathrm{NMR}\left(\mathrm{CDCl}_{3}, 300 \mathrm{MHz}\right)$
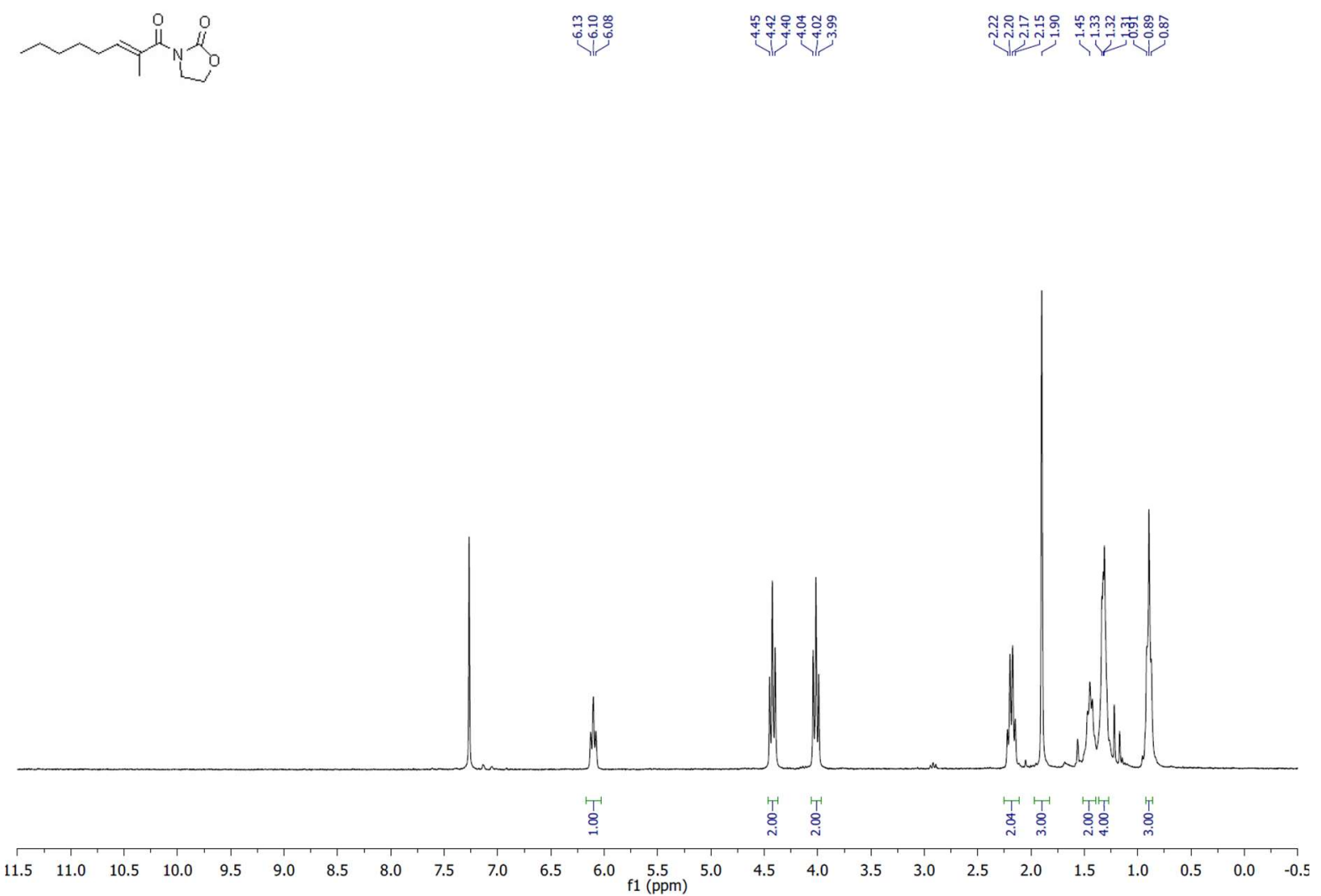
${ }^{13} \mathrm{C} \mathrm{NMR}\left(\mathrm{CDCl}_{3}, 75 \mathrm{MHz}\right)$
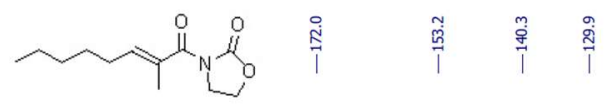

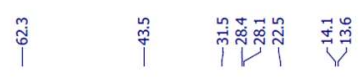
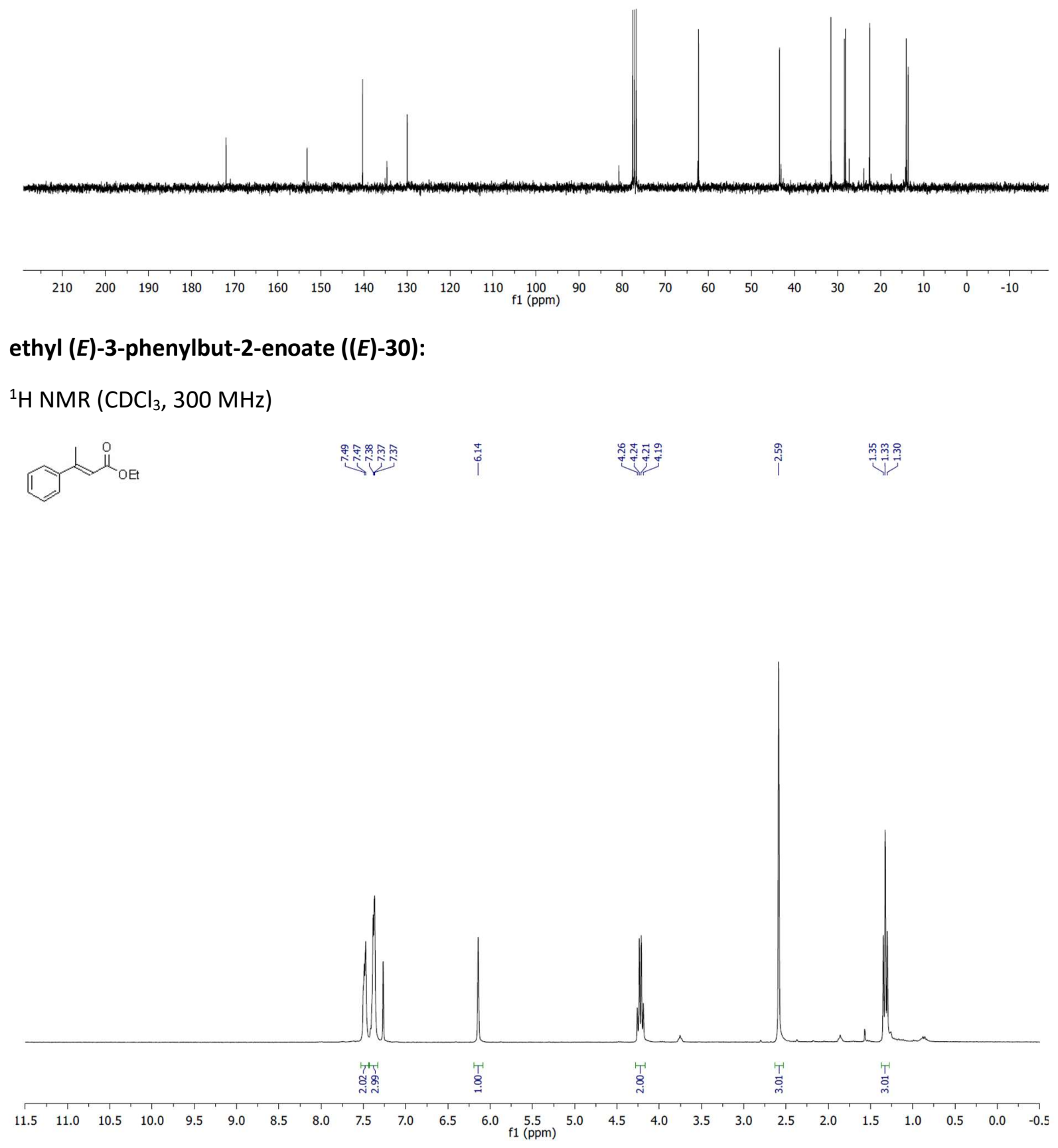

147 
${ }^{13} \mathrm{C} \mathrm{NMR}\left(\mathrm{CDCl}_{3}, 75 \mathrm{MHz}\right)$
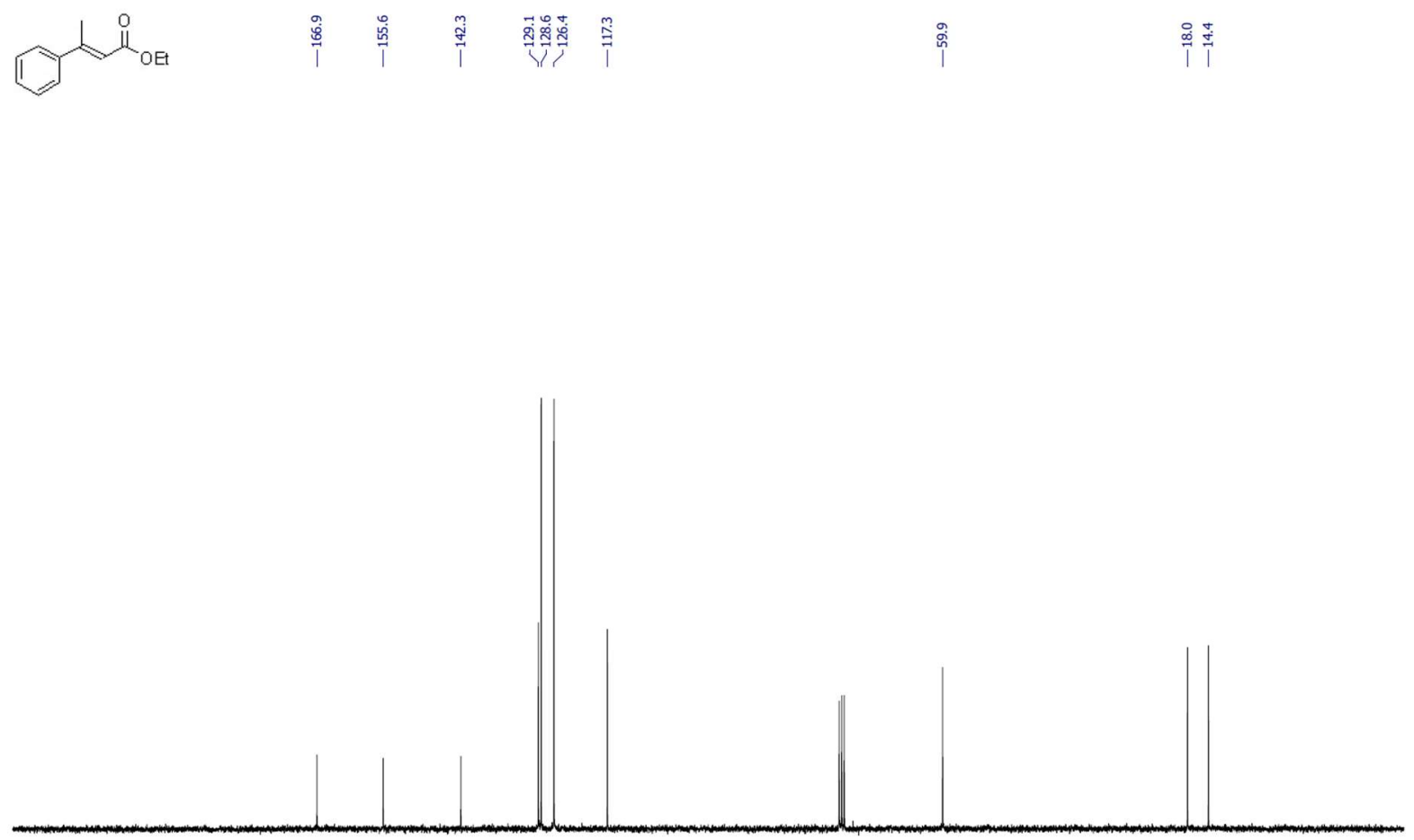

$\begin{array}{lllllllllllllllllllllllllll}210 & 200 & 190 & 180 & 170 & 160 & 150 & 140 & 130 & 120 & 110 & \begin{array}{c}100 \\ \mathrm{f} 1(\mathrm{ppm})\end{array} & 90 & 80 & 70 & 60 & 50 & 40 & 30 & 20 & 10 & 0 & -10\end{array}$

ethyl (E)-3-phenylpent-2-enoate ((E)-32):

${ }^{1} \mathrm{H} \mathrm{NMR}\left(\mathrm{CDCl}_{3}, 300 \mathrm{MHz}\right)$
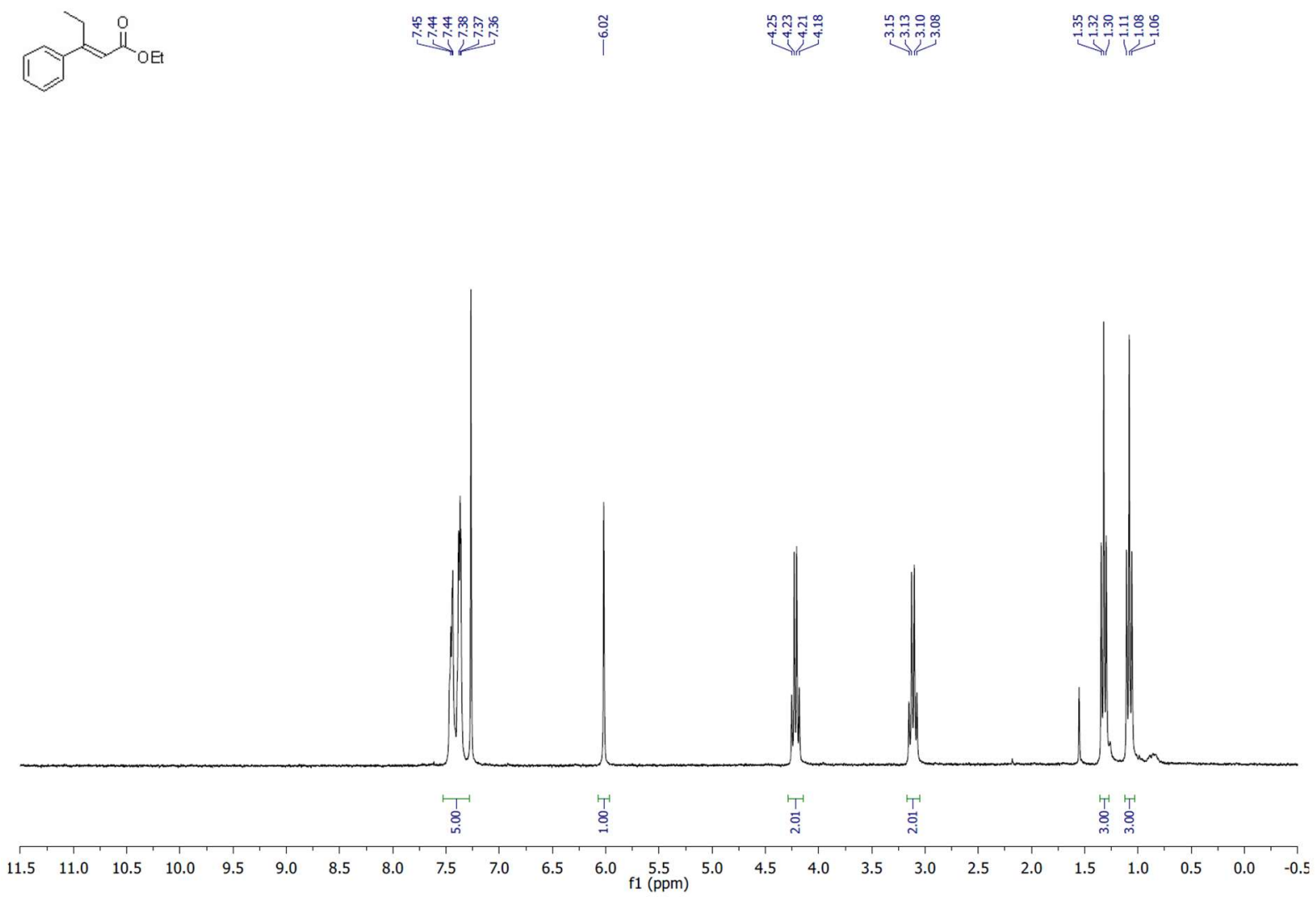
${ }^{13} \mathrm{C} \mathrm{NMR}\left(\mathrm{CDCl}_{3}, 75 \mathrm{MHz}\right)$

10 年

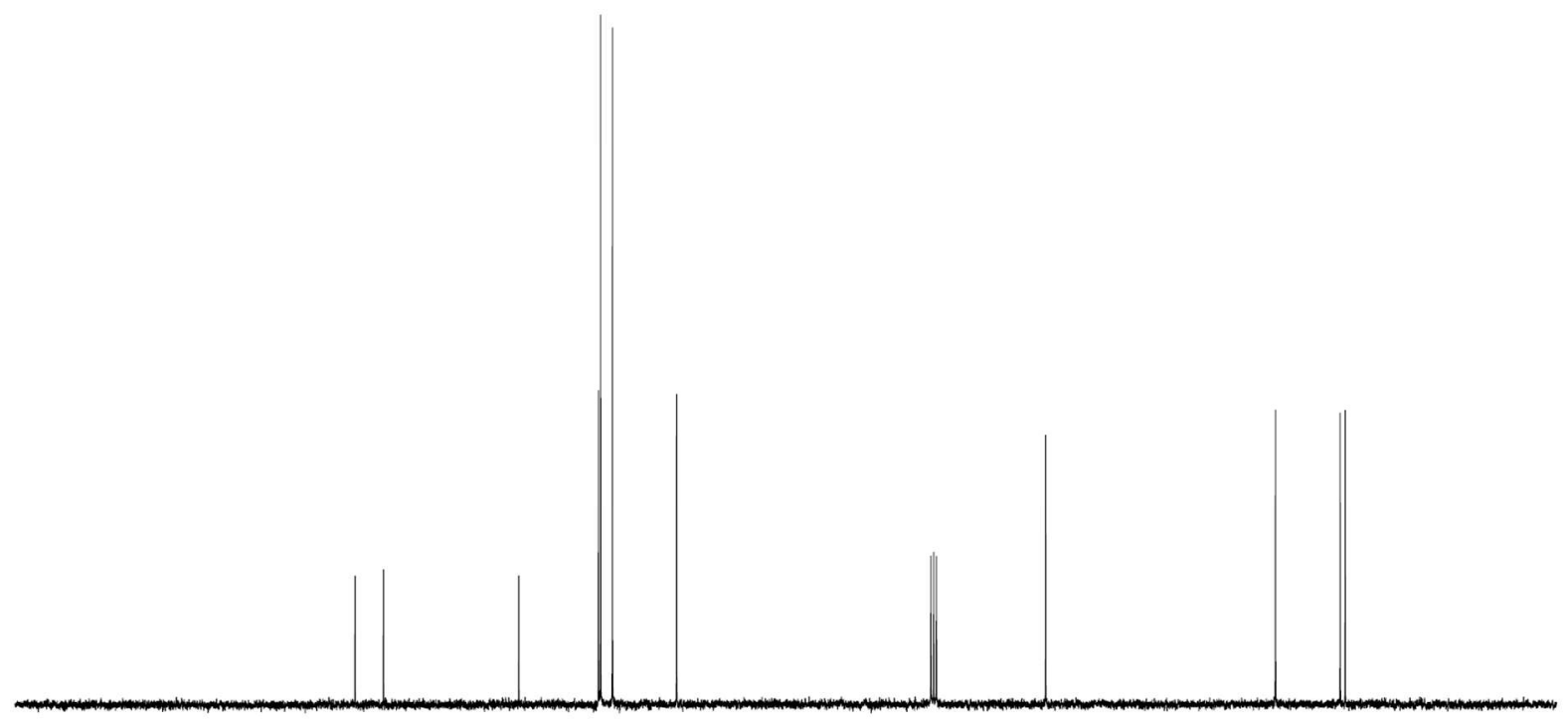

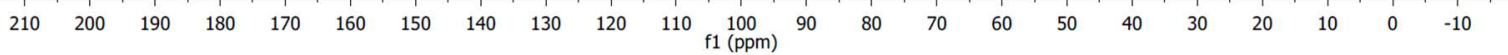

ethyl (E)-3-(p-tolyl)but-2-enoate ((E)-34):

${ }^{1} \mathrm{H} \mathrm{NMR}\left(\mathrm{CDCl}_{3}, 300 \mathrm{MHz}\right)$

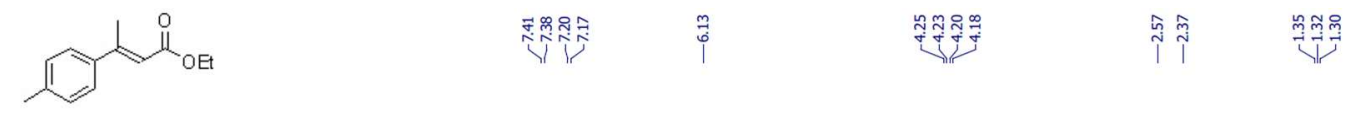

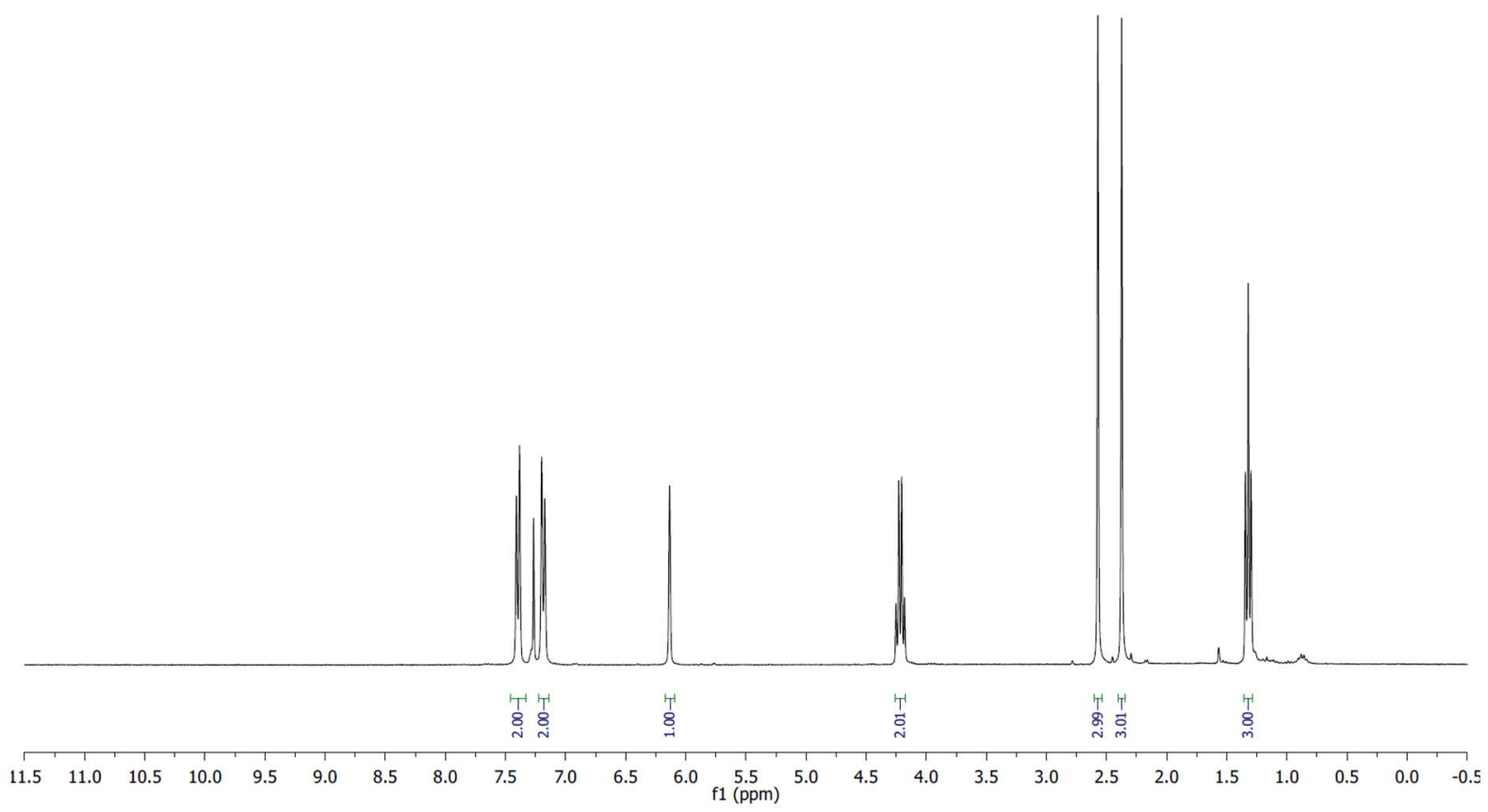


${ }^{13} \mathrm{C} \mathrm{NMR}\left(\mathrm{CDCl}_{3}, 75 \mathrm{MHz}\right)$
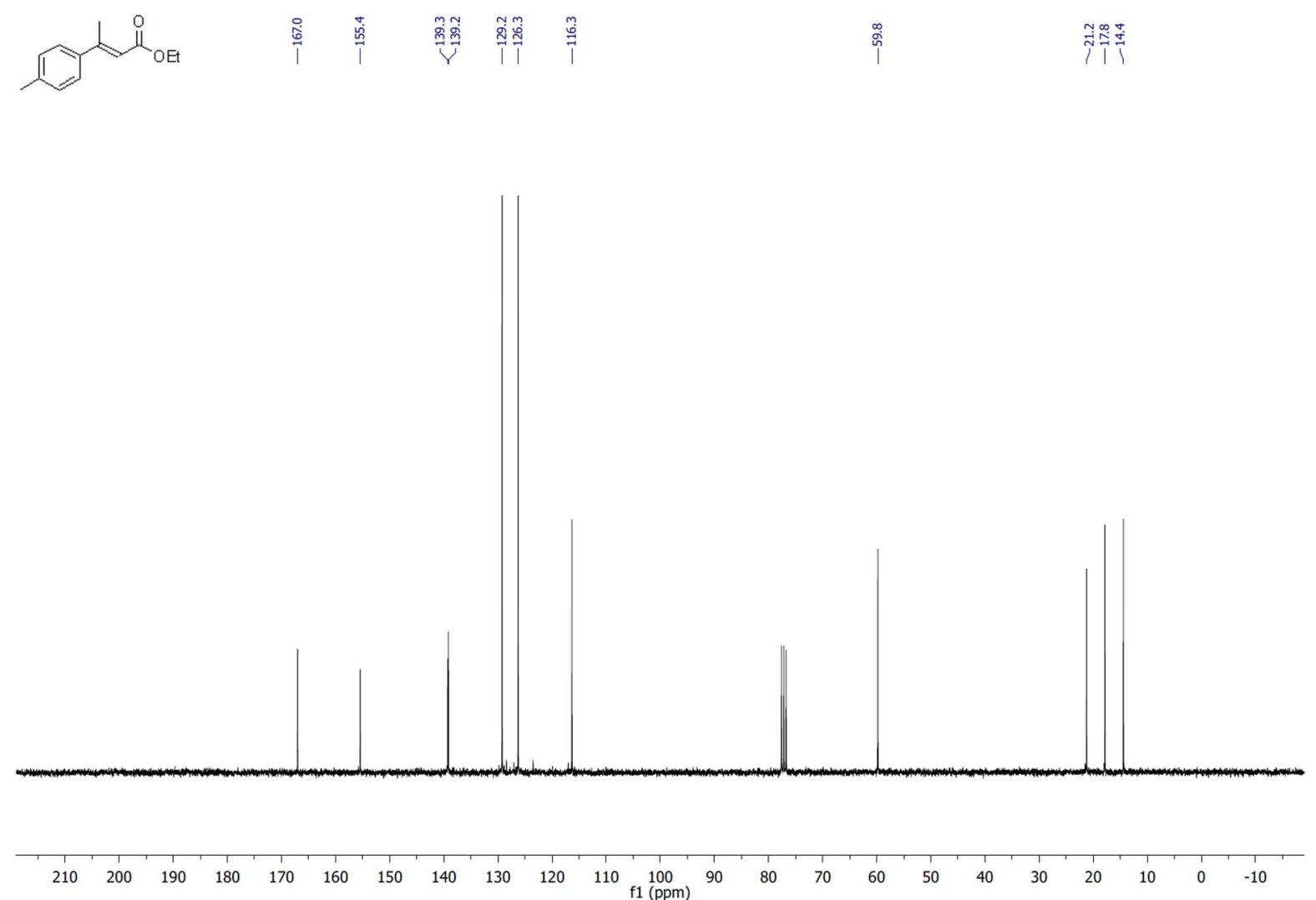

ethyl (E)-3-(m-tolyl)but-2-enoate ((E)-36):

${ }^{1} \mathrm{H} \mathrm{NMR}\left(\mathrm{CDCl}_{3}, 300 \mathrm{MHz}\right)$
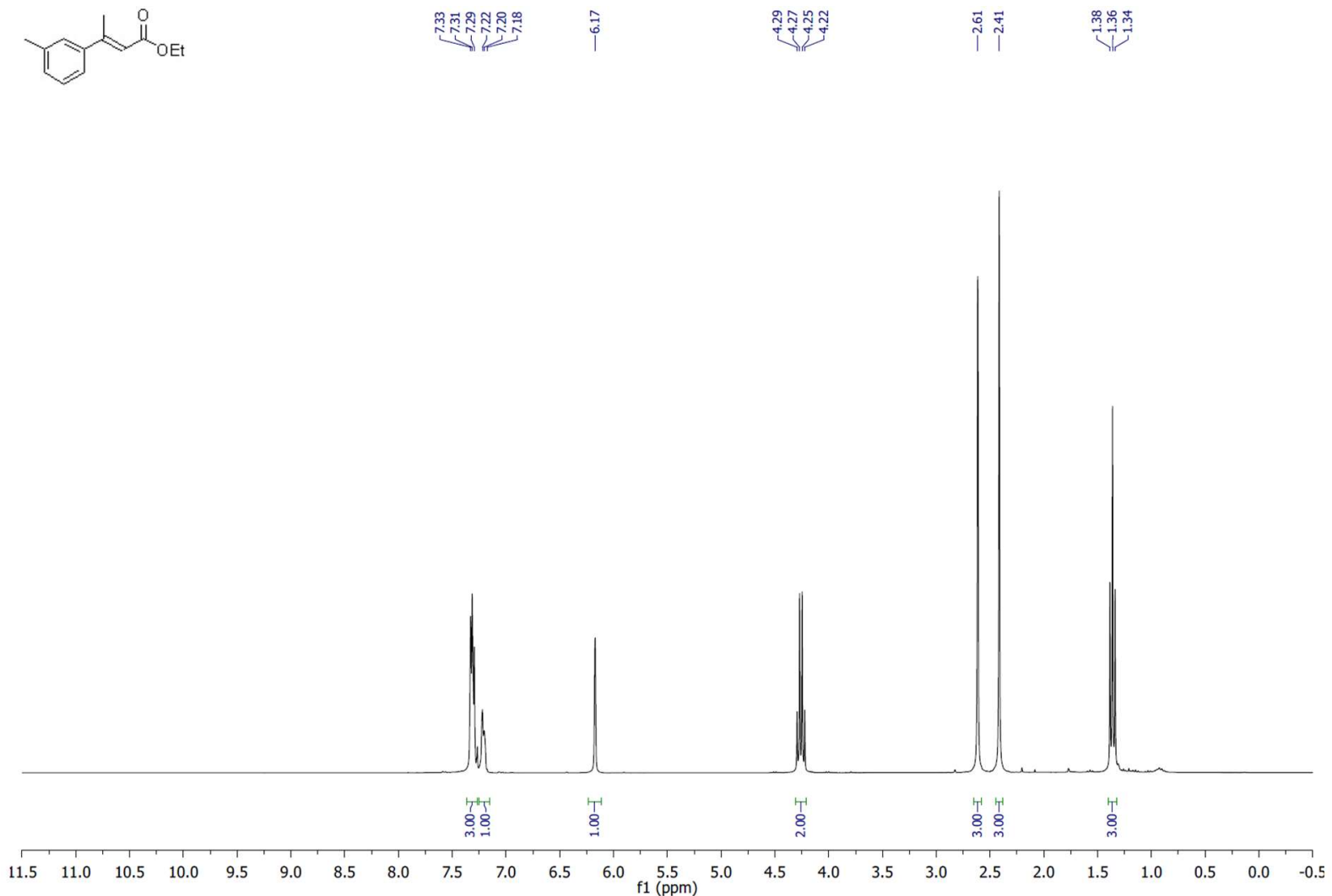
${ }^{13} \mathrm{C} \mathrm{NMR}\left(\mathrm{CDCl}_{3}, 75 \mathrm{MHz}\right)$
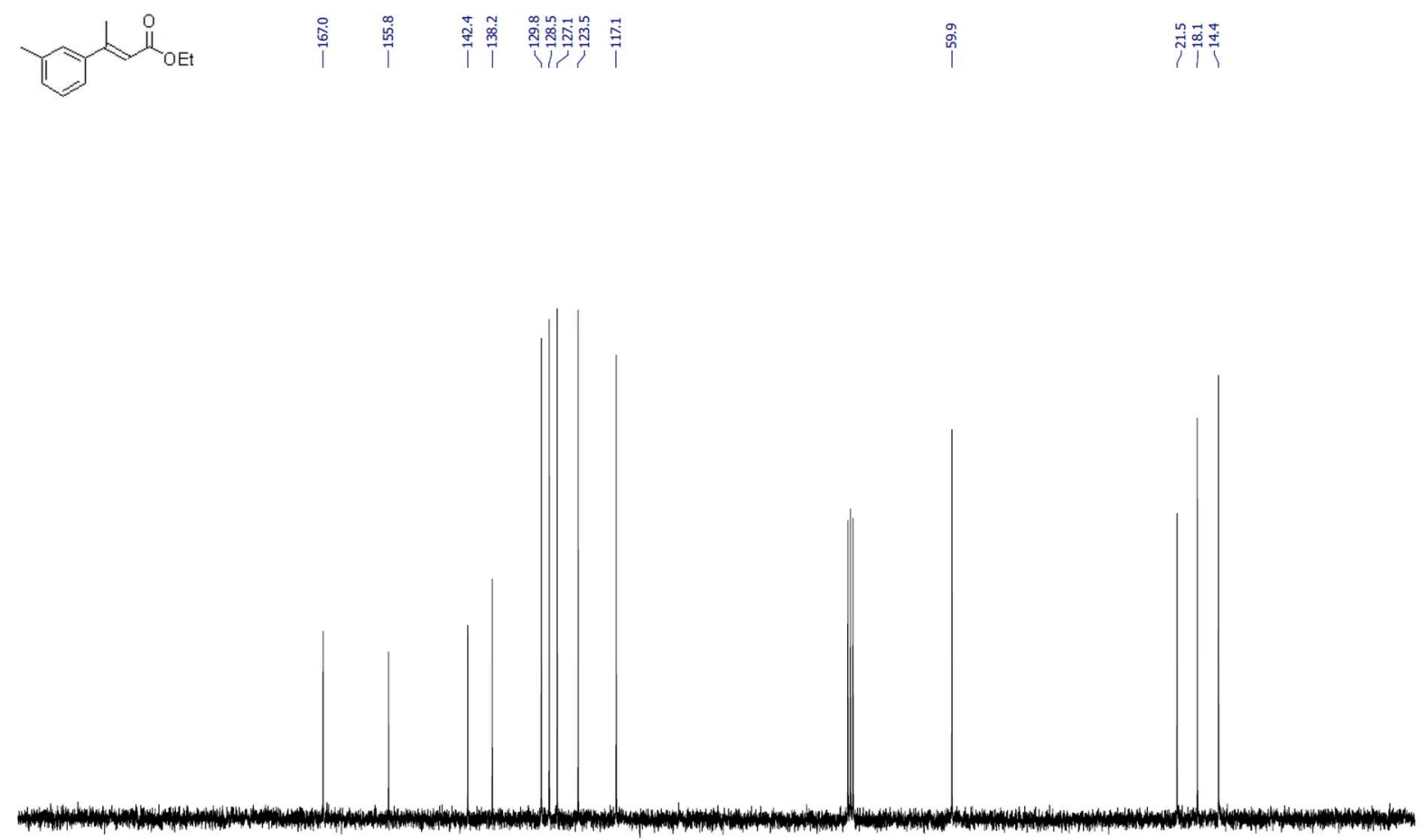

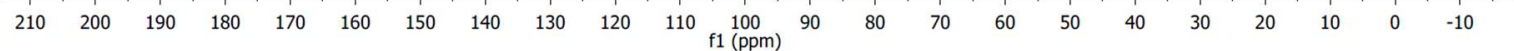

ethyl (E)-3-(o-tolyl)but-2-enoate ((E)-38):

${ }^{1} \mathrm{H} \mathrm{NMR}\left(\mathrm{CDCl}_{3}, 300 \mathrm{MHz}\right)$

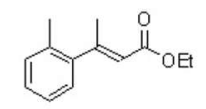

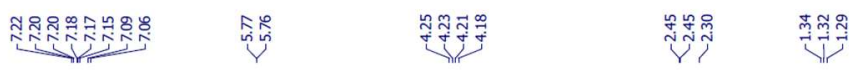

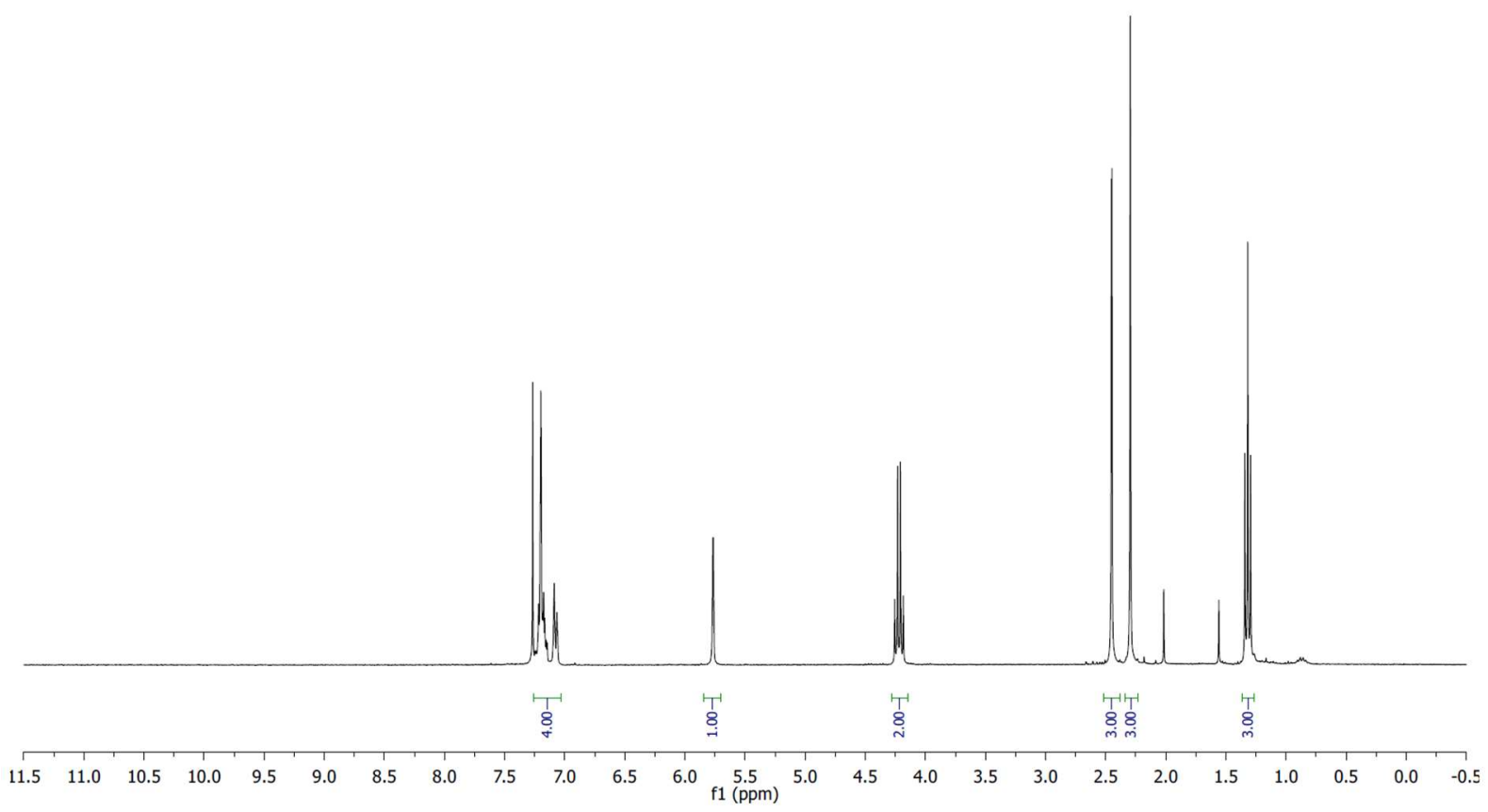


${ }^{13} \mathrm{C} \mathrm{NMR}\left(\mathrm{CDCl}_{3}, 75 \mathrm{MHz}\right)$
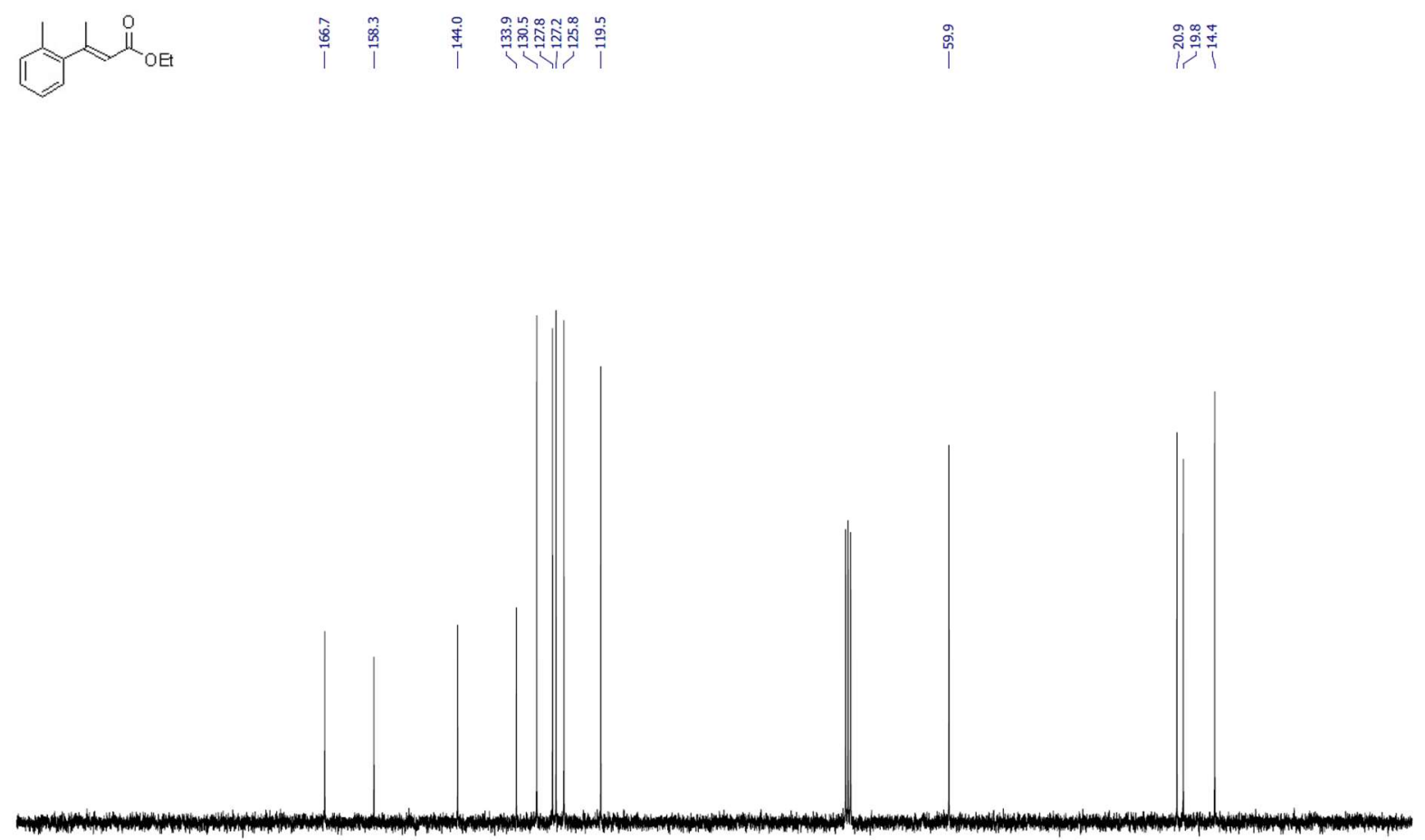

$\begin{array}{llllllllllllllllllllllllllll}210 & 200 & 190 & 180 & 170 & 160 & 150 & 140 & 130 & 120 & 110 & 100 & 90 & 80 & 70 & 60 & 50 & 40 & 30 & 20 & 10 & 0 & -10\end{array}$

ethyl (E)-3-(4-(tert-butyl)phenyl)but-2-enoate ((E)-40):

${ }^{1} \mathrm{H} \mathrm{NMR}\left(\mathrm{CDCl}_{3}, 300 \mathrm{MHz}\right)$

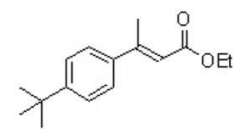

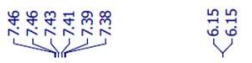

过的罚

踣弯

U.

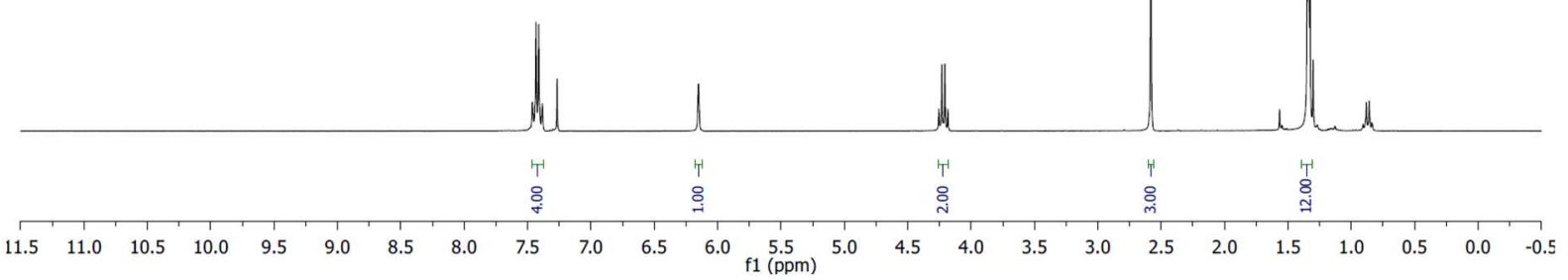


${ }^{13} \mathrm{C} \mathrm{NMR}\left(\mathrm{CDCl}_{3}, 75 \mathrm{MHz}\right)$

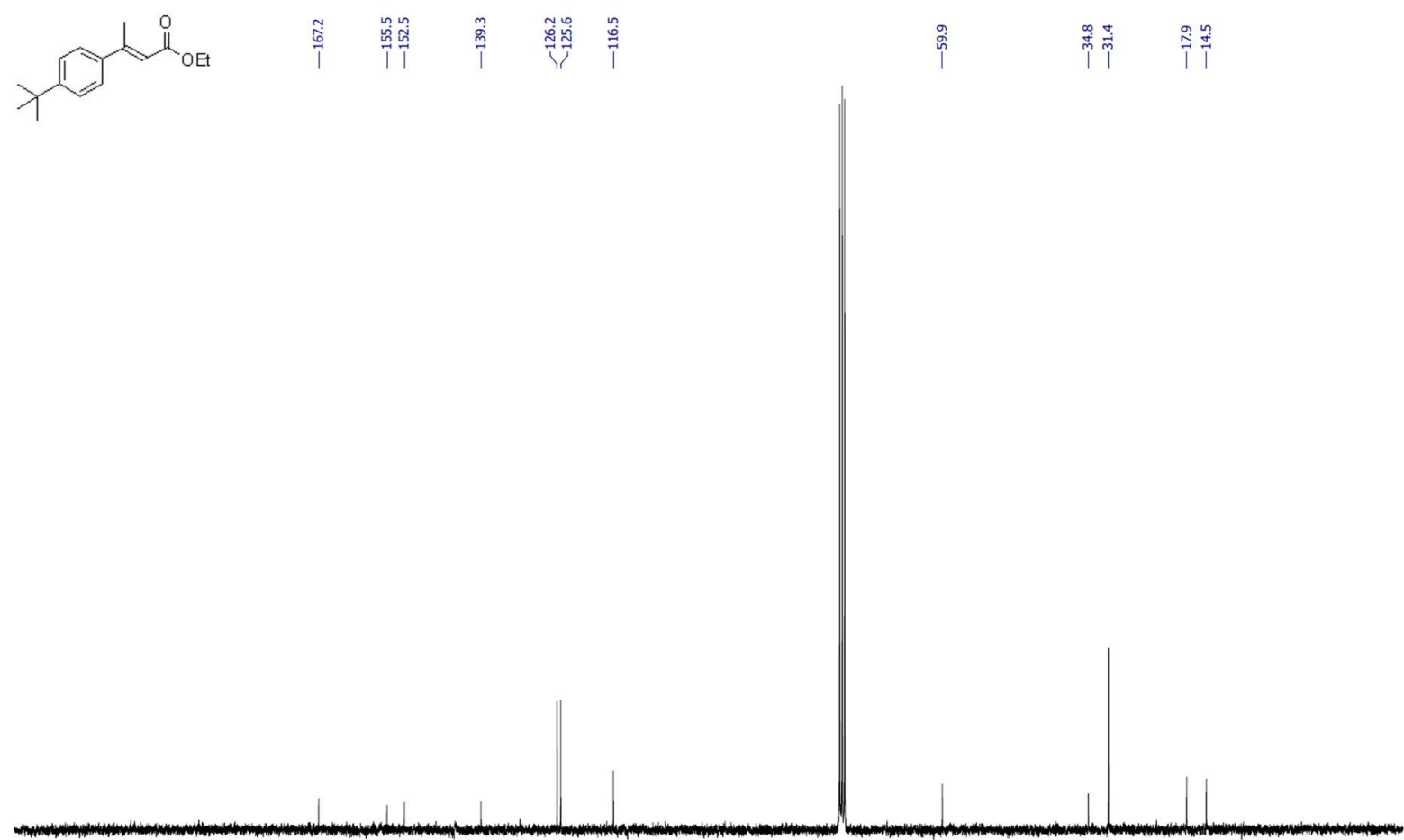

$\begin{array}{lllllllllllllllllllllll}210 & 200 & 190 & 180 & 170 & 160 & 150 & 140 & 130 & 120 & 110 & \begin{array}{l}100 \\ 100\end{array} & 90 & 80 & 70 & 60 & 50 & 40 & 30 & 20 & 10 & 0 & -10\end{array}$

ethyl (E)-3-(4-methoxyphenyl)but-2-enoate ((E)-42):

${ }^{1} \mathrm{H} \mathrm{NMR}\left(\mathrm{CDCl}_{3}, 300 \mathrm{MHz}\right)$
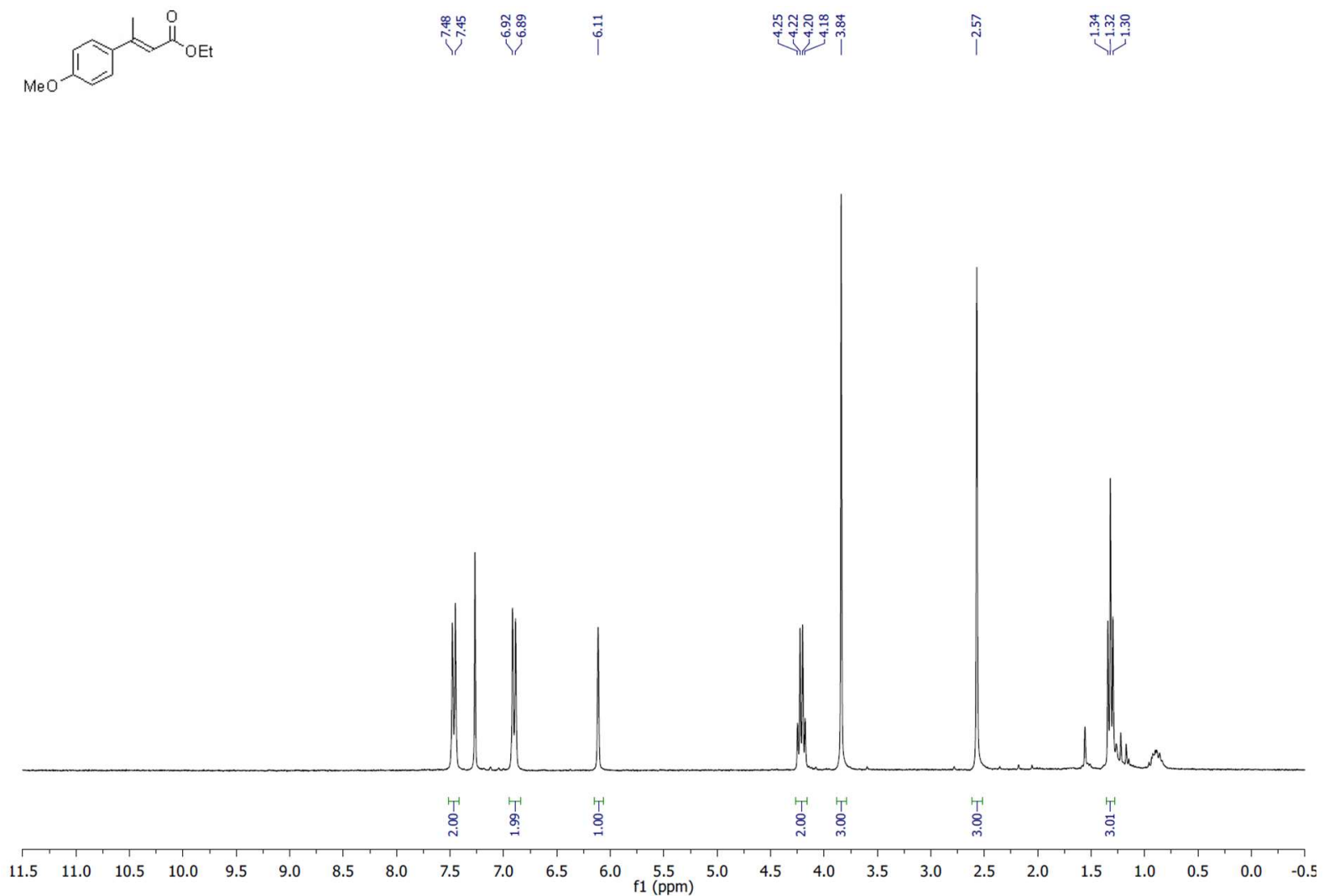
${ }^{13} \mathrm{C} \mathrm{NMR}\left(\mathrm{CDCl}_{3}, 75 \mathrm{MHz}\right)$

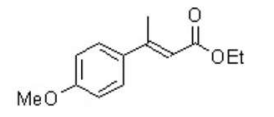

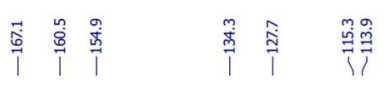
总触

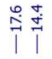

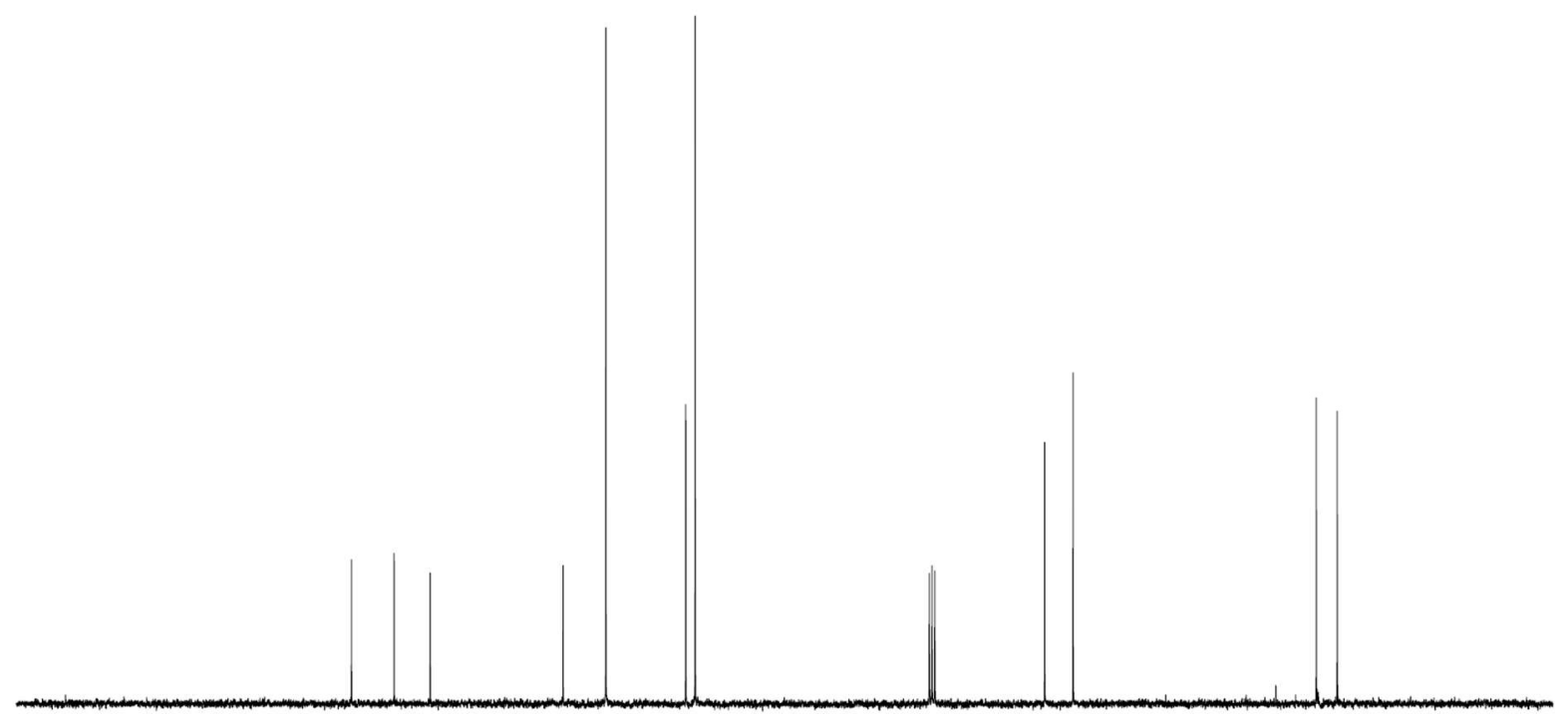

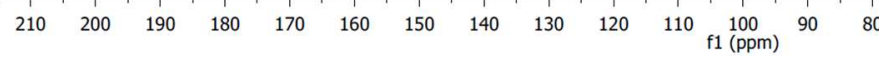

ethyl (E)-3-(4-fluorophenyl)but-2-enoate ((E)-44):

${ }^{1} \mathrm{H} \mathrm{NMR}\left(\mathrm{CDCl}_{3}, 300 \mathrm{MHz}\right)$

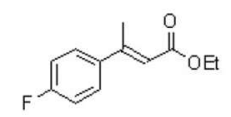

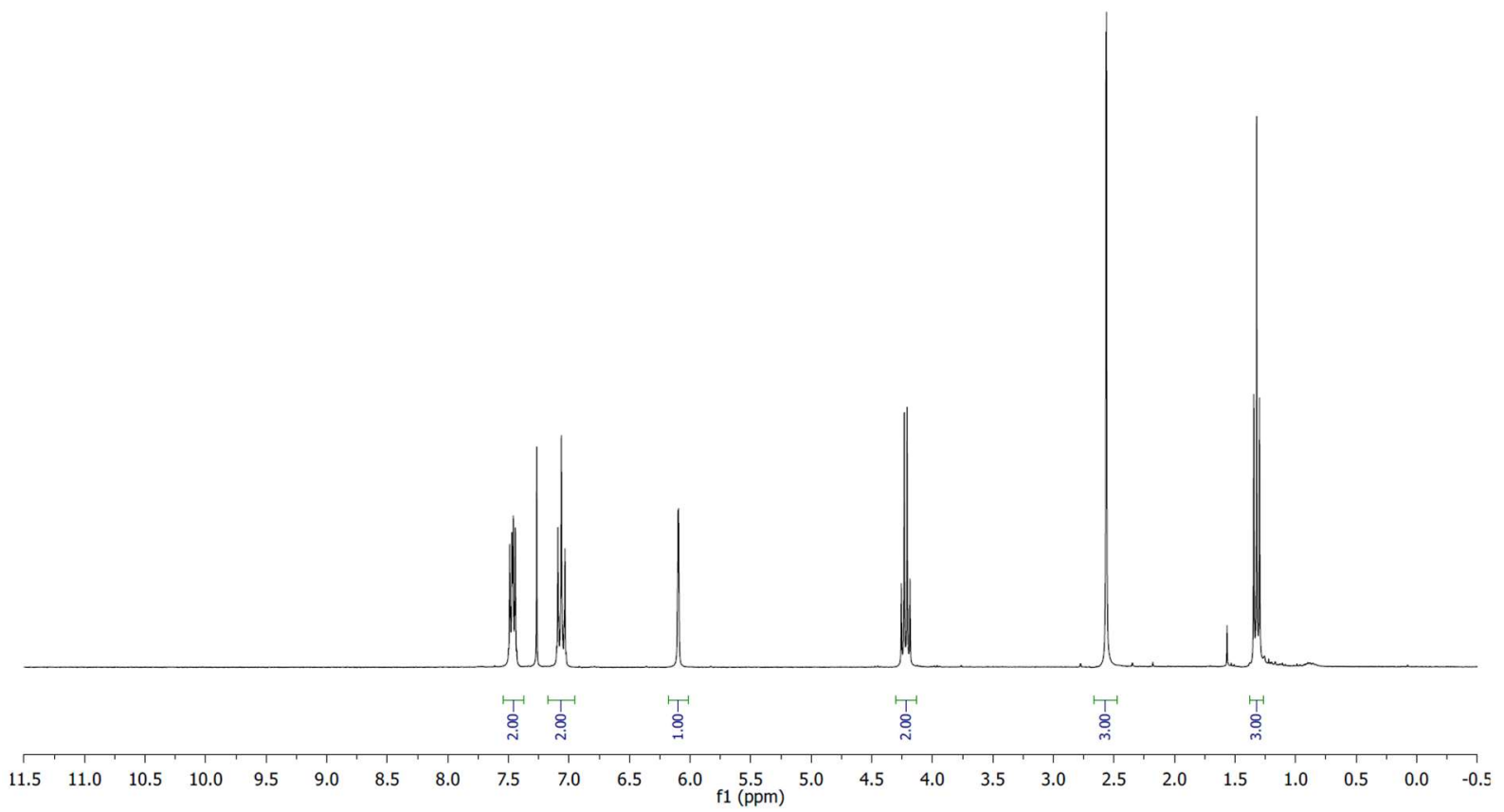


${ }^{13} \mathrm{C} \mathrm{NMR}\left(\mathrm{CDCl}_{3}, 75 \mathrm{MHz}\right)$

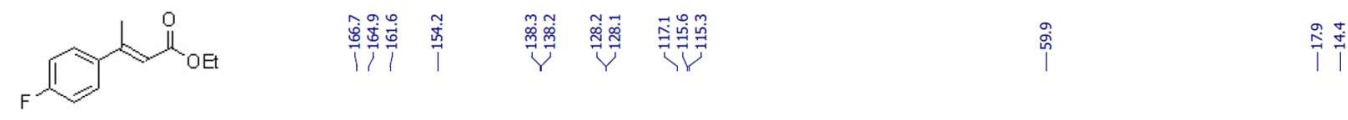

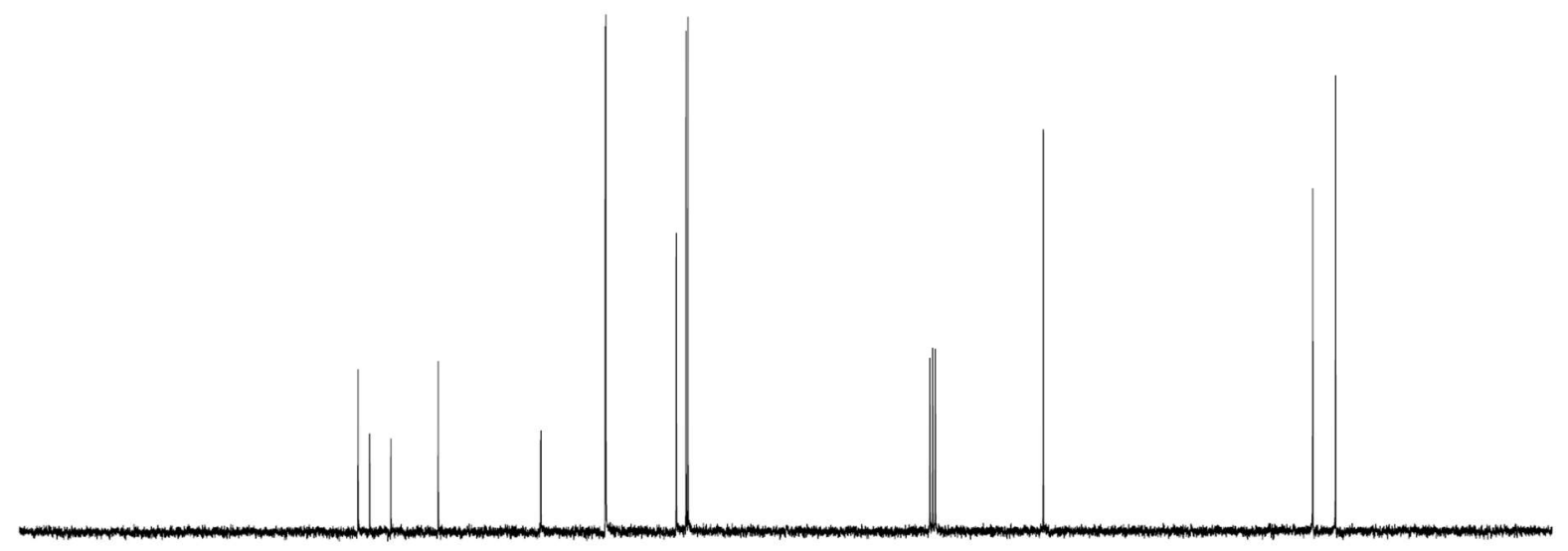

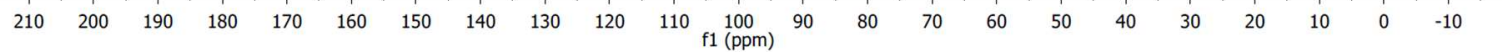

${ }^{19} \mathrm{~F} \mathrm{NMR}\left(\mathrm{CDCl}_{3}, 282 \mathrm{MHz}\right)$

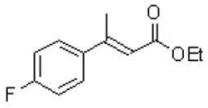

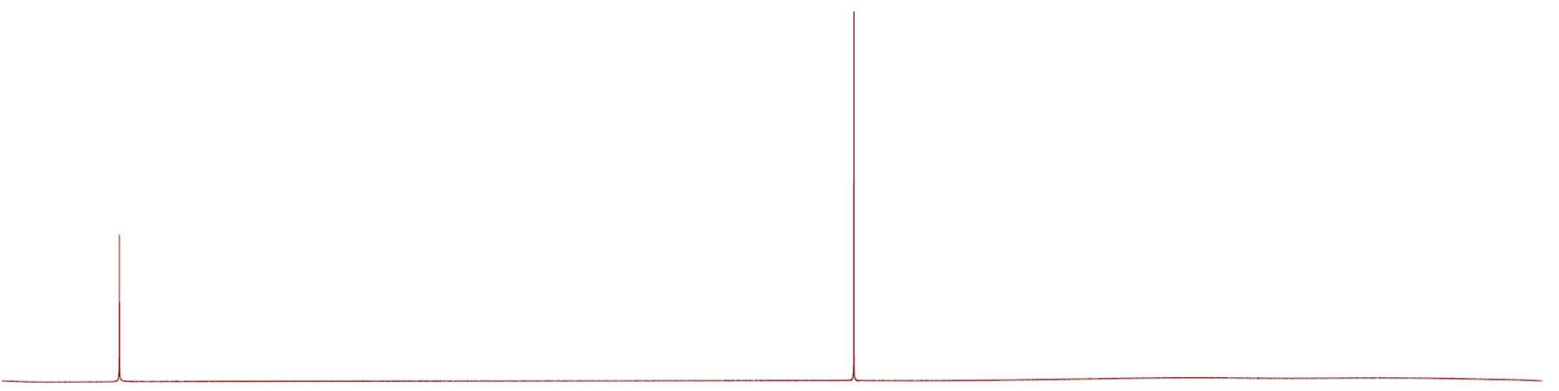

$\begin{array}{llllllllllllllllllllllll}10 & 0 & -10 & -20 & -30 & -40 & -50 & -60 & -70 & -80 & -90 & -100 & -110 & -120 & -130 & -140 & -150 & -160 & -170 & -180 & -190 & -200 & -210\end{array}$ 
ethyl (E)-3-(4-bromophenyl)but-2-enoate ((E)-46):

${ }^{1} \mathrm{H} \mathrm{NMR}\left(\mathrm{CDCl}_{3}, 300 \mathrm{MHz}\right)$<smiles>CC(C)=CC=C1C=CC(Br)C1</smiles>

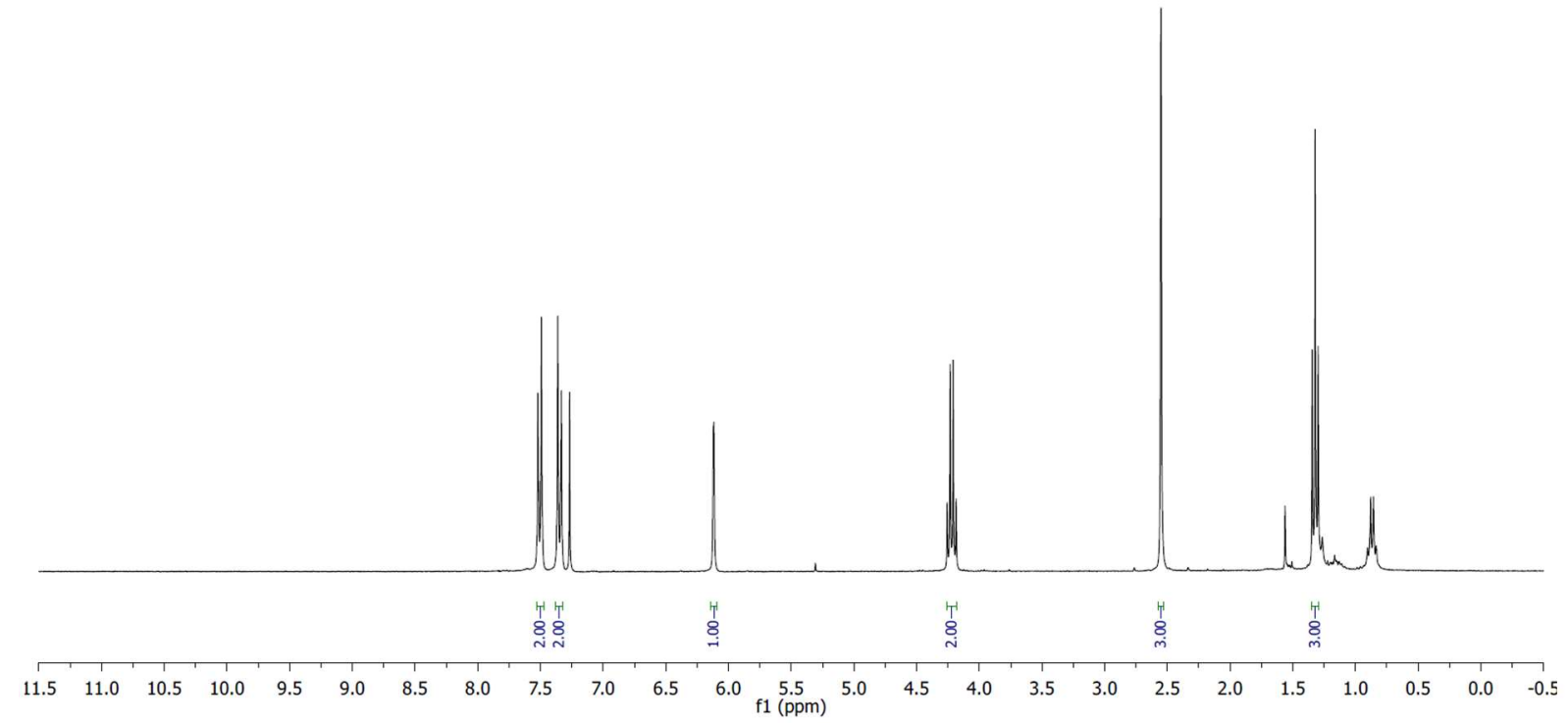

${ }^{13} \mathrm{C} \mathrm{NMR}\left(\mathrm{CDCl}_{3}, 75 \mathrm{MHz}\right)$

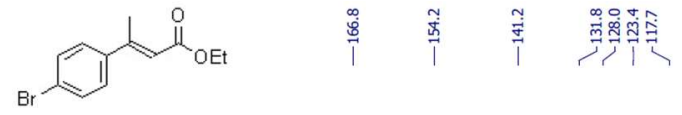

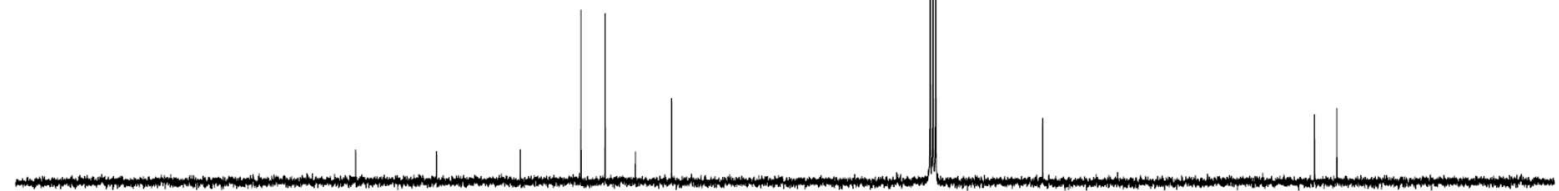

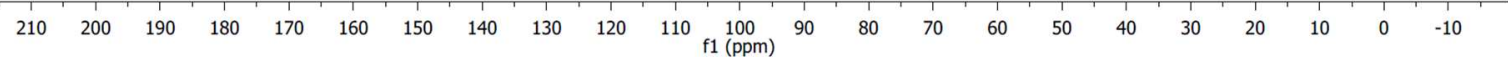


ethyl (E)-3-(4-iodophenyl)but-2-enoate ((E)-48):

${ }^{1} \mathrm{H} \mathrm{NMR}\left(\mathrm{CDCl}_{3}, 300 \mathrm{MHz}\right)$

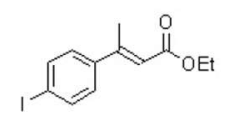

ฟกำ

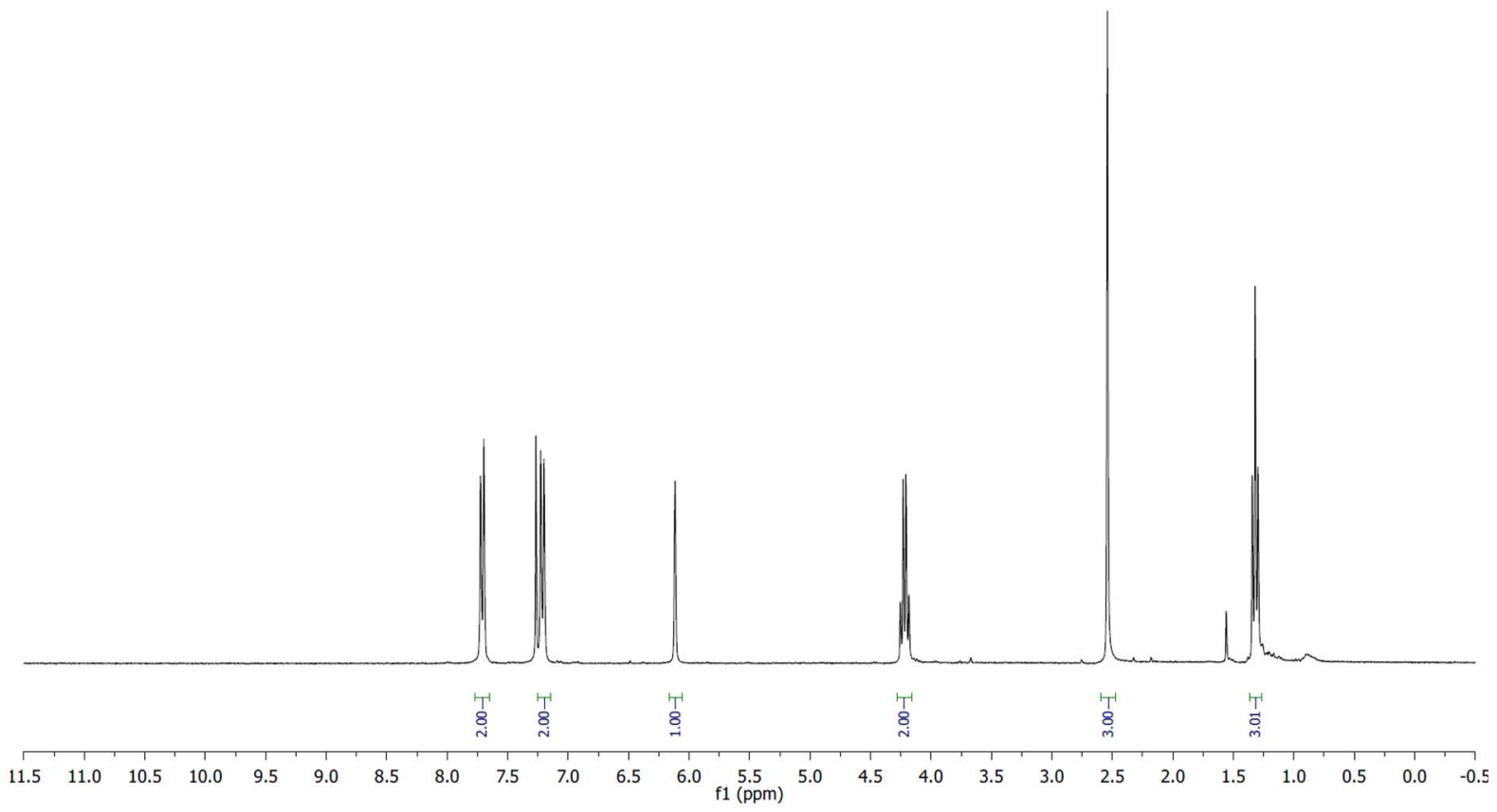

${ }^{13} \mathrm{C} \mathrm{NMR}\left(\mathrm{CDCl}_{3}, 75 \mathrm{MHz}\right)$

篮

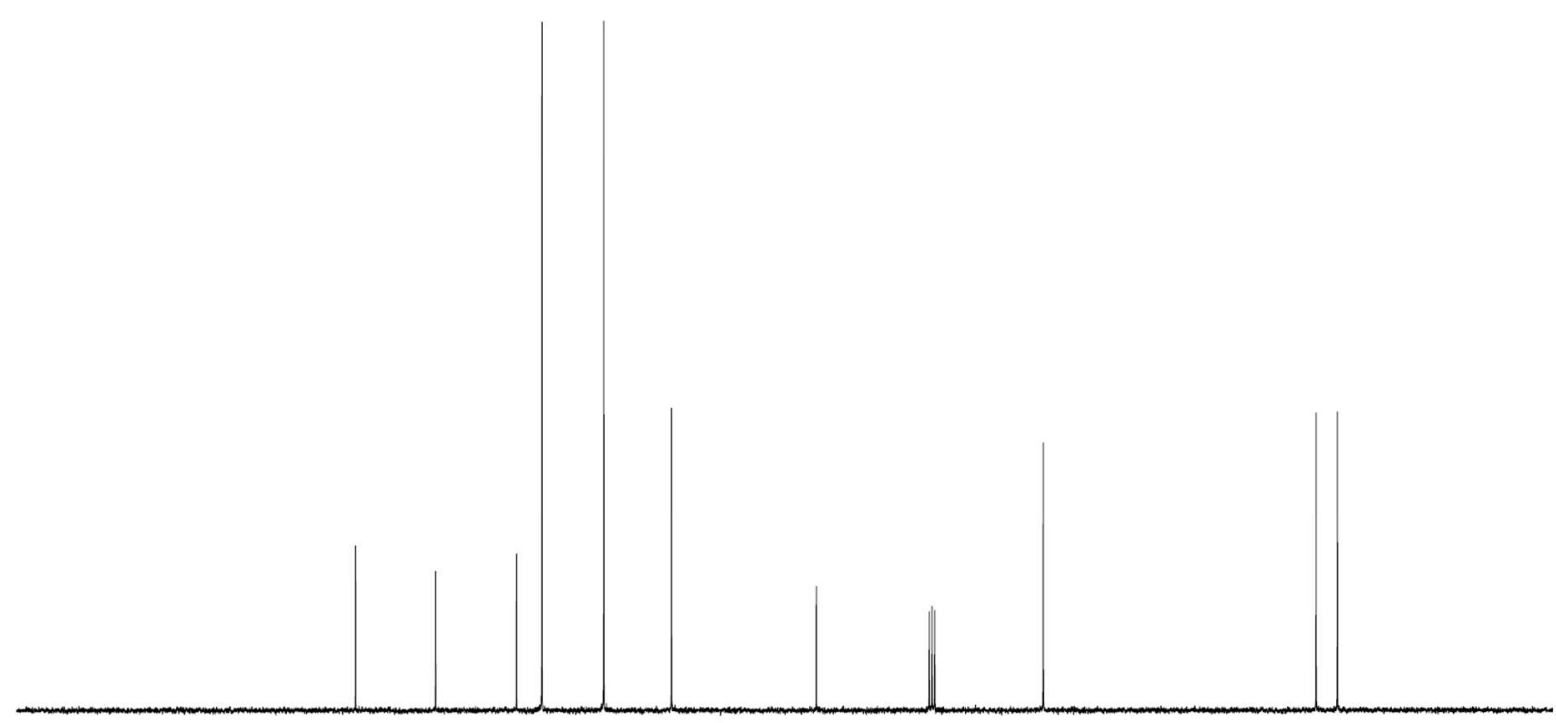

$\begin{array}{llllllllllllllllllllllll}210 & 200 & 190 & 180 & 170 & 160 & 150 & 140 & 130 & 120 & 110 & 100 & 90 & 80 & 70 & 60 & 50 & 40 & 30 & 20 & 10 & 0 & -10\end{array}$ 
ethyl (E)-3-(4-nitrophenyl)but-2-enoate ((E)-50):

${ }^{1} \mathrm{H} \mathrm{NMR}\left(\mathrm{CDCl}_{3}, 300 \mathrm{MHz}\right)$
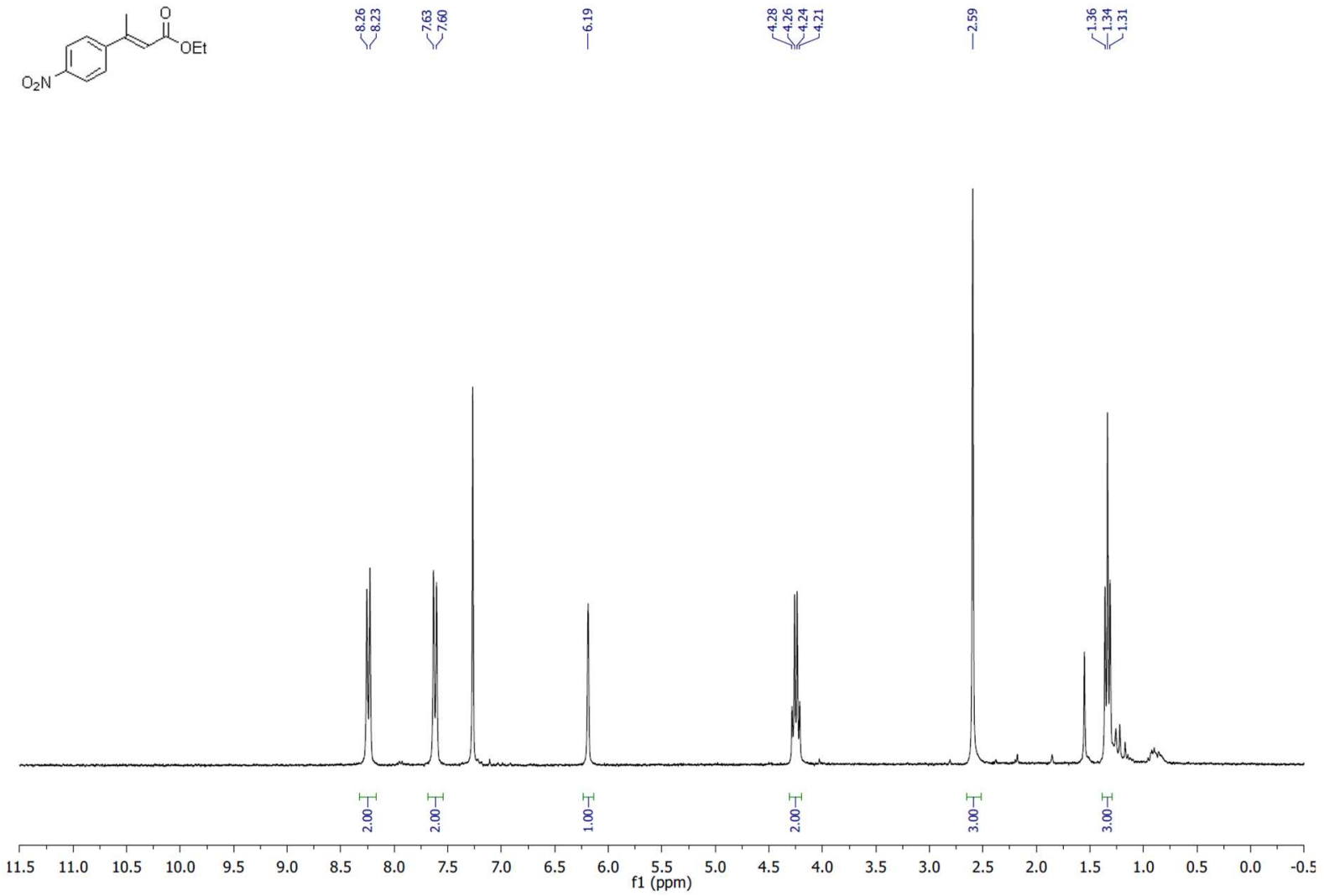

${ }^{13} \mathrm{C} \mathrm{NMR}\left(\mathrm{CDCl}_{3}, 75 \mathrm{MHz}\right)$
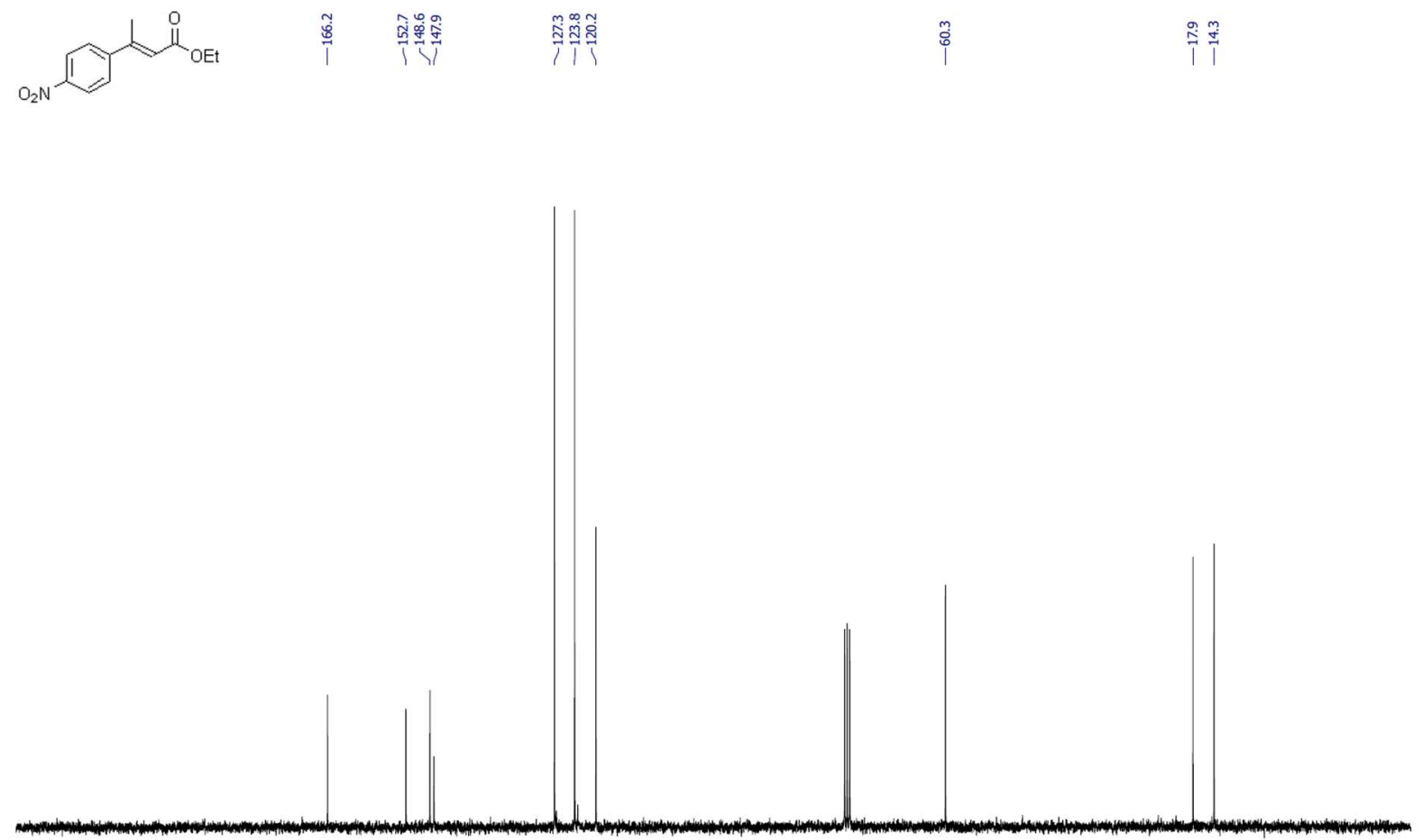

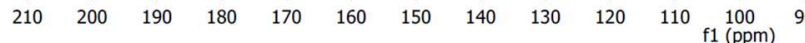


ethyl $(E)-4,4,4-$ trifluoro-3-phenylbut-2-enoate $((E)-52)$ :

${ }^{1} \mathrm{H} \mathrm{NMR}\left(\mathrm{CDCl}_{3}, 300 \mathrm{MHz}\right)$<smiles>CCOC(=O)/C=C(/c1ccccc1)C(F)(F)F</smiles>

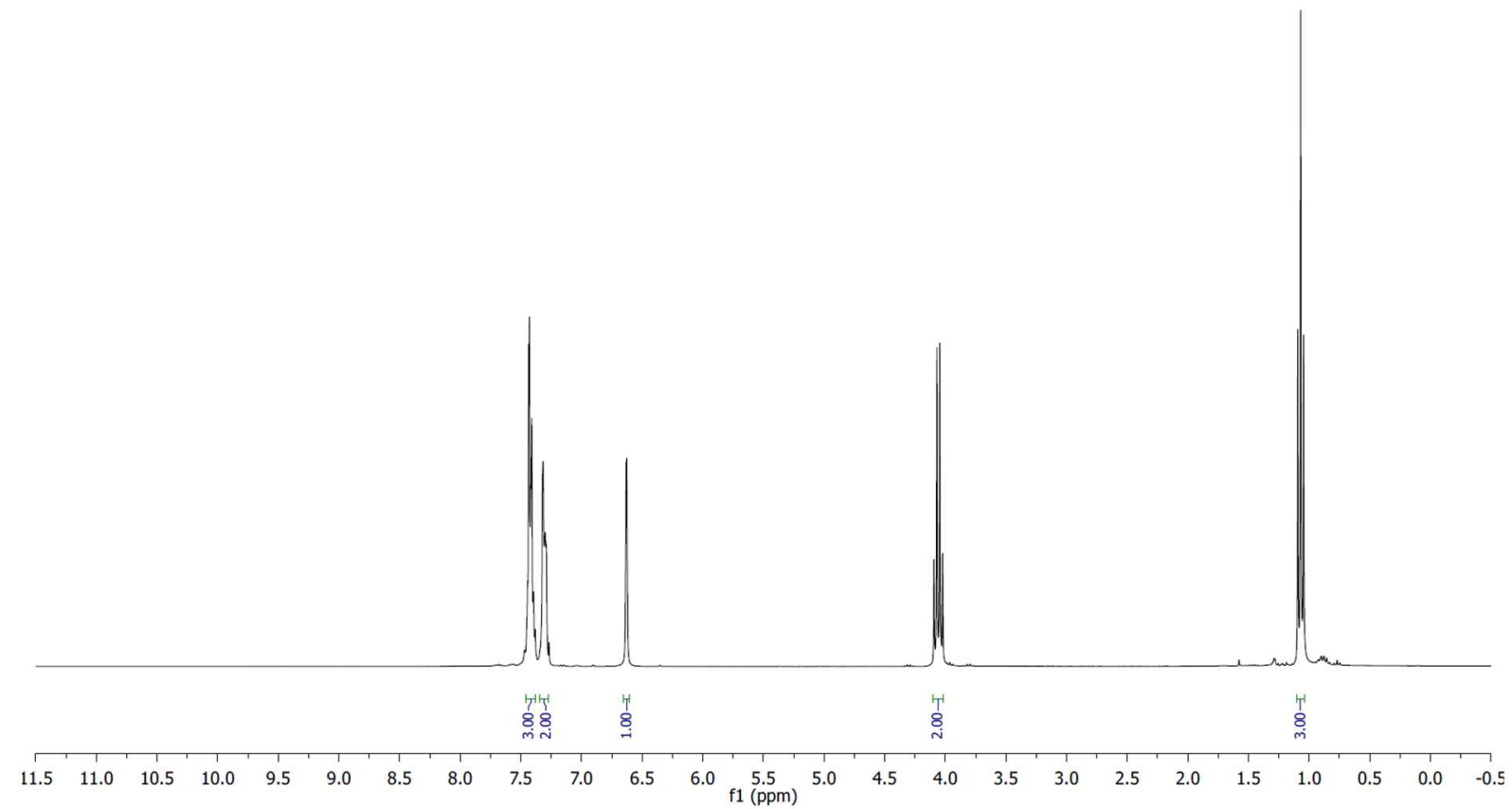

${ }^{13} \mathrm{C} \mathrm{NMR}\left(\mathrm{CDCl}_{3}, 75 \mathrm{MHz}\right)$

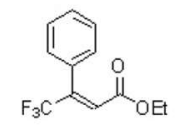

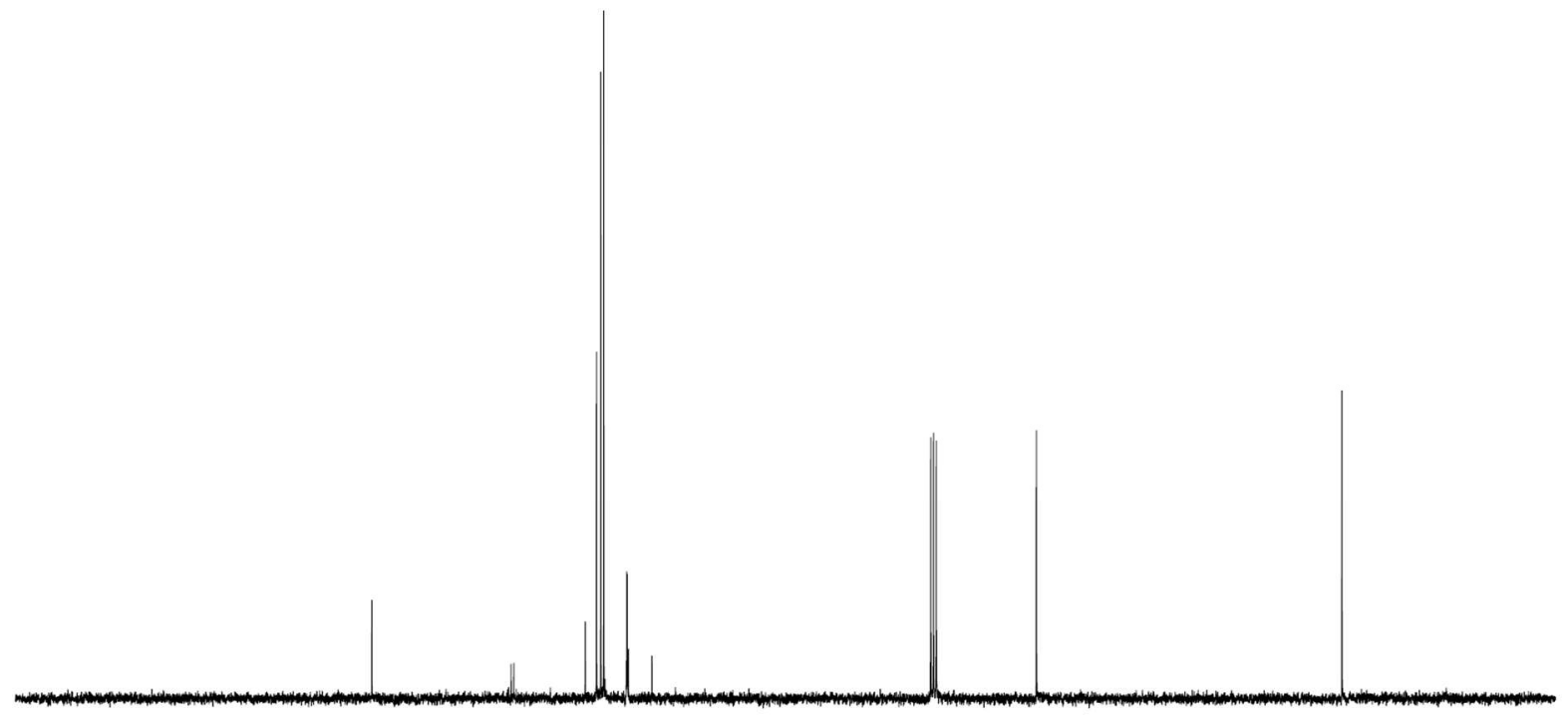

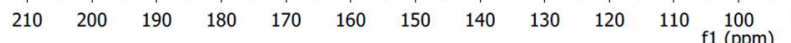


${ }^{19} \mathrm{~F} \mathrm{NMR}\left(\mathrm{CDCl}_{3}, 282 \mathrm{MHz}\right)$

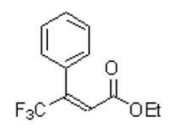

$\overrightarrow{\mathrm{p}}$

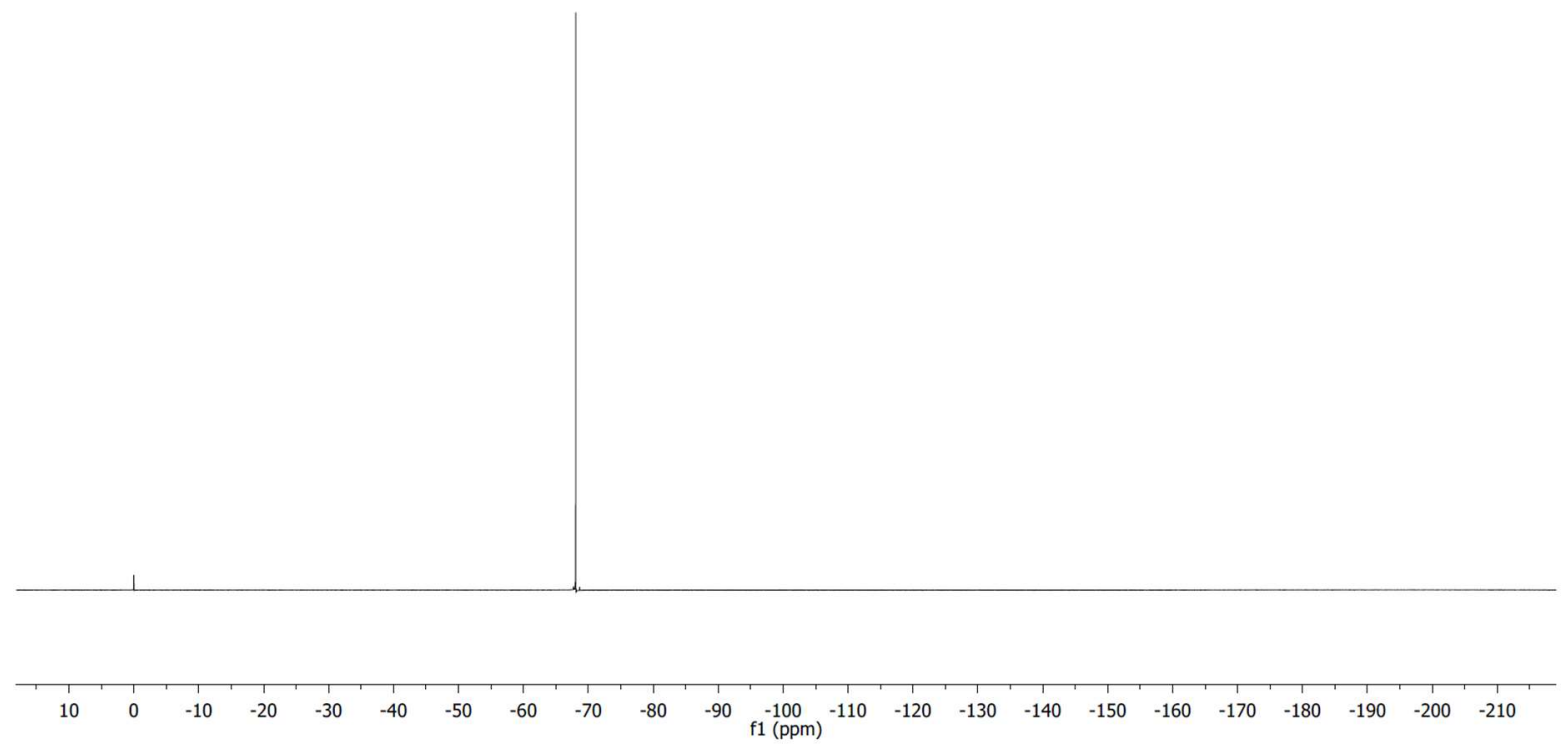

(E)-3-phenylbut-2-enoic acid (Ac-29):

${ }^{1} \mathrm{H} \mathrm{NMR}\left(\mathrm{CDCl}_{3}, 300 \mathrm{MHz}\right)$

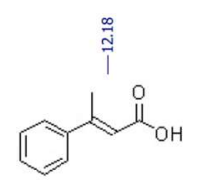

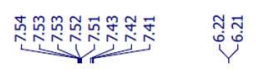

产

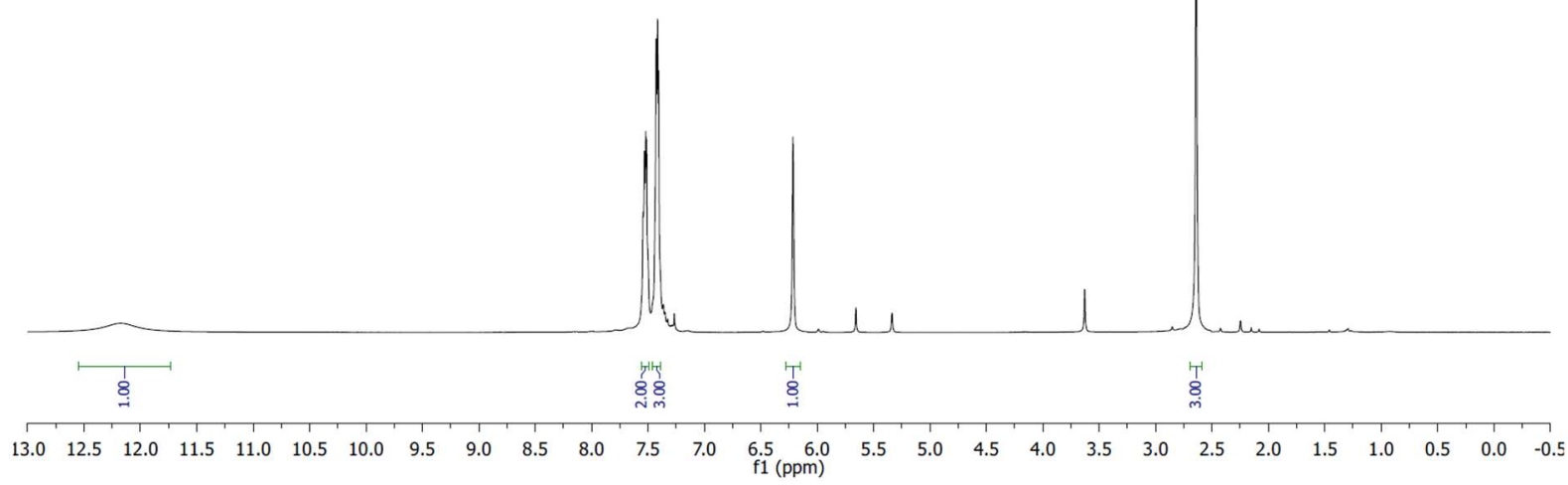

160 
${ }^{13} \mathrm{C} \mathrm{NMR}\left(\mathrm{CDCl}_{3}, 75 \mathrm{MHz}\right)$

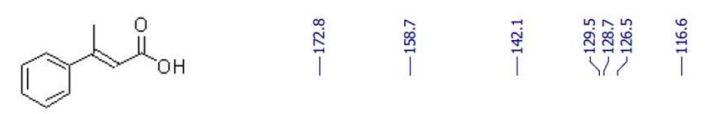

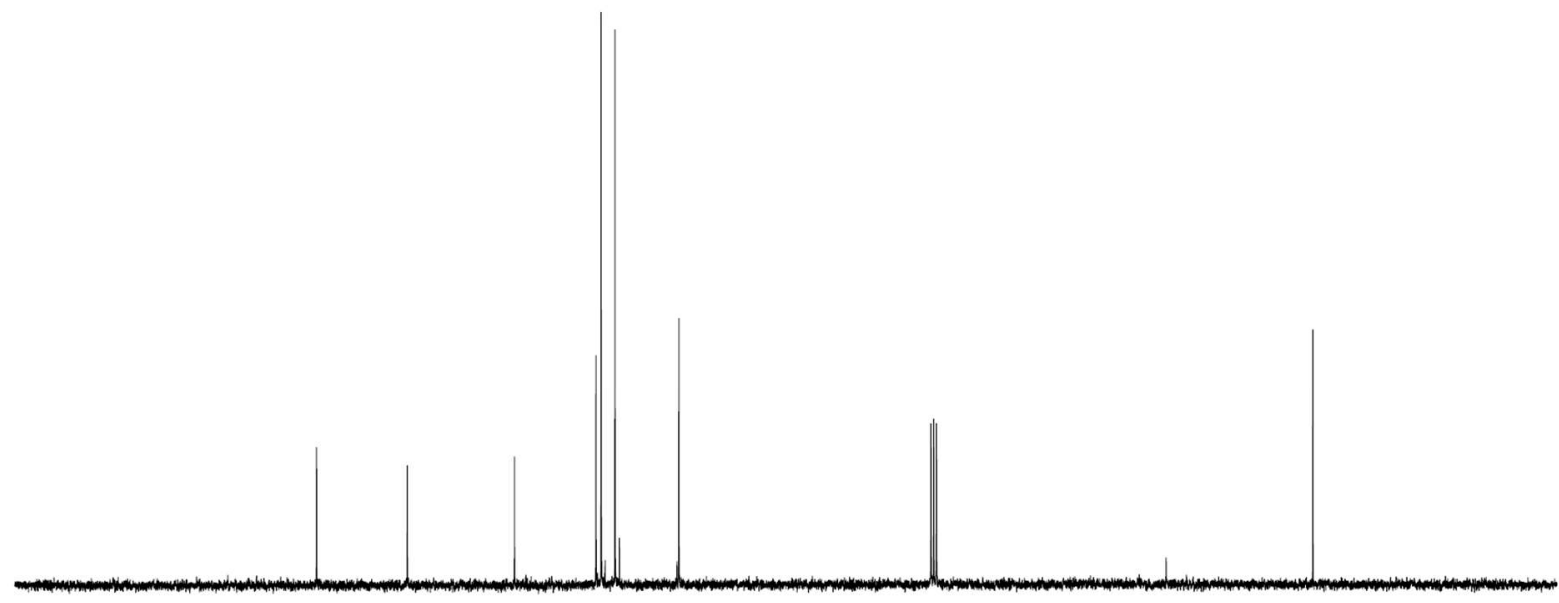

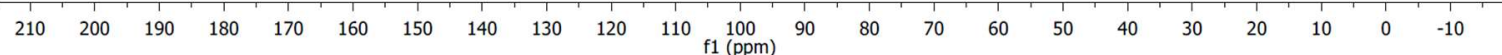

(E)-3-phenylpent-2-enoic acid (Ac-31):

${ }^{1} \mathrm{H} \mathrm{NMR}\left(\mathrm{CDCl}_{3}, 300 \mathrm{MHz}\right)$
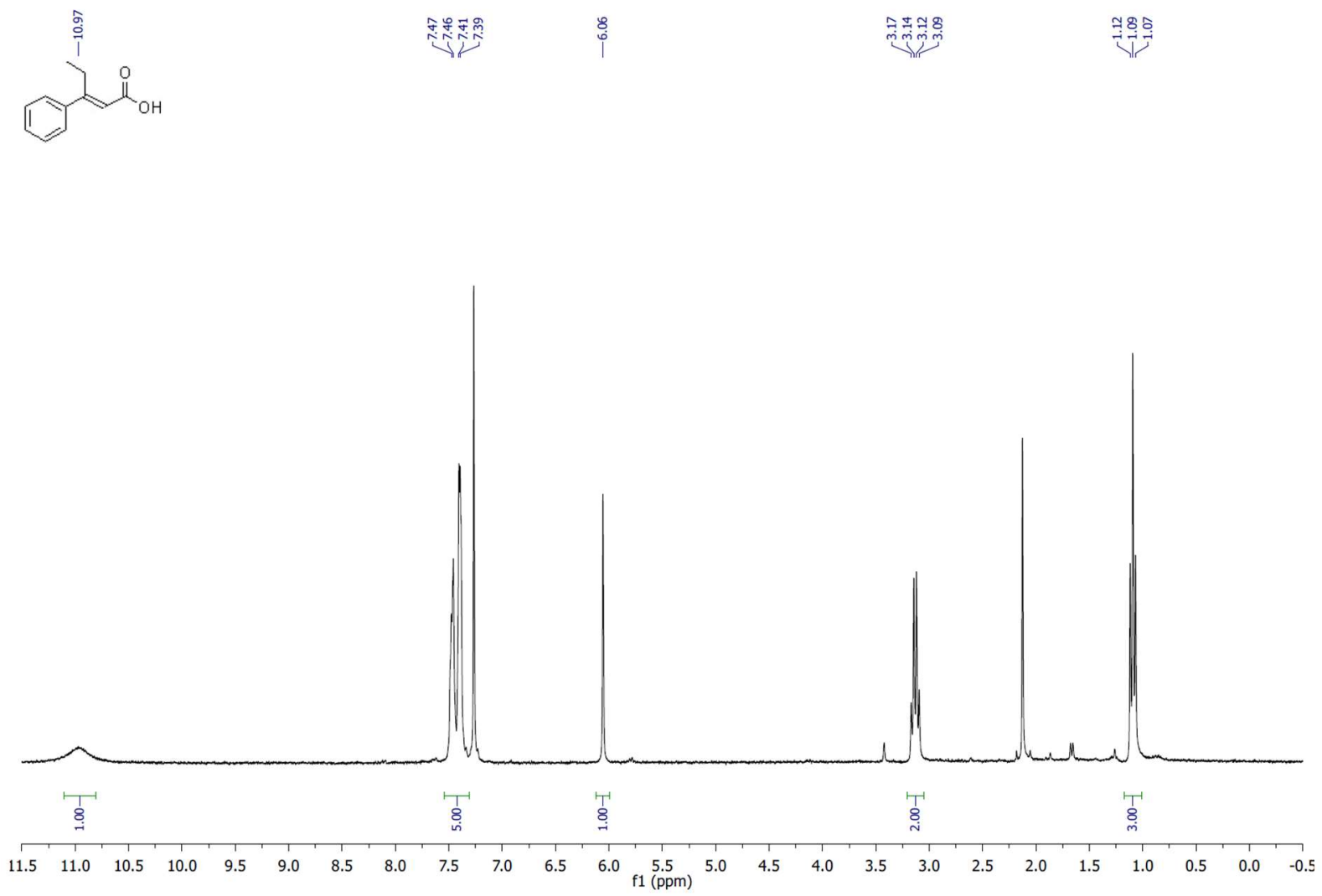
${ }^{13} \mathrm{C}$ NMR (DMSO-d6, $75 \mathrm{MHz}$ )
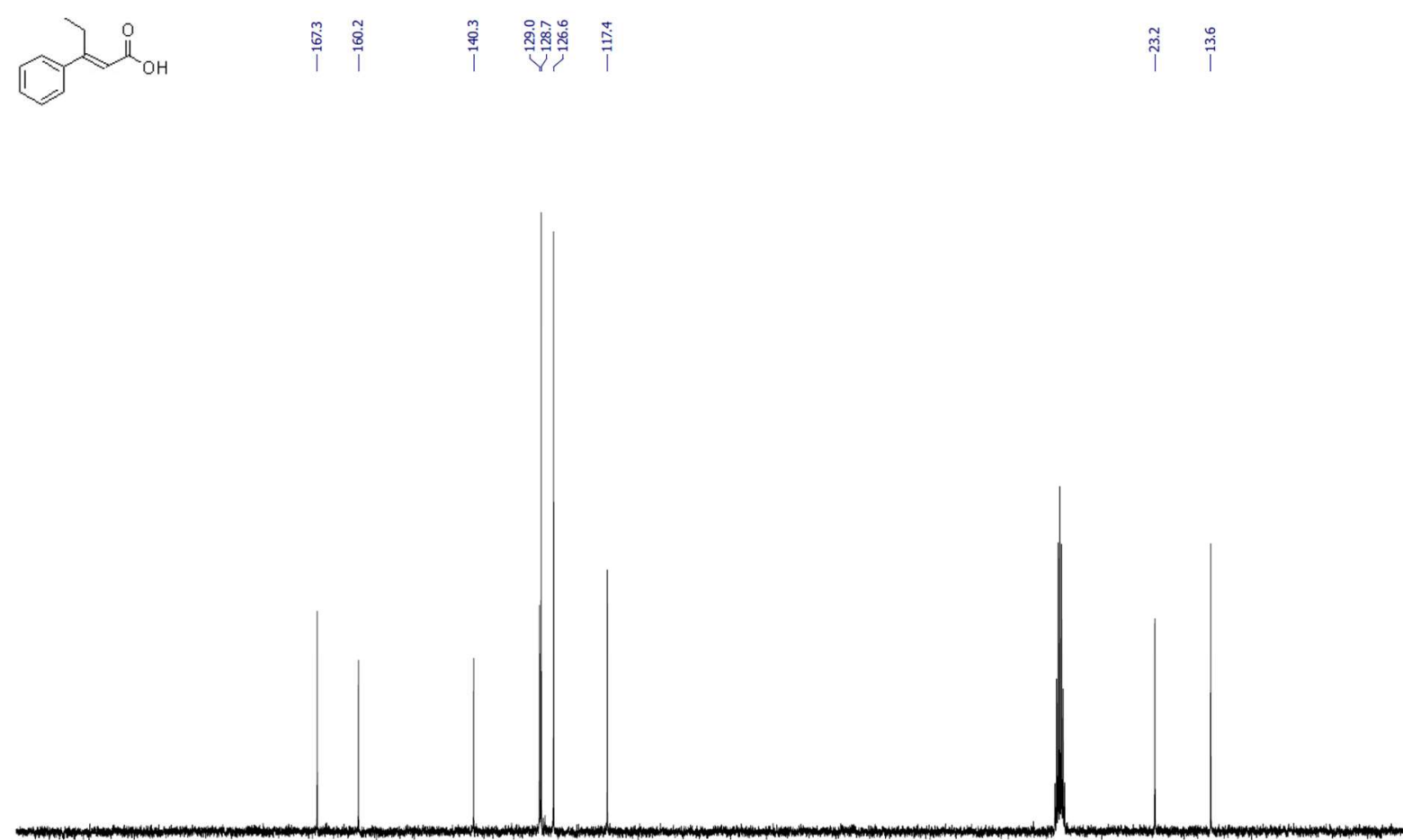

$\begin{array}{llllllllllllllllllllllllll}210 & 200 & 190 & 180 & 170 & 160 & 150 & 140 & 130 & 120 & 110 & \begin{array}{c}100 \\ \mathrm{f} 1(\mathrm{ppm})\end{array} & 90 & 80 & 70 & 60 & 50 & 40 & 30 & 20 & 10 & 0 & -10\end{array}$

(E)-3-(p-tolyl)but-2-enoic acid (Ac-33):

${ }^{1} \mathrm{H} \mathrm{NMR}\left(\mathrm{CDCl}_{3}, 300 \mathrm{MHz}\right)$
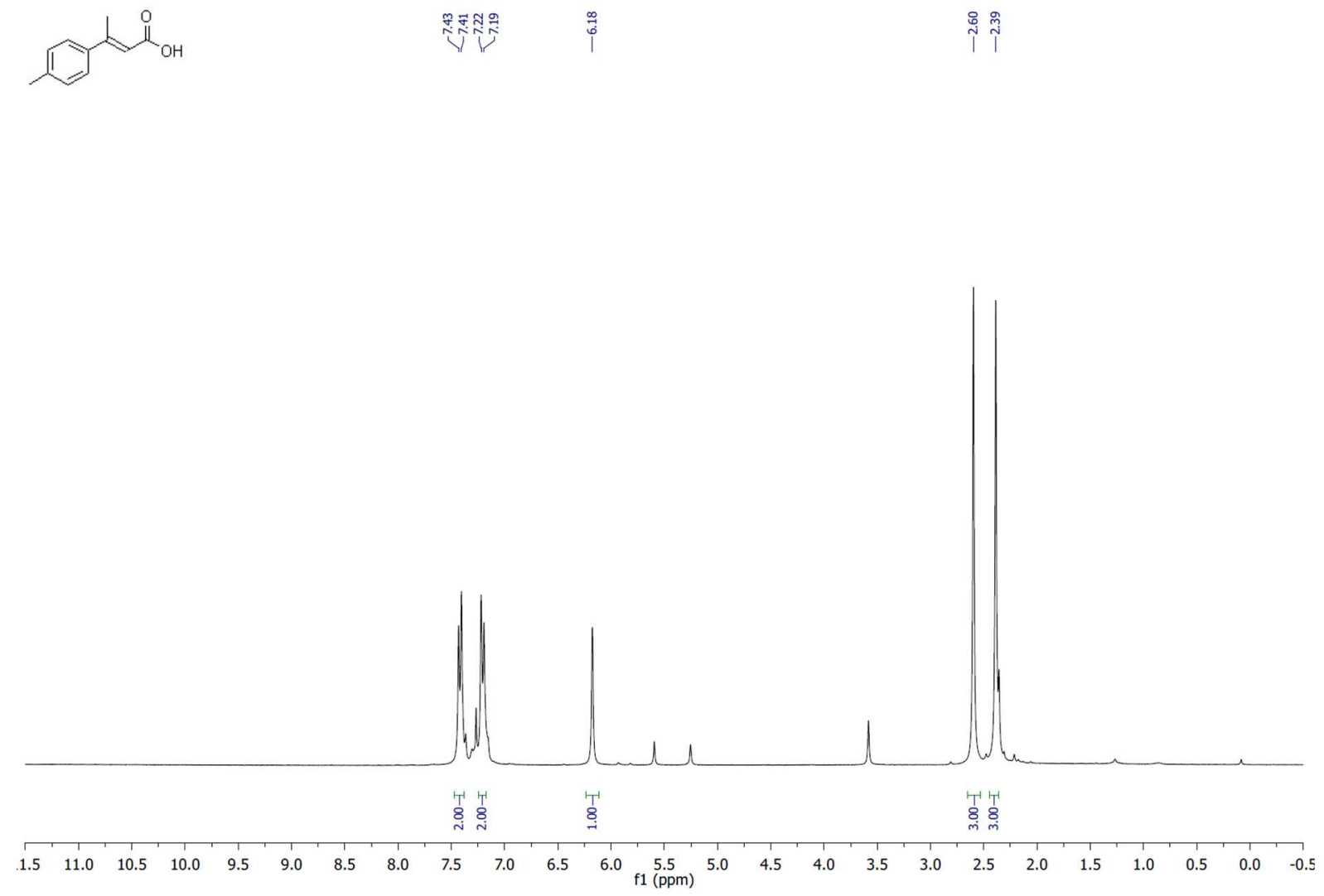
${ }^{13} \mathrm{C} \mathrm{NMR}\left(\mathrm{CDCl}_{3}, 75 \mathrm{MHz}\right)$

管

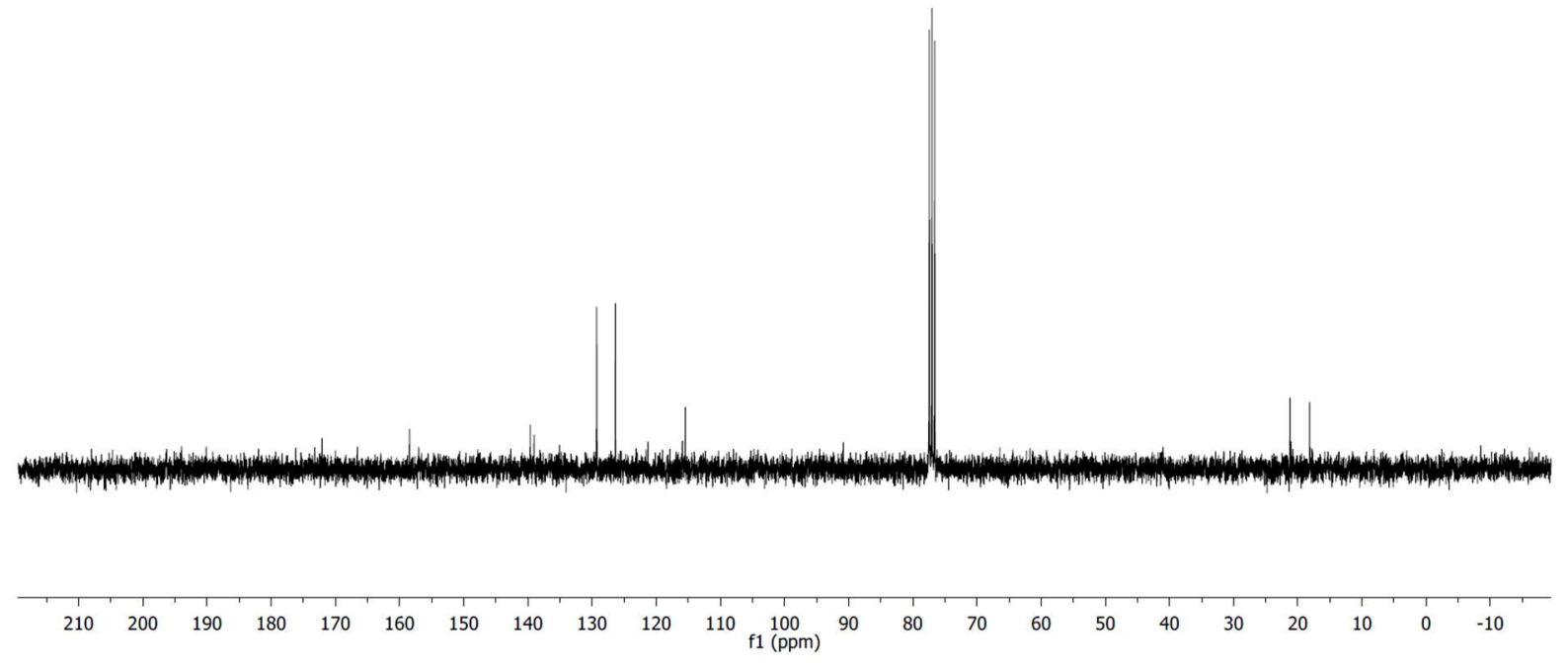

(E)-3-(m-tolyl)but-2-enoic acid (AC-35):

${ }^{1} \mathrm{H} \mathrm{NMR}\left(\mathrm{CDCl}_{3}, 300 \mathrm{MHz}\right)$
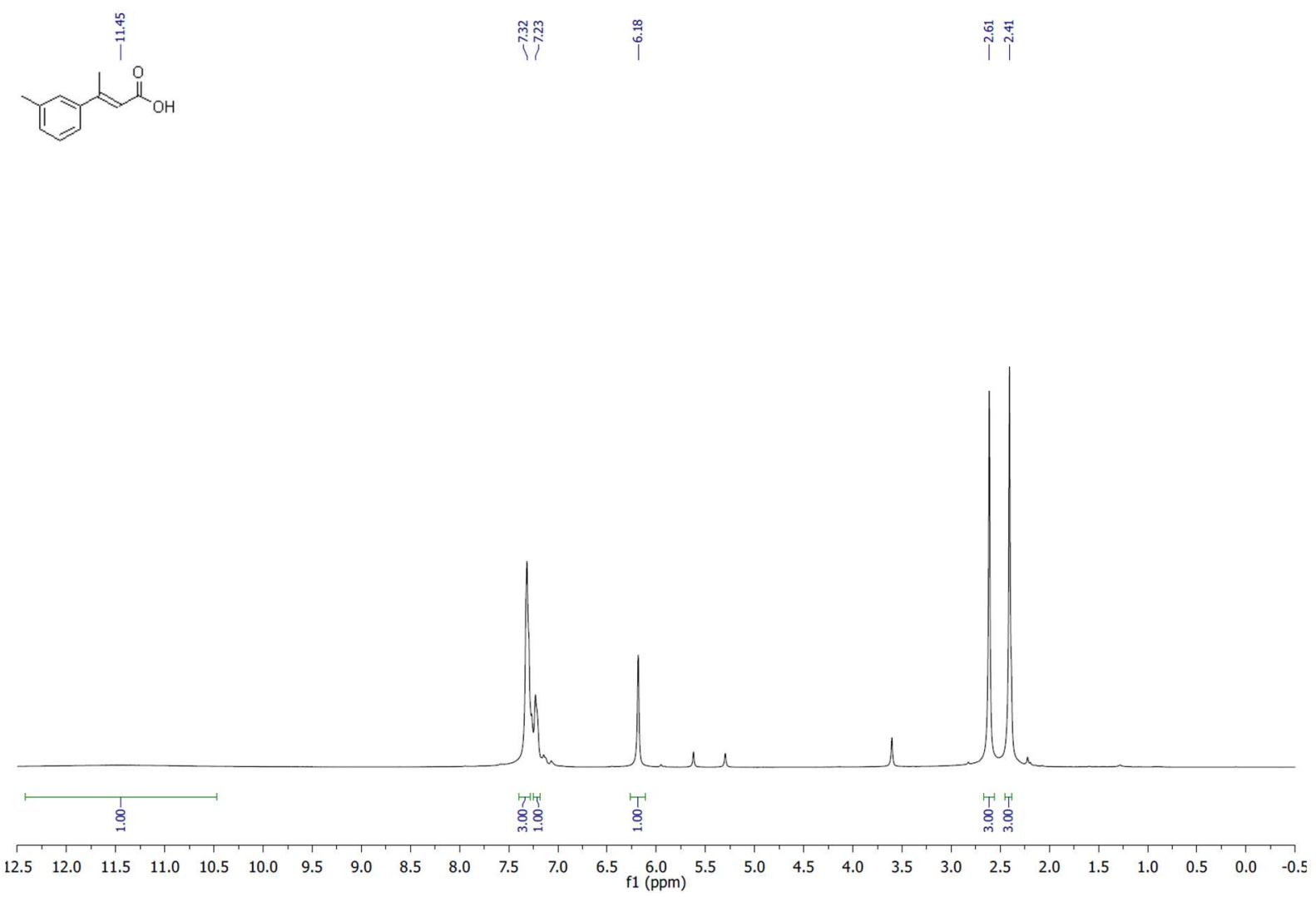
${ }^{13} \mathrm{C} \mathrm{NMR}\left(\mathrm{CDCl}_{3}, 75 \mathrm{MHz}\right)$

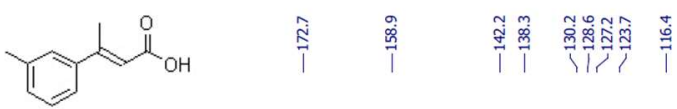

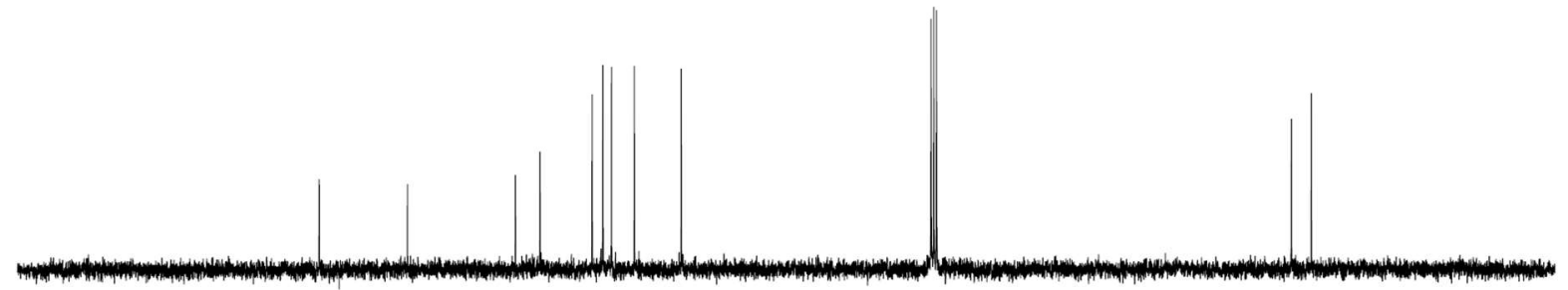

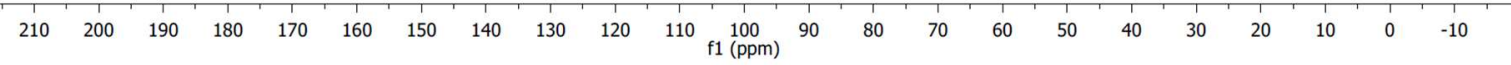

(E)-3-(o-tolyl)but-2-enoic acid (Ac-37):

${ }^{1} \mathrm{H} \mathrm{NMR}\left(\mathrm{CDCl}_{3}, 300 \mathrm{MHz}\right)$
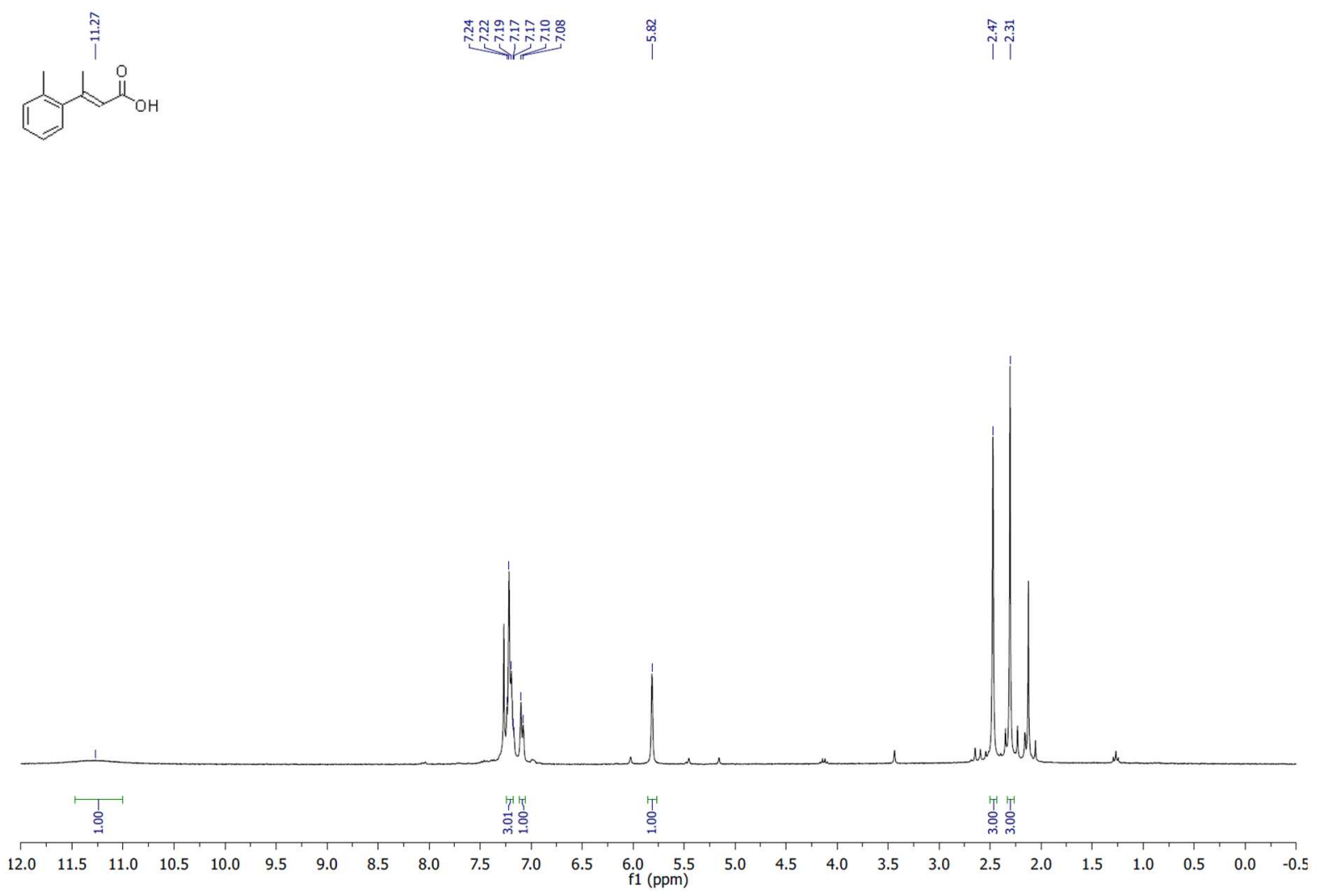
${ }^{13} \mathrm{C} \mathrm{NMR}\left(\mathrm{CDCl}_{3}, 75 \mathrm{MHz}\right)$

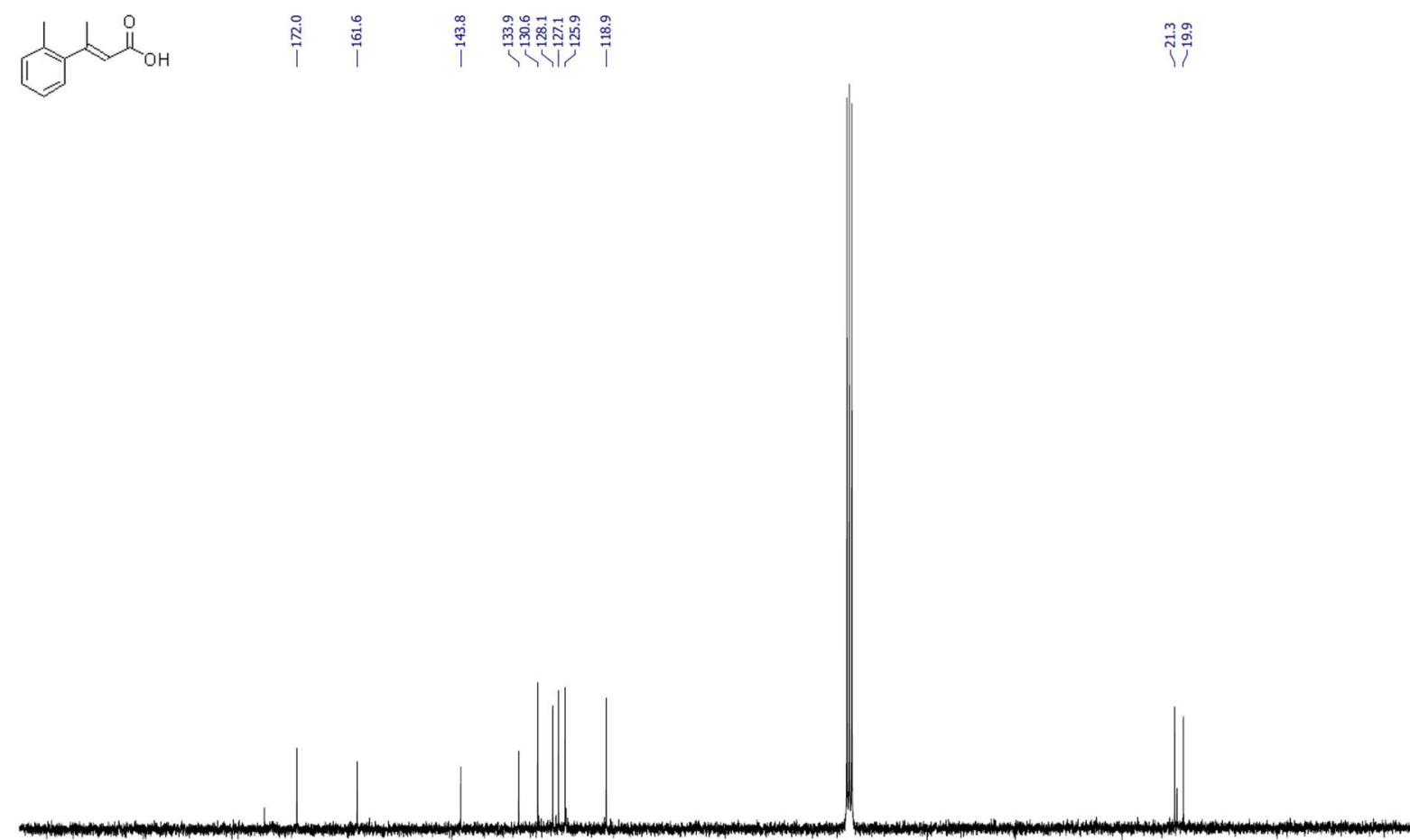

$\begin{array}{llllllllllllllllllllllllllllllll}210 & 200 & 190 & 180 & 170 & 160 & 150 & 140 & 130 & 120 & 110 & 100 & 90 & 80 & 70 & 60 & 50 & 40 & 30 & 20 & 10 & 0 & -10\end{array}$

(E)-3-(4-(tert-butyl)phenyl)but-2-enoic acid (Ac-39):

${ }^{1} \mathrm{H} \mathrm{NMR}\left(\mathrm{CDCl}_{3}, 300 \mathrm{MHz}\right)$

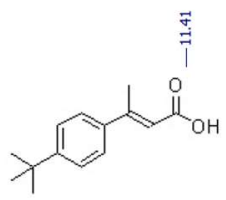

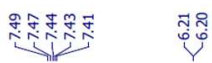
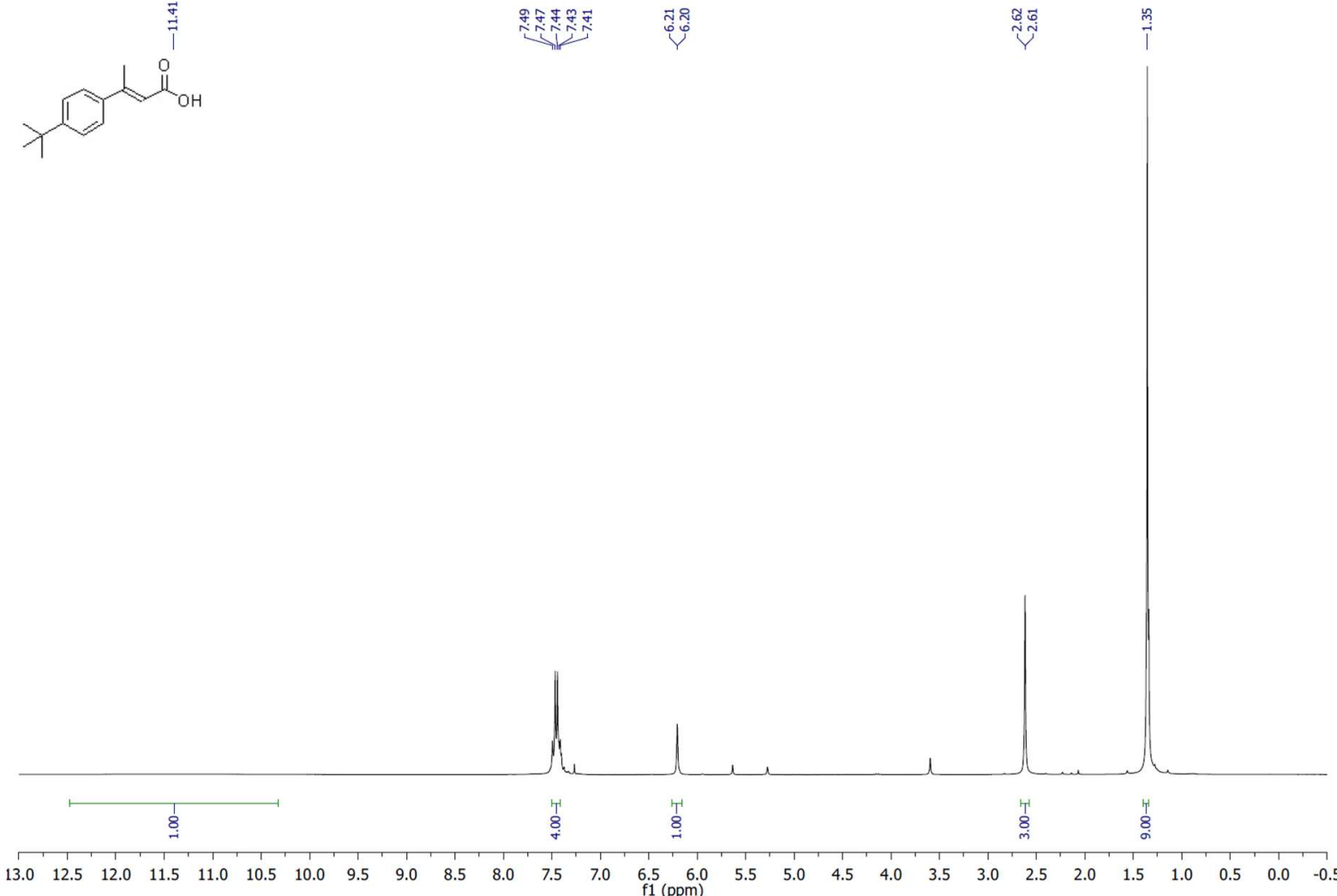
${ }^{13} \mathrm{C} \mathrm{NMR}\left(\mathrm{CDCl}_{3}, 75 \mathrm{MHz}\right)$
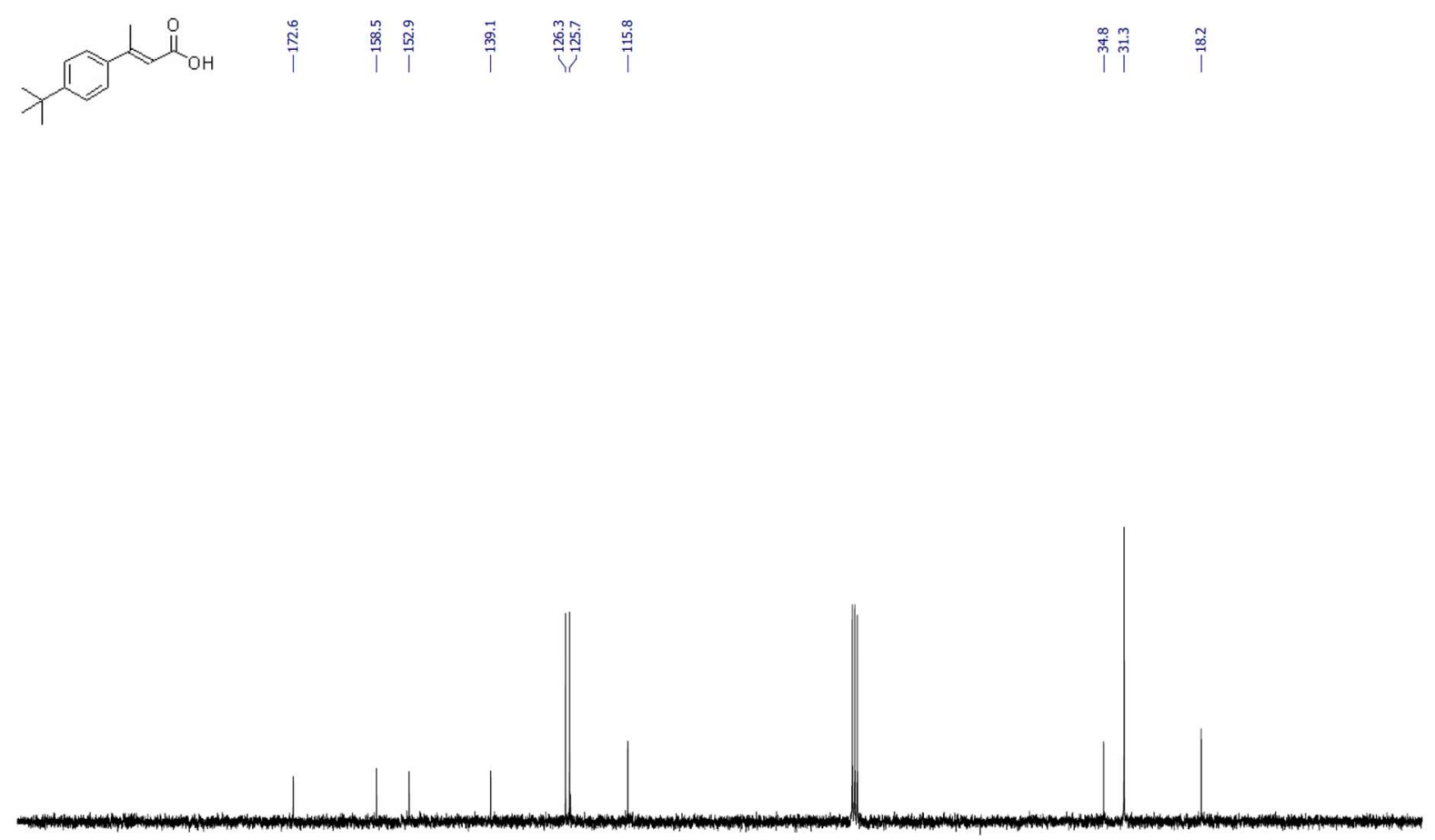

$\begin{array}{lllllllllllllllllllllllll}210 & 200 & 190 & 180 & 170 & 160 & 150 & 140 & 130 & 120 & 110 & 100 & 90 & 80 & 70 & 60 & 50 & 40 & 30 & 20 & 10 & 0 & -10\end{array}$

(E)-3-(4-methoxyphenyl)but-2-enoic acid (Ac-41):

${ }^{1} \mathrm{H} \mathrm{NMR}\left(\mathrm{CDCl}_{3}, 300 \mathrm{MHz}\right)$
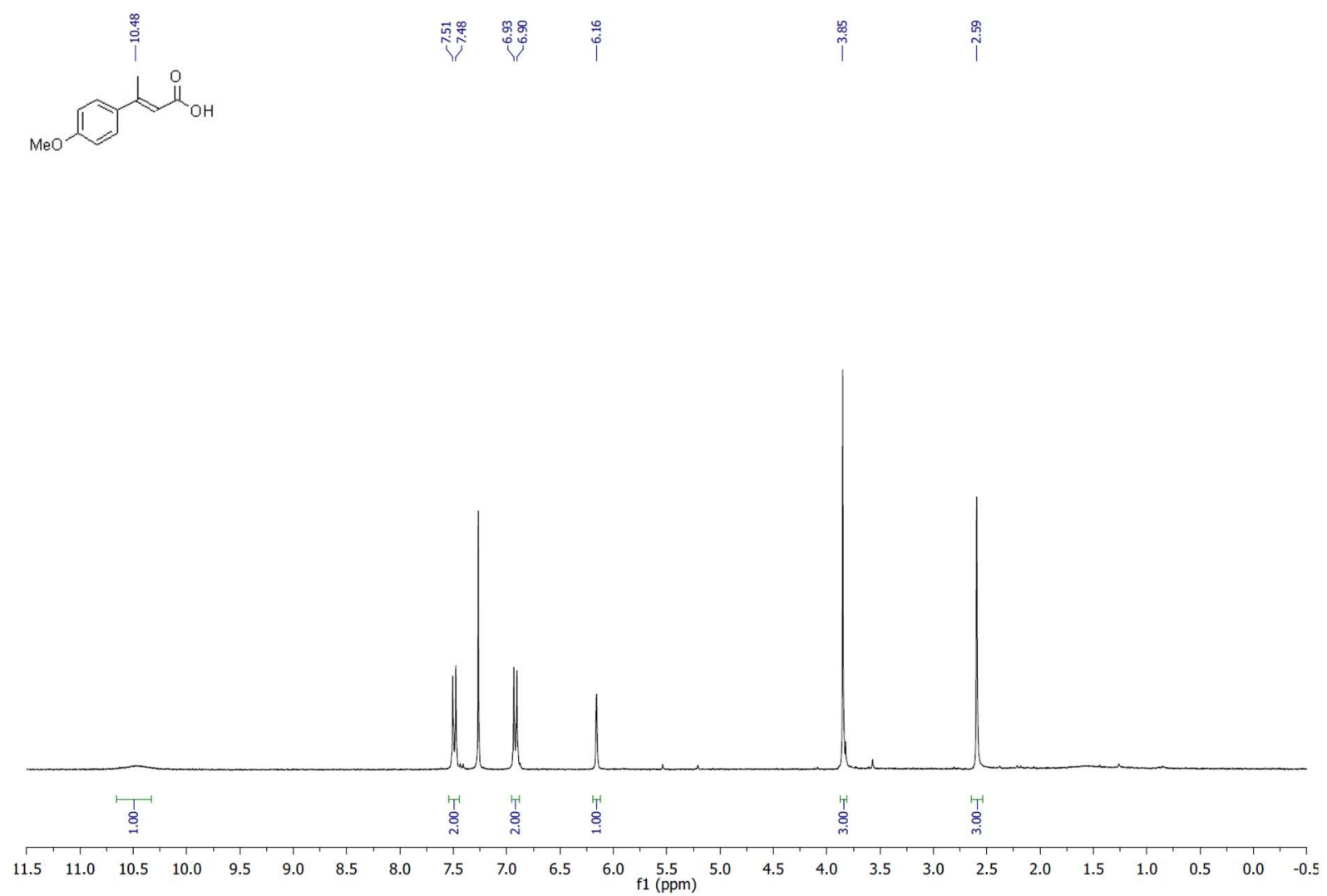
${ }^{13} \mathrm{C} \mathrm{NMR}\left(\mathrm{CDCl}_{3}, 75 \mathrm{MHz}\right)$
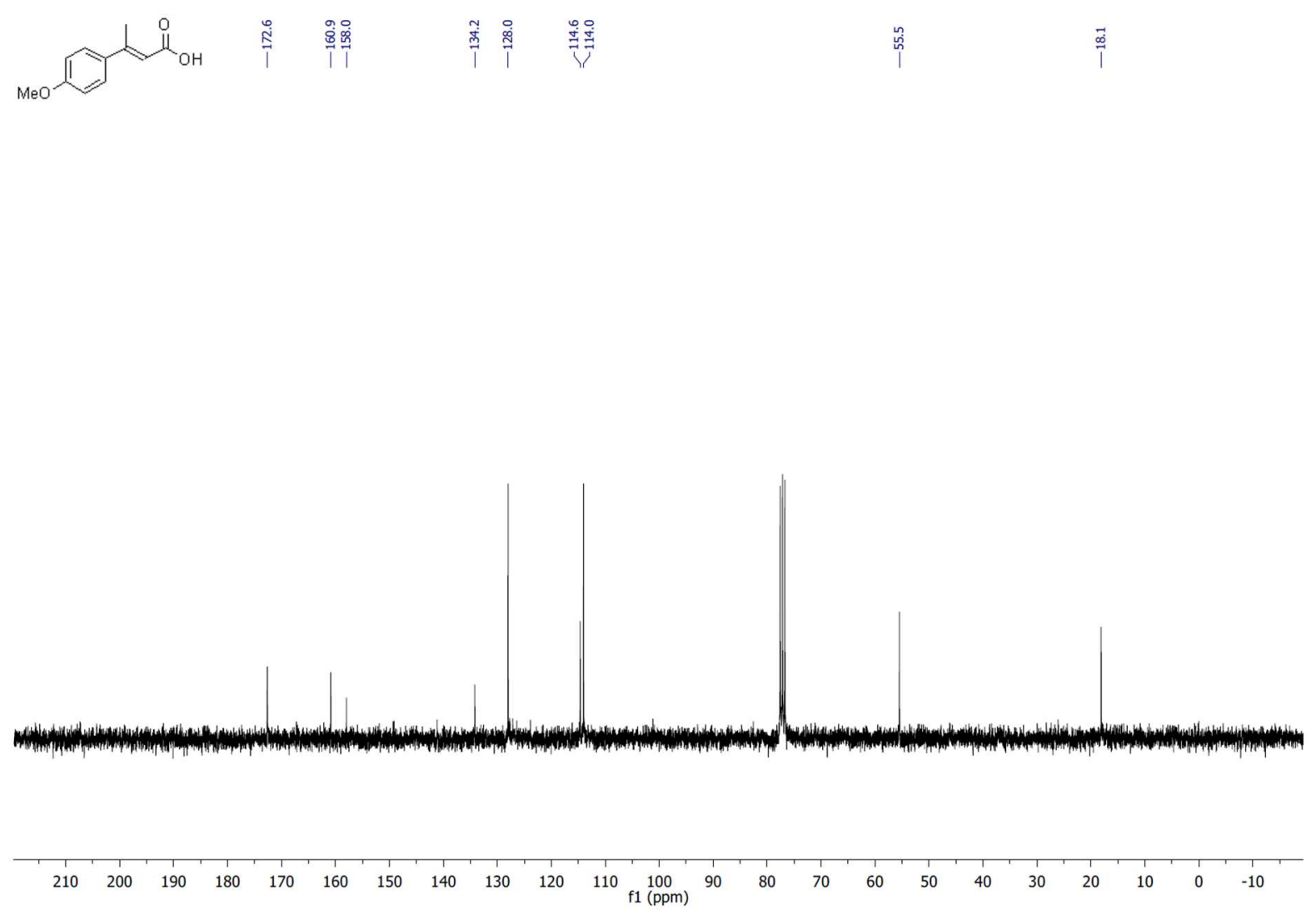

(E)-3-(4-fluorophenyl)but-2-enoic acid (Ac-43):

${ }^{1} \mathrm{H} \mathrm{NMR}\left(\mathrm{CDCl}_{3}, 300 \mathrm{MHz}\right)$
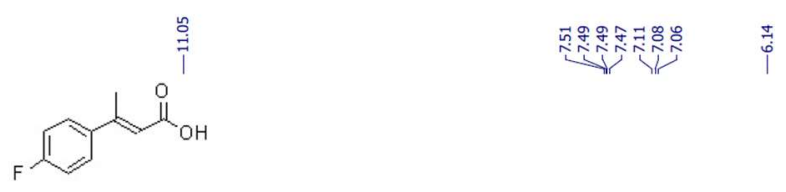

$\stackrel{\leftrightarrow}{\text { I }}$

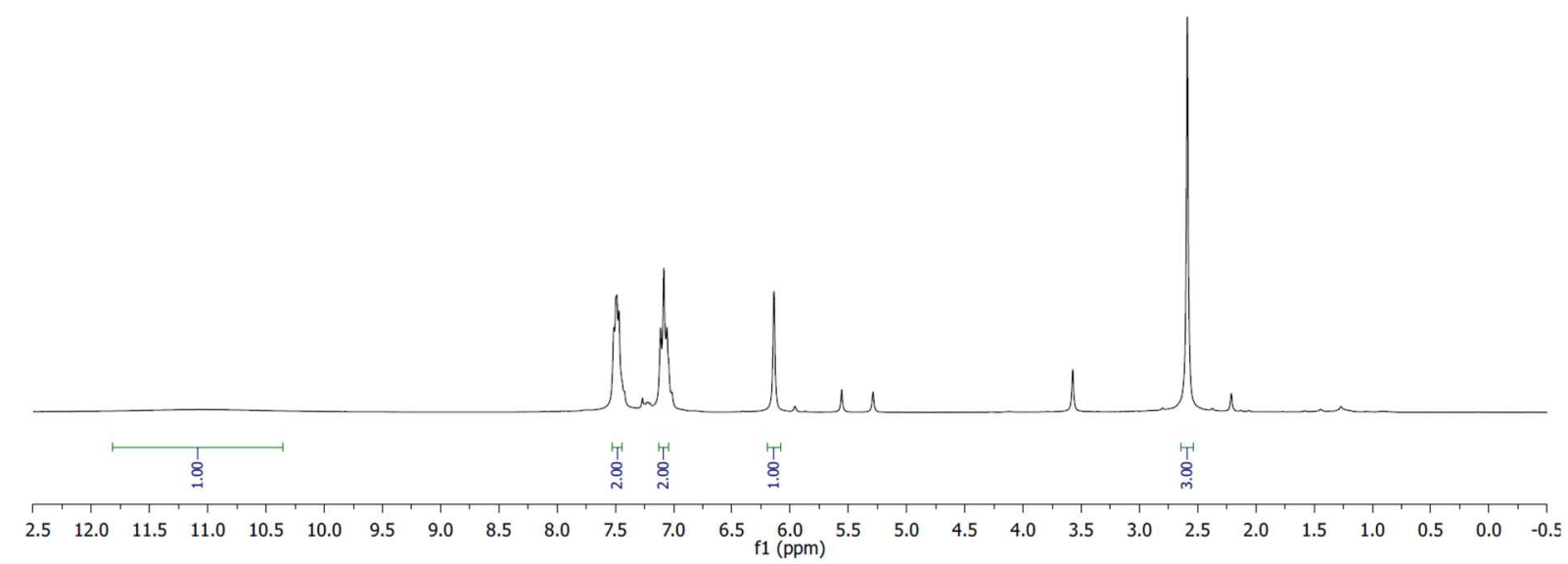


${ }^{13} \mathrm{C} \mathrm{NMR}\left(\mathrm{CDCl}_{3}, 75 \mathrm{MHz}\right)$

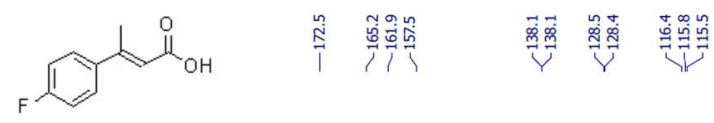

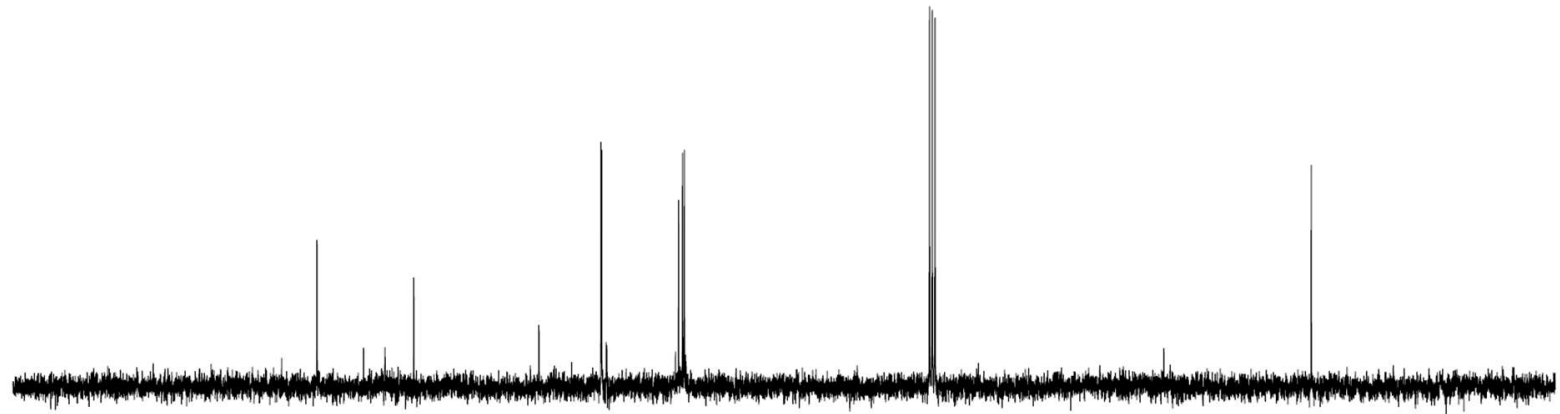

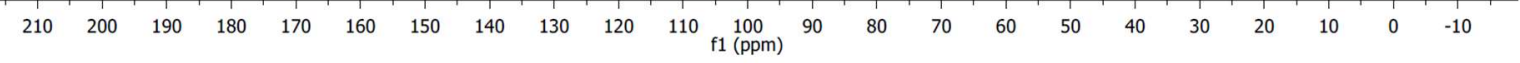

${ }^{19} \mathrm{~F} \mathrm{NMR}\left(\mathrm{CDCl}_{3}, 282 \mathrm{MHz}\right)$<smiles>CC(=O)C=C(C)c1ccc(F)cc1</smiles>

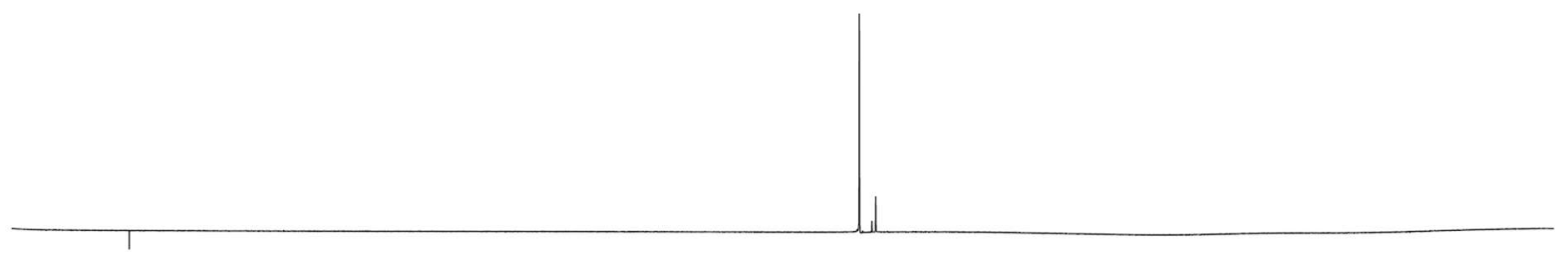

$\begin{array}{llllllllllllllllllllllll}10 & 0 & -10 & -20 & -30 & -40 & -50 & -60 & -70 & -80 & -90 & -100 & -110 & -120 & -130 & -140 & -150 & -160 & -170 & -180 & -190 & -200 & -210\end{array}$ 
(E)-3-(4-bromophenyl)but-2-enoic acid (Ac-45):

${ }^{1} \mathrm{H} \mathrm{NMR}\left(\mathrm{CDCl}_{3}, 300 \mathrm{MHz}\right)$

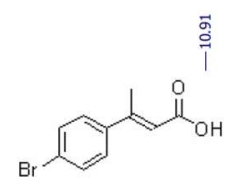

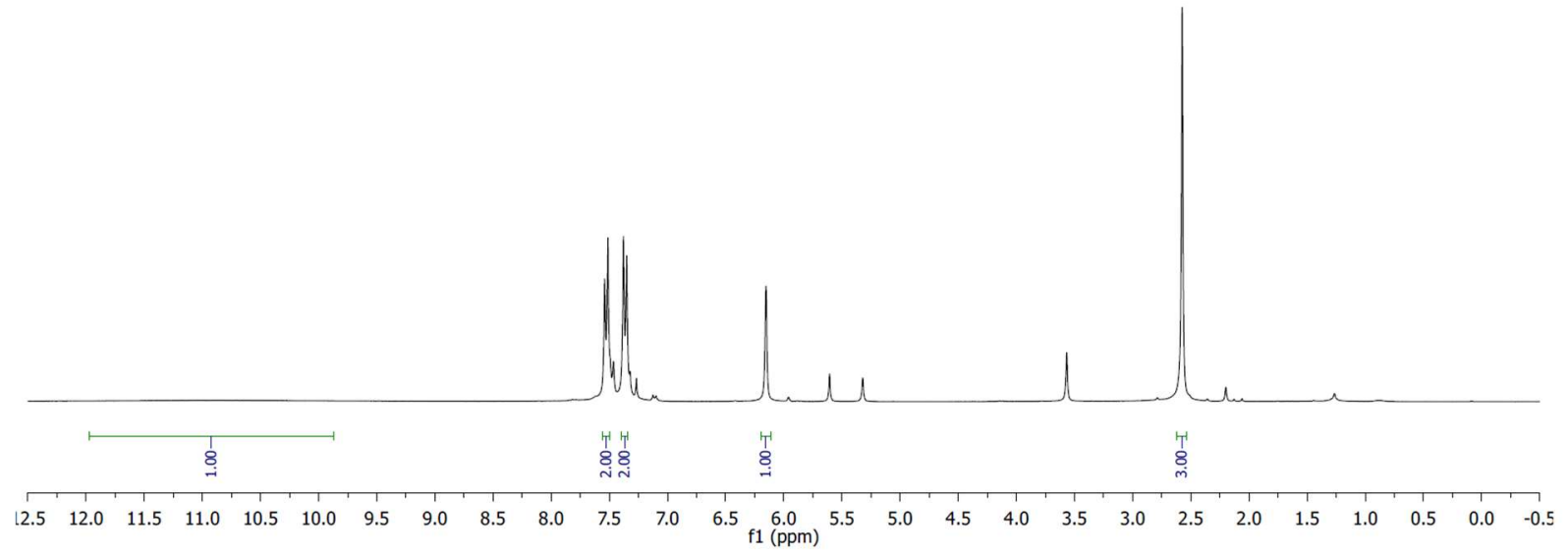

${ }^{13} \mathrm{C} \mathrm{NMR}\left(\mathrm{CDCl}_{3}, 75 \mathrm{MHz}\right)$

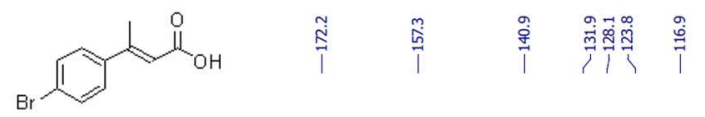

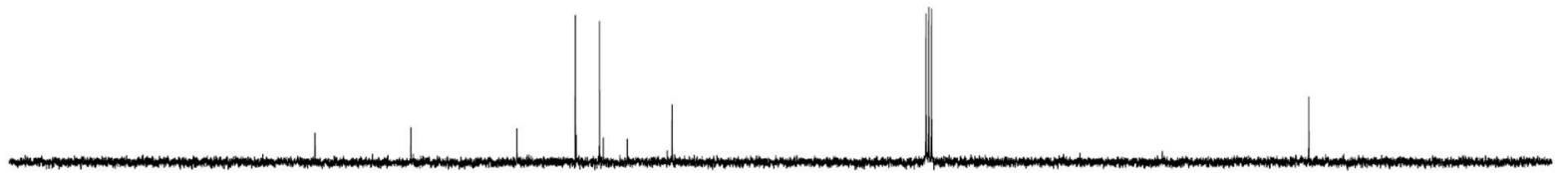

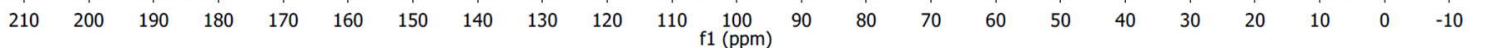


(E)-3-(4-iodophenyl)but-2-enoic acid (Ac-47):

${ }^{1} \mathrm{H} \mathrm{NMR}\left(\mathrm{CDCl}_{3}, 300 \mathrm{MHz}\right)$
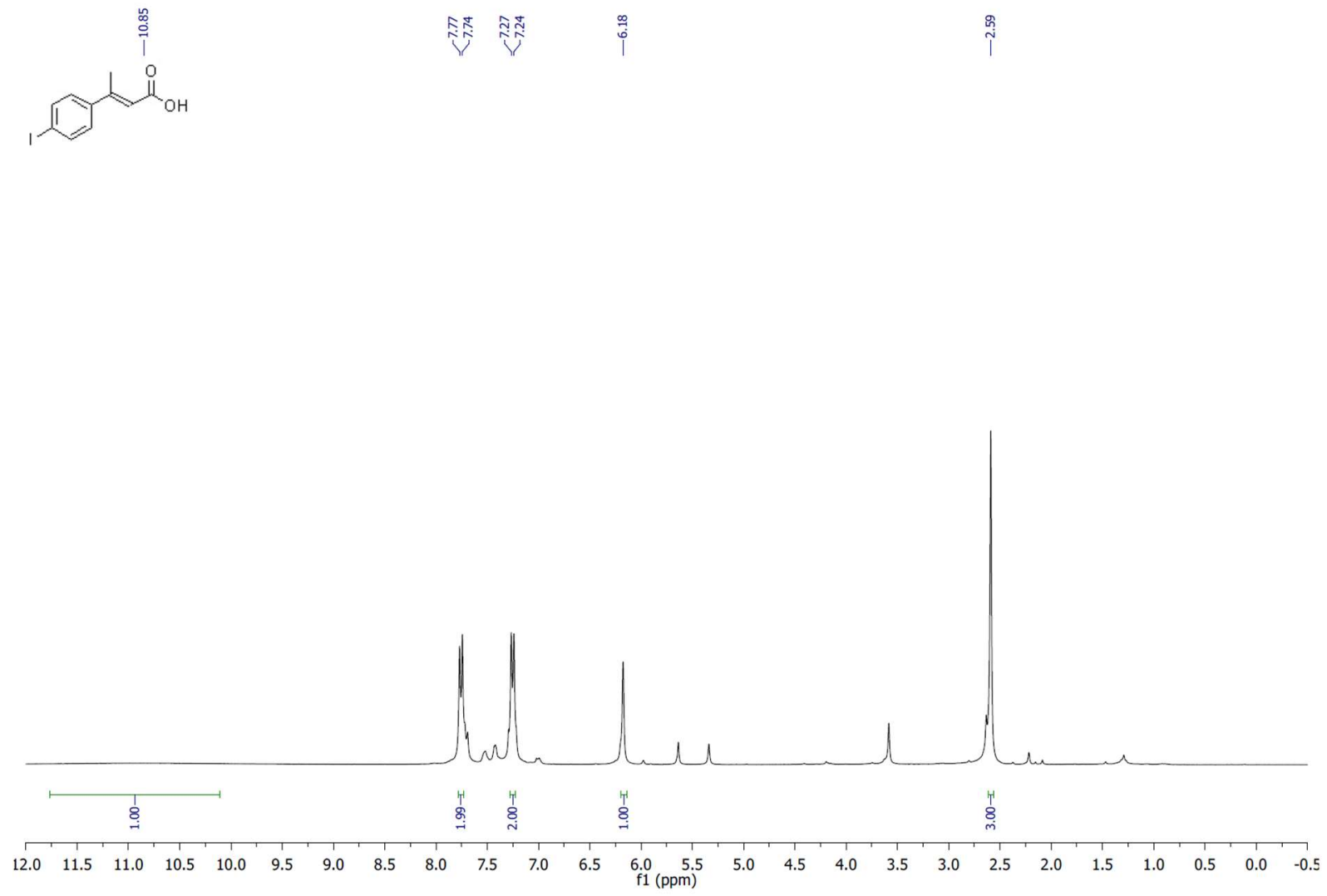

${ }^{13} \mathrm{C} \mathrm{NMR}\left(\mathrm{CDCl}_{3}, 75 \mathrm{MHz}\right)$

مـ

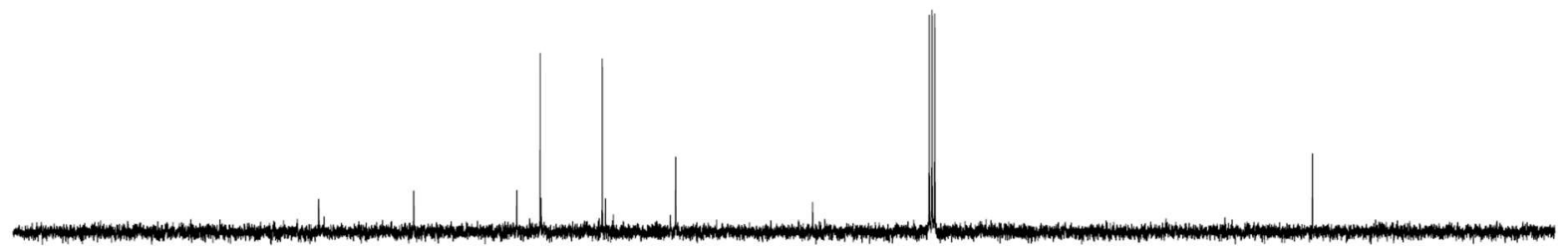

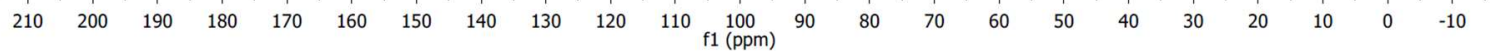


(E)-3-(4-nitrophenyl)but-2-enoic acid (Ac-49):

${ }^{1} \mathrm{H} \mathrm{NMR}\left(\mathrm{CDCl}_{3}, 300 \mathrm{MHz}\right)$
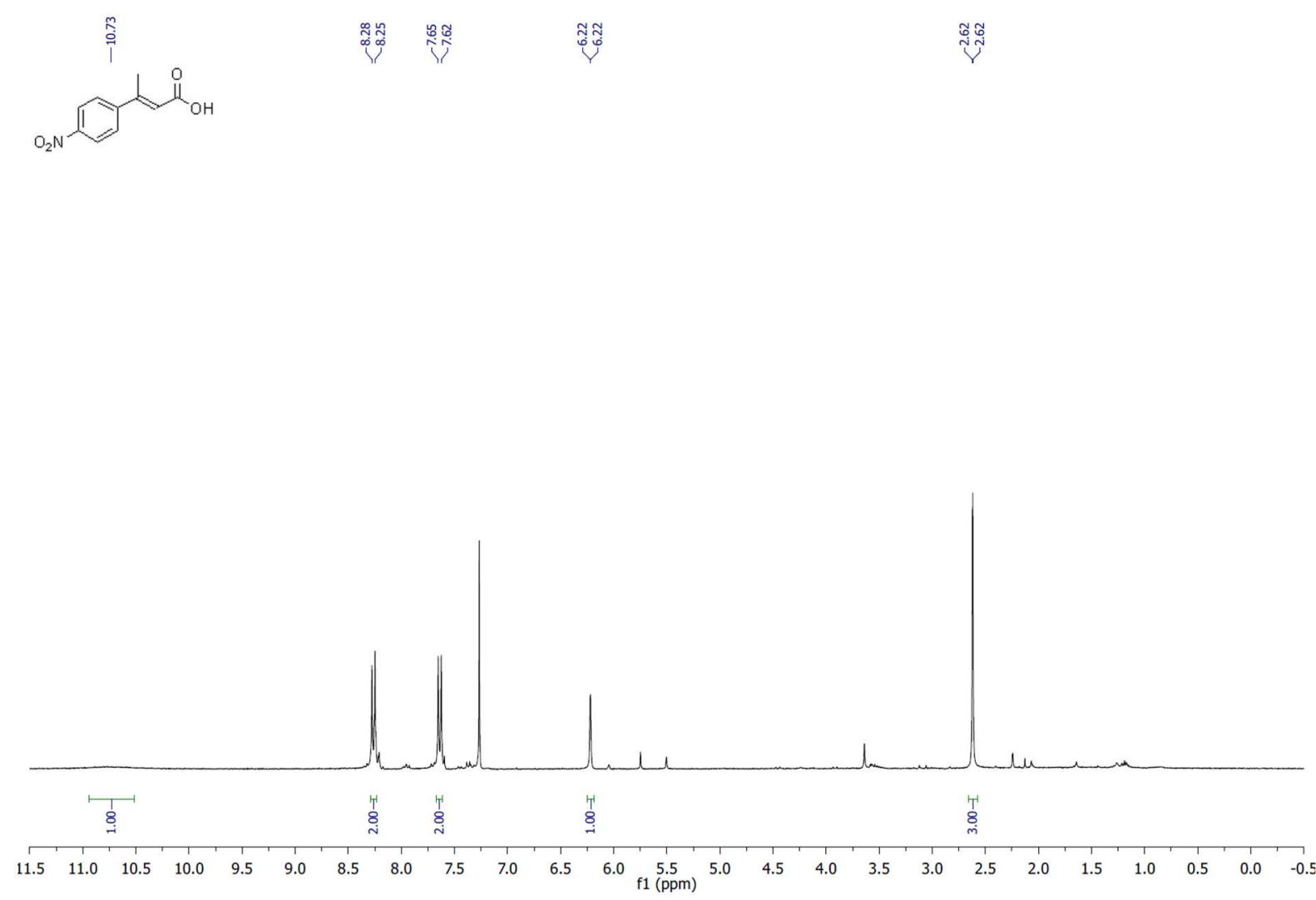

${ }^{13} \mathrm{C} \mathrm{NMR}\left(\mathrm{CDCl}_{3}, 75 \mathrm{MHz}\right)$

O

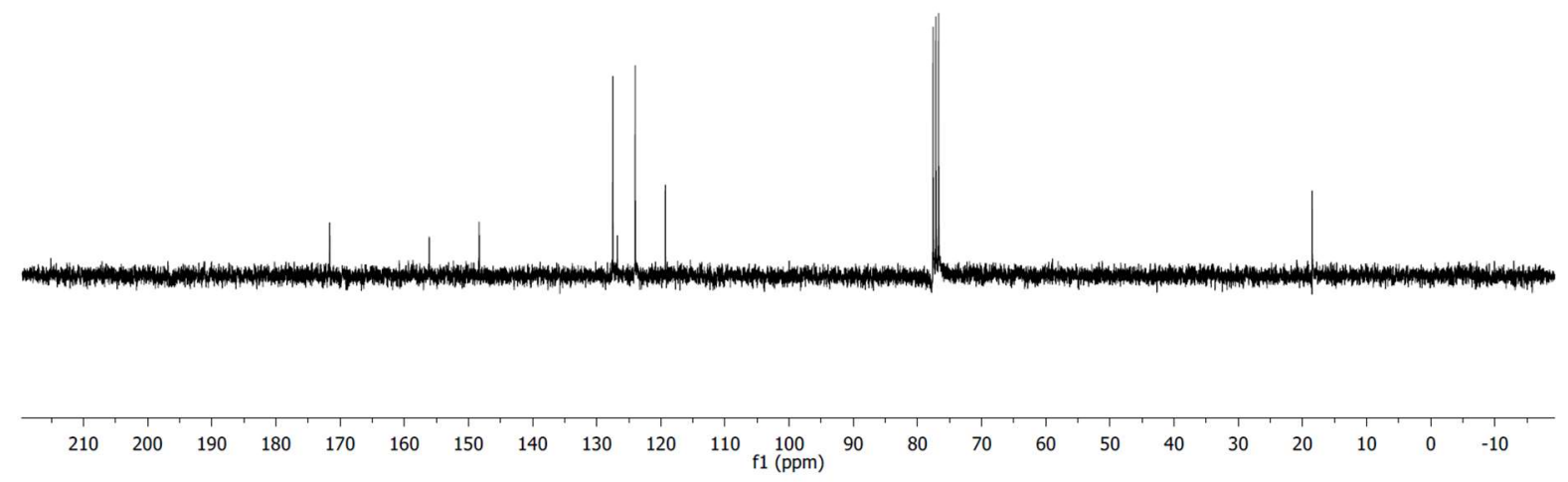


(E)-4,4,4-trifluoro-3-phenylbut-2-enoic acid (AC-51):

${ }^{1} \mathrm{H} \mathrm{NMR}\left(\mathrm{CDCl}_{3}, 300 \mathrm{MHz}\right)$

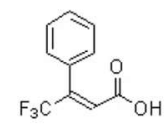

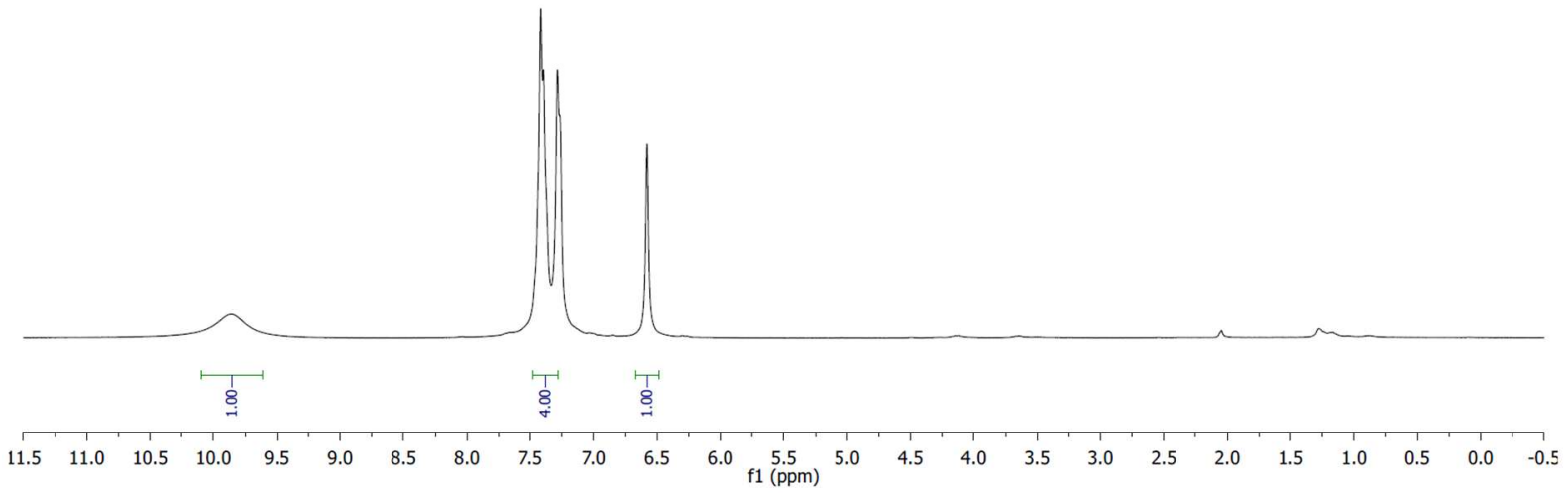

${ }^{13} \mathrm{C} \mathrm{NMR}\left(\mathrm{CDCl}_{3}, 75 \mathrm{MHz}\right)$
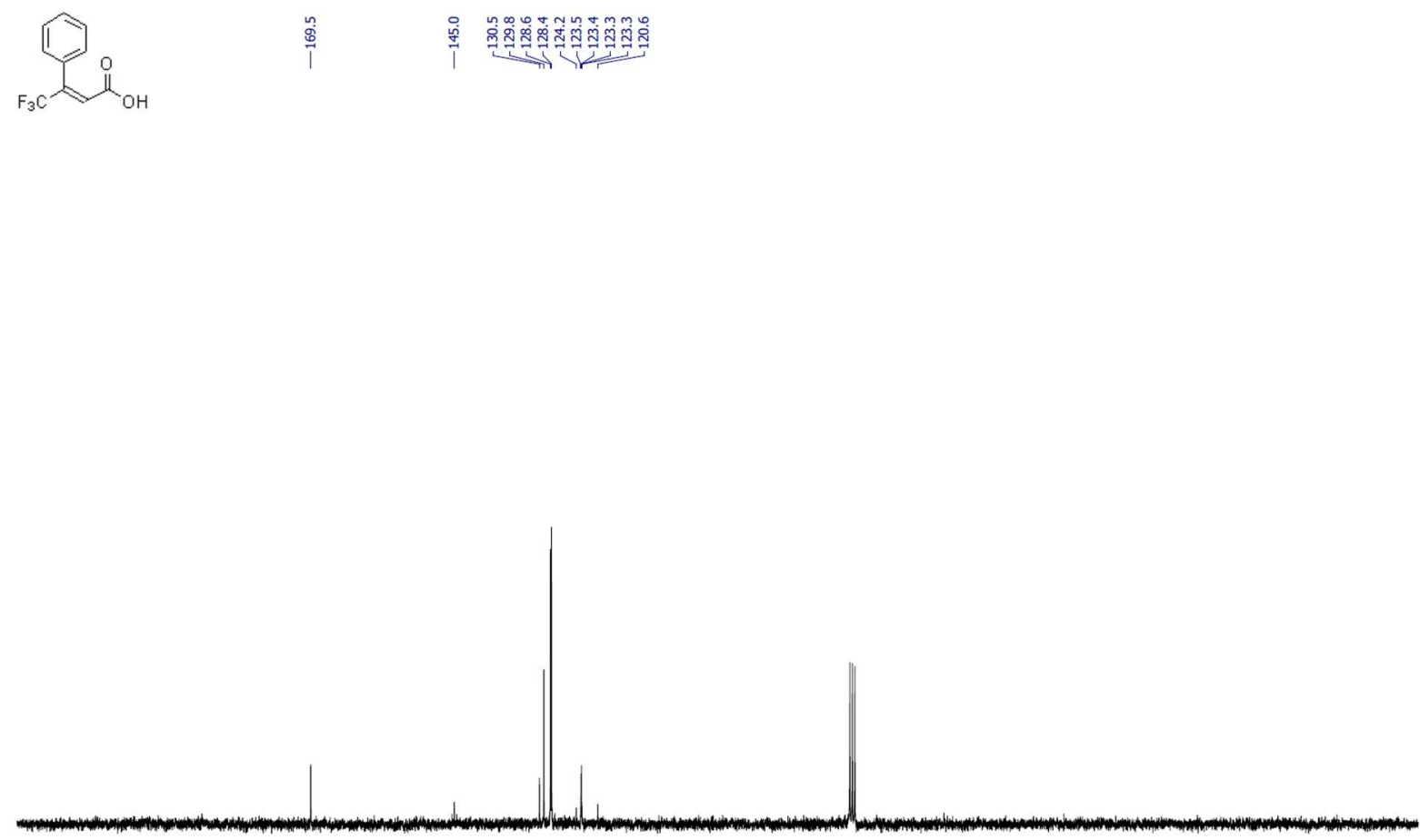

$\begin{array}{lllllllllllllllllllllll}210 & 200 & 190 & 180 & 170 & 160 & 150 & 140 & 130 & 120 & 110 & \begin{array}{c}100 \\ \mathrm{f} 1(\mathrm{ppm})\end{array} & 90 & 80 & 70 & 60 & 50 & 40 & 30 & 20 & 10 & 0 & -10\end{array}$ 
${ }^{19} \mathrm{~F} \mathrm{NMR}\left(\mathrm{CDCl}_{3}, 282 \mathrm{MHz}\right)$

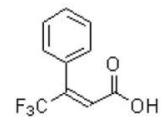

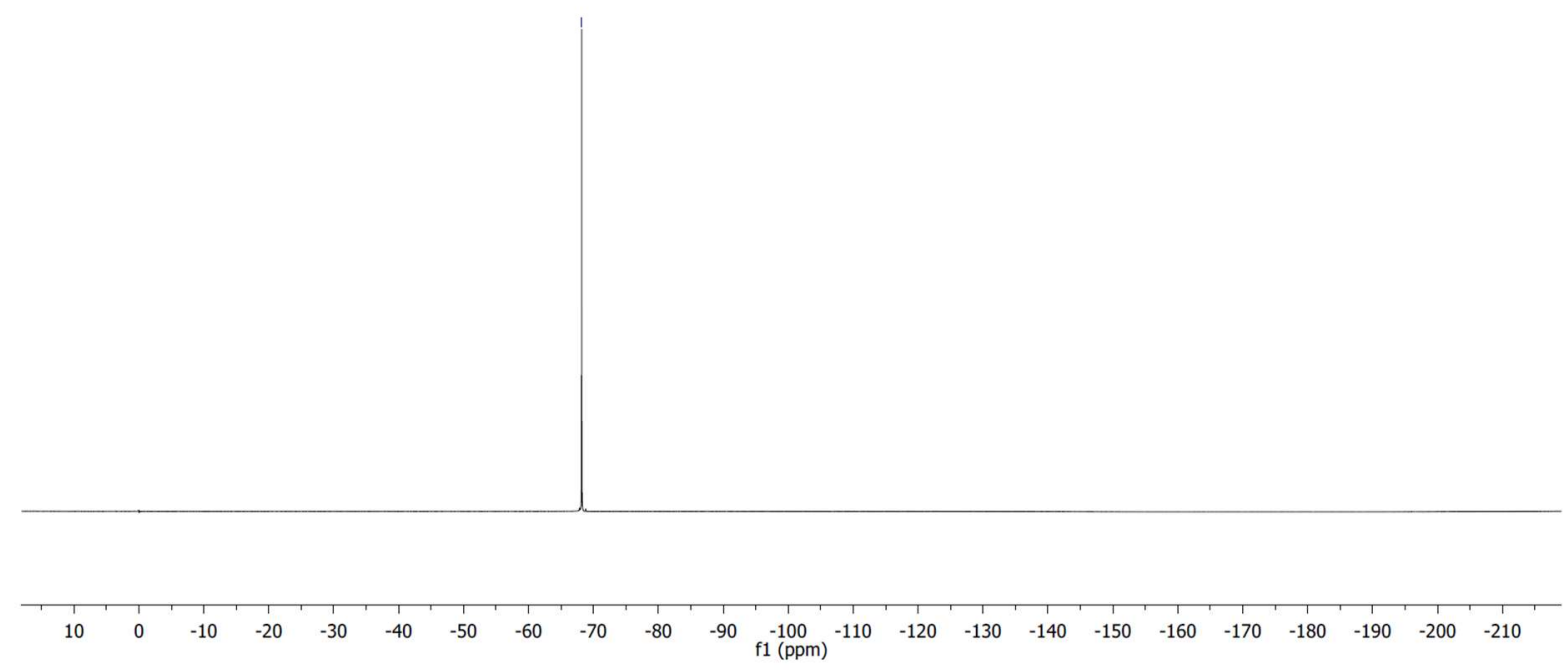

(E)-3-(3-phenylbut-2-enoyl)oxazolidin-2-one ((E)-29):

${ }^{1} \mathrm{H} \mathrm{NMR}\left(\mathrm{CDCl}_{3}, 300 \mathrm{MHz}\right)$<smiles>C/C(=C\C(=O)N1CCCC1)c1ccccc1</smiles>

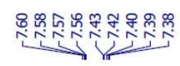

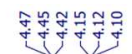

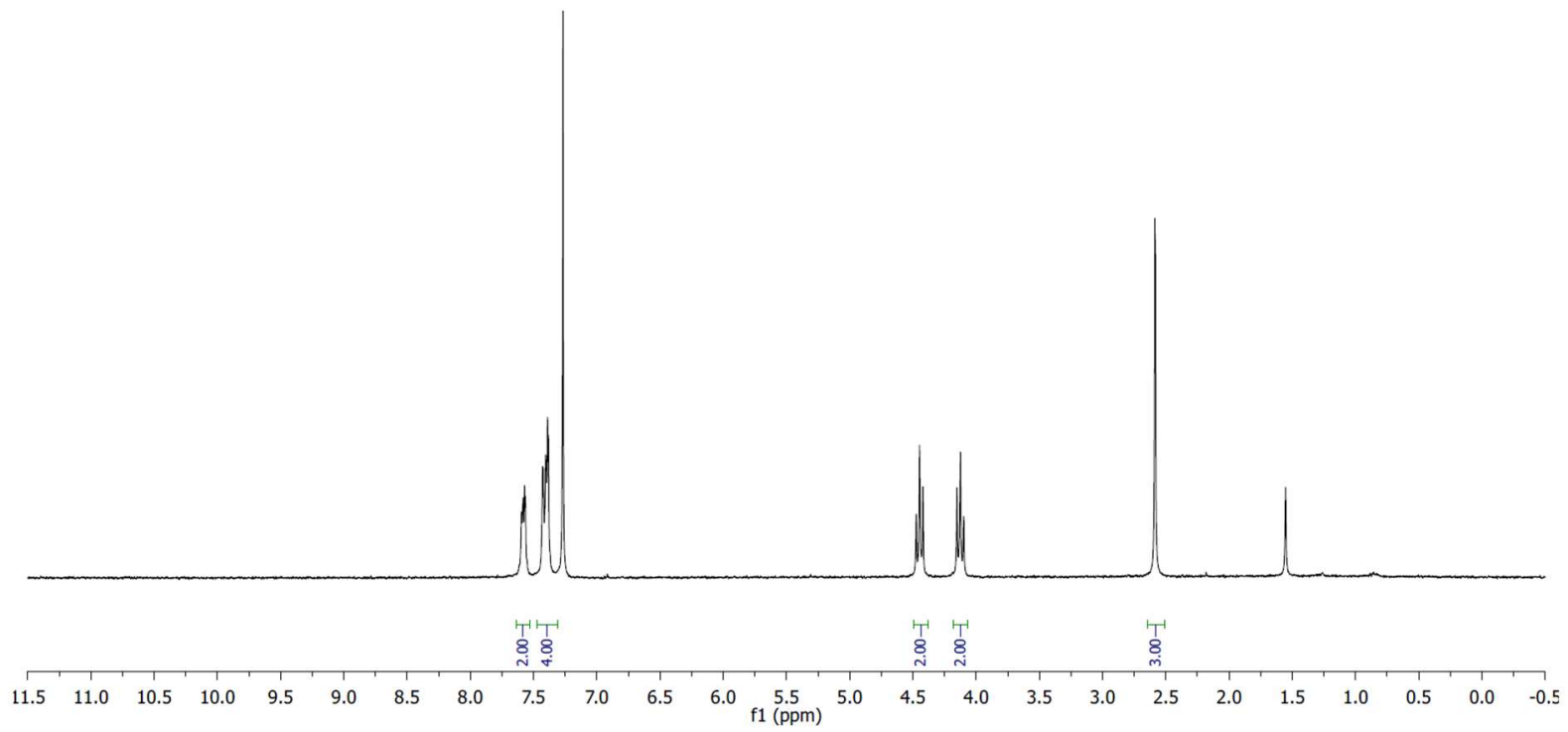


${ }^{13} \mathrm{C} \mathrm{NMR}\left(\mathrm{CDCl}_{3}, 75 \mathrm{MHz}\right)$

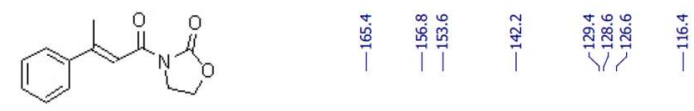

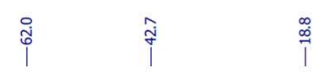

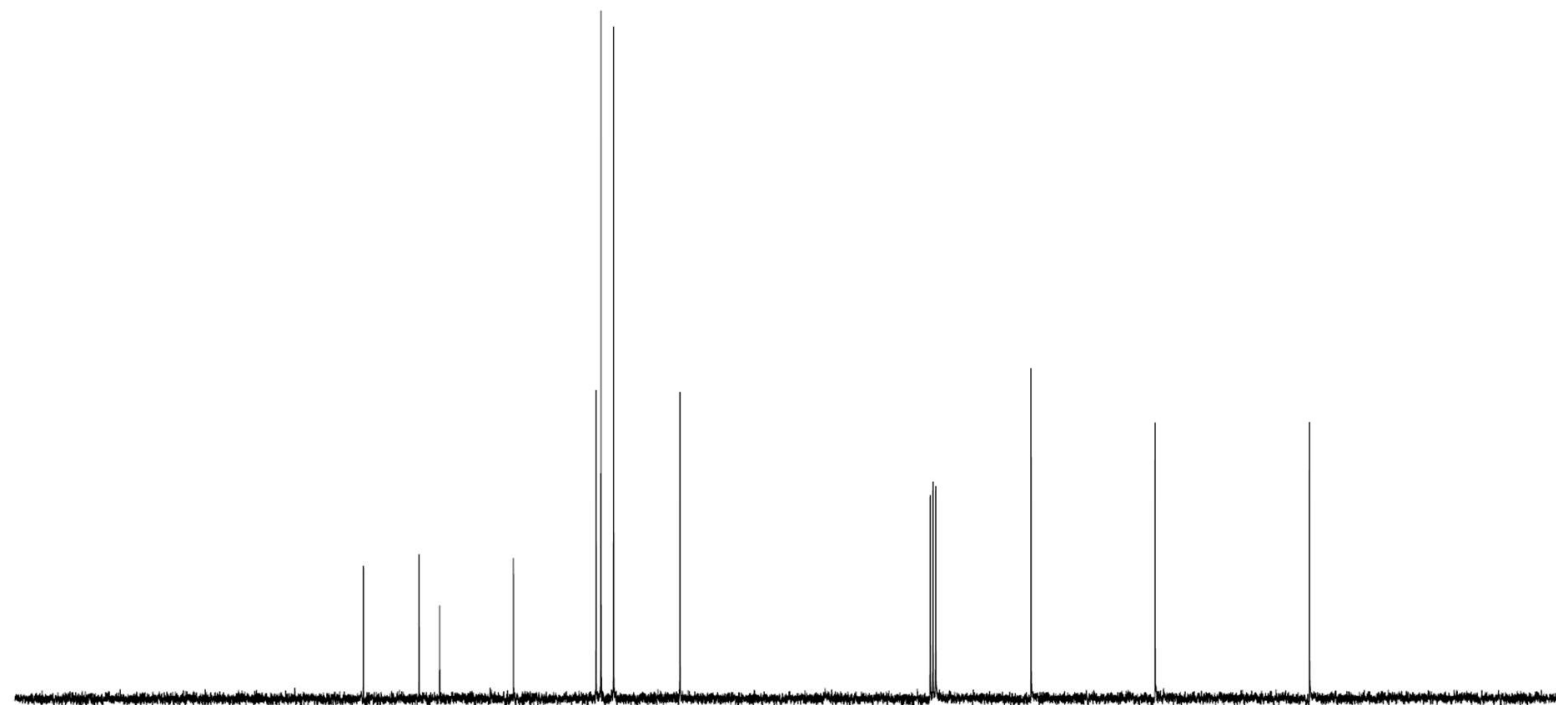

$\begin{array}{lllllllllllllllllllllllll}210 & 200 & 190 & 180 & 170 & 160 & 150 & 140 & 130 & 120 & 110 & 100 & 90 & 80 & 70 & 60 & 50 & 40 & 30 & 20 & 10 & 0 & -10\end{array}$

(E)-3-(3-phenylpent-2-enoyl)oxazolidin-2-one ((E)-31):

${ }^{1} \mathrm{H} \mathrm{NMR}\left(\mathrm{CDCl}_{3}, 300 \mathrm{MHz}\right)$<smiles>CCC(=CC(=O)N1CCCO1)c1ccccc1</smiles>

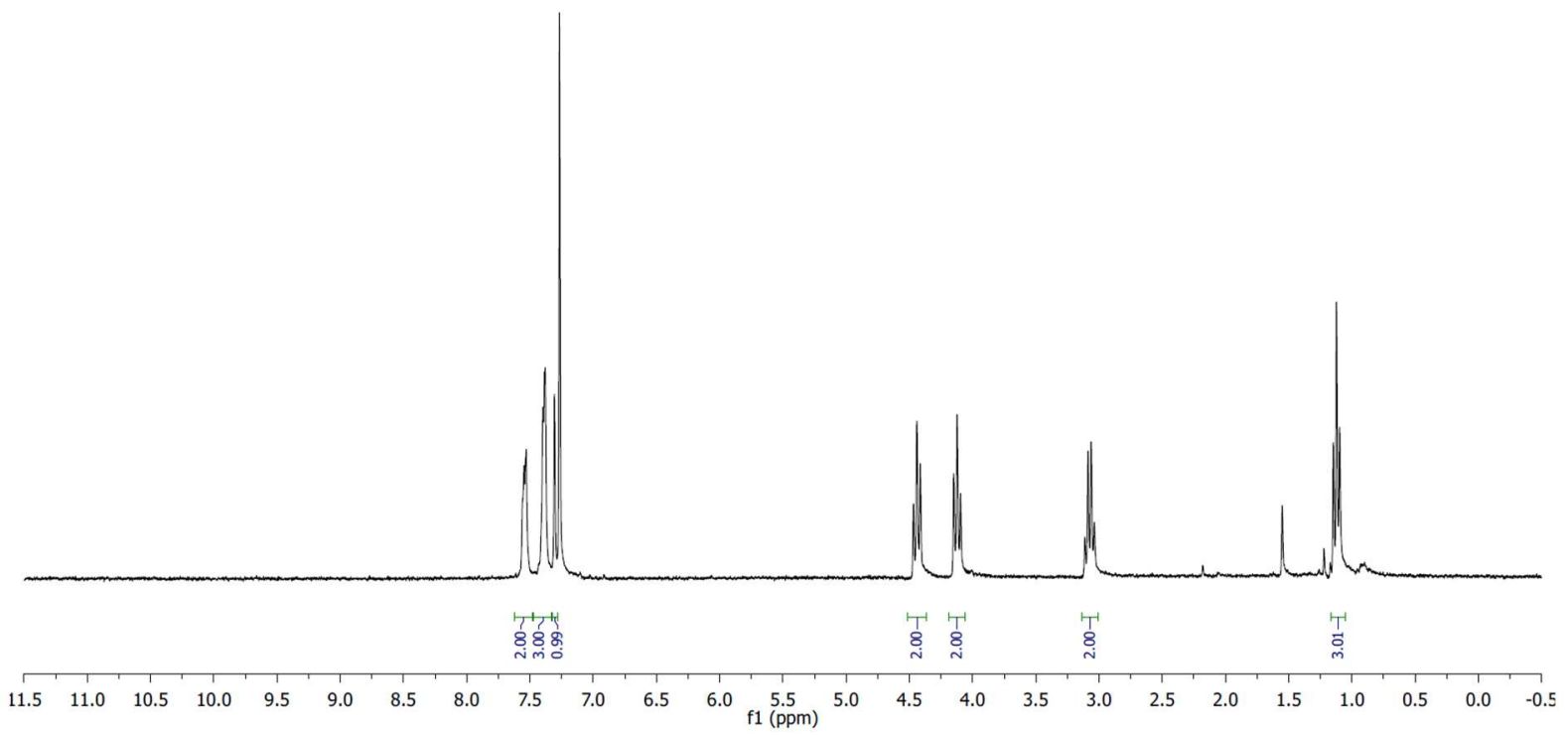


${ }^{13} \mathrm{C} \mathrm{NMR}\left(\mathrm{CDCl}_{3}, 75 \mathrm{MHz}\right)$
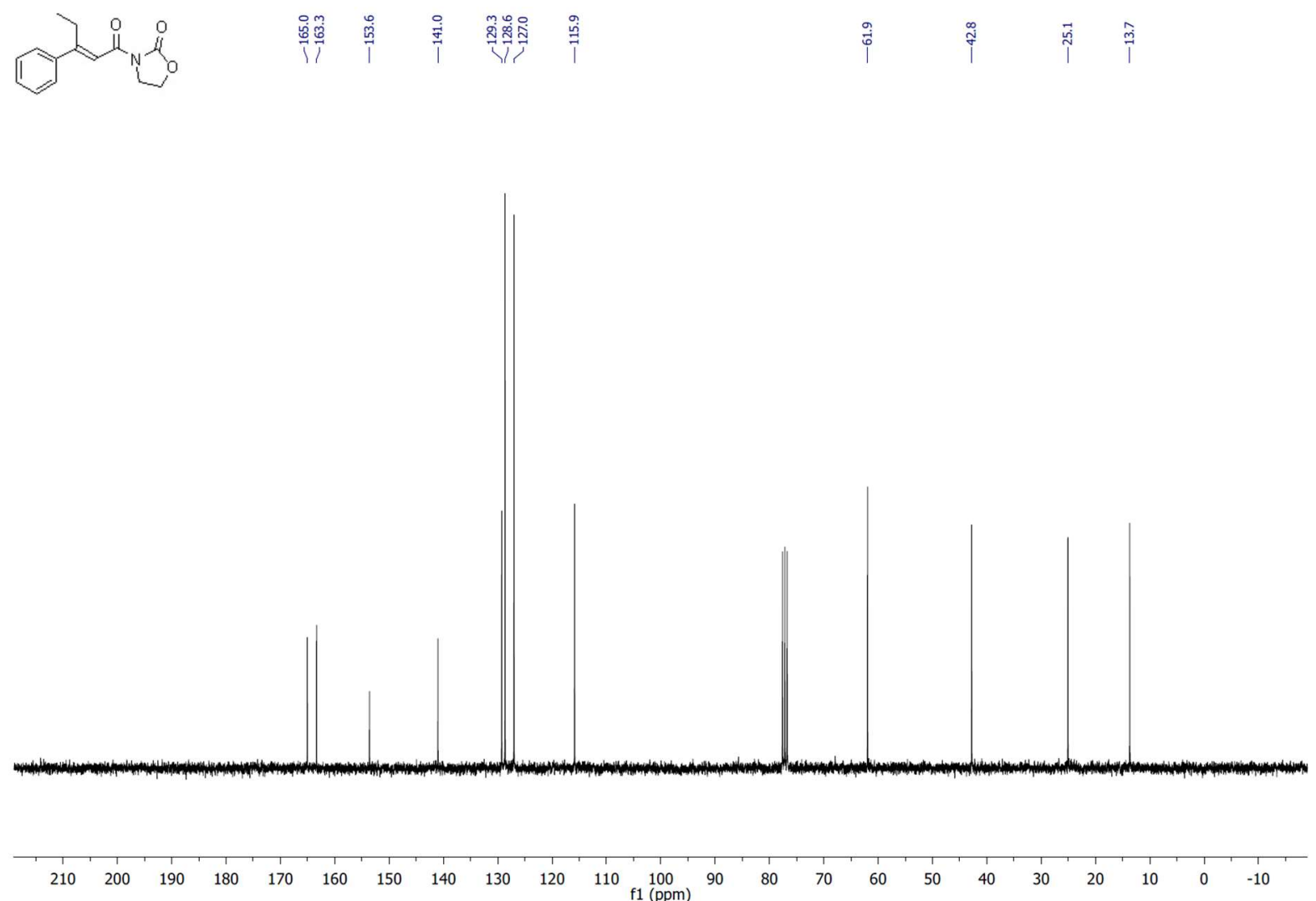

\section{(E)-3-(3-(p-tolyl)but-2-enoyl)oxazolidin-2-one ((E)-33):}

${ }^{1} \mathrm{H} \mathrm{NMR}\left(\mathrm{CDCl}_{3}, 300 \mathrm{MHz}\right)$<smiles>CC(=CC(=O)N1CCCO1)c1ccc(C)cc1</smiles>

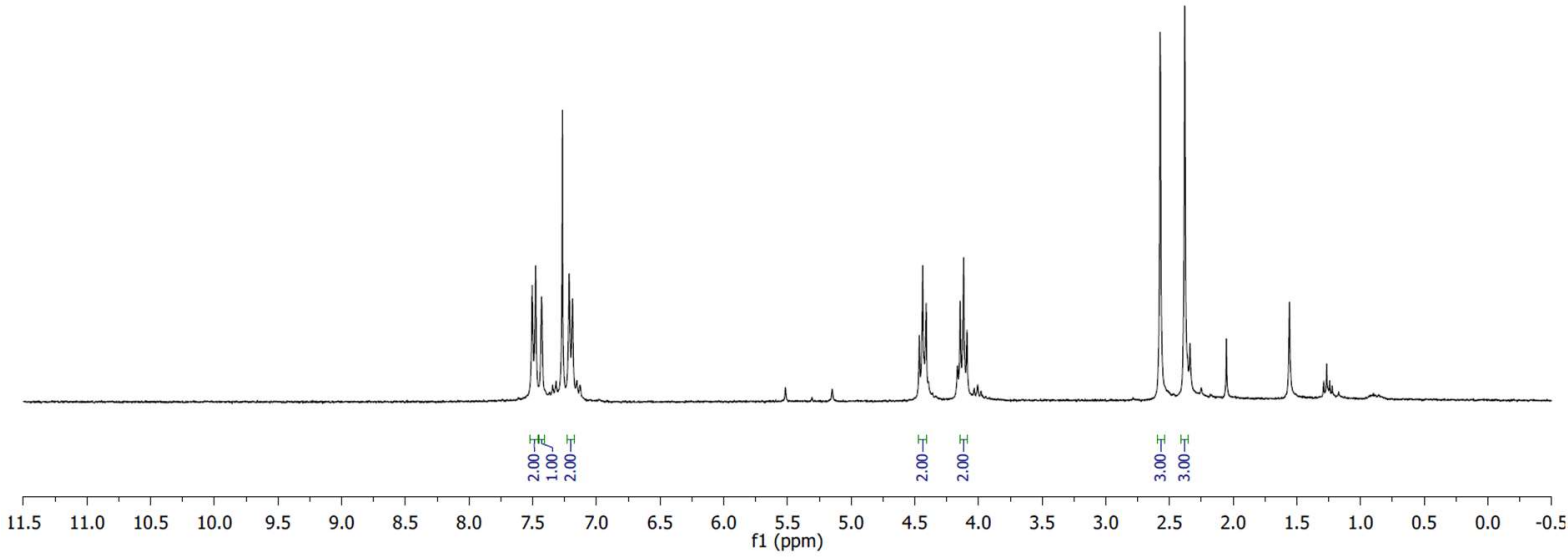


${ }^{13} \mathrm{C} \mathrm{NMR}\left(\mathrm{CDCl}_{3}, 75 \mathrm{MHz}\right)$

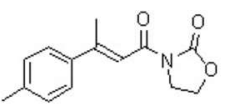

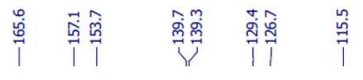

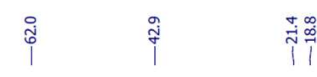

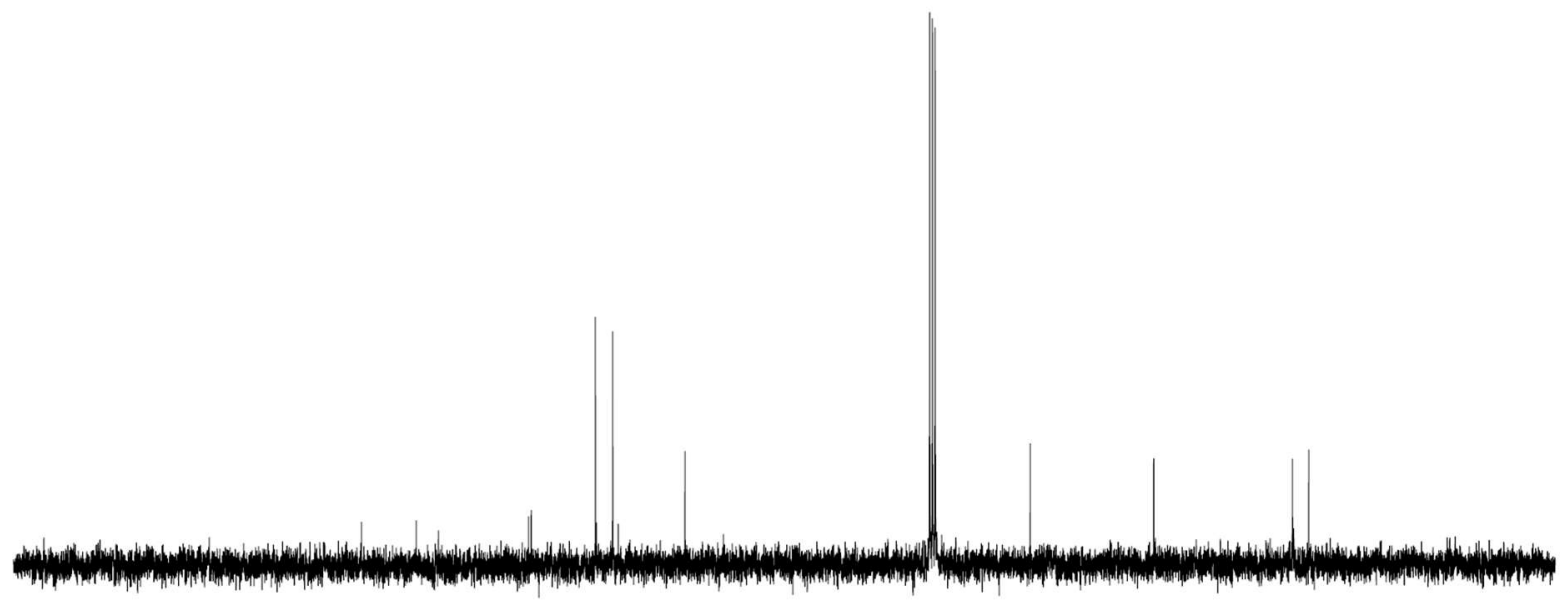

$\begin{array}{lllllllllllllllllllllll}210 & 200 & 190 & 180 & 170 & 160 & 150 & 140 & 130 & 120 & 110 & \begin{array}{l}100 \\ \mathrm{f} 1(\mathrm{ppm})\end{array} & 90 & 80 & 70 & 60 & 50 & 40 & 30 & 20 & 10 & 0 & -10\end{array}$

(E)-3-(3-(m-tolyl)but-2-enoyl)oxazolidin-2-one ((E)-35):

${ }^{1} \mathrm{H} \mathrm{NMR}\left(\mathrm{CDCl}_{3}, 300 \mathrm{MHz}\right)$

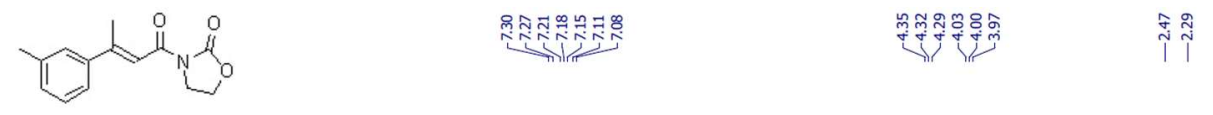

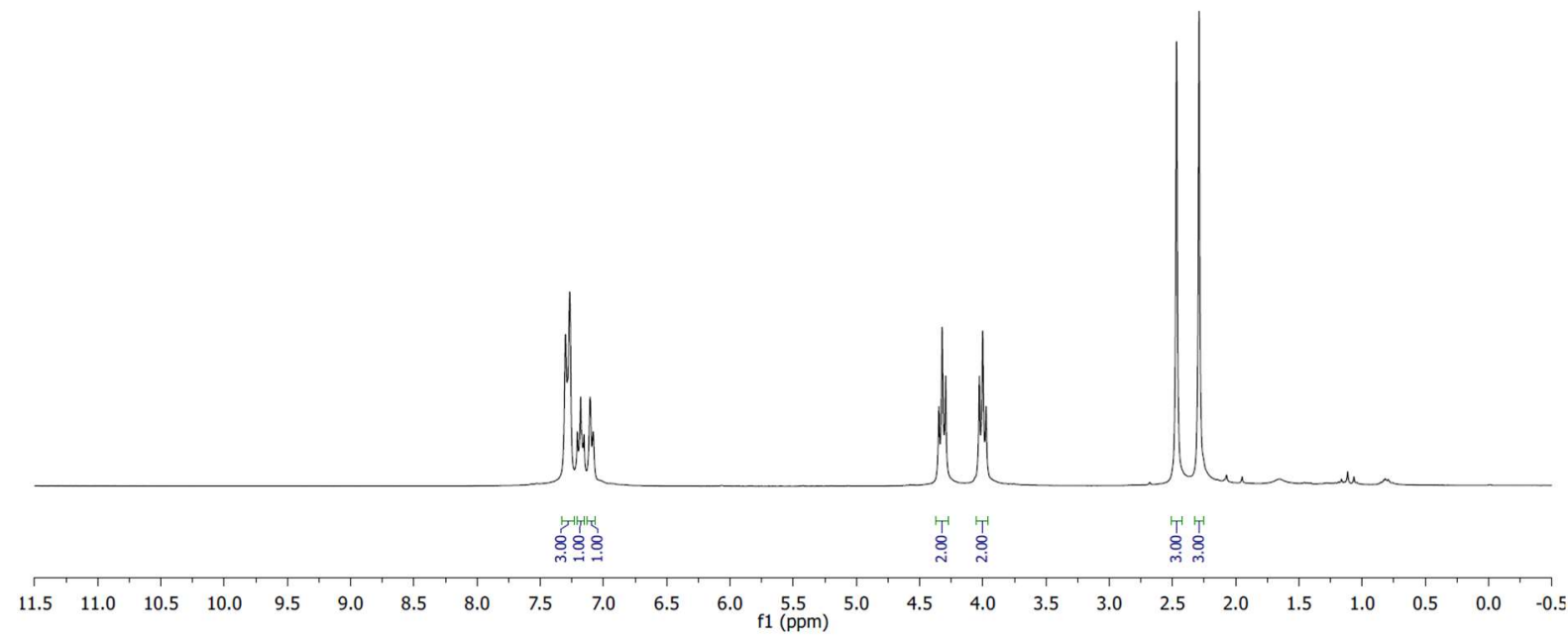


${ }^{13} \mathrm{C} \mathrm{NMR}\left(\mathrm{CDCl}_{3}, 75 \mathrm{MHz}\right)$

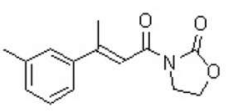

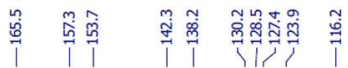

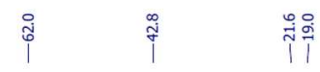

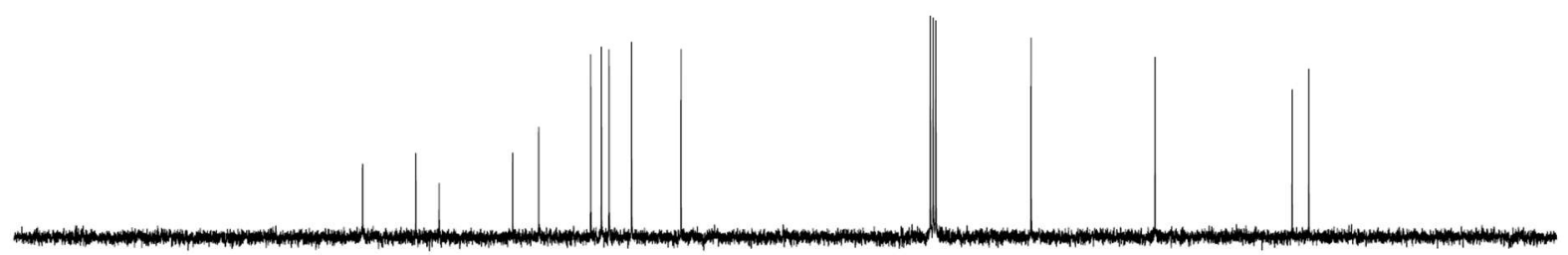

$\begin{array}{lllllllllll}210 & 200 & 190 & 180 & 170 & 160 & 150 & 140 & 130 & 120 & 110 \\ \mathrm{f} 1(\mathrm{ppm}) & 90\end{array}$

(E)-3-(3-(o-tolyl)but-2-enoyl)oxazolidin-2-one ((E)-37):

${ }^{1} \mathrm{H} \mathrm{NMR}\left(\mathrm{CDCl}_{3}, 300 \mathrm{MHz}\right)$<smiles>C/C(=C\C(=O)N1CCOC1=O)c1ccccc1C</smiles>

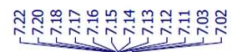

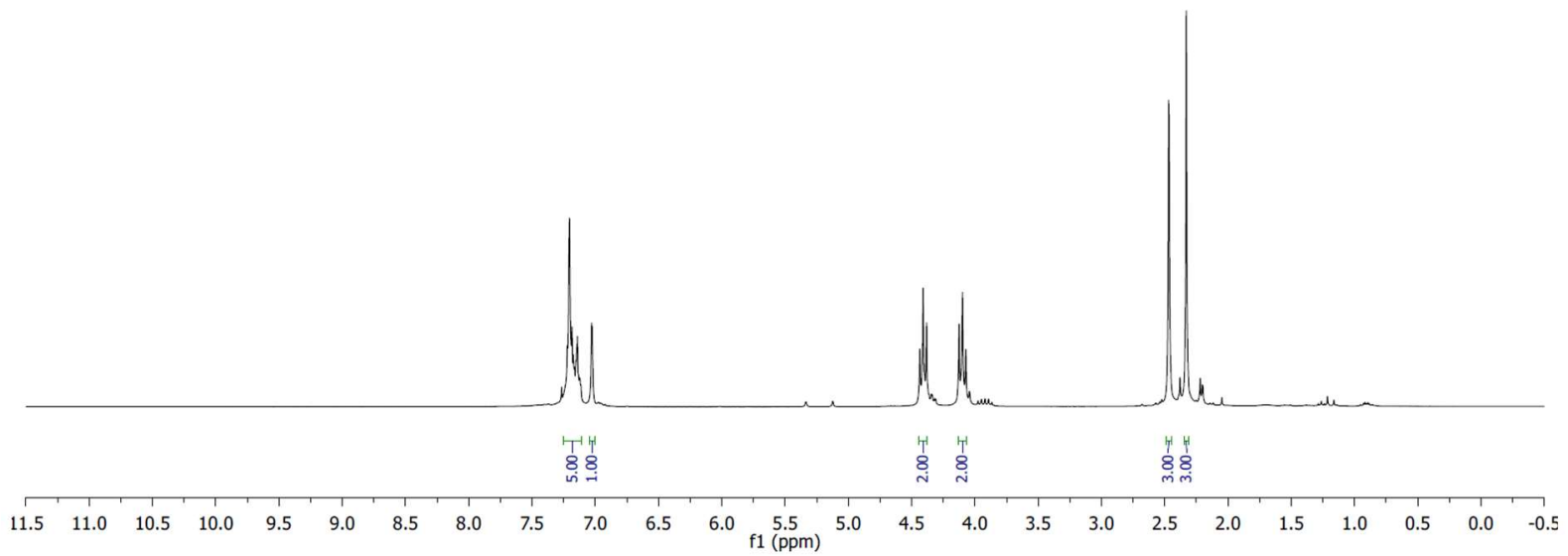


${ }^{13} \mathrm{C} \mathrm{NMR}\left(\mathrm{CDCl}_{3}, 75 \mathrm{MHz}\right)$
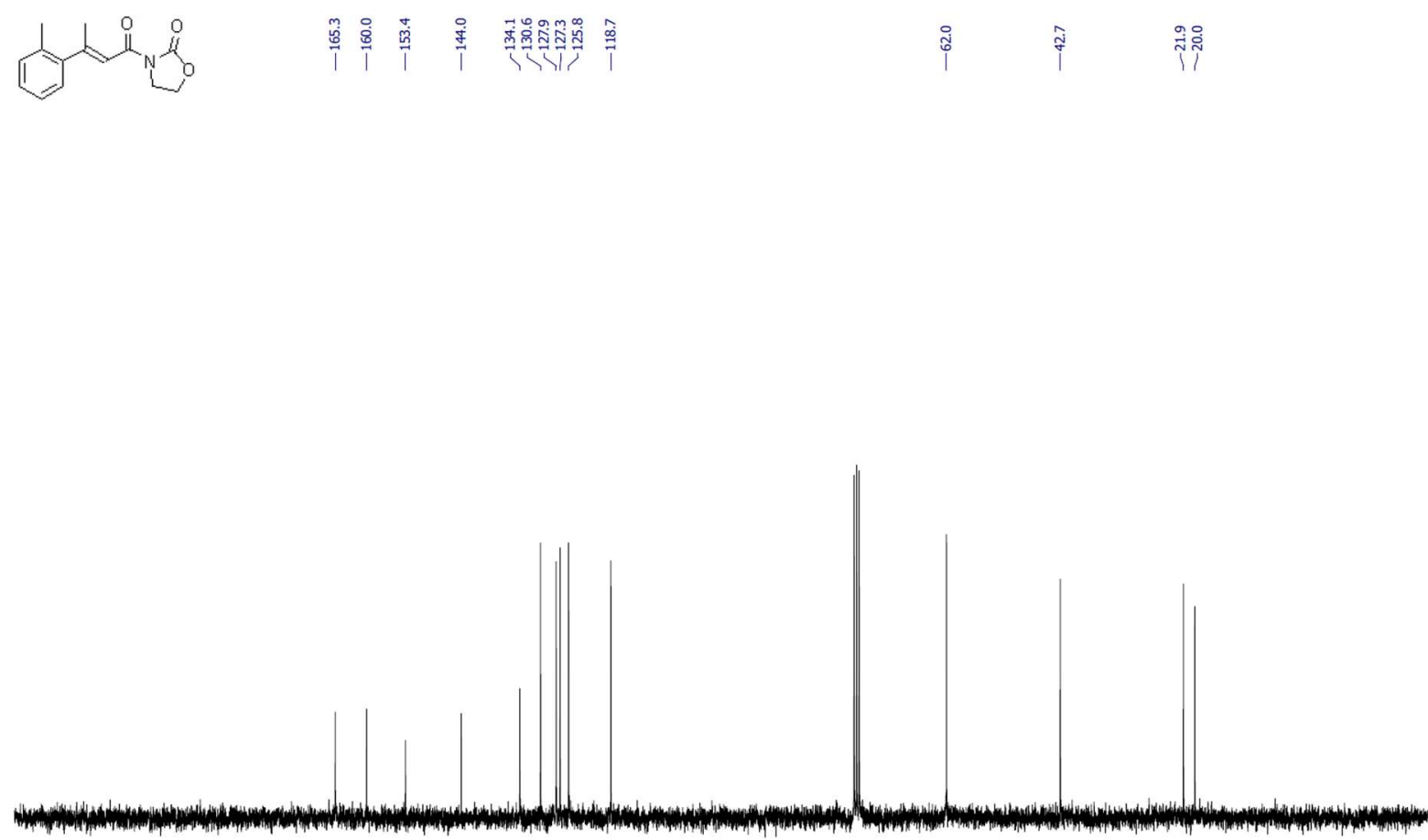

$\begin{array}{llllllllllllllllllllllll}210 & 200 & 190 & 180 & 170 & 160 & 150 & 140 & 130 & 120 & 110 & 100 & 90 & 80 & 70 & 60 & 50 & 40 & 30 & 20 & 10 & 0 & -10\end{array}$

(E)-3-(3-(4-(tert-butyl)phenyl)but-2-enoyl)oxazolidin-2-one ((E)-39):

${ }^{1} \mathrm{H} \mathrm{NMR}\left(\mathrm{CDCl}_{3}, 300 \mathrm{MHz}\right)$

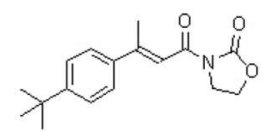

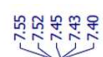

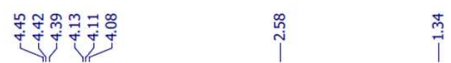

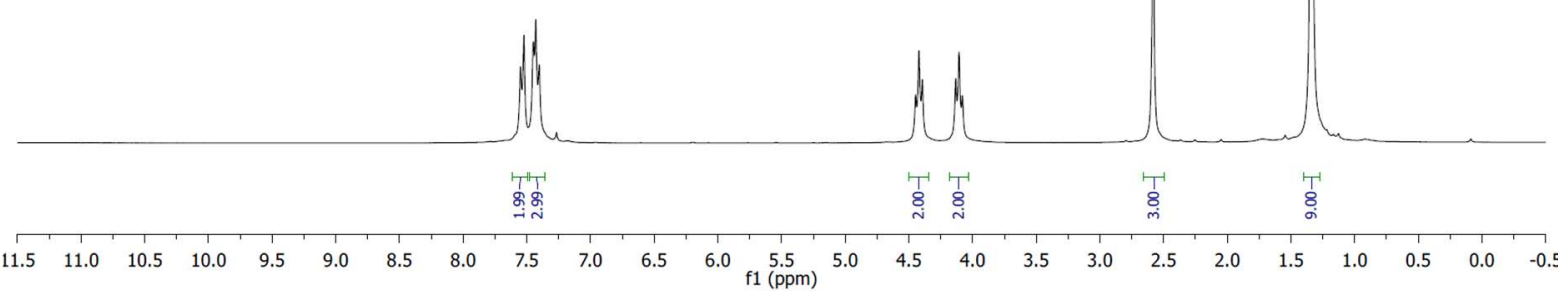


${ }^{13} \mathrm{C} \mathrm{NMR}\left(\mathrm{CDCl}_{3}, 75 \mathrm{MHz}\right)$
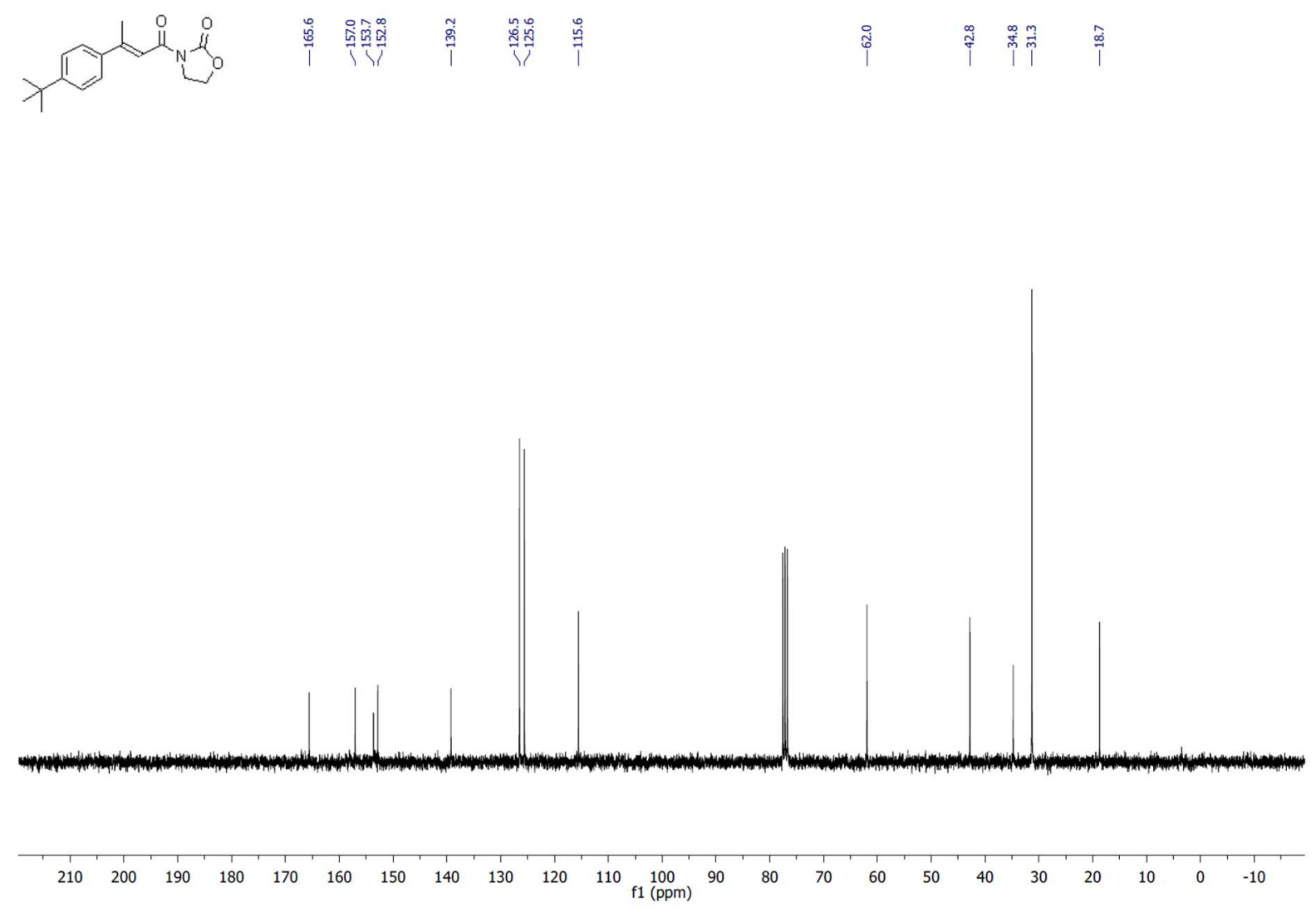

(E)-3-(3-(4-methoxyphenyl)but-2-enoyl)oxazolidin-2-one ((E)-41):

${ }^{1} \mathrm{H} \mathrm{NMR}\left(\mathrm{CDCl}_{3}, 300 \mathrm{MHz}\right)$
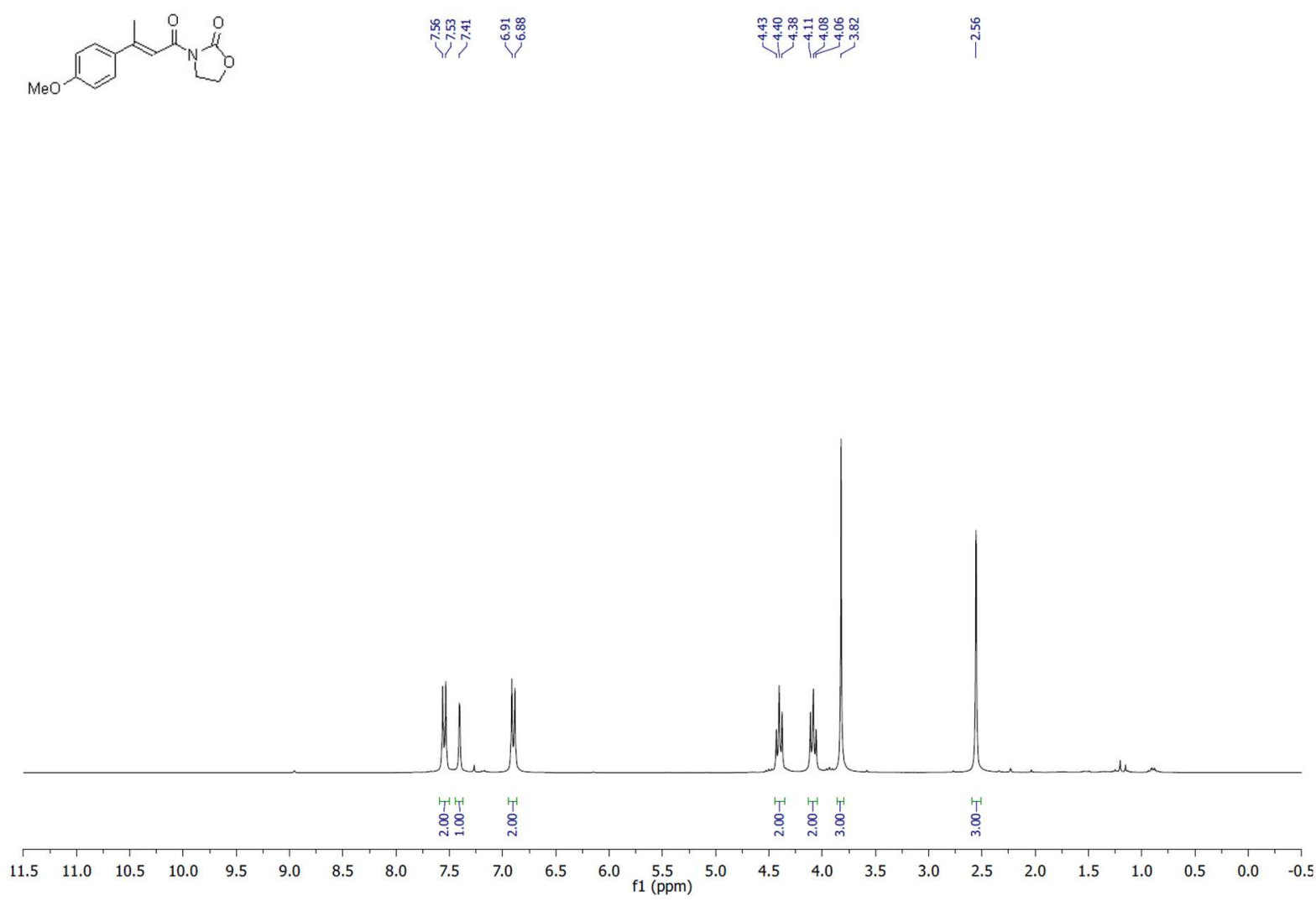
${ }^{13} \mathrm{C} \mathrm{NMR}\left(\mathrm{CDCl}_{3}, 75 \mathrm{MHz}\right)$
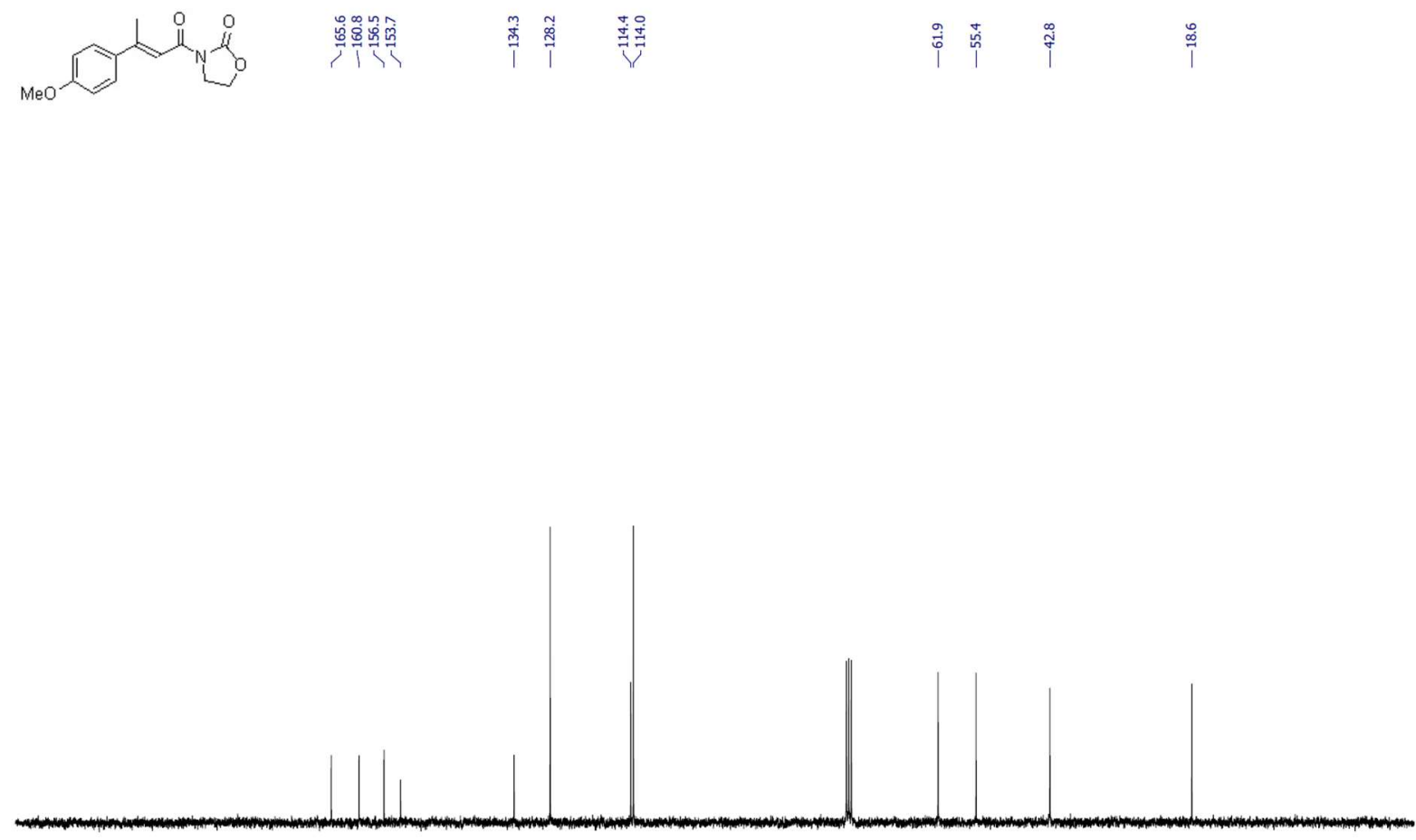

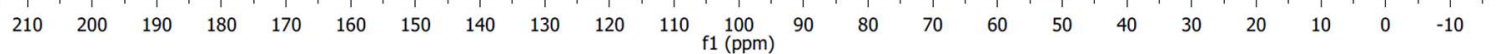

(E)-3-(3-(4-fluorophenyl)but-2-enoyl)oxazolidin-2-one ((E)-43):

${ }^{1} \mathrm{H} \mathrm{NMR}\left(\mathrm{CDCl}_{3}, 300 \mathrm{MHz}\right)$
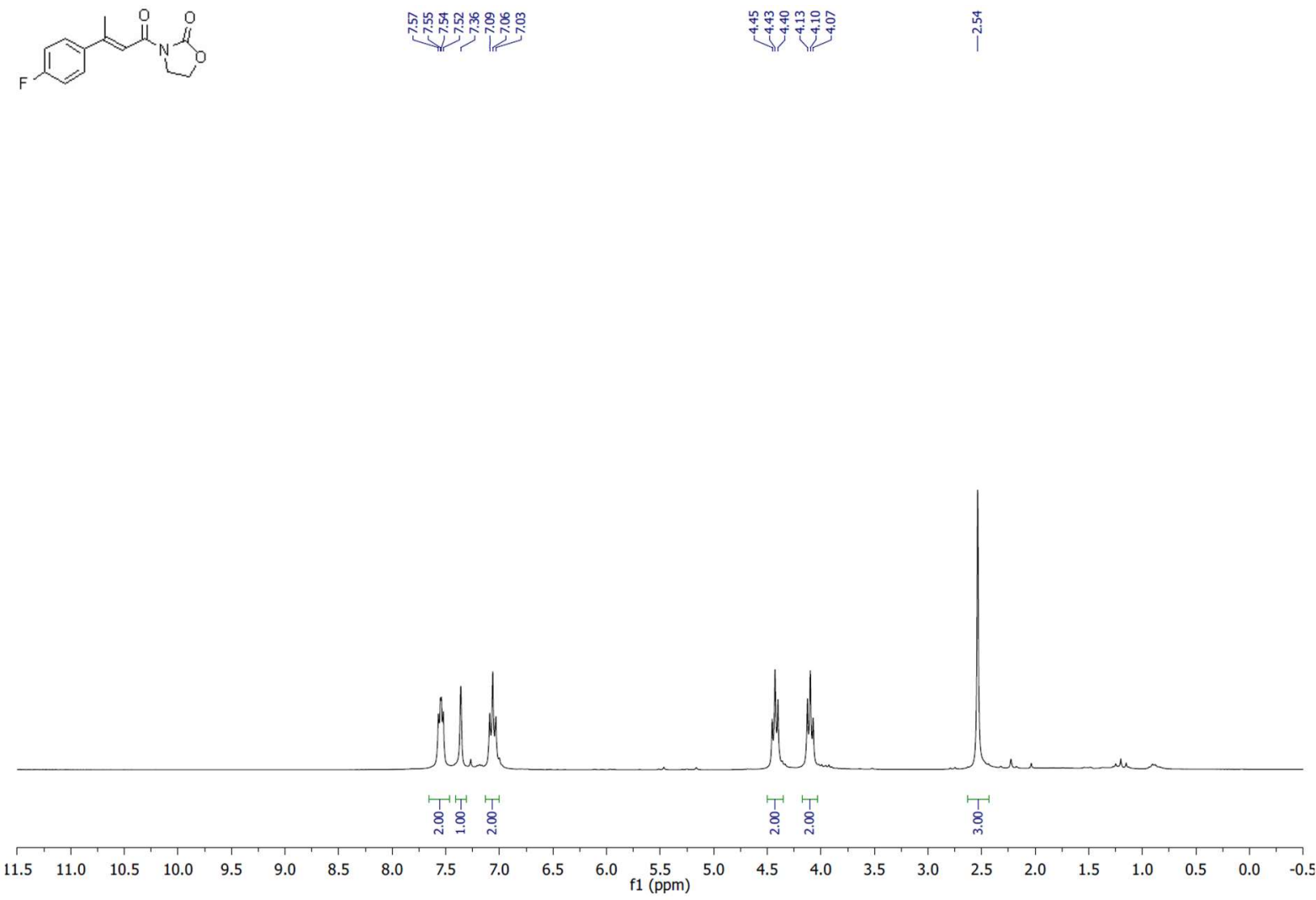
${ }^{13} \mathrm{C} \mathrm{NMR}\left(\mathrm{CDCl}_{3}, 75 \mathrm{MHz}\right)$

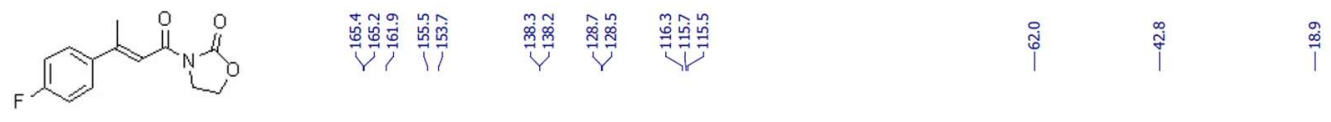
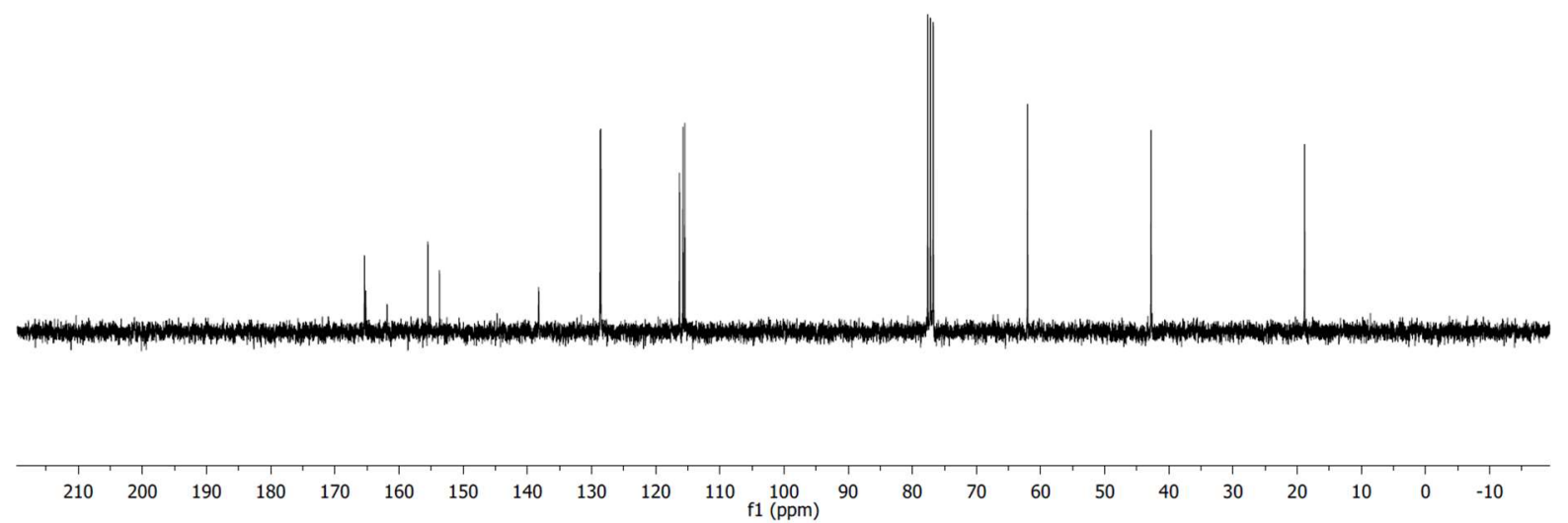

${ }^{19} \mathrm{~F} \mathrm{NMR}\left(\mathrm{CDCl}_{3}, 282 \mathrm{MHz}\right)$
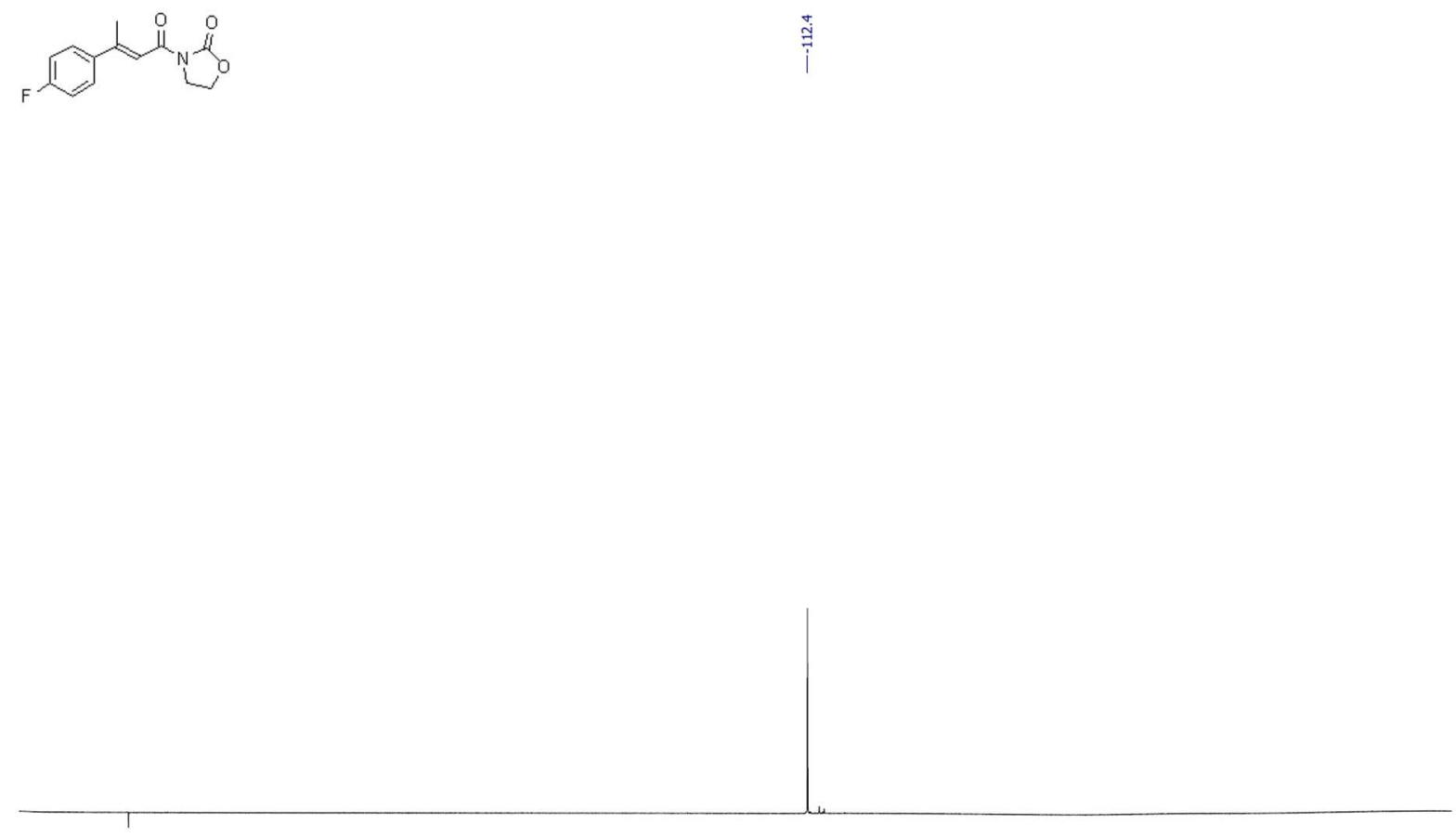

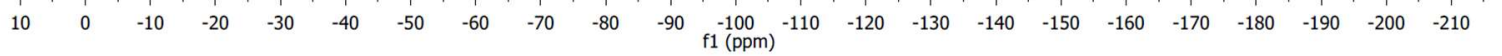


(E)-3-(3-(4-bromophenyl)but-2-enoyl)oxazolidin-2-one ((E)-45):

${ }^{1} \mathrm{H} \mathrm{NMR}\left(\mathrm{CDCl}_{3}, 300 \mathrm{MHz}\right)$

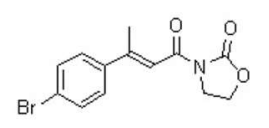

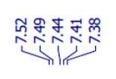

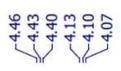

$\stackrel{\dddot{3}}{1}$

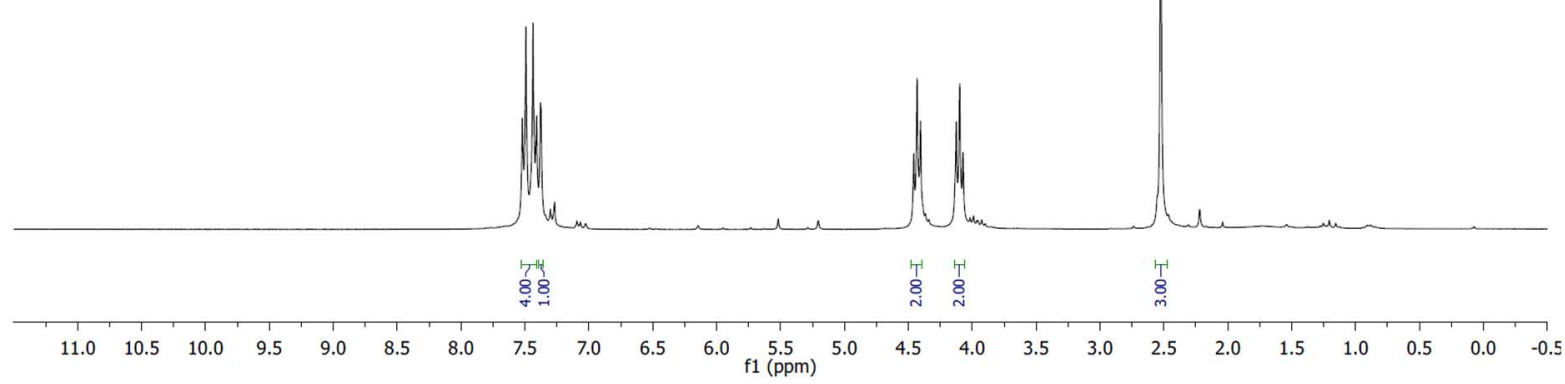

${ }^{13} \mathrm{C} \mathrm{NMR}\left(\mathrm{CDCl}_{3}, 75 \mathrm{MHz}\right)$
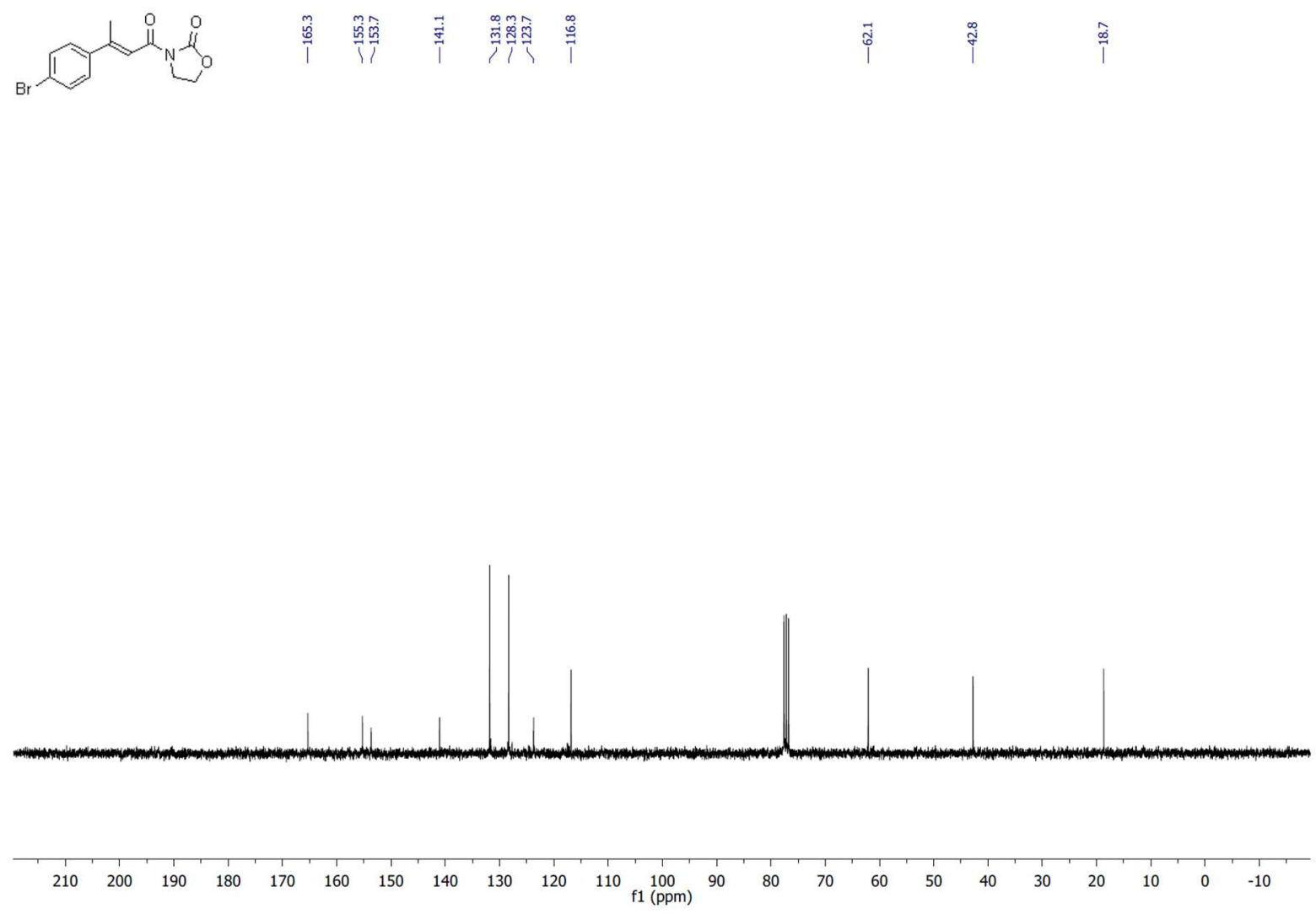

182 
(E)-3-(3-(4-iodophenyl)but-2-enoyl)oxazolidin-2-one ((E)-47):

${ }^{1} \mathrm{H} \mathrm{NMR}\left(\mathrm{CDCl}_{3}, 300 \mathrm{MHz}\right)$<smiles>CC(=CC(C)c1ccc(I)cc1)N1CCO1</smiles>

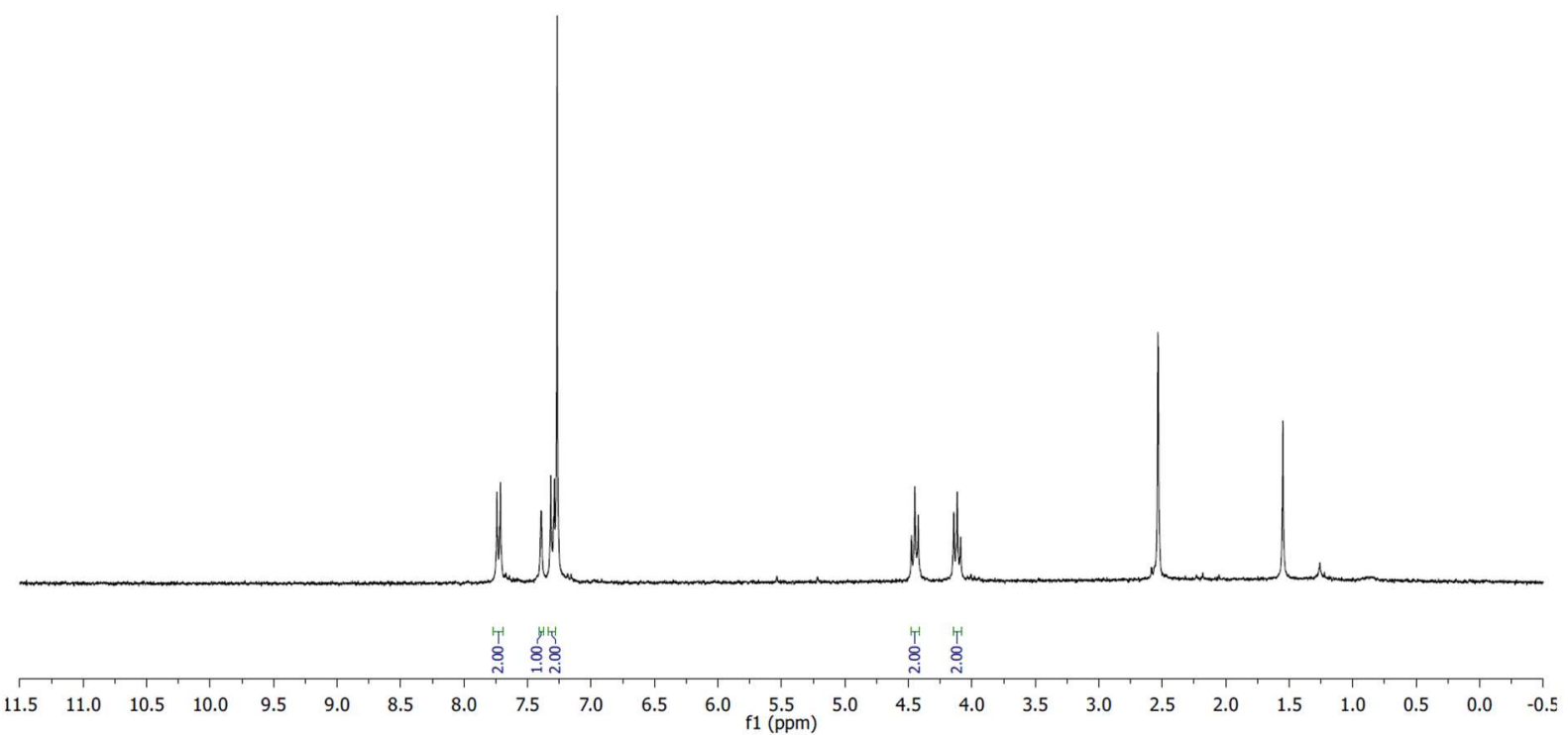

${ }^{13} \mathrm{CNMR}\left(\mathrm{CDCl}_{3}, 75 \mathrm{MHz}\right)$<smiles>C/C(=C\C(=O)N1CCOC1=O)c1ccc(I)cc1</smiles>

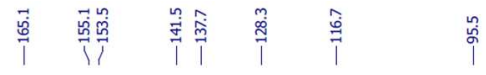
ֻृ

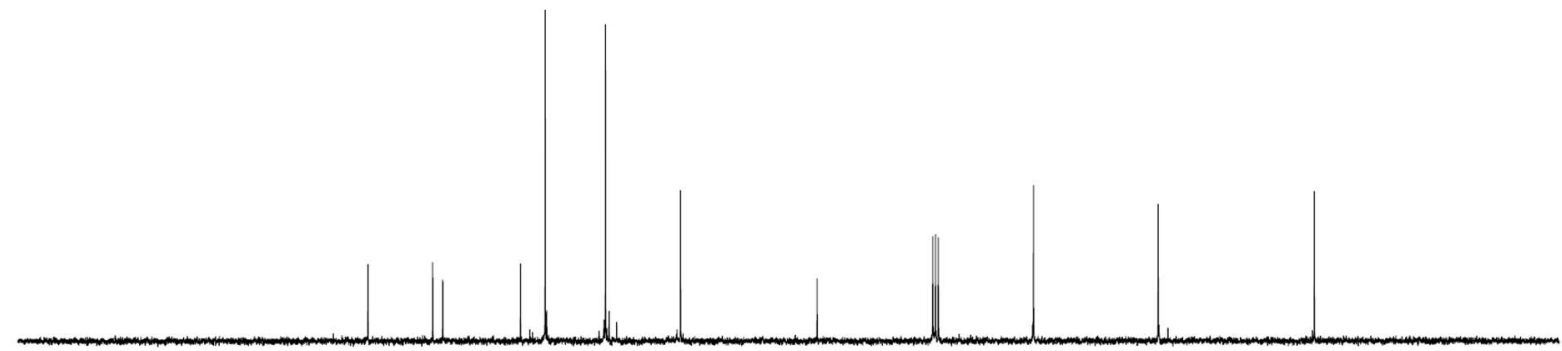

$\begin{array}{lllllllllllllllllllllll}210 & 200 & 190 & 180 & 170 & 160 & 150 & 140 & 130 & 120 & 110 & 100 & 90 & 80 & 70 & 60 & 50 & 40 & 30 & 20 & 10 & 0 & -10\end{array}$ 
(E)-3-(3-(4-nitrophenyl)but-2-enoyl)oxazolidin-2-one ((E)-49):

${ }^{1} \mathrm{H} \mathrm{NMR}\left(\mathrm{CDCl}_{3}, 300 \mathrm{MHz}\right)$<smiles>CC(=C(C(=O)N1CCCO1)c1ccc([N+](=O)[O-])cc1)c1ccccc1</smiles>

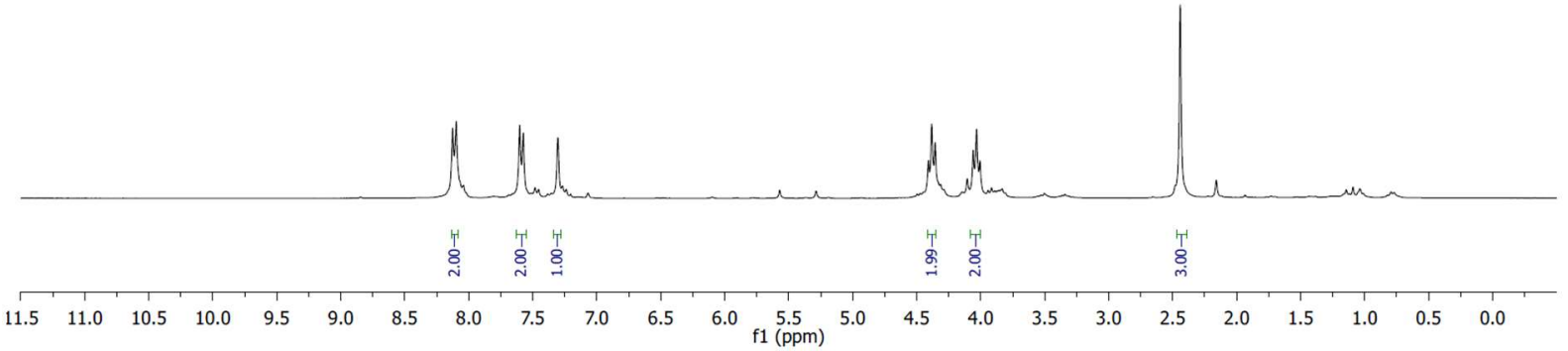

${ }^{13} \mathrm{C} \mathrm{NMR}\left(\mathrm{CDCl}_{3}, 75 \mathrm{MHz}\right)$

至N

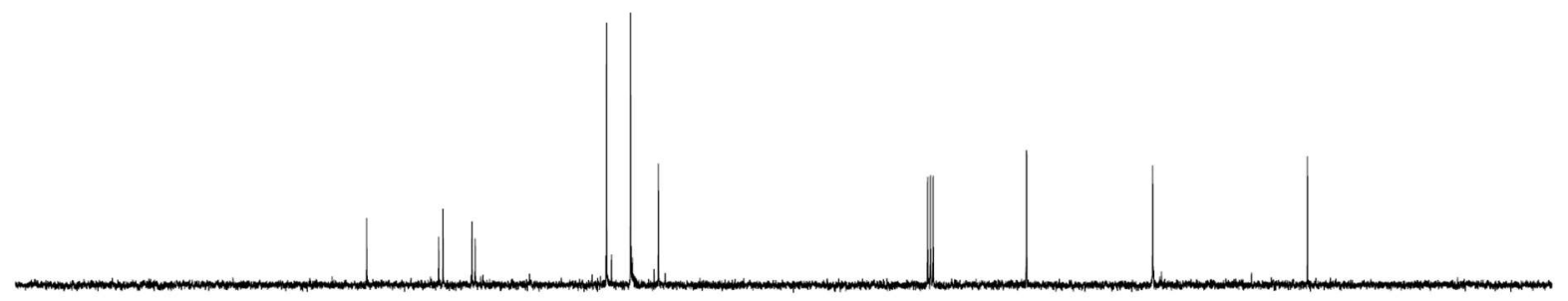

$\begin{array}{lllllllllllllllllllllll}210 & 200 & 190 & 180 & 170 & 160 & 150 & 140 & 130 & 120 & 110 & 100 & 90 & 80 & 70 & 60 & 50 & 40 & 30 & 20 & 10 & 0 & -10\end{array}$ 
(E)-3-(4,4,4-trifluoro-3-phenylbut-2-enoyl)oxazolidin-2-one ((E)-51):

${ }^{1} \mathrm{H} \mathrm{NMR}\left(\mathrm{CDCl}_{3}, 300 \mathrm{MHz}\right)$
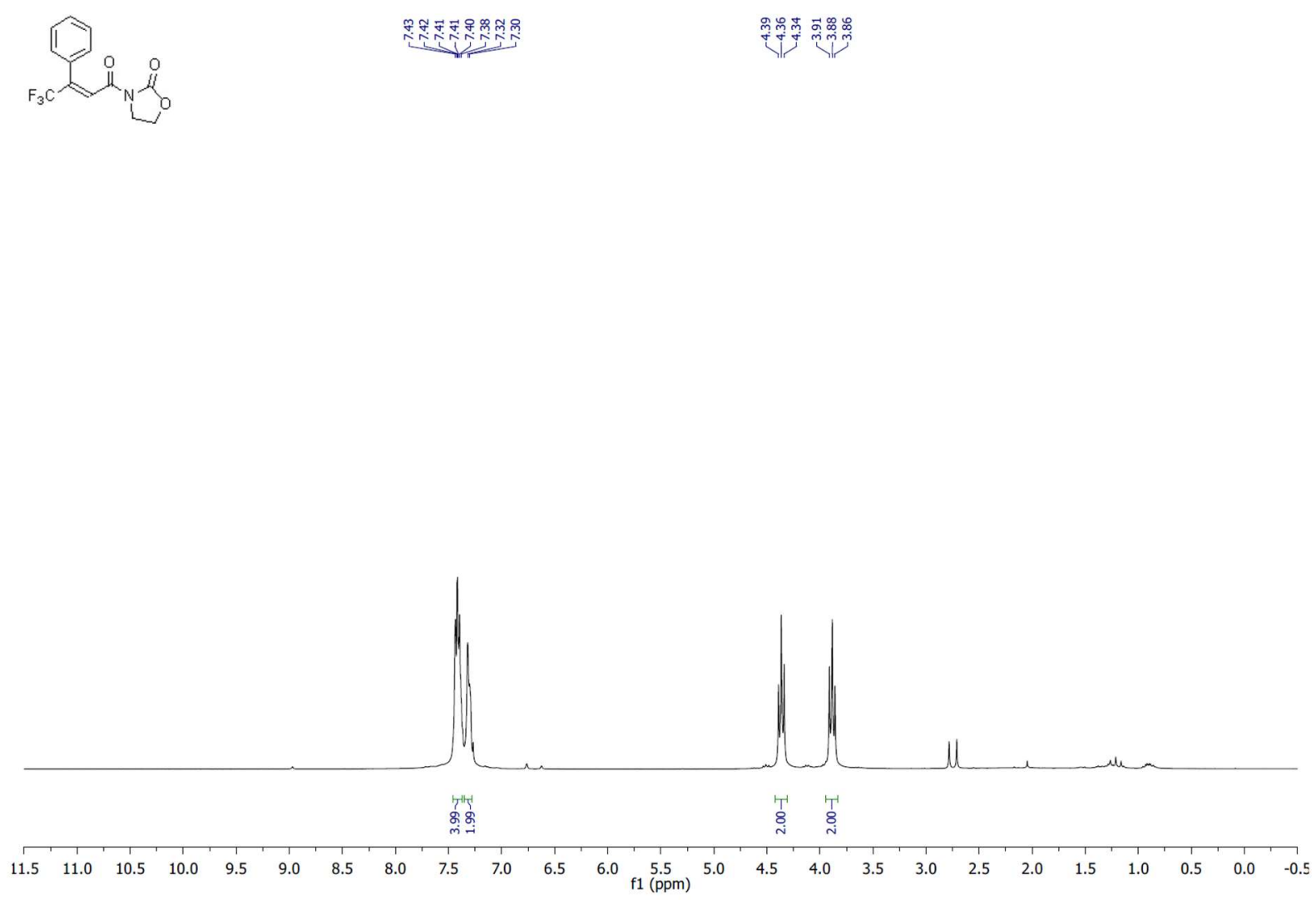

${ }^{13} \mathrm{C} \mathrm{NMR}\left(\mathrm{CDCl}_{3}, 75 \mathrm{MHz}\right)$
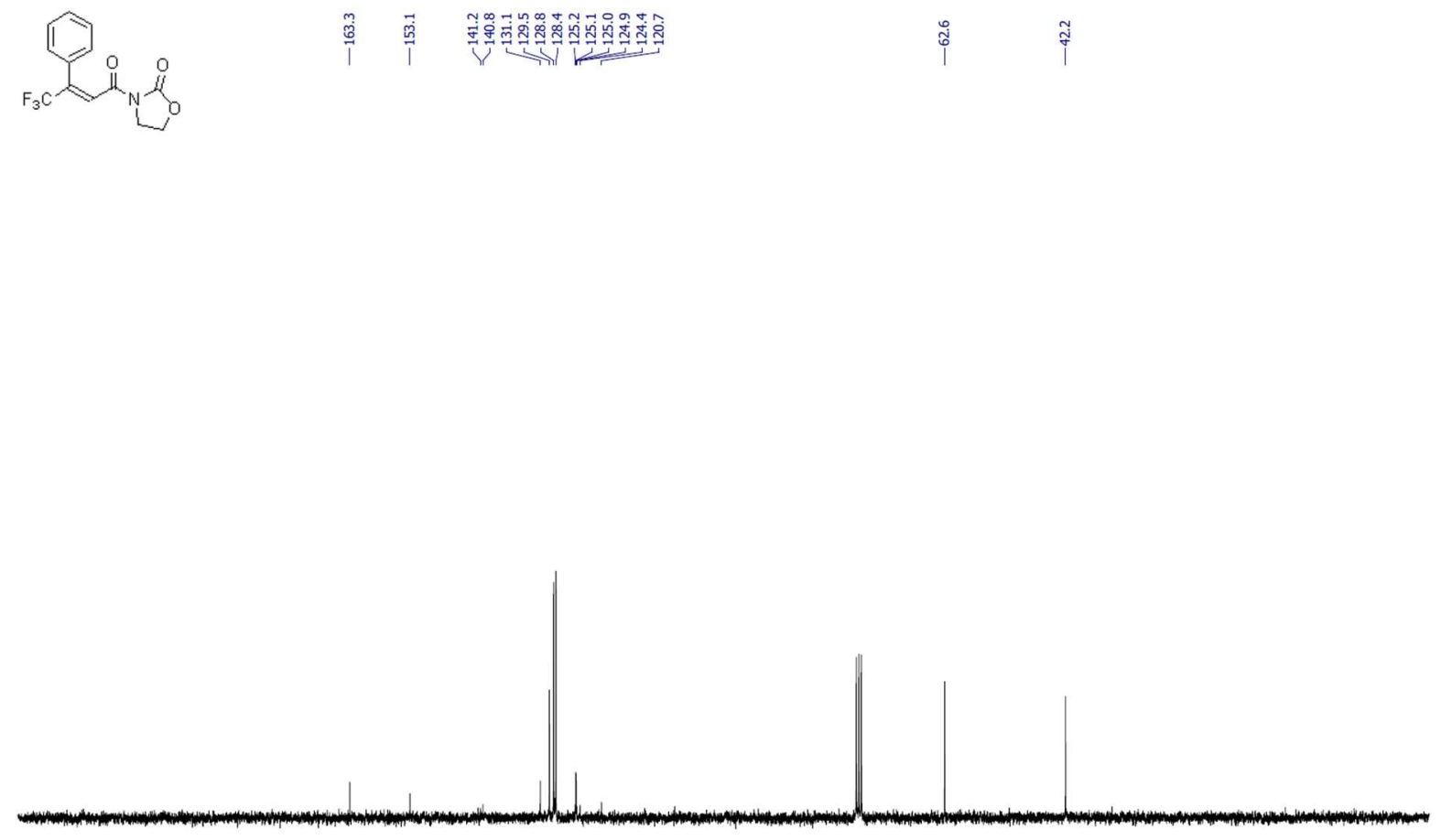

$\begin{array}{llllllllllllllllllllllllllll}210 & 200 & 190 & 180 & 170 & 160 & 150 & 140 & 130 & 120 & 110 & \begin{array}{c}100 \\ \mathrm{f} 1(\mathrm{ppm})\end{array} & 90 & 80 & 70 & 60 & 50 & 40 & 30 & 20 & 10 & 0 & -10\end{array}$ 
${ }^{19} \mathrm{~F} \mathrm{NMR}\left(\mathrm{CDCl}_{3}, 282 \mathrm{MHz}\right)$<smiles></smiles>

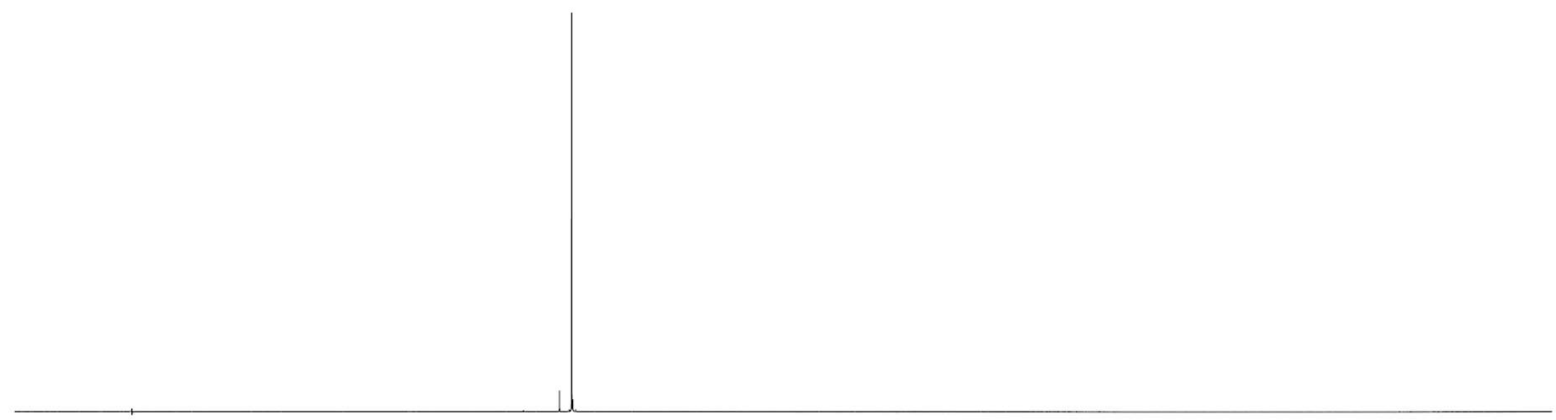

$\begin{array}{lllllllllllllllllllllll}10 & 0 & -10 & -20 & -30 & -40 & -50 & -60 & -70 & -80 & -90 & -100 & -110 & -120 & -130 & -140 & -150 & -160 & -170 & -180 & -190 & -200 & -210\end{array}$

(E)-3-phenylbut-2-enenitrile ((E)-53):

${ }^{1} \mathrm{H} \mathrm{NMR}\left(\mathrm{CDCl}_{3}, 300 \mathrm{MHz}\right)$
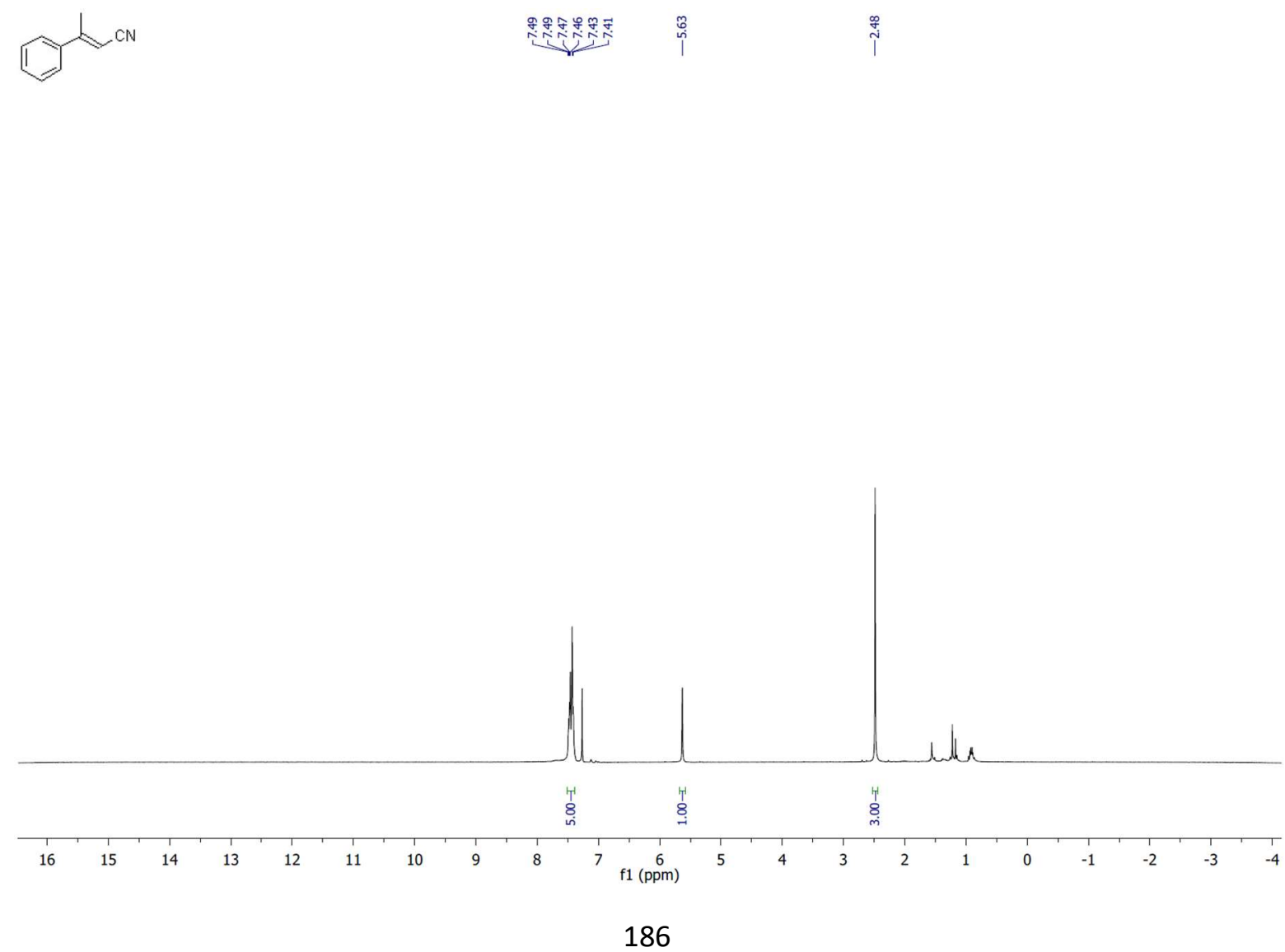
${ }^{13} \mathrm{C} \mathrm{NMR}\left(\mathrm{CDCl}_{3}, 75 \mathrm{MHz}\right)$

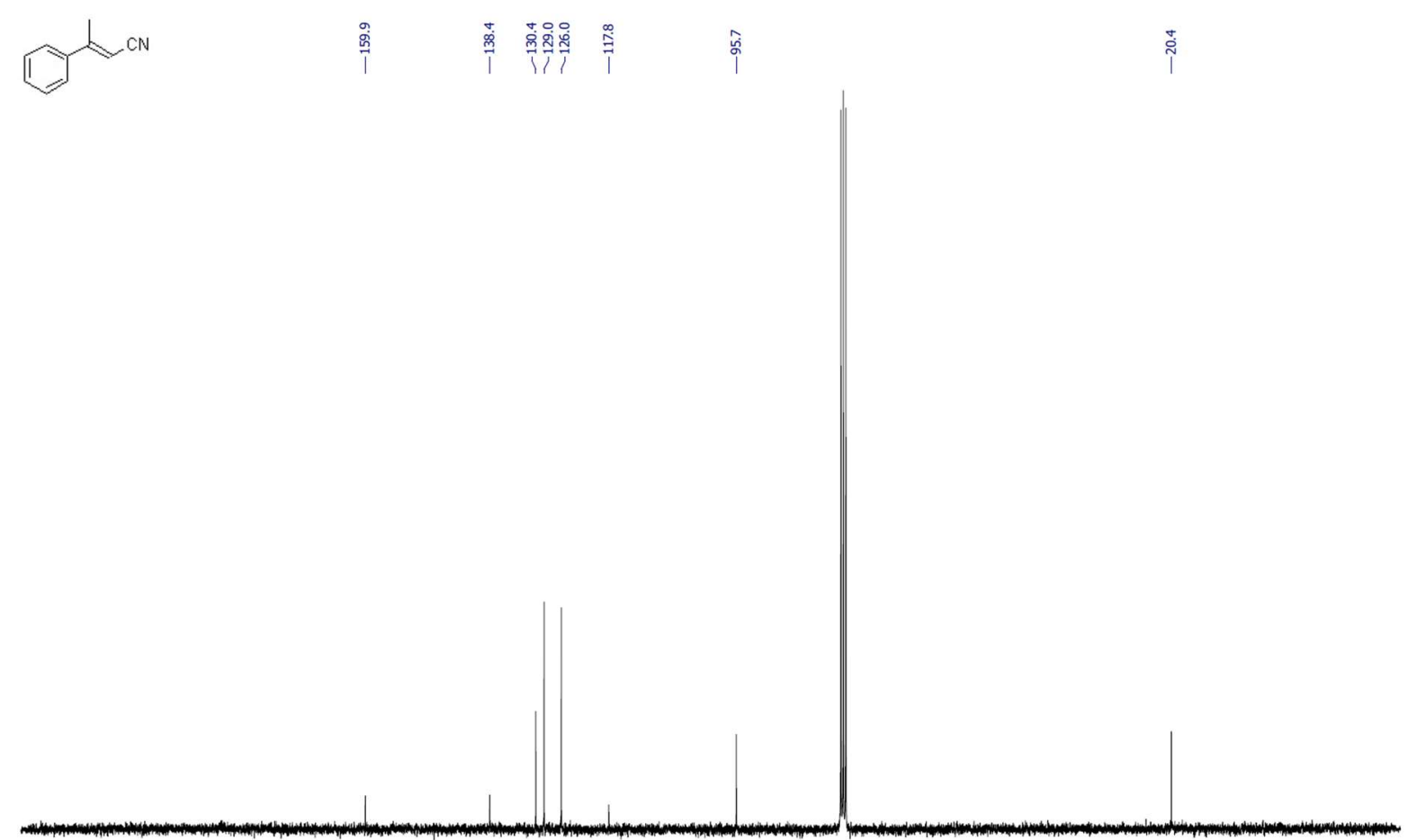

$\begin{array}{lllllllllllllllllllllll}210 & 200 & 190 & 180 & 170 & 160 & 150 & 140 & 130 & 120 & 110 & 100 & 90 & 80 & 70 & 60 & 50 & 40 & 30 & 20 & 10 & 0 & -10\end{array}$

diethyl (E)-(2-phenylprop-1-en-1-yl)phosphonate ((E)-54):

${ }^{1} \mathrm{H} \mathrm{NMR}\left(\mathrm{CDCl}_{3}, 300 \mathrm{MHz}\right)$

$1 \mathrm{CPO}^{\mathrm{POEt})_{2}}$

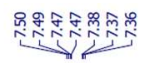

茄要

题赾是是

สูก

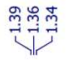

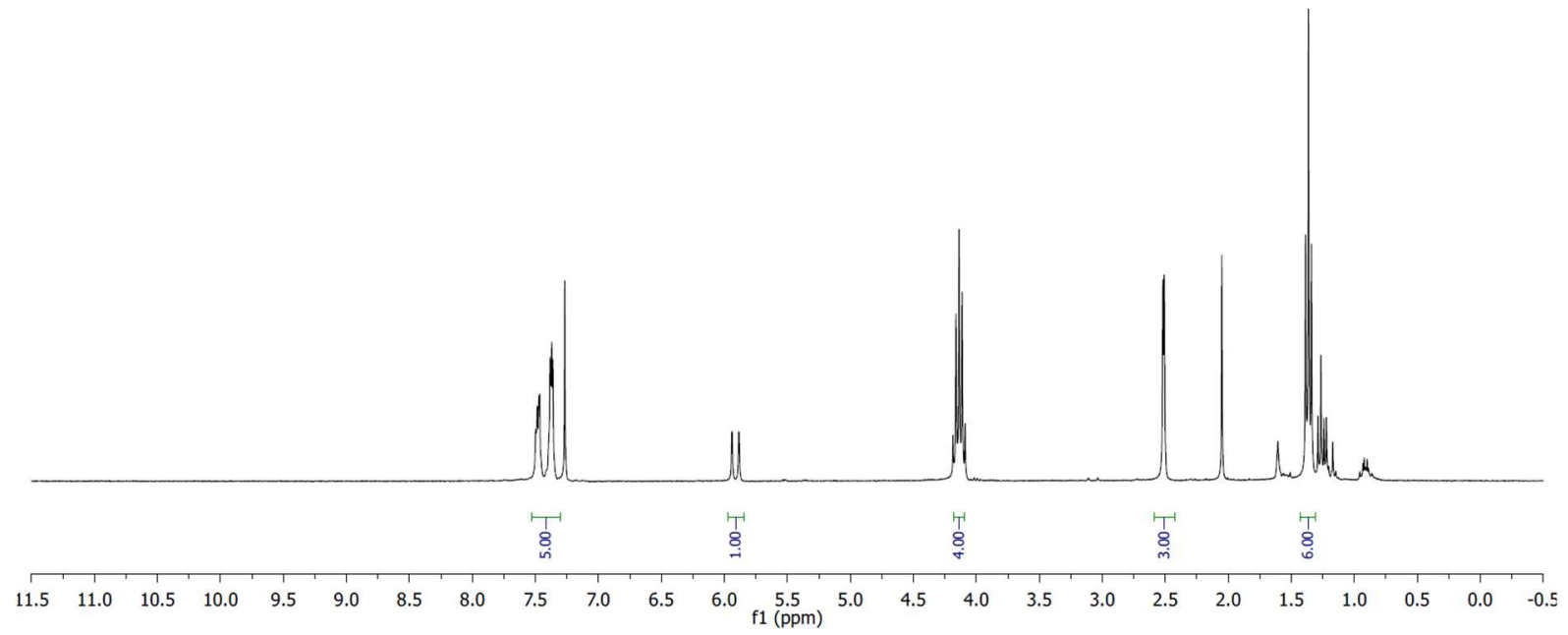


${ }^{13} \mathrm{C} \mathrm{NMR}\left(\mathrm{CDCl}_{3}, 75 \mathrm{MHz}\right)$

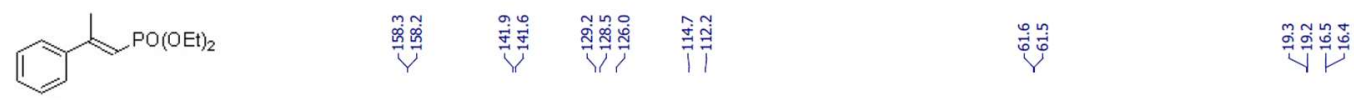

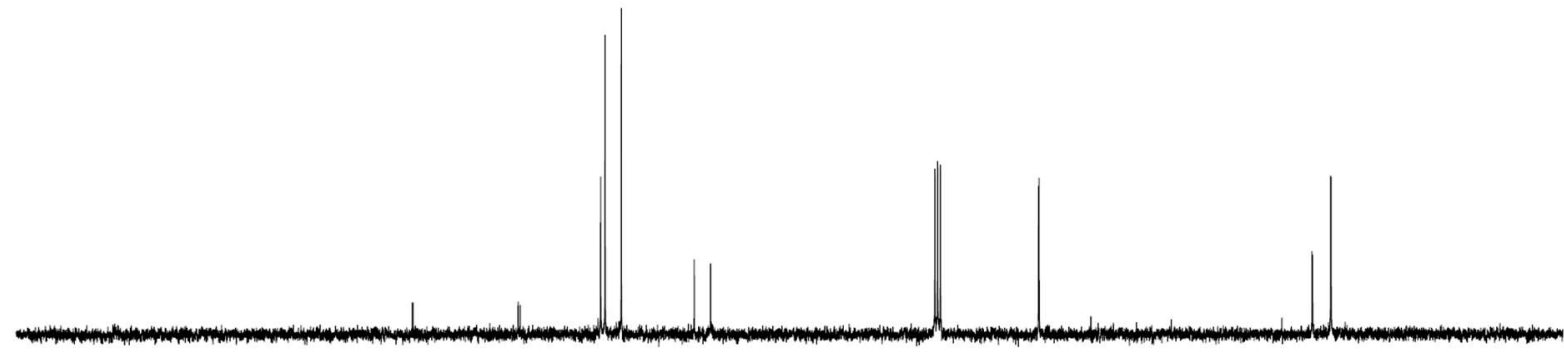

$\begin{array}{llllllllllllllllllllllll}210 & 200 & 190 & 180 & 170 & 160 & 150 & 140 & 130 & 120 & 110 & \begin{array}{l}100 \\ \mathrm{f} 1(\mathrm{ppm})\end{array} & 90 & 80 & 70 & 60 & 50 & 40 & 30 & 20 & 10 & 0 & -10\end{array}$

${ }^{31} \mathrm{P} \mathrm{NMR}\left(\mathrm{CDCl}_{3}, 121 \mathrm{MHz}\right)$

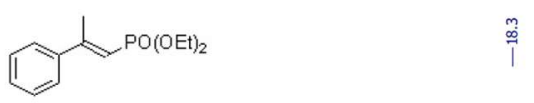


(E)-((2-phenylprop-1-en-1-yl)sulfonyl)benzene ((E)-55):

${ }^{1} \mathrm{H} \mathrm{NMR}\left(\mathrm{CDCl}_{3}, 300 \mathrm{MHz}\right)$

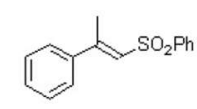

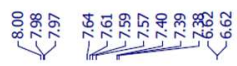

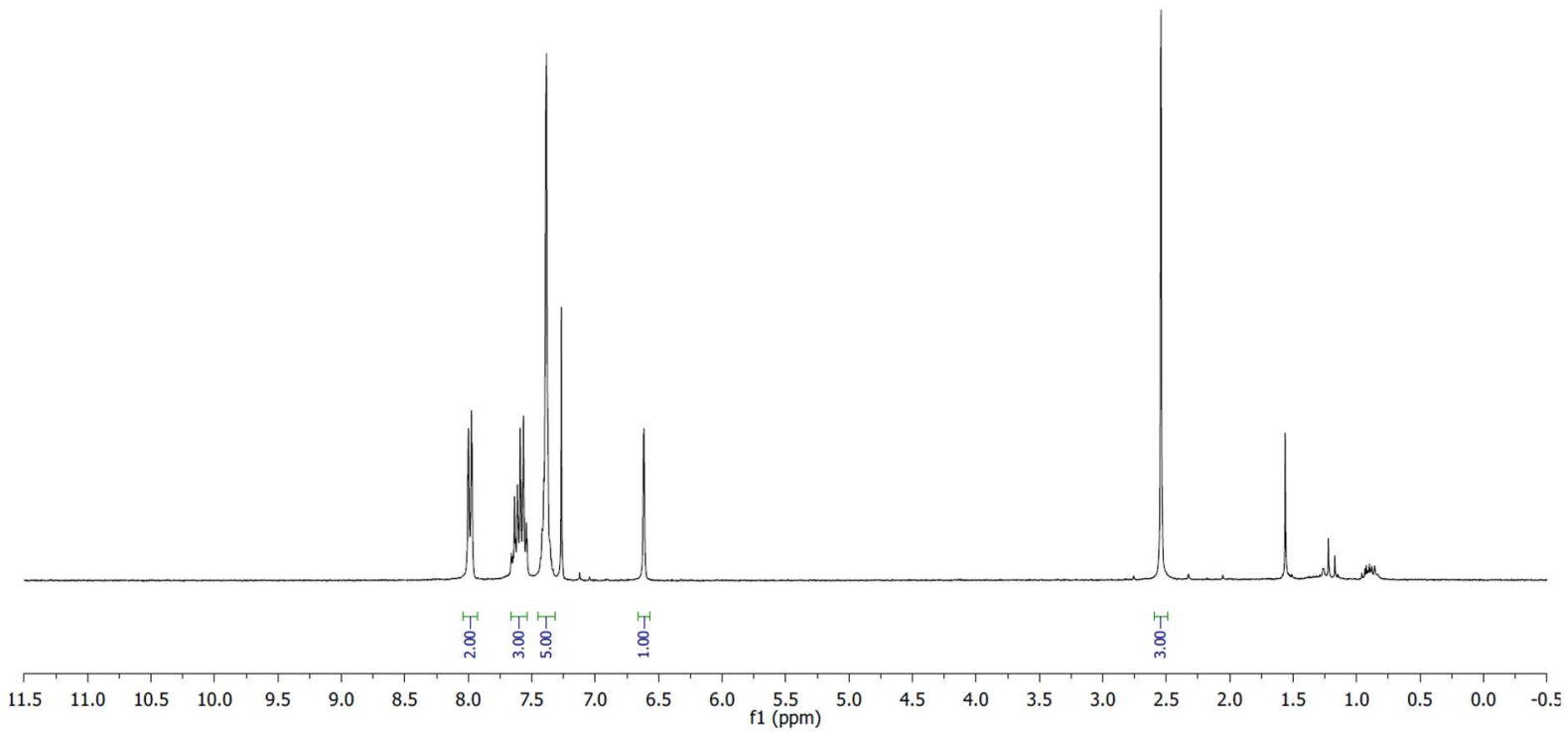

${ }^{13} \mathrm{C} \mathrm{NMR}\left(\mathrm{CDCl}_{3}, 75 \mathrm{MHz}\right)$

$1 \mathrm{SO}_{2} \mathrm{Ph}$

兽

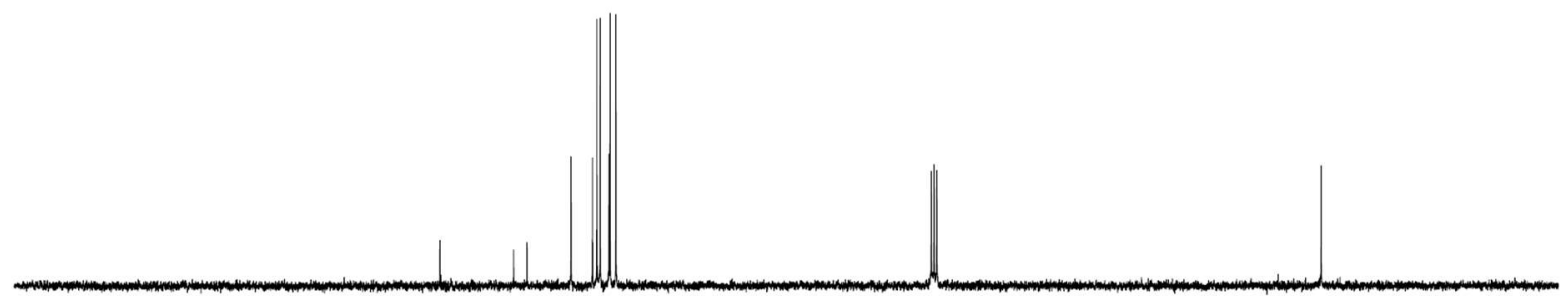

$\begin{array}{lllllllllllllllllllllll}210 & 200 & 190 & 180 & 170 & 160 & 150 & 140 & 130 & 120 & 110 & \begin{array}{l}100 \\ 100\end{array} & 90 & 80 & 70 & 60 & 50 & 40 & 30 & 20 & 10 & 0 & -10\end{array}$ 


\section{2. (Z)-isomers}

methyl (Z)-2-methyl-3-phenylacrylate ((Z)-1):

${ }^{1} \mathrm{H} \mathrm{NMR}\left(\mathrm{CDCl}_{3}, 300 \mathrm{MHz}\right)$
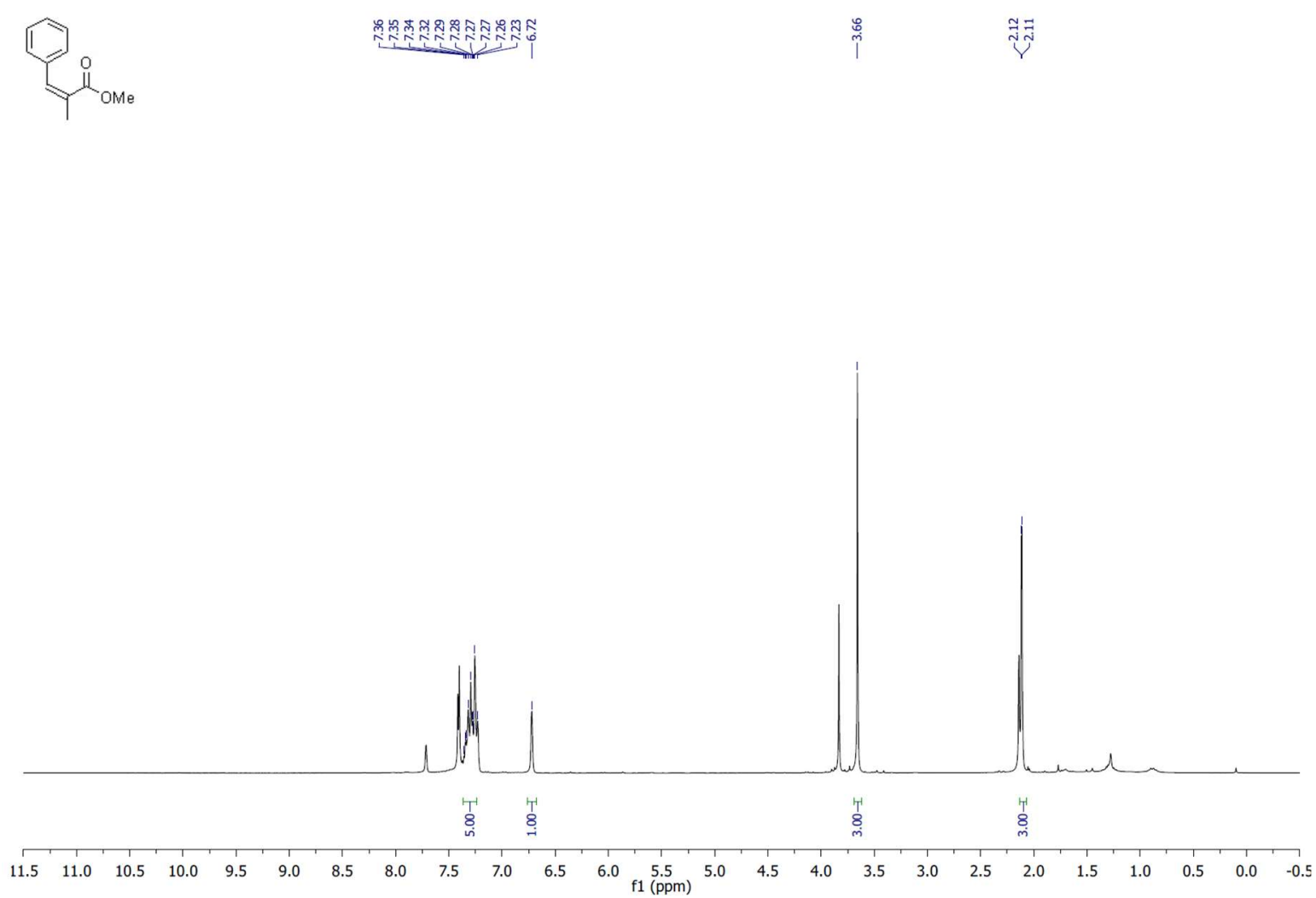

${ }^{13} \mathrm{C} \mathrm{NMR}\left(\mathrm{CDCl}_{3}, 75 \mathrm{MHz}\right)$
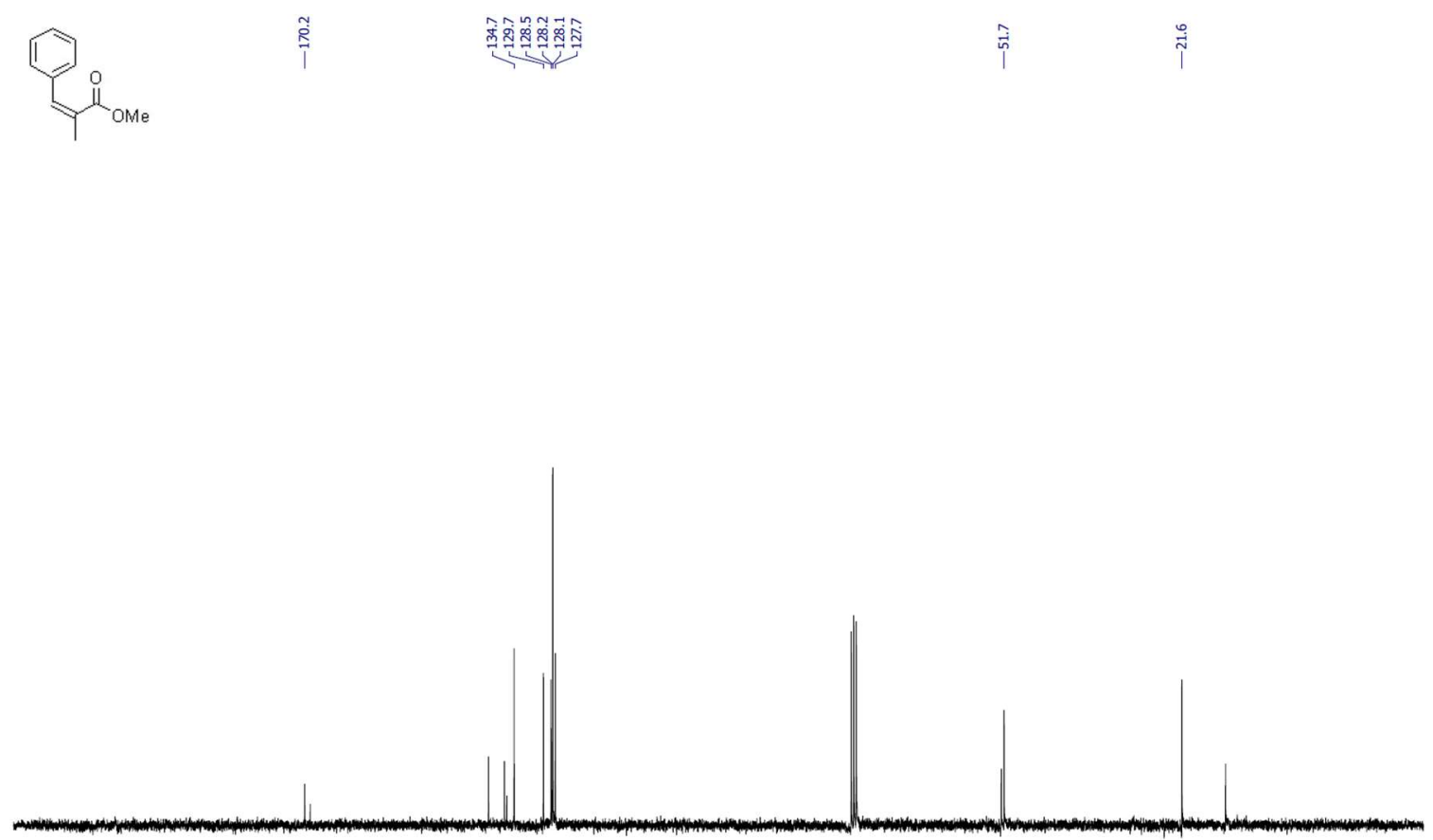

$\begin{array}{llllllllllllllllllllllllll}210 & 200 & 190 & 180 & 170 & 160 & 150 & 140 & 130 & 120 & 110 & \begin{array}{c}100 \\ \mathrm{f} 1(\mathrm{ppm})\end{array} & 90 & 80 & 70 & 60 & 50 & 40 & 30 & 20 & 10 & 0 & -10\end{array}$ 
(Z)-N,N,2-trimethyl-3-phenylacrylamide ((Z)-2):

${ }^{1} \mathrm{H} \mathrm{NMR}\left(\mathrm{CDCl}_{3}, 300 \mathrm{MHz}\right)$
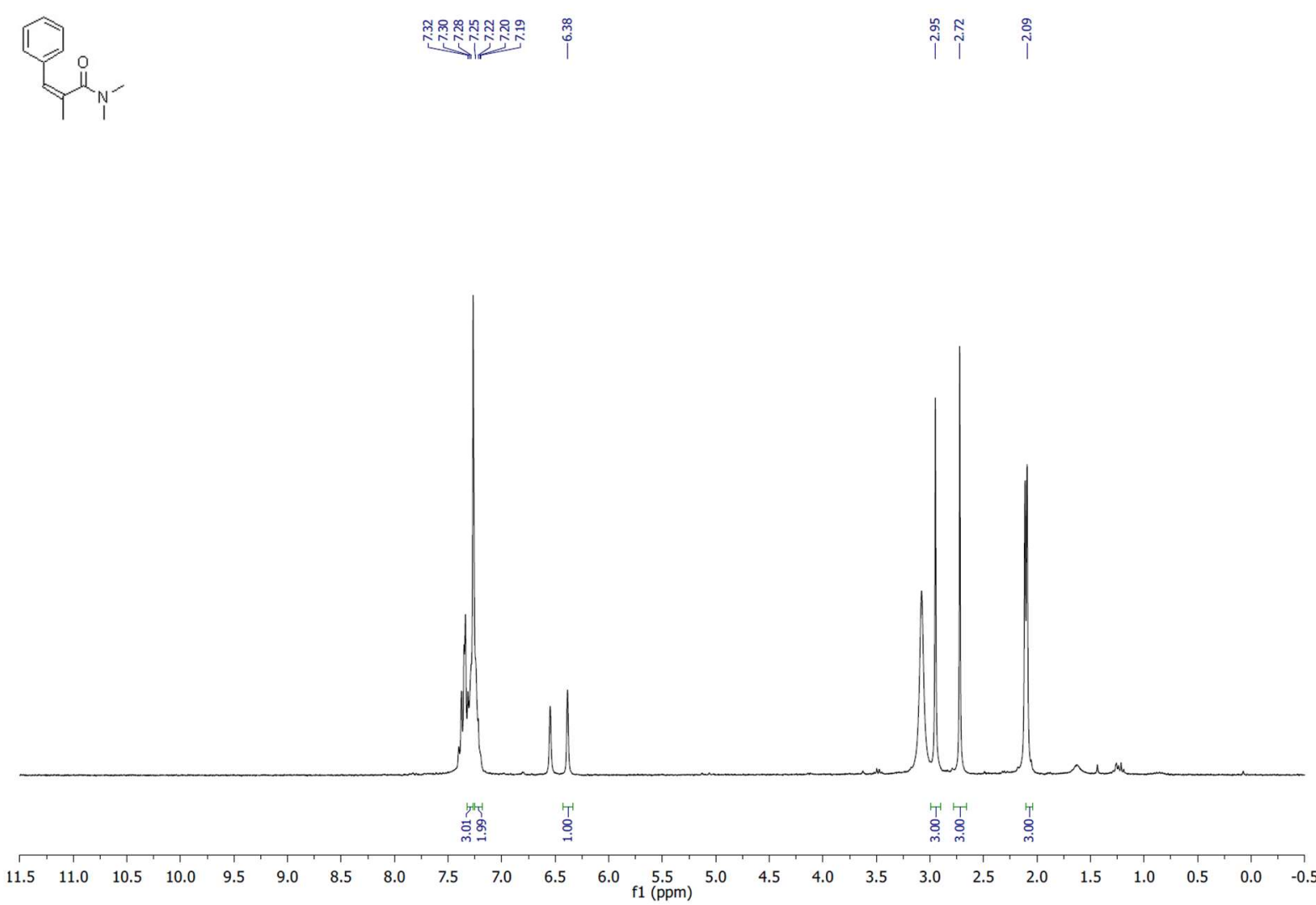

${ }^{13} \mathrm{C} \mathrm{NMR}\left(\mathrm{CDCl}_{3}, 75 \mathrm{MHz}\right)$
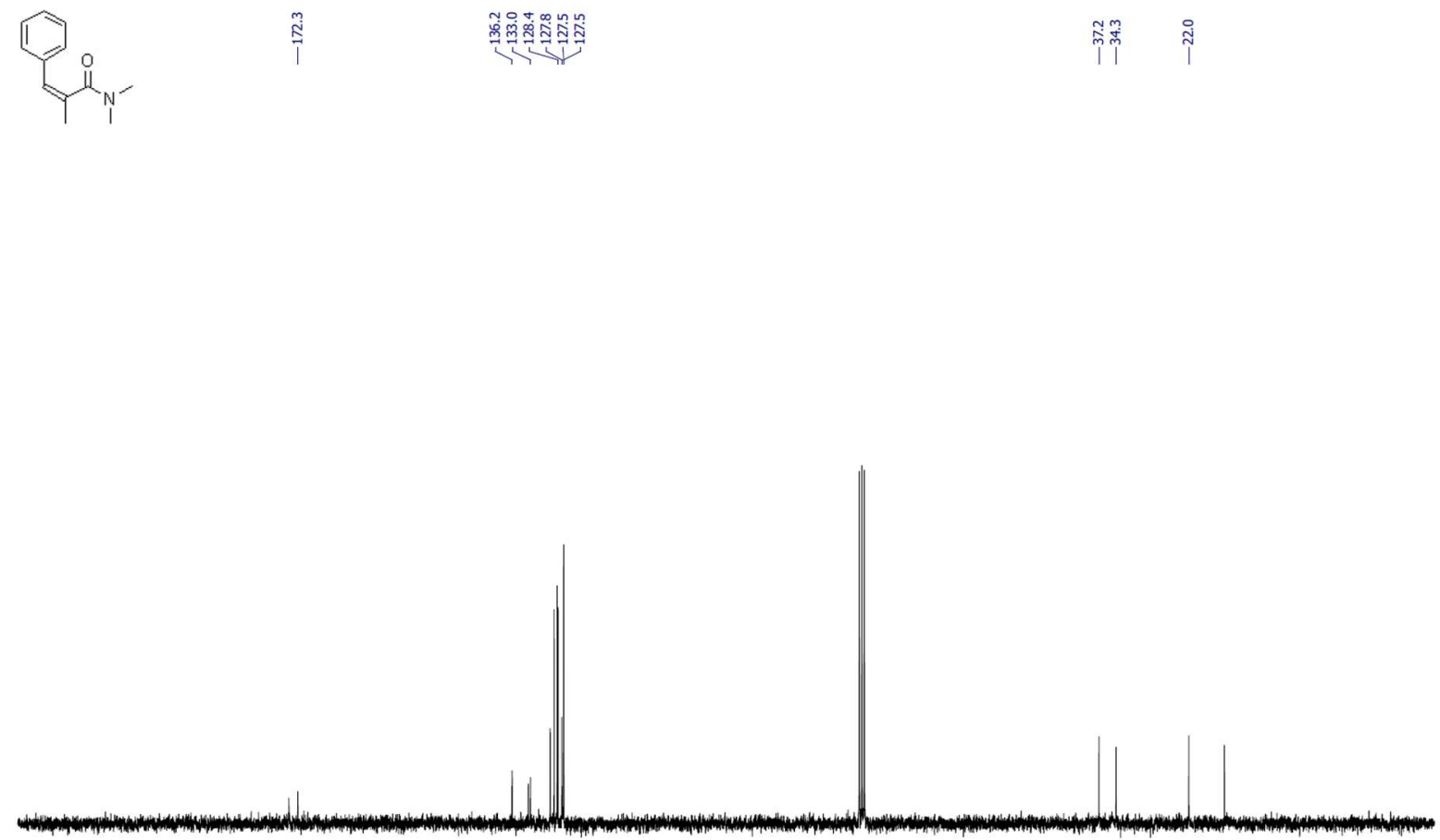

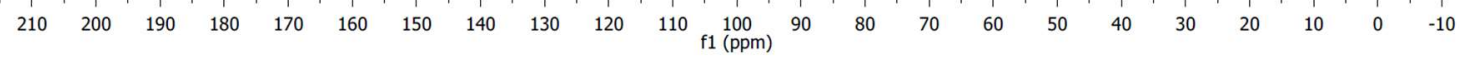


diethyl (Z)-(2-methyl-3-phenylacryloyl)phosphonate ((Z)-3):

${ }^{1} \mathrm{H} \mathrm{NMR}\left(\mathrm{CDCl}_{3}, 300 \mathrm{MHz}\right)$
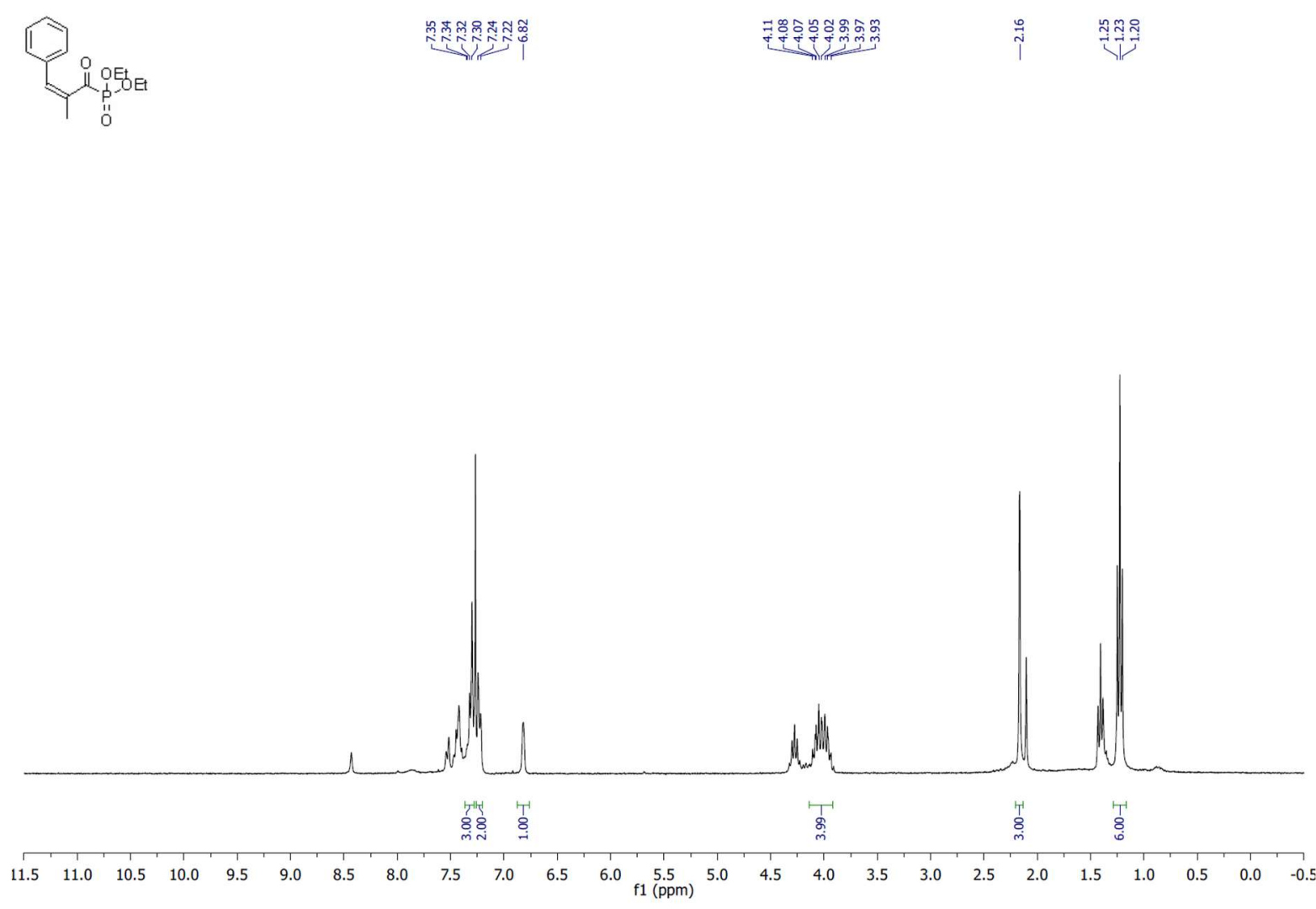

${ }^{13} \mathrm{C} \mathrm{NMR}\left(\mathrm{CDCl}_{3}, 75 \mathrm{MHz}\right)$
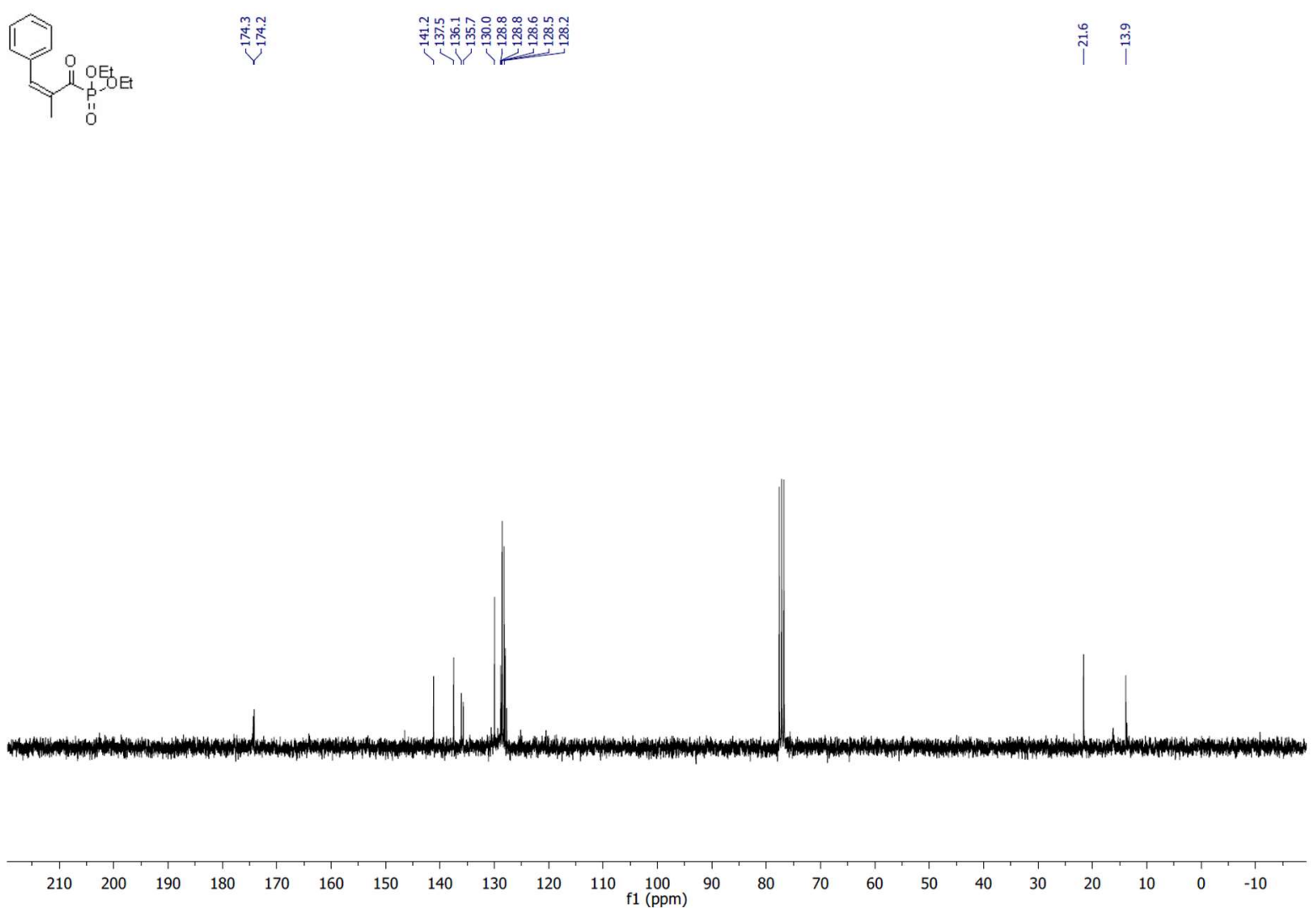
${ }^{31} \mathrm{P} \mathrm{NMR}\left(\mathrm{CDCl}_{3}, 121 \mathrm{MHz}\right)$<smiles></smiles>

\section{(Z)-3-(2-methyl-3-phenylacryloyl)oxazolidin-2-one ((Z)-4):}

${ }^{1} \mathrm{H} \mathrm{NMR}\left(\mathrm{CDCl}_{3}, 300 \mathrm{MHz}\right)$
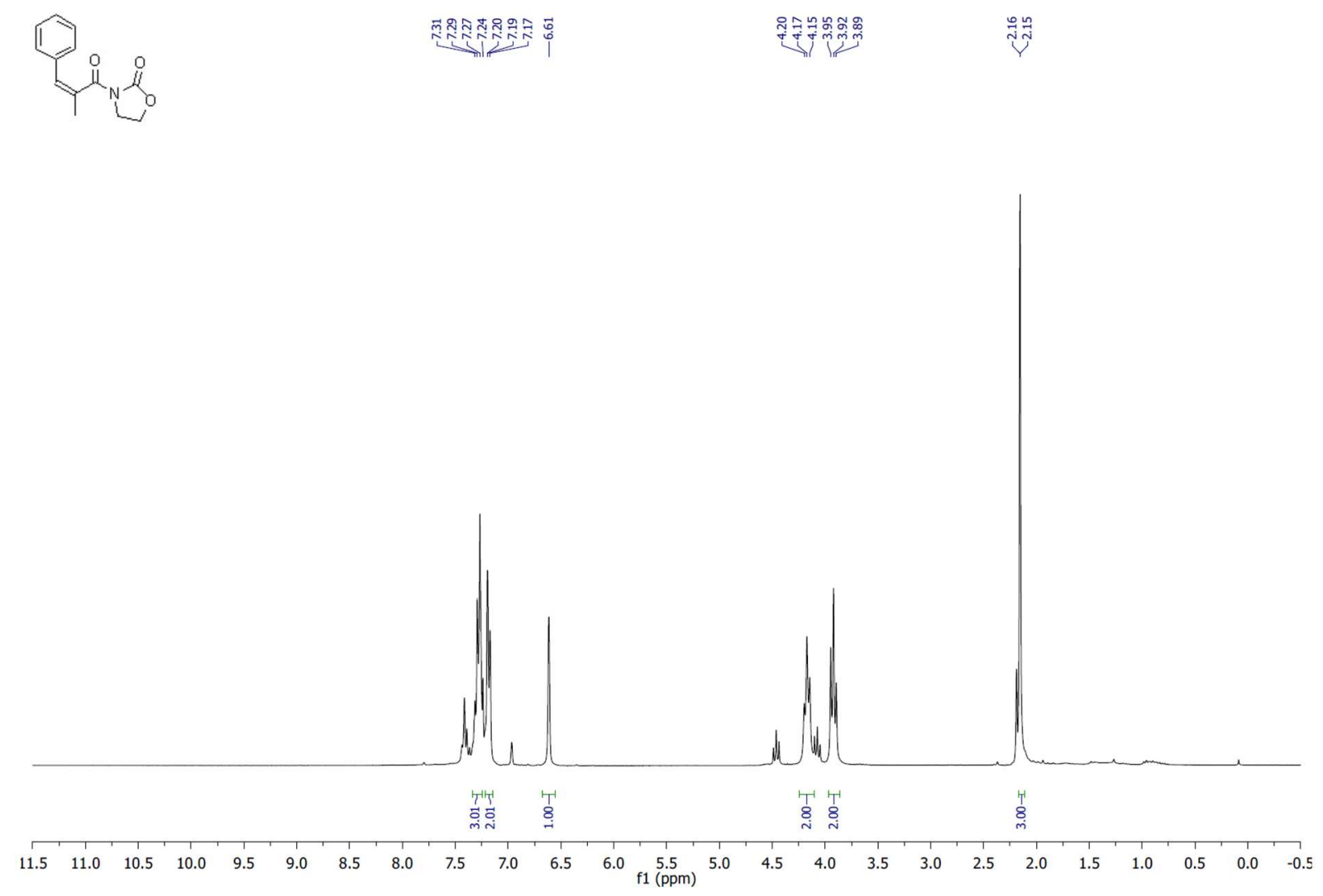
${ }^{13} \mathrm{C} \mathrm{NMR}\left(\mathrm{CDCl}_{3}, 75 \mathrm{MHz}\right)$
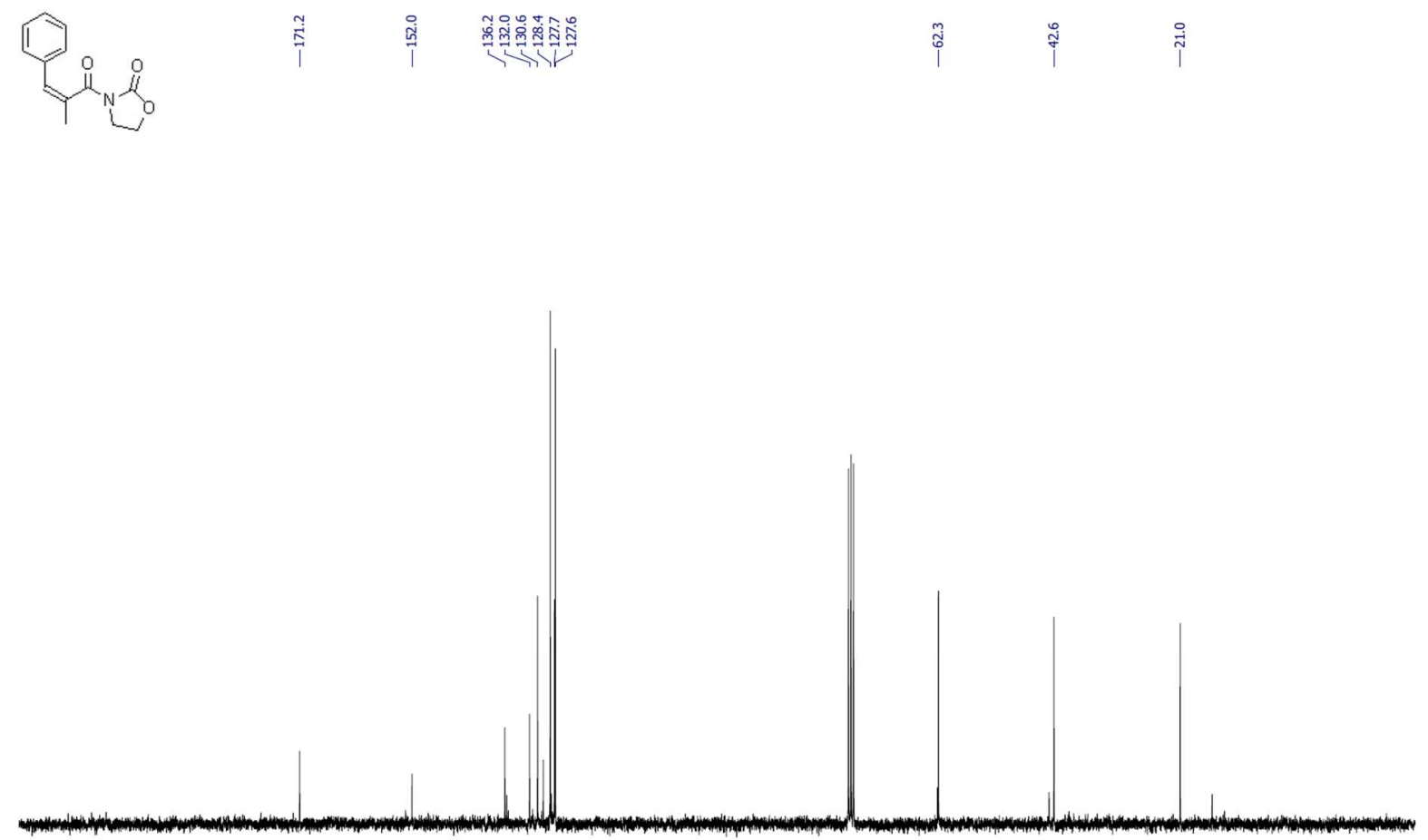

$\begin{array}{llllllllllllllllllllllllll}210 & 200 & 190 & 180 & 170 & 160 & 150 & 140 & 130 & 120 & 110 & \begin{array}{c}100 \\ \mathrm{f} 1(\mathrm{ppm})\end{array} & 90 & 80 & 70 & 60 & 50 & 40 & 30 & 20 & 10 & 0 & -10\end{array}$

(Z)-N,2-dimethyl-3-phenyl-N-(pyridin-2-yl)acrylamide ((Z)-5):

${ }^{1} \mathrm{H} \mathrm{NMR}\left(\mathrm{CDCl}_{3}, 300 \mathrm{MHz}\right)$
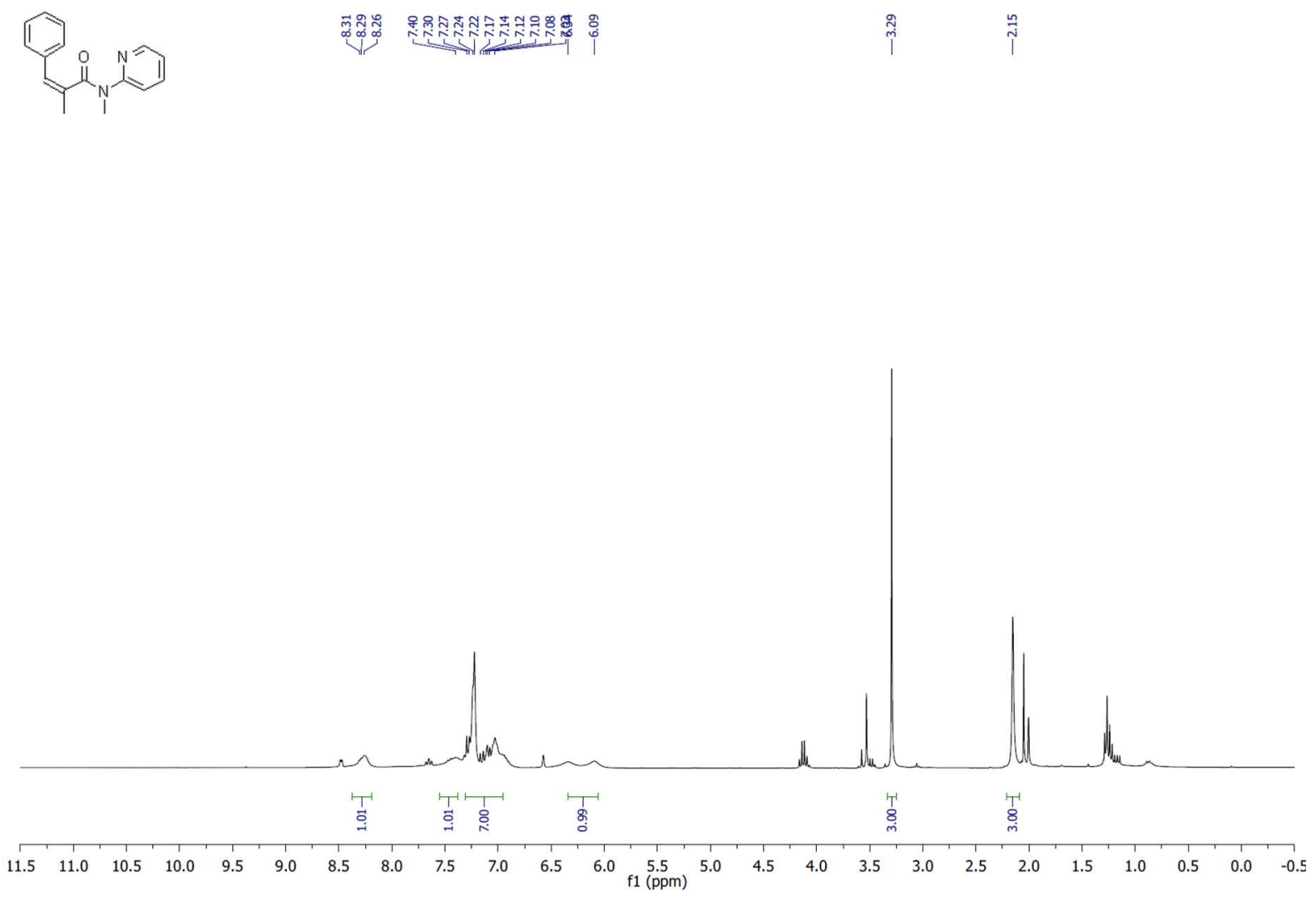
${ }^{13} \mathrm{C} \mathrm{NMR}\left(\mathrm{CDCl}_{3}, 75 \mathrm{MHz}\right)$
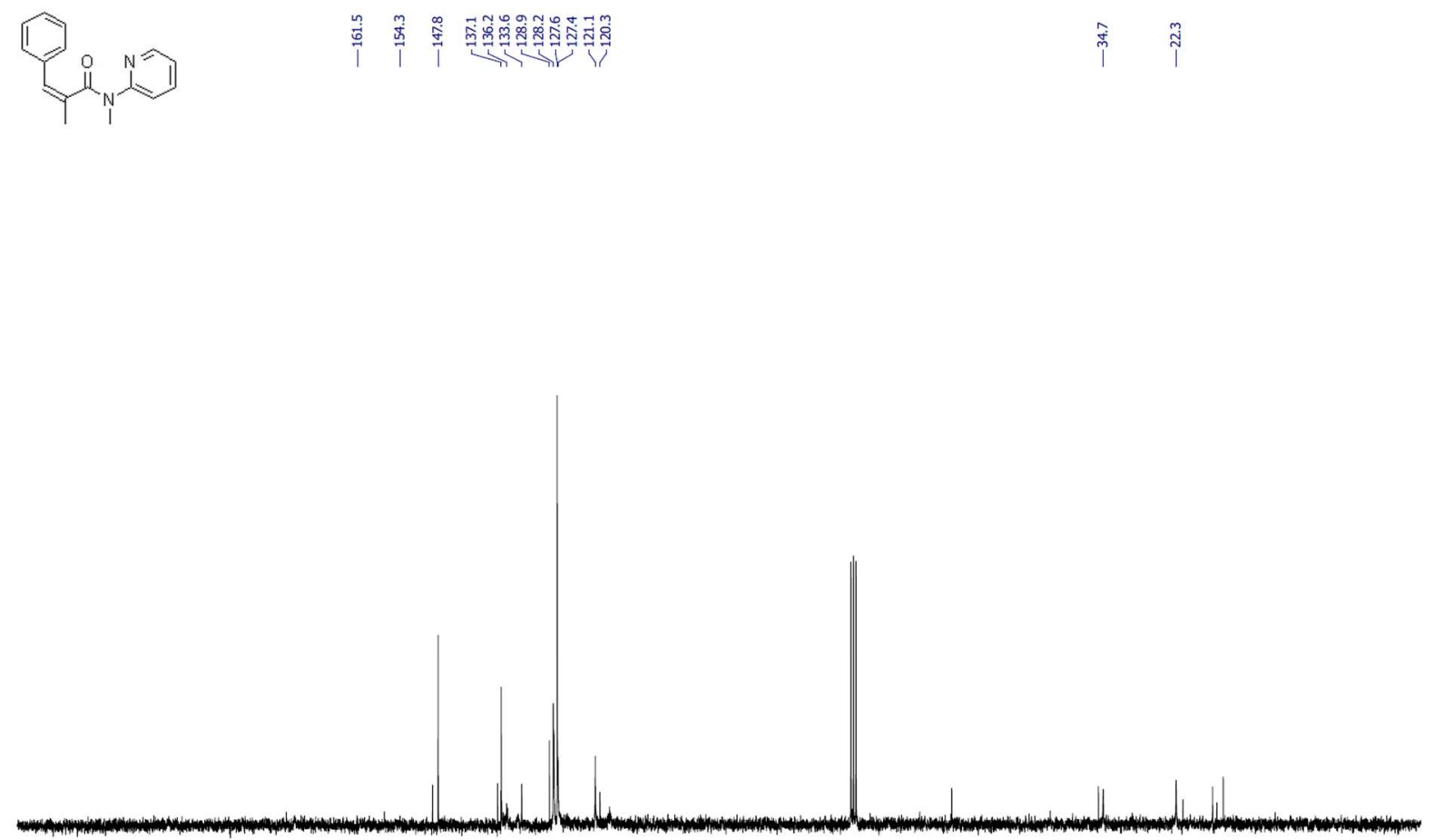

$\begin{array}{lllllllllll}210 & 200 & 190 & 180 & 170 & 160 & 150 & 140 & 130 & 120 & 110 \begin{array}{c}100 \\ \mathrm{f} 1(\mathrm{ppm})\end{array}\end{array}$

(Z)-1-(2,3-dihydro-1H-pyrrolo[2,3-b]pyridin-1-yl)-2-methyl-3-phenylprop-2-en-1-one ((Z)-6):

${ }^{1} \mathrm{H}$ NMR (benzene-d6, $300 \mathrm{MHz}$ )

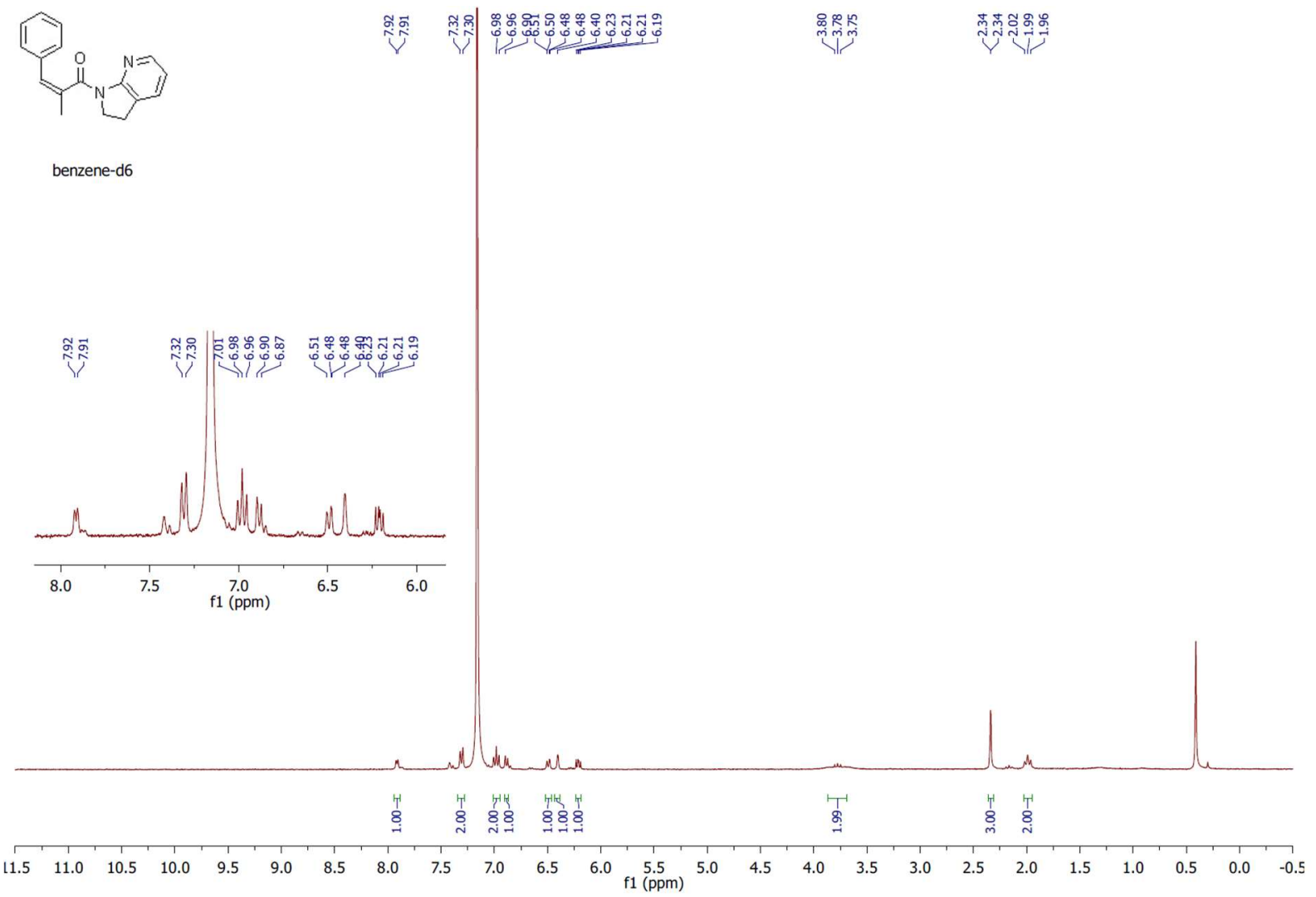


${ }^{13} \mathrm{C} \mathrm{NMR}\left(\mathrm{CDCl}_{3}, 75 \mathrm{MHz}\right)$
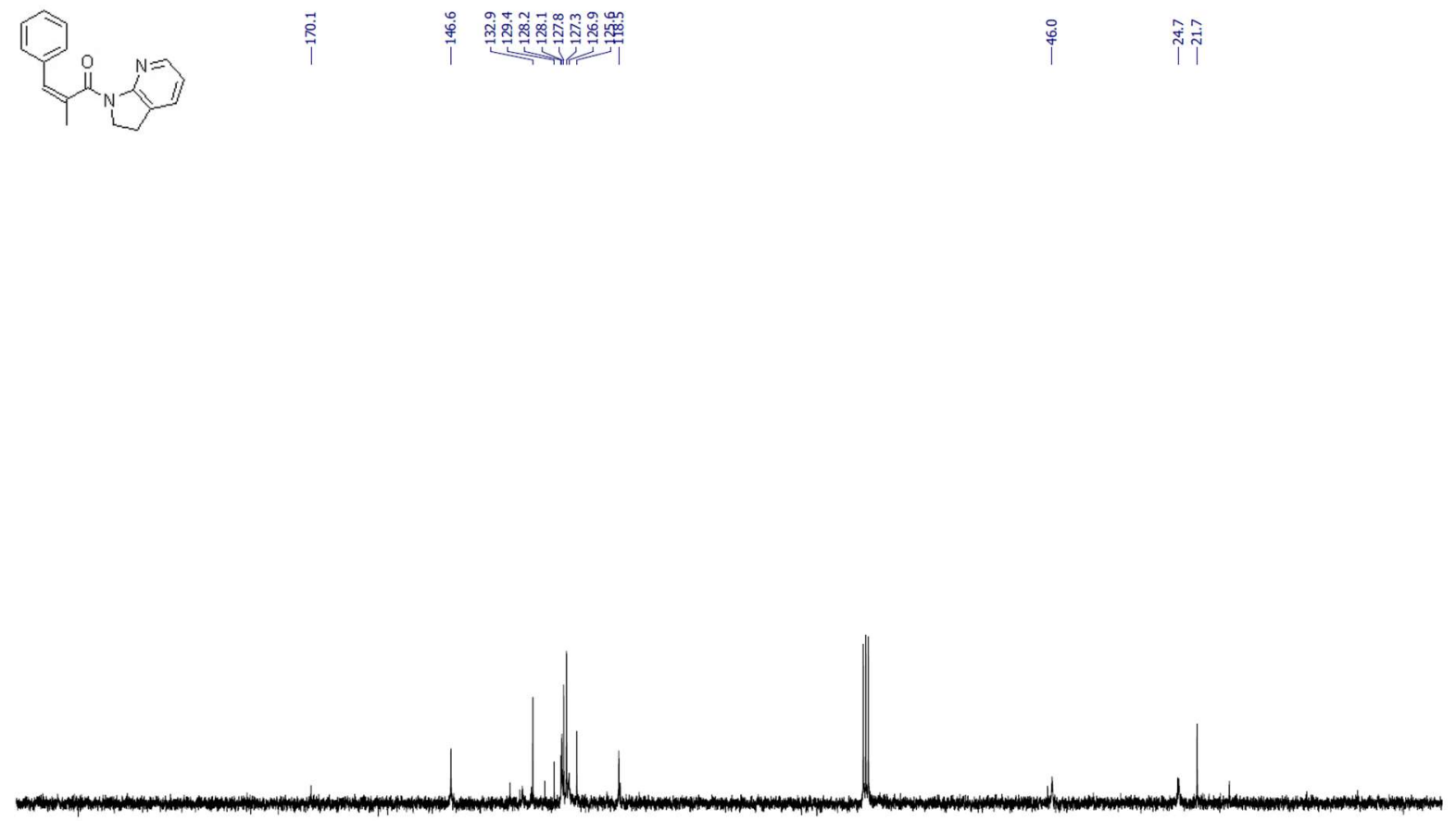

$\begin{array}{lllllllllllllllllllllll}210 & 200 & 190 & 180 & 170 & 160 & 150 & 140 & 130 & 120 & 110 & \begin{array}{c}100 \\ \mathrm{f} 1(\mathrm{ppm})\end{array} & 90 & 80 & 70 & 60 & 50 & 40 & 30 & 20 & 10 & 0 & -10\end{array}$

(Z)-2-methyl-3-phenyl-1-(1H-pyrazol-1-yl)prop-2-en-1-one ((Z)-7):

${ }^{1} \mathrm{H} \mathrm{NMR}\left(\mathrm{CDCl}_{3}, 300 \mathrm{MHz}\right)$
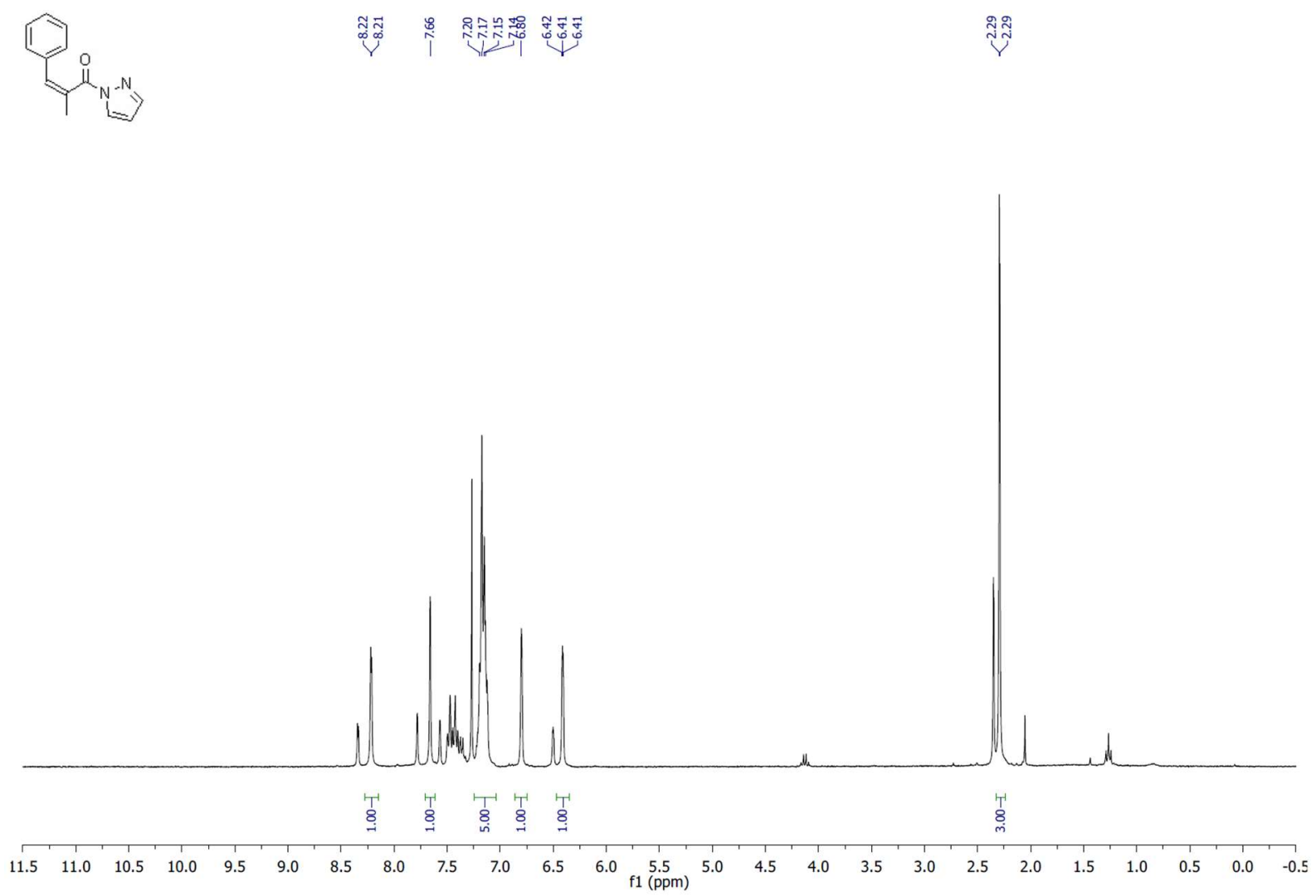
${ }^{13} \mathrm{C} \mathrm{NMR}\left(\mathrm{CDCl}_{3}, 75 \mathrm{MHz}\right)$
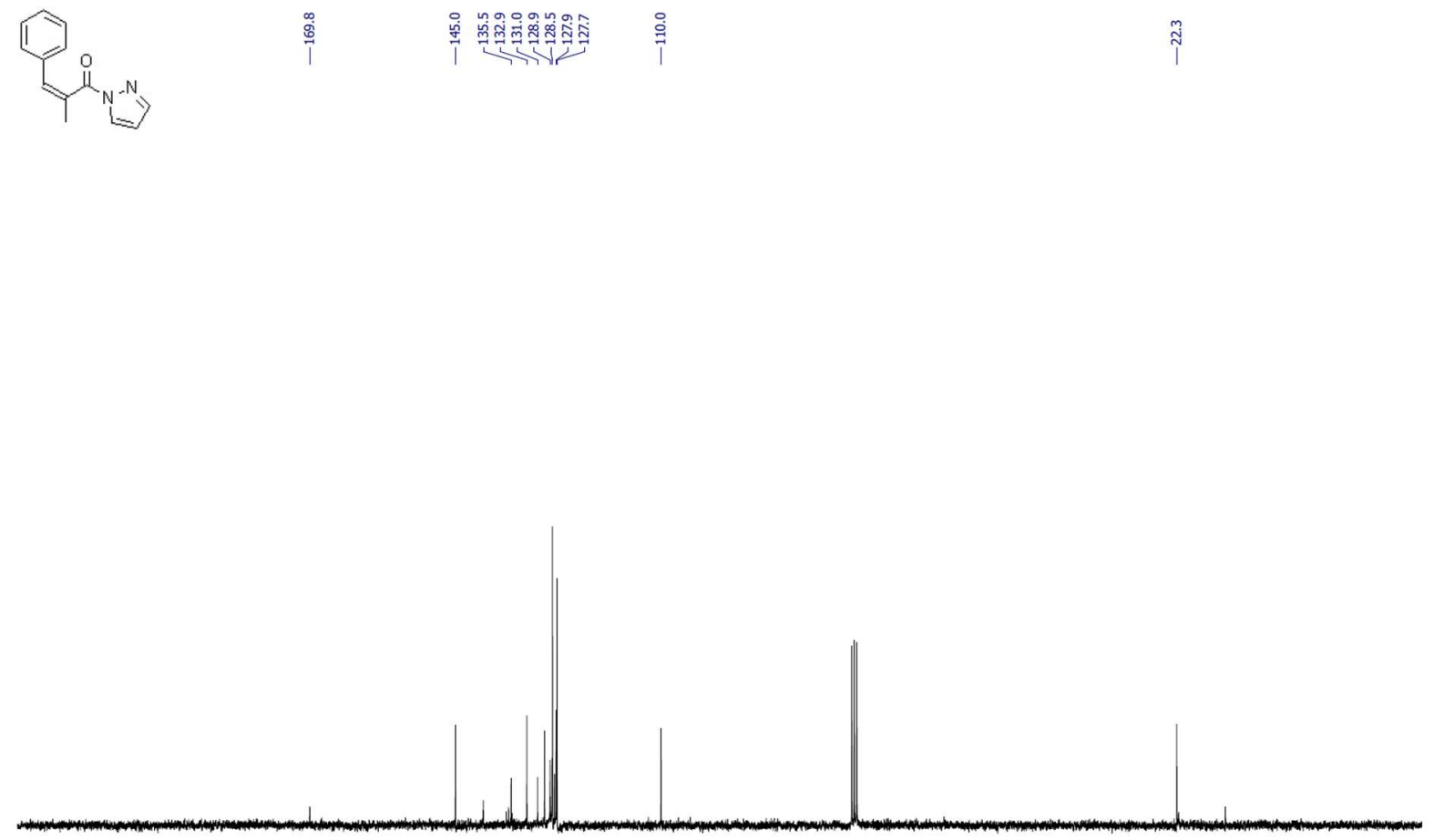

$\begin{array}{llllllllllllllllllllllll}210 & 200 & 190 & 180 & 170 & 160 & 150 & 140 & 130 & 120 & 110 & \begin{array}{c}100 \\ \mathrm{f} 1(\mathrm{ppm})\end{array} & 90 & 80 & 70 & 60 & 50 & 40 & 30 & 20 & 10 & 0 & -10\end{array}$

\section{(Z)-3-(2-methyl-3-phenylacryloyl)benzo[d]oxazol-2(3H)-one (T1):}

${ }^{1} \mathrm{H} \mathrm{NMR}\left(\mathrm{CDCl}_{3}, 300 \mathrm{MHz}\right)$
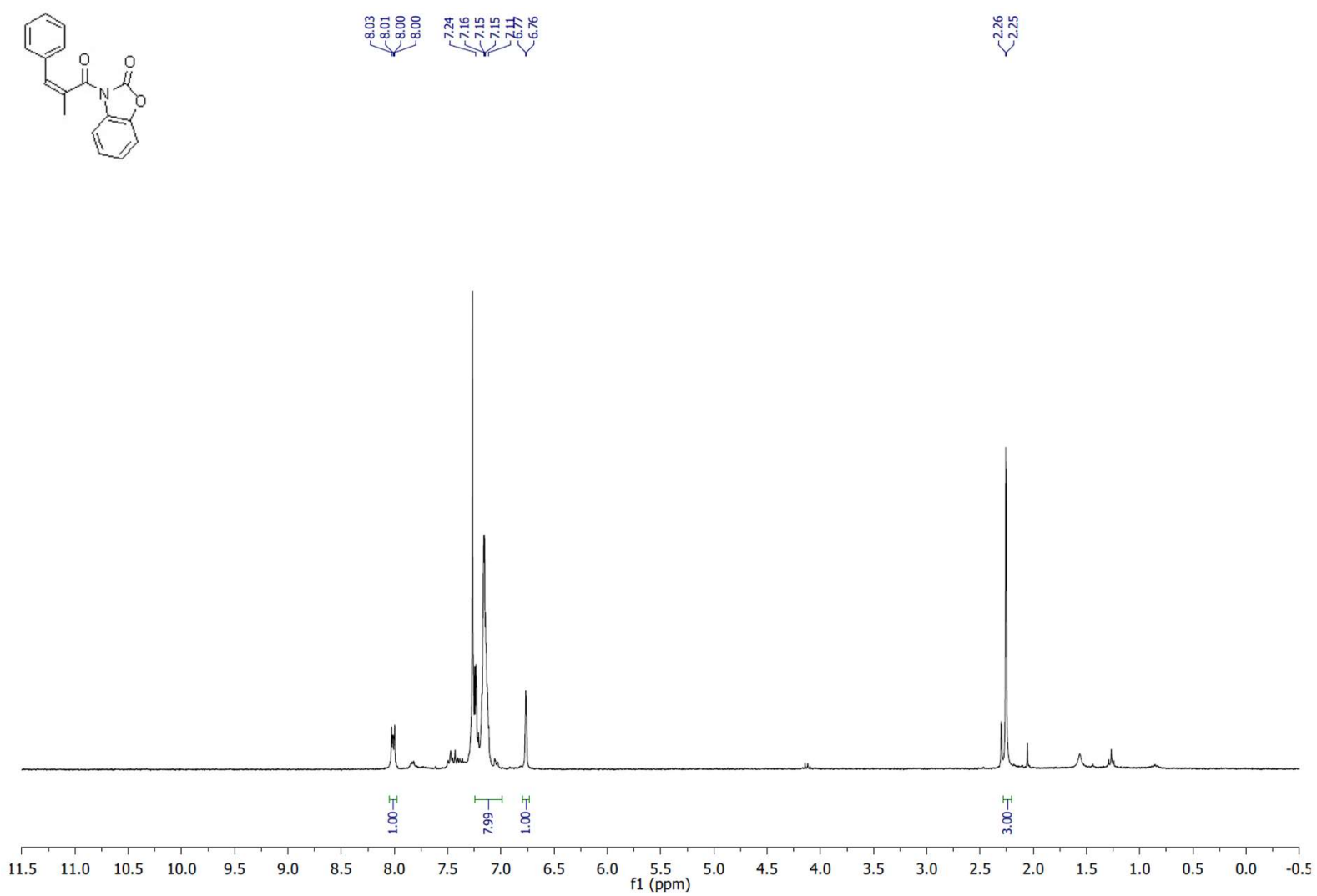
${ }^{13} \mathrm{C} \mathrm{NMR}\left(\mathrm{CDCl}_{3}, 75 \mathrm{MHz}\right)$

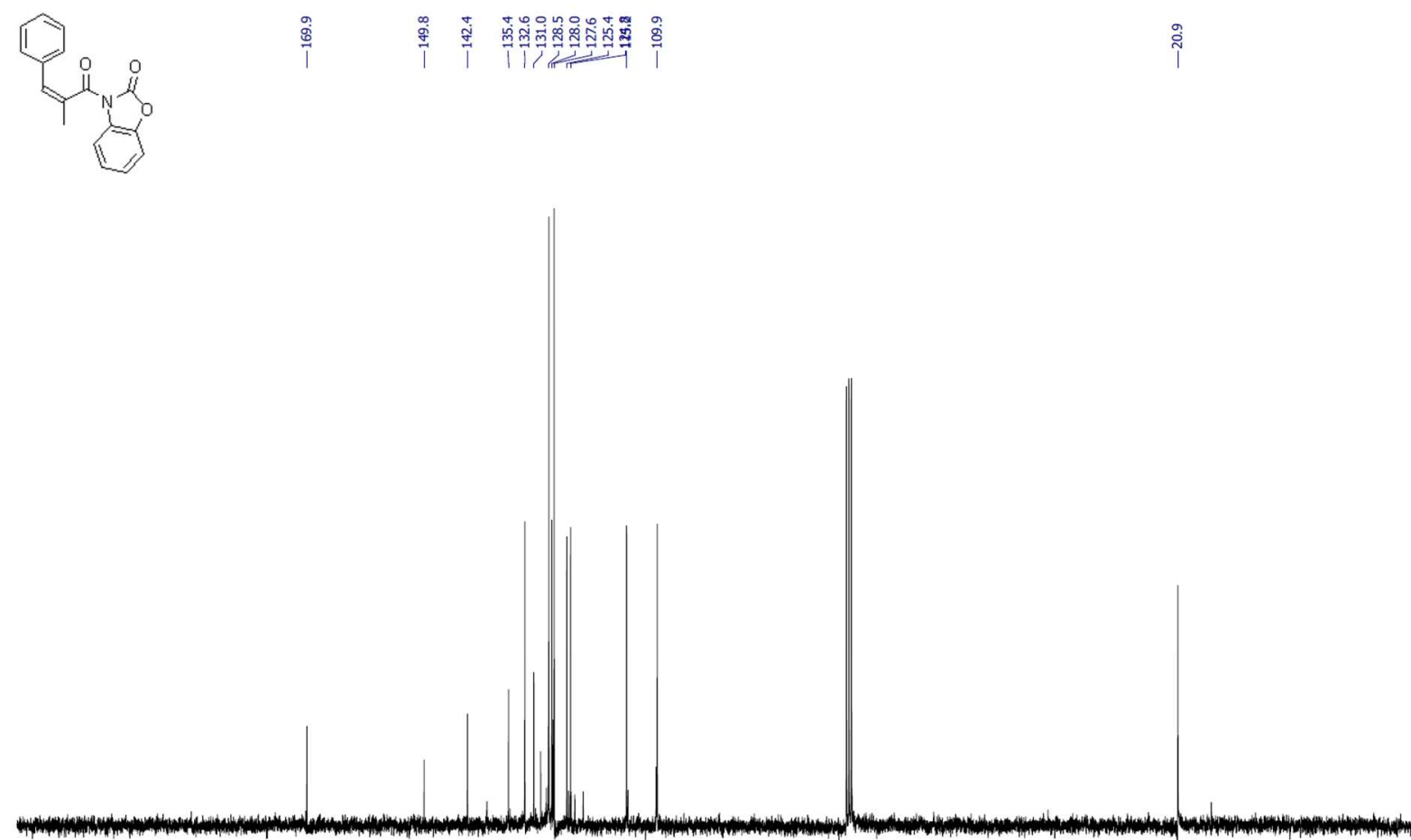

$\begin{array}{llllllllllllllllllllll}210 & 200 & 190 & 180 & 170 & 160 & 150 & 140 & 130 & 120 & 110 \underset{\mathrm{f} 1}{100}(\mathrm{ppm}) & 90 & 80 & 70 & 60 & 50 & 40 & 30 & 20 & 10 & 0 & -10\end{array}$

(Z)-1-(2-methyl-3-phenylacryloyl)pyrrolidin-2-one (T2):

${ }^{1} \mathrm{H} \mathrm{NMR}\left(\mathrm{CDCl}_{3}, 300 \mathrm{MHz}\right)$
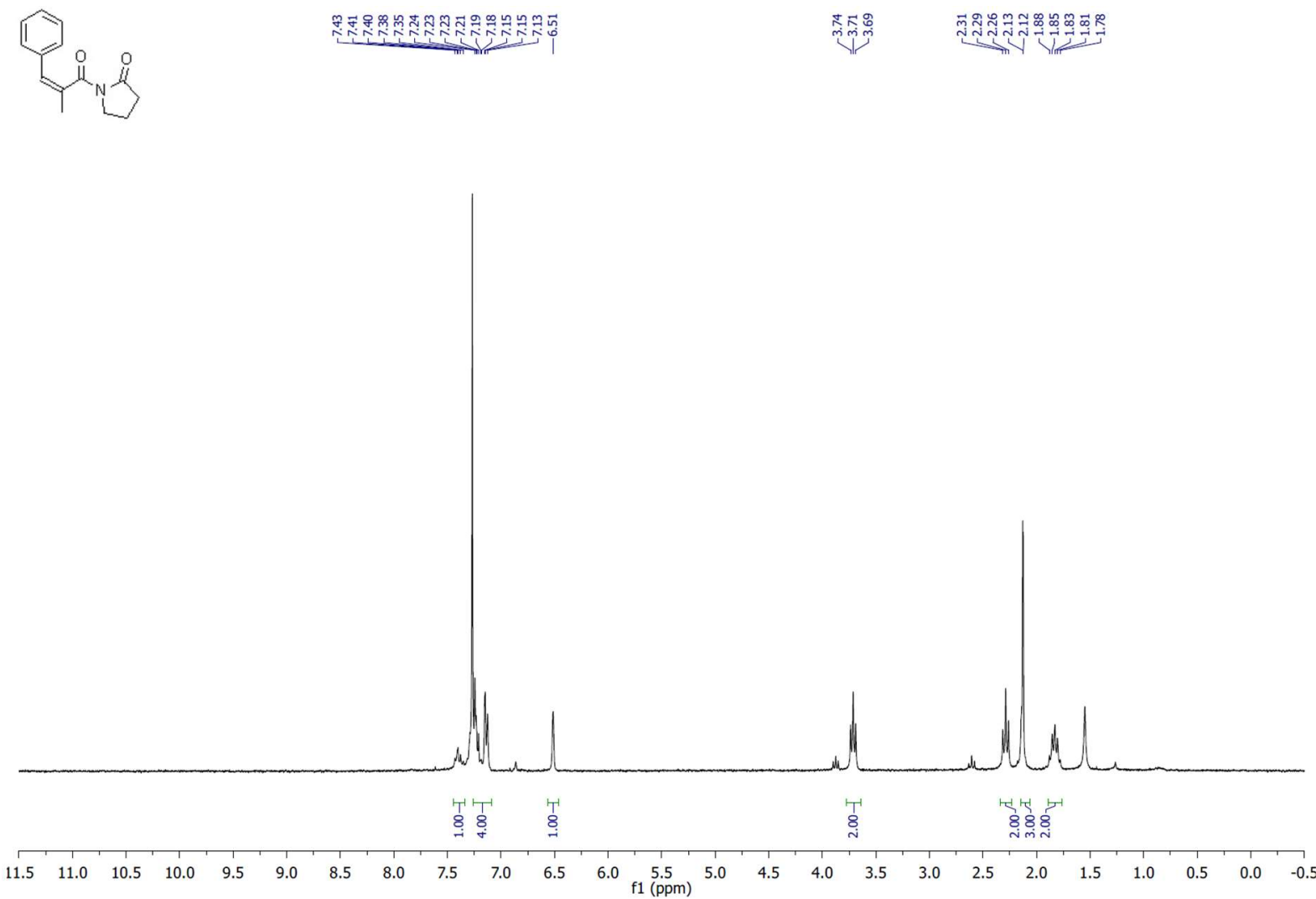
${ }^{13} \mathrm{C} \mathrm{NMR}\left(\mathrm{CDCl}_{3}, 75 \mathrm{MHz}\right)$
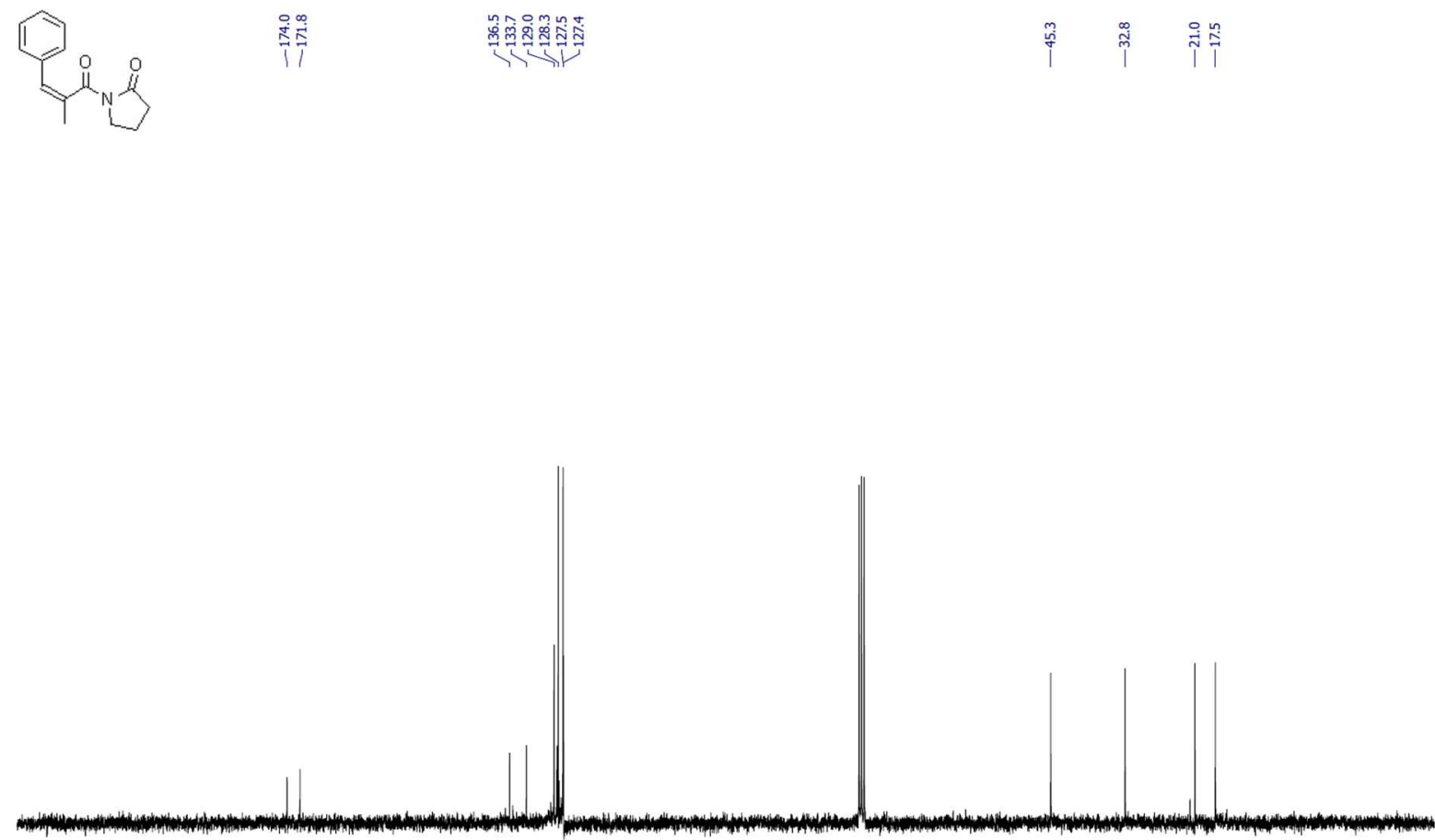

$\begin{array}{lllllllllllllllllllllll}210 & 200 & 190 & 180 & 170 & 160 & 150 & 140 & 130 & 120 & 110 & \underset{\mathrm{f} 1}{100}(\mathrm{ppm}) & 90 & 80 & 70 & 60 & 50 & 40 & 30 & 20 & 10 & 0 & -10\end{array}$

(Z)-1-(2-methyl-3-phenylacryloyl)piperidin-2-one (T3):

${ }^{1} \mathrm{H} \mathrm{NMR}\left(\mathrm{CDCl}_{3}, 300 \mathrm{MHz}\right)$
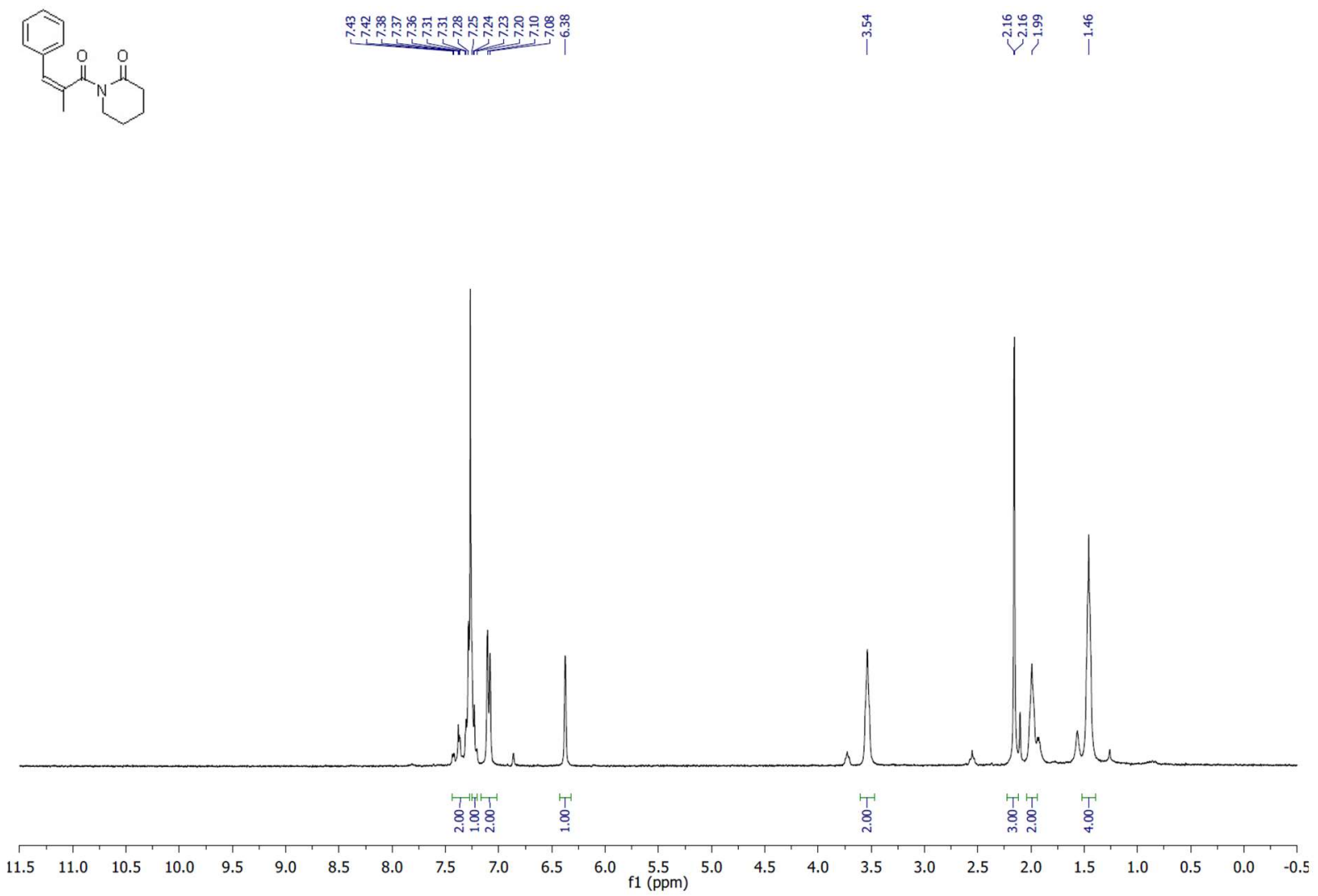
${ }^{13} \mathrm{C} \mathrm{NMR}\left(\mathrm{CDCl}_{3}, 75 \mathrm{MHz}\right)$
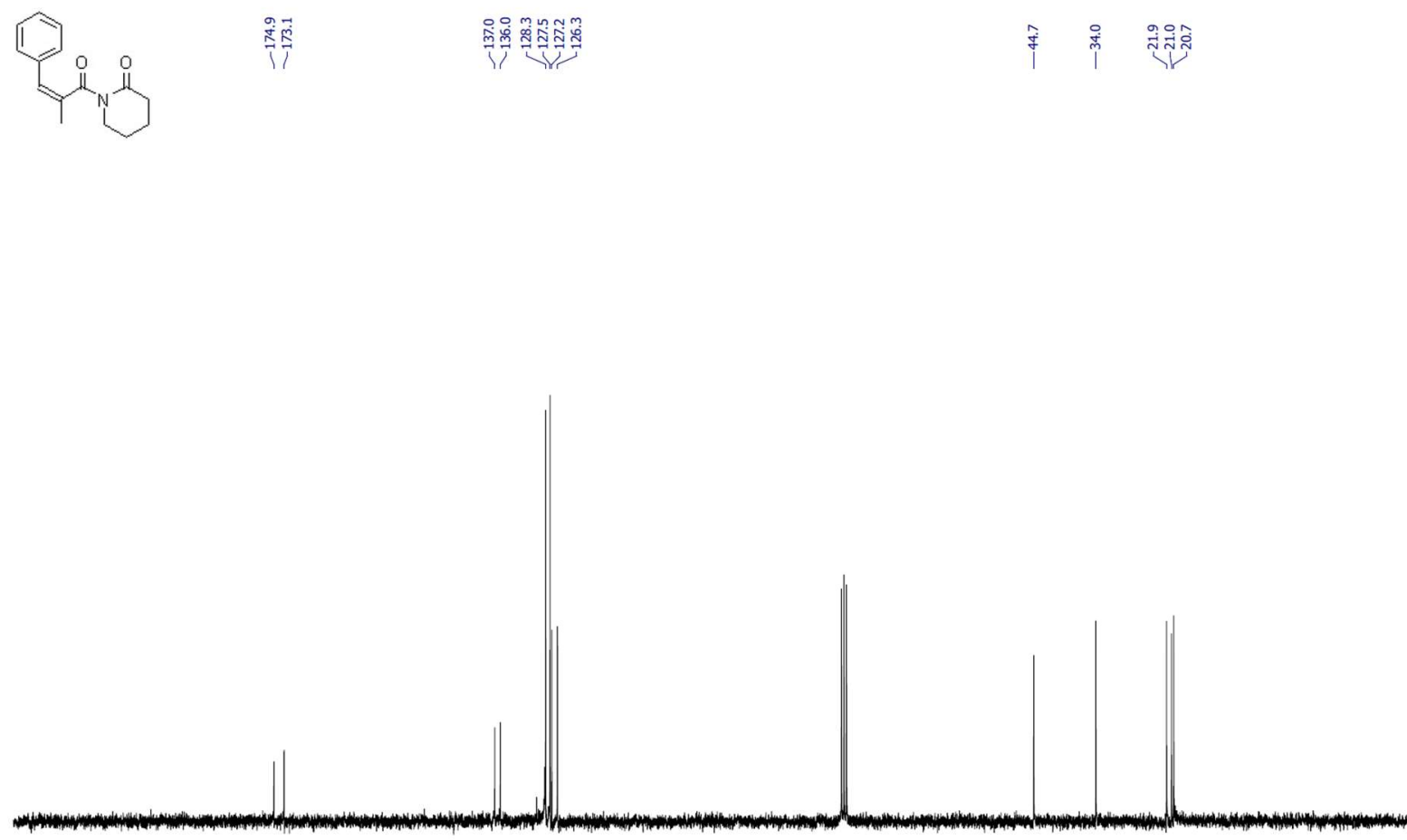

$\begin{array}{llllllllllllllllllllllll}210 & 200 & 190 & 180 & 170 & 160 & 150 & 140 & 130 & 120 & 110 & 100 & 90 & 80 & 70 & 60 & 50 & 40 & 30 & 20 & 10 & 0 & -10\end{array}$

(Z)-2-methyl-3-phenyl-1-(2-thioxooxazolidin-3-yl)prop-2-en-1-one (T4):

${ }^{1} \mathrm{H} \mathrm{NMR}\left(\mathrm{CDCl}_{3}, 300 \mathrm{MHz}\right)$
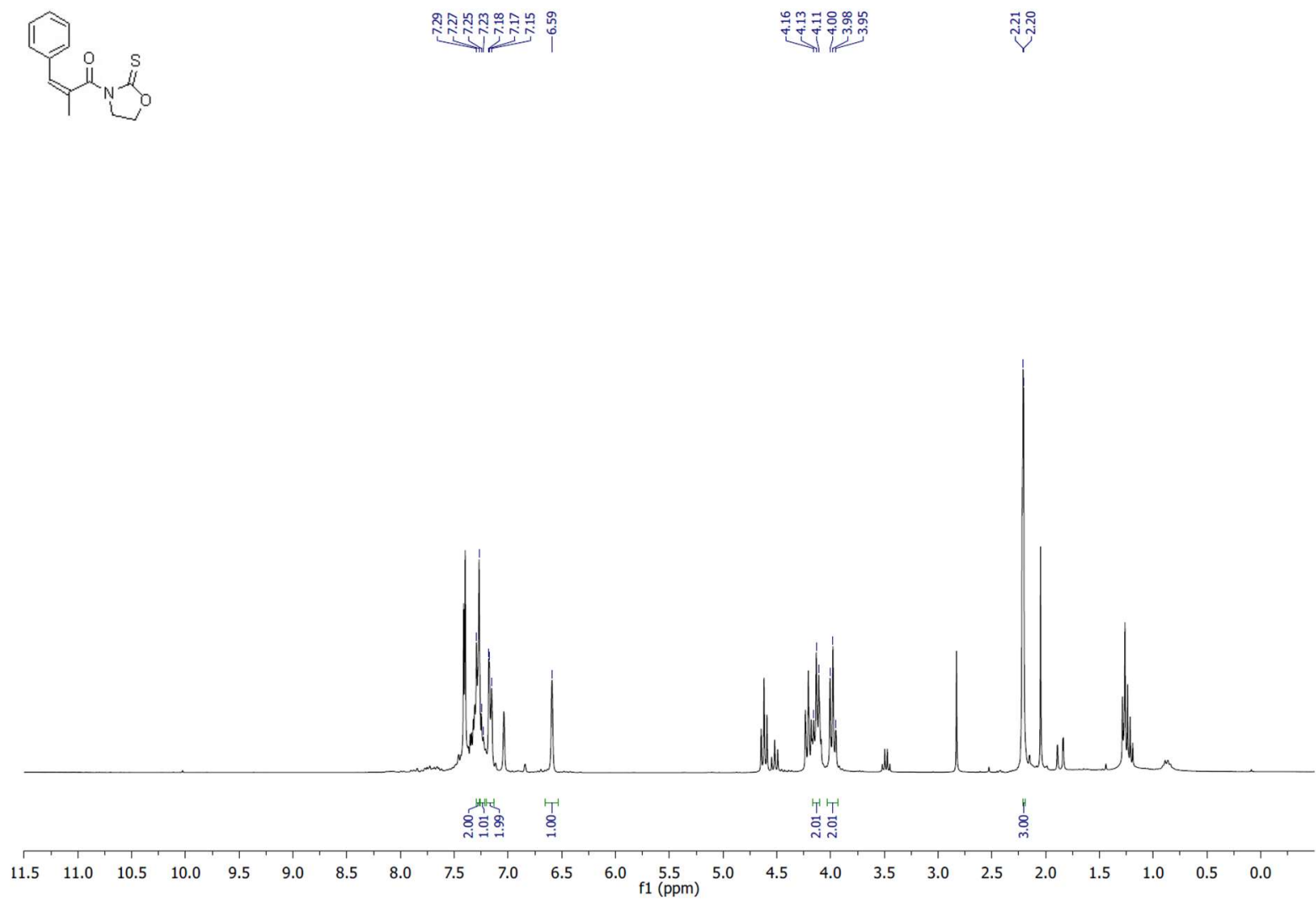
${ }^{13} \mathrm{C} \mathrm{NMR}\left(\mathrm{CDCl}_{3}, 75 \mathrm{MHz}\right)$
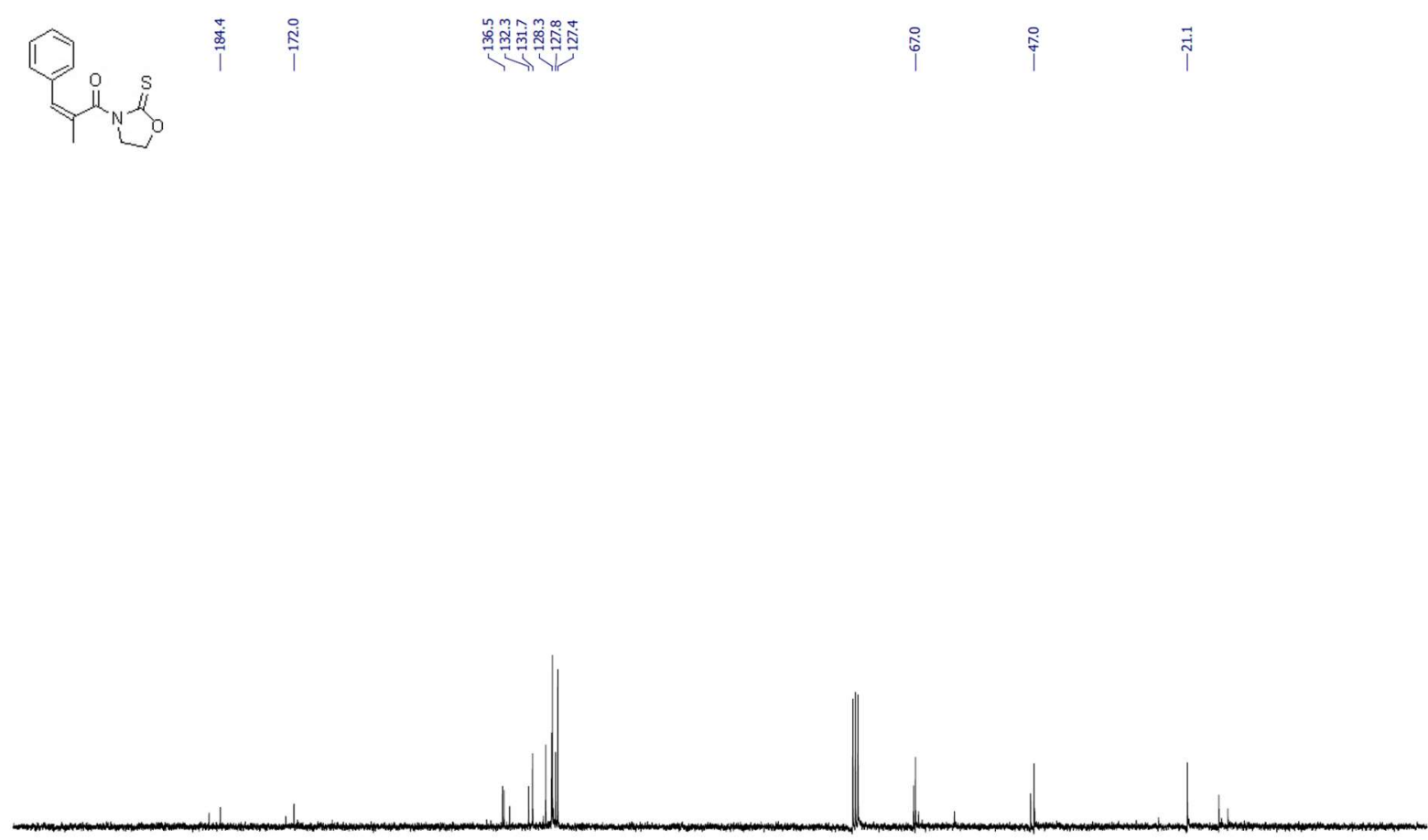

$\begin{array}{lllllllllllllllllllllll}210 & 200 & 190 & 180 & 170 & 160 & 150 & 140 & 130 & 120 & 110 & \begin{array}{c}100 \\ \mathrm{f} 1(\mathrm{ppm})\end{array} & 90 & 80 & 70 & 60 & 50 & 40 & 30 & 20 & 10 & 0 & -10\end{array}$

(Z)-2-(2-methyl-3-phenylacryloyl)-1-phenylpyrazolidin-3-one (T5):

${ }^{1} \mathrm{H} \mathrm{NMR}\left(\mathrm{CDCl}_{3}, 300 \mathrm{MHz}\right)$
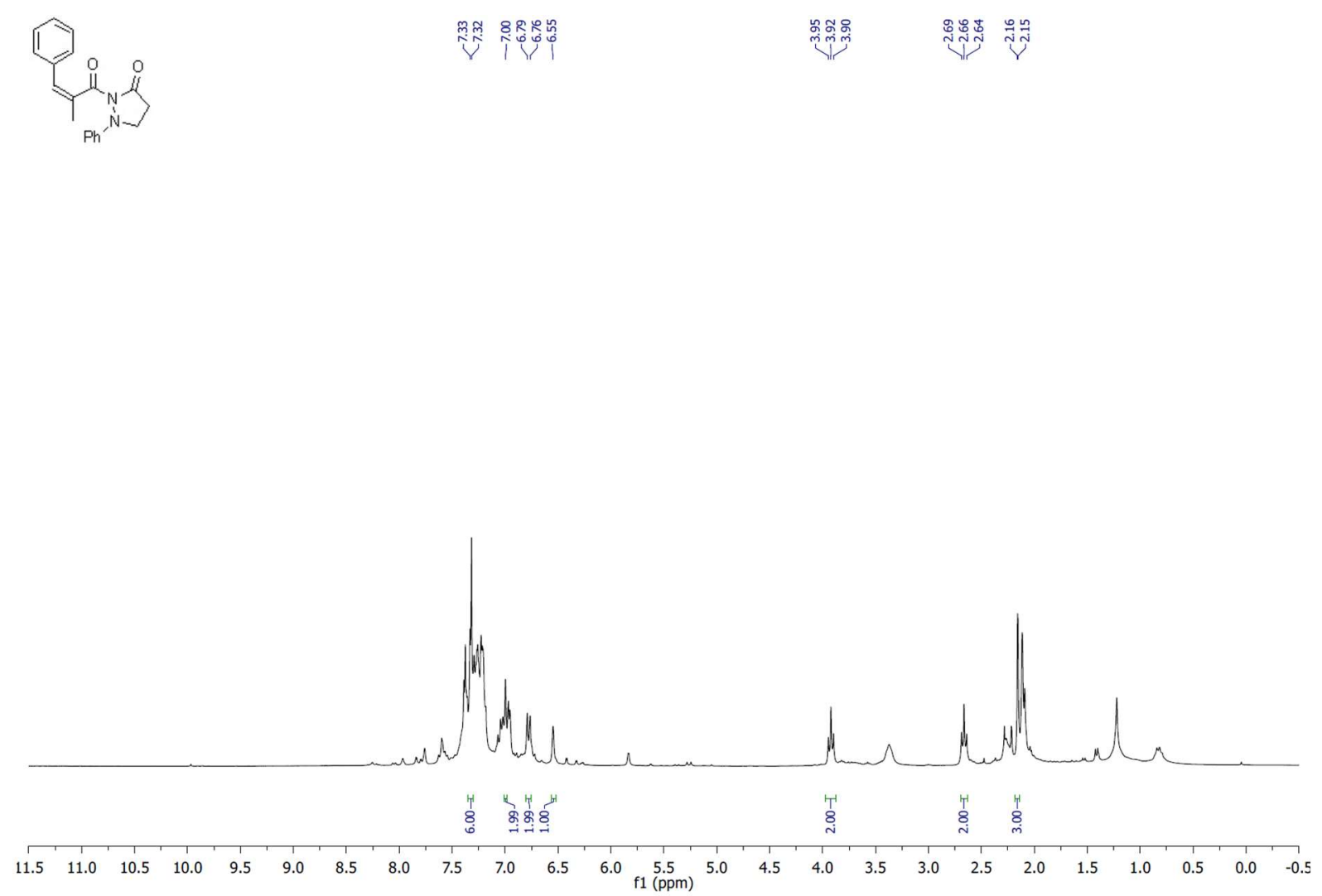
${ }^{13} \mathrm{C} \mathrm{NMR}\left(\mathrm{CDCl}_{3}, 75 \mathrm{MHz}\right)$
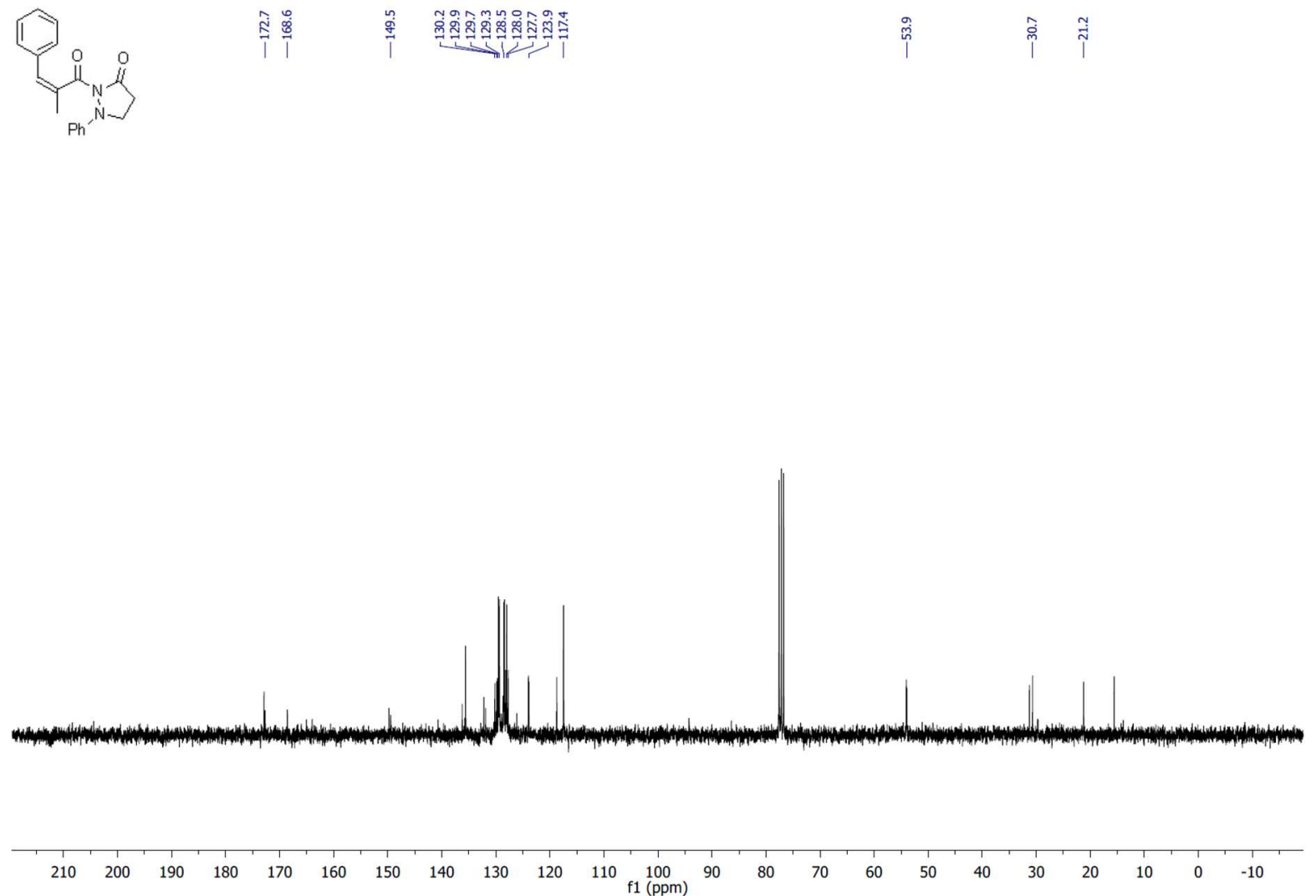

(Z)-3-(2-benzylidenebutanoyl)oxazolidin-2-one ((Z)-8):

${ }^{1} \mathrm{H} \mathrm{NMR}\left(\mathrm{CDCl}_{3}, 300 \mathrm{MHz}\right)$<smiles>C/C(=C/c1ccccc1)[C@H]1OCN1C</smiles>

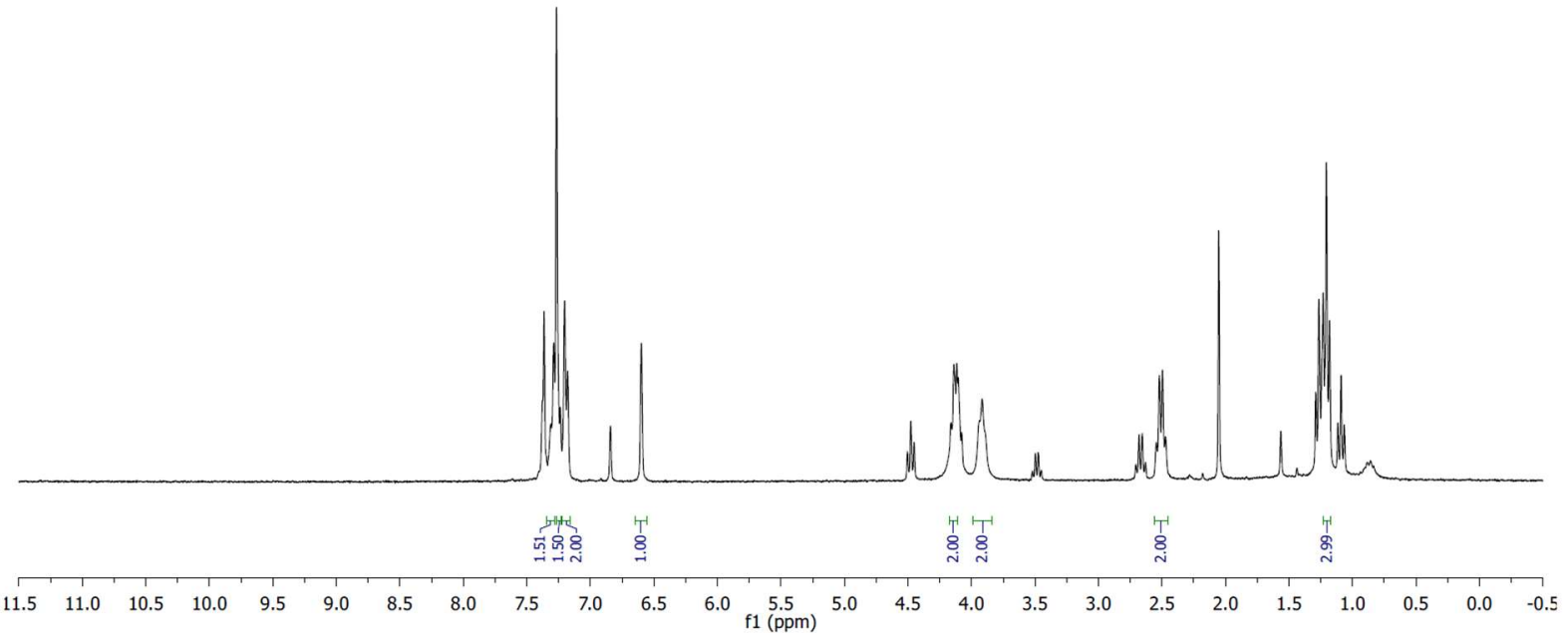


${ }^{13} \mathrm{C} \mathrm{NMR}\left(\mathrm{CDCl}_{3}, 75 \mathrm{MHz}\right)$
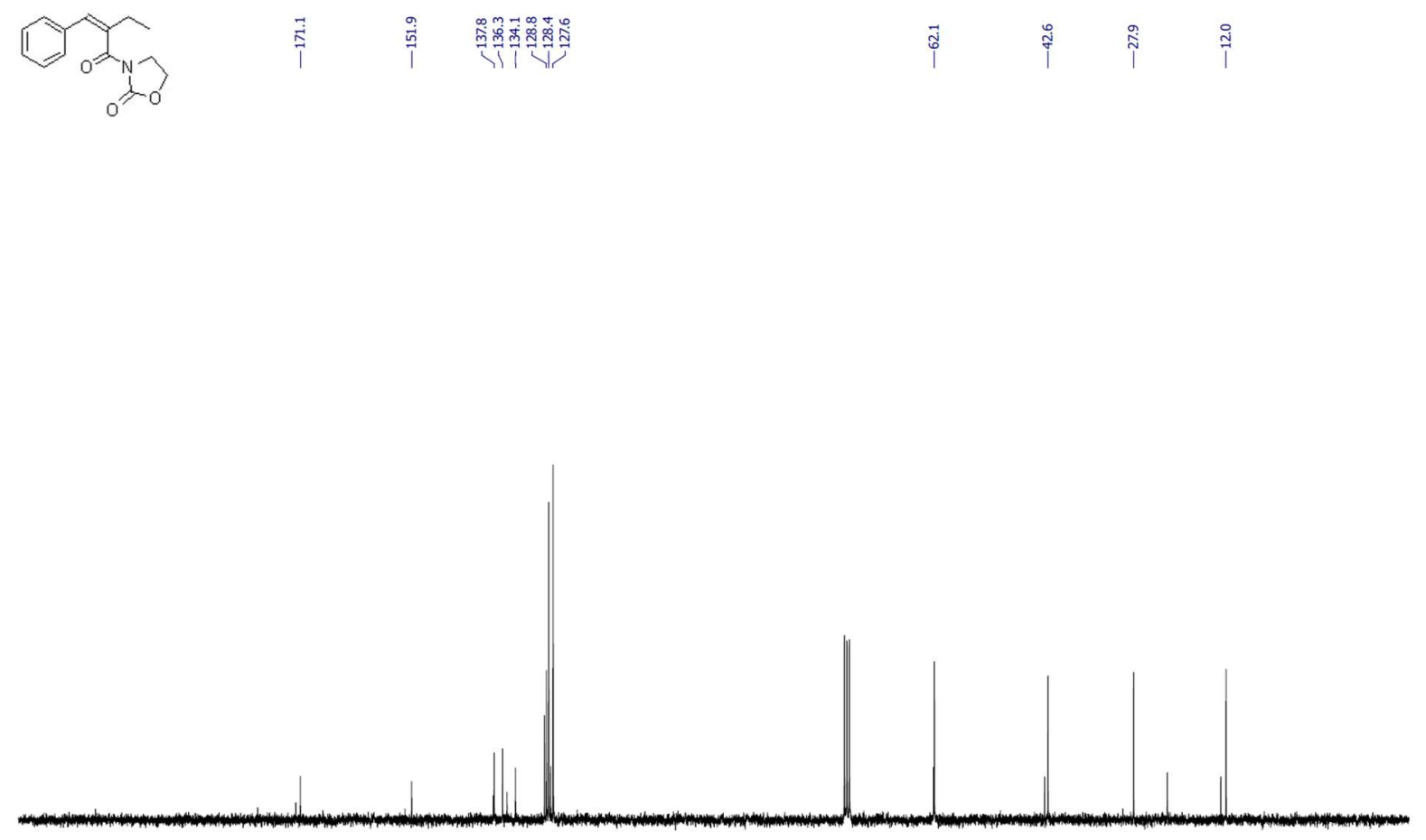

$\begin{array}{lllllllllllllllllllllll}210 & 200 & 190 & 180 & 170 & 160 & 150 & 140 & 130 & 120 & 110 & \begin{array}{c}100 \\ 100\end{array} & 90 & 80 & 70 & 60 & 50 & 40 & 30 & 20 & 10 & 0 & -10\end{array}$

(Z)-3-(2-methyl-3-(p-tolyl)acryloyl)oxazolidin-2-one ((Z)-9):

${ }^{1} \mathrm{H} \mathrm{NMR}\left(\mathrm{CDCl}_{3}, 300 \mathrm{MHz}\right)$
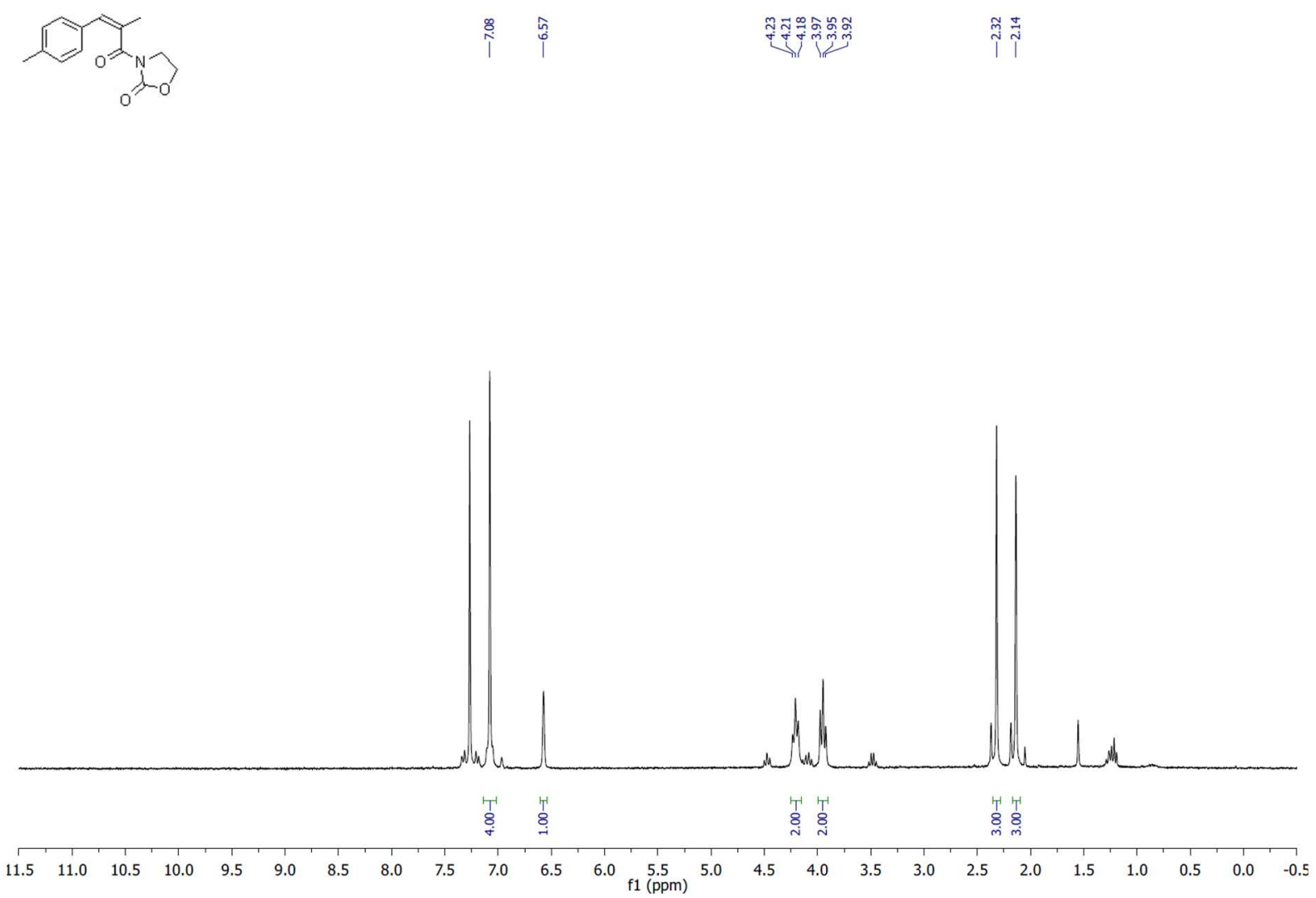
${ }^{13} \mathrm{C} \mathrm{NMR}\left(\mathrm{CDCl}_{3}, 75 \mathrm{MHz}\right)$
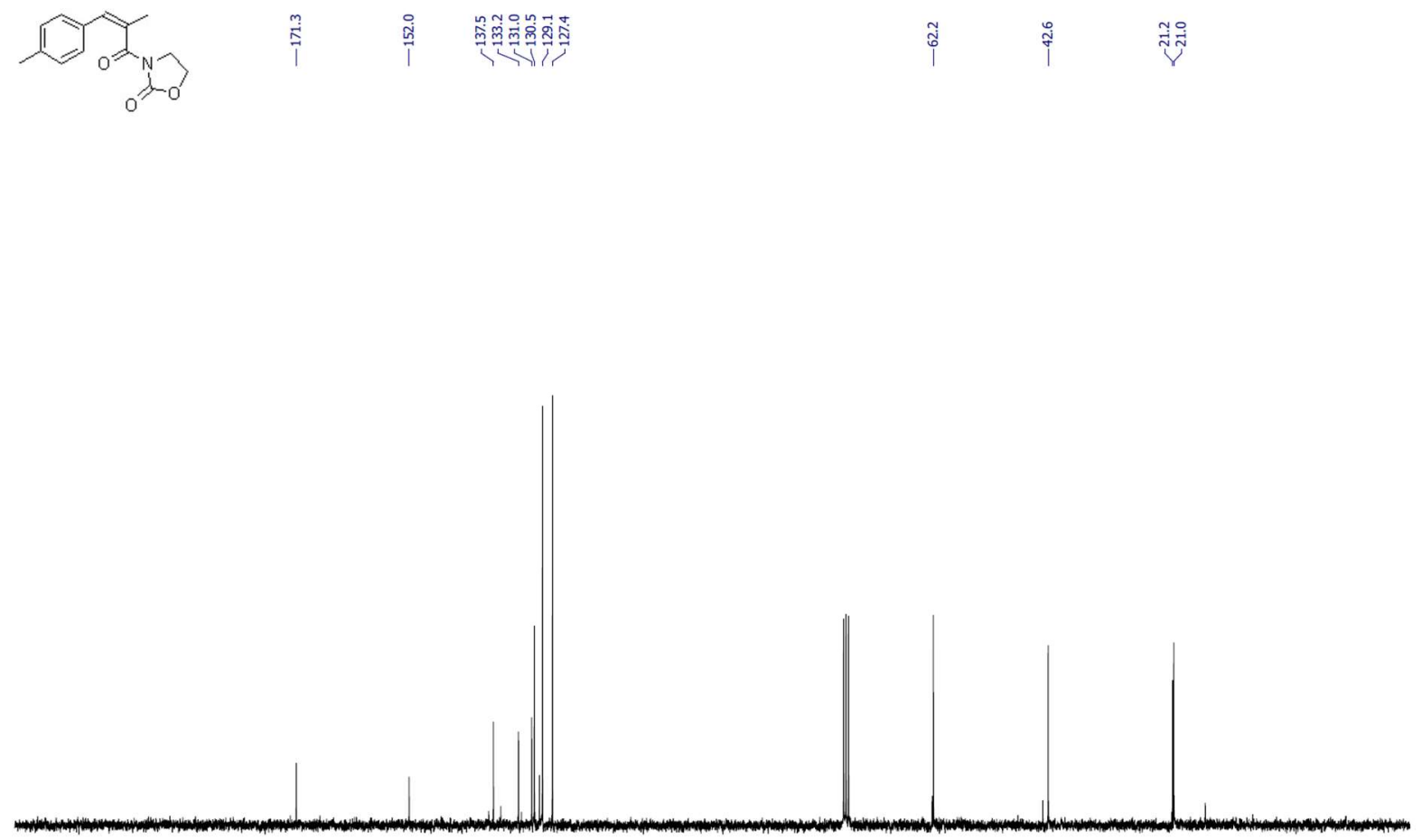

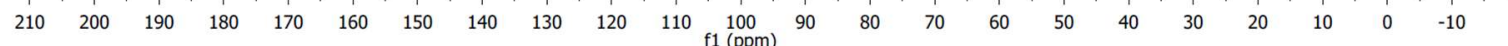

(Z)-3-(2-methyl-3-(m-tolyl)acryloyl)oxazolidin-2-one ((Z)-10):

${ }^{1} \mathrm{H} \mathrm{NMR}\left(\mathrm{CDCl}_{3}, 300 \mathrm{MHz}\right)$

$\mathbb{2} x_{0}>0$

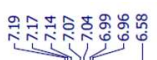

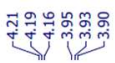

ํํำ

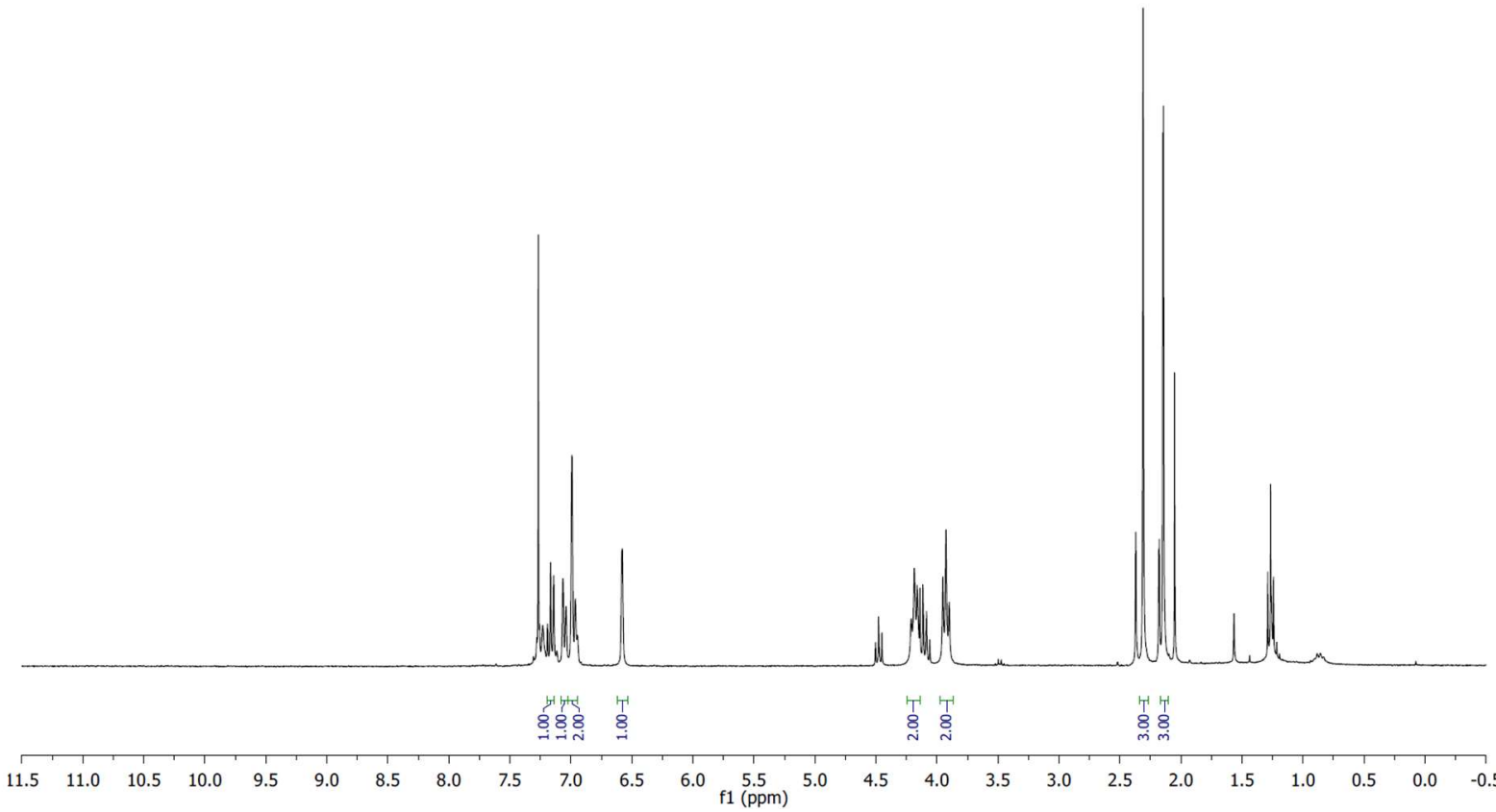


${ }^{13} \mathrm{C} \mathrm{NMR}\left(\mathrm{CDCl}_{3}, 75 \mathrm{MHz}\right)$

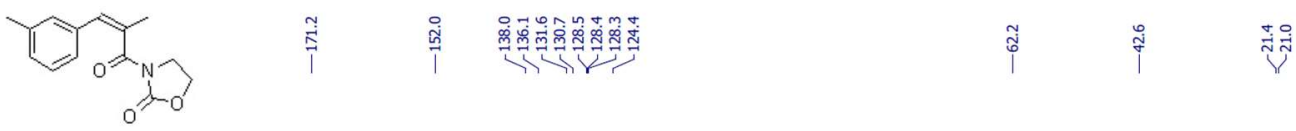

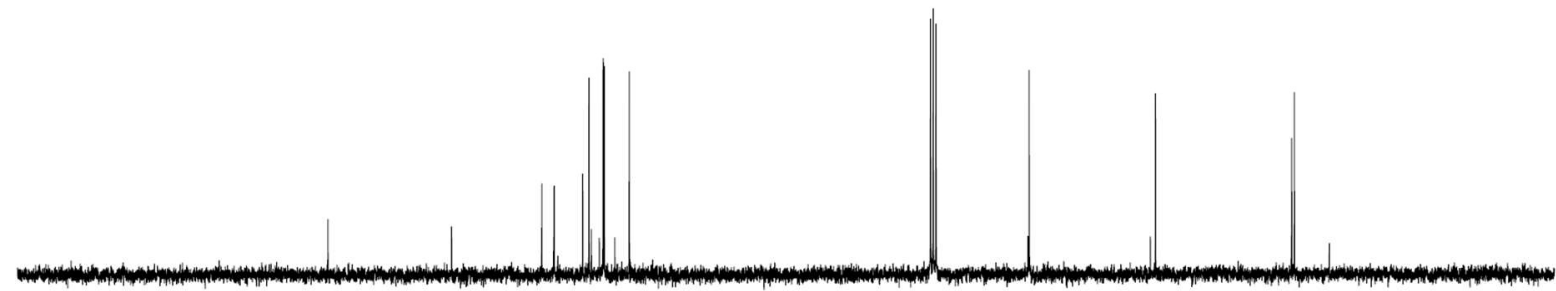

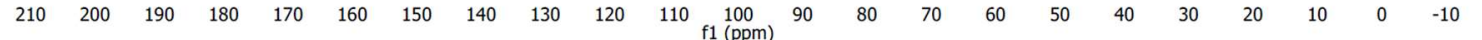

(Z)-3-(2-methyl-3-(o-tolyl)acryloyl)oxazolidin-2-one ((Z)-11):

${ }^{1} \mathrm{H} \mathrm{NMR}\left(\mathrm{CDCl}_{3}, 300 \mathrm{MHz}\right)$<smiles>Cc1cccc(C(C)C(=O)N2C3CCCC2C3)c1</smiles>

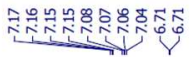

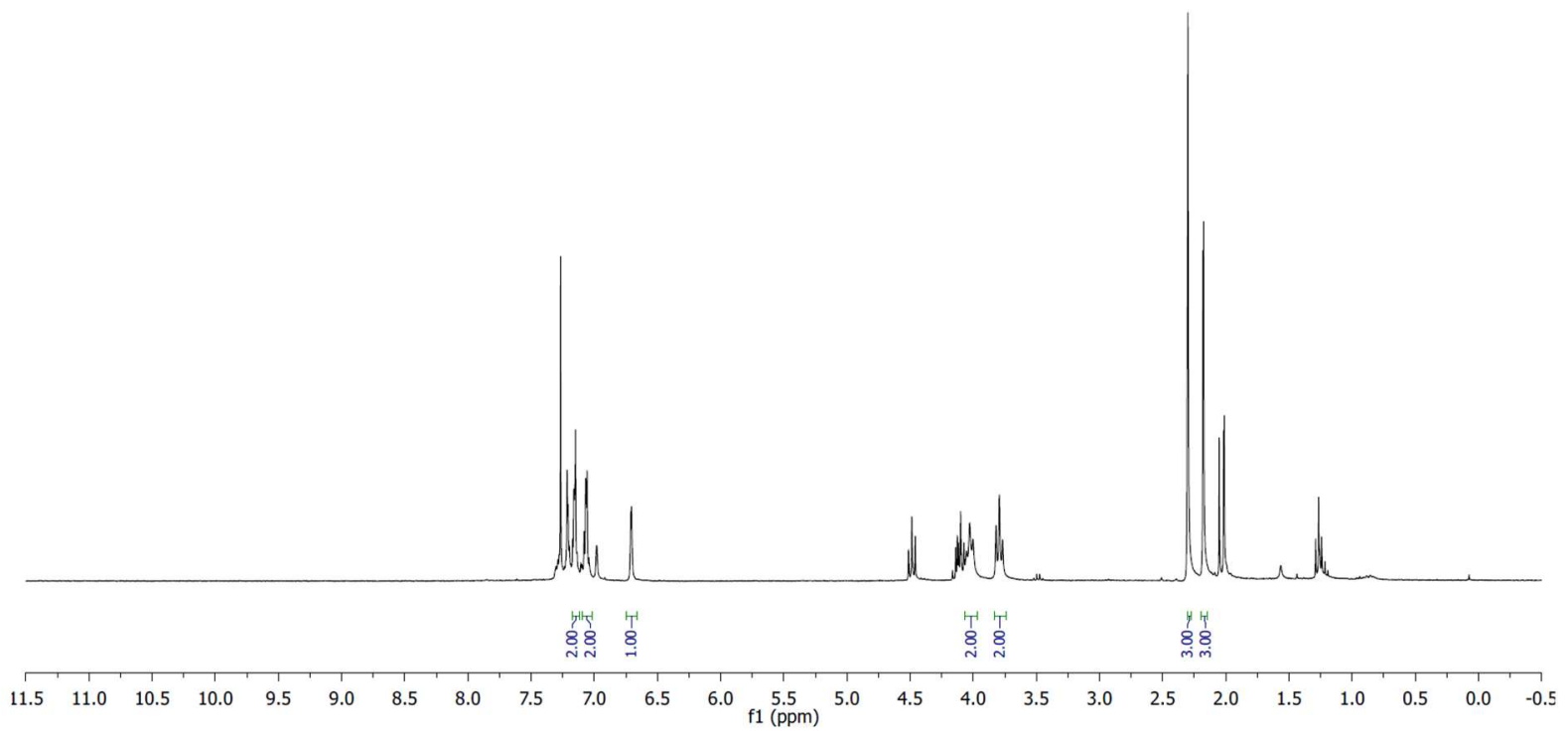


${ }^{13} \mathrm{C} \mathrm{NMR}\left(\mathrm{CDCl}_{3}, 75 \mathrm{MHz}\right)$
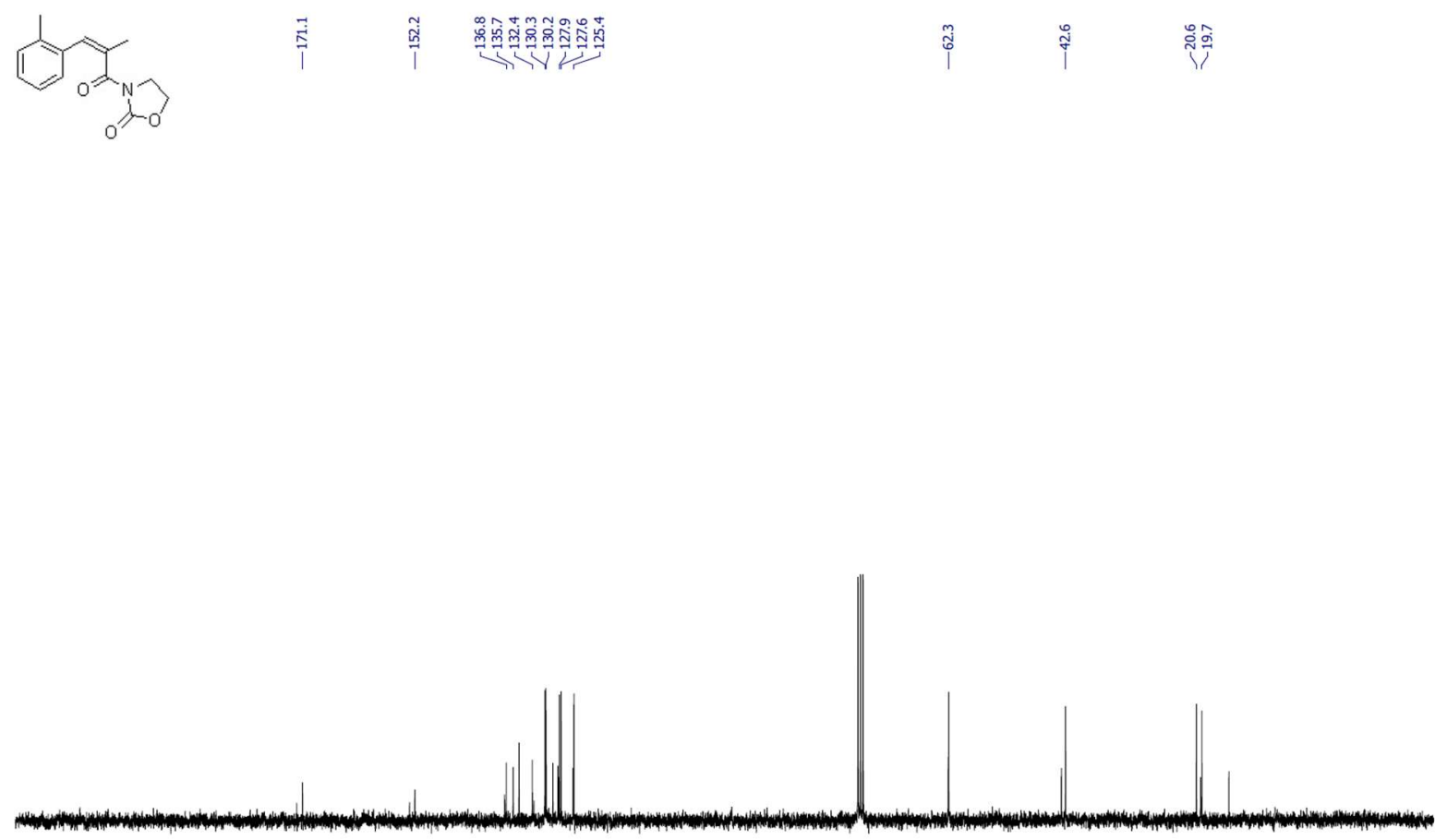

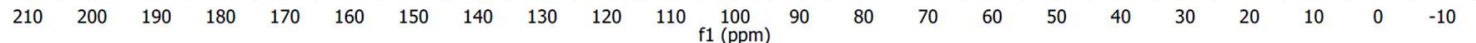

(Z)-3-(3-(4-(tert-butyl)phenyl)-2-methylacryloyl)oxazolidin-2-one ((Z)-12):

${ }^{1} \mathrm{H} \mathrm{NMR}\left(\mathrm{CDCl}_{3}, 300 \mathrm{MHz}\right)$
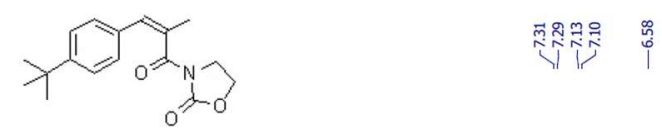

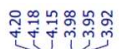

$\stackrel{ \pm}{i} \stackrel{\stackrel{0}{i}}{i}$

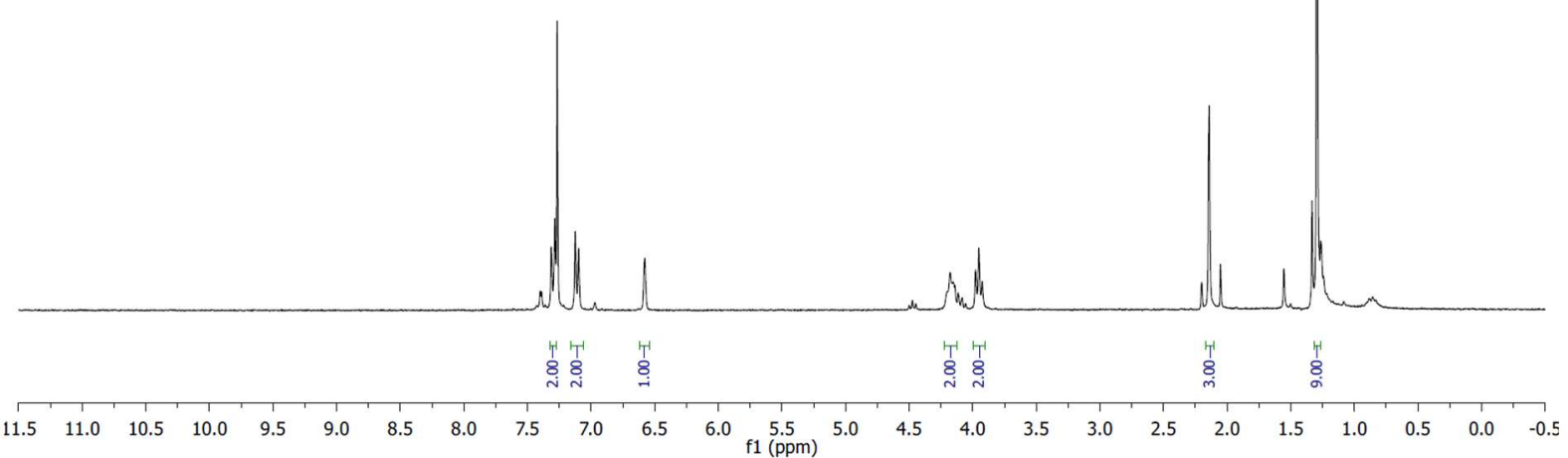


${ }^{13} \mathrm{C} \mathrm{NMR}\left(\mathrm{CDCl}_{3}, 75 \mathrm{MHz}\right)$

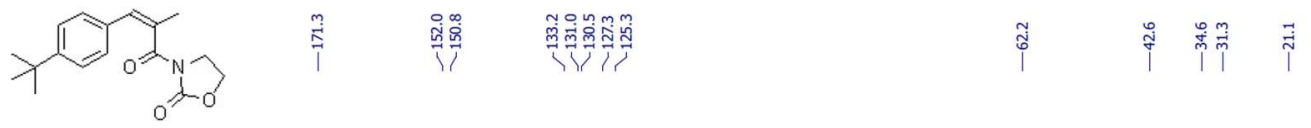

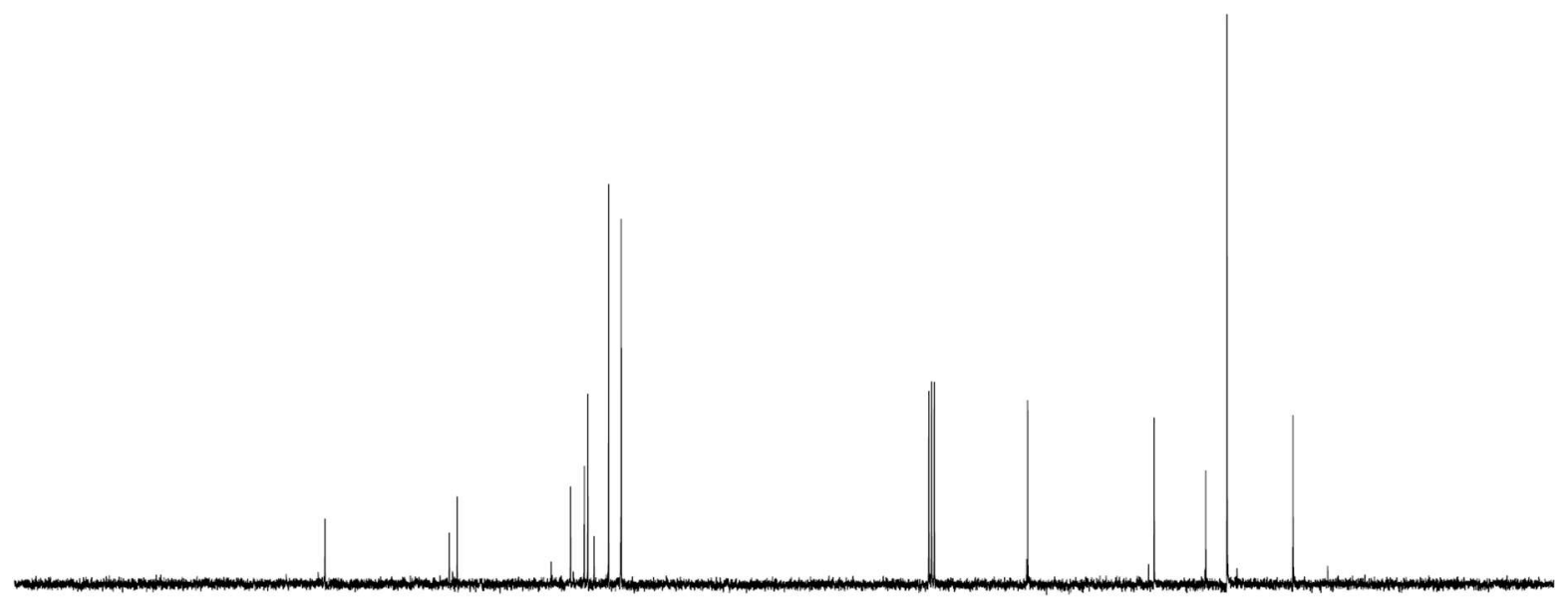

$\begin{array}{lllllllllllllllllllllll}210 & 200 & 190 & 180 & 170 & 160 & 150 & 140 & 130 & 120 & 110 & 100 & 90 & 80 & 70 & 60 & 50 & 40 & 30 & 20 & 10 & 0 & -10\end{array}$

\section{(Z)-3-(3-([1,1'-biphenyl]-4-yl)-2-methylacryloyl)oxazolidin-2-one ((Z)-13):}

${ }^{1} \mathrm{H} \mathrm{NMR}\left(\mathrm{CDCl}_{3}, 300 \mathrm{MHz}\right)$

舟

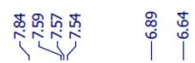
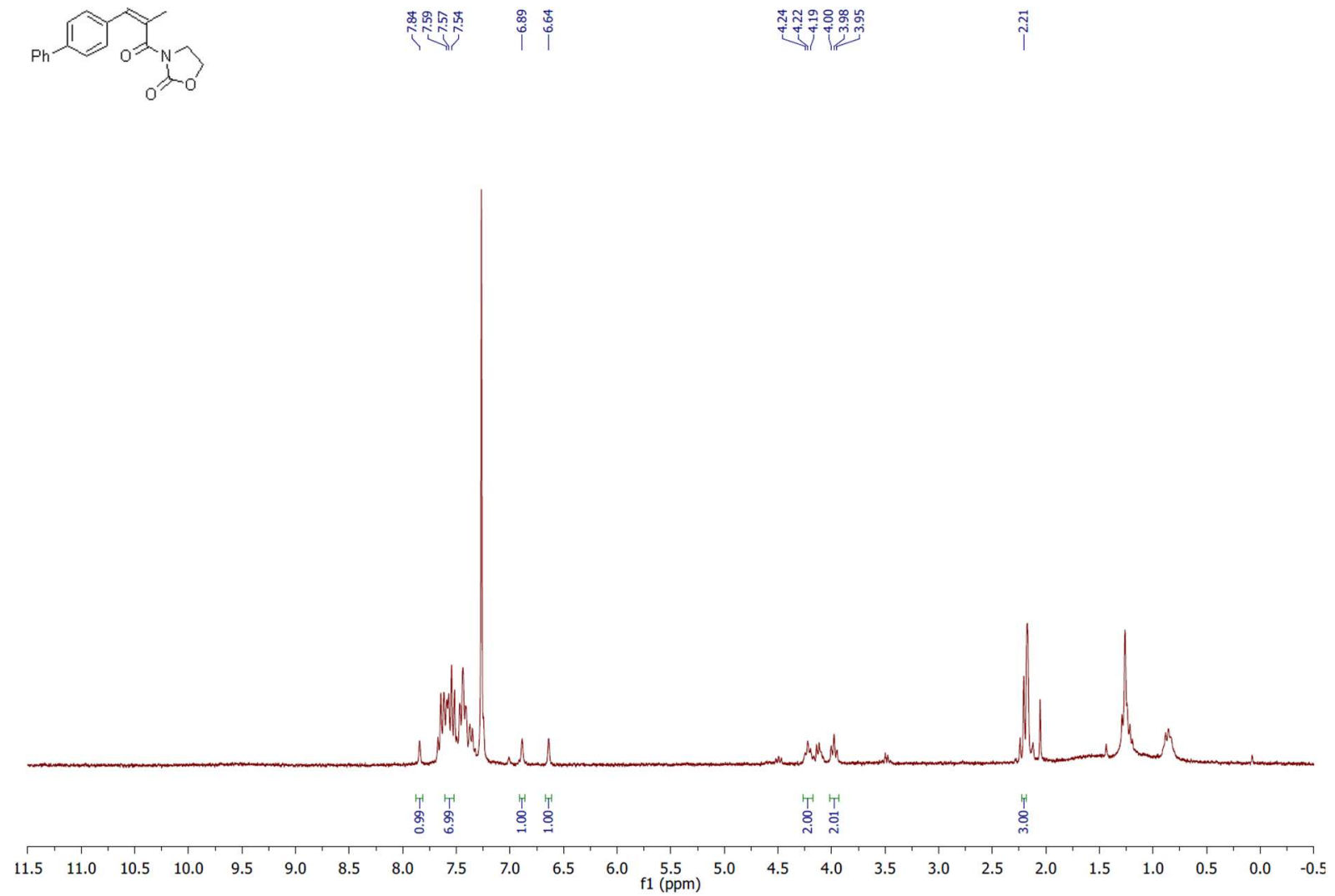
(Z)-3-(3-(4-methoxyphenyl)-2-methylacryloyl)oxazolidin-2-one ((Z)-14):

${ }^{1} \mathrm{H} \mathrm{NMR}\left(\mathrm{CDCl}_{3}, 300 \mathrm{MHz}\right)$
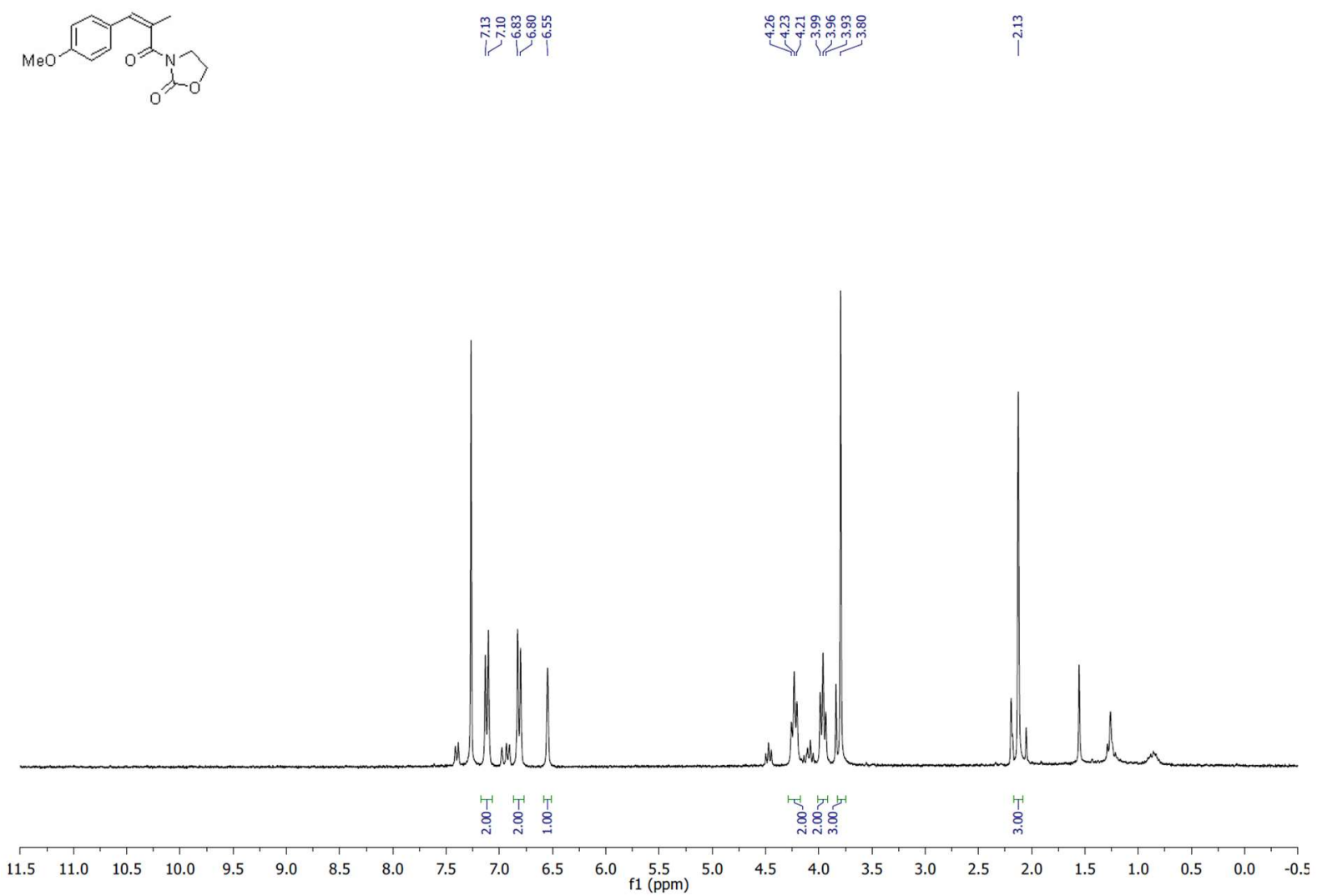

${ }^{13} \mathrm{C} \mathrm{NMR}\left(\mathrm{CDCl}_{3}, 75 \mathrm{MHz}\right)$

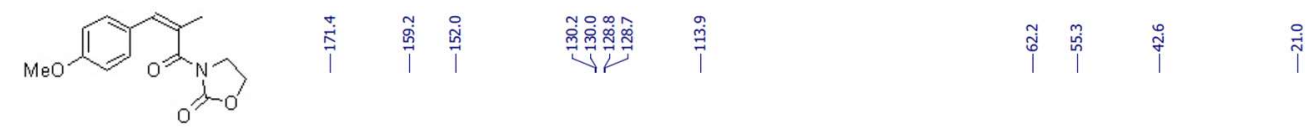

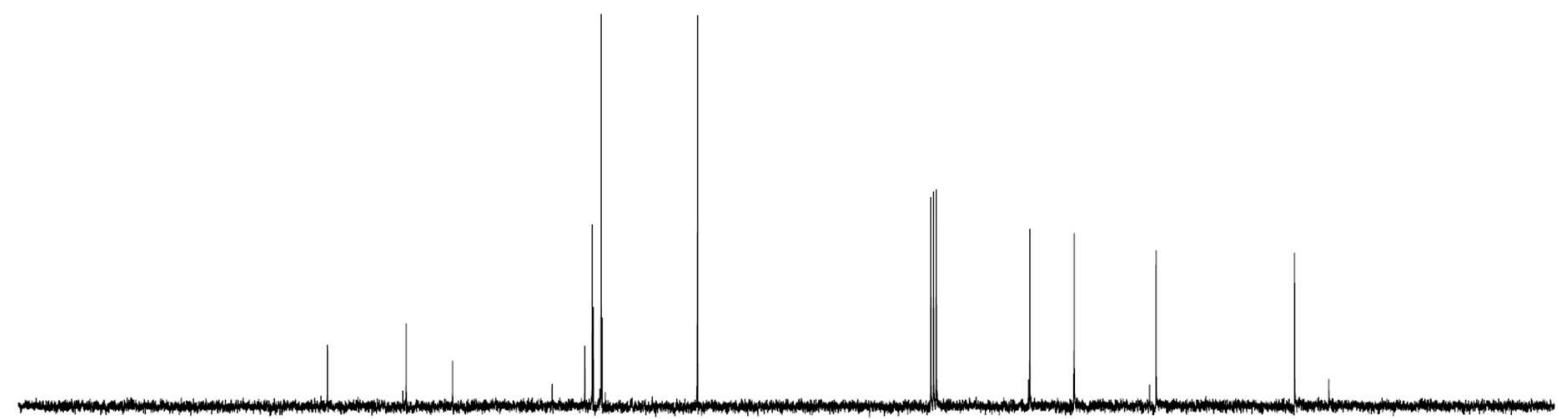

$\begin{array}{lllllllllll}210 & 200 & 190 & 180 & 170 & 160 & 150 & 140 & 130 & 120 & 110 \\ \mathrm{f} 1(\mathrm{ppm})\end{array}$ 
(Z)-3-(2-methyl-3-(naphthalen-2-yl)acryloyl)oxazolidin-2-one ((Z)-15):

${ }^{1} \mathrm{H} \mathrm{NMR}\left(\mathrm{CDCl}_{3}, 300 \mathrm{MHz}\right)$

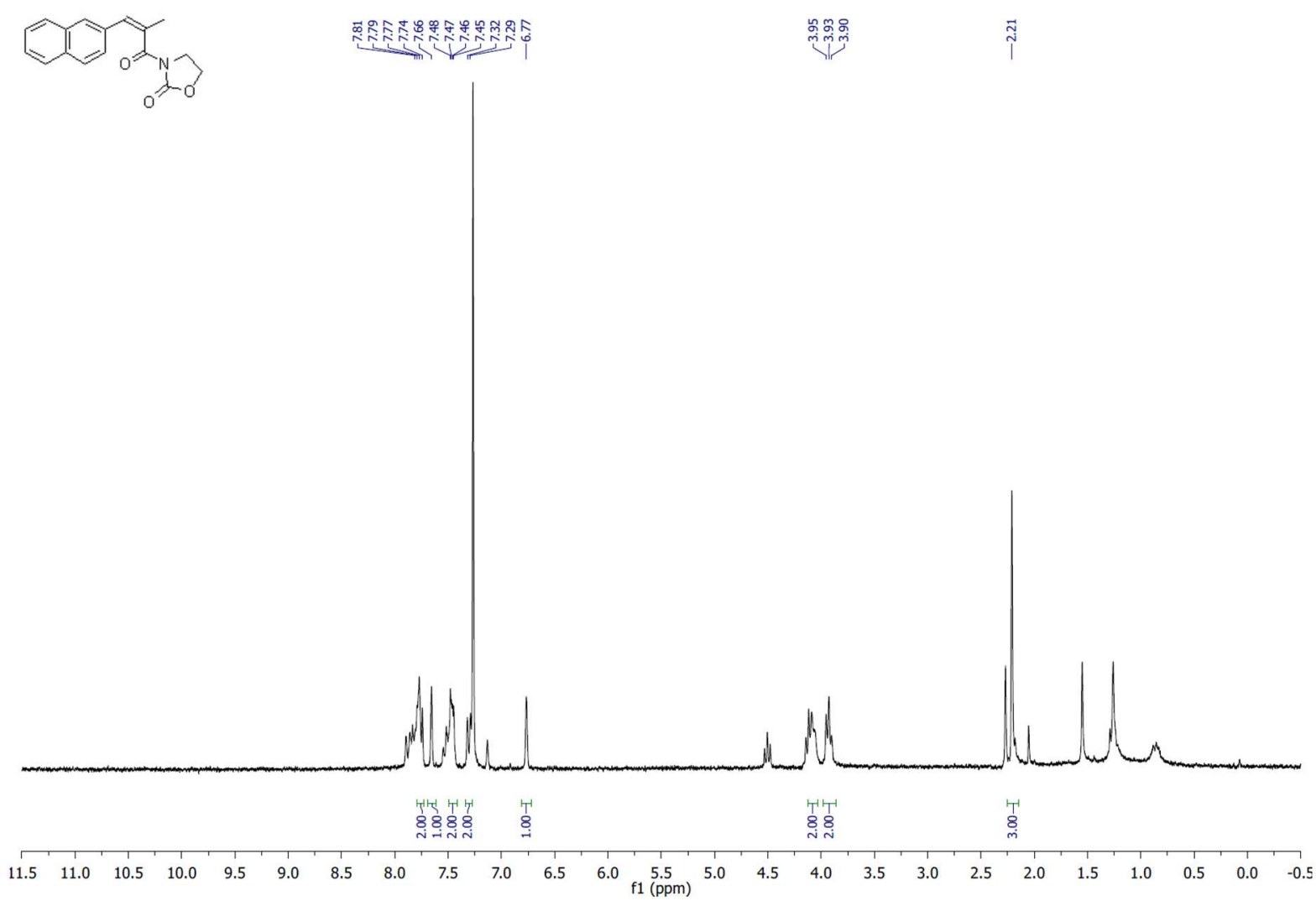

${ }^{13} \mathrm{C} \mathrm{NMR}\left(\mathrm{CDCl}_{3}, 75 \mathrm{MHz}\right)$

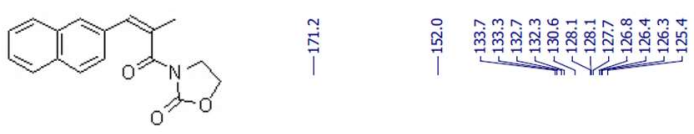

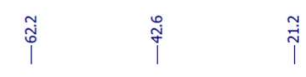

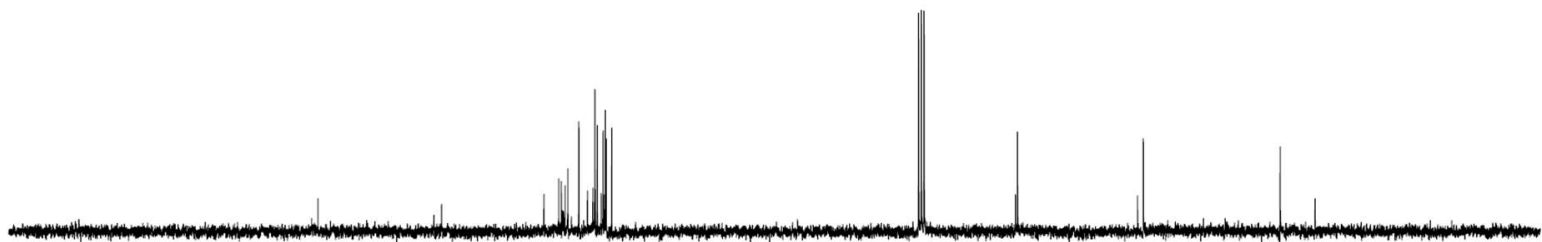

$\begin{array}{lllllllllllllllllllllllllll}210 & 200 & 190 & 180 & 170 & 160 & 150 & 140 & 130 & 120 & 110 & 100 & 90 & 80 & 70 & 60 & 50 & 40 & 30 & 20 & 10 & 0 & -10 & \end{array}$ 
(Z)-3-(3-(4-fluorophenyl)-2-methylacryloyl)oxazolidin-2-one ((Z)-16):

${ }^{1} \mathrm{H} \mathrm{NMR}\left(\mathrm{CDCl}_{3}, 300 \mathrm{MHz}\right)$
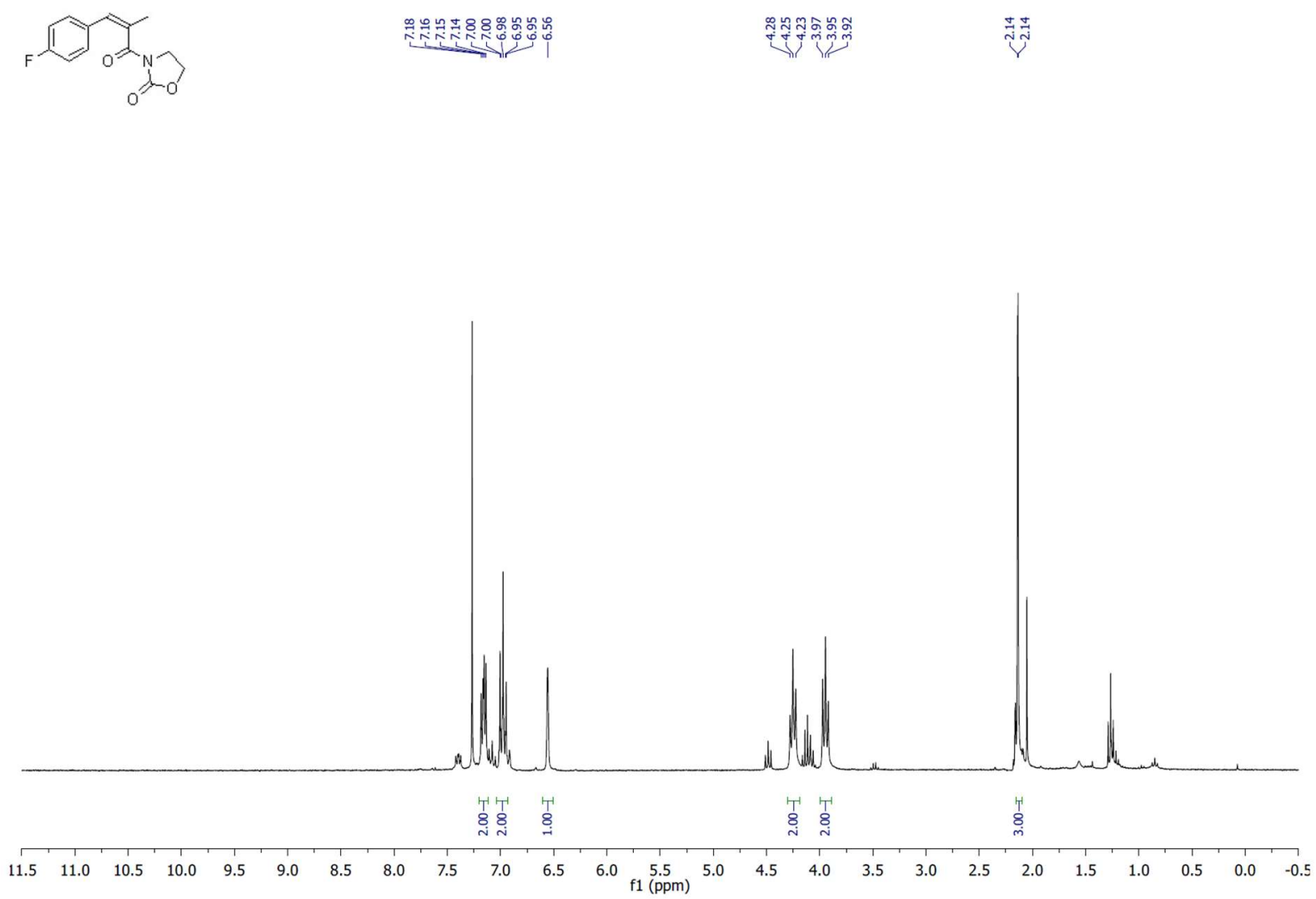

${ }^{13} \mathrm{C} \mathrm{NMR}\left(\mathrm{CDCl}_{3}, 75 \mathrm{MHz}\right)$
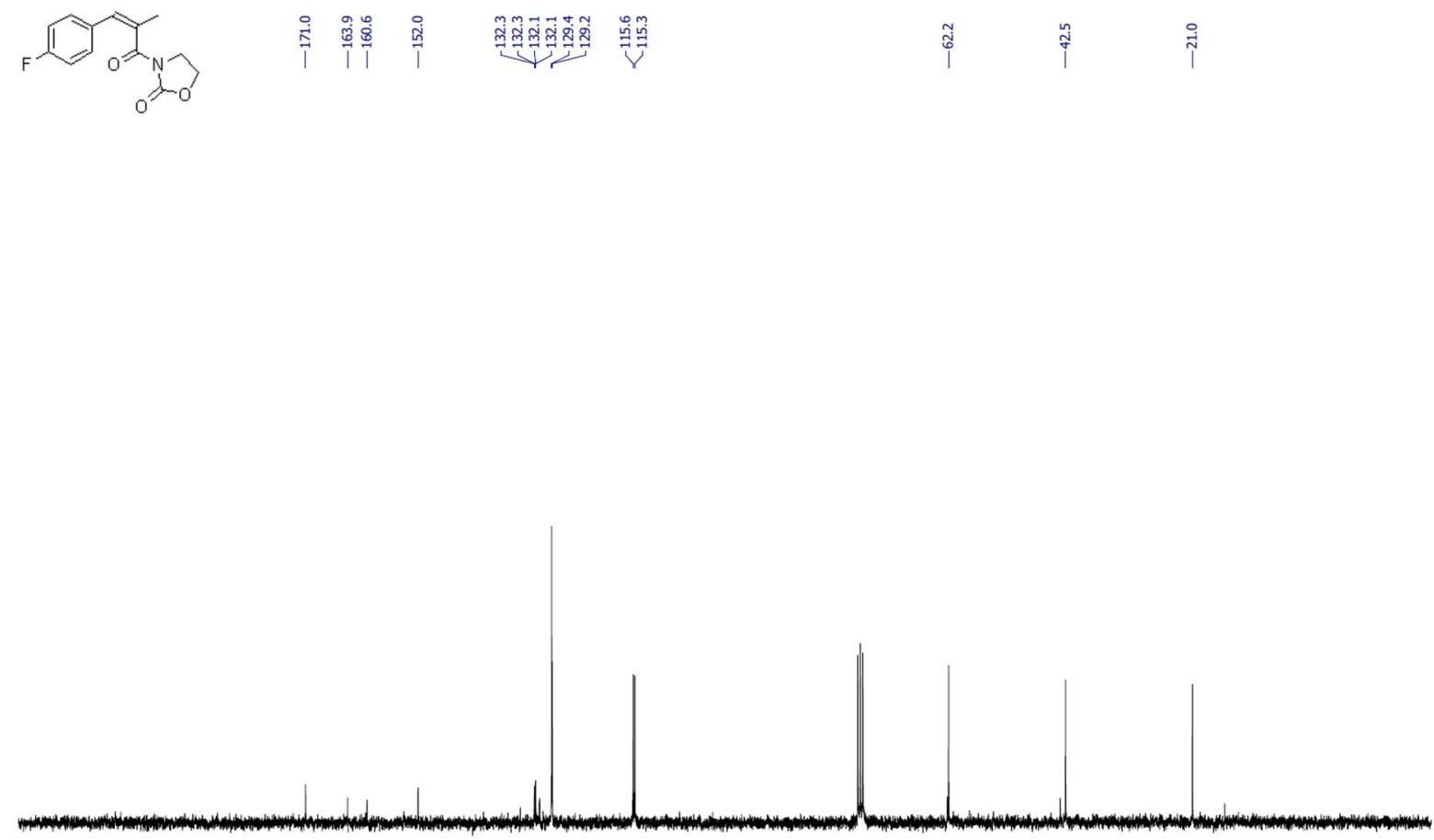

$\begin{array}{lllllllllllllllllllllll}210 & 200 & 190 & 180 & 170 & 160 & 150 & 140 & 130 & 120 & 110 & \underset{\mathrm{f} 1}{100}(\mathrm{ppm}) \\ \end{array}$ 
${ }^{19} \mathrm{~F} \mathrm{NMR}\left(\mathrm{CDCl}_{3}, 282 \mathrm{MHz}\right)$

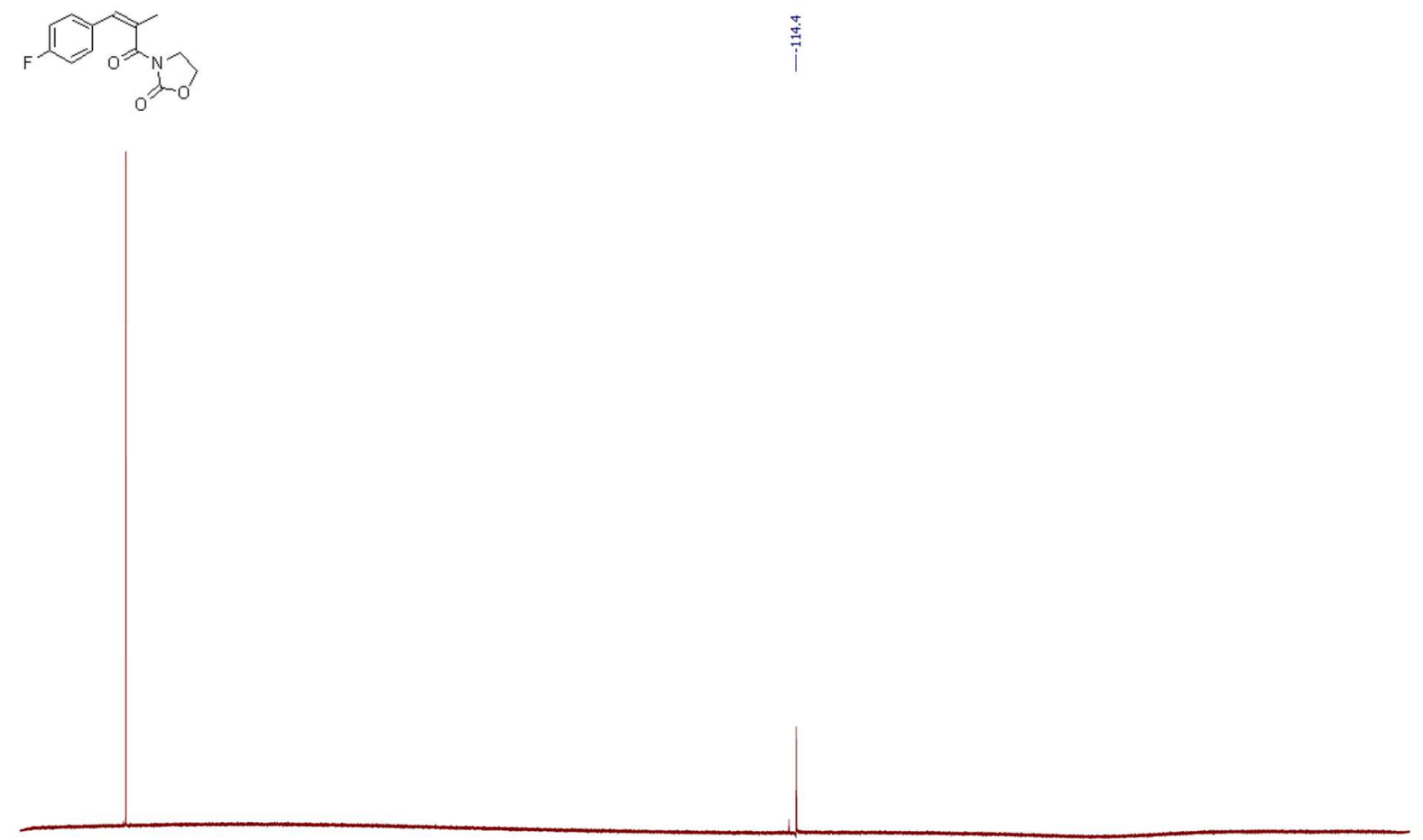

$\begin{array}{lllllllllllllllllllllll}10 & 0 & -10 & -20 & -30 & -40 & -50 & -60 & -70 & -80 & -90 & -100 & -110 & -120 & -130 & -140 & -150 & -160 & -170 & -180 & -190 & -200 & -210\end{array}$

(Z)-3-(3-(4-chlorophenyl)-2-methylacryloyl)oxazolidin-2-one ((Z)-17):

${ }^{1} \mathrm{H} \mathrm{NMR}\left(\mathrm{CDCl}_{3}, 300 \mathrm{MHz}\right)$
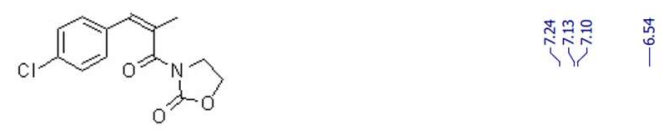

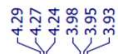

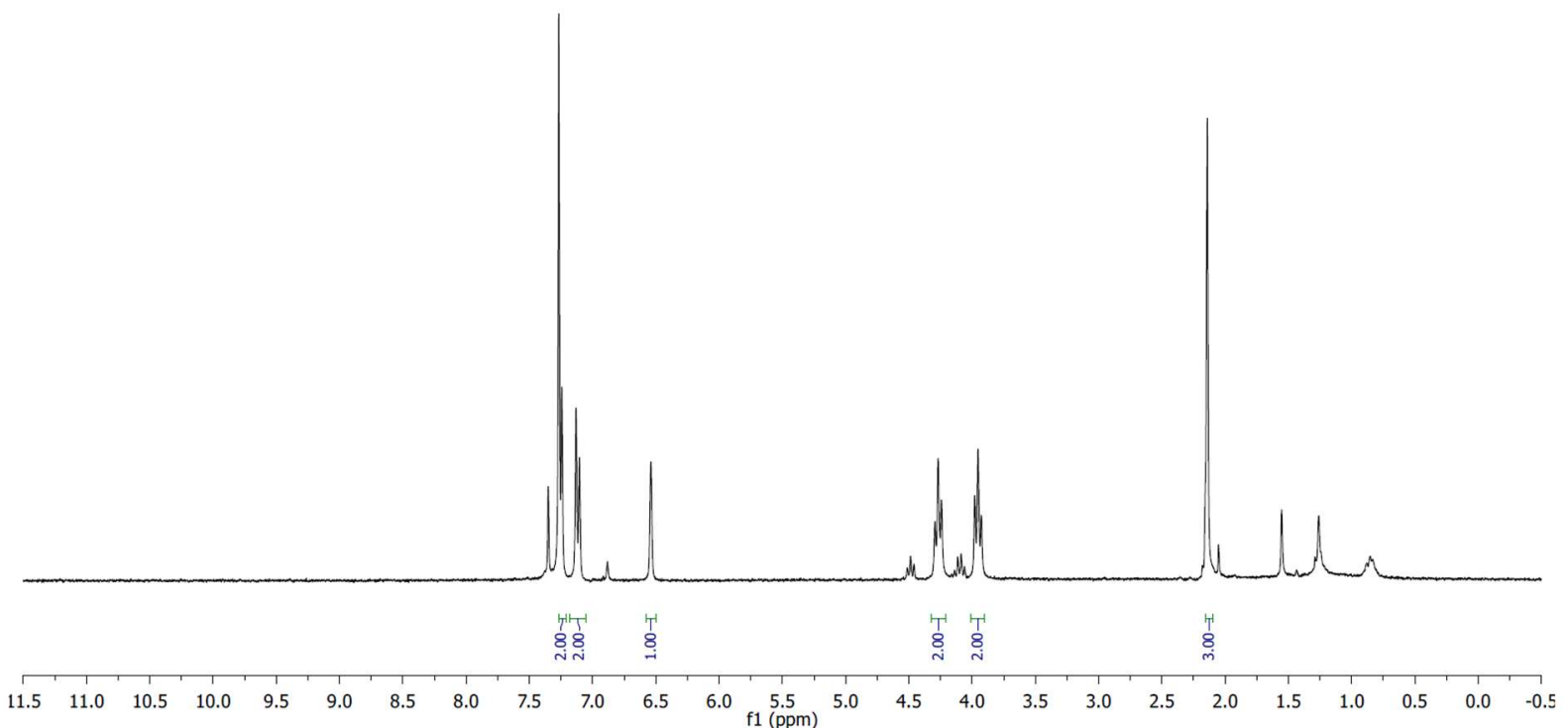


${ }^{13} \mathrm{C} \mathrm{NMR}\left(\mathrm{CDCl}_{3}, 75 \mathrm{MHz}\right)$
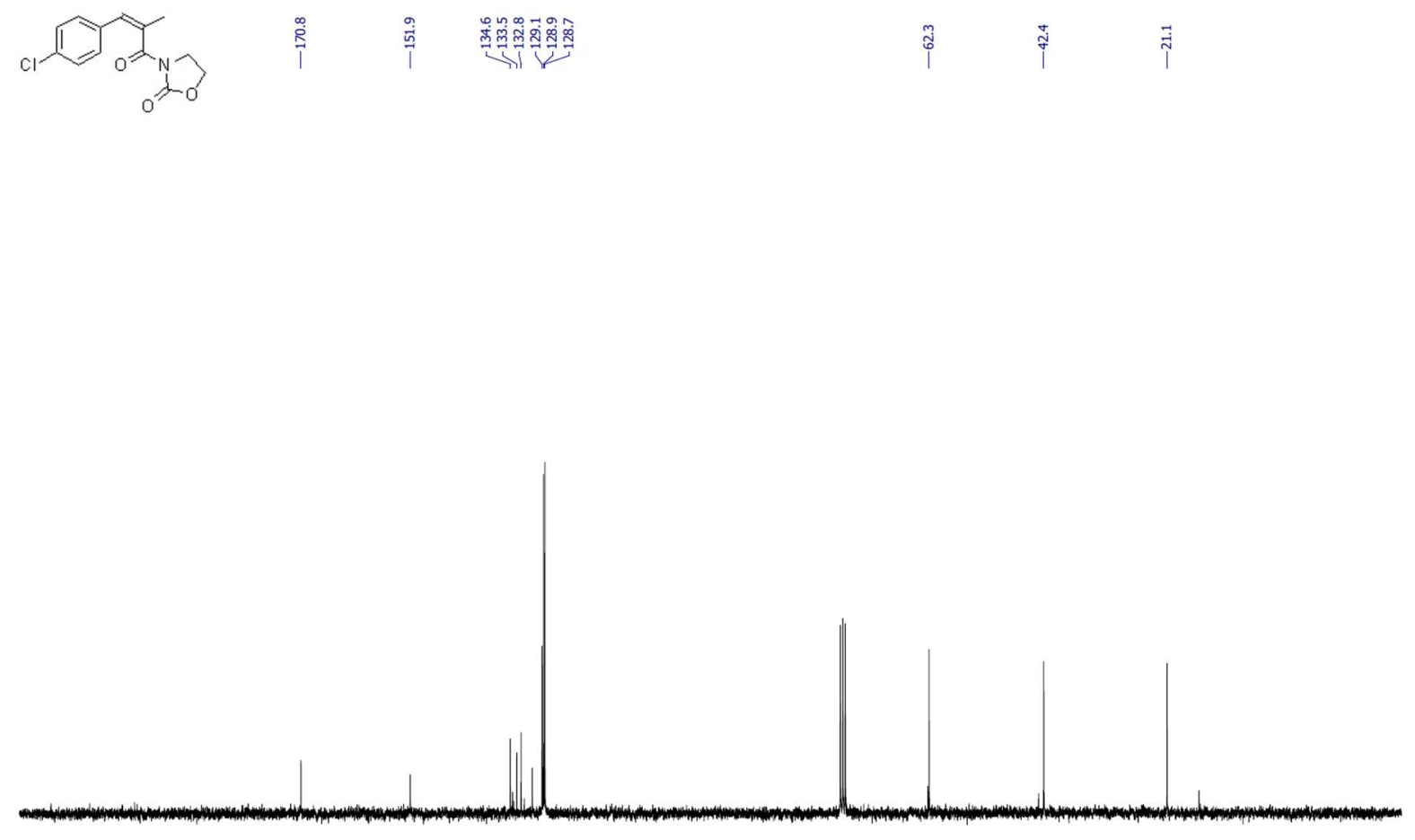

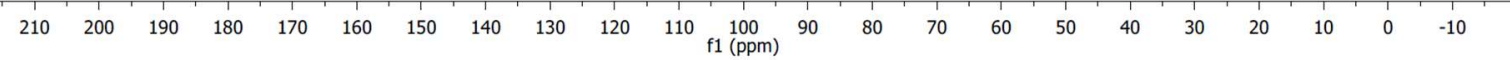

(Z)-3-(3-(4-bromophenyl)-2-methylacryloyl)oxazolidin-2-one ((Z)-18):

${ }^{1} \mathrm{H} \mathrm{NMR}\left(\mathrm{CDCl}_{3}, 300 \mathrm{MHz}\right)$
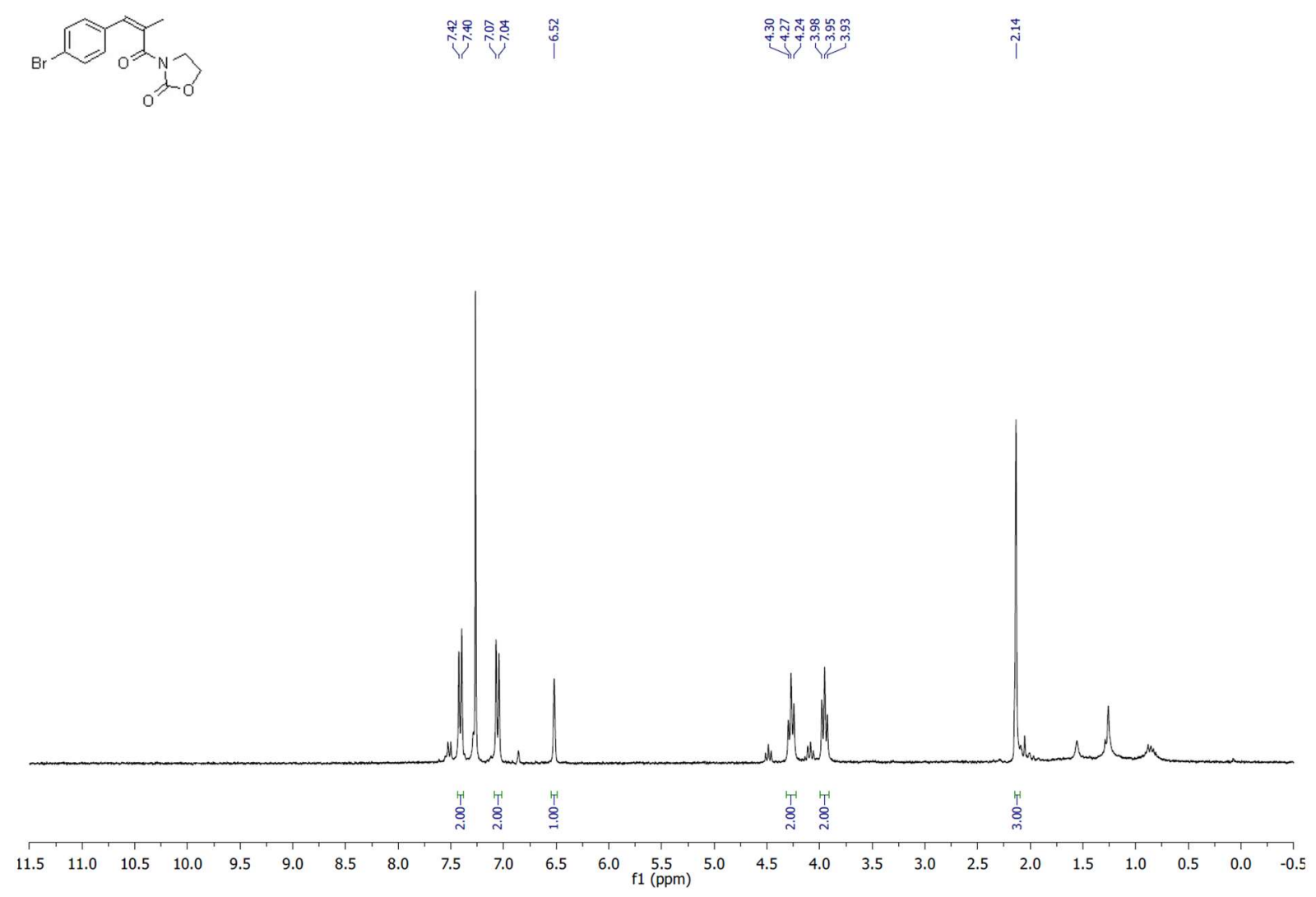
${ }^{13} \mathrm{C} \mathrm{NMR}\left(\mathrm{CDCl}_{3}, 75 \mathrm{MHz}\right)$

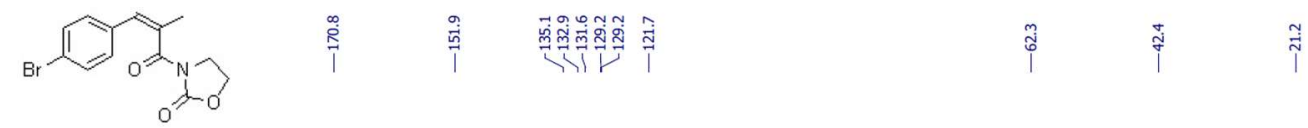

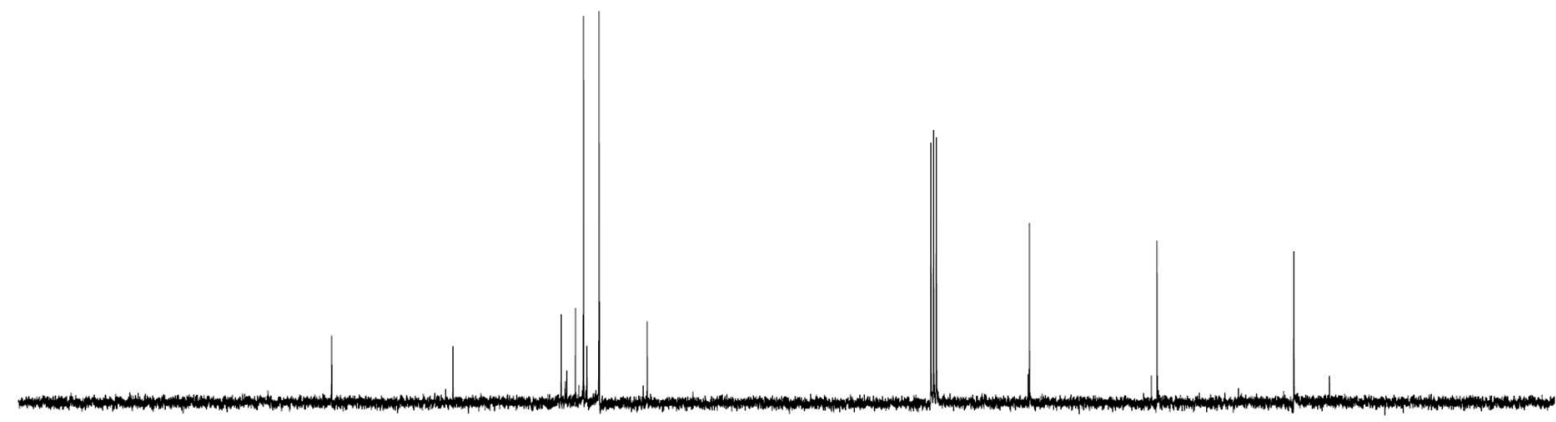

$\begin{array}{lllllllllllllllllllllll}210 & 200 & 190 & 180 & 170 & 160 & 150 & 140 & 130 & 120 & 110 & \begin{array}{c}100 \\ \mathrm{f} 1(\mathrm{ppm})\end{array} & 90 & 80 & 70 & 60 & 50 & 40 & 30 & 20 & 10 & 0 & -10\end{array}$

(Z)-3-(3-(4-iodophenyl)-2-methylacryloyl)oxazolidin-2-one ((Z)-19):

${ }^{1} \mathrm{H} \mathrm{NMR}\left(\mathrm{CDCl}_{3}, 300 \mathrm{MHz}\right)$
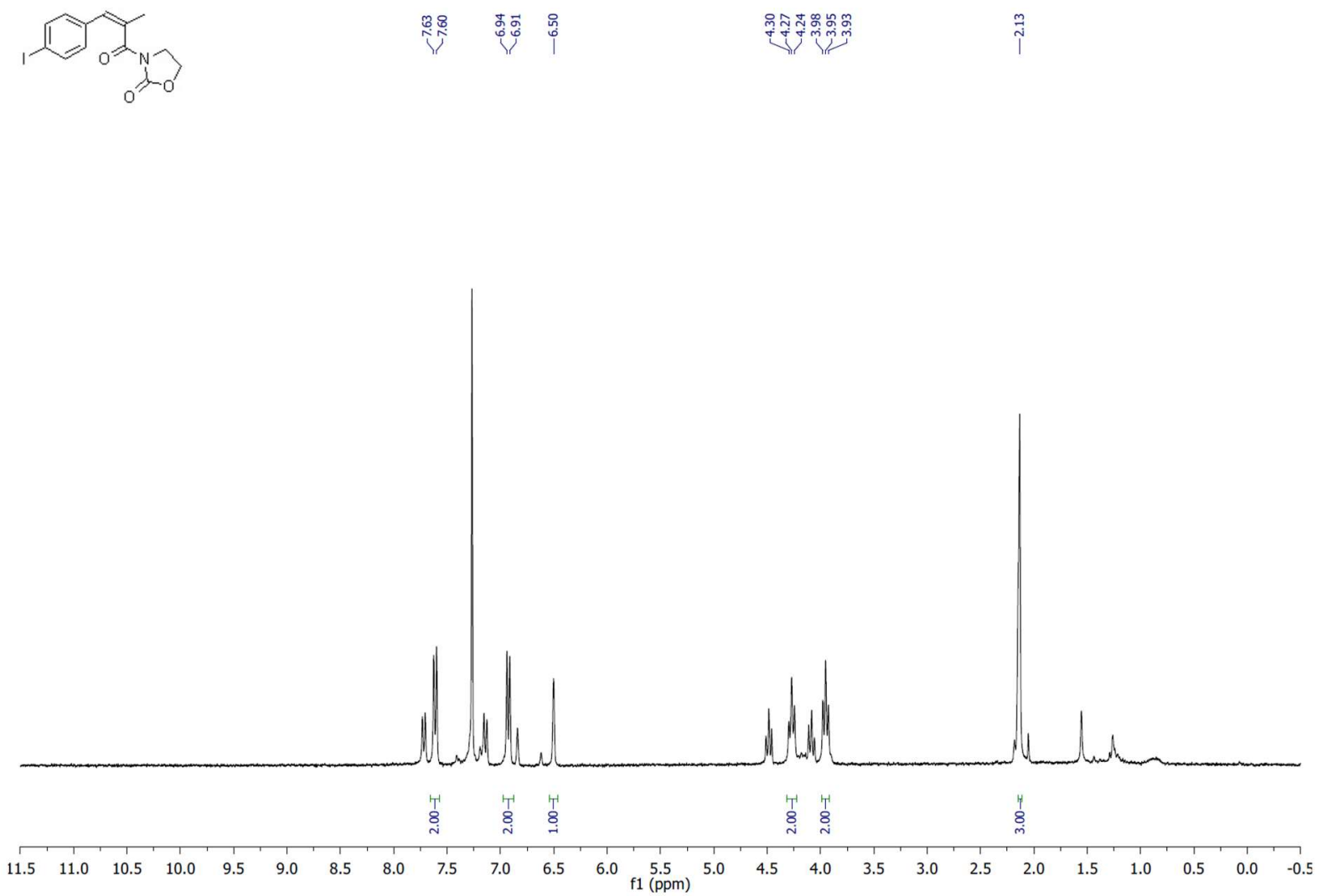
${ }^{13} \mathrm{C} \mathrm{NMR}\left(\mathrm{CDCl}_{3}, 75 \mathrm{MHz}\right)$

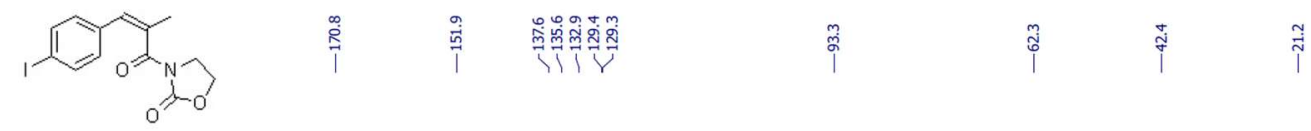

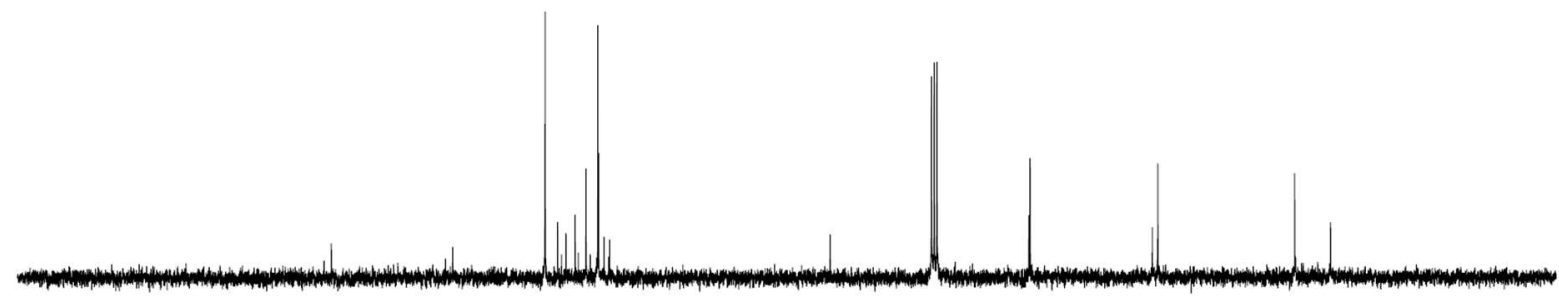

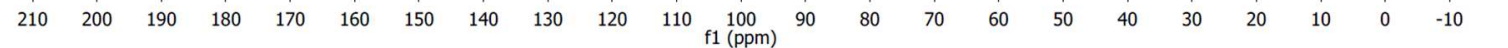

(Z)-3-(2-methyl-3-(4-(trifluoromethyl)phenyl)acryloyl)oxazolidin-2-one ((Z)-20):

${ }^{1} \mathrm{H} \mathrm{NMR}\left(\mathrm{CDCl}_{3}, 300 \mathrm{MHz}\right)$
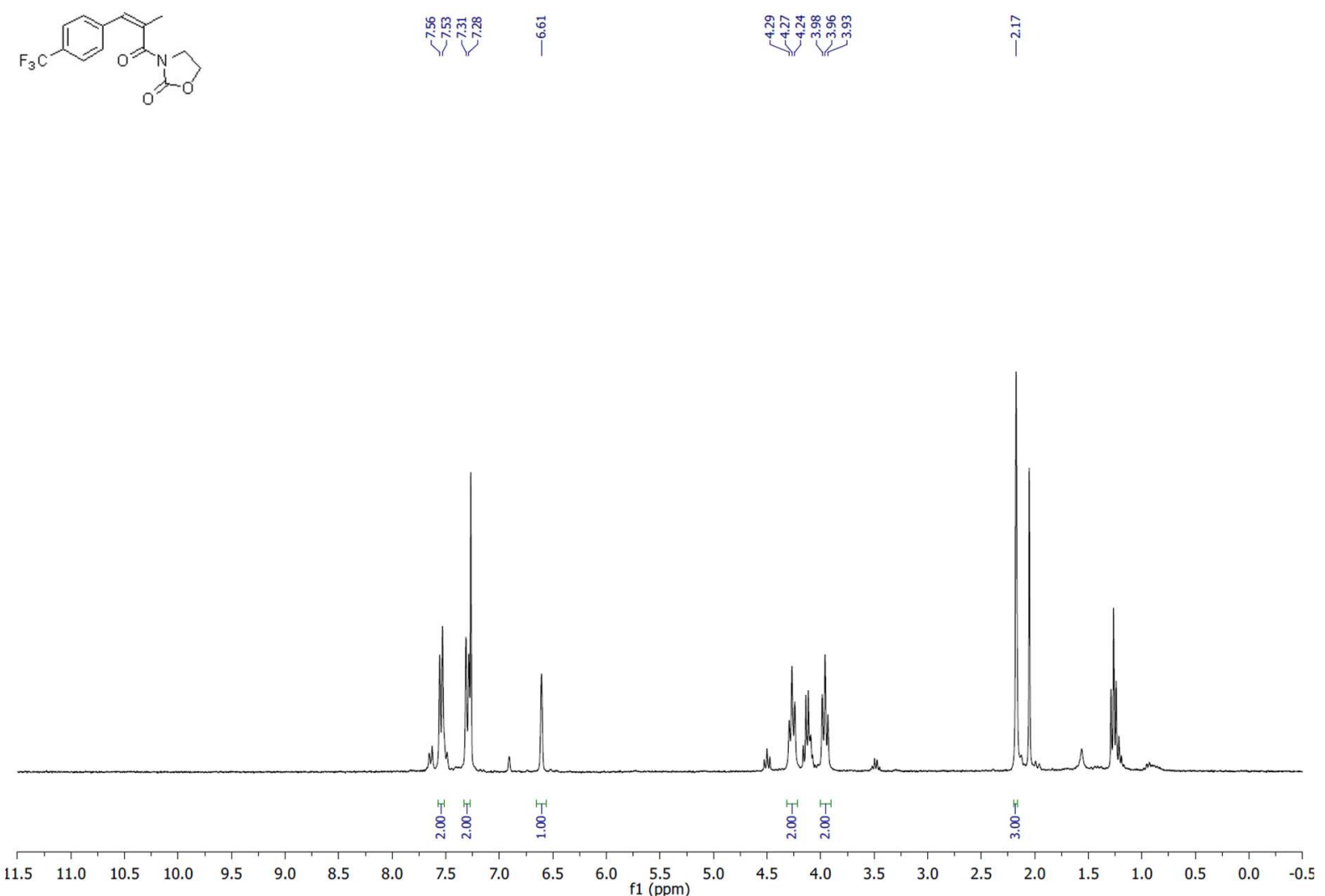
${ }^{13} \mathrm{C} \mathrm{NMR}\left(\mathrm{CDCl}_{3}, 75 \mathrm{MHz}\right)$

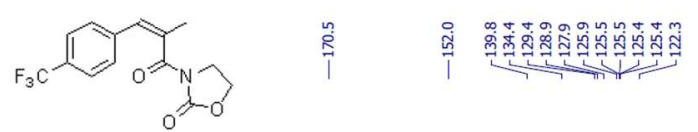

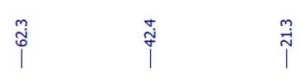
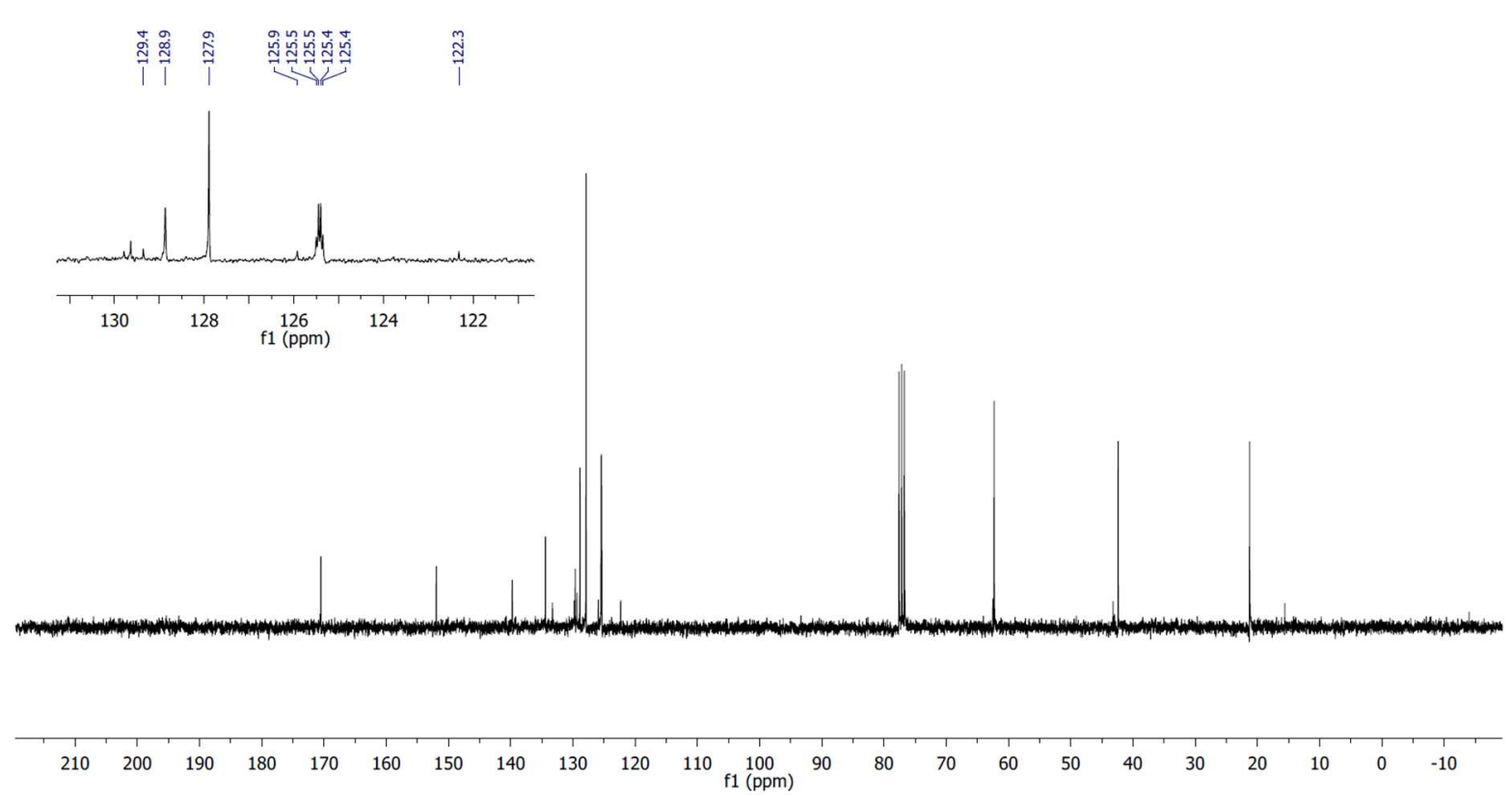

${ }^{19} \mathrm{~F} \mathrm{NMR}\left(\mathrm{CDCl}_{3}, 282 \mathrm{MHz}\right)$

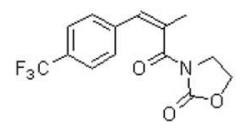

$\vec{\phi}$

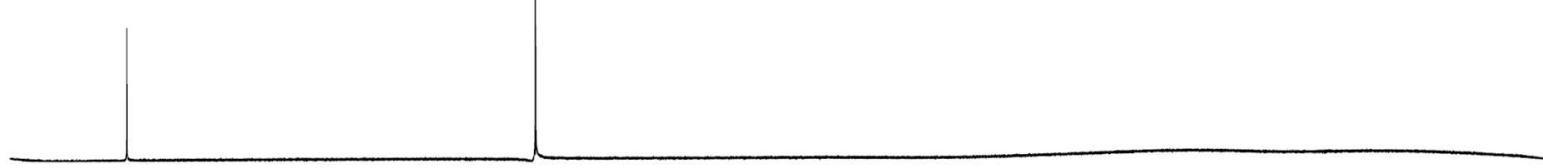

$\begin{array}{llllllllllllllllllllllll}10 & 0 & -10 & -20 & -30 & -40 & -50 & -60 & -70 & -80 & -90 & -100 & -110 & -120 & -130 & -140 & -150 & -160 & -170 & -180 & -190 & -200 & -210\end{array}$

215 
(Z)-3-(2-methyl-3-(4-(4,4,5,5-tetramethyl-1,3,2-dioxaborolan-2-yl)phenyl)acryloyl)oxazolidin-2-one $((Z)-21):$

${ }^{1} \mathrm{H} \mathrm{NMR}\left(\mathrm{CDCl}_{3}, 300 \mathrm{MHz}\right)$

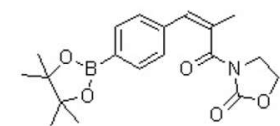

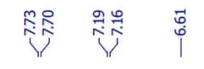

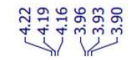

ה้ำ

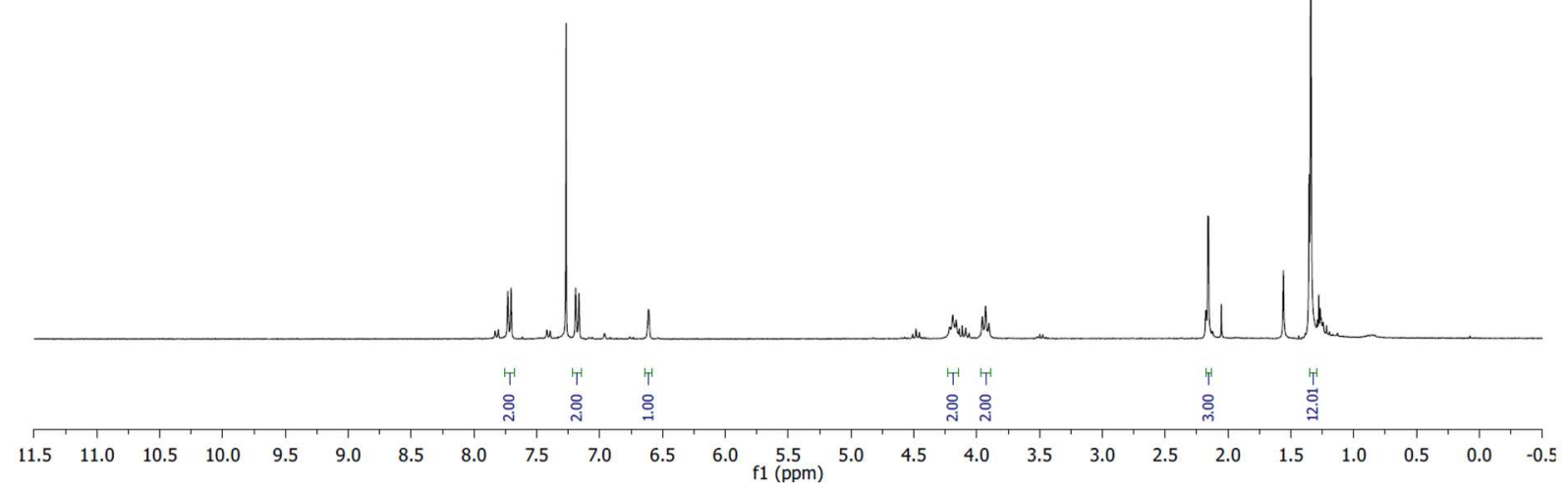

${ }^{13} \mathrm{C} \mathrm{NMR}\left(\mathrm{CDCl}_{3}, 75 \mathrm{MHz}\right)$

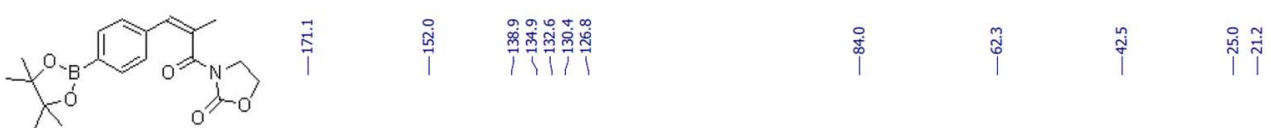

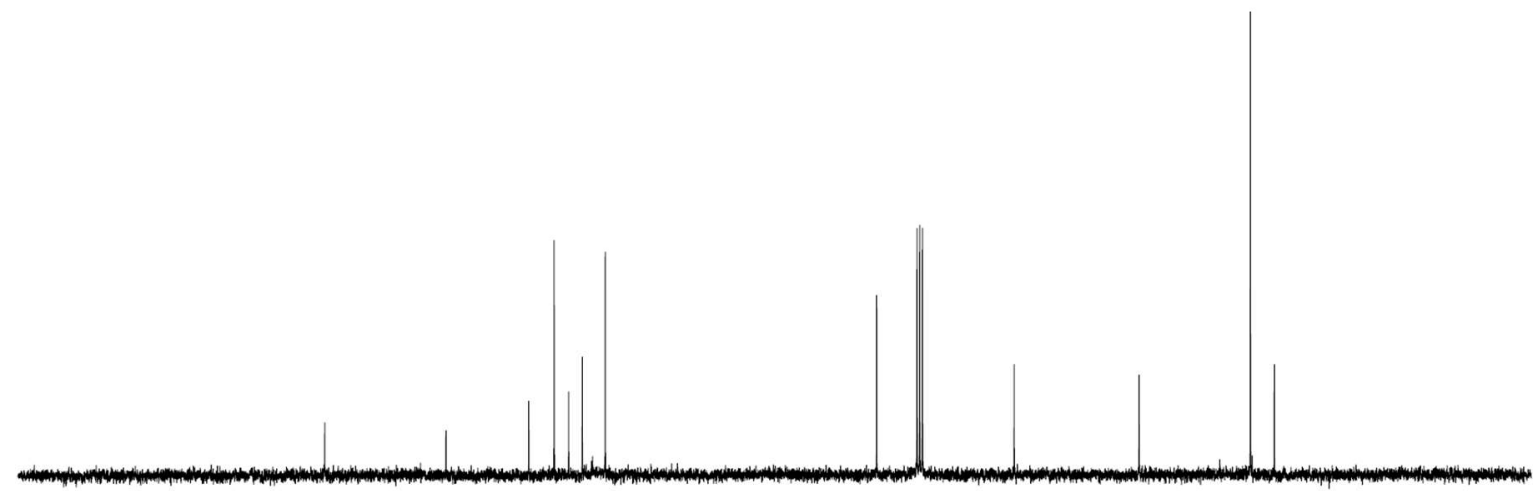

$\begin{array}{lllllllllllllllllllllll}210 & 200 & 190 & 180 & 170 & 160 & 150 & 140 & 130 & 120 & 110 & \underset{\mathrm{f} 1}{100}(\mathrm{ppm}) \\ 90 & 80 & 70 & 60 & 50 & 40 & 30 & 20 & 10 & 0 & -10\end{array}$ 
(Z)-3-(2-methyl-3-(4-(methylsulfonyl)phenyl)acryloyl)oxazolidin-2-one ((Z)-22):

${ }^{1} \mathrm{H} \mathrm{NMR}\left(\mathrm{CDCl}_{3}, 300 \mathrm{MHz}\right)$
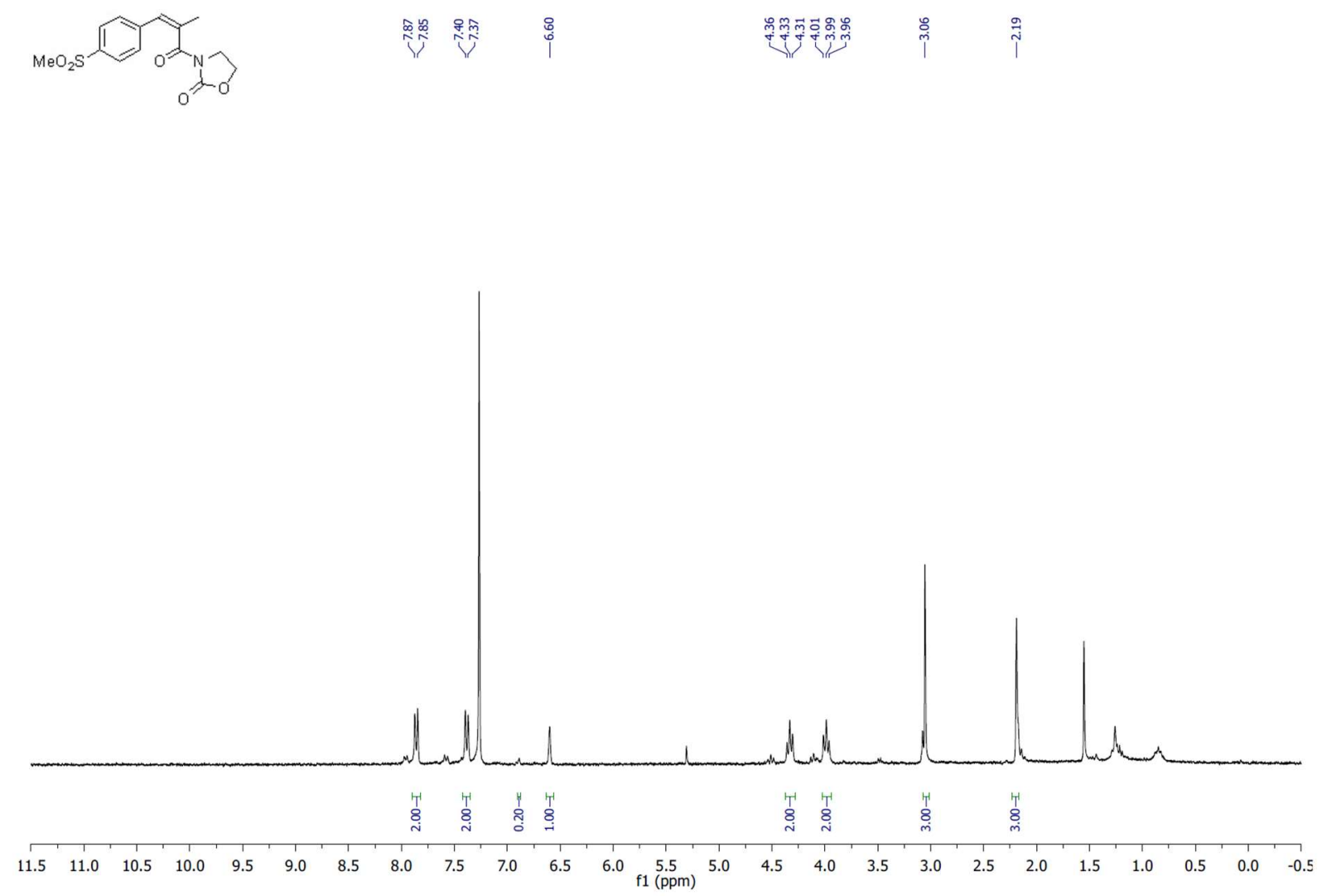

${ }^{13} \mathrm{C} \mathrm{NMR}\left(\mathrm{CDCl}_{3}, 75 \mathrm{MHz}\right)$

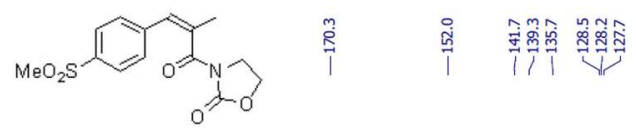

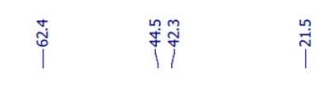

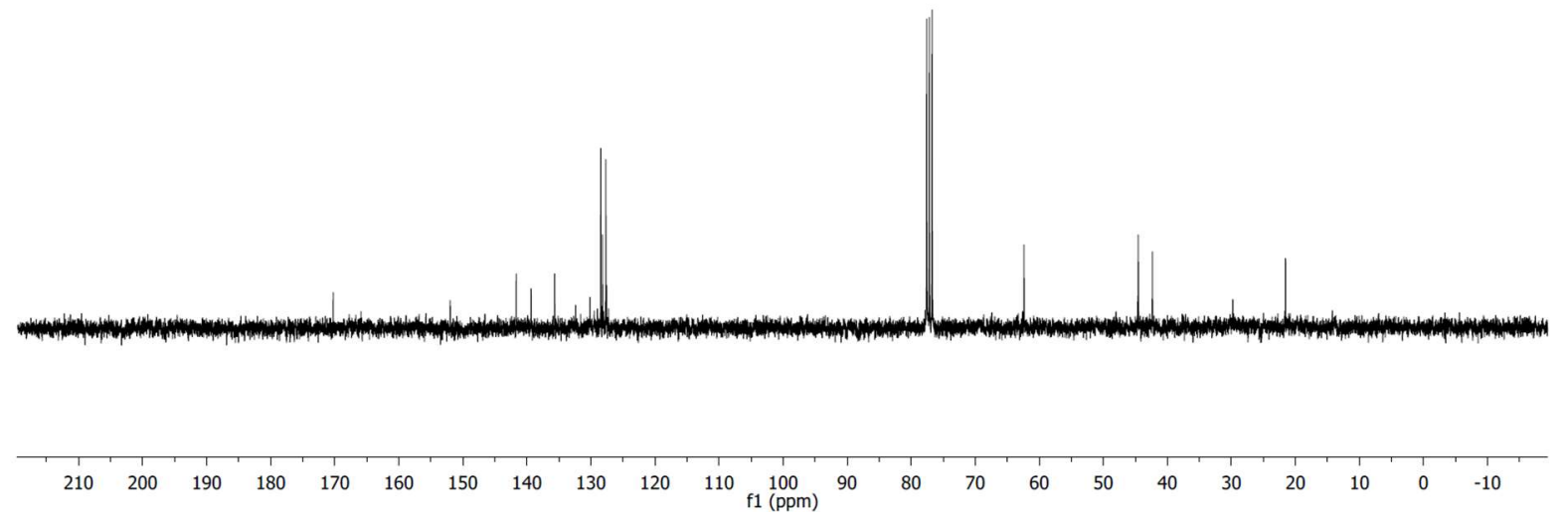


(Z)-3-(3-(4-acetylphenyl)-2-methylacryloyl)oxazolidin-2-one ((Z)-23):

${ }^{1} \mathrm{H} \mathrm{NMR}\left(\mathrm{CDCl}_{3}, 300 \mathrm{MHz}\right)$
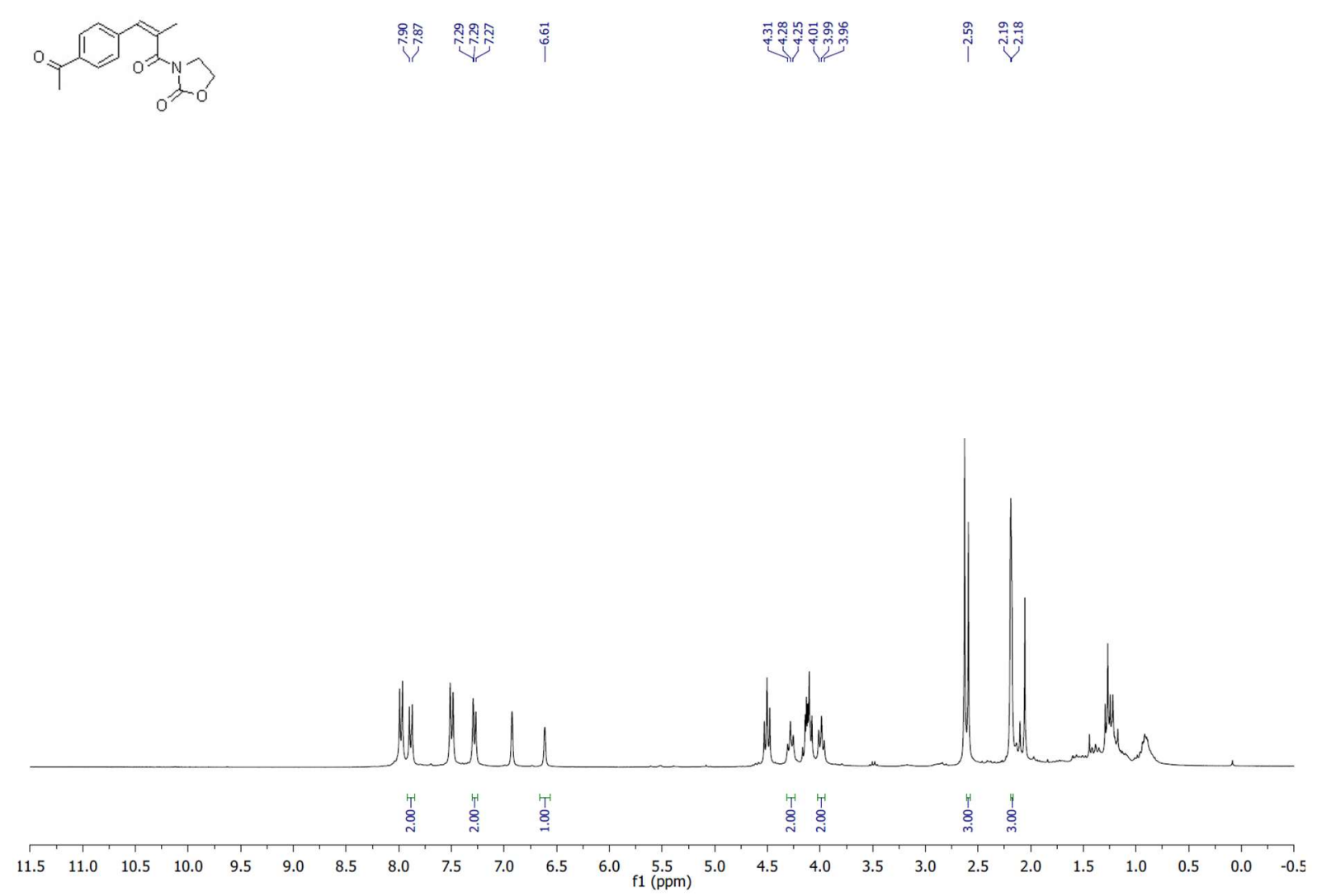

${ }^{13} \mathrm{C} \mathrm{NMR}\left(\mathrm{CDCl}_{3}, 75 \mathrm{MHz}\right)$
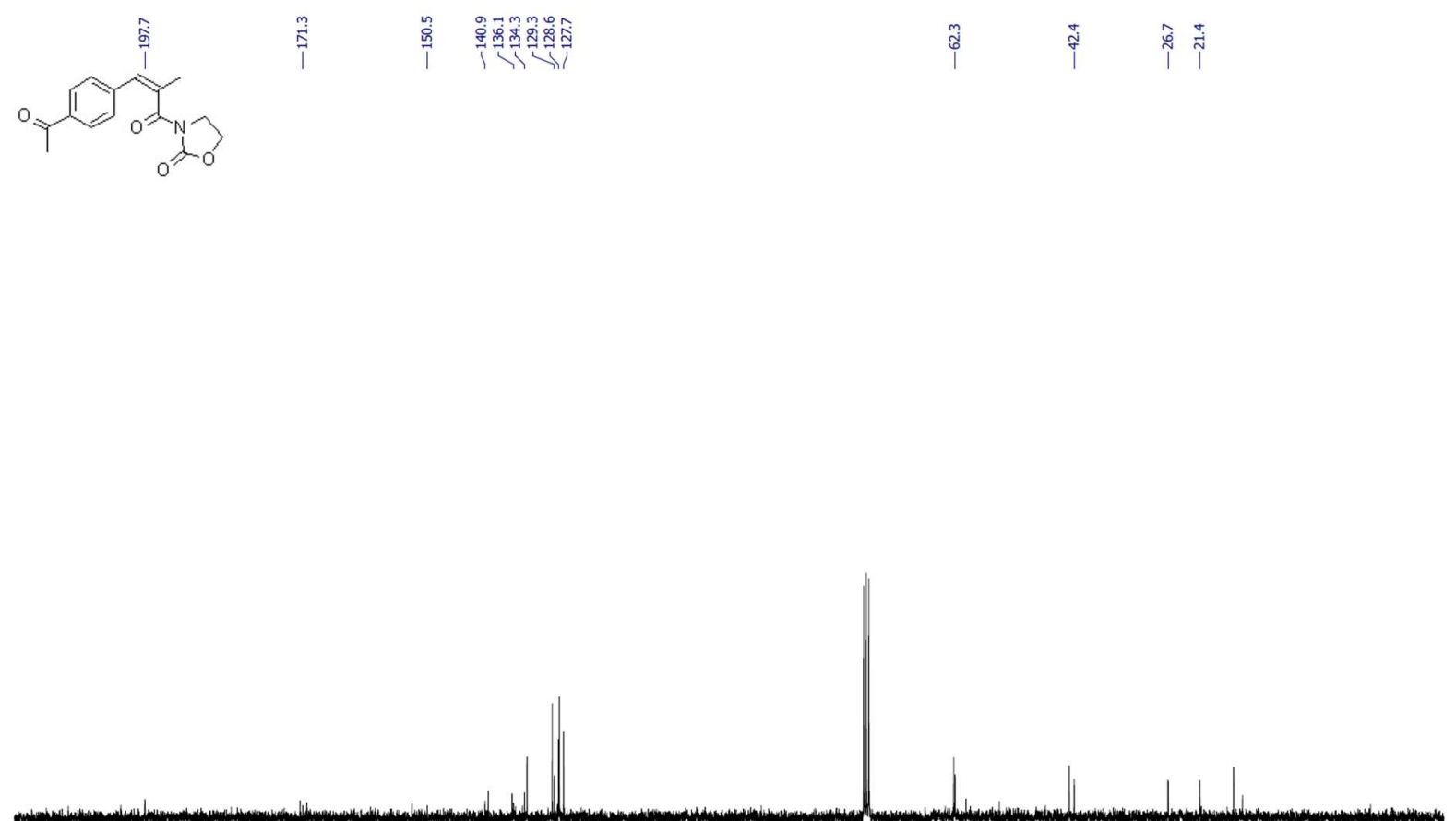

$\begin{array}{llllllllllllllllllllllll}210 & 200 & 190 & 180 & 170 & 160 & 150 & 140 & 130 & 120 & 110 & \begin{array}{c}100 \\ 100\end{array} & 90 & 80 & 70 & 60 & 50 & 40 & 30 & 20 & 10 & 0 & -10\end{array}$ 
(Z)-3-(3-(furan-2-yl)-2-methylacryloyl)oxazolidin-2-one ((Z)-24):

${ }^{1} \mathrm{H} \mathrm{NMR}\left(\mathrm{CDCl}_{3}, 300 \mathrm{MHz}\right)$
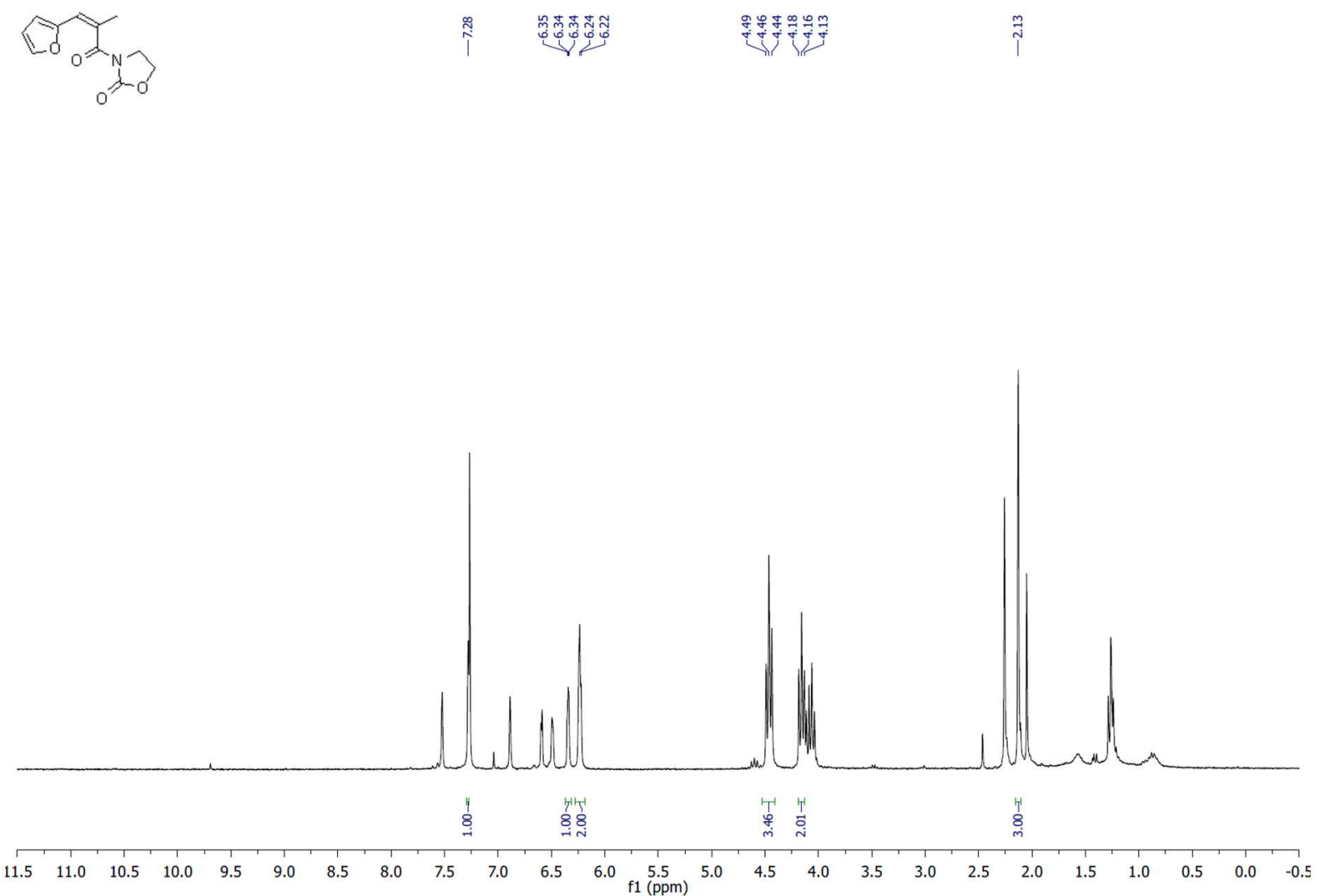

${ }^{13} \mathrm{C} \mathrm{NMR}\left(\mathrm{CDCl}_{3}, 75 \mathrm{MHz}\right)$

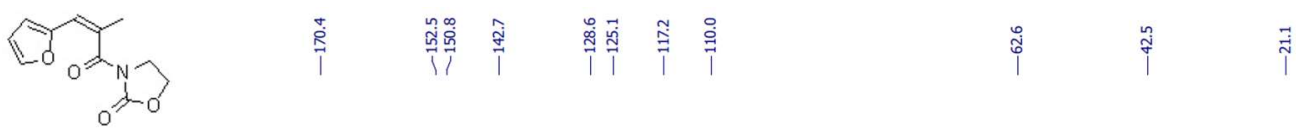

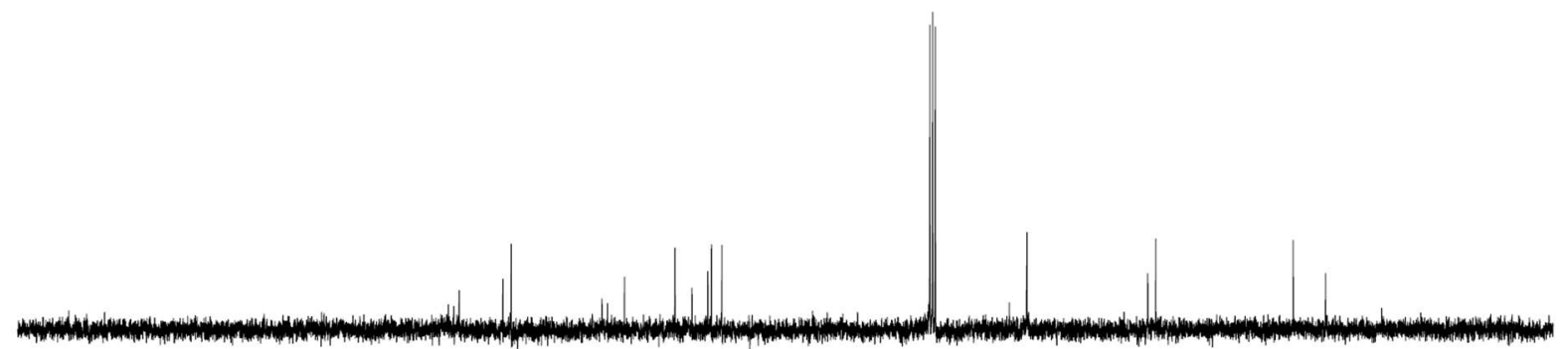

$\begin{array}{llllllllllllllllllllllllll}210 & 200 & 190 & 180 & 170 & 160 & 150 & 140 & 130 & 120 & 110 & 100 & 90 & 80 & 70 & 60 & 50 & 40 & 30 & 20 & 10 & 0 & -10\end{array}$ 
(Z)-3-(2-methyl-3-(thiophen-2-yl)acryloyl)oxazolidin-2-one ((Z)-25):

${ }^{1} \mathrm{H} \mathrm{NMR}\left(\mathrm{CDCl}_{3}, 300 \mathrm{MHz}\right)$

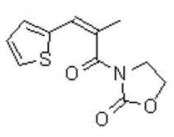

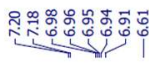

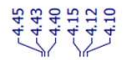

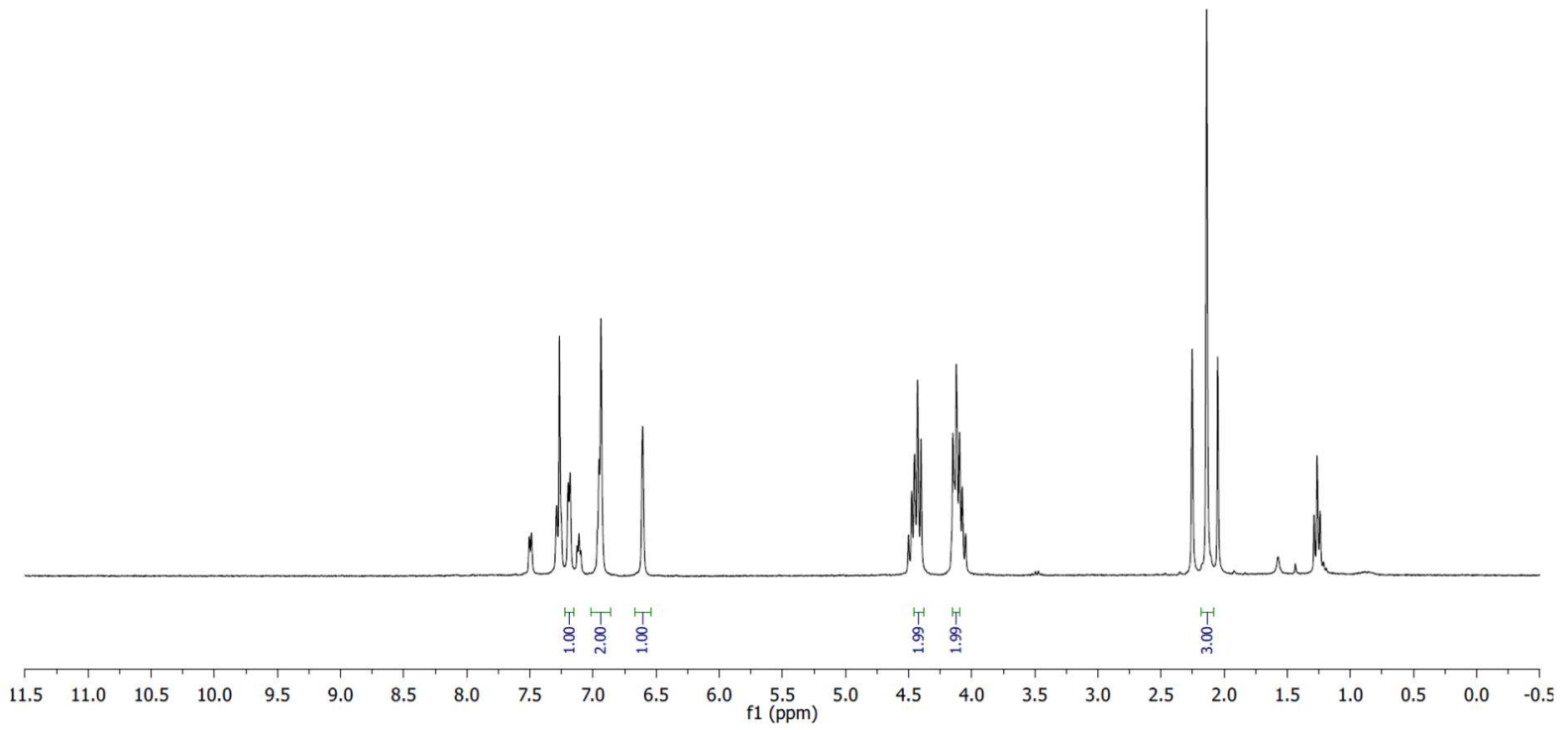

${ }^{13} \mathrm{C} \mathrm{NMR}\left(\mathrm{CDCl}_{3}, 75 \mathrm{MHz}\right)$
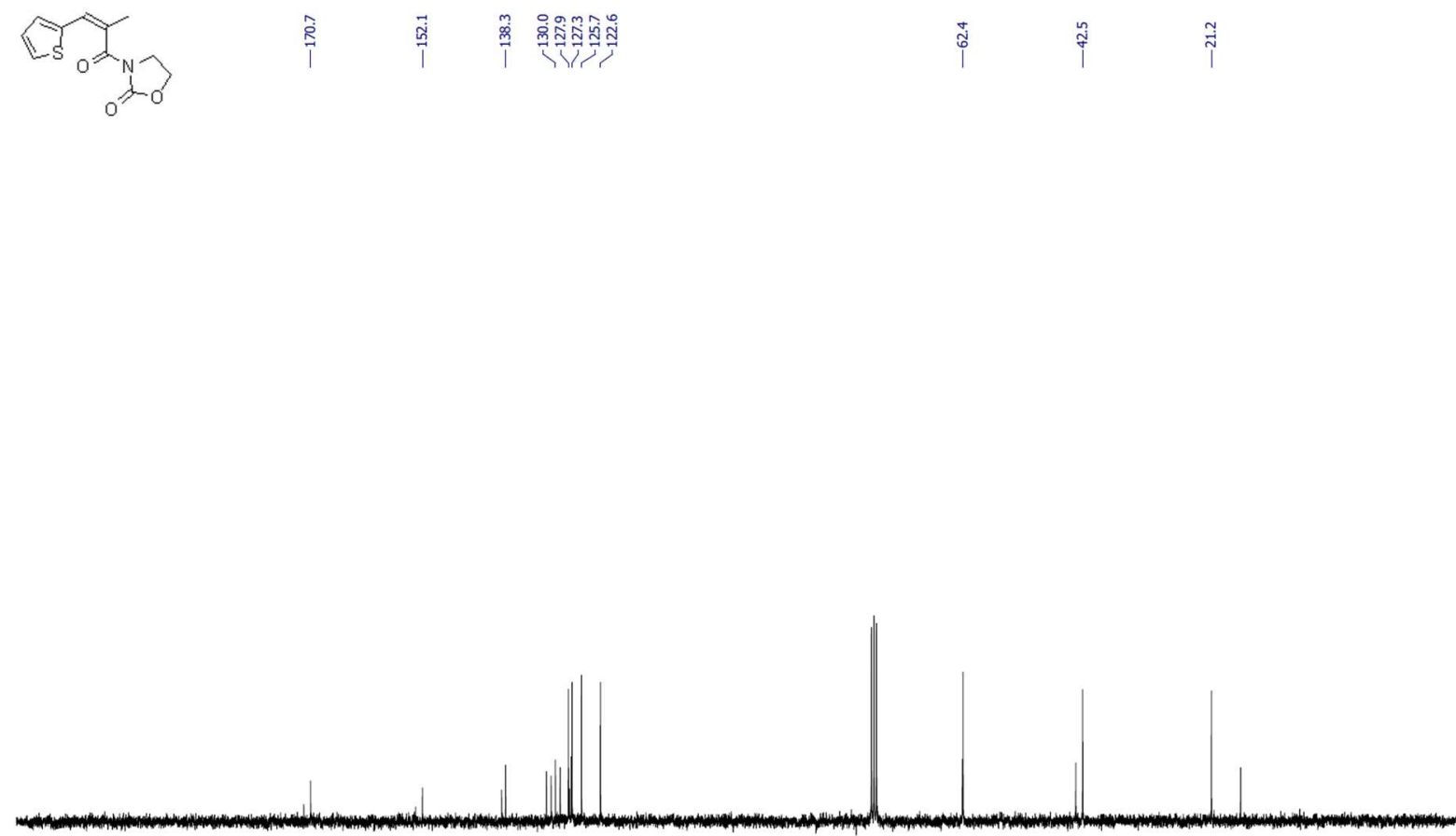

$\begin{array}{lllllllllll}210 & 200 & 190 & 180 & 170 & 160 & 150 & 140 & 130 & 120 & 110 \\ \mathrm{f} 1 & \begin{array}{l}100 \\ (\mathrm{ppm})\end{array}\end{array}$ 
3-((Z)-3-(4-(cinnamyloxy)phenyl)-2-methylacryloyl)oxazolidin-2-one ((Z)-26):

${ }^{1} \mathrm{H} \mathrm{NMR}\left(\mathrm{CDCl}_{3}, 300 \mathrm{MHz}\right)$
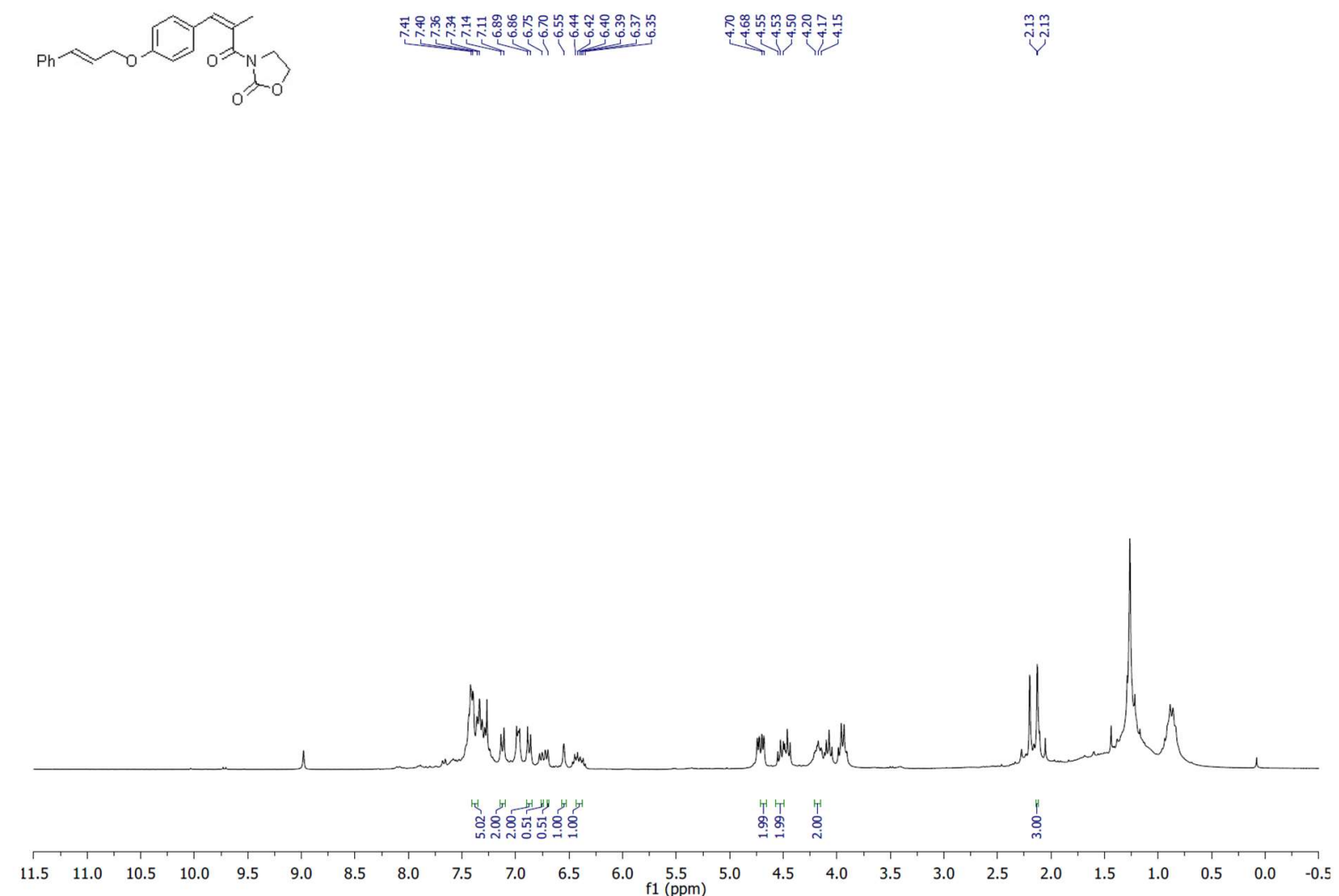

${ }^{13} \mathrm{C} \mathrm{NMR}\left(\mathrm{CDCl}_{3}, 75 \mathrm{MHz}\right)$
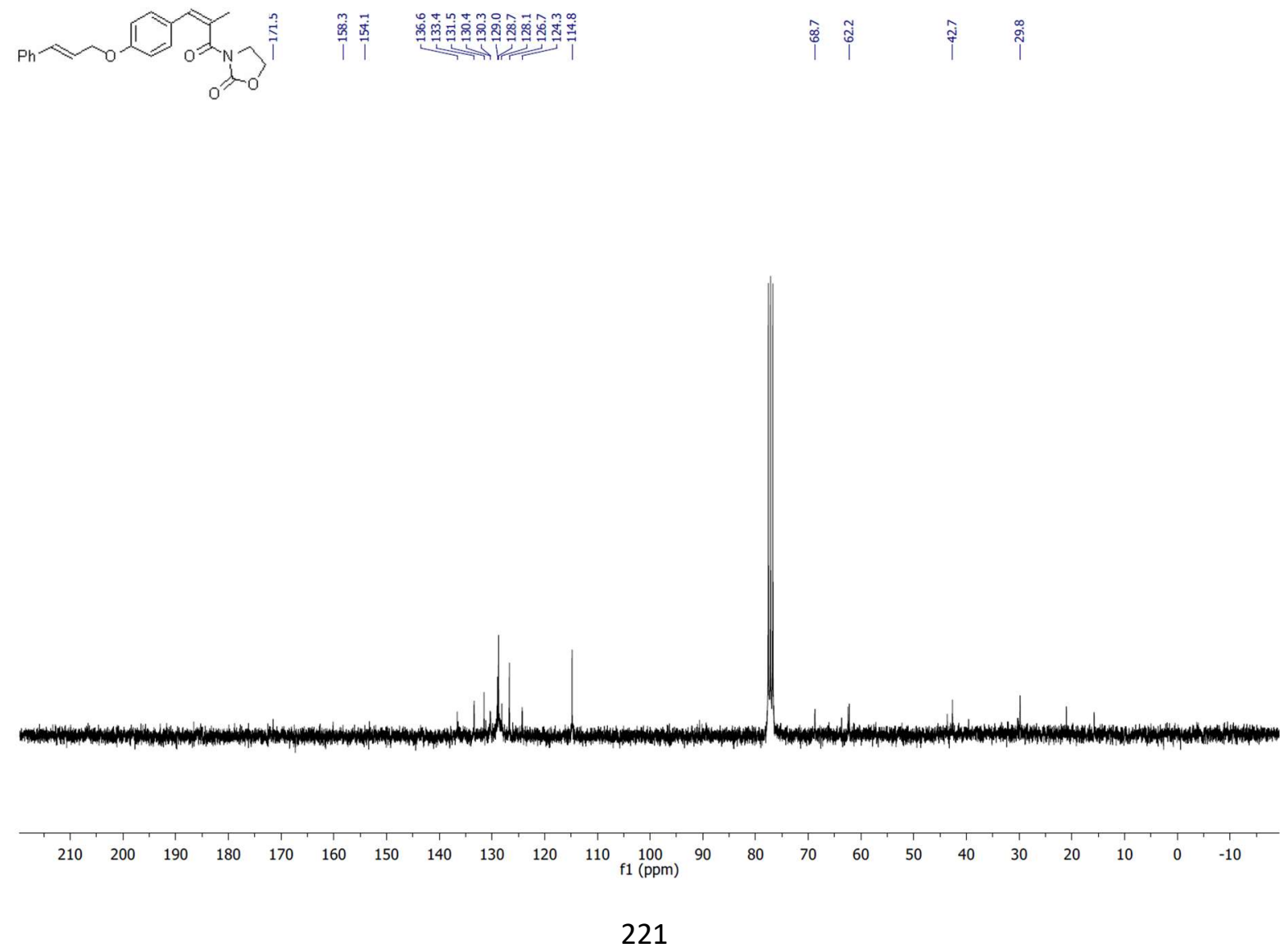
(Z)-3-(3-phenylbut-2-enoyl)oxazolidin-2-one ((Z)-29):

${ }^{1} \mathrm{H} \mathrm{NMR}\left(\mathrm{CDCl}_{3}, 300 \mathrm{MHz}\right)$
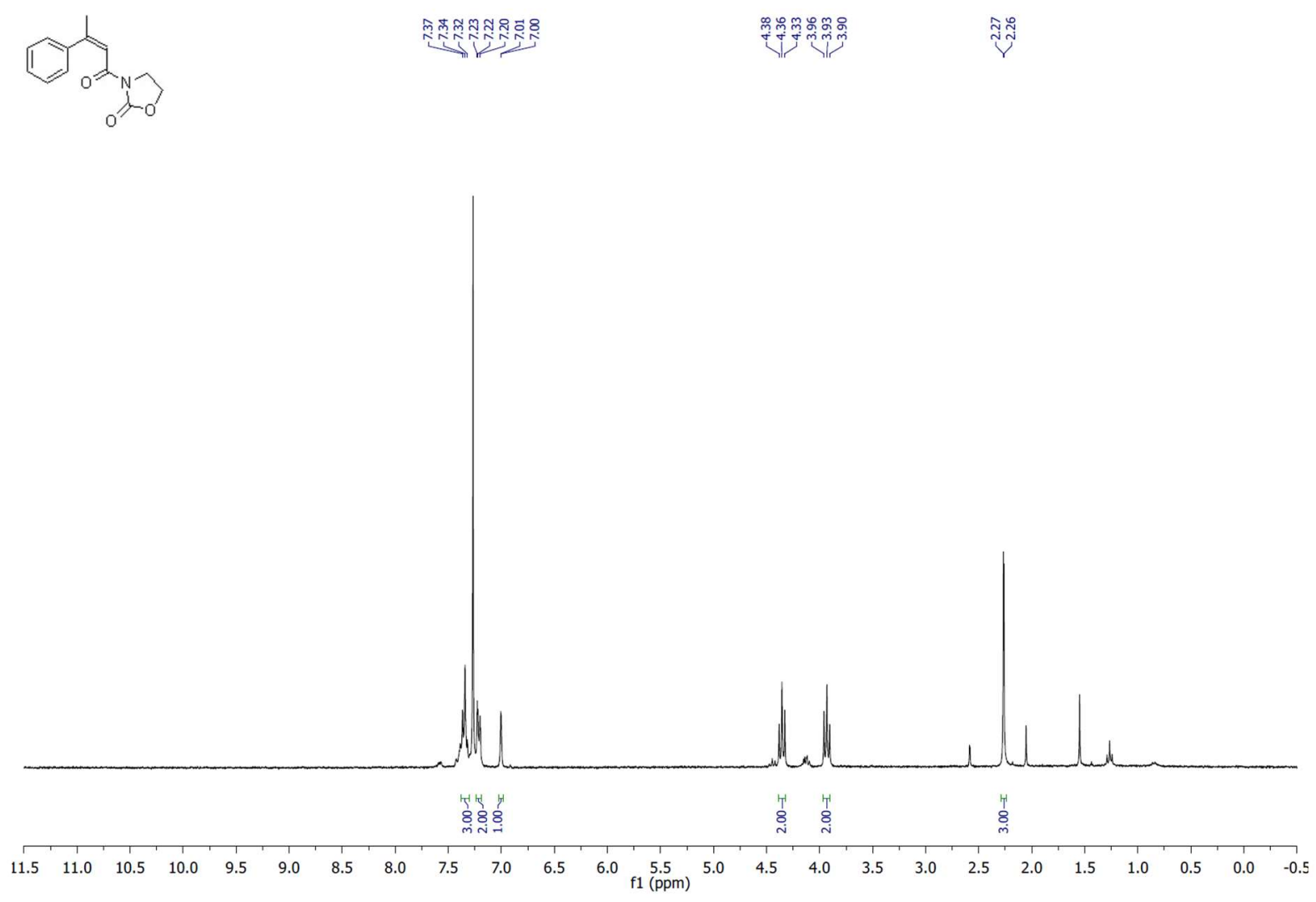

${ }^{13} \mathrm{C} \mathrm{NMR}\left(\mathrm{CDCl}_{3}, 75 \mathrm{MHz}\right)$
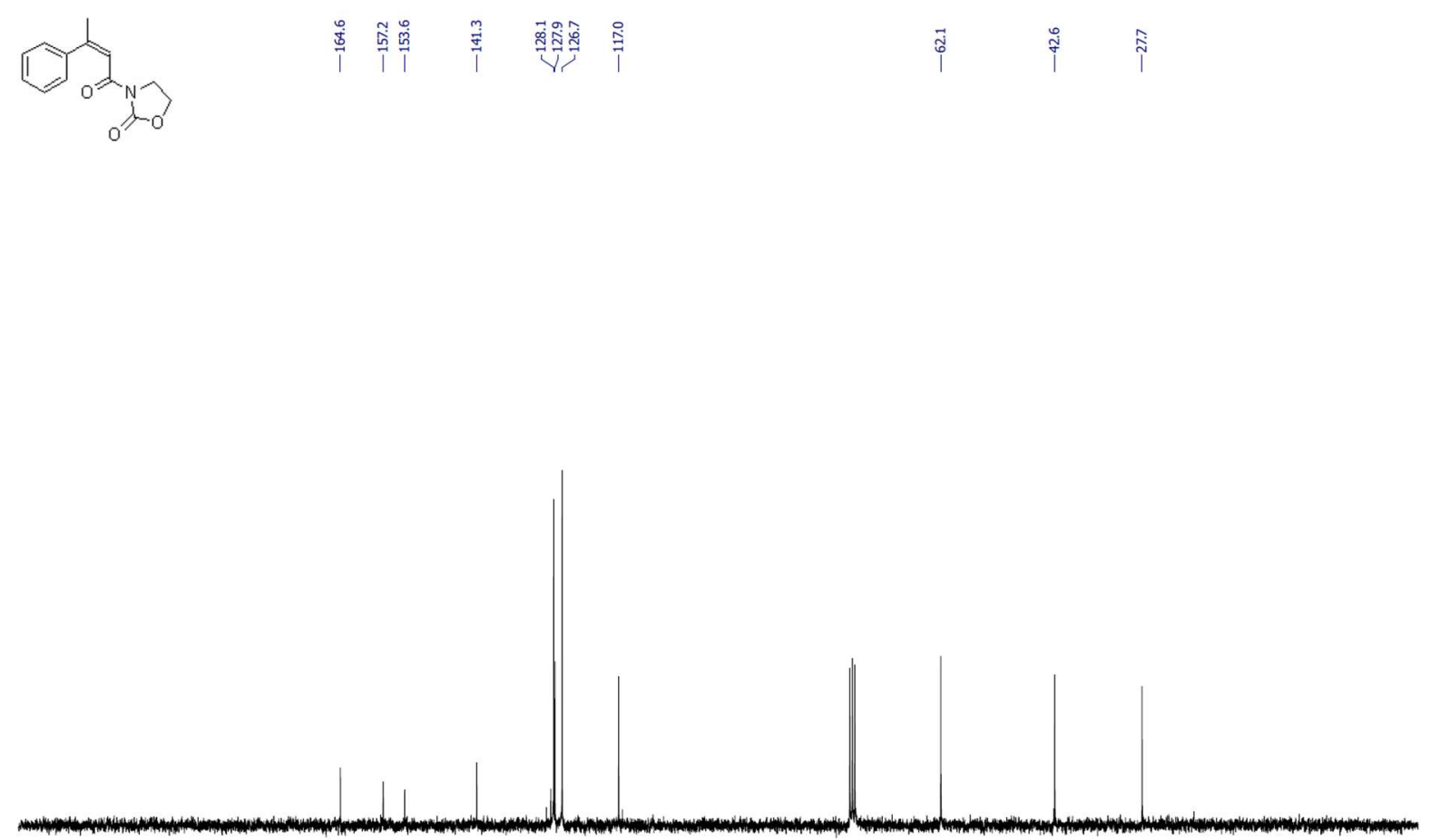

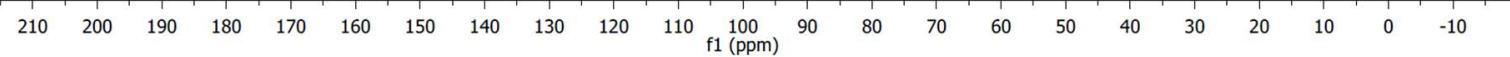


ethyl (Z)-3-phenylbut-2-enoate ((Z)-30):

${ }^{1} \mathrm{H} \mathrm{NMR}\left(\mathrm{CDCl}_{3}, 300 \mathrm{MHz}\right)$<smiles>CCOC(=O)/C=C(/C)c1ccccc1</smiles>

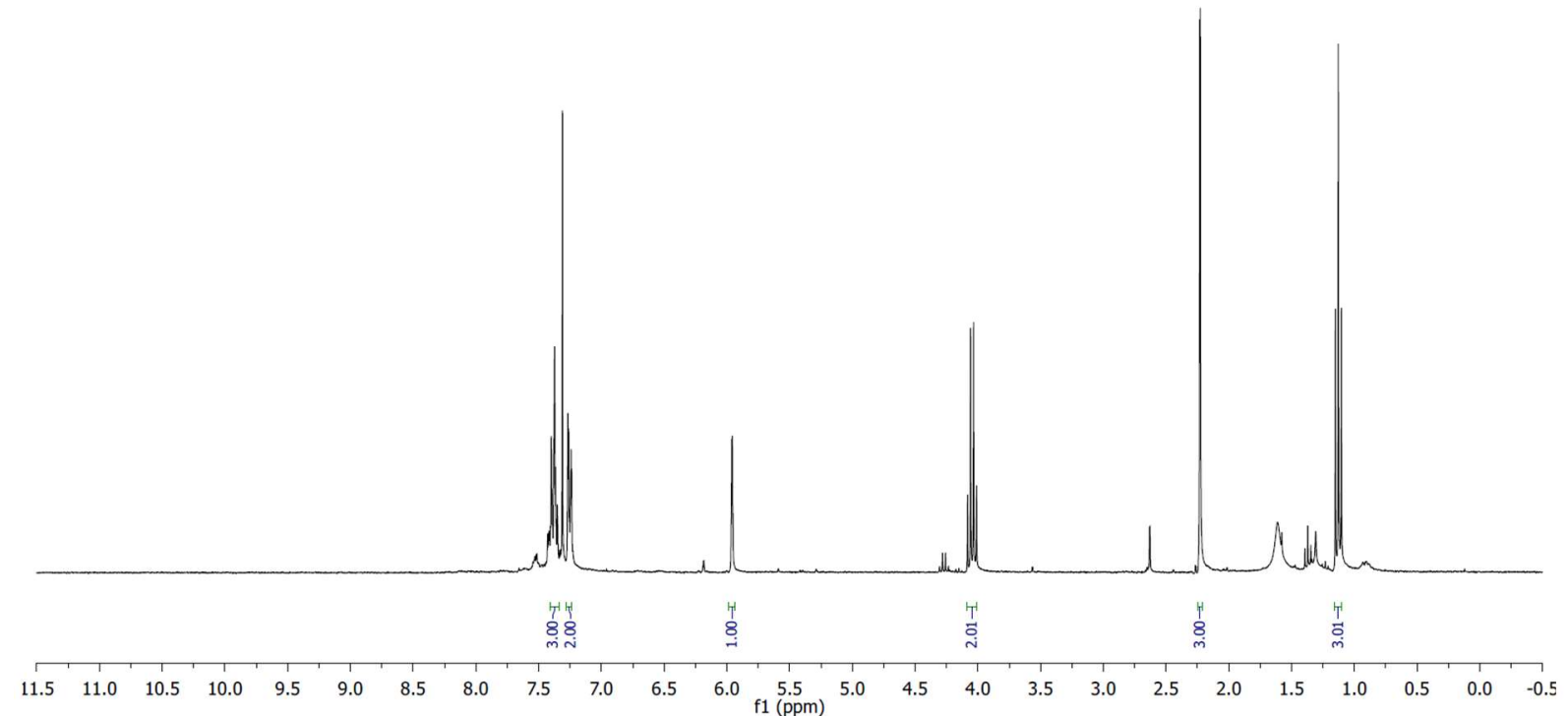

${ }^{13} \mathrm{C} \mathrm{NMR}\left(\mathrm{CDCl}_{3}, 75 \mathrm{MHz}\right)$<smiles>CCOC(=O)/C=C(/C)c1ccccc1</smiles>

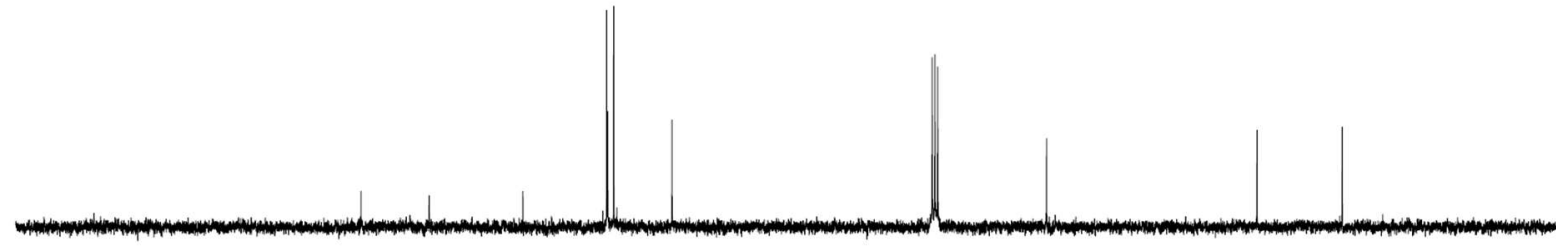

$\begin{array}{llllllllllll}210 & 200 & 190 & 180 & 170 & 160 & 150 & 140 & 130 & 120 & 110 & \begin{array}{l}100 \\ \mathrm{f}(\mathrm{ppm})\end{array}\end{array}$ 
(Z)-3-(3-phenylpent-2-enoyl)oxazolidin-2-one ((Z)-31):

${ }^{1} \mathrm{H} \mathrm{NMR}\left(\mathrm{CDCl}_{3}, 300 \mathrm{MHz}\right)$
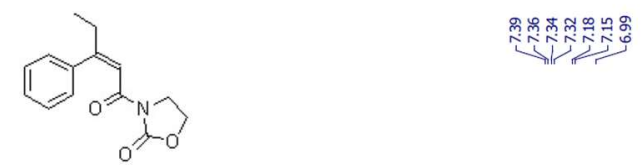

$i_{0 \rightarrow 0}$

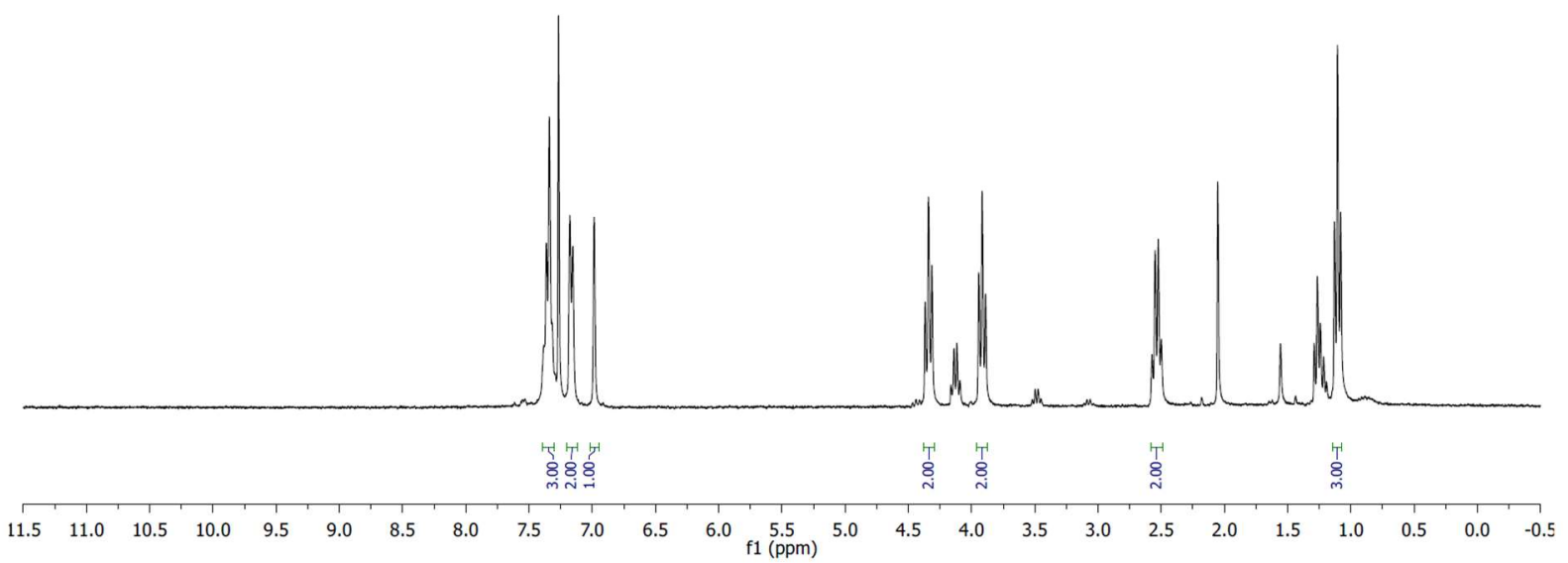

${ }^{13} \mathrm{C} \mathrm{NMR}\left(\mathrm{CDCl}_{3}, 75 \mathrm{MHz}\right)$<smiles>CC/C(=C/C(=O)N1CCCO1)c1ccccc1</smiles>

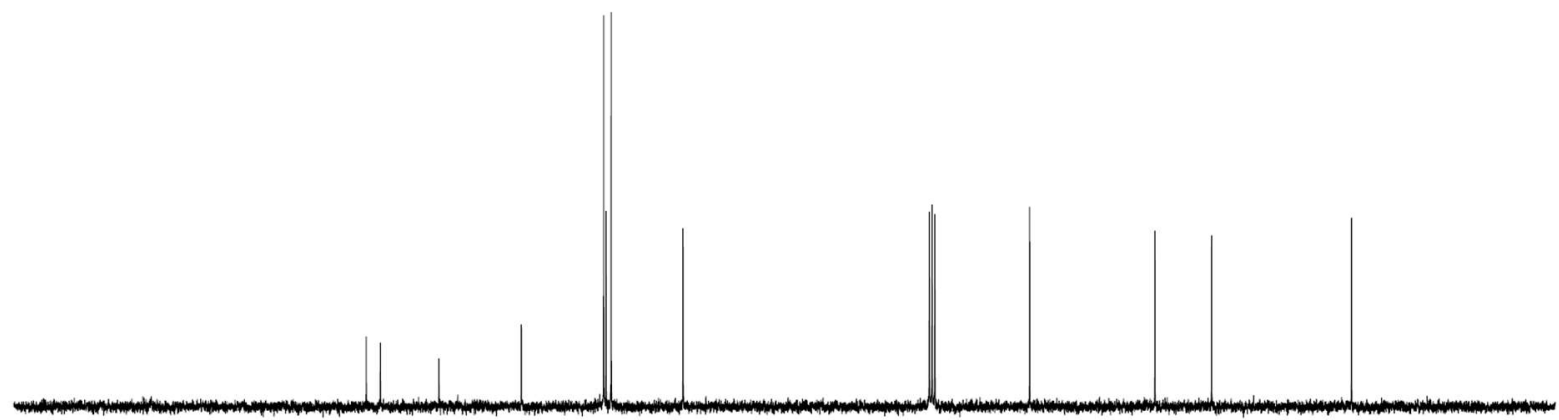

$\begin{array}{lllllllllll}210 & 200 & 190 & 180 & 170 & 160 & 150 & 140 & 130 & 120 & 110 \\ \mathrm{f} 1(\mathrm{ppm})\end{array}$ 
ethyl (Z)-3-phenylpent-2-enoate ((Z)-32):

${ }^{1} \mathrm{H} \mathrm{NMR}\left(\mathrm{CDCl}_{3}, 300 \mathrm{MHz}\right)$
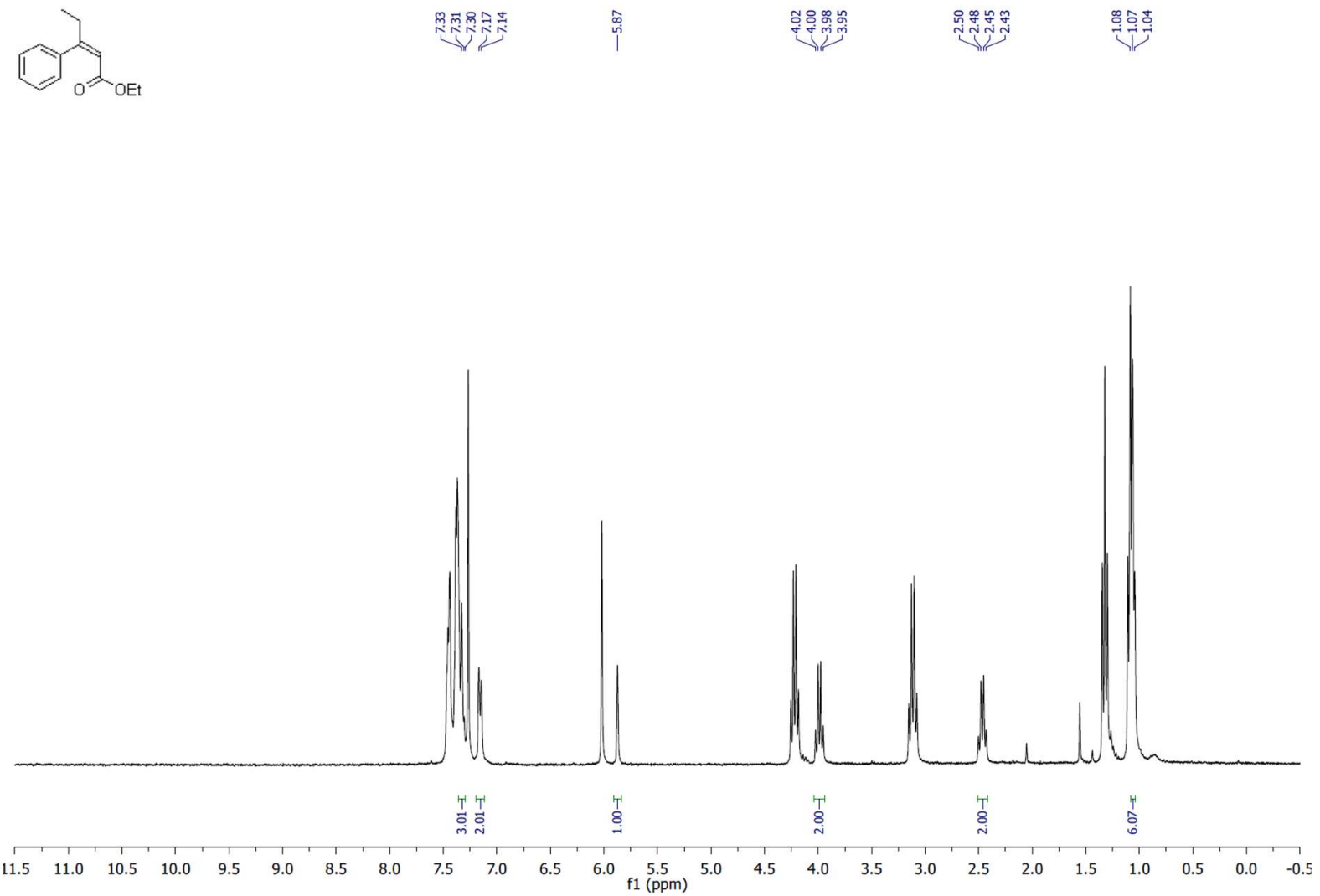

${ }^{13} \mathrm{C} \mathrm{NMR}\left(\mathrm{CDCl}_{3}, 75 \mathrm{MHz}\right)$

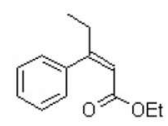

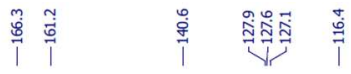

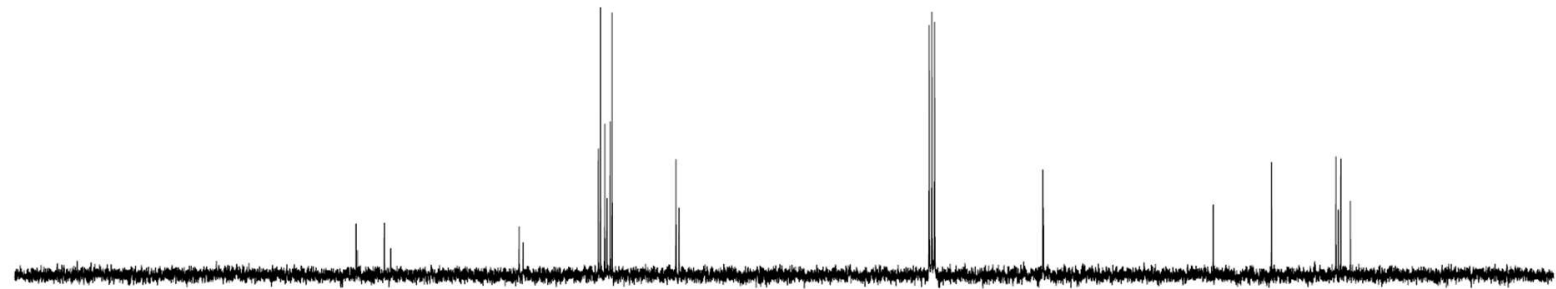

$\begin{array}{lllllllllll}210 & 200 & 190 & 180 & 170 & 160 & 150 & 140 & 130 & 120 & 110 \begin{array}{c}100 \\ \mathrm{f} 1(\mathrm{ppm})\end{array}\end{array}$ 
(Z)-3-(3-(p-tolyl)but-2-enoyl)oxazolidin-2-one ((Z)-33):

${ }^{1} \mathrm{H} \mathrm{NMR}\left(\mathrm{CDCl}_{3}, 300 \mathrm{MHz}\right)$
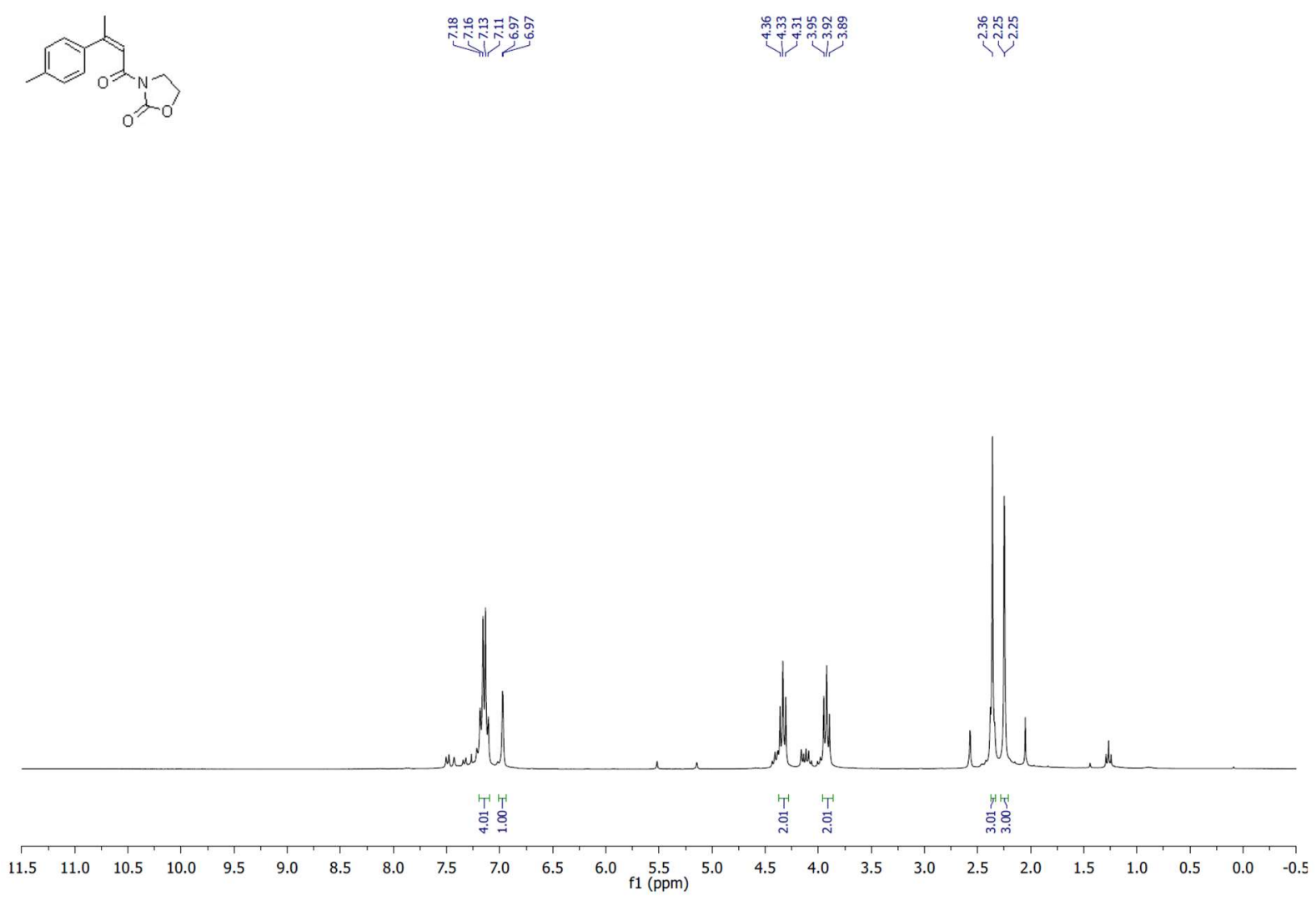

${ }^{13} \mathrm{C} \mathrm{NMR}\left(\mathrm{CDCl}_{3}, 75 \mathrm{MHz}\right)$

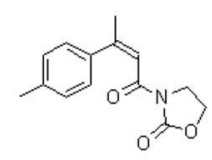

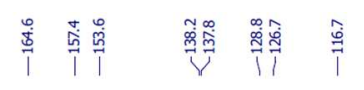

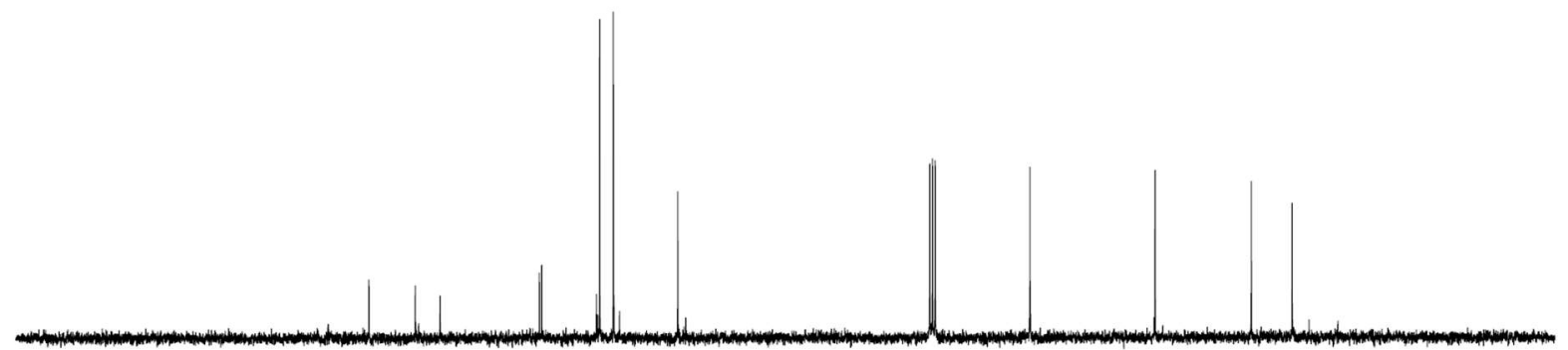

$\begin{array}{lllllllllll}210 & 200 & 190 & 180 & 170 & 160 & 150 & 140 & 130 & 120 & 110 \\ \mathrm{f} 1(\mathrm{ppm})\end{array}$ 
ethyl (Z)-3-(p-tolyl)but-2-enoate ((Z)-34):

${ }^{1} \mathrm{H} \mathrm{NMR}\left(\mathrm{CDCl}_{3}, 300 \mathrm{MHz}\right)$
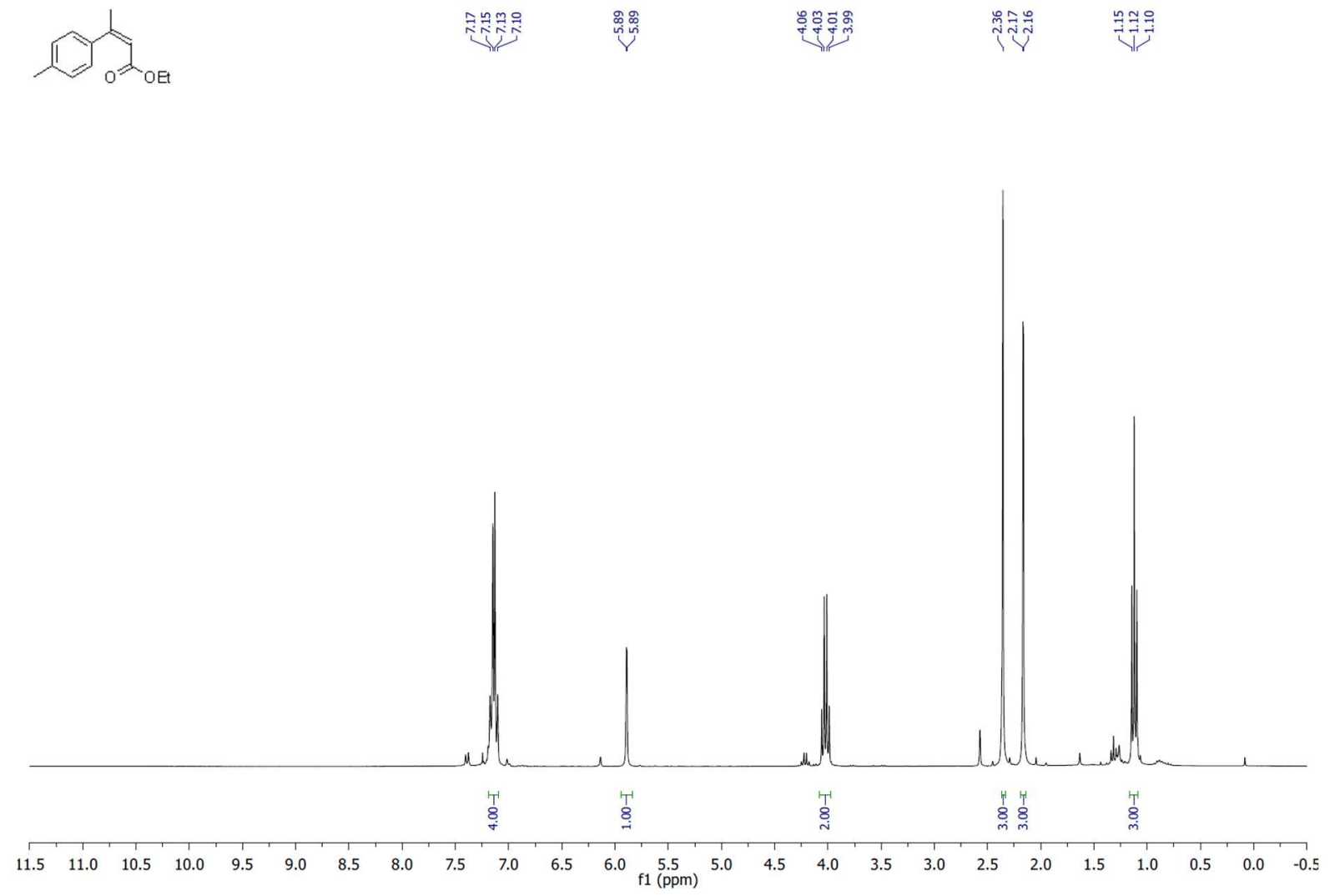

${ }^{13} \mathrm{C} \mathrm{NMR}\left(\mathrm{CDCl}_{3}, 75 \mathrm{MHz}\right)$
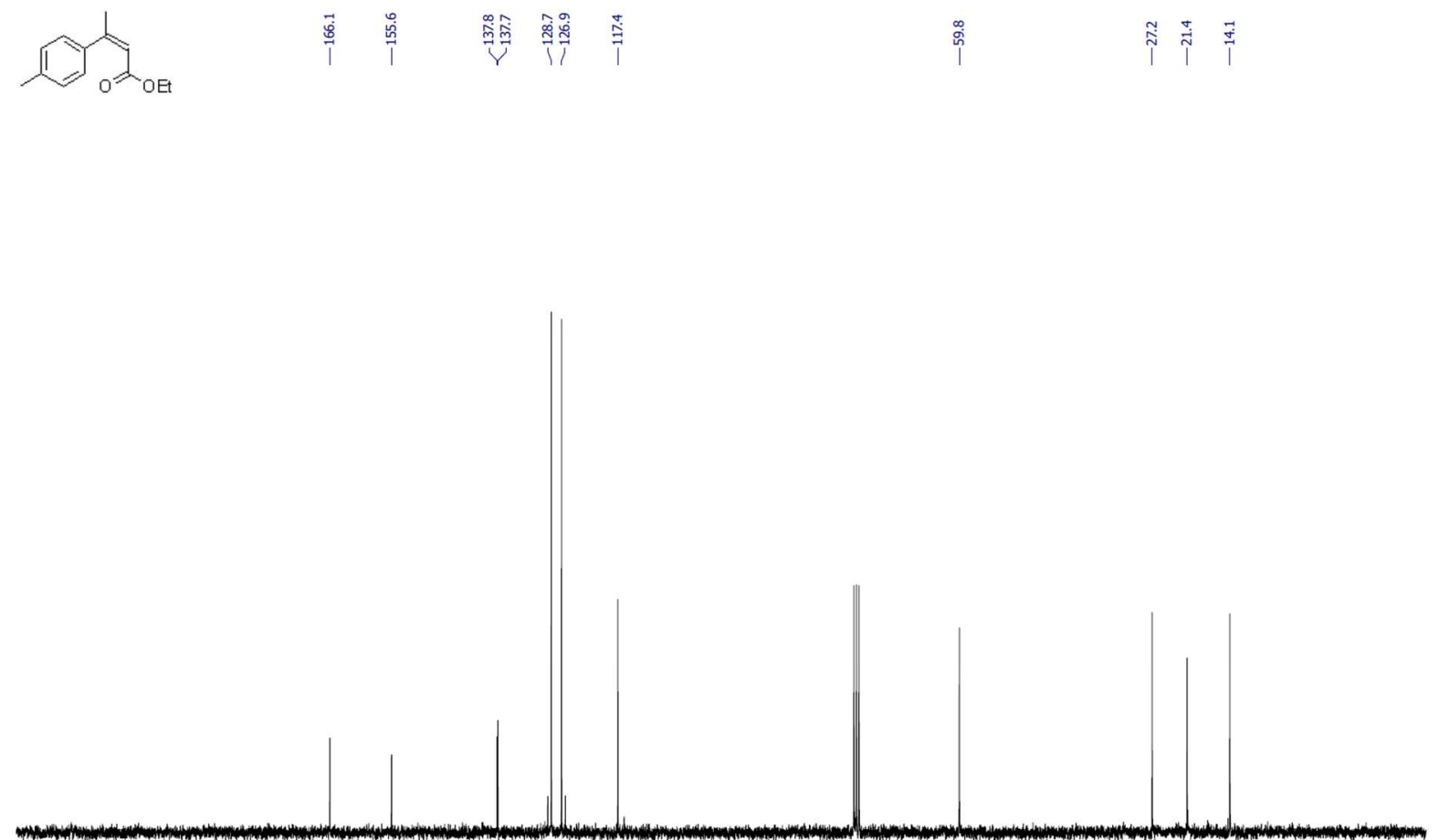

$\begin{array}{lllllllllll}210 & 200 & 190 & 180 & 170 & 160 & 150 & 140 & 130 & 120 & 110 \\ \mathrm{f} 1(\mathrm{ppm})\end{array}$ 
(Z)-3-(3-(m-tolyl)but-2-enoyl)oxazolidin-2-one ((Z)-35):

${ }^{1} \mathrm{H} \mathrm{NMR}\left(\mathrm{CDCl}_{3}, 300 \mathrm{MHz}\right)$<smiles>Cc1cccc(C(C)C)c1NC(=O)C1CC2CCC1C2</smiles>

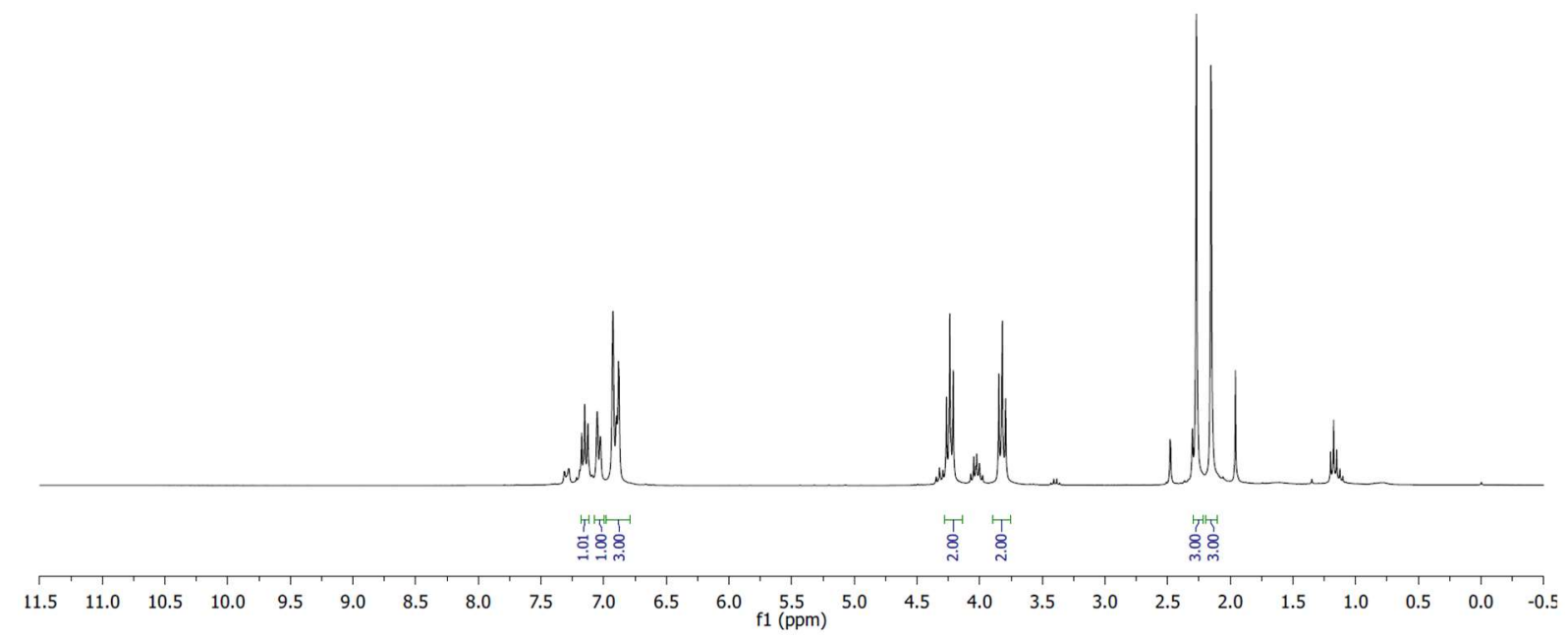

${ }^{13} \mathrm{C} \mathrm{NMR}\left(\mathrm{CDCl}_{3}, 75 \mathrm{MHz}\right)$
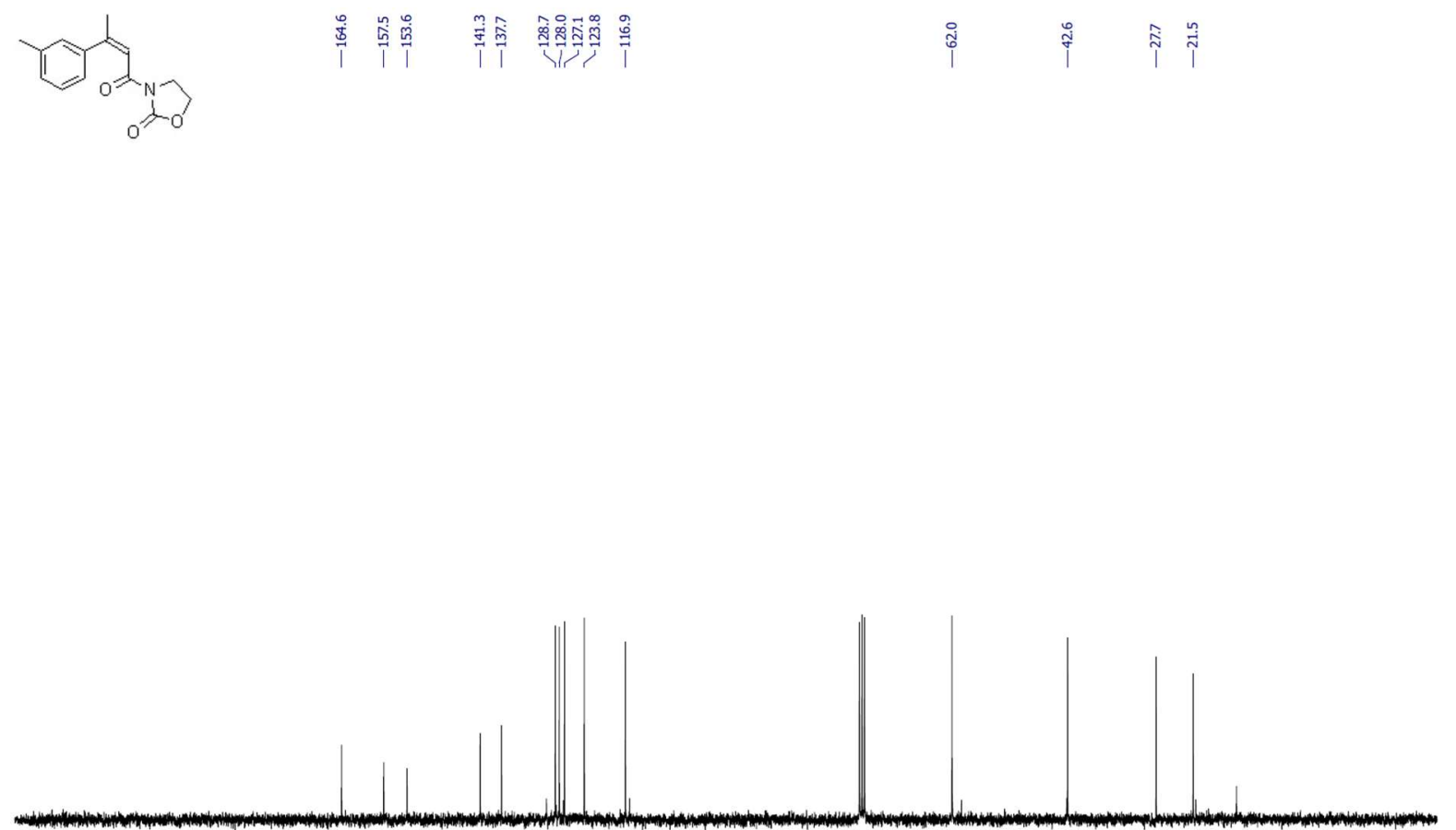

$\begin{array}{lllllllllll}210 & 200 & 190 & 180 & 170 & 160 & 150 & 140 & 130 & 120 & 110 \begin{array}{c}100 \\ \mathrm{f} 1(\mathrm{ppm})\end{array}\end{array}$ 
ethyl (Z)-3-(m-tolyl)but-2-enoate ((Z)-36):

${ }^{1} \mathrm{H} \mathrm{NMR}\left(\mathrm{CDCl}_{3}, 300 \mathrm{MHz}\right)$

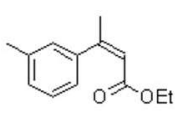

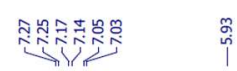

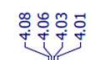

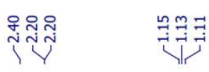

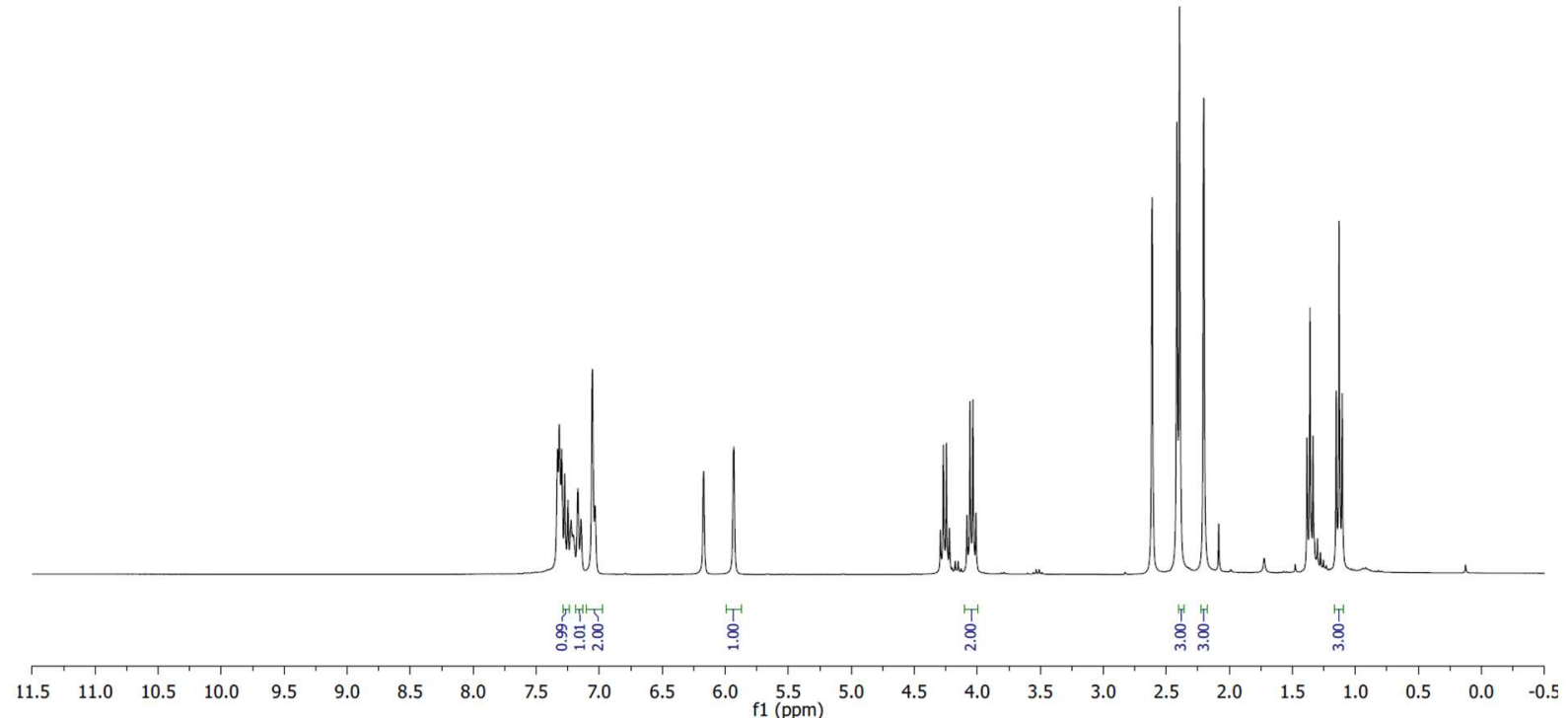

${ }^{13} \mathrm{C} \mathrm{NMR}\left(\mathrm{CDCl}_{3}, 75 \mathrm{MHz}\right)$

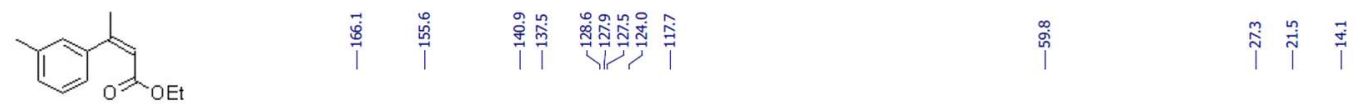

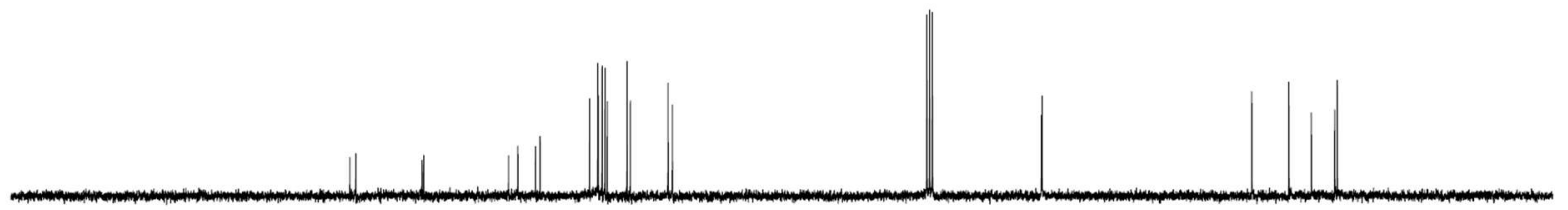

$\begin{array}{lllllllllllllllllllllll}210 & 200 & 190 & 180 & 170 & 160 & 150 & 140 & 130 & 120 & 110 & 100 & 90 & 80 & 70 & 60 & 50 & 40 & 30 & 20 & 10 & 0 & -10\end{array}$ 
(Z)-3-(3-(o-tolyl)but-2-enoyl)oxazolidin-2-one ((Z)-37):

${ }^{1} \mathrm{H} \mathrm{NMR}\left(\mathrm{CDCl}_{3}, 300 \mathrm{MHz}\right)$<smiles>C/C(=C/C(=O)N1CCOC1=O)c1ccccc1C</smiles>

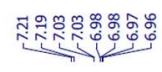

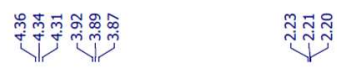

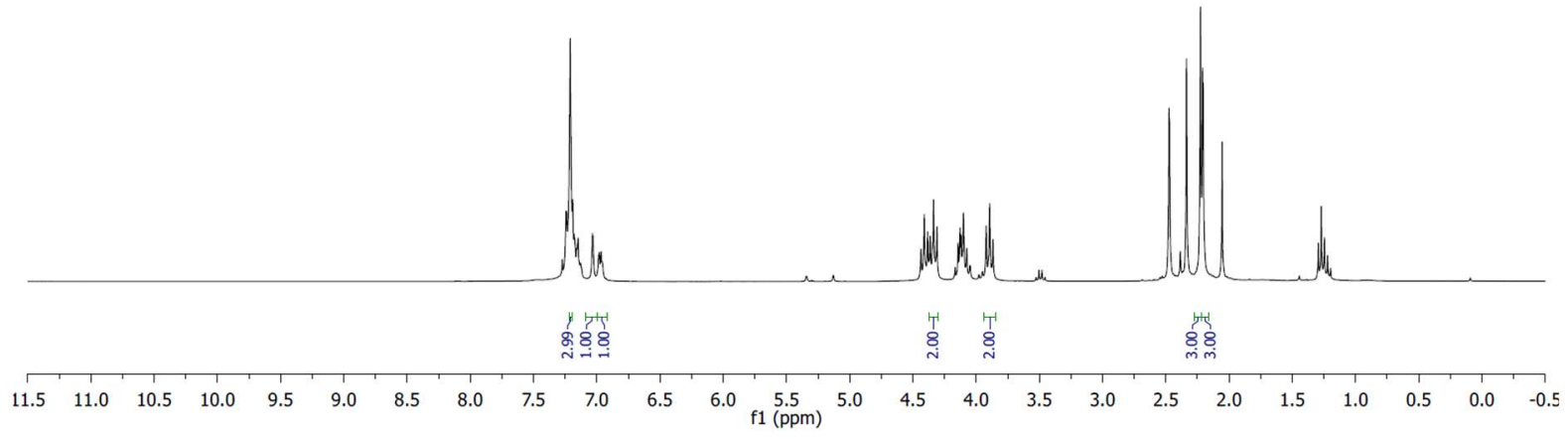

${ }^{13} \mathrm{C} \mathrm{NMR}\left(\mathrm{CDCl}_{3}, 75 \mathrm{MHz}\right)$<smiles>Cc1ccccc1C(=O)N1CCCC1</smiles>

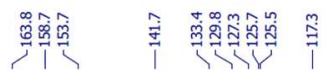

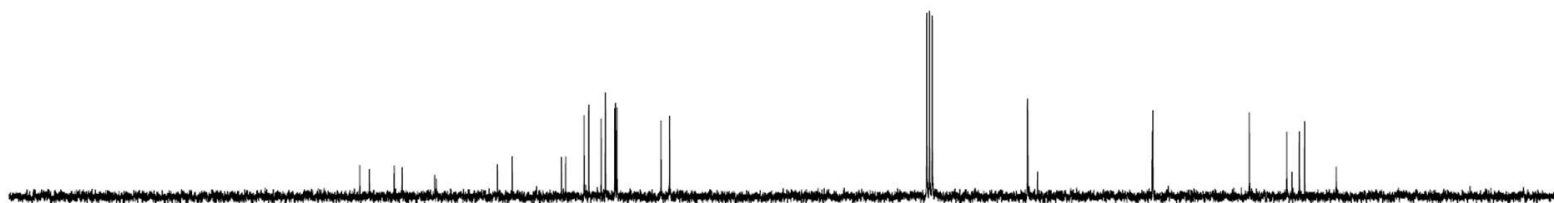

$\begin{array}{lllllllllll}210 & 200 & 190 & 180 & 170 & 160 & 150 & 140 & 130 & 120 & 110 \\ \mathrm{f} 1(\mathrm{ppm})\end{array}$ 
(Z)-3-(3-(3-(tert-butyl)phenyl)but-2-enoyl)oxazolidin-2-one ((Z)-39):

${ }^{1} \mathrm{H} \mathrm{NMR}\left(\mathrm{CDCl}_{3}, 300 \mathrm{MHz}\right)$
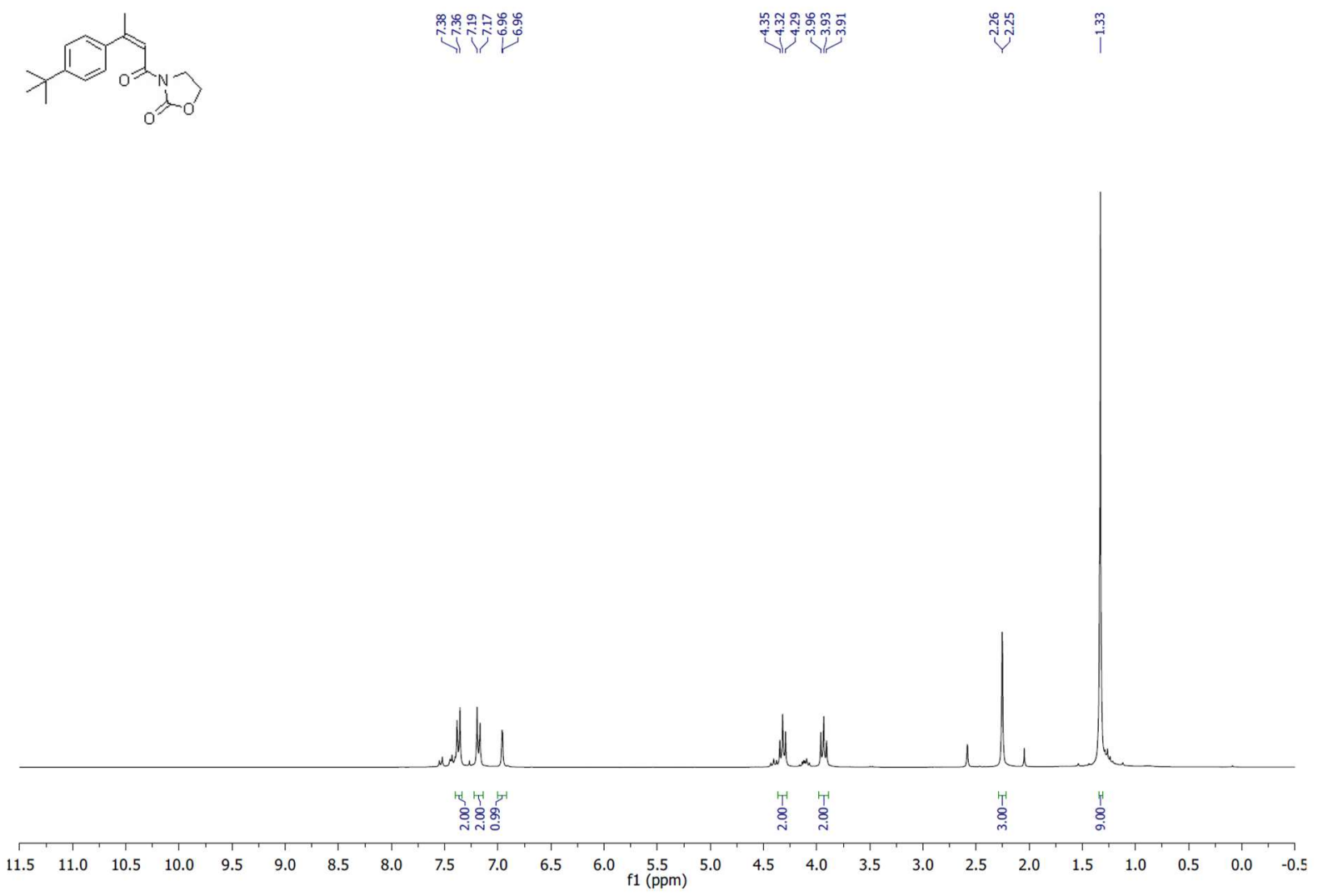

${ }^{13} \mathrm{C} \mathrm{NMR}\left(\mathrm{CDCl}_{3}, 75 \mathrm{MHz}\right)$
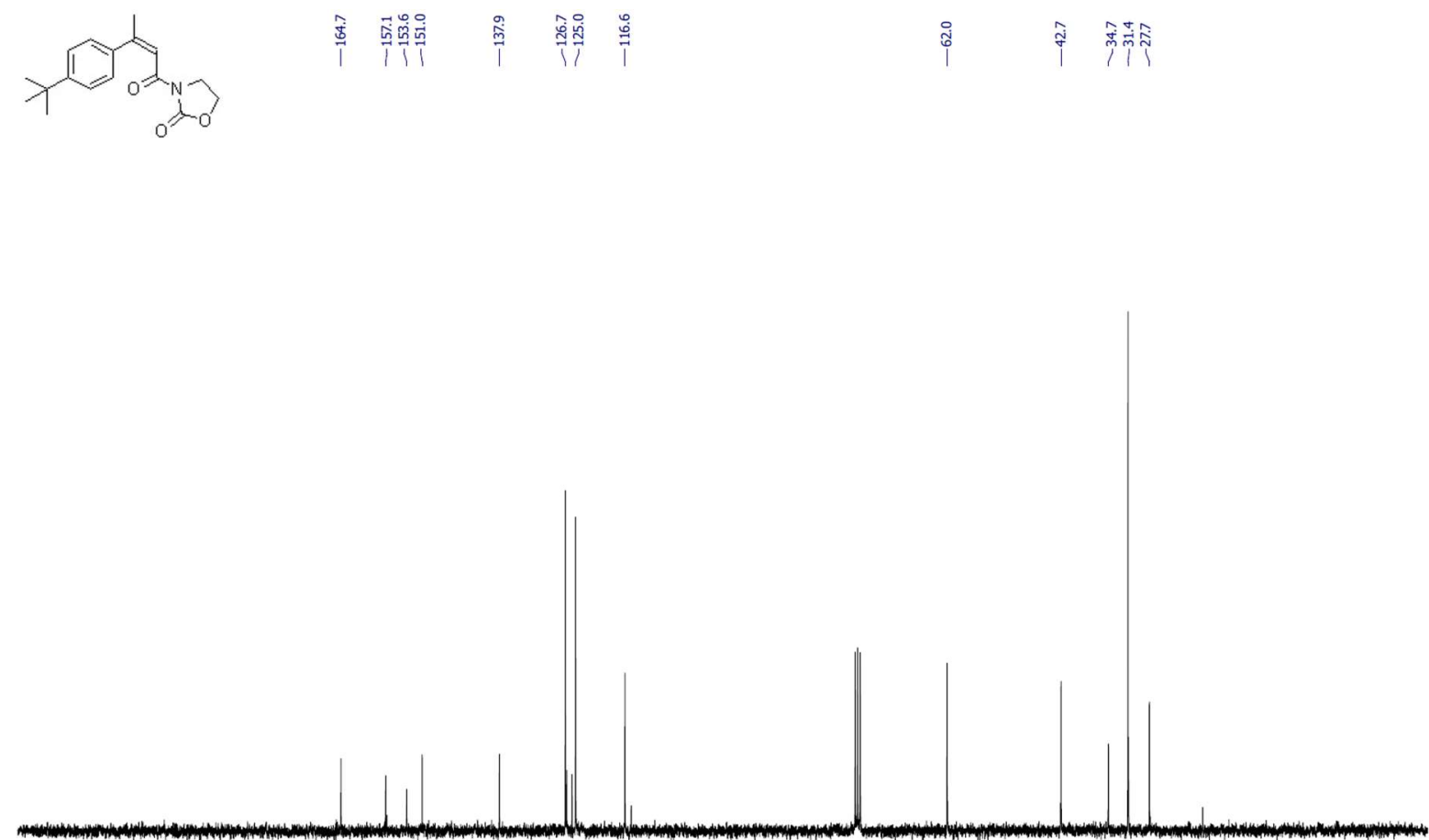

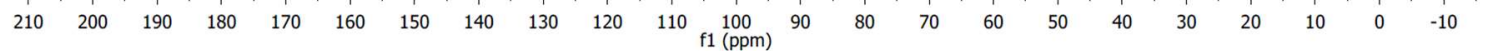


ethyl (Z)-3-(3-(tert-butyl)phenyl)but-2-enoate ((Z)-40):

${ }^{1} \mathrm{H} \mathrm{NMR}\left(\mathrm{CDCl}_{3}, 300 \mathrm{MHz}\right)$

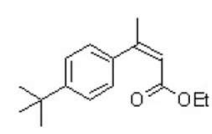

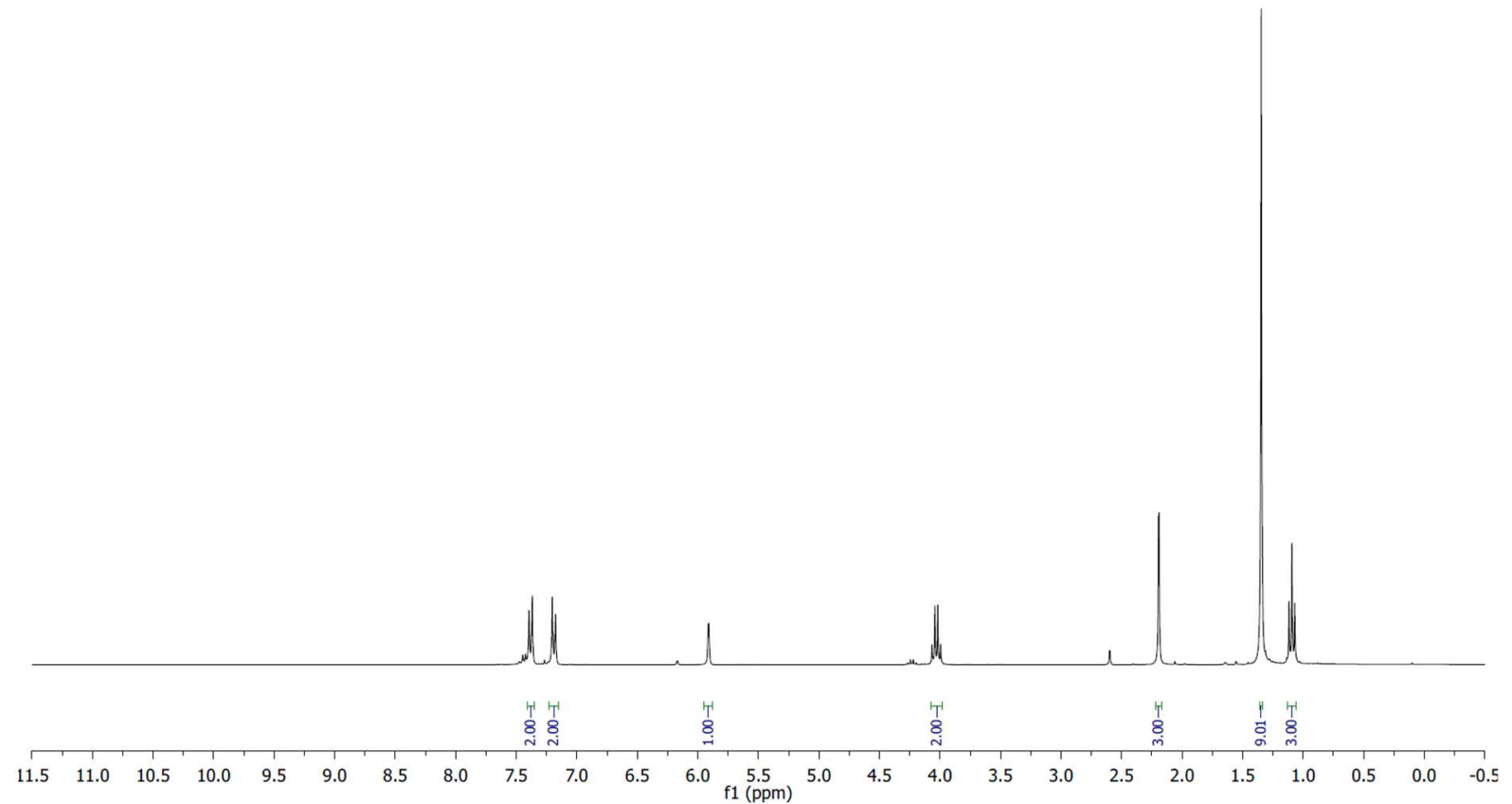

${ }^{13} \mathrm{C} \mathrm{NMR}\left(\mathrm{CDCl}_{3}, 75 \mathrm{MHz}\right)$

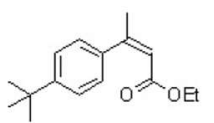

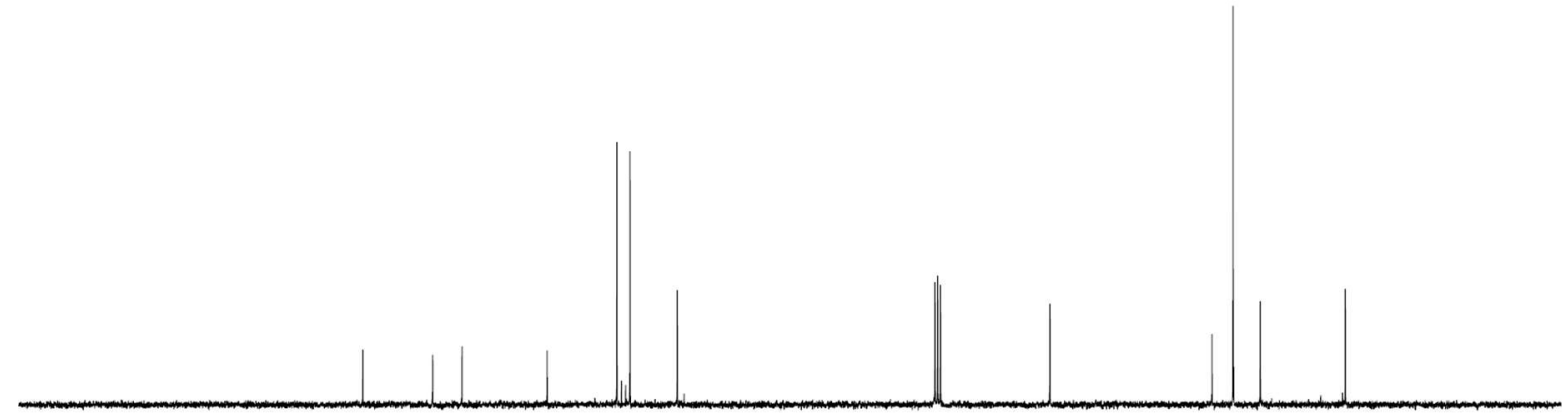

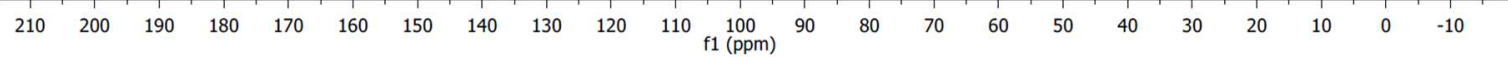


(Z)-3-(3-(4-methoxyphenyl)but-2-enoyl)oxazolidin-2-one ((Z)-41):

${ }^{1} \mathrm{H} \mathrm{NMR}\left(\mathrm{CDCl}_{3}, 300 \mathrm{MHz}\right)$
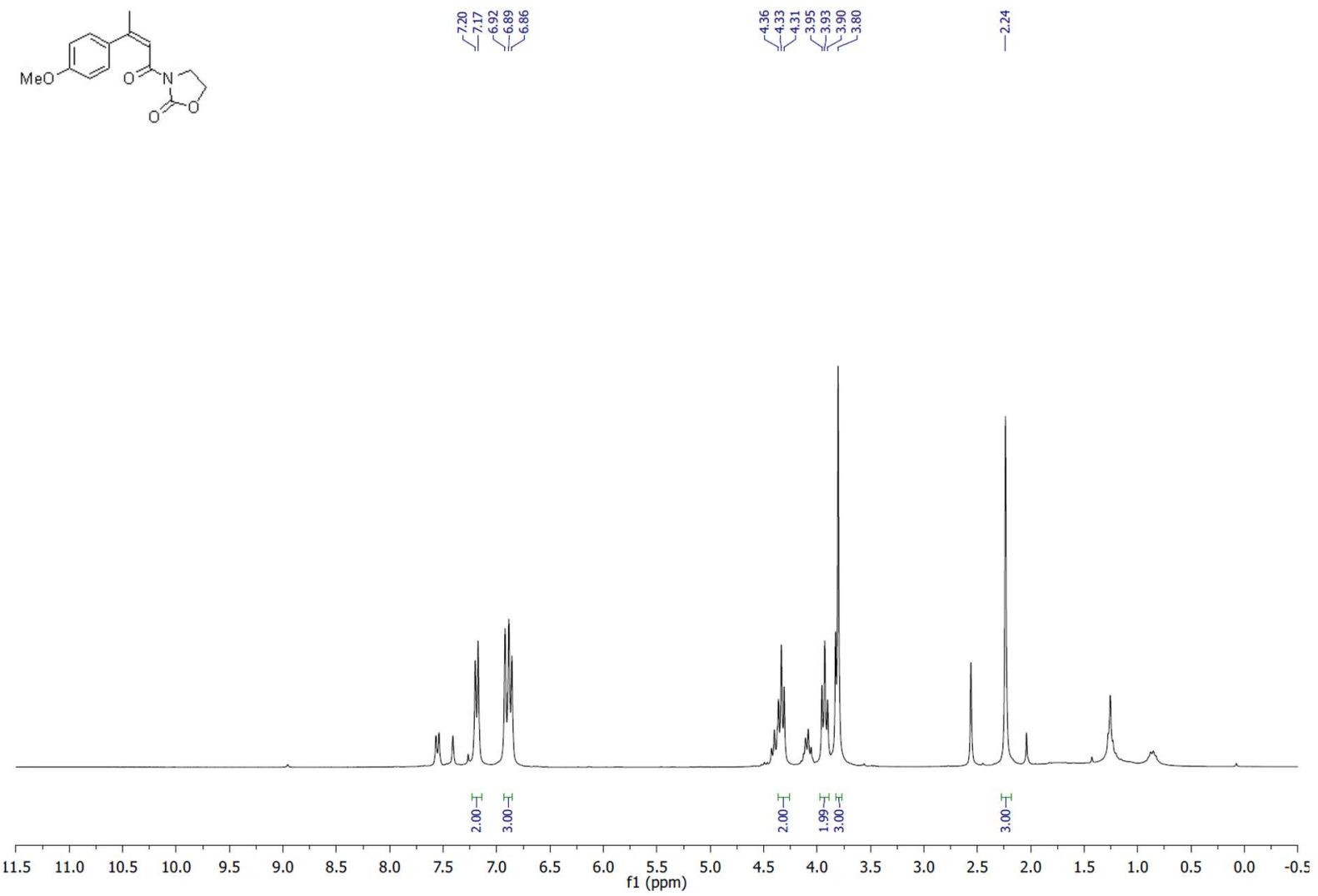

${ }^{13} \mathrm{C} \mathrm{NMR}\left(\mathrm{CDCl}_{3}, 75 \mathrm{MHz}\right)$
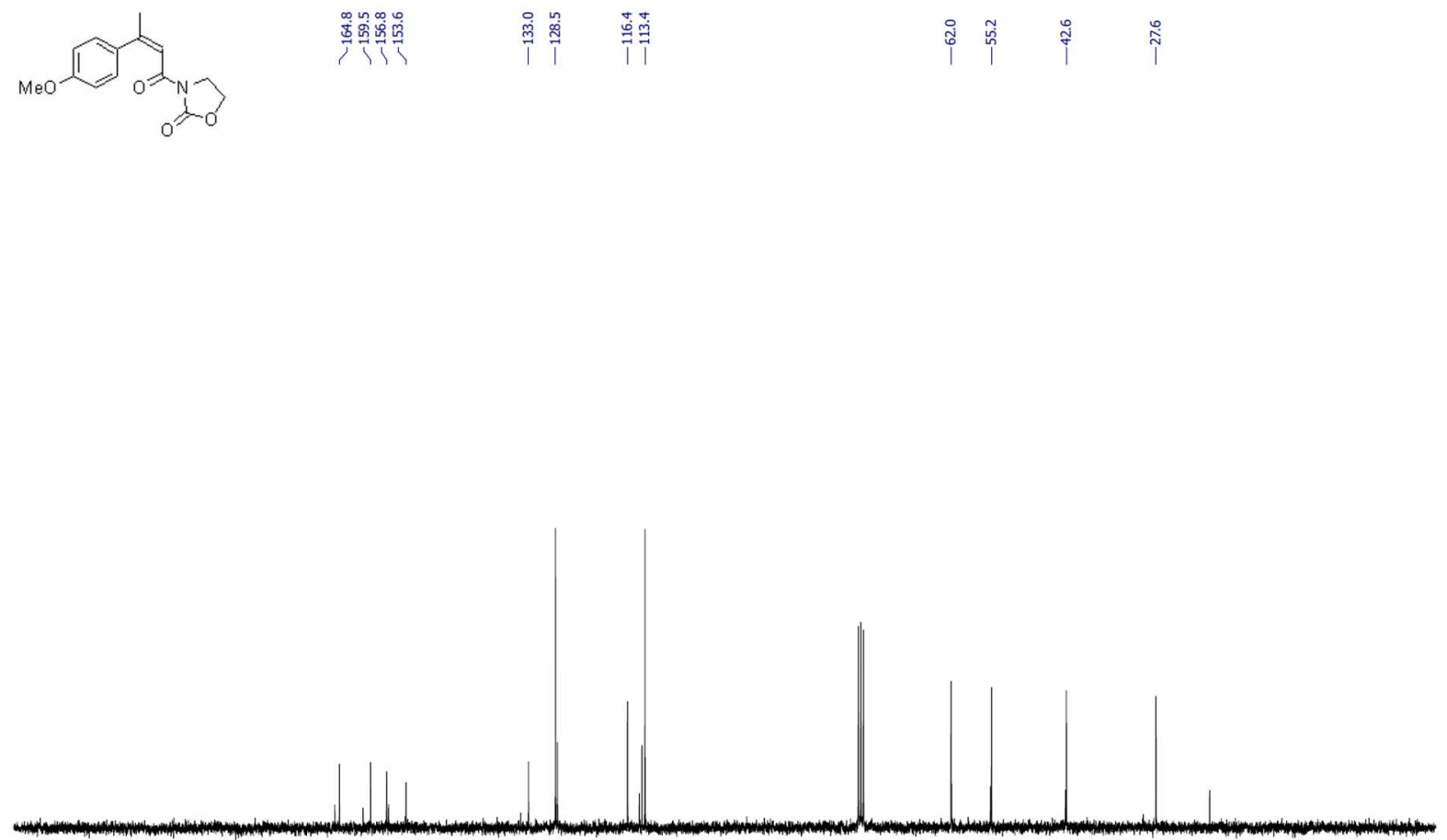

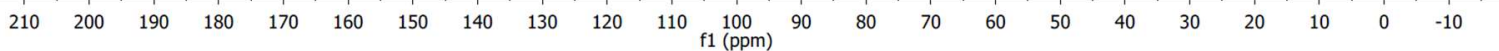


ethyl (Z)-3-(4-methoxyphenyl)but-2-enoate ((Z)-42):

${ }^{1} \mathrm{H} \mathrm{NMR}\left(\mathrm{CDCl}_{3}, 300 \mathrm{MHz}\right)$

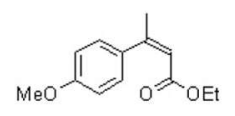

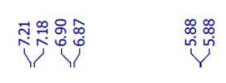

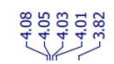

ำ

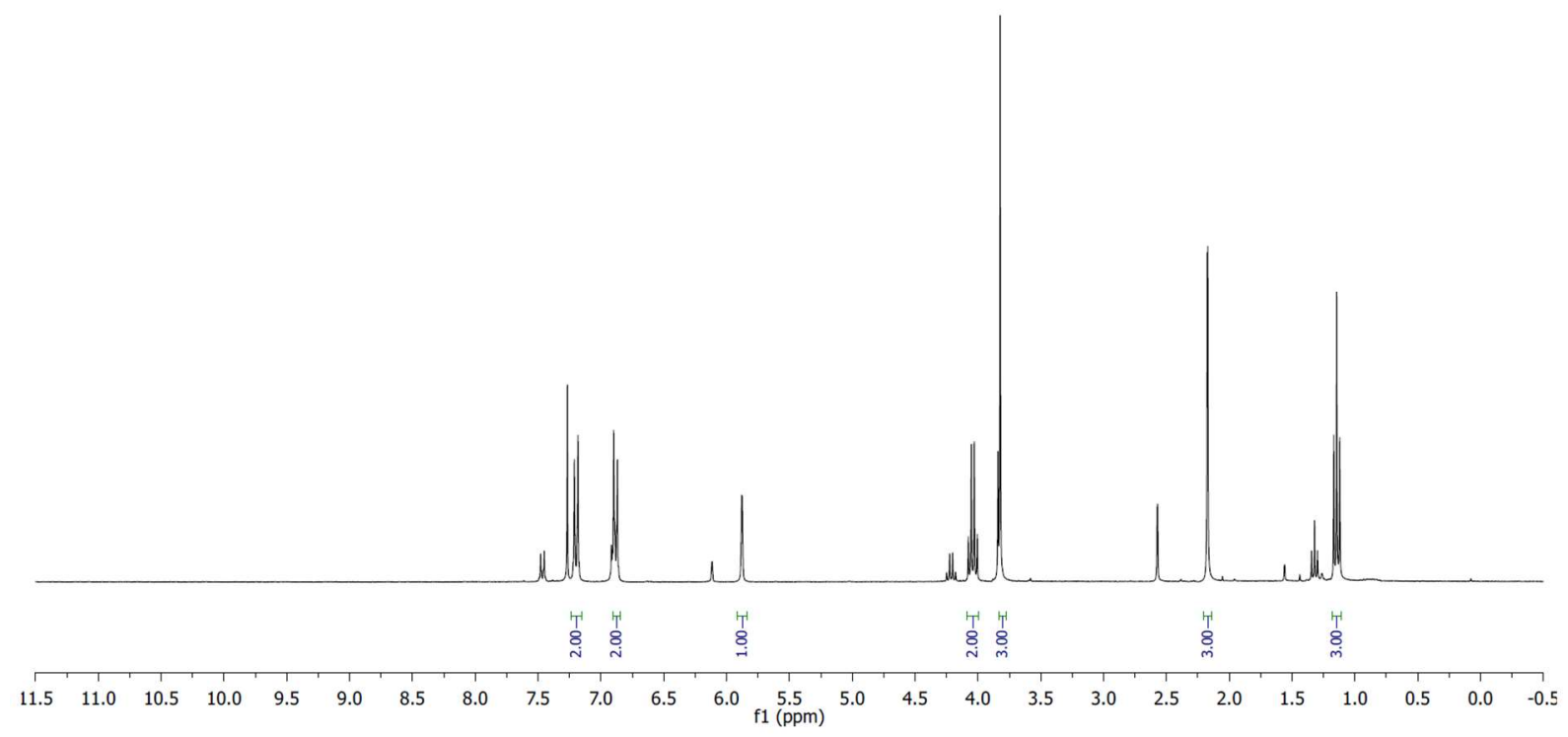

${ }^{13} \mathrm{C} \mathrm{NMR}\left(\mathrm{CDCl}_{3}, 75 \mathrm{MHz}\right)$

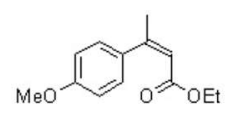

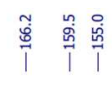

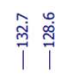

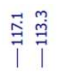

$\stackrel{\infty}{\infty}$

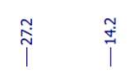

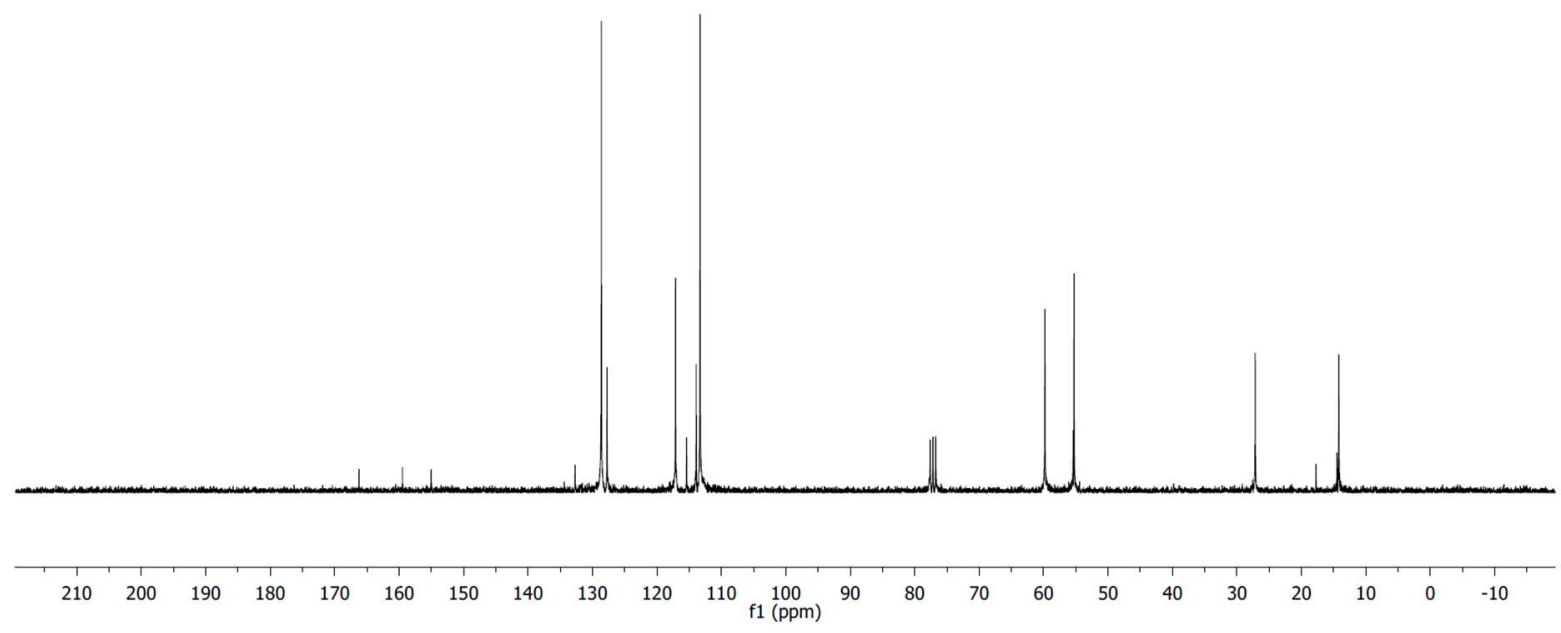

234 
(Z)-3-(3-(4-fluorophenyl)but-2-enoyl)oxazolidin-2-one ((Z)-43):

${ }^{1} \mathrm{H} \mathrm{NMR}\left(\mathrm{CDCl}_{3}, 300 \mathrm{MHz}\right)$

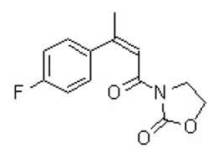

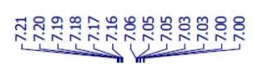

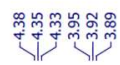

รูก บี

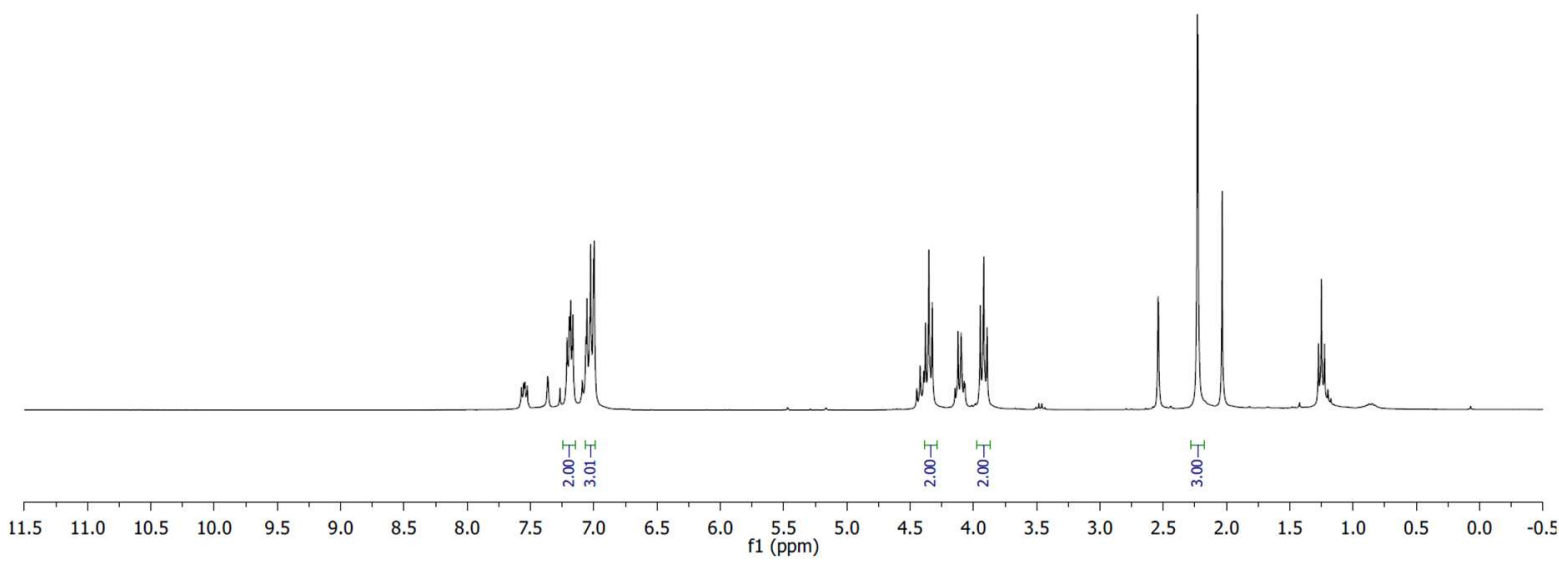

${ }^{13} \mathrm{C} \mathrm{NMR}\left(\mathrm{CDCl}_{3}, 75 \mathrm{MHz}\right)$

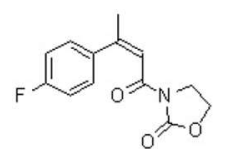

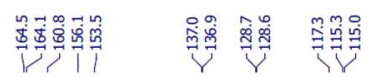

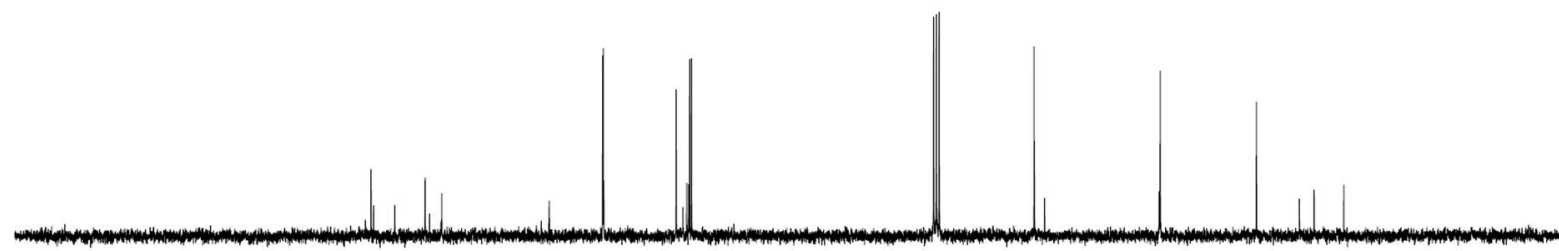

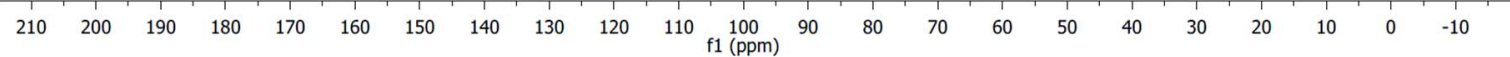


${ }^{19} \mathrm{~F} \mathrm{NMR}\left(\mathrm{CDCl}_{3}, 282 \mathrm{MHz}\right)$<smiles>Cc1cc(N2CCCO2)ccc1N=O</smiles>

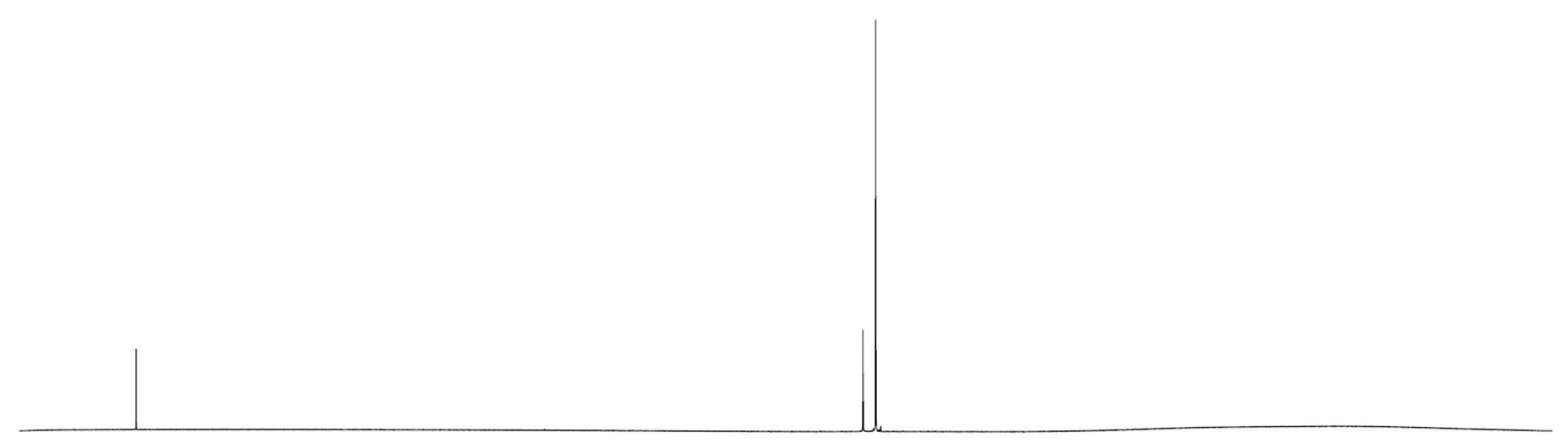

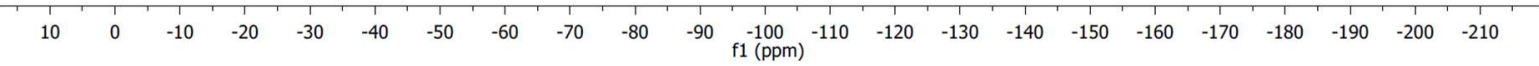

ethyl (Z)-3-(4-fluorophenyl)but-2-enoate ((Z)-44):

${ }^{1} \mathrm{H} \mathrm{NMR}\left(\mathrm{CDCl}_{3}, 300 \mathrm{MHz}\right)$

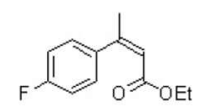

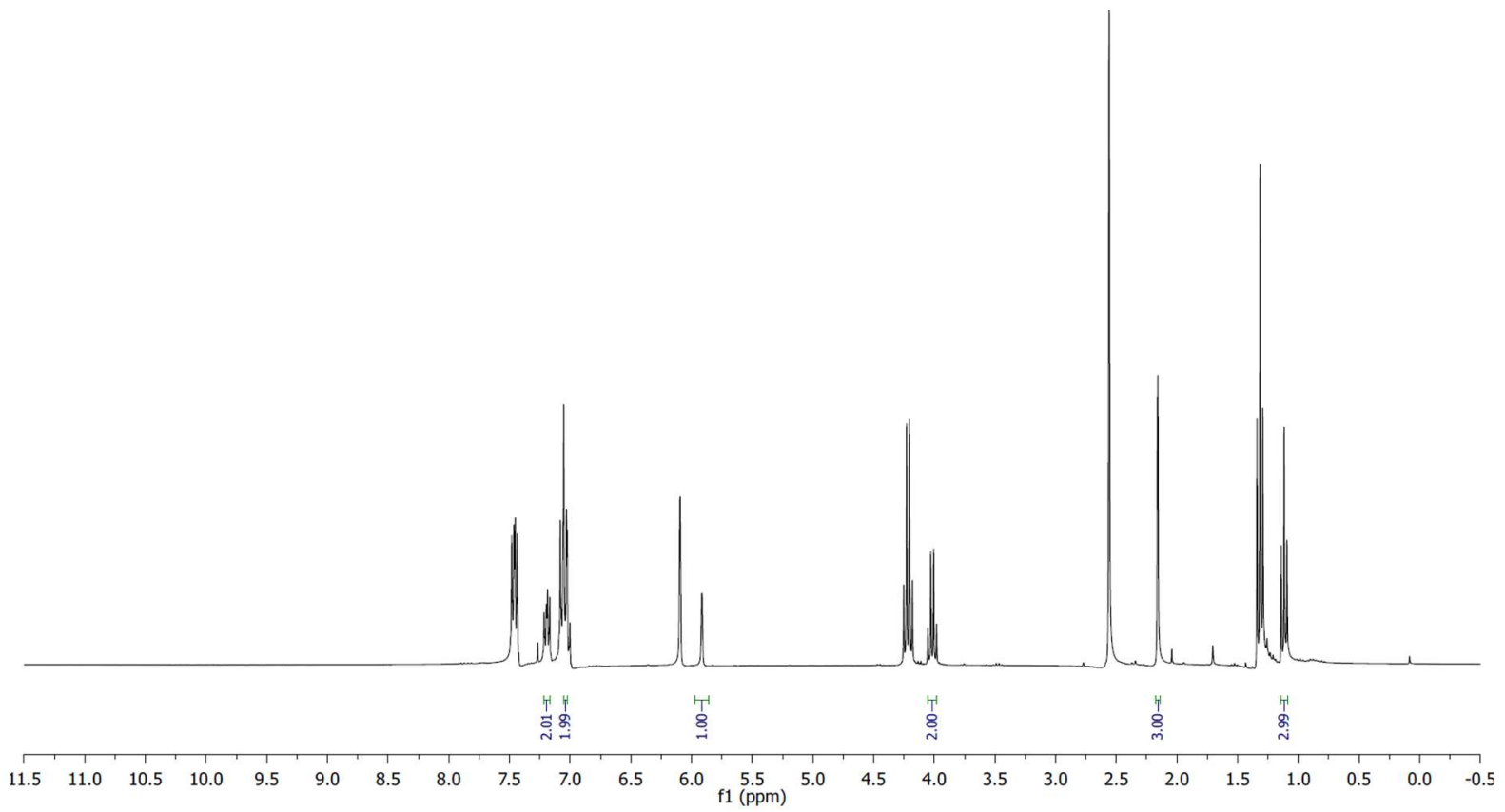


${ }^{13} \mathrm{C} \mathrm{NMR}\left(\mathrm{CDCl}_{3}, 75 \mathrm{MHz}\right)$

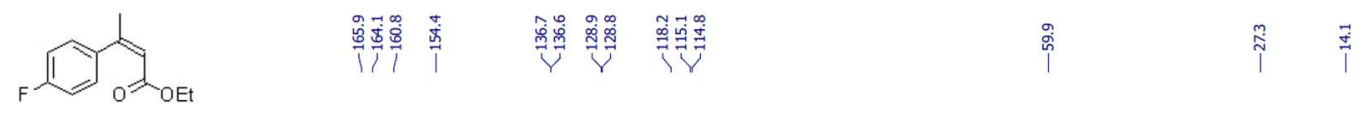

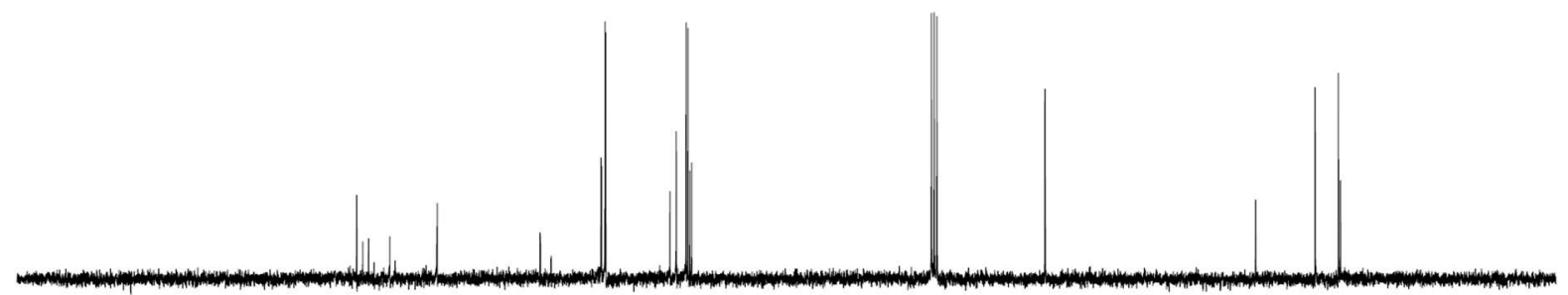

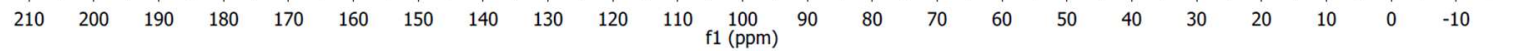

${ }^{19} \mathrm{~F} \mathrm{NMR}\left(\mathrm{CDCl}_{3}, 282 \mathrm{MHz}\right)$

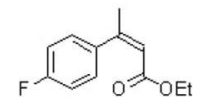

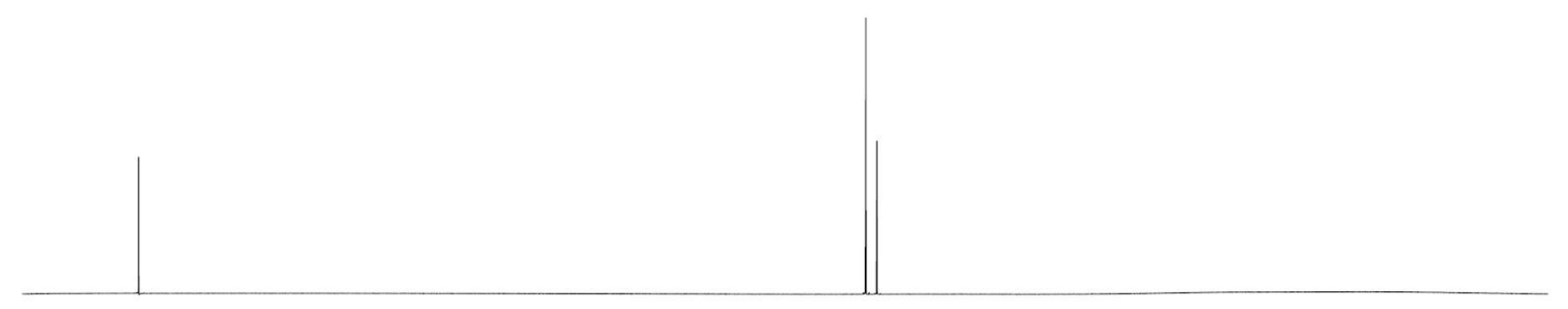

$\begin{array}{llllllllllllllllllllllll}10 & 0 & -10 & -20 & -30 & -40 & -50 & -60 & -70 & -80 & -90 & -100 & -110 & -120 & -130 & -140 & -150 & -160 & -170 & -180 & -190 & -200 & -210\end{array}$ 
(Z)-3-(3-(4-bromophenyl)but-2-enoyl)oxazolidin-2-one ((Z)-45):

${ }^{1} \mathrm{H} \mathrm{NMR}\left(\mathrm{CDCl}_{3}, 300 \mathrm{MHz}\right)$

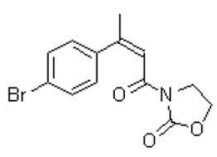

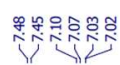

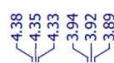

สิ

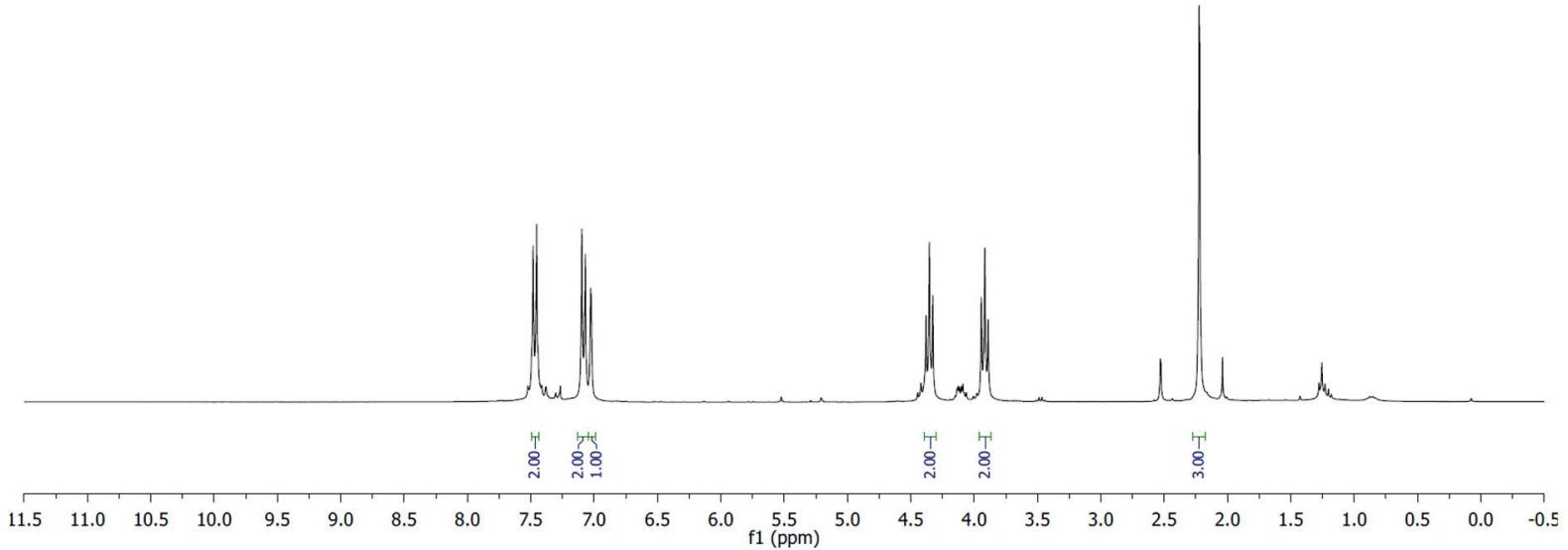

${ }^{13} \mathrm{C} \mathrm{NMR}\left(\mathrm{CDCl}_{3}, 75 \mathrm{MHz}\right)$

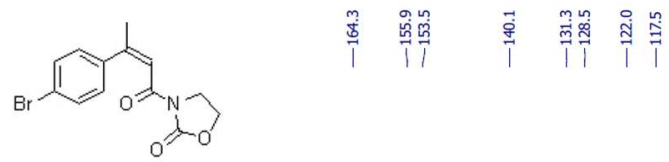

药

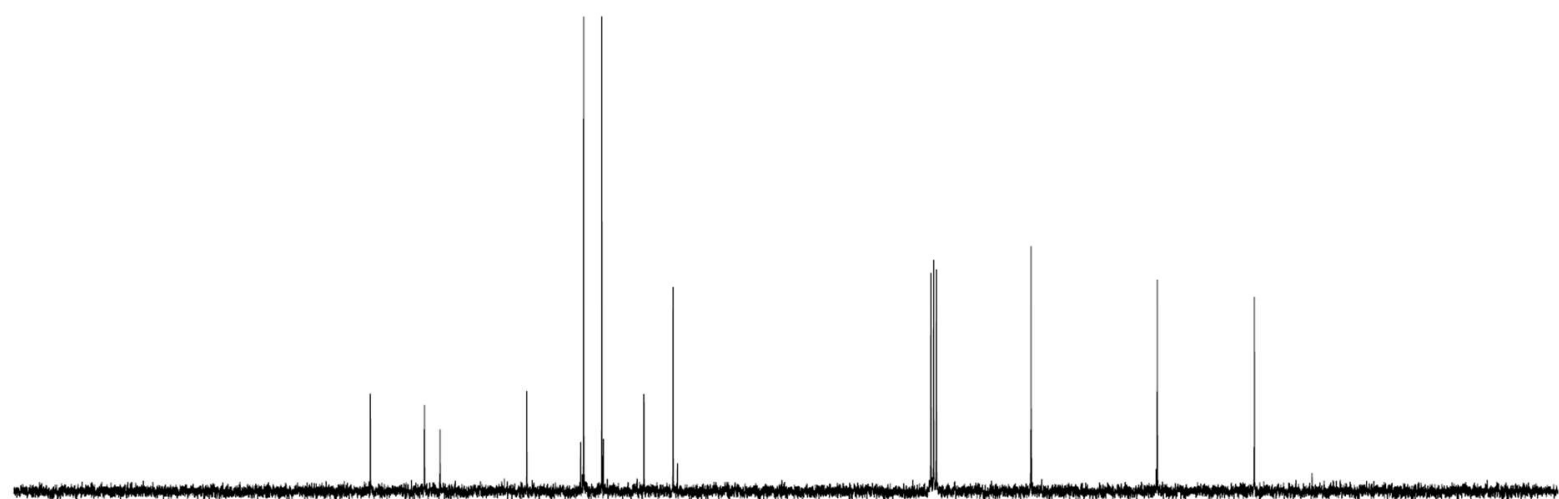

$\begin{array}{lllllllllllllllllllllll}210 & 200 & 190 & 180 & 170 & 160 & 150 & 140 & 130 & 120 & 110 & 100 & 90 & 80 & 70 & 60 & 50 & 40 & 30 & 20 & 10 & 0 & -10\end{array}$ 
ethyl (Z)-3-(4-bromophenyl)but-2-enoate ((Z)-46):

${ }^{1} \mathrm{H} \mathrm{NMR}\left(\mathrm{CDCl}_{3}, 300 \mathrm{MHz}\right)$

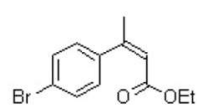

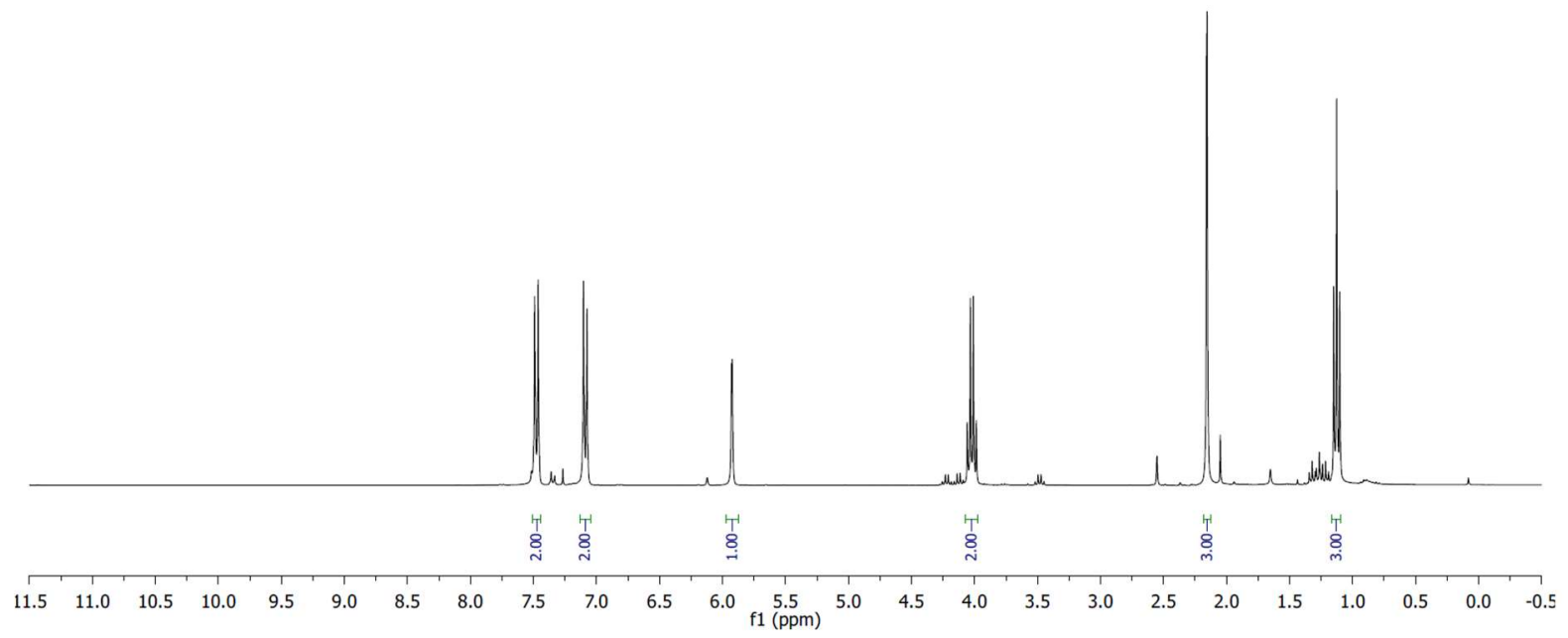

${ }^{13} \mathrm{CNMR}\left(\mathrm{CDCl}_{3}, 75 \mathrm{MHz}\right)$

Br

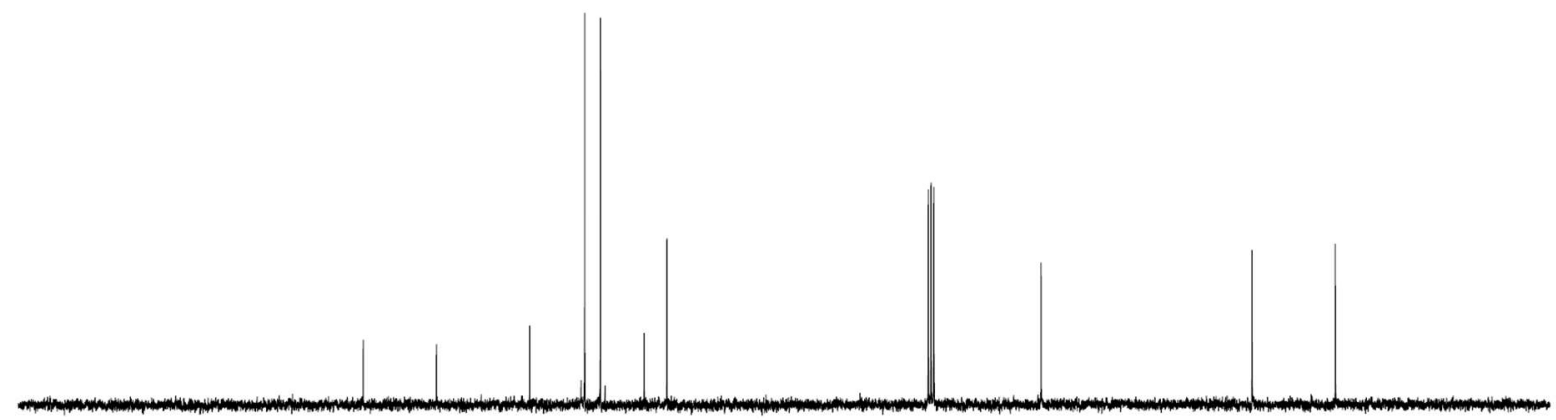

$\begin{array}{lllllllllll}210 & 200 & 190 & 180 & 170 & 160 & 150 & 140 & 130 & 120 & 110 \\ \mathrm{f} 1(\mathrm{ppm}) & 100\end{array}$ 
(Z)-3-(3-(4-iodophenyl)but-2-enoyl)oxazolidin-2-one ((Z)-47):

${ }^{1} \mathrm{H} \mathrm{NMR}\left(\mathrm{CDCl}_{3}, 300 \mathrm{MHz}\right)$
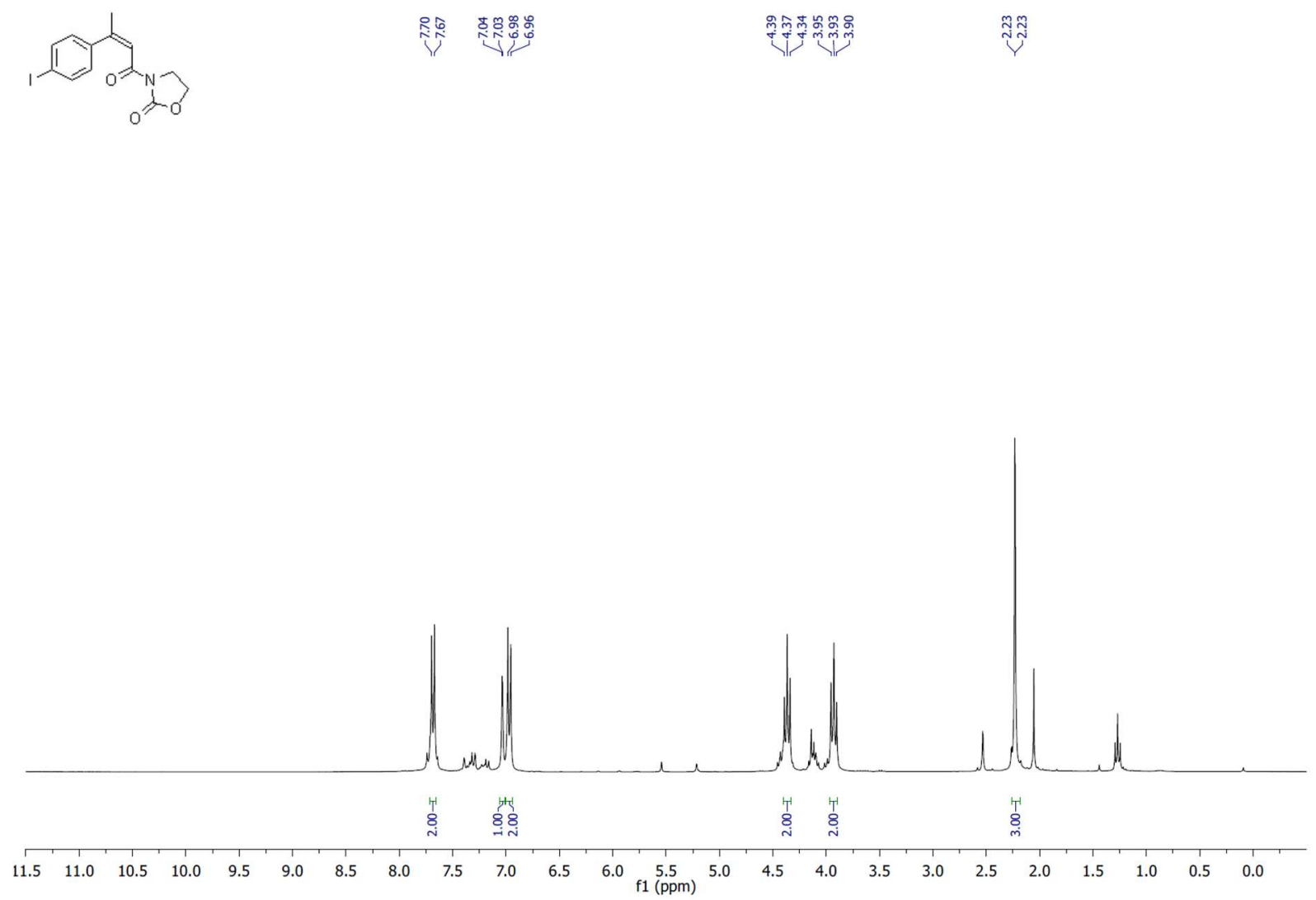

${ }^{13} \mathrm{C} \mathrm{NMR}\left(\mathrm{CDCl}_{3}, 75 \mathrm{MHz}\right)$

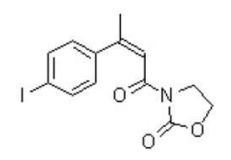

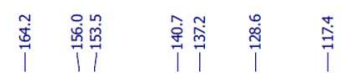

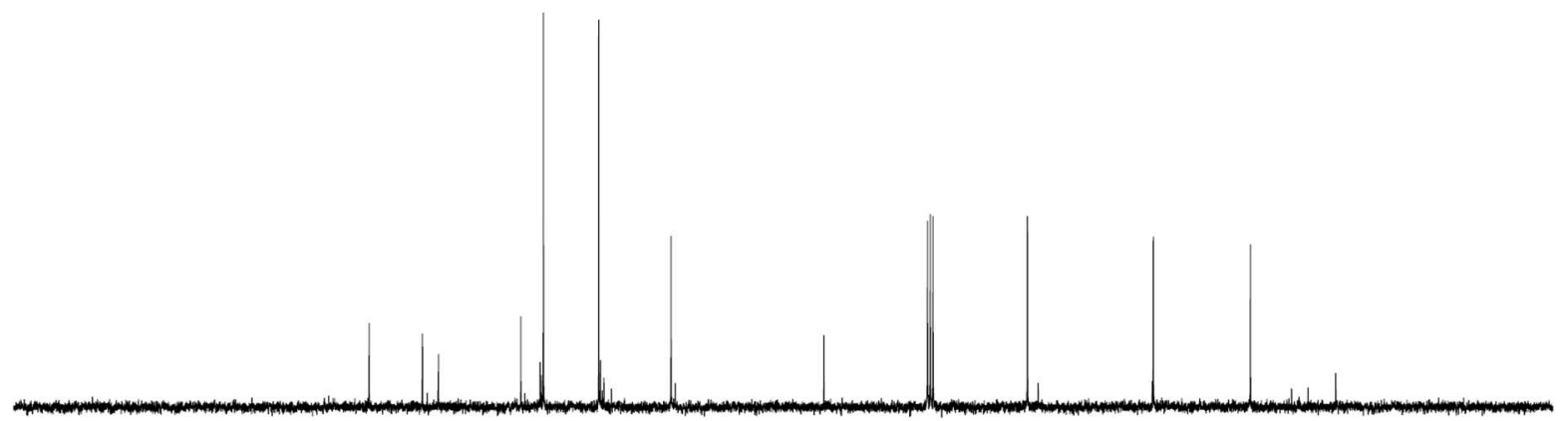

$\begin{array}{llllllllllllllllllllllllll}210 & 200 & 190 & 180 & 170 & 160 & 150 & 140 & 130 & 120 & 110 & 100 & 90 & 80 & 70 & 60 & 50 & 40 & 30 & 20 & 10 & 0 & -10\end{array}$ 
ethyl (Z)-3-(4-iodophenyl)but-2-enoate ((Z)-48):

${ }^{1} \mathrm{H} \mathrm{NMR}\left(\mathrm{CDCl}_{3}, 300 \mathrm{MHz}\right)$<smiles>CCOC(=O)/C=C(/C)c1ccc(I)cc1</smiles>

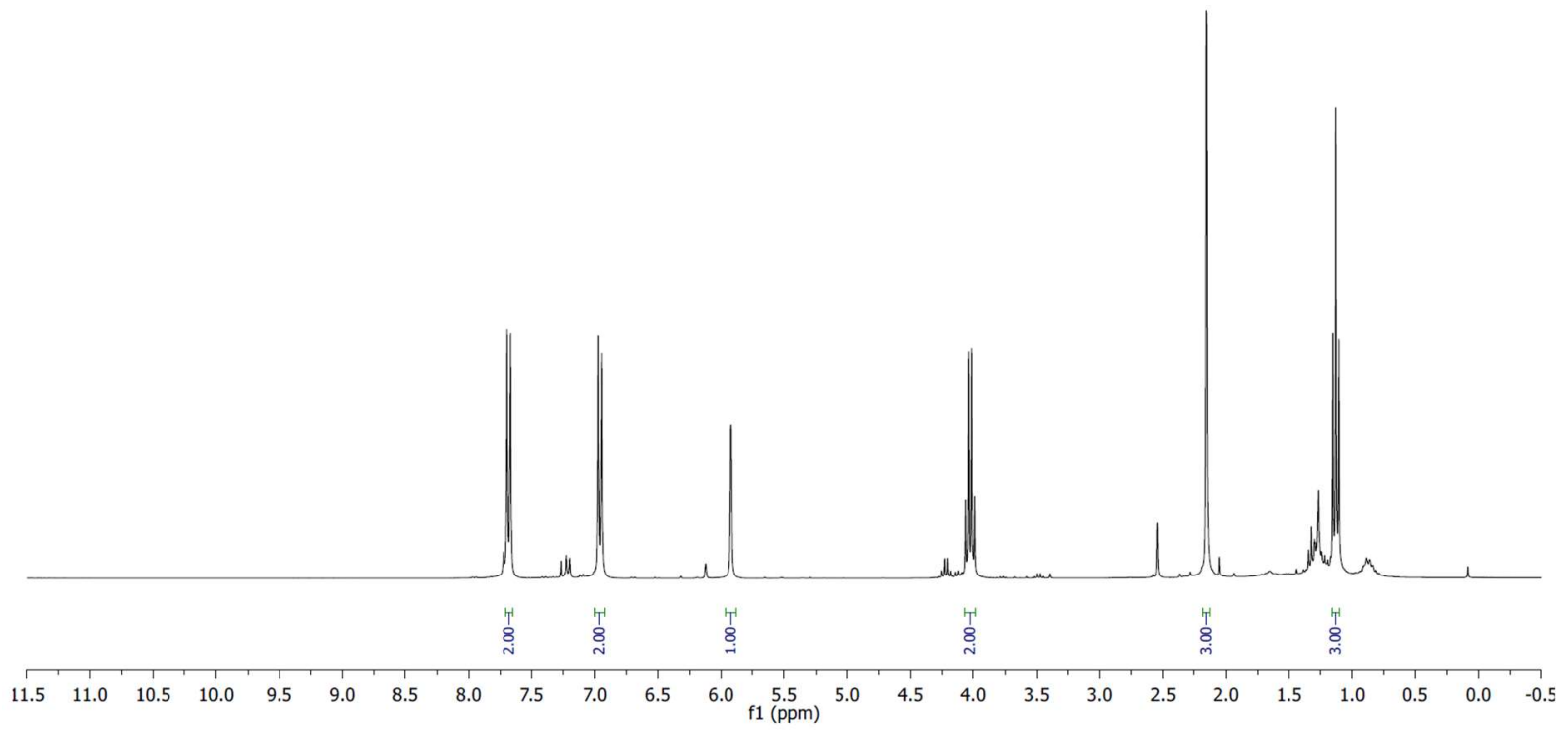

${ }^{13} \mathrm{C} \mathrm{NMR}\left(\mathrm{CDCl}_{3}, 75 \mathrm{MHz}\right)$

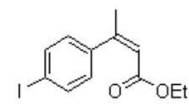

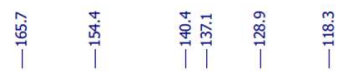
$\stackrel{\substack{0 \\ \text { i }}}{2}$ $\stackrel{0}{\circ}$ $\vec{i}$

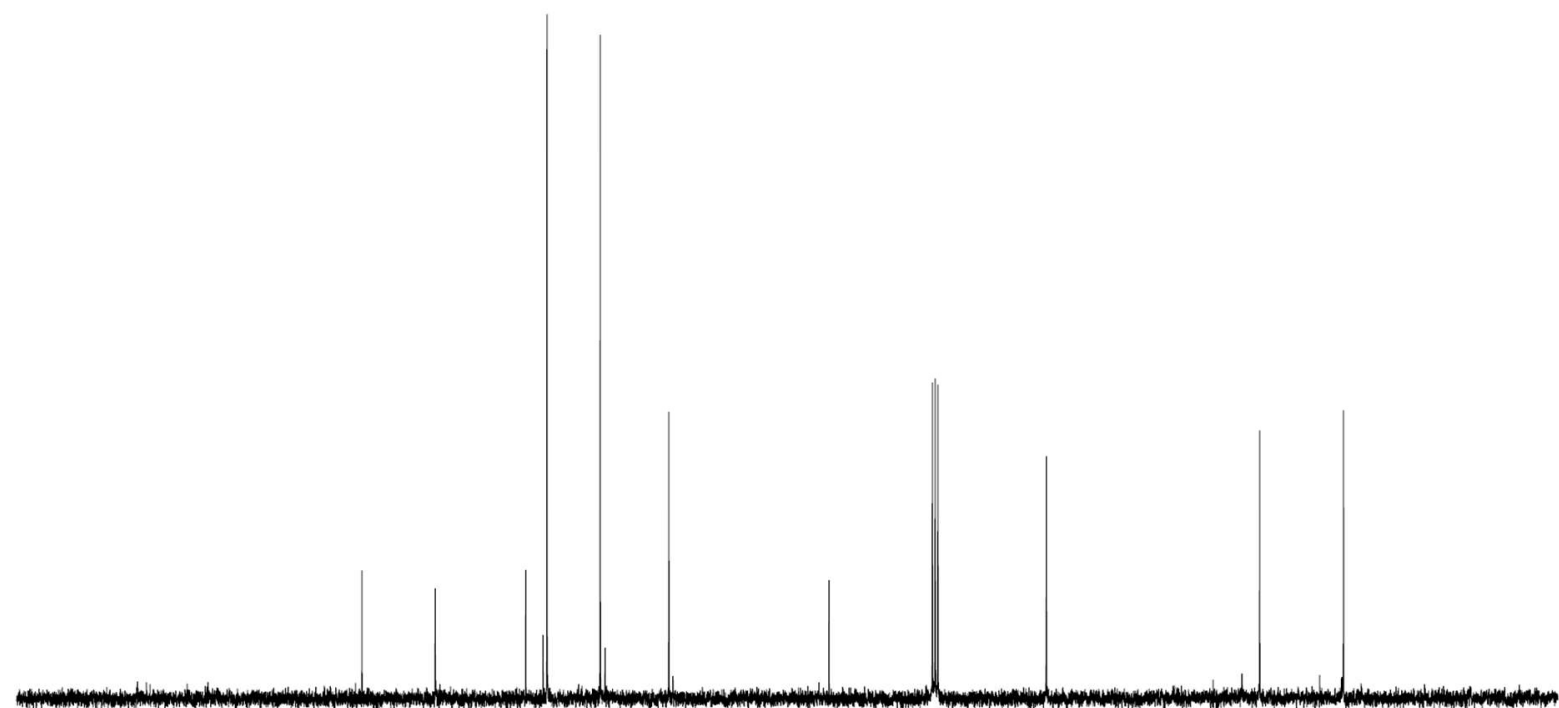

$\begin{array}{lllllllllll}210 & 200 & 190 & 180 & 170 & 160 & 150 & 140 & 130 & 120 & 110 \\ \mathrm{f} 1(\mathrm{ppm})\end{array}$ 
(Z)-3-(3-(4-nitrophenyl)but-2-enoyl)oxazolidin-2-one ((Z)-49):

${ }^{1} \mathrm{H} \mathrm{NMR}\left(\mathrm{CDCl}_{3}, 300 \mathrm{MHz}\right)$
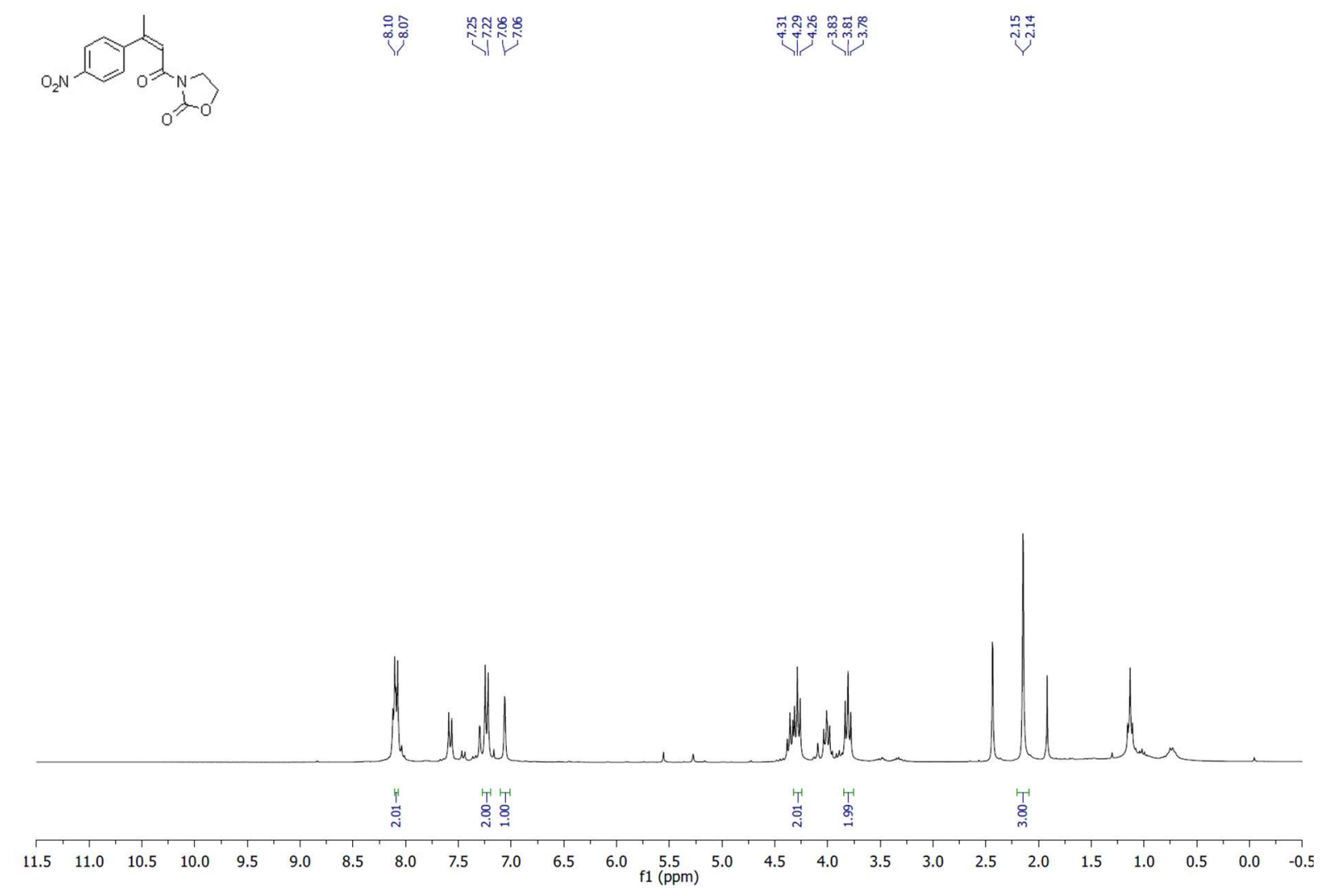

${ }^{13} \mathrm{C} \mathrm{NMR}\left(\mathrm{CDCl}_{3}, 75 \mathrm{MHz}\right)$
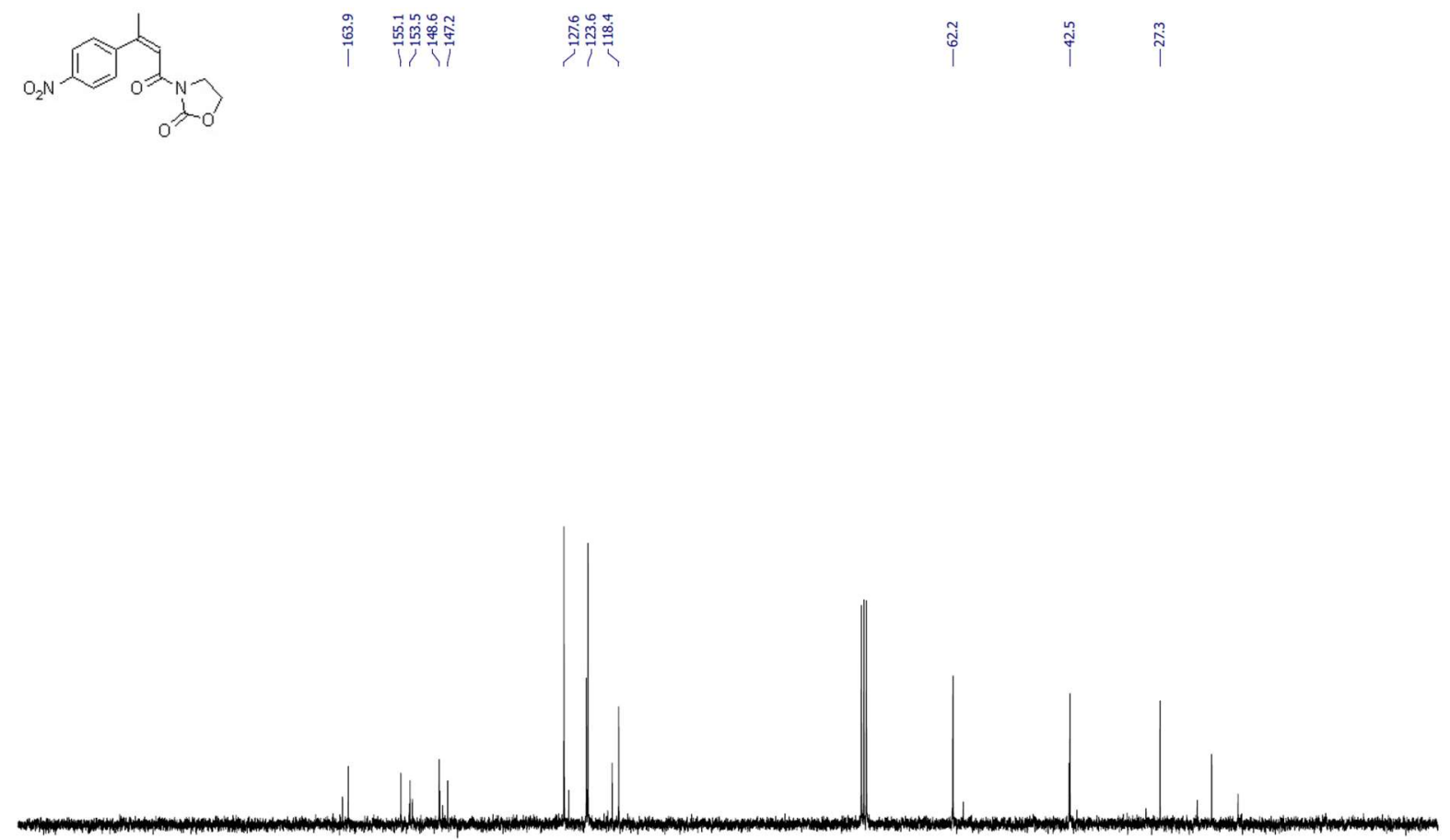

$\begin{array}{lllllllllllllllllllllllll}210 & 200 & 190 & 180 & 170 & 160 & 150 & 140 & 130 & 120 & 110 & \begin{array}{c}100 \\ \mathrm{f} 1(\mathrm{ppm})\end{array} & 90 & 80 & 70 & 60 & 50 & 40 & 30 & 20 & 10 & 0 & -10\end{array}$ 
ethyl (Z)-3-(4-nitrophenyl)but-2-enoate ((Z)-50):

${ }^{1} \mathrm{H} \mathrm{NMR}\left(\mathrm{CDCl}_{3}, 300 \mathrm{MHz}\right)$<smiles>CC1CCC(C)C(C)C1</smiles>

ํํำ

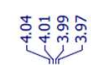

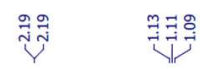

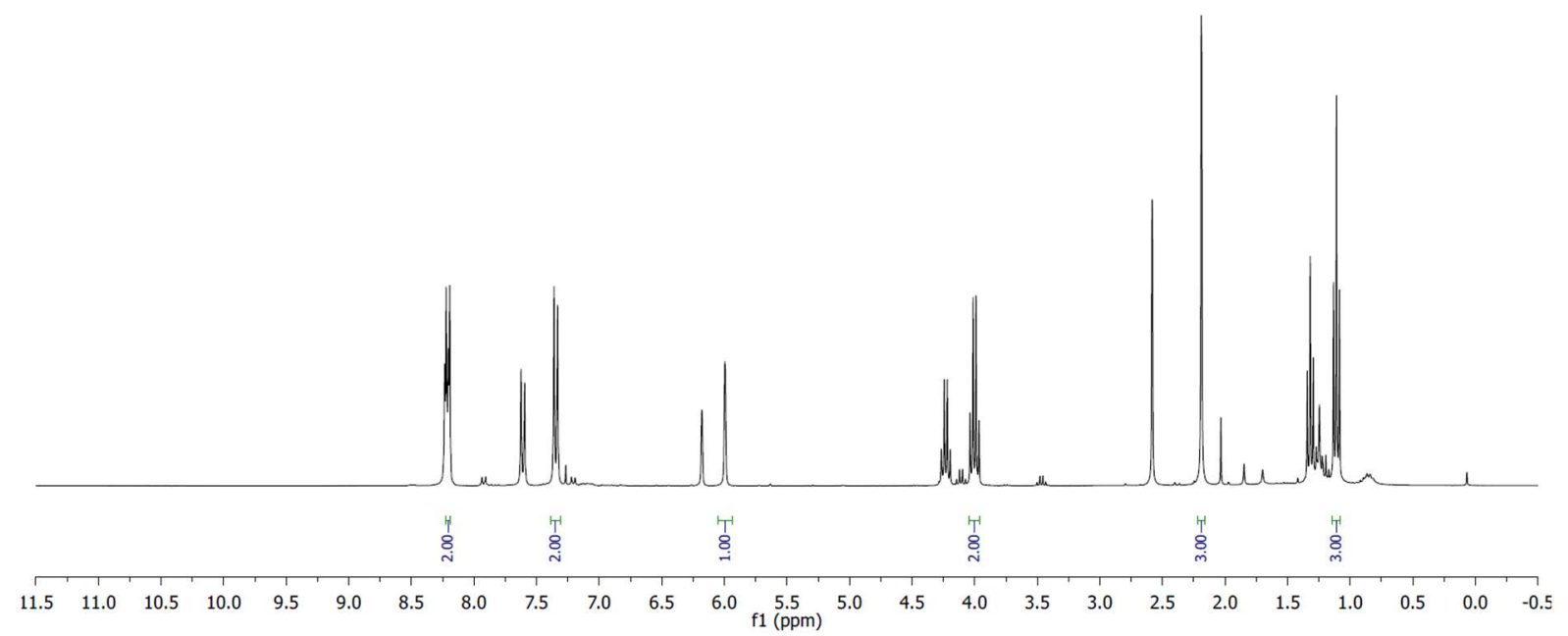

${ }^{13} \mathrm{C} \mathrm{NMR}\left(\mathrm{CDCl}_{3}, 75 \mathrm{MHz}\right)$<smiles>Cc1cc(C)c([N+](=O)[O-])cc1[Ge]</smiles>

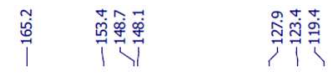

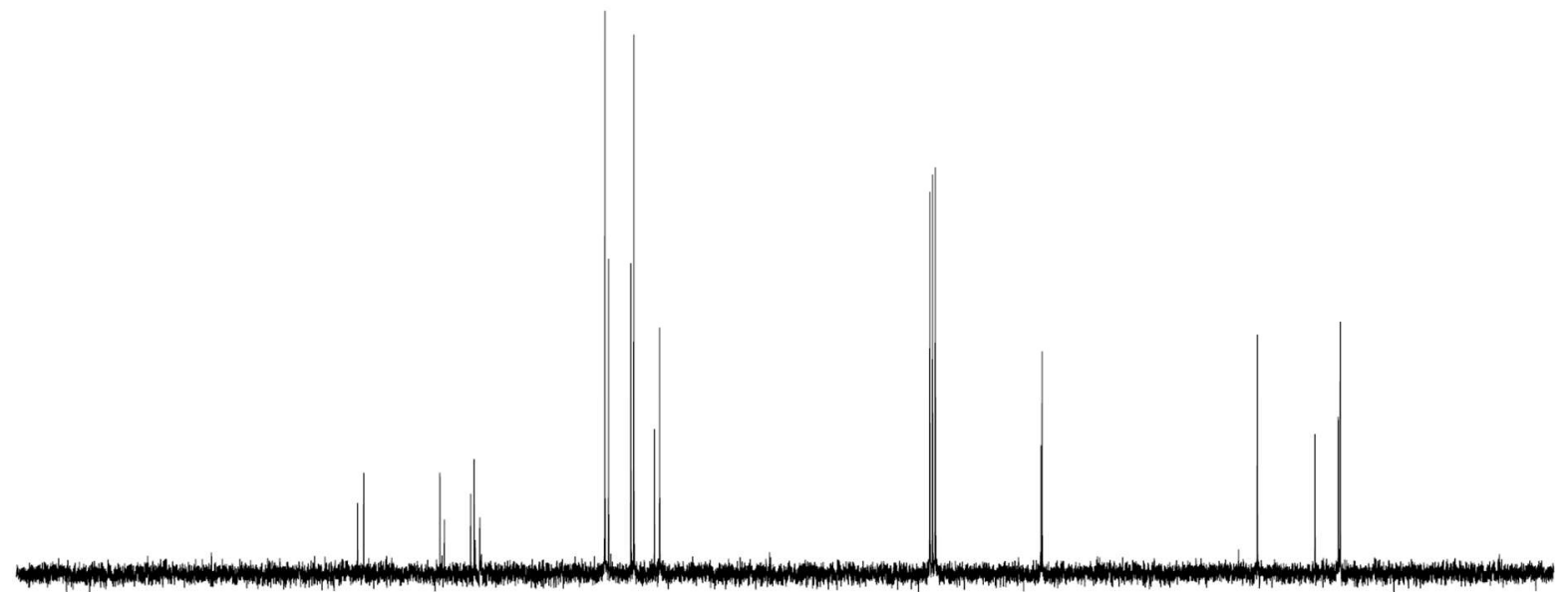

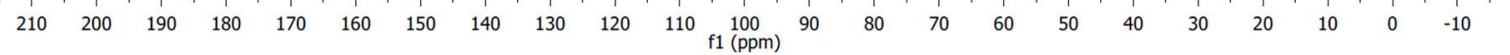


(Z)-3-(4,4,4-trifluoro-3-phenylbut-2-enoyl)oxazolidin-2-one ((Z)-51):

${ }^{1} \mathrm{H} \mathrm{NMR}\left(\mathrm{CDCl}_{3}, 300 \mathrm{MHz}\right)$
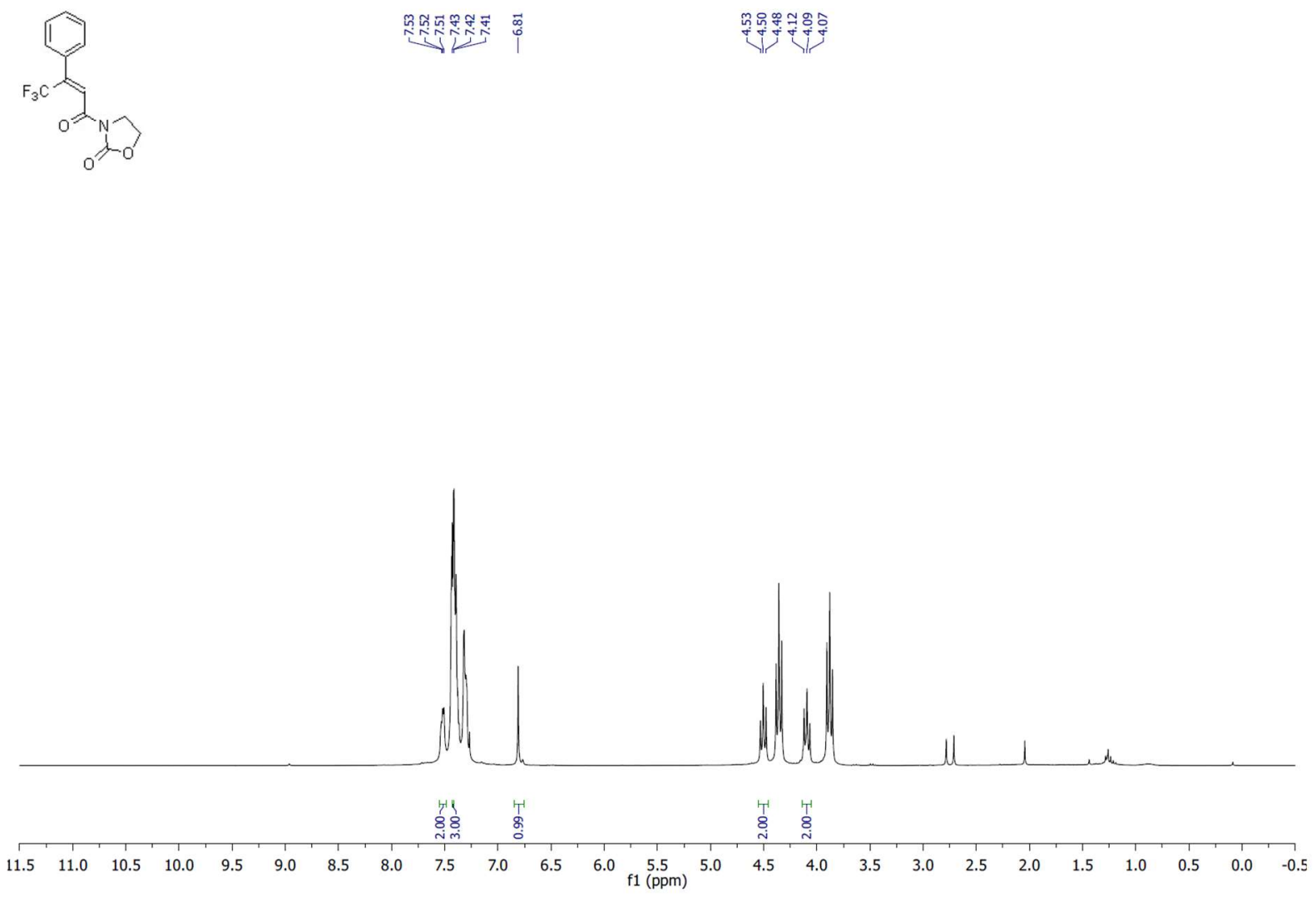

${ }^{13} \mathrm{C} \mathrm{NMR}\left(\mathrm{CDCl}_{3}, 75 \mathrm{MHz}\right)$
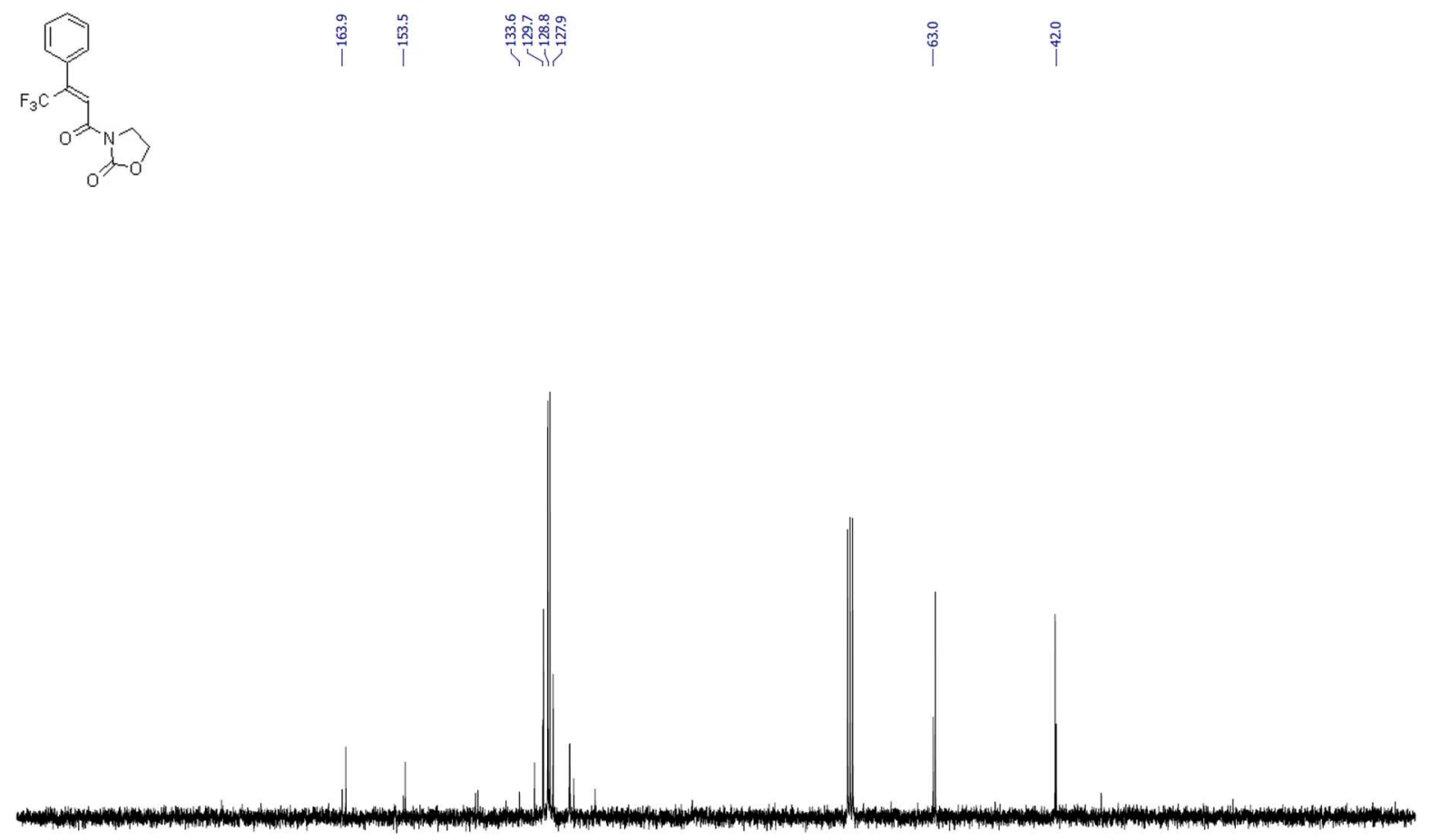

$\begin{array}{lllllllllllllllllllllll}210 & 200 & 190 & 180 & 170 & 160 & 150 & 140 & 130 & 120 & 110 & \underset{\mathrm{f} 1}{100}(\mathrm{ppm}) & 90 & 80 & 70 & 60 & 50 & 40 & 30 & 20 & 10 & 0 & -10\end{array}$ 
${ }^{19} \mathrm{~F} \mathrm{NMR}\left(\mathrm{CDCl}_{3}, 282 \mathrm{MHz}\right)$

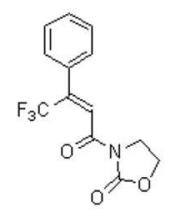

$\stackrel{+}{i}$
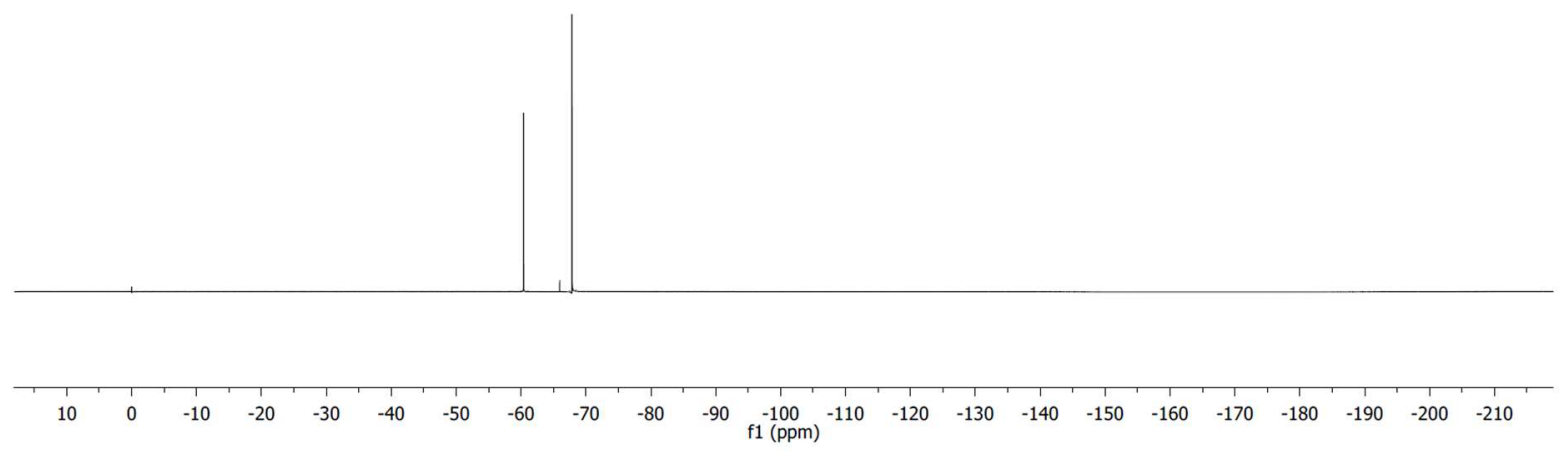

ethyl (Z)-4,4,4-trifluoro-3-phenylbut-2-enoate ((Z)-52):

${ }^{1} \mathrm{H} \mathrm{NMR}\left(\mathrm{CDCl}_{3}, 300 \mathrm{MHz}\right)$
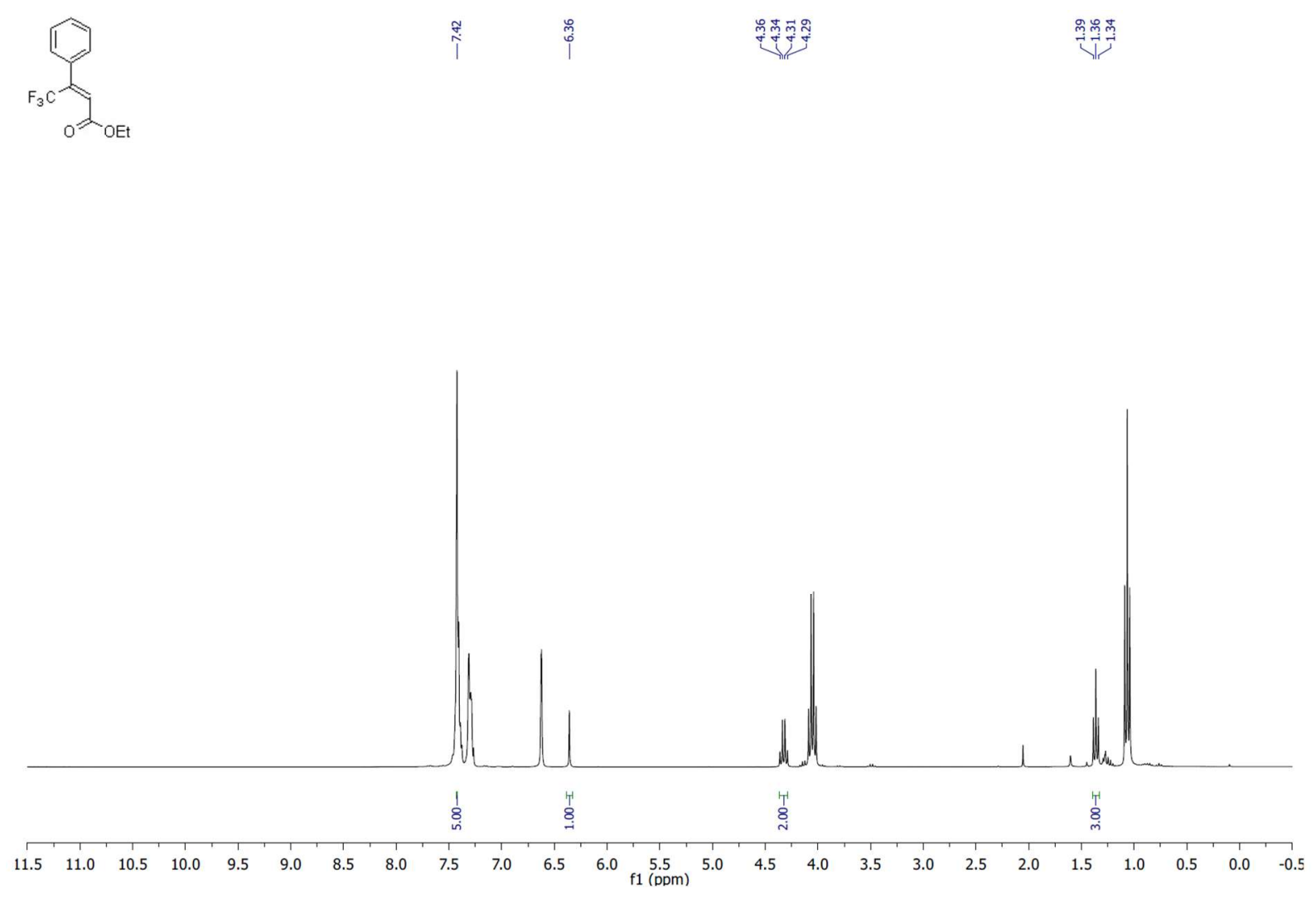

245 
${ }^{13} \mathrm{C} \mathrm{NMR}\left(\mathrm{CDCl}_{3}, 75 \mathrm{MHz}\right)$

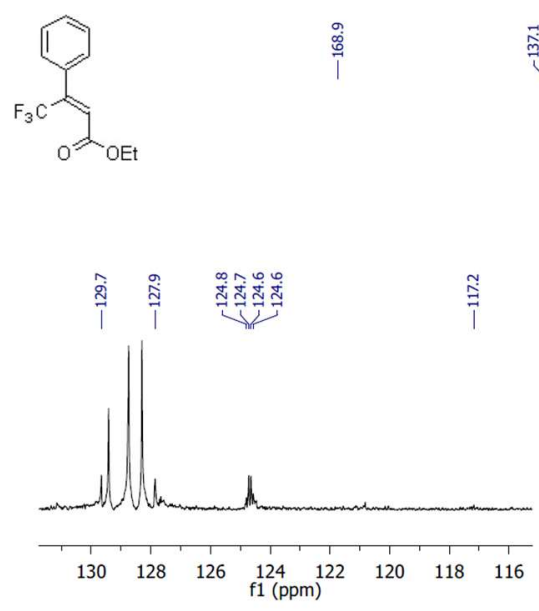

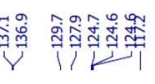

ì

$\stackrel{F}{i}$
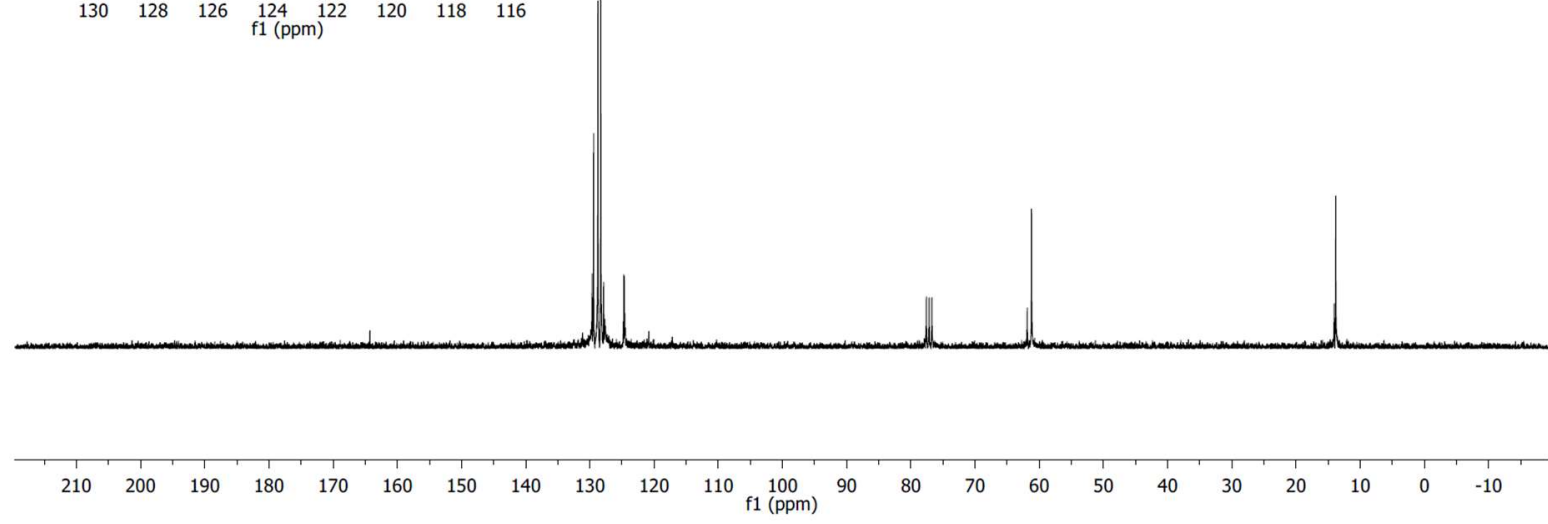

${ }^{19} \mathrm{~F} \mathrm{NMR}\left(\mathrm{CDCl}_{3}, 282 \mathrm{MHz}\right)$

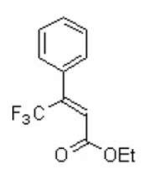

$\stackrel{8}{8}$
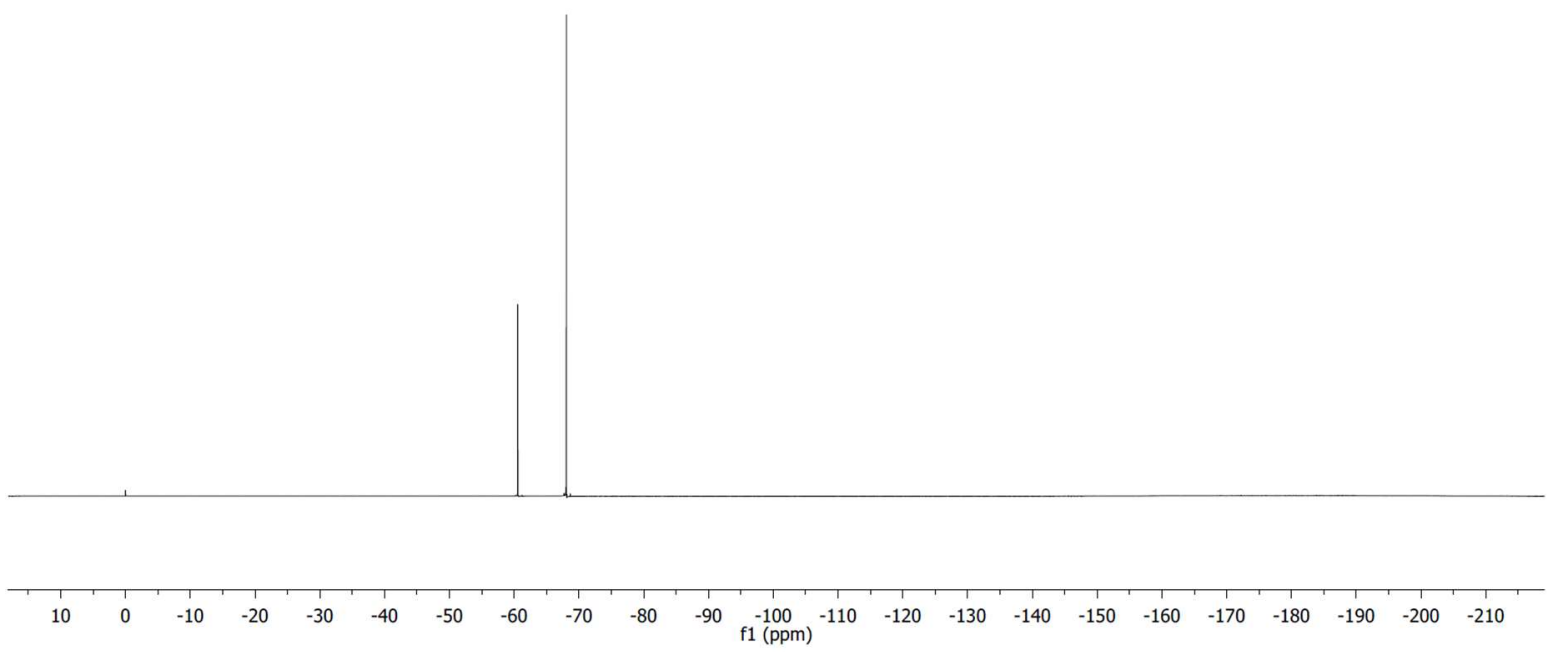

246 
(Z)-3-phenylbut-2-enenitrile ((Z)-53):

${ }^{1} \mathrm{H} \mathrm{NMR}\left(\mathrm{CDCl}_{3}, 300 \mathrm{MHz}\right)$<smiles>CC(C)c1ccccc1</smiles>

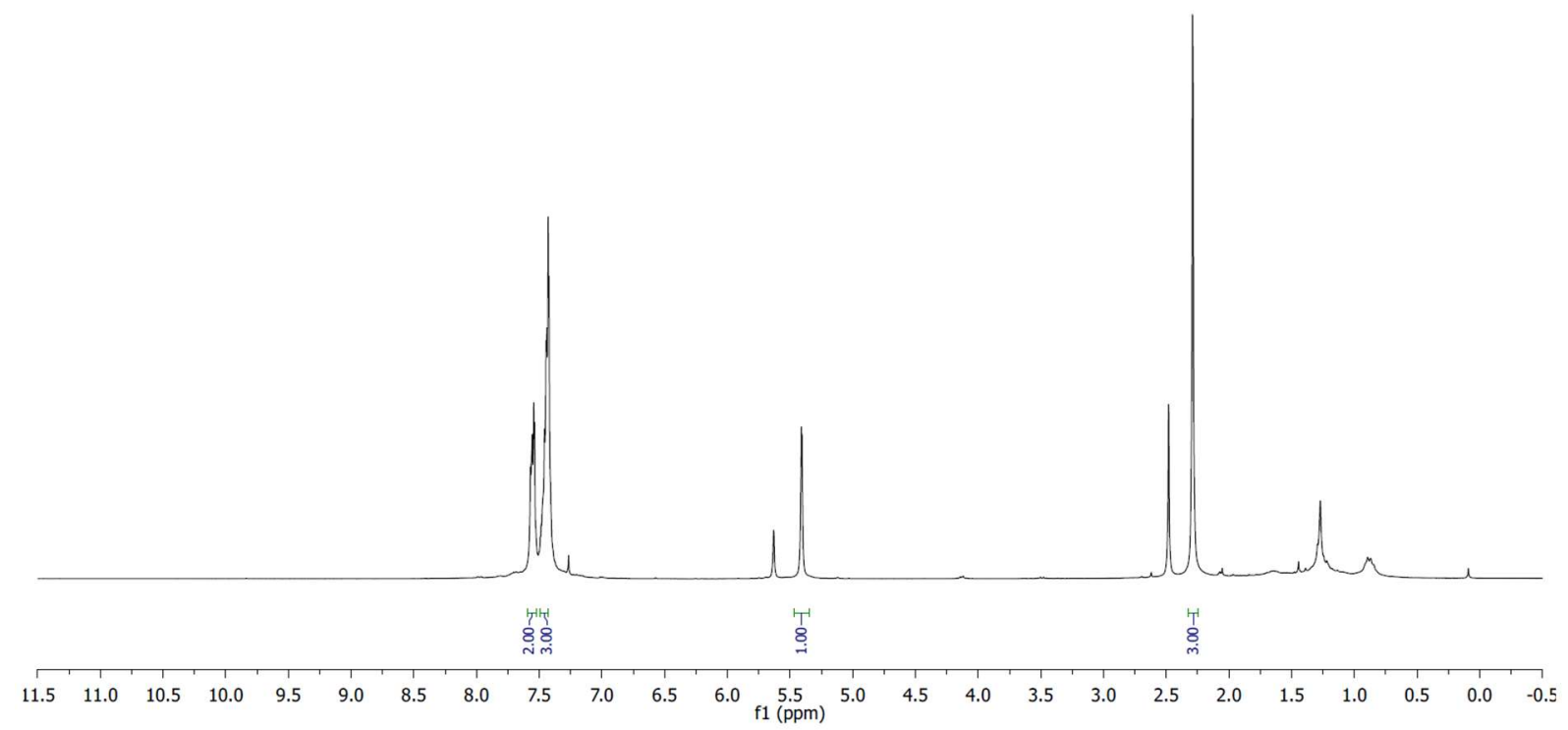

${ }^{13} \mathrm{C} \mathrm{NMR}\left(\mathrm{CDCl}_{3}, 75 \mathrm{MHz}\right)$<smiles>CC(C)=C(C)c1ccccc1</smiles>

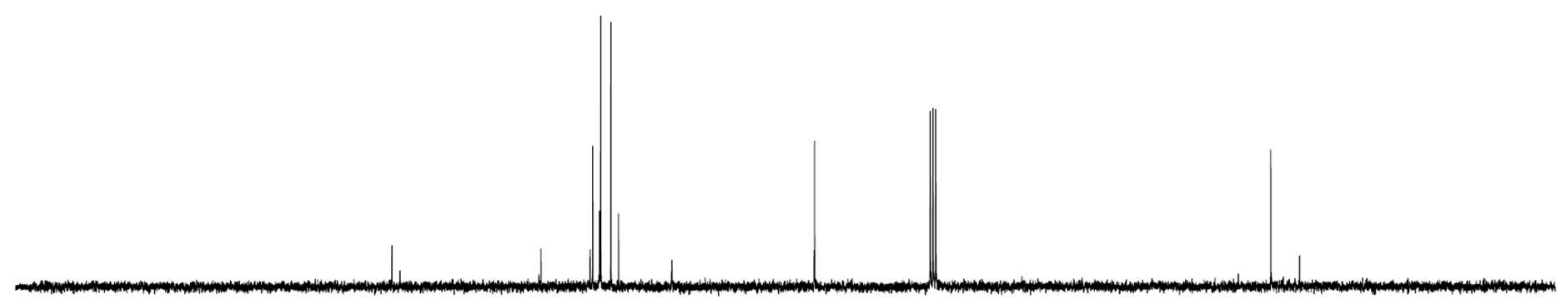

$\begin{array}{lllllllllllllllllllllll}210 & 200 & 190 & 180 & 170 & 160 & 150 & 140 & 130 & 120 & 110 & \begin{array}{c}100 \\ \mathrm{f} 1(\mathrm{ppm})\end{array} & 90 & 80 & 70 & 60 & 50 & 40 & 30 & 20 & 10 & 0 & -10\end{array}$ 
diethyl (Z)-(2-phenylprop-1-en-1-yl)phosphonate ((Z)-54):

${ }^{1} \mathrm{H} \mathrm{NMR}\left(\mathrm{CDCl}_{3}, 300 \mathrm{MHz}\right)$
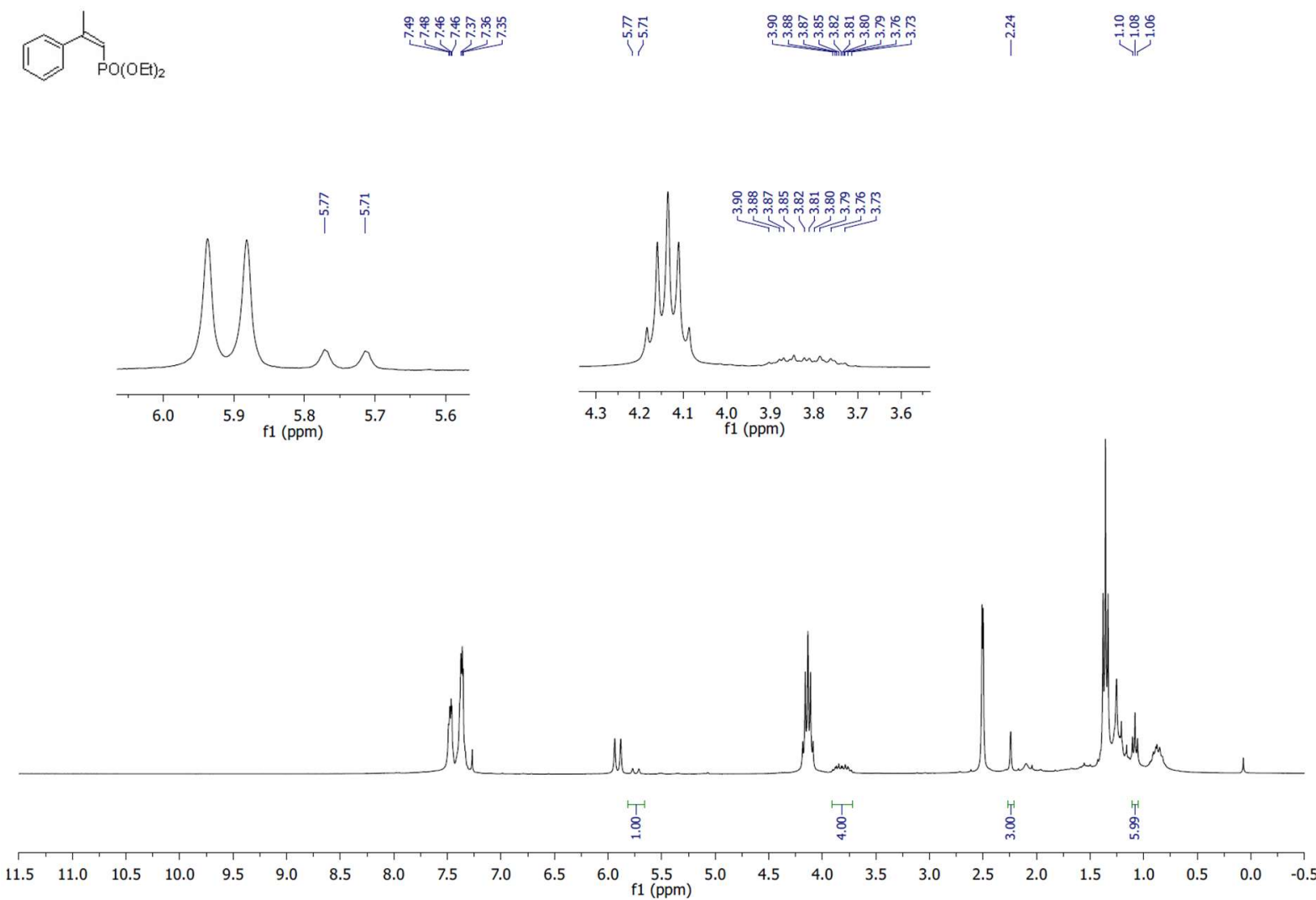

${ }^{31} \mathrm{P} \mathrm{NMR}\left(\mathrm{CDCl}_{3}, 121 \mathrm{MHz}\right)$
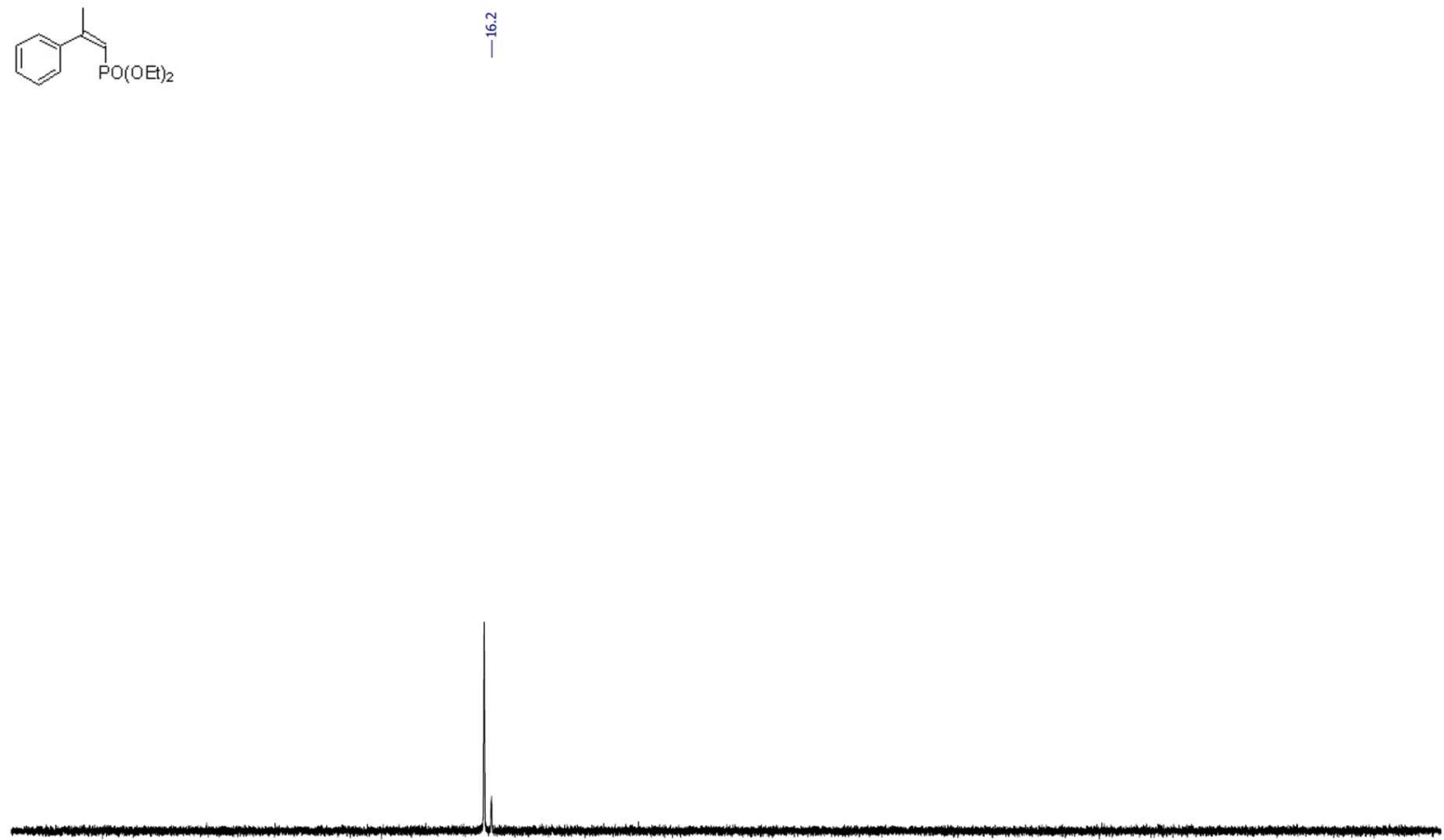

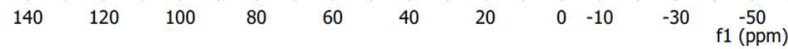


(Z)-((2-phenylprop-1-en-1-yl)sulfonyl)benzene ((Z)-55):

${ }^{1} \mathrm{H} \mathrm{NMR}\left(\mathrm{CDCl}_{3}, 300 \mathrm{MHz}\right)$<smiles>C/C(=C/[SbH])c1ccccc1</smiles>

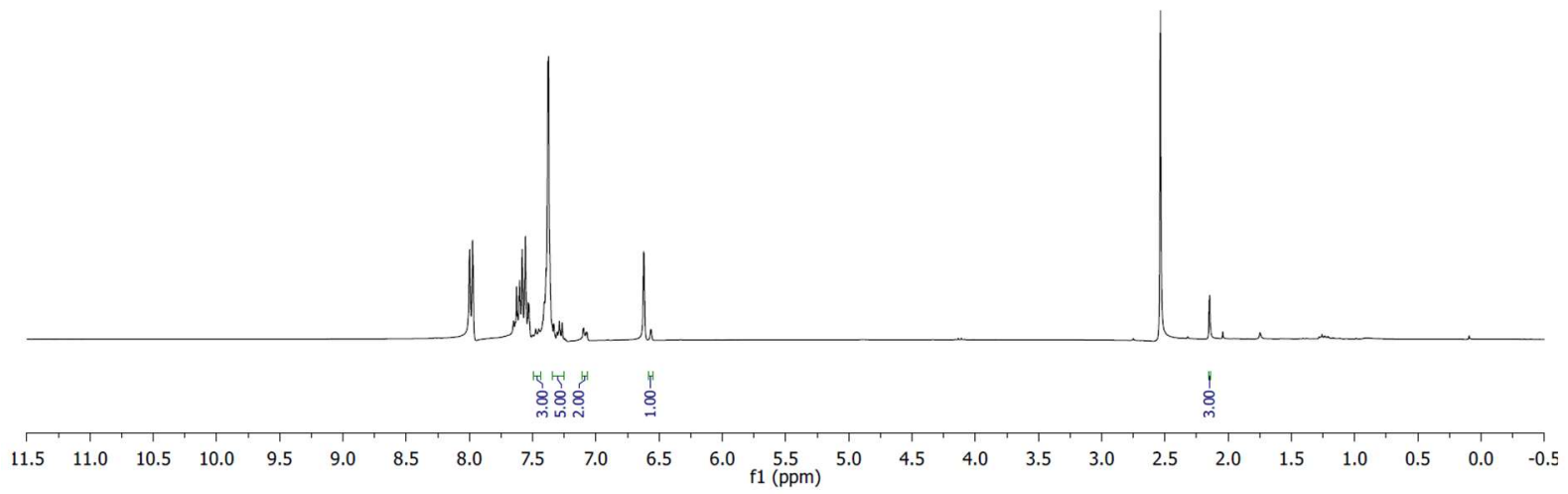

${ }^{13} \mathrm{C} \mathrm{NMR}\left(\mathrm{CDCl}_{3}, 75 \mathrm{MHz}\right)$<smiles>CC(=O)c1ccccc1</smiles>

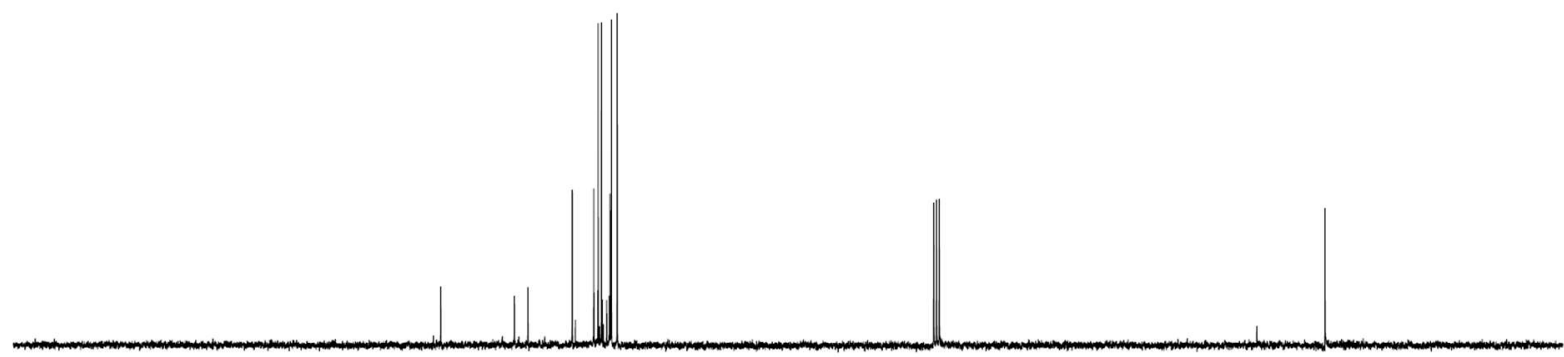

$\begin{array}{llllllllllllllllllllllll}210 & 200 & 190 & 180 & 170 & 160 & 150 & 140 & 130 & 120 & 110 & \begin{array}{c}100 \\ \mathrm{f} 1(\mathrm{ppm})\end{array} & 90 & 80 & 70 & 60 & 50 & 40 & 30 & 20 & 10 & 0 & -10 & \end{array}$ 


\section{GC-FID Spectra}

\section{(Z)-3-(2-methyl-3-(p-tolyl)acryloyl)oxazolidin-2-one ((Z)-9)}

$(E)$-isomer $((E)-9)$

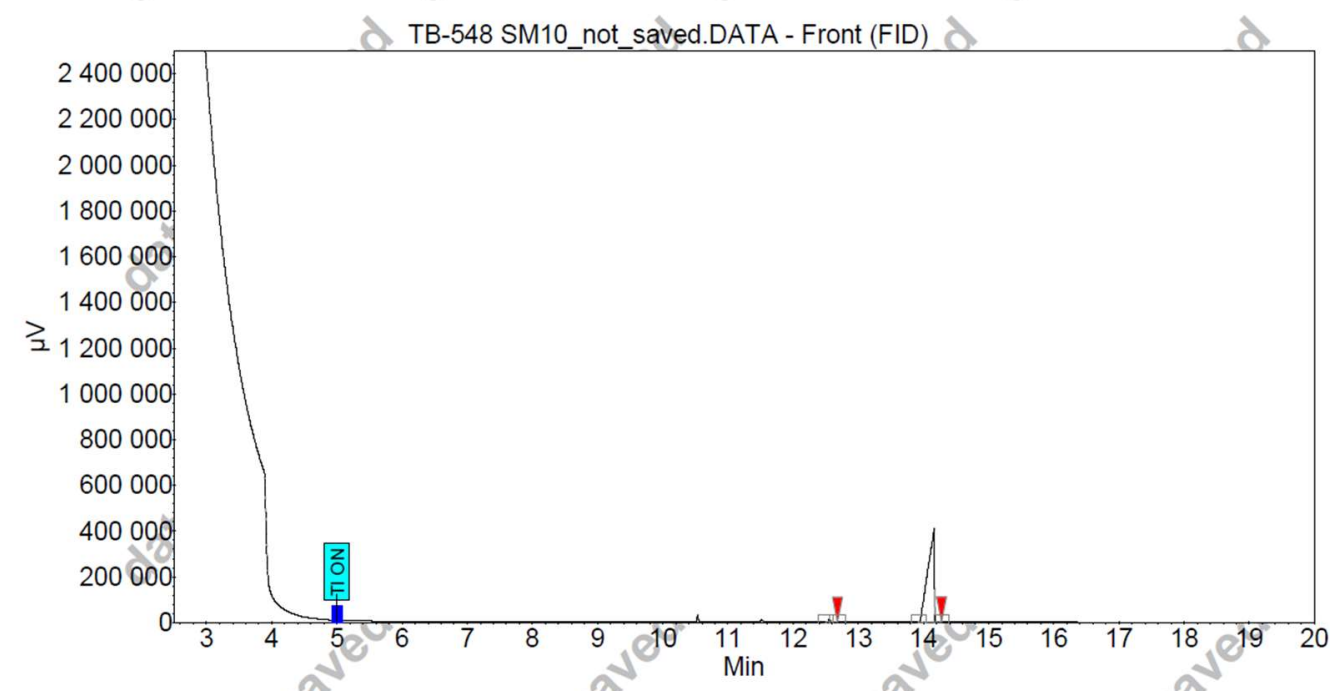

\section{Peak results :}

\begin{tabular}{|c|l|r|r|r|r|r|r|r|}
\hline Index & Name & $\begin{array}{r}\text { Time } \\
{[\mathrm{Min}]}\end{array}$ & $\begin{array}{r}\text { Area \% } \\
{[\%]}\end{array}$ & $\begin{array}{r}\text { Quantity } \\
{[\% \text { Area] }}\end{array}$ & $\begin{array}{r}\text { Height } \\
{[\mu \mathrm{\mu V}]}\end{array}$ & $\begin{array}{r}\text { Area } \\
{[\mu \mathrm{V} . \mathrm{Min}]}\end{array}$ & As. PE. & Res. HW \\
\hline \hline 1 & UNKNOWN & 12.557 & 0.857 & 0.86 & 13534.4 & 415.7 & 2.19 & 0.00 \\
\hline 2 & UNKNOWN & 14.168 & 99.143 & 99.14 & 410385.5 & 48110.3 & 0.52 & 13.29 \\
\hline & & & & & & & & \\
\hline Total & & & 100.000 & 100.00 & 423920.0 & 48526.0 & & \\
\hline
\end{tabular}

(Z)-isomer ((Z)-9)

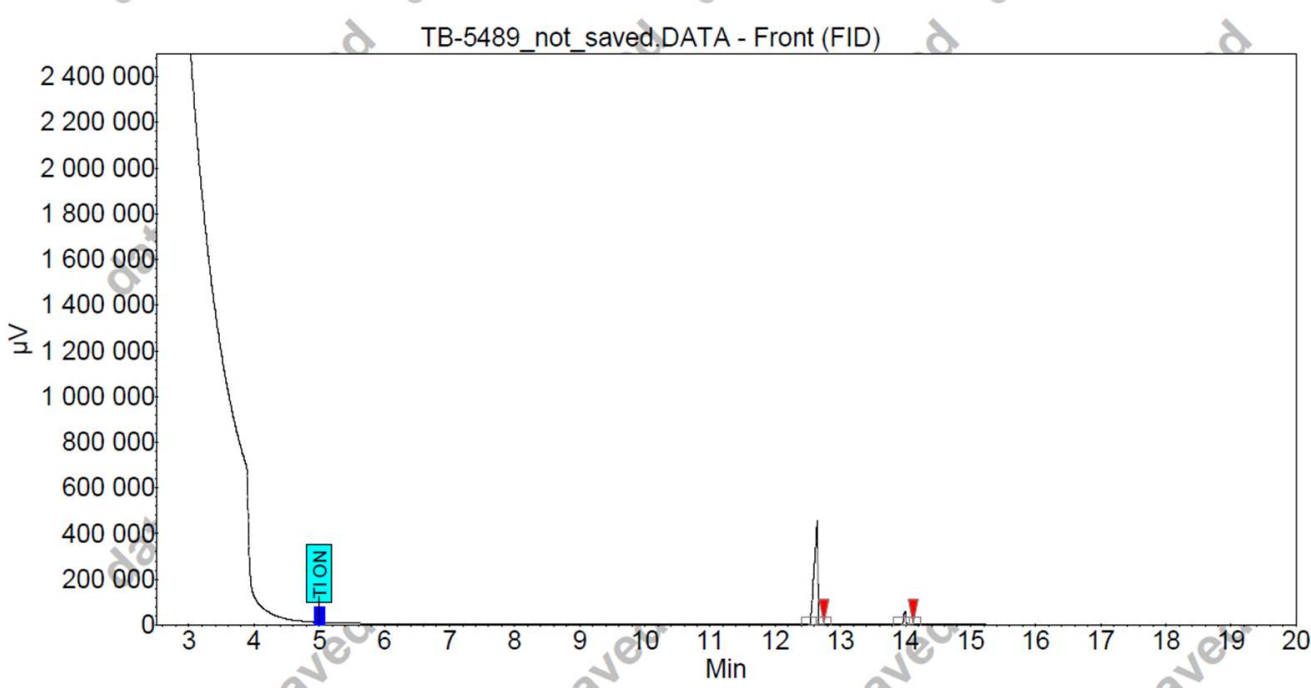

Peak results :

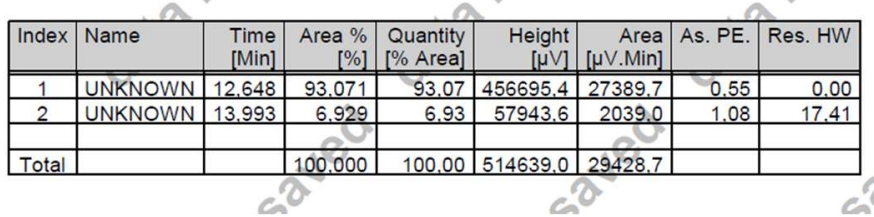


(Z)-3-(2-methyl-3-(m-tolyl)acryloyl)oxazolidin-2-one ((Z)-10)

$(E)$-isomer $((E)-10)$

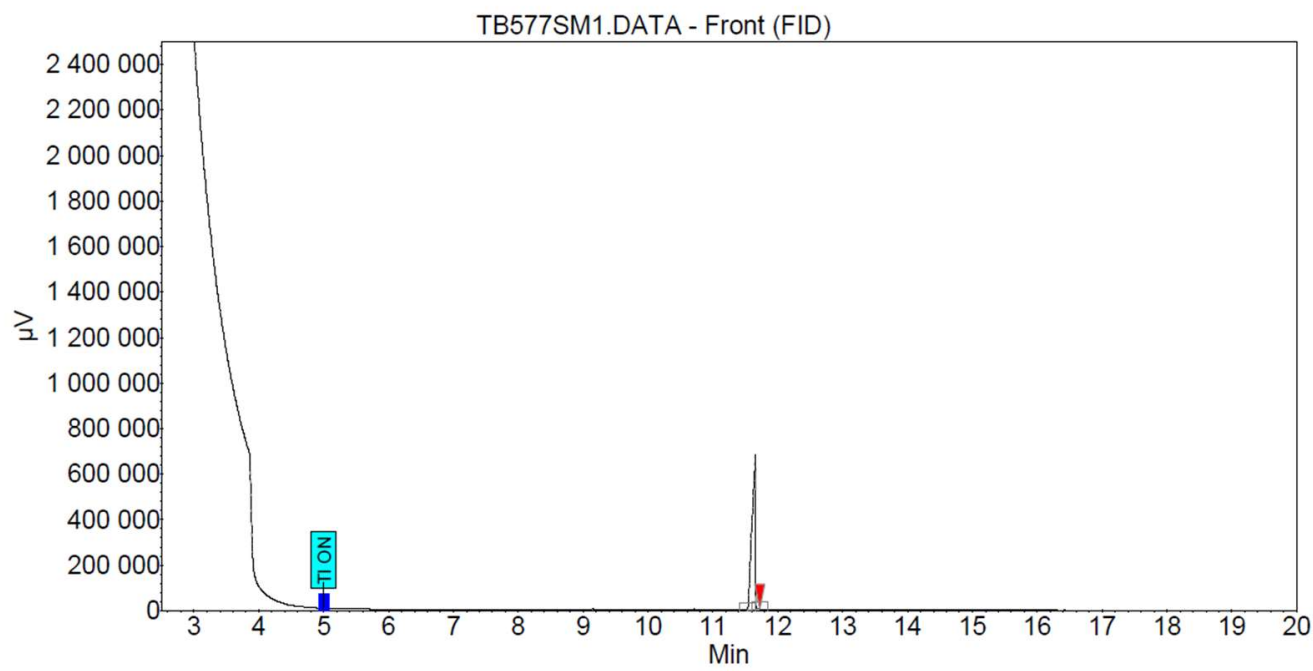

Peak results :

\begin{tabular}{|c|l|r|r|r|r|r|r|r|}
\hline Index & Name & $\begin{array}{r}\text { Time } \\
{[\mathrm{Min}]}\end{array}$ & $\begin{array}{r}\text { Area \% } \\
{[\%]}\end{array}$ & $\begin{array}{r}\text { Quantity } \\
{[\% \text { Area] }}\end{array}$ & $\begin{array}{r}\text { Height } \\
{[\mu \mathrm{V}]}\end{array}$ & $\begin{array}{r}\text { Area } \\
{[\mu \mathrm{V} \text {.Min] }}\end{array}$ & As. PE. & Res. HW \\
\hline \hline 1 & UNKNOWN & 11.655 & 100.000 & 100,00 & 681397.7 & 42618.8 & 0.54 & 0.00 \\
\hline & & & & & & & & \\
\hline Total & & & 100,000 & 100,00 & 681397,7 & 42618,8 & & \\
\hline
\end{tabular}

(Z)-isomer ((Z)-10)

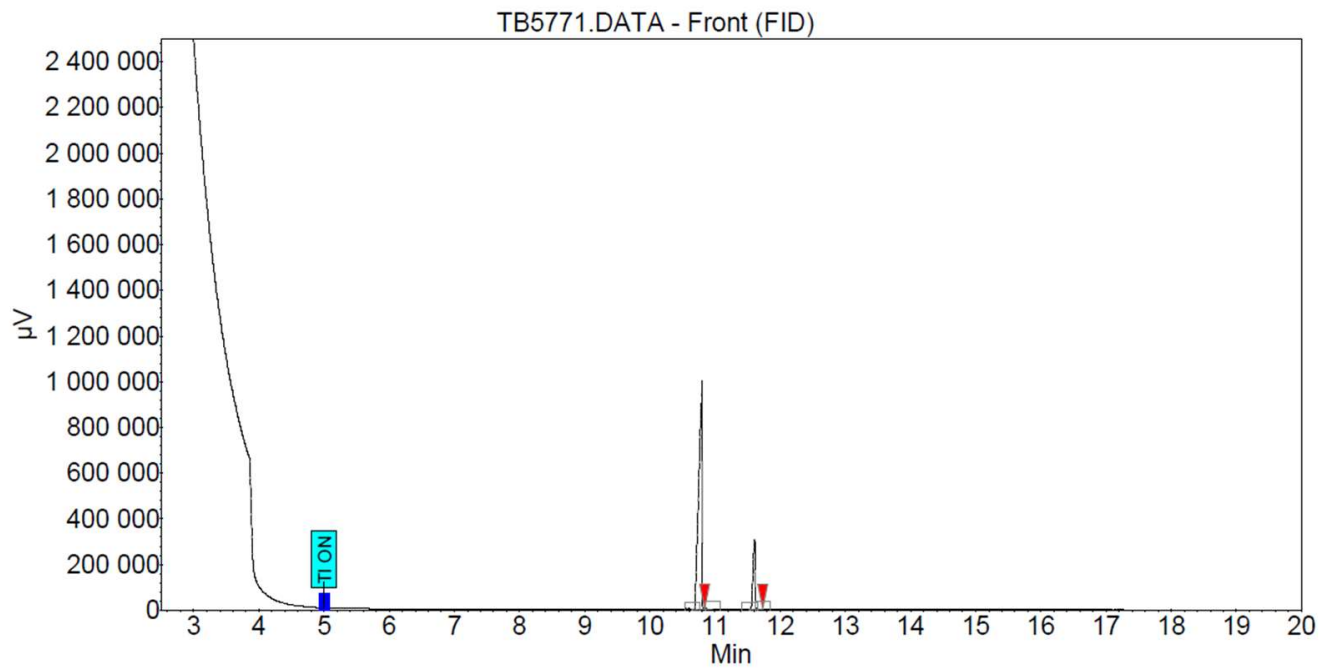

Peak results :

\begin{tabular}{|c|l|r|r|r|r|r|r|r|}
\hline Index & Name & $\begin{array}{r}\text { Time } \\
{[\mathrm{Min}]}\end{array}$ & $\begin{array}{r}\text { Area \% } \\
{[\%]}\end{array}$ & $\begin{array}{r}\text { Quantity } \\
{[\% \text { Area] }}\end{array}$ & $\begin{array}{r}\text { Height } \\
{[\mu \mathrm{V}]}\end{array}$ & $\begin{array}{r}\text { Area } \\
{[\mu \mathrm{V} \text {.Min] }}\end{array}$ & As. PE. & Res. HW \\
\hline \hline 1 & UNKNOWN & 10.800 & 85.184 & 85.18 & 1001843.0 & 59342.9 & 0.53 & 0.00 \\
\hline 2 & UNKNOWN & 11.608 & 14.816 & 14.82 & 306492.9 & 10321.2 & 0.61 & 10.27 \\
\hline & & & & & & & & \\
\hline Total & & & 100.000 & 100.00 & 1308335.9 & 69664.1 & & \\
\hline
\end{tabular}


(Z)-3-(2-methyl-3-(o-tolyl)acryloyl)oxazolidin-2-one ((Z)-11)

$(E)$-isomer $((E)-11)$

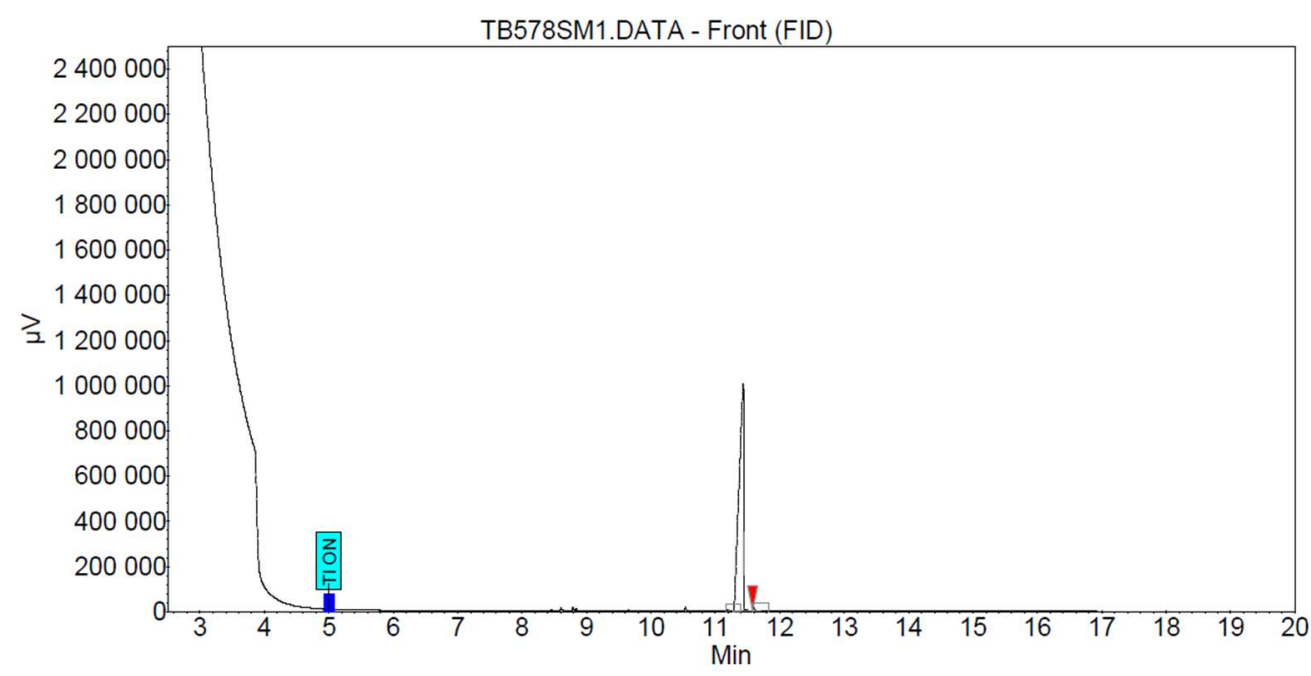

Peak results :

\begin{tabular}{|c|l|r|r|r|r|r|r|r|}
\hline Index & Name & $\begin{array}{r}\text { Time } \\
{[\mathrm{Min}]}\end{array}$ & $\begin{array}{r}\text { Area \% } \\
{[\%]}\end{array}$ & $\begin{array}{r}\text { Quantity } \\
{[\% \text { Area] }}\end{array}$ & $\begin{array}{r}\text { Height } \\
{[\mu \mathrm{V}]}\end{array}$ & $\begin{array}{r}\text { Area } \\
{[\mu \mathrm{V} . \mathrm{Min}]}\end{array}$ & As. PE. & Res. HW \\
\hline \hline 1 & UNKNOWN & 11.437 & 100,000 & 100,00 & 1005417.1 & 81708.0 & 0.53 & 0.00 \\
\hline & & & & & & & & \\
\hline Total & & & 100,000 & 100,00 & 1005417.1 & 81708,0 & & \\
\hline
\end{tabular}

(Z)-isomer ((Z)-11)

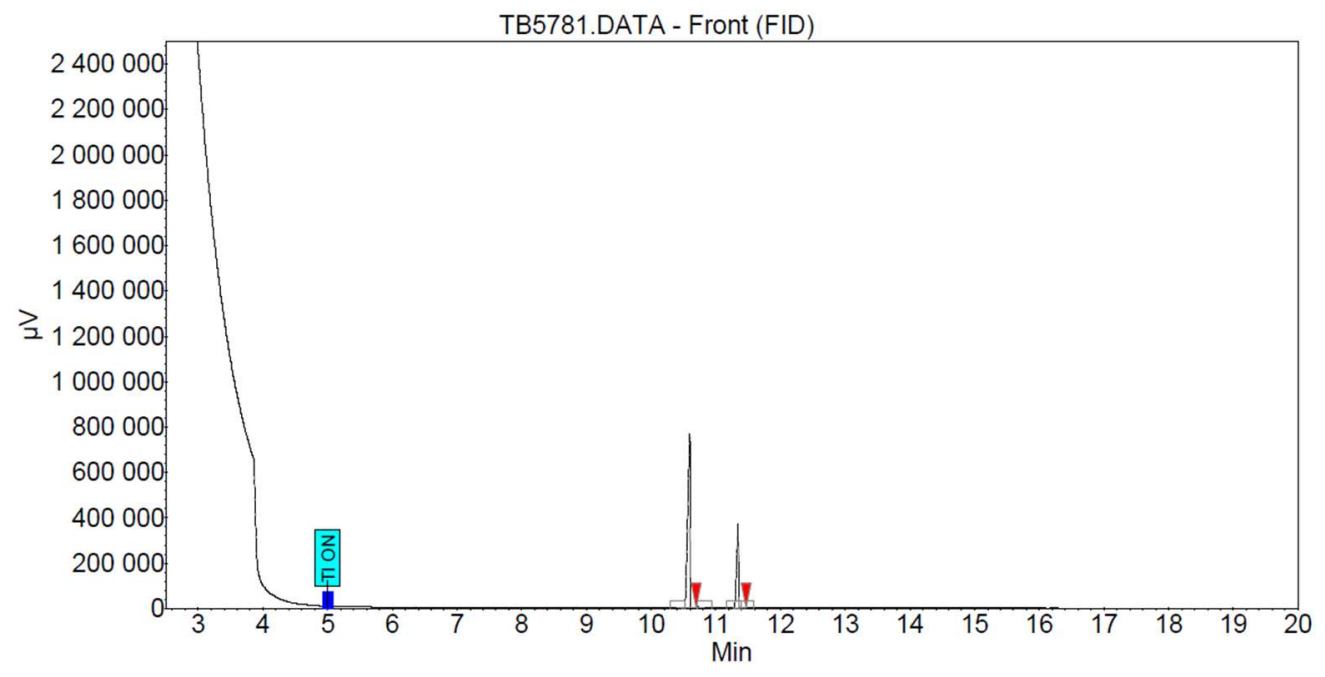

Peak results :

\begin{tabular}{|c|l|r|r|r|r|r|r|r|}
\hline Index & Name & $\begin{array}{r}\text { Time } \\
{[\mathrm{Min}]}\end{array}$ & $\begin{array}{r}\text { Area \% } \\
{[\%]}\end{array}$ & $\begin{array}{r}\text { Quantity } \\
{[\% \text { Area] }}\end{array}$ & $\begin{array}{r}\text { Height } \\
{[\mu \mathrm{V}]}\end{array}$ & $\begin{array}{r}\text { Area } \\
{[\mu \mathrm{N} \text {.Min] }}\end{array}$ & As. PE. & Res. HW \\
\hline \hline 1 & UNKNOWN & 10.597 & 71.134 & 71.13 & 765183.8 & 30088.0 & 0.56 & 0.00 \\
\hline 2 & UNKNOWN & 11.347 & 28.866 & 28.87 & 371237.3 & 12209.9 & 0.60 & 12.55 \\
\hline & & & & & & & & \\
\hline Total & & & 100.000 & 100.00 & 1136421.2 & 42298.0 & & \\
\hline
\end{tabular}


(Z)-3-(3-(4-(tert-butyl)phenyl)-2-methylacryloyl)oxazolidin-2-one ((Z)-12)

$(E)$-isomer $((E)-12)$

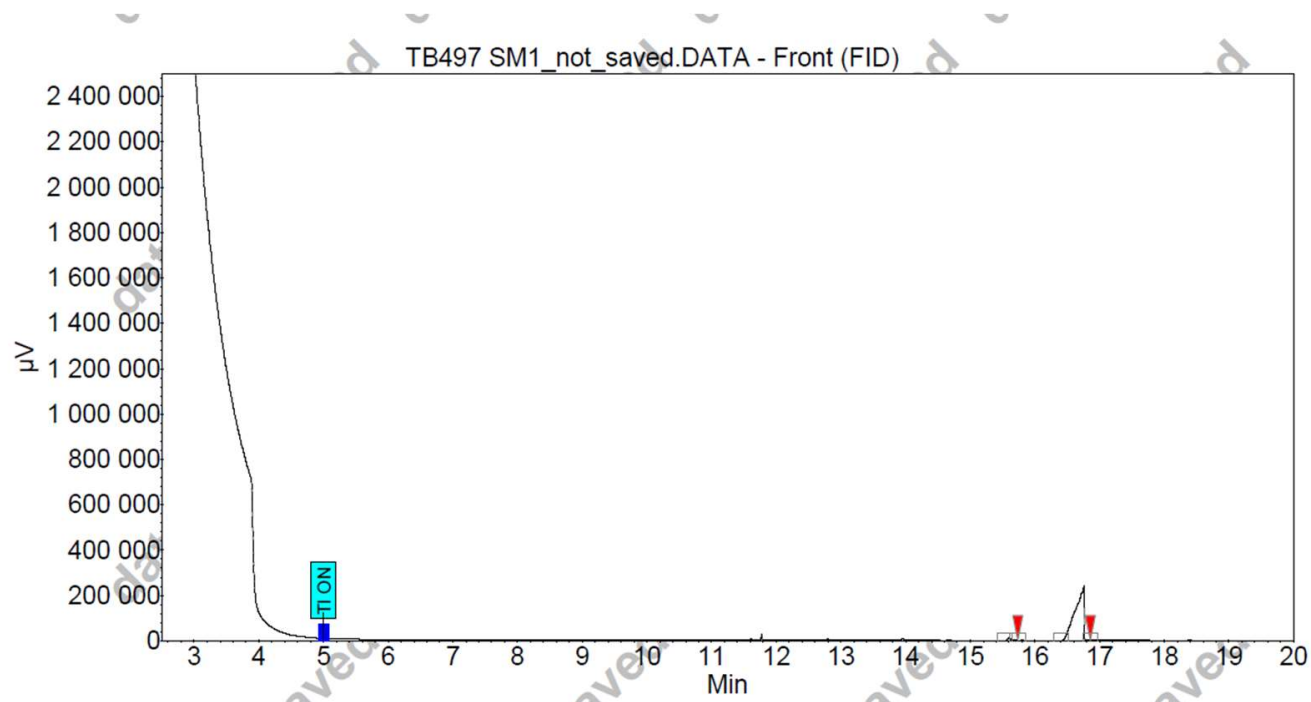

Peak results :

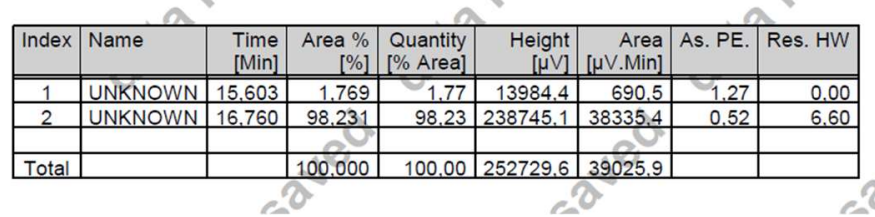

(Z)-isomer ((Z)-12)

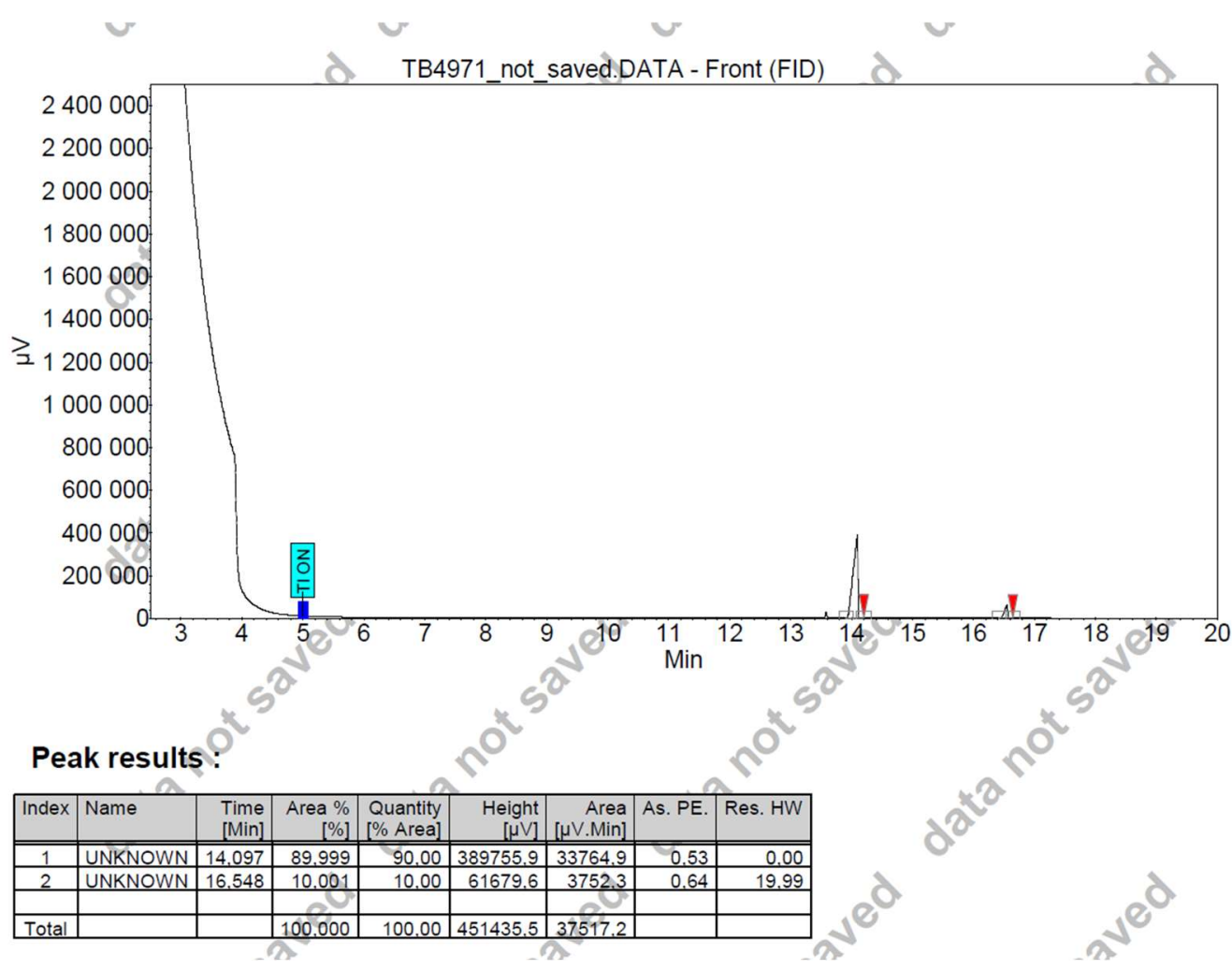




\section{(Z)-3-(3-(4-methoxyphenyl)-2-methylacryloyl)oxazolidin-2-one ((Z)-14)}

$(E)$-isomer $((E)-14)$

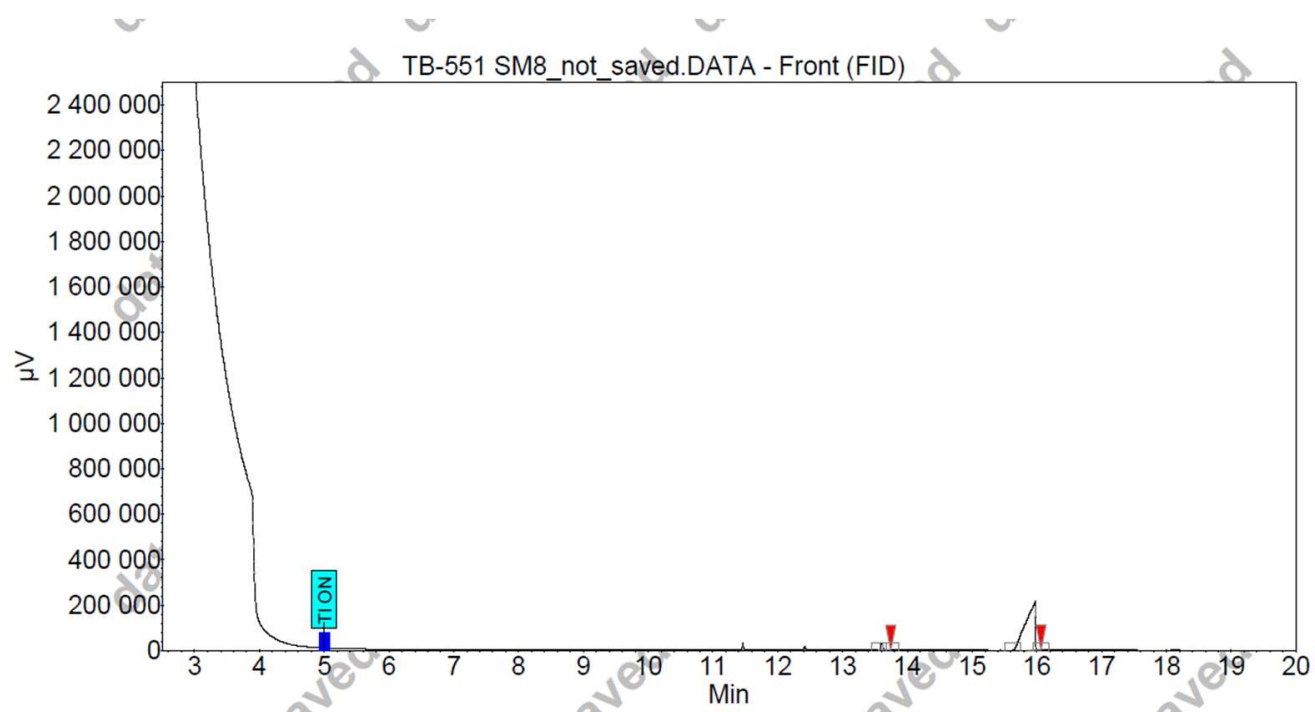

Peak results :

\begin{tabular}{|c|l|r|r|r|r|r|r|r|}
\hline Index & Name & $\begin{array}{r}\text { Time } \\
{[\text { Min] }}\end{array}$ & $\begin{array}{r}\text { Area \% } \\
{[\%]}\end{array}$ & $\begin{array}{r}\text { Quantity } \\
{[\% \text { Area] }}\end{array}$ & $\begin{array}{r}\text { Height } \\
{[\mu \mathrm{V}]}\end{array}$ & $\begin{array}{r}\text { Area } \\
{[\mu \mathrm{V} . \mathrm{Min}]}\end{array}$ & As. PE. & Res. HW \\
\hline \hline 1 & UNKNOWN & 13.605 & 3.107 & 3.11 & 34191.2 & 1181.0 & 1.49 & 0.00 \\
\hline 2 & UNKNOWN & 15.973 & 96.893 & 96.89 & 214196.1 & 36836.7 & 0.52 & 13.96 \\
\hline & & & & & & & & \\
\hline Total & & & 100.000 & 100.00 & 248387.3 & 38017.7 & & \\
\hline
\end{tabular}

(Z)-isomer ((Z)-14)

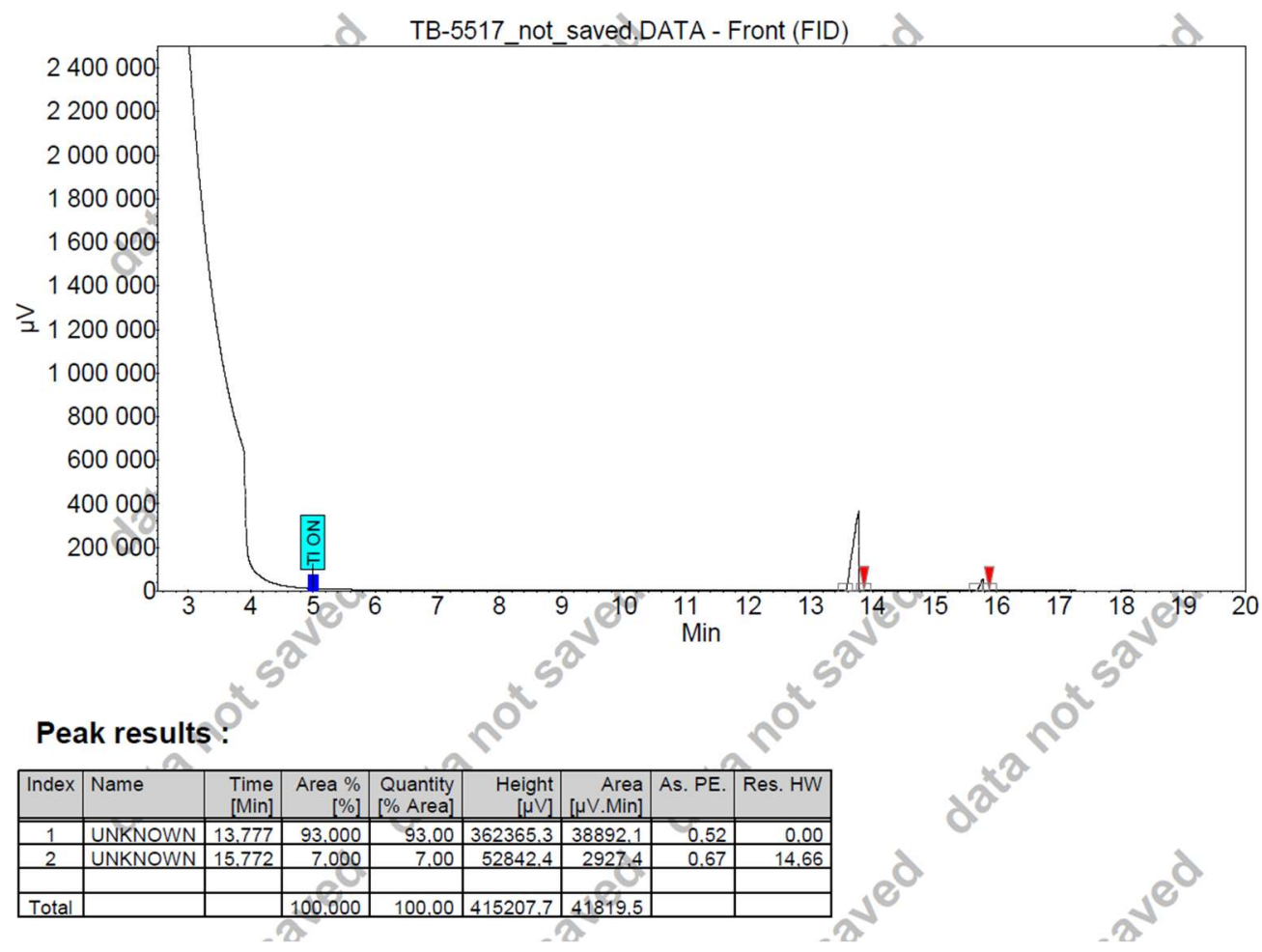


(Z)-3-(3-(4-fluorophenyl)-2-methylacryloyl)oxazolidin-2-one ((Z)-16):

$(E)$-isomer $((E)-16)$

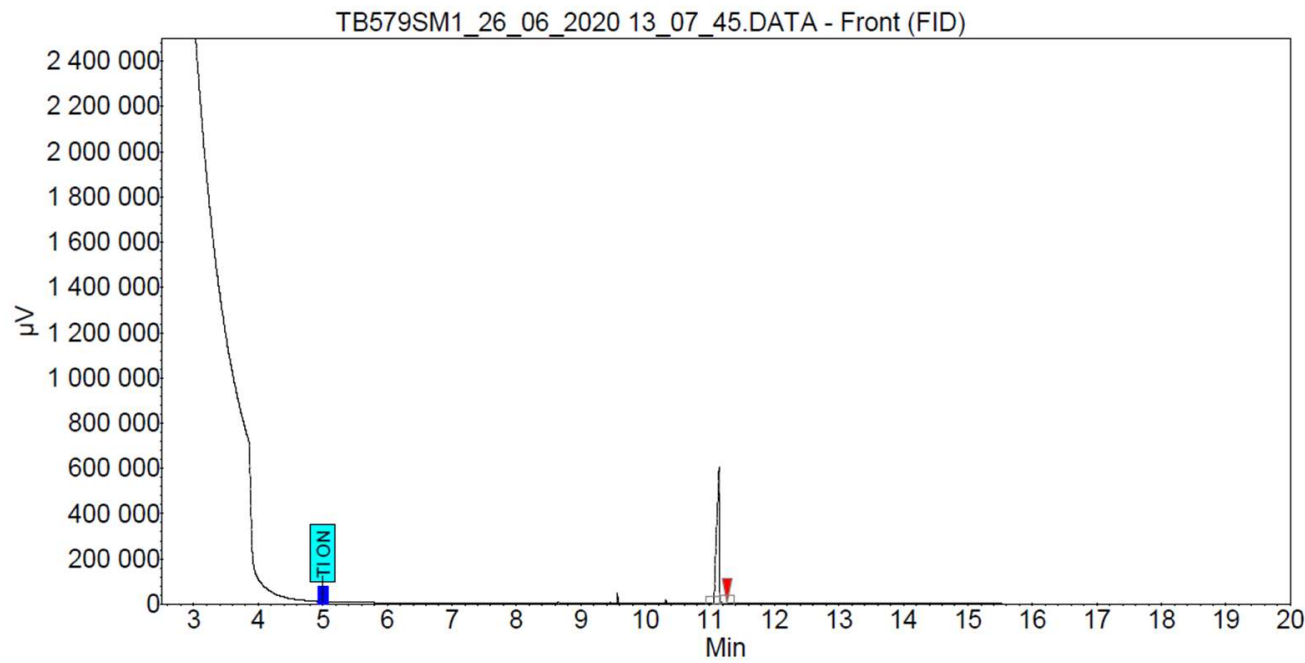

Peak results :

\begin{tabular}{|c|l|r|r|r|r|r|r|r|}
\hline Index & Name & $\begin{array}{r}\text { Time } \\
{[\text { Min] }}\end{array}$ & $\begin{array}{r}\text { Area \% } \\
{[\%]}\end{array}$ & $\begin{array}{r}\text { Quantity } \\
{[\% \text { Area] }}\end{array}$ & $\begin{array}{r}\text { Height } \\
{[\mu \mathrm{V}]}\end{array}$ & $\begin{array}{r}\text { Area } \\
{[\mu \mathrm{V} . \text { Min] }}\end{array}$ & As. PE. & Res. HW \\
\hline \hline 1 & UNKNOWN & 11.142 & 100.000 & 100.00 & 604135.1 & 28059.5 & 0.56 & 0.00 \\
\hline & & & & & & & & \\
\hline Total & & & 100.000 & 100.00 & 604135.1 & 28059.5 & & \\
\hline
\end{tabular}

(Z)-isomer ((Z)-16)

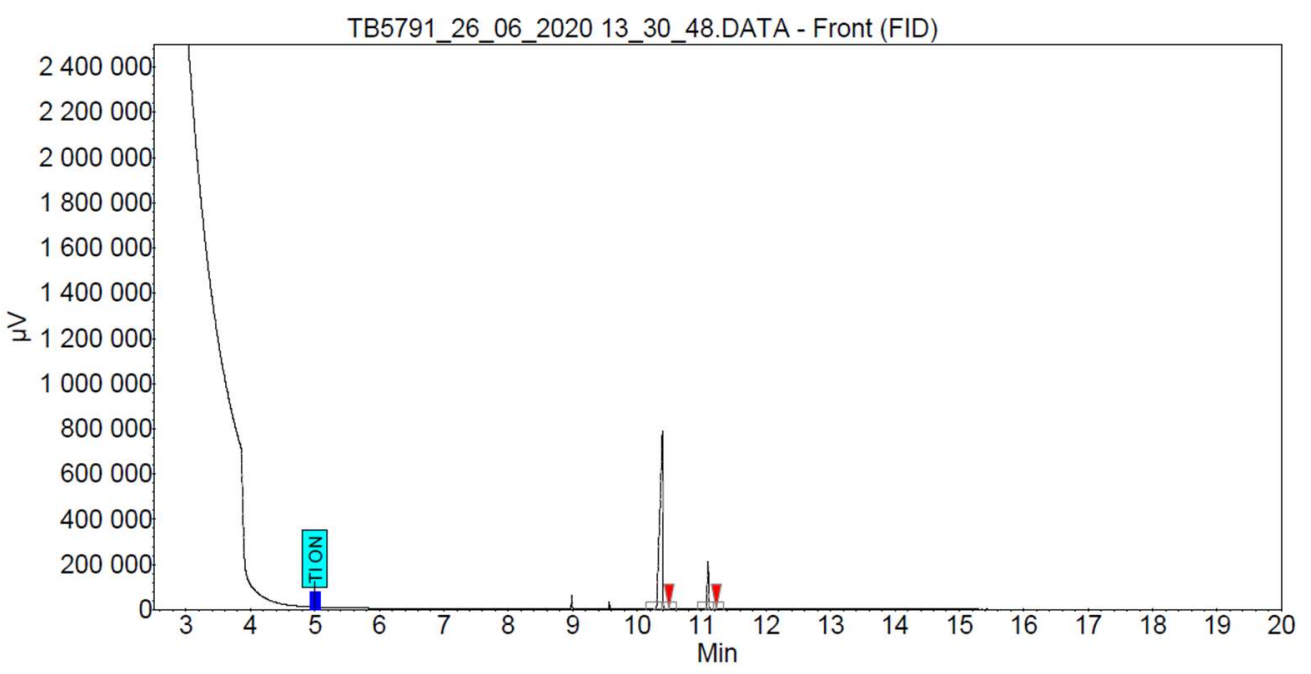

Peak results :

\begin{tabular}{|c|l|r|r|r|r|r|r|r|}
\hline Index & Name & $\begin{array}{r}\text { Time } \\
{[\mathrm{Min}]}\end{array}$ & $\begin{array}{r}\text { Area \% } \\
{[\%]}\end{array}$ & $\begin{array}{r}\text { Quantity } \\
{[\% \text { Area] }}\end{array}$ & $\begin{array}{r}\text { Height } \\
{[\mu \mathrm{VV}]}\end{array}$ & $\begin{array}{r}\text { Area } \\
{[\mu \mathrm{V} . \mathrm{Min}]}\end{array}$ & As. PE. & Res. HW \\
\hline \hline 1 & UNKNOWN & 10.397 & 89.766 & 89.77 & 786803.4 & 42856.5 & 0.55 & 0.00 \\
\hline 2 & UNKNOWN & 11.102 & 10.234 & 10.23 & 208047.0 & 4886.1 & 0.73 & 10.85 \\
\hline & & & & & & & & \\
\hline Total & & & 100.000 & 100.00 & 994850.3 & 47742.7 & & \\
\hline
\end{tabular}


(Z)-3-(3-(4-chlorophenyl)-2-methylacryloyl)oxazolidin-2-one ((Z)-17)

$(E)$-isomer $((E)-17)$

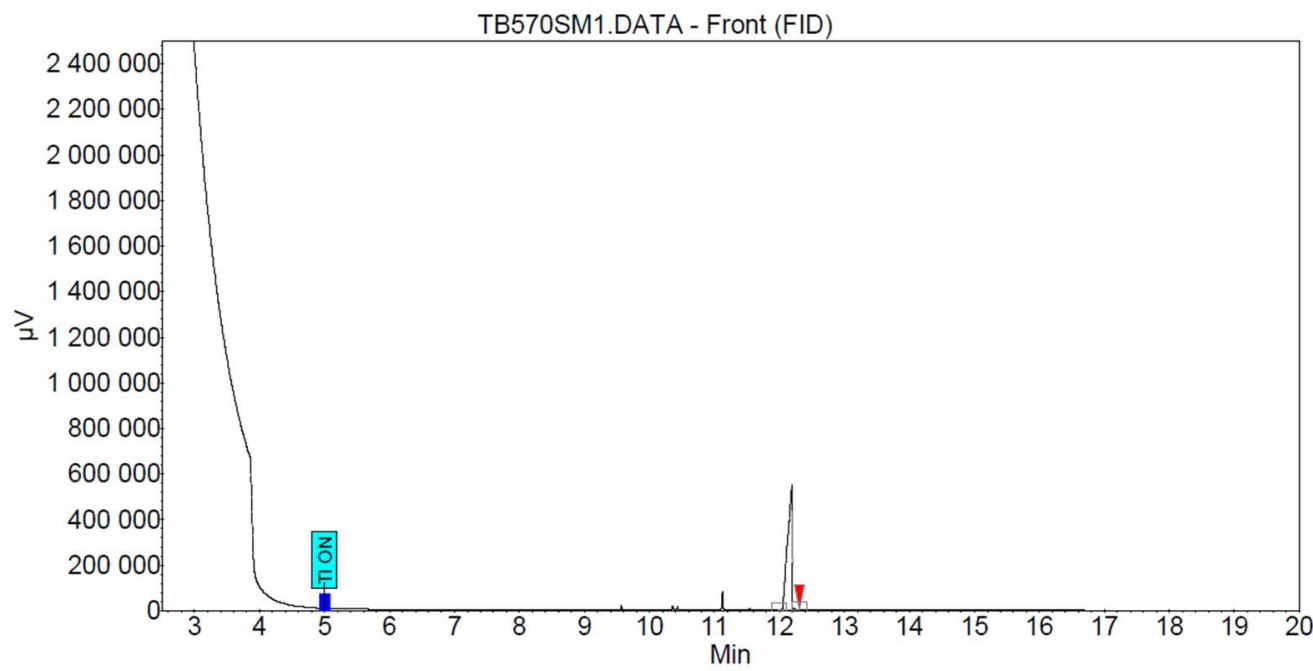

Peak results :

\begin{tabular}{|c|l|r|r|r|r|r|r|r|}
\hline Index & Name & $\begin{array}{r}\text { Time } \\
{[\mathrm{Min}]}\end{array}$ & $\begin{array}{r}\text { Area \% } \\
{[\%]}\end{array}$ & $\begin{array}{r}\text { Quantity } \\
{[\% \text { Area] }}\end{array}$ & $\begin{array}{r}\text { Height } \\
{[\mu \mathrm{V}]}\end{array}$ & $\begin{array}{r}\text { Area } \\
{[\mu \mathrm{V} . \mathrm{Min}]}\end{array}$ & As. PE. & Res. HW \\
\hline \hline 1 & UNKNOWN & 12.193 & 100,000 & 100,00 & 546105.5 & 44670.4 & 0.53 & 0.00 \\
\hline & & & & & & & & \\
\hline Total & & & 100,000 & 100,00 & 546105,5 & 44670,4 & & \\
\hline
\end{tabular}

(Z)-isomer ((Z)-17)

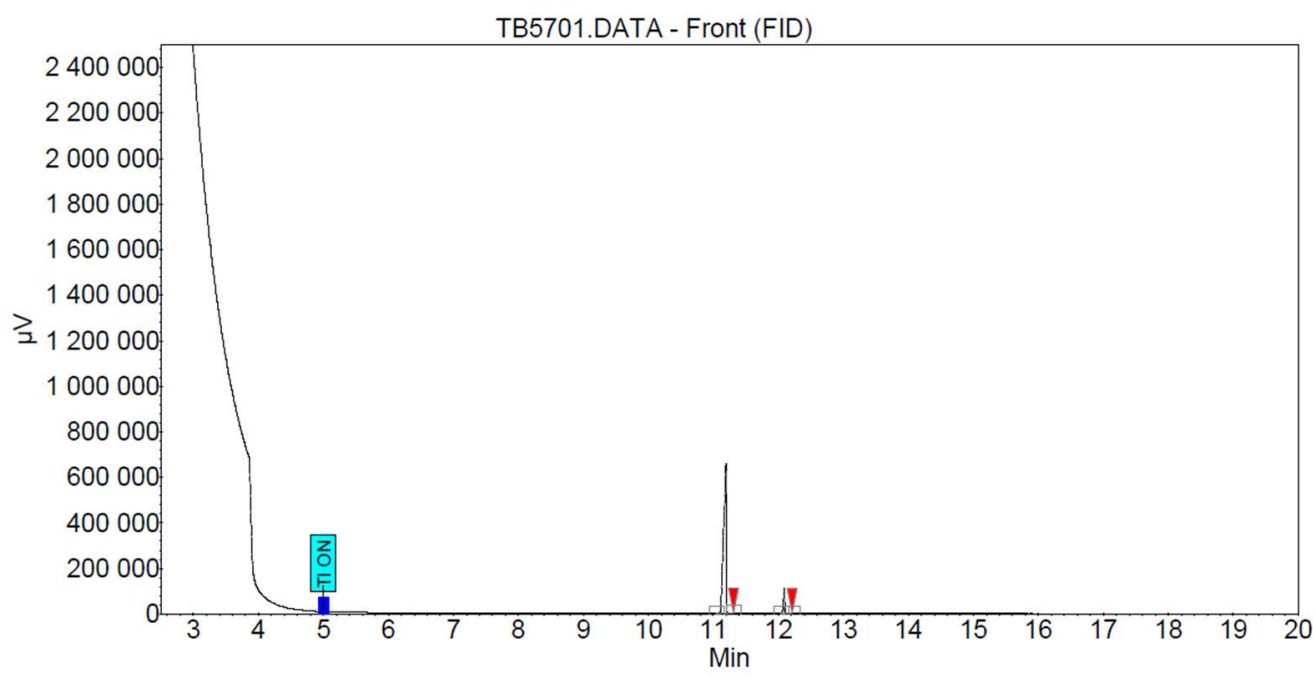

Peak results :

\begin{tabular}{|c|l|r|r|r|r|r|r|r|}
\hline Index & Name & $\begin{array}{r}\text { Time } \\
{[\mathrm{Min}]}\end{array}$ & $\begin{array}{r}\text { Area \% } \\
{[\%]}\end{array}$ & $\begin{array}{r}\text { Quantity } \\
{[\% \text { Area] }}\end{array}$ & $\begin{array}{r}\text { Height } \\
{[\mu \mathrm{V}]}\end{array}$ & $\begin{array}{r}\text { Area } \\
{[\mu \mathrm{V} . \text { Min] }}\end{array}$ & As. PE. & Res. HW \\
\hline \hline 1 & UNKNOWN & 11.195 & 91.605 & 91.61 & 659492.8 & 31214.0 & 0.55 & 0.00 \\
\hline 2 & UNKNOWN & 12.092 & 8.395 & 8.39 & 111583.3 & 2860.5 & 0.80 & 15.22 \\
\hline & & & & & & & & \\
\hline Total & & & 100.000 & 100.00 & 771076.1 & 34074.5 & & \\
\hline
\end{tabular}


(Z)-3-(3-(4-bromophenyl)-2-methylacryloyl)oxazolidin-2-one ((Z)-18)

$(E)$-isomer $((E)$-18)

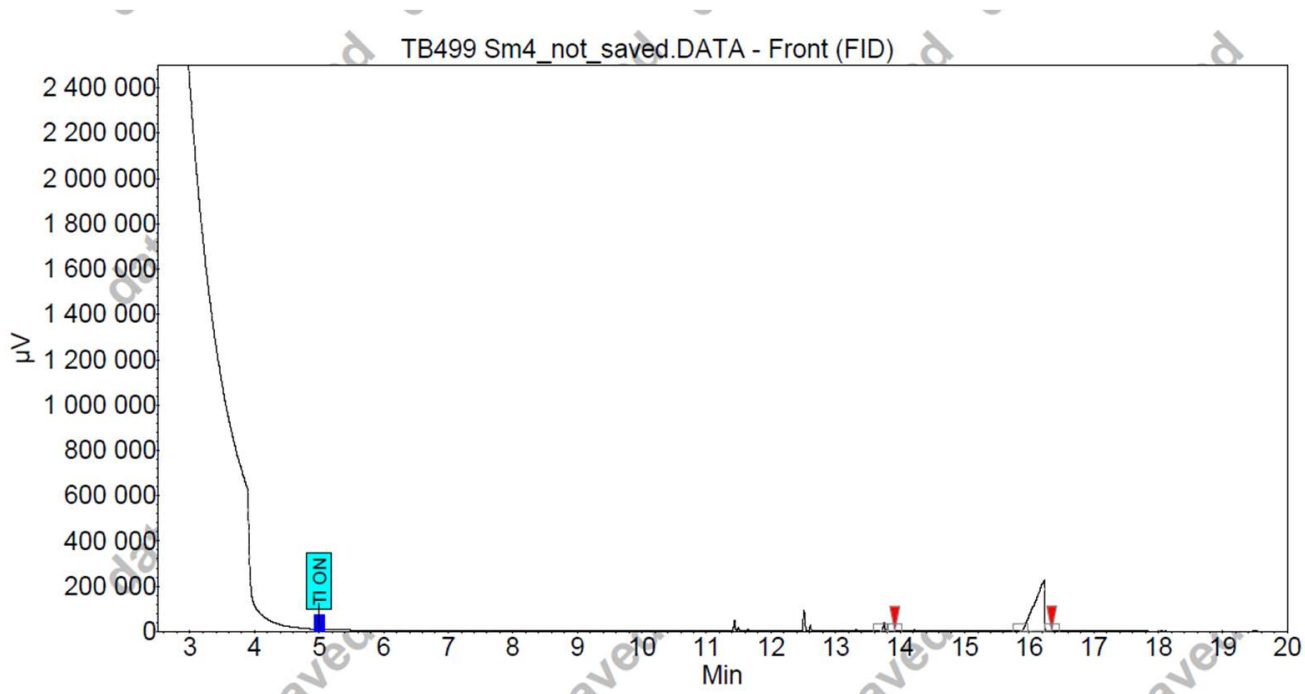

Peak results :

\begin{tabular}{|c|l|r|r|r|r|r|r|r|}
\hline Index & Name & $\begin{array}{r}\text { Time } \\
{[\text { Min] }}\end{array}$ & $\begin{array}{r}\text { Area \% } \\
{[\%]}\end{array}$ & $\begin{array}{r}\text { Quantity } \\
{[\% \text { Area] }}\end{array}$ & $\begin{array}{r}\text { Height } \\
{[\mu \mathrm{V}]}\end{array}$ & $\begin{array}{r}\text { Area } \\
{[\mu \mathrm{V} . \text { Min] }}\end{array}$ & As. PE. & Res. HW \\
\hline \hline 2 & UNKNOWN & 13.755 & 2.712 & 2.71 & 36254.7 & 1109.3 & 1.01 & 0.00 \\
\hline 1 & UNKNOWN & 16.237 & 97.288 & 97.29 & 223461.3 & 39793.6 & 0.51 & 14.92 \\
\hline & & & & & & & & \\
\hline Total & & & 100.000 & 100.00 & 259716.0 & 40902.9 & & \\
\hline \multicolumn{70}{|c|}{} \\
\hline
\end{tabular}

(Z)-isomer ((Z)-18)

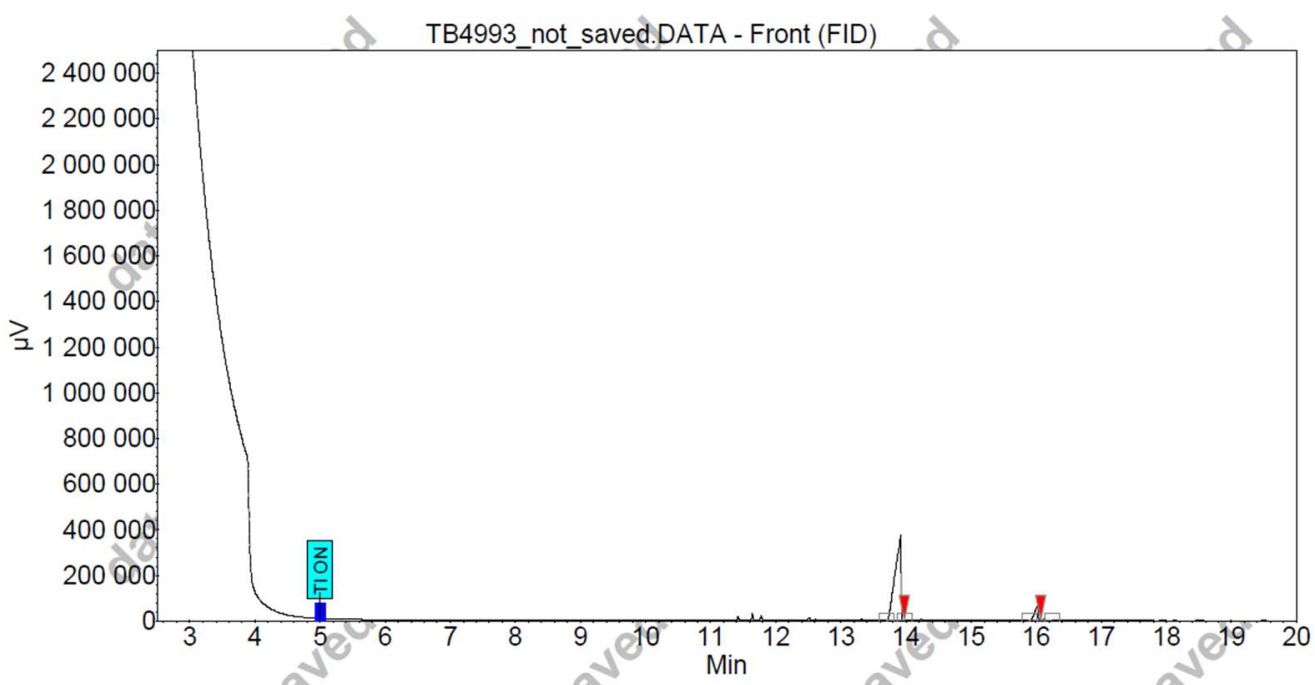

\section{Peak results :}

\begin{tabular}{|c|l|r|r|r|r|r|r|r|}
\hline Index & Name & $\begin{array}{r}\text { Time } \\
{[\text { Min] }]}\end{array}$ & $\begin{array}{r}\text { Area \% } \\
{[\%]}\end{array}$ & $\begin{array}{r}\text { Quantity } \\
{[\% \text { Area] }}\end{array}$ & $\begin{array}{r}\text { Height } \\
{[\mu \mathrm{V}]}\end{array}$ & $\begin{array}{r}\text { Area } \\
{[\mu \mathrm{V} . \mathrm{Min}]}\end{array}$ & As. PE. & Res. HW \\
\hline \hline 1 & UNKNOWN & 13.923 & 90.451 & 90.45 & 372519.8 & 39054.7 & 0.52 & 0.00 \\
\hline 2 & UNKNOWN & 16.023 & 9.549 & 9.55 & 64038.4 & 4122.9 & 0.61 & 14.65 \\
\hline & & & & & & & & \\
\hline Total & & & 100.000 & 100.00 & 436558.3 & 43177.6 & & \\
\hline
\end{tabular}


(Z)-3-(3-(4-iodophenyl)-2-methylacryloyl)oxazolidin-2-one ((Z)-19)

$(E)$-isomer $((E)-19)$

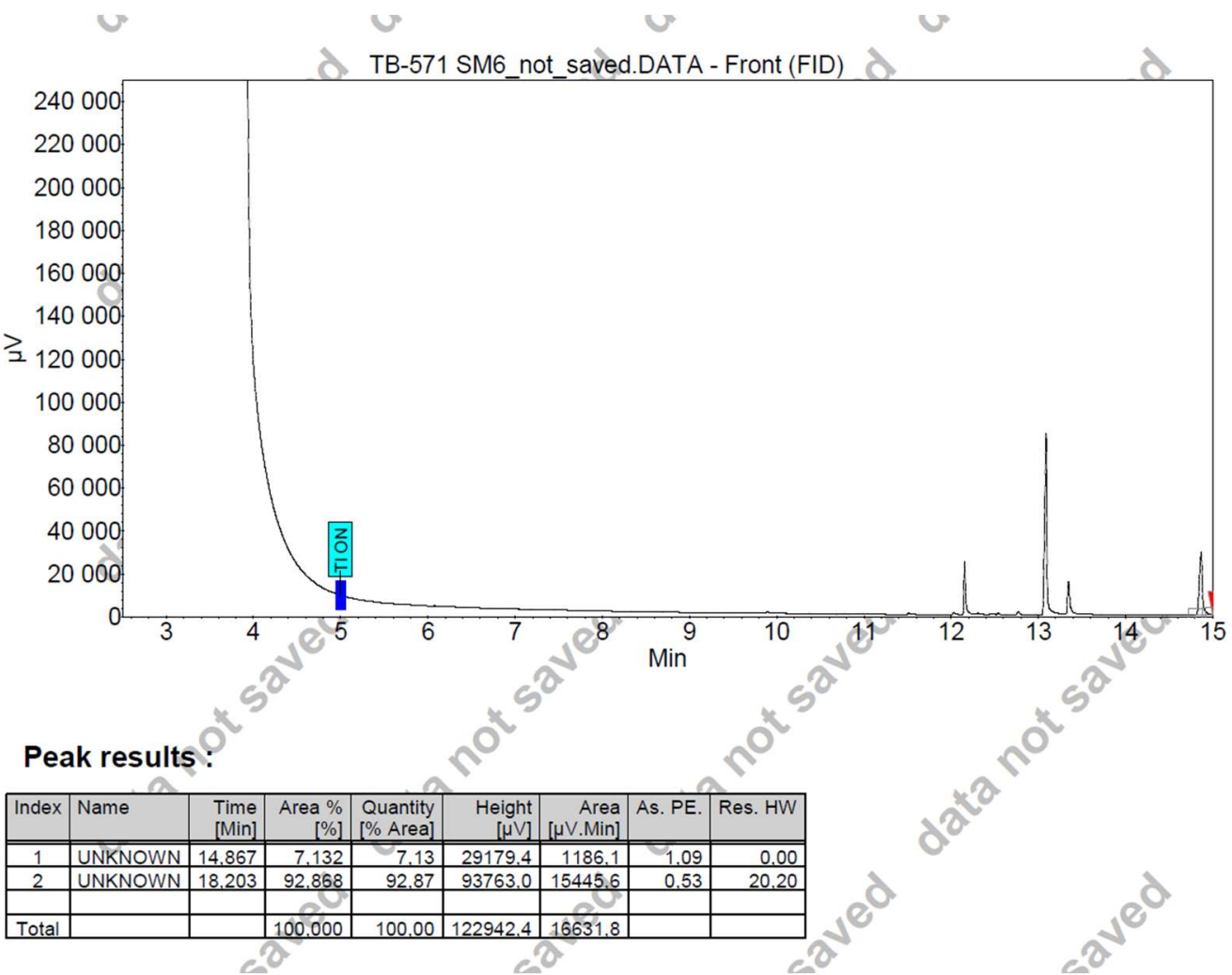

(Z)-isomer ((Z)-19)
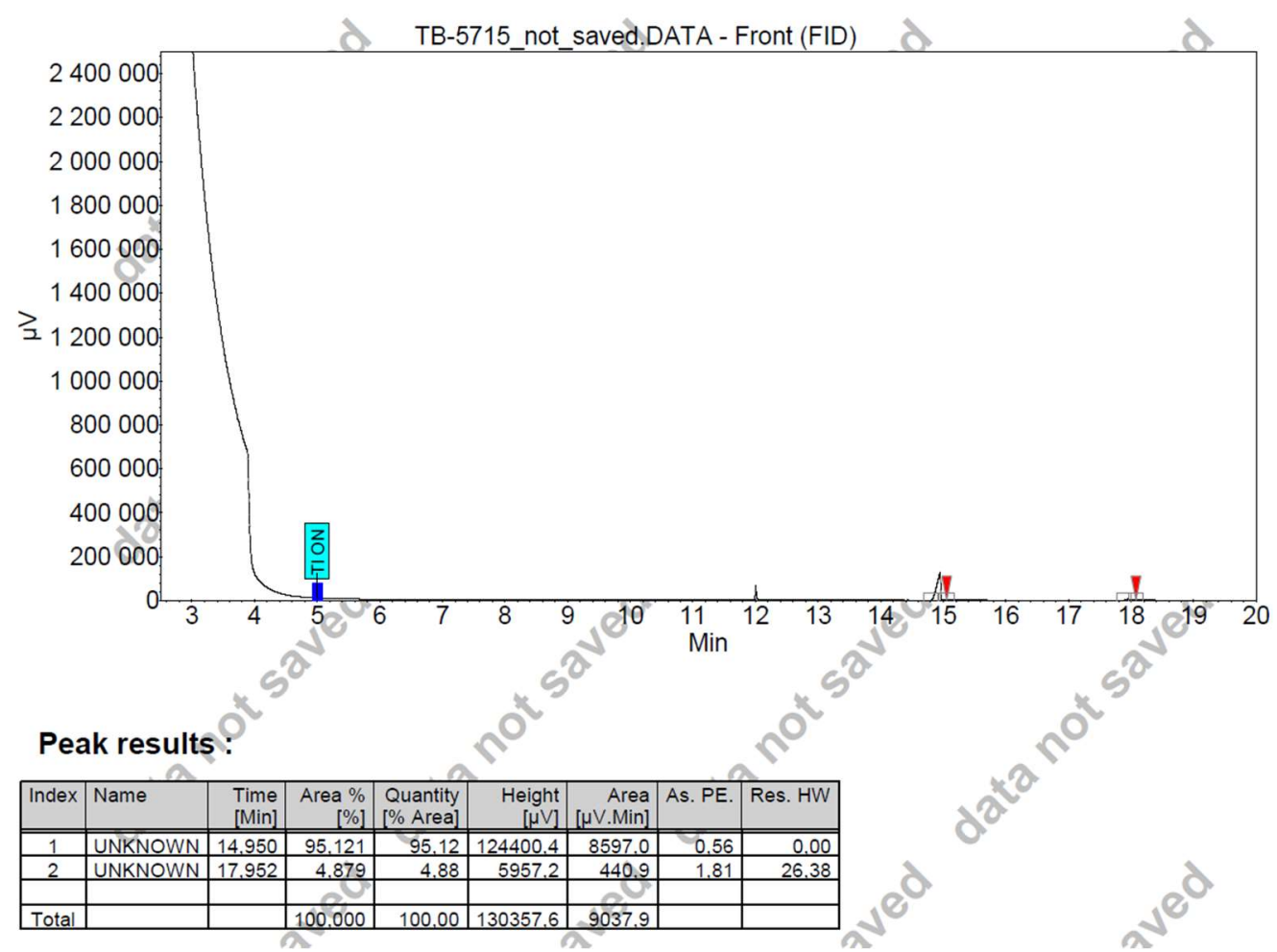
(Z)-3-(2-methyl-3-(4-(trifluoromethyl)phenyl)acryloyl)oxazolidin-2-one ((Z)-20)

$(E)$-isomer $((E)-20)$
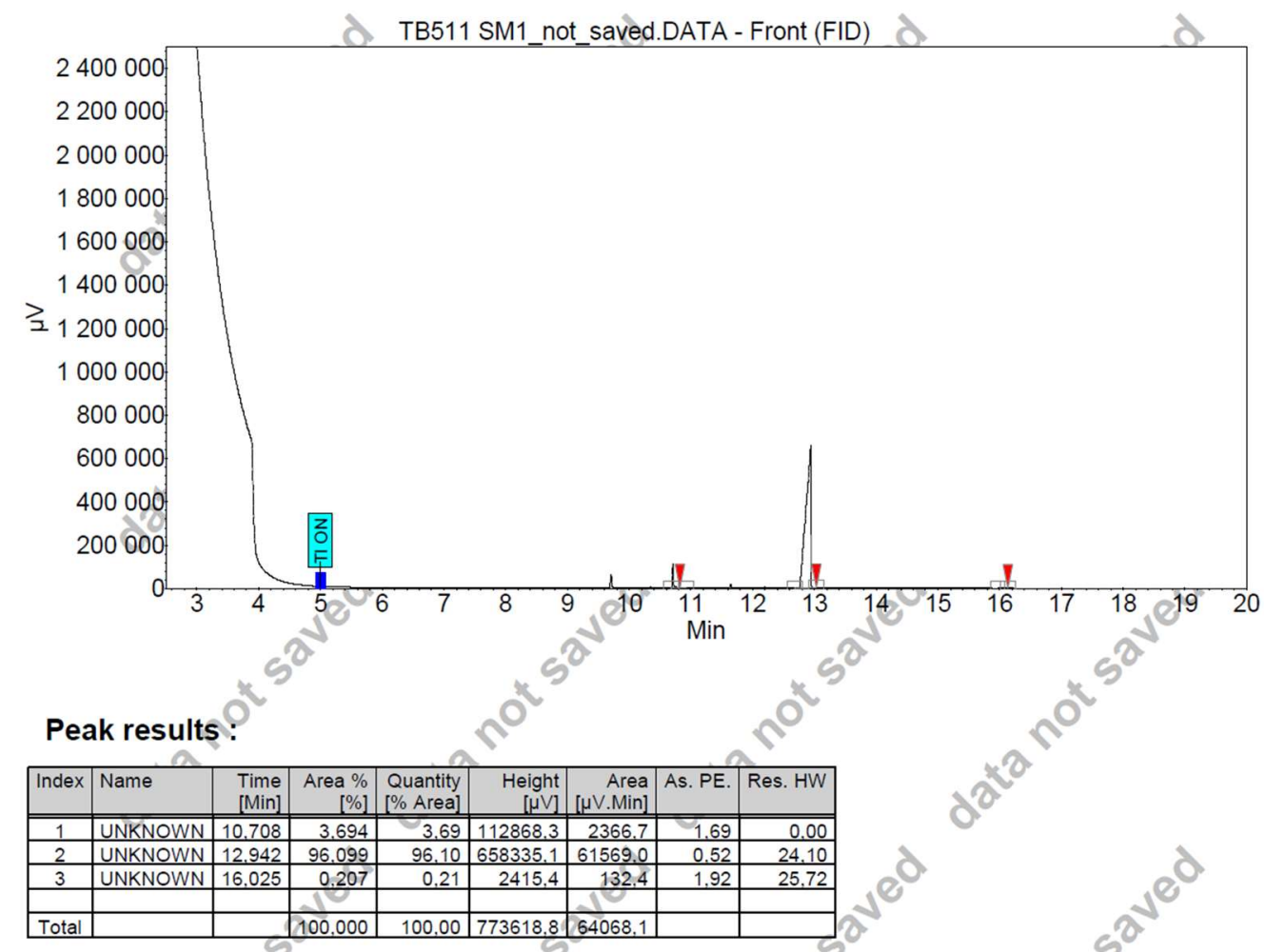

(Z)-isomer ((Z)-20)

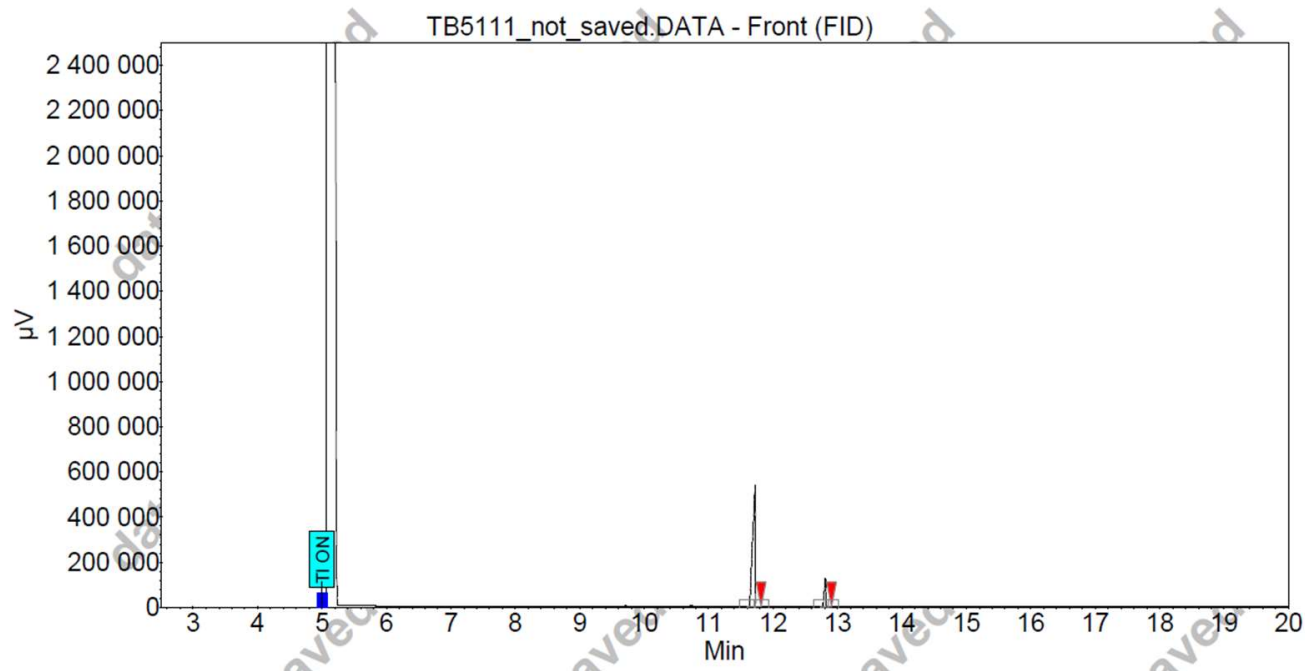

Peak results :

\begin{tabular}{|c|l|r|r|r|r|r|r|r|}
\hline Index & Name & $\begin{array}{r}\text { Time } \\
{[\text { Min] }}\end{array}$ & $\begin{array}{r}\text { Area \% } \\
{[\%]}\end{array}$ & $\begin{array}{r}\text { Quantity } \\
{[\% \text { Area] }}\end{array}$ & $\begin{array}{r}\text { Height } \\
{[\mu \mathrm{V}]}\end{array}$ & $\begin{array}{r}\text { Area } \\
{[\mu \mathrm{V} . \text { Min] }}\end{array}$ & As. PE. Res. HW & \\
\hline \hline 1 & UNKNOWN & 11.722 & 88.255 & 88.25 & 540610.4 & 26148.8 & 0.55 & 0.00 \\
\hline 2 & UNKNOWN & 12.810 & 11.745 & 11.75 & 125674.4 & 3480.0 & 0.72 & 17.31 \\
\hline & & & & & & & & \\
\hline Total & & & 100.000 & 100.00 & 666284.8 & 29628.8 & & \\
\hline
\end{tabular}


(Z)-3-(2-methyl-3-(4-(4,4,5,5-tetramethyl-1,3,2-dioxaborolan-2yl)phenyl)acryloyl)oxazolidin-2-one ((Z)-21):

$(E)$-isomer $((E)-21)$

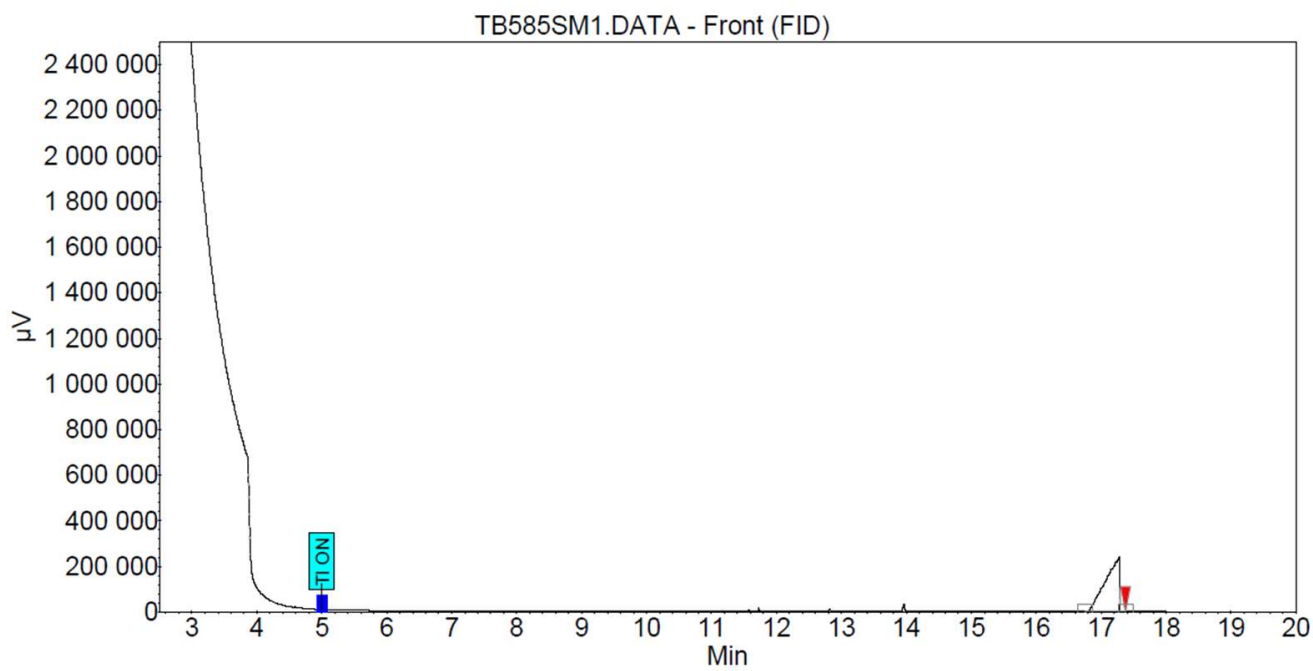

Peak results :

\begin{tabular}{|c|l|r|r|r|r|r|r|r|}
\hline Index & Name & $\begin{array}{r}\text { Time } \\
{[\mathrm{Min}]}\end{array}$ & $\begin{array}{r}\text { Area \% } \\
{[\%]}\end{array}$ & $\begin{array}{r}\text { Quantity } \\
{[\% \text { Area] }}\end{array}$ & $\begin{array}{r}\text { Height } \\
{[\mu \mathrm{V}]}\end{array}$ & $\begin{array}{r}\text { Area } \\
{[\mu \mathrm{V} . \mathrm{Min}]}\end{array}$ & As. PE. & Res. HW \\
\hline \hline 1 & UNKNOWN & 17.280 & 100.000 & 100.00 & 241388.4 & 59507.3 & 0.51 & 0.00 \\
\hline & & & & & & & & \\
\hline Total & & & 100.000 & 100.00 & 241388.4 & 59507.3 & & \\
\hline
\end{tabular}

(Z)-isomer ((Z)-21)

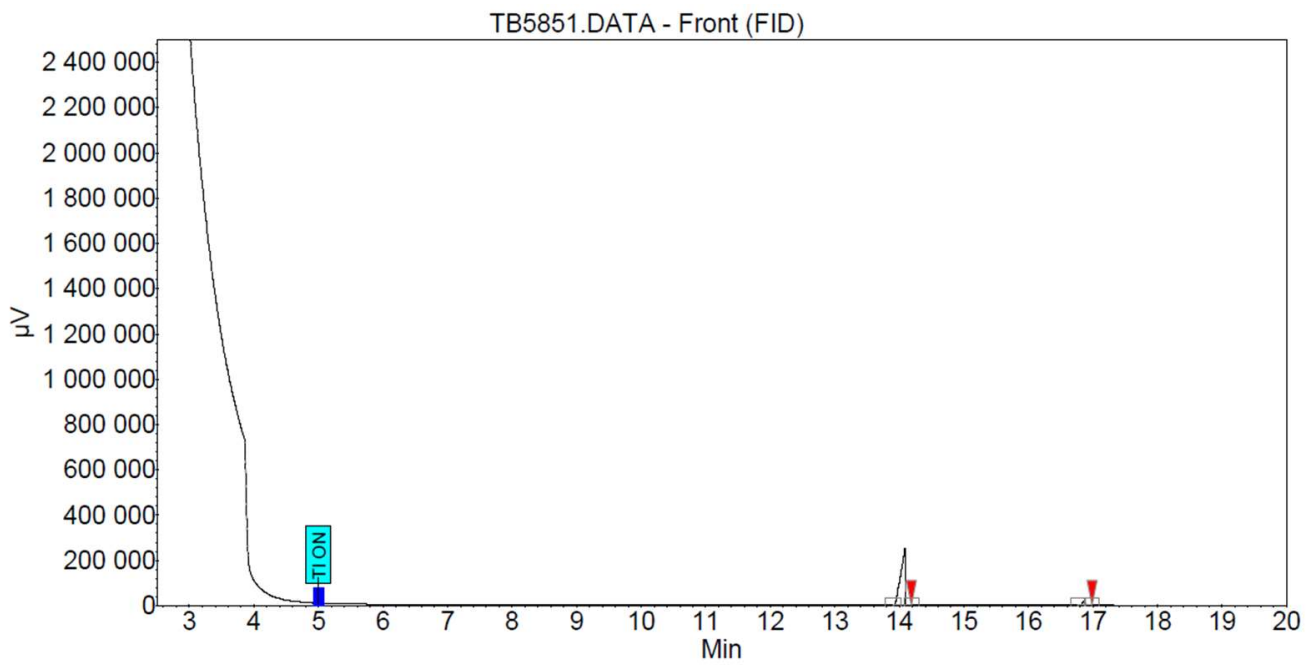

Peak results :

\begin{tabular}{|c|l|r|r|r|r|r|r|r|}
\hline Index & Name & $\begin{array}{r}\text { Time } \\
{[\text { Min] }}\end{array}$ & $\begin{array}{r}\text { Area \% } \\
{[\%]}\end{array}$ & $\begin{array}{r}\text { Quantity } \\
{[\% \text { Area] }}\end{array}$ & $\begin{array}{r}\text { Height } \\
{[\mu \mathrm{V}]}\end{array}$ & $\begin{array}{r}\text { Area } \\
{[\mu \mathrm{V} . \mathrm{Min}]}\end{array}$ & As. PE. & Res. HW \\
\hline \hline 1 & UNKNOWN & 14.092 & 95.349 & 95.35 & 249768.7 & 21267.8 & 0.53 & 0.00 \\
\hline 2 & UNKNOWN & 16.863 & 4.651 & 4.65 & 18483.9 & 1037.4 & 0.88 & 24.55 \\
\hline & & & & & & & & \\
\hline Total & & & 100.000 & 100.00 & 268252.5 & 22305.1 & & \\
\hline
\end{tabular}


(Z)-3-(2-methyl-3-(4-(methylsulfonyl)phenyl)acryloyl)oxazolidin-2-one ((Z)-22):

$(E)$-isomer $((E)-22)$

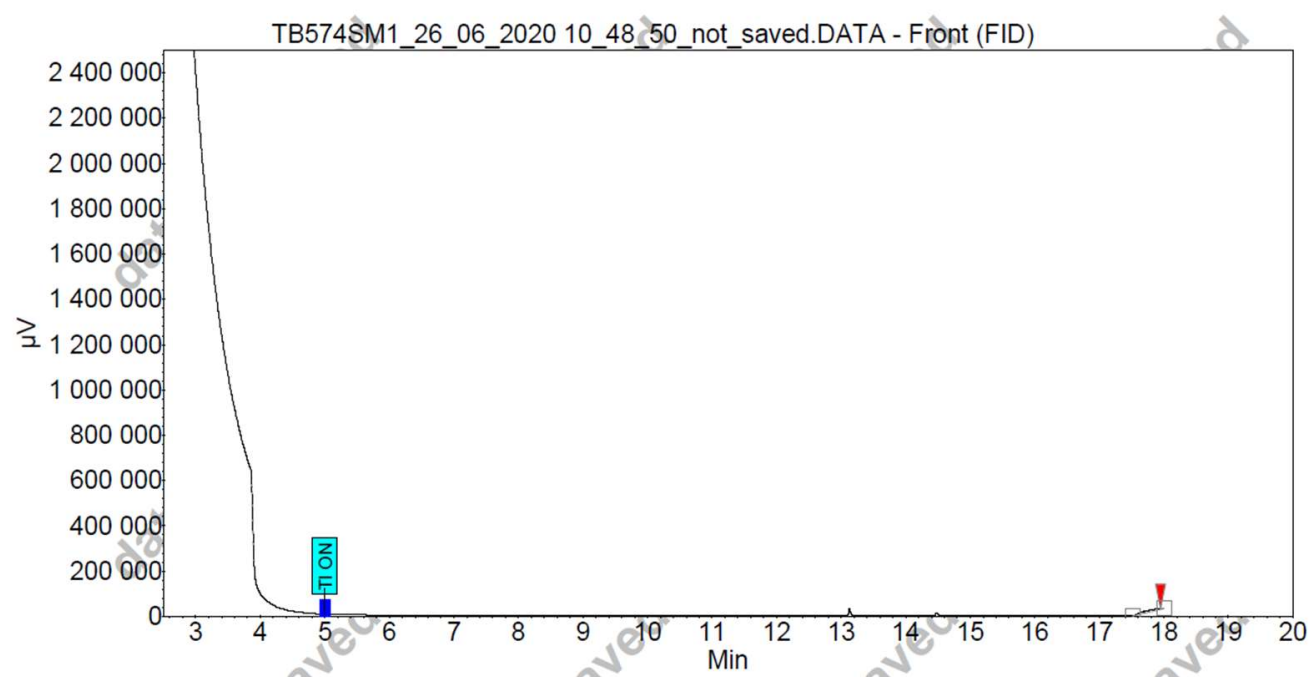

Peak results:

\begin{tabular}{|c|c|c|c|c|c|c|c|c|}
\hline Index & Name & $\begin{array}{l}\text { Time } \\
\text { [Min] }\end{array}$ & $\begin{array}{l}\text { Area } \% \\
{[\%]}\end{array}$ & \begin{tabular}{|l|} 
Quantity \\
{$[\%$ Area] }
\end{tabular} & $\begin{array}{c}\text { Height } \\
{[\mu \mathrm{V}]}\end{array}$ & $\begin{array}{r}\begin{array}{r}\text { Area } \\
{[\mu \mathrm{V} \text {.Min] }}\end{array} \\
\end{array}$ & As. PE. & Res. HW \\
\hline$\overline{11}$ & UNKNOWN & 17.673 & 100.000 & 100.00 & 8927.8 & 2614.6 & 2.40 & 0.0 \\
\hline otal & & & 100.000 & 200 & 2 & 2614 & & \\
\hline
\end{tabular}

(Z)-isomer ((Z)-22)

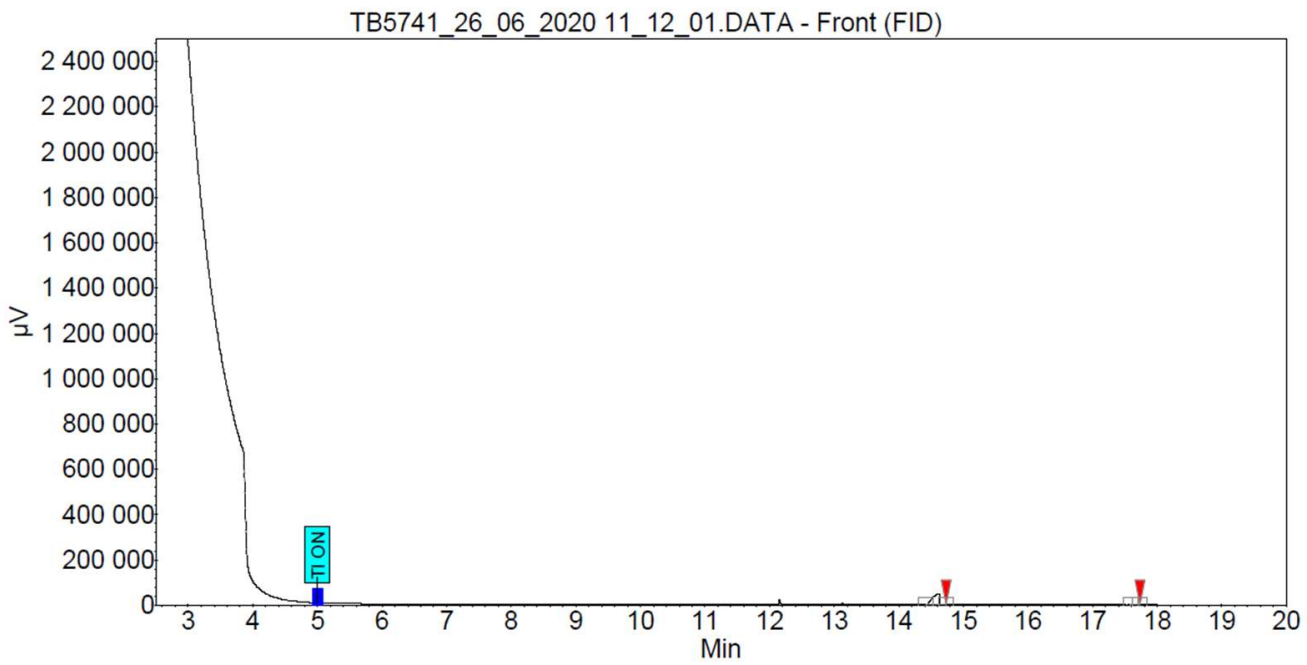

Peak results :

\begin{tabular}{|c|l|r|r|r|r|r|r|r|}
\hline Index & Name & $\begin{array}{r}\text { Time } \\
{[\text { Min] }]}\end{array}$ & $\begin{array}{r}\text { Area \% } \\
{[\%]}\end{array}$ & $\begin{array}{r}\text { Quantity } \\
{[\% \text { Area] }}\end{array}$ & $\begin{array}{r}\text { Height } \\
{[\mu \mathrm{V}]}\end{array}$ & $\begin{array}{r}\text { Area } \\
{[\mu \mathrm{V} . \mathrm{Min}]}\end{array}$ & As. PE. & Res. HW \\
\hline \hline 1 & UNKNOWN & 14.620 & 97.626 & 97.63 & 49027.1 & 6062.1 & 0.56 & 0.00 \\
\hline 2 & UNKNOWN & 17.637 & 2.374 & 2.37 & 2014.3 & 147.4 & 1.79 & 16.71 \\
\hline & & & & & & & & \\
\hline Total & & & 100.000 & 100.00 & 51041.4 & 6209.5 & & \\
\hline
\end{tabular}


(Z)-3-(3-(4-acetylphenyl)-2-methylacryloyl)oxazolidin-2-one ((Z)-23)

$(E)$-isomer ((E)-23)

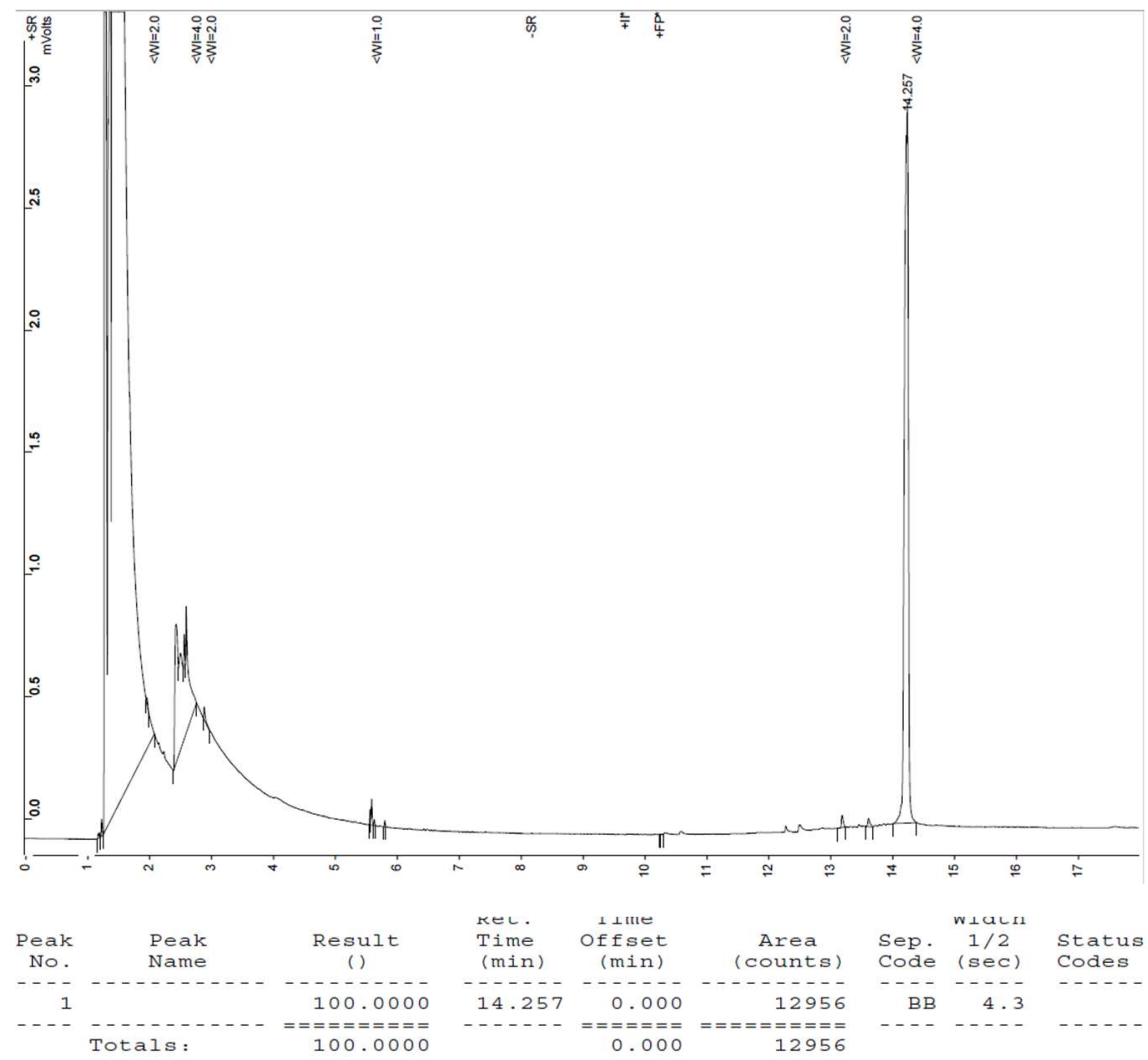

(Z)-isomer ((Z)-23)
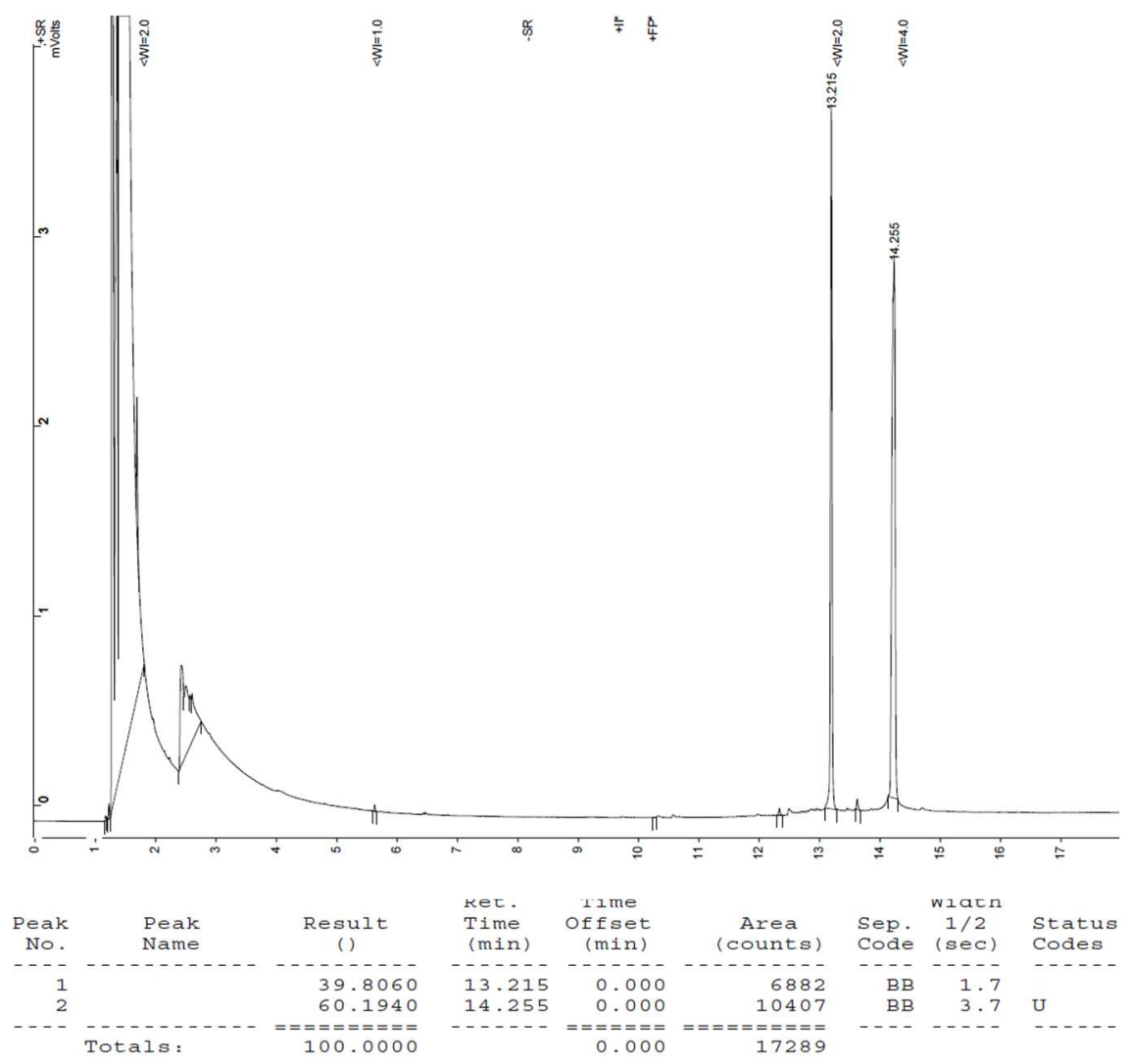


\section{(Z)-3-(3-(furan-2-yl)-2-methylacryloyl)oxazolidin-2-one ((Z)-24)}

$(E)$-isomer $((E)-24)$

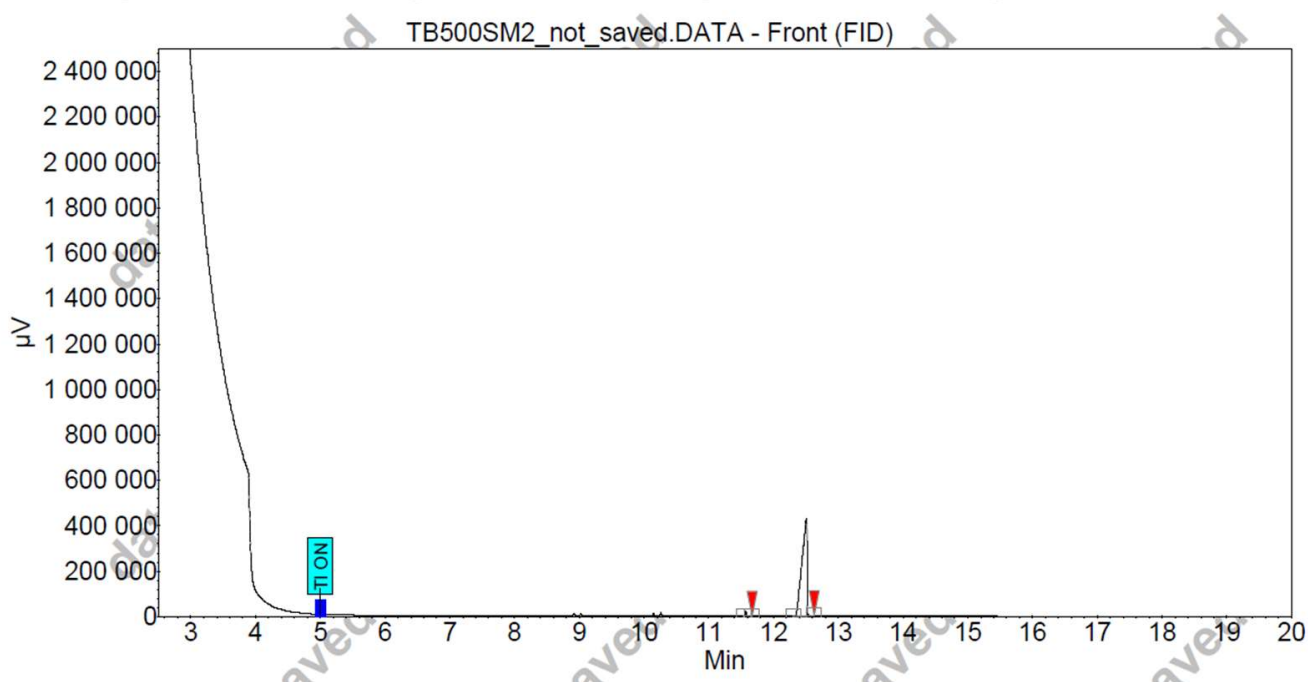

Peak results:

\begin{tabular}{|c|l|r|r|r|r|r|r|r|}
\hline Index & Name & $\begin{array}{r}\text { Time } \\
{[\text { Min] }]}\end{array}$ & $\begin{array}{r}\text { Area \% } \\
{[\%]}\end{array}$ & $\begin{array}{r}\text { Quantity } \\
{[\% \text { Area] }}\end{array}$ & $\begin{array}{r}\text { Height } \\
{[\mu \mathrm{V}]}\end{array}$ & $\begin{array}{r}\text { Area } \\
{[\mu \mathrm{V} . \text { Min] }]}\end{array}$ & As. PE. & Res. HW \\
\hline \hline 1 & UNKNOWN & 11.570 & 1.495 & 1.50 & 22079.3 & 578.6 & 1.68 & 0.00 \\
\hline 2 & UNKNOWN & 12.507 & 98.505 & 98.50 & 428890.6 & 38117.9 & 0.55 & 10.06 \\
\hline & & & & & & & & \\
\hline Total & & & 100.000 & 100.00 & 450969.9 & 38696.5 & & \\
\hline
\end{tabular}

(Z)-isomer ((Z)-24)

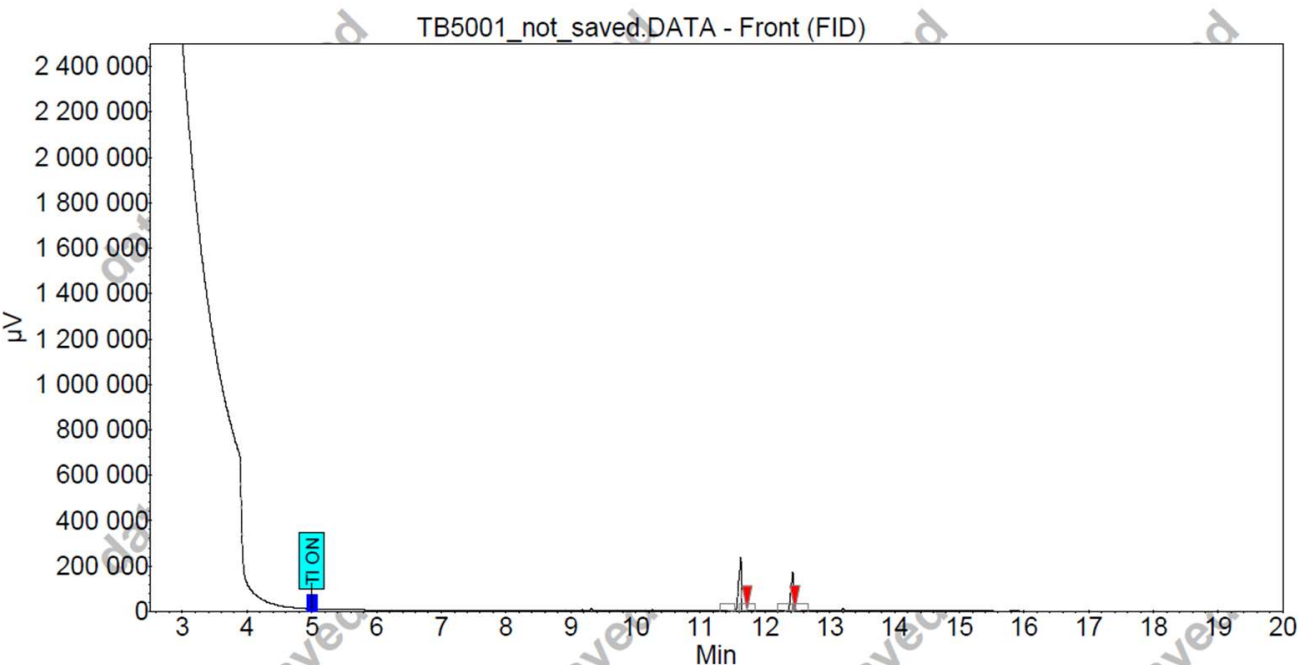

\section{Peak results :}

\begin{tabular}{|c|l|r|r|r|r|r|r|r|}
\hline Index & Name & $\begin{array}{r}\text { Time } \\
{[\mathrm{Min}]}\end{array}$ & $\left.\begin{array}{r}\text { Area \% } \\
{[\%]}\end{array}\right] \begin{array}{r}\text { Quantity } \\
{[\% \text { Area] }}\end{array}$ & $\begin{array}{r}\text { Height } \\
{[\mu \mathrm{V}]}\end{array}$ & $\begin{array}{r}\text { Area } \\
{[\mu \mathrm{V} . \text { Min] }}\end{array}$ & As. PE. & Res. HW \\
\hline \hline 1 & UNKNOWN & 11.627 & 60.234 & 60.23 & 236518.0 & 10004.0 & 0.58 & 0.00 \\
\hline 2 & UNKNOWN & 12.428 & 39.766 & 39.77 & 169611.2 & 6604.5 & 0.60 & 11.96 \\
\hline & & & & & & & & \\
\hline Total & & & 100.000 & 100.00 & 406129.2 & 16608.5 & & \\
\hline
\end{tabular}




\section{(Z)-3-(2-methyl-3-(thiophen-2-yl)acryloyl)oxazolidin-2-one ((Z)-25)}

$(E)$-isomer ((E)-25)

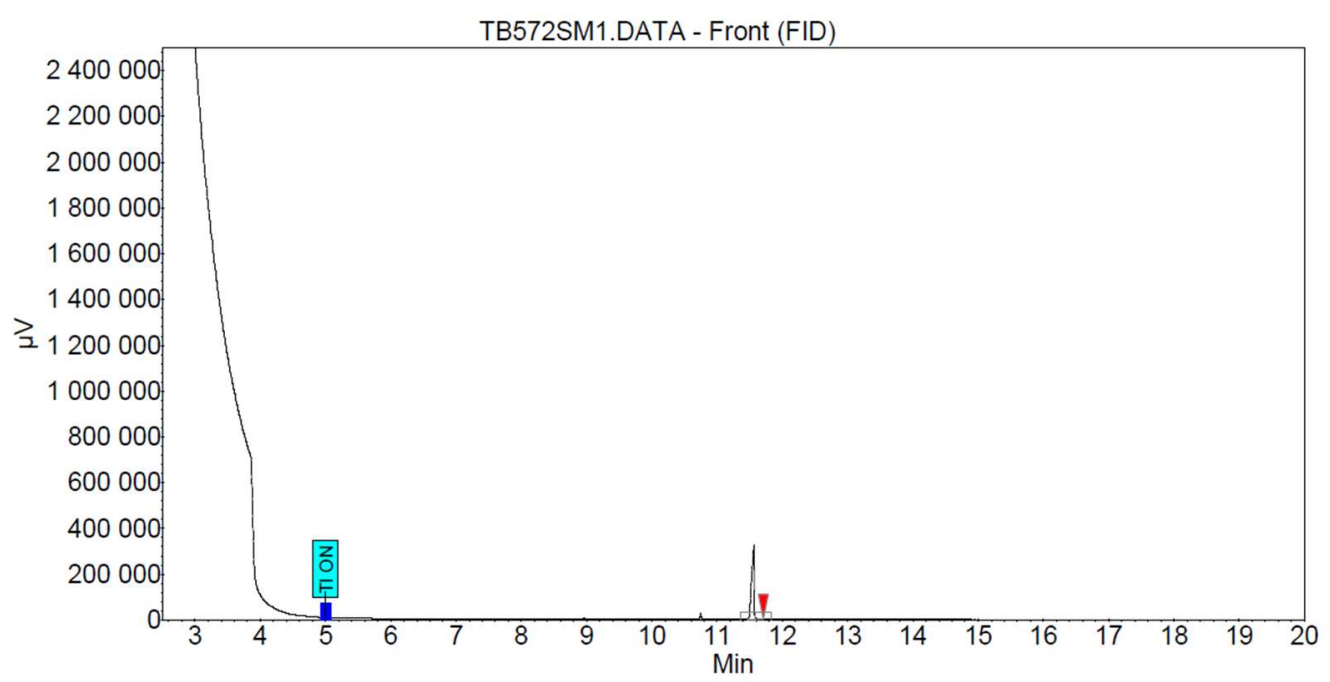

Peak results :

\begin{tabular}{|c|l|r|r|r|r|r|r|r|}
\hline Index & Name & $\begin{array}{r}\text { Time } \\
{[\text { Min] }}\end{array}$ & $\left.\begin{array}{r}\text { Area \% } \\
{[\%]}\end{array}\right] \begin{array}{r}\text { Quantity } \\
{[\% \text { Area] }}\end{array}$ & $\begin{array}{r}\text { Height } \\
{[\mu \mathrm{V}]}\end{array}$ & $\begin{array}{r}\text { Area } \\
{[\mu \mathrm{V} . \text { Min] }}\end{array}$ & As. PE. & Res. HW \\
\hline \hline 1 & UNKNOWN & 11.565 & 100.000 & 100.00 & 324767.9 & 15360.5 & 0.58 & 0.00 \\
\hline & & & & & & & & \\
\hline Total & & & 100.000 & 100,00 & 324767.9 & 15360.5 & & \\
\hline
\end{tabular}

(Z)-isomer ((Z)-25)

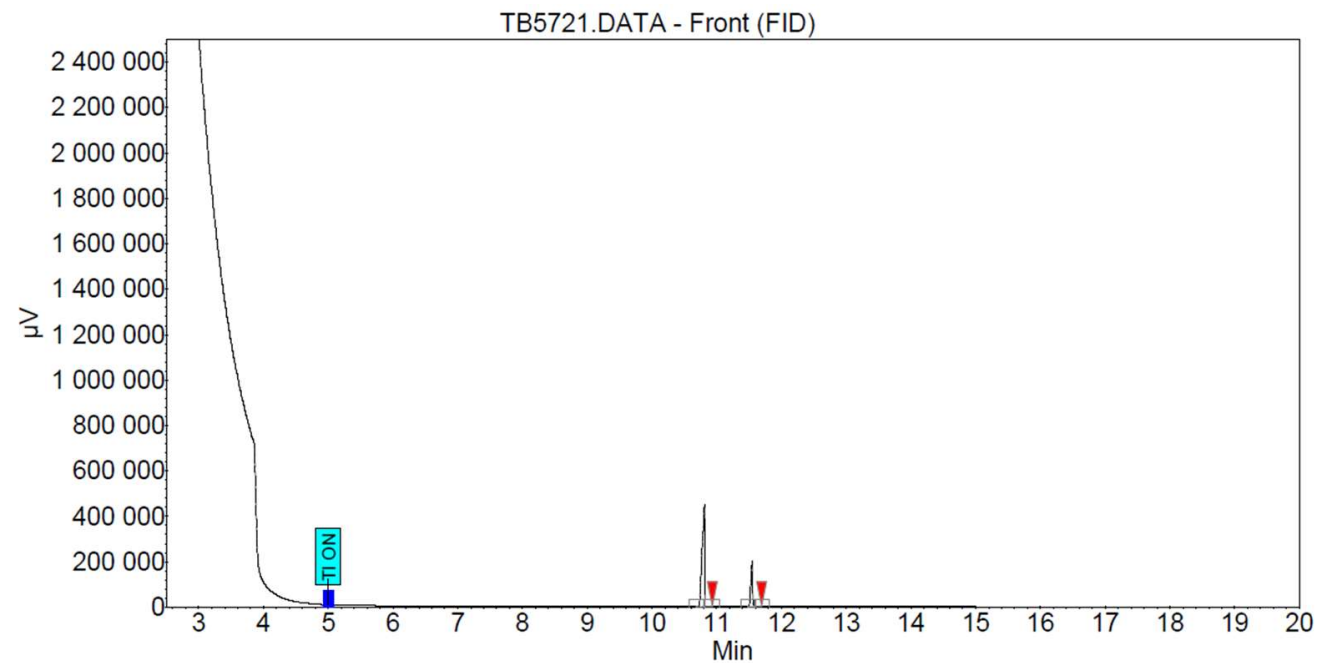

Peak results :

\begin{tabular}{|c|l|r|r|r|r|r|r|r|}
\hline Index & Name & $\begin{array}{r}\text { Time } \\
{[\text { Min] }}\end{array}$ & $\begin{array}{r}\text { Area \% } \\
{[\%]}\end{array}$ & $\begin{array}{r}\text { Quantity } \\
{[\% \text { Area] }}\end{array}$ & $\begin{array}{r}\text { Height } \\
{[\mu \mathrm{V}]}\end{array}$ & $\begin{array}{r}\text { Area } \\
{[\mu \mathrm{V} . \mathrm{Min}]}\end{array}$ & As. PE. & Res. HW \\
\hline \hline 1 & UNKNOWN & 10.812 & 76.101 & 76.10 & 449783.3 & 20670.0 & 0.56 & 0.00 \\
\hline 2 & UNKNOWN & 11.548 & 23.899 & 23.90 & 199945.4 & 6491.2 & 0.65 & 11.28 \\
\hline & & & & & & & & \\
\hline Total & & & 100.000 & 100.00 & 649728.8 & 27161.2 & & \\
\hline
\end{tabular}


(Z)-3-(3-phenylbut-2-enoyl)oxazolidin-2-one ((Z)-29)

$(E)$-isomer ((E)-29)

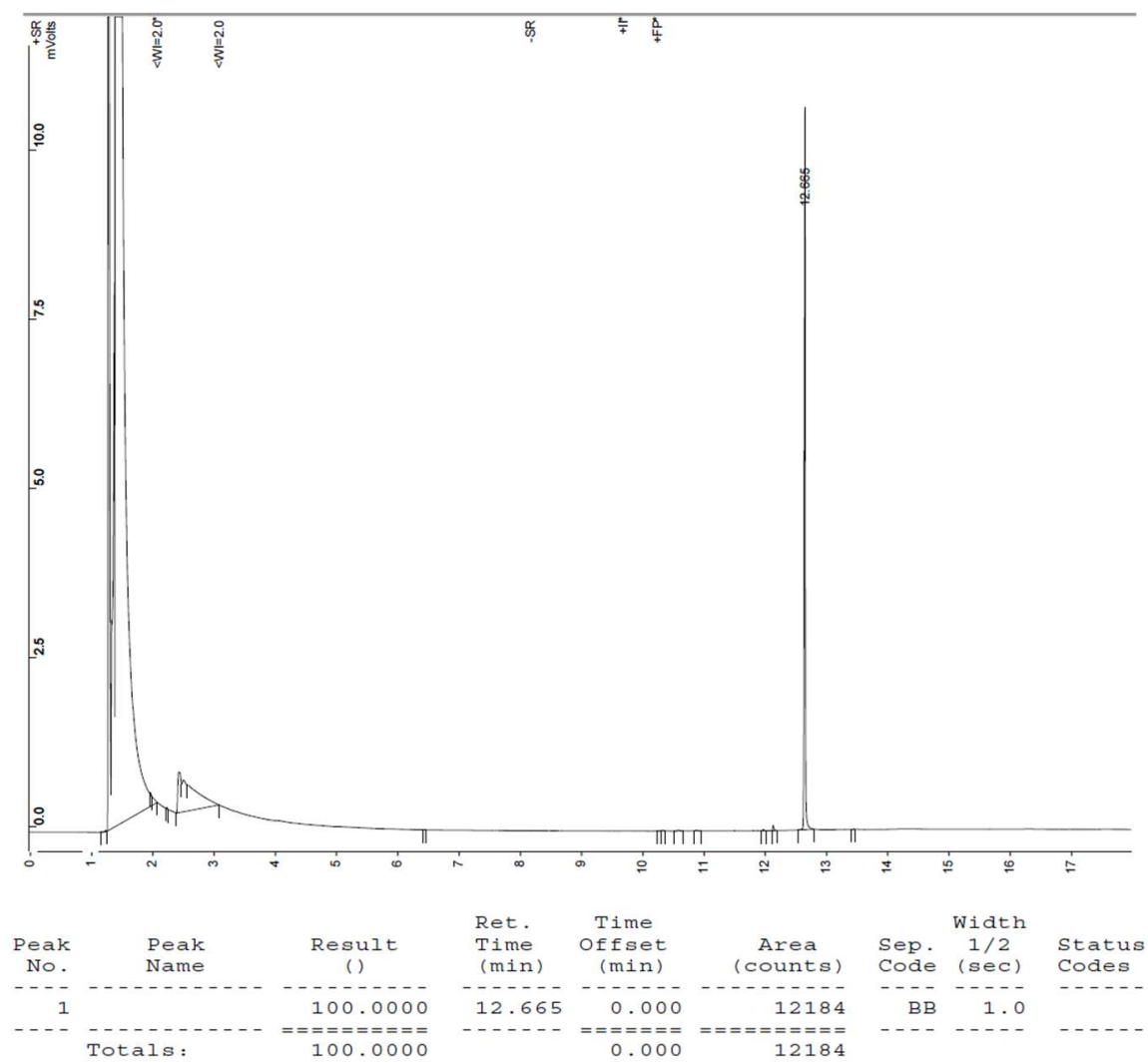

(Z)-isomer ((Z)-29)

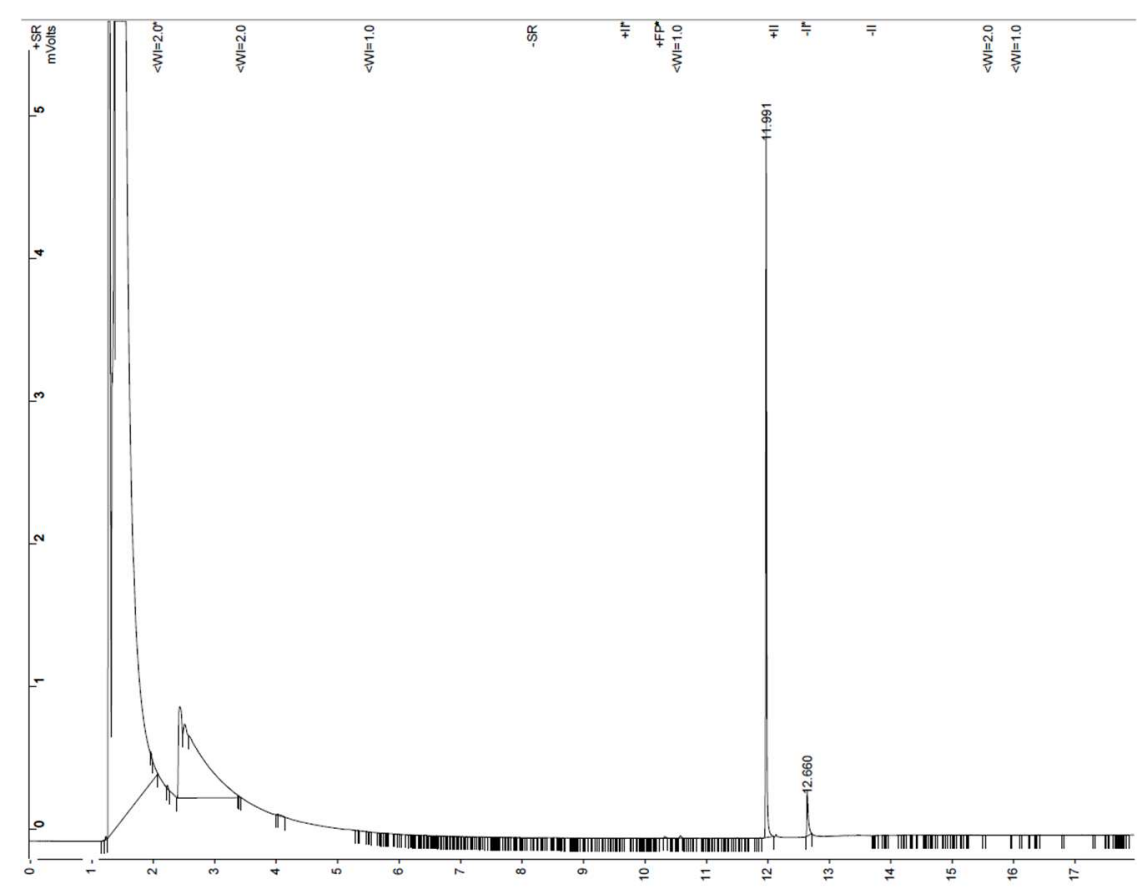

\begin{tabular}{|c|c|c|c|c|c|c|c|c|}
\hline \multirow{2}{*}{$\begin{array}{l}\text { Peak } \\
\text { No. }\end{array}$} & \multirow[b]{2}{*}{$\begin{array}{l}\text { Peak } \\
\text { Name }\end{array}$} & \multirow[b]{2}{*}{$\underset{()}{\text { Result }}$} & \multirow{2}{*}{$\begin{array}{l}\text { Ret. } \\
\text { Time } \\
\text { (min) }\end{array}$} & \multicolumn{2}{|l|}{ Time } & \multicolumn{3}{|c|}{ Width } \\
\hline & & & & $\begin{array}{l}\text { Offset } \\
(\min )\end{array}$ & $\begin{array}{c}\text { Area } \\
\text { (counts) }\end{array}$ & $\begin{array}{l}\text { Sep. } \\
\text { Code }\end{array}$ & $\begin{array}{l}1 / 2 \\
(\sec )\end{array}$ & $\begin{array}{l}\text { Status } \\
\text { Codes }\end{array}$ \\
\hline 1 & & 91.3842 & 11.991 & 0.000 & 5402 & $\mathrm{~PB}$ & 0.9 & \\
\hline 2 & & 8.6158 & 12.660 & 0.000 & 509 & BB & 1.3 & \\
\hline & Totals: & $\begin{array}{l}======= \\
100.00000\end{array}$ & & 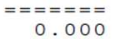 & $\begin{array}{l}==== \\
5911\end{array}$ & & & \\
\hline
\end{tabular}


ethyl (Z)-3-phenylbut-2-enoate ((Z)-30)

$(E)$-isomer $((E)-30)$

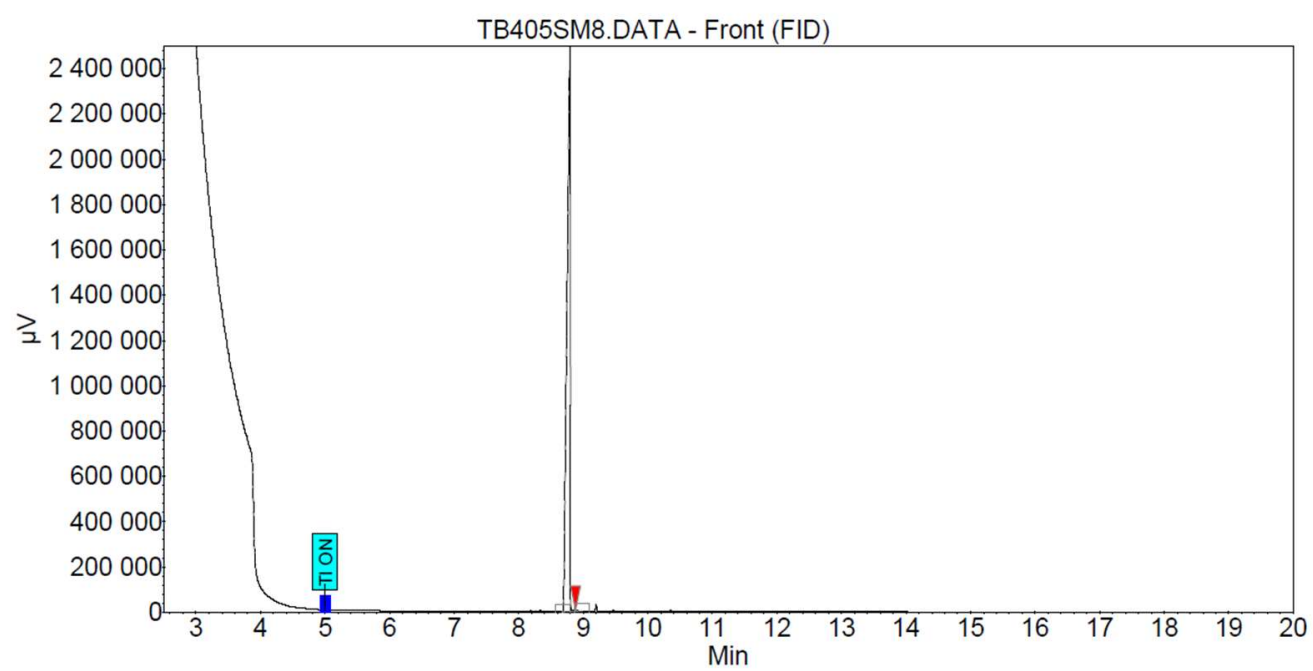

Peak results :

\begin{tabular}{|c|l|r|r|r|r|r|r|r|}
\hline Index & Name & $\begin{array}{r}\text { Time } \\
{[\text { Min] }]}\end{array}$ & $\begin{array}{r}\text { Area \% } \\
{[\%]}\end{array}$ & $\begin{array}{r}\text { Quantity } \\
{[\% \text { Area] }}\end{array}$ & $\begin{array}{r}\text { Height } \\
{[\mu \mathrm{V}]}\end{array}$ & $\begin{array}{r}\text { Area } \\
{[\mu \mathrm{V} . \mathrm{Min}]}\end{array}$ & As. PE. & Res. HW \\
\hline \hline 1 & UNKNOWN & 8.795 & 100.000 & 100.00 & 2582158.2 & 145892.6 & 0.55 & 0.00 \\
\hline & & & & & & & & \\
\hline Total & & & 100.000 & 100.00 & 2582158.2 & 145892.6 & & \\
\hline
\end{tabular}

(Z)-isomer ((Z)-30)

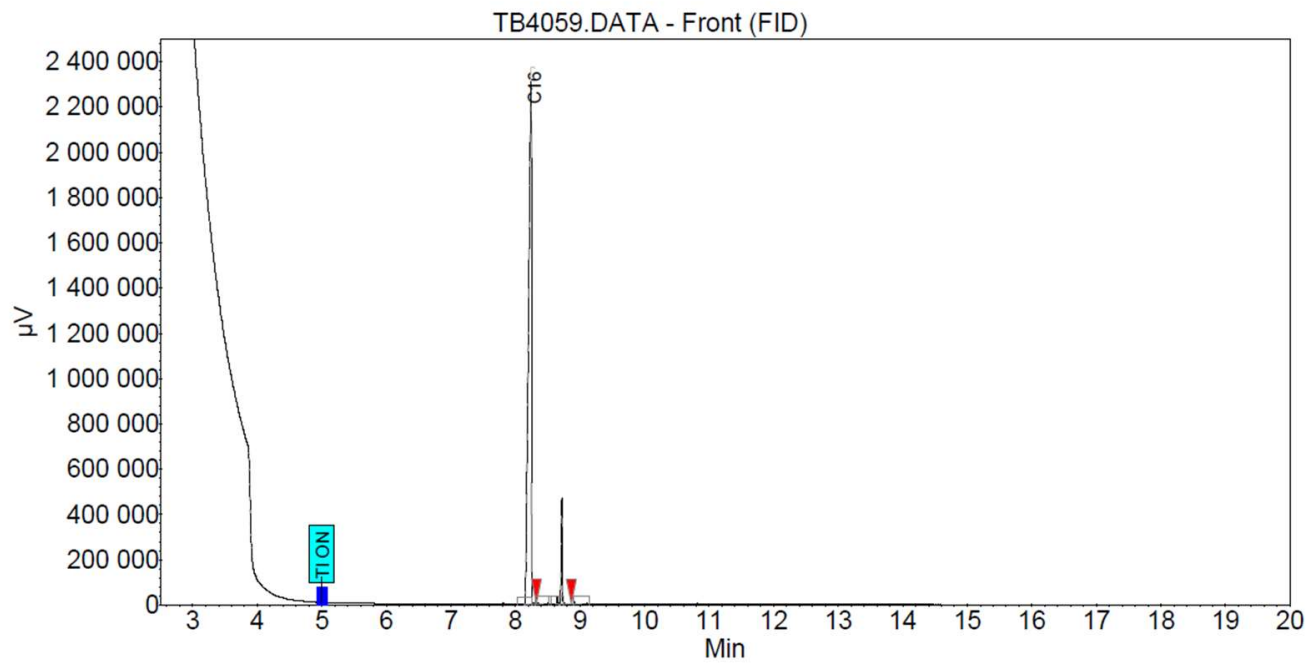

\section{Peak results :}

\begin{tabular}{|c|l|r|r|r|r|r|r|r|}
\hline Index & Name & $\begin{array}{r}\text { Time } \\
{[\text { Min] }]}\end{array}$ & $\begin{array}{r}\text { Area \% } \\
{[\%]}\end{array}$ & $\begin{array}{r}\text { Quantity } \\
{[\% \text { Area] }}\end{array}$ & $\begin{array}{r}\text { Height } \\
{[\mu \mathrm{V}]}\end{array}$ & $\begin{array}{r}\text { Area } \\
{[\mu \mathrm{V} . \mathrm{Min}]}\end{array}$ & As. PE. & Res. HW \\
\hline \hline 1 & $\mathrm{C} 16$ & 8.242 & 92.921 & 92.92 & 2313277.1 & 114928.1 & 0.55 & 0.00 \\
\hline 2 & UNKNOWN & 8.720 & 7.079 & 7.08 & 469713.1 & 8755.2 & 0.75 & 8.59 \\
\hline & & & & & & & & \\
\hline Total & & & 100.000 & 100.00 & 2782990.3 & 123683.3 & & \\
\hline
\end{tabular}




\section{(Z)-3-(3-phenylpent-2-enoyl)oxazolidin-2-one ((Z)-31)}

$(E)$-isomer $((E)-31)$

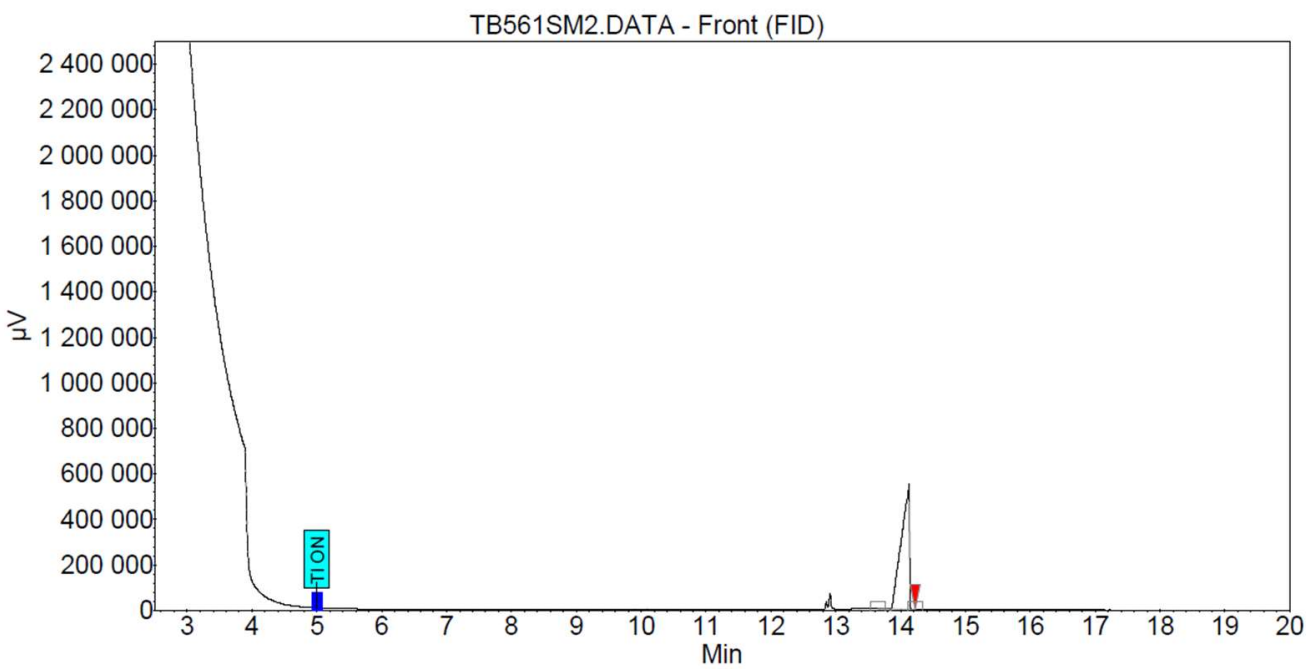

Peak results :

\begin{tabular}{|c|l|r|r|r|r|r|r|r|}
\hline Index & Name & $\begin{array}{r}\text { Time } \\
{[\text { Min] }}\end{array}$ & $\left.\begin{array}{r}\text { Area \% } \\
{[\%]}\end{array}\right] \begin{array}{r}\text { Quantity } \\
{[\% \text { Area] }}\end{array}$ & $\begin{array}{r}\text { Height } \\
{[\mu \mathrm{V}]}\end{array}$ & $\begin{array}{r}\text { Area } \\
{[\mu \text { V.Min] }}\end{array}$ & As. PE. & Res. HW \\
\hline \hline 1 & UNKNOWN & 14.132 & 100.000 & 100.00 & 549207.0 & 77429.2 & 0.52 & 0.00 \\
\hline & & & & & & & & \\
\hline Total & & & 100.000 & 100.00 & 549207.0 & 77429.2 & & \\
\hline
\end{tabular}

(Z)-isomer ((Z)-31)

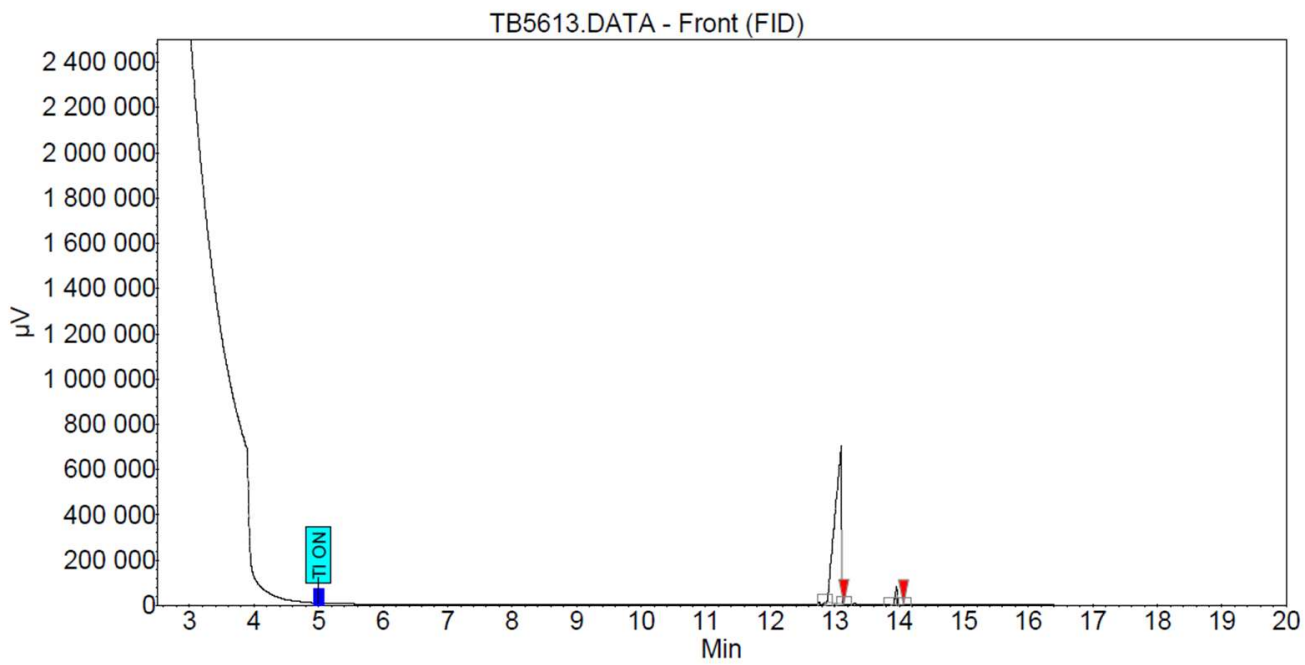

\section{Peak results :}

\begin{tabular}{|c|l|r|r|r|r|r|r|r|}
\hline Index & Name & $\begin{array}{r}\text { Time } \\
{[\text { Min] }}\end{array}$ & $\begin{array}{r}\text { Area \% } \\
{[\%]}\end{array}$ & $\begin{array}{r}\text { Quantity } \\
{[\% \text { Area] }}\end{array}$ & $\begin{array}{r}\text { Height } \\
{[\mu \mathrm{V}]}\end{array}$ & $\begin{array}{r}\text { Area } \\
{[\mu \mathrm{V} . \mathrm{Min}]}\end{array}$ & As. PE. & Res. HW \\
\hline \hline 2 & UNKNOWN & 13.105 & 96.785 & 96.78 & 699241.3 & 81216.5 & 0.51 & 0.00 \\
\hline 1 & UNKNOWN & 13.958 & 3.215 & 3.22 & 81241.3 & 2698.3 & 0.70 & 6.88 \\
\hline & & & & & & & & \\
\hline Total & & & 100.000 & 100.00 & 780482.7 & 83914.7 & & \\
\hline
\end{tabular}


ethyl (Z)-3-phenylpent-2-enoate ((Z)-32)

$(E)$-isomer ((E)-32)

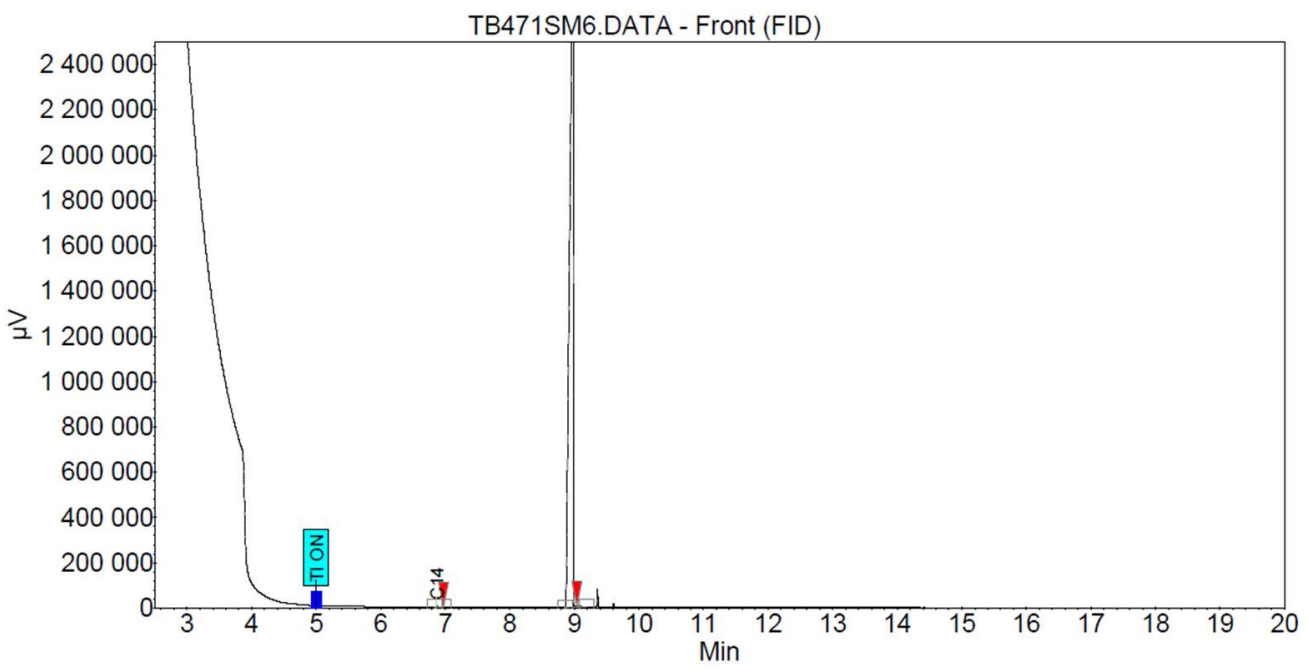

Peak results :

\begin{tabular}{|c|l|r|r|r|r|r|r|r|}
\hline Index & Name & $\begin{array}{r}\text { Time } \\
{[\text { Min] }]}\end{array}$ & $\begin{array}{r}\text { Area \% } \\
{[\%]}\end{array}$ & $\begin{array}{r}\text { Quantity } \\
{[\% \text { Area] }}\end{array}$ & $\begin{array}{r}\text { Height } \\
{[\mu \mathrm{V}]}\end{array}$ & $\begin{array}{r}\text { Area } \\
{[\mu \mathrm{V} . \mathrm{Min}]}\end{array}$ & As. PE. & Res. HW \\
\hline \hline 1 & C14 & 6.863 & 0.071 & 0.07 & 2981.3 & 145.4 & 3.51 & 0.00 \\
\hline 2 & UNKNOWN & 8.983 & 99.929 & 99.93 & 3417711.2 & 205203.0 & 0.54 & 24.05 \\
\hline & & & & & & & & \\
\hline Total & & & 100.000 & 100.00 & 3420692.5 & 205348.3 & & \\
\hline
\end{tabular}

(Z)-isomer ((Z)-32)

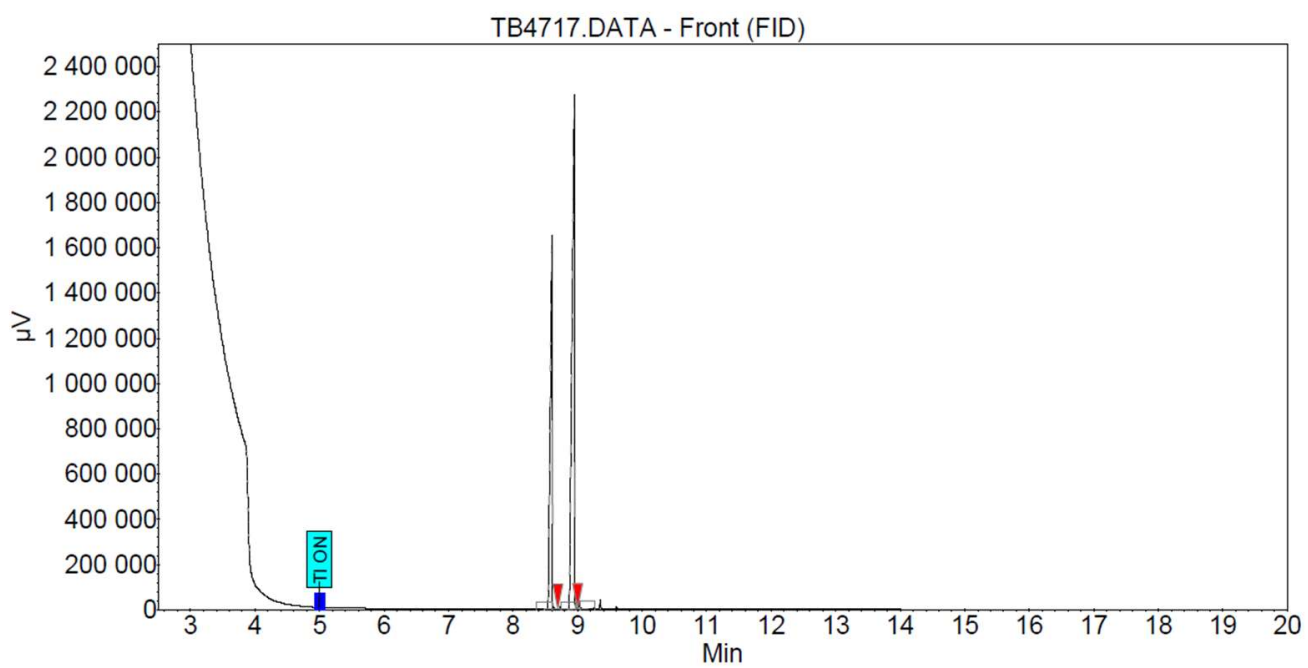

Peak results :

\begin{tabular}{|c|l|r|r|r|r|r|r|r|}
\hline Index & Name & $\begin{array}{r}\text { Time } \\
{[\text { Min] }]}\end{array}$ & $\begin{array}{r}\text { Area \% } \\
{[\%]}\end{array}$ & $\begin{array}{r}\text { Quantity } \\
{[\% \text { Area] }}\end{array}$ & $\begin{array}{r}\text { Height } \\
{[\mu \mathrm{V}]}\end{array}$ & $\begin{array}{r}\text { Area } \\
{[\mu \mathrm{V} . \mathrm{Min}]}\end{array}$ & As. PE. & Res. HW \\
\hline \hline 1 & UNKNOWN & 8.603 & 37.968 & 37.97 & 1651368.4 & 60231.5 & 0.58 & 0.00 \\
\hline 2 & UNKNOWN & 8.948 & 62.032 & 62.03 & 2271669.6 & 98406.2 & 0.56 & 5.17 \\
\hline & & & & & & & & \\
\hline Total & & & 100.000 & 100.00 & 3923038.0 & 158637.7 & & \\
\hline
\end{tabular}


(Z)-3-(3-(p-tolyl)but-2-enoyl)oxazolidin-2-one ((Z)-33)

$(E)$-isomer $((E)-33)$

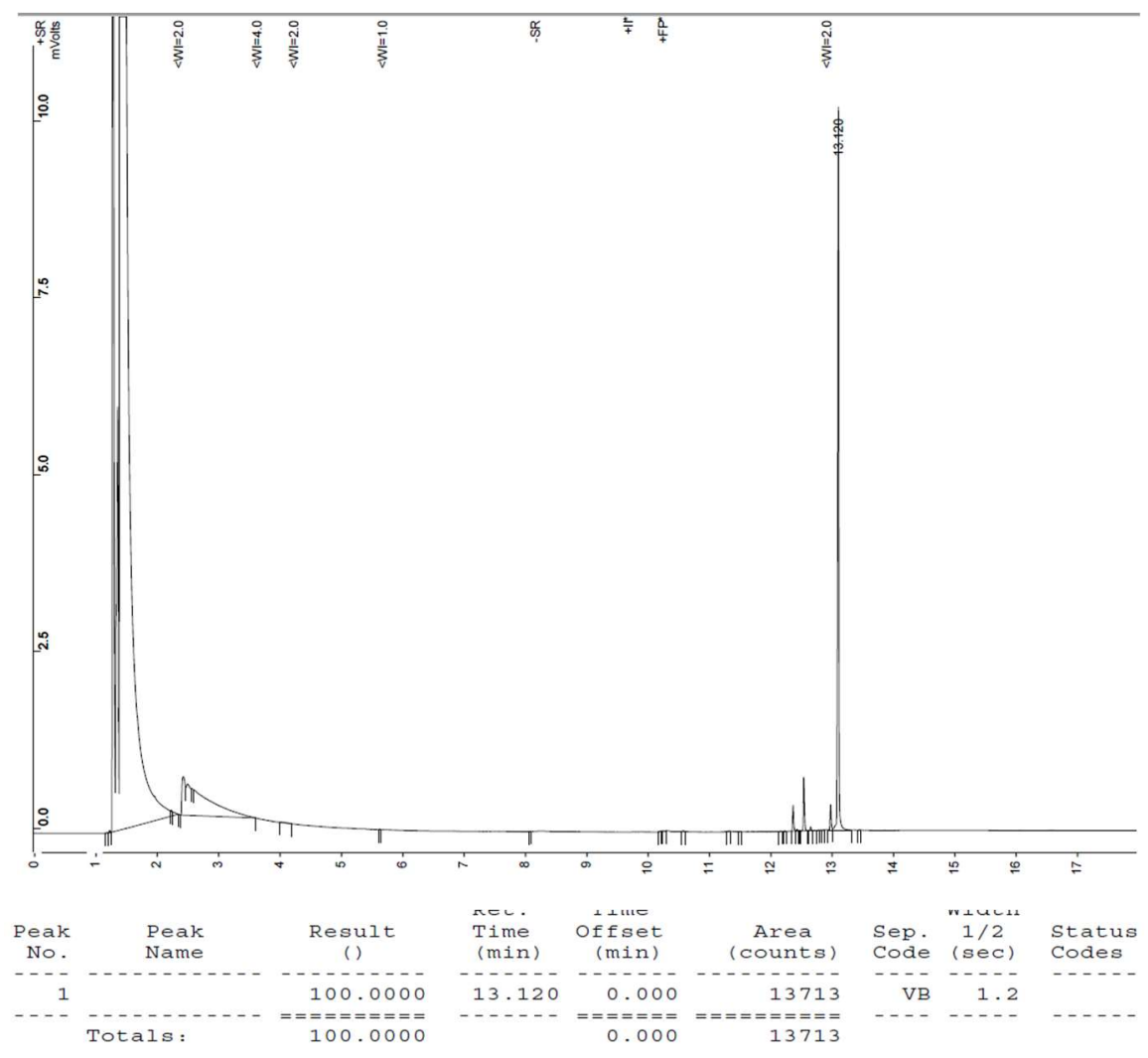

(Z)-isomer ((Z)-33)

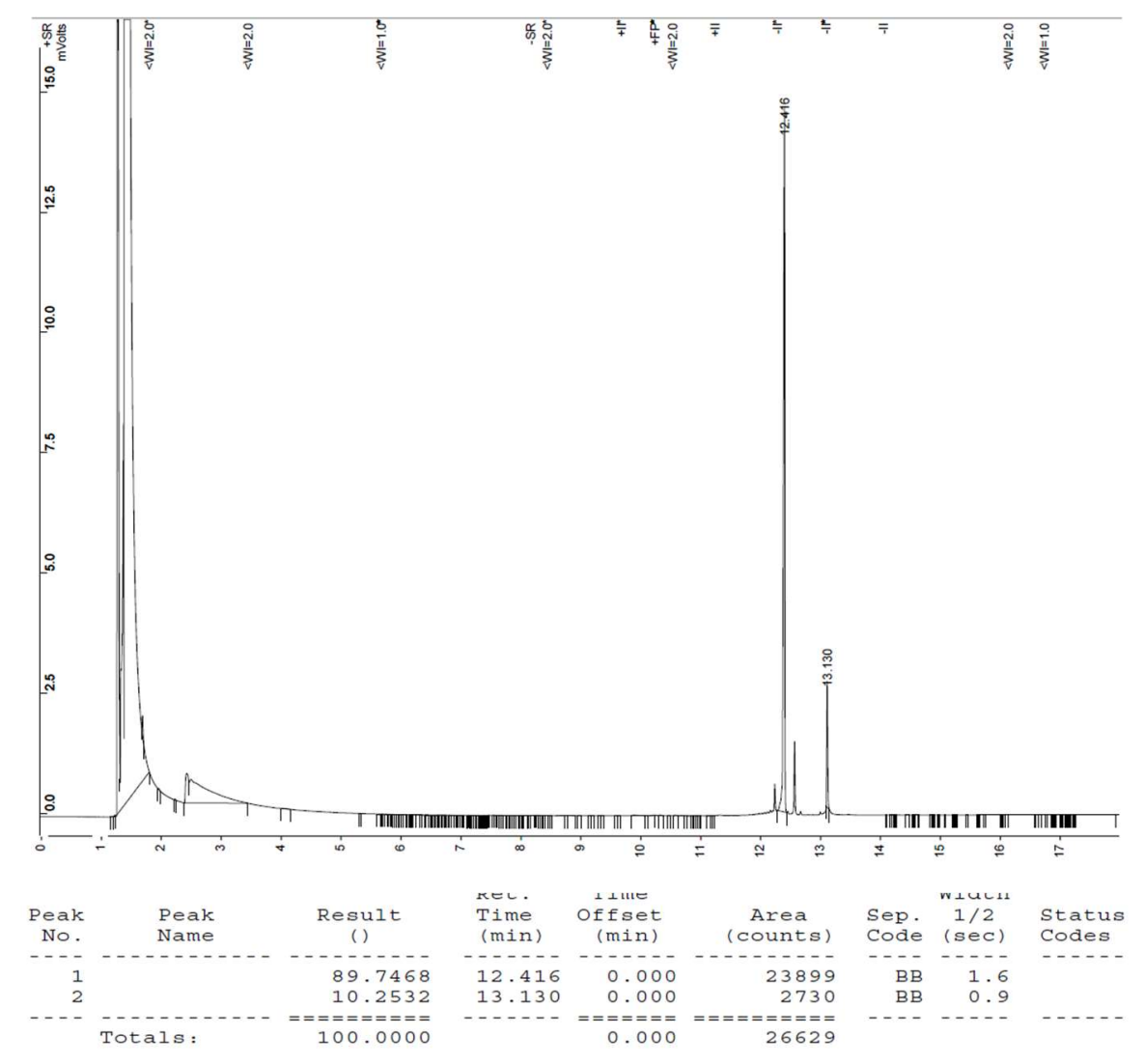


ethyl (Z)-3-(p-tolyl)but-2-enoate ((Z)-34)

$(E)$-isomer $((E)-34)$

TB598SM2.DATA - Front (FID)

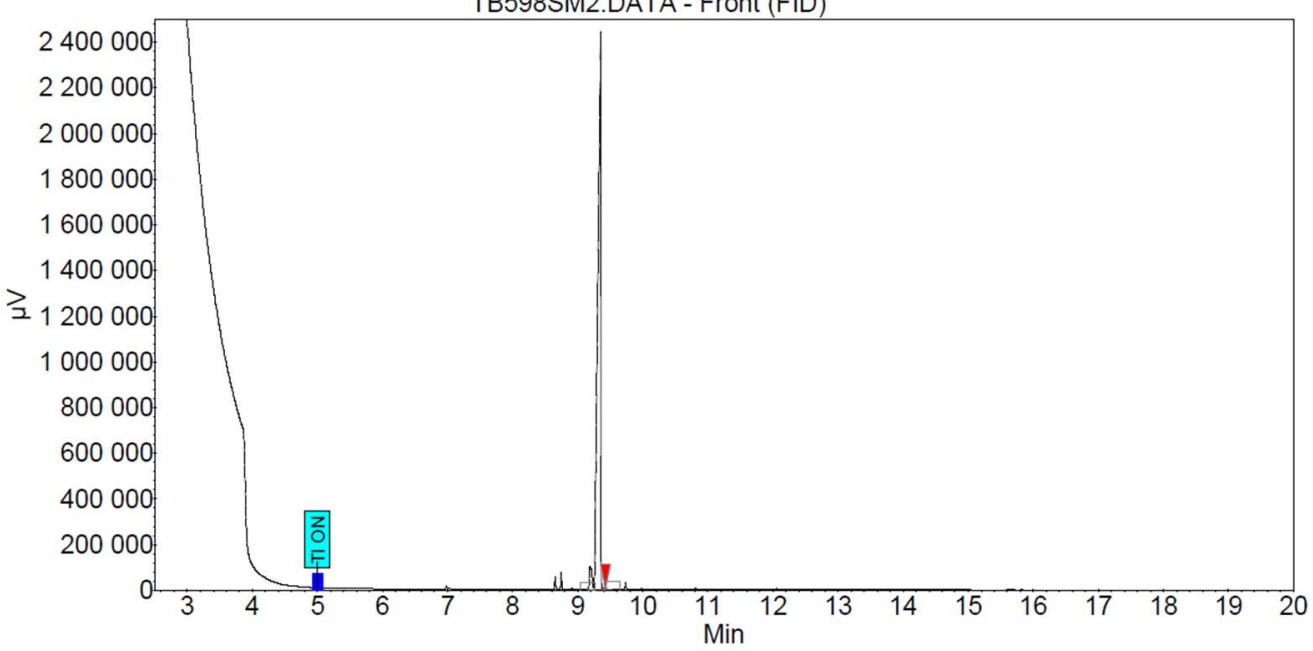

Peak results :

\begin{tabular}{|c|l|r|r|r|r|r|r|r|}
\hline Index & Name & $\begin{array}{r}\text { Time } \\
{[\mathrm{Min}]}\end{array}$ & $\begin{array}{r}\text { Area \% } \\
{[\%]}\end{array}$ & $\begin{array}{r}\text { Quantity } \\
{[\% \text { Area] }}\end{array}$ & $\begin{array}{r}\text { Height } \\
{[\mu \mathrm{V}]}\end{array}$ & $\begin{array}{r}\text { Area } \\
{[\mu \mathrm{V} . \mathrm{Min}]}\end{array}$ & As. PE. & Res. HW \\
\hline \hline 1 & UNKNOWN & 9.352 & 100.000 & 100.00 & 2442007.0 & 130834.9 & 0.55 & 0.00 \\
\hline & & & & & & & & \\
\hline Total & & & 100.000 & 100.00 & 2442007.0 & 130834.9 & & \\
\hline
\end{tabular}

(Z)-isomer ((Z)-34)

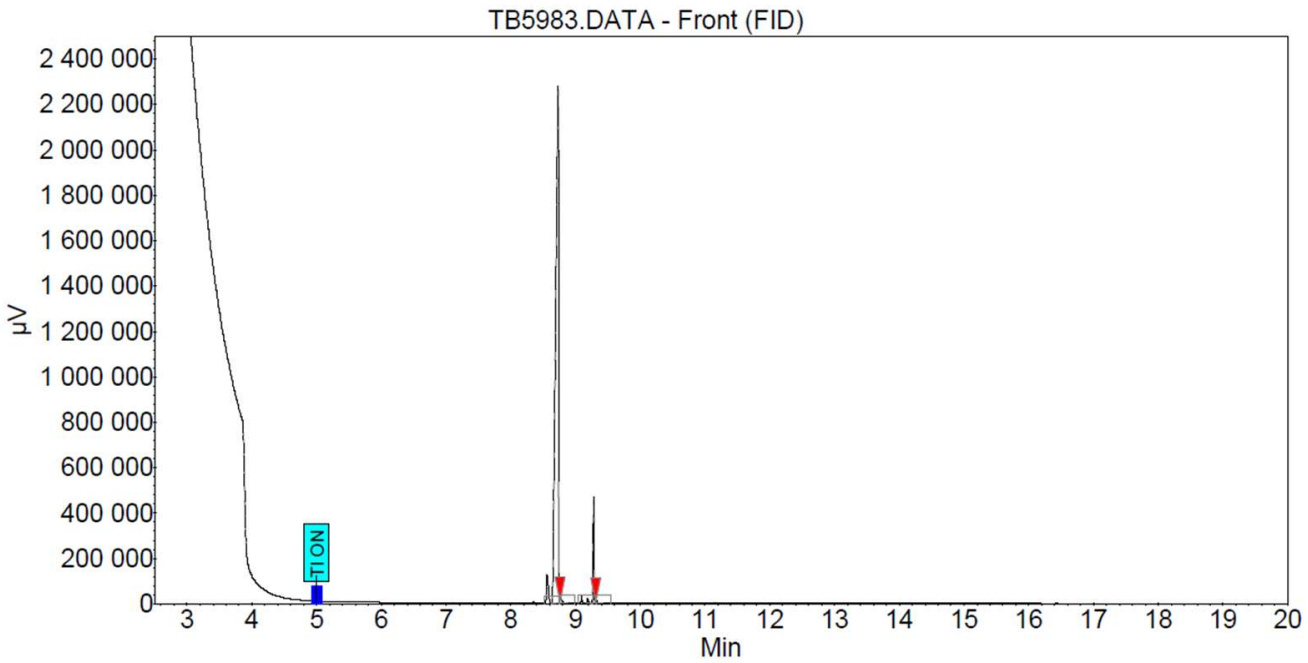

Peak results :

\begin{tabular}{|c|l|r|r|r|r|r|r|r|}
\hline Index & Name & $\begin{array}{r}\text { Time } \\
{[\text { Min] }}\end{array}$ & $\begin{array}{r}\text { Area \% } \\
{[\%]}\end{array}$ & $\begin{array}{r}\text { Quantity } \\
{[\% \text { Area] }}\end{array}$ & $\begin{array}{r}\text { Height } \\
{[\mu \mathrm{V}]}\end{array}$ & $\begin{array}{r}\text { Area } \\
{[\mu \mathrm{V} . \mathrm{Min}]}\end{array}$ & As. PE. & Res. HW \\
\hline \hline 1 & UNKNOWN & 8.732 & 93.139 & 93.14 & 2279342.9 & 112243.6 & 0.55 & 0.00 \\
\hline 2 & UNKNOWN & 9.282 & 6.861 & 6.86 & 465357.7 & 8268.5 & 0.73 & 9.90 \\
\hline & & & & & & & & \\
\hline Total & & & 100.000 & 100.00 & 2744700.6 & 120512.1 & & \\
\hline
\end{tabular}


(Z)-3-(3-(m-tolyl)but-2-enoyl)oxazolidin-2-one ((Z)-35)

$(E)$-isomer $((E)-35)$

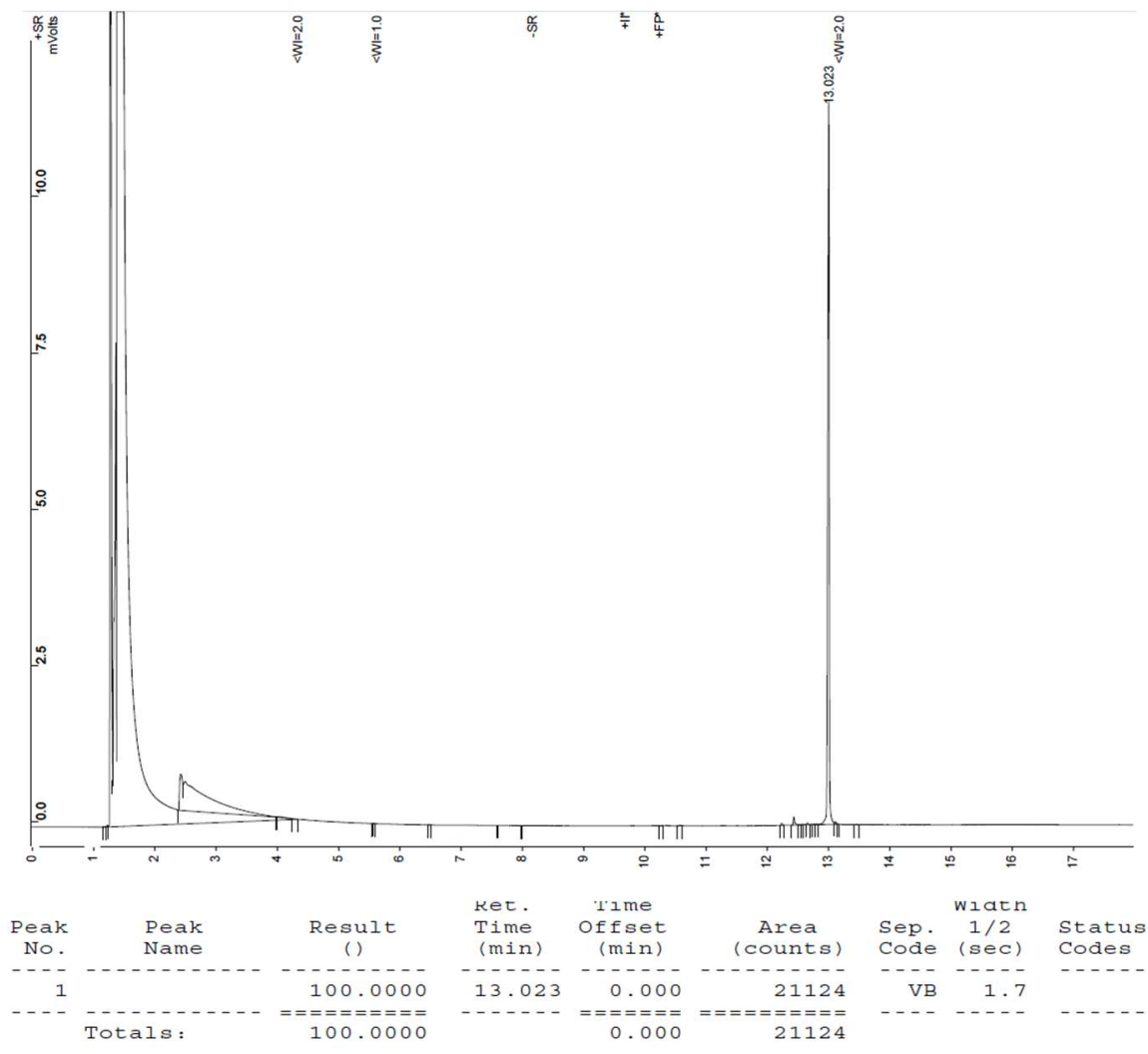

(Z)-isomer ((Z)-35)

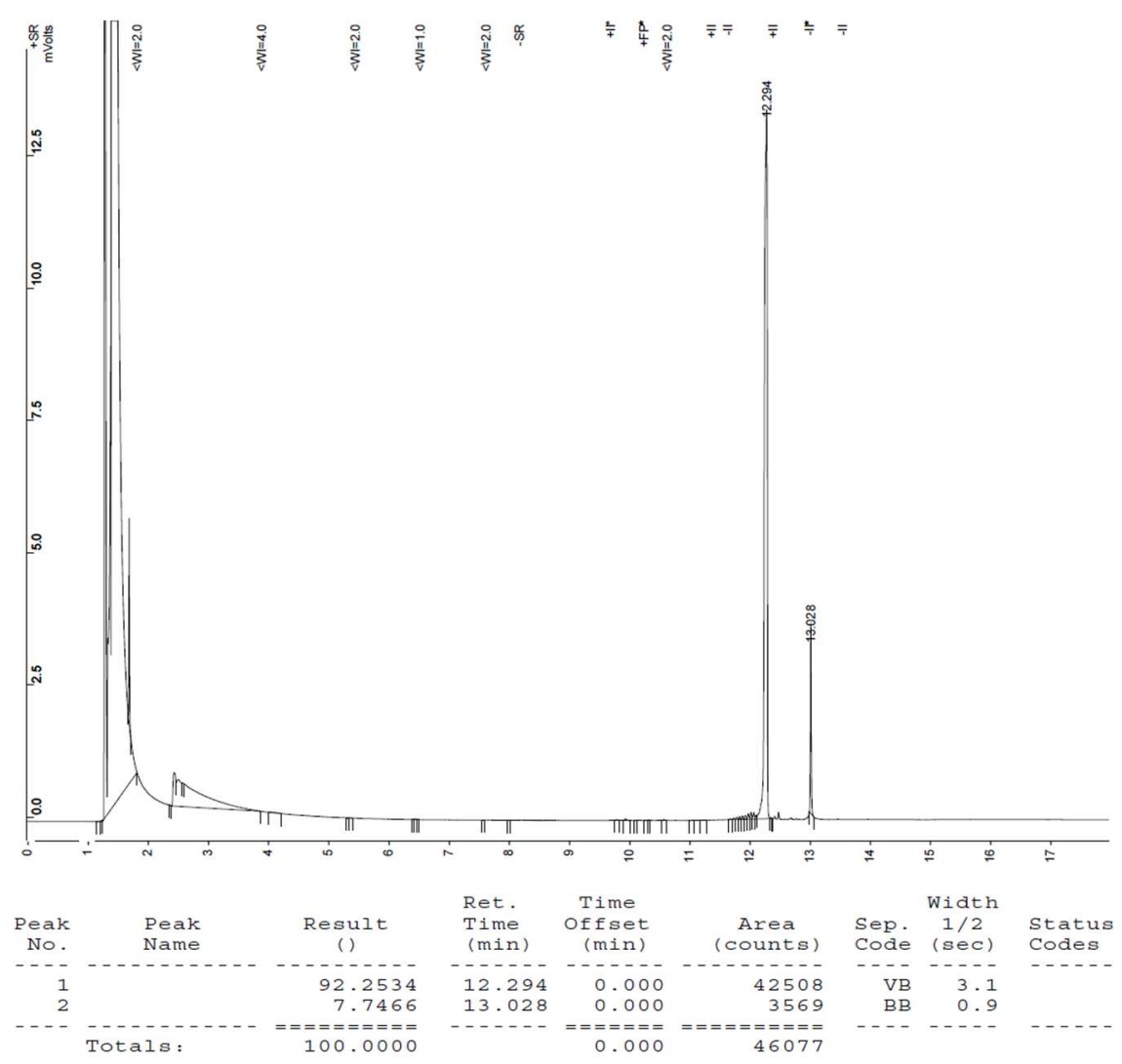


ethyl (Z)-3-(m-tolyl)but-2-enoate ((Z)-36)

$(E)$-isomer ((E)-36)

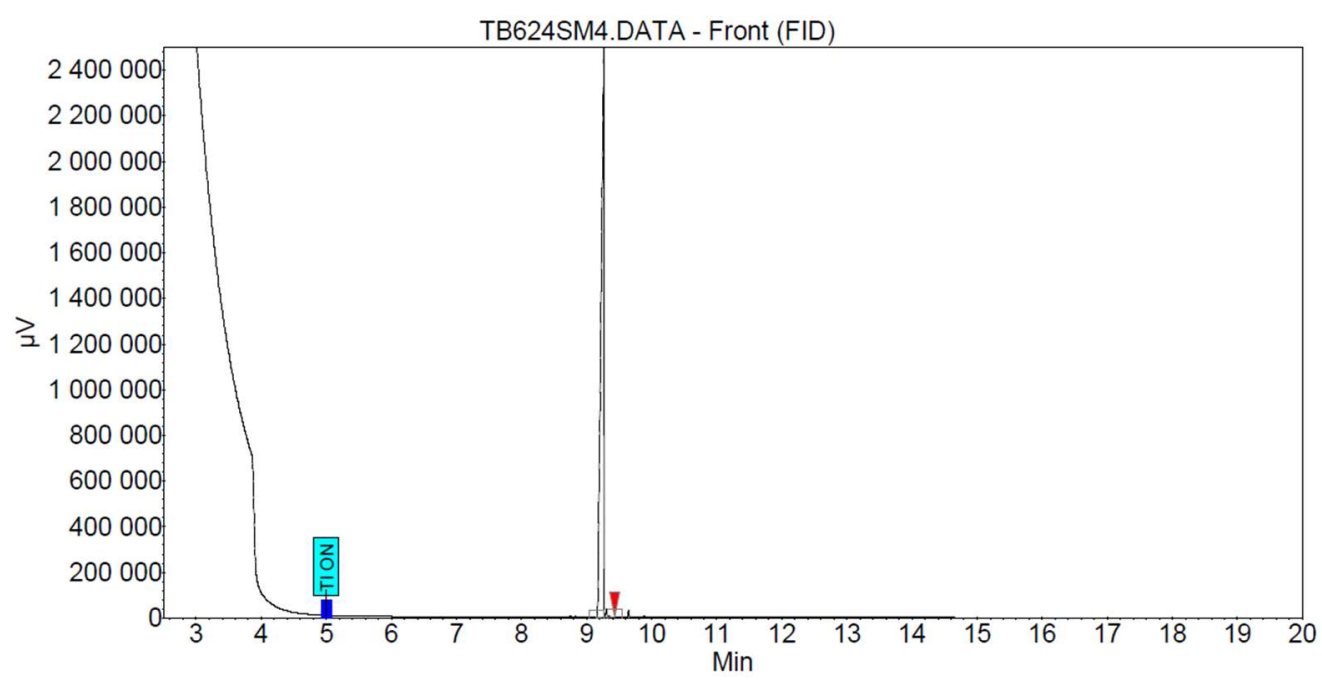

Peak results :

\begin{tabular}{|c|l|r|r|r|r|r|r|r|}
\hline Index & Name & $\begin{array}{r}\text { Time } \\
{[\text { Min] }]}\end{array}$ & $\begin{array}{r}\text { Area \% } \\
{[\%]}\end{array}$ & $\begin{array}{r}\text { Quantity } \\
{[\% \text { Area] }}\end{array}$ & $\begin{array}{r}\text { Height } \\
{[\mu \mathrm{V}]}\end{array}$ & $\begin{array}{r}\text { Area } \\
{[\mu \mathrm{V} . \mathrm{Min}]}\end{array}$ & As. PE. & Res. HW \\
\hline \hline 1 & UNKNOWN & 9.265 & 100,000 & 100.00 & 2559040.4 & 134116.7 & 0.54 & 0.00 \\
\hline & & & & & & & & \\
\hline Total & & & 100,000 & 100,00 & 2559040.4 & 134116.7 & & \\
\hline
\end{tabular}

(Z)-isomer ((Z)-36)

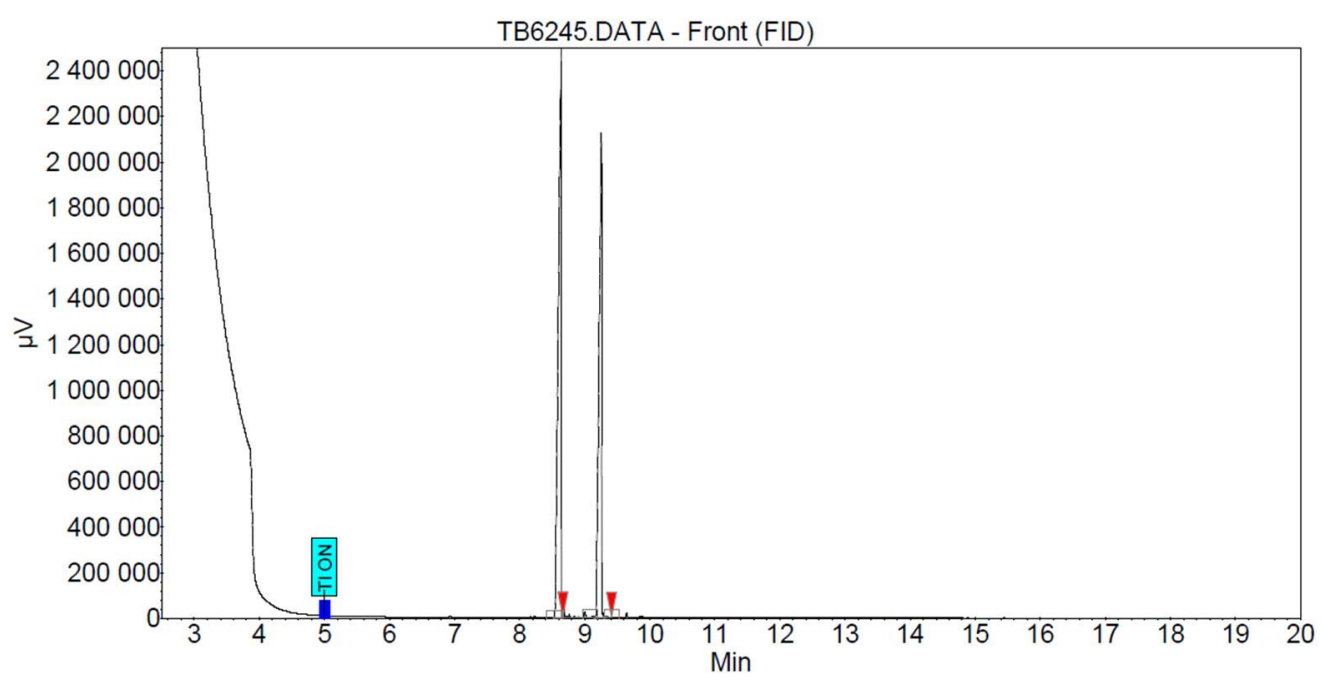

Peak results :

\begin{tabular}{|c|l|r|r|r|r|r|r|r|}
\hline Index & Name & $\begin{array}{r}\text { Time } \\
{[\text { Min] }]}\end{array}$ & $\begin{array}{r}\text { Area \% } \\
{[\%]}\end{array}$ & $\begin{array}{r}\text { Quantity } \\
{[\% \text { Area] }}\end{array}$ & $\begin{array}{r}\text { Height } \\
{[\mu \mathrm{V}]}\end{array}$ & $\begin{array}{r}\text { Area } \\
{[\mu \mathrm{V} . \text { Min] }]}\end{array}$ & As. PE. & Res. HW \\
\hline \hline 1 & UNKNOWN & 8.635 & 57.070 & 57.07 & 2555538.9 & 126644.0 & 0.55 & 0.00 \\
\hline 2 & UNKNOWN & 9.255 & 42.930 & 42.93 & 2125020.9 & 95265.2 & 0.55 & 7.77 \\
\hline & & & & & & & & \\
\hline Total & & & 100.000 & 100.00 & 4680559.8 & 221909.2 & & \\
\hline
\end{tabular}


(Z)-3-(3-(o-tolyl)but-2-enoyl)oxazolidin-2-one ((Z)-37)

$(E)$-isomer ((E)-37)

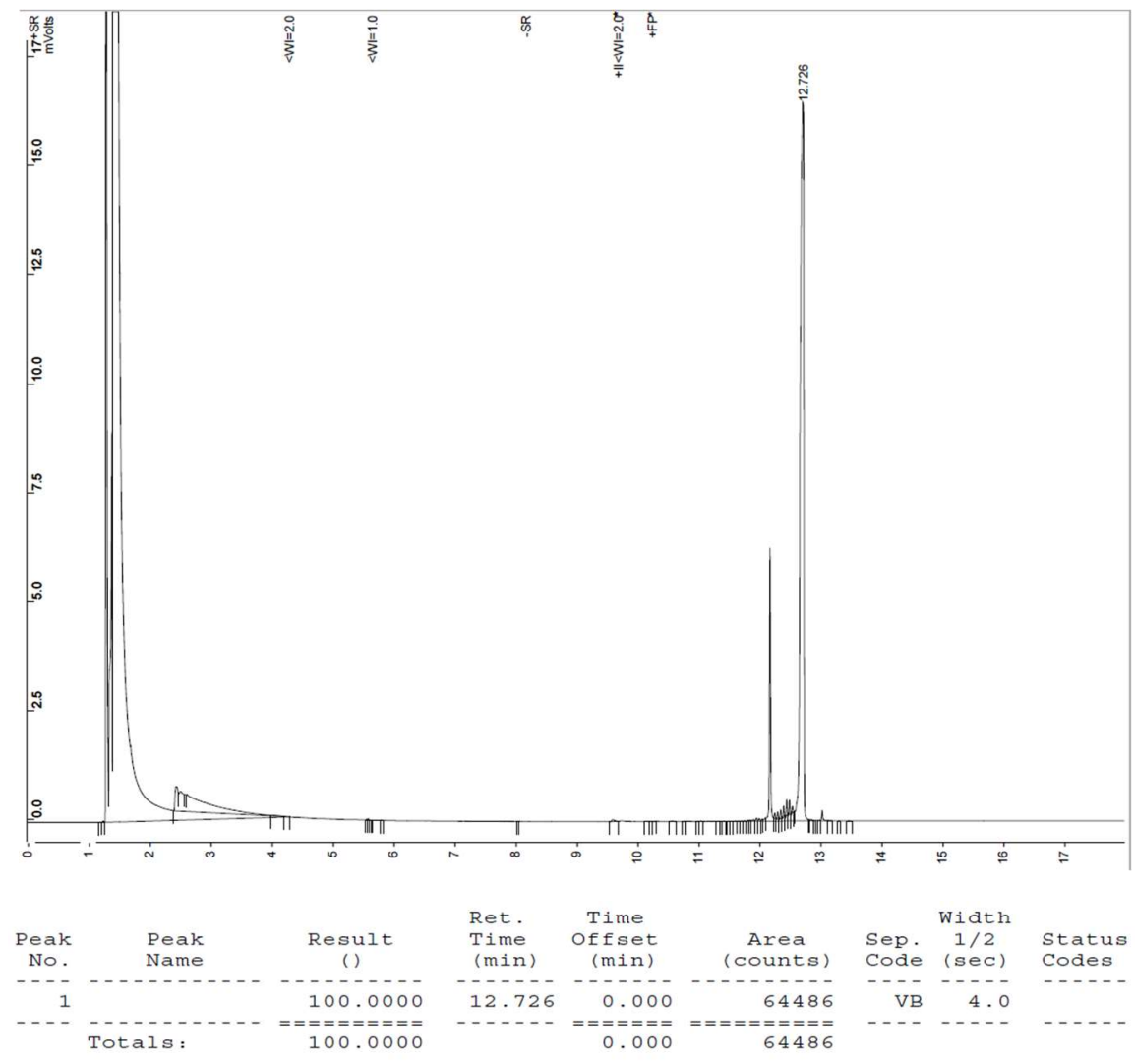

(Z)-isomer ((Z)-37)

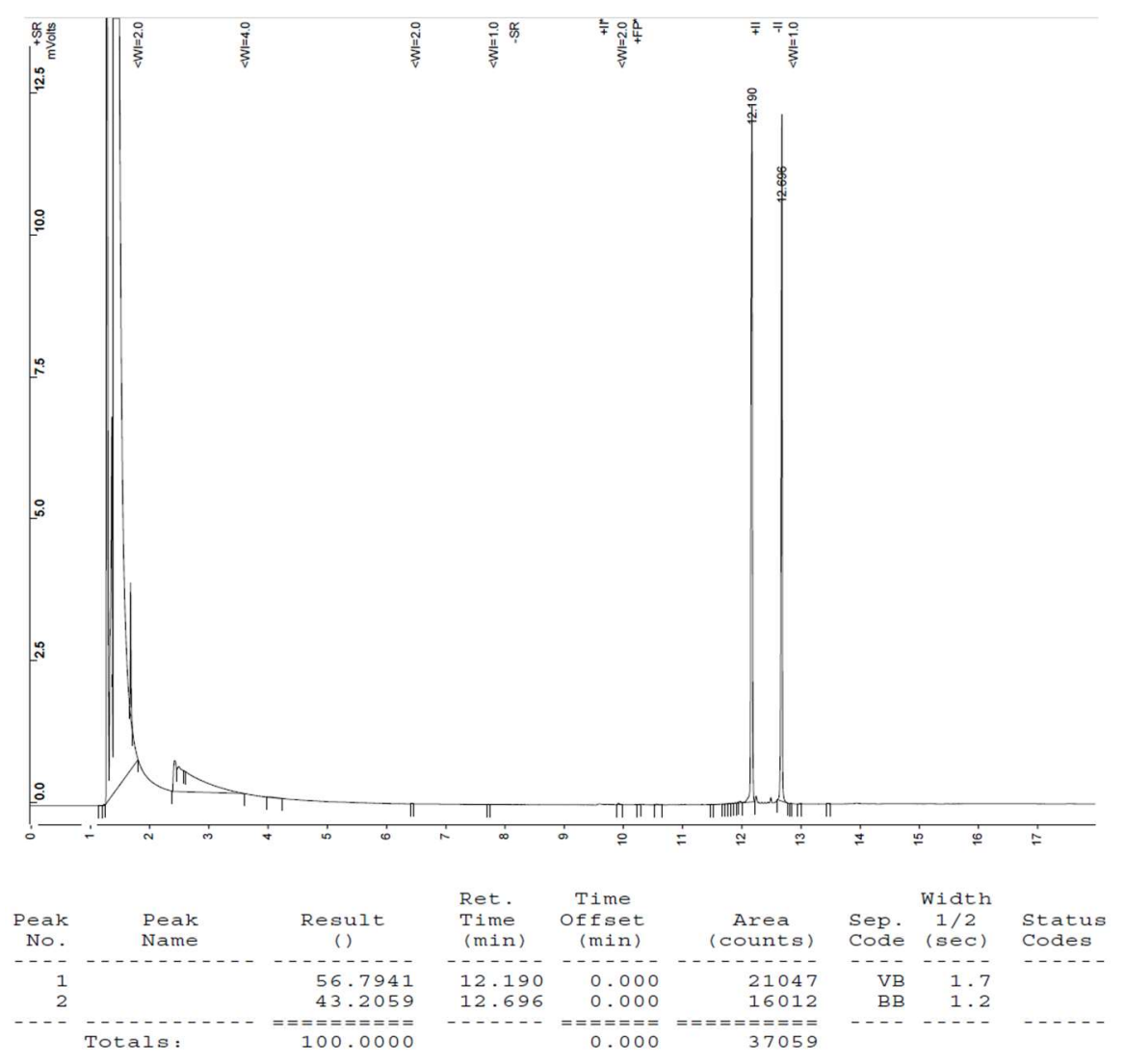


(Z)-3-(3-(3-(tert-butyl)phenyl)but-2-enoyl)oxazolidin-2-one ((Z)-39)

$(E)$-isomer ((E)-39)
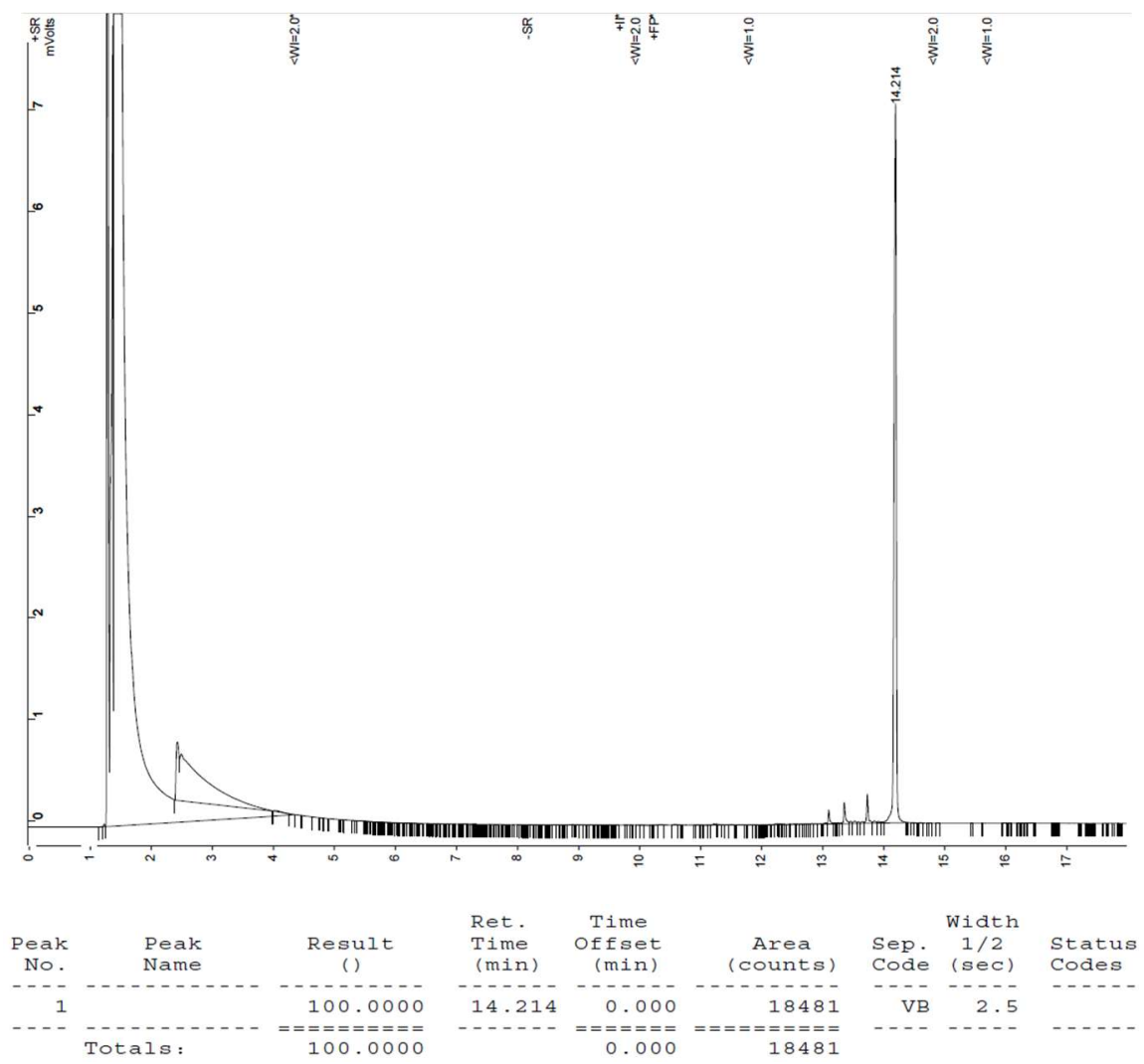

(Z)-isomer ((Z)-39)

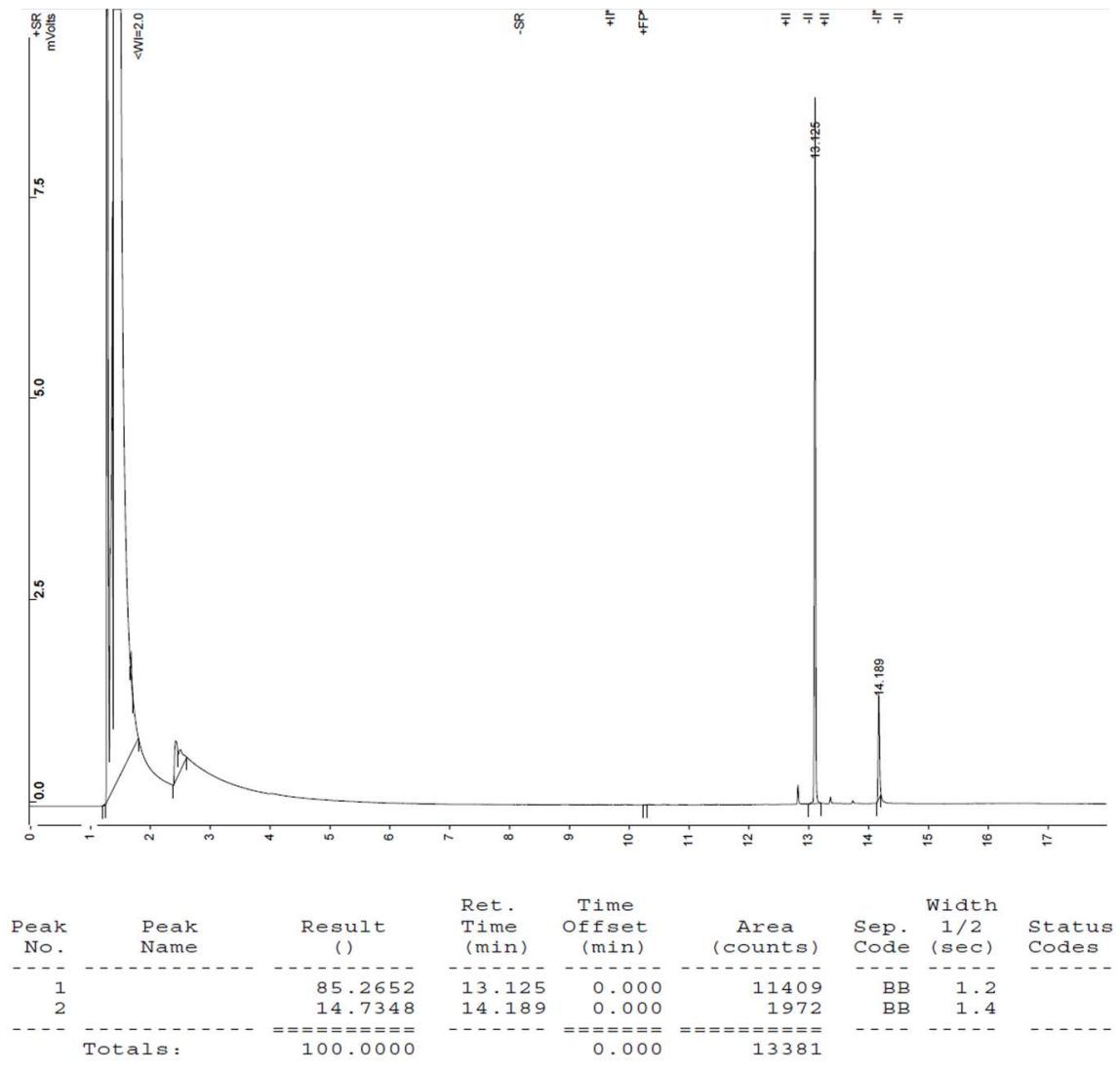


ethyl (Z)-3-(3-(tert-butyl)phenyl)but-2-enoate ((Z)-40)

$(E)$-isomer ((E)-40)

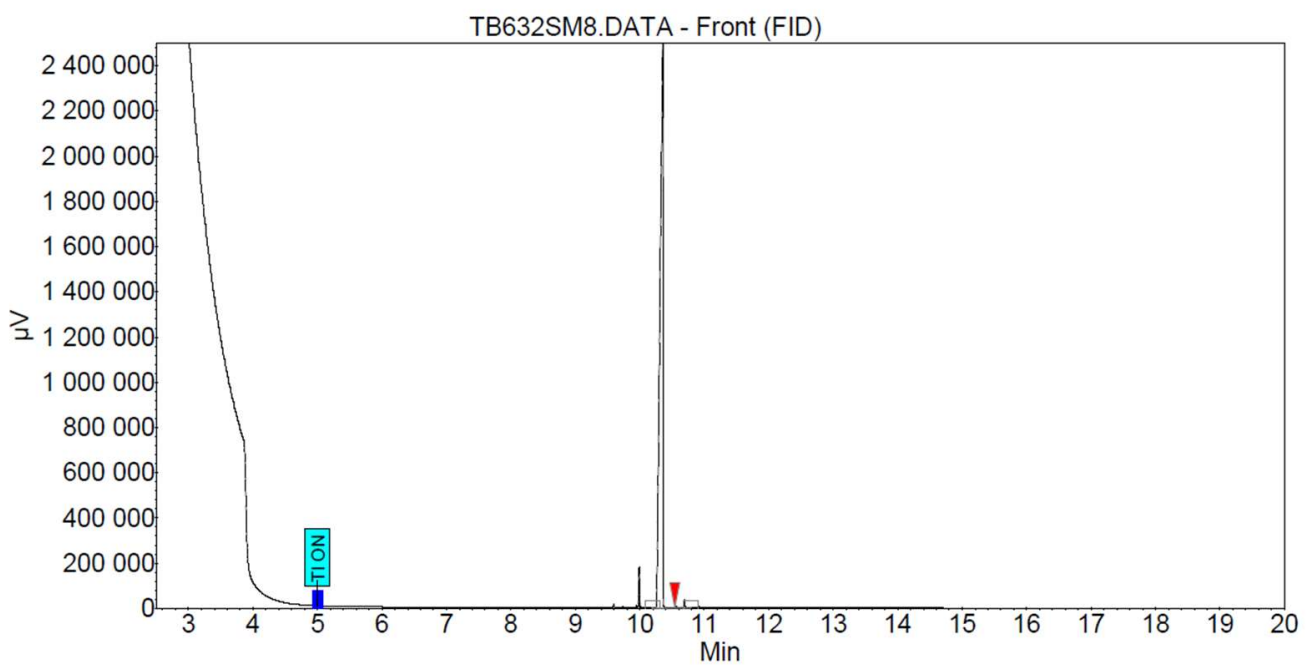

Peak results :

\begin{tabular}{|c|l|r|r|r|r|r|r|r|}
\hline Index & Name & $\begin{array}{r}\text { Time } \\
{[\text { Min] }}\end{array}$ & $\begin{array}{r}\text { Area \% } \\
{[\%]}\end{array}$ & $\begin{array}{r}\text { Quantity } \\
{[\% \text { Area] }}\end{array}$ & $\begin{array}{r}\text { Height } \\
{[\mu \mathrm{V}]}\end{array}$ & $\begin{array}{r}\text { Area } \\
{[\mu \mathrm{V} . \text { Min] }]}\end{array}$ & As. PE. & Res. HW \\
\hline \hline 1 & UNKNOWN & 10,358 & 100,000 & 100,00 & 2602214.9 & 138040.1 & 0.54 & 0.00 \\
\hline & & & & & & & & \\
\hline Total & & & 100,000 & 100,00 & 2602214,9 & 138040,1 & & \\
\hline
\end{tabular}

(Z)-isomer ((Z)-40)

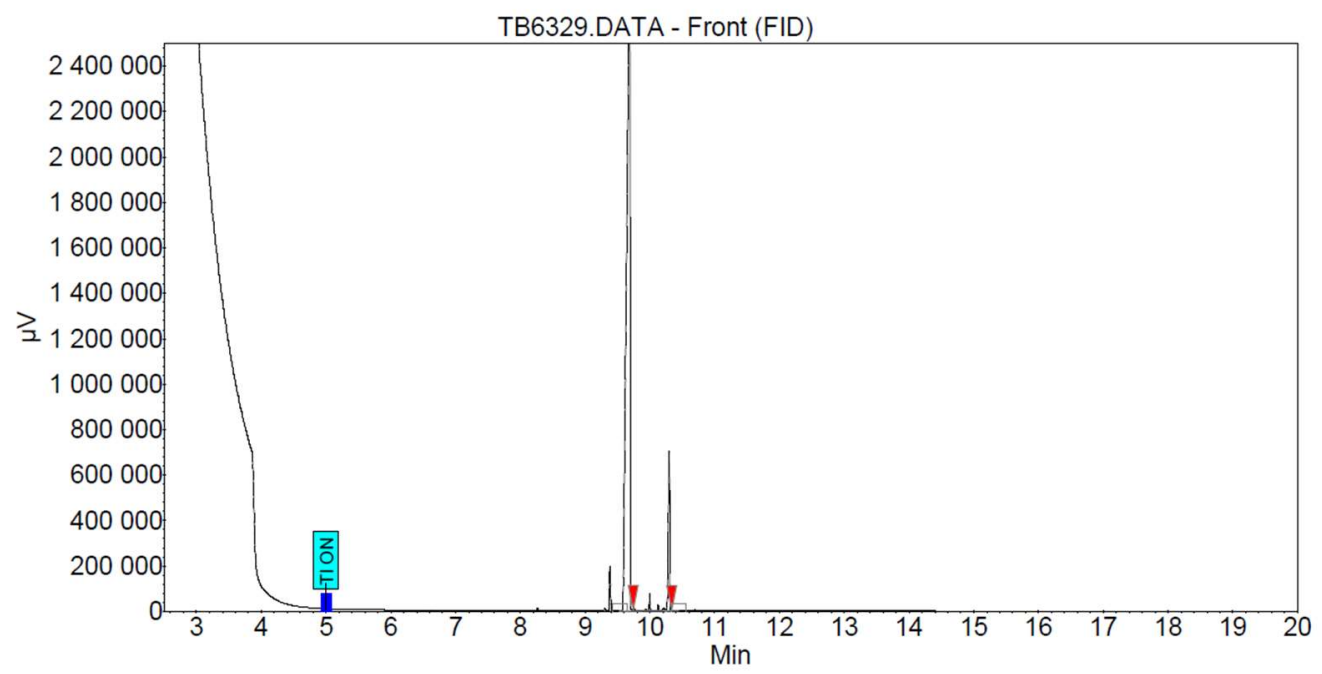

Peak results :

\begin{tabular}{|c|l|r|r|r|r|r|r|r|}
\hline Index & Name & $\begin{array}{r}\text { Time } \\
{[\text { Min] }}\end{array}$ & $\begin{array}{r}\text { Area \% } \\
{[\%]}\end{array}$ & $\begin{array}{r}\text { Quantity } \\
{[\% \text { Area] }}\end{array}$ & $\begin{array}{r}\text { Height } \\
{[\mu \mathrm{V}]}\end{array}$ & $\begin{array}{r}\text { Area } \\
{[\mu \mathrm{V} . \text { Min] }]}\end{array}$ & As. PE. & Res. HW \\
\hline \hline 1 & UNKNOWN & 9.690 & 91.672 & 91.67 & 3001087.0 & 169867.4 & 0.53 & 0.00 \\
\hline 2 & UNKNOWN & 10.298 & 8.328 & 8.33 & 702966.6 & 15431.6 & 0.65 & 9.25 \\
\hline & & & & & & & & \\
\hline Total & & & 100.000 & 100.00 & 3704053.6 & 185299.0 & & \\
\hline
\end{tabular}


(Z)-3-(3-(4-methoxyphenyl)but-2-enoyl)oxazolidin-2-one ((Z)-41)

$(E)$-isomer $((E)-41)$

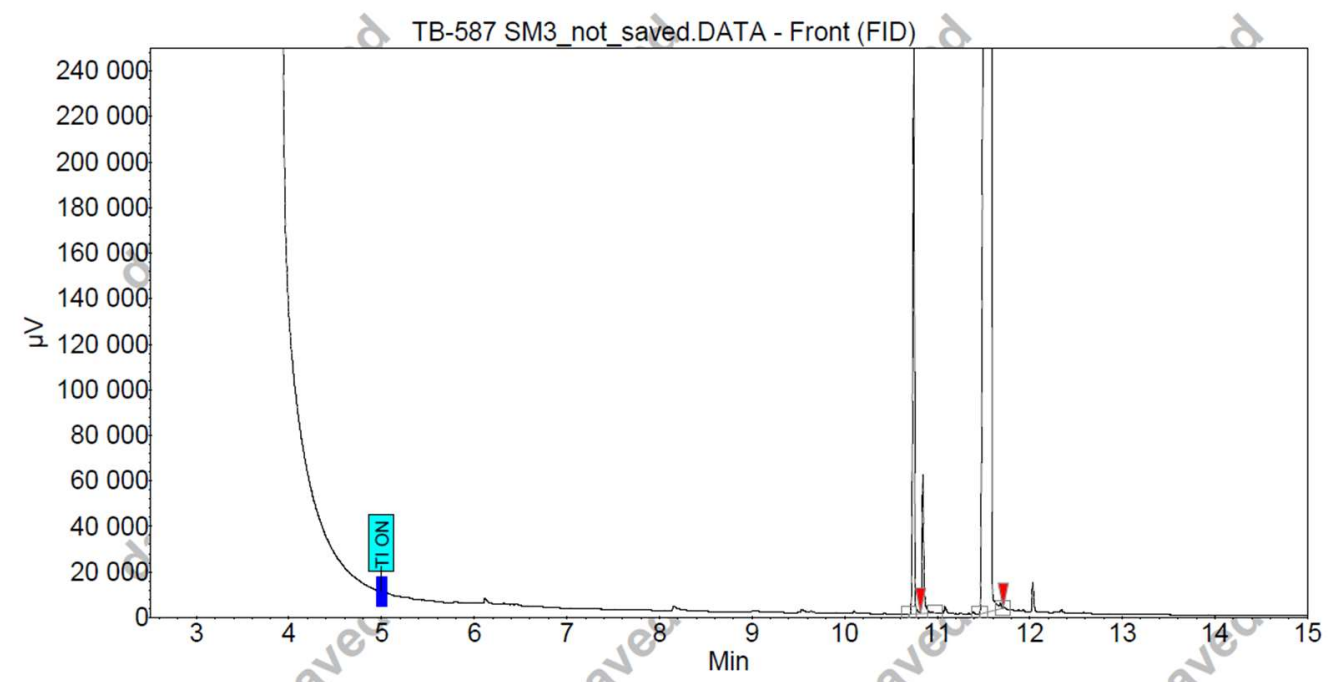

Peak results :

\begin{tabular}{|c|l|r|r|r|r|r|r|r|}
\hline Index & Name & $\begin{array}{r}\text { Time } \\
{[\text { Min] }}\end{array}$ & $\begin{array}{r}\text { Area \% } \\
{[\%]}\end{array}$ & $\begin{array}{r}\text { Quantity } \\
{[\% \text { Area] }}\end{array}$ & $\begin{array}{r}\text { Height } \\
{[\mu \mathrm{V}]}\end{array}$ & $\begin{array}{r}\text { Area } \\
{[\mu \mathrm{V} . \text { Min] }]}\end{array}$ & As. PE. & Res. HW \\
\hline \hline 1 & UNKNOWN & 10.748 & 5.206 & 5.21 & 253510.4 & 4943.2 & 0.75 & 0.00 \\
\hline 2 & UNKNOWN & 11.587 & 94.794 & 94.79 & 1446965.5 & 90018.4 & 0.54 & 12.45 \\
\hline & & & & & & & & \\
\hline Total & & & 100.000 & 100.00 & 1700475.9 & 94961.6 & & \\
\hline
\end{tabular}

(Z)-isomer ((Z)-41)

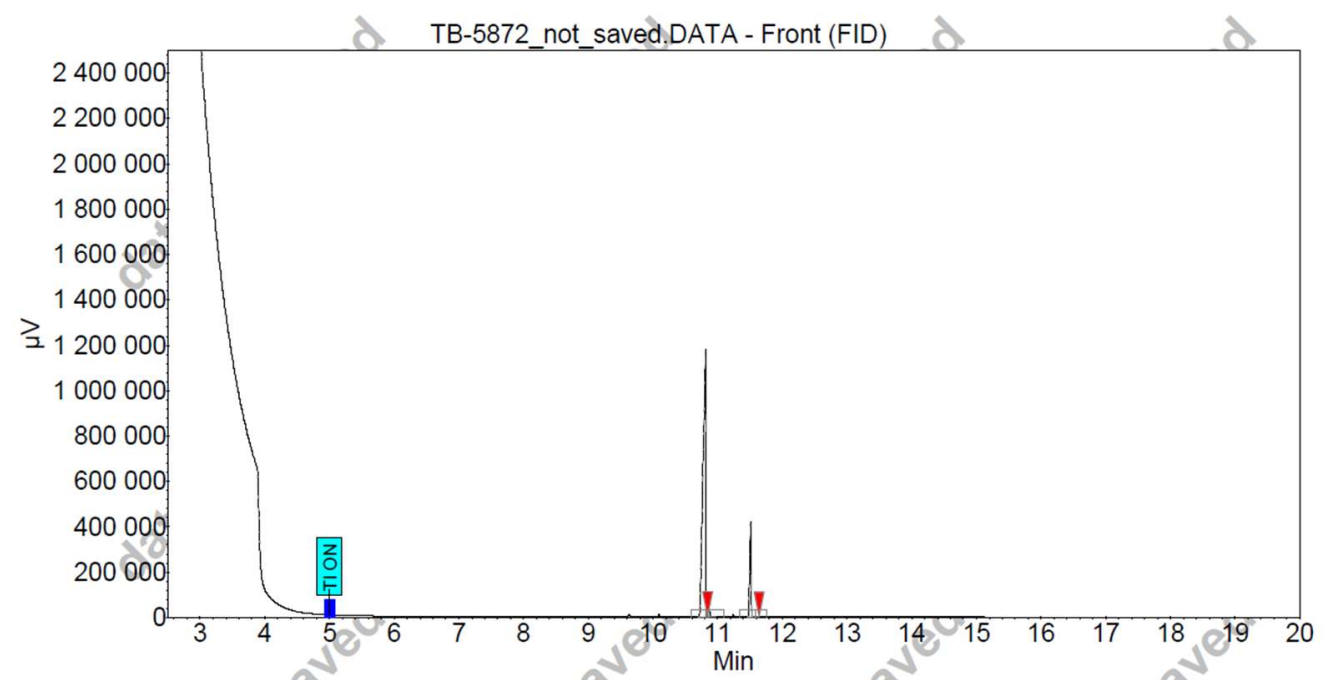

Peak results :

\begin{tabular}{|c|l|r|r|r|r|r|r|r|}
\hline Index & Name & $\begin{array}{r}\text { Time } \\
{[\text { Min] }]}\end{array}$ & $\begin{array}{r}\text { Area \% } \\
{[\%]}\end{array}$ & $\begin{array}{r}\text { Quantity } \\
{[\% \text { Area] }}\end{array}$ & $\begin{array}{r}\text { Height } \\
{[\mu \mathrm{V}]}\end{array}$ & $\begin{array}{r}\text { Area } \\
{[\mu \mathrm{V} \text {.Min] }}\end{array}$ & As. PE. & Res. HW \\
\hline \hline 1 & UNKNOWN & 10.817 & 85.172 & 85.17 & 1181452.1 & 60524.9 & 0.54 & 0.00 \\
\hline 2 & UNKNOWN & 11.512 & 14.828 & 14.83 & 419438.9 & 10537.2 & 0.67 & 10.97 \\
\hline & & & & & & & & \\
\hline Total & & & 100.000 & 100.00 & 1600891.1 & 71062.1 & & \\
\hline
\end{tabular}


ethyl (Z)-3-(4-methoxyphenyl)but-2-enoate ((Z)-42)

$(E)$-isomer ((E)-42)

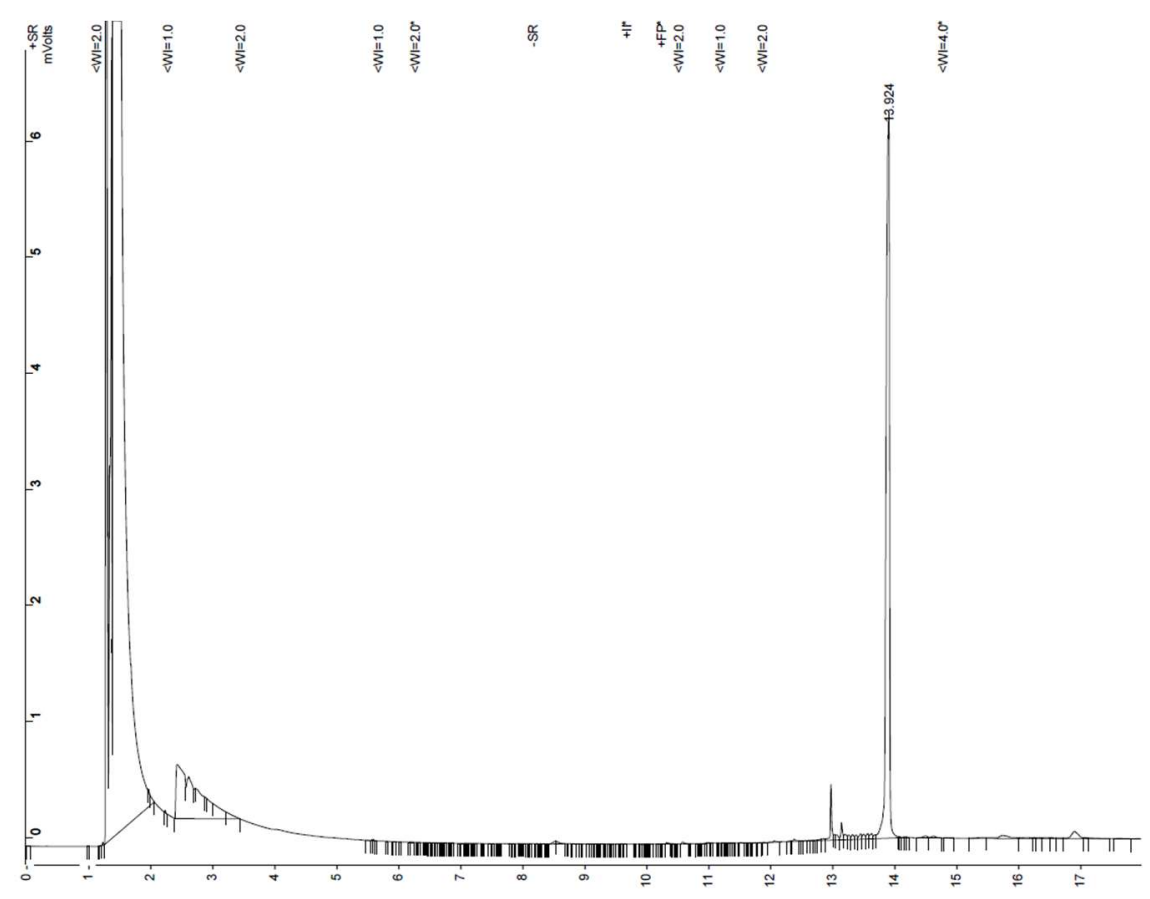

\begin{tabular}{|c|c|c|c|c|c|c|c|c|}
\hline & & & Ret. & Time & & & Width & \\
\hline $\begin{array}{l}\text { Peak } \\
\text { No. }\end{array}$ & $\begin{array}{l}\text { Peak } \\
\text { Name }\end{array}$ & Result & $\begin{array}{l}\text { Time } \\
(\min )\end{array}$ & $\begin{array}{l}\text { Offset } \\
\text { (min) }\end{array}$ & $\begin{array}{c}\text { Area } \\
\text { (counts) }\end{array}$ & $\begin{array}{l}\text { Sep. } \\
\text { Code }\end{array}$ & $\begin{array}{c}1 / 2 \\
(\mathrm{sec})\end{array}$ & $\begin{array}{l}\text { Status } \\
\text { Codes }\end{array}$ \\
\hline 1 & & 100.0000 & 13.924 & 0.000 & 23664 & $\mathrm{VB}$ & 3.7 & \\
\hline & als: & $\begin{aligned}== \\
100\end{aligned}$ & & $\begin{aligned} &===== \\
& 0.0000\end{aligned}$ & $\begin{array}{r}====== \\
23664\end{array}$ & & & \\
\hline
\end{tabular}

(Z)-isomer ((Z)-42)
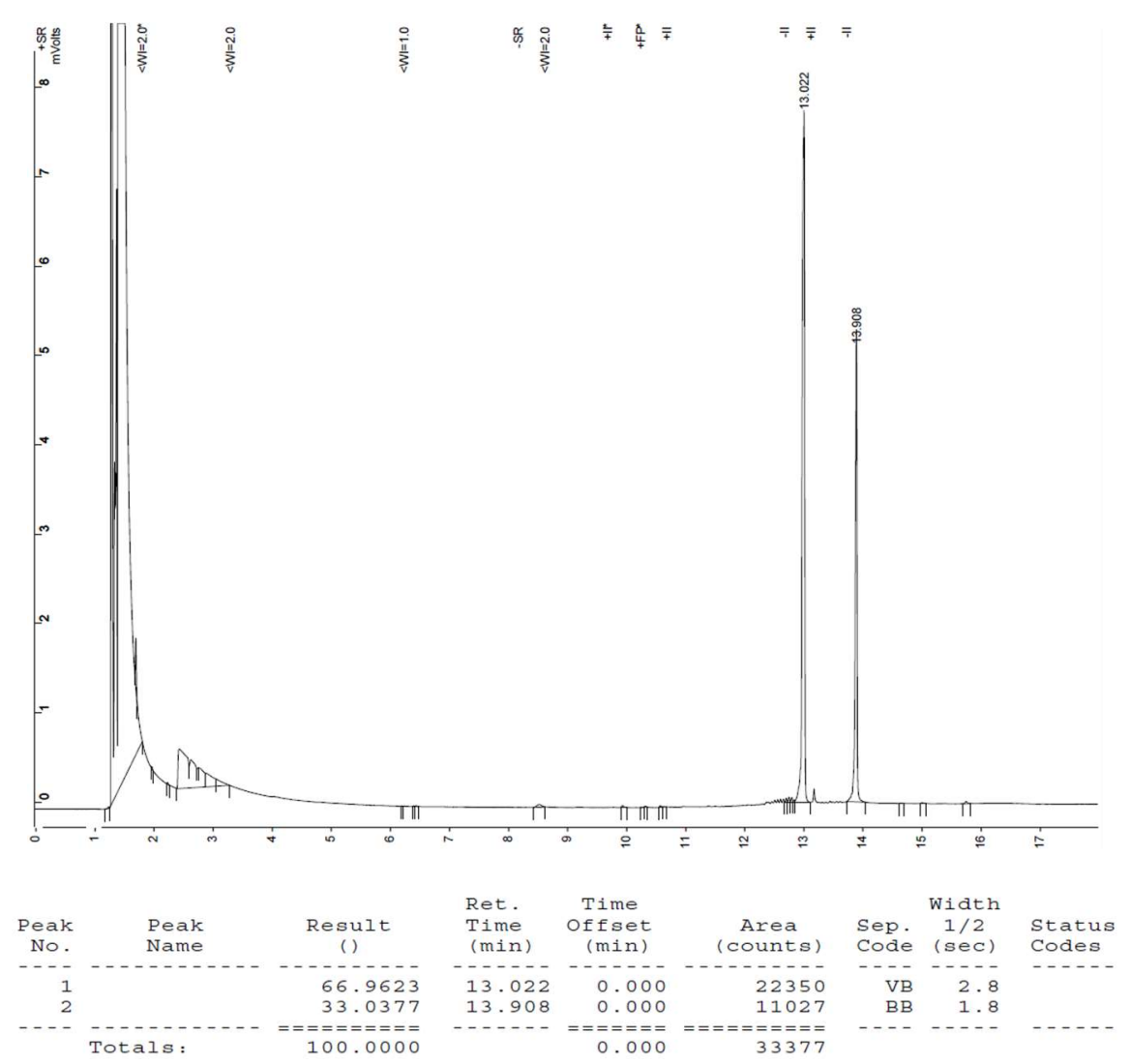
(Z)-3-(3-(4-fluorophenyl)but-2-enoyl)oxazolidin-2-one ((Z)-43)

$(E)$-isomer ((E)-43)

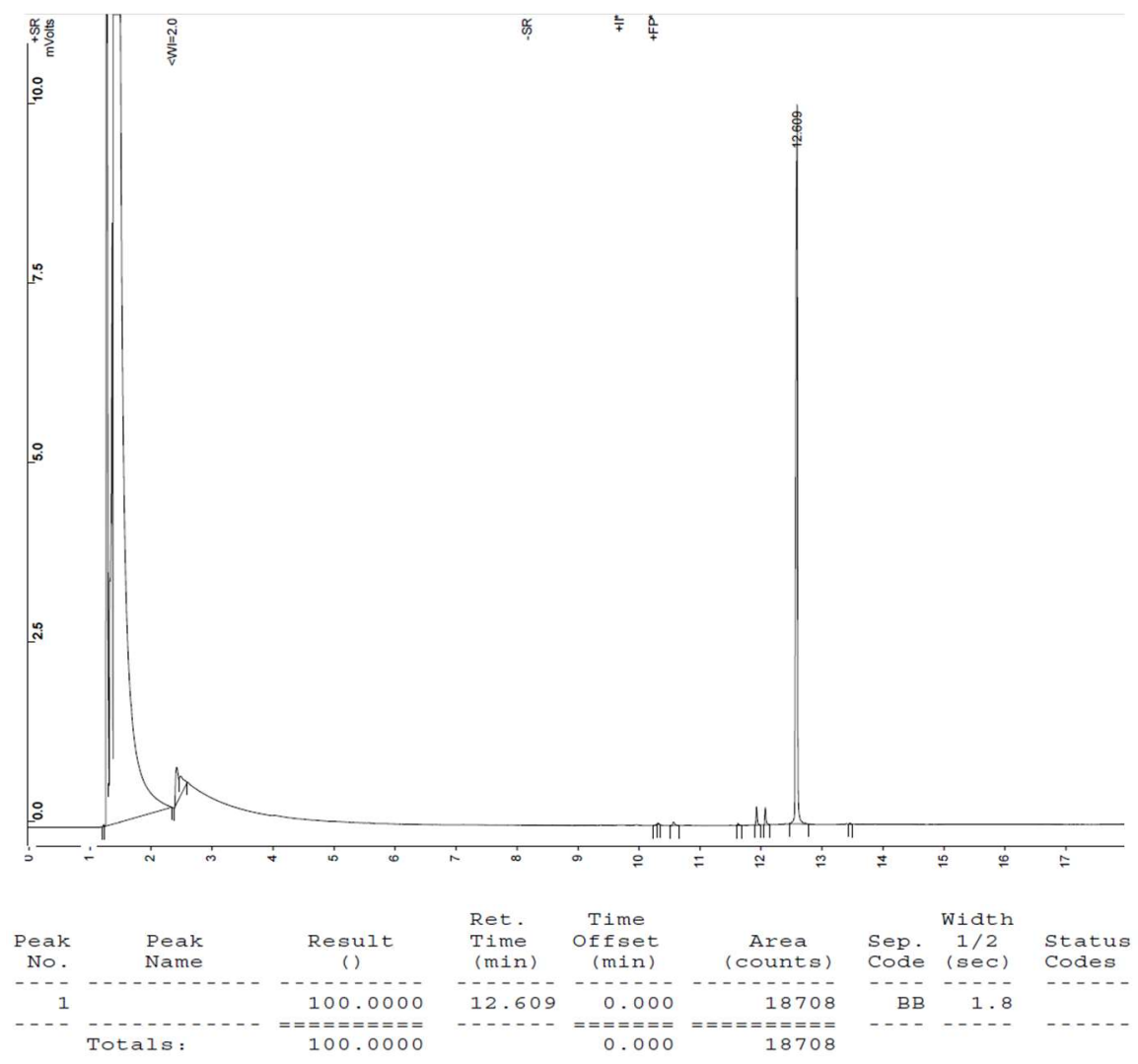

(Z)-isomer ((Z)-43)
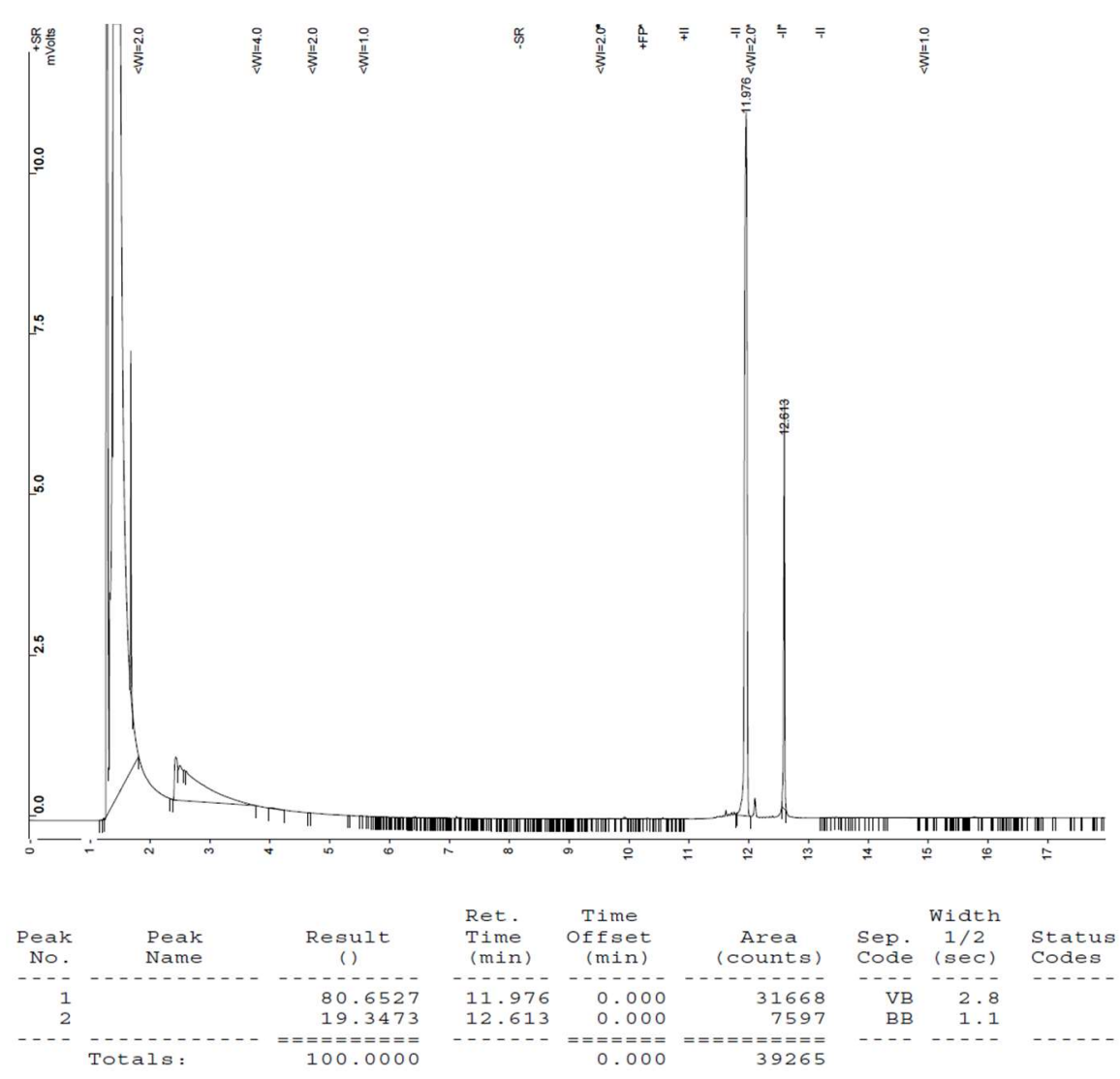
ethyl (Z)-3-(4-fluorophenyl)but-2-enoate ((Z)-44):

$(E)$-isomer ((E)-44)
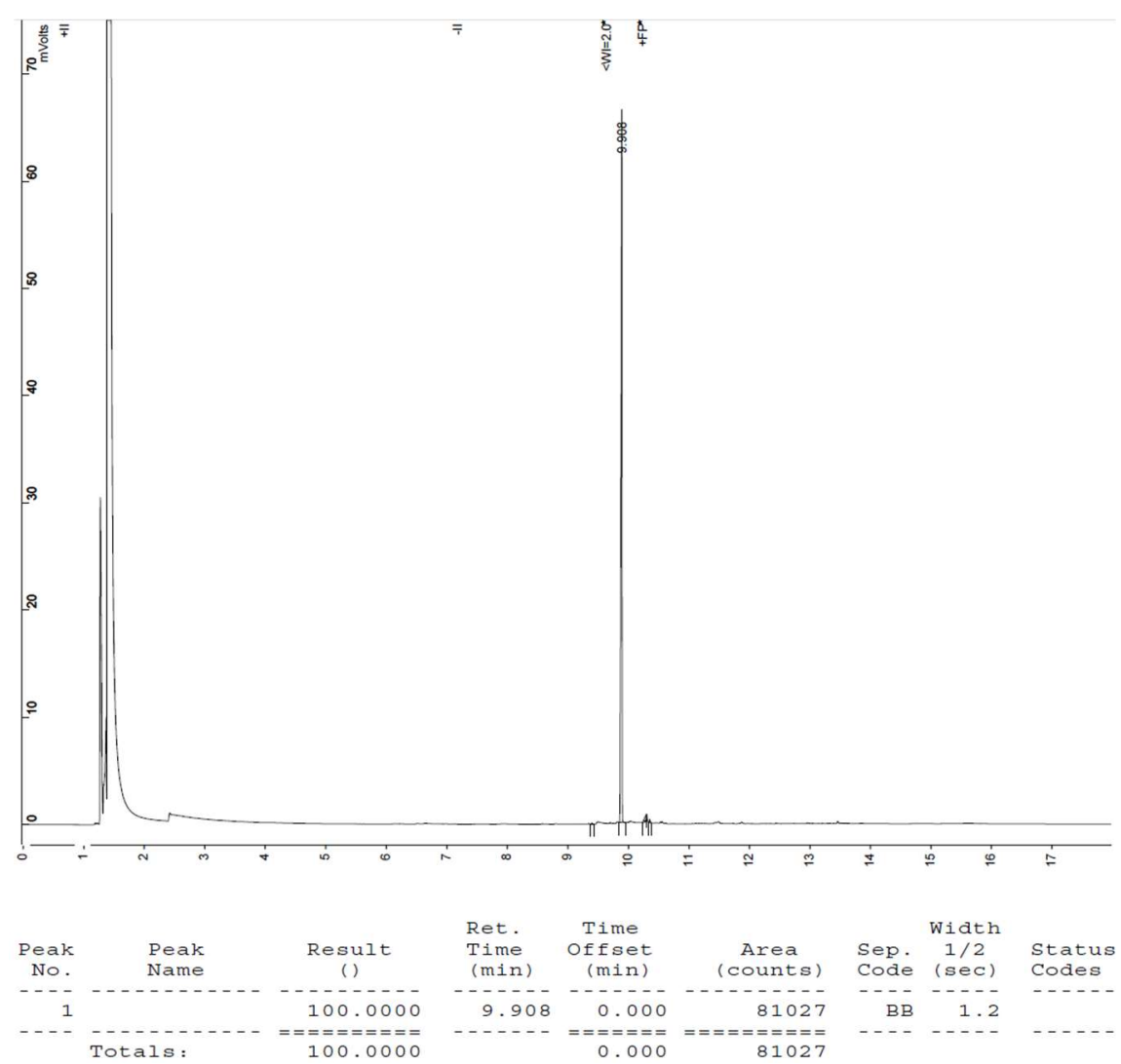

(Z)-isomer ((Z)-44)
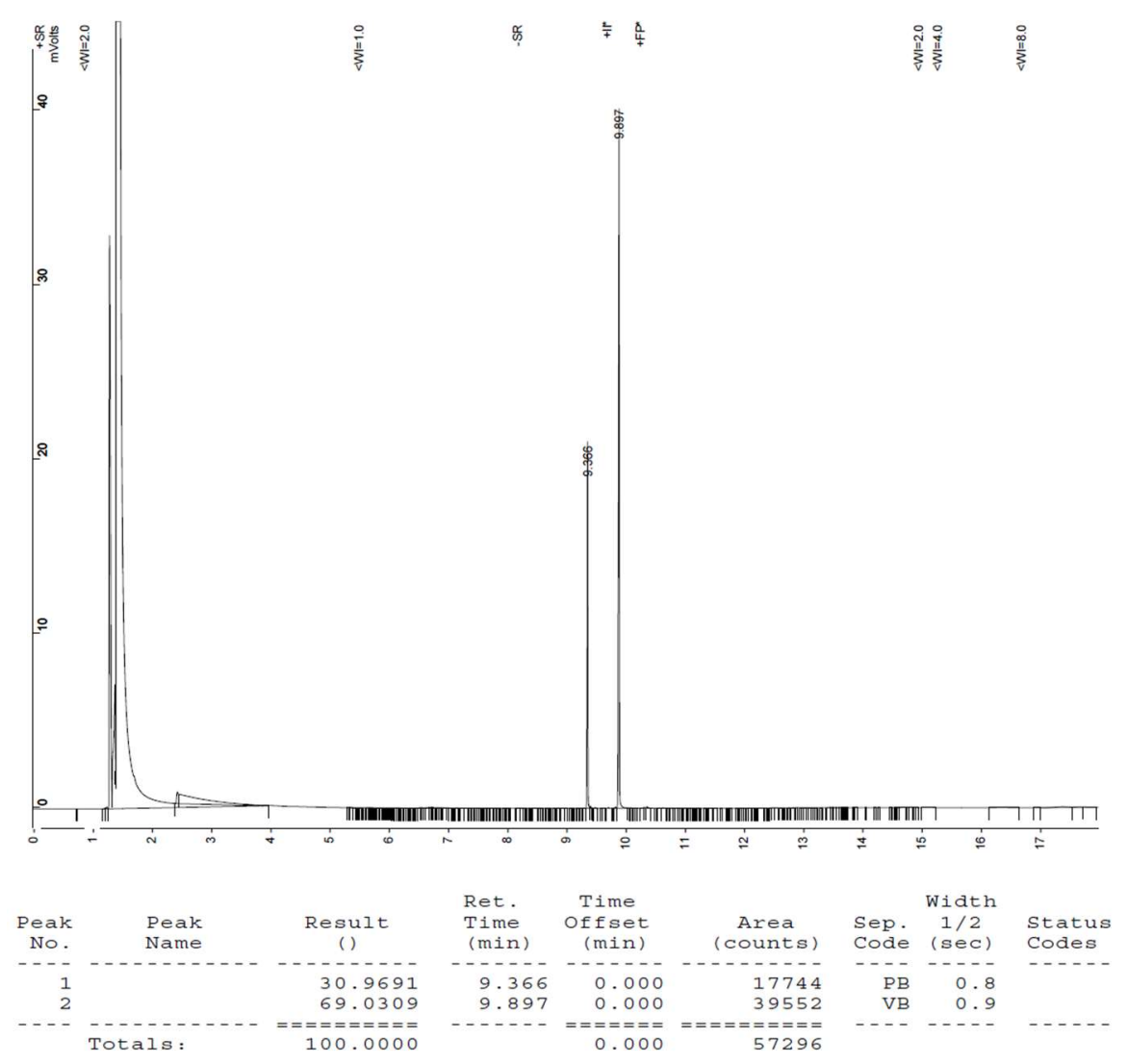
(Z)-3-(3-(4-bromophenyl)but-2-enoyl)oxazolidin-2-one ((Z)-45)

$(E)$-isomer ((E)-45)

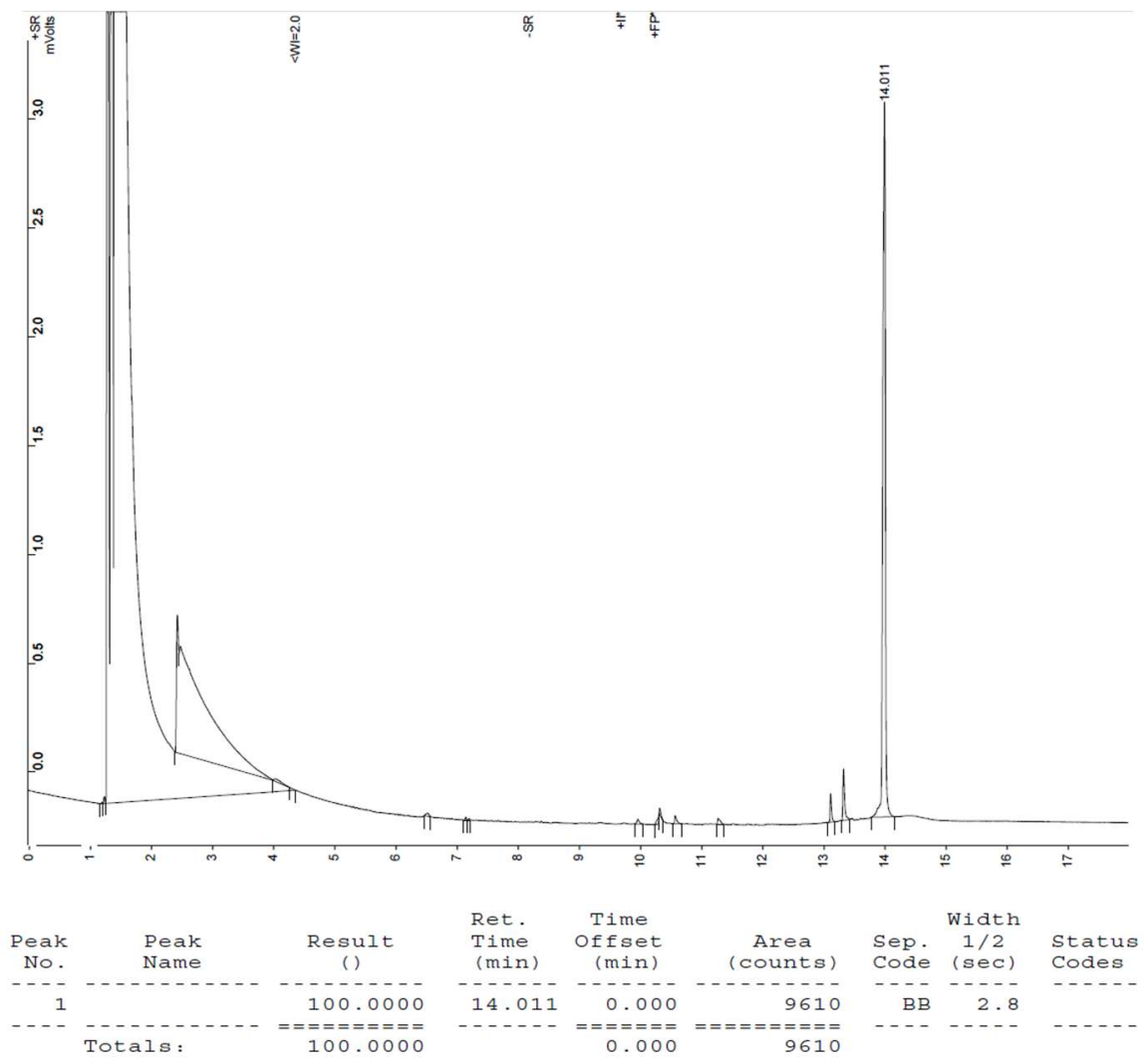

(Z)-isomer ((Z)-45)

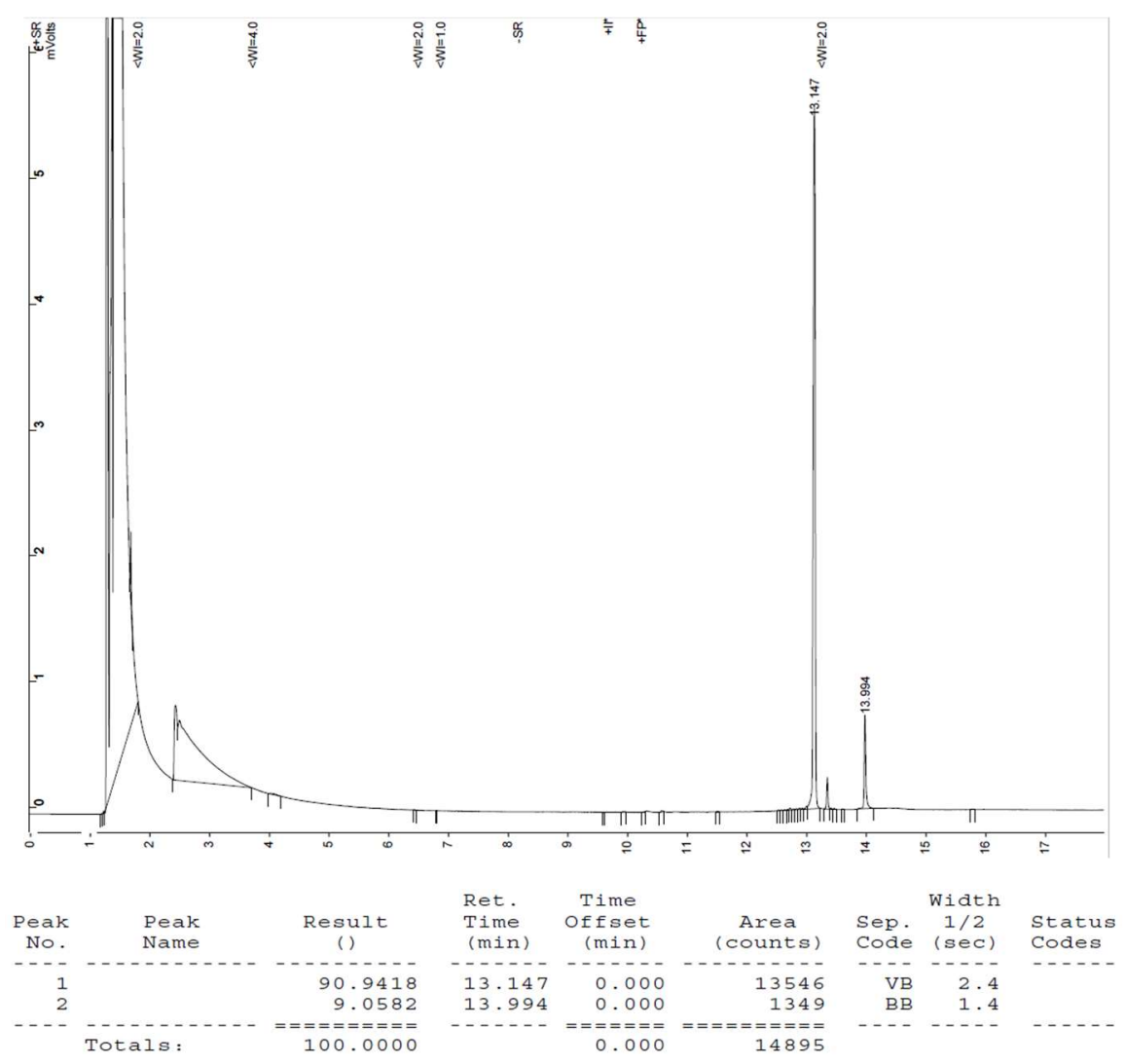


ethyl (Z)-3-(4-bromophenyl)but-2-enoate ((Z)-46)

$(E)$-isomer ((E)-46)

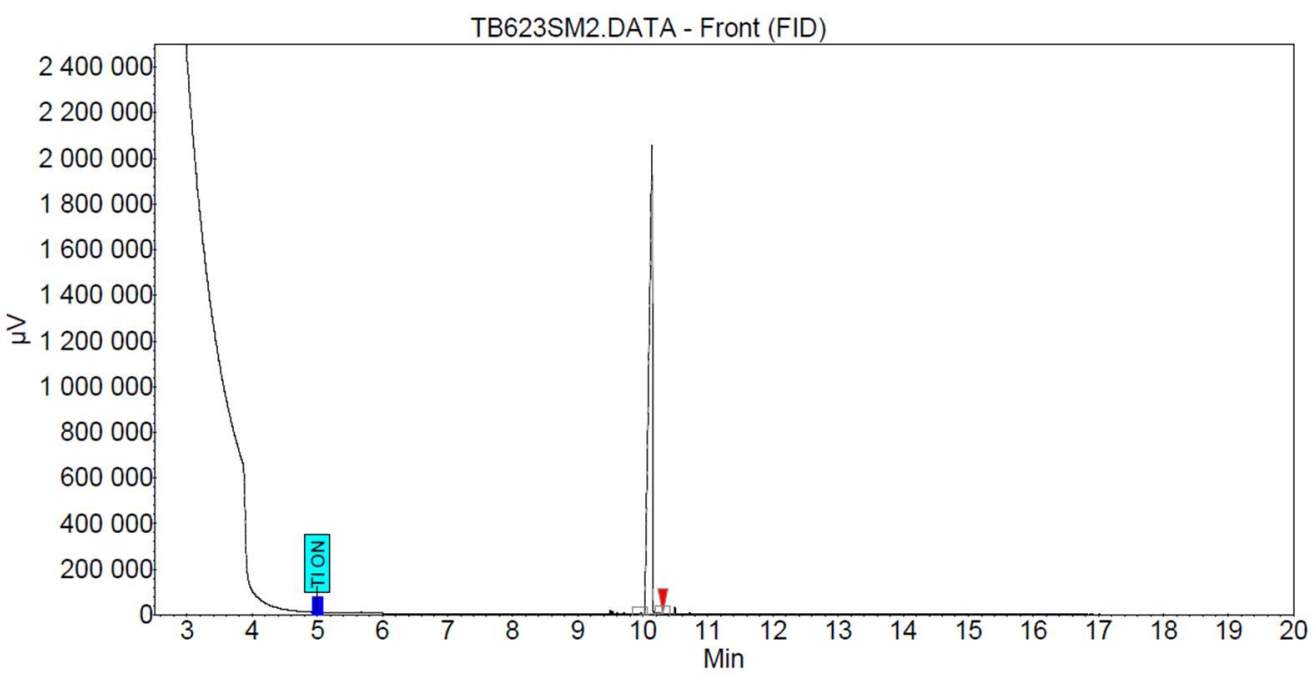

Peak results :

\begin{tabular}{|c|l|r|r|r|r|r|r|r|}
\hline Index & Name & $\begin{array}{r}\text { Time } \\
{[\mathrm{Min}]}\end{array}$ & $\begin{array}{r}\text { Area \% } \\
{[\%]}\end{array}$ & $\begin{array}{r}\text { Quantity } \\
{[\% \text { Area] }}\end{array}$ & $\begin{array}{r}\text { Height } \\
{[\mu \mathrm{V}]}\end{array}$ & $\begin{array}{r}\text { Area } \\
{[\mu \mathrm{V} . \text { Min] }]}\end{array}$ & As. PE. & Res. HW \\
\hline \hline 1 & UNKNOWN & 10.145 & 100,000 & 100,00 & 2052367.0 & 128708.7 & 0.53 & 0.00 \\
\hline & & & & & & & & \\
\hline Total & & & 100,000 & 100,00 & 2052367.0 & 128708.7 & & \\
\hline
\end{tabular}

(Z)-isomer ((Z)-46)

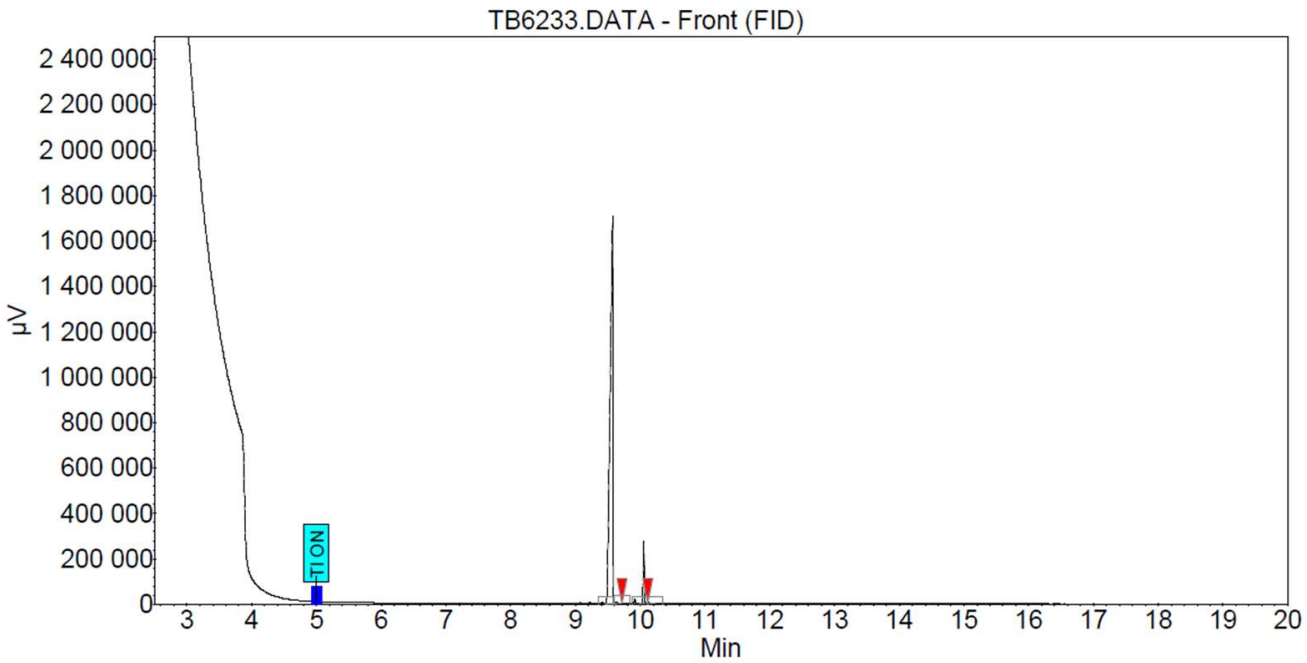

Peak results :

\begin{tabular}{|c|l|r|r|r|r|r|r|r|}
\hline Index & Name & $\begin{array}{r}\text { Time } \\
{[\mathrm{Min}]}\end{array}$ & $\begin{array}{r}\text { Area \% } \\
{[\%]}\end{array}$ & $\begin{array}{r}\text { Quantity } \\
\% \% \text { Area] }\end{array}$ & $\begin{array}{r}\text { Height } \\
{[\mu \mathrm{V}]}\end{array}$ & $\begin{array}{r}\text { Area } \\
{[\mu \mathrm{V} . \mathrm{Min}]}\end{array}$ & As. PE. & Res. HW \\
\hline \hline 1 & UNKNOWN & 9.575 & 94.703 & 94.70 & 1706407.1 & 84249.5 & 0.55 & 0.00 \\
\hline 2 & UNKNOWN & 10.057 & 5.297 & 5.30 & 272474.3 & 4712.0 & 0.78 & 8.78 \\
\hline & & & & & & & & \\
\hline Total & & & 100.000 & 100.00 & 1978881.3 & 88961.5 & & \\
\hline
\end{tabular}


(Z)-3-(3-(4-iodophenyl)but-2-enoyl)oxazolidin-2-one ((Z)-47)

$(E)$-isomer ((E)-47)

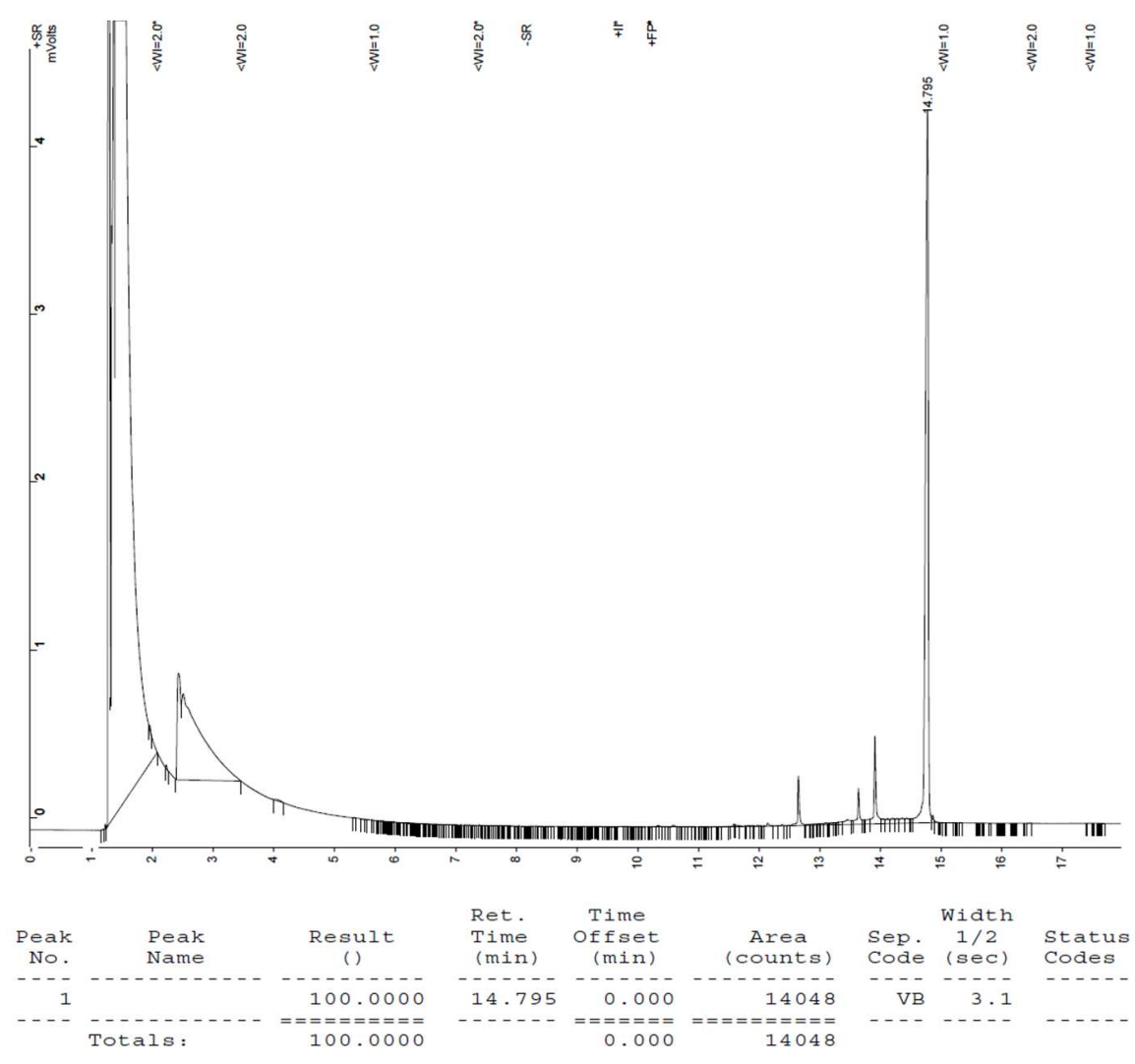

(Z)-isomer ((Z)-47)

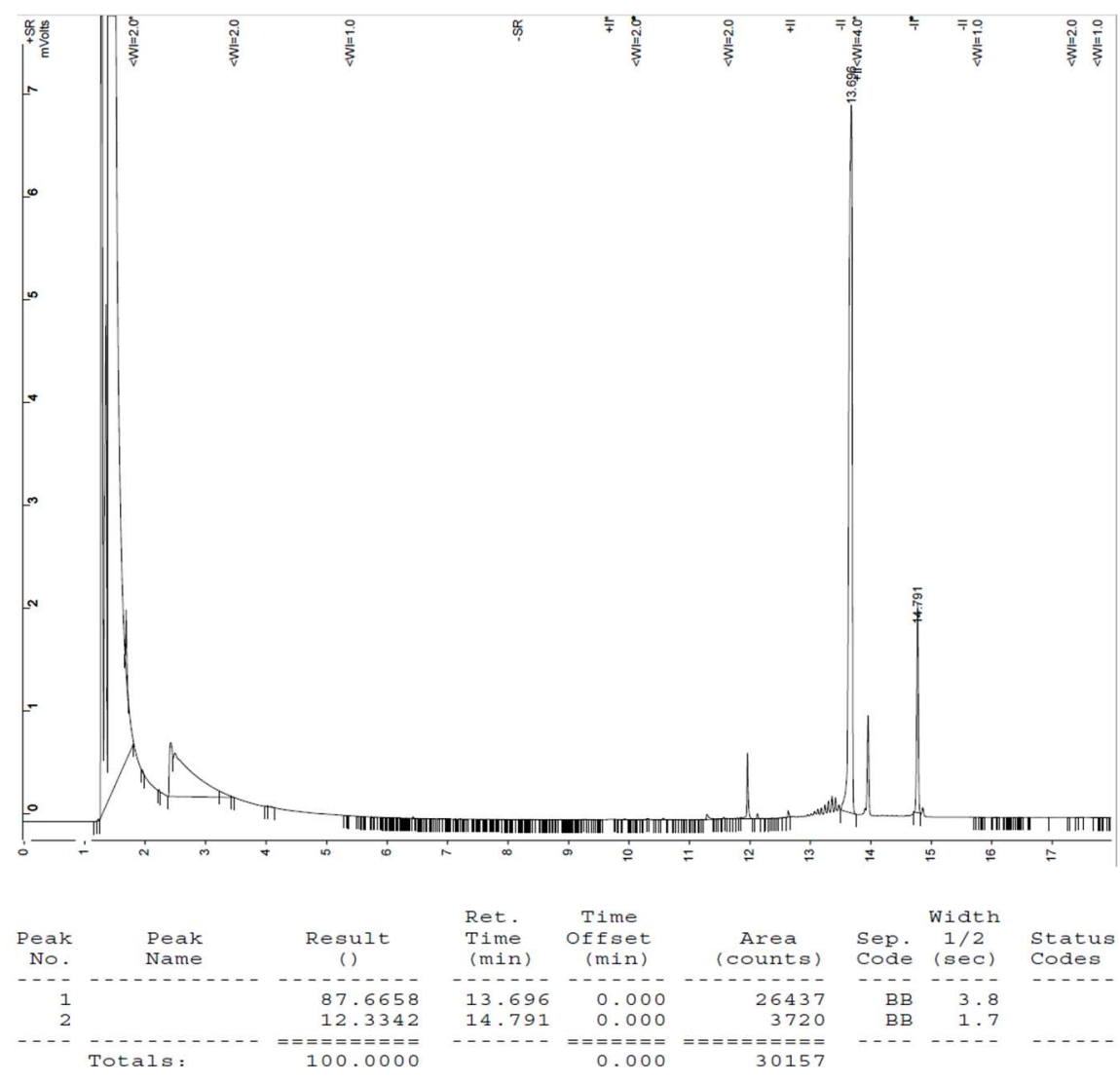


ethyl (Z)-3-(4-iodophenyl)but-2-enoate ((Z)-48)

$(E)$-isomer ((E)-48)

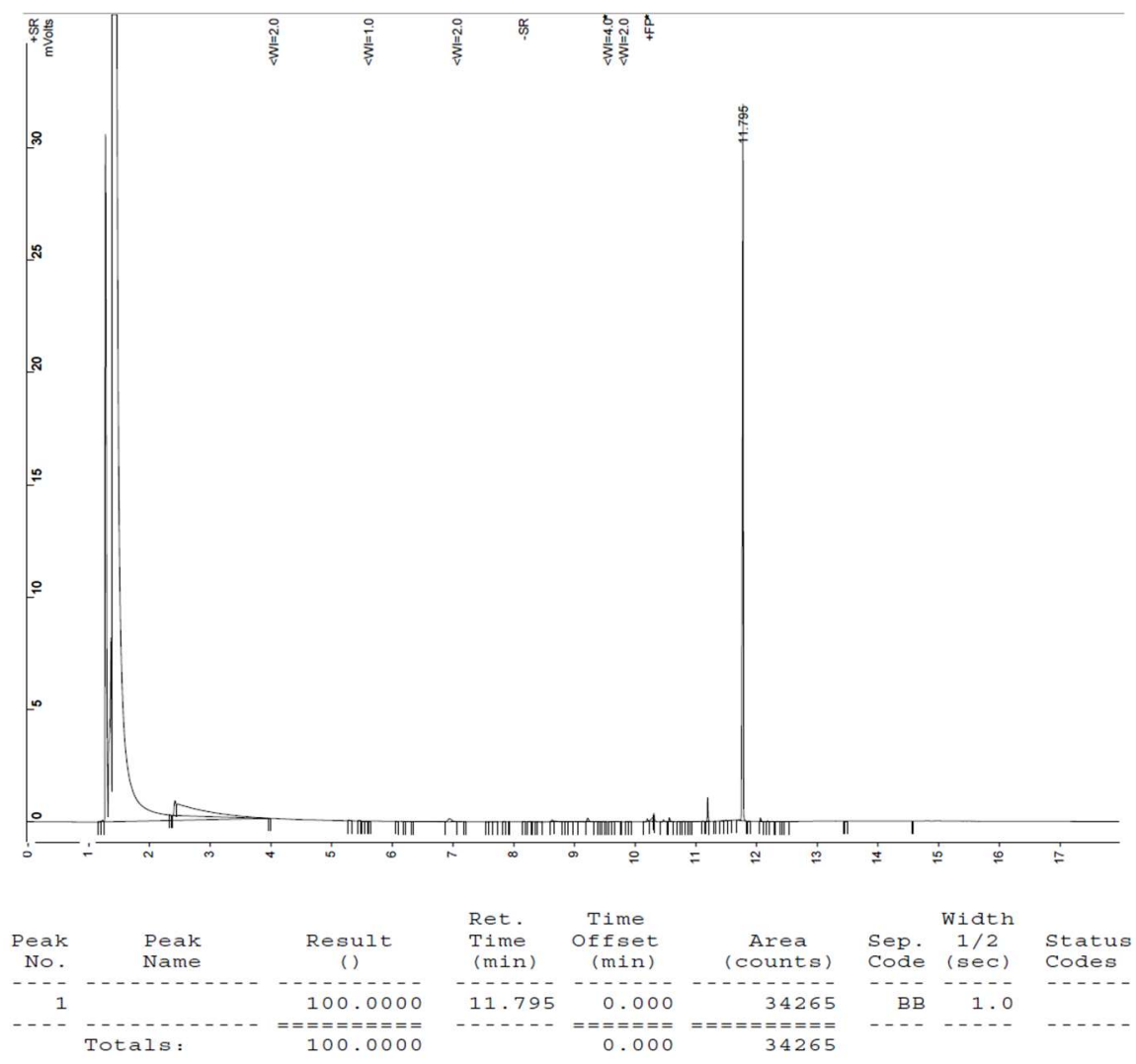

(Z)-isomer ((Z)-48)

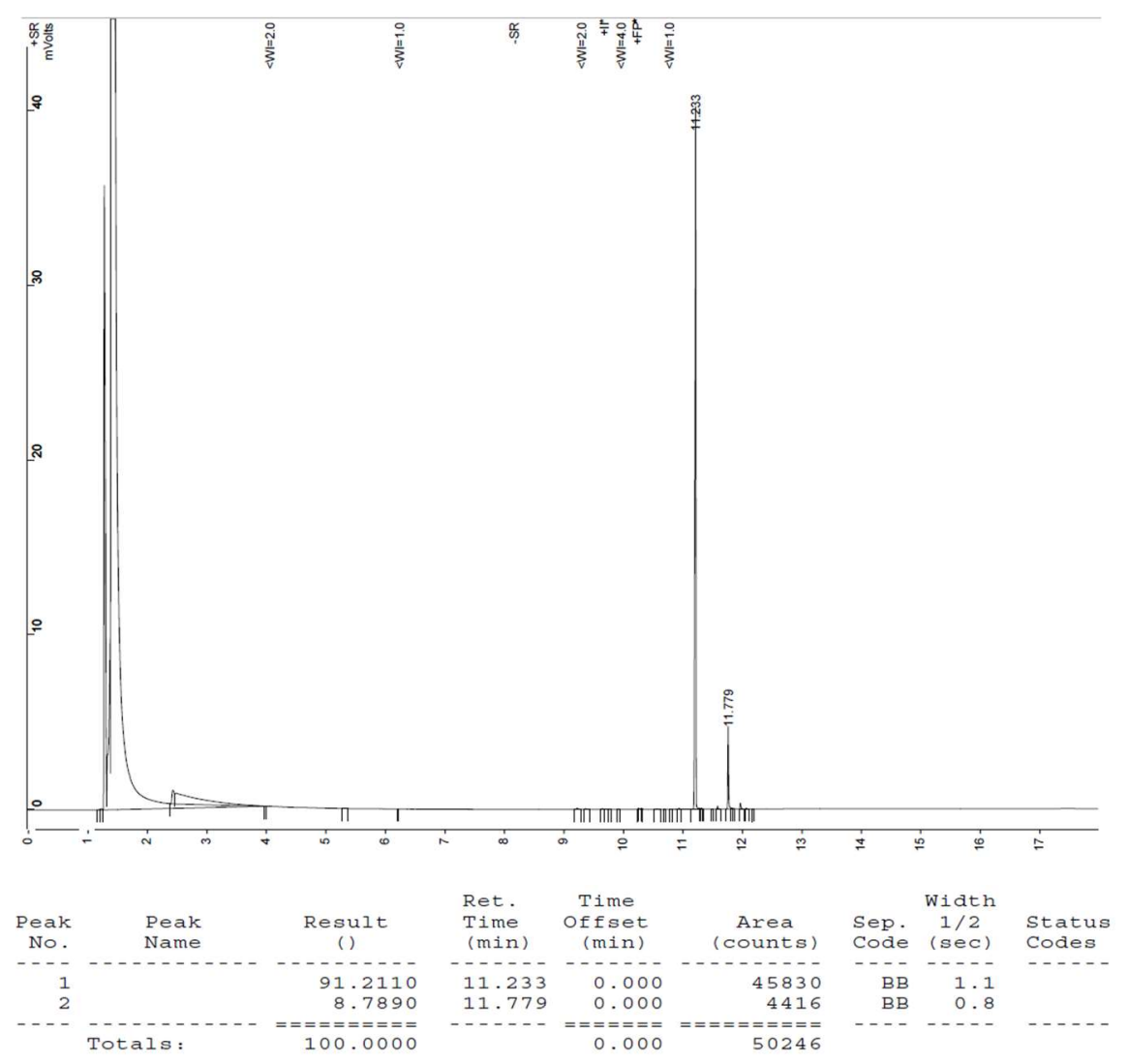


(Z)-3-(3-(4-nitrophenyl)but-2-enoyl)oxazolidin-2-one ((Z)-49)

(Z)-isomer ((Z)-49)

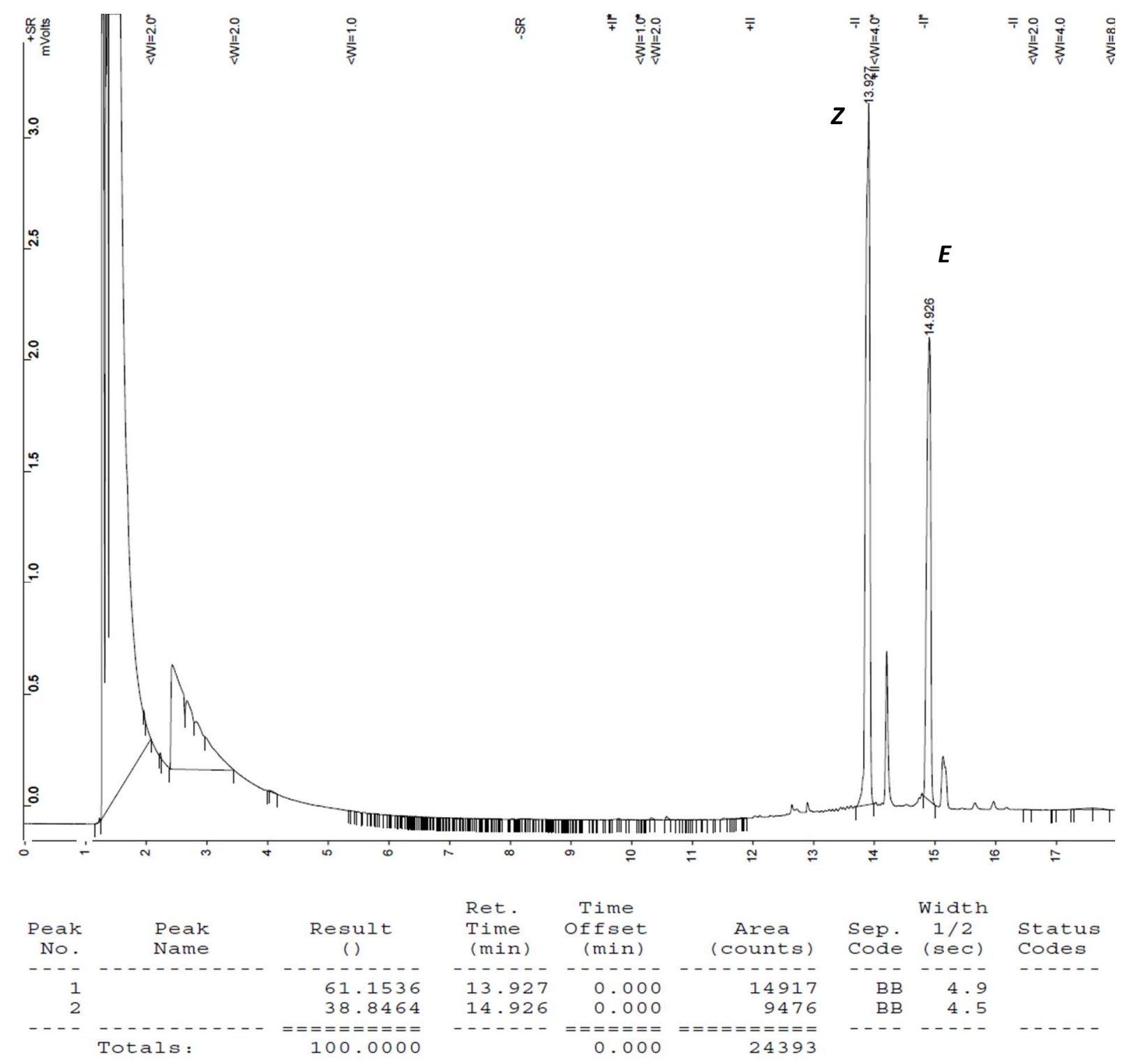


ethyl (Z)-3-(4-nitrophenyl)but-2-enoate ((Z)-50)

(Z)-isomer ((Z)-50)

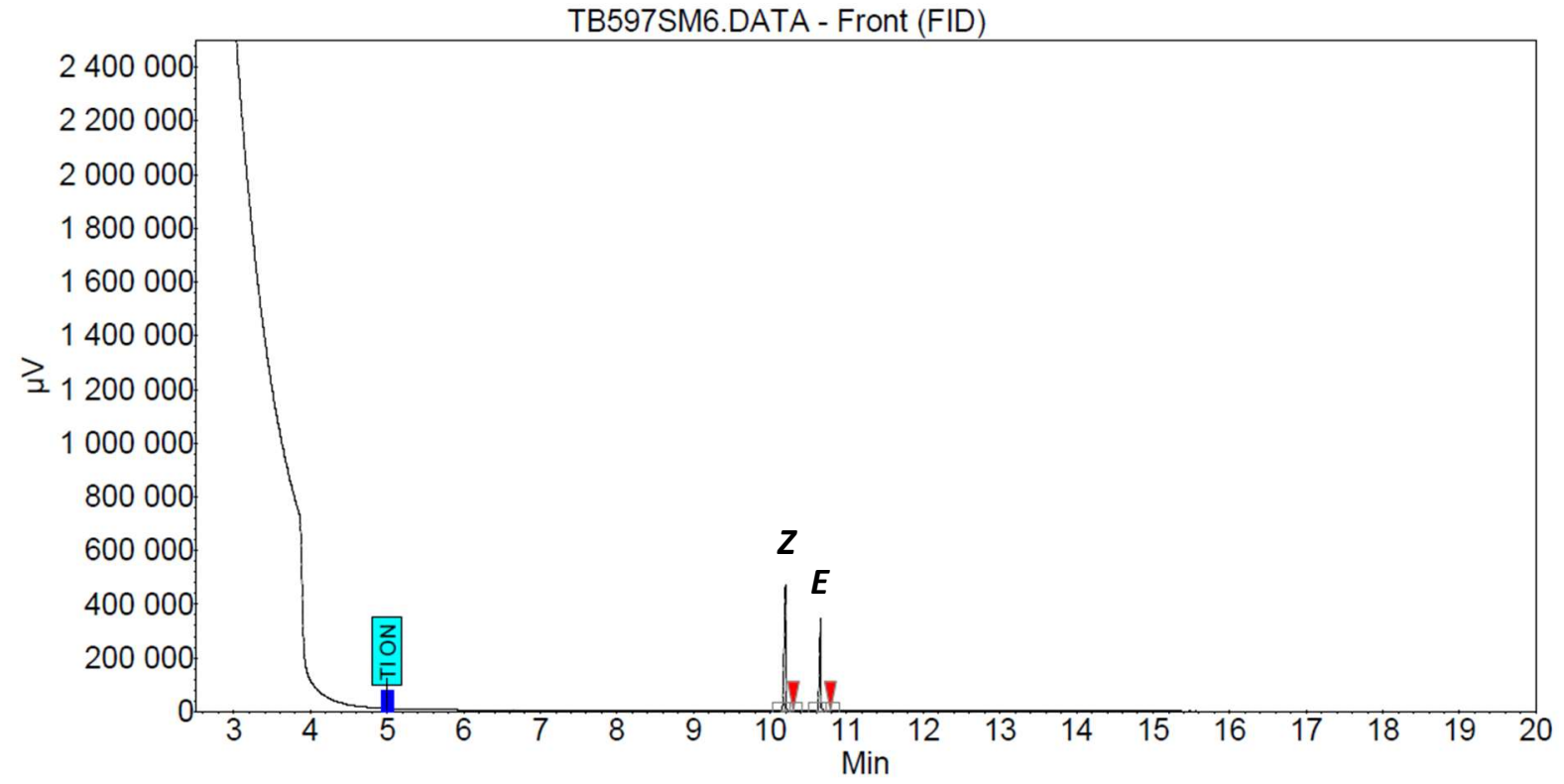

Peak results :

\begin{tabular}{|c|l|r|r|r|r|r|r|r|}
\hline Index & Name & $\begin{array}{r}\text { Time } \\
{[\mathrm{Min}]}\end{array}$ & $\begin{array}{r}\text { Area \% } \\
{[\%]}\end{array}$ & $\begin{array}{r}\text { Quantity } \\
{[\% \text { Area] }}\end{array}$ & $\left.\begin{array}{r}\text { Height } \\
{[\mu \mathrm{V}]}\end{array}\right]$ & $\begin{array}{r}\text { Area } \\
{[\mu \mathrm{V} . \mathrm{Min}]}\end{array}$ & As. PE. & Res. HW \\
\hline \hline 1 & UNKNOWN & 10.202 & 63.162 & 63.16 & 468438.1 & 12253.4 & 0.63 & 0.00 \\
\hline 2 & UNKNOWN & 10.657 & 36.838 & 36.84 & 342052.3 & 7146.4 & 0.70 & 12.06 \\
\hline & & & & & & & & \\
\hline Total & & & 100.000 & 100.00 & 810490.4 & 19399.8 & & \\
\hline
\end{tabular}


(Z)-3-(4,4,4-trifluoro-3-phenylbut-2-enoyl)oxazolidin-2-one ((Z)-51)

$(E)$-isomer ((E)-51)
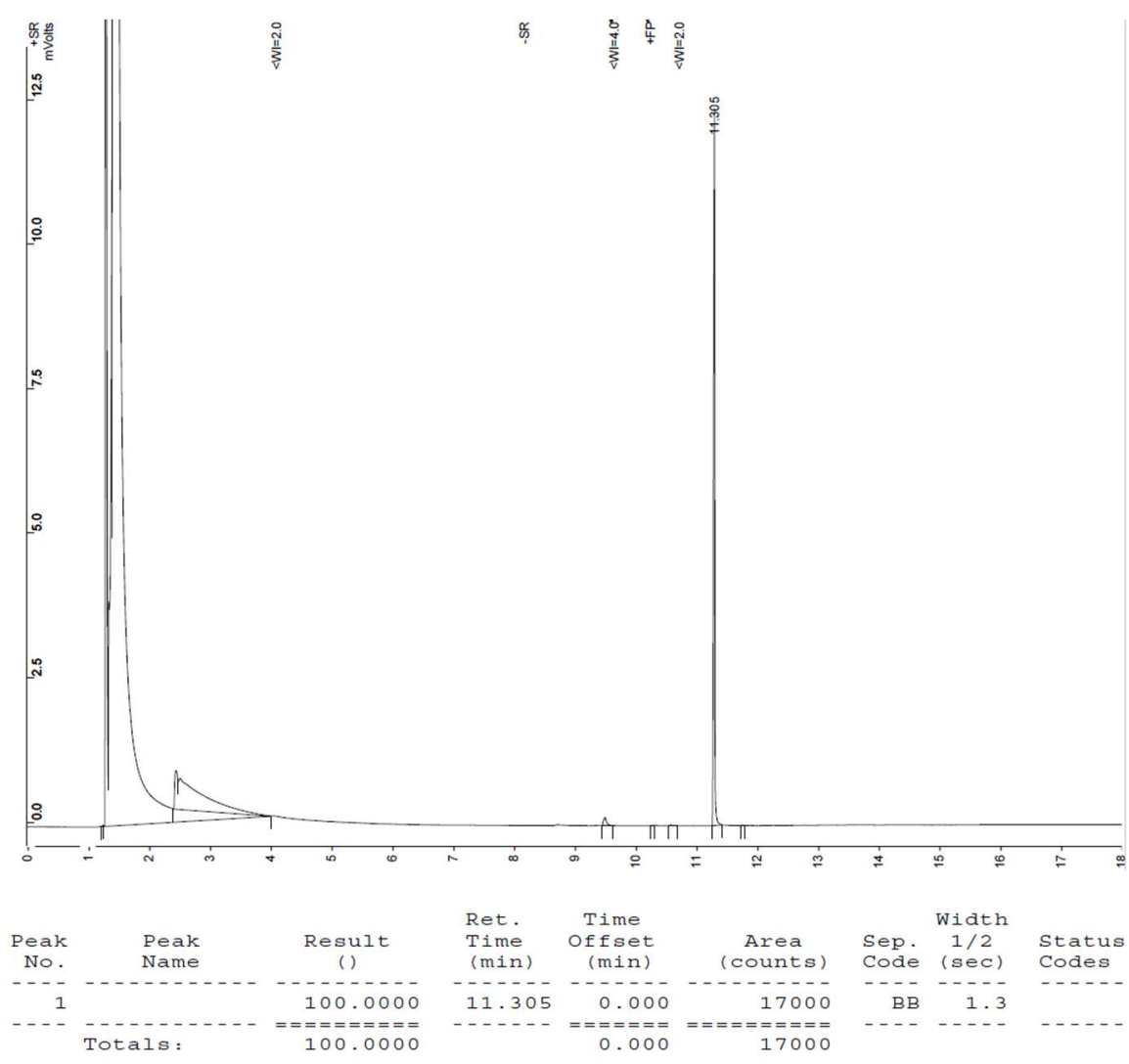

(Z)-isomer ((Z)-51)
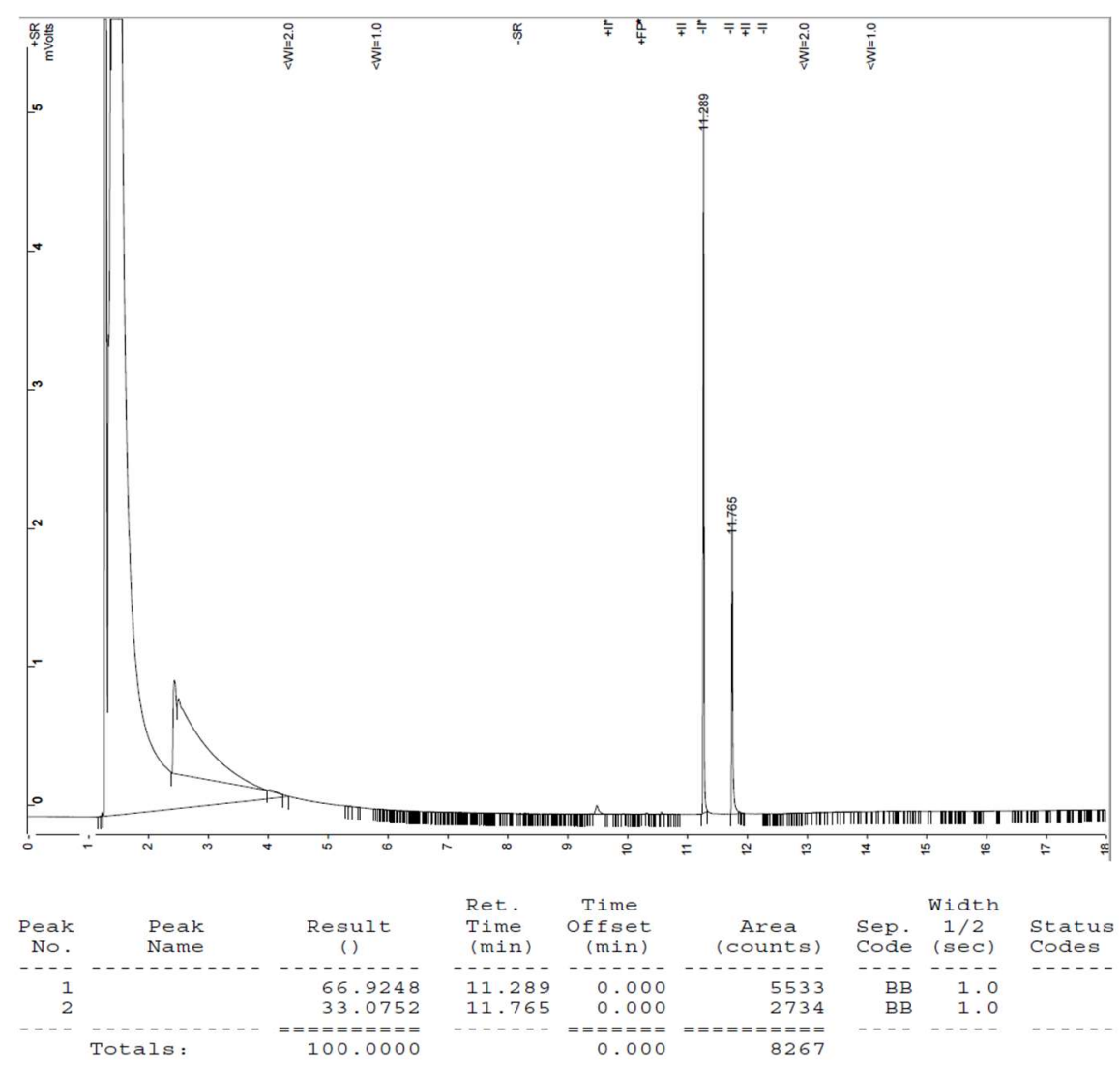
ethyl (Z)-4,4,4-trifluoro-3-phenylbut-2-enoate ((Z)-52)

$(E)$-isomer $((E)-52)$
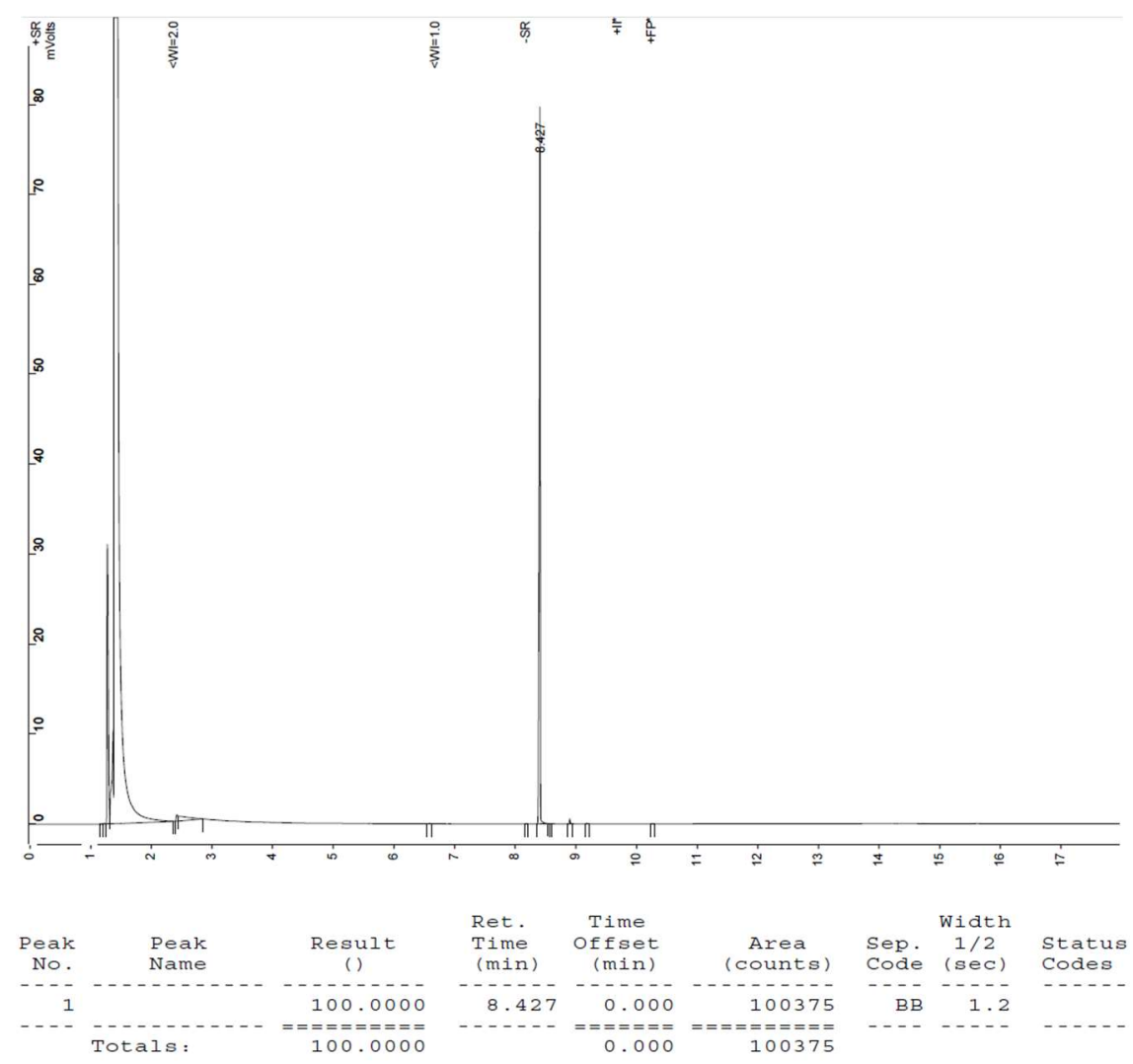

(Z)-isomer ((Z)-52)
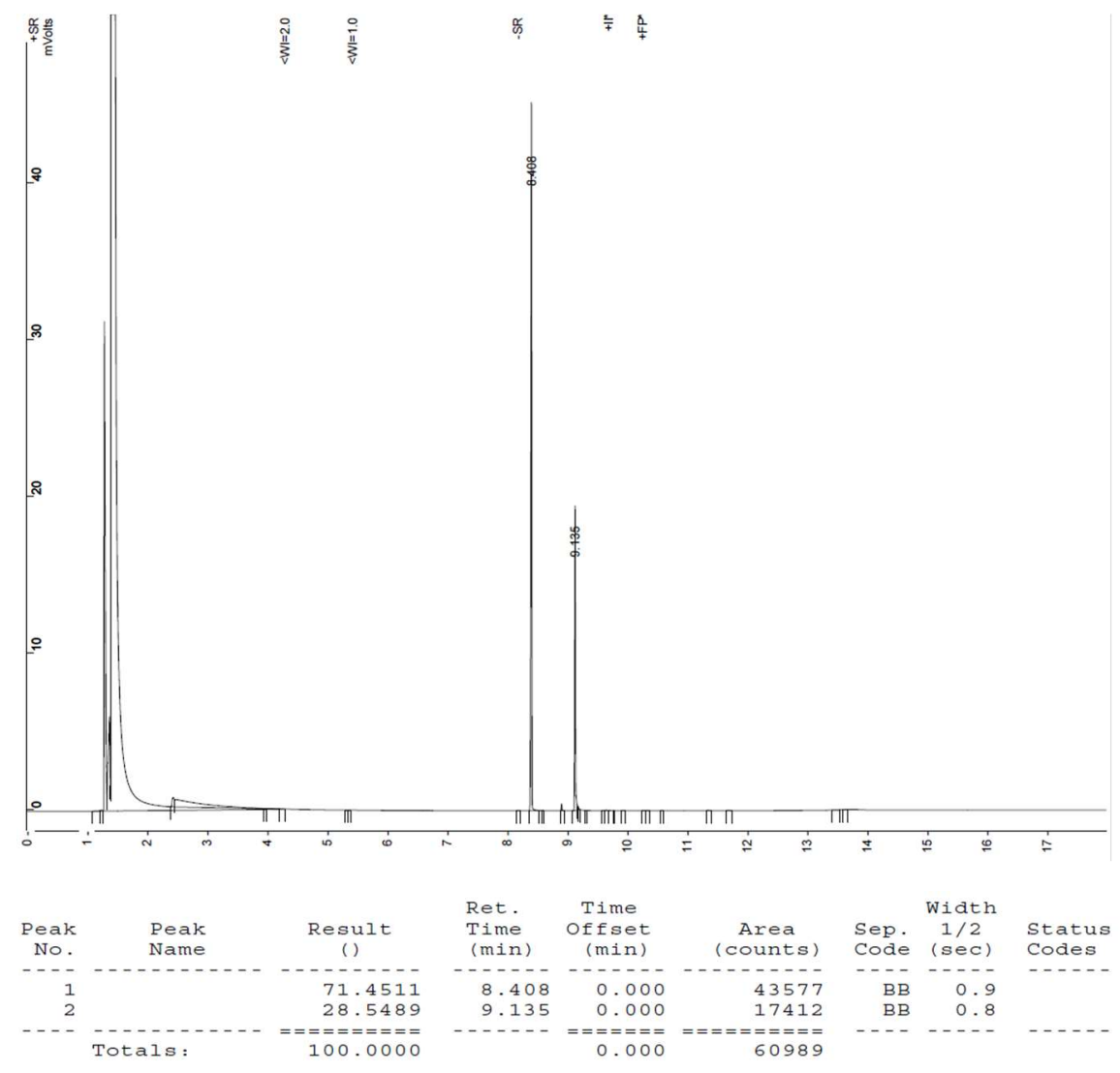
(Z)-3-phenylbut-2-enenitrile ((Z)-53)

$(E)$-isomer $((E)-53)$

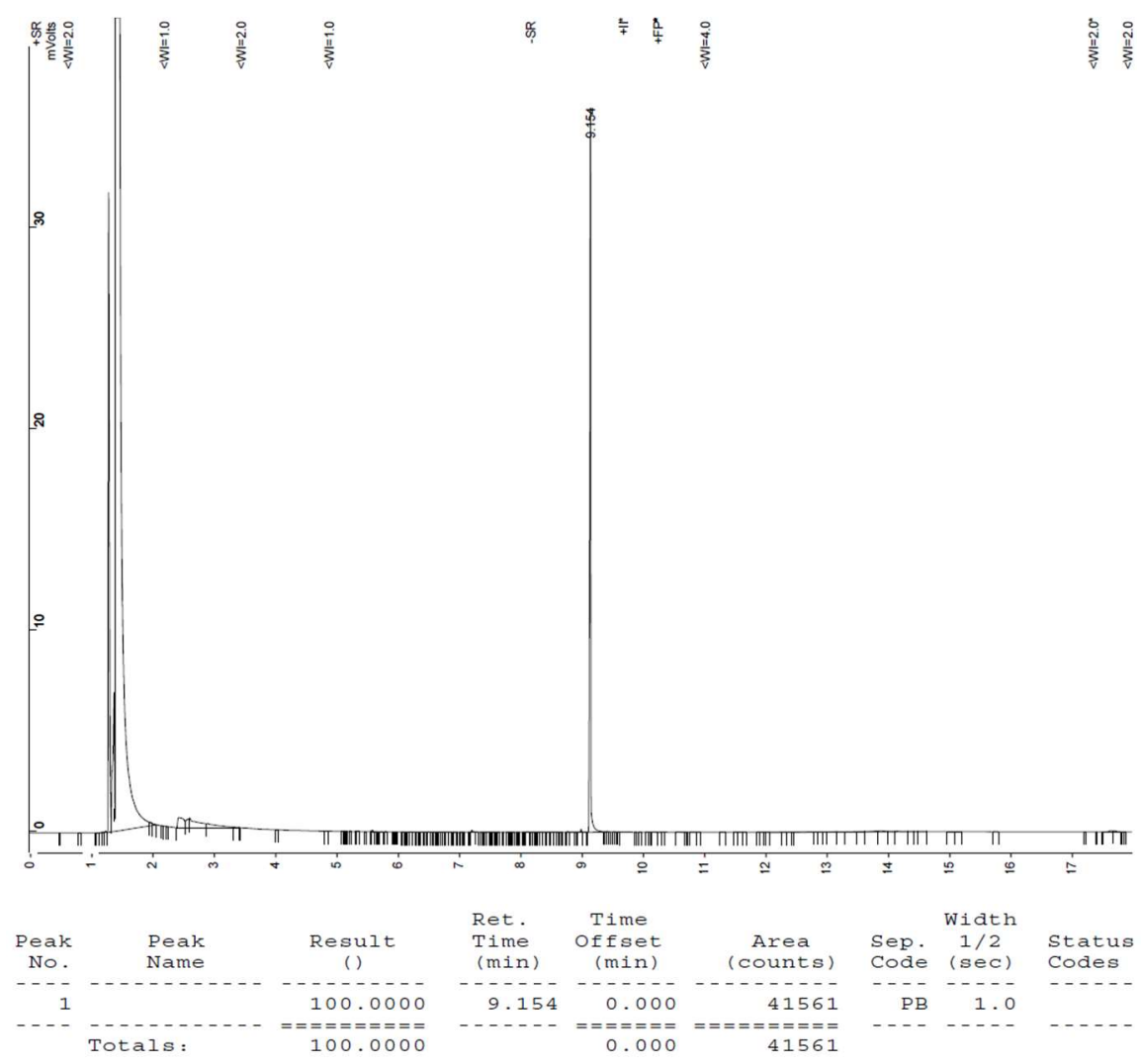

(Z)-isomer ((Z)-53)
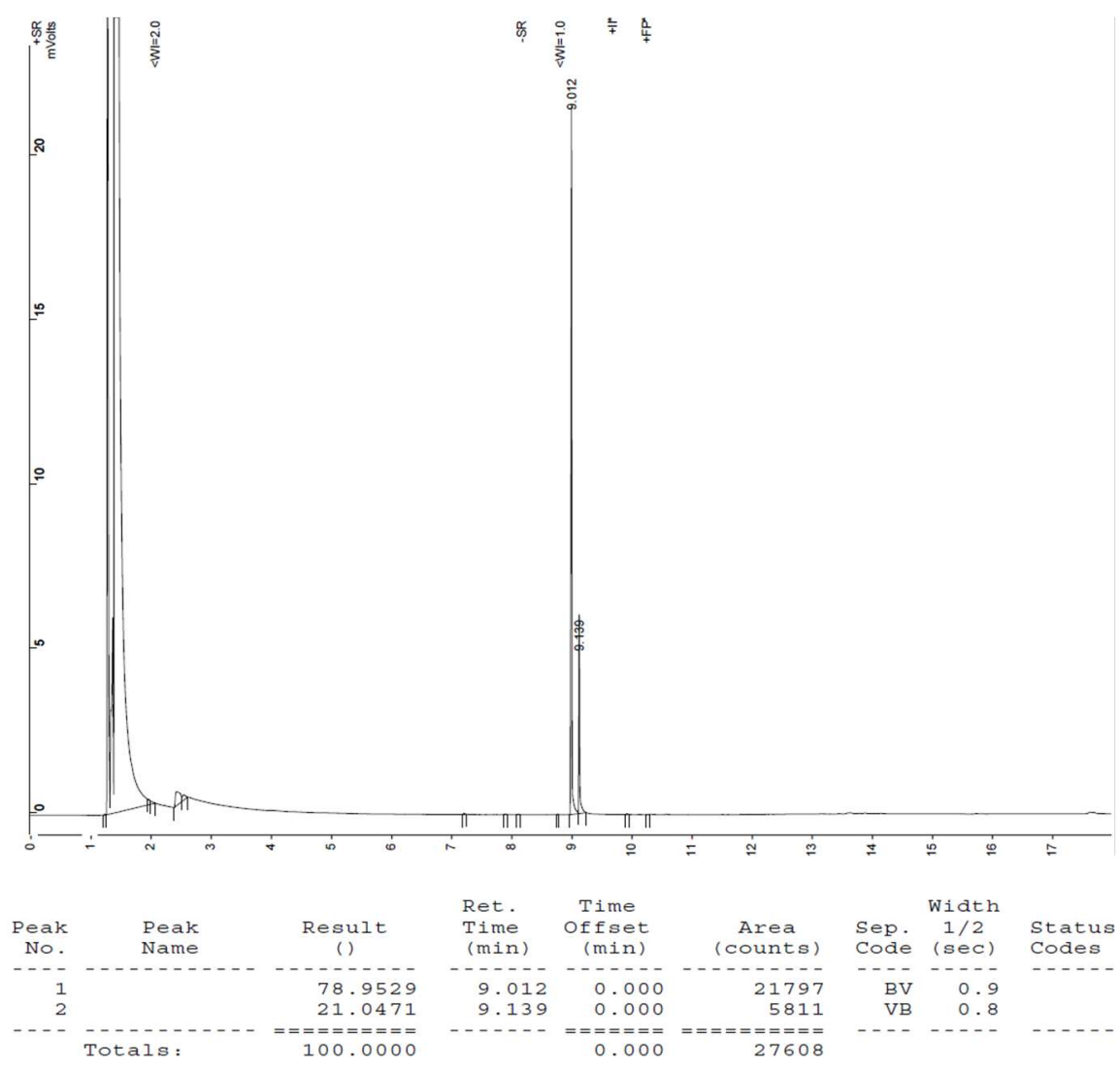
diethyl (Z)-(2-phenylprop-1-en-1-yl)phosphonate ((Z)-54)

$(E)$-isomer ((E)-54)
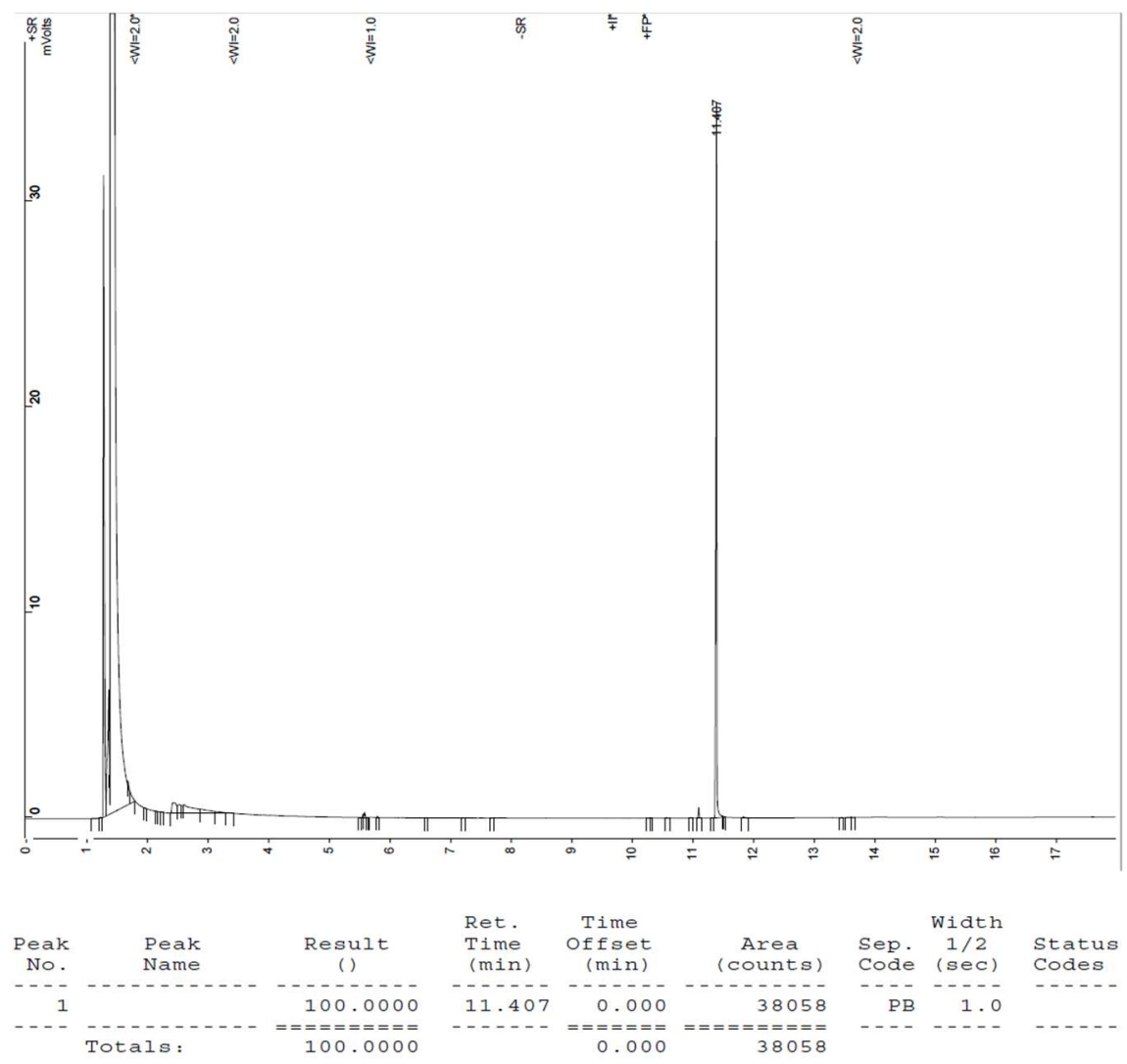

(Z)-isomer ((Z)-54)
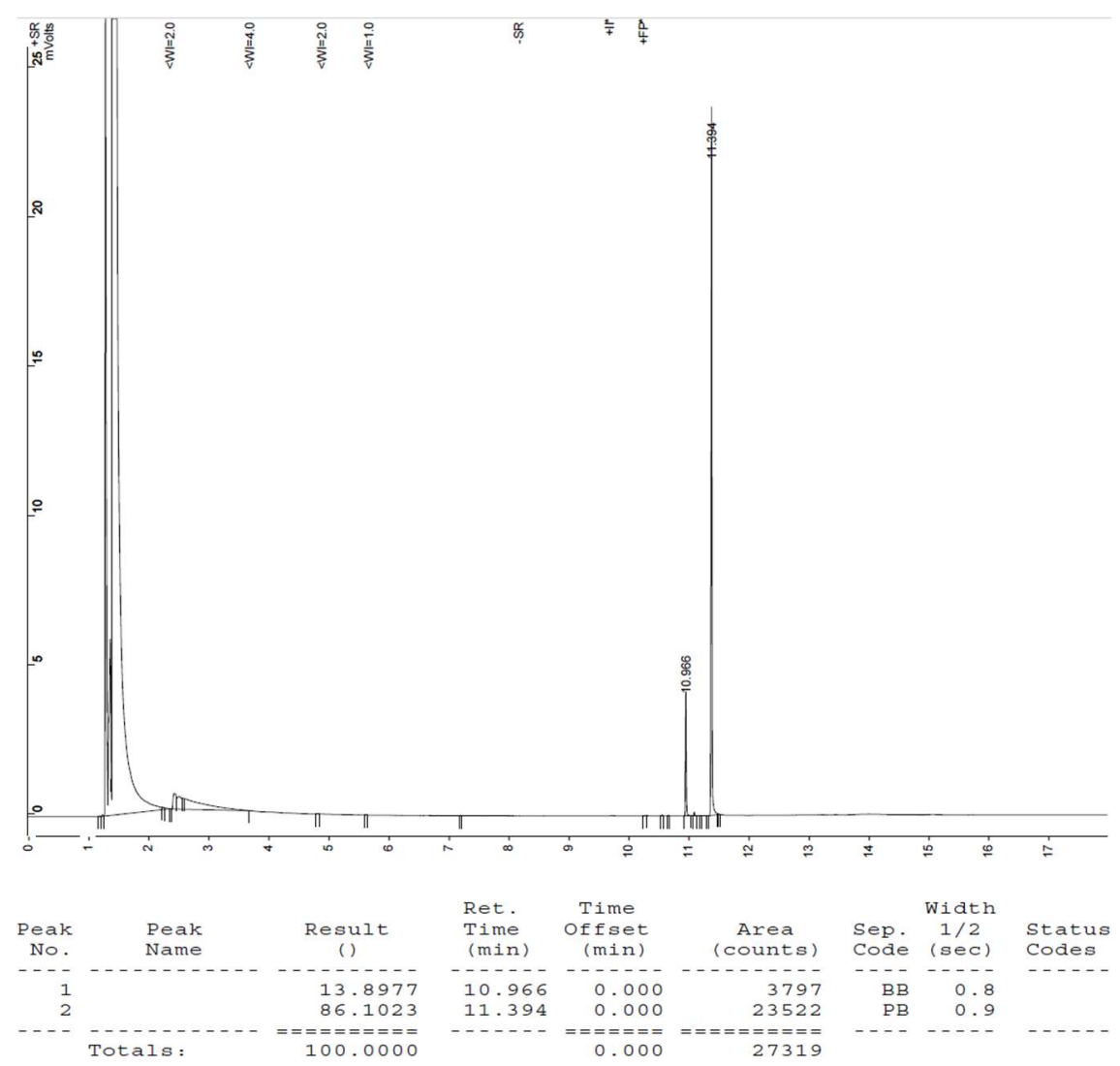


\section{(Z)-((2-phenylprop-1-en-1-yl)sulfonyl)benzene ((Z)-55)}

$(E)$-isomer $((E)-55)$

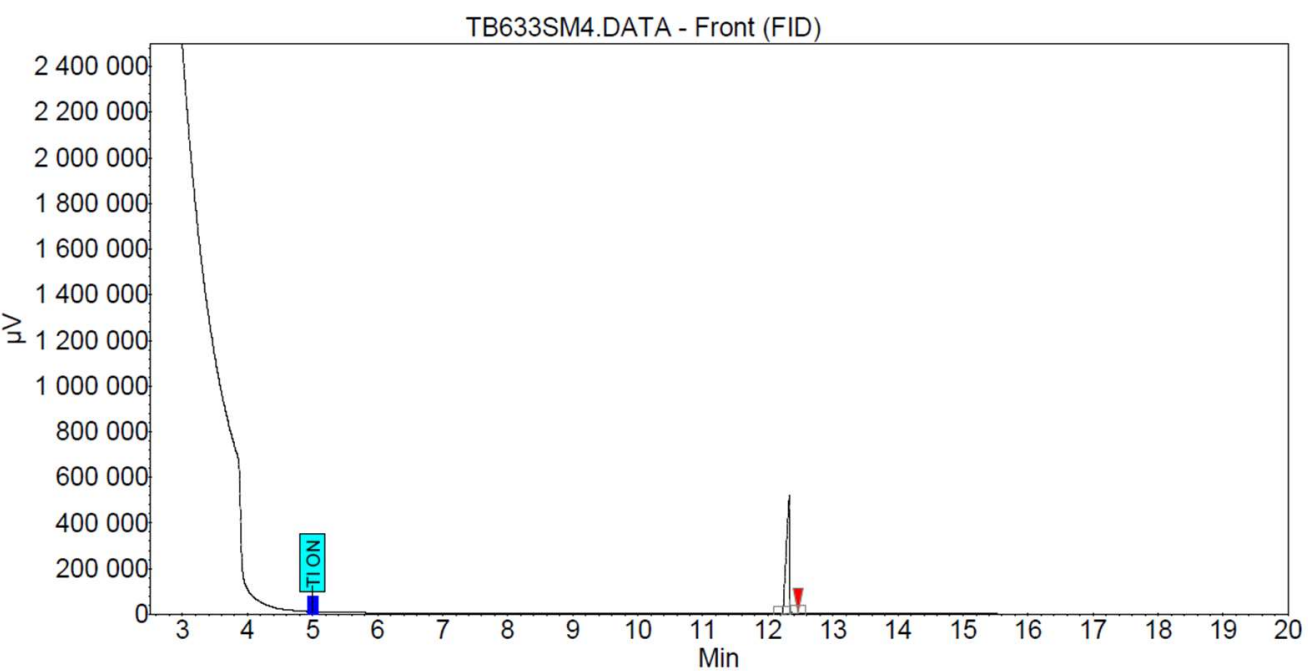

Peak results :

\begin{tabular}{|c|l|r|r|r|r|r|r|r|}
\hline Index & Name & $\begin{array}{r}\text { Time } \\
{[\text { Min] }]}\end{array}$ & $\begin{array}{r}\text { Area \% } \\
{[\%]}\end{array}$ & $\begin{array}{r}\text { Quantity } \\
{[\% \text { Area] }}\end{array}$ & $\begin{array}{r}\text { Height } \\
{[\mu \mathrm{V}]}\end{array}$ & $\begin{array}{r}\text { Area } \\
{[\mu \mathrm{V} . \mathrm{Min}]}\end{array}$ & As. PE. & Res. HW \\
\hline \hline 1 & UNKNOWN & 12.328 & 100.000 & 100.00 & 516833.8 & 28941.1 & 0.55 & 0.00 \\
\hline & & & & & & & & \\
\hline Total & & & 100,000 & 100,00 & 516833,8 & 28941.1 & & \\
\hline
\end{tabular}

(Z)-isomer ((Z)-55)

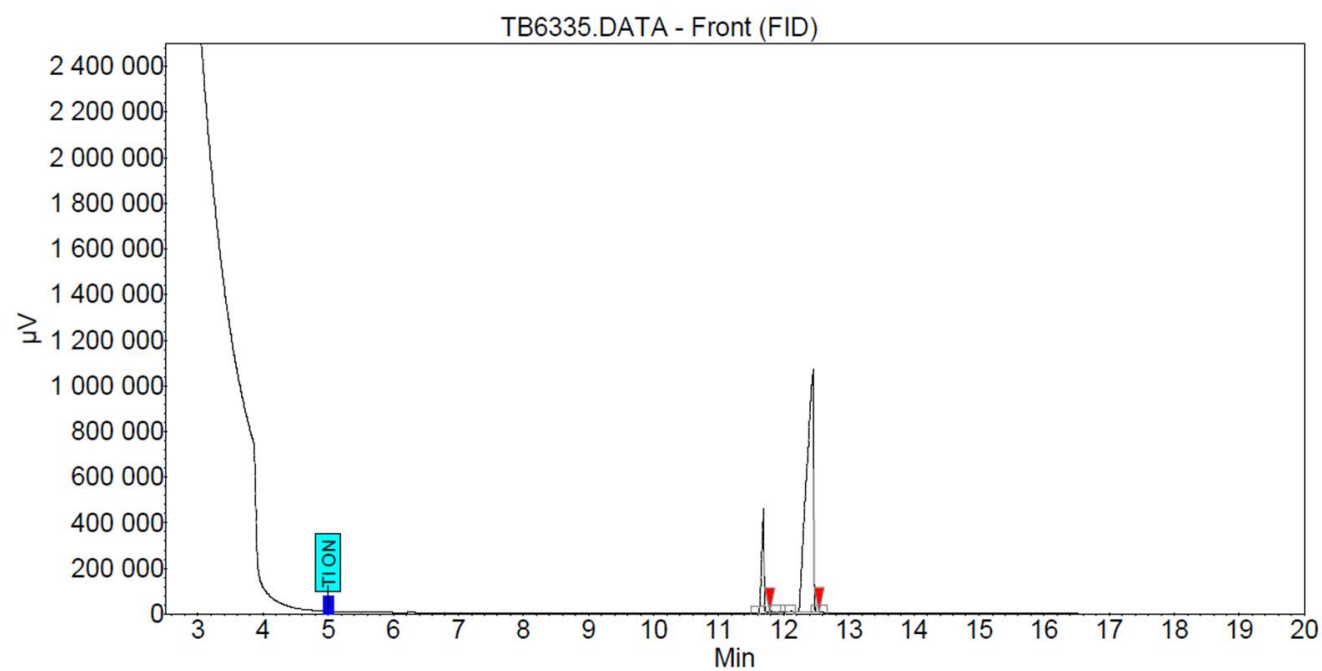

Peak results :

\begin{tabular}{|c|l|r|r|r|r|r|r|r|}
\hline Index & Name & $\begin{array}{r}\text { Time } \\
{[\mathrm{Min}]}\end{array}$ & $\begin{array}{r}\text { Area \% } \\
{[\%]}\end{array}$ & $\begin{array}{r}\text { Quantity } \\
{[\% \text { Area] }}\end{array}$ & $\begin{array}{r}\text { Height } \\
{[\mu \mathrm{V}]}\end{array}$ & $\begin{array}{r}\text { Area } \\
{[\mu \mathrm{V} . \mathrm{Min}]}\end{array}$ & As. PE. & Res. HW \\
\hline \hline 1 & UNKNOWN & 11.688 & 12.097 & 12.10 & 456418.6 & 18378.9 & 0.62 & 0.00 \\
\hline 2 & UNKNOWN & 12.458 & 87.903 & 87.90 & 1064909.0 & 133545.3 & 0.51 & 5.41 \\
\hline & & & & & & & & \\
\hline Total & & & 100.000 & 100.00 & 1521327.6 & 151924.3 & & \\
\hline
\end{tabular}

